- 


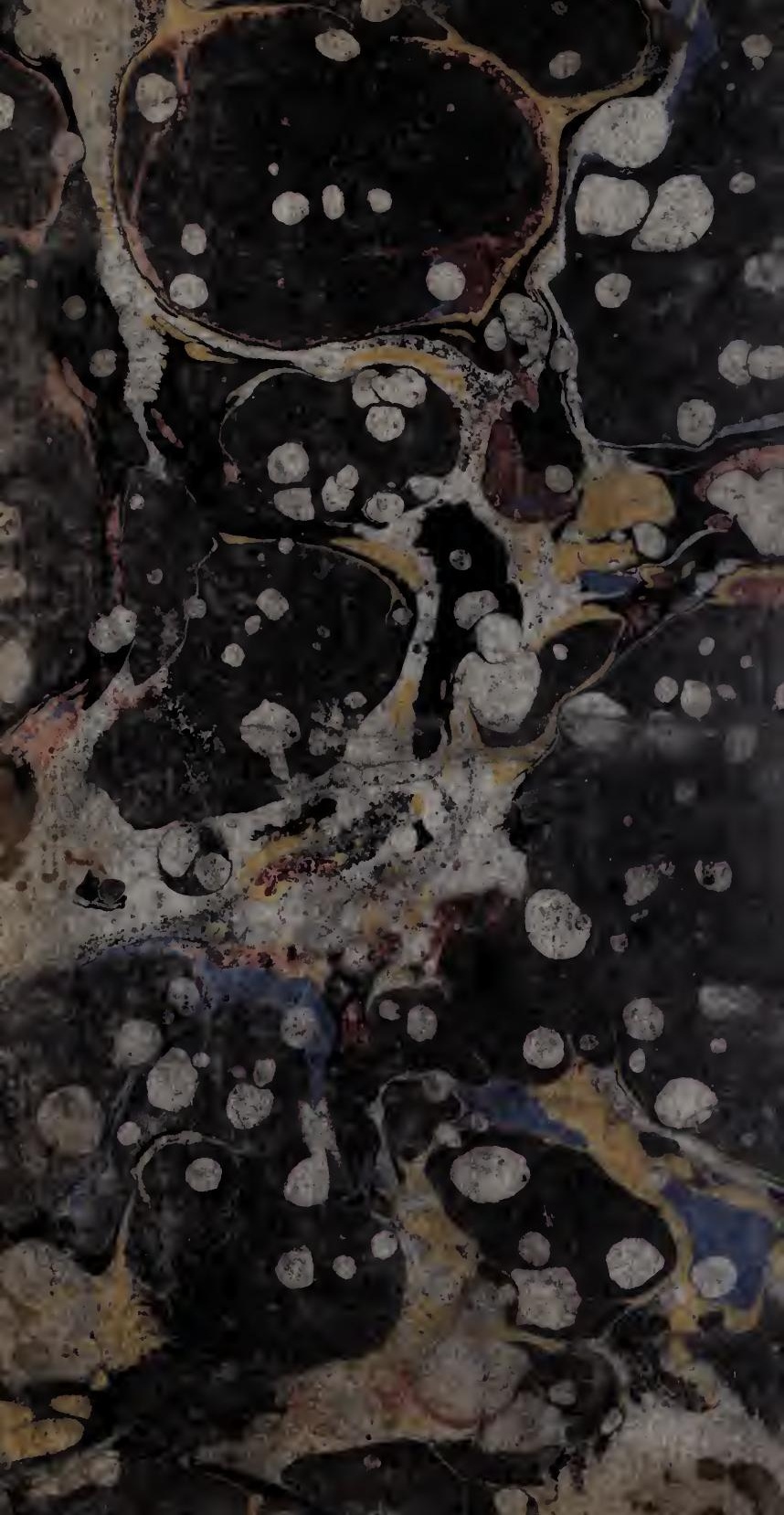


$y^{\circ} 0_{0 .}^{\circ}$

-

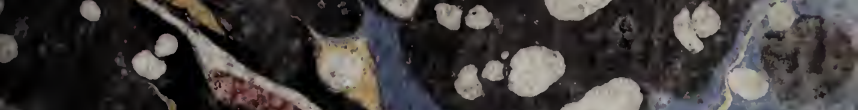

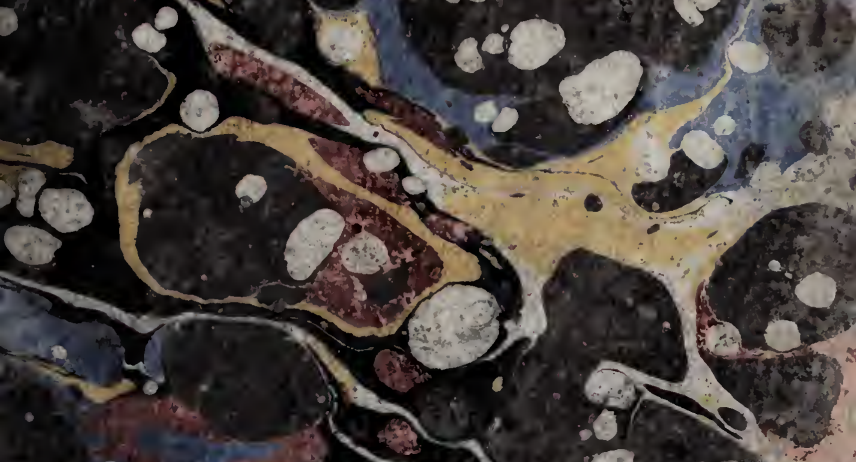

-6.

e.r. ibon

: $:$ con

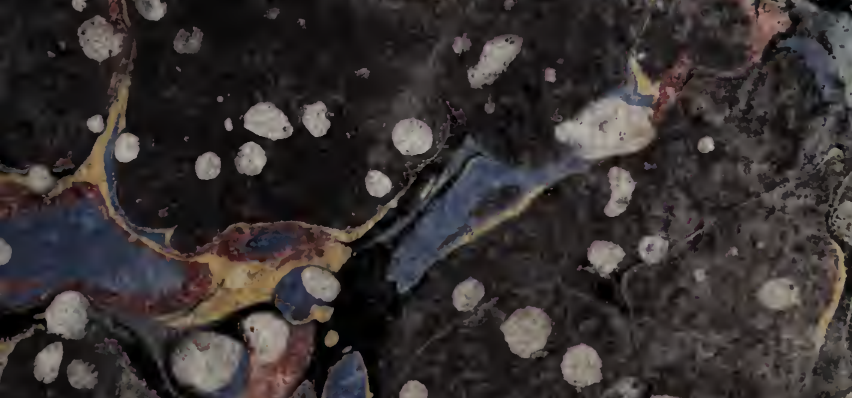
(N) 


$$
d
$$




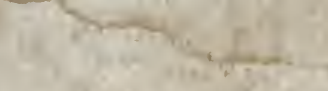

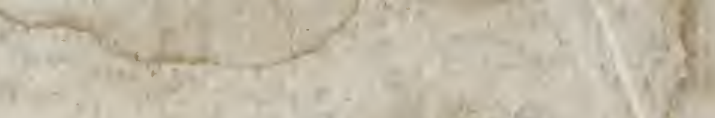

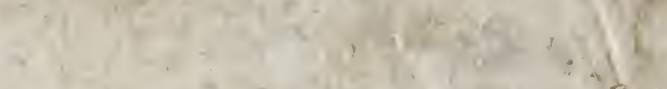

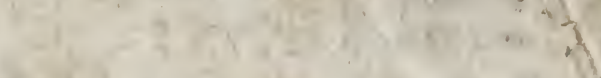

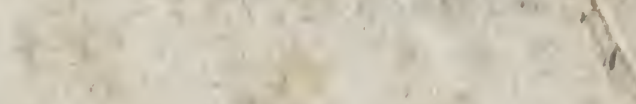

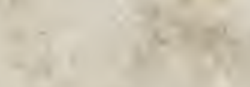

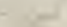
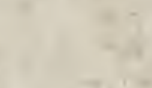

(1)

(n)

$=$

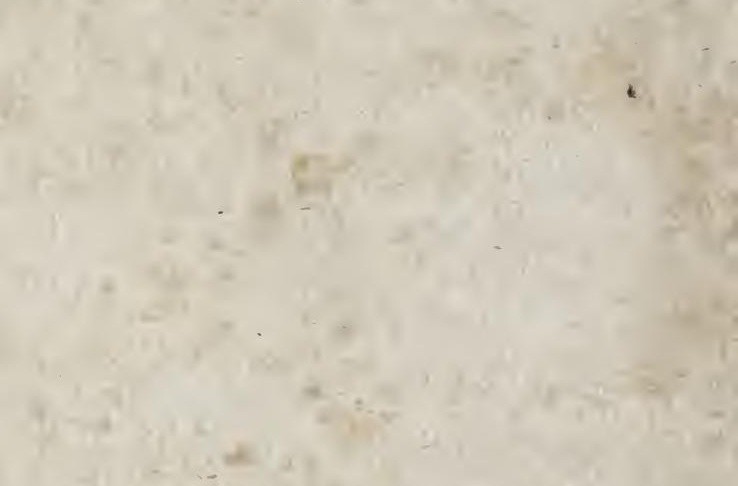

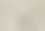

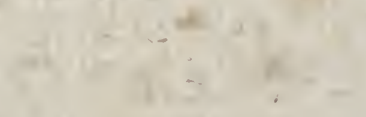

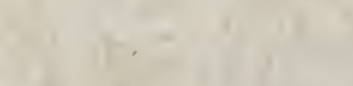

1<smiles>CC1CCC1</smiles>

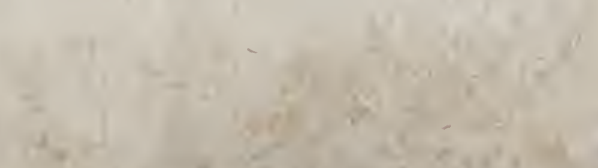

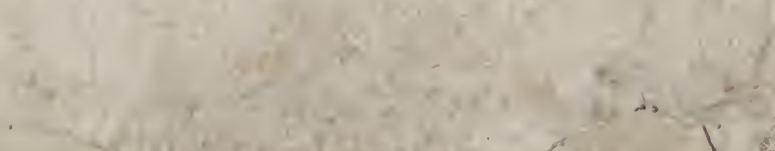

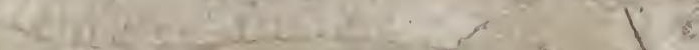




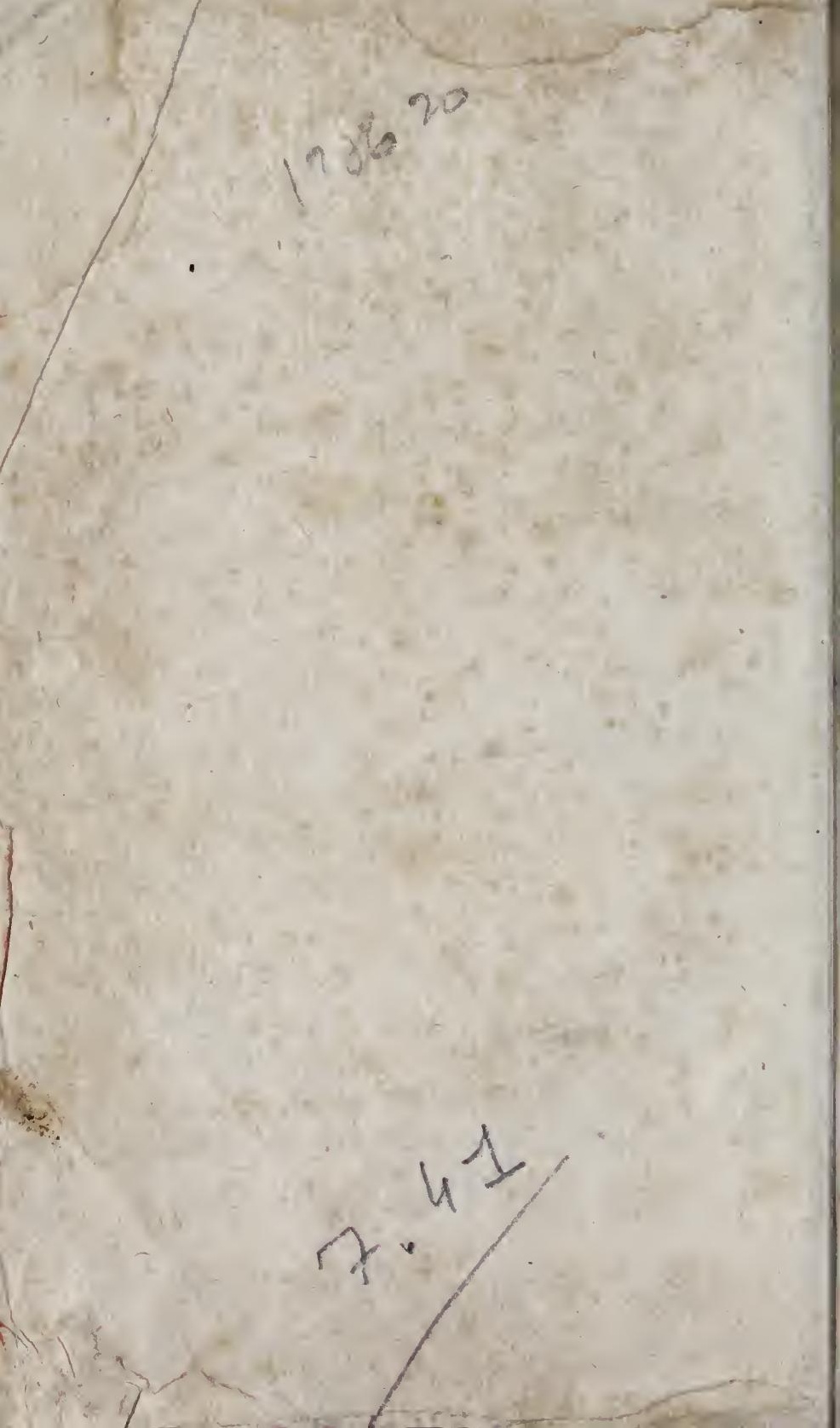




\section{MVSEVM GEVERSIANVM}

\section{S I V E}

\section{N D E $\mathbb{X}$}

RERVM N A T.VRALIVM CONTINENS

INSTRVCTISSIMAM COPIAM PRETIOSISSIMORVM O M N I S G E N E R I S , E X

TRIBVS REGNIS NATVRA OB JE C TOR V M

$\int \begin{gathered}\text { DVMIN GIVISERAT MAGNADILIGENT } \\ \text { MVLTAQVECVRA COMPARAVIT } \\ V I R \text { AMPLISS IMVS }\end{gathered}$ $\triangle B R A H A M V S$ GEVERS - OLIM CONSILIARIVS PRIMVSQVE VRBIS ROTTERODAAENSIS CONSVL PREFECTVS STL VARVM HOLLANDIE \& WESTFRISIE SOCIETATIS INDIE ORIENTALIS DI RECTOR ACADEMIAE, CESAREA NATVRE CVRIOSORVM SOCIVS ETC. ETC.

Publice diftrahendam

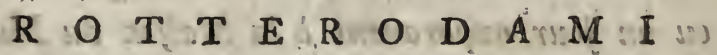
in Atdibus Nobiliffimi Defuncti

Diebus I2. Septembris \& reqq. I $78 \%$.

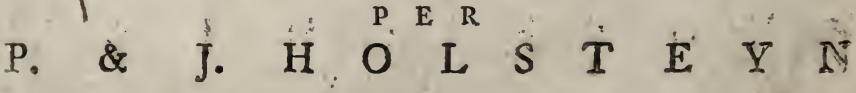
Bibliopolas.

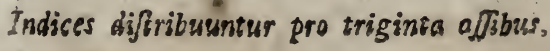


Men zy verdagt gehouden te zyn het gekogte in grof: Zilver Geld, of goude Ryders, en 5.per $C_{t}$. aan opgeld te betaalen; alles zal I4. dagen yoor de Verkooping, of zelfs yroeger, konnen worden gezien op vertooning yan Billetten, welke, met opgeeving van de Naamen en Qualiteiten der Perzoonen, en de daartoe verkiezende tyd, yan te yooren te beyraa. gen zyn ten Huize yan het Mufeum.

On eft adyerti, que le payement fe doit faire en grolfe Monnoye d'argent, ou bien des Ryders d'or; Eg pour Surplus 5. par $C_{t}$. on pourra voir le $C_{a}$ ininet du moins quinze jours ayant la Vente, au meme plutot en montrant les Billets avec les Noms, E Qualités des Perfonnes, E choififfant un tems conyenable, à condition qu'on s'addreffe la veille, ou wn jour auparavant is la Maifon du MuJeum: 


\section{ELENCHVS CAPITVM.}

Mammalia.

Amphibia Reptilia.

Ainphibia Serpentia.

Pirces, Nantiaque.

Infecta, Vermes, Zoophy-

ta, Planta.

Pifces Siccati.

Amphibia Siccata.

Partes Avium, aliorum. que Arimalium.

Aves \&c.

Infecta Exotica.

Coleoptera.

Scarabæi.

Lucani.

Caffrdes.

Sylphz.

Cryfomela.

Coccinella.

Curculiones.

Actelabi.

Cerambyces.

Lepturæ.

Eleatres.

Bupreftides.

Cicindelæ.

Carabi.

Tenebriones.

Ditifci.

Hemiptera.

Blatta.

Mantides.

Giylli.

Fulgoræ.

Cicadæ.

Cimices.

Nepr.

Lepidoptera.

Papiliones.

Sphinges.

Phalena.

Neuropiers.

Libellula.

Myrmeleonęs.
2. Fymenoptera.

$20 \%$

6 . Tenthredines.

202.

10. Ich eumones.

204.

18. Spheges.

Vefpa,

Chryfides.

28. Apes.

32. Diptera.

Mufcæ.

204.

200.

288.

208.

2 I2.

212

212.

212

254.

214.

$2 I 6$.

222.

222.

222.

222.

222.

224.

$220^{\circ}$

84. Scolopendre.

86. Juli \&c.

88. Infecta Indigena in $\mathrm{Ar}$ -

94. mentario.

228:

96. Schediasma Teftaceorum. 233:

96. Univalvia. C!atr. I. 236.

98. Vermiculi. Gen. I. 236.

100. Tubuli.

300. Scutigeruli. Gen. 3. 240.

102. Patellæ.

ro2. Ancilia.

Gen. 4. 240.

Gen. 5. $24 c$.

102. I14. Auriformes. Gen. 6. 248.

102. Argonautz. Gen. 7. 250.

I04. Nautili. Gen. 8, 25 ż.

114. Angarii. Gen. 9. 254.

II6. Rhinocerotes. Gen. I0. 252.

II8. Neritæ. ... Gen. I I. 26a.

26. Neritæ formes. Gen. 12. 274.

I28. 'Turbines. Gen. 13. 270.

128. Trochi. Gen. 14. 280.

180. Monocerotes. Gen. I5. 290.

186. Strombi. Gen. 16. 290.

200. Buccina. Gen. 17. 296.

200. Purpure, Gen. 18. 308.

202. Muriciformes. Gen. 19. 3r6.

Murices. 
Murices:

Nlatæ.

Coni.

Cylindri.

Globolx.

Calizies.

Bulle.

Porcellana.

Bivalvia

Tamarindi for-

mes.

Pectines.

OAtrez.

Arca.

Hippopodes.

Macerophylla.

Spondyli,

Lampades.

Cardia.

Chamæ formes.

Chama.

Tellina.

Capifteria.

Mytuli.

Riparix.

Baphix.

Solenes.

Pholades.

Anatifera.

Parafiti val. via.

Balani.

Convexi valvia.

Anadema.

Cidares.
Gen. 20. 322. Umbilici Ma-

Gen, 2I. 334. rini formes. Gen. 51. 484.

Gen. 22. 344. Cordi formes, Gen, 52.484. Gen. 23. 368. Gale3. Gen. 53. 486. Gen. 24. 380. Olivæ formes. Gen. 54. 286. Gen. 25. 386. Artocrex. Gen. 55. 488. Gen. 26. 396. Stribilitz. Gen. 56. 488. Gen. 27. 398. Lagani. Gen. 57. 488. Claff. II. 4 40. Difci rransfizi. Gen. 58 . 490. Rotulæ. Gen 59. 490., Gen. 20. 412. Birita. Gen. 60. 490. Gen, 30. 414. Echini fpinis

Gen. 31. 420. armati. 492. Gen. 32. 424. Partes Conchiliorum. 494. Gen. 33. 428. Zoophyta. Madreporæ.

Gen. 34. 430. Milleporæ. Gen. 35. 432. Jfides. Gen. 36. 438. Tubipora. Gen. 37. 438. Efcharz. Cellularix.

Gen. 38. 444. Sertularix. Gen. 39. 450. Gorgonix. Gen. 40. 455. Antipathes. Gen. 4I. 462. Spongix. Gen. 42: 464. Alcyonia. Gen. 43. 468. Corallinz. Gen. 44. 472. Vegetabilia. 49\%. 446 . 508. 514. 516. 516. 516. 518. 518. 526. 528 . 532 . 532. 536. 542. 542. 544. 574. 618. $6_{40}$ Ger. 45. 4i4. Forilia. Gen. 45. 476. Terra. Gen. 47. 476. Lapides, Mineræ. Clatf IIr. 478. Petrificata. Gen. 48. 478.' Lapides figurati.

Lapides varii, Calculique. 644 . Claff. IV. 480. Artefacta. Gen. 49. 48:. Armentaria. Gen. 50. 482. Omiffa. 646. 650. 054.

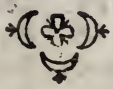


INDEX SYSTEMATICVS

\section{$M V S E I$ GEVERSIANI}

$$
\text { S I V E : }
$$

\section{$\begin{array}{llllllll}E & L & E & N & C & H & V & S\end{array}$}

RER $R$ NATVRALIVM

$$
\text { T A M }
$$

ANIMALIVM \& PLANTARVM

$$
\text { Q V A M }
$$

FO S S I LIVM.

DE S C R I P TAR V M

EIC: S SECVNDVM SYSTEMATA.

$\dot{A} L I N N \dot{E}, P A L L A S I I, W A L L E R f t$

A C Q V O A D

$T E S, T, A, C E A$

PRAESERTIM I V X T A

SCHEDIASMA. STSTEMATIS

\& $M V$ S C HENII. 


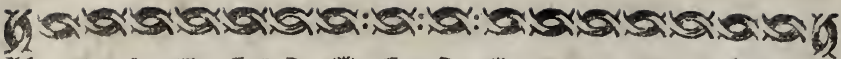

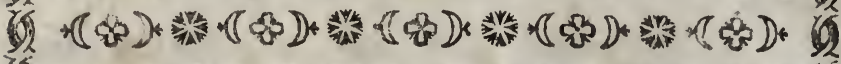

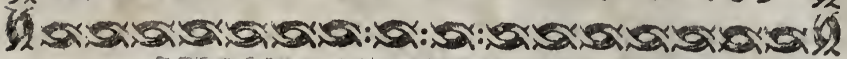

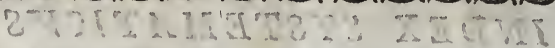
A $N I M A L I A$

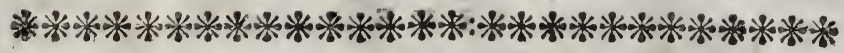

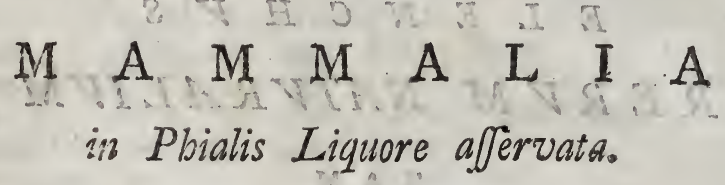

1 oetus humanus, 4 Menfium ex Africa. (Mujeum n². Is

2 Foetus humanus, 5 Menfium ex Africa. (2.

3 Foetus humanus, 7 Menfium ex America. (3:

4 Foetus humants, 8 Menfium ex Africa. 4.

5 Simia Midas Linin. Syftem. Natur. Edit. XII. pag. 42. 27 : Buff 30, t. 13. (.22.

6 Simia Mulatta? Linn. o., Zimmermo Geogr. Zool. Iox fpecies rara. (28.

7 Simia Mulatta, r. xarietas, Linn. O. ( 29.

8 Simia Mulatta, f. foetus. ( 46.

9 Simia Mulatta, minor. ( 47 .

30 Lemur Volans, rariffinum animal in paucis Mufeis obvium

Linn. 45. 5. ( 15 )

II Lemur Tardigradus, Linn: 44. 1. ( 32.

12 Lemur Tardigradus, Linn. 44. I. ( 33 .

I3 Lemur Spettrun, Linn. o. rarum animal. (34.

I4 Lemur Spectrum, Cranio antice denudato ut pateat crifta frontalis fingülaris, $\operatorname{Linn}$. O. ( 35 .

15 Vefpertilio Spectrum, Limn. 45. 2. (16.

16 Vefpertilio SpeEtrim, Limn. 1. c. pulcripr \& major (Iy:

17 Verpertilio Exoticus, fufcus, Linn. O. (50.

18 Bradypus Didaltylus, Linn. 5I. 2. cum pullo fugente (25t

I9 Bradypus Didaltylus, Linn. 5I. 2. (37.

20 Bradypus Didactylus, Linm. 51. 3. (38. 
\%
A
N
I
M A
U X.

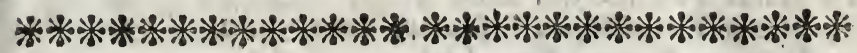

\section{A M E L L E S}

\section{en Liqueur.}

$\mathrm{U}_{n}$

2 Un foetus humain de 5 mois, de l' Afrique (2.

3 Un foetus humsin de 7 mois, de l'Amérique ( 3.

4 Un foetus humain do 8 mois, de l' Afrique (4.

5 Le Tamarin, Buff. tom. 30. tab: I3. (22.

6 Le Singe Mulatta efpece trés rare, Zimmerm. Geogr. Zool. ( 28.

7 Variété du Mulatte. (29.

$8 U_{n}$ Foetus de singe fans poils ( 46.

9 Un Singe Mulatte plus feune ( 46 .

so Un Maki Volant, Seb. Th. 1. tab. 34. f. 1. 2. Espéce très rare, E̊ qu'on trouve gueres dans les Cabinets. (15.:

II Le Loris, Seb: Th. I. tab. 35. f. I. 2. (32.

12 Un Loris. ( 33.

I3 Le Tarfier, Linn. 0. Buff, 26. tab. 9. animal rare des Indes. (34:

I4 Le Tarfier, mais la partie anterieure du crane eft déceuverte $E^{2}$ montre une crete ofleufle; comme dans les Animaux carnaciers. ( 35.

I5 Le Vampyre, Buff. 20. Seb. Th. I. tab. 58. I. (i6.

I6 Le Vampyre, plus beau \& plus grand. "(27.

17 Chauvefouris étrangère, Buff. 20. tab 18. ( 50 .

I8 l'Unau, Buff. 26:tab. 1. avec un jeune aux Mamselles. (25.

19 l'Unau, Seb. Th. I. tab. 34. S. I. (37.

20 l'Unau. ( 38. 
21 Bradypus Didactylus, foetus Linn. 5I. 2. ( 54.

22 Bradypus Tridactylus Linn: 50. I. ( 72.

23 Brądypus Tridactylus, Limm. 50. 1. (85.

24 Myrmecophaga Liciactyla, Limn. 5I. I. (26.

25. Myrmecophaga Didactyla, Linn. I. c. (40.

26 Myrmecophaga Didactyla, foetus, Linn. 5I. I. (42.

27 Manis Pentadactyla, Linn. 52. x. ( 20.

28 Manis Pentadactyla, Linm. 52. I. (I4.

29 Manis Tetradactyla, f foetus, Lime 53.2. ( 55.

30 Dafypus Novemcinetus, Linn. 54. 6 (31.

3I Felis Tigris, $f$ foetus, Linn.61.2. ( 48.

32 lielis Catus, pedibus fex monftrofis. ( 7 .

33 Felis Biceps, monftrofus. (49.

34 Felis Catus, capite monftrofo. (8.

35 Felis Cati, foetus in membranis. ( 13 .

36 Viverra Nafua, cujusdam pullus, Linn. 62.2. ( 27.

37 Viverra Na/ua, f. pullus, Linn. 64. 2. (57.

38 Viverra Nafua, cujusdan foetus, Linn. 64. 2. ( 52.

39 Viverra, Nafua, cujusdam foetus, Linn. 64. 2. 106.

40 Didelphis Opolfum, femina cum pullis, Linm.72.3. ( 18.

4I Didelphis OpoJum, mas. Linn. 72. 3. 1 (19.

42 Didelphis Orientalis, Limn.o. Pallas Mifcell. Zool. (44.

43 Didelphis Dorfigera, Linn. 72.-5. ( 4 I.

44 Didelphis Dorfigera, $[$. foetus Linn. 72.5 . ( 52.

45 Didelphis Opolfum, f. foetus, Linn. 72. 3. ( 56.

46. Didelphis Fufcus, cauda tota villofa, Linn. 0. ( 69.

47 Didelphis Dorfigera, Linn. 72. 5. ( 70.

48 Didelphis Dorfigera, cum pullis. ( $7 \mathrm{r}$.

49 Didelphis Dorfigera fufcus, f. foetus. ( $c$.

50 Didelphis Murina, Linn. 72.4 . ( 76.

51 Didelphis Dorfigera f. foetus, Linn. I. c. ( 75 .

52 Hyftrix Criftata Linn. 76. I. rarum animal. (2r.

53 Lepus Brafilienfis Tapeti, Linn. 78. 4. (43.

54 Lepus, cujusdam foetus. ( 65.

55. Lepus Cuniculus, Linn.-77. 2. (67.

56 Lepus Cuniculus, Linn. 77. 2. (68.

57. Mus Paca, Linm. 81. 6. ( 30.

58 Mus faculis, Linn. 85. 20. ( 6.

59 Mus Sylvaticus, albus, Linn. 84. 17. ( 59.

60 Mus Americanus, Linn O. $(73$.

6I Sciurus Vulgaris, Linn. 86. I. (24.

62 Sciurus Volans Limn. 88. 10. ( 36.

б3 Mofchus Tragulus, Mencinna, Linn. o. (45.

64 Morchus Traguhes, א. varietas Ceilonica nigro friata, Linn. O. ( 62 . 
2I Unau ou foetrus. ( 54.

22. l'Ai, Edw. tab. 220. Buff. tab. 26. fig. 5. 6. $C 72$.

23 l'Ai. ( 85

24 Le Fourmiller, Buff, 20 tab. 30. Edw. 228, ( 26 .

$25 U_{n}$ Fourmiller. Edw. 220. (

26 Foetus d'un Fourmiller. (42.

27 Pangolin, Buff. 20. tab. 34. (20.

28 Pangolin. (I4.

29 Foetus di Panzolin. ( 55 .

30 Un Cachicame ou Taton a neuf bandes, Buff, $20 . t a b .37$ ( $3 \mathrm{I}$.

3 I Feetus d'un Tigre. ( 48.

32 Chat a fix pattes monftreufes. ( 7 .

33. Chat a deux têtes: (49.

34 Chat ì tête monftreufe. (8.

35 Foetus de Chat dans Jes Membranes. (I3.

36 Feune Belette. (27.

37 Un Feune Coati. ( 57 .

38 Foetus de jeune Belette.

39. Un Foetus de jeune Belette. ( 66 .

40 Sangue femelle avec Jes petits, Buff, tab.46. (I8.

4I Sangue male, Buff. tab. 45. (I9.

42 Phalanger Buff. tab. IO. ( 44 .

43 Philandre de Surinam., Merian. tab. 66, (4I.

44 Foetus de Philandre de Surinam. ( 52.

45 Foetus de Sangue. ( 56.

46 Philandre brun a queue toute touffue, Seb. Th. I. tab. 3I. f. 6. ( 69 .

47 Pbilandre de Surinam. Merian. tab. 66. ( 70.

48 Philander de Surinam. avec ces petits. ( 78 .

49 Une Marmole, Buff. 20. tab. 52: ( 60.

50 Un Sangue, Buff. 20, tab. 45. ( 76 .

5I Philandre de Surinam. ( 75 .

52 Herifon Afiatique, Buff. I6. tab. 6. (21.

53 Lievre d'Amérique Tapeti Jonfth. Quadr. tab. 63 ( 43.

54 Foetus d'un Lievre. (65.

55 Le Lapin, Buff. 20. tab. 5 r. (6).

56 Le Lapin. ( 68.

57 Le Paca, Buff. 20. tab. 43. (30.

58 Le Gerboa, Edw. I. tab. 219. (6. Animal rare.

59 Souris blanc. ( 59.

60 Souris d'Amérique. ( 73 .

6r Ecureuil, Buff, 14. tab. 32. (24.

$62 U_{n}$ Polatouche, Buff. 20. tab. 22. 23. ( 36.

63 Chrevotain des Indes Orientales, Buff. 24. tab. 42 ( 45.

64. Variété du Chrevotain de Ceilon ì firies roires. ( 62. 
65 Cervus Pygmaus, fubtus albus, Linn. 92.3. (6r. 66 Cervus $P$ ygmaus, f. varietas, foetus, rarifimum fpecimen; Linn o. ( 53.

67 Cervus Guineenfis, Linn. 94. 7. (23.

68 Cervus Guineenfis, C. foetus, Limı. 94. 7. (39\%

69 Equus, ejusdem foctus. ( 64 .

7o Equus, fo foetus minor. ( 74 .

71 Sus Hydrocharidis, f. foetus, Linn. O. ( 52.

72 Delphinus Phocana vitulina, fofoetus, Likn. I08. I. raruma (pecimen. $\quad 63$.

73 Balæria Myfticetus, f. ejus Oculus, Linn. 105. I. (12.

74 Ovis Foetrus. ( 5 .

i5 Alcedo I pida, zudis roftro flavo, Linn. 179. 3. (15i

76 Anas, Pullus, pedibus. 4. (9.

77 Gallina Pullus, pedibus 4. (10.

78 Gallina Pullus, pedibus 4. (II.

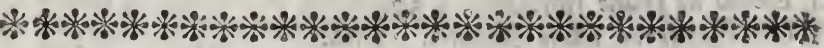

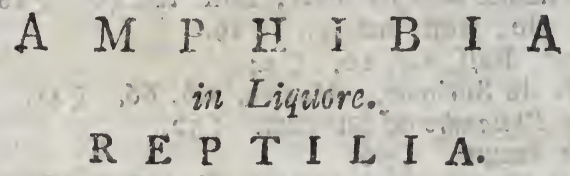

I Teftudo Scabra, Linn. 351.6. (19.

2 Teftudo Mydas, Lini. 350. 3. ( 20.

3 Tefludo Mydas, fo varietas. Linn. 350. 3. (2I*

4. Rana Pipa, mas, Linn. 354. I. (2.

5 Kana Pipa, fœmina. Linn. 354. I. ( $\mathrm{r}$,

ó Rana $P_{\hat{i}} a$, mas, Linn. 354. I. ( 3 .

7 Rana Fipa, mas, Linn. 354. 1. (4.

8 Rana Pipa, mas, Limn. 354. 3. (5.

9 Rana Paradoxa, Linn. 356. 13. hæc pedibus. 2. 66.

Io Rana Paradoxa, Linn. 1. c. pedibus. 4. (8.

I r Rana Paradoxa, Linni. I. c pedibus. 2. ( 10.

I2 Rana Paradoxa, Limm. 1. c. pedibus. 2. (9.

I3 Rana Paradoxa, Linn. I. c. pedibus nullis. (Ir.

I4 Rana Arborea, Linn. 357. I6. (I 2.

I5 Rana Bicolor, Linn c. Boddaert Monogr. tab. I. (7.

I6 Rana Paradoxa; Linm. 1. c. pedibus nullis. (I2*.

17 Rana Aquaqua, Limn. 356. I2. 1. O. (13.

I8 Rana -Arlorea alba, Linn 357. I6. ( 16 .

Ig Rana Ameiva, fubsus brunneo maculata, Lims, o, ( 7 . 


\section{Amphibies.}

65 Cerf Figmée au deffous blanc. (6r..

66 Foetus dun Cerf Pigmée très rare. (6).

67 Le Cerf de Guienne. ( 23.

68. Un pius jeune Cerf de Guienne.

69 Foetus d'un Cineval. ( 64 .

70 Foëtrus d'ün Cheval; plus petit. (74.

7 I Foetus d'un Porc. ( 52.

72 Le Phoque, Foetus, rare efpece, ( 63.

73 l'Oeil de Baieine. ( 12.

74 Lo foetus d'un Mouton.

75 l'Alcede ifpide ou Maxtin Pecheur de bec jaune. ( $I$.

76 Un jeune Canard à 4 pieds. ( 9 .ivy

y7 Un jeune Poule à quatre pieds: (ro.

78 Un jeune Poule d quatre pieds. (II.

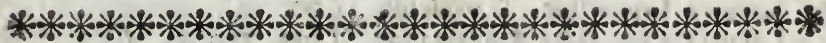

A. $\mathrm{M}$ P $\mathrm{H}: \mathrm{I}$ I. E S

est Liquewr.

R.EP T I E S.

I Tortue de Riviere, Seb. Th. I. tab. 79 f. I. 2, (I9.

2 Tortue de Mer a bords denielés, Seb. Th. I. tab. 80. f. 9. ( 20 .

3 Varieté de Tortue de Mer, Seb Th. I.tab. 79, f. 5.6. (2x.

4 Pipa male. (2,

5 Pipa femelle. ( $I$.

6 Pipa male. ( 3 .

7 Pipa male. (4.

8 Pipa male. 5.

9 Grenouille a queue $\varepsilon^{2}$ deux pattes. ( 6 .

Io Grenouille a queue $\mathscr{c}^{\circ}$ quatre pattes. ( 8.

II Gremouille a queue $\mathcal{E}^{\circ}$ deux pattes. (10.

I 2 Grenouille a queue $\mathcal{E}^{2}$ deux pattes. ( 9.

I3 Grenouille a queue Jans pattes. (II.

I4 Grenouilleblanche a doigts ronds. (12.Seb. Th. I. tab. 73. f. 3.

I5 Grenouille bleue a ventre d'ocre; Boddaen epift. ad Oofterd.

tab. 1 .

I6 Grenouille à queue fans pattes. ( $12 *$.

I7 Grenouille Americaine Aquaqua. (13.

I8 Grenouille blanche a doigts ronds. ( 16.

19 Grenouille grile brune en defous brune tachetée. ( 17.

A 4 20 Grenouille 
20 Rana Plicata, f. varietas, plica aculeata, fronte \& dorfo maculis duabus fufcis, Linn $\circ$. ( 18.

2I Rana Depreffa, Aquaquæ affinis, Limn. O.) ( 15.

22 Rana Lactea, Linn. O. ( 22.

23 Rana Lactea, f. varietas. (23.

24 Lacerta Crocodilus, Américanus, Linn. 359. I. (29.

25 Lacerta Crocodilus, Americanus, Limn. 1. c. (3I."

26 Lacerta Crocodilus; longiroftris, Linn. O. rariffima fpecies: (33.

27 Lacerta Crocodilus Orientalis, Linn. 359. I. (44.

28 Lacerta Crocodilus, major, Linn. 1. c. (204.

29 Lacerta Crocodilius, minor, Linn. 1. c. (64.

30 Lacerta Crocodilus, in) ovo. (69.

3I Lacerta Crocodilus, maculis quadratis.) ( $8 \mathrm{I}$.

32 Lacerta Crocodilus, f. varietas. (109.

33. Lacerta Crocodilus, E varietas. (90.

3 Lacesta Crocodillis Americanus, Linn. 759 . I. (55.

35 Lacerta Gecko, absque cauda, Linn. 365. 2 r. ( 14 .

36 Lacerta Geçko, maculis niveis, rará fpeçies. (80.

37 Laceita Gecko, cauda fafciịs albis nigrisque. Limn.1. c. (83.

38 Lacerta Gecko, ut N8:35. ( 108.

39 Lacerta Gecko, f. varjetas. ( 71 .

40 Lacerta Iguna, Linn. 306.-26.

4I Lacerta Agama, Linn. 307. 28. (5I.

42 Lacerta Agana alba Tingularis. Linn. $367.280^{\circ}$ ( $570^{\circ}$ I

43 Lacerta Iguana, 'fed minor.' Linn 1.' c. (68.) C

44 Lacerta Iguana. Linn. 1. c. ( 75.

45 Lacerta Monitor, Linn -360. 6. 62 .

46 Lacerta Monitor, i. varietas. Limn. I. c $\mathrm{C}_{3}$.

47 Lacerta Azurea, Linn. 352. I2. (i1d.

48 Lacerta Ameiva, Linn. 362. 14. (9.1.

49 Lacerta Ameiva, f. varietas. Linn. 326. I4. - (93.

50 Lacerta Ameiva. Linn. I. c. (79.

5 L Lacerta Agilis, Linn 363 . i5. (74.

52 Lacerta Chamaleon, Linn. 364. 20. (- 106.

53 Lacerta Chamaleon, Capenfis, cum variis Crocodilis parvis;

Linn. 1. c. ( 24.

54 Lacerta Chamaleon, Linn. 1.c. (46.

55 Lacerta Chameleon, Capenfis, Linn. 364. 20. (9\%

56 Lacerta Hiftrio, Linn o. (67.

57 Lacerta Hiftrio, f. varietas, Linn. O. (73.

58 Lacerta Hiftrio, fo varietas, Linn. O. (88.

$\$ 9$ Lacerta Hiftrio, f. varietas, Linn. 0. (89.

60 Lacerta Hiftrio, f. varietas, Linn. O. ( 94. 
20 Grenouille à bords dentelez variété le front Es :e dos cे ditux taches brunes. ( 18 .

2i Semblant à l'aquaqua. (I5.

22 Grenouille grife is taches blanches. (22.

23 Varieté de ta Grenouille grife. (23.

24 Ur Crocodile d'Amérique, Merian. fgg. 69. (29.

25 Crocodile d'Amérique. ( 30.

26 Crocodile à long bec, très rare. Edw. tab. I. f, 2. (33, Gron. Zooph. pag. II. $n^{\circ} \cdot 40$.

27 Crocodile des Indes Orientales. (24॰

28 Crocodile plus grand. (204.

29 Crocodile plius petit. ( 64

30 Crocodile plus petit dans Son œuf. (69.

3I Un Crocodile à taches quarríes. (8I.

.32 Un Crocodile variété. ( IO9.

33 Un Crocodile variété. ( 90.

34 Crocodile Américain. ( 55.

35 Gecko fans queue, Seb. Th. I. tab. I08. f. I. 3. 5. (14..

36 Gecko rare à taches blanches. $\{80$.

37 Gecko à queue bandée de noir, Ė de blanc. (83.

38 Gecko à taches blanchies le mềme que. 35. (IOS.

39 Gecko variété. (7I.

40 Leguan, Seba Th.I. tab. 95. f. I. (27.

4I Leguan, a crete fous la Gorge, Seb. Th. I. tab.96. f. 4. (5I,

$42^{\circ} U_{n}$ Agama blanc, Seb. Th. I. tab. I07. f. I. (57.

43 Un petit Leguan. ( 68.

44 Le petit Leguan. ( 75 .

45 Un Lefard Sauvegarde, Seb. Th. II. tab. 86. (62.

46 Une variété de la S7uvegarde. ( 63 .

47 Un Lezard à bande bleues Eo noires la queuse epinarifo, Seb. Th. II. tab. 62. 6. (IIO.

48 Lezard Ameivé à double collier, Edw, tab. 202. (2I.

49 Lezard Ameive, ou varieté du mồme. (93.

50 Le Lezard Ameive. ( 79.

5I Lezard Agile le ventre tacheté de noir E ecailiss pointises, Edw. 247. 2. (74.

52 Un Cameleon, Seb. Th. I. tab. 82. (106.

53 Un Cameleon du Cap avec quelgues petits Crocodiles, (24.

54 Un Cameleon le pareil de 52. (46.

55 Cameleon du Cap. (97.

56 Lefard tacheté de blanc \&o de noir. (67.

57 Varieté du même. (73.

58 Le pareil. (89.

5o Le pareil. (89.

to Le pareil. ( 94. 
6 I Lacerta Chamaleon, lateribus nigris, Linn, 364. 23. ( 100.

62 Lacerta Lineata, Linn. 306. 24. ( 98.

63 Lacerta Lineata, Linn. I. c. ( 104 .

64 Lacerta Calotes, Limn. 307. 27. (95.

65 Lacerta Calotes, macu'ata. Linn. o. ( 76.

66 Lacerta Calotes, f. varietas. Linn. 1. c.. (77.

67 Lacerta Calotes, maculata. (107.

68 Lacerta Agama, Linn. 367. 28. (72.

69 Lacerta Agama, Linn. 1. c. (96.

70 Lacerta Stincus, fafciatus albus, fafciis ig. nigris, Línw. 365. 22. ( $48 \mathrm{I}$.

71 Lacerta Stincus, Linn. 365. 22. (82.

72 Lacerta Stincus, Linn. I. c. (ros.

73 Lacerta Paluftris, huic junctus Polytmus, Linn. 370. 44. ( 35.

74 Lacerta, ex ovo prodiens. ( 70.

75 Lacerta Teguixin, Linn. 368. 34. (78.

76 Lacertæ Teguixin, cujusdam foetus. Linn. 1. c. (84.

77 Lacerta Salamandra, Linn. 371. 47. (85.

78 Lacerta Anguina, Linn 371. 48. (100.

79 Lacerta, an Pandang Valentini? Linn. O. ( 102.

80 Lacerta Verrucofa, nigro grifea, Linn. O. (10!.

81 Lacerta Aurato Linn. 368. 35. (86.

82 Draco Volans, Anericanus, Linn. 358 . T. (8\%.

83 Draco Volans, f. varietas. Limn. 1. c. (99.

\section{S E R P E N T I A.}

84 Crotalus Horridus Limn. 372. 2. (25.

85 Crotalus Horridus, nulto major. Linn. I.c. (40.

86 Crotalus Duwifius, Limi. 372. 4. ( 32.

67 Boa Hortulana fufca triangulis atris, Limn. 374.10 ? ( 34 .

88 Boa Conftrietor. Linn. 373. 4. (4I.

89 Boa Murina, Linn. 374. 5. ( 54 .

90 Boa Fufca, maculis nigris altio marginatis, Linno $\delta_{0}$ ( III.

9r Boa Pullus, maculis purpureis, Linn. O. (I37.

92 Boa Ophrias, marmorata, Linn. 374. 8 (I 45.

93 Boa Enydris, plumbea annulis fufcis, Lim. 374. 9: ( 146.

94 Boa Hortukana fufco purpurea, Linn. 274. Io? (169. 
61 Le Cameleon à cotés noirs, Seb. Th. I. tab. 84. (102.

62 Le Lezaird d lignes blanches. ( 98.

63 Le pareil. (IO4.

04 Lezard bleuatre a queue trís longue a tête $\mathcal{E}^{\circ}$ dos dentelées,

Seba I. tab. 95. (95.

65 Une varieté à tackes. ( 76.

66 Un pareil. ( 77.

67 Le pareil. (IO7.

68 l. Agami, Seb. Th. I. tab. 107.f. 1. (72.

69 Le pareil, Seb. Th. I tab. 107. $f .2$ (96.

$70 U_{n}$ Seine à bandes Ig noires d'Amboine efpéce rare. (48I.

7 I Un Seine blanche, Seb. Th. I. tab. 105. 3. (82.

72 Le pareil. (105.

73 Un Lezard Aquatique, Seb. Th. II. tab. I2. f. 7. ( 35. avee un Colibri.

74 Un Lezard fortant de fon cuf. (70.

75 Us Lezard a côtes pliez, Seb. Th. I. 98. 3. ( 78.

$76 U_{n}$ Foetus d'un Lezard (84.

77 Un Salamandre. Seb.Th.II. tab: 15. f. 5. (85.

78. Un Lezard en forme de Coleuvire a très petites pattes le Corps effilé la queue longue, Seb. Th. II. tab. 68. f. 7. 8. (100.

79 Le Lezard Pandang de Valentini brun a raye bifarcie blanche d'Amboine. ( 102.

so Lezard Gekko gris \& noir. ( $10 \mathrm{r}$.

81 Seine doré, Seb. Th. I. 89. 3. (86.

82 Lezard Volant d'Amérique, Seb. T7. I. 102. f. 4. (87. 83 La Variété du même. (20.

\section{$S E R \quad P \quad E N S$.}

84 Serpent à fonnettes. (25.

85 Pareil beaucoup plus grand. (40.

86 Serpent à fonnettes le Corps à Rhombeaux brins, Seb.Th.II. $t a b .95 \cdot f \cdot 2 . \quad(32$.

87 Boa oil Serpent a ecuffons Anales, lruns a triangles noirs, Seb. Th. II. tab. 74. I. ( 34 .

88 l'Idole des Negres, Seb. Th. II. tab. 98. (4. $\mathrm{r}$.

89 Serpent Boa dे taches rondes, Seb. Th. II. tab. 29. I. la bout cilla ou ce Serpent eft entortillé eft haute de 26 pouces. ( 54.

90 Boa Tachetée, à taches qoires cerclées de blanc. (IIx.

9r Boa brun a taches pourprées. (137.

92 Boa Marbré, Knorr. Delic. Nat. tab. 9. (145.

93 Boa couleur de plomb a anneaux bruns. (140.

34 Boe brun pourpré. ( 169. 
95 Boa Enydris, Linn. 374. 9. ( 170.

96 Coluber Maculatus, ex albo fuicoque, Limn. o. an Padera? ( 26.

97 Coluber Caftanea, fquamis dorfalibus furco caftaneis, Linn. ०. ( 36.

98 Coluber Cobella r. varietas, Linn. 378.) 26 ( 37.

99 Coluber Vittatus, Linn. 380. 36. (39.

roo Coluber Naja, pulcerrima, Linn. 382 . 50. ' ( 38.

IOI Coluber Calanarius, Linn. 375. 5. ( 42.

I02 Coluber Typhlus, Linn. 378. 22. (49.')

I03 Coluber Coeruleus, Linn. 377. 19. (50.

104 Coluber Angulatus, Linn. 377. 18. ( 52.

I05 Coluber Melanocephalus, Linn. 378. 25. ( 53.

I06 Coluber Melanocephalus? an Typhlus?. Linn 3?8.22. $25 ?$ (56.

107 Coluber Ahatulla, Linn. 387.83. (58.

108 Coluber Lemniscatus, Linn. 386. 74. ( 60.

109 Coluber Striatus, fafciis ftriis obliquis albis capite. Box, Linn. o. ( 59.

110 Coluber Mytterifans, f, varietas, Linn. 389.95. (6r.

II I Coluber Typhlus f. varietas, Linn. 378. 22. (65.

II 2 Coluber Tyria, Linn. 386. 78. ( 65.

II 3 Coluber Naja, parva, Limn. 382. 50. (92.

114 Coluber Plumberss, an Saturninus? Linn. 384. 6r. Mus. Adolph. tab. 9. f. I. ( 103.

II Coluber Naja, vid. No. 100., Linn. I. C. (II2.

116 Coluber facapara, Linn. O. (I 3 .

II Coluber Nebulatus Linn 383. 59. (II5.

II 8 Coluber Maurus, Limn. 379. 34. (II4.

II Colube: Maurus, f. varietas, Linn. 1. c. (II6.

I20 Coluher Ahatulla. Linn. 387. 83. (II7.

I2t Coluber Ahatulla, Linn. 1. c. (125.

I22 Coluber Ahatulla, f varietas, Linn. I. c. (133.

I23 Coluber Pallidus, Linn. 381. 48. (I 18.

124 Coluber Miliaris, Limn 380. 37. (II

125 Coluber Grifeus, faíciis abdominalibus 2 fufcis. Lim. 0. ( 120

126 Coluber AEfculapii, f. varietas, Limm.38c.38. (I21.

\27 Coluber Canus, Linn. 382. 52. ( 122.

y 28 Coluber Regine, fufcus fubtus albo nigroque varius, Linn. 378. 27 . ( 127.

129 Coluber Myderizans, Linn. 389. 95. (129. 
95 Boa Gris varié. ( 170 .

96 Coubeuvre tacheté de blanc EO de noir. (26.

97 Couleuvre a dos coleur de Chataigne ( 36.

98 Couleuvre varité de la Corbelle de M. Linné. (37.

99 Couleuvre à ecuffons dentelés, Seb. Th. II. tab. Co. $f$ 2.3. ( 39 .

Ico Couleuvre a lunettes Seb. Th.II. tab.90. (38. fort belle. 10 C Couleuvre à ventre tacheté de brun Muf. Ad. tab.6. $f \cdot 3$. (42. 102 Couleuvre plombé bleuatre. 149.

I03 Couleuvre bleua:re Seb Tb. II, tab. 13. f. 3. ( 50.

104 Couleuvre gris brun à bandes noires Seb.Th. Ir. tab.12.f.3. ( 52 .

IOS Couleuvre a tête noire. ( 53.

100 Couleuvre plombé lleuatre. (99.

107 Couleuvre long, vert bleuatre. Seb. Th. II. iab. 82. f. r. 158.1

108 Couleuvre annulé de blanc E' de brun Seb. Th. I. tab. Ic. (60. È varieté.

109 Couleuvre a taches transverfes blanches la tête de Boa. ( 59.

1 Io Couleuvre gris a grandes blanches, Catesb. tab 47. ( $\sigma I_{0}$

II Couleuvre varieté de Dille. ( 15.

II Couleuvre blancheatre a trois lignes de taches carrées brunes. ( 66.

II Couleuvre pet it Serpent a lunettes. ( 92.

114 Couleuvre plombe de grands yeux. Mur. Adoliph, tab. 9. f. г. (. O3.:

115 Couleuvre à iunettes, vid. $n^{2}, 100$. (IIr.

II6 Coubuvre Tachetée, è grandes taches noir \& blanc. ( 113 . II7 Couleuvre Marbrée de brun Ẽ cendré en defJous brun है blanc Muf. Adolph, tab. 17.7. ( 115 .

118 Coulenvre brun de deux bandes fur le dos en deffous noir les cotez à fries noires. (II 4 .

II Couleuvre sarieté du precedent. ( 46 .

120 Couleuvre le pareil de n ${ }^{\circ}$. Io7.Seb. Th. II. tab.82 f. I. (Ii Te

21 Couleuvre le pareil. (125.

122. Couleuvre variété du meme. (:33.

123 Couleuvre Tacherée, à taches grifes EO points bruns Mur. Adolob. tab. 7. f. 2. ( I 18 .

124 Couleuvre brun a points blancs. (IIg.

125 Couleuvre gris a deux bandes brunes au ventre. ( 120.

I26 Couleuvre varieté de l'Efculape. (121.

I 27 Couleurre grife $a$ bandes brunatres \&o points blancs aux cotez Muf. Adolpin. tab 12. $f$ (122.

128 Couleuvre brun en defous brun \& blanc. (127.

129 Couleuvre le pareil de $n^{\circ}$. 110 . ( 129 . 
130 Coluber Getulus, Linn. 382. 53. (130.

I3! Coluber Hepaticus, punctis nigris, Linn。 O. (I $3 \mathrm{I}_{\text {. }}$

132 Coluber Piumbers, pundtis albis, Linn. O. (134.

133 Coluber Grijeus, Linn. O. ( 135 .

134 Coluber Dipjas, Linn. 386. 76. (140.

135 Coluber Pullus. ( 14 ?

136 Coluber Trifcalis, Linn. 385. 72. (143.

137 Coluber Tyria, Linin. 386. 78. (I 44 .

138 Coluber Fufcus, ftriis nigris, fcutis albis, nigro margipatis, Linn. O. ( 147.

139 Coluber Dipfas, C. varietas, Linm. 386.' 76. (148.

140 Coluber ESfculapius, Linn. 380. 38 . (149.

I4r Coluber Cinereus, annulis fufcis ad abdomen paucioribus, I.inn. ○. ( $15 \mathrm{I}$.

${ }_{42}$ Coluber Plumbers, abdominis fquamis albo fufcoque variis, Limn. O. ( 152 .

143 Coluber Dipfas, Linn. 386. 76. ( 150.

144 Coluber Awnulatus, Linn. $3860^{\circ} 75$. ( 163 .

145 Coluber Cinereus, Linn. 388. 90. ( 153.

146 Coluber Padera, Linn. 382. 51. ( I54

147 Coluber Striatus, albido rubefcens ftriis argutis nigris, Linn: O. ( 155.

148 Coluber Carinatus, Linn. 384. 65. (156.

149 Coluber Regina, Linn. 378. 27. (157.

150 Coluber "Padera, cum Rana in ore, Limn. 282. 5 \% ( 158.

151 Coluber Mycterizans, Linn. 389. 95. (159.

152 Coluber Ordinatus, Linn. 379. 29. (I6I.

I53 Coluber Fafciatus, Linn. 378 23. ( 162.

54 Coluber Fafcictus, Linn. 1..c. ( 165.

155 Coluber Miliaris, Linn. 380. 37. ( 156.

156 Coluber Miliaris, Linn. 1. c. ( 174.

Is7 Coluber Lineatus, pallide fufcus linealis aibidis, Linn. . $_{0}$ ( $18 \mathrm{x}$.

158 Coluber Hippocrefis, Linn. 388. 88. ( 17 r.

I59 Coluber Nebulatus? vel Regina? Limm. 383 59? 27 ? ( 172.

I60 Coluber Sibilans, Linn. 383, 54. C. 175.

I61 Coluber Purpureus, lineis 2 albis nigro punckatis, Linn. O. ( 173 .

162 Coluber Sipedon, Linì. 379. 33. ( 174.

I6 3 Coluber Natrix, Lian. $380.4 \mathrm{I}^{\circ}$ ( 76.

164 Coluber Niger, albo guttatus, Limn: O.
(105 Coluber 


\section{Serpens.}

Ijo Couleuvre noir bleuatre a lignes jaunes fur le dos. Catesjo. tab. 52. ( 130.

13I Couleuvre couleur de foye a points noirs ( $13 \mathrm{~T}$.

I 32 Couleuvre plombe a points blancs. (I34.

I33 Couleuvre gris cendré ( 135 .

I34 Couleuvre Aurore Seb. Th. II. tab. 24. 3. ( 44 J.

I35 Couleuvre jeune brun noiratre. ( 142 .

I 36 Couleuvrs bleu plombé à trois lignes brunes fur le dos. (I 43.

उ37 Couleuvre le même que $\mathrm{n}^{\circ}$. I I2 ( 144 .

138 Couleuvre hrun à lignes noires E écuffons blancs bordés de noir. ( 147 .

139 Couleuvre variété du Serpent Aurnee n. 134. (I48.

I 40 Couleuvre Efculape. Seb. Th. II. tab. 18.f. 4. (I49.

I4r Couleuvre Cendré a anneaux bruns. (I5I

142 Couleuvro plombé les ecrilles de l'anus noirs \& bruks. ( 152.

I43 Couleuvre Aurore Seb. Th. II. tab. 24. 3. ( 150.

344 Cosleuvre blanc d taches rondes brunes, qui $\int e$ touschers Seb. Th. II. tab. 38. 2. (163.

145 Couleusvre Cendré, blanc en deffous. ( 153.

$\$ 46$ Couleuvre blanc d taches trunes ( 154 .

147 Couleuvre roux blancheatre a firies fones noires. (155.

148 Couleuvre plomeé les bords des ecailles blanches. (I56.

149 Couleuvre brun. ( 157 .

150 Couleuvre blanc d taches brunes, avec une grembouille dans fon bec. ( 158 .

151 Coulezvre pareil dे n०. 129. (159,

152 Couleuvre bieuatre tacheté \& narbré de noir. Catesb. t. ‘3. '16r.

153 Couleuvre noiratre $\dot{a}$ bandes bbanches. Catesb. $t .58$. ( 62

154 Couleuvre pareil. ( 155.

155 Couleuvre brun d points blancs, ( 165.

156 Couleuvre pareil. ( 174 .

157 Couleuvre brunatre à ftries blanches. (18r.

158 Couleuvre plombé a taches tranes, Eे une bante brane depe rieve la tete. Mur. Adolph, tab 16. 2. (17:.

I59 Couleuvre marbré de brun Ẽ de cendré. ( 172 .

160 Coulervre bleuatre d bandes noires en deffous blane, Seb. Th. II. tab. 52.f: 4 . ( 175 .

161 Couleuvre pourpré a deux lignes blanches pointillées de noir. ( 173.

162 Couleuvre brun. ( 174 .

163 Couleuvre noir a deux taches blanches an corb. (i 70 . I6. Couleure noir pointillé de blanc. ( $17 \%$. 
265 Cọluber. Vioiaceus, Linn. O. (18c. 166 Coluber Sipedon, Linn. 372. 33. (181.

167 Coluber Sipedon, Linn. 1. c. (189.

I68 Coluber Hippocrepis, f. varietas, Linn. 388. 88. ( $182 \%$

169 Coluber Carulefcens, Linin. 389. 96. (133.

I;

I71 Coluber Annulatus, C. varietas, Linn.386. 75. ( 185.

372 Coluber MyEterizans, Linn, 389. 95. ( 186.

173 Coluber Fufcus, fubtus albus, Linn. C. (18\%.

174 Coluber Ovivorus, Limn. 385. 67. (190.

175 Coluber Fufcus, fubcus brunneus, Linn. O. (I I91.

176 Coluber Padera, Lin\%. 382. 51. ( 193.

177 Coluber Severus, Limn. 379. 31. (197.

I78 Coluber Doliatus, Linn. 379. 28. (20r.

$x 79$ Coluber Brumnefcens fafciis fufco rufis, Limns, O. (203.

I80 Coluber Coeruleus, Linn. 377. ID. (199.

I8r Coluber Dipjas, Linn. 386. 76. ( 198.

I82 Coluber Marginatus, maculis albis rotundis, nigro marginatis, Limn. O. (6I.

183 Anguis Colubrina, Linn 390. 4. (12?.)

.184 Anguis Lumbricalis albus fufco annulatus, Limm. 59y 9? f.०. ( 188.

185 Anguis Alba, fafciis rigris, Linn. O. ( (136.

186 Amphisbæna Fuliginofa, Linn. 392 1. (179.

387 Amphisbæna Fuliginofa, Linn. 1. c. (I39.

188 Amphisbæna Fuliginofa. Linn. 1. c. (128.

I89 Amphisbrna Fuliginof?, Linn.1 c. (124.

x90 Amphisbæna Fuliginofa, Linn. 392. I. (47.)

29r Amphisbæna Fuliginoja, Linn. 1. c. (I41.

192 Amphisbana Fuliginofa, Linn. 1. c. ( 160.

93 Amphisbana Fuliginofa, Linn. I. c. (179).

194 Amphisbæna Fufca, fubtus albo maculata, Linn. 0. (196.

J95 Amphisbana Fufca, abdomine albo nigroque annulato, Limn. о. ( 168.

Igs Amphisbana Alba annulata, Linn. O. (138.

I97 Amphisbrna Alba, Linn 393. 2. ( I67.

I98 Amphisbana Alba, Linn. I. c. (43.

199 Cæcilia Tentaculata, Linm. 393. I. (178.

200 Boa Scytale, Linn. 374. 7. ( 194.

201 Boa Scytale, Livin. 1. c. (I 95.

202 Boa Scytale, annulis albo fufcisque, Limm. 374 7. (22.

203 Caput cinerei Serpentis loris nigris. (194.

204 Draco Volans, Orientalis nigro maculatus, Linn. 358 . I. (1 26.

Cetera Amphibia ficcata, inter ficcorum Animalium elenchurin comparebunt. 
305 Couleuvre Violet: ( 180.

166 Couleuvre, Noir pareil de 162. (181.

167 Couleuvre pareil. ( 189.

168 Couleuvre Plombé yarieté de 158, Muf.Ad.tab. 19. f. 2. (182.

169 Couleuvre Bleuatre. ( 183

170 Couleuvre Noir yarieté Americaine de 163, (184.

i 7 Couleuvre Blanc varieté de $144 . "$ ( 185.

172 Couletwvre Brunatre varieté de 157: Muf: Adolph, tab. It.

$f$ 2. ( 186 .

173 Couleuvre Brun d̀ ventre blanc. (18\%.

174 Couleuvre Blanc à points noirs. (190.

I75 Couleuvre Brun noiratre en deffous brun. (191.

176 Couleuvre Blanc yarieté de 46 , ( 193 .

177 Couleuvre Cendré d bandes blanches, Seb. Th. II. tab. 54:

f. 4 . $\quad(197$

I78 Couleuvre (petite) blanche ì anneaux bruns. (20:.

I 79 Couleuvre Brunatre a bandes brunes rouges. ( $\approx \mathrm{co}$.

I8c, Couleuvre Bleue, Seb. Th.II. tab I3 3. (199

I8I Coulenvre Aurore, Seb. Th II. tab. 24. $f$ 3. (198.

182. Couleuvre Bandé d taches blanches. ( 61.

I 83 Couleuvre Vipere. ( 123.

184 Couleuvre Blanche à anneaux bruns. ( 188 .

185 Couleurre Blanche d arneaux noirs. ( 136.

186 Amphisbene. Noire blanche." ( 172.

187 Amphishene påreil. ( 139 .

188 Amphisbenze pareil. ( 128.

189 Ampbisbene pareil. ( 124.

Igo Amphisberie pareil. (47.

19I Amphisbene pareil. (14I.

192 Amphisbene pareil. ( 160 .

193 Amphisbene pareil: ('79.

194 Amphisbene Brune en def(Jous tachetée de blank. (Ig6.

195 Amphisbene Blanche. (168.

I96 Amphisbene Blanche. (138.

197 Amphisbene le pareil d anneaux, Seb. Th. II. tab. 6.f. 4 (169.

198 Amphisbene le pareil. (43.

I99 Serpent ridé à deux barbillons. ( 178.

200 Serpent Boa gris brun id taches randes noires tilgole des Negres de Juida. (194.

20 I Serpent le pareil. (195.

202 Serpent a taches blaizhes cerclées de noir. ( 23.

203 Tete a'un grand Serpent cendré ¿ bandes noires. (193.

$2 \approx 4$ Lezard Volatit des Indes. ( 126.

Les autres Ampinibies feches, feront mis

dans l'elerctius dès Animaux feches 


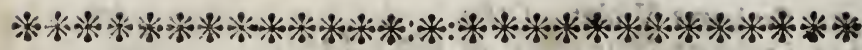

$$
\begin{aligned}
& P \text { I S C E S } \\
& \text { V T } \mathbf{T} \\
& \text { N A N T I A } \\
& \text { in Liquore. }
\end{aligned}
$$

I Wurena Helena, alba punctis fufcis, Linn., 425. I.

2 Murena Angrilla, Linn. 426. 4. (20.

3 Murena Anguilla, f. varietas. ( 32.

4 Murena Brunnea, maculis fufcis, Linn. 0. (9?.

5 Gymnotus Electricus; Linn. 427. 2. (90.

6 Gymnotus Carapo, Linn. 427. I. ( 56.

7 Gymnotus Albifrons, f. varietas, Linn. 428. 3. (57.

8 Gymnotus Albifrons, Linn. 428. 3. (25.

9 Echeneis Neucrates, Linn. 446. 2. (2.

Io Echeneis Neucrates, Linn. 1. c. (36.

II Echeneis Remora, Linn. 446. r. (7x.

12 Echeneis Remors, Linn. 1. c. ( 61.

I3 Gobius Eleotris, Linn 449. 3. (70.

14 Gobius Alepidotus, an Anguillaris? Linn. 450. 8 (73.

15 Scorpæna Porcus, Linn. 452. I. ( 53.

16 Cottus Grunniens, Linn. 451. 3. ( 15.

17 Cottus Scaber, Linn. 45t. 4. ( 19.

18 Cottus Scaber, Linn. 1. c. ( 29.

Ig Cottus Scorpius, Capite Præmagno indicus, Linn. 452.5. ( 24.

20 Cottus Scorpius, f. varietas, Linn. 452. 5. (8.

21 Cottus Serratus, Linn. O. ( 26.

22 Cottus Grunniens, Linn. 1. c. ac 5. ( 28.

23 Cottus Masmoratus, Linn. O. indicus. ( 43.

24 Chatodon Striatus, Linn. 464. 16. (12.

25 Chætodon Acuminatus, Linn. 460. 3. (35.

26 Chætodon Capiftratus, Linn. 465. 18. (46.

27 Chatodon Capiftratus, Limm. 1. c. ( 62.

28 Chatodon Fufcus, fafciis 2 albis, Linn. O. ( $\sigma_{3}$.

29 Chxtodon Roftratus, Linn. 462. 9. ( 64.

30 Sparus Dentex, Linm, 471. 20. (4. 


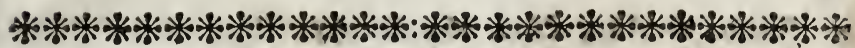

$$
\begin{gathered}
\text { P I S S N S } \\
\text { N A T E A N T S } \\
\text { en Liqueur. }
\end{gathered}
$$

I A nguille Helene blanche d points bruns. Seb. Th. II. tab̆, A 69. 4. (16.

2 Anguille Commune. (20.

3 Anguille varieté de la même. (32.

4 Anguille brune aे points noirs. ( 92.

5 Gymnote E'ectrique Gronov. Zooph. tab. 8. I. (90.

6 Gymnote Carapo. Seb. Th. 1II. tab. 32, f. 4. ( 50.

7 Gymnote aे front blanc. (25.

8 Gymnote varieté du même. ( 57.

9 Remora, Catesb. Carol. tob. 26. (2. .

10 Remora. ( $3 \sigma$.

II Le Sucet. ( $7 \mathrm{I}$,

12 Le Sucet. (6r.

13 Goujon blancheatre da nageoires dorjales egabes. ( 70 .

14 Goujon fans Ecailles. (73.

15 Rafcafle à barbillons aux narines Seb.Th.III. tab.28.3. (15. 16 Cabot a barbillons. Seb. Th. III. 28. 4. (15.

17 Cabot à tubercules. ( 19.

18 Cabot pareil. (29.

I9 Cabot à tete fort grande. ( 24 .

20 Cabot varieté. (8.

21 Cabot à deux barbillons. ( 26 .

22 Cabot le pareil. ( 28.

23 Cabot Marbré, des Indes. ( 43.

24 Chatodon à bandes. Seb. Th. III. tab. 25. 9.) (I2.

25 Chatodon a Epine dorfale en fil long. Mur. Adolph. tah. 33. $f$. 3. ( 35 .

26 Cbetodon avec un ceil vers ke quewe. Muf. Adolph. tab. 33.

f. 4. Seb. Th III. tab., 25. $f,-16$. ( 46.

27 Chatodon pareil. (62.

28 Chatodon brun, \& deux bandes blanches. ( 63 .

29 Chatodon d deux bandes drunes, le mufear long. Seb. Tb. III. $t a b .25 .17).(64$.

30 BrEme dे gros dents Willugb. tab. XI. f. G. (40 
3: Sparus Oiphus, Lirn. 469 8. (35.

32 Sarus Sapenne, fufcus, Linn. O. ( 69 .

33 Sparus Cantharus, Linn. 470. 13. (78.

34 Sparus Cantharus, f. varietas. Linn.1 c. (81.

35 Labrus Fulis, Limn. 476. 15. ( 6.

36 Labrus Hiatula, Linn-475. 12 . ( 7.

37 Labrus Lineatus, linea laterali elevata, Linn. o. (9.

38 Labrus Fulvus, Linis. 479. 38. (33.

39 Labrus Falcatus, Linn. 475 10. ( 52.

40 La rus Cromis, Lirin. 479. 35. (3.

41 Labrus Lumaris. Limm. 474. 6. (65.

42 I,abrus Coeruleus Lmm. O.

43 Labrus Teres, Linn. 0 ( 66.

44 Labrus Fulis, Linn. 476. 15. (67.

45 Labrus Fulvus, fo varietas, Linn. 479. 33. an Sciena? Linn. 18c? ( 75.

45 Labrus Alepidotus, Linn. 0. ( 76.

47 Labrus Dentsx, officulis, pinnarum omnibas Capillaceis. ( 5

48 Perca Amboinenfis, alba pinnis nigro marginatis fafciisque duabus nigris, Linn. O. huic ad politus Diodon echinatus. ( 13.

49 Perca Amboinenfis, alter, Linn. O. (10.

50 Perca Guttata, Linn. 485. 21. (14.

5I Perca Strinta, Linn. 487. 28. (3r.

52 Perca Atrari3, Linn 485. I6. (38.

53 Perca Nobilis, Linn. 484 Ir. (47.

54 Perca Megalophralma, ftriis fex albicantibus, Linn. 0. ( 54 .

55 Perca Guttata, Linn. 485. 21. ( 59.

56 Scomber Pelamis. Linn. 492. 2. (60.

.57 Trigla Volans, Linn. 498. 8. (34.

58 Trigla Volans, Linn I. c. ( 44 .

59 Silurus Galeatus, Linn. 503. II. (27.

60 Silurus Amia, an Calva? Maxilla inferiore longiore; Linn. O. ( 55 ?

6i Sciana Cappa? Linn. 480.' I? ( 77.

62 Salmo Saurus, Americanus, Linn. 511. I4. (48.

63 Fiftularia Tabacaria, Linn. 515. I. (II.

64 Fiftularia Tabacaria, per magna, Lim. I. c. (58.

65 Efox Belone, Linn. 517: 6. (18.

66 Efox Belone, Linn. 1. c. (39.

67 Efox Belone, Linn. 1. c. (74.

68 Efox Belone, Linn 1. c. (91.

69 Pleuronectes Plate $\int a,-$ Linn. 456. 6. \& varii alii parvi. (72. 70 Argentina Sphyrana, Linn. 518. I. ( 79. 
3r Brême à ail proche de la queue. (30.

32 Brème à dos brun. (60)

33 Brême à bandes longitudịnales. ( 78 .

34 Brême varieté du même. (8I.

35 Perroquet Paon Maris. ( 7.

36 Perroquet à fix bandes noires. ( 7 .

37 Perroquet à ligne laterale elevée. ( 9.

$3^{8}$ Perroquet à corps brun jaunatre, Catesb. tab. 10. f. 2. ( 33 .

39 Perroquet à nageoires dorfales vafcale en faulx. ( 52 .

40 Perroquet à bandes brunes. ( 3 .

4 I Perroquet à bande pourprée fur la nageoire dorfale. Gronov. Zooph. tab. 6. f. 2. ( 65 .

42 Perroquet Bleuatre. ( $4 \mathrm{r}$.

43 Perroquet Cylindrique. ( 66.

44 Perroquet Paon Marin. ( 67.

45 Perroquet varieté de 38 . ( 75 .

46 Perroquet fans ecailles. ( 76 .

47 Perroquet à gros dents, les offelets des nageoires, fileux ( 5.

48 Perche blanche de nageoires bordees de noir $\&$ deux bandes. noires d'Amboine, avec un poifJon HerifJon. ( 13.

49 Perche blanche d'Amboine variete. ( 10.

50 Perche à gouttes, Catesb. I4. (I4.

5 I Perche Striée. (3I.

52 Perche Noire à nageoires tachetées. ( 38.

53 Perche blanche d huit bandes brunes, Seb. Th. III.tab. 25.

I2. (47.

54 Perche à grands yeux $E$ fix bandes hlanches. (54.

55 Perche le pareil de 50. ( 59.

$56^{\circ}$ Maquereau à quatre lignes noires. ( 60.

57 Milan Sapphirine Volant. ( 34.

58 Milan Sapphirine Volant. (44.

59 Silure à fix barbillons, Seb. Th. III. tab. 19.f.70 (27.

60 Amie d machoire interieure longue. ( 55.

6I Daine? la tête à doubles ecailles. ( 77 .

62 Saumon d'Amerique. ( 48.

63 La pipe à tabac;, Cates S. II tab. 17. f. 2. (Ir.

64 La pipe à tabac pareille tres grande. ( 58.

65 Brochet dे long bec ou Orphie. (18.

66 Brochet à long bec pareil. (39.

67 Brochet d long bec pareil. ( 74 .

68 Brochet iे long bec pareil. ( 91 .

69 La fole avec plufieidrs petits. ( 72 .

70 Ablet. ( 79. 
71 Cyprinus Auratus, Linn. 527. 7. (40.

72 Clupea Sprattus, Linn 523. 2. (80.

73 Clupea Sternicla, Linn 524. 8. (82.

74 Squalus Canicula, Linn. 399. 8. (17.

75 Squalus Galeus, Linn. 399. 7. ( 21.

76 Squalus Tiburo, Linn. 399. 6. (22.

77 Squalus Galeus, Linni. 1. c. (49.

78 Squalus Galeus, Linn. 1. c. (50.

79 Squalus Carcharias, Linn. 400. I2. (29.

80 Lophius Hiftrio, Linn. 403. 3. ( 42.

81 Tetrodon Lineatus, Linin. 4 II. 3. (37.

82 Tetrodon Lineatrs, Linn. I. c. (5r.

83 Tetrodon Lineatus, Linn. I. c. (45.

84 Baliftes Hispidus, Linn. 405. 2. (68.

85 Baliftes Aculeatus, Linn. 406. 6. ( 84.

86 Syngnathus Acus, Isinn. 416 2. (1.

87 Syngrathus Hippocampus, Linn. 417. 7. (26.

Ceteri Pifces.jiccati poftea resenfebuntur.

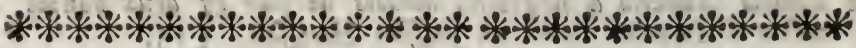

$$
\begin{gathered}
\text { I N. S E C } \\
\text { in Liquore. }
\end{gathered}
$$

I Hruca spinoja. (I.

2 Eruca Spinofa Cpinis ramofis, rara. (5.

3 Eruca Fufca fafcirs pallidis lineis transvertis junctis. 66 .

4 Eruca Papilionis Menelar, Linn. 748.26. (4.

5 Eruca Papilionis magna fuifca. ( 3.

6 Eruca Papilionis alba non villofa. ( 2 .

7 Eruca Papilionis Pierix, Linn. 754. 52. (15.

8 Eruca Papilionis cujusdam glabra. (25.

- Eruca Papilionis Indici. (28.

1.0 Eruca Sphingis Indica. ( 30 .

II Eruca Spinis quatuor in quogue annulo. ( 32.

12 Eruca Lichene quafi veftita. ( 34 .

13 Eruca Apirodita aculeatæ fimilis. ( 35 .

14 Eruca Fufca \& alia. ( 36.

15 Eruca in folin convoluto. ( 37.

I6 Eruca Sphingis Indica. (38.

37 Eruca an Papilionis P/idii? (40. 
7: Poifon doré de la Chine, l'Haarl. Verh. VIII. tab. 17. ( 40.

72 Hareng Sardelle. ( 80.

73 Hareng d poitrine \&5 ventre Aigues Gronov. Zooph tab. ?. f. 5. ( 82 .

74 Requin Chien de Mer. ( 17.

75 Requin a Narines proches de la bouche. (2r. I sur.)

76 Requin Tibure de tête large ( 22.

77 Requin pareil de 74. (49.

78 Requin pareil ( 50.

79 Requin Cáıcharias. ( 23.

80 Diable de Mer tacheté Petiv, Gaz. 20. (42.

81. Heriffon de Mer à bandes. ( 37.

82 Le parei!. ( 5 I.

83 Le pareil. (45.

84 Balifte Vetu, Seb. Th. III. 34. 2 ( 68.

85 Balifte, Epineufe, Seb. Th. III. tab. 24. f. 15. ( 84 .

86 Poiffon Aiguille. ( 1.

87 Cheval Marin. (26.

Les autres Poiffons fechez feront rommé après.

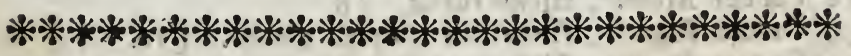

$$
\text { I I S } \underset{\text { en Liqueur. }}{\mathrm{C}} \mathbf{T} \mathrm{E} \text { S. }
$$

I henille Epineure. (I.

2 Chemille Epineufe à epines branchues, fort rare. (5.)

3 Chenille Brune à bandes claires, jointes par des lignes trans. verfes. ( 6.

4 Chenille du Papillon bleu fatiné de Surinam. Merian Surin. tab. 53 . (4.

5 Chenille du Papillon (grande) brune. ( 3.

6 Chenille du Papillon blanche non velue. ( 2.

7 Chenille du Papillon vitré, Merian Surin. 16. ( 15.

8 Chenille rafe du Papillon. (25.

9 Chenille d'un Papillon des Indes. (28.

ro Cherille d'un Papillon Belier des Indes. ( 30 .

II Chenille à quatre epines fur chaques anneau. ( 32.

I2 Chenille conme couverte d'un Lichen. ( 34 .

13 Chenille refJemblant au Souris de Mer. (35.

$r_{4}$ Chemille brune \& des autres. ( 36.

Is Chenille dans urse feuille roulée, ( 37.

I6 Chenille d'un Sphinx des Indes. ( 38.

If Chenille du Papilloil du Pfidium? Merian Surin tab 19. (40. B 4

I8 Chenille 
If Eruca annulis comprefiu gibbofis. ( 43.

19 Eruca Albida, ftigmatibus nigris. (45.

20 Eruca Albida, ac I2: (46.

21 Eruca $A b b i d a$, annulis nigro marginatis. (59.

22 Eruca Sphingis labrufce.- (48.

23 Eruca Magraa, fulva. (47.

24 Eruca Exotica. eum duabus aliis. ( 49.

25 Eruca, duæ glabre. ( 44 .

26 Eruca Striata, quatuor nigra ftriis albidis. (4).

27 Eruca Spinofa, rainofa cum alia quadrata. ( 39.

28 Eruca, dur Scarabei. (26.

29 Eruca Scarabiai indiei. ( $3 \mathrm{r}$.

30 Eruca $A p o d a$, capite nigro an curculionis palmarum? (33.

3T Lagina cum variis Erucis. (24.

32 Fulgora Laternaria, Linn. z०3. x. ( 16.

33 Formica Abba iǹ nido ${ }^{\circ}$ ( 7 .

34 Scotpin pectine- 9 dentato, Linn. 0." ( 12 .

35 Scorpio pectine ro dentato. ( $2 \mathbf{r}$.

36 Formica Cephalotes. Linn. 964. I5. ( 42.

37 Cancer Mantis, Linis I054. 76 . ( 7 .

38. Cancer Mantis, fafciis fufcis. (8

39 Cancer Mantis, C. variet is thorace glabro. (10.

40 Cancer Sqquilla, Livn. IOSI 66. (9.

41 Cancer Pelogicus, Linn. \$O42. I9 (II, I

42 Cancer ArEzus, Linn. 1053.75. C 17.

43 Cancer Squilla Americana, Linn. IOSI 66. (18.

44 Cancer Depurator, Linq3. 1043. 23.) (20.

45 Cancer Planiufcuhus, thorace utrinque fpina, fez dentato Ixvi, chelis teretebus. Limn. O. ( 19.

46 Cancer Diogenes Magnus, Linn. 1049. 58. (13.

47 Aranea Scolopendra, f.Gigantéa Occidentalis, Linn.1003. 4.' an Diadema? Lim. I030, I? (22. 29.

48 Julus Complanatus, Linn. 1065 4. (14.

49 Myrmileon Formicarium, r. Lạva Linn. 9I4 3. (23.

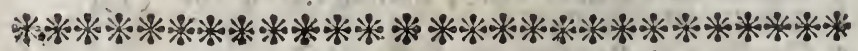

\section{VERMES MOLLVSCA}

in Liquore.

50 Aphrodita Carunculata, Linn. O. (I.

5.I Aphrodita Carunculata. ( 5 .

52 Aphrodita Flava. Linn. O. (8. 
I8 Chenille à Anneaux comprimes $\mathcal{E}^{\circ}$ bo/Jus. I. ( 43.

I9 Chenille Blancheatres a ftigmates noires. ( 45 .

.20 Chenille pareil de I2. ( 46.

21 henille blanche à Anneaux bordez de noir. ( 50 .

22 Chenille du S, hinx du Labrufcnm, Merian Surin. 34. (48.

23 Chenille (grande) fauve ( 47.

24 Chenille Exotique, avec deux autrés. (49.

25 Chenille $\mathrm{Rafe}$, avec une pareille. (44.

26 Chenille quatre noires à fries blanches. (4t.

27 Chenille epineufe branchue, \&ै une-quarrée. (39.

28 Chesille deux des fcarabé. ( 26.

29 Chenille d'un Scarabé des Indes. (3I.

30. Chenille fans pieds a tete noire de l'Elephant, Merian Surin. tab. 48. f. 3. ( 33 .

31 Bouteilles avec plufieurs Chenilles. ( 24.

32 Un porte lanterne, Merian Surin pl.49.Holl.Acad.I. I. (i 6. ,

33 Fourmi blanche dans fon nid (27.

34 Un Scorpion a peigne de I9 dents. (I2.

35 Le Scorpion a peigne de 19 dents. ( 21 .

36- Fourmi a grnffe tete, Merian Surin. tab. I8. (42.

37 Ecriviffe Mante, Ramph. Amboinfch Rarit, tab. 3. l. c. (7.

38 Ecriviffe Mante d bandis brunes. . ( 8 .

39 De l'Ecrivi $\iint e$ Mante varieté. (10.

40 Chevrette d'Anerique., Seb. Th. III. tab 21. f. 9. (9.

4 I Crabbe de Mer, Rumph. tab. 7. $f$ I2. (II.

42 Crabbe, nommée Ours, Seb. Th. III. tab. IO. f. I. ( 17.

43 Crabbe d'Amerique, Seb. Th. III. tab. 2I. f. 10. (13.

44 Crabbe a pattes nageoires, Seb. Tb. III. tab. i 8. f. 9 . ( 20.

45 Crabbe platte a deux epines laterales $\mathcal{E}^{2} f(x$ pointes d mains cylindriques. (19.

46 Crabbe Diogene tres grand, Romph. 5. f. c. l. (13.

47 Araignée ou Diademe. ( 22.

48 Scolopendre plat. ( 14.

49 Larve da Fourmillion, Rös. Inf. T. III. tab. I7. (23.

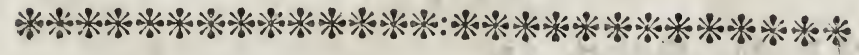

\section{E R S M O U X.}

\section{en Ligueur.}

50 Aphrodite à caroncules, Pall. Mifcel Zocl. tab. 8. f. Iz. I3. (I.

5 I Aphrodite à caruncules pareille. ( 5 .

52 Aphrodite Jaure, Pall. l. a. f. I-II. ( 3. 
53 Aphrodita Aculeata, Linn. 1084. I. (6.

54 Holothuria Frondofa, Linn. 1089. r. (II.

55 Aiterias Ophiura, Linn. Iroo. II. ( 3 .

56 Afterias Multiradiata Linn. 1101. 15.

(4.

57 Sepia Media, Linn. 1095. 3. ( 2.

58 Sepia Media, Linn. 1. c. ( 9.

59 Sepia, an Nautilo permagna, Linn. 1095. ( 7.

60 Tænia Lata, Linn. 1324. 3. ( 10.

\section{E S T A C E A in Liggore.}

6I Chiton Fafcicularis, cum animale, Linn. I106. 4. (1. 62 Lepas Mitella, Linn. 1108.15. (3.

63 Lepas Anatifera, fucus veficularis \&c., Linm. II09. 18. (4.

64 Voluta Cymbium, cum animale, Linn. I196. 436 . (3.

\section{$\begin{array}{lllllllll}Z & \mathrm{O} & \mathrm{O} & \mathrm{P} & \mathrm{H} & \mathrm{Y} & \mathrm{T} & \mathrm{A}\end{array}$ in Liquore.}

65 Pennatula Grifea, Linn. I32r. J. (2. o6 Pennatula Grijea minor, Linn. l.c. (r. 67 Tubutaria Ramea absque Polypis, (3.

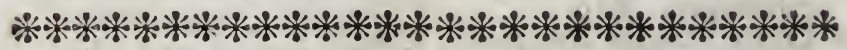

$$
\begin{aligned}
& \text { P L A N } \quad \text { T } \\
& \text { in Liquorie. }
\end{aligned}
$$

68 Fructus Alkekengi. ( $\mathrm{I}$.

69 Fructus Mammea? (2.

70 Fructus Aurantiorum. (4.

7 I Flores Aloes. ( 5 .

72 Malus Punica. ( 6 .

73 Fructus Theobros:e. ( 7.

74 Fruetus Anacardii. ( 16.

75 Fructus Anacaraiii. (9.

76 Frictus Amacardii. $(10$. 


\section{Vermiffeaux, $E^{2} c$.}

53 Aphrodite Souris de mer, Pall. l.c. I. Baft. Subfec, tab. 6. $f$. 12. ( 6 .

54 Holothurie à tete branchue, Schwedifch. Abh. 1;67. (II.

55 Etoile de Merà cinq pointes, Seb. Th. III. tab.9.f. 5. 6. (3. 56 Etoile de Mer a plufieurs pointes, Link Stell. Mar. tab. 2i. f. 33. (4.

57 Seiche platte. ( 2.

58 Seiche platte. ( 9.

59 Polype du Nautile, Rumph. tab. I7. D. (7.

60 Vers fulitaire. (10.

\section{O Q U I.L L A G E S. en Liquesr.}

6r Un Chiton a huit articulations avec fon Animal, Rumph. pl. $10^{\prime \prime} f^{\prime}$ 4. (I.

62 Tulipe de Mer. (3.

63 Lepade Anferifere. (4.

$64 \mathrm{La}$ Couronne d'Ethiopie aveejon Animal, Adanf. Seneg. tab. 3. $f$. 2. ( 2 .

\section{Z O O P H Y T E S.}

\section{en Liqueur.}

65 Penne Marine grife très grande, Seb. Th. III. tab.x6.f. 8. (2. 66 Penne Marine plus petite. ( $x$.

67 Tubulaire fans Polypes. (3.

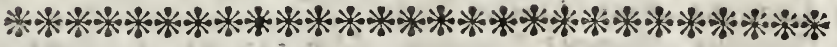

\section{P L A N T E S. \\ en Liqueur.}

68 Fruit d'Alkekengi. ( 1 .

69 Fruit du Mammea de Surinam 3 - (2.

70. Fruit Pomme d'Orange. (4.

7 I Fleur d'Aloe. ( 5 .

72 Fruit Pomme de Granat. ( $\sigma$.

73 Fruit du Cacao. ( 7.

74 Fruit de la Cajou (I6.

75 Fruit de la Cajou. (19.

76 Fruit de la Cajou. (Io. 
77 Fruetus Arsacardii. (8.

18 Fructus Limonum. (II.

79. Fructus $T: i, n g u l a t o$ rotundus linea rubra in dorfo. ( 13.

80 F uêus Dature. ( 14.

81 Fruetus Pomum venenatum. (15.

82 Auranius Malus, cum alio inmato. (12.

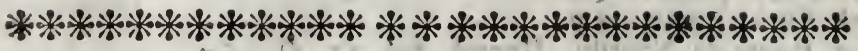

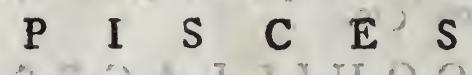

Siccati.

1 parus Magalodon, dentibus magnis, Linus. o. ( 23.

2 Sparus Erythrinus, f. varietas, Linn. 469. 10.

3 Sparus Megalodon, f. varietas, Linn. o (ro4.

(99.

4 Sparts Striatus, caudx appendicibus.-2. (106.

5 Sparus Argyrops, Linn. 47x. 19. ( 124.

6 Sparus Melanurus, Linn. 468. 4 (I26.

7 Labrus Fulis, Linn. 476 . 15. ( 94 .

8 Labru: Lunaris? Linn. 474. 6? (107.

9 Labrus Maçulatus, dorio maculato abdomine fafciis duabus, cauda lanata, Linn. c. ( 109.

Io Labrus Gibbus, officulo fecundo ventralium longiffime fitis formi, Linn. O. (II6.

II Labrus Fulvus. Linn. 479. 38." ( 125.

12 Labrus Maculatus, idem ac.9. (115.

13 Labrus Microlepedotus, Linn. O. ( 155.

34 Labrus Punctatus, Linn. 477. 23. ( 166.

15 Cyprinus Albrs: Linn: O. ( 95.

ys Cy prinus Alburnus? f. varietas, Linn. 53r. 24. B. (Iоr.

17 Cyprinus Vimba, Linn. 531 25. (103.

18 Cyprinus Dipterygius. Linn.0, (II7.

I9 Perca Vittata, Linn. 485. 19. ( 97.

20 Perca Teres, linea laterali interrupta. Linn. 0: ( 100.

21 Perca Teres, linea interrupta. Linn. 0. ( 105.

22 Perca Diagramma, Linn 487. 27. (IIO.

23 Perca Diagramma, Linn. 1. c. (II3.

24 Perca Fufca. Linn. O. ( 120.

25 Perca Fufca. Linn. O. ( I 20.

26 Perca Unicolor marina. Linn. O. ( I 22.

27 Perca Nobilis, Linn. 484. II. (I 23.

28 Chætodon Pinnatus, radiis quą̧uor dorfi \& ani longifimis, Limn 46 . . 4. (II 4 .

2) Chatodon 


\section{Poifons Sechez.}

77 Frrit pareille. ( 8.

78 Fruit de Limon. (II.

79 Fruit Triangulaire a parties rondes, le dos a ligne rouge. ( 13. 80 Fruit de la Datura. (I 4.

81 Fruit venimeufe nommé Banc Priffon. (15.

82 Fruit Poinme d'Orange dans la quelbe une autre eft crue. (I2.

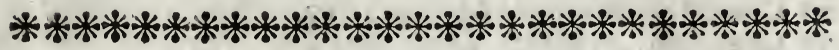

P O I S S O N S.

Sechez.

I Breme à grandes dents. (93.

2 Breme ou varieté rouge: ( 99.

3 Breme varieté de $\mathrm{n}^{2}$. I. (104.

4 Breme Strié d deux appendices do la queuse. ( 106.

5 Breme d̀ œil argenté. (I 24.

6 Breme à queue noire. ( 126 .

7 Paon de Mer. ( 94.

8 Peiroquet de Mer, à queue fourchus. ( 107.

9 Perroquet de Mer, d̀ dos tacheté Ej $^{\circ}$ à ventre de desx bandes. ( 109.

Io Perroquet de Mer, à dos boflu, E' l'offelet de la nageoine ventrale treslong. (i I 6 .

II Perroquet de Mer' jaune. (I25.

I2 Perroquet de Mer tacheté, le meme que nº.9. (II5.

I3 Perioquet de Mer à petites ecailles. (155.

14 Perroquet de Mer à lignes pointillées. ( 106.

15. RofJe blanche. (95.

16 Varieté de l'Alburnus. (IOr.

$17 \mathrm{Le} \mathrm{Vimba} \mathrm{(} 103$.

I8 Roffe a grandes nageoires pectorales. (II7.

I9 Perche Strié. ( 97.

20 Perche à ligne laterale rompue. (1003.

2 I Ptrche à ligre laterale rompue. ( 105.

22 Perche d innes jaunes. (110.

23 De la Perche Jaune varieté. (II $3^{\circ}$.

24 Perche brune. ( 120.

25 Perche biune. (120.

26 Prrche marine. d un couleur. (122.

27 Perche nob'e. 123.

28 Chatodon, a nageoires de quatre offelets du dos EO de l'arass tres longs Seb. Th. III. tab. 25. 15. ( 114 . 
29 Chatodon Acuminatus, Linn. 46x. 3. (i5 .

30 Cbxtodon Acuminatus, Limn. I. c. (I57.

31 Cbætodon scuminatus, Linn. L. c. ( 163.

32 Cinatodon Pinnatus, Linn. 46r. 4. ( 125.

33 Chætodon Pinnatus, Linn. 1. c.- ( 153.

34 Chrotodon Roftratus, Linn 462.9 (is4.

35. Chætodon Nigricans, Linn. 462. 10. ( 160.

36 Chatodon Aruanus, Linn. 464. 17. ( 161 .

37 Chxtodon Arcuatus, Linn. 462. 8. ( $: 62$.

38 Chatodon Strjatus, Linn. 404. 16. ( 164.

39 Chatodon Serratus, feriebus fex macularum fufcarum; Linn. O ( 107.

40 Chæiodon Macrolefidatus, Linn. 464. 14. (15J.

4I Chætodon Macroletidotus, Linn. I. c. (156.

42 Chrtodon Capiftratus, Limn: 467. 18. (108.

43 Sciæna Umbra, Limn. 480. 40. (iI8.

44 Efox Gymnocephalus, Limm. 517. 9. (98.

45 Mullus, maxilla in feriore longiore dorfo gibbo, Linn.o ( 112.

45 Gafterofteus Spinachia, Linn. 492. 10. ( 129.

47 Trigla Volans, Linn. 498. 8. ( 133.

48 Trigla Volans, Linn. I. c. (134.

49 Trigla Volans, Linno l. c. ( 130 .

5o Trigla Volens, Linn. !. c. (I $3 \mathrm{I}$.

5I Exocctus Volans? Linn. 520. 1? ( 132.

52 Echeneis Neucrates, Limm. 446. 2. (I2\%.

53 Echeneis IVeucrates, Linn. 1. c. ( 148.

54 Cottus Quadricornis, Linn. 45i. 2 . ( 123.

55 Cottus Qundricornis, Linn. 1. c. ( 144 .

56 Cottus Quadricornis, Linm. I. c. (I49.

57 Fiftularia Tebacaria, Linn 525. I. (I4r.

58 Silurus Tibiceri, corpore plano, lato, cauda longa teres,

Linn. O. ( 142 .

59 Silurus Tibicen, ut fupra. ( 193.

co Teuthis Fonus, Linn. 507.2. ( 96.

Gr Lcricaria Cataphraeta, Linn. 508. I. (136.

62 Chimxia Monftrofa, Linn. 401. I. (I35.

63 Syngnathus Typhle, Lim. 416.1. (137.

64 Syngnathus Typhle? Linn. 1. c. (138.

65 Syngnathus Arrs, Linn. 4 16. 2. (150.

66 Syngnathus Hippocampus, Linn. 417. 7: (195.

67 Syngnathus Hippocampus, Linn. I. c. ( 146 .

68 Syngnathus Hippocampus, Linn. 1. c. ( 147.

60 Baliftes Vetula, Linn. 406. 7. ( 1 II.

70 Baliftes Papillojus, Limn. 405. 4. (I58.

7 Balifes Tomentofus, Limm 405. 3. (I59. 


\section{Poiffons Seches.}

29 Chatodon pointu a troifieme offelet du dos tres long. Mur. Adolph. tab. 33. 3. (III.

30 Chetodon pointu. ( 157 .

3I Chatodon pointu. (I03.

32 Chatodon à nageoires le même que nD.25. ( 152 .

33 Chatodon à nageoires. (153.

34 Le Chatodon à nageoires, Ser. Th III. tab. 25. I7 (I54. 35 Chatodon à long bec, Seb. Th. III. tab. 25 2. ( 160.

36 Chatodon à trois bandes brunes, ( 161 .

37 Chetodons quatre arcs blancs, Seb. Ih. III. tab.25 5. (162. 38 Chatodon Strié a cinq lignes brunes. Seb. Tl. IIl.tab.25.9. ( 164.

39 Chatedon tachetées d̀ bandes brunes. (197.

40 Lhatodon à crandes ecalles. ( 155 .

41 Chatodon à grandes ecailles ( 156 ,

42 Chatodon ou Capiftrate. ( I08.

43 Sciane Ombre. tacketée de noir. ( 118.

44 Brochet à tête nue. ( 98.

45 Barbeau boffu a macheoire inferiense longus. (i12.

46 Epinoche, Blocha Gefcb. der Fisfchen. tab. 53. ( 129.

47 PoifJon Volant. Seb. Th. III. 28. 7. (133.

48 Poifjon Volant. ( 34.

49 Poilfon Volant, (130.

so Poiffor Volant. (13 I.

II V crieté du Volant exocetus. ( 132.

52 Le Remora ou arrete nef. Seb. Th. III. :ab. 33, f. 2. (127.

53 Le Remora ou arrete nef, ( 448 .

54 Chabot à quatre cornes. (I28.

55 Chabot à quatre cornes. (1 44.4 .

56 Chabot à quatre cornes. ( 149 .

57 Le PoifJon pipe: (14x.

58 Pai Jon Trompette a corps large E' plat, la qquerse longue. ( 142.

$59 \mathrm{Le}$ Poifon Trumpette. (143.

6o Teuthie de Java. ( 96.

6r Le Cuirafier armé. (136.

62 Le Diable de Mer. (135.

63 Poifon aiguille dे cotes. ( 137.

64 Poiffon aiguille a fax cotes. ( 138.

65 Poiflon aiguille d fept cotez. (150.

66 Cheval marin. (191.

67 Le Cheval marin. ( 466.

68 Le Cheval marin. ( 147.

69 Balifte Gronard. (III.

70 Balifte à tubercules. (158.

7I Balifte Velu. SI59. 
72 Baliftes Hispidus, Linn. 405. 2. ( 165.

73 Tetrodon Lagocepinalus, Linn. 4 10. 2. ( 178.

74 Diodon Atringa, Linn. 412 1. ( 175.

75 Diodon Atringa, fovarietas, Li*n. 1. c. (176.

76 Diodon Atringa, f. varietas, Linn. l. c. ( 177 .

77 Diodon Atringa, f variotas, Linn. 1. c. (102.

75 Diodon Atringa, magnus, Linn. 1. c. (180.

79 Diodon Atringa, Linn. i. c. (181.

80 Diodon Fufcrts, macu is albis, Linn. O. ( 182.

81 Diodon Hyftrix, Linn 413. 2. ( 183.

82 Diodon Hyftrix, Linn 1. c. (I79.

83 iodon Atringa, Livin. I. c. (185.

84 Oll racion Bicaudalis, Lim 408. 3. (168.

85 Oftracion Quadricornis, Linn, 409. 5. (169.

86 Oftracion Cormutus, Limm. 400 6. ( 170.

87 Otracion Quadricornis, Linn. 409. 5. ( $77 \mathrm{r}$.

88 Oftracion Cormutus? f. varietas, Liwn. 1. C. ( 172.

89 Ottracion Quadricornis, f varietas, Linn $4 C 9$ 5. ( 173.

90 Oftracion Depreffis, corpore açuleáto, Linn. O. (174.

9I Pieuronectes Aculeatus, nigro fufcus, Linn. O. ( 187 .

92 Sparus Megalodon, f. varietas, Linn. O. (IIg.

93 Sparus Megalodon, f. varietas, Linn c. (121.

94 Gafterofteus Spinachia, Linn. 492. 10. (139.

95 Gafterofteus Spinachia, Linn. 1. c. ( 140.

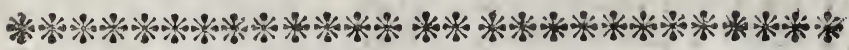

\section{A M P H I B I A}

\section{Sicca.}

96 Laceita Ameiva, Limm. 362. 14. (202.

s7 Lacerta Iguana. C. varietas, Linm. 368. 26. (203.

98 Lacerta Crocodilus, Linn. 359. 1. (204.

99 Lacerta Crocadilus, Linn. 1. c. (205.

Ico Draco Volans, Americanus, Linn. 358. x. (208.

101 Draco Volans, Orientalis, Linn. 1. c. (207.

\02 Lacerta Iguana, Limn. I. c. (209.

103 Lacerta Agilis, Linn. 363. 15. (208.

104 Draco Volans, Linn 1. c. (210.

105 Lacerta Stincus, Linn 365. 22. (2: I.

106 Lacerta Cordylus, Linn 361. . . ( 212.

107 Lacerta Stellio, Lim. 361: IO. (213. 
72 Balifte à pointes. ( 165.

73 Poilfon Soustleor. ( 178.

74 Yierifon Attringa. ( 175 .

75 Herilfon istringa, ou varieté. ( 176.

76 Heriljon, ou autre arieté. (17).

77 Herillori, ou varieté. ( 102.

78 Herillon de Mer, très grama. ( 180.

79 HerijJon de Mer. ( $18 \%$.

80 Heriffun Brun à taches blanches. (182.

8I Porcipic de Mer. ( 183.

82 Porcipic de Mer. ( $\$ 79$.

83 Heriffon de Mer. ( 185.

84 Poifjon Coffir $=\ddot{a}$ deux epines à la queue. (168.

85 Poiflou Coffre à quasre cornes. ( 16 ).

85 Poilfon Coffre cornus. (170

87 Poiffon Coffre d quatre cornes (I7I.

88 Poiffon Coffre cornu, ou varieté. (I 72.

89 PoijJon $\mathrm{C}$ ffre, ou varieté à quatre cornes.

so Poiffor Coffre, plat à pointes. (174.

9: Turbot a pointes brun noiratre. (187.

92 Breme, a gros dents ou varieté. (IIg.

93 Breme, a gros depts, ou autre variete.

94. Epinoche. ( $139^{\circ}$.

95 Epirioche. (140.

(179.

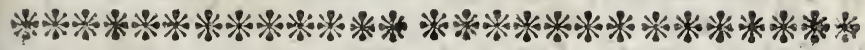

\section{A M P H I BIE S.}

Sechez.

96 Lezard Ameiva. ( 202.

97 Lezard Leguan, ou varieté. (203.

98 Lezard, ou petit Crocodile. (204.

99 Lezard, ou petit Crocodile. (205.

100 Lezard Volant d'Ameriqus. (206.

101 Lezard Volant des Indes. $(20 \%$.

102 Lezard oa Leguan. (209.

103 Lezrrd à Collier. (2e8.

$\mathrm{IC}_{4}$ Lezard Volant. (2io.

105 Lezard ou le Seine (2Is.

106 Lezard Cordyle. (21 2

Io7 Lezard à queue epineufe, (213. 
108 Lacerta Stellio, Linn. 1. c. (214.

109 Lacerta Chameleon, Linn. 364. 20. (215.

Iro Lacerta Chalcides, maculis nigris, fubtus a ba, Lisn. 369. 41. ( 216.

III Lacerta Ameiva, dux, Linm. 1. c. ( 217.

112 Lacerta Iguana, parva, Linn. i. c. (2 I8.

113 Lacerta Agilis, Linn. 1. c. (219.

II4 Serpentes Colubri, aliquot ficcati. ( 220.

115 Crotalus, fo ejustem Crepttaculum. (22r.

116 Serpens Siccatus, long. 15 ped. (222.

I17 Serpens Typhlus, Lins. 378. 22? (223.

118 Lacerta Iguana, Linn. 1. c. (224.

II 9 Lacerta Iguana, Linn. 1. c. ( 238.

120 Lacerta Monitor, Li, n. 360. 6. (225.

121 Teftudo Geometrica, Linn. 353. 13. ( 226.

122 Teftudo Geometrica, f. tefta, long. $\mathrm{I}^{\mathrm{I}} \frac{1}{2}$. poll. lat. 7 poll: Linn I. c. $\$ 2: 7$.

123 Teftudo Geometrica, f. tefta ejusdem, Linn. 1. c. long. I5 poil. lac. 9 poli. ( 228.

124 Teftudo Mydas, Linn. 350. 3. long. II poll. ( 229.

125 Teftudo Scabra, Linn. 351. 6. (230.

126 Teftudo Lutaria Linn 352. 7. (23r.

127 Teltudo Geometrica, Linn. I. c. ( 232.

128 Teft: do Scabra, Linn. 1. c. (233.

129 TElfudo Orbicularis. Linn. 351. 5. (234.

130 Teftado Caretta, Linn, 351. 4. long. I9 poll. lat. I4 poll. (235.

I3T Tertudo Caretta. Linr. 1. c. 236.

132 'Teftudo Mydas, Linn. I. c. long. Io, lat. $7 \frac{1}{2}$ poll. (237.

133 Rana Pipa, Linn. 354, r. (239.

134 Rana Bufo, Linn. 354. 3. longa 9. poll. ( 240.

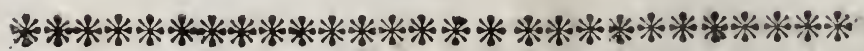

\section{P A R T E S A V I V M}

aliorumque Animalium.

1 vum Cafuaris. (442.

2 Roftrum Platalea. (444.

3 Roftrum Bucerotis Hydroceratis. ( 443.

4 Roftrum Bucerotis Criftati. (445.

5 Roctrum 


\section{Parties des Oifeario}

108 Lezard à queue epineufe ou varieté. (214a

IOg Lezard or Cameleon. (215.

110 Lezard Brun à taches noires deffous blanc. (2ro.

IIr. Lezard ou deux Aneiva. (Ir7.

I12 Lezard ou petit Leguan. ( 218.

II 3 Lezard à Collier. (219.

II 4 Quelques Serpents feches. (220."

II La jonnette d'un Serpent d Connettes. (22r.

Ii6 Serpent feché de la longueur de I5 pieds. (222.

II 7 Serpert ou le Typhlus? (223.

Ir 8 Lezard ou Leguan. (224.

IIg Lezard ou Leguan ou varieté. (288:

120 Lezard Sauvegarde. (235.

I2I Tortue à Carreaux hexagones bordez de jaune. ( 226 .

122 Tortue, ou tefte da même, long $11 \frac{1}{2}$ pouc. larg. 7 poucc. ( 22 G

I2 3 Tortue à Carreaux, long. Is pouc, larg. 9 pouc. ( 228.

124 Tortue Noire, liffe, long. I I pouc. (229.

125 Tortue a Ecailies boffues. ( 230 .

126 Tortue dे teite plat, d troís ecailles en arretes. (231.

127 Tortue, ou la même que n. I2I. (

I28 Tortue, ou la même que.n. I25. ( 233 .

I29. Tortue Ronde. (234.

I30 Tortue Carette, long. In pouc. larg. 4 pouc. (235.

I3I Tortue Carette, (236.

I 32 Tortue Carette, la même que n. I24. long. 1o pouc. lärg, $7 \frac{2}{2}$ pouc. (237.

133 Gremouille Pipa. ( 239.

I 34 Grenouille Bufo, très grand Crapaud de 9 pouces. (240.

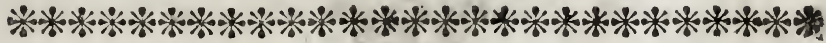

\section{PARTIES DES OISEAUX,}

E2 d'autres Animaux.

3 Bec d'ur Calao. (445.

4 Bec autre d'un Calao. (445. 
5 Roftram Ramphafti- : (446.

6 Roftrum Ramphafti, cum duobus aliis. (447.

7 Nidus Avis Indica. ( 448.

8 Nidus Avis Indica, r. varietas. (449.

9 Nidus Avis Indice, f. varietas. (450.

Io Oraria Raye. (I84.

II Cornu f. Dens Pifcis Monoceri (f. Narwal) 6, pedibus \& ultra. (187.

12 Cornu f Dens Pifcis Monoceri. (186.

13 Cornua Dama Cervi. (107.

I4 Cornua Ibicis Capre. (104.

Is Cornua Kevil!ce. (I I s.

I6 Cornua Grimmic. (106.

17 Cornua Cervi Ramus. (97.

I8 Cornu Rhinecerotis bicornii. (Ior.

I9 Cornu Rhinocerotis. ( 96.

20 Cornua Bovis Mufci. (I12.

iI Caput suis Babyruffe. (109.

22 Caput Sisis Babyruffae. (110.

23 Ca ut Suis Babyrul/ae. (III.

24 Dentes Amphibii Hippopothami. (I08.

25 Ofra \& Auditus Hippopothami. (113.

26 Ofra \& Auditus Balana. (I'4.

27 Foetos Humani Americani ficcatus. (105. 


\section{Parties des Ofeaux Ẽc.}

- Bec d'un Taucan. (446.

6 Bec d'un Taucan Eo deux autres. (447.

7 Nid d'un Oifeau des I'des. ( 448.

8 Nid' d'un Orfear des Indes. (449.

9 Nid d'un Oifeau des Indes, ou varieté. (450.

Io Oeufs d'une Raye. (184.

II La Corne ou dent d'un Monocere de 6 pieds, $\sigma^{\circ}$ plus. (186.

I 2 La Corne ou dent du neême ( 187 .

13 Les Cornes d'un Cerf $d^{3}$ Ame. (107.

I 4 Les Cornes d'un Chevrette. (104, is

I5 Les Cornes d'an Cheville. (105.

16 Les Comes a"un Grimmean Taurreau. (106."

17 Les Cornes d'un Cerf branché. ( 97.

38 Les Cornes $d$ inn Rhenocere à deax cornes, (xos:

I9 Les Cornes d'un Rhenocere. ( 96.

20 Les Cornes d'un Bœuf Mufchi. ( 112.

21 La tểse d'un Sanglier Babyruffe. (rog.

22 La tête d'un même. ( $x 10$.

23 La tête d'un même. (IIr.

24 Les dens de l'Amphilie du Cheval de Rivtere ou Hippotame: ( 108

25 Les Offemens de l'ouz̃ de l'Hippotame. (II3.

26 Les Offemens de l'oriz du Baleine. (II 4 .

27 Le Fotus bamain Jeché d'un Americain. (IIs.

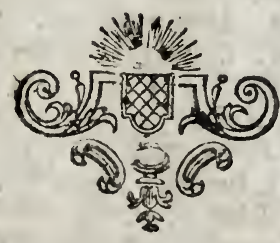




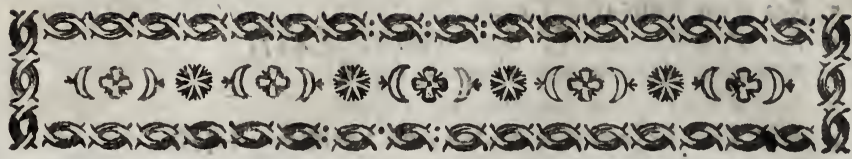

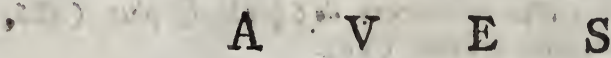

AC NONIVLLA

\section{$M$ A $M$ M A I I A.}

Que omnes in naturali earam pofitione, artificiofa manu farite, cumque Speciebus differentibus, ex yariis Generibus conjunzte, fub Campanis nitidis, a $N^{*}$. I. ad Ir 4. vel in Ciftis vitro teitis, ac vivis colomibers, Jecurdum habitationis earum Regionem pitas, a $N^{2}$. 115 . ad 157 confervantur: quare ece mullo modo in connexione fubfequentium Generum defcribi potuerunt, fed abfolute Secundum Campararun E Ciftarum numeros, qui omnes poft quemque objectorum numcrum adscripti, annotand des fwrnt.

I Dicus Lineatus f. Criftatus, corpore atro, crifta rubra, Linn. 174. 4 (Campana I.).

2 Picu Ferrugineus, capite nigro, albo punctato, Linn $\mathrm{O}$. (I.

3 Mótacilia Rubretra Linn. 332. I6. (2.

4 Motacilla Rubretra . I. femina, Linn. 1. c. (2.

5 Taragra Atrata, Linn. 319. 9. (2.)

6 Taragra Militaris, Linn 3 36. 17. (2.

7 Merops Apiafter, Linn. 182. 1. (3.

8 Merops Apiafer, f. femina, Limn. 1. c. (3.

9 Merops Apicfer, Linn. 1. c. (3.

Io Merops Apiafter, nondum adultus, Limmo 1. c. (4.

II Merops Ater, fubtus albidus, Linn. o. (4.

I2 Merops Ater, f. fermina, Linn. O. (4.

13 Upupa Promerops, Linn. I84. 2. (5.

14 Upupa Promerops, Linn 1. c. (5.

15 Fringitia Crythrophthalma, f. Banana, dorfo caerulefcen'i, humeris albido violaceis, alis caudaque cyaneis, Linn. 3:8. 6. is

I6 Fringulld Crytrophthaima, f. Banana, foemina, Linn. !. c. ( 6 .

17 Ralus 


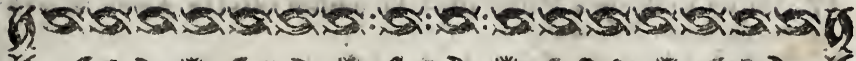

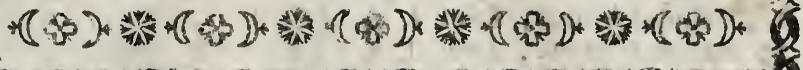 H.}

\section{O I S E A U X}

ET QUELQUES

\section{A M M E L L E S.}

Lesquels tous daris leur pofition naturelle, remplis (ouforcies) par une main tres habile, Ẽ groupé enfemble avec des Efpécès differentes de pluffeturs Genres, fe repofent, ou fous des Clocbes d'un verre pure, de $N^{0}$. 1. jufquia $N^{8}$. 114: ou dans des Caiffes, couvertes de vitres, ED peintes par derrière avec des couleurs des plus yives felon la Region de leur habitation de N॰. Ir4. jufqu'à 157: Ainfi qu'on ne les a tut d'écrire dans une con nexion de Gentes qui fe fuivent, mais qu'on s'eft trouvé obligé de bes annoter felon les nombres des Cloches $\&^{2}$ Caifes qui fe trousent immédiatement après chaque nombre des objets.

$\sim$

I Pe de Cayenne, rayée, Buff, tab. 596. (ClocheI.

2 Pie Rouillé - pointé de blanc de tête noire. ( 1 .

3 Hocbe-queue rouge, ou Tarfier, Buff, $t a b .678 .2$. (2.

4 Hocbe-queue rouge ou Tarfier femelle. (2.

5 Tangara Negre, Buff. I 74. 3. (2.

6 Tangara Cardinal, Edw. 82. (2.

7 Guepier Commun, Buff. 938. (3.

8 Guepier Commun, ou femelle. (3.

9 Guepier Commun. ( 3.

10 Guepier Commun, encore jeure (4.

II Guepier Noir, blanc ens deffous. (4.

32 Guepier Noir, femelle. (4.

: 3 Houpe, Prowérops, Briff. 2. tab 43. 2. (5.)

14 Houpe, Promérops ( 5.

15 Pircon Banane d dos bleuatre, ailes $E^{2}$ queue bleues. (5: 16 Pinfon Banane femelle. ( 6 .

$c_{4}$

17 Rale 
17 Rallus Cinereus, palearibus criftaque fufcis, Linn. 'O. Nas turforfcher XI. tab. 2. ( 6.

I8 Rallus Cinereus, Linn. 1. c. ( 6.

Ig Rallus Cinereus, Linn. 1. c. (6.

20 Manucodia Sexpernis, Linn O. (7.

21 Alcedo Criftata, Linn. 178. I. (8.

22 A'cedn Viridis. longuicaudæ, Linm. O. (8.

23 Alcedo superciliofa, cauda longa, gula alba, Linn. I79 6:.(8.

24 Alcedo Cuprea, collari antice albo, Linn. O. (8.

25 Alcedo Cupres an femina præcedentis? Linn. O. (8.

26 Trochilus Pella; Linn. 189. 2. (8.

27 Trochilus Pella, Linn. 1. c. ( 9.

28 Certhía Famofa, Lints 187.20. (20.

29 Certhia Famofa r. varietas, Linn. I. c. (9.

30 Loxia Colius Linn. 301. 12. (10.

31 Oriolus Hemorrhous, niger \& dorfo infimo cinnabarino; Linn 6r. 6. (I0.

3: Fringilla Cineren, a is rectricibus alarum rutis, Linn. $0 .(10$.

33 Alcedo Ifpida, cerrulea, Limn. I79. $3^{\circ}$ (Ix.

34 Alcedo Supercilinga, fubtus alba, fafciis viridibus Linn. 179. 6. (in.

35 Alcedo Superciliofa, Lins 1. c. (Ir.

30 Alcedo Viridis, alboque varia, Linn. O. (Ir.

37 Alcedo Cyaneus, linea fulva, corpore cyaneo, Linn. O. (Ir.

38 Alcedo Smyrnenfis, viridis, fubtus fulva, Limn. 18I. "II. (Ir.

39 Alcedo Rudis Linn. 181. I2 (I1.

40 Cuculus Criftatus, grifeus, fubtus albue, Linn. O: (I2.

4l Mufcicapa Surinama, corpore grifeo \& vertice aureo, Linn. 325.5 (13.

42 Múcicapa Pracan , pectore \& gula incanis, alis caudaque nigris, macula alba alarum transvería, Limn. O. (I 2 .

43 Lux!a Nigra, macula alarum doffique tlavis, Linn. 0. (12.

44 Picus Lineatus, f. varietos, Limis. 174. 4. (12.

45 Lanius Fuivus, remigitus fubtus nigris, Linn. O. (I3.

46 Pfittacus Accipilrinus, Linn 148. 38. (I4.

4) Pfittacus Garrulus, Linn. 144. 25 (15.

48 ffiticus Lory, Linn. $45.270^{\circ}$ (16.

49 Pfittacus Coccineus Lory, remigibus apice cœruleus Linn. O $\quad 17$.

50 Pfictacus Coeruleus, vertice coeruleo, dorfo \& corpore viridi, colore pallido viridi, fubtus azureo rubro ftriato, Linn. O (18.

5. Pfictacus Domicella, fo mas Garruli, colore flavo, Limno 145. 20. ( 19. 


\section{Diffearix Ẽc.}

17. Rale Cendré, àcrete Ẽ pendants bruns, Natuuxfor fcher $X I$. tab. 2. ( 6 .

18 Rale Cendré. ( 6.

19 Rale Cendré. ( 6 .

20 Sixfilet, o» Oifeau de Paradis, Buff..... (7..

2x Martin Pecheur Huré, B:If. 65:

22 Martin Pecheur Verd, à longue queue. (8.

23 Martin Pecheur, à Soufcils verd dorè, d̀ queue longue $\mathcal{E}^{\circ}$ gorge blanche, Edw. 245. (8.

24 Martin Pecheur Bronzé, d collier blanc. ( 8.

25 Martin Fecheur Brorzé, ou femelle du précedent. n.24. (8. 26 Oifear Colibri Topaze, Buff. 599. ( 9.

27 Oifear Colibri Topaze. (9.

28 Grimpereau Fameux de longue queue, le corps verd brillant. Buff. $83=1$. ( 9.

29 Grimpereau Fameux, ou varieté a long queue. (9.)

30 Gros-bec Coliou, Buff. 282. (10.

. I Carange Hemeroide, noir defjous ronige, Buff. (Io.

32 Pinçon Cendré, da ailes Eे épaules roulfes. (IO.

33 Martin Pecheur Bleu, d bande roulfe. (II.

34 Martin Pecheur d̀ Sourfils, verd blanc end defJous, d̀ bande verte. Edw. 245. (Ir.

35 Martin Pecheur à Sourfils verd. (II.

35 Martin Pecheur Verd É blanc, Buff 59!. (Ir.

37 Martin Pecheur Bleuatre noir, fauve en defous, Buff. 216. ( 37.

S8 Martin Pecheur de Smirne, verd, en defJous jaune-roux." (ir.

39 Martin Peckeur, ou varié du mềne. (I I.

40 Coucou Hupś, gris, à ventre blanc. ( 12 .

41 Gobemouche de-Surinam, le corps gris de/jus d'oré. (12.

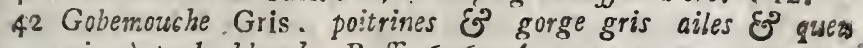
noir. à tache blanche. Buff. 676 (12.

43 Gros-bec Noir, à taches, des ailes jaunes. ( I 2.

44 La Pié Rayée, ou varieté, Buff. 13. (6r2.

45 Pie-Griechs Roux jaune, ì ailes noires èn defous." (13.

46 Perroquet Einaille. Buff 626 . (14. 47 Perroquet Lory des Molucces, Daubeton IIIg. 84.

48 Perroquet Lory, Buff. 368 . ( 16.

49 Perroquet Lory, rouge dे ailes. bleu au bout. (I7)

50 Perroquet ds $S$ mmet b!eu, dos verd, en defJous azur. Strie de rouge. $(-8$.

5 I Perroguet Dancifeau, mále àu Lory des Molucces, Baff. 84. (19. 
ta Trogon Brafilienfis, capite nigto, gula fufca, abdomine fanguineo, dorfs fulvo, remigiun marginis atris, lateralibus albis, Linn o. (20.

53 Trogon Ater. abdomine albo, rectricibus lateralibus albls nigro ftriatis, Linn. 167.3 .3$. ( 21 .

54 Oriolus Cayanenfis, Limn. 163. is (22.

55 Trogan Coeruleus, capite \& pectore coeruleo, alis nigris, ut et rectrices medii lateralibus nigris, extremi albis Linn. O. ( 23.

- 56 Trogon Viridis, aureus fubtus albus, Linn. 167.3. (24.

57 Lanius Ablis, capite fupernus, remigibus rectricibusque nigris, Limm. O. (25.

58 Lanius Cineretus, maculis nigris, cauda alifque atris, Lim o. $(25$.

59 Lanius Curviroftris, ftriis collaribus \& fafciarum albis, fronte olivaceo, fubtus albus Linin. 135. 8. (25.

co Columba Grifea, fafciis maculis fufcis, Linn. 0. ( 26.

61 Columba Minuta, máculis chalybeis, Linn. 285. 35. ( 26.

62 Columba Cinerea, albo cinefefcens corpore, fronte, gula \& pectore atris remigibus nigris, Linn. o. (26.

63 Certhia Spiza, Limi. J86. I2. (27.

64 Certhia Spiza, f. femina, vel varietds. (27.)

65 Certhia Chalybes, f. varietas, iemigibus reetricibusque fuscis, Linm. 186. 10. (27.

66 Certhia Purpurea, capite cupreo, Linn. O. (27.

67 Manucodia Aurea, C. Oriolus Aureus, Linn. 163. Ig? ( 28.

18 Ampelis Mayñarta, Limn. 298. 5. (29.

69 Ampelis Cotingh, Letrin. 298. 4. (29.

70 Ampelis Pompadora. Lim. 398. 2. ( 30.

ir Oriolus Guienenfis, corpore nigro, pectore \& humeris coccineis, Lin\%. 162. 9. ( 30.

72 Paradifea Regia, Limn. I66. 2. ( 31 .

73 Fsingilla Paroara, rigra, reetricibus albo marginatis capite gula que cuccineis, Limm, O. ( 3 t.

74 Fringilla Paroas a, r. femint, Linm. O. (3r.

75. Múcicapa Rubra, Limn. 326. 8. (32.

76 Oriolus Sparius, Linn 162 . II. ( 32 .

77 Loxia Cardinalis, Lim. 300. 5. ( 33 .

78 Oriolus Spurius, Linin. 162. Ir. (33.

79 Fringilla Aurantia, alis caidaque nigris, Limn. o. (34.

80 Pipra Pareola Linn. 339. 2 ( 34.

8I Pipra Leucocilla, teftacess, temporibus fanguineis, Linn. $340.9 . \quad$ ( 34.

82 Motacilia 


\section{-iffeaux Ẽc.}

52 Courarcou de Brefil, à ventre gouge, dos noir cotes blarches. Buff. 452 . (20.

53. Couraucou Noir, à ventre blanc, les rectrices des cotes blansches a firies noires. ( $2 \mathrm{I}$.

54 Carange de Cayenne, Buff. 484. (22.

55. Couraucou Bieu, do têtte bleue Ẽ ailes noires. (23.

56. Coureucou Verd, de ventre bleu defouis blanc, Bur. 452. (24.

57 Pis-Grilche Blanche, à tete, queue Eo ailes noires. (25. 58 Pie-Griéche Cendré, d taches noires \& queue noire. (25.

59 Pie-Griéche Ecorcheur, ou Vanga, à ftries $\mathcal{E}^{2}$ bandes blanche, front olivatre, Buff. 228. ( 25.

60 Pigeon Gris-brun, à longues taches bruns. (26.)

6I Pigeon Brun, tres petit â taches d'acier, Daubeton 243. (25.

62 Pigeon Cersdré, à plaftron noir. ( 26.

63 Grimpereau Guit guit, yerd, ḋ tête noire, Bưfi. 578. (27.

64 Grimpereau Guit-guit, femelle. (27.

65 Grimpereau couleur d'acier, ou varieté da queue hrwre. ( 27.

60 Crimperecu Pourprê, à tête bronzée. (27.

67 Traupiale Dorée, Buff. 79. $0: 6$ carange dort. (28.

68 Cotinga de Mainas, Buff. 12). ( 29.

69 Coting $a$ Cordon bleu, Buff. 188. (29.

70 Coting a Paupac, (Pompadore) Daubeton f. Buff. 21 ? ( $300^{\circ}$

7I Carange de Guienne, noir-rouge on diefjous, Buff. $53 \%$ (30.

72 Manucode Roïal, Birf. 499. (3r.

73 Pingon Paroare, noir à téte $5^{\circ}$ gorge rouge. Buff. 55.2. (31.

74 Pinçon Paroare, orl femelle. ( $3 \mathrm{~T}$.

75 Goberwouche Rouge, Edw. 239. 2. (32..

76 Baltimore Batard, Buff. 506. I. ( 32.

77 Grosbec ou Carcinal Huré. (33..

78 Balimore Batard, Buff 506. ( 33.

79. Pimgon Orange, \& ailes \& queue noire, Buff, 156. I. (34.

30. Manakin Noir, do dos bleu Ẽ crête rouge. Edw. $26 \mathrm{r}$. Daubiton. $68 \%$ 2. ( 34.

8 I Manakin à tête blanche, Brifr. 4, tub. 35.2. ( 34 . 
- 82 Motacilla Nigra, maculis pennular um cauda $\&$ fecunda rum alæ albis, Litin. O. ( 34 .

83 Oriolus Capenfis, olivaceus, fubtus flavus, f. femina Limn. 163. 18 . 35.

84 Picus Undatus, Linn. 175. I1, ( 35.

85 Oriolus Melanoceplaalus, Linn. J60. 3. ( 36.

86 Fringilla Banana, virefcens, tectribus alarum opalinis, Linn. o. ( 37.

87 Fringilla Nigra, capite viridi, corpore nigro, fafcia a arum albida, Linn. $\bigcirc$. ( 37.

88 Fringilla Nigra, f. femina, Linn. O. ( 37.

89 Todus Niger, fubtus albus, Linn. Q. ( 38.

90 Todus Fuscus, maculis aiarum albis, Linn. ०. ( 38 ,

91 Tndus Fuscus, 1. femina, cinerefcens, maculis, albis nigro purctatis, Linn. o. ( 38 .

92 Todus Niger, fubtus flavus, marginibus pennarum rectricibus, extremibus albis, Linn o." ( 38

93 Motacilla Varia, cauda bifida, Linn."333. 23. ( 38.

94 Motacilla, r. cjusdem Nidus. ( 38.

95 Pipra Aureola, capite coccinea, Linn. 339. 7. (39.

96 Pipra Aureola, Linn.. 1. C. (39.

97 Pipra Aurcola, r. varietas. ( 39.

98 Pipra Nigra, capice pectoreque flavo, Linn. o. (39.

99 Pipra Rubra, $f_{0}$ varietas pracedentium, Linn. 0. (39.

Ico Loxia Sulphurata, fusco olivacea, fubtus ferruginea, Linn. 305. 30. ( 40.

ror Loxia Olivacea, fufca, fubtus brunnea, Limn. O. (40.) 102 Loxia Olivaces, fulva, fubtus aiba, collari nigro, Limn. O. $(40$.

I03 Loxia Olivacea, gula fulva, an Canedenfis? in Nido, Linn. 304. 29 ? (40

104 Oriolus Bananz, capite \& colla caftanea, dorfo reEtricibusque nigris, Linn. 16r. 4 ? ( 4I.

Ies Oriolus Banana, Lim I. c ( 4 .

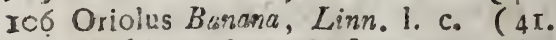

107 Mufcicapa Senegalenfis; atra, abdomine fulva, dorio infim) albo. Linn. 327. I 5 . (43.

I08 Mifcicapa Paradifi, fulva, Linn. 24. I. (42.

I09 Fringitita Autumnalis, capite caftaneu, pectore cœruleo, Linn. 320. I5. (43.

I10 Fringilla Auturnalis, f femina, Lirn. 1. c. (43.

III Emberiza Nigra, fascia a'arum alba, Linn. O. ( 43 . I12 Enberiza Nigra, Linn. O. (43.

II3 Emberiza Olwacca, Linn. 309. 6. (43. 


\section{Oifeanx Eैc.}

82 Hoche-queu Noir, dे ailes \& queue blänchice. (54.

83 Baltimore du Cap, olivatre defJous jaune, femelle. ( 35 .

84 Pie Ondée, teftace les côtes de la tête rouge, Edw. Gleani 334. ( 35 .

85 Carange d̀ tête noire, Buff. 79. ( 37.

86 Pingon Banane, vera' dे tectrice, d'aibes blascheatred (37.

87 Pingon Noir, dè tête verte. (37.

88 Pinçon Noir, ou femelle. ( 37.

89 Todier Noir, blane en defJous. ( 38.

90 Todier Brun, d̀ taches bianches, blanc en defous. (38.

9I Todier Brun, ou femella du mêmed tache blanches pointée de noir. ( 38.

92 Tndier, Noir jaune en deffous les bord's dus plumes Eo pointes blanches. ( 38 .

93 Figuier de St. Domingo, de qusue fendu Edw. Glean. 300: ( 38.

94 Lavardine, ou fon Nid. ( 33.

95 Manakin d̀ tête rouge, Buff. 34. 3. (39.

96 Marakin 2 iête rouge. ( 39.

97 Manakin Noir, à tête jaune de citron. (39.

98 Manakin Noir, tête Ẽ poitrine jaune. (39.

99 Manakin Rouge, varieté des preceìess. (3).

100 Gros-bec O'ivatre, en deffors brwn. (40.

ror Gros-bec Olivatre, defJous brun. ( 40.

102 Gros-bes Fauve, de collier noir. (40.

103 Gros-bec Olivatre, a gorge fau»e, dans fon Nid. (40.

$\mathrm{IO}_{4}$ Carange Banane, dète $\mathcal{E}^{\circ}$ colbrun, le dos noir. (4I.

I05 Carange Banane. (4I.

Io6 Carange Banane. ( 41 .

107 Gobemouche de Senegal, noir, deffous brum, lo dos bleu, Buff. 567 . ( 42 .

I08 Gobenouche de Paradis, dlongue qusue, Buff. 284. ( 42.

109 Pinçon de Surrinam, dểte trun, poitrine bleu. (43.

iro Pingon de Surinam, ou femelle ( 43 .

II Ortolan Noir, à bande, des ailes blanches. ( 43.

II2 Ortolan Noir. (43.

II3 Ortolan Olive, Brif.J. 3. tab. 23.5. (43.) 
Ir4 Pfittacus Azureus, capite viridi, dorfo alisque nigfis; fascia alarum flavą, azureo \& cauda violacea nigra maculata, Linn. O. (44.

Ir5 Pfiteacus Viridis, fronte cæruleo, dorfo interro \& pectore rubris, Linn. O. (44.

II6 Pipra Rubicolla, criftzt , aurantia, pu'cerrimum exemplar, Buffonni, Ecisvartii diverfum, Linn. 338. , Naturforfcher $X I . t=b$. I. (45.

II Pfittacus Chryfoptetus, virídis maculis aurantiis, Linn: 149. 43. ( 46 .

I18 Pfittacus Viridis, tectricibus alarum azureis, Linn. o. $(46$.

Iró Pipra Leucocephala, Lirn. 340. 8. . (47.

120 Pipra Lercocephala, Limo. 1. c. 147.

I2 I Pipra Gutturalts, nigra, Linn 340. 10 . (47.

I22 Fringilla Nigra, alis albis ftriatis, fubtus alba, Linn。 o. ) 47 .

123 Fringilla Tricolor, viridis, pectore punctis nigris, fubtus albz, Linn. 323. 34 . ( 47.

I24 Turdus Virinis, humeris criftaque cyaneis, loris nigris, Linn. O. ( 48.

125 Turdus Viridis, Lim. O. (48.

I26 Fringilla Melba, fuperne ferruginea, inferne maculis nigro albis, Linn. 319. 8: (49.

I27 Emberiza Butyracea, ferruginea, capite colloque atris, Littn. 304. 28. (49.

128 Loxia Aftrild, pectore coccineo, Linn. 303. 2r. (49.

129 Loxia Senegalenfis, punctulata, Linn. O. ( 49.

130 Emberiza Senegalenfis, f. varietas, collari albo, Linn. 0. (49.

$1_{2}$ Pringilla, Sanguiniroftris, capite nigro cinereo, genis aurantiis, roftro rubro, Linn 303. 20. ( 50 .

I32 Loxia Sanguiniroftris, f. varictas, Linn. 303.20. (50.

133 Fringilla Amandava, fufca alto punctata, Linn. 319. Io (50.

x34 Fringilla Amandava, f. femina, Lirn. 1. c.. (50.

135 Frirgilla Amondaya, ro varietas, pectore luteo, Limin. 1. c. $(50$.

I36 Fringillae Amcrdava, f. femina, Limm. 1. c. (50.

I37 Fringilla Crythrophthalma, macula alaruin alba, Linn. 3 r 8 : 6 ( 50.

I28 Fringila Crythropinthalma, Limn. I. c. (50.

I39 Picus Bengalenfis, f femina, dorio purpureo fulgedo; pectre ex nigro \& albo variegato, Linn. 175 . 13 ? (51. Iso Cuculus Honoratis, "fafciatus, Limb 169.7. (152. 


\section{Oideaux E̊c.}

114 Perroquet Azuré, is tete verte, dos EO ailes noires, avec sinu bande jaune Jur les aiies. (44.

115 Perroquet Verd, a front bleu, 8 poitrine rouge. (44.

I 6 Manakin ou Cocn de la Roche à crete., couleur d"orange; très belle piece differente de celle de Mr. Edw. Bufton, \&e. Naturforfcher, XI. tab. I. (45.

117 Perroquet à ailes dorées, di baches oranges. (46.

I18 Perroquet Verd, \&paudes dorées. (46.

It) Manakin à tête blanche , Buff. 34. 2. (47.

120 Manakin dà tête dorés. (47.

121 Manakin Noir, à gorge blanche, Buff. 324. I. (4\%

I22 Pingon Noir, à ailes firiées de blanc. (47.

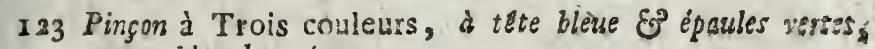
ventre blanche. ( 47.

124 Crive Verte, à croupion È épanies bleues. (48.

125 Grive Verte. ( 48.

126 Pirgon Melba, verd di taches noires, d̀ tête rougre. Edä: 128. ( 49.

127 Ortolan couleur de Rouille, à tête É col roire. (49.

128 Gros-bsc Senegale, rouge la poitrine rouge, Buff. 157 , 2a (49.

129 Gros-bec Senegale, pointillte. (49.

130 Ortolan Senegale, pointillé varieté, à collier blanic. ( 490

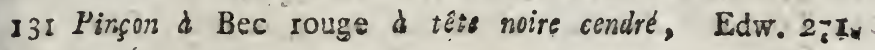
2. $(50$.

132 Gros-bec Rouge. (50.

I33 Pinçon Bengale, brunse piqqusttí de.blanc, Buff. II5. 2; ( 50.

134 Pinçón Bangale, ou feimelle. (50.

35 Pingon Amandave; ou varieté d ventre jaune. (50.

136 Pinģon Amandave, ou femelle. ( 50.

I37 Pinģon Brunet, les ailer à taches blanches. (50.

138 Pingon Brunct. ( 50.

I30 Pie de Benale, ou femelle, dd dos pourpré, la poitrine, E⿱ gorge varie de blanc, Eै noir. ( 51 .

140 Coucou tachété de Malabar, Natuurforicher. Buff. 294. (52. 
I\$I Cuculus Honoratus, corpore nigricante, Linn. 1. c. (\$2.

I42 Cuculus Cinereus, nigricans, maculis atrís, Limn. ०. ( 52.

143 Motacilla Aurocapilla, in Nido. ( 53 .

I44 Motacilla iurocapilla, fo mas, Linn. 334. 29. ( 53.

I 45 Motacilla Petechta, viridi olivaces, loris nigris, Linn.

334. 30. $(54$.

I46 Motacilla Maderaspatane, fubtus grifeus, Limn. 33.4. 28. ( 54 .

147 Motacilla Aurocafilla, olivacea, Linn. 334. 29. ( 54.

I48 Motacilla Carolinenjis, an Fringil a? capite fufco, Linn. 318. 18. ( 54 .

I49 Motacilla Perechia olivacea, Linn. 334. 30. ( 54.

150 Muscicapa Cayanen/ts, virers, Limn。 327. 11? ( 55.

151 Muscicapa Cayanensis, vireris, Linn. 1. c. ( 55.

152 Mufcicapa Cayanemass, fo femina, Linn 1. c. ( 55.

I 53 Mufcicapa Olivacea, fufco virens, Lisn. 327 . I4. (55.

154 Motacilla Troglodytes, gुrifea, Linn. 337. 46. ( 56.

155 Certhia Pufilia fuperne fufca, Linn. 185. 3. ( 56.

156 Certhia Carulea, cum Nido, Linn. 185. 3. ( 56 .

I57 Certhia Carrucaria , olivacea, fubtus chalybea, laterum ma cula, flava, Linn. 185. б. ( 50.

I58 Trochilus Mofquitus, viridi aureus, Linn. 192.14. (57.

I59 Trochilus Mellifugus, Linn. 92.15. ( 57 .

I60 Trochilus Mellijugus, Linn. 1. c. (57.

I6I Trochilus Tomineis, furcus, Linn. 191. 12. ( 57.

162 Trochilus Pegajus, corpore virefcente aureo, Linn. 192. $16 .(57$.

163 Trochilus Topaziguta, Linn. O. ( 58.

167 Trochilus Colidris, Linn. I9r. I2. ( 58.

Ip5. Trochilus Auratus, viridi-aureus, gula rufa, Linn. 0. ( 58.

I66 Trochilus Auratus, Limn. O. (58.

I67 Certhia Familiaris, cinerea, Linn. I84. I. ( 58.

I68 Trochilus Mango, abdomine atro, Lim. 191. 10. ( 59.

169 Trochilus Colubris, viridis, cauda atra, Linn. I9I, 12.

I 70 Trochilus Colubris, Limn. 1. c. (59.

17 I Trochilus Colubris, in Nido, Linn. 1. c. (59.

172 Truchilus Smaragdinus, viridis, pectore \& collo cosruleis, Linn. O. ( 59 .

173 Trochilus Holofericeus, fafcia pectorali coerulea, Linn. Igr II. $C 60$.

174 Trochilus Holojericeus, albo guttatus, Linn. I. c. ( 60 .

175 Trochilus Varius, viridi, remigibus fulcis, rectricious rufis apice, Linn. 0 . ( 60.

I76 Trochilus Varius, Lim. 1. ce $(60$ 
i4t Coucou Tachete, le corps noiratre. ( 52 :

142 Coucou gris Cendré, a taches noires. ( 52.

${ }_{4} 3$ Figuier à Tête dorée, dans fon Nid Edw. 252。 (53v

144 Figuier à Tête dorée, male. ( 53 .

45 Figuier d Tête rouge, vert olivatre, dl liens noirs. Edw. Glean. 256. 2. ( 54 .

146. Figuier Cheri, deJous gris, Brifr. III. 28. 2. (54.

147 Fiquier à Tête dorée obivatre. (54.

148 Fauvette à Téte noire, ou Pinfon de Caroline. (54.

$x_{49}$ Figuier à Tête rouge, olivatre, Edw. Glean. 256. 2. (54.

150 Gobemouche de Cayenne, verdatre. Buff. 574. 2. (55.

I5I Gobemouche de Cayenne, verdatre. Buff. l.c. (55.

I52 Gobemouche de Cayenne, verdatre, ou fentelle. ( 55.

I53 Gobemouche de Cayenne, olivatre, brun.vert, Buff. 454. ( 55.

154 Hoche-queue, or le Roitelet, gris, Abbin Cit. I. tab. 53. ( 56.

155 Petit Grimpereau, defus brun, Edw. 26. ( 56.

I56 Crimpereau Bleu, dans fon Nid, Edw. 2I. (56.

I57 Grimpereau des Philippines, olivatre, dedous couleur' d'acier, d large tache joune, Buff. 576. 2. ( 50 .

I58, Colibri Rubis topaze, Buff. 22\%. 2. (57.

I59 Colibri Succe-miel, vert dort, Buff. 276. 3. ( 57.

160 Colitri Succe-miel. ( 57 .

16r Colibri D mine, rubis brun, Edw. 138. (57.

102 Colibri Pegare, gris en defjous, Brill. III. 36. 4s ( 57.

163 Colibri Topaze. ( 58 .

104 Colibri de Caroline, 1 ubis. ( 38.

165 Colibri Doré, a gorge rouge. ( 58.

160 Colibri Doré, ou varieté. ( 58.

I67 Grimpereau Familiere, cendrt. ( 58.

I08 Colibri Cravatte doré, d yentre noir, Buff. 672. 2. ( 590

169 Colibri Verd, d queke noire, Catesb. tab. 65. ( 59.

170 Colitri Verd. ( 59

I 7 i Colibri Verd, dans fon Nid. ( 59.

I $7^{2}$ Colibri Emeraude. cus Amethifte? Edw. 35. ( 59.

I 73 Colibri Verd, de ventre roir; la poitgine d bande bieso. Buff. 680.1. ( 60 .

174 Colibri Verd, a ventre noir. 160.

i 75 Colibri Verd, ou varie, a rearices rouges. $(60$. I 76 Colibri Verd. (60. 
177 Todus Fufcus, albo gutcatus, crila transverfa rubra nigrn marginata, Linn o. (61.

178 Bucco Viridis, fronte rubra, gula aurantia, Linn. o: (6:.

179 Trochilus Cupreus, collo rufo, collari nigro, Linn. o. (62:

180 Trochilus Cusreus, f, varietas, Limn o: (6?.

181 Trochilus Criftatus? pennis lateralibus colli ereetis, Linm. O. 102'。

182 Trochilus Cupreus, collari albo, Linn, o. C 62.

183. Trochilus Cupreus, reetricibus lateralibus longioribus.. apice tantum pennatis, raristimus, Linn. o. (62.

184 Trochilus Cupreus, f. femina, Linn. 0 . ( 62.

185 Trochilus, Mellivora? gula rubra, Linn. o. ( 63.

186 Trochilus Smaragdinis, Linin. O. (63.

187. Trochilus Smaragdinus, in Nido, Linn. 0... ( 63.

388 Trochilus Viridis, abdomine albo, Linn. 0 . ( 63 .

189 Trochi us Viridis, Linn; 0 . ( 63 .

190 Trochilus Viridis, L varietas, rectricibus lateralibus apice, Linn. o. $(63$.

391 Trochilus Dominicus, viridis, fubtus grifeus, rectricibus acutis, albo marginatis, intermediis longisfimis, Linn. 191. 9. (64.

192 Trochilus Viridis, collo azureo, Linn. O. ( 64 :

193 Trochilus Viridis, fubtus cinereus, rectricibus albo dimidiatis, Linn. 0. $(64$.

194 Certhia Currucaria, olivacea, Linn. 185. 6. ( 65.

105 Cerbia Currurcaria, f. femina, Linn. l. c. (65.

196. Certhia Pinus, purpureo nitens, abdomine flavo, Limm. 187. 16. ( 65

197 Trochilus Viridis, gula rufa, rectricibus. intermedis. albis. Linn. O. C 65 .

198 Trochilus Viridis, f. varietas, Linn. 0. $(65$,

399 Trochilus Virsdis. f. femina, in Nido, Linn. o, $(65$.

200 Certhia Afra, viridis, peetore rubro, Linn: 186. 11 . (66.

201 Certhia Spiza, violacea, Linn, 186. x2. (66.

202, Certhia Spiza, f. femina, Linn. 1. c.

203 Certhia Afro, Linn. 186, Ix. (66.

204 Certhia Afra, Linn. I. c. (66.

205 Hirundo Purpurea? Chinenfis, alis caudaque nigris, corpore chalybeo, fubtus albo, peetore maculato. Linn. 344. $5 \%(67$.

206 H ruado Nigra Indica collari albo, cumNido, Linn.0. (67. 207 Certbia Cerubs, Linn, 185. 8. (68, 


\section{Oiffeaux Eัc:}

177 Todie Brun, goutte de blanc, \&hape transyerfe rouge, Naturforfch. XVII t. Cor.

278 Barbu Verd, \& front rouge, 89 gorge orangke. ( $6 \mathrm{r}$.

179 Colibri Bronze. a cous roux, EO cullier noir. ( $\sigma_{2}$.

180 Colibri Bronze, ou variets. ( 62.

18. Colibri Crete, $\partial$ plumes du cou, allongles en crlte. (62:-

182 Colibri Bronze, ou Jacobine, d cou blanc. Buff. 6jo. ( 62.

183 Colibri Bronze, d plumes laterales de la queue, barbues bers pointe, trts-rare. $(02$.

184 Coibri Bronze ou femelle. ( 62 .

is 5 Colibri Rubis, dineraude, Buff. 276.2. ( $\sigma_{3}$.

${ }_{186}$ Colibri Emeraude, Edw. 38.83 . ( 63 .

187 Colibri Smaragdıne, dans fon Nid. (63.)

188 Colibri Verd d ventre blanc. Eidw. 35. 1. ( 63 .

189 Colibri Verd. $\left(\sigma_{3}\right.$.

rgo Colibri Verd, ou varieté, d redrices laterales. $(63$.

Ior Colibri de St. Domingo, verd, deffous gris reatrices aigués d deux bords blancs, trés long. Buff. 680. (64.

192 Colibri Verd, a collier d'azur. (64.

193 Colibri Verd, defjous cendre, d reatices de moitie blanchas. BrifT. III. 36. 4. C 64 .

194: Crimperears Philippine, jarne, d dos olivatre, les giles blewes, d deux bandes blanches, Edw. Glean. ( 6 j.

1.95 Grimpereau Philippine, ou femelle, Daubiton. 276.2. (6s.

196 Grimpereau Pourpré,' dentre jaune. (65.

I97 Colibri Verd, d gorge rouge, la queue au milieu blanche, (65.

198 Colibri Verd, ou variete. (65.

199 Colibri Verd, ou femelle, Africaine dans fon Nid. C $66 . ?$ 200 Grimpereau Verd, d poitrine rouge, E' croupion bleu, Butfo. 246. 3. Edw. 81. ( 60 .

20 r Grimperears de Brafile, bleu. Buff. 246 1: (66. 202 Grimpereau de Brafile, ou femelle. ( 66.

203 Grimpereu Noir, d col, dos 89 croupion rouges, Edw. 8x. ( 60.

204 Grimpereas Noir. (66.

205 Hirondelle Pourpree de la Chine, les ailes, E quew noire, le corps couleur à acier, deflous blanc, d poitrine tachetde, Buff. 546. 2. $\quad 67$.

206 Hirondelle Noire, des Indes, d collier blanc, avec fon Nid. (67. 207 Grimperear Bleu, Edw. 2I. (68. 
208 Certhia Cerulea, . mas, Linn. 1. c. (68.

xon Ceithia Viridis, ubiique, Linn. o. 168.

210 Certhia Celcfina, viridis, fubtus flava, pectore viridi, Linn. 0 . (68.

21 I Cuculis Ceractus, nigro undulatus, fubtus albus, Linn. o. $(69$.

212 Cuculás Cinereus, longuicaudus, capite nigro, alis caudaque fuscis, $r \in$ Ctricibus lateralibus longisfimis, fubtus albus, Linn. O. (70.

283 Loxia Atra, corpore gulaque suxantiis, pectore \& abdomine atris, alis caudaque grifeis, Zirm. O. ( 70.

214 Tanagra Brafilicnfis? cyaneaque atra, fronte, peêore, gula azureis himeris, fubtus alba, Lirm. 316. 15? (70.

215 Tanagra Brafslienfis? f. varietes, Linn. I. c. (70.

216 Pfittacus Pullarius. Linn. 149. 45. (7\%.

217 PGttacus Pullariwa, f. ysrietas, cauda viridi, Linn. l. c. (71.

118 Pfitacus Viridis, crifto rubro, Linn. O: (78.

219 Pfittacus Viridis, fronte flavo: Linn. 0. ( 71 .

220 Turdus Ater, an Oriolus Xanthornus? corpore nigro, alis, pettore \& abdomine albo guttatis, Linn. O. (72.

221 Fringilla Sinica? viridi-olivacea, Linn. 321. 20 ? ( 72 . 222 Emberiza Olivacea, fubtus dava, alis caudaque fuscis, apice albo. Linn. O. ( 72 .

223 Emberiza Olivacea, Linn. O. ( 72.

224 Loxia Longicauda, atra, dorfo \& abdomine albo, Linn 0. $(73$.

225 Loxia Nigra, humeris dorfogane favis, Linn. O. (73.

226 Certhia Cyares, Linn. 188. 24. ( 74.

227 Cerchia Familiaris, Linn. 184. 1. (24.

228 Merops irsfia, viridis, capice nigro, maxilla inferiori alba, Linn. c. ( 74.

229. Merops Prafia, f varictas, Linn. O. ( 74.

230 Frisgilla Butvracea? Lins 321. 22 ? ( 75.

331 Fringilla Berylina, viridis, dorfo caudague nigro, Linn 0. $(75$.

232 Fringilla Beryllina, f. femina, an Tanagre Brafilienfis? Linn. $0 . \quad(\div 5$ :

233. Tanagra Brefiliengs., Linn 358. 15. (75.

234 Tal agra Atr 3 humeris hololericis, Jinn. $0 . \quad$ ( 75 .

235 Greculs Qifrula lonsicuda. Limn. 105. 7. C 76

230 Cuculus C yanus, corpore calianco pupurefcente, Linro 170. 4 ( 37

297 Frinilla Cayna? ? Tantgera, Lim. O. R. 316 14. (78

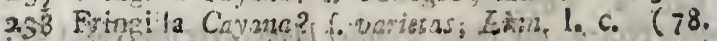

\$39 Fringilla Caychas? Likm, Lo c.

rinis - : 
240 Fringilla Cayana? r. mas, Linn. 1. c. ( 78.

241 Fringilla Maja Linn, 323. 35. ( 79.

242 Frin illa Maja, f. fémina, Linn. I. c. (79.

243 Lanius Curviroftris, niger, teetricibus alarum albo guttatis, abdomine albo, Linn. 135 8. (80.

244 Lanius Cürviroftris albus, r. varietas, Linn. I. c. (80.

245 Oriolus Mexicanus, Linn. 162 13. ( 81 .

246 Oriolus Chryjocephalus, Linn. 164. 20. (81.

247 Loxia Aurantia, capite \& abdumine atris, remigibus fufcis Linn. 0. (82.

248 . Coxia Oryzivora Linn. 302. 14. ( 82.

249 Loxia Oryzivora. f. femina, Linn 1. C. (82.

20 Coxia Ajtrild, Linz. 3c3.21. (82.

251 Motacilla Ficedala? grifeo-virens, campe?tris, Limm. 329. 5.' $(82$.

252 Todus Iatericus, vertice flavo, fubtus albus, Linn. C 183.

253 Parus Fuscus, alarum fascia interrupta, duplici vertice, \& pectore albo Linn. o. (83.

254 Parus Olivaceus, fufcus, Atria verticis flava, Linn. O. (83.

255 Pans Viridis, olivaceus fubtus albicans, vertice zureo, Linn o. 183.

256 Muscicapa Fujca cauda longa, Linn o. (83.

257 Orio us Spurius, Linn. 162. 11. (84

753 Larius Albus. fo femina, nigro varlus. Limm. o. (85.

259 Lanius? Niger, $f$ mas, fubtus albus, Linn. C. (85.

260 Lanius? Ater, gula alba, Linn. o. (86.

26. Alcedo Ispida, Afrátic Linm. 163. 16. (86.

262 Alcedo Afratica f Ispida? Linn 1, c. (86.

269 Orist is Jacrocephalus, Limn $163 ; 16 .-187$.

264 Frungisila Chalybea, cauda longa, f. Tanagra, Linn. o. 188.

265 P ocellaria Pelagica, uropygio nigro, Limn. 212. r. (89. 266 O iolus Niger, Isscia vertici \& dorfo iuferiori flavercentibus, uriopygia rubro, Linn. $0^{\circ}(90$

257 Lanius Collaris, fàicia alarum \& reetricibus lateralibus albis Linn 135. 9. " ( 91.

268 Ori lus Bonana, Linn. 162. 12 ' ( 92.

769 O iolus Icterocephalus, Linn. 1. c. ( 23 .

270 Oriolu: Niger, bumeris albis, Linn. O. (93.

¿71 B cco Niger, frotite \& collari aibio, Lipn. O. (94.

272 Loxia Curviroftra, Linn. 299. I. " 95.

273 Loxia Curvirofira, fo femina Linn 1 c. $(95$.

274 Emberiza Viridis, loris fubtus que lavis, Linn. ०. ( 96.

275 Fringilla 


\section{Oiffedut Eेc.}

240 Pinçon Cayenne, ou male. ( 78.

241 Pinçon Maja, pourpré. Buff. 109. 2. (79. 242 Pinçon Maja, ou femelle. (79.

243 Pie-grieche d Bec-courbe, voir, les ailes tachetes it blanc, d ventre blanc, ou Vanga Buff. 228. ( 80.

244. Pie-grieclie d Bec-ccurbé, ou varieté. blanc. (80.)

245 Carange de Mexique, Edw. Glean. tab. 243. Daubeton, 5. 3. . $(8 \mathrm{r}$.

246 Carange Noire, d tete jaurie. Brifr. App. 2. tab. 2 ( $8 x_{\text {. }}$. 247 Grós-bec Orange, la tete Eे verstre noire dorte. Buff. IT. 12. 2. ( 82.

248 Gros-bec de la Chine.

249 Gros-bec de la Chine. ou femelle. (82.'

250 Gros-bec Aftrild, ou Senegale rouge. Buff. 157.2. (82.

251 Fauvette, Gris verd, chompetre, ou de $3 a$ Fameique Edw 1 22. 2. ( 82 .

252 Todier jaunatre, a fommet joune, blanc en defous. ( 83.

253 Mefange Brune, d bandes blanches interrumpus, a fommet E' de tete blanches. (83.

254 Mefarge Verd, olivatre, fommet is frie jaune. ( 83.

255 Mefange Verd, olivatre, blancheatre, o fonimet dore. (83.

256 Gote-mauche Brun, la queue longue. ( 83.

257 Carange Batard, Buff. 5c6. 1. ( 84.

258 Pie-grieche Blanc, varie de noir, ou femelle. (85.

259 Pie-griche?' Noir, deffous blanic, or male. (85.

260 Pie-grie ke? Noir, à gorge Banche. (85.

26I Martivi-pecheur des Indes:' (86:

$2 \sigma_{2}$ Martin-pecheur de l'Afie: (86.)

263 Carange Noir, d tette janne. Buff. 443. (87.

264 Pinçon Couleur d'acier, a longue queuse. (88.

265 Petril, Edw. 90. I. (89.

206 Carange Noire, ¿ Jommet \& dos pofierieür jaune, E crous pion rouge ( 90 .

267 Pie grieche Collier, $d$ corps noir, en defous blanc d bandes, les ailes, EF rebrices laterales blanches. Baff. pl. enil. $477^{\circ}$ i. ( $(9 x$.

268 Carange Bonane, d tête Ẽ poitrine moire dorèe, Britr. II. 12. 2. ( 92.

260 Carange Noir, à tête jauñe: $(93$.

270 Carange ou Traupiale Noire, d epaules blanches. (93:

271 Barbi Noir, a front fo $^{2}$ collier blanc. ( 94.

272 Gros-bec Croire, Buff. 2:8: (95.

273 Gros-bec Croile, ou femelle ( 95.

274 Ortolan Verd, deffous joune, Bciff, 659. 1. (96. 
275 Fringilla Viridis f. Loxiz? Linn. O. ( 96.

276 Mntacilla Troglodytes; undique brunnea, Linn. 337. 46. $(96$.

277 Emberiza Nivalis, Linn. 308. I. ( 96.

278. Látrius Excubitor, Linn. 135. I1. (97.

279 Emberiza Hortulana, Limn. 300. 4. 198.

280 Fringilla Hortulana, 1, Emberiza. Linn. I. c. $(98$.

281 Oriolus Galbula, f. femina, Lim. 160. 1. ( 99.

282 Ampelis Garrulus, Bohemicus. Linn. 297. I. ( 100.

283 Loxia Coccothrauftes, Linn. 299. 2. ( 100.

284 . Parus Biarmicus, vertice cano, cauda corpore longiore, capite barbato, Linn 342. 12. ( Ior.

285 Motacila Rubicola. Linn. 337. 45. (ror.

286 Yunx Torquilla, Linn. 172. 1. ( 102.

287 Yunx Torquilh, Linn. 1. c. (102.

288 Lanius Excubitor, Linn. 135. I1. (rc3.

289 Oriolus Galbula. r. femina, Linn. 160. 1. (104.

290 Eurberiza Helvola, Linn: O. ( 105.

291 Einberiza Helvola . r. varietas, Linn. o. ( 105.

292 Loxi! Pyrrhula, Linn. 3004 (106.

293. Loxia Pyrrhula, Linn. 1. e. (106.

294 1.0xia Pyerhula, f. varietas, Linn. 1. c. (106.

295 Enve:iza Nivalis, C. AE/tiva, Linn. 308. 1. ( 107.

296 Oriolus Xanthurnus, fulcus, vel Galbula? f. mas, Lim. 160. $)$ ? ( 108.

297 Ardea Stellaris, Linn. 239. 21. (Cifta rc9.

298 Pelicanus Carbo, Linn. 216 3. ( 109.

299 Vucur Papa, pulcerrimus, Linn. 122, 3. ( 110.

300 Scolopax Caural? f. Solaris, Limm. O. (111.

301 Crax Alector, Linn 209. !. (III.

302 Pfitcacus Viridis, Limm. O. (III.

303 C. lumba Cineren, gula alba, Limm. 0 . (IIT.

.304 Phafianus Fuscus, nigro maculatus, Linn. O. (IIr.

305 Pfitacas Guineenfis. Linn. O. (I 2.

300 Tetrao Urogallus, r. mas Linn. 273. I. (II2.

307 Tetrao Urogallus, f. femina, Linns. l. c. (I i 2.

308 Tetrao Marilandicus? fuscus, nigro undulatus, an $\mathrm{Cu}$ pido? Linn. 277. 17. ( II2.

309 Tetran Mnrilandicus, f. femina, Linn. 277.5 ? 17. (I 2.

310 Pfitacus Viridis Linn. O. ( $1: 2$ :

311 Falca Nobilis, Linn. O. ( I 12.

312 Vuliur Papa, pulcerimus, Linn. I22. 3. ( 113.

313 Prafiarus Hybridus, Linn o. (II3.

314 Tetrao Frincolinus, Lnn. 275. 10. (Ir3.

3 is Ardea Grifea, maculis nigris, Linn, o (113. 


\section{Oifleaux Eैc.}

275 Pingen Verd, ou Gros-bec. ( 96.

276 Fouvette Troglodyte, brune. ( 96 .

277 Ortolan de Neige, Buff. 497. 1. ( 96.

278. Pie grieche Dos gris. (97.

279 Ortolans Jardinier, Buff. 247. I. (98.

280 Ortolen, ou Pincon Hortalana. (98.

281 Carange Garrule, ou le Lorcot femelle. (99.

282 Carange Garrule, de Boheme. ( 100.

283 Gros-bec Brun, d bande blanche fur les ailes, Edw. 188. (100.

284 Parus Biarmique, le Jommet chauve, EO la queue plas grande que le corps, bo tite barbue. (Iox.

285 Hoche-queue la poitrine couleur de Ronille. 79. (ror.

286 Torcol, Buff. pl. 698. ( 102.

287 Torcol, ou variett. ( 102.

$288 \mathrm{Pis}$ Grieche Cendré. ( 103.

289 Lorcot Jaune, ou fomelle. (104.

290 Ortolan Jaunatre. ( 105.

291 Ortolan Jaunatre. (105.

292 Bouvreuil, Buff. 145. 1. ( 106.

293 Bouvreuil, Buff. 145. 2. (106.

294 Bouvreuil, ou variete. ( 106.

295 Ortolan de Neige, en habit d'étt, Buff. 497. (107. =

296 Lorcot male, ou Xanthorne, Buff: pl. 26. (108.

297 Butor Etoillé, Buff. 189. (Caiffe 109.

$2 \subset 8$ Cormoran, Buff. 927 . (IC9.

299 Roi des Vautours, trés bears. Buff. 428. Edw.1I. tab. 2. ( 110.

300 Caural ou l'Oifearu au Soleil, Buff. (III.

301 Hocco Mltu, Buff, 86. (Irir.

302 Perroquet Munier, Buff. pl. 861. (ixr.

${ }_{30} \mathrm{O}_{3}$ Pigeon Cendré \& gorge blanche. (III.

304 Phaijan Brun, a taches noires. (IIr.

305 Perroquet Moineau de Guinée. (I12.

306 Cocq de Bruyere, ou male, Buff. 173. (II2.

307 Cocq de Bruyere, ou femelle. (II2.

308 Coca de Bruyeie, brun, onde de noir, Buff. 174? (112.

309 Cocq de Bruyere, ou femelle. II2. (122.

310 Perroquet Verd, Daubeton 861. (I12.

3:I Faucon Noble. (II2.

31 2 Roy des Vautours, tres bear. (II3.

$3 \times 3$ Phaifant Coguard. (113.

3 I 4 Perdrix Frar colin. Buff. 147. (ir3.

IIs Héron Gris, d taches moires.

D 5 
316 Cosvus Glandarius, Linn. 156 7. (1:3.

317 Columba Caronata, pulcerrima, Lim. 282. 17. (1) 318 Falco Chryjaétos, fúfcus, ftriis nigris, Linñ. 125. 5. (115. 319 Anas Nigre, Linn. O. (Irb.

320 Tetrao Marilendicus, maculis albis, Limm. 277. xt. (II7.

321 Tetrao Marilandicus, fo femina, Linn, 1. c. (II7),

$\$ 22$ Phafianus NyEhtmeries, r. mas, pulcertimus, Linn. 2 j. 6. (irg.

323 Phafianus Nyahemerus, C. femira, pulcerrima, Linn. 1. c. ( 110.

324 Phafianus Pitus, f. mas, pulcettimus, Linn. 272. 5. (II). 325, Phafianus Piatus, C. femina, pulcerima, Lim. 1. $\varepsilon_{\text {. }}$ ( Irg.

326 Anas Dominica, fulvo-lutea, Speculo alarum alto, Linn. 201. 22. (122.

327 Anas Fusca, collari \& abdomine ajbo, Linn. O. (122. 328 Falco Gentilis, Linn 126. 13 . ( 123

329 Anas Domirica fulvo lutea, Linn 201. 22. (I24.

330 Anas Autumnalis, Linn 205 39. (124.

331 'Hirundo Arus, nigricars, Linn. 344. 6. (124.

332 Anas Capenfis, capite collo, alisque digris, abdomine fulvo, Linn. O. ( 125.

333 Anas Crifta, lutea, alis nigricantibus, Linh. o. ( \pm 26 .

334 Anas Crifta, r. femina, an n8.332. Linn.o. ( $1=6$.

335 Anas Nigra, alis nigris, maculis albis, capice collogue albis, Linn. O. ( 127.

.336 Caprimulgus Americanus, maximus, Limm. 346. ふै. ( 128.

337 Phafianus, Colchichus, rufo fulous, Linn.270 3. (128.

333 Platalea Leucorodic alba, Linn. 231. I. (128.

339 Ardea Cayanenfis, varia, Linn o. (T28.

340 Ardea Minuta, C. Ardeola, Linn. 240. 26. \& (128.

341 Pfittacus Macao, ruber, Linn. 139. I. (129.

342 Pfittacus Paradifi, luteus, Limn. 147. 34. (129.

343 Platalea Ajaja, fanguinea, Linn. 231. 2. (129.

344 Ardea GrifeG, corpore fuscefcente, Linn. 339. 22. (129.

245 Paradifea Apoda, cum pedibus fuis, Linn 166.I. ( 129.

346 Rallus Grifeus, fupra grifeus nigro maculatus. macula alarum alba, fubtus cinerefcens, Linn. O. (129.

347 Ardea Virgo, Linn. 234. 2. (120.

$34^{8}$ Cuculus Fulvus, fubtus aureus, Linn, 0. ( 130.

349 Merops Apiafter, albus, capite nigro, Lirn. I82. I. (I3O.

350 Merops Apiafter, fulvus, Linn. I82. I. ( 130. 358 Tringa 


\section{Oifleaux E?C.}

316 Corbeau Glande: (113.

3I) Pigean de Banda, tres bears Buffi. II8. (II4.

318 Aigle Doré, brun, d ftries noires, Buff. 410. (irs.

319 Conard Noir. ( 116

320 Cocq de Bruyere, d taches blanches. (Ix6.

321 Cocq de Bruyere, ou femelle. (117.

322. Phaijant Tricolor, ou male, trés beau. Biff. 1:3. (NI9.

323 Phaifant Tricolor, ou femelle. Buff. 124. (II

324 Phailant dila Chine, ou male, tre's bear, Buff $2: 2$. ( 1 ig. 325 Phaijant de la Chine, ou femelle, très belle. (1ise. 326 Canard Fauve, dache dos ailes blonches. B ff 003. ( 122. 327 Canard Sarceile, d collier E en deffous blanc. (122. 328 Faucon Noble Buff. 47 c. ( 123.

329 Canard D misique fruive. (1 124 .

330 Comard Sifleur, ou Conard d'Amerique, farcelle at cusomis: Edw. r94. : (124.

331 Hirondelle Apus, noir atre, \& gorge binnche noir cendree. Biff. 543 . ( 124

33 Oye du Cap, tęte cor $\mathcal{E}^{2}$ siles noires, $d$ ventre fouve, ( 125.

333 Canard Hupe, joune, à ailes noiratres. ( 126: ; s?

334 Canard Hupé, ou femelle. \& 126 .

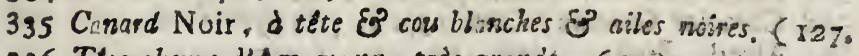

336 Tte-chevre d'Am rique, tres-grande. ( 128

337 Faijant Commun, d'un brun rouge Buff. 121 . ( 128 .

338 Spatule Banche, Buff 405. ( 128.

339 Onoré de Cayente, bariole. Ci28.

340 Petit Heron, ou Blongios, Buff. 325. ( 228 .

34I Perroģuet Ara, rouge, Buff. 12. ( 129 .

342 Perroquet Paradis, joune, de CubB, Buff. 336. (I29.

343 Spatule Rouge, Buff. 165. (12).

344 Heron Gris, brunatre (129.

345 Heran Gris, ou Oifeau de Paradiș, avec fes pieds (120. 346 Ralle Gris, tacheté de noir defcus cendrs. ( $129 \%$

347 Demoijelle de Numid e Buff. 241 . ( 130 .

\$4\$ Coucou Jaune, en defJous dore. ( 130 .

349 Mangeur des Abeilles blanc, tête noire. (I3C. 350 Mangeus des Abeilles, brun. ( 130 . 
351 Tringa Squatarola? grilea, cauda alba, nigro dimidiata capite, gula, colloque vigris, fubtus albus, Limm. 252. 23? ( 130.

332 Scclo ax Niger, albo guttatus, Linn. O. (130.

353 Proenicopterus Ruber. pulcerrimus, Linn. 230. I. (r31.

354 Tantalus Ruber, wulcerrimus, Linn. 24I. 5. (13x.

355 Scolopax Solaris, Limn O. (131.

356 Parra facana, nigra. Linn. 259. 3. (13r.

357 Tringa Grifea, f. Strenaria, pluvialis, cauda alba, nigro dimisiata, capite, gula, colloque nigris, fubtus albus, Linn. o. f. 251. 16. (13x.

358 Tringa Brunnea, an varia? albo guttata, fubtus alba, Linn. 252. 21.? ( 132.

352 Anas Fufcrn, ipeculo \& macula poft aures alba, Linn. o. ( 133 .

360 Pfittacus Parodifus, f. varietas, Linn. o. (134.

301 Anas Fufca, alis nigris, fpeculo ferrugineo, corpore nigro guttato, genis, gula \& collo albis, Linn. O. ( $135^{\circ}$ 362 Rallus Fuscus, infra fulvus, Linn. O. (136.

363 Xanthorous Decumanus, Linn. O. Naturrforf. XVIII. tab. I. ( 138.1

364 Crotophaga Ani, Linn 154. T. ( 138.

365 Ramphastus Aracari, Limn. I5I. 3. (139.

366 Ramphartus Tucanus, niger, gula alba, Linn. 151.5. (139. 367 Picus Martius, Linn. 173. 1. (I 39

368 Coracias Garrula, Linn. 159. 1. ( 139.

369 Alcedo Rudis, Linn. 181. 12. (140.

370 Alca Torda, Linn. 210. 1. (140.

37 I Phafianus Gallus, f. pullus tripes, Limn. O. (iq0.

372 Ampelis Chinensis, Linn. $0^{\prime}$ (148.

373 Crotophaga Ambulatoria. Linn. 155. 2. (14).

374 Alcedo Ispida, Linn. 179. 3. (14\%.

375 Pfittacis Cacatua, crifta flava, Linn. 143.22. (142.

376 Pfittacus Lory, remigibus azureis, cauda apice flava, Linn. C. ( $1+2$.

377 Alcedo Nigra, fubtus fulva, linez collari alba, Linn. C. (143.

378 Oriolus Xanthornus, f. Decumamus, Linn. O. Naturforfcher XVIII. tab. I. (I 43

379 Pfittacus Guineenjis, Linn. O. ( 144.

380 Pfittacus Aeftirus? capite rubro, corpore flavo, alis viridibus, flavo maculatis, cauda longa, Linn. 146. 32 ? ( 44.

38 I Pfittacus Viridis, capite nigro, collari, fubtusque aurantius, Linn, c. ( 144. 


\section{Oifeaux EC.}

351 Pluviep Varié, gris, \& queue blanc, d moitt noir, lä tlle Es. gorge noires deffous blanc. (130.

352 Becalle Noire, d taches blanches. ( 130.

353 Flammant Rouge, trds beais. Buff. 63 . ( $13 x_{\text {. }}$

354 Courli Rouge, trés bear, Buff. 8I. ( $r_{3} x_{0}$.

355 Becaffe Solaire. (131.

356 Chlrurgien Jacare, noire. 322 . (r31.

357 Chevalier Gris, as Coulier Chaud, d quene blanche, tete \& cou noir. (13I.

358 Chevalier Brun, ou Varié? moucheté de blanx, defous blanc. Buff. 923. (132.

359 Canard Brun, noiratre d ailes noires \& tashe blanche. (133. 360 Perroquet de Paradis, orb varieté. (134.

36. Canard Brun, d aibes noires, be corps noir pointillte, bo ttte blanche. (135.

362 Rale Brun, en defous farre. ( 136.

363 Cadique Noire, quewe joune, Pall. Mifceli. Zool. (138.

364 Oifear Ani, ors des Savannes, Buff. 102. I. (138.

365 Taucan Grigri, Buff. 166. ( 139.

366 Taucan Toco, noir, d gorge bionshe. (139.

367 Pie Noire, d tlte rouge. Buff. 117. (139.

368 Geay de Strasbourg, Buff. 501. ( 139.

36. Martin-pecheur Hupe, noir, en deJous fauve, Buff. 62. (140;

370 Macareau d'Europe, Kdw. Glean. 358. (140.

371 Pbaifant Cocq, jeune, 1 trois piods. ( 140.

372 Cotinga de la Chine. (1 4 I.

373 O jears Ambulante, ors la petit Ani, Buff. ibid. 2. (14t;

374 Martin-pecheus des Indes. ( 141.

375 Perroquet Cacathoe, d tele joune, Buff. pb. 14. (142.

376 Perroguet Lory. plumes d'azur, be bout de quieue jaune. (142.

377 Martin-pecheur; ou Alcion Noir. (143.

378 Carorge Xantorne, ou Decumain, Naturforfch. XVIIT. tab. r. (ra3.

379 Perroguet Moineau de Guinte, Edw. 237. I. (1444.

380 Perroquet d'ifite? ou Perruche, d ttte rouge, corps jasne E ailes verizes, dquese bongue. (144.

38: Perroquet Verd, the noire, deffous orange. (144. 
38.2 Pfittacts Viridis, minor, genis aurantiis, Linn. 0.1 I44 383 Numida Meleagris, cum pullo, Linn. 273. 1. ( 146. 384 Tetrao Alchata, Linn. 276. It. (146.

385 Anas Fujca, fuperne fusco-brunnea, alis nigris, fpeculo alba, Linn. O. ( 147.

386 Anas Brunnes, fuhtus alba, Linn. O. (14\%

387 Crex Hocco I Globicera Linn. 270. 4? 148.

388 Numida Crißzata, 'Linno o. Pall. Specil.'Zool. IV. tab, 2. 2. ( 48

389 Numida Alba, Linn. o. ( 49 .

300 Tetra Marilnnasicus Linn 277. 17. ( 149.

391 Tetrao Capenfis, Linn o. (149.

392 Tetrao Capenfis varietas, Linn. O. C 149.

393 Numida Cinerea. Linn. O. ( I $\subset$.

394 Tetrao Francolinus. f mas, Linn 275. 10. ( 150.

395 Tetras Francolinus , fo fe mina, Linn. I.c (150.

396 Tetrao Americana, f Coturnix, Linn: 278. 20. (150.

397 Columba Oenas, Linn. 279. (absque Cifta.

398 Faico Fulvus, maximus, Linn. 125 6. (0.

399 A as Flivn, (peculo albo, Linn o. (0.

400 A'cedo Ispida, Linn 179. 3. ( 152.

401 Alacda Calandra, fafcia pectorali fusca, Linn. 288. 9. ( 152.

402 C rvus Corax, Linn. 155. 2: ( 152.

403 Turdus Itiaczs, Linn. 292. 3. ( 153.

404 Curvus Criftaties? f. Ampelis Garrulus, Linn. 157. 8 ? (I53.

405 Fingilla Tigrina, pectore nigro albo maculato Linn. - ( 154

406 Fringilla Tigrina, f. varietas, Linn. O. C 154.

407 Fringilla Senegala? pectole fulvo, Linn. 320.18 ? (154) 408 Emberiza Lisdovicia; Linn. 310. 10. ( 154.

4 C9 Motacilla Hippolais, Limn. 330. 7. C 155.

410 Motacilla Alba, Limn. O. ( 155.

4 Ir Motacilla Alba, Linn. O. ( 155.

fr2 Molacilla Alba, fovarietas, Linn. O. (155.

$4^{\text {T } 3}$ Pipra Aureola. Linn. 339. 7. ( 156.

44 Pipra Gutturalis, nigira, alba gula, Linn. 340:10. (156:

415 Trochilus Smapagdus Lirn. O. ( 156.

4.6 Trochilus Mosquitus, Linn 192. 14. (156.

417 Phafianus Nyohemerusi f $\mathrm{f}$ mas. pulcerrimus, Limo. 272. 6.: 6. (absque Cifta cum feguentibus:

418 Phafianus Nydhemerus, f. femina, pulcerrima, Linn. 272.6.

49 Cuculus Niger, cauda longa lineata, Linn. 170. 2 ? 


\section{Oiffeaux EF?}

982. Perruche Verd id jeunes orangkes. (144.

389. Poulepintede de Guienne, avec la jeune, Buffo rogt ( 146 .

384 Cocq Alchata, Buff, 130 . (i46.

385 Canard Brun, par deffus brunatre, dailes noires. (247\%

386 Canard Brun, bianc en defous. ( $1477^{\circ}$.

387 Hocco Globulaire, a ailes noires, tache blanche glemorte.

Edw. 295. ( I 48 .

288 Poulepintade d Crete, Pall. Specil Zool. IY. tab. 2. (343.

389. Poulepintade Blanche. ( 449.

390 Perdrix d'Amérique, Albin. 28. (14,9.

39r Perdrix du Cap. (149.

392, Perdrix da Cap, ou varieté (149. .

393 Poulepintade Cendree. ( 150.

394 Perdrix Esaneolin, ou male. Buff. 147. Cr50d.

395 Perdrix Francolin, ou femelle. (150:

396 Perdrix d'Amerique. ( 550.

391 Pigeon Commun; Buff. sio. ( Sans Caiffe.

398 Faucon Brun, tres grand. 10.

399 Canard Rauve, d tache. des ailes blenches. ( 0 :

400 Martin-pecheur Commun. (I52.

40I Alowiete Calandre, d bande brine, Buff. 363. 2. (152)

402 Corbeau Choucas des Alpes, Buff. 435. ( 152.

403 Grive xlinaire, Buff. 406. (I53.

404 Corbeas Hupe. (153.

405 Pingon Tigrine, d poitrine tachltte. (154.

406 Pingon Tigrine, ou varieté. ( 154 .

407 Pingon Senegali, a gorge farwe. Buff. 223. (154

408 Ortolan de la Louñiana, Buff. 158. X. (254.

409 Fauvette Blanche. (155.

410 Fauvette Blanche, Buff. 580 . (I55.

4II Faswette Bianche. (155.

412 Fouvette Blanche, ou yarieté. (155.

4 I3 Manakin Noir, d. tete rouge. Buff. 34. 3.. (I56.

414 Manakin Noir, a gorge blanche. Buff, 276. 2. (I56.

415. Colibri Emeraude, Buff, 276. 2. (156.

416 Colibri Rubis Topaze, Buff, 227. 2. (156.

417 Faifant Tricolor, ou male, tress bears, ou Phaijant blans:

de la Chire. (les Juivans fans Caifle.

418. Phaijant Tricolir ou femelle, trés belle. (157. 419 Coucous Nuir, a, queue longue disagie. 
420 Emberiza Rubra, capite \& abdomine nigro, Linn. 0. $42 f$ Oriolus Cayanenfis, niger, maculis luteis; Linn. 163.15.

422 Oriolus Decumanus? f. Xanthornus? Limn. 0.

423 Picus Lineatus, niser, Linn 174. 4.

424 Pipra Aureola, capite coccineo, Linn. 339. 7.

425 Alcedo Aureata, ex albo nigraque varia, Limn. 0. 426 Taragra Pompadora, unicolor, Linn. 0.

427 Alcedo Caleftinus, capite, collo \& abdomine rubris, gula alba, dorfo coleftino, Linn. $\odot$.

428 Oriolus Babtimore, nigricans, fubtus fulvus, Linn. 162. 10.

429 Alcedo Nigricans, ex albo nigroque varia, Linn. c, 430 Tanagra Chlorotica Linn. 317.23.

431 Tanagra Atrata, futtus \& fronte flava, [Linn. 315. 9.

432 Tanagra Gularis, Linn, 316. 13.

433 Loxia Nigra, bumeris dorfoque flavis, Linn. 0.

434 Tanagra Pompadora, unicolor. Linn. o.

435 Pfittacus Galgulus, ubique concolor, Linv. x50. 46.

436 Emberiza Carulefcens, humeris albis, remigibus carue leis, Linn. e.

437 Trochilus Viridis, Linn. c.

438 Pipra Pareola, crifta Ranguinea, Linn. 339. 2.

439 Pipra Pareola, C. femina, Linn. 1, c.

440 Tantalus Albrs, remigibus atris, Linn. o.

44I 'Árdea Cerulea, occipite criftato, Linn. 238. 17.'

442 Muftela Erminea, Linn 68. 10. (Cifta Ir 3. 77.

443 Lemur Spedrum, f. Tarfier Buffoni, Linn. O. rarum (100**.

444 Lepus Brafilienfis, f. Tapeti Buffoni, Linn. 78. 4 (118. 78. 445 Vivora Nafua, C. Coati Buffoni, Linn: 64. 20, (120.79. 446 Didelphis. OpolJum Linn. 72.3. ( 120.79.

447 Lemur Tardigradus, fo Loris Buffoni, Linn. 44. 1.? (120.81. 448 Muftela Erminea, Linn. 6\%. 10. (120. 82.

449 Cerrus Dama, ninimus, Linn. 92. 3. (120. 82.

450 Simia Pithecta. Linn. 40. 22 ( 137.84 .

451 Didelphis OpofJum, Linn. 72. 3. ( 137.85.

452 Bradypus Didactytus Linn. 5 1. 2. (137.80.

453 Sciurus Cinerews; minor, gula siba, Linn.86. 3. (r $37^{\circ}$ 87.

454 Myrmecophaga Didacibla. Linn. 5I I. (138. 88.

455 Canis Pyramus, nieer, Linn o. (145. 80.

456 Sciurus Singitta, f. Betaunitta Taguan Buffuni, Linn. 88. II (I51. 90; altus 27. látus 2u. poll. 


\section{Oi\eaux E'Gi}

420 Ortolan Rouge, \& tete Eे ventre noire.

421 Carange de Cayenne, noir, bes ables d tacke jaune. Edw. Glean 322.

422 Carange Decumain, ou Xantorne.

423 Pie Rayée, noir, de Cayenne Buff. 596.

424 Manakin Doré, à tzte rouge.

425 Facamar ou Martin pecheur Dors, varié de blanc E̊ noir. 426 Tangara Pompadour, d'un coulewr.

42? Martin pecheur Celeftin, tete, col, Ev ventre rouges à dos bleue.

428 Corange Baltimore, noiratre, deJjous fauve.

429 Martin pecheur Noir, vorié de noir E blanc. Buff. 4 I6.

430 Tangara Noir, a front $\mathcal{E}^{\circ}$ poitrine jaunes, Briff III. isb. 2. $f$. 3 .

431 Tangara No'r, defous joune.

432 Tangara, ou Cardinal d'Amérique, Buff. 155. 2.

433 Gros-bec Noir, d dos jaqre.

434 Tangera Pompadour, d'un couleur.

435 Perruche Verce, partout d'un couleur.

436 Ortolan Bleuatre, d aibes bleues.

437 Colubri Verd.

438 Manakin, à Dos bleu, Ẽ creté rouge, Edw.26r.1.

430 Manakin, ì Dos bleu, our femelle.

$44^{\circ}$ Courli Blanc, d ailes noires.

44I Heroiz Bleuacre, creté par devant la téte. Buff. 859.

$4 \div 2$ Beletic Hermine. (113. 77 .

$4+3$ Makivolant, ou Tarlier Buff. 26. 9. animal rare. (100*k.

444 Lievie de Brafile, ou Tajete Buff. (118. 78.

445 Belette Feune, ou Coati Buff. 8. 48. (I 20.79.

446 Philendie Sangue, Buft. 10. (120. 80.

$447^{\circ}$ Makivolant, 0u Loris Buff. I3. 30. (I20. 81.

448 Belette Hermine. (I2C.82.

4) Le Cerf, ou Chevistain Buff. 12.42. (121. 83.

450 Singe, equ Jacci Buff. 15.17. (137. 84.

451 Philandre Sangue. ( 157.85 .

452 L'Unas Buff. 13.5. 6. (137. 86.

453 Ecuteuil Gris, très gesit, de col wharche. Buff. 10. 2a

(: $: 37.87$.

454 Le Futrmillier, Buff. 10. 30. (137. 88.

455 Le Chier Piramus, noir. $\quad 1: 45.89$.

450 Ecaretil Volant, or Vetaurifte, Buff, Supp. VI. SI. (ISI. 90. hast. 27. large, 20 pouses. 
457. Simia Midas, minutisfimus, Limi. 42. 27. (Camp. 152. 9 I.

458 Capra Cervicapra, f. Strepficeros, pullus, Linn. 96. 8 ? (absque Cista 92.

459 Canis Lupus, Linn. 58. 2. ( $\$ 4$.

460 Lemur Catts, Linn. 45. 4. $(95$.

46r Vivera Zibetha, Linn. 65. 5. ( 99.

462 Bos Muscus, Capenfis, an Caffra? i. ejusjem caput, Linn. O. $(98$.

463 Capra Cervicapra. f. ejudem caput, Linn. $968 ?$ (io2. 464 Manis Pentadactyla, magnus, Linn. 52 1. (103. 465 Lemur Tardigradus, Linn. 44. I. (100*.

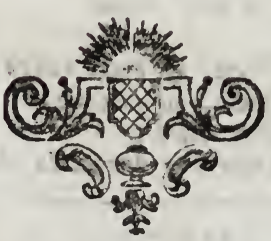




\section{Diffeaux E⿱宀:}

457 Singe ou Tamarin, trespetit, Buff. I5. I3. (Camp. I52. 9r, 458 feune Chevre, ou Strepficere Buff. Suppl. VI. 2. Jans Caifje. 92.

459 Le Chien Loup, Buff. 7. I. (94.

460 Le Makivolant, ou Mococo Buff. 13. 22. (95.

46I La Belette, ou Civette Buff. 2. 34. (99.

462 Vache du Cap, ou tête de Vache. ( 98.

403 Cerf ou Feune Chevre, ou la tette dü méme. (102. 464 Le Pangolin Grand, Buff. 10. tab. 34. (100. 46) Le Makivolant, ou Loris Buff. 13. 30. (100\%.

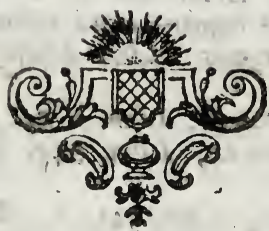




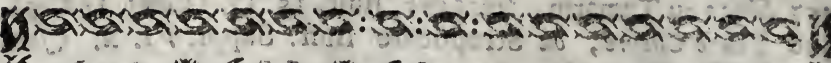

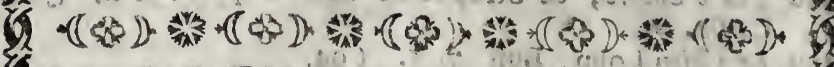
Y

\section{INSECTA EXOTICA. \\ C OLE.OPTERA.}

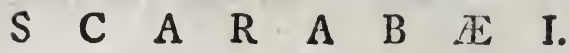

I Gcarabxus Hercules, Linn. 541. 1. Voet Scarab, tab. I2. D fig. 98 . (179.

2 Scarabæus Hercules, Linn. 1. c. ( 179 .

3 Scarahxus AEteon, Linn. 54r.3. Voet 15. 18. (i 76.

4 Scarabxus AEcon, Limn. I. c. ( 177.

5 Scarabæus Ailas, Linn. 542. 6. Voet 15. 19. ( 178.

6 Scarabzus Ailas? capitis cornu tricorni, Linnz. O. (849.

7 Scarabaus Dichotomus, Linn. Mantij]. I. 529. Viet I4. 107. (183.

8 Scarabæus Dichotomus, Linn. 1. c. (183.

9 Scarabrus Rhinoceros, Linn. 544. 14. Röjel Scarab. II. tab. A. ( 180.

Io Scarabæus Gideon, Linn. 54I. 2. Röf. Scarab. II. A.5. ( 184.

II Scarabaus Gideon, Linn. I. c. (184. bis.

12 Scarabrus Gideon, f. feurina, Limn. 1. c. ( 846.

I3 Scarabaus Gideon, f. varietas, Linn. I. c. (846.

I4 Scarabæus Rhineccros? f. varietos, cornu obtufo bifido, Linn. O. ( 181 .

15 Scarabæus Aloc̈us, Linm. 542. 7. Röj. Scarab. II. tab. A. f. 6. ( 182 .

16 Scaralaus Naficornis, vel potius foraicenfis, Limn. 544. 15? Fabricius Spec. Inject.pag. I2. $n^{n} .43$. ( 8 17.

17 Scarabous Naficornis, f. varietes, Linn. \& Fabric. I. c. ( 847.

I8 Scarabæus Naficornis, f. varietas, cornu minori, Linn. \& Fatric. 1. c. (1024.

19 Scarabaus Molof/ess, Linn 543. 8. Drury tab. 32. f. 2. Voet 23. 2. (883. bis.

20 Scarabxus Ligiceps, Limn. O. Voet 27. 34. C 053 .

2I Scarabreus 


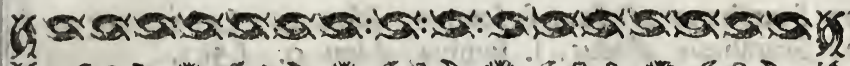

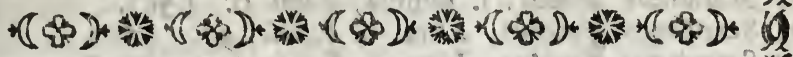

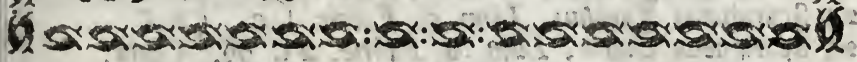

INSECTES ETRANGERES

C OLEOP TERES.

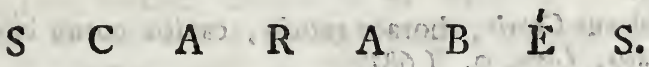

I Carabe Hercule, Linn. 54!. I. Voet Scarab. tab. I2. S. . 98. ( 179.

2 Scarabé Hercu'e, le pareil, Linn. l. c. (I 79.

3 Scarabé Adẹon, Linn. 54r. 3. Voet 15. 18. (176.

4 Scarabé Acteon, le pareil. Linn. l: c. : (177).

5 Scarabé Atlas, Linn. 542. 6. Voet 15. 19. (178.

6 Scarabe Atlas, la tet te d trois comies. Linn. o. ( 845 .

7 Scarabé Doublé, ou Divifé, Linn. Mantif. I. p. 529. Voet 14: 107. ( 183 .

S Scarabé Doublé, le pareil, Linn. l. c. ( 183 .

9 Scarabé Rhinocere, Linn. 544. I4. Rör. Scarab. II. tab. A. $\left(x 80^{\circ}\right.$

10 Scarabé Gideon, Linn. 541. 2. Rör. Scar. II.. A. 5. (184.

II Searalé Gideon, le même, Linn. l. c. . (184. bis.

12 Scarabé Gideon, oufemelle, Linn. l. c. ( 846 .

13 Scarabé Gideon, ou varieté, Linn. l. c. ( 846 .

14 Scsrabé Rhinocere; ou varieté, la corne rebouche E fondus Linn. O. ( 181 .

I5 Scarabé Aljé, Linn. 542. 7. Rör. Scarab. II. A. 6. (182.

15 Scarabé Naficorne ou plut6t de Jamaique, Linn. 544. 15 ? Fabricii Spec. InfeE p.I3. no. 43. (847.

17 Scaratié Naficorne, ou varieté, Linn. E Fabric. l. c. ( 847.

is Scarabé Naficorne, variete, la corne plus petite, Linn. EQ Fabric. l. c: (roz4.

Ig Scarabé Molofre, Linn. 543. 8. Drury tab. 32.f.2. Voet 23. 2 C\$83. bis.

25 Scarabé Ligicipe, Linn. O. Voet 27. 34. (653. 
2I Scarabreus Aloerus, f. femina, Linn. O. (583.

22 Scarabæus Aloëus, f. femina, Linn. O. (583.

23 Scarabxus Facchus, Linn. O. Fabric. 97. (680.

24. Scarabxus facchus, Linn. O. Fabric. I. c. $(848$.

25 Scarabeus Capris, Linn. O. Fabric. 23. 99. ( 962.

26 Scarabaus Lunaris, Linn. 543. 10 Doet 23. 6. (17 r.

27 Scarábxus Lusnaris, an feminá? Linn. 1. c. ( $56 \mathrm{r}$.

28 Scarabæus Sacer, Linn. 545. 18. Voet 27. 4. (i68.

29 Scarabxus IJjequeboënfis, Linn. O. Voet 24. 10. (109.

30 Scarabæus Capris 1. Feftivus, Lim, 552, 52. Vost 23. 5. ( 170.

31 Scarabaus Cappis, thorace retufo, capitis cornu lato, tridentato, Linn. O. (681.

32 Scarabaus Ligiceps, fed minor, Linn. o. Voet 26. 33. (849.

33 Scarabaus Capris, clypeo elytris ftriatis, Linm. 0. ( 1026.

34 Scarabzus Stephanardes, Linn. O. Vost 27. 41. (roz7.

35 Scarabæus . Capiris, clypeo oblongo ovato punetato, Linn. O. (1028.

30 Scaratzus Marginatus, clypeo marginato fufco rubens, Linn. O. ( 172 .

3f Scarabaus Vacca, Americani, Linn, 547. 25. (657.

38 Scarabæus Auratus, capite vix cornuto, aurato, elytris \& margine thoracis auro pialis, Linn. 0 ( 963.

39 Scarabius Auratus, ut in no. pracedenti, Linn O. ( 963.

40 Scarabaus Thyphreus, Indicus, Linn.543. 9. Voet 12. 4.

- $(173$.

41 Scarabaus Aier, clypeo corniculis, Linn. O. (682

42 Scarabaus Ater, fubtus rufo pilofus tharace gibbo returo, capite corniculato, Linn. O. ( 560.

43 Scarabæus dter, pracedenti affinis, Linn, O. (560,

44 Scarábæus Ater. fimilis prxcedenti, Linzo. O. (545.

45 Scarabaus Brevicornis, thorace retufo emarginato, capitis corna brevi incurvo, Linn. 0. (654.

40 Scarabaus Caftaneus, thora lato fưlcato, capitis cón na ad thoracem incarvo, Linn. 0 . (102s.

47 Scarabaus Caftaneus, fimilis pracedenti, Linis O. (1025. 48 Scarabæus Gibbofus, caftaneus, vertice plano, Liris. 0. ( 47 t.

49 Scarabæus Laticollis, r. varietas, elytris ftriatis albo maculatis, I.inn 549. 38. (867.

50 Scarabaus Laticollis, fumilis pracedenti, Linn. I. c. (ort. 


\section{Injectes.}

2 Scarabé Aloé, ou femelle, Linn. o. $\mathrm{C}_{53} \mathrm{~S}$.

22 Scarabé Aloé, ou femelle, Linn. O. $(583$.

23 Scaralé Jacchus, Linn. O. Fabric. 97. (680:

24 Scarabé Jacchus, le pareil, Linn. O. Fabric. l. c. ( 848.

25 Scarabé Capre, Linn. O. Fabric. 23. 99. (962.

26 Scarabé Lunaire, Linn. 543. 10. Voet 23. 6 . (I7I.

27 Scarabé Lunaire, ou femelle? Linn. l. c. (56r.

28 Scarabé Sacre, Linn. 545. 18. Voet 27. 4. (168.

29 Scarabé d'Ireguebe, Linn. O. Voet 24. 10. (169.

30 Scarabé Feftive, Linn. 552. 52. Voet. 23. 5. ( 170.

3I Scarabé Capre, le corcelet emoudsé, d corne de tite large,

E' tridenté, Linn. O. (68I.

32 Scarabé Ligicipe, mais plus petit, Linn. l. c. Voet 26. 33. $(849$

33 Scarabé Capre, l'ecufJon, delytres ftriées, Linn.0. (1026.

34 Scaralie Steptanoide, Linn. O. Voet 27. 4r. (1027.

35 Scarabé Capre, à bruclier allongé ovale pointé, Linn. 9. (1028. ¿ 6 Scarabé Marginé, à bouclier bordé d'un bring rouge. Linn. $\odot$.

( 172 .

37 Scarabé, dit Vache Americaine, Linn. 547. 25. (657.

38 Scarabé Doré, la tête d peine cornue elytres, EO le bord du corcelet doré, Linn. o. $(063$.

3) Scarabé Doré, comredans le nombre precedent, Linn. 0. ( $9 \sigma_{3}$.

4 O Scarabé Thyphaus, des Indes, Linn. 543. 9. Voet 12. 4. ( 173.

4I Scarabé Noir, le bouclier d dsux cornss, Linn. O. (682. 42 Scarabe Noir, defous le poil rouge, le corcelet boffu emouffé, la tête do large corne, Linn. 0 . ( 560.

43 Scarabe Noir, le pareil, Linn. O. ( 560 .

44 Scarabe Noir, Semblable au precedent, Linn. O. ( 545.

45 Scarabé Ccurte - Coine, le corcelet emousfé deborde, ba corne de la tete un peru courbé, Linn. O. ( 654 .:

40 Scarabé Chataigne, le corcelet large, fillonné la corne de la tete courbé veris le corcelet, Linn. O. ( 1025.

47 Scar iké, Chataigné, le pareil, Linn. O. (1025.

48 Scarcué Bolfu, ou Chataigne, \&̀ Jommet de la tête uni, Linn. O. (47r.

49 Scrirabe d Large teftre, ou varieté, les elytres ftriés, Eo tacheté de blanc, Linn 549.38. (867.

50 Scarabe d Large teftre, ou Collin, Semblable ars precedent; Linn. b. c. (914. 
5I Scarabrus Laticollis, præcedenti affinis, Linn. 1. e. ( 9 '4.

52 Scarabæus Laticollis, groffus, elytris fulcatis fusco punctatis, Linn. I. c. (964.

53 Scarabaus Laticollis, ,. varietas, ac $n^{\circ} .49$ ? Limn.1.c. (103.

54 Scarabxus ...., elytris cerinatis, porcatis fcabris, clypeo thoracis latimimo, lateribus plano, Linn. 0. (683.

55 Scarabzus ..... fimilis, Linn. O. ( 728 .

56 Scarabæus Amicioides, planus, ater fufco ciliatus, anno Cimex? Linn. o. ( 678.

57 Scarabxus Amicioides, pracedenti affinis, Limn. o. (678.

58. Scarabæus Ater , fubtus rufo pilofus, thorace gibbo, capite corniculato, Linn: C. ( 560 .

59 Scarabzus Ater, fimilis præcedenti, Limn. O. (543.

60 Scarabæus...., parvus, rubens pedibus porticis uniangulatis, Linn. O. ( 898 .

or Scarataus ....., fimilis, Linn. o. ( 915.

62 Scarabsus Caftaneus, pedibus anticis tricuspidatis, Lim. o. (103I.

63 Scarabeus Caflaneus, fimilis pracederti, Linn. O. (1029. 64 Scarabæus" Niger, elytris bafi macula sulva, Linn. ${ }^{\circ}$ ( 1032 .

65 Scarabrus Picets, thorace fubglobofo punctato, Linn. O. ( 1033.

66 Scarabæus Bilobus, Linn. 144. 12. ( 174 .

67 Scarabrus Bilobus, fimi' is præcedenti, Limn. I. c. (174.

68 Scarabrus Melolonithn, fervida, Limn. o. Falric. 32. 4. (767.

69 Scarabrus Melolortha, fimilis, Linn. O. Fabric. 1. c. $\{767$.

70 Scarabeus Melolontha, f. varietas, ferruginea, Linn. 0. ( 770.

71 Scarabxus Melolontba, Linn. O. an Refexs Fabric. 57. $6 ?$ ? $(770$.

72 Scarabaus Melolontha, Indica, Limn. O. ( IO34.

73 Scarabæus Melolontha, brunrea, Linn. 554.60. Voet 7. 53. 54. ( 1035.

74 Scarahxus Oculatus, ater nitèns, capite bituberculato. oculis albis, Limı. O. ( 1100 .

75 Scarabaus Oculatus, præcedentiafinis, Limn. O. (Iroo.

76 Scarabæus Mus, cinereus, Linn o. Voet 9.75. (10).

77 Scarabaus Viridis, villofus, elyeris planiufculis, Limm. 0. (889.

78. Scarabaus Lineola, Linn. 552. 53. Rốf. Scarab. II. tab. B. 7. ( 197 .

79 Scarabaus Lineola, fimilis, Linn. 1. c. (197.

80 Scarabaus 
5I Scarabe 2 Large teftre, ou Collin, le pareill au precedent: Linn. l.c. ( 914.

52 Scarabé, \Large teftre, ou Collin, grofJe, elytres fillonnées, pointées de brun, Linn. lo. C. (964.

53 Scarabe 2 Large teftre, ou Collin, le mémede n ${ }^{\circ} .49$ ? Linn.o.

( 103.

54 Scarabé...., les elytres creux, raboteux le bouclier dus cortelet très large, les coios unis. Linn. $0 . \quad(683$.

55 Scarabé...., ou pareil, Linn. 0. ( 728 .

56 Scarabé Amiciode, unis, noir, melé de brun, Linn. O. (678. 57 Scarabé Amiciode, le paseil du precedent, Linn. o. ( 678 .

58 Scarabé Noir, defous poil rouge, le corcelet bo/Ju, la têto cormu, Linn. o. ( 500.

59 Scarabé Noir, Jemblable au precedent, Linn. 0. (543.3

oo Scarsbé ....., petit, rougeatre les pattes derrieres avec un ongle, Liñ. O. ( 898.

oi Scarabé..... le pareil, Linn. o. (915.

62 Scarabe Chataigne, les pattes devant à trois points, Linn: 0. ( $103 x$.

63 Scarabe Chataigne, le pareil dib precedent, Linn. o. (ro29. 64 Scarabé Noir, les étuis de la baje à tache fauve, Limn. $0_{0}$ (IO32.

65 Scarabe Comme-poix, le corcelet usi pers rond, EO pointu, Linn. O. C 1033.

66 Scarabé Bilobé, ou nivifé? Linn. 544. 12. (I 74.

67 Scarabé Bilosé? femólable aus precedent, Linn. l. c. (I744. 68 Scarabé Melolonte, fervide, Linn. O. Fabric. 32. 4. ( 767.

69 Scarabé Melolonte, le pareil Linn. o. Fabric. l. c. (-67.

70 Scarabe Melolonte, ou varieté; couleur de ruzilise. Linn: 0.

( 770 .

7I Scarabé Melolonte, le même, Jinn. o. ou le Recowibé de Fabric 57. 6? ( 770.

72 Scarabe Mulolonte, des Ixdes, Linn. O. (Inza.

73 Scarabé Melolonte, bruine, Linn. 554. 60. Vout 7. 53 , 54. (1035.

74 Scarabé Oculaire, noir, la tête d tubercales, les y:ux blancs, Linn. O. . ( I 100.

75 Scarabé Oculaire, femblabie ar precedent, Linn. C. (1100.

76 Scarabe. Souris candré, Linn. O Voet 3. 75. (109.

77 Scarabé Verd, velu, les eitytres unis, Linn. o, (889.

78 Scarabé Petite Ligne, Linn. 552. 53. Rör. Scarab. II. B. 7. ( 197.

79 Scaraké Peticu Ligne, le pareil, Linn. l. c. ( 197. L 5

85 Scarabe 
- 80 Scarabaus Livoola, f. yarietas, Linn. 1. c. Voot ro. 85. ( 198.

81. Scarabæus Lineola, fimllis, Linn. \& V'oet l. c: (108.

8 i Scarabrus Lineola, f. femina, Linn. O. (470.

83 Scarabaus Lineola, finilis præcedenti, Linn, o. ( 199.

84 Scarabæus Lineola, f. præcedenti affinis, Limm. O. (199.

85 Scarabaus Piceus, thurace fubcylindrico Itria transverfa, elytris friatis, Linn. o. ( 586.

86 Scarabæus Politus, ater nitens, elytris fubrugolis, Linn. O. ( $(96 \mathrm{r}$.

87 Scarabaus Lanius, ruber, punckis nigris, Linn. 557. 77. Voet 2. 16. ( 203.

88 Scarabaus Lanius, frmilis pracedenti, Linn. \& Voet. l. c. $(203$.

89 Scarabaus Copenjis, punctis abis, Lim. 556. 73. Voet. 2. II. (205.

9o Scarabaus Capenfis, finilis pracedenti, Linn. \& Voet. 1. c. $(205$.

9r Scarabaus Lenius, f. varietas, Linn. 1. c. Voet 2. I6. (203.

92 Scarabrus Lonius, pracedenti fimiliș, Linn \& Vobt. l. c. $(203$.

93 Scarabsus Capenges, Lins. \& Vout 1. c. ( 204.

94 Scarabaus Niger, elytrorum futura poftice elevata, Ipina bifida, Linn. 0. ( 859 .

95 Scarabaus Niger, pracedenti affints, Linis. 0. (859.

s6 Scarabatis Niger, elytris macula oblonga rubra, maculis aureis, Linn. (1040.

97 Scarabaus Nigar, maculis oblongis albidis, Linn. $\odot$. ( $104 \mathrm{r}$.

98. Scarabaus Niger, pracedenti affinis, Linn. O. (104r.

99 Scarabaus Niger, finilis, Linn O. ( $1 \mathrm{C}_{42}$.

xoo Scarabaus Fafcicularis, lineolis quacuor albis, Linn. $557^{\circ}$. $75 \quad 655$

¿O1 Scarabæus Fafcicularis, fimilis, Linn.1. c. (655.

102 Scarabaus Fafcicularis, pracedenti affnis, Linn. 1, c. (656. bis.

103 Scarabaus Srriatus, thorace viridi aneo convexo, elytris convexis, caftaneis, ftriatis, femoribus torofis Linn. $\odot$. ( 923.

104 Scarabxus Striatus, capite tuberculato, an femina? Limm. O. $(966$.

I0S Scarabæus Piceus, fubtomertofus, Limm. O. (087.

106 Scarabæus Micans, Linn 0. Fabric. 50. Viet 40. 5. ( 768.

\pm 07 Scarabaus Bicolor, Linm. O. Fabric. 38, 16. Voet 8. 66. (101. bis. 
80 Scarabé Petite Ligne, ou varieté, Lipn. l. c. Voet $30.85 \%$ (198.

81 Scarabe Petite Ligne, le pareil du precedent, Linn. \& Ve t b. c. ( 198.

82 Scarabé Petite Ligne, femblable, ou femelle ? Linn.0. (470

83 Scarabé Petite Ligne, le pareil, Linn. O. ( 199.

84 Scarabé Petite Ligne, le pareil du precedent, Linn. 0. (199.'

.85 Scarabe Noiratre, le corcelet cylindrique une firie transyerfe. les étuis ftries, Linn. O. ( 586.

86 Scarabe Poli, noir, les étuis un peu ridés, Linn. 0. (96r.

7 Scarabe Lanius, rouge, d points noirs, Linb. 557. $77^{\circ}$ Voet 2. I6. (203.

88 Scarobé Lanius, le meme du precedent, Linn. E Voet 1.6. ( 203.

8g Scarabe du Cap, d points blarics, Linn. 556. 73. Voet 2 . II. ( 205.

90 Scarabé du Cap, le meme du precedent, Lina. E Voet l. c. $\left(205^{\circ}\right.$.

9x Scarabe Lanius, ou varieté, Linn. l.c. Voet 2. X6. (203. I 92 ScarabelLanius, le meme du precedent) Linn. EO Vaet l.c. (203. 93 Scarabe du Cap, Linn. EO Voet l. c. (204.

94 Scarabe Noir, la fusture de derriere des étuis elevé, lepine ferdu, Linn. 0. ( 859 .

95 Scarabe Noir, le méme du precedent, Linn. 0. (859. 96 Scarabé Noir, les étuis \& longue tache rouge, avẹe des taches dorées, Linn. O. (1040.

97 Scarabé Noir, longues taches blanches Linn. O. ( $104 \mathrm{r}$.

98 Scarabé Noir, le meme du precedent, Linn. O. (104\%.

99 Scarabé Noir, Jemblable au precedent, Linn. O. (1042.

joo Scarabé Bandé, dquatre petites lignes noires, Linn. 55\%: 75. $(655$.

ror Scarabé Bandé, le niême du précedent, Linn. $l$ e. $(655$. 102 Scarabé Bandé, Jemblable du piécédent, Linn. b.c. (656. bis

103 Scanabé Strié, le corcelet bron cuiverux, les ituis courbés chataigne les criaffes mufculeufes. Linn. O. (923.

104 Scarabe Strie, ou fernelle, la tête d tubercules, Linn. O. ( 960.

Ics Scarabé Noiratre, Linn, 0. $(687$.

Io6 ScarabéR eluifant, Linn. 0. Fabric. 50. Voet 40.5. (768. 107 Scarabé à deux Couleurs, Linn. O. Yabric. 38. I6. Voek 8. 66. (161. bis. 
108. Searabzus Bicolor, 1. varietas, parva, Linn. O. ( 684 . Iog Scarabæus Chry/ss, Linn. 551. 49. Voet 8.63. (585, bis. wo Scarabæus Luteus, pedibus anticis villolis, villo luteo, Linn. 0. ( 187.

711 Scarabæus Longimanus, Linm. 549. 39. Voet iI., 97. (i 85.

112 Scarabxus Atreon, r. femina, Linn. 54t. 3? Voet 16. (186.

II 3 Scarabæus Magnus, ater elytris glabris, Linn O. Voet 140. fed major. (IIr 5 .

114 Scarabæus'Magnus, f. varietas, Linn: 0 ( 768.

I15 Scarabaus Bicolor, Linn. 0. Fabric. 38, 10.V Voet 8. 66. (190. bis.

110 Scaribxus Pannipes, Linn. O. Goetz. Enttom. 77. Voet. 8. 64. ( 193.

Ii) Scarabaus Vitris 2. Linn.0. Fabric 41. 34. Voet 7. 56. 57 ( 1043 .

II8 Scarabaus Viridis, elytris ftriatis, Linn, O. (195.

119 Scarabæus Marginatus, Linn. O. Fábric. 25. 20. Vook 4. ( 212.

I 20 Scarabaus Elegans, Linni o. Fabric. 56. 33.i (19). ter?

121 Scarabaus Ele gans, f:varietas, ac na.ur 8? Linn. 0. (1098.bis.

122 Scarabæus Signatus, Linn. O. Fabric. 54 19. (685. bis.

123 Scarabaus Signatus. f. varietas, Limn. o. ( 686.

124 Scarabaus Capenfis? R. varietas, Linn. 556. 73 ? Voet 2. 11. $(730$.

125 Scarabæus Lunaris, elytriș ferrugineis, apice acuto, ma. culis cuabus atris albo lunatis, Linn. o. ( 858 .

126 Scarabaus Niger, femoribus pofticis fubtus villofis. Linin o. ( 860 .

I 27 Scarabxus Ligiceps, Indicus, Limn. O. Voct 4. 32 . (206. bis.

128 Scarabxus Ater, thorace tereti elytris rugofis, Linn. 0. ( 658 .

I29 Scarabzeus Hiftrio, fulvus, Limn. O. Voet 3. I8. (1039 bis. 130-Scarabass Fiavus, thcrace nigro luteoque, elytris luteis. Linn ०. ( 1036.

$13 \mathrm{I}$ Scarabæus Flavus, f. varietas, Linn. O. (1037.

132 Scarabaus Puntatus, capite atro elytris luteis, punets . nigris paucis fparfis, Linn 0. Voet 21. 145. (10;8. bis. 133 Scarabaus Luteus, parvus, Linn. O. Voet 2 r. 150. ( 200.

I34 Scarabaus Luteus; an mas? Linn. O. ( 472.

135 Scarabaus Grifeus, elytris grifeis, maculis 4 atris ad bafin elytrorum, Linn. O. ( 473 . 


\section{Insectes.}

se 8 Scarate a deux Couleurs, ou variett, tres petit, Linn: 0 : (684.

Ic9 Scarabe Chryfis, Linn. 55I. 49. Voet 8. 63. (585. bis: ro Sccrabé Jaunatre, les pattes devant velouté d'un veiu journ Liñ. O. ( 187.

III Searabé Longuemain, Linn. 549. 39. Voet 2. 97. ( 85.

I:2 Scarabé Acteon, ou femelle, Linn. o. Voet. 16i ( 186.

I1 3 Scarabe Grand, noir, les etuis fons poils, Linn. C. Voet 140. mais pius grand. (IIIS.

II4 Scaraib Grand, ou varieté, Linn. O. ( 768.

Ix 5 Scarabe devx Couleurs, Linn. O. Fabric. 38. 16. Voet 8. 66. (190. bis.

II6 Scarabt Pannipes; Linn. O. Goetz. Entom. 77. Voet 8. 64. $(193$.

II 7 Scarabe Vitris? Linn 0. Fabric. 41. 34. Voet 7. 56.57. ( 1043 .

I 8 Scaraté Verd, elytres flriss, Lino. O. (195.

119 Scarabé Bordé, Linn. O. Fabric. 25. 20. Voct 4. (282. $x 20$ Scarabe Elegant, Linn. O. Fabric. 56. 32. (192. ter.

I2 I Scarabe Blegant, ou le metmeque $n^{\circ}$. I I 8? Lind. o. (1098. bis.

122 Scarabe Scellè, Linn. O. Fabric, 54. xo. (685. bis.

123 Scarabé Scellé, ou varieté, Linn. c. (086.

124 Scarabé du Cap? on variete, Linn. 556. 73 ? Voet a. II. ( 730.

I25 Scarbé Lunaire, 'les étuis couleur de rouille, d deux taches. noires luné de blanc, Linn. O. (858.

I26 Scarabe Noir, les cuiffes derriere par defous veloutús, Linn. o. $(860$.

$x 27$ Scarabe Ligicipe, des Indes, Linn.o. Voet 4. 32. (206. bis. I28 Scarabe Noir, d corcelet roid Ė long, les étuis ridés, Linñ. o. $(658$.

129 Scarabél'Hiftrion, farve, Lin. o. Voet 3. 18. (1039 bis. 130 Scarabé Jaune, le corcelet noir Eं juune, les étuis jaznneatres, Linn. o. 1036.

131 Scarahé Jaune, on varieté Linn. O. (1037.

132 Scarabé Pointu, à tête noir, elytres jaumes, avec des poin. tes noirs Jparfes, linn. O. Voet as. 345. (IC38. tis.

I33 Scarabe Jaune, petit, Linn. C. Voet 2r. 150. (200.

J34 Scarabé Jaure, ou male? Linn. o. ( 472.

I35 Scaraic Gris, a elytres grifes, avec 4 taches noires dia bofe des etuis, Linn. o. (473. 
$78 \quad$ Injecta.

I36 Scarabaus Griferus, pracedenti affinis, Linn. O. (474:

137 Scarabæus Fufcus, elytris complanatis rufo fufcis, parvus, Linn. 0 ( 201 .

238 Scarabxus Virens, viridi cupreus, Linn, o. Gronov. Zooph. II tab. XV. f. II (194.

139 Scarabaus Niger. faf́cicularis, Limn. 0. 20. $(965$.

740 Scarabus Ater, magnus, tibiis duplico ferie fpinarum, Limn. O. (854.

I4t Scarabius Plinctatus, f. varietas, Linn. 557. 73. Voet 43. 18. (21. 593 . bis.

\section{U C A N I.}

142 Lucanus Interruptus, Linn. 560.4. Voet 29. I. (175.ter. 143 Lucanus Interraptus? Linn. 1. c. Voet 29.2. (IC97.

I 44 Lucapus Megolopthalmus, capite \& thorace atris, maxillis incurvis craffis, Linn. O. (85I.

145 Lucanus Tafferanius, Linn. O. Voet 30. 6. ( 852.

146. Lucanus Dorias, f. femina, Linn. O. Voet 29 4 (88r.

147 Lucanus Ater, maxillis compreffis fubtus carinatis lon. gitudine thoracis, Linn. 0. (189. bis.

\section{A S S I D E S.}

248 Gaffida GroJa, Linn. 577. 17. Sultz. Infect. tab. 3. I. ( 476.

I49 Caffida Grofla, an fimilis? Linn. 1. c. (534. bis.

150 Calinda Grolle? I. verietas, lutea, Linn. 1. co Voet 2. 42. 9 ( 535 . bis.

I5t Calfida Viridis, lineis coccineis media dimidia, Linn. 0. Voet 42. 14 (208. bis.

152 Caffida Grofjo, f. varietas, an ne. 150 ? Linn. 1. c. (207.

753 Caffida Groffa? ro varietas altera, Linn. O. ( $2 \mathrm{Ir}$.

154 Caffida Bipuftula, Linn. 5;8, 30. Naturforfch. VI. tab. 4. (209. bis.

I55 'affi la Bipsatula, Linn. 1. c. (210. bis.

1.56 Cafita Cymea. Linn. 577.22. Voet 42.13. ( 690.

157 Caffid Hebrea, Linn. O. Fabric. 109. 10? ( 856.

158 Caffida Sexpunctata, Linn. O. Fabric. 109. I3. Voet 43. 10. $(772$.

159. Caffida Septgutata, fimilis præcedenti, an no.157? Linn. $57 \% 19$ ? ( 563. 160 Calfida Bicornis, Linm. 576. 8. Voet 42. 5. (223. bis.
I6I Caffija 
36 Scarabé Gris, femblable au précedent, Linh. 0. (474:

137 Scarabé Brun, petite elytres réunis bruns, EO rouges, Linn. o. ( 20 r.

138. Scarabé Verdatre, ou verd-cuivreux, Linn. O. Gronov: Zooph. II. $t a b$. 15. f. II. ( 964.

139 Scarabé Noir, à fafcicules, Linn. O. (195.

140 Sarabe Noir, grand, de double ordre de fibres de l'efpine, Linn. o. ( 854.

14 Scarabé Pointé. 0u variétt, Linn. 557. 73. Vaet 43. 18. (21. 593. bis.

\section{U C A I N E S.}

142 Lucaine Interrompu, Linn. 560.4. Voet 29. 1. (175. ter. 143 Lucaine Interrompu, Linn. l c. Voet 29. 2. ( 1097. 144 Lucaine Mogolopthalme, la tbte 8 corcelet noirs, les machoires courbés épaijes, Linn. 0 . (851.

145 Lucaine Quarré, Linn. o. Voet $30.6 .<852$.

146 Lucaine Doreas, ou femelle, Linn. O. Voet 29. 4. (881. 147 Lucaine Noir, les machoires comprimez deffous caves, de la longitude du corcelet, Linn. O. (189. bis.

\section{A S S I D E S.}

148 Cafjide Grolte, Linn. 577. 17. Sulz. Infec. tab. 3. fig. I: ( 476.

x49 Caldide Groffe. Linn. l.c. (534. bis.

150 Caffide Groffe, ou varieté jaune, Linn. l. c. Voet 42. 19 ( 535 . bis.

151 Cafide Verd, dignes rouges de la moitie, Linn. o. Voet 42. I4. (208, bis.

152 ceffide Groffe, ors varieté de $n^{2} .150$ ? Linn. l. c. (207.

153 Caffide Groffe, ou varieté de la méme, Línn. o. (2ir.

254 Caffide Verd a deux puftules, Linn. 578. 11. Nacurforfch. II.tab. 4 (209. bis.

255 Cafide Verd a deux puftules, la meme, Linn. l. $c_{0}$ ( 2 10 bis.

156 Calfide Bleue, Linn. 577.22 . Voet 42. 13. (690.

157 Calfide Hebre, Linn o. Fabric, 109. 10? ( 856.

158 Cafide à fix Points, Linn. o. Fabric. 109. 13. Voet 43. 16 ( 772 .

159 Calbide à fept Points, la meme du precedent, ou $\mathrm{n}^{\circ} .157$. Jinn. 577. 19 ? ('563.

360 Coglide à deux Cornes, Linn. 576. 8. Voet 42. 5. (.223. bis. 
I6r Caffida Cruciata, Linn. 576. 9. Voet 44. 27. (214. I62 Caffida Inequalis, Linn. 578. 24. Naturforfch. XI. tab. 2 f. 5. (213. bis.

163 Caffida Lateralis, Linn. 578. 26. Sultz. Infet. tab. 3.2. (2).

164 Caffida Discoides, Linn. 578. 27. Voet 4I. 2. (212 bis. Ios Cafiida Lateralis, f. varietas, Linn. 1. c. Voet 43. 24. (roir.

166 Caffida Punctata, viridi chalybea, maculis Aavis nigro, punctatis, Linn. O. an Decudfata Fabric. 115. 48? (689. bis.

167 Caffida Punitata, fimilis precedenti, Linn. \& Fabric. I. c. (6or.

168 Calfida Puntata? Linn. O. Fabric. 110. 20.

I69 Caffida Cruciata, Linn. 576. 9. (590. bis.

170 Cafirda Purpurea, Linn. 575. 12. (591. bis.

171 Caflida Marginata Linn. 576 14. ( 592.

172 Caffida Atra, fronte humeris \& macula irregulari flavis, Linn. O. ( 1 roz.

173 Callda Spinifex, Linn. 576. 7. Voet 43. 19. (216. bis.

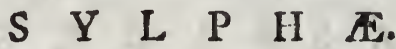

174 Sylpha Fndica, Linn. 570. 6. Linn. 3. (2x\%. bis. 175 Sylpha Bimaculata, Linn. O. Voet 48. 3. (482. b's. i75 Sylpha Nigra, labio emarginato, elytris porcatis, Lini。 o. $(967$.

\section{H R Y S O M E L E.}

I77 Chryfomela Unicolor, fusco rubra, Linn, o. ( 239.

178 Chryfomela Marginata, Linn. 591. 39. (24I.

I 79 Chry fomela Cordata, elytris rufis, maculis flavis cordi$\therefore$ formibus, Linn. O. Voet 31. 18. (242. bis.

180 Chryfomela Punctata, elytris rubris puizctis nigris $\mathbf{2} 2$. Linn. O. Voet 3r. 15. (Iros bis.

I8 Chryfomela Caftanea, rubro caftanea, capite atro, Linn. o. $(240$.

182 Chryfomela Gigantea, Linn. 586. I. Voet 33. 40 (233. bis.

I83 Chryfomela Gigantea, 1. varietas, flava, Linm. \& Voet. l.c. $(232$.

184 Chryfomela Gigantea? Linn. \& Voet. lo c. (231. bis. I85 Chryfomela 
161 Caflde Croiree, Linn: 576. 9. Voet 44. 27. (214.

162 Calfide Inegale, Linn. 578. 24. Naturforfch. $X I$. tab. 2. f. 5. (213. bis.

163 Cafide Jaterale, Linn. 578. 26. Sulcz Infect: tab. $3 \mathrm{f}$. 2. (214.

164 Cafsule Difcoide, Linn. 578. 27. Voet 4r. 2. (212. bis. 165 Colfide Laterale, ou varieté, Linn. l. c. Voet 43.24. ( 1 orr.

166 Cafjide Pointée, verd d'acier, da taches jaunes pointes de noir. Linn. O. ou Deculfate? Fabric. 115. 48? (689. bis. I67 Cafide Pointée de noir, la mêra de la precedente, Linn. Ẽ Fabric. l. c. ( 09 r.

I68 CaJjide Pointée? Linn. O. Fabric I10. 20. (215.

rog Caljide Croiré, Linn. 576. 9. (590. bis.

170 Co Jide Pourprée, Linn 576. 12. (59r. bis."

17 I Creflite Marginée, (Bordée) Linn. 576. I4. ( 592 .

172 CaJjide Noire, la tête éparles jaunes, d'une tacbe ircegu-: liere. Linn. O. ( 1102.

I73 Cafide Portant epines, Linn. 576. 7. Vcet 43. 19. (216. bis.

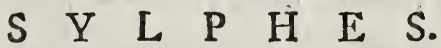

174 Sylphe des Indes, Linn. 570. 6. (217. bis.

175 Sylphe à Deux taches, Linn. 0. Voet 48 . 3. (482. bis. I 76 Sylphe Noir, d̀ levre bordé, d̀ elytres elevées, Linn. O. (267.

\section{H R Y S M. E L E S.}

177 Chryfomele d'un Couleur, bs un rougeatre, Linn. O. (239. bis.

178 Chryfomele Bordé. Linn. 59r, 39. (24I.

179 Chryfomele à Cœur, elytres où étuis rouges d taches jaunes. linn. O. Voet. 3r. 18. (242. bis.

180 Chryjomele Poincée, les elytres rorages, \& 12. points noirs, Linr. O. Voot 31. 15. Cros. bis.

$18 \mathrm{I}$ Chryfonele Chaitaigne, rouge, la tete roire, Linn. O. (́) 240 .

182 Chryfomeic Geante, Linn. 586. I. Voet 33. 4. (233. bis.

I83 Chryfonnele Gannte, ou varieté jaune de la mémé, Linno. Ẽ Voet l. c. (232.

I84 Chryomele Geante, Linn. \& Voet l. c. ' (231. bis. $x^{2}$ 
195 Chryfomela Gigantex, f varietas, Linn. l. c. (230.

186 Chryfomela Pustatas, Linn. 586. 3. Gronov. Zooph. IT. tab. I6.f. 7. (234. bis.

187 Chryfomela Nigra, lineis undulatis rubris', Linn. O. Voet 33. r. (235 bis.

183 Chryfomela Nigra? f. varietas lutea, Linn, C. (IIO4.

189 Chryfomela Nigra? C.varietas alia, Linn. O. (233.

Igo Chryfomela Gibbofa, Limib. 586. 2. Grorsv. Zooph. Il. tab. I4. f. 5. ( 237.

191 Chryfomela Gibbofa, fimilis precedenti, Linn. I. c. (238. bis.

192 Chryfomela Gisbofa? f. varietas lutea, Linn. 1. c. (238. bis.

193 Chryfomela Atra, fasciis 2 undatis luties, Linn. a ( 229

194 Chryfomela Atra, pracedenti fimilis, Linn. O. (228. bis. 195 Chryfomela Atra, f. varietas, Linn. O. Voet 44. (IIO3. bis.

ro6 Chryfomela Pronctata, oblonga, punctis 8 aurantiis, Lisin. ०. ( $\Sigma$ z6.

197 Chryfomela Feftiva, Linn. c. Fabric. 124. 47" Voet 31. 19. $(227 .$.

198 Chryfomela Feftiva, finilis pracedenti, Linn. ๑. (225. ter.

I99 Chry Tomela Atra, maculis marginalibus 4 flavis, Linn. O. $(243$.

200 Chryfomela Viridis, nitens, Linn. c. ( 696.

201 Chryfomela Annularis, oslonga, annulis 4 nigris, Linn. o. $(426$.

202 Chryfomela Giverfa, lutea, Lixm o. (694.

$\$ 3$ Curyfomela Oúlonga, lineis tribus undatis rubris, Linn. O. ( 1099.

204 Chryfomela Lineata, an fimilis pracedenti? Linn. O. (769. 205 Chrylomela Lineata, f. varietas, Linn. O. Voet 3I. 12. $\therefore$ (594. bis

\section{O C C I N E L L}

206 Coccinella Fusca, luteo punctis numerofis atris, Linn. o. (478. bis.

207 Coccinella Punctata, fusco brunnea 6 punctata, maculis atris, Linn. O. ( $77 \iota$.

208 Coccinella Macklata, Linn. O. Fabric. I. 98. \& 22. ( 896. 


\section{Injectes.}

I85 Chryfonele Geante, ou varieté de la méme, I.inn. l.c. (230.

186 Chryfomele d Cing points, Liun. 586. 3. Gronov. Zooph. II. tab. I6. fig. 7. (234 bis.

I87 Chryfoinele Noire, d̀ lignes rouges en ondes, Linn. $Q$. Voet 33. (235. bis.

188 Chryfomele Noire? ou varieté jaunes de la même, Linn. o. ( IIO4.

189 Chryfomele Noire? ou autre varieté de la même. Linn. C. (233. 190 Chryfomsle Boflu, Linn. 586. 2. Gronov. Zooph. II.tab. I4. $f .5$. ( 237.

I9I Chryfonnele Boffu, la même du precedent, Linn. l. c. (236. bis.

192 Chryfomsle Boflu? ou varieté jaune de la meme, Linn. l.6. (?38. bis.

193 Chryfomele Noire, d deux bandes ondés jaunes, Linn. O. ( 229 .

194. Chryjomele Noire, la même du precedent, Linn. O. (228. bis. 195 Chryjomele Noire, ou varieté, Linn. 0, Voet 44. (1103. bis.

I96 Cory fomele Pointé, allongée, à huit pointscouleurd'orange, Linn. ( 226.

I 97 Chryfonele Feftive, Linn. O. Fabric. I 24. 47? Voet 3I. I9. ( 227.

198 Cliryfomelie Fufive, la wême duprecedent, Linn. 0. (225 ter. I99 Chryjonele Noire, d guatres taches jatines margizales, Lini?. C. $(243$.

2co Chryjomele Verte, be!!?, Linn. O. (696.

201 Clirylome le Cerclé, longue, dq squaire ammeaux noirs, Linn. 0. (420.

202 Chryjomele Bolrue, jaune, I.inn. o. (694.

203 Chryfomele Allongés, d trois lignes cnderes rouges, Linn. 0. ( 1099 .

204 Chryfomele Raysé, ou la même de la precedente? Linn. 0. ( 760.

205 Chryfomele Rayée, ou varieté, Linn. O. Voct 3I. I2. (s94. bis.

\section{O C H I N E L E S.}

200 Cochinelle Brune. jcune, avec beaucoup de points noirs, Lian. O. $14 ; 8$. bis.

20? Cochineibe Pulutilies, Lrune, à fix points, taches noires, Linn. O. $77 x$.

208 Cochnelle Tachece, Linn. 0. Fabric. 98. 29.

$(8)^{\circ}$

CHARAN. 


\section{V R C V L I O N E S.}

209 C.irculio Palmarun, Lima 606. 1. Me ian. Swrin, tab. 49. f. 3. $(775$ bis.

2 ro Curculio Hemipterus, Linn. 606. 3. Voet 36. 9. ( 776. 857 . bis.

2 Ir Curculio Niger, fascia thoracis auiartia, Limm. 0. (706. bis.

212 Curculio Niger, Linn. O. an Cruentatus? Fabric. 162. 3. (778. bi:

213 Curculio Palmarum, f. varietas, Linn. I. c. (58:. bis. 214 Curculio Comatus, roftrovillofo, Limm. o. f. Barbiroftris? Fabric. 172. 65? Seb. IV. teb. 25. f. 5. (245. bis.

215 Curculio Bimzoculatus, thorace trituberculato elytris nigris, maculis 2. citreis, Linn. O. (2.77.

216 Curculio Scater, Linn. O. Fabric. 17\% 77. (60) bis.

217 Curculio Scaver, fo varietas, Limm. O. (21,6.

218 Curculio Punetulatus, thorace globofo, elycris nigris, puretis 4 albis, Linn. O. (477.

219 Curculio Punctatus, elytris muricatis, thorace punctato, Linn. o. an Scraber? Fabric. .... (972.

220 Curculio Hebes, Limn. O. Fabric. i74.80. (700.

221 Curculio Annulatus, Linn. 613.54. (913.

222 Curculio Muricatus, abdomine roturciato muricato, Linn. 9 . ( 734.

223 Curculio Muricatus, fo varietas, Linn. O. (974

224 Curculio Ater, albido maculatus, Limm. O. ( 732.733 . bis. 225 Curculio Nebulojus, Limm. 617.84. ( 248.

226 Curculio Nebulofus. fimilis, Linn l. c. (1107. bis.

227 Curculio Fuscus, albo marginatus, Linn. O. (gII.

228 Curculio Dispar? f. varietas, Linn. 613. 55? ( 696.

229 Curculio Dispar? an pracedenci affinis? Linm.l.c. ( 92 .

230 Curculio Breviroftris, thorace atro, elytris grifeis, Limon. 0. ( 249 .

23x Curculio Grifers, thorace albo magginato, elyuts punctis 2 niveis. Linn. O. (253.

232 Curculio Griftus, præcedenti affinis, Lim . O. (254. bis. 233 Curculio Grifeus, r. varietias, Limno. O. (255.

234 Curculio Betula, Linn. 6Ir. 39. (625.

235 Curculio Fuscus, Linn. O. (256.

236 Curculio Niger, ano albo, Linn o. (259. bis.

237 Curculio Niger. fimilis, Lim. O. (257.

238 Curculio Niger, fimi is pracedenti, Lin?. o. (258.

239 Curculio Varius, elytris purpureis, Linn. O. (618.bis. 


\section{H A R A N S O N S.}

209 Charanfon Pd!miere, Linn. 606. r. Merian Surin., tab. 4.f. 3. (775. bis.

210 Charanjon Heiniptere, Linn. co5. 3. Voet 36. 9. ( 776. 857 . bir.

2 I Chararjoin Noire, bande du corcelet eft orange, Linn. $\bigcirc$. (706. bis.

212 Charangon Noire, ou Ciuentatus? Linn. O. Fabric. 162.3. ( 778 . bis.

2 I 3 Chara gan Palmiere, ou varicté, Linn. l. c. (581. bis. 214 Charanjon Barbue, à bec velouté, Linn. O. Fabric. 172. 05\% Seb. IV.tab. 95. f. 5. (245. bis.

215 Charanfon Tacheice, le corcelet d trois tubercules, étuis noirs, do deux taches jaunaties, ou citrines, Linn. 0. (247.

216 Ciaaranfon Raboteux, Linn. o. Fabric. 174.77. (669.bis. 217 Charanfon Raboteux, ou varieté, Linn. O. ( 246.

2 r8 Chrranfon Pointille, le corcelet rond, étuis noirs, d 4 pointes, blunckes. Linn. O. (47\%.

2 Ig Charangon Pointée, les elytres froilis, ou chiffonne, le corcelet pointé, Linn. O. ou le Rajoteux? de Fabric. ... ( 972.

220 Charanfoiz Emoufte, Linn. O. Fabric 174. 80. (700.

22 I Charangon à Anneaux, Linn. 613. 54. (973.

222 Claranjon Herifte d eventre ronde, froiffi, Limn.o (734.bis.

223 Charanjon Herilfé, ou varieté, Linn. O. ( 974.

824 Chsranjon N sir, tacheté de blanc, Linn. O. ( 732.733.

225 Charais ois Nebuleux, Linn. 617. 84. (248.

226 Chararifon Nebuleux . le fareil, Linn. l.c. (1107. bis.

227 Charanfon Brun, bordé de blave, Linn. O. (orI.

228 Chura!fon Dispar? ou varieté, Linn. 613. 55? (696.

229 Charanfon Dirpar? ou varieté du precedent? Linn. loc. ( 9 ?6.

2 jo Charanjon Courte-bec, le corcelet noir, les étuis gris, Linn. O. ( 249.

23I Charanfon Gris, le corcelet bordé de blane, les elytres de deux bointes blancbes, Linn c. (253.

2.32 Cluardrjon Gris, le même du precedent, Linis. o. (254. bis.

233 Ch:ranjon Gris, ou varieté du méme, Linn. o. ( 255.

234 Charanfor Betule, Linn. 6ri. 39. (6)5.

235 Charanson Brun, Linn. O. ( 256.

236 Charanfon Noir, le derriere blanc, Linn. o. (259.bis.

237 Cbaranfon Noir, le pareil, Linn. 0 ( 257.

233 Charanfon. Nuir, le pareil du precedent, Linn. C. (258.

239 Claranjon Varié, les elytres pourpres, Linn. O. (618. bis. $\mathrm{E}_{3}$ 2,40 Charanjon 
240 Curculio Varizss, fimilis, Linn. 0. ( 617 . bis.

241 Curcuio Va:ius, fimilis, Linn. $0 . \quad(6,6$.

242 Curculio Varius? r. varietas, Linn o. (250. bis.

243 Curculio Varius, f. pracedentiaffinis? Limn. O (25:. bis.

244 Curculio Anckoraco, Linn. 613. 56. Gronov. Zooph. II. tab. 5 f. 4. (266. bis.

245 Curculio Apchoraco, Linn. \& Gronon. 1. c. (265. bis

246 Curculio Linearis. Linn O Fabric. 173. 69. ( 264.

247 Curculio Varius? f. varictas, Linn O. ( 202.

248 Curculio Variws, an fimilis pracedenti? Linn. O. ( 263.

242 Curculio Dispar, Limn. 613. 55. (260. bis.

250 Curculio Dispar, f. femina, Limn. 1. c. (26r.

25 I Curculio Breviroftratus, thorace fpinofo tuberculato, elytris rubris punctatis, atris numerofo, Linn o. (218.

252 Curculio Breviroftratus, f. fimilis. Linn. O. (219. bis.

253 Curculio Fafciatus, brevis thorace fulcato elytris nigris rubro fafciatis punetatis, Limn. O. ( 692 .

254 Curculio $F_{a}$ fciatzs, fimilis, Linn. O. (73\%. bis.

255 Curculio Ater, futura elytrorum dentata. Linn, O. (97r.

256 Curculio Fuscus, bievi elytris muricatis" fufcis, roft:o muricato, Linn. o. ( 925 .

257 Curculio Piceus, elytris fuicatis, punctis eleratis femoribus, fubtus villo tefiaceo, Liru. c. ( 568 .

258 Curculio Palmarum, f. yarictas, Linm.606.1? (587. bic.

259 Curculio Longiroftris, thorace tuberculato, elytris niģris, bafi \& apice maculis citreis fulratis, carinis fubmuricatis, abdomine lateralibus flavis, Linn. 0 . ( 247 .

260 Curculio Ater, elytris friatis albido marginatis, Linm. D $(26.4$.

261 Curculio Niger, albo pundtatus, Linm. O. ( 252.

\section{A $T$ T $T$ T E L A A}

2.62 Attc'abus Bifasciatus, fafciis 2 flavis, Linn.c. (920. bis. $20 ́ 3$ Attelabus Trifasciatus, fasciis fulvis. Linn.c. (295. tis. 204 Aitelabus Trifisciatus. r. yzrietas. Linn D. ( 294 265 Aitelabus Ater, macuia lunari faritis 2, Linn. 0. ( 660. 266 Attelabus Ater, fasciis 2. ut is. Lim C. (rio. bis. 26- Atrelabus Ater. P. varietas Linn. O. ( $7 \mathrm{r}$. bis. 268 Attelabus Lter, finilis, Linn. O (623. bis.

250 Attelabus Ater, an pracedenti afinis? Linn. C. ( $: 3^{5}$. bis. $27^{\circ}$ Attelabus Flazus, pu Ets elytrorum azticis $\sigma$. fasciajue nigris, Linn. c. 9980. 
27 Attelabus Flavus? f. varietas, Linn. c. (713.

272 Attelabus Niger. maculis flavo luteis, Linn. O.

273 Attelabus Fafcintus, e ytris anticis flavis pofticis luteis, tafcia atra. Linn. C. ( 872.

274 Attelabus Striatus, fasciis 2. luteis, Linn. o. ( 930.

275 Attelabus Siriatus, fimilis præcedenti, Linn. O. (293.

276. Artelabus Pimelia? elytris ellipticis carinatis, Linn. 0. (292. bis.

27.7. Artelabus Pimelia? pracedenti affinis, Linnı. O. (932. 278 Attelabus Pimeli. ? an fimilis precedenti? Limn. 0 . ( $95:$. 279. Artelabus Inordinatus, fafciis irregularibus, Linn 01979. 280 Attelabus Ater, thorace reflezo, Linn. o. ( 186 . $28 \mathrm{I}$ Attelatus Maculatus, thorace dilatato, maculiz grifeis, elyuis ramofis: Linn. O. ( 622 .

282 Attelabus Fulvrs, fafcia fulva, Linn。 O. (573. 929. bis. 283 Attelabus Fulvus, fimilis, Limn. O, (709. bis.

28.4 Atcelabus Fulvus, faiciis rubris, Linn. 0. (87I.

285 Attelabus Fulvus? an præcedenti fimilis? Limn: 0. (372.

\section{E R A B Y C E S.}

286 Ceramhyx Mirimus, elytris chalybeis ftramineo marainatis, Lins?. O. (48?.

287 Cerabbyx Furpureus, elytris purpureis nitertibus, fafçis 2. margineque flavis, Linn.o. (4i9.

288 Ceramby $r$ unctatus, elytris teftaceis, punctis 3. aureis, Livin. O. ( $48 \mathrm{r}$.

289 Ceranbyx Viridis, minimus. Linn. O. (483.

290 Cerambyx Pulex, elytris teftaceis, Lim. O. ( 307.

29 I Cerambyx Varius, vertice atro elytris fuscis, Linn. 0. ( 301 ? 937.

292 Cerambyx fuvencus, Linn. 6.31. 53. ( 302.

293 Cerambyx fuvencus? an firnilis piredenti? Linnn 1. c." (43?.

294 Cerambyx Ebtlinus? f. varietas, Linn. 637. 83? (625. bis.

295 Cerambyx Luteus, elytris 5. notatis, an Stigma? Limn. 635. 72 ? (306.

296 Cerambyx Serraticomis, Linn. 634. 65. (321.

297 Cerambyx Ramphygeus, teftaceus, elytris maculis 2. Havis, Linn. 633. 6T. ( 304 .

228 Cerambvx Surinamus, Linn. 632. 54. ( 320.

299 Cerambyx Surinamzus, fimilis. Linn. l. c. (3!9.

300 Cerambyx Surinamus? an præcedenti affinis? Linat. 1. c. (IIIC. 
271 Efcarbot Jaune, ou varieté du mêne? Linn. o. (7r3.

272 Efcarbot Noir, d taches jaunatres. Linn. O. ( 859 .

273 Efcarbot Bandé, les eiytres de devant jaunes. $E^{\circ}$ derriere jauneatres (couleur de boue) d bande noir, Linn. C. 1872.

274 Efcarbot Strié, d deux bandes jaunes, Linn. O. (930.

275 Efcarbot Strie, Semblable auprecedent, Linn. O. (:23.

276 E carbot Pime le, eiyzres elleptiques cavés, Linn. O. (292. bis.

277 Efcarbot Pimelis? le mlme du precedent. Linn. O. (932. 278 Efcarbot Pimelie? ou le pareil du precedent? Linn. 0 . (93. 279 Efcarbot Inordiné, bandes irregublieres, Limn. O (9i9. 280 Efcarlot Noir. le corcelet recourbe, Linn. O. (186. 281 Efcarbot Tacheté, le corcelet etendus daches griffes, elyeres branchues, Ling. O. $\quad(622$.

$282 E$ fcarbot Fauve, à bandes jarnes, Linn. 0. (573.220.6bis. 283 Efcarbot Fauve, ou le meme, Linn. 0. (709. bis. 284 E/carbot Fauve, d bandes rouges, Linn. O. (87i. 285 Efcarbot Fùve? ou femblable dus precedent? Linn. Q. (372,

\section{A P R I C OR N E S.}

286 Copricome tres Petit, elytres couleur d'acier bordés, pribía, Linn o. ( 480 .

287 Capricome Pourprè, elytres bordées de 2 bandes jaunes. Linn. 0. (479.

288 Capricome Pointé, les elytres de trois points dorés, Linn. 0. (481.

289 Capricorie Vurd, tres petit, I.inn. O. $(483$.

290 Capricarne Puce, elytres tefacćs, Linn. o. ( $10 \%$

291 Copricorne Varié, le Jonmet noir, les étuis bruzis, Linn o. ( 301 ? 937.

292. Capricome Juvenceau, ou Linn 631. 5. (302.

293 Capricorne Juvenceau? ou le ind me que le precedert ? Linn. O. ( 433.

294 Capricorne Ebuline? ouvariete, Linn. 637. 83? (625. bis.

295 Capricome Jaune, les elytres insrqués, cu Stigma? Lina. 635.72 ? $\quad 306$.

296 Capricome d Corne-dentelé, cu cu forme de fiié, Linn. 65. ( 321 .

297 Capricorne Rhamphyge, is teface dure, les clytres à cieux taches jaurzes, Linn. 633. 61. ( 30 .

298 Capricome de Surinam, Lim. 632. 54. ( 320 .

299 Capriconne de Surinan, lo meme Linn. l c. (3rg.

300 Capricorne de Susinam? o:l le muline da precedent? Linn. l. c. ( I I I10. 
30r Cerambyx Oceliatus, elytris luteis, ocellis albis, Limm ○. $(303$.

302 Cerambyx Raciris, (Bruchus), Linn. 605. 4. ( 878. 303 Ceramby $x$ Badris, f. femina, Linn. 1. c. (880. bis. 304 Cerambyx Bactris? an fimilis pracedenti? Linn. 1. $c_{\text {e }}$ 1879.

305 Cerambyx Ater, fascia coccinea, Linn. O. f. Rhagium Ornatum, Fabric. 230. 5? Noet 25. 136. (297.

300 Cerambyx Ater, punetis 3. Aavis, Linn. o. (334.

307 Cerambyx ....., elytris azureis, pedibus luteis, Linn. 0 , (307. bis.

308 Cerambyx Cyaneus, viridis, maculis 2. luteis in thorace ftriis 2. Juteis, Linn. O. ( 714.

309 Cerambyx Feftivus, Linn. 523. Ir. (484. bis.

310 Cerambyx Fefítivs? an fimilis præcedenti? Linm. l. c. (305. bis.

3r I Cerambyx Delinestus, elytris teftaceis albido marginatis, Linn. O. ( 318 bis.

312 Cerambys Moculatus, elytris fuscis, macula utrinque atra, $L i n n$. C. ( $\mathrm{C}_{62}$

313 Cerambyx Fuscus, thorace globofofufco, Linn o. (... 314 Cerambyx Crioceris, f. Chry?onela Polygina? tefaceus, Limi. 589. 24? ( 78 j.

315 Cerambjx Brwnneus, maculis atris, ad apicem 2. niveis, Linn. O. (49ז.

316 Ceranbyx Voetii, Limm. o. Voet 14. 39. (337. \& 595,

3I 7 Cerambyx Nitens, Linn. O. Fabric. 21x. II? (315.

318 Cerambyx Auricomus? C. varietas, Linn. 629. 40? $(: 09$.

319 Cerambyx Auricomus, fimilis, Linm. 1. c. (311. bis. 320 Ceranbyx afer, Linn. O. Fabric. 12. (940.

321 Ceramby Vittatus, Linn. o Fabric. 14. (310. bis.

322 Cersmbyx Aurricomus, Lins. 629. 40. ( 312.

323 Cerambyx Vitiatus? r. varietas, Limne 0 . ( 574.

324 Cerambyz Furinofus. Linn. 626. 24. Merian. Surin. tab. 2. f. 24 . (3) 3. bis.

325 Cerambyx Forinofus, fimilis, Linn. 1. c. (3I 4. b's.

326 Ceramby's Futinofus ? an ámills pracedenti? Linn. I. c. Voet 6. 12. (2 6. \& 48 \& bis.

327 Cerambyx Succinctus, Linn. O. ( 322 : bis.

328 Caramby x Succindus, Limn. O. ( $>23$. bis.

329 Cerambyy Succindus f. femina, Limin o. ( $48 \%$.

330 Cerambrx Ru'er, thor"ce fpinclu xubro, elytis nigris bafí rubis, Linn. O. Intt 8. 24. (490.

33I Ceranby x Rulier, Linno. O. Vuet l. c. (489. 


\section{Insectes.}

sor Capricorne Oeillé, elytres jaunes, à youx blaiches, Linn.'o. ( 303.

302 Capricorne Bactra, (la Bruche,) Linn. 605. 4. (878. 303 Capricorne Bactra, ou femelle, Linn. I c. (880. bis 304 Capricome Baftra? ou le metme diu precedent? Linn. I c (879. 305 Capricorne Noir, à bande décarlase, Linn. o. ou le Rhaugium Orné de Fabric. 230. 5 ? Voet 25. 136. (297.

306 Capricarne Noir, à trois points jaunes, Linn. o. ( 334.

307 Capricorne ...., de elytres azures, les pieds jaunes, Linn. o. (307. bis.

308 Capricome Verd bleu, $d$ deux tachles jannes, au corcelet deux firies jaunes, Linn. $O$ ( $7 I_{4}$.

309 Capricorre Feftive. Linn. 623.11. (484. bis

3ro Capricorne Feftive? ou le ineme du precedent? Linn. l.c. (305. bis.

3 II Capricome Defineé, elytres teftacés bordé de blanc, Linn. 0. ( 318 bis.

3 I 3 Capricorne Tacheté, elytres bruns à chaque coté tache noire. Linn. o. (c,62.

3I 3 Capricome Brun, le corcelet bo:fu rond, Linn. O. (...

3I4 Capriconze Criocere, tefacice, Linn. o. ou Chryfomele Polichon:e? Linn. 589. 24? ( 783 .

3 Is Capricorne Brun, à deux taches noires, ou fonnmet 2 blan. ches, Linn. O. (49r.

316 Cappricorne de Voet, Linn. O. Voet I4. 39. (337. 595.

317 Cepricorne Luifant, Linn. o. Fabric 211. I1? (3is.

318 Capricorne B!ondin? ou vurieté, Linn. 629. 40? (309.

319 Capricorne Blondin, Linn. l. c (3Ix.

320 Capricorne Afre, Linn. O. Fabric. II.12. ( 940.

321 Capricome Bandé, Linn. O Fabric. I4. (310. bs.

322 Capricorne Blondin, Linn. 629 40. ( 312 .

323 Capricorne Bandé? oib uarieté, Lim. O. 574.

324 Capricome Farineux. Linn. 626. 24. Merian Surinan. tab. 2. f: 24. ( $3 \pi_{3}$. bis.

325 Capricorne Farineux, le même, Linn. l c. (3r4. biss. 326 Copricorne Farineux'? ous varieté du precedcat? Linn. l. c. Voet, 6. I2. (316. 486. bis.

327 Capricorne Troufié, Linn. O. (322. bis.

$3 \geq 8$ Capricome Trouffe, Linn. 0 . (223 bis.

329 Capricorne Troufte, ou femelle, Linn. o. (437. bis.

330 Carpicorre Rouge, corcelet epine rouge, elytres noires, Linn. o. Voet 8 2s. (490.

33 I Cepricone Rouge, Lisr. O. ( $4 \& 9$. 
332 Cerambvx Ruber, fimilis pracedenti, Lim. o. Voet !. c (483. bis.

333 Cerambys Depreffus, Linn. O Voet 15.63. ( 332.

334 Cerambyx Scorpio, Limn. o. Fabric. 210.6. (333.

335 Ceranbyx Scorpio? f. varietas, Linn. O. ( 327

336 Cerambyx Ferrugineus, elycris piceis, punetis ferrugi neis, Linn. 0. (317.

337 Cerambyx Glaucus, Linn. 626. 28. de Geer. Inf. V. tab. 14. f. 4. ( 326 .

338 Cerambyx Fuvencus, fusco olivacaus, Linн. 631. 53. (329.

339 Cerambyx Depreffus, ro varietas, Linn, o. Voet tab. 15 62. ( 336.

340 Cerambys Mccobatus, elytris rufo fuscis, maculis atris, Livin. O. ( 325.

34 Cerambyx Lineatss, Linn. o. Fabric. 238. I3. (488 bis.

342 Cerambyx Nigtr, eiytris carinis 2. atris, Linn. 0. (324. bis.

343 Cerambyx Niger, fimilis pracedenti. Linn. o. (325. bis. 344 Cerambyx Niger? an fimilis pracedenti? Lim. 0 . (491. 345 Cerambyx Niger, C. mas, Linn. O. (492.

346 Ceramby $x$ Niger? f, yarietas, Lim o. (403.

347 Cerambyx Cinnamomets, Linn. 023. 10. Drury tab. 40. f. 2. ( 339 494. bis.

348 Cerambyx Thome, niger, margine luteo, Linn. 623. 9. (651. bis.

349 Cerambyx Ater, fasciis puncatis 2. albis, Linn. $\circ$. ( 78,5

350 Coranbyx Rubus, thorace fpinofo, elyeris maculatis, Linn. 625 21. Voet:3.54. ( 1060.

35 I Cerambyx Oculator. Linn. O. Fabric. 219. 19. Voet. 7. 21. $(557.626$ bis.

352 Cerambyx Hottentotus, Linn, O. Fabric. 220. 22. Voct $826 . \quad(737$.

353 Cerambyx Capenfis, elytris fafciis q̨uatuor rufis, Linn. 6:8. 36 Drury tab. 39. f. 3? (115.715 bis.

354 Cerambyx Niger, flavo maculatus, antennarum articulis pilofis, Linn. O. Vout 10. 39. (718.

355 Cerambyx Piceus, macu'is atris, antennarum articalis Ce- $^{-}$ cundis fpinofis, Linn. 0 . (504.

356 Cerambyx Pulcerrmirs, Linn o. Fabric. 217. 8: ( 576.

357 Cerambjx Sanguiners, corpoie nigro, Lirn. 635.80. Voet 9. 35. $(704$.

358 Ceramhyx fter, turciis 2.aurantis, Linn. O. (ro6ı. bis.

359 Cerambyx Ater, fimillis pracedenci, Linn. C. ( 1062.1064 . 360 Ceramby $x$ 
360 Cerambyx Armillatus, elytris ferrugineis nigro margina tis, Linn 622. 4. Gronov. Zooph. II.tab. 15. f. б. $\mathrm{C}^{6} \mathrm{OO}_{3}$

36I Cerambyx Varius, thorace cornuto, elytris cinereis antennis ́pinofis, Linn. O. ( 338.

302 Ceramb Fasciatus, Linn. O. Fabric. 21. Voet II. 49 A. ( 309 .

363 Cerambyr Hottentotus, Linn O. Fabric. 220. 22. (736. bis. 364 Cerambyx Hottentotus, fimilis, Limn. O. Fabric. I. c 1717.

365 Cerambyyx Hottentotus? C. varietas, Linn. O. ( 577.

360 Cerambyx Stigma, Linn 635.72. de Geer. Infect. V. tab 14. f. 3. ( 597 . bis.

367 Cera sibyx Stigma? an præcedenti affinis? Linn. \& de Geer. l. c. ( 244 .

368 Cerambyx Stigma? f. varietas, Linn. 1. c. ( 599.

369 Corambyx Stigma? an præcedenti fimilis? Linn. 1. c. $(596$.

370 Cerambys Miles, Linn. O. Voet I. 3. (...

378 Ceram yx Coriarius, corpore piceo, Linn. 622. 7. Voet 3.9. $(340$.

372 Cerambys Coriarius, fimilis præcedenti, Linn. l. c ( 328.

373 Cerambry Brusmeus, thorace unifpino?o, elgtris apice bifpincfis caffeis, Linn. O. (1057. 1058. bis.

374 Ceramoyx Ater, elytris bifpinolis, maculis tribus albis, Lim 0. ( ..66\%. bis.

375 Crraubyx Voetii, Limn. O. Voet 8. 26. (350. bis.

376 Ceramby $\times$ Piceus, th race şuadrifpinoli incurvis, ant nnis ferrats, Linn. O. ( 340.

377 Cerambys Longimanus, Linn. 62I. I. Röf.II. tab. I. A. ( 344 . bis.

378 Ceranbyx Longimanus, f. femina, Linn 1. c. (143. bis. 379 Ceranbyx Cervicorris, Linn 622. 3. Merian Surin. tab. 43. ( 33 i.

380 Cerambyx Cerviconis, f. femina, Limm 1. c. (627.

38 I Ceramtyx Cervicornis, f mas, Linn. 1. c (345. b.s.

382 Ceramby Cervicomis? f. varietas, Linn. 1. c. ( 345 . 34 . ter.

\section{E $\quad P \quad T \quad V \quad R \quad A E$.}

383 Leptura Viridis, tharace cylindrico, Limm. c. (1050. bis $38+$ Leptura Viridis, firnilis piacede:ti, Linn. o. ( 1051 . 385 Lepiura Parpurea, Linzs. O. (1052. bis. 
360 Capricorne \& Bagues ou Braffelets, elybres couleur de rouille, burdés de noir, Linn. 622. 4. Gronov. Zooph.II. tab. 15. f. $6 \quad(603$.

30 I Capricorne Variś, d corcelet cornu, les étuis cendrés, Eे les unternes épineuses, Lirn o. ( 339 .

362 Capncorne Bande, Linn. c. Fabric. 220. 2r. Voet Ir. 49. A. ( $3<8$.

363 Capricorne Huscenote, Linn.o Fabric.220. 22. (736. bis. $50+$ Capricorne Hottentote, le mêne du precedent, Linn. 0. Fabric 1. c. $\quad(717$.

365 Cepricurne Hotterstote? ou variésé, Linn. O. ( 577.

360 Capricome Stigma, Linn. 635. 72. de Geer Infeet. V. tab. I4. f. 3. (597. bis.

367 Capricorne Srigma? ou Jemulable du precedent? Linn. EP de Geer l.c. (244.

363 Capricorne Stignaa? ou varieté, Linn. l. c. (598.

369 Capriccrne Stigma? ous Jemblable du precederst? Linn. l. ca. (596. bis.

370 Capricone Militaire. Linn. O. Voet r. 3. (...

37 I Capriconne Corroyeur, à corps noiratre, Linn. 622. 7. Voet 3. 9 . $(340$.

372 Capricorne Corroyeur, Jemblable an precedent, Linn. b. c. 3. Q. $(328$.

373 Capricorne Brun. le corcelet d wne epine, d formet, \& les etuis à deux epines trusies. Linn. O. (1057. $10 \$ 8$.

374 Capricorne Noir, les etuis à deux epines, EO trois taches Hanches, Linn, O. ( ...66ri. bis.

375 Cappicome de Voat, Linn. O. Voei 3. 26. (350. bis.

376 Capricorne Couleur de Poix., le corcelet à quatre epines recuurtetes, bes antennes en frie, Lian. O. (340.

377 Cap̣ricorne Longue main, Linn. 621. Rôs. II. tab. I. A. (344. bis.

378 Cupricome Longue main, ou femeile, Linn.l.c (343 bis. 379 Capricorne Corne de Cerf, Linn. 023. 3. Merian. Surin. tab. 48. ( 331 .

350 Capricome oine de Cerf, ou femelle, Linn. 1. c. (627. 38: Cipricome Corne de Cerf, our quale, Linn. l. c. (345.bis. 382 Capricome Come de Cerf? ou varieté, Linn. b. c. (345. 346. ter.

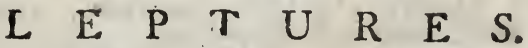

383 Lepture Verte, d corcelet cylindrigune, Linn. o. (1050 bis. 384 Leptuie Verte, la méme du precederit, Linn. O. (105:. 385 Lepture Pourpiée, Linn. O. (1052. bis. 
396 Leptira Purpurea? an precedenti affinis? Linn. 0. (ros3. bis.

387 Leptura Nigra, fo varietas, Linn. 639. 14? (1054. bis. 388 Leptura Parva, an varietas pracedenti? Linn. 0. (1055. 339 Leptura Ariatis, Indicus, Linn. 0. (298. bis.

390 Leptura Arcuata? Indica, Limm.640. 2I? Sulz Inj. 5. 31. ( 186.

391 Leptura Notata, maculis 2 fulphureis, Linn. O. (I loga 392 Leptura Flava, thorace atro, eigtris luteis, Linn. o. (296.

393 Leptura Flava? f. varietas præcedenti, Lirn. Г. (296. 394 Leptura Flava? an præcedenti fimilis? Linn. 0. ( 624.

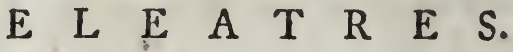

325 Eleater Micans, viridi aureus, Linn. o. (1047.

396 Eleater Phosphoreus, fuscus, Linn. 652. 5. de Geer IV. tab. I. 2. ( 272.

397 Eleater Ligneus, ferrugineus, Linn. 652. 7. ( 273.

398 Eleater Niger, thuracis lateribus flavefcentibus, Lirn. 0. (274.

399 H.leater Aterrimus, pusetatus, Limn。653. 17. ( 562. bis. 400 Eleater Aterrimus, fed major, Linn. I. c. (1045. bis. 4or Eleater Maximus, Linn. o. ( 588. bis.

402 Eleater Porcatus, fuscus, Limm. 652. 6. (270. bis. 403 Eleater Porcatus? f. varietas, Linn. I. c. (70r. 404 Eicater Porcatus? an pracedenti aftinis? Linn. 1. c. (269. bit.

405 Eieater Porcntus? \{. varitas præcedenti, Linn l.c. (589. 406 Eleater Speciofus, albus, maculis \& lineis purctatus, Linn. 65\%. 2. Voet 45. 32. (267. bis.

407 E'eater Speciofus? f. varieias, Linn. l.c. (1046.

408 Eleater Speciojus? an fimilis pracedenti? Linn. 0. (268.

409 Eleater Nocilucus, Limin. 65r. 4. ( 27 r.

\section{B V P R E T I D E S.}

410 Buprefts Gigantea, corpore inaurato, Linn. 659. I. Merian Surin. tab. 50. (279: bis.

4I Kuproftis Gigantea, præcedenti affinis, Linn. l. c. (280. bis.

412 Bupreftis Gigantea? r. varietas, Linn. 0. (62I. bis. 413 Bupreftis 
386 Lepture Pourprée? ou femblable de la precedente? Linn. O: (IO53. bis.

387 Leplure Noire? ou variete, Linn. 639. 14? (1054. bis. 388 Lepture Petite? ou varieté de la precedent $?$ Linn. 0 . (1055. 389 Lepture du Belier, des Indes, Linn. O. (298. bis. 390 Lepture Courbée en Arc, des Indes, Linn. 640. 21. Sulz. Int. tab. 5. f. 31. (186. bis.

$39 r$ Lepture Marquée, de derax taches jaunes, Linn. O. ( 110 . 392 Lepture Jaunatre, de corcelet noir, elytres jaunes, Linn.o. (296.

393 Lepture Jaunatre? ous varieté de la precedente? Linn. 0. ( $57 \mathrm{r}$.

394 Lepture Jaunatre? ou femblable d̀ la precedent? Linn. 0. (624.

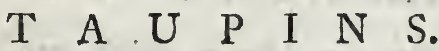

395 Toupin Re'uifant, verd-dore, Linn. O. ( 1047.

396 Taupin Phosphore, brun, Lirn. 652. 5. de Geer IV. tab. 18. f. 2. $(272$.

397 Taupin De bois, couleur de Rouille, Iinn. 652. 7. (273. 398 Taupin Noir, les cbtes du corctlea jounatres, Linn. 0. ( 274 . 399 Taupin Pointé, tres noir, Linn. 653. I7. (552. bis. 400 Taupin Pointé, tres noir, mais plus grand; Linn. lo c. (1045. bis.

401 Taupin Tres Grand, Linn. O. (588. bis.

403 Taupin Percé, brun, Linn. 652. 6. (270 bis.

403 Taupin Perces? ou varieté. Linn. l. c. (70r.

404 Taupin Percé? ou femblable aib precedent? Linn. l. c. (269. bis.

405 Taupin Percé? ou varieté du precedent? I.inn.l.c. (589

406 Taupin Specieux, blonc, à taches, Eै lignes pointées, Linn. 65i. 2. Voet 45. 32. (267. Lis,

407 Taupin Specievx? ou varietb, Linn. o. ( 1046.

408 Toupin Specieux, femblable au precedent, Linn. O. ( 268. 409 Taupin Eclairant la suit, Linn. 651.4. (271.

\section{B U P R E S T E S.}

410 Buprefle Geant, le corps doré, Linn. 659. I. Merian. Suin. $t a b .50$ (279. bis.

41 Buprefle Gear, , le mềne du precedent, Linn.l.c. (280.bis. 412 Bupreftc Geant? ou varieté, Linn. O. (62I. 
413 Bupreffis Gigantea? an ptacedentiaffinis? Linn 0. (70) bis.

114 Bupreftis Fascicularis, corpore inaurato hirfuto, Linn 66:. 12. Seb. IV. tab. 20. f. 5. (7a3. bis.

4I5 Bupreftis Fascicularis, fimilis præcedenti, Lim. 1. c. (564. ter.

416 Buprettis Fascicularis? f. varietas, pilis albis, Linn 66r. 12 ( 704. his.

417 Bupreftis Fascicularis? an pracedenti aflinis? Limn. I. c. (649.

$\$ 18$ Bupreftis Fascicularis, finilis præcedenti, fed minor; Linn. 1. c. ( 975.

419 Buprefti, Fascicularis? f. varietas, Linn. o. (976.

420 Bupreftis Hirta, corpore hiriuto, Linn 06$)$ I3? $(620$. 421 Bupreftis Mariana, Linn. 660. 6. Petiv. Gazopb. 2. 2. (976. bis

422 Bupreftis Depressa, Linn. o. Fabric. 276 12. (697. 423 Bupreftis Bicolor, Limn. O. Fabric. 273. 4. (291.

424 Bupreftis Obfiura, Linn. o. Fabric, 274. 9. ( 278. 425 Bupreftis Lineata, Linn. o. Fabric. 275. 13. (ro48. bis 126 Bupreftis Lineata? fo varietas, Linn. 0. Fabric. 1. c ( 1049. bis.

427 Bupreftis Chryfis, Linn。 o. Fabric. 275. 13. de Geer IV. tab. 17. f. 25 . ( 928.

428 Bupreftis Sternicornis; corpore inaurato, Linn. 660. 5. (275. bis.

429 Bupreftis Rutilaris, Linn. o. Fatric. 274. Ir. (277. 430 Bupreftis Fascicularis, Linn. 661. I2. (346. ter. 43 I Bupreftis Lineata? f. yarietas, fed major, Chinenfis, Limm o. (...

\section{I C I $N$ D E E L. AE.}

432 Cicindela Aequinoctialis, Linn. 658. 7. Voet. 40. 5. ( 289.

433 Cicindela Lurida, Linn. o. Fabric. 284. 9. (570. bis. 434 Cicindela Aequinozialis, eadem ac n². 432 . Linn. I. c ( 918 . bis.

435 Cicindela Capenfis, Linv. 657. 3. \&c Geer. VII. tab. 47. f. 3. (869. bis.

436 Cicindela Capenfis, fubienea, Linn. 1. c. ( 870.

437 Cicindela Interrupta, Linn. ๑. ( 780 . bis. 438 Cicindela Virginica, Limm. 657. 5. (I108. bis. 


\section{Infeates:}

13 Buprefte Geant? ou Jemblabic du precedent? Linn. O. (702. bis.

14 Buprefte à Bandes, le corps doré, velu, Lirn. 661. 120 Seb, IV. tab. 20.f. 5. (703. bis.

I5 Buprefte Fafciculaire, ou le meme du precedent, Linn. l.c. (564. ter.

I6 Buprefte Fafcié? ou varieté, velublanche, Linn. 601.12 ? $(704$ bis.

i7 Buprefte Fafc:é? ou femblable du précedent? Linn. l.c. (649. 118 Buprefte Faicié, le pareil du precedent, mais plus petit, Linn. ( 975 .

II Buprefte Fafcié? ou varieté, Linn. O. (976. t20 Buprefte Velu, à corps veloute, Linn. 66r. 13. (620. 2 I Buprefte Mariane, Linn. 660. 6. Petiv. Gazoph. 2. 2. (976 bis.

122 Buprefte Deprimé, Linn. 0. Fabric. 276. 19. (697.

123 Buprefte de deus Couleurs, Linn. 0. Fabr. 273. 2. (29.1. 424 Buprefle Obfcure, Linn. O. Fabric. 274. 9. (278. 425 Buprefte Ligné. Linn. O. Fabric. 275. 13. (1048. bis. 426 Buprefte Ligné? ou varieté das même, Liun. O. Fabr. l.c. ( 1049 bis.

427 Buprefte Chrylis, Linn. O. Fabric. 275. 13. de Geer IV. tab. 17. f. 25. (928.

428 Butrefte Sternicorne, le corps doré, Lind, 660. 5. (275.' bis.

429 Buprefte Refplendiffant, Linn. O. Fabric. 274. II. (27\%. 430 Buprefte Bandé, Linn. 661. 12. (346. ter.

431 Buprefte Ligne? or yarieté, plus grande, de la Chine, Linn. o. (...

\section{I C I N D E L E S.}

432 Cicindele Figuincetiale, Linn. 658. 7. Voct 40.5. (289)

433 Cicindele Livide, Linn. O. Fabric. 284. 9. (570. bis. 434 Cicindele Aquinoctiale, la même que n.432. Linn. bo ce (Q18, bis.

435 Cicindele du Cap, Linn. 657. 3. de Geer VII. tab. 47. 3. ( 869 bis.

436 Cicindele du Cap, un pers cuivreux. I.inn. I. c. (870.

437 Cicindele Interrompu, Linn. O. (780. bis.

438 Ciciridele de is Virgine, Linn. 647. 5. (s r 108. 
C A R A B I.

439 Carabus Mylaris, giganteus, Linn. o. Pall. Injezt. T. IV. ${ }^{2} a b$. 3. f r. (916.

440 Carabus Viridis, parvus, Limn. o. ( 977.

441 Carabus Tobidus, Linn o. Fabric, 300. 2. $186 \mathrm{r}$.

442 Carabus Tabidus? fo varietas, Linno o. Fabric. 1. c. ( 863.

143 Carabus Tabidus? f. varietas, Linn. o. Fabric. 1. c. F $78 \mathrm{r}$. 444 Carabus Fufciatus, a'is atris, falcia alba, Limmo. (485. bis

445 Carabus Rubra, Linr. O. (ro56.

440 Carabus Scrutator? fo varietes, Linn. O: Falric. 239. I9. ( 717.

447. Carabus Ruficornis, Linn. O. Fabric. 24r. 17. ( 198.

448 Carabus Ruficornis? 1. varietas, Linn. O. Fabric. 1. c. (863.

449 Carabus Ruficornis? an varietas precedentis? Lixn. 0. (881.

450 Carabus Tabidus, Linm. o. Fabric. 3c0.8. (569) bis.

45. Carabus Sexgutratus, Linn.0. Fabric. 236.4. (549. bis.

452 Carabus Sexguttatus? f. varietas, Linin: O. ( 567.

453 Crrabus Quáriguttatus, Linno. O. Fabric, 236. 5. (850.

454 Carabus Untguttatus, Linn. 0. ( 707 bis.

455 Carabus Unigutatus? an fimilis pracedenti? Linn.: 0. Linn. O. ( 283 bi. .

456 Carabus Decemguttatus? Linn. 669. 10? Fubric. 236. 3. (284. bis.

457 Crabus Gernmatus, Indicus, Limm。 O, Falric. 300.9. (285.

458 Carabus Moxillatus, ruber, latus pedibus nigris, Linm. 0. ( 770 .

459 Carabus Saccharivora, Linn. O. Voet 33. 1. (802.

\section{TENEBRIO NES.}

460 Tenebrio Gigas, nigreicens, Linn.674. 1. (282. 46. 'Tenebrio Molitar. Linn, 674.2. (299.

462 Tenebrio Molitor? f. varietas Limn I c. (853.

463 Tenebrio Molitor? an finilis? Linn.1. c. (782.

404 Tenebrio Chalybeus, violaceus, Linn. 674. 3. (917. 


\section{A R A B E S.}

439 Carabe, ou Milaire gearte, Linn. o. Pall. Infect. ... Seb IV tab $3 \cdot f \cdot \cdot$. ( 916.

$440 C_{a}$ abe Verit, tres petit, Linn. o. (977.

441 Caraile Pourri, linn c. Fabr. 300. 8. (861. 442. Cralle Peurit? ou variete, Linn. O. Fabr. l. c. (863. 443 Carabe Pourn? ou varieté, Linn. O. Fabr, l. c. C (58 r. 414 Carabe Fafcié, d ailes noires, bande blanche, Linn 0. (4.5. bis.

445 Carahe Roune, linn o. ( 1056.

446 Carabe Scrutataire? ou varieté, Linn. O.Fabric. 239.19. ( 717 .

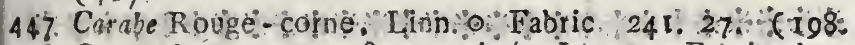
448 Carrobe Rouge-corne? ou varieté, Linn: o. Fabric. l. c. (863. i

449 Csrabe Ruge-corìe? ou varieté du precedent? Linn. 0. ( $78 \mathrm{I}$.

450 Carabe Paurí, Linn. O. Fabric. 3co. 9. (569. bis. 45 I Carabe a 6-Gouttes. Linn - O Fabric 236.4 . (549. bis. 452 Carabe äb'Gouttes? ou variete, Liño, 0. ( 567. 453 Cabe à quatre Gouttes, Linn. . Fabric. 236.5. (850. 454 Carabe dun Goutte Linn o. C $70 \%$. bis.

455 Carabe d'un Goucte? ou Semblakle du precedent? Liñn. C. (283. bis.

456 Carabe à dix Gouttes? Linn. 069.10? Fabric. 236.3. (284. bis.

457 Carabe Pretieufe, des Indes, Linn. O. Fabric. 300. 9. ( 285.

458' Carabe a Macheoire, rouge dy pattes noires, Linh, 0 . . 6779 .

459 Carate Sucre Vorant, Limni o. Voet 33. I. (862.

$$
\text { I E N E B I O N S. }
$$

460 Terel, ion Geant, noiratre, Linn. 674. r. (282;

461 Tenebrion Meunier, Linn. 674. 2. (299.

462 Tenelyion Meunier? ou varicté, Linn. l. c. ' (853.

4.53 Tenebrion Meunier, cu le pareil , Eimri, l. c. (732. 404 Tenebrion d'Acier, couleur, de viotette, Linn. 674.3. (917. 


\section{I I I S C I.}

465 Dytifcus Piceus, Linn. 664. 1. De Geer IV. p. 371.tabै. I. 4.12 . ( $5 n 7$.

566 Dytilcus Piceus, Linn. EO de Geer 1. c. $(010$.

467 nytifcus Marginatis, niger Linn. 665. 7. Röf.II. tab.9. $f$. 10. (9I1. bis.

408 Dytifcus Marginalis? f. varietas, margine rufo, Linn. 0. (96i.

469 Dytilicus Marginalis? an varietas præcedentis? Linn. 0. (rogr.

470 Dytifcus Latisfimus, niger, linea Hava, Limn, 665. 6. (84I, 47. Dytifcus Ater, Linn. O. (1023.

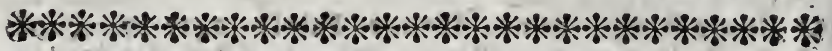

\section{$\begin{array}{lllllllll}H & E & M & I & P & T & E & R & A\end{array}$}

\section{B L A $\quad$ T $T$ T}

472 Blatta Americana, forrucinea, parva, Limn. 687. $40^{\circ}$ de Geer IV. tab. 44. 1. 3. (110.

473 Blatta Nivea, antennis flavis, Linn. 688. 5. Drury II. 1ab.36.f. I: $(532$.

474 Blatta Heteroclita Pallafii, Linno O. ( 924.

475 Blatta Atra, maculis 3 luteis, Li:n. O. (4i5. "Vide infra $n 3^{\circ} \cdot 800-667$.

\section{$M A \cdot N$ T I D E S.}

476 Mantis Gigas, Linr.689. 1. Roj. II.tab. 19. (59.60. bis. 477 Mantis Gigas? perfeclus, Linn. \& Röj. I. c. ( 512.

478 Mazatis Necydaloides, Linm. 1091. I4. R8j. II. tab. 19: (57. bis.

479 Mantis Iinearis, Linn. C.Petiv. Gazoph. 60. 2. (118, bis. 480 Mantis Spinofa, corporetrigono, Spinofo, cáuda latiore, Liño. 0. ( $6 \mathrm{I}$.

48 I Mantis Linearis? r. varietas. Linn. o: ( 570.

482 Mantis Bifpins/a, Linn. O. Fabric. 274 4. (456. 483 Mantis Atroptzica, Linn. o. Pall. Spicil..... (812. 484 Mantis Necydaloiles, Linn. 69I. I.f. Röf. II tab. 19. (58. 485. Mentis Irrorata, Linas 690. 7. ( 520.

486. Niantis Aromatica? F. varzetas Giga? minor, viridis, Lim. 689. I? (642. bis.

487 Mantis Siccifulia, Linn. 689. 3. Edww.258. (4n.510. bis. 488 Mantis 


\section{I $T$ I $S$ Q U E S.}

465 Dytisque Couleur de poix, Linn. 664. 1. de Geer IV. $p$ 371.tab. 14.f. 12. ( 507.

466 Dytisque Couleur de poix, Linn. Es de Geer l.c. (910.) 467 Dytisque Bordé, noir, Linn. 665. 7. Roef. II. tab. 9. $f$. 10. ( 9 II bis

468 Dytisque Bordé? ou varieté, bord rouge, Linn - (96I. 460 Ditisque Burdé? ou varieté dus precedent?'Linn. o. (109r. 470 Dytisque Tì ès large, noir, \& bande jaune, Linn. 665. 6 . (84r.

475 Dytisque Noir, Lian. O. (102):

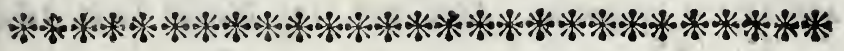

$\begin{array}{lllllllllll}H & E & M & I & P & T & E & R & E & S .\end{array}$

B $L A$ A $T$ T E S.

472 Blatte Americaine, coubleur de rouille, petite, Linn. 087.4. de Geer $l V . t a b .4 \overline{4} . f \cdot 1-3$. (110.

473 Blatte Blanche, antennes jaunes, Linn. 588, 5. Duvry

I II. tab 36.f. I. ( 532 .

474 Blatte Hetaroclite de Pallas, Linn. o. (924.

475 Blatte Noire. \& trois tacbes jaunes, Linn. 0. ( 475 .

"Voyes deffous n०. 660.-667.

\section{A N T E S.}

476 Mante Gaate, Roer. II. tab. 19. ( 59.60 . bis.

477 Munte Geante, le neme, parfait, Linn. EO Roer. l. c. (512.

4,8 Mante Nicidaloide, Linn. 691. I4. Roef. II. f. I9. (57. bis.

479 Mante à Lignes, Linn. O. Petiv. Gazoph. 60.2. (118. bis. 480 Marte Epineure, dicorps triangulaire, la queue plus large que l'epine, Linn. o. (6r.

$48 \mathrm{r}$ Mante à Lignes? ou varieté, Linn. o. ( 570.

482 Marte d deux Epines, Linn. O. Fabric. 274. 4. (456. 483 Marte Atrophique, Linn. o. Pall. Spicil. 274. 4. (812. 484 Mante Necydaloide? Linn. 691.14. Roef. II.tab. 19. (58. 485 Mante Arrorée, Linn. 690. 7. ( 529.

486 Mante Aromatique? ou variete de la Geante? plus petite verte, Linn. 689. I? (6.2. bis.

487 Mante Sechefeuille, Linn.689.3. Edw. 258. (10.654 bis. 
488 Mantis Siccifolia, élytris latioribus, Linn. 1, c. (530.

489 Mantis Siccifolia, f. Larva. ( 48.

490 Mantis Sicrifolia? [. varistas, alis confolutis, Linn.0. (509.

49r Mantis Pelinicornis? defunt antennæ, Linn. 691. 10? (47.

492 Mantis Gongylodes, Limm, 690. 4. Röf. II, tab. 7. f. I. 3? (1012. 1044.

493 Mantis Siccifolia? r. varietas, Linn. o: ( 56.

494 Mantis Precaria Linn. 69r. 8. (50. bis

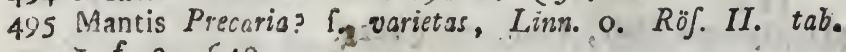
I. $f$. 2. ( 49.

496. Mantis Strumaria, Linn. 691. 13. Merian Surin. tab. $2 \%{ }^{\circ}$ ( 45.

497 Mantis Strumaria, r. Larva. ( 108.

493 Mantis Precaria? f. varietas, Linn o. (5r. 584. bis.

499 Mantis Oratoria, Lingi. 695. 6. (53,54. bis.

500 Mantis Oratoria? f. varietas, Linn. O. (723.

501 Mantis Oratoria, C. Larva. $\mathrm{C}_{3}$.

502 Mastis Orateria, f. Larva, fed. minor. ( 528.

503 Mantis Oratoria, f. Larra. (607. bis.

504 Mantis Oratoria, f. Larva. (ror3.

505 Mantis Oratoric? \& variétas, Limn.o. ( 527.

5006 Mantis Fufca, alis fufcis, Limm. O. (515. bis."

507 Mantis Perfêicus, alis diaphanic, macula marginali,

Linn c. Pall. Spicil ... (kg). bis.

508 Mantis Bifpinofa, Linq. O. Fabric. 374 3. (55.

509. Mantis Mendica, Lammo. Fabric. 275 7. ( 52.

sio Mantis Mendica? f. varietas. Linn o. Fabric. I. c ( 813 .

5 II Mantis Anvulata, alis late carinatis pedibus annulatis, Linn. 0 ( $5: x$.

512 Mantis Oratoria? alis ftramineis, Linn. 6006 ? ( 670 . bis.

513 Mantis Oratoria. Iniica, Linn. 1, c. ( 668 . bis.

5 rit Mantis Oratoria? fo varietas, Linn. O. ( 608.

\section{G R Y L L I.}

515 Gryllus Najutus, corpore viridi, Linn. 692. I. Röf. II.tab. 4. $(730.745 ., 785.817 .8: 9$ 1004. bis ; 8. Jpecim. 516 Gryllus Nafutus? f. varietas, Linn. I. c. (70.

5 I.7 Gryllus Fiafciotus, Limo o. ( 72.

5I8 Gryllus Faforatus? To varietas, Limm o. (82r. 
485 Mante Sechefeuille? ors variett de la meme, les étuis.plus. lasges, Linn. l.c. ( 530 .

489 Nante Sechefeuille, ou fa Larve. ( 48.

490 Mante Sechefeuille? ou varieté, les ailes enveloppés, Linn.

o. ( 509 .

491 Mante Corne d peigne? les antennes manquent, Linn.69r.

Io? (47.

492 Mante Gongylode, Linn. 690. 4. Roef. II. tab..7.f. I-3.

( IOI2. 1044.

493 Mante Sechefeuille? ou yarieté Linn. o. ( 56.

494 Mante Precaire, (Emprunié) Linn."69r. 8. (50.

495 Mante Precaire? ou varieté, Linn. o. Roef. II. tab. I. f. 2. $(49$

496 Mante, Ecrouellée, Linn. 69̣1, 13, Merian. Surin. tab. 27. $\{45$.

497 Mante Ecrouellée, ou Ja Larre. ( 108.

498 Mante Precaire? ou varicté, Linn. o. (5r. 584. bis.

499 Mante Oratoire, Linn. 690. 6. (53.54, bis.

500 Mante Oratoire? ou yariete, Linn. 0. (723.

$50 I$ Mante Oratoire, ou Jarve. ( 663.

502 Mante Oratoire, ou Lavve, mais plus petit. ( 528.

503 Mante Oratoire, ou Larve. (607. bis.

504 Mante Oratoire, ou Larve. ( $\mathrm{rOI} 3$.

505 Mante Oratoire? ou variete, Linn. o. ( 527.

506 Mante Brune, da ailes brunes. Linn. o. ( 515 .

507 Mante Diaphane, da ailes trailparentes, à tache marginale,

Linn. o. Pall. Specil... (609. bis,

508 Mante à deux Epir es, Linn. O. Fabric. 274. 3. ( 55 .

5с9 Mante Gueule, Linn. O. Fabric. 275. 7. ( 52 .

5 Io Mante Gueufe? ou varieté, Linn. o Fabric. l. c. (8 3 .

5 II Mante ì Ailes Cavées, les pattes avec des annearux,

Linn. o. (5il.

512 Mante Oratoire? ailes cosleur de paille, Lirn. 690. 16 ? Cerclé. (670. bis.

5I 3 Mante O atoire? des Indes, Linn. l.c. (668. bis. 514 Mante Oratoire? ou variete, Linn. o. (608.

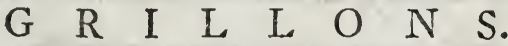

515 Grillon, ì Grand nez, (Moqueur) à corps yerd, Linn: 692. I. Roef. II. tab. 4. (730. 745. 785.817.819: ro 4 . Lis; 8 pieces.

516. Grillor M qu ur? ou varieté, Linn. l. c. (70.

$5 I 7$ Grillon Bandé Lirn. O. ( 72.

518 Grillon Bandé? ou varieté, Linn. o. (821.

G 5 
5ig Gryllus Fafciatus? f. varietas, Linn. o. (818. 520 Gryllus Brevicornis, Limn. 692. 2. (555. bis. 521 Grgllus Brevicornis? Linn. 092. 2 ? (106. 522 Gryllus Erevicornis? f. varietas, Lirn. 0. (724. 523 Grgllus Turritus, Lim. 692, 1. B. (749. 524 Gryllus Turritus? f. varietas, Linn. 0. (748. bis. 525 Gryllus Turritus, f. Larva. (1085. 526 Gryllus Striatus, Linn. O. (667. bis, 527 Grvllus Faftigiatus, Linn. 097. 29. (1113. 528 Gryllus Virens, pedibus viridibus, Limn. 0. (881. bis. 529 Gryllus Virens? f. vorietas, pedibus aurantiis, Linn. o. (74,

530 Gryilus Succinctus? f. varistas, Limm. 699. 36? ( 756. 53x Gryllus Reticulatus, Linn. 0. Fabric. 362. 7. (1005. 532 Gryilus Coriaceus, Linn. 697. 30. (753. 755. bis. 533 Gryllus Conocepbalus, Limn. 696. 19. (50r.

534 Gryllus Umhracula:us, viger, Linn. 695. 14. ( 946.

535 Gryllus Maculatus, alis macu'is quadratis nigris, Lim. c. $(675$.

536 Gryllus Maculatus? f. varietas, Linn。 o. (645.

537 Gryllus Orbiculatus, fcuiello orbiculari fubdentato, Linn. 0. 76 .

538 Gryllus Punttatus. Linn. O. Fabrici 364. 14. Drury II. tah. 4\%. f. 4 ( 46 I.

539. Gryllus Lamellofus, Linn. 696. 21. ( 75.

540 Gryllus Laniger, thorace trifido. femoribus lanatis, Linn. O. (822.

54 I Gryllus Sulcatus, thorace trifulco, Linn. O. (815.

542 Gryllos Sulcatus? f. varietas, Linn. 0 . (...

542 Gryllus Rubrefcens, thorace quadifulco rotundo, tibiis rubris, Linn. O ( 823.

544 Gryllus Migratorius, Linn. 700. 4'. Röf. II. tab. 24. ( 824 .

545 Givllus Tataricus? P. sarietas, Linn. 700.42 ? (751 bis. 546 Gryllus Tataricus? an varietas præcedentis? Linm. I. c. ( 752 .

547 Gryllus Coeruleus? alis nigro maculatis coeruleis, Liqun. $708,48 ?$ ( 1014.

\$48. Gryllus Rofius, thorace 4 fulcato, alis rofulis quadripitatis, Liun. O. ( 582.

549 Gryllus Criftatus? f. varietas, Linn. 699. 37? ( 752 .

550 Grulus Tatoricus, Limm. 700. 42 . (78.

55I Gryllus Succindzus, Linm. 699 36. (77. bis.

552 Gryllus 


\section{Infeates.}

519 Grillon Bandé? ou varieté, Linn. Q. (818. 520 Grillon à Courte-Corne, Linn. 692. 2, (555. bis.

521 Crillon à Courte-Corne, Linn. l.c. ( $: 06$.

522 Grillon à Courte-Corne? ors varieté, Linn. O. (724.

523 Grillon Klevé en Your, Linn. 692. 1. $\beta$. ( 749.

524 Grillon Elevé en Tour? ou varieté, Linn. 0. (748. bis

525 Grilion Elevé en Tour, ou Larve. ( 1085.

526 Grillon Striéz, Linn. 0. (667. bis.

527 Grillon Aigu1.é en pointes, Linn. 697. 29. (1113.

528 Grillon Verdeatre, d pattes vertes, Linn. 0.' (881. bis.

529 Grillon Verdeatre? ou variete, dd pattes d'orange, Linn.

O. $(74$.

530 Grillon Succincte? ou yarieté, Linn. 699. $36 ?$ (756.

53: Grillon en forme de Refeau, Linn. O. Fabric. 362. 7. (1005.

532 Grillon De Cuir, Lion. 697. 30. (753. 755. bis,

533 Grillon Corocephale, Linn. 696. 19. (501.

534 Grillons Ombragé, noir Linn. 695. 14. (946.

535 Grillon Tacbeté, les ailes d̀ taches quarrez noires, Lian. (645.

536 Crillon Tacheté? ou varieté, Linn. O. (645.

537 Grillon Otbiculaire, \& bouclier rond denté, Linn. 0. (76. 538 Grillon Pointéz, Linn. O. Fabric, 364. 14. Drury. II. tab. 41. 4. (46I.

539 Grillon dे Lammelles, Linn. 696. 23. (75.

540 Grillon Agneau, le corcelet fendu en trois, les cuis en laine, Linn o. ( 822 .

54x Grillon Sillonne, le corcolet a trois pointes, Linn. c. ( 875.

542 Grillon Sillonne? ou varieté, Linn. $\circ$ (...

543 Grillon Rougeatre, le corcelet rond à quatre pointes, Linn. c. $(833$.

544 Grillon Migratoire, Linn. 7c0. 41. Roef. II. tab. 24. 1824.

545 Grillon Tatariçue? ou varieté, Iinn. $7004: ?$ (751. bis.

5.46 Grilion Tataiigue? cuvarieté, de la precedente? Linn.l.e. ( 752 .

547 Grillon Bleu? les aibes tacheté de noir bleuatres, Linn. 70r: $48 \%$ ( III 4 .

548 Grillon Rougeatre, le corcelet à quatre points, ailes rouges quadruplez. ( 582 .

549. Grillon à Crece ? où varieté, Lir,n. 699. 37? (752.

550 Grillon Tatarique, Linn. 700.42. ( 78.

551 Grillon Succince, Linn. 699. 36 . (77.bis. 
552 Gryllus Succinctus? an præcedenti fimilis? Linm. 1. c.. (i 79.

553 Gryllus Succinctus? f. yarietas, Linn. O, (750.

554. Gryllus Maculatus, alis nigro maculatis, Linn o. (528.) 555 Gryllus Maculatus? f. varietas, fasciis 2 hya!inis, Linn. $\therefore$. ( $\mathrm{II}_{4}$.

556 Gryllus Serratus, abdomine coeruleo, Linn. 693.5. (459.

557 Gryllus Striaius ro Brevicornis? Linn. 692. 2? (901.

558. G!yllus Cariratus, Linn 093 6. (754.

$559 \mathrm{Grypl}$ us Quadrangulatus, alis maculis magnis, quadratis nigris, Linn, O. ( 644

500 Gryllus Tararicus? f varietas, Linn. 700. 42 ? ( 751 bis. 56:-Gryllứs Carinätus, cazina compreffa, ftris 2 atris, Linn? - ( 752 .

562 Gryllus Laurifolius, Linn. 695. 17. de Geer III. tab. $38 \div f .2$. (63.)

503 Gryllus Fufcus, anternis corpore longioribus, corpore fusco, Limo. O. (7I.

564 Gryllus Myrtifolius, Linn, 696. 18. de Geer III. tab. 38. $f$. 4. ( 816

565 Gryllus Maculatus, thorace maculis ovalibus impreffis, Linn. O. ( 647 .

566 Grŷllus Faftigiaius, Linn. 697. 29. ( 68 .

567 Giylus Elongatus; Linm. 606. 20. Röf. II. tab.. I8.-f. 7. $(65$.

508 Gryllus Myrtifolius, Limo. 696, 18. de Geer $I I I$. tab. :38. f 4 . (64 bis.

569 Gryllus Myrtifolius. P. Larva. ( ...

570 Gryllus Myrtifolins? f varietas, Linn. 1. c. (672) his?

571 Gryilus Myrtifolius, idem ac no. 565. Linn. 1. c. $(820$.

572 Grylus Papiltofus, thorace conico, Lirn o. Fabric.

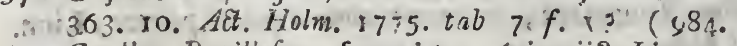

573 Gryllus Papiliofus, 1. varietas Aninarii? Linm. O. Fabrici 574 Gryllus Serratus, Linn. 693. 5. Sulz. Ins. tab. 8. f. 5. 8. $(559$.

575 Gryllus Acriminatus, Limn 696. 23. Sulz. Inj. tabi g. f. I. $(814,100$ o. bis.

575, Gryllus Coriaceus, Linn. 697. 3^. $(67$.

577 Grylius Rufofuscus, maculis pallidioribus, Linn o. (69. 578 Grylus Lobatus, femoribus lobatis, Limn. c. ( 646.

579 Gryllus Serratus, abdomine coe u'eo Linn.693. 5. (459. 580, Gryllus, Myrtifolius, Limn. 695. 18. (516. bis. 581 Grylles Tettigonia, f. Larva ejusdem, (... 582 Gryllus Myrifolius, Limm. 1: c. ( 820. 
552 Grillon Succinte? ou fewblahle du precedent ? Linn. l.c. ( $>79$. 553 Grilion Succirte? ou varieté, Linn. O. ( 750.

554 Grillon Tacheté, les ailes d taches noires. Linn. o (528. 555 Grillor: Tacheté? ou varieté, à deux bandes bleuses, Linn. 0. (II 4.

556 Grillon Serié, ventre bleu, Linn. 693. 5. (450.

557 Grillon Striéz, or Cource-Corne? Linn..692. 2? (gor.

558 Grillon Cariné, ou Cavé, Linn. 693. 6. (754.

559 Grilion Quadrangulaire, les ailes à grande toches quarres noires, Linn. O. (644.

560 Grillon Tatarique? ou varieté, Linn. 700.42 ? (751. bis. 561 Grillon Cavé, comprimé, Ė à deux ftries noires, Linn. O.' ( 752 .

562 Grillon ou Feuille de Laurier, Linn. 695. 17. de Geer III, ta\}. 38. 2. (63.

563 Grillon Brun, les antennes plus grandes aue le corps, ie corps brum, Lini. O. ( $7 \pi_{0}$.

564 Grillon. Myrthe Feuille, Linn. 696. 18. de Geer III. zab. 38. f. 4. ( 816.

565 Grillon Tacbeté, le corcelet imprimé de taches ovales. Linn. 0. (647.

566 Grillon Aiguité en pointe, Linn. 697. 29. (68.

567 Grillon Alongé, Linn. 696. 20. Roef. II. tab. 18. f. $7^{\circ}$ ( 65.

568 Grilion Myrthe Feuille, Linn. 696. 18. de Geer III. tab. $38 . f .4 . \quad$ ( 54 . bis.

509 Grillon Mlyrthe Feuille, on Ja Larve. (....

570 Grillon Myrthe Feuille? ou varieté, Linn.l.c. (672.bis. 57 I Grillon Myrthe Feville, ous be meme que $n^{\circ} .565$. Linn. l.c. $₹ 820$.

572 Grillon Boutonne, a corcelet conique, Lina. O. Fabtic. 363 10. Act. Hoim. 1775. tab, 7. f. I? (984.

573 Grillon Butonné, ou varieté des Aninaire? Linn. 0. Fabric. 369. 9. (....

574 Grillon Serté, Linn. 693 5. Sulz. Inf, tab. 8. f. 58. (559.

575 Grillon Pointu, Lian. 669. 23. Sulz. Inf. tab. 9. $f$. I, (8r4, roco bis.

576 Grillion de Cuir, Linn. 697 30. $(67$.

577 Grilion Brun Rouge, taches pales, Linn. o. (69.

578 Grillun Lobé, les cuifjes lobées. Linn o. ( 646.

579 Grillon Scrré, d̀ ventre bleu celefte, Linn. 693. 5. (459.

580 Grillon Myrthe Feuilie Linn. C96. 18. (516. bis.

581 Grillon Tettigone, ou fa Larve. (....

582 Crillon Mgrthe Feuilie, Linn. l. c. ( 820 . 
583 Grgllus Veficularis, viridis, albo guttatus, Linn. (558. 609. 984. bis.

584 Gryllus Succindus, Linn 699. 36. (610, bis.

585 Gryllus Succinctus? 1. varietas, Linn. 1 c. ( 727.

586 Gryllus Serratus, Linn. 1. c. Sulz. Inf. tab. 8. f. 5. 8. (458.

587 Gryllis Serratus? an præcedenti affinis? Linno 1. c. (66. bis.

588 Gryllus Serratus? alis expanfis, Linn. O. (987.

589 Gryllus Citrifolius, Linn. 695. 16. Röj. II. tab. 16.f. x. (62. bis.

s9o Gryllus Elethas, Linn. 699. 35. Röf. Inf. II. tab. 6, f. 2. (II).

59r Gryllus Tataricus, Linn. 700. 42. (87.

592 Grylius Viridisfimus? thorace rotundato, alis rubris, elytris viriditus, Linn. 698. 31? (460.

593 Gryl us Oculatus, minor, Lim. 696. 22. (ro88.

594 Gryllus Oculatus, fed major, Limn. 1. c. (1087.

595 Gryilus Oculatus? C, varietas, Linn. O. (1086.

596 Gryilus Verrucivorus, Indicus, Linn. 698. 33. ( 986.

597 Gryllus Carinatus? thozace tricarinato, alis maculatis, l.imn o. $(98$.

598 Gryllu Sulphureus, Linn O. Fabric. 369. 30. (5\$3.

599 Gryl us Aquilinus, Limm. 697. 27. Drury. II. tab. 44. (85.

600 Gryllus Carolinus, Linm. 701. 49. de Ceer III. tab. 4I. f. 2. 3. 282 .

601 Gtyllus Carolinus? f. yarietas, Linn. o. (...

602 Grylus Carinatus, Limm. 693. 6. (1007.

603 Gryllus Viridiflsmus, f. Larva (95I.

$60 \%$ Gryllus Fuscus, f Larva. (950.

6os Grylius Ater, C. Larva. (949.

606 Gryllus Fuc cus, f. Larva. ( 348

607 Gryllus Ferrugineus, thorace haftiz formi, alis fufcis, macuits ferrugiseis, Linn 0 . (I089.

608 Giy!lus Flavus, thorace fubcarinata, carina \& margine flavis, Lirar.o, (150r.

.609 Gryllus Fafciatus, thor ce fafciis 2. lateralibus marginaque a arum flavis, Linn c. (675.

6in Gryllus Miliaris, punctatus, Linn. 7 co. 30. (86.

6I I Gryllus Spinofus, thorace fipinofa, alis virefcentibus alb pur ctatis, Linn 0. (97.

612 Giyllus Spinofus? I varietas, Linn. O. (96. bis.

6I3 Gryllus Migratorius, Linno 700.4I. Röf. II. tab. 24. 1462.

614 Grylius Tataricus? f. varietas, Linn. 700, 42 ? (84. bis. 


\section{Inferter:}

583 Grillon Veficulaire, verte andé de blane, Lian. $0,(558$,

(609. 984. bis.

584 Grillon Succinte, Linn. 699. 36. (6to. bis.

585 Grillon Succince? ou variete, Linn. l.c. $(727$.

$586^{\circ}$ Grillon Serré, Linn. l.c. Sulz. Inf. tab. 8. f. 5. 8. (458.

587 Grillion Serré? ou varieté du precedent? Linn. l.c. ( 06. bis.

588 Grillon Serré? d ailes etendues, Linn. O. (987.

589 Grillon Feville de Citron, Linn.695. 16. Roef. Inf. II. tab. 10. f. r. (62. bis.

590 Grillon IElephant, Linn. 699. 35. Roef. Inf. II. tab. 6.f. 2. (119.

59r Grillen Tatarique, Linn. 700. 42 ( 87.

592 Grillons Emeraude? corcelet arrondé, les ailes rouges, ètuis vertes, Linn. 698. 31 ? (460.

593 Grillon Ocule, plus petit, Linn. 696. 22. (1088.

594 Grillon Oculé, plus grand, Linn. 1.c. (1087.

595 Grillon Oculé? ou varieté, Linn. O (1086

596 Grillon Gobe-Vers, des Indes, Linn. 698. 33. ( 986.

597 Grillon Caré? le corcelet d trois caves, les ailes tachetes Linn. 0. ( 98.

598 Grillon Sulphure, Lins. O. Fabric. 369.30 ( 513.

599 Grillon Aquilire, Linn. 697. 27. Drury -11. tab. 44 ( 85.

600 Grillon Caroline, Linn. 701. 49. de Geer III. tab. 4Li f. 2. 3. ( 282.

6or Grilion Caroline? ou varieti, Linn. c. (....

602 Grillon Cavé, (Cariné) Linn. 693. 6. (1007,

003 Grillon Emoraude, os ja Larve. (95x.

6.) Grillon Brua, ou Larye. ( 950.

605 Grillon Noir, ou Larve. (949.

006 Grillon Brun, ou Larve. ( 348.

007 Grillon Rougeatre, corcelet en forme de lance, ailes bruns it taches rowilleufes, Linn. O. (1089.

608 Grillon Jaune, le corcelet cavé, bordé de jaune, Linn. 0. (I508.

609 Grillon Bandé, le corcelet $E \geqslant$ deax bandes $\& 2$ bord des. ailes jarne, Lino. $0 . \quad(675$.

610 Grillow Miliaire, poinse, Linn. 700. 39. (86.

6I I Grillon Ep neus, be corcelet epineus, les ailes vertes poino tées de blanc, Linn. O. 697 .

6:2 Grillon Epineus? ou yarieté, Linn. o. (96. bis.

613 Grillon Migratoize, Linn. 200 41. Röf. II. Aab. 24. ( 452.

It Griban Tatarique? os variete, Ling. $730.4^{2}$ (84. bis. 
- I5 Gryllus Oculatus, oculis magnis, pallide marginatis, Linn. o. (8s.

616 Grgllus Striatus, elytris ftriis 3 obliquis, Linn. O. (80.) 617 Gryllus Hyacynthus, elytris hyalinis nigro punetatis, Linn. O. (82.

6r8 Grgllus Hyacynthes? f. varietas, Linn. O. (83.

619 Gryilus Indicus, zermanici varietas, Linn o. (90. bis: 620 Gryllus Flavws, Linn. 702.52. de Geer III. tab. 4I. f. 5. (89. bis.

62 I Gryllus Exoticus, germanici varietas, Lirn. 0. (898. bis. 622. Gryllus Morbillofus, Limm. 700. n 38. ( 648.

623 Gryllus Morbiblofws? f. varietas, Linn. 1. c. (649.

624 Gryllus Obfcurus, Lisn. 701. 50. de Geer III. tab. 4. f. 4. $(88$.

625 Gryllus Diaphanus, alis diaphanis, Linn 0. ( 95.

626 Gryllus Miliaris, Linn. $700.39 . \quad$ ( 882.

627 Gryllus $P$ undtutus, elytris fusco virentibus, alis rubris nigro punctatis, Limn. O. (99. 897 . ter.

628 Gryllus Striatus, thorace criftato, crifta nigra, elytris - fufcis, fasciis 5 flavis, Linn. O. ( 160.

629 Gryllus Aurifiacus, alis rubro aurantiis, Linn. O. (502.) 508.

630 Gryllus Flavus, Linn. o. (259.

63I Gryllus Miliaris? I varietas, Linn o. (288. ror4 bis.

632 Gryllus Succindus: Linn. 699.36. ( 93.

633 Gryllus Fuscus, tibiis portiris aurantiis, Linn. o. (1008.

634 Gryllus Italicus? [. varietas, Liтn. 701. 46? Rôf. II. tab. 2I. f. $\sigma$. ( $\sigma$.

635 Gryllus Hyalin 2 ? R. varietas altera, Linn. O. ( 92.

636 Gryllus Exoticus, Linn o. (884.

637 Gryilus Indicus, Linn. O. (94.

638 Gryllus Coerulefiens, Linn. 7co. 44. Sulm. Inj. tab. 9. f. 60. (673. 674.

639 Grullus Marginatus, thorace alis flavis nigro marginatis, apice hyacinis, Linn. 0 .

640 Grvilus Umbractiatess. Linn 695 14. (ror. 726. bis. 64 I Gryllus Umbraculatus? f, varietas, Linn o. (650.

642 Gryllus Campeltris, Linn. o. (102. 103.

643 Giyllis Pupus, Linn 699. 34. Röf. II. tab. 6. f. 3. (104. 557.602. bis.

044 Gryl'us Acheta, capite genubusque flavis, Linn. $0.65 \mathrm{I}$. 645 Gryllus Acheta. C. Larva. ( 463.

646 Gryllus Muricatus, crifta Cpinufa, Linn. o: (947. bis 047 Gryllus Tettigomis. R. Larva. (Ins.

648 Giyllus Tettigonia, f. Larva. (II $\sigma$. 


\section{Infertes.}

615 Grillon Oculaire, de grands yeux bordéz de blanc, Linn. 0. (81.

6I6 Grillon Strié, étuis d 3. Atries obliques, Linn. o. (80.

617 Grillon Hiacinte, étuis bleues pointés de noir, Linn.0. (82. 618 Grillon Hacinte? ou varieté, Lits 0 (83.

6:9 Gillon Indien, ou yariete de la germanique, Linn. 0. (90. bis.

620 Grillon Jaune, Linn. 702. 52. De Geer III. tab. 4I. f. 5 . (89. his.

621 Grillon Exotique, ouvarieté germanique, Linn. $0 .(898$. bis.

622 Grillon Rougeole, Linn. 700. 38. ( 648.

623. Grillon Rougeole? ou varieté, Linn. l. c. ( 649 .

624 Grilión Obfcure, Linn. 701. 50. De Geer 'III, tab. 4r. f. 4 . ( 88.

625 Grillon Diaphane, da ailes transparentes, Linn. 0. (95.

626 Grillon Miliaire, Linn. 700. 39. ( 882.

027 Grillon Pointé, à étuis brun verts, les ailes rouges pointees de blanc, Linn. O. (99.897. ter. 1

o2s Grillon Strié, à corcelet creté, d'un crete noir, les elytres truns d cin bandes jaunes, Linn. O. (soo.

629 Grillon Orange, les ailes oranges Lian, 0 . (502.508.

ถ3० Grillon Jaune, Linn. O (299.

63 I Grillon Miliaire? ou variete, Linn. o. (288. 1014. bis.

632 Grillon Troufre, Linn. 699.'36. (93.

033 Grillon Brun, les patses derrieres orangés, Linn.0 (1008.

634 Grillon Italien? ou yarieté, Linn. 7or? 46: ? Roef. II. tab. 2r. 6. (16

635 Grillon Hialine? os autte varieté, Linn. 0. (92.

636 Grillon Exotique, Limn. O. (894.

037 Grillon Indien, Linn. $0 . \quad$ ( 94

638 Grillon Bleuatre, Linn. 700. 44. Sulz. Infect. tab. 9. f. 60 . (673. 674.

639 Grillon Bordé, le corcelet, E' les ailes jaunes bordées de noir, Linn. o. (

640 Grillon Ombragé, Linn. 695. 14. (101. 726. tis.

641 Grillon Ombrage? ou variete, Linn. $0 . \quad$ (650.

642 Grillon Champetre, Linn. O. ( $\mathrm{IO}^{2} .103$.

643 Grillon Pupus, Linn. 699. 34. Roef. II. tab. 6. f. 3 . (104.557.002. bis.

644 Grillan Achete, la tête Eg genoux jaunes, Linn. o. (651.

645 Grillon Achete, ou Larye. (46?.

$6 \$ 6$ Grillon Aiguillion, ou forme de cliaulfe trappe, d'un crete epinerux, Linn o. (947. bis,

647 Grillon Tettigone, ou Larve. ( 105.

645 Grilion Tertigone, ou Larve. ( 106. 
64. Gryllus Pupus, f. Larva. (ro\%.

650 Gryllus Talpa, f. Larva. ( 613 .

65 I Gryllus Talpa, r. Larva, (608.

652 Grylius Onos vel Laxmanni? T. Preumora Thunbergit? Linn o (108.'. $80 \mathrm{r}^{3}$.

653 Gryllus Cinereus, nigro punctatus, Linn. o ( 757 .

654 Gryllus Indo-Domefticus, Linn. O. ( 826 bis.

655 Gryllus Indo-Domefticus? r. varieths, fascia flava, Litim. o. (827. bis.

656 Gryllus Indo-Domejticris? r. varietas pracedentis? Linn. o. $(6 \leq 2$. bis

657 Gryllus Indo-Domefticus? C, varietas, Linin. O. (rob. 952. bis.

658 Gryllus Capentis, f Talpa? Linn. 690. 34 ? (53т. 659 Gryllus Indo-Domeficus, f. Latva. (rog. Co1. bis.

\section{B $\quad$ L $\quad$ A $\quad$ T $\quad$ T $\quad$ E.}

660 Blatt2 Gigantea, Linn. 687. 1. Drury II. tab. 36. f. 2 (1092.

66r Blatta Aigyptiaca, atra, Linn. 687. 2? (953.

662 Blatta Americana, Linn. 687.4. Merien Surin. tab. т. (938.

663 Blatta Flava, thorace marginato flavo; Limb. O. (roro. 664 Blatta Maculata, flavo maculata, Linn. O. (IOII. ter. 665 Blatta f. Lorva cujusdam Blatte. ( 939.

666 Blatta r. Larva, Indica (1090.

657 Blatta f. Larva, alia. ( 1016 ror 7. bis. vide fupra $N^{\circ} \cdot 47^{2-475}$.

\section{F V L G O R $\mathbb{E}$.}

668 Fulgora Laternaria, Linn. 703. t. Roef. Inj. II. tab 28. f. 29. ( $\mathrm{x}$. bis.

669 Fulgora Candelaria, Limn. 703. 3. Rö. Inf. II. tab. 30. (2. ter.

670 Fulgora Flanmea, Linn. 704. 7. Stoll. Cisad. II. tcb. 2. (3.

671 Fulgora Tenebrofa, Linn. 0. Fabric. 674: \&. ( 6. 
649 Grillon Papus, ou Larve. (107.

650 Grillon Talpa, (Taupe?) ou Larve. (613.

65 I Grillon 'Talpa, (Taupe?) ou Larve. (608.

652 Grillon Onos, ors de Laxman? ou Pneumore de Thun. berg? Linn. o. (108 ou 80r.

053 Grillon Cendré, pointé de noir, Linn. O. ( 757 .

654 Grillon Domertique des Indes, Linn. o. (826, bis.

655 Grillon D imeftique des Indes? ou varieté, a bande jassre, Linn o. (827. bis.

656 Crillon Domettique des Indes? ou autre varieté, Linn. 0. (652. bis.

657 Grillon Domeftique des Indes?" ou vasieté, Linn. 0.) (iog. 952. bis.

658 Grillon du Cap, ou Ta'pa? Linn. 699. 34? (531. bis. 659. Grillon Dom=ftique des Indes, ou Larve, (109.601. bis.

\section{B L A T T E S.}

600 Blatte Geante, Linn. 687, I. Drury II. tab. 36. 2. (:092.

66r Blatte Egyptierne? noire, Linn. 687.2?' (953.

002 Blatte Americaine, Linn. 087.4 . Merian. Surin, tab. I. ( 938.

663 Blatte Jaune, à corcelet bordés de jaune, Limn. o. (10ro. 664 Blatte Tacheté, \& taches jaunes, Linn. $n$. (rorr. ter. 665. Blatte ou Larve de certaine Blatte. (939.' o66 Blatte ou Larve, des Indes. (ingo.

667 Blatte ou Larve, autre. (1016. 1017. bis."

"Voyez en haut, $N \cdot .472-475$.

\section{F U L G O R E S.}

668 Fulgore Lanterne, Linn. 7๑3. 1. Roef.InC. II, tab, 28. $f .>9$. (I bis.

969 Fulgore Chandellere, Linn. 703. 3. Roef. Inf. II. tab. 30. (2. ter.

670 Fulgore de Flamme, Linn. 704. 7. Stoll. Cicad. 1I. t. b. 2. (3.

671 Fuigore Tencbreux, Linn, 0. Fabric. 674. 8. (6. 


\section{I C A D A E.}

672 Cicada Fronditia, Linn. 705. 3. (5. bic.

673 Cicada Sangrinolenta, Lirn. 708. 22: (10.)

674 Cicada Carnifex, Linn. 0. Fabric. 088. 4 ( 128.

675.Cicada Spumaria, Indica, Linn. 708 24. (11.

076 Cicada Osellata?' f. varietas, Linn. O. Fubric. 682. r. (14.

677 Cicada Tibicen? f. varietas, Linn. 707.19? Merian Surin. táb. 49. (20.

6,8 Cicada Globulifera, Linn. O. Pall. Specil. Zoob. VII.f. I2. (420́.

679 Cicada Lanata, Linn. .7Ix. 42. Drury II. tab. 37. f. 3. ( 4 .

680 Cicada Nigra, alis fuscis, ocello albo, Linn. o. (5. bis. 68I Cicada Alba, ano lanato, elycrorum apicibus albo punctatis, Linn. O. (552. bis.

682 Cicada Prnztata, alis fuscis albido punetatis, Linn. o.

683 Cicada Nigra, thorace cornu incurvo, Linn.0. (7.8. ter 684 Cicada Carrulea, alis albidis cœrulescentibus, panctis nigis ad apicem, Linn. O. ( 19.

$685^{\circ}$ Cicada Phalenoides, Linno. 710: 40. Stoll. Cicad. II. tab. 2 f. 9. (15.

686 Cicada Crux, Linn. 705. 5. Stoll. Cicad.II.tab. 2. f. Is. (12. bis.

687 Cicada Sanguinea, Limi o. de Geer III. 'tab. 33. F. I7 ( $74 \mathrm{r}$. ter.

688 Cicnda Aurantia, dorfo flavefcente, alis fascia tiava, cr llari aurantio, Linn. O. (773.

689 Cicada Fusca, alis hyalinis, frontis fascia-rubra, Limn. O. $(17$

690 Cicada Fufca? I. varietas, abdomine fasciato, Linn. 0. (. I8.

6gr Cicada Subaurita, capite \& thorace viridibus, alis fuscc maculatis Linn. 0. (1082.

- 692 Cicada Grifea, alis maximis grifeis, textura nitidisfima Linn. n. ( 746.

693 Cicada Purictata, alis fuscis, albido punctatis, Linn. o. (...

694 Ci $a d a$ Guttata, alis carneis, maculis atris, Linn. 0 . (5)4. bis.

695 Cicada Tibicen, Linn. 707. 19. Merian Surin. tab. 49 (... bis.

6,6 Cicadi 


\section{I G A L E S.}

i72 Cigale Feuillue, verdoyante, Linn. 705. 3. (5. Bis.

i73 Cigale Sanglante, Linn. 708.22. ( 10.

j74 Cigale Bourreau, Lirn. 0. Faluric. 688. 4. ' 428.

75 Cigale Spumante, des Indes, Linn. 708. 24. (11.)

¡j6 Cigale Oculée? ou varieté, Linn. o. Fabric. 682. I. (14.

577 Cigale Tibicine? ou varieté, Linn. 70\%. 19.3 Merian. Surin. tab. 49 (20.

578 Cigale Globulaire, Linn. o. Pall. Spec. Zool. VII. $f$. I2. ( 429.

579 Cigale à Laine, Linn. 71 r. 42 , Drury Il.tab. 37.f. 3. (4. 580 Cigale Noire, les ailes bruns, yeux blancs, Linn. o. (5. bis. i8i Cigale Blanche, l'anus en laine, le Jommet des étris poirntée de blanc, Linn. O. (552. bis.

is 2 Cigale Pointée, des ailes brune pointées de blanc, Linn. 0.

i8 3 Cigale Noire, le corcelet à copne curbé, Linn. 0. (7. 8. ter.

84 Cigale Bleuatre, dailes blanches tirant fur le blew, des points noirs vers le foimmet, Linn. 0 . (19.'

85 Cigale Phaleonide, Linn. 710.40. Stoll. Cicad. II, tab. 2. $f .9 .-136$.

i86 Cigale Croix, Linn. 705. 5. Stoll. Cicad. II. tab. 2. f. 18. (12. bis.

is7 Cigale Sanguiné, Linn. o. De Geer III. tab. 33. f. 17. (74r. ter.

is8 Cigale d’Orange, d dos, \&9 ailes jaunes, Linn. 0. (773. i89 Cigale Brune, les ailes bleues, d bande rouge, Linn. 0. ( 17.

igo Cigale Brune? ou variete, d ventre bandé, Linn. o. . (i8.

in I Cigale Subaurite, la tête, Eg corcelet verd, les ailes tachetes de brun, Linn. o. (I082.

i92 Cigale Grife, a tres grandes ailes grifes, la texture tres propre, Linn. o. ( 746 .

io3 Cigals Pointée, les ailes brunes, pointées de blanc, Linn. 0.

ig4 Cigale Tacheté, les ailescharnels à taches noires, Linn. 0 ; (514. bis.

ig5 Cigale Tibicine, Linn. 707. 19. Meriąn. Surin, tab. 49. C... bis. 
695 Cicada Septemdecim, Linm. 708. 20. Philos. Trarsact. 1765. tab. 8. C igri, an 798. bis.

697 Cicada Capenjis, Linn. 706. 1 3. Petiv. Gas, tab 4. f. 1. (55c. 6) 8 Ciçada Capen fis? $\mathrm{f}$ varietas. Limn. 706. I3 ? (194. bis. 699 Cicada Capenfis? f. varietas pracedentis? Linn. O. ( $72 \mathrm{I}$. 7co Cicada Rubrescens, cospore belvolo, alis diaphanis, Lìn. 0. (21.

I01 Cicada Fusca, capite viridi, thorace fusco, alis hyalitis, Linn. 0: (s19. bis.

702 Cicada Fulca, fed majnr, nitida, alis hyalinis elytris ruei caltareis, Linn. co, (086. bis.

i03 Cicada Virens, alis diaphanis, Linn. o (...

7.04 Cikada $V$ eficularis, brunnea, clypeis veficularibus, Limn. O. $(553$.

705 Cicada Gijea, alis diaphapis margine punctato, Linn, 이 0.$)(500$

7os Cicada Grifea , 1. femina, Linn. o. (...

707 Cicada Virescens, capice clypeoque viridi, alts diaphanis, ○. Kivilin. 0. ( $\therefore$...

"C I M I C E $\quad \mathbf{E}$.

7c8 Cimex Gigas, thorace rotundato, elytris ferrugineis, Lizbial o. (.758. bis.

709 Cimex Feflivus, thorace purpureo rugofo, elytris medio viridibus, Linn. o. ( 750 , bis.

710 Cimex Purpureus, alis maculis viridibus, Linn o ( 759.

7 If Cimex Giboojus, luteus, a tis grifeis, apice albis, Linm. 0. ( 10.8 .

7:2 Cinex Truncotus, thorace aurito, cornubus truncatis, Lim. o. ( 1019.

7 r 3 Cinex Rotunius, capice, aliş̧ue atris, thorace fcutellogue flavis, Linm. o. ( 828 bis.

714 Ciasex Rotundrs, vel Grijeus, f. varietas, fucco nebulofus, alìs ftriatis, \& inacula atra trizona, Linn. o. ( 830 ,

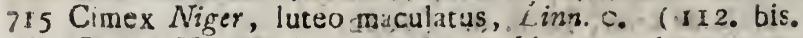

726, Ctmex Niger, thorace tubro, Linn. O. (11:3.)

717 Cimex Auritas, fusco ferrugineus, Linn. 0 . (814.

718. Cusex Auritus, viridis, pedibus flavis, Linn. c. (903.

7 ig Cinex Hirfusus, teltaceus, thurace fpinofo, Linn. 0. (120. bis.

720. Cimex Auritus, nitens, elytris luteo friatis, Limm. 0. (1021.

721 Cinex Luteus, alis viridibus, Limn. O. (117. 
5:6 Cigale Dix-fept, Linn : 708. 20. Philor. Transact 1765. tab. 8. ( 91 ou 793. bis.

697 Cigale du Cap, Linn. 7 \%. 1 3. Petiv, Gaz. tab. 4. f. Y. ( 550 . 098 Cigale du Cap? cil varieté, Linn. 708. 13 ? (194. bis.

6.99. Cigale du Cap? ou autre varieté de la méme, Linn. O (72r. 700 Cigale Ruseacie, le corps noir, . les ailes diaphanes, Linn. O. (21.

7or: Cigate Brune, la tête verte, le corcelet brun, les ailes blews, Linn. O. (519. bis.

zon Cigale Brune, mais pius grande, propre les ailes bleues, elysres chataignes; Linn. 0. ( 686 . bis.

703 Cigale Verdatre, d ailes transparentes, Linn. o. (... ${ }_{604}$. Cigale Veficulaire, brune, eculJons veficulaires, Linn., 0 . ( 553.

705. Cigale Grife, les ailes transparentes, le bord pointé, Linn. 0 . ( 500 .

706 Cigale Grife, on femelle, Linn. 0. (...

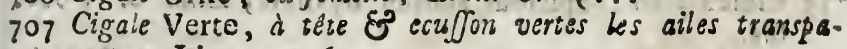
renites, Linn. 0. (...

\section{$P$ U N A I S E S.}

703 Punaife Gcante, s corcelet ronde les étuis couleur de rouille, Linn. O. (758. bis.

700. Punaife Feftive, corcelet pourpré, les etuis au milieu vertes, Linn o, $(760$. bis.

7 ro Punaife Pourpre-violet, les ailes d taches vertes, Linn 0. ( 759 .

7.I I 'Punoife Boftu, jaune, à ailes grifes, au point blanc. Linn. o. (ro18.

7r2. Punaife Tronqué, le corcelet doré, les cornes tronquées, Linn. o. ( 019.

713 Punaife Ronde, la tête E2 ailes noires, le corcelet, E'? l'ecusJon jaunes, o. (8z8. bis.

$7{ }^{4}+$ Punaife Ronde? ou Grife, ou varieté, brune mebuleufe is ailes ftriés. de tache noire triangulaire, Linn. o. ( 830.

715 Punaife Noire, borảe de jzune, Linn. O. (112. bis. 716 Panaife Noire, à corcelet rouge, Linn. O. (II3. 717 Punaije Aurite, brune rouillee, Linn. O. (814. 718 Punaise Aurite, verte, d pattes jatmes, Linn, O. ( 903. 719 Punaife Hiriute, teftacée, d corcelet epineux, Linn. 0. (120. tis.

720 Punaife Aurite, propre les clytres ftriez de ja:tne, Linn. 0. (102r.

721 Punaife Ja unatre, à ailes vertes, Linn. o. (II7. $\mathrm{H}_{4}$ 722 Punaife 
722 Cimex Viridis, a'is aqueis, Linn. o. (954. bis.

$723 \mathrm{C}$ mex Bengalenfis, Linn. O. (831.

724 Cimex Notatus, fuscus fubauritus, maculis pallidis, Linn.

o. $(76,832$. bis.

726 Cimex Flavrs, teftaceus, fcutello luteo, Linn. O. (464.)

727 Cimex Ruber, thorace \& alis rubris, puneto atro, Linn. 0. (121.

728 Cimex Striatus, teftaceus, elytris nigro ftriatis, Linn. 0. (115. bis.

729 Cimex Marmoratus, chalybeus flavo marmoratus, Linma c. (116. bis.?

730 Cimex Marmoratus? f. varietas, Linm. 0. (1094. bis.

$73 \mathrm{I}$ Cimex Ater, thorace \& alis luteo marginatis, Linn. 0. ( 956.

732 Cimex Punctatus, teftaceus, nigro punctatus, Linn. ¿00 (8:9

733 Cimex Ater, apicibus alarum hyalinis, Linn. O. ( 960. bis.

734 Cimex Marginatus, thorace enrarginato, fpinis fcutello perpendicularibus, Linn. O ( 833.

735 Cimex Marginatus? f. varietas, fed nigra, Linn. O. ( 834

736 Cimex Aurantius, elytris connatis inflatis, antennis in apice labii Linn o. ( 122.

737 Cimex Argus, leftaceus, thorace friis 2. undatis flavis, elytris ocellatis nigris, Limm. O. (123.

738 Cimtx Maculatus, teftaceus, thosace naculato, Linn.o. (958.

739 Cimex Stockerus, corpore viridi, maculis nigris, Llmm. 7 I5. 2 (IO22, ter.

740 Cimex Compreffus, antennis clava compreffa, Linn. 0. $(614$

741 Cimex Oblongus, thorace curito, lateribus fpinofis, Linn. o. (13r. bis.

742 Cimex Ollongus, thorace inermi, lateribus abdominis recurvis, Linn o. (28. bis.

743 Cimex Biguttatus, fed Indicus, Linn. o. ( 469.

744 Cinex Subniger, oblongus, fuscus, Linn. O. (127. bis:

745 Cimex Fuscus? [ varietas pracedentis? Linn. 0. (905.

746 C mex Oblongus, thorace aurito fpinofo, Linn. o. ( 388.

747 Cimex Muricatus, abdomine lateribus muricato, femoribus craffis bifpinofis, antennis clavatis, Limm. $O$. (240. bis.

748 Cimex Violaceus, fem ribus pofticis craflis, abdomine violaceo, Lism: O. ( I 42 . 
722 Punaje Verte"; di oiles aquatiques, Linn. 0. ( 954 . bis. : 723 Punaife de Bengale, Linn. o. '(831.

724 Punaife Marçuée, brune, de ailes pales, Linn. o. (76). 832, bis.

726 Punaife Jaune, teftacée, di ecuffon jaune, Linn o. (464.

727 Punaife Rougeatre, le corcelet , $\mathcal{E}^{2}$ ailes rouges, à point noir. Linn. O. C $: 2 \mathrm{I}$.

728 Punaife Striée, teftacée, les étuis ftriés de noir, Linn. 0. (II5. bis.

729 Punaife Marbré, conleur d'acier tacheté de jaune, Linn. O. (II 6. bis.

730 Punaije Marbrée? ou varieté, Linn. O. (1094. bis.

73r Punaije Noire, corceltet, $\mathbb{E}^{\circ}$ ailes bordés de jaune, Linn. 0. (956.

732 Punaife Pointée, tejtacée, pointée de noir. Linn. O (829.

733 Punzaife Noire, les pointes des ailes bleus, Linn. 0. (960. bis.

734 Punaife Bordée, corcelet debordé, ecuffon de epines perpendiculaires, Linn. o. ( 833 .

735 Punaife Bordée? ou varieté noire, Linn. o. (834.

736 Punaife Orange, les étuisjointes, antennes enflees. Linn. 0. ( 122.

737 Punaife A:gus, teftacée, le corcelet d deux ftries ondés jaunes, les étuis noirs. Linn. $O$. ( 123.

738 Punzife Tachetée, teftacée, le corcelet tacheté, Liiss. 0. ( 958

739 Punaije de Stoker, le corps verd à tackes noires, Linp. 715 2. (IO22. ter.

742 Punaife Comprimé, les antennes d'une clave conprimée. Linn. o. Coit.

74I Punaife Allongée, le corcelet auré, d̀ laterales épinersjes, Linn. C. (13!. bis.

742 Punaife Allongée, à corcelet inerme, les cotés du ventre recourbées Limn. o. (28. bis.

743 Punaife d deir Gourtes. Indienne, Linn. O. (460.)

744 Punaije Noiratre, alıongé, brun, Linn. o. (127. bis. 745 Punaije Bruse? ou varieté de la precedente? Linn. 0. ( 905.

746 Punaife Allongée, de corselet epinuex, Liun. o. (388.

747 Punase Poincue, le ventre courbé, des cuiffes épaifjes a deru épines, les antenmes pointues, Linn o. (240.) bis.

748 Punaife Vialerte, les cuilfes derriure épaifjes, ventre violet, Linn. 0. (142. bis.

$$
\text { H } 5
$$


249 Cimex Niger, tibiis unifpinofis, alis nigtis, Linn. 0. (144. bis.

750 Cimex Cinereus, abdomine luteo, Limn. O. ( 766.

75 I Cimex Striatus, thorace nigro rubro friato, Linn. O. ( 130 .

752. Cimex Muricatus? f. varietas, an idem acn ${ }^{\circ} .747$ ? Linn. O. (1095. bis.

753 Cimex Acanthinus, tibiis trifpinofis, thorace lateribus fpinofo, Linn. o. ( 12 ) bis.

754 Cimes Rufus, thorace rufo, bafi elytrorum puneto luteo, Linn. o. ( 763 .

755 Cimex Fuscus, femoribus repandis, thorace aurito, Linn. o. (677. bis.

756 Cimex Ferrugineus femoribus craffisfimis repandis, dente valido fpinofis, Lirmo. (

757 Cimex Ferrugineus? f. varietes, tibiis fublamellatis, Linn. ०. (1 26.

758 Cimex Phyllopus? f. varietas, Linn. O. ( 764.

750 Cimex Phyllopus? f. varietas, Linn. O. (14I.

760 Cimex Brunneus, nitens, auratus, 'pinis nigris, e'gtris ftriis 6. qui alternis luteis, Linn. c. ( 1020 .

761 Cimex Niger, abdomine rubru \& nigro marginato, Linn. o. $(83$ r. bi:.

762 Cimex Ater. totus niger, Linn. 0. (1093. bis.

763 Cimex Ater? f. varietas, Linn. o. (II4. bis.

764 Cimex Stockerus, Linn. 715. 2. ( 836 . bis.

765 Cimes Scarabcoides? Indicus, Linn. 716. 4? (606 bis.

766 Cimex Nobilis, Linn. 716. 3 (124. bis.

767 Cimex Nobilis? f. varietas, Linn. 1. c. (125.

708 Cimex Oblongus, thorace aurito, lateribus fpinolis, Linn. o. (...

769 Cimex Subniger, oblongus fuscus, Linn o. $\quad 905$.

770 Cimex Subniger? f, varietas. Linn. o. (....

77 I Cimex Viridis, thorace alisque viridibus luteo margina. tis, Linn o. (1 46 .

772 Cimex Ferrugineus, thorace ferrugines, elgcrorum lateribus fulvis, Linn. o. (134.

773 Cimex Ater, fascia lunulisque fulvis, Linn. o. (I 43 .

774 Cimex Virefcens, viridis, collari, \& margine thoracis rubris : Linn o. ( 147.

775 Cimex Niger, elytrorum bafi luteo, Linn. O. (133.

776 Cimex Niger? f. varietas, puncto atro in elytris, Linn. o. (i 38.

777 Cimex Coromandus, Linn. O. (904.

778 Cimex Perfonatus, pedibus anticis nigris, ceteris lureis, Linn. O. (145. ter. 
742 Punaife Noire, les pattes at une epine, les ailes noires, Lion. O. (144. bis.

750 Punaife Cendré, de ventre jaune, Linn. 0. ( 766.

751 Punaife Striée, le corcelet noir, à ftries rouges, Linn. 0 . (130.

752 Punaife Pointue? ou le méme de $n^{\circ} .747$ ? I.inn 0 . (IC95. bis. 753 Punaife diantine, pattes a troìs epires, corcelet epineux, Linn. 0. (129, bis.

754 Punaife Rouge, a corcelet rougeatre, la bafe des étuis a. point jaune, Linn. O. ( $>63$.

755 Punaife Brune, bes. cuiffes recourbèes, le carcelet aurite, Linn. O. $\mathrm{C}_{77}$. bis.

756 Punaife Rouillée. les cuiffes très epaifjes recourbées, d epines d'un dent fort, Linn. O. (702. bis.

757 Punaife Rouillée? ou $\int a$ varieté, les pattes d lanmelles, Linn. O ( 126

758 Punaife Phillope? ou varieté, Linn. $0 . \quad\left(764^{\circ}\right.$

759 Punaije Phillope? ou varieté, Linn. O. (14T.

760 Punaife Brune, de epines noires les ettuis à 6 Jtries, alterno tif jaunes, Linn. 0. ( 1020.

761 Punaife Noire, a ventre bordé de rouge, Linn. O. (831. Bis. 762 Punaile Noire par tout, Linn. o (1093. bis. 763 Punaife Noire? ou varieté. Linn. O. (II4. bis. 764 Punaife de Stokerus, Linn.715. 2. ( 836 . bis 765 Punaije Scarabeide. des Indes, Linn 756. 4? (606. bis. 766 Punaije Nobls, Linn. 716, 3 ( 124 . bis.

767 Punsife Nob' ? ou varieié, Linn. l. 6. (125. 768 Punaife Allongée, corcelet aurité, les côtés epineujes, Lino. o. (...

769 Punaife Noiratre, allongée brune, Linn. 0. (905.

770 . Punaife Noiratre? ou variete, Linn. O (...

77 Puizajje Vtrre, corcelet EO ailes vertes, borldees de jaunz, Linn i. ( 146.

772 Panaife Rouillée à carcelet acier, lescotes des étuis jaunes, Linn. o. (134.

773 Punaife Noire, $d$ bande jaune, Linn. O. ( 148.

774 Punaife, Verdeatre, do collier verd, $\mathcal{E}^{3}$ bord du corcelet rouge Linn. O. ( 1,47 .

775 Punaife Noire, la baje de l'etuis jaume, Linn. O. (133.

770 Prnaije Noirs? ou varicté, poirtés cic noir dans les êtuis g. Linn. 0. (133.

777 Panaife Coromandus, Linn. O. ( $(04$

778 Puraife Perfonnée, à paties de dsyant noires, bes autres jast nes, Limn, O." (145. ter.

779 Punaife 
779 Cimex Auranteus, thoracis margine rubro, elytris aurantiis, fascia atra, Linn. 0. ( 132.

280 Cimex Ater, fascia fulva, Linn. O. (135. bis.

$78 \mathrm{I}$ Cimex yirens, oblongus, elytris rabris, maculis 2. viridibus, Linn. O. (137. bis.

782 Cimex Ruber, elgtrorum macula nigra, Linn. O. (139.

783 Cimex Ruber, macula rotunda atra, Linm o. (839.

784 Cimex Ruber? f varietas, Limm. o. (83\%. bis.

785 Cimex Ruber, alis apice hyalinis, Linn o. (957. bis. 786 Cimex Ruber? f. varietas, macula alarum fulca, Linn: 0 . ( 840.

787 Cimex Ruber? f. varietas rrrecedentis? Linn. o. (837. bị. 788 Cimex Maculatus, antennis compreflis t iarticulatis, ca: pite \& pectore nigris, flavo maculatís, Linn. o. ( 149.

789 Cimex Luteus, thorace bicarina:o bispinof, Linn. 0. (533.

790 Cimex Spinula, teftaceus, Rpinulis horizontalibus, Linn. o. (465.

7.9r Cimex Grifeus, Linn. 0. ( 466 .

792 Cimex Punctatus, niger, thorace quadrifpinofo atro, aiis fuscis, punctis 2. aurantiis, Linn. o. ( 152.

793 Cimex Rubrescens, niger, bafi alarum rubro, Linn. 0. ( 153.

794 Cimex Planorbis, thorace plano, brunneo iubirigono, Linn. o. (615.

795 Cimer Punctatus. thorace convexo atro, bafi alartm puncto rubro, Linn. o (143.

796 Cimex Planus, thorace plano, rugoro, alis fuscis, Limm. o. (154.

797 Cimex Aculeatus, fusco virens, alis atris, ano aculeato, Linn o (765. bis.

798 Cimex Larvat s, cinereus, pedibus anticis longioribus, Linn. 0. ( $35 \mathrm{r}$.

799 Cimex Spinofus, capite \& thorace fpinis 2 erectis, Linn. o. ( 153.

800 Cimex Ater, pedibus villolis, Linn. o. (740. bis.

Sor Cimex Ater? fo varietas, Linn o. (136. bis.

802 Cimex Nebulatus? cinerescens, f. varietas præcedentis? Linn. o. (Iso.

803. Cimex Clavigerus, f. Larva Subaptera. ( ${ }_{\$} \$ 9$; his.

804 Cimex Clavigerus, f. Larya, vel vasietas precedentis? ( 988.

805 Cimex Cruciger, thorace luteo, cruce nigra, abdomine fulvo fasciato, Limn. 0. (150. bis. 
779 Punaife Orangée, 'le bord du corcelet rouge ; les iruis oranges d bande noire, Linn. O. ( 132.

780 Punaife Noire, da bande jaune, Linn. o. (135. bis.

781 Punaije Verte, allongee, les étusis rouges, d deusx taches, yeries, Linn. 0. (137. bis.

782. Punaife Rouge, les étuis d tache noire, Linn. o. (139.

783 Punaije. Rouge, at tache ronde noire, Linn. o. (839.

784. Punaife Rouge? 016 varieté, Linn. O. (838. bis. 785 Punaife Rouge, les siles au point, bleus, Linn. 0 . ( 957. bis.

780 Punaifo Rouge? ou varieté, les ailes d toche brune, Linn. O. (840.

787 Pamaife Rouge? ouvarieté de la precedente? Lino. o. (837.bis. -88 Punoife Tachetcee, ontennes comprimés a trois articles, le rtte, EO poitrine noires, d tacbes jounes, Linn. 0. (149.

789 Punaife Jaune, le corcelet à deux caves, E' deux epines, Linn. O. ( 533.

790 Punaife Epineufe, tefaccée, à epines horizontales, Linn. o. (465.

igl Punaife Grife, Linn. o. ( 466.

79. Punaife Pointée, migire, le corcclet dे 4 epines noires, is deux pointes brunes, 2 diorange, Linn. o. ( 52.

793 Punaife Roujeatre, noire, ba baje des ailes rouge, Linn. 0. (x53.

234. Punaife Applati, d corcelet plat, brune, triangulaire, Linn. o. (615.

795 Prnaife Pointé corcelet courbé noire, la bafe des ailes d point rouge, Lino. o. (143.

796 f unaife Platte, dorcelet applati, ridéz, les ailes brunes, I.ion. O. (154.

797 Punais: Puintue, brun yeste, les asilesnoiges, l'anus pointé, Linn. O. (765. bis.)

798 Punaife Larvée, cendrée, d pattes longes de devant, Liñ. o. (851.

299 Punaife Epineufe, la tete, EO corcelet deux epines drojtes, Linn. o, ( 153

8 on Punaife Noire, les pattes veloutés, Lino. O. (740. bis.

Bor Punnije Noire? ou varieté. Linn. o. ( 136 . bis.

802 Punaije Nebuleufe? cendrée, varieté de la precedente? Linn. ". ( 150.

803 Punnife Porte-cloux, f. Larve, ow Subaptere, (I59. bis. 814 Punaife Porte-cloux? ou variete de le precedente? ( 988. 805 Funaife Porte Croix, le corcelet jaune, d croix noir, le vestre bandez de jaune, Linn. o. (156. bis. 
806 Cimex Cruciger? t. varietas præcedentis ? Linn. 0. (162: 807 Cimex Pillo/us, thorace lanato, abdomine incifo, Linm. i. $(467$.

808 Cimex Criciger, thorace luteo, abdomine fulvo marginato, Linn. o (157. bis.

809 Cimex Ater, thoi ace \& capite nigro marginatis, Linn. 0. (158. bis

810 Cimex Flarus, thorace \& elytrorum margine atro, Limo. - 0. C C 24 .

8 ir Cimex f. Larva. ( 955.

812 Cimex Bengalenzis, f. Larva. ( 906.

813 Cimex f. Larva. ( 806.

8 if Cimex f Larva. (164. 801 . bis.

8 is Cimex f. Larva, thorace rubro ( 163.

816 Cimex f Larva, 2. parva. ( 105.

817 Cimex f. Larva, füsco nigra, an Blatta? (164.

818 Cimex f. Larva, on Biatta? (907.

819 Cimex f Larya, luiea plane depreffa. (160. bis.

820 Cimex f. Larva eadem (I95. 261.

821 Cimex f. Larva. (rog6.

\section{N E P E.}

822 Nepa Pulligera, Linn. o. (842. bis.

823 Nepa Pulligera, r. mas, Linn. o. ( 913.

824 Nepa Grandis, maculata, Linn. 713 I. Rof. III. tab. 26. ( I 66.

825 Nepa Cinerescens? Indica, Linn. 714! 5? Rôj. Append. tab. 22. f. 6-8. (844.

826 Nepa f. Larvæ quædam forfan Blattarum. (679. $788^{\circ}$ 9i2. ter.

827 Infectum quodfam Acari formis, cauda fetis corpore quintuplo longioribus, veficulis 2. albis. ( 877 .

828 Onifcus Oceanicus? Indicus, magnus, Linn. 106r. 12 ? AE. Helv. V. tab. 's. ( 787 . 
or6 Punaife Porte Crois ? ou veriett de lo méme, Linn. o, (162. 807 Punaife Velue, le corcelet à laine, le ventre fendu, Linn. o. $(467$.

Sos Punaife Pórte Croix, “d corcelet jaune, ventre bordé de joune Linn. O. (157. bis.

809 Puritife Noire, à corcelet, Ė têté bordẻes de noir, Linn. 0. (Is8. bis.

8:0 Punaife Jaune, à corcelet, Ẽ bord des étuis noirs, Linn.o. (224

BI I Punaife ou Larve. (955.....

8I2 Punaife ou Larre de Bengale. ( 906.

813 Punaije ou Larve. ( 806.

814 Punaife ou Larve. (164. 801. bis.

815 Punaife ou Larve, à corcelet rouge. ( 163. 816 Punaife ou Larve, spetits. (165.

817 Punaife ori Larve, brun-noire, ou Blatte. ( 164 is 818 Punaife ou Larve, ou Blatte? ( 907.

819 Punaife ou Larve, jaune, deprimée. (I60. bis.) 820 Punaife ou Larve de la même. (195. 26I.

82r Punoife ou Larve. (1096.

\section{SCORPIONS AQUATIQUES.}

822 Scorpion Aquat. Portant des petits, Linn.o. ( 842 . bis. 813 Scorpion Aquat. Portant des petits, male, Linn. 0 , (9:3

824 Scorpion Aquat. Grande, tachetée, Linn. 7ז3. I. Roer. III. tab. 26. ( 168.

225 Scorpion Aquat. Cendrés, des Indes, Linn.714. 5 : Rosi. App tab. 22. $f$ 6-8. (844.

826 Scorpions Aquat. ou Larves peutetre des Blattes? ( 679 . 788. 912 . ter.

827 Certain Infecte, la forme d'un Acare, la queue cing fois plus grande, que le corps d deux veficules blanches. (877.

828 Onifce Aquat. de lOocean, des Indes, grand, Linm. 10ọt. I2? Act. Helv. V. tab. 5. ( 787 . 


\section{LE PIDOPTERA. \\ P A P I L I O N E S.}

828 Papilio Leilus, alis caudatis concoloribus nigris, fascia lineisque viridibus, Linn. 750. 31. Merian. Surin. tab. 29. ( 917.

820 Papilio Leilus, Linn. Eo Merian. l. c, (819.

830 Papilio Protefilaris, . alis caudatis fasciis fuscis, angulo ani rubro, Linn. 752. 39. Merian Surin. tab. 43. ( I046.

83 I Papilio Podalirius, alis flavefcentibus, fasciis nigricantibus, fubcus linea fanguinea, Linn. 751, 36. Merian. Surize tab. 44. (1516.

832 Papilio Machaon, Indica, alis flavis, limbo fusco, lunulis flavis, angulo ani fulvo, Linn. 750. 33. Edw. Glean. 343. ( 1515.

833 Papilio Thoas, Limn Mantid. II.pag. 536. (815.

834 Papilio Thoas? fovarietas, Linn. I. c. (1464.

835 Papilio Thoas. f. fernina, Linn. 1 c. (II94

836 Papitio Podalirius? f. yarietas, Linm: O. ( 1463.

837 Papilio Nireus, alis nigris, fascia inaurato viridi, fubtus nigris, Linn. 750. 28. Drury Inject. II. tab. 3. f. I. 2. (1329.

838 Papilio Thoas? f. varietas, Linn. 1. c. ( 1464.

839 Papilio Riftus, alis dentatis fuscis, virefcente maculis, Linin. 754 48. Cramer. 106. C. D. C $(814$ bis.

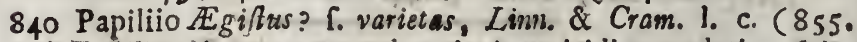

84 I Papilio Agamemnon, alis nigris, visidi maculatis, fubtus ocello lunato, maculisque rubris, $\operatorname{Linn} 748.22$. ( 1326 . tis.

842 Papilio Agamemron? f. varietas, Linn 1. c. (1123. bis. 843 Papilio Xuthus, alis nicris, albido ftriatis maculatis, Linn. 751. 34. Cram 73. A. B. (856 bis.

844 Papilio Xuthus? fovarietos, Linn \& Cram I.c. ( 867 , bis.

845 Papilio Paris, alis nigris, pofticis macula cyanea, ocello purpureo, fubtus ocellis feptem, Linn. 745. 3. Cram. 103. A. B (847. bis.

846 Papilio Lurus? Linns. 8 ro of Cram 199. A. B. ( 1047. 847 Papilio Lunus? i. varictas, ex America, Linn. E' Cram. L. c. $(: 327$. 


\section{$L \begin{array}{llllllllllll}L & E & P & I & D & O & P & T & E & R & E & S\end{array}$ \\ P A P I L L O N $\mathrm{N}$.}

828 Papillon Leilus, $d$ ailes pointues noires, d'un même couleur d bande, \&े raye vertes, Linn. 750. 31. Merian. Surin, tab. 29. ( 917.

829 Papillon Leilus, le pareil, Linn. E Merian. l.c. (819.

830 Papillon Protefilae, da ailes pointues, bandes brunes, E' l'anus à angle rouge, Linn. 752, 39. Merian. Surin. tab. 43. ( 1046 .

831 Papillon Podalire, à ailes jaunatres, bandes noires, desJous d ligre couleur de sang, Linn.75I. 36. Merian. Surir. tab. 44. ( 1510.

832 Papillon Machaon, des Indes, à ailes jaunes, bord brun, lunules jaunes, lanus d angle fauve, Linn. 750.33 . Edw. Glean. 343. ( 1515.

833 Papillon Thoas. Linn. Mantiff. II. pag. 536. (815.

834 Papillon Thoas? ou variesé, Linn. l. c. (1464.

835 Papillon Thoas, ou femelle, Linn. l. c. (rig4.

836 Papillon Podalire? ou varieté, Linn. O ( 1463.

837 Papillon Nireu', da ailes noires, d bande vert-doré, delJous noires, Litn. 750. 28. D. ury Infect. II. tab, 4. f. 1. 2. ( 1329.

838 Papillon Thoas? ou varieté. Linn. l. c (1 464.

839 Papillon $1 E$ gyfte, $d$ ailes dentees brunes, $d$ taches yertes, Linn. 754. 48. Cram. 106. C. D. (8I4. bis.

840 Papillon Egyfte? ou variete, Linn. Ẽ Cram. l c. (855. 84I Papillon Agamemnnn, d ailes no:res, tachetées de verd, deffous osil luné, Ẽ taches rouges, Linn. 748. 22. (1.326. bis.

842 Papillon Agamemnon? ou varieté, Linn.l.c. (I I 23, bis.

843 Papillon Xuthus, d ailes noires, tacheté, Eo frie de blanc; Linn. 751. 34. Cram. 73. A. B. (856. bis.

${ }_{44}$ Papillon Xuthus? ous variete, Linn. E Cram. i.c. (867. bis. 845 Papillon Paris, d ailes noires, les pofterietsies d tache bleue, oeil pourpre, \& de (jous fept petits yeux, Linn. 745. 3. Cram. 1C3. A. B. (847. bis.

846 Papillon Lunus? Linn. 810, 6. ? Cram. 19). A. B (1047.

847 Papillon Lunus? ou variett, d'Amerique, Linn. EO Cram. b. c. $(132 \%$. 
848 Papilio Punctata, alis catidatis obliquis fuscis, lineisque ferrugineis, Linn. o. d'Aubet. . . . ( 1405.

849 Papilio Pranctata? fovarietas, Lim. O. SI 1048.

8so Pditilio Argiatus, Linn. o. (814. bis.

85I Papilio Helena? fo arietas, an Gambrifusu? Linn. o. (822. bis.

852 Papi io Helenas, a is rigris, ponticis macula alba, lunulis feptem fersugineis, Linn. 745: 4. Cram. 153. A.B. (II27.

853 Pspilio Helenus? f. varietas, Linn. \& Cram. 1. c. (II 44: bis.

857 Papilio Meneftheus., Linn O. Cram. I42 A. B. ( 1328.

855 Papilio Hedor, alis cautatis nigris; primoribus farcia alba, pofticis maculis rubris, Linn. 745.2. Cram. 12. A. B. (is68.

856 Papilio Polydorus, alis nigris, macula alba, lunulis fep. tem rubris, Linn. 746. 10. Cram. 128. A. B. (1445. bis.

857 Papilio Polydorus? C. varietas, Liriro \& Cram. L c. (II 49. bis.

858 Papilio Polydorus? f. varietas, Linn. O. (1617. bis.

859 Papilio Caudata. Linn. o. (845.

$800 \mathrm{~Pa}$ jilio Deiphobus, a ais nigris, fubtus baff rubro maculatis, Linn. 746. 7. ( 821 .

861 Papilio Ulijes, aiis nigris, pofticis oculis feptem, Linn. $748.21 .(820$.

862 Papilio Pammon, alis nigris, omnibus margine maculatis, pofticis fascia, maculis feptem albis, Linn.746. 8. Cram. I4I. B. $\quad(8 ; 6$.

863 Pajilio Pammon? f. Yarietas, Linn. \& Cram. 1. c. (817. bis

864 Papilio Fabius, Linn, O. Cram. ... ( $133^{2}$

865 Papilio Petreus, Linn. C. d' Aubet ... Suiz. Infeat tob. 13. f. 4. ( 14 彳. bis.

866 Papilio Piranthus, Linn. O. Cram. 204. A. B. (II)5. 867 Papilio Pyrrhus, alis fuscis, fascia alba, Lirn. 749. 25. Crann 220. A. (824. bis.

868 Pa:ilio Nijus Limn o. Cram. 150. A. (823.

809. Papilio Laërtes, Linn. O. Cram. 74. C. (133r.

870 Papilio Teucer, alis fubre andis lividiusculis, fubcus nebul fis. pofticis ocellis tribus, Linn. 753. 44. (1110. 1627. bis

87. Papilio Euryatus, Linn. O Cram. 74. A. B. (825. bis.

872 Papilio Euryalus? f. varietts. Linn. o. (...

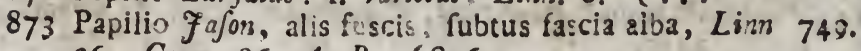
26. Cram. 86. A, B. $(826$. 


\section{Infectes.}

348 Papillon Pointu, les ailes pointés, "obliques brunes, lignes couleur de rouille, Linn. O. d'Aubet. ... (1405. 349 Papillon Pointu? ou varieté, Linn. O. ( 1048.

3 so Papillon Argiate, I.inn. o. ( 814 bis.

35x Papillon Helene? ou variete, ou Gambrife? Linn. 0. (822. bis.

852 Papillon Helens, d ailes noires, tacbe blanche, de fept lunubles, couleur de rouille, Linn. $745.4 \mathrm{Cram}$. I53. $A$. $B$. (1127. bis.

853 Papillon Helene? ou varieté, Linn E Cram l.c. (1144. bis.

854 Papillon Menefthée, Linn. O. Cram. 142. A. B. (1 328. 855 Papillon Hector, d ailes noires, les anterieures d fascie blanche, les poffericures de taches rouges, Linn 745. 2 . Cram. 12. A. B. ( 1568.

856 Papillon Polydore, d ciles noires, tache blanche da feps lunules rouges, Linn. 746. 10. Cram. 128. A. B. (i445. bis.

857 Papillon Polydore? ou varieté, Limn. E Crain l: c. (I 149. bis.

858 Papillon Polydore? ar varieté? Linn o. (16r7. bis. 859 Papillon dे Queue, Linn. O. ( 845 ."

860 Papillon Deiphobe, à ailes noires, deffous ls base tacheté de rouge. Linn. 746. 7. (821.

861 Papillon Uliffe, à ailes roires, les pofrerientres deflows d fept yeux, Linn. $74^{8}, 21$. ( 820 .

862 Papillon Pammon, à ailes noires. 1 le bord tacheté, ies? pofterieures is fajcie, E e fept taches blanches, Linn. 7.40 .8 ; Cram. 141. B. (816.

8.63 Papillon Paminon? ouvarieté, Linn. $\mathcal{O}^{\circ}$ Cram l. c. (85\%) bis.

864 Papillon Fabius, Linn, O. Cram..... (1332.

865 Papillon Petreus, Linn. O, d'Aubet... Sulz. Infeat tab. 13. f. 4 (1465. bis.

806 Papillon Piranchus, Limn. O Cram. 204. A B. Cirgs.

867 Papillon Pyrthe, da ailes brunes. fafcie blanche, Linn。 749. 25. Cram. 220.' $A$. (824. bis.

868 Papillon Nife, Linn. C. Cram iso: A. (823.

869 Papillon Laërres, Linn O Cram. 74. C. (1331. bis.

\$70 Papillon leucre, ta ailes recoustées. couleur de plomb, deffous nebraleux, les polterieures a trois yeuax, Linn. 753. 44. (IIIO 1627. bis. 87 T Papillon Euryale, Linn. o. Cram. 7\%. A. B. (825.

872 Papillon Euryale? ou varieté, Lirn. 0

873 Papillan Jafon, da ailes brumes, delfous lande do caragteres blanches, Lini). 749. 26. Cram. 85. A. B. (826. 12

874 Papillon 
874 Papilio Flava, alis luteis, lineis obilquis atris, Linn. 0. ( $8+4$.

875 Papilio Arfinoe, Linn. o. Cram. 160. B. C. (842.

876 Papilio Sarpedion, alis dentatis nigris, fascia viridi, (ubtus maculis quinque rubris, Linn. 747. 15. Cram. 122. 'D. E. ( 1125 .

877 Papilio Sarfedon? C., varietas, Linn. \& Cram. 1. c. (iIs3. bis.

878 Papilio Interrupta, Linn. O. (858. bis.

879. Papilio Polydamas, alis dentatis nigris, fascia interrupta flava, fubtus maculis linearibus flexuofis rubris, Linn. 747. 12. Drury 17. $f$. I. 2. (I 393.

880 Papilio Athamas, Linn. O. Cram -89. C. D. (859. 881 Papilio Penthefslea, Linn. O. Cram. 145. B. (r606. 882 Papilio Varanes, Limn. O. Cram. 160. D. (1124. 883 Papilio Decius, Linn. O. Cram 114. A. B. (1330. 884 Papilio Charinaus, Linn. o. Fabric. Syft. Entom. ... (ro49.

885 Papilio Philoctetes, alis fuscis, pofticis ocellis duabus cceruleis, pupilla nigra, punctis tribus albis, Linn. 750. 29. Cram. 20. A-C. ( 838 . bis.

886 Papilio Lena, alis fubdentatis fuscis, pofticis atris, albo corruleoque punctatis, Linn. 784. 2c6. Cram. 198. D. E. (839. 1334. bis.

887 Papilio Beroë, Linn. o. Crann. 78. C. D. (rr78.

838 Papilio Beroë, pracedenti fimilis, Linn。 o. Cram. 78. C. D. ( ...

889 Papilio Lena? f. varietas, Linn. 1. c. (839. Irjt. 1206 bis.

890 Panilio Demoleus. alis dentatis fuscis, maculis flavis, pofticis ocellis binis, Linn. 753. 46. Cram. 231 . $A$. $B$. C 1468 .

89r Papilio iDemoleus? f. varietas pracedentis? Linn. $80 \mathrm{Cram}$. (1313. bis.

892 Papilio Demoleus? f. yarietas, Linn. o. (868. bis. 893 Papilio Demoleus? f varietas, alia, Linn. C. ( 870.

894. Papilio Panope, Linn. O. Cram. 295. E. F. $(920$.

895 Papilio Idomeneus, alis fubcrenatis, fubtus nebulofis, ocellis duobus, Linn. 753. 45. ( 929 . bis.

896 Papilio Brummea, alis fuscis, porticis fascia maculari flavo lutea, Linn. O. ( 857 .

897 Papilio Similis, alis repandis nigris, punctis albidis, bafin verfus lineatis, Linn. 732. 193. Cram. 59 D. E. (8900 
874 Papillon Jauneatre, dailes jaunes, E lignes obliques noires, l.inn. o. (844.

875 Papillon Arfinoe, Linn. o. Cram. 160. B.C. (842.

870 Papillon Sarpedon, d ailes dentées noires, deffous à cinq taches rouges, Linn. 747. 15 Cram. 22. D. E. (1125.

877 Papillon Sarpedon? ou varieté, Linn. E Cram. l. c. (ris3. bis.

878 Papillon Inrerrompu, Linn. o. (858 bis.

879 Papillon Polydamas, à ailes dentées noires, d̀ bande interrompue jaune: deffous à taches \& lignes rouges, Linn. 747. 12. Drury 17. f.1.12. ( 1393 .

880 Papillon, Athamas, Linn. O. Cram. 89. C. D. (859. 881 Papillor Penthefilea, Linn. O. Cram. I45 B. (1606. 882 Pupillor Varanes. Linn. O. Crám. 160. D. (II24. 883 Papillon Decius, Linn. O. Cram. 114. A. B. (I 330. 884 Papillon Charinaus, Linn. o. Fabric. Syß. Entom. .... ( 1049.

885 Papillon Philocletes, à ailes brunes, les pofterieures à deùx yeux bleues celefte. prunelle noire, a trois points blancs, Linn. 750. 29. Cram. 30. A-C (838 bis.

886 Papillon Lena, d ailes dentées brunes. les pofterieures noires, pointées de blane, E bleu celefte, Linn. 784. 206. Cram. 108. D. E. (839. 1334 bis.

837 Papillon Beroë. Linn o. Cram. 78. C. D. ( I 178.

888 Papillon-Beroë, Semblable au precedent, Linu. o. Clam. 78.C. D. (...

889 Papillon Lena? ouvarieté, Linn. l.c. (839. II5t. 1206. bis.

890 Papillon Demoleus, da ailes dentées brunes, taches jounes, les pofterieures d deux yeux, Linn. 753. 46. Cram. 23I. A.B. (1 468. bis.

$89 s$ Papillon Demoleus? ou varieté du precedent? Linn. \& Cram: l. c. (1313.

892 Papillon Demoleus? ou varieté, Linn. 0 . (868. bis.

893 Papillon Demoleus? ou autre varieté, Linn. o. (870.

894 Papillon Panope, Linn. O. Cram. 295. E. F. ( 920.

895 Papillon Idomenée, $i$ ailes un peu cochées, dedfous nebuleufes, a deux petits yeux. Linn. 753. 45. ( 929. bis.

896 Papilion Brun, à ailes pofterieures brunes, a bande tacheté de jaune, Linn. o. (857.

897 Papillon Pareil. da ailes un peu courbées noires, da pointes blanches tirant jur le blene. EO rayée vers la baje, Linn. 782. 193. Crain. 59. D. E. (890. 
898 Papilio Anchifes, alis dentatis nigris, maculis feptem: rubris ovatis, Limn. 746. Ir. Seba IV. tab. 7. f. 27. 28. Merian Surim. tab. 17 . (1209. bis.

899 Paplllo Eneas, alis primoribus puncto albo, Linn. 747 16. Cram. 29. C. D ( 1059. bis.

soo Papilio Hypprfon, Linn. O. Cram. 29. E. (1060. bis. got Papilio Hypprenon? f. yarietas, alia, Linn. o. Cram. 1. c. ( 1470.

902 Papilio Hypprson? $f_{0}$ yarietas, Linn. o. Cram. 1. c. ( 1002.

so3 Papilio Hyppufon? C, yarietas, Linn. O. ( 1063. 904 Papilio Hyppujon? f. varietas, Limn. O. (1210. 905 Papilio Veriumnus? r varietas, Linn. o. (1340. 906 Papilio Anchijes, vid. n०. 898 \& Linn 1. c. (1339.bis. 901. Papilio Aineas? . yarietas, alis atris, Linn. 1. c. (898. 9:8 Papilio Sefoltris, Linn. O. Cram. 211. F. G. (12r1. bis. geg. Papilio Sejoftris? f, varietas, Limn, o. Cram l. c. (I2I2. bis.:

910 Papilio LyGidas, Linn. o. Cram. I03. ... (134r. bis. 91 Papilip Lycidas? fo varietas:, Lirin o. Cram. l. c. (1469 bis.

gr2 Papilio Belus, Limn o. Cram. Ir.2. A. Crgor.

913 Papilio Fasciatus. alis fuscis, fascia incurva flava, pos. ticis viridibus, Linn. O. ( $x_{4} 67$.

914 Papilio सEneas? alis bafi brunneis, Linn 747. 10? Cram. 29. C. D. ( 849.

915 Papilio Aárez, fupra azureus; infra cinereus," Lims. 0. (1501.

916 Papilio Mennon, alis detutatis nigris, omnibus, baff fubcus subró notatis, Linar. 747 13. Crann. 93 C. ( 1047 .

9I7 Pajilio Memnon? T. varietas, Linn \& Cram. 1. c. (846. 918.Papilinj. \&genor, alis dentatis rigris, bafi funguineis; primorib:s Atriazis. pofticis discon albo, maculis nigris Zinm 747. I4, Cram, 32 A. B. (1, 52:

9!9 Papilio Anceus, Linn. O. Cram, 222. A. B. (1602. 920 Papilio Ancous? R. varietas, Linn. O C 1603.

प्र21 Papilio Memnon? Linn. 747. 83. B? Cram. 49. A. B (II 14.

922 Papilio Meminon? \& varietas, Linn. \& Crami! c C (II48 923 Papilio Memmon? "r, varietas, Linno \& Cram. I. ( (II50.

924 Papilio Panthous; alis dertatis nigris, primoribus alb meculat!s, peftieis naculis abbis nigra cetis, Lirn. $74^{\mathrm{f}}$ 17. Cram. 133. A. (854. 
898 Papillon Anchife, $d$ ailes dentées moires, di fept taches rouges oyales, Linn. 746. II. Seb. IV. tab. 7: f. 27. 28. Merian Surin. tab. 17. (1209., bis.

897 Papillon Er éa, les ailes devant pointées de blanc, Linn. i47. 16. Cram. 29. C. D. (1059. bis.

goo Papillon Hyppufon. Linn 0. Cram 29: E. (1060. bis.

901 Papillon Hyppufon? ou autre varieté, Linn o. Cram.

l. c. $(1470$.

goz Papillon Hyppufon? autre varieté du même, Linn. o.

Cram. l. c. (1062.

903 Papillon Hyppufon? ou varieté femblable, Linn. 0. (1063.

乌o4 Papillon Hyppulon? ou varieté, Linn. O. ( 1210.

yo5 Papilion Verturnne? ou variete, Linn. O. ( 1340.

go6 Papilion Anchife, voyez n. 898. Linn. l. co (1339. bis

907) Papillon Enée? ou varieté, do ailes noires, Linn. $l$. c. ( 898.

908. Papillon Sefoftris. Linn, O. Cram 2 I I F. G. (1211 bis. 909 Papillon Sefoltris? ou varieté, Linn. o. Cram. l. c. (1212. bis.)

910 Papillon Lycidas, Linn. O. Cram, 103 .... (1341. bis. 9. 1 Papillon Lycidas? ou yarieté, Linn. O. Cram. b.c. (1469. bis

912 Papillon Be'us, Linn. O. Cram. Ii2. A. (rgor.

913 Papillon Bandé, de ailes brunes, bande courbée jaune, les ailes poflerieures yertes, Linn. O. ( 1467.

9:4: Papillon Enée? les ailes de la baje brunes, Linn. 747. 16? Ciam. 29. C. D. ( 849 .

915. Papillon Bleu; deflus azuré, deffous cendré, Linn. (rsos.

9is Papillon Memnon, à ailes denties noires, de la baje toutes marquées de rauge, Linn. 747.13. Cram. 91. C. (ro47. 'i 7 Papillon Memnon? ou variete, Linn. E' Cram.l.c. (846. 9.8 Papillon Agenor, de ailes dentées noires, do la baje rouges, les anterieures ftriés, les pofterieures de disque blanche, taches noires, Lina. 747. 14. Cr3m. 32. A. B. (II52.

grg Papillon Anceus, Linn. O. Cram. 222. A.B. (1602. 920 Púpillon Anceus? ou varieté, Linn. O. Cram. b. c. ( 1603 . y2r Papillon Memnony Linn. 747. 13 B.? Cram. 49. A. B: (1149.

922 Papillon Meinnon? ou varieté, Linn. Eo Cram. l.c. (1)48. - 923 Papillon Memnon? ou varieté, Linn. $8^{2}$ Cram. l.c. (I i 50. 924 Papillon Panihous, a ailes dentées noires, les premieres - tachetés de blanc, les pofterieures à tashes blanches, melées de noir, Linn. 748. 17. Cram. 133. A. (854.

I $4 \quad 925$ Papillon 
925 Papilio Ainphimedon, Lim o. Cram. I49. A. (863.

926 Papilio Priamus alis dentatis, fupra viridibus, pofticis maculis fex nigris, Linn. 744. 1. Cram. 24. A. B. (837. bis.

927 Papilio Helena, alis dentatis atris, pofticis disco com. múni aurato, Lirn. 748. 19. Cram. 140. A. B. (88r. bis.

928 Papilio Hyppolitus, Linn. o. Cram. .. (853.

. 229 Papilio Amphitryon, Linno o. Crain. 7. A. B. (862, 930 Papilio Ilioneus, Linn, o. Cram. 53. A. (16rg.

93 I Papilio Hélenor, Linn. o. Cram. 86. A. B. (927.

932 Papilio Neftor, alis dentatis, fupra fuscis, maculis dis. coque coruleis, fubtus ocellis tribus, Linn. 752. 40. Cram. 19. A. B. (1616.

933 Papilio Achilles, alıs dentatis fupra nigris, fascia ccerue léa, fubtus fuscis, ocellis tribus, Linn. 752. 42. Cram. 27. A. B $(928$ bis.

934 Papilio Achilles? I. varietas, Linno \& Cram. 1. c. (936. ter.

935 Papilio Helenor, Linns. O. Cram. 86. A. B. ( 935.

936 'Pápilio Menelaus, alis dentatis fupra coeruleis, fubtus - netulofis, punctis fuscis, Linn. 748. 20. Cram. 21. A. B. $(93 \mathbf{I}$.

937 Papilio Rethenor, Limn. O. Cram. 15. A. B. $(932$.

938 Papilio Neftor, Liı.n. 752. 40. Cram. 19. A.:B. ( 1377 . bis

939 Papilio Achilles? f. varietas, Limn 752. 42 ? Cram. 27. A. B. $(1506$.

945 Papilio Menelaui? f. varietas, Linn 748.20 ? (934. bis. $94 \mathrm{I}$ Papilio Cerulea, a'is fuscis, disco cyareis, Linn. $\circ$. (14) 4.

942 Papilio Striata, alis fuscis, ad apicem aurantio ftriatis, fascia flava, Linn; o. ( 1260.

943 Papilio Fasciata, alis atris, fa cia alba, Linn o. (666. bis. 944 Papilio Maculata. alis atris, fasciis maculofis 2. albis, polticis fuscis, Linn. O. (II39. bis.

945 Papilio Atra, alis atris, fascia maculora, maculaque fuperiorum albis, polticis fuscis, apice albis, Linn. $0^{\circ}$ (1347.

946. Papilio Horta, alis oblongis rubris, primoribus apice hyalinis: pofticis fubtus albidis nigro punclatis, Linn. 755 . 54. Sulz. Inf. tab. I5. f. I. (1349. bis.

947 Papilio Horta? f. varietas, Linn I. c. (13r4. bis. 948 Papilio Horta? C, varictas, Limm. O. (897. bis. 
925 Papillon Amphimedon, Linn. 0. Cram 149. A. (863.) 926 Papillon Priam, da ailes deritées, defousvertes. polterieures ă fix taches noires, Linn. 744. I Cram. 24. A. B. (837. bis. 927 Papillon Helene, $d$ ailes dentees noires, les pofterieures disque commune do ée, Linn. 748.19. Cran. 140. $A, B$. (86r. bis.

928 Papillon Hippolite, Linn. o. Cram. :... ( 853 .

929 Papillon Amphitryon, Linn. O. Cram. 7. A B. (862.

930 Papillon llioneus, Linn. 0 . Cram 53. A. (1619.

931 Papillon Helenor, Linn. O. Cram. 86. A. B. (927.

932 Papillon Neftor, à ailes dentées defus brunes, dे taches E disque bleu celefte, defous d trois petits yeux, Linn. 752. 40. Crain. 19. $A$, B. (16:6.

933 Papillon Achille, $\dot{d}$ ailes dentées, defus noires, a bande bleue deffous brunes, à trois yeux, Linn. 752. 42. Cram. 27. A. B ( 928 bis.

934 Papillon Achille? ou varieté L Linn. ê Cram. l.c. (63G. ter.

935 Papillon Helenor. Linn. o. Cram. 86. A B. (935.

936 Papillon Menelaus, da ailes dentées, de Jus bleues celefle, deffous nebulersjes, à points bruns, Litin. 748. 20. Cram. 21. A. B. 93 I.

937 Papillon Rethenor, Linn o. Cram. 15. A. B. (932.

938 Papillon Neftor, Linn. 752. 40. Cram. 19. A B. ( 1377. bis.

939 Papillon Achille? ou varieté, Linn. 75242 \% Cram. 27. $A B . \quad(1506$

940 Papillon Menelaus? ou varieté, Linn. 748. 20? ' 934. bis.

24I Papillon Bleue, d̀ ailes brunes, difque bieue, Linn. 0. ( 1414 .

942 Papillon Strié, à ailes brunes, vers le poiitt ftriées d’urange. d bande jaune, linn. 0 . (126r.

943. Papillon Bandé, à ailes nires, Ê bainde blanché, Linn. 0 . (606. bis.

$944 P a r i l l u n$ Tacheté, à ailes noires, d̀ deux bandes blancbes, les pofterieures brunes, Linn. O. (II39 bis.

945 Papillon Noirtite, à ailes noires, bande tachasté, les fupe. rieures blanches, pofterieures brunes au point, Linn 0. ( I 347 .

946 Papillon Horta, d ailes allongées rouges, les anterieures au point hyalines. Les pofzerieures defjous bianches, poinatéos de noir, Linn. 755.54. Sulz. Inf. tah. I 5 f. I (1349. bis. 94? Papillon Horta? ou v riete, Linn. l c. (13 r 4. bis. 948 Papillon Hurta? ou varieté, Linn. o. (397. bis. 


\section{$13^{*}$

949 Papilio Fusca, ponticis disco fulvis, maculis atris, Linn. O. $(350$.

950 Papilio Stelenes, alis fuscis, fasciz virefcente obtufa interrupta, Linn 750. 30. Cram. 196. E. F. (998.

951- Papilio Cameena? Limn o. Drury. II tab. 7. f. 2. (933. 952 Papilio Maculata, alis fusois, pofticis rubris, maculis atris, Linn. o. (rit 28 bis.

953 Papilio Maculata? f, varietas, nigra, Linn. O. (II 40 bis.

954 Papilio Thalia, alis oblongis integerrimis fuscis, primioribus flavo maculatis, pofticis ftriatis, Linn. 757.67. Seb. IV. tab. 7. f. I. 2. (1037.

955 Papilio Horta? f. varietas, Linn 1. c. (1429.

956 Papilio Horta? 1. yarietas, Linn. o. (1406. 1786.

957 Pazilio Horta? f. varietas precedentis? Linn. O. (1407.

958 Papilio Maculata, eadem ac $n^{\circ} .944$. Linn. O. ( 1345 .

959 Papilio Atra, eadeni ac no .945 , Linn。 O. ( 1346 .

960 Papilio .... 2'is nigris, maculis magnis albis, Linn. o. ( 1348 .

961 Papilio Melpomene, ali, oblongis nigris, primoribus fascia rubra, pofticis bafi fu tus punetis fanguineis, Linn. 758 . 7I. Röf. IV. $t a b .3, f .6$. ( 983 . bis.

962 Papilio Melpomene? an fimilis precedenti? Linn. \& Räf. 1. c. ( 1411 . bis.

963. Papilio Melpomene? Jan yarietas præcedentis? Linn. I. c. (1367. bis.

964 Papilio Erato, alis oblongis atris, primoribus flavo maculatis. bafi rubris, pofticis rubro ftriatis, Linn. $75 \%$. 70. Crams Irg A. (1278. bis:

965 Papilio Erato? an fimilis pracedenti? Linz. \& Cram. I. c. (1401.

966 Papilio Melpomene? f. varietas, Linn. O. (1507. bis.

967 Papilio Myrthi, Lim. O. Cram. I77. E. ( ( $6_{3} \mathrm{C}$.

968 Papilio Myrthi? f. varietas, Linn. o. (984. bis.

960 Papilio Melpomene? f. varietas, Linn. O. (I612.

970 Papilio Erato? f. varietas, Linn \& Cram. 1. c. (rog9.

$97 \mathrm{I}$ Papilio Pfdii, alis oblongis fuscis, primoribus fasciis tribus, pofticis duabus hyalinis, Linn. 756. 64. Merian. Surin. tab. 15. (1002. bis.

972 Papilio Pfidii, præcedenti affinis, Limm. \& Merianz. I. c. (1003. bis.

972 Pap.lio Pjdidi $?$ f. varietas, Linn. C. ( 1279.

974 Papilio Pfidi? fo varietas, Linn. 0 . (1000. 
9.49. Papillon Brun, derriere fauve; $\dot{a}$ tackes noires; Linn. O. (1350.

950 Papillon Stelenes, les ailes dedus brunes, interrompue d'uns 1 bande verdeatre, Linn. 750. 30. Cram. 196. E. F. (998. 951 Papillon Camoena? Limn. O. Drury II. eab.7. f.2. ( 933. 952 Papillon Tacheté, à ailes brunes, derriere rouges, àtaclies noires, Linn. o (II28. bis.

953 Papillon Tacheté? ou varieté noire, Linn. O. (1 140. bis. 954 Papillon Thalie d ailes ollongẹes, entieres brunes, les premieres tachetées de jarine, les pofferieures à fories, Linn. 757. 67. Seb. IV. tab. 7. f. I. 2. (1037.

955 Papillon Horta? ou varieté, Linn. l. co (1429.

956 Papillon Horta? ou variett, Limn. o. ( 1406. I 786.

957 Papillen Horta ? ouvarieté de la precedente? Linn. O. (I $40 \%$. 958 Papillon Tacheté, le nême que n॰.944. Linn. o. (1345.

959 Papillon Noirette, le même qus n. 945. Linn. o. (1 346. 960 Papillon .... a ailes noires, grandes tachises blanches, Linv. o. i 1348.

96I Papillon Melpomene, à ailes allongées roires, les preanieres à fafcié rouge, les poftericures cie la bafe deffous à points couleur de jang, Linn. 758. 71. Roef. IV. tab. 3. f. 6. ( 983. bis.

962 Papillon Melpomene? ou Jemblable du precedert 3 Linn: E'Roel. l. c. (1411. bis.

963 Papillon Melpomene? ou varieté du precedent ? Lìno. l.c. (1367. bis.

964 Papillon Erato, $\ddot{a}$ ailes allongées noires, les premieres tacbetées de jaune. de la boje rouges, ies pofarieurres है Atries rouges, Linn. 757. 70. Cram. I19. A. (1278 bis. 965 Papillon Eato, or Jemblable du precedent? Lirn. EO Uram. l. c. ' (1 $40 \mathrm{r}$.

966 Papillon Melpomene? on varieté, Linn. O. (150\%. bis. 967 Pấillon Myrthe, Linn. O Crain 177. E. (I630. 968 Papillon Myrithe? ou varieté, Linn. 0. (984. bis. s69 Papillon Melpómerse? ou varieté, Linn. o. (I612. 970 Papillon Erato? os verieté, Linn $\mathcal{E}^{\circ}$ Cran. l. c. (rogs. 971 Papillon Pfidie, is ailes allongées trunes, les anterieures $a$ trois bandes, les pollerieures a deiix bandes hyalines. Linn. 756. 64. Merian. Surin. tab '19. (rooz. bis.

972 Papilion Pifie, Jemblable as piecelient, Limn. $0^{\circ}$ Merian. l. c. (roo3. bis.

973 Papillon Plidit? ou varieté, Lińn. o. (1 279. 974 Papilion Pfidie? ou varieté, Linn. O. C1000. 
-975 Papilio Ricini, alis oblongis fuscis, primoribus utrinque fasciis duabus flavis, Linn. 756. 63. Merian Surin. tab. 30. ( 986. bis.

976 Papilio Ricini? 1. varietas, Linn. \& Merian, 1. c. (985.

977 Papilio Ricini? an pracedenti affinis? Linn. 1. c. (987. 978 Papilio Ricini? f. varietas, Limn. O. (1410.

979 Papilio Antiocbus, Linn. Mantif. I. p. 12. Cram. 38. E. F. $($...

980 Popilio Atra, fasciis 2. albis obliquis, Linn. 0. (.988.

98 I Papilio Mneme, alis ob!ongis nigris, bafi fulvis, primoribus fascia, maculisque tribus tiavis, Linm. 756. 59. Sulz. Inf. tab. 15 f. 3. 4. (1269. bis.

982 Papilio Mneme? an pixcedenti affinis? Linn. \& Sulz. I. c. (127r.

983 Papilio Mneme? f. varietas, Linn. o. (989. bis.

ఏ84 Papilio Mneme? f. varietas, altera, Linn. o. Ci2?2. bis.

985 Papilio Melite, alis oblongis flavis, primoribus fupra nigris, lineis duabus, fasciaque flavis, Linn 755. 57. Cram. 153. C. D. ( $1>73$ bis.

986 Papilio Mop $\int a$, alis ublongis. luteis nigrisque, fubtus punetis marginalibus octo albis, Linn. 756. 59. $\beta$. Cram. 190. D. ( 1409 bis.

987 Papilio Melites ? f. sarietas, Linn. O. (99r. bis.

988. Papilio Melites? C. varietas, Linn. o. ( 1397.

98y Papilio Mneme? r. varittas, Linn. O. (990. 1274. bis.

990 Papilio Fulva, alis polticis fasciis duabus dentatis fulvis, Linn. O. (992. bis.

991 Papilio Polymnia, a'is oblongis luteis, primoribus fascia Hlava, pofticis fasciis tribus nigris, Lirn. 755. 58. Cram I. 191. E. ( 1270 .

992 Papilio Polymnia? an pi xcedenti affinis? Linn. \& Cram. 1. c. (1398. bis.

993 Papilio Polymnia? r. varietas, Linn. I. c. (1399.

994 Papilio Polymnia ? r. varietas alia, Linn. O. ( 400.

\$95 Papilio ..... alis fulvis, apice, margineque nigro, Linn. O. ( 1276.

926 Papilio .... an præcedenti affinis? ILinn. ०. ( 993. 1095: bis.

997 Papilio . . . alis luteis, fascia flava, Linn. ○. ( 1097. 998 Papilio .... alis flavis fubdiaphanis, Linn. ○. (1096. 999 Papilio 
ois Papillon Ricine, a ailes allongées branes, les premieres de chaque coté da deux bandes jaunes, Linn. 756.63. Merian. Surin. tah. 30. (986. bis.

976 Papillon Ricıne? ou varieste, Linn. EO Merian. l. c. ( 985.

977 Papillon Ricine? ou Semblable az precedent? Linn. loca (987.

978 Papillon Ricine? ou varieté, Linn. O. ( 14 ro.

979 Papillon Antioche, Linn. Mantid. I. P. 12. Cram. 38. E. F. (....

980 Papillon Noire, dd dersx bandes obliques, Linn. O. (988. 981 Papillon Mneme. à ailes allongées noires, de ba bafe farbves, les premieres dे bonde, $\mathbb{E}^{\circ}$ trois taches jaumes, Linn. 756. 59 Sulz. In . tab. 15.f. 3. 4. (1269. bis.

982 Papillon Mneme? ou Jemblab!e au precedent? Linn. E Sulz. l. c. (1271.

983 Papillon Mneme? ors variet , Linn. O. ( 989 kis. $98+$ Papillon Mneme? ou autre varieté, Linn. O. (1272, bis. 985 Papillon Melite, d̀ ailes allongées jaunes, les premieres deffus noires, d deux rayes, $0^{\circ}$ bande jaunes, Linn. $755^{\circ}$ 57. Cram. 153. C. D. (1273. bis.

286 Papillon Mopfe, da ailes allongées, jaunes \& noires, desfous a luuit points marginales blancs, Linn. 756. 59. $\beta$. Cram. 190. D. (1409. bis

987 Papillon Milites ? ou varieté. Linn. O. (99r. bis. 983 Papillon Mi'ites? ou autre varieté, Linn. O. (1397. 989 Papillan M eme? ou varieté, Linn. o. (990. 1274 . bis. 990 Papillon Fauve, les ailes de derriere d derux bandes farbes. dentelées, Linn. o. (992. bis.

$99 r$ Papiilon Polymnie, à ailes allongées jaunes, les premieres d bande jaune, les pofterieu, es d trois fafcies qoires, Linn. 755. 58 Cram I. 191. $E^{\circ}$ (1270.

992 Papillon Polymnie? ou semblable du precedent? Linn. ED Cram. l. c. (1398. bis.

993 Papillon Polymnie? ous yarieté, Linn. l. c: (139).

994 Papillon Polymnit? ou autre varieté, Linn O ( 1400.

995 Papillon. . . . à ailes fauves, le fommet, 'E le bord roir. Linn o. ( 1276 .

996 Papillon.... ou Jemblable dus preceder:t? Linn. 0. (993. 10)5, bis.

997 Papillon . . . a ailes jaunatres, Ė bande jarne, Lina. o. $(1097$.

998 Papillon ... d aibes jaunes, un peu transparantes, Linr: o. $(1096$. 
999: Papillo Mneme? maculata, alis nigris, Linn. 756. 597 ( $\therefore . .$.

1000 Papilio Melites? f. varietas, Linn. O. 6. . .

Ioor Papilio Necrea, alis dentatis fufcis albo maculatis, ac linea rubra cinctis, Linn. 782. I90. Edw. tab. 33. $\therefore \quad$ (20067. bis.

1002 Papilio Nearea? fi varietas, Linn. 1. c. (1214. bis.

1003 Papilio Necerea? f. varietas, macula viridi, Linn. o. (926. Ir99. bis.

1004 Papilio Iphicla, alis dentatis, fafcia communi alba, Linn. 780. 181. Cran. 188. B. D. E. F. (910. bis.

I005 Papilio Elea, alis dentatis, fufco nebulofis, fafcia flava, Linn. 781. 183. (606. 1620. bis.

1006 Papilio Idmone, alis dentatis fufcis, utrinque fafcia alba, Linn. 78 1. 182. (840. bis.

1007 Papilio Demophon, alis dentatis nigris, fafcia viridi, fubtas grifeo maculatis, Linn. 753. 47. Cram. 158. E. (1064. I159. bis.

1008 Papilio Demophon? f. varietas, Linn. \& Crann. 1.c. ( $15 \mathrm{co}$. I009 Papilio Demophon? f. varietos precedentes? Linn. o. (885. bis.

1oro Papilio Midanus, alis nigris, albo punctatis, Limm. 765. 108. Ehret tob. 3. ( 1167 .

Ior I Papilio Obrinus, alis nigris, fafcia cyanea, fubtus viridibus, fafcia alba, Linn. 766. II3. Cram. 49. E. (1213. bis.

I012 Papilio Obrinus? C. varietas; an femina? Linn. 0. (1066.

1013 Papilio Hyperia, Linn. o. Cram. 236. E. (I574.

1014 Papilio Sibilla, alis dentatis fufcis, fafcia alba, Linm. 78\%. 186. (1521. bis

Iors Papilio Sibilla? f. varietas, Limn. 1. c - (893.

Ior6 Papilio Iphigenia, Linn. o. Cram. 67. D. E. (I584.

1017 Papilio fiimena, alis dentatis nigris. fafcia cnerulea irterrup/a, albo punctata, Linn. 780. 178. Cram. 121. A. B. C. $(88 \mathrm{I}$.

Ior8 Papilio Dirce: alis fufcis, fafcia flava, nigro undula. tis, Linn. 773. 171. Cram. 212. C. D. (9I6. bis.

sorg Papilio Átorion, alis fufcis, fafcia lutea, macula cæ: rulea, Linn. 794. 262. Cram. 49 C. D. (I I g6. bis.

rozo Papilio AEtorion, præcedenti affinis, Limn. \& Cram. 1. c. (II97. bis, 
999 Papibion Mneme? tachete, dailes noires, Linn. 756. 59 ?2 (....

1000 Papillon Melites? ou varieté, Linn. o. (. . .

1001 Papillon Nox rea, dailes dentées brunes, tacketées de blanc, Eे entourées d’une ligne rouge, Linn. 783. 196. Edw. tab. 33. ( 1067 bis.

1002 Papillon Neærea? ou varieté, Linn. $l$ c. (I214. bis. 1003 Papillon Ncærea? ou variete, à tache verte, Linn. - o (926. II 99. bis

1004 Papillon lpticla, à ailes dentées, fafcie commune blanche, Linn. 780. I81. Cram. 188. B. D.: E. F. (910. bis.

1005 Papillon Elea, is ailes dentées, brun-rebrileufes, à bande joune, Linn. 781, 183. (606. 1620. bis.

Ic06 Papillon idrone, dे ailes dentes brunes, à fafcie blanche de chaque roté, Linn, 781 . 182 ( 840 . bis.

1007 Papillon Demophon, à ailes dentelies noires, fafcie verte. deffous tachetées de gris, Lirn. 753. 47. Cram. 158. E. (1064. 1159. bis.

1008 Papillon Demopbon? ou varieté, Linn. E Cram. lo co ( 1500.

I009 Papillon Demophon? ou varieté du precedent? Linn. $0_{0}$ (885. bis.

1010 Papillon Midamus, d ailes noires pointées de blanc, Linn. 7C5 108 Fbret tab. 3. ( 1367 .

sori Papillon Obrinus, \& ailes noires, bande breu, deffous vertes, $a$ bande blancbe, Linn. $766: 113$. Cram. 49. $E_{0}$. (I213. bis.

IOI 2 Papillon Obrinus? an variete, an femelle? Linn. O. (ro66.

1013 Papillon Hyperia, Linn. O. Cram. 236. E. ( 1574 .

1014 Papillon Sibille, d ailes dentées brumen, d bande blanche. Linn. 78\%. 186. (1521. bis.

rors Papillon Sibille? ou varieté, Linn. l. c. ( 893 .

10r6 Papillon 1 phigenie, Linn. O. Cram. $6 \%$ D. E. C 1584. 1017 Papillon Alimene, ailes dertíes noires, à bande bleue incerrompue, pointées de blanc; Linn. 780. I78. Cram. I21. $A$ B. C. (88I.

1018 Fapillon Dirce, da ailes trunes, do fáfcie jaune, ondées de noir. Linn. 778. 171. Cram. 212. C. D. (gi6. bis.

rorg Papillon Actorion, dailes brumes. fafcie jarme, tache blew celefte, Linn. 794. 266. Cram. 49. C. D. (1196. bis.

1020 Papillon Actorion, femblable au precedent, Linn. Ef Cràm. b. c. (II97. bis. 
ro21 Papilio Attorion? C. varietas, Linn. o. Cram. I. a (i198. bis.

1022 Papilio ..... alis fufcis, bafi corulefcentibus primoribus macula albida ad apicem, pofticis caudatis, ocellis 2. ad anum, Linn. o. ( 1335 .

1023 Papilio .... alis fubfalcatis ferrugineis, bafi cœiruleis, apice atris, Linn. o. (1564.

1024 Papilio . . . a alis ocraceis falcatis, excifis apice fufcis, Linn. o. (873. bis.

3025 Papilio Urtica, Indica, alis fulvis, nigro maculatis, terpunctata, Lirn. 777. 167. Schaff. tab. 142. f. 1.2. (i519. bis.

1026 Papilio C. album, Capenfis, alis luteis 'atro maculatis, C. albo ornatis, Linn. 778. 168. Röj. I. tab 5. (1518. bis.

1027 Papilio .... alis luteis apicibus ftriaque fufcis, pofticis fubtus ocellis 4. Linn. o. ( 843.

1028 Papilio Ifidora, Linn. o. Cram. . . . (1057. bis. Jo29 Papilio Ifidora? f. varietas, Linn. o. ( 466 . bis. to30 Papilio .... alis aurantiis in rofeum mutantibus, pofticis fupra punctum, fubtus ocellis fex minimis, Linn. 0. ( 1336 .

1031 Papilio .... . . varietas precedentis? Linn. 0. ( 1058. 1032 Papilio .... alis fufcis, falcatis caudatis, apicum macula atra, fulvo cincta, maculis duabus albis, Linn. o. ( 1598.

I033 Papilio Echion, alic fúcis, fafcia rubra, ocello rubro Linn. 788. 224 Drury ... (1200. bis.

1034 Papilio Almana, alis fufcis, pupillagemina, Linn. 769. 132. Cram. 58. F. G. (874. bis.

ro35 Papilio Lemoraias, alis grifeis, punetis flavis, unico ocello, Linn. 770. 136. Crans. 35. D. E. F. (876. II 94 . bis.

1036 Papiiio .... alis a'bidis, primoribus ocello uno, pofticis duobus, Linn o. (841. I 20I. bis.

ro37 Papilio Charonia, Linn. o. Cram. 47. A. B. C. (860. 1038 Papilin Antiopa, alis nigris, limbo albido, Linn. 776. $165 R \ddot{0} \int . I, t a b$. I. ( $15\{0$.

1039 Papilio Laomedia, alis cinereis, primoribus fex oculis, pofterioribus quinque, Limm. 772 . 145. Cram. I. 8. E. G. (III3. 
1021 Papillon Actorion? ou varieté, Linn. o. Cram. l. c. (II98. bis. 5 ?

ro22 Papillon .... à ailes brunes par devant bleues, ds tache blanche, en derriére deux yeux vers l'anus, Linn.o. ( I 335 .

1023 Papillon ..... a ailes couleur de rousille, la cbafe bleue, au fommet noir, Linn. Q ( 1564.

ro24 Papillon .... d ailes feuilletées, d laintes trunes, Linn. 0. (873, bis.

1025 Papillon Urtice, des Indes, da ailes fauves, tachetes de noir, d trois points. Linn. 777. "167. Schaef. táb. 142. f. 1. 2. (1519. bis.

1026 Papillon C. Blanc, du Cap, ailes jaunes, täehetées de noir, orné d'un C. blant, Linn. 778.168. Raef. I. tab. 5. (1518, bis.?

1027 Papillon . . ad ailes jaunes, les pointes frries brunes, d quatre yeux, Linn. 0. ( $843:)$

1028 Papillon Ifidore. Linn. o. Cram. . . (1057. bis.

1029 Papillon Ifidore? ou varieté, Linn. O. (1466. bis.

1030 Papillonz .... les ailes oranges changeants en rouge par derridre, fur ke point Jont $\int \mathrm{x}$ tres petits year, Linn: 0 . ( 1336.

I03 I Papillon .... ou variete du frecedent? Lina. 0 . ( 1058.

1032 Papillon .... a ailes brunes, courbees, ar point une tache noire, entouré de jaune, \& deux taches blanches, Lirin o. ( 1598.

1033 Papillon Echion, dailes brumes, bande rouge, oeil rouge, Linn. 788. 224. Drury . . C (1200. bis.

1034 Papillon Almana, d ailes brunes, double prunelle, Linn. 769. 132. Cram. 58. F. G. (874. bis.

1035 Papillon Lemonias, d ailes grijes, points jaunes, ac wn œil, Linn. 770.136. Cram. 35. D. E. F. (876.11194. bis.

1035 Papillon.... d ailes blanches, les premieres d uns oeil, Eo les derrieres d deux yeux. Linn. O. (841: (120r. his.

1037 Patillon Charonia, Linn. O. Cram. 47. A. B.C. (880 : $\mathrm{r}=38$ Papillon Antiope, d ailes moires, le bord blanc, Linn. 776. 165. Roef. I. tab. I. CI549: .

ro39 Papillon Laomedie, les ailes cendries, les premieres do $\int_{2} x$, les poffericures à cing yeux, Linn. 772. 145. Cram. 1.8. s. G. (II) 
I040 Pavilio . .... alis ac te dentatis, luteis nigro marginztis, bafi \& margine anteriore fuscis, nigro maculatis, Linn: O. ( 1333.

I04i Papilio Aferie, alis dentatis, luteo variis, Linn. 769. 133. Cram. 58. D. L. (875. bis.

1042 Papilio Orithya, alis fuscis, fupra oce!lis duobus, fubcus unico, Linn. 770. 137. Cram. 32. F. (137. 1205. bis.

1043 Papilio Orithya? fo varietas, Linn. \& Crann. L c. (1206. bis.

1044 Papilio Orithya? fo, varietas, Linn. I. c. (I207. bis,

1045 Papilio Fidia, alis fuscis cœraleo micantibus, duobus ocellis, duobusquie punctis albis, Limn. 770. 138. Espe tab. 49. (1157: bis.

1046 Papilio Fidie? f. varietas, Linm. 1. c. ( 1208 . bis.

1047 Papilio Oenone; alis albido macalatis, Linn. 770.135. Cram. 35 A. B. C. (1336. 1408 bis.

1048 Papilio Oenone, f. mas, Linn. \& Cram. I. c. (1156. bis.

1049 Papilio Orithya, fo mas, Linn. 770. 137. Cran. 19. $C$ D. ( 1185 . bís.

I050 Papilio Oenone, f. fenina, Linn. \& Cram. 1. c. (880. I05I Papilio Clelia, Linn. O. Cram. 21. E. (877. 879. bis. 1052 Papilio Cardui, alis fulvis, albo nigroque variegatis, Linn. 774. 157. Cram. 26. E. F. (ris5. bis.

053 Papilio Cardui? f. varietas, Linn. \& Cram. I. c. (1337. bis.

1054 Papilio Cardui? r. varietas, Linn. \& Cram. 1. c. ( $15 \mathrm{I} 2$. bis.

I055 Papilio Cardui ?. Varietas, Linn. \& Cram. I. c. ( 1220. 1056 Papilio Cardui? C. varietas, Linn 0. (120).

1057 Papilio Amathea, alis angulatis fuscis albo punetatis, fascia rubra, linea atra undata, Linn. 779. 174. Cram. 209. $A B$. (1419. bis.

1058 Papilio Atalanta, alis atris albo maculatis, fascia purpurea, Linn. 779. 175. Cram. 84. E. F. (1551.

1059 Papilio Prythea? f. varietas, Linn. O. (879. bis.

1065. Papilio Cardui? f.' varietas, Linn. O. ( 1202 .

1001 Papilio Atalanta? an fimilis præcedenti? Linł. I. c. Cram. 30. D. 59. D.E. (890. bis.

$10 \sigma_{2}$ Papilio Atalanta.? fo varietas, Linin \& Cram. I. c. (889. bis. 


\section{Infertes.}

10 jo Papilion . . . les ailes dentélés jaunes, tordées de noir a la baje, $\mathcal{E}^{2}$ aú bord devant brunes, taclieties de noir, Linn. o. (1333.

104I Papillon Afterie, d ailes dentées varides de jaune, Linn. 709. 133. Cram. 58, D. E. "(8.75. bis,

$\mathrm{XO}_{4} 2$ Papillon Orithya, $d$ ailes brunes defus à deux yeux. defJous a un oeil. Linn. 770. 137. Cuam. 32 F. (137. I205. bis.

1043 Papillon Orithyz? ou varieté, Linn. E Cram. l. c (1206. bis.

Ic44. Papillon Orithya? ats varieté, Linn. E Cram. t. c. (1207. bis.

ro45 Papillon Fidia, d ailes brunes reluifantes de bleze, de deu. yeux, E' dersx points blancs, Linn. 770. 138. Efpe tat. 49. (IIs:- bis.

ros 6 Papilion Fidia? ou varieté, Linin. l. c. (12c8. bis.

1047 Papillon Oenone, a ailes tacbetées de blanc, Linn. 770. 195. Cram. 35 A B. C. (1335.016 1408. tis

1048 Papillon Venone, ou male, Lint. Ë Cram. l.c. ( 1156. bis

1049 Papillon Orithya, ou male, Lirn. 770. 137. Cram. I9. C. D. (1I85. bis.

Ioso Papillon Oenone, ou femelle, Linn. E Cram. h. c. (880. Iost Papillon Clelia, Linn. o. Cram. 2 I. E (877. 878. bis.

I0s2 Papillon Cardui a ailes fauves, variées de blane, $\varepsilon^{2}$ de noir, Lian. 774. 157. Cram. 26. E. F. (II55. bis.

1053 P'apillon Cardui? ous variz:é, Limn. EO Cram. b.c. (1337. bis.

ro54 Papillon Cardui? ou variete, Linn, 80 Cram. l. c (1512. 1055 Papillon Cardui 3 ou variete, Linn. 8 Crail. b.c. (1520. 1056 Papillañ Cardui? a:s variesé Linis. U. (1202.

1057 Pupillon A mathea, à ailes anglées brunes, pointées de blanc, à fafcie rouge. È ligne noire ondée, Linu. 779. 174. Cram. 209. A. B. ( $\$ 419$, Wis.

1058 Papillon Atalanta. a ailes noires, tachetées de blane, d tande pourpre, Linn. 779175 Cran. 84. E. F. (155I. -3059 Paillon Pryteus? ous varieté, Linn. 0. (879. bis. 1060-Papillon Cardui? az yarieté, Irisn. O. (1202. Ic6I Papillbon Atalantas ou femblabie du precedent? Linn. b. c. Cram 30 D. 59. D.E. C 890 bis 1062 Papillon Atulanat ou variete, Litn. E Cram. l. c. (339. bis. 
1063 Papilio Atabonta? f. yarietas, Linn. o. (1338, bis: 1064 Papilio Atalanta, f. femina, Linn lo c. (1599. bis. 1005 Papilio... alis fuscis, maculis córuleo viridibus Linn 0. (1132. bis.

1066 Papilio .... 2n fimilis? ex China, margo poftice ma culis rubris. Linn o. "Cram. 154. A. (871.

1067 Papilio .... f. varietas pracedentis? Linn.0 (III 2 bis 1068 Papilio .... alis nigris . ftriis viridibus, fubtus fusce nebulofis, Linn. O. ( 872 .

1069 Papilio Bolina; Ampericana, alis:nigris, duabus macu lis, Linn. 781. 188. Cram 65. E F (1342. bis.

1070 Papilio Balina? ex China, Linn. O Cram. 290. A. B (882.

I078 Papilio Midamus? C. yorietas, Linn. 765. Ic8? (1604 bis.

1072 Papilio Midamuss? C. varietas, alia, Linn. 1. c. (1166.

1073 Papilio Midamus? Linn. 1. c. Sul3.tab. 16. f. 4. ( $110 \mathrm{c}$ bis.

1074 Papillo Midamus? absąue maculis, Linn. I. c. (IgI) bis.

I075 Papilio Midamus? absque maculis, Lirn. 1. c. ( 1165 ro76 Papilio Midamus? f. varietas, Linn. o. (II63.

1077 Papilio Midamus? f, vorietas, absque maculis, Linn. c. (1164. bis.

I078 Papilio Midamus? f. yarietas, absque maculis, Limm. c. (II65.

I079 Papilio Midamus, f. femina, alis pofticis albo ftriatis Linn. o. (

L080 Papilio Midamus? f. varietas, vel femina, Linn. 6 (II17. bis.

108 I Papilio Midamus? f. varietas, Linn. O. (I167.

1081 Papilio Camilla, alis fuscis albo fasciatis, annulo an rubro, Linn, 78 r. 187. (883. bis.

1082 Papilio Chione. Linn. O. Cram. 90. E. F. (888. bi=. IC83.Papilio Leucothoé, alis fupra fuscis, fubtus luteis, fas ciata, maculis albis nigro diftinctis, Linn. 780. i7s Crasm. 203. E. F. (.988. bis.

1084 Papilio Leucothoè, præcedenti fimilis, Linn. \& Cran 1. c. (887. bis.

1085 Papilio Leucothoë? f. varietas, Linn. I. c. (1607. bis 1086 Papilio Leucothoë ? f. Hylas ? Lim, 780. 179, B (884. bis.

x087 Papilio Hylas? '. Leucothoë? Lina. 1. c. ( I608.

1088 Papilio Dia? f. varievas, Indica, alis fuscis maculatis Linn. 785. 207 ? ESp. tab. I6. f. 4. (1523. 
1063 Papillon Atalanta : ou variete, Linn.l.c. (1338. bis. 1064 Papillon Acalánta, ou femelle. Linn. l. c. (1599. bis. t965 Papillon .... d ailes trunes, taches vertes, Linn. 0. (II3\%. bis.

loos Papillon .... ou pareil? de la Chine, le bord d taches rouges, Linn. o. Cram, 154:A. (87I.

1067 Papillon... o ou variete du precedent? Linn. o. (111 2. bis. roos Papillon . ... à ailes noires; Aries vertes, deforis brune nebuleufes, Linn, o. ( 872 .

069 Papillon Boline, Americaine, a ailes noires, fo deux taches, Linn. 781. 188. Cram. 05. E. F. (1343. bis.

oyo Pspillon Boline? de la Chine, Linn. O. Cram 190. A.B. $(882$.

071 Papillon Midame? ou variete, Linn. 765 . 108 ? ( 1604. bis:

072 Papillon Midame? ou autre varieté, Linn. l c. (II66.

o73 Papillon Midame? Linn. l.c. Sulz. tab. Io.f. 4. (Ir6o. bis.

074 Papillon Midame J fans taches, Linn. l.6. IIIII. bis. 975 Papillon Midame?: Jans tackes, Linn. l.c. (1165. ... o76 Papillon Midame? ou varieté, Linn; O. (1163:

077 Papillois Midame? ou varieté, fans taches, Linn. lo co (I164. bis.

O78 Papillon Midame? ou raricté, Jans taches, Linn, l.c. c. (IIS5.

079 Papillon Midame, or fermelle; a ailes friées de blane, Linn. 0. ( 1118.

080 Papillon Midame? ou varieté, ou femelle, Linn. O. (ir17. bis.

081 Papillon Midame? ou varieté, Linn. O. (1r67.

08 1 Papillon Camilla, de ailes brunes, bandées de blanc, l'anews. d anneau rouge, Linn. 781. 187. (883. bis.

082 Papillion Chione, Linn. o. Cram. 90. E. F. (888. bis.

083 Papillon Leucothoë. dे ailes deffus brunes, defJous jasnes, bandées, d taches blancbes, deftinguées de noir, Linn. 780. 179: Cram. 203. E. F. (988. bis.

U84 Papillon Leucothoë, Fewblable als precedent, Linn. EO Cram. lic. (887. bis.

oS5 Patillon l.eucothoë? ou varieté, Linn. l.c. (1607. bis. 086 Patillon Leucothoê? 'ou Hylss? Linn. 780. 179. B.? ( 884 bis

087 Papillon hylas? ou Leucotheë? Linn. l.c. (1608. Wis. $=88$ Papillon Dia? des. Indes. our varieté, dailes brunes tache: tées, ELinn. 785. 2c7.? Efpe tab.: 16. f: 4. (1523. K 3 I089 Papillow 
1082: Papillo Dia 9 6. varietas, Linn. 1. C. (1524.

Icoj Pa iilo Venilia, Linn. 780. 177. Seb. III. tab. $16 f$. .2. 34. 89. (II 70. bis.

I091 Papilio Vanille? Linn. 787. 216. (II71. bis.

1092 Papilio Vanille? \&. varietas, Linn. I. c. ( 1605.

I'93 Papilio Vanille? f. varietas: Linn. I. c. (898.

1094 Papilio Vanilla? f. varietas, Linn. O. (1217. bis.

1095 Papilio Vanilla? P. varietas, Linn. 0. (899. bis.

jogs Papilio Niphe, alis dentatis luteis, fascia albida, Limn 785. 208: Cram. 14. B. C. (II7I.

Ito7 Papilio Cindra, alis fulvis nigro maculatis, fubtis luteis, punctis nigris, rufo cinctis, Linn. 0. ( 1169 . bis.

1098 Papilio Punctata, alis fulvis nigro maculatis, pofticis flavis fascia aurantia, Linn o. de Geer. II. ... (1552.

I099 Papilio Pundtata? fo varietas, Linn. C. (1522. bis.

I so Pa ilio Maculata, alis luteis auro maculatis, Linm. o . 2 (123) bis.

1131 Papilio Inmaculata, alis fulvis immaculatis, margine \& apice macula aurantia, nigris, Linn: 0. (1232.

İo2.Pupilio Fulva, alis fuscis, fubtus fulvis, Linm. o ( 1621.

Iİo3 Papilio Lydia, Linn, o. Cram. 148. D. (II72. bis. 1104 Papilio Lydia, Linn. O. Cram. 1. C. (ril4. bis.

II05 Papilin Ariadne alis nigro undulatis, Linn. 778. 170. $\therefore$.Cram. $x_{44}$ G. H. (1235.

I IC6 Papilio Ariadne? f. varietas, Linn. \& Cram. I. c (isos.

Iro7 Papilio Cinamomeus, nigro ftriatus, Linn. o. ( 1236.

IIC8 Papilio Plexipprs, alis fulvis, venis nigris, margine ni gro. punctis albis, Linn. 767.117. Cram. 206.E F. (1168. bis.

Iico Papilio Plexipprs? r. varietas, Linn. \& Cram. I. c (892. bis.

I ro Papilin Egifipprus, f. femina, Linn. c. Cram. 180. A (12) 1219 . bis.

fin I.Papilia Egefippus, f. mas. Linn o. (891. bis. III Papilio Chryfippus, alis fulvis, margine ngro, puncti: albis, Limn 767 . II). Cram. 118 B. C. ( 846 .

sir3 Papilio Alcippus, Linn: O. Cram. 127. E. F. (89; r 343 . 1344.125. 
I089 Papillon Dia ? ou variete, Linn. l. c. (1524.)

1092 Papillon Venille, Linn. 780. 177. Seb. III. tab. 16. f. 34. 89. ( 1170. bis.

109: Papillon Vanille, Linn. 787. 216. (ri71. bis.

1092 Papillon Vanille? ou variete, Linn. l. c. ( 1605.

1093 Papillon Vanille? ou varieté, Linn. l.c. ( 898.

1094 Papillon Vanille? or varieté, Linn. O. (1217. bis.

1095 Papillon Vanille? ou varieté, Linn. O. (890. bis.

1096 Papillon Niphe, d ailes dentées jaunes, d bandé blonchea.

tre, Linn 785. 208. Cram, 14. B. C. (II7I.

1097 Papillon Bandé, $d$ ailes fauves tachetées de noir, defous jaunes, d pointes noires, cinglez de rouge, Linn. 0. (1109. bis

1098 Papilion Pointé, c̀ ailes fauves, tachetées de noir, au bout jarnes, à bande orangée, Linn. 0 . de Geer II. (155.2.

1099 Papillon Pointé? ou varieté, Linn. o. (1522. bis. Iroo Papillon Tacheté, dailes jaunes, tacbetées d'or, Linn. 0. (123r. bis.

I 1 I Pasillon Sans taches, ailes fauves, fans taches, le bord, E' fommet d tache orange. noirs, Linn. O. ( 1232.

1102 Papillon Fauve, do ailes brunes, deffous fauves, Linn. 0. ( $162 \mathrm{r}$.

x103 Papillon Lydia, Linn. O. Cram. If8. D. ( II72 bis.

IIO4 Papillon Lydia, Linn. O. (IOr 4.

1105 Papillon Ariadne, à ailes ondées de noir, Linn. 778.170. Cram. I44. G H. (1235.

1106 Papillon Ariadney ou varieté du méme, Linn. \& Cram. $l$ c. ( 1505 .

I 107 Papilion Canel'e, ou Cinamome, forié de noir, Linn. o. ( 1235.

II08 Popillon Plexippus, $d$ ailes farves, yeines noires, bord noir, points blancs, Linn, 767.117. Cram. 206, $E . F$. (II68 bis.

Irog Papillon Plexippus? ou variets, Linn. EO Cram. I.c (802, bis.

I 10 Papillon Egefippe, ou femelle, Linn. O. Cram. I80. 1. (1215. 1219. bis.

III Papilion Egefippe, cu male, Linn. o. (894. bis.

I1I2 Papillon Chryfippe, d ailes fauves, bord noir, points blancs. Linn. 767 rig. Cram. I 18. B. C. (896.

III 3 Papillon Alcippe, Linn. o. Cram. I27. E. F. ( 895. I343. I344. ter. 
II I4 Papilio Phorrefa, alis dentatis fulvis, fasciis tribus fusa cis, Linn. 780. 180. Cram. 130. B. C. (891. bis.

II 15 Papilio PunEtata, alis fulvis nigro maculatis, pofticis punctis fex albis, Linn. O. (1569. bis.

II 6 Papitio Nigrescens, alis fulvis, apice nigro, macula fulva, pofticis fulvis, nigro maculatis, Linn. O. ( 126.

II Pailio Oculata alis fuscis micantibus, porticis ocellis qu lique atris, Linns: C. ( 848 . bis.

1118 Papilio Oculata? f. yarietas, Linn. O. (1218.

I I $9^{\circ}$ Pabilio Lena, idemac no. 886. Linn. 784. 206. Cram. 198. D. E. ( 850 bis.

I1 20 Papitio Piere, atis oblongis diaphanis, poftice binis ocellis, Linn. 754: 52. Merian. Surin. tab. I6. $f$. I. .0. (85)

II2I Papilio Piera? ᄃ. varietas, Linn. \& Merian. 1. c. - 852 .

II 22 Papilio..... alis fusco piceis, fascia alba, porticis fascia rubra, Linn. o. (I I13.

I 23 Papilio Amphinome, alis nigris, cæruleo nebulofis, fasr. cix alba, Linn. 779. 176. Cram, 54, E. E. (1575.bis. II24 Papilio Feroria, alis dentatis, cœruleo fuscoque unduJatis: Lim 770. 140. Cram. I92. E. F. (g18. bis.

I125 Papilio Licus, alis, fufcis, fascia alba, Linno o. Cram. 223. ( 914 . bis.

I 26 Papilin Icarus. Linn o. Cram. 18. A. (14?r.

I 27 : Papilio Icarus? f. varietas, Linn. O. Cram. I. C. (I 234. 1544 . bis.

II 28. Parilio Licus? f. varietas, Linn. O. Cram. 1. c. (1622. bis.

II 29. Pavilio licus? fovarietas, fasciis tlavis, margine purctis rubri, Linn o. \& 1626.

u3a. Pullin Licus? if yariatas. alis furcis . ftrin alba post.cis fuscis, margine-maculis feptem rotundis aureatis, Linn: 0 (1 233.

III I Paptiso Lucille, , f. mas, Linn. 0.: Cram. 156. $A$. (13 52 . bis.

I132 Papilio Lucille I? f. varietes, Linn. O. Cram. 1. c. ( 1355 .

IL 3 Papitio Lucille? f. varietas, Limn e. (1 353 .

II 34 Papilie Lucille? f. varietas, vel mas, Linn. o. (II 31. his::

Ir34 Paplio Cypdrifra Lixn o. Cram: 39. D E. (II32. bs. II 35 Papilio Cyporiffa? r, varietas; Litrmo O. (I35'. I354. 130 Papilio Sylvia, Lim. C. (gI2. bis. 
1137 Papilio Ocraeea, alis fufcis, fascia fuperiorum ex 4. maculis albis, pofticis ocraceis, apice fuscis, Linn 0. Cori.

1138 Papilio Idmone? alis fulvis, apice fuscis, fascia alba, pofticis purpurafcentibus, Linn 781. $182 ?$ (864. .

II 39 Papilio Idmone? f. varietos, Linn. 1. c. (865.

1140 Papilio Cypreffe, Linn. O. Cram 39. DE. (rr32.

II 4 I Papilio ..... alis fuscis, primoribus fascia lata ocracea, pofticis fascia pallida, Linn. O. (908. bis.

Ir42 Papilio Lucille? f. varietas, Linn. o. (1356. I357. r56r. ter.

II43 Papilio .... alis fuscis, fascia alba, bafi fulva, Linn. 0. (998.

1144 Papilio Eropus, alis fuscis, fascia lutea, Linn. 708. 128. Cram. III. F. G. ( 906.

II45 Papilio ..... alis fuscis, macula apicis ocracea, fubtus punctis 4. flavis, Linn. O. ( 1264 .

1146 Papilio ..... alis fuscis, fascia obliqua ocracea, pofticis rufis, fubtus cinereis, ocellis cocceis 4. Linn o. (1418.

II 47 Papilio ..... alis fuscis, disco fuperiorum luteo, pofticis bafi luteis, Linn. o. ( 900 bis.

Ir 48 Papilio ..... alis fuscis, fuperioribus ad apicem albidis, Linn. o. ( 1106.

II49 Papilio ..... alis fuscis, fubtus ftriis duabus rufis, punetisque alb:s, Linm. o. (II39. bis.

1150 Papilio.... f. varietas, Linn. 0. (1392. bis.

II5I Papilio ..... alis fuscis, fascia maculari alba, pofticis corruleis, ocello ad anum, Linn. O. (913. bis.

1152 Papilio Cafire, alis fuscis, fascia ferruginea, Linn. 767. 1 20. Cram. IC5. A. B. ( $44 \mathrm{C} 8$.

II53 Papilio Sephore, alis fuscis, ccello unico, Linn. 767. I21. Merian. Surin. tab. 35. (1240. bis.

II54 Papilio Sophora? C. varietas, Linn \& Merian. I. c. ( I242. bis.

1155 Papilio Cafjie, Limm. 767. 120. Cram. I05. A.B. ( 905. bis.

II56 Papilio Xanthus, alis fupra fuscis fascia ferruginea, Linn. 767. I22. Cram. 183. A. B. ( 904.

II57 Papilio Xanthus? f. varietas, Limo. \& Cram. 1. c. ( 1478.

IIs8 Papilio Xanthus, præcedenti affinis, Linn. \& Cram. 1. c. $(1477.1479$. bis. 
II 37 Papillon Ocrée, a ailes brunes, la bandes des fuperieures de 4. taches blanches, les pofterieures ocrées, au point brune, Linn. 0. (9I I.

1138 Papillon Idmone? d ailes fauves ar point brumes, d bande blanche, aub bout pourpreses, Linn. 781 1. 182? ( 864 .

II 39 Papillon Idmone? ou varieté, Linn. l. c. ( 865.

I 40 Papillon Cypreffe, Linn. o. Cram. 39. D E. (rr 32.

II4I Papillon.... d ailes brunes. les anterieures bande ocrie, les pofterieures d bande pale, Linn. O. ( 908. bis.

II 42 Papillon Lucille? ou variete, Linn. O. (1356. 1357. I56r. ter.

1143 Papillon ..... aे ailes brunes, bande blanche, de la bafe farve, Linn. O. ( 998.

1144 Papillon Eirope, dे ailes brunes, bande jarne, Linn. 768. 128. Cram irx.F. G. (006.

II 45 Papillon .... à ailes brunes, tache du fommet ocrée, des: fous 4 points jarnes, Linn. 0 . (1264.

II 46 Papillon .... à ailes brunes, bande ocrée, obligue, les pofterieures rougatres deffous cendrees, da 4 yeux rouges, (Linn. O ( 1418.

II 47 Papillon .... dे ailes trunes, le palet des fupsrieurs jarnes, les pofterieures de la bafe jaunes, Linn. c. ( 900 bis,

II 48 Papillon .... à ailes brunes, les fuperieures blanchatres d'en haut, Linn. 0 . ( I ro6.

1149 Papillon ...d ailes brunes. deffous 2 ftries rougatres, Eg poirts bleues, Linn. O. (I139. bis.

II 50 Papillon ..... ou varieté, Linn. O. (1302. bis.

Iis I Papillon ... \& ailes brunes, bande tachetée de blane, les pofterieures bleues, ur oeil d l'anus, Linn. o. (9I3. bis.

II52 Papillon C:Sie, à ailes brunes, bande couleur de rousille, Linn. 767. 120. Cram. 105. A. B ( 1408 .

II 53 Papilion Sophore, à ailes brunes, Eo un oeil, Linn. 767. 121. Merian. Surin. tab. 35. (1240. tis.

It54 Papilion Sophore? ou varieté, Linn. EO Cram. b. t. (1242. lis.

1155 Papillon Caffie, Linn. 767. 20. Cram Io5. A. B. ( 909 . bis.

II 6 Papilion Xanthus, da ailes deffus brabies, $d$ bnande couleur de rouille. Linn. 767. 122 Cran. 183. A. B. (904.

1157 Papillon Xanthus? ou varieté, Linn. ô Cram. l. c. ( 1478.

1158 Parillon Xarthus, femblable as precedent, Linn E Cram, b. c. (I 477 . I479 bis. 
I159 Papilio Odius Fabrici Lina O. (1239.

Ir60 Papilio .... alis fuscis, fubtus ocellis grifeis, Limn. 0. ( I 243.

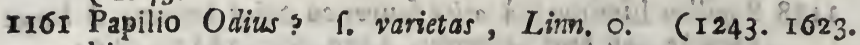
bis.

I 62 Papilio .... alis fuscis, fubtus oculis grifeis, Linn. 0.

$\therefore$ ( 1243 .

II63 Papilio ..... alis fuscis , fubrus ad marginem ocellatis, primoribus ocellis 4. pofticis quinque, Limn. O. (907. bis.

II64 Papilio ..... alis fuscis, fubeus grlfeis, ftriis duabus rufis, pofticis ocellis quinque, Linn. $00^{*}$ ( 1247.

II65: Papilio .... ᄃ. varietos, vel pracedenti aftinis? Linn. 0. ( 1068.

II $66^{\circ}$ Papilio Fusca, pofticis ocello, pupilla argentea ad anum, Linn. O. ( 1173.

1167 . Papilio Fusca. fubtus fascia obliqua alba, primoribus oce lis 2:, Linn. O. ( 1476.

r 108 Papilio CalJus, alis fucis, Linn. 768. 125. (I361. bis.

I 99 Papilio Clytus, alis fufcis, fascia flsva, Linn. 768. 124 Cram. 86. C. U ( 1360.

1170 Papilio ..... alis fa!catis, fubcaudatis, fuscis, primo-

i ribus macula atra, aurantio cineta. Limn. o. ( 1586.

I 7 I Papilio.... f. yarietas, pofticorum ocelis fex ex$\because$ ternis maximis, Limn. o. C...

1172 Papilio .... alis fuscis, fubtus forticis ocellis 6. , Linir. o. (1069. bis.

I 173 Papilio... r. varietas, Linn. o. (1070.

II74 Papilio Mara, a is fuscis, pofticis fupra tribus ocellis, Linn. 771. 141. ( 1528

II75 Papilio Fanira, alis fuccis, nrimnrihus luteis, unico utrirque ocello; Lim. 774. I56. (I589.

II76 Papilio .... alis luteis, uncici, ocello bipupillato, I.inn, o. ( 1529.

1177. Papilio .... alis luseis, fusco undatis, ocello uno, pofticis tritus, Linn. 0. ( 1525 .

Ir78 Papilio .... . variatos; Limm o ( 526 .

II 19 Papilio.... alis fuscis, ad marginem villofis, Limn.

is: 0.0 ( 1530 .

I 80 : Papilio .... alis cinereis, macula fulva, pofticis ocellis feptem. Linn. O. ( 1532 bis

Ir8r. Papiio ..... f varietas, vel femina, Linn. o. (Is53. II\&2 Papilio Furtina? exotica, f. varietas, Limm. 774. I55? (1527. bis. 
1159 Papillon Odie de Fabrice, Linn. -. (1239

1160 Papillon ... a ailes brunes, deforus des yeux gris, Lim. o. ( 1243.

II I I Pajillon loudie? ous varieté, Linn. O. (1243. I623. bis. I 162 Papillon, a ailes brunes, défjous des yeux gris, Linn. 0 . ( 1243.

I 63 Papillon ... a ailles brunes, des yeux au bord, tes anterieures, de $4 . E^{\circ}$ les pofteriewrés de 5 yeur, Linn. $\bullet$ ( 907 . bis.

1164 Papillon . . d ailes brunes, defous grifes, of deus ftries rougatres, bes pofterieures ont 5 yeux, Linn. 0 . ( $1247^{\circ}$.

1165 Papillon ... ous voriet, ou Jemblalle ars precedent? Linn. o. ( I068. .

II66 Papillon Brun, Jes pofterieuspes de un oeil, dont la pruneile argentee, vers l'anus. Liñ. O ( 1173

II67 Papillon Brun, defjous d bande oblique blanchie, hes anterieures de deux yeux , Lien. O. (1476.

1168 Popillon Caftus, Linn. 768. 125. (1361. bis.

riog Papillon Clytus, dे eiles fawves, bande jawne, Linn. 768. 124. Cram 86.C. D. (1360.

II 70 Papillon.... à ailes courbees, en queue, brüres, tes anterieures, dine tache noire, cirglées do arange, Linn. 0. ( 1586.

I17I Papilion .... ou voriete, is 6 yeux entericures trids gronds, Lisn. o. ( . . .

1172 Papillon .2 a aibs brwnes, defjous 6 yeux pofte vieures, Linn. c. (1069. bis.

I I 3 Papillon .... ous variets, Linn. O. ( 1070.

II74 Papillon Mara, d ailes brunes, les poflerieures de Jus a trois petis yeux, Linn. $77 x$ 141. (I 528 .

I175 Papillon Janira, à ailes bnunes, les premieres jaunes, de chaque côté un petit oeil, Linn, 774.156 ( $1589^{\circ}$

I 76 Papillon ..... à ailes jaunes anterieures, wn oeil d deux pruneazx, Linn o. ( I 529.

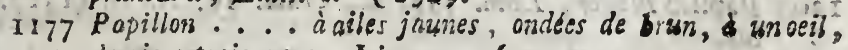
derriere trojs yeux, Linn. O $\$ 1525$.

I 78 Papillon..... ou varieté Linn o. (I526.

1179 Papillon . . i. a ailes brunes, as bord velue, Linn. o. $\left(1330^{\circ}\right.$

1180 Papilion .... à ailes cendres tacbe fawve, pofteriewrs de 7 yeux, Linn. O. (1532. Lis

118 r Papillon ..... ou variete, on femelle, Linn, O. (1553. II82 Papillon Jurtine? ois varieté, etranger, Linn. 734 . 155 ? (1527: bis. 
1183. Papilio Hermione? Indica f. mas, alisfu!cis, falcia pallida Linn. 773. 149? Ejpe tab. 8. $f: 20^{\circ}$ (1531. bis. 3184 Papilio Hermione? C. yarietas, Linm. . c. (1556. 1185 Papilio Hermione, If femina, Linn. I. c. (1533. bis. rr80 Papilio Hermione? f. varietas, Linn. 1. c. (1554.bis. 1 187. Papilio Hermione? f. varietas, Linn. I. c. (1535. bis. 1188 Pa ilio ..... alis fufcis, primoribus ocellis duobus, fubtus fafcia undata lata communi, Linn. 0 . ( $1557^{\circ}$

1189 Papilio Hermione? f. varietas, Linn. o. (I554.

1190 Papilio Hermione? f. varietas, Linn o. (I555.

1191 Papilio Hermione, r. mas Linn 1. c. (1531. bis.

1192 Papilio Caffe, eadem ac $n^{\circ}$. II52. Limm. 767. 120. Cram, 105. A. B. (I24I bis.

1193 Papilio Teucer, eadem ac no. 870. Linn. 753. 44. (iIIO.

Ir94 Papilio Teucer? fo varietas, Linn 1. c. (1627.

I195 Papilio Teucer? \& vari,tas, Linn. l. c. (930.

I 96 Papilio Idomensus, eadem ac n०.89j. Linn. 753. 45. (929. bis.

II97 Papilio.... alis fuscis, disco cyaneis, margine punctis aurantiis, Lism o. (I 4 I 4 .

Ir 98 Papilio Helenor, eadem ac no 931 . Linno. O. Cram. 86. A. B. an Linn. 745. 4? (927.

irg9 Papilio Neftor, eadem ac n 932 . Limn. 752. 40. Cram. 19. A. B. (1415.

I 200 Papilio Achilles, eadem ac n०. 933. Limn. 752. 42. Cram. 27. A. B. (228. bis.

1201 Papilio Achilles, Lirin. I. c. (936. ter.

1202 Papilio Helenor, Linn. o. Cram. 86. A. B. (935.

1203 Papilio Menelaus, eadem ac $n^{2} .936$. Linn. 748. 20. Cram. 2r. $A$ B (93r. bis. :

1204 Papilio Rethenor, eadem ac $n^{\circ} .937$. Linn. O. Cram. 15. A. B. (932. bis.

I205 Papilio Neftor, Linn. 752. 40. Crams. 19. A. B. (I3I7. bis.

1200 Papilio Achilles? r. varietas, Lirn. \& Cram. I. c. ( 1505.

r207 Papilio Merelcus, Linn. \& Cram. l. c. ( 934 bis.

1208 Papilio Idea, alis albir, venis \& maculis nigris, Linn. 758. 73. (937. bis.

I209 Papilio Idea \& f. varietas, maculis atris, Linn. I. c. ( 1609.

г2 ro Papilio ..... alis fulvis inmâculatls, margine nigro, punctis 2. arjenteis, Linn. O. (9+1. bis. 
1183 Papillon Hermione? Indien, ou male, d ailes brunes, bande pale, Li in 773. 149? Lifpe tab. 8. f. 2. (I53I bis.

1184 Papillon Hermi ne? ou variete, Linn b. c. (1556.

1105 Papillon Hermione, oufemelle, Linn. l c. (1553. bis.

Ir 86 Papillon Hermione? au variete, Linn. l. c. (1554. bis.

1187 Papillon Hermione? ou varieté, Linn l.c. (1535. bis.)

II 88 Papillon .... d ailes brunes, les anterieures d deuse yerbx, defous large bande ondée, Linn. O. (1557.

II89 Papillon Hermione? ou variete, Linn. O. (1534.

II90 Pafitlon Hermione? ou autre variete, Lino. 0. (1555.: IIgi Papillon Hermione, ou male, Linn. . . c. (1531. bis.

I1 92 Papillon Caffie, le meme que no. I155, Linn. 767. 120' Cram, 105. A. B. (1241. bis:

Ir 93 Papillon Teucre, le méme que ñ.. 870. Linn. 753. 44. (Irio.

1194 Papillon Teucre? ou variet है, Linn. J. c. ( $1 \sigma_{270}$

II95 Papillon Teucre? ou varieté, Linn. l. c. ( 930.

I 96 Papillon Idomenée, le mémé que $n^{\circ}$. 895. Linn. 753. 45. ( 929. bis.

II97 Papillon .... a ailes brunes à difque blcu, le bord a pointes oranges, Linn. O. ( 1414 .

II98 Papillon Helenor, le meme que n?. 931. Linn. O. Cram. 86 4. B. ou Linn. 745. 4.? (927.

I199 Papillon Neftor, le méme que n०.932. Linn. 752. 40, Cram. I9. A B. (1415.

1200 Papillon Achille, le méne que no. 933. Linn. 752.42. Cram. 27. $A$ B. (928. bis.

201 Papillon Achiles, Linn. \& Cram. l. c. (936. ter.

122 Papillon Helenor, Linn. O. Cram 86. A. B. ( 935.

1203 Pápillon Menelaus, le meme que n. 936. Linn. 748 . 2o. Cram. 2r. A. B. (93r. bis.

t204 Papillon Rethenor, le meme que, $n^{\bullet}$. 937. Linn. O. Cram. I5. A. B. (932. bis

1205 Papilion Neftor, Linn. 752. 40 Cram. 19. A. B. (13r7. bis.

1206 Papillon Achilles? ou varieté, Linn. E Cram. l. c. (1506.

1207 Papillan Menelaus, Linn. E Cram. l. c. (934. bis. 1208 Papillon Idea, aiies blanches, veines \& taches noires, à bord noir, pointé de blanc, Linn. $758,73$. (937. bis.

I209 Papillon Idea? ou variete, d taches noires, Linn. $7 . t_{0}$ ( 1609.

I210 Papillon .... à ailes fauves fans taches, d̀ bord noire, 82 pointes argenties, Linn. 0 . (941. bis. 
I2II Papilio :. . . f. varietas, Linn, o. (I482.

I2I2 Papilio ... alis: flavis, margine \& apice nigris. fubtus primoribus macula ferruginea, polticis duabus argenteis, Line o. (I250.bis.

I2I3 Papilio ..... r. varietas, Linn. o. ( 1249.

I2I 4 Papilio Lyjippus, f. mas, alis ats is , fafcia lineari rubra. Limm. 793. 250: (946.

1215 Papilio Lysippus, f. femina, Linu. 1. c. ( 945.

216 Papilio Euippe, alis flavis, primoribus apice atric, pofticis fubtus albis Linn. 762. 87. (I I 74 .

1217 Papilio Phleas, alis fulvis nigro punctatis, fubcus albo marginatis, Liqm. 793.252. ( 1 IO4.

I218 Papilio .... alis flavis, apice nigro, macula aurantia, Linn. 0. ( II77. bis.

rèrg Papilio .... alis albis, apice \& margine atris, macula aurantia, Linn. O. ( II38.

I220 Pàpilio Hyparețe, alişalbis, pofticis fubtus venis maculifque rubris allian. 763. 92 . ( 1570 .

1221 Papilio Hyparete, Livin. I. c. (I 587.

I222 Papilio Hyparete? \&. varietas, Limn. I. c. (I 20.

I223 Papilio Hecabe, alis flavis, eximo acris, fubcus punetis Iren pallidis, Linn. 763.96. (953. bis.

I224 Papilio Hecabe? an præcedenti affinis? Linn. 1. c. (952.

I225 Papilio Hecabe? f. varictas Linn 1. c. (954. bis.

1220 Papilio .... alis fivis, margioe nioro, fubtus puncta nulla, Linn. O. ( 1396.

1227 . Papilio ..... alis fulvis, fubeus non punctatis, Linn, o. (942.

I228 Papilio.... alis fulphureis, margine \& apice atris, macula atra, dentatis oblongis, Linn. ?. (II33.

2229. Papilio Chboris, albs, Cubius flava, margine nigro, Linn. o Fabric. 183. Crsm 207. F. G. ( 1256.

x.2zo Papillo Sylvia, Linn. O. Fabric. I66. (1 255 . bis.

123I Papilio Hyale? als fisvis, fubtus primoribus punctc ferruginto po?ticis duabus argenteis, Linn. 764. I00? Cram. I4.F.G. ( 943.

r232-Papilio ... alis, Hbis, bafi flavis, margine nigris, Libin. O. ( 1572 .

1233 Pap:lio .... alis albis, bafi flavis, puncto medio atro, Lin?. B. ( I 25 I. bis.

.234 Papilióo.... varietas. Linn o. (148r. bts.

I 235 Papilio.... alis alts apice late nigris polticis fubtus flavis, ferie punctorum nigrorum, Linn. o. (939. bis. 
3211 Papillon .... ou varieté, Linn. o. ( 1482.

2212 Papillon .... d ailes jaunes, be bord, EO Sommet noirs, par defous les anterieures d'une tache rouillee, les pofterieures di deux taches argentées, I.inn. 0 . (I 250 . bis.

I 213 Papillon . . . or varieté, Linn. O. (1249.

1214 Papillon Lyfippus, ou male, do ailes noires, bande rouge, Linn 793.250. (946.

1215 Papillon Lyfippus, ou femelle, Linn. l. c. (945.

1216 Papillon Euippe, d ailes jaunes, les premieres d fommet noires, les pofterieures deffous blanches, Linn. 762. 87. ( 1174.

I217 Papillon Phlæas, à ailes fauves, pointées de noir, dedjous berdées de blanc, Linn. 793. 252. (Iro4.

1218 Papillon . ... d ailes jaunes, fommet noir, tache orange, Linn. O. ( II 77 bis.

1219 Papillon .... d ailes blanches, be fommet \& bord noir. d tache orange, Linn o. (1138.

1220 Papillon Hyparete, d ailes blanches, les pofterieures des. Jous veines, EO taches rouges, Linn. 763. 92. ( 1570 .

122 I Papillon Hyparete, Linn. l. c. (1587.

1222 Papilinn Hyparete? ou varieté, Linn. l.c. (II 20.

1223 Papillon Hecabe, d ailes arrondies jaunes, noires au bout, deffous a poirts pales, Linn. 763. 96. (953. bis.

1224 Papillon Hecabe, ou pareil, Linn. l. c. (952.

1225 Papillon Hecabe? ou varieté dus precedent? Linn. I 6 . (954. bis.

1226 Papillon .... d ailes jaunes, bord noir, defous aucune pointe, Linn. o. ( 1396.

I227 Papillon ....d ailes farives, de Jous Jans points, Linn. O. $\left(94^{2}\right.$.

1228 Papillon .... d ailes couleur de foufre, le fommet, E le bord noir, les pofterieures a tacbe noires, E dentelseses, Linn. o. ( $x+33$.

1229 Papillon Chloris, blanche, defous jaune, d bord noir, Linn. O. Fabric. 183. Cram. 207. F G. (1256.

1230 Papillan Sylvia, Linn. O. Fabric. 106. (1255. bis.

123 r Papillon Hyale, d̀ ailes jaunes. defous de point rouillé, les pofterieures 2 argenties, Linn. 764. 100. Cram. I4. F. G. ( 943.

1232 Papillon .... les ailes blanches, de la bafe jaunes, ars point burd noires, Linn. o. ( 1572.

1233 Papillon .... les ailes blanches. de lo baje jaunes, d noir ou milieu, Linn. O. (125\%.bis.

1234 Papillon .... ou varieté, Linn. O. ( $148 \mathrm{r}$, bis.

1235 Papillon.... d ailes blanches. les pofserieures noires. def]ous jaunes, à nombre de points noirs, Linn. o. (939 bis. 
1236 Papilio Rhamni ?. C. varietas, Indica, alis primoribus aurantis, marpine flavo, Linn. 765. 106? (I536. bis.

I2.37 Papulio Rhamni? Indica, Linn. 1. c. ( 1562.

1238 Paprilio Hyale, Linn. 764. 100. Cram. 14. F. G. (1315 bis.

1239 Papilio Hyale? fo varietas, Limn '. c. (1362. 1537. 1538. ter.

I 240 Papilio Hyale? fo varietas, Linn. 1. c. (1363. bis.

I24I Papilio Hyale? f. varietas, Linn. O. ( I 423 . bis.

1242 Papilio.... alis Aluis, margine nigris, fubtus pallidis, Itría coximuni rufa, Linn. O. ( 944 bis.

1243 Papilio .... alis flavis, margine atro, Linn. 0. (1366.

1244 Papilio .... alis flavicantibus, margine lato atro, Linno o. (1254. 1610: bis.

1245 Papilio .... alis flavis, unicoloribus, Linn. 0. (1248. ter.

1246 Papilio.... alis albis, bafi flavis, Linn o. (rro5. 1247 Pap.lio .... alis albis, nervis, \& apice nigris, Limm. o. (II76.

1248 Papilio.... alis albis, apice nigro, fubtus margo niger, maculis cordatia Aivis, Limm o. ( 1383 .

1240 Panilio .... r. rovieias, Linn. o. (1354.

1250 Papilio.... alis albis, maella apicis atra, Linn. 0. (1539. bis.

325 Papitio.... alis albis, bafl flavis, margine atro, Linn. O. ( 1571 .

1252 Papilio.... alis flavis macula fubtus ferruginea, duabus peficon argenteis, Lim. o. (1248,

I253 Papilin Lybithis, Limb. O Fabric. I72. (1175. bis. 1254 Papilio ... alis albis apics a femifascia atris, fubtus fu cis, Lim. O. ( 938.

I255 Papilio... alıs alhis, apice macula deniata aira, Limn. O. ( 1285 .

256. Papllio .... alis fupra igris, fascia incurva alba, bafi croceis, Linn o. (1365.

I 257 Papalio... . alis albis, apice atıis, Linno o. (I54ว. bis.

I258 Papilio ... alis albis, macula \& apice nigris, Lim. O. C 1542 .

1259 Papilio ...., . varietas, Linn. 0. (II37.

I260 Puullio Cardomines fo mae, alis albis, primoribus fu'vis, polticis viridi nebulolis, Lim. 701. 85. Eaw. 125. ( $5040^{\prime}$ bis. 
236 Papillon Rhamni ? ou varieté, des Indes, les ailes premieres a'orange d bord jaune, Linn. $765.106 . ?$ ( 536. bis.

237 Papillon Rhamni? des Indes, Linn. b. c. '(1562.

238 Papillon Hyale, Linn. 764. 100. Cram. 14. F. G. ( 1315.

239 Papillon Hyale? ou varieté, Linn. l. c. (1362, 1537. 1538. ter.

240 Papillon Hyale? ou varieté, Linn. l. c. ( 1363 . bis.

zar Papillon Hyale? ou varieté, Linn. O. ( 1428 bis.

242 Papillon .... à ailes jawnes, le bord noires, delfous pales, à frie conmune rouge, Linn. O. (944. bis.

243 Papillon . ... à ailes jaunes, à bord noir, Linn. O. ( 1366. 244 Papillon ... d ailes jaunatres, à large bord noir, Linn. o. (1254. 16io, bis.

245 Papillons.... à ailes jaunes d'un couleur, Linn. 0. (II 48. ter.

246 Papillon.... à ailes blancbes, de la baje jaunes, Linn. o. (1105.

247 Papillon.... à ailes blanches, les nerfs EO fommet noirs, Linn. o. (1176.

248 Papillon ... do ailes blanches, le fommet noir, deffous le bord noir, de tacbes jounes en ceur, Linn. o. ( 1363 .

249 Papillon .... ou ja varieté, Linn o. ( $\$ 364$.

150 Papillons.... à ailes blanches, le point à tache noire, Lirin. O. ( 1539.

25 I Papillon .... da ailes blanches, de la baje jaunes, à bord noir, Linn. O. ( $157 \%$.

252 Papillon .... di ailes jounes, defjous tache couleur de rouilie, pofterieures deux argentées, Linn. 0 . (1248.

253 Papillon Lybythie, Linn. O Fabric. 172. (II75 bis. :54 Papillon .... à ailes blanches, le jommet, Ẽ demi bande noirs, defJous brumes, Linn. o. (938.

255 Papillonz .... à ailes blanches, le Jommet d tache noir, dentelé Linn. o. ( 3285 .

256 Papillon .... les!ailes deffus noires, bande courbée, blanche de la bas:, couleur de Jaffran, Linm. O (1365.

257 Papillon .... à siles blanches, au point noires, Linn. o. (154\%, bis.

55 Papillon... d ailes blanckes, tache EO fommet noires; Linn. O. (1542.

259 Papillon ..... or varieté, Linn o. (II37.

:60 Papillon Cardamines, ou male da ailer blanches, les premieres fauves, les pofterieures verd-nebuleufes, Linn. 761. 85. Edw: 125. (1564. bis. 
1261 Papilio Cardanines? f. varictas, Linn. \& $E d w .1$. c. ( 1076.

1262 Papilio Cardamines? f. varietas, Linn. \& $E d w$. I. c. (1679.

I263 Papilio..... alis albis, primoribus apice, pofticis margine nigris, Linn. O. (1259 bis.

1264 Papilio Belia, alis albis, grifeo fasciatis, Linn 76r. 84. (1513. bis.

1265 Papilio Belia? f. varietas. vel mas, Linn. I. c. (940. bis. 1266 Papilio ..... nis oblongis albis, apice fu:cis, Linn. ०. (996. bis.

1267 Papilio Belia? r. varietas, Linn. O. (1265. 1685. bis. 1208 Papilio ... . alis fuperioribus macula flava, Linn. $0^{\circ}$ ( 955.

1269 Papilio . ... alis fuperne fuscis, disco ad bafin fulvo, primorum apicibus macula ovali alta, Linn. $0 . \quad$ (905.

1270 Papilio ..... alıs albis, 2pice nigris, fubcus margıne, \& cofta flavis, Linn 0. Cram.2s. I. H. (1260. bis.

1271 Papilio.... alis albis, margine nigro, ad nervos ftriato, an Sphinx? Linn. O. ( 1499.

1272 Papilio ..... a'is rigris, disco flavo, Linm. o. ( 956.

1273 Papilio ..... alis fupra albis, apice atro, pofticis flavo fuscis, Linn. no. (I 34 . bis.

1274 Papilio ..... alis albis, atice lato atro, macula alba, fubius flavis maculis, pofticorum quatuor argenteis, Lini3. O. ( I 135 .

1275. Papilio ..... alis albis, cona nigris, Linn, o. ( 1257.

1276 Papilio Brafica, alis albis, primoribus maculis, \& apice duabue atris, Linn. 759. 75. ( 1186.

1277 Papilio Brafica, f. mas, Lirn. I. c. (154I.

1278 Papilio . . alis fuscis, primoribus ocello, pofticis 7. Linn. O. Cram. 12. G. H. ( $107 \mathrm{I}$.

1279 Papilio.... alis brunneis, fuperiorum macula, pofticorum fascia alba, Linn. O. (1496. bis.

1280 Papilio . . . . r varietas præcedentis, Limn. O. (r498. bis.

128, Papilio ..... alis pullis, oceilo ad marginem, pofticis oceliis ublcuris, Linn o. (1237. bis.

1282 Papilio .... . . varietas, vel $n^{\circ}$. 1279. Linn. 0. (I)74.

2283 Papilio Pkylleus, Linn. O. Cram. 20. E, 64. D. E? ( 1474.

1284 Pspilio Fhylleus? C. varietas, Limn.o O. Cram. 282. A. B. ( 1497 .

1285 Papilio thylleus? f. varietas . Linn o. Cram. l.c. (915. 1286 Papilio Lucinde, Limn. O. Cram. I. E. F. (1079. 
125r P.pillon Cardamines? ou variete, Linn. E Edw. I. c. (:076.

I262 Papillon Cardamines? ou variete, Linn. \& Edw. l.c. (1679. 1263 Papillon . . . . les ailes blancbes, au premiers d'un point, les pofterieures d'un bord noir, Linn. O. (i259. bis.

1264 Pnpillon Belie., da ailes blanches, bandées de gris, Linn. 761 84. (1513. bis:

1265 Papillon Belie? oumale, ou varieté, Lian. l.c. (940.bis. I266 Papilion.... les ciles allongées blanches, d Jommet brunes, Linn. O. (996. bis.

1267 Papillon . . . or varieté, Linn o. (1 265. 1685. bis. 1268 Papillon.... les ailes fuperieures d'un tache jaune, Linn. 0. (955.

1269 Papillon .... d asles deffus brunes, la baje fauve, d tacke ovale blanche, Linn. 0 . ( 905 .

1270 Papillon .... d ailes blanches, ar point noires, le bord E cote jaunes, Linn. o. Cram. 20. I. H. ( 1260 . bis.

$127 \mathrm{r}$ Papillon ..... dailes blanches, le bord noir, ftrié vers les nerfs, os Sphinx.? Linn. o. (1499.

272 Papillon .... d ailes noires, palette jasune, Linn. 0. (956.

273 Papillow .... à ailes defjus blancbes, d̀ point noir, les pofterieures $j$ aunzes, E9 brunes, Linn. O. (I134. bis.

274 Papillon .... a ailes blanches, d'un point barge noir, d tache blancbe, les pofterieures d quatre taches argenties, (Linn O. (II35.

275 Papillon .... à ailes blanches, du côté noires, Linn. 0. (1257,

276 Pajillon das Choux, \& ailes blanches, les premieres E 16 fommet à deux tackes noires, Linn. 759. 75. (1186.

277 Papillon des Choux, ou male, Linn. l.c. (I54r.

278 Papillon .... les ailes brimes, les devants d un oeil, le pofterieures d 7 yeux, Linn. O. Cram. 12. G. H. (ro7x.

279 Papillon....d ailes brunes. les fuperieures d'une tache, le pofterieures d bande blanche, Linn O. (1496. (bis. 280 Papillon .... ou varieté, Linn. O. (1498. bis.

28 i Papilion .... d ailes noires, un oeil au bord, les pofserieures ont des yeux obfcures, Linn. O. (1237. bis.

282 Papillon .... ous varieté, ou no. 1 279. Linn. 0. (1074. 283 Papilion Phylleus, Linn. o. Cram. 20. E, 64. D. E? ( I 474.

284 Papillon Phylleus? ouvariett, Linn. O. Cram. 282. A. B. ( 1497.

85 Papillon Phylleus? ou varieté, Linn. O. Cram. l.e. (g15. :86 Papillon Lucinde, Linn. O. Cram. I. E. F. (1079.

$$
\text { L } 3 \text { I287 Papillon }
$$


165

I287 Papilio Ariadne, alis nigro undulatis, antice puneto a/. b.), Linn 778, I 70. Cram. 86. E. F. (II15.

1288 Papilio.... ceinereó fusca, macula alba ad apicem, Linno O. C 922 .

1289 Papilio Grilea, punctis flavis, pofticis ftria aurantia, Linn. o. ( Ic8 r.

I290. Papilio .... fuperne fusca, inferne ocracea, ftria rufa, Linn. o. (1080.

I291 Papilio .... alis cinnamomeis, primorum fascia alba, 4... nofticorum macula disci, Linn. O. ( 1475 .

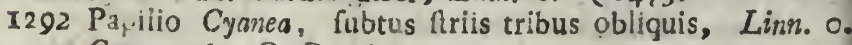
Cram. I69. C D. ( IOgI.

1293 Papilio Fusca-Cinerea, ad anum coruleo micans, pofticis frris tribus, primoribus uno ocelio, pofticis 3 . intimo 2 pupillaco, fubtus cyaneis, Limn. o. Cram. 10. D. E. ( 1214 .

1294 P pilio Fusco-Cinerea, fasciis obliquis 5. pofticis ocellis 4. Linn 0. Cram.... B. C. ( 1628 .

I295 Papilio... alis fupra albis, apice fuscis, interne ftriis 4. ocellis 5. pupillis argenteis, Linn. O. Cram. 194. D. E. ( 1073 .

I296 Papilio ..... alis fuscis cœruleo micantibus, fubtus, Itriis tribus, ocellis quinque, Linn. o. ( 1072.

1297 Papilio'..... alis fuscis, prim rribus maculis, fascia alba, polticis ocelli atris, Linn. O. ( I 353 .

I298 Papilio . . . . alis pallidis, maculis fuscis, pofticis ocellis 4. ultimo azureo, Linn. O. ( 1472 ,

I299 Papilio . ... alis fuscis, primoribus fex maculis albis, pofticis ocellis 3. piimo tripupillato, Linn. O. Cram 24. $D$ ? $(923$.

1300 Papilio.... alis albidis, margine nigris, maculis lunaribus , Linn. O. (1 259.

I 3 І Papilio .... alis fuscis violacen micantibus, fubtus albis, fasciis 5 rufis, purtice ocello azureo, Limn. O (924.

I 302 Papilio .... alis albidis, pifticis nriis duabus fu cis, ocellis tribus, Limm. O. ( 225 .

1303 Papilio .... alis fuscis, fupra ocello I. pofticorum 2. pupillis argenteis, infra ftriis duabus fuscis, Limn. o, (1103.

1304 Papilio ..... a'is fuscis, fascia femicirculari alba, Linn. o. (I233.

I30'5 Papilio ..... alis nigro cyaneis, porticis ad anum albis, Linn. O. Cram. 184. D. E. (1075.

1306 Papilio .... alis fuscis, bafi ocellatis, fubtus pnfticis albis, Limo. o. Cran. 184, F? G? (1473. 
1.887 Paritlon Ariadne, à ailes noires, ondées, devant d pointe blanche, Linn. 778170 Cram. 86 . E. F. (IIIS

I 288 Papillon.... cendré brun, à tacke blanche vers le point, Linn o. ( 922.

I 289 Papillon Gris, à pointes jounes, poflerieures d'une firie crangée, Linn. O ( 108 I.

129 Papillon .... defJus brum, deffous ocrée, d̀ ftrie rougeatre, Linn. o. 11080.

1291 Papillon .... da ailes canellées, lespremieresd bande blanclie, les pofterieures a tache da disque, Linn. 0. ( 1475. $12 \ni 2$ Papillon B!eu, deffous dio trois fries obliques, Linn. 0. Cram. Iog. C. D. (ro)?.

I293 Papillon Bleu-Cendré, vers l'anus bleu luijant , a 3 ftries, les anterieures à un ceil, les pofterieures trois, desfous blens Linn. O. Cram 10. D. E. (1244.

1274 Papillon Brwu-Cendré, à cinq bandes obliques, les pofterieures à quatre yeux Linn, o. Cram... B. C. (16?8.

1295 Papillon.... les ailes deffur blanches, ars point brunes, en de dans 4 fries, 5 yeux, iprunelles argentées, Linn. 0 . Cram. :94. D. E. (1073.

1206 Papillon....d ailes brures, luifante de blers, defjous 3 Aries $80^{\circ}$ cinq yeux, Linn, o. ( 1072 .

1297 Papillon.... a ailes brunes, les anterieures $d$ taches, EO bande blaniche, les pofterieures à yeux noirs, Limn o, ( 1358.

I 298 Papillon... a a ailes pales, taches brunes, les poftericures à quatre yeux, la dernicre azuré, Linn. O. ( 1472.

1299 Papilion .... ì ailes bruntes, les anterieures as 6 taches blanclues, les pofberieures à 3 yeux, dont le premier d 3 prunear x, Linn. O. Cram. 24. D? (923.

1300 Papillon.... ¿ ailes blancbeatres, bord noir, à taches lunaires, Linn. O. (1359.

I 3 I Papillon .... à ailes brunes, inifantes violet, defous blanckes, à cinnq bandes rouggeatres, le derriere un oeil azuré, Linn. o. ( 934 .

I302 Papilion .....a ailes blanches, les derrieres d deux ftries brunes 3 yeux, Linn. O. i 925.

${ }_{13} 3$ Papilion, .... à ailes brines, defjus unoeil fur les derrieres 2 prunelles argentées, en bas, 2 ftries brunes, Linn, 0. $(\mathrm{I} s \mathrm{C}$.

I 304 Papilion . . . à ailes brunes, bande demicirculaire blanche, Linn. o. (1 238.

1305 Papillon .... d ailes noires bleus, derriere vers l'anus blanches, Linn. O. Cram. 184. D. E. (1075.

I 306 Papillon.... d ailes brunes, de la baje d̀ yeux, defjous lis derrieres blanches, Linn. O. Cram. 184. F? G? (1473. L 4 1307 Papillon 
1307 Papilio .... f. varietas, Linn. o. Cram. I. c. (967. I3C8 Papilio .... alis nigris, azureo micantibus, infra fuscis, macula atra, Linn. O. Cram. 328. E? ( 962. bis

1309 Papilio .... alis azureis, macula rocunda alba, Linn. n. ( 1624 .

1310 Papilio ... . alis fuscis, fascia femicirculari alba, eadem ac $n^{\circ}$. 1304. Lirn. O. ( 1629 .

13 ! 1 Papilio.... alis fuscis, albo punctatis, pofticis ad anum albis, Linn. o. ( 966.

I3Ir Papilio .... alis fuscis, fascia violacea, pofticis albis, Linn. o. ( 965.

I212 Papilio.... alis fuscis, ocello zurantio ad anum, Linn. O. ( 1082.

13 '3 Papilio Argus, alis caruleis, pofticis fubtus limbo ferrugineo , Linn. 789. 232. RöJ. III. tab. 37. f. 4. (i543. bis.

1314 Papilio Argiolus, alis fupra coeruleis, margine atris, fubtus punctis disperfis nigris, Linn. 790. 234. (I559.

I3I5 Papilio Argiolus? f. varietas, Linn. I. C. (1560.

I 3 I6 Papilio ..... alis primoribus fuscis, margine maculis rubris, fubtus ocellatis, Linn. o. Cram. 270. D. E ? (1545. bis.

1317 Papilio .... alis fuscis, fubtus ftriis duabus obliquis, ocellis quinque, Lins. O. (957.

I318 Papilio ..... alis fuscis, fubeus ftria rufa, ponticis ocello marginali 6. Linn. o. (958.

$13 \times 9$ Papilio .... . alis fuscis, fubtus ftriis 2. ocelilis 6. duobus mediis cœecis, Linn. o. (1245. bis.

I320 Papilio Pamphilus, Indicus, alis flavis, primoribus ocello, pofticis cinereis ocellis quatuor, Linn. 79r. 239. (1544. bis.

1321 Papilio Pamphilus? f. varietas, Linn. I. c. (I561.bis:

1322 Papilio Danis, Linn. o. Cram. 7o. E. F, (947. bis.

I323 Papilio Carica, alis fulvis, disco albo, margine cinereo ocellato, Linn. 792. 244. Merian Surin. tab. 40. $f$. I. (950. bis

1324 Papilio Carica? f. varietas, Linn. \& Merian. 1. c. (1394. bis.

1325 Papilio Carica? fo varietas, Linn. \& Merian. l. c. (126r. bis.

I326 Papilio Lamis, Linn. O. Cram 235 G. (921. bis.

1327 Papilio .... alis nigris, disco albo. Limn. o. (1388. bis.

1328 Papilio ..... alis nigris, disco fulphureo, Lirn. o. (1262. bis. 
1307 Papillon . . . . ou varieté, Linn. 0. Cram. l. c. (967. 1308 Papillon .... d ailes noires, de bleu luifantes, en bas brunes, d taches noires, Linn. 0. Cram. 328. E ? (902.bis.

1309 Papillon ....d ailes azurées, ì tache blanche rondée, Linn. o. ( 1624.

I3ro Papillon .... d ailes brunes, bande demi-cercle blanche, le même que $\mathrm{n}^{\circ}$. 1304. Linn o. (1629.

33 II Papillon....d aibes brunes, poirtie de blanc, les pofterieures blanches vers l'anus, Linn. 0 . ( 966.

I3II Papillon ..... a ailes brunes, d bande violette, les pofterieures blanches, Linn. o. ( 965 .

I312 Papillon.... à ailes brunes, otil.orange vers l'anus, Linn. o. (ros2.

1313 Papillon Argus, à ailes bleues, les pofterieures defous couleur de rouille, Lisn. 789.232 . Köt. III. tab. 37.f.4. (1543. bis.

1314 Papillon Argio'e, d siles deffus bleus, au bord noires, deffous plufieurs pointes noires. Linn. 796. 234. (1559.

1315 Papillon Argiole? ou varieté, Linn. l. c. (1560.

1316 Papillon.... à ailes de devant brunes, d̀ bord des taches rouges, deffous d yeus, Linn. O. Crain. 270. D. E.? (I545. bis.

1317 Papillon .....d ailes brunes, defjous 2 ftries obliques, it cing yeux, Linn. o. ( 957.

1318 Papillon ..... d ailes brunes, defjous une ftrie rougea. tre, derriere un oeil au bord, Linn, n. ( 958.

13:9 Papillon ..... d ailes brunes, deffous a deux ftries, fix yeux, les deux ou milieu aveugles, Linn. 0 . (1245. bis.

1320 Papillon Pamohile, des Indes, da ailes jounzes, les premieres d) un oeil, pofterieures condrées à 4 yeux, Linn. 791. 239. (1544.bis.

1321 Papillon Pamphile? ou variete, Linn. l. c (156r. bis.

1322 Papillon Danis, Linn. O. Cram. 70. E F. ( $9: 7$ bis

I323 Papillon Carice, d ailes fauves, difque blanche, is bord cendre, oculé, Linn. 792. 244. Merian Surin. tab. 40. f. I. C (950. bis.

\324 Papillon Carica? ou varieté, Linn. E Merian. l. c. (I394:: lis.

1325 Papillon Carica? ou variete, Linn. ED Merian. l c. (126r. bis.

I 326 Papillon Lamis, Linn. o. Cram. 235. G. (921. bis.

1327 Papillon $\ldots$ à ailes noires, palette biancke, Linn. 0. (1388. bis.

1328 Papillon .... d ailes noires, difgue couleur is joufre, Linn. 0. (1262. bis. 
$\$ 329$ Papilio . . . . alis nigris, fascia transzerfa alba, Linn. o. ( 949.

1330 Papilio Danis? f. varietas, Linn. a. ( 948 .

I33r Papilio .... alis coccineis fusco marginatis, Linm. o, ( 1448.

1332 Papilio ..... alis fuscis, ad anum cocćineis, Linn. o. (1083.

I333 Papilio .... alis fuscir, ftria cœrulea, Linn. o. ( 908. I334 Papilio .... alis nigris, cœrulco micantibus, fubtus ferie - duplici argentea, Linn o. (1502.

1335 Papilio ..... T. varietas, Linn. 0. (1084.

I336 Papilio ..... alis fuperne fuscis, poltice rufis, ft:ia argentea, Lifm. o. (961.

I337 Papilio . ... alis utrinque fuscis, Limn. o. ( 960.

I338 Papilio Hecabe, alis flavis, extimo atris, fubtus plurimis punctis pallidis, Linn. 753. 96 . ( 1573.

r339 Papilio .....alis flavis, fuscoyue fasciatis, Limn 0 . ( 1389.

1340 Papilio . ... alis fuperne fuscis, macula nivea, intert e fulphureis, maculis rubris auratis, Linn O. ( 1480.

$\$ 34$ I Papilio .... alis fu cis, primorum fascia flava, Linizo o. $(964$.

${ }_{1} 342$ Papiito .... alis fuscis, Linn. o ( 959.

1343 Papilio.... alia fu cis, dicco albo, Limb. o. (ro7s. 1344 Papilio .... alis cinereis, albo maculatis, Linm. o. (1390.

3345 Papilio Carica? f. varietas, Linn 792, 24.4? ( 1483.

I 346 Papilio .... alis aurantiis, margine atro, Linn. 0. ( 1107.

I347 Papilio Nauplia, alis fuccis, primoribus fupra maculis quatuo:, polticis fascia albidis, Linn. 783.197. Cram. 3IG. D E. ( $\mathrm{I}_{2} \mathrm{C}_{3}$ bis

I348 Papilio Nauplia? f. varietas, Liin. l. c. Cram. 3 I6. F. G. (995. 1264 . bis.

1349 Papilio... alis oblongis fuscis, maculis duabus culphureis, fubtus margine rubro, Limo. O. (994.

I350 Papilio.... f. varietas, Lim. 0. (10,8.

\$351 Papilio .... alis fuscis, macu'a alba, Limn. O. ( 1001 .

$\$ 352$ Papilio.... alis fuscis, coeruleo micantibus, fascia transverfe alba, Limn. O. (1106. 
I329 Papillon .... à ailes noires, bande transverfe blanche, Linin. o. $(949$.

I330 Papillon Dasis? ar varieté, Linn. O. (948.

1331 Papillon ... . à ailes rouges, bordez de brun, Linn.

( 1448.

1332 Papillonz .... à ailes brunes, vers l'amas rouges, Linn. - (1083.

I333 Papillon .... à ailes brunes, firie bleu celefte, Linn. o. (968.

1334 Fapillon .... d siles noires, luijantes de bleu, defous double raye argentée. Lins. O, (150?.

1335 Papillon .... ou varieté, Linn O. (ro34.

1336 Papillon .... à ailes deffus brunes, dernieres rougeatres, à Ptrie argemtine (nacre) Linn. O. (gor.

I337 Papillon .... à ailes brumes de chaque coié, Linn. 0 . Co6o.

I 339 Papillon Hecabé, à ailes jaunes, su bout noires, deflous a plufieurs pointes pales, Linn. 763.96 . (1573.

1339 Papillon . . . a ailes jaunes, bandées de brum, Linn. $0_{0}$ ( 1389.

I $340^{\circ}$ Papillon. . . d ailes defris brues, tache blanche; en dedans couleur de foufre, è taches rouges dorées, Linn. o. $(1480$.

I34I Papillon ..... d ailes brunes, bes devans à bande jaun, Linn. o (964.

I342 Papillon .... à ailes bris:es, Linn. o. (959.

1343 Papillon .... d ailes brines pallette blanche, (Iinn. O. (1078.

ir 44 Papillon .... à ailes cendrees, tacbettes de Ubane, Linn. $0 . \quad$ ( I390

I345 Papillon Caricx? on6 varieté, Linn. 792 244? ( 1483 . 1346 Papillon .... के aibes oranges, à bord voir, Litin.o. (ixo7.

I347 Papillon Nauplia. da ailestrumes, les premieres deffus à ğrsz. tre tackes, les pofterieures, à bande blanche, Linn. 783 . 197. Cram. 316́. D. E. (I263.'bis.

1348 Papillon Naupia? ou varieté, Linn. l. c. Cram. zró, F. G. (995. I264. bis.

1349 Papillon .... ì ailes allonghes brunes, à deux taches cosileur de fonfre, aefJous borl rouge, Linn. O. (99ł.

1350 Popillon .... ou varieté, t.inu. 9. (1098.

I35I Papillon.... à ailes brunes, d̀ tache blancbe, Linn.o. (1001. bis.

I352 Papillon .... à ailes linunes, luifantes de lleu, à bande transyerfe llancke, Linn. c. (1106. 
1353 Papilio .... a alis fuscis, pofticis fascia alba, Linn. o. (962.

1354 Papilio Ammon, Linn. o. Cram. 146. B. (1395.

I355 Papilio Flora, Linn. O. Cram. 257. B. C. (1004. bis.

1356 Papilio Flora? r. varietas, Linn. 0. Cram. I c. (1005. bis.

1357 Papilio Flora? f. varietas, cooruleo micans ad inarginem interiorem, Linn. o. ( I006.

I358 Papilio.... alis diajhanis, ftriis fuscis, pofticorum margine aurantio, Linn. O. ( 1434.

1359 Papilio..... mas . alis oblongis, maculis duabus diaphanis, pofticis 1. Limn. o. ( 1007.

I360 Papilio Euterpe, alis oblorgi; atris, albo punctatis, fascia fulva, Liinn. 756 6r. (r ro9. bis.

1361 Papilio Euterpe? an pracedenti fimilis ? Linn. 1. c. ( $)$ 2 2. bis.

1362 Papilio .... alis fu'vis, apice nigris albo maculatis, pofticis fulvis nigro marginatis, Linn. 0. ( 1267.

1363 Papilio ..... f. varietas, Linn O. ( 1268.

1364 Papilio Helicon, alis fuscis, ad apicem aurantio ftriati, fascia flava, Linn o (1260.

1365 Papilio .... alis nigris, maculis aurantiis, Linn. 0. Cram 142. G. ( 1310 .

I366 Papilio Fusca, maculis primoribus duabus, polticis fascia alba, Lirn. O. ( 1222.

I367 Papilio Cinereo-Fusca, alis albo maculatis, Linn. 0. ( 122 r. bis.

I368 Panilio Cinereo-Fusca? f. rarietas, alis fuscis, maculis punclisque diaphanis marnoratis, Linn. O. ( 1220. bis.

1369 Papilio Nivea, Linn. O. Cram. 22. C. (95 r.

$137^{\circ}$ Papilio .... alis fuscis, nigro rivulatis, Linn. 0. (ro89.

I37I Papilio .... alis luteis, nigro marginatis, margine flavo, Linn. O. ( 1517.

1372 Papilio ..... alis fuscis, macula aurantia ad apicem, maculis 2 in medio, 3 ad apicem hyalinis, Linn. O. ( 1223.

I373 Papilio.... alis anticis, macuilis hyalinis, pofticis azureo ftriatis, Linn. O. (1224. bis.

1374 Papilio .... f. varietas, Linn 0. ( 1225.

1375 Papilio ..... alis fuscis, maculis diaphanis, pofticis ftriis 2. cyareis, fubtus caruleis, Linn. O. ( 1087. 
1353 Papillon .... a ailes brunes, fofterieures d bande blanche, Linn. O. (962.

1354 Papilion Ammon, Linn. o. Cram 146. B. (1395.

1355 Papillon Flora, Linn. O. Cram. 257. B. C. (1004. bis.

1356 Papillon Flora? ou varieté, Linn. o. Cram. l. G. (1005.

$\$ 357$ Papillon Flora? ou variete, luifant de bleu, vers le bord interieur, Linn. 0 . (1006.

1358 Papillon....d ailes transparantes, d fries brunes, les derrieres d bord orange. Linn. O. (1434.

1359 Papillon .... ou male, d ailes allongees, ì deux taches transparantes, derrieres d une tache, Linn. O. (1007.

I 360 Papillon Euterpe, d asles allongées noires, pointées de blanc, d bande fause. Linn. 756. or (1109. bis.

1361 Papillon Euterpe? ou Semblable du precedent? Linn. l. 6. (902. bis.

1362 Papillon.... d ailes fauves, d̀ point noir tacheté de blanc, derriere fauves, bordées de noir, Linn. O. ( 1267.

$13 \mathrm{C}_{3}$ Papillon .... ou variete, Linn. O. (1268.

1364 Papillon Helicon, d ailes brunes, vers le point firiées dorange, d bande jaune, Linn. 0 . (1260.

1365 Popillon ....d ailes noires, taches oranges, Linn. 0. Cram. 142. G. (1316.

1366 Papillon Brune, par devant dersx taches, derriere une bande joune blanche, Linn. C. ( 1222.

1367 Papillon Brun-Cendré, les ailes tachetées de blane, Linn. C. (122t. bis.

I368 Papillon Brun-Cendré? aus varieté, $d$ ailes brumes, d taches, E points transparantes, marbrées, Linn. O. (1220. bis.

1369 Papillon Blanc, Linn. O. Cram. 22. C. (951.

1370 Popillon .... d ailes brunes, melies de noir, Linn. 0. ( 1 r.8.

1371 Papillon .....d ailesjounes, bordées de noir, dbord jaune, Lirin. O. ( 517.

$137^{2}$ Papillon ......d ailes brunes, d tacke orangie, vers le point, derux taches au milieu, couleur de verro, Linn. $c$. 1223.

1373 Papilion .... d ailes de devant, d tache couleur de verre, les dersieres ftrie d'azure, Linn. o. (12240 bis.

1374 Papillon .... ou variete, Linn. O. (1225.

1375 Papillon .... dites brunes, d taches transparantes. les pofterieures $a$ deux taches perfes bleu, deffous blew celefte, Linn, o. ( 1087. 
I376 Papilio Hebrus, alis fuscis, coruleo marmoratis, pofticis ftria nivea cyaneo marginata, Linm. 0. Cram.50. E. F. (1226.

I377 Papilio . . . alis nigricantibus, fubtus cyareeis, Linn. O. (1227.

1378 Papilio .... alis primoribus fuscis, bafi, fasciz \& apice flavis, pofticis luteis, margine nigro, Linn. o. (973.

I 379 Papilio .... alis fusco nigris, fascia aurantia ad apicem in qua macula flava, Linm o. (972. bis.

1380 Papilio .... alis fuscis, fascia diaphana, Linn. 0. ( 836 . bis.

1381 Papilio .... alis rigro fuscis concoloribus, Limr. O. (1402. bis.

1382 Papilio .... f. varietas, Limn: $\odot$. (I6: 4 .

1383 Papilio Cafius, Lings. O. Cram. 20. C. D. (97I.

I384 Papilio Phidias? alis viridi cupreis, bafi nervicque fuscis apicibus luteis, capite ancque aurantiis, Linn. 795. 263? Cram. 244. A. B. ( 974 bis.

1395 Papilio .... alis nigris, viridi ftriatis, apicibus luteis, Lim. O. (1368.

I 386 Parilio .... alis nigris, fubtus viridi flavis, Linn. $\circ$. (975.

I 387 Papilio ..... r. varietas, Linn. O. (976.

1388 Papilio .... alis fuscis, bafi viridi aureis, Linn. o. $(982$.

1389 Payilio .... alis fubangulatis piceis, maculis fasciзçue atris, margo pofticarumluteus, Linn. O. (1203. bis.

т 390 Papilio .... . . varietas, Limmo. ( 204.

139 I Papilio ..... . varietas, fed fusca, Linn c. (1085.

1392 Papilio .... alis fuscis, fascia media albida, Linn. O. ( 970.

1393 Papilio .... alis fuscis, fuperioribus maculis diaphanis, Linn. O. (-ros6.

I394 Papilio .... f. varietas, eadem ac nº I390. Linn. 0. ( 960.

1395 Papilio .... r. varietss, Lim. O. (10S8.

1396 Papilio .... alis fuccis, fascia interrupta, fene?ra:a flava. fubus masula argentea, Linn. o. (II7s. bis.

1397 Papiiio Pileas, alis fubceudatis, arris, bals lutes, maculis 4 fencftratis. Liq\%. O. Cram. 283. F. (II80. 1398 Papilio ... al is atris, thorace cæruleo, maculis fasciague feneflutis, Jim o. ( $118 \mathrm{r}$. 
1376 Papillon Hebrus, i ailes brumes, marbrées de bleu, der. riere d Jtrie blanche, bordées de couleur de perfe, Linn. O. Cram. 50. E. F. ( 1226.

1377 Papillon. ... a ailes noiratres, deffous bleues, Linn. 0. (1227.

1378 Papillon ... à ailes anterieures brunes, la bafe, bande E2 point jaunes, les pofterieures jaunatres, à bord noir. Lirn. 0 . $(973$.

1379 Papillon .... à cilles brunn noires, à bande orange, vers le point dans lequel une tache jaune, Linn. o. (972. bis.

1330 Rapillon . . . a ailes brunes, $\mathcal{E}^{2}$ bande transparente. Linn. O. ( 836 bis.

138 I Papillon.... à ailes brans noires, Linn. O. (1462.bis. 1382 Papillon .... ou variete, Linn. O. ( 614.

I 383 Papillon Caffius, Linn. o. Cram. 20, C. D. (97r.

3384 Papillon Phidias, à ailes, verd-cuivreufes, de la bafe, E̋ nerves bruites, à fommet jaune, latte, 'E' l'anus oranges, Linn. 795. 263 Cram. 244. A. B. (9:4. bis.

3385 Papillon .... a ailes noiratres, les ftries vertes, pointes jaunes, Linn. O. ( 1368 .

I386 Papillon.... a ailes noires, defjous, verte jaunes, Linin o. ( 975.

3387 Papillon .... ou varieté, Linn. O. (976.

$\$ 388$ Papillon ..... à ailes brines, de la bafe vertes dorée, Linn. o. ( 982.

1389 Papillon .... à ailes en angles, coulear de poix . à taches, Eg bande noirs, le burd des pofterieures jaune, Linn. o. ( 1203 bis.

I3so Papillon . . . . ou varieté, Linn. o. ( 1204 ;

391 Papilion .... ou variete, mais brune, Linn o. (1085.

I392 Pepillon . . . . à aites brunes, bandie bulanche are miliew, Linn. 0. 670 .

1393 Papillon .... à ailes brunes, les fuperietires à tacbes diaphanes, Linn. o. ( 1686 .

J394 Papiblon .... ou varieté, le méme que I390. Linn. o. ( 950.

$\$ 305$ Vapillon . . . ou varieté, Lirn. O. (ros8.

$1396 \mathrm{P}$ cpillon .... à ailes brunes, à bande interrompu, fenes. trée jauns, deffous tache argentee, Linn. O. (II79. bis.

I 327 Papilion Pileus, à ailes en queue, noives, de la b̆nfe jaunes, al 4 taches fereftrées, 1 inn. O. Cram 283. F. (I 180. ¿393 Papillon... à ailes roires, le corcelet bleu, de taches Ẽ bande fenefirées, Lisn. O. (118r. 
1399 Papilio Apaftus, f. Pibeus, Linn. O. Cram. III, D. ( 1325.

I400 Papil $0 . .$. alis coruleis nigris, fascia diaphana, Linn. O. ( $x_{4} 16$.

I 40 I Papilio Atra, maculis tribus diaphanis, Linn. O. ( I45I. bis.

1402 Papilio .... thoracis Ariis 3 albidis, alarum maculis cœruleis, Linn. O. (980. bis.

r403 Papilio ..... varietos, Linn. O. (1029. bis.

1404 Papilio Bixa? alis fuscis, pofticis fubsus fascia alba, Linn. 795. 26t? Cram. 199. C. D. (1228. bis.

I405 Papilio Bixa? f. varietas, Linn. \& Cram. 1. C. (ro93. bis.

I405 Papilio Bixa? f. varietas, Lin». \& Cram. 1. c. (1229. bis.

I407 Papilio Bixa? f. varietas, alia, Linn. o. (1460. bis.

1408 Papilio .... alis viridi nigris, ano barbato, rubromarginatis, Linn. O: (1230. bis.

1409 Papilio .... f. varietas, Linn. o. ( I6r6.

$\Upsilon_{410}$ Papilio .... alis fuscis, maculis fulvis, Linn. O. (I 183. bis.

I4II Papilio ..... alis fuscis, maculis quinque feneftratis, fubtus fascia alba, Linn. O. ( 1323.

I4I2 Papilio ..... f. varietas, fubtus maculis argenteis, 4 diaphanis pofticis, maculis 2 argenteis, Linn. 0. (r503.

I4I3 Papilio .... alis fuscis, maculis gamofis fuscis, Linn. o. (1324.

I4I4 Papilio.... alis nigris, lineis maculisque diaphanis, Linn. O. ( 1461 .

I4I5 Papilio.... alis bruneis, maculis luteis, Linn. o. $^{\circ}$ (1219. bis.

I4I6 Papilio .... alis fuscis, maculis 9 diaphanis, poiticis fubtus albidis , Linn. O. (1402.

1417 Papilio ..... is luteis, fuscia longitudinali, maculisque hyalinis, Limn. o. (I 494.

1418 Papilio Proters, alis fuscis, maculis feneftratis artennis uncinatis, Linn. 794. 259. Cram. 240. D. F. G. (i055. bis.

1419 Papilio Proteus? \& varietas, alis feneftratis, Limn. \& Cram I. c. (1056. bis.

1420 Papilio Proteus? f. varietas, Linn. \& Cram. I. c. ( IOS4.

1421 Papilio Protens? C. varietas, 2lia, Linn. O. ( 1459. 
1399 Papillon Apafte, or Piieus, Linn. O. Cram. Ir $:$. (i325.

1400 Papillon ....d ailes blerb celeftes, noires, à bande diaphäne, Lins. O. (1416.

I 401 Papillon Nuir, à truis saches transparantes, Linn. o. (1451. bis

1402 Papillon.... les ailes à trches bleu celeftes, ED trois ftries du corcelet, Linn 0 . ( 980 . bis.

1403 Papillon.... ou varieté, Lnn. O. (ro:9. bis.

I404 Papillon Bixa, à ailes brunes. Les pofferiecires de $\int_{c}$ cus $d$ bande blanche, Linto 795. 264? Cram. 199. C. $D$ : (1228. bis.

1405 Papillon Bixæe ? ouvarieté, Linn. E Cran l. c. (Ic93. bis.

$I_{406}$ Papillon Bixa? ou variete, Linn. E Cram. i. c. (2z). bis.

1407 Papillon Bixæ? ou cutre varieté. Linn. o. (itro. bis.

$x_{+} 08$ Papillon.... a ailes ver:-noires, l'anus barbu rouge, les derrieres bordées ae rouge , Linn. O." (1 230." bis.

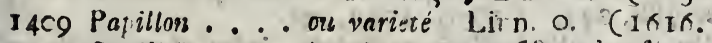

1410 Papillon ... à ailes Irunes, È tachesfarves, Linn. o. (1183. bis.

I4I Papillon...$d$ ailesbrunes, cing taches ferieftrées, des. Jous à bande blanche, Linn. 0 . ( 1,23 .

I41 2 Papillon. Oou varieté, de Jous à quatre taches argentines, les pofterioures diaphanes, à deux taches argentines, linn o: ( 503 .

I4'3 Papillon.....à ailes branes, \& taches en forme de $G$. Linn. 0 . (1324.

I4I 4 Popillon .... a ailes noires, à lignes, $\mathcal{E}$ taches transparantes, Linn. 0 ( I $46 \mathrm{r}$.

1415 Papillon..... à ailes brunes; taches jaunes, Linn. 0 . (1219. lis.

I 416 Papillon.... d ailes brunes, 9 tacbes traniparentes, les derrieres deffous blanchéatres. $\mathrm{L} \mathrm{p}^{\mathrm{n}} \mathrm{O}$. ( $\mathrm{I}+\mathrm{O} 2 \mathrm{2}$

I4I Papi.lon.... à aiies $j$ unes, bande longit radinale, E taches hyalines, Linn 0 . (494.

I 48 Papillon Pr teus, à ailes branes, taches ferieftrées. les antennes ecochues, Linn. 794. 259. Cran. 240. D.F.G. ( 1055 bis.

1419 Papillon Proteus? ou varieté, d ailes feneftrées, Linn Eे C.am. $i$ c. ( I056. bis.

1420 Pafillon. Proceus? uu varieté, Linn. ED Cram. l. c. I IC54.

1421 Papilion Proteus? ou autre varioté, Linn. O. (I 159. 
1422. Pap lio Proteus 2 fr varietas, alis fuscis, cauda \& margine pofteriorum alb's, Linn. 0 ( $98 \mathrm{r}$.

I23 Pap:lio .... ai s fuscis, magine pofticorum \& ano flavo, Linn. O. (I 182 bis.

1424 Papilio .... alis cacdatis, caudis recurvis, fusccis fas. ciis $2^{2}$ recurvis. Linin. O. (II85.

1425 Papilio .... alis albo punetatis, linea curva rubra, Linns o; (1383.

1426 Papilio Rethus, Lim, o. Cram. 63. C. ( I184.

1427 Papilio .... alis.tigris, bafi azureis, pofticis recurvis, caudatis, raculis duabus coccineis, Linn. 0 . (1179)

I428 Papilio Charineus, Linn. O. Cram. 59. A. (835.

1429 Papilio Cupido, alis fubtus albidis, maculis argenteis, Linn. 787. 217. C 1385 . bis.

1430 Papilio Cupido? T. varietas, Linn. \& Cram. 1. c. (827. 828 . bis.

I430 Papilio Cupido? fo varietas, alia, Linn. O. (IISIs I189. ter.

1431 Papilio Morfyas, alis cœruleis, fubtus punctis atris, Linn. 788. 223. (IOSO IO52. ter.

I432 Papilio .... alis caudatis cœruleis, infra marmoratis ex fusco \& albo, Linn. o. ( 830 . bis.

1433 Papilio .... alis cœruleis, primorum macula, \& apice atris, fubtus fuscis, albo punctatis, Linn. o. (1186.

1434 Papilio .... alis fupra coruleis, infra fuscis, maculis zuro marginatis, Linn. O. ( 832.

I435 Papilio .... alis fupra cœruleis, pofticis ocello brunnco humerali, Linm. O ( 1187.

I 436 Papilio.... alis caudatis azureis, pofticis fubcus nigris, prope caudas viridi maculatis, Linn. O. ( 829.

I437 Papilio Regalis, bicaudalis, Linn. o. ( 495 .

1438 Papilio ..... alis nigris, ad marginem interiorem 2zureis, Linn. O. ( 1093.

I439 Papilio .... f. varietas, Linn. O. ( 1458.

1440 . Papilio .... . . varietas, Linn. O. (1404. bis.

I44I Papilio .... alis azureis, infra fuscis, linea alba com. muni, Linn. O. (1457. bis.

I.442 Papilio ..... alis rubviolaceis, ocellis caudalibus atris, viridi argentea, Linn. 0. (1550. bis.

I443 Papilio .... alis cœeruleis, maculis atris, fubtus albis punct is nigris, Linn. O. ( 5596.

1444 Papilio $\because .$. alis toruleis, apice nigris, infra cinereis, tascia alba, Limn. O. ( II88. 
1422 Papillon Proteus? ou varieté, da ailes trunes, la queue $E^{\circ}$ bordes derrieres bl nches, Linn. $O$. (9881.

I423 Papillnn .... a ailes brunes, le bord des pofterieures, E l'anus jaunes, Lirn. o. (118', bis.

1424 Kapillon .... à ailes en querse, la queue courbé lrures, $d$ deux bandes courbées. Liin. 0 . ( 1185.

1425 Pupillon ... à ailes pointées de blanc, d ligne courbée rouge, Linn o. $(1383$.

1426 Papillon Rethus, Linn. O Cram. 63 C. ( $\times 184$.

1427 Papillon . ...d ailes noires, de la base azurées, derrieres courbées en queue, à 2 taches rouges. Linn O. (11 79.

1428 Papillon Charineus, Linn. O. Cram 59. A. (835.

I429 Papillon Cupidon, ì ailes defus blanches, d taches argentines, Linn. 787 217. ( 385 . bis.

1430 P pillon Cupiton? ou varieté, Linn. El Cram. l. c. (827. 828. bis.

I430 Papillon Cupidon? ou autre varieté, Lirn. O. (II5I. II89, ter.

143I Papillon Marfyas, à ailes bleues celeffes, deffous pointes noires, Linn. 788.223. ( 1050. 1052. ter.

1432 Papillon .... à ailes en queus bleues, en bas morbrées de brun E⿺ blanc, Linn. O. ( 8 jo.

1433 Papillon..... à ailes bleues, les premieres di tache, \& pointe noires, deffous brunes, pointees de blanc, Lirn. $\mathrm{O}^{\circ}$ (I) 86.

1434 Papilion .... à ailes defJus bleues, en bas brunes, à taches bordée d'or, Linn. O. ( 832 .

i435 Papillon .... d ailes de (Jus bleues, les derrieres à oeil brun, Linn. O. (I:87.

436 Papillon.... à ailes en queue azurées, les pofterieures deffous noires, proche de la queue tacheté de vert, Linn. o. (829.

437 Papillon Royalle, à deux queues, Linn. o ( 1495.

438 Papillon .... à ailes noires, au bord interieur azurées, (Linn. o. (rogo.

439 Papillon ... o ou varieté, Linn. O. (1458.

(440 Papillon .... . ou varieté Linn. o. (1 04. bis.

441 Papillon .... à ailes azurées, en bas brunes, d̀ ligne blanche commune. Linn. o. (i457. lis.

442 Papillon....a ailes violettes, les yeux fur la queue noirs, verd: argentines, Linn. 0 ( 1550 . bis.

443 Papillon.... à ailes bleues celefte, taches noires, defjous blanches, do pointes neires, Linn. O. ( 596

444 Papilion .... d ailes bleu celeftes, au point noires par on bus cendrées, à bande blanche, Linn. o. (i 188. 
1445 Parilio ..... varietas, Linn. 0. ( 1377.

14.6 Papilio .... f. varietas, fdscia, ocellisque caudalibus rubris, Linn. o. ( 1378.

I 447 Papilio ..... f. varietas, alia, Limm. o. ( I 1 ) o.

1448 Papilio .... r. varictas, Lirn. c. (83.3.

I449 Papilio .... fascia obliq̧ua alba, Linn. O. ( 1379.

1450 Papilio .... . . variecas, eadem ac $11^{\circ}$. 1447. Linn. 0. (I 193.

I45 I Papilio ..... varietas, Linn. o. ( 1382.

1452 Papilio.... alis caudacis, fusco cinereis, infra albis maculis ferrugineis, Linn. O. ( I 380.

1453 Papilio .... alis fuscis, porticis uceilatis, Linn. o. ( 1384.

1454 Papilio Phaleros, alis cuudatis, fupra nigro coruleis, Linn. 796. 272. Cram. 51. F. G ílo5I.

455 Papilio Phaleros? f. varietas, Linn. \& Crann. 1. c. (831.

I456 Papilio Phalexos? f. varietas, alia, Linn. o. (1191.

I 457 Papilio Phaleros? f. varietas. Linn. o. ( $\mathrm{I}_{4} \mathrm{I} 7$.

I 458 Papilio.... alis albis caudatis, apice tuscis, macula atra, Linn. O. ( 8 I 8 .

I459 Papilio ..... alis fuscis, pofticis albis latentibus, infra fusco maculatis, Linn. O. ( I192.

I460 Papilio .... alis giffeis, fubtus nebulofis, Linn. 0 (I38I.

I46I Paplio ..... alis albidis, apice pallide fuscis, ocellc atro, fub:us lineis 2 undulatis. Linn. o. ( 834 .

I462 Papilio.... alis fubcaudatis fuscis, primoribus fasci: fulva, maculis atris, fubtus ucraceis, ocellis nigı is viridi paliida, Linn. O. (1514.)

\section{S $\quad P \quad H^{\prime} I \quad N \quad G \quad E$ S.}

1463 S.hinx ..... a'is integris, pal'ide grifeis, ftriiz obli. quis paralellis, Linn. O. ( 1301 .

1404 Sphinx Cinerea, maculis duabus fuscis, ǫusdratis, apici bus obfcaris. Linn. O. ( 1302.

I465 Sphinx Ruffica, Linn. 0. Fabric. 28. Sulz. Inf. tab 20. f. 2. (1296. bis.

I465, S, hinx ..... alis grifeis concoloribus, Linn. O (II43

1467 Sphinx Alecto, Linn. O, Fabric. 92. ( 808.

1468 Sphinx Oldenlandia, alis viridi grifeis, corpore arg?n teo, trifariam punctato, ftriis 2 argenteo marginatis Linti. 0. Fabric. 37. (1309. bis. 
1445 Papillon . . . ou varieté, Linn o. ( 1377 .

$44^{6}$ Papillon .... ou varieté, à bande, \& les yeux de la queue rouge, Linn. o. ( 1378 .

1447 Papillon .... autre varieté, Linn. o. (Irgo.

$144^{8}$ Papillon .... ou varieté Linn O. (833.

1449. Papilnn ..... à bande oblique blanche, Linn. O. (1379.

$45^{\circ}$ Papillon .... ou varjeté le memeque $n^{\circ}$. 1447. Linn. 0.

(1193.

491 Papillon .... ou varieté, Lirn. O. ( 1382.

1452 Papillon.... à ailes en queue d'un brun cendré, par en thas blanches, à taches couleur àe roulllée, Linn. 0 . $(380$.

1453 Papillon. . . a ailes brunes, les pofterieures à yeux, Lirn 2. ( 1384.

1454 Papilion Phaleros, à ailes en queue, deffus noir-blezs celefte, Linn. 796. 272. Cram sI. F. G. (1051.

1455 Papillon Phaleros, ou varieté, Linn. E Cram. l. c. (831.

1456 Papition Pbaleros? ou autre varieté, Linn. O. (IIgr.

1457 Papilion Phaleros? ou varieté, Linn. O (I4I7.

-458 Papillon.... à ailes blanches, au point brune, à toche noire, Linn. 0 ( 818.

1459 Papillon .... à ailes brisnes, les pofterieures blanches, en bas tachetées de brun. Linn. 0. (1192.

1450 Papillon .... à ailes grifes, defous nuancées, Linn. 0. ( I ; 81 .

1461 Papillon . . . à ailes blancheatres, au point pale - brunes. à oeil neir, deffous 2 lignes ondées, Linn. 0 . (834.

1462 Papillon.... à ailes un peu en queue brunes, anterieures à bande fauve, taches noires, defous ocrackes, yeux noirs, pale vertes, Linn. C. (1514...

\section{$\begin{array}{llllll}S & P & I & N & X & S\end{array}$}

1463 Sphinx .... à ailes entieres pale grifes, $a$ fries obliques paraleilles. Linn. O. (I zor.

1464 Juhinx $\mathrm{C}=$ ndré, à deux taches brunes, quarrées à pointes olifrures, Linn. O. (I 302.

1455 Sphina Champetre, Linn. o. Fabric. 28. Sulz. Inf. tab. 20 f. 2. (1269. bis.

1406 Sphinx .... à ailes grijes de même couleurs, Linn. 0. $(1143$.

1467 Spnivx Aiedo, Linn. C. Fabric. 52: (808.

1468 sphinx d'Oldelande, à ailes verd-grifes, le corps argemtine, pointé de trois diverfes manierés, hordée de deux ftries arguntines, Linn, O. Fahr. 37. (1309.bis. 
I469 Sphins Grifeo-Fulva, rivulis nigris, puncto albo, Linms o: (608. bis.

I 470 Sphinx.... alis cinereis, macula magna pallidiore, nigro punctata, margine frris fuscis, Linn. O. (812.

I 47 I Shinx Atropos? alis pofticis flavis, fusco fasciatis, abdomine luteo, cingulis atris, Linn. 799. 9 ? ( 1490. bis

$I_{4} i^{2}$ Sphinx . . . alis viridi olivaceis, pofticis rubris, Limm. - (1308.

$\mathrm{x}_{473}$ Sphinx .... a'is albis, nigro denfe ftriatis, ftria media alba. Linn. O. ( 1455.

1474 Sphinx Boerhavia Limm. O. ( 1582.

I475 Sphinx Cocus, Linn. 0. (ram. 54. E. (1298. 1299. bis.

1476 Sphinx Cocus? f. varietas, Linn. O. Cram. I. c. (1300. 1477 Sphind Hydaspus Linn o. Cram. 118 C. (813.

1478 Sphinx Hydaspus? f. varietas pracedentis? Linn. 0. Cram I. c. (1490.

1479 Sphinx Hydaspus? f. varietas, Linn. 0. Cram. 1. c. (is 85.

I480: Sphinx Hydasprus, fo mas, Linn. o. Cram. 1. c. (8ro. 148I Sphinx Elpenor, Indicus, alis viridibus, fasciis purpureis, polticis rubris, bafi nigris, Linn. 801. 17. ( 1303. ter.

1482. Sṕhinx Atropos, eadem ac. n०. J47I. Linn 799. 9. ( $8 \mathrm{Ir}$. bis.

I783 Sphinx Oleander, Levantinus, Limu. O. Cram. 224. $D$. (IIY3. :447. bis.

$148+$ Sphinx Cocrs, ut in $n^{\circ}$. 1475. Limm. O. Cram. 45. E. ( $1: 83$ I 454 . bis.

I483 Sphinx. . lis nigro fuccis ponticis flavis. margine fusco, an Pagana? Linn. O. Fabric. 29? ( 1422 .

1485 Sphinx .... alis rigris, pofticis fascia flava, Linn. 0. (1044.

I 786 Sphinx.... alis viridibus, macula trigona obfcura, Lim 0. (1297.

I 48,7 Sphinx Clotho, Linn. o. Fabric... ( $\mathbf{r}+2 \mathbf{I}$.

4888. Sphinx .... alis ferrugineis, macula purpurea, ocello ad marginem, Linn. O. ( 1100

1489 Sphinx . . . . alis fuscis, fasciis deabus ex puncts albidis, ano bartato, Linn. o. ( 807 .

149? Sphinx Zygana alis brunneis, primorum maculis 3 fereftratis, pofticic unica, cofta primorum rubra, Linn 0. Fabric.... (794. bis. 
r.4.o Sphinx Gris-Fauve, a rives noires, pointe blanchs, Linn. 0. (008. bis.

$T_{4}$ o Sphinx... a ailes cendrees, grande tache pale, pointée do noir, le tord d fries brunes, Linn. O. ( $81_{2}$

\471 Sphinx Atropos? à ailespofterieures jaunes, be ventre jau. ne, à bandes noires, Lnn. 79y. 9 ? (I 490. bis.

I 472 S hinx.... à ailes verd-olivatres, les pofterietures rouges, Linn. O. (1308.

1473 Sphinx.... ä ailes blanches. Striées de noir, ou milieu do ftrie blanche, Linn. 0. ( 1455 !

I 474 Sphinx de Boerhaven, Linn. O. (I582.

- 475 Sphinx Cocus, Linh. 0. Cram. 54. E. (1298. 2299. bis. 1476 Sphinx Cocus, ou yarieté, Linn. O. Cram. l. c. (1 300.

1477 Sphinx Hydaspe, Linn. O. Cram, I 8. C. (813.

I 478 Shpirux Hydaspe? ou varieté du precedent ? Linn. 0. Cram. 1. a : (I 490 o.

1479. Sphinx Hydaspe? or varieté; "Linn. o. Cram. lo $c_{0}$ (i) 85 .

I480 Sphinx Hydaspe, ou male, Linn. c. Cram. l.c. (8ro.

I48 I Sphinx Elpenor, des Indes, à ailes vertes, fascies pour. trées, les pofterieures rouges, de la bafe noires, Linn. 801. 7. 7. (I303. ter. ?

I 482 Sphitix Atropos, le méme que n०. 1471. Linn. 799. 9. (8II. dis.

I 483 Sphinx O'eandre, du Levant, Linn. 6. Cram. 224. D. (rr 83. I447. bis.

I484 Sphinax Cocus, le même de n ${ }^{\circ}$ 1475. Linn. O. Cram. 45. E. ('1183. 1454. bis.

1495 Sphinx .... a a ailes noio brunes, les pofterieuresjaunes, ì bord brin, ou Pagana? Linn. O. Fabr. 29? 1422.

${ }^{1} 485 S_{p}$ himx $\ldots \ldots d$ ailes noires, pofterieures à bande jaune, Linn. c. (1044

x+86 Sphinx .... à ailes vertes, taches triangulaires obfcures; Linn. O. ( 1297.

T487 Sphinx Clotho. Linn. o. Fabric. ... (142!

I488 Sphinx.... à ailes rouillée, tache pourpre, wn oeil vers le bord, Lirn. o. (Itco.

148, Sphinx.... à ailes brunes i deux bandes à pointes blanches lamus barbu, (Linn. o. (807.

I 490 Sphinx Zygene. à ailes brunes, les anterieures à 3 taches feneftrẹes, pofterieures d une tache, le côsè des premieres rouge, Linn. o. Fabric.

( 794. 
I 49 I Sphinx . . ... alis nigris, bofi aureis, maculis duabus albis, Linn. O. ( 795 .

1492 S.hinx Ergeneus? f. varietos, alis aureis, macula fluva, Linn: 0. Fabric.. - 798.

1493 Sphirs.... alis nigris, macula sotunda dia, hana, Linin. 0. ( 795

I494 Sphunx Eryx, Linn O. Fabric. 22. Cram 140. E. F. (797. bis.

I495 Sphinx.... alis fuscis, maculis hyalinis, punetoque cœruleo, Linn. o. ( 10 ? bs.

I496 Sphinx spercheus, fed abdomen caret fascia albida, Linn. o. Cram. 146. C ( 800

1497 S. hisx Spercheus?, fovarietas, Linn. O. Cram.1 c. (I32I. bis.

I498 Sphinx .... alis n'gro viridibus, Linn. O. ( 799 .

r 499 S, hinx .... dis nigris, collari fanguineo, Linn. 0. ( I3C4.

Isco Sphinx ..... alis fusc's, abdomine cœruleo viridi, thorace ranguineo, Linn. O. ( 801 . bis.

I50r Sphi, Z Zyanı, alis 2 a:ris, corpore fa!ciis 3 riveis, Linn o. Cram. 11. D. ( 1486 .

1502 Sphinx Cerbera, irid $s$, alis punctis feneftratis fub. tus ci gulis rubris, Lirn. 800. 38. Cram ... Fabric. 12. (1322. bis.

I503 Sphinx Cerbera? f. varietas, Linn. I. c. Cram. 7. 83. F. $(802$ bis.

1504 Sphinx Cerbera? f. varietas præcedentis? Linn. 1. o. (1427.:430. ter.

1505 Sphinx Cerbira? f. varietas, Linn. \& Cram. l. c. (13ั12. tuis.

I506 Sphinx Fuci formis? pedibus antennisque luteis, Linn. 803. $28 \%$ ( 1042.

1507 Sphinx Api-formis? Indicus, thorace viridi aureo ftriata, Linn. 804. 29?. ( $10_{43}$.

$1508 \mathrm{Sp}$ inx Api-formis? I varietas, Indica, Linn. o. (805.

150y Suhirix Tipuli-formis? Indicus, ais ferieftatis, abdomine barbato, arro, Linin 804. 32? (806.

I510 Sphnx Hamorrhoidalis, Linn. 0. Cram. 52. C. D. (803. bic.

J5II Sphi x Hgemorthoidnlis? fo varietas pracedentis? Linn. - Crain i. c. (804

I5I2 Sphirx Hamorrhoidalis? f. varietos, Linn. o. Cram 1. c. ( 1595 .

ISI3 Sphinx Fuci formis, Indicus major, abdomine flavo, Linn. O. ( 1456.

PHALENAE. 
149r Sphinx . . . a ailes noires , de la bafe d"ories, à deux tasihes blancbes, Linn. 0. ( 796.

1492 Spninx Ergetieus? ou varieté, à ailés dorées, tache jaunes. Linn. O. Fabric ...' 798

1493 Sphinx ... a ailes noires, tache onde transparante. Linn o 179 .

1494 Sphinx Eryx, Linn. O. Faluric. 22. Cram. 140.E F. (70) bis

1495 Sphinx . . a à ailes hrunes taches inyalines, do pointe bleu reiefte, Linn. u. ( 0 (9) bis.

I496 Sphinx Spercheus, mais le ventre ria paint de lande blanche, Linn. O Cram. 4r. C ( 800 .

I497 Sphinx Spercheus\% ou varieté, Limn.o.Cram.l.c. (rj2r. bis.

1498 sphinx . . . . a ailes noir-"ertes, ${ }^{2}$ Lirin. $0 . \quad$ (799.

1499 Sphinx .... à ailes voires. gorge rouge, Linis. O ( 1304.

1500 Sphinx . a ailes brunes, le ventre blew verd le corcelet rouge Linn. c." (8or. bis

I 501 Sphinx Z.vgxne à deux ciles noirer, le corps à deux bandes blanches, Linn. O Cram 11: D. 14 .

1502 S, hinx Certiere, verd, à ailes pointées feneftres deffous bances rouges, Linn. 806.38. Cram... Fabric. 12. (1322. bis.

1503 sphinx Cerbere? ou varieté, Linn. l. c. Cram, 7. 83. F (802. bis.

I504 Sphinx Cerhere? ou varieté du precedent? Linn. E Cram. l. c. (1427. 1430 , ter.

1505 Sphinx Ceiberc? ou varieté, Linñ. EO Cram.l.c. (i3iz. is.

r506 Sphinx Fuci-forme? les pattes antennes jaunes, Linna 8c3. 28 ? (104?.

j5or Sphinx Api-forme? Indien, le corcelet yerd, d'or friés, linn. 804. 29.? ( 1043 .

1508 Sphinx Api-forme f ou var:eté Indienne, Linn. O (805.

Isug Sthinx Tipuli-forme, des Indes, à ailes fenefirées, le ventre barine noir, Linn. 804.32. ( 800 .

I sio Sphinx Hemorrhoidale, Linr. o. Cram. 52. C.D. (803. tis.

3511 Sphinx Hemorrhoidale? ouvarieté du precedent? Linn.o.

I512 Splinx Hemorrhoidale? ou varieté, Linn o. Cram. l.c. ( 1595

15:3 Sphinx Fuci-forme? des Indes plus grand, à venttre joune, Linn. O. ( 1456. 


\section{P $\quad H \quad A \quad L \quad E \quad N$.}

I5 14 Phalens Atlas, alis falcatis, luteo variis, macula feneftrata, Limn. 808. 1. Merian Surin. tab. 52. ( 779. bis.

I515 Phalena Atlas, fimilis præcedenti, Linn. 1. c. (... bis."

- I5I6 Phalera Atlas? f. varietas, Linn I. c. (.. bis. I5I 7 Phalena Aurata. Linn. $\odot$ Cram, 8. A. (I4I2.

1518 Phalena Gurata? f. varietas pracedentis? Linn. o. Cram. l. c. $(778$.

1519 Phalena Hesperus, Linn. 0. Cram. 68. A. (1375. bis.

I520 Phalena Hesperus? f. varietas, Linn. O. Cram. 1. c.

i521 Phalena NeEtitans, Linn. O Fabric. 196. I2. (156,5.

1522 Phalena Nectitans? f. varietas, Linn. o. Fabric. I. C. (1318.

1523 Phalena Eurinome, Linn. o. Cram. 70. A ( 1489.

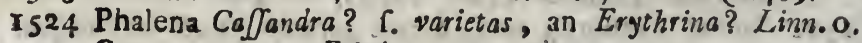
Cram. 197.... Fabric. 9. (1491.

1525 Phalena Lumo? alis caudatis, virentibus concoloribus, ocello luuato, Linn. 810. 5. Cram. 31. A. B. ( I583. bis.

1526 Phalena Mylitta, Linm. O. Drury II. tab. 5 f. I. $(777$.

I527 Pha ena Cytherea, Linn. o. Subz. Infect. tab. 21. $f$. I. (1305. bis.

1528 Phalena Cytherea? f. varietas, Limm. C. Sulz.l c. (1306. bis

1529 Phalena Cytherea? an præcedenti affinis? Linn. \& Sulz. o. (... bis.

I530 Phalena Cytherea? f. varietas, Limin. O. (1510. bis.

I53I Phalena Paphia, alis falcatis, concoloribus, ocello feneftratis Linn. 809. 4. (I3I7 bis.

1532 Phalena Paphia? an finilis precedenti ? Linn. l. c. (1423. ter.

.1533. Phalena Paphia? r. varietas, Linn o. ( 1588.

I534 Pha!ena Erythrina, Linn. o. Mtrian. Surin. tab. II. (776. bis.

1535 Phalena Militaris, Linn. 811. 12. Cram. 29. B.

1536 Phalena Liberia, Linn. O. Cram 268. F. (1022. bis. 1537 Phalena Abas, Linn. O. Cram. 37. A. B. ( 1024. 
1538 Phaiena Liberia, fo femina, Limm O. Cram. l. c. ( 1442.

1539 Phalena .... alis fusco ciner eis, ftriis quatuor pallidioribus, duabus incurvis, Linn. 0. ( 1294 .

I540 Phalena .... alis fuscis infinis, ocello tiavo, pupilla vitrea, Linn. o. ( 1286.

I54I Phalena.... alis tusco purpureis, Atriis 3 nigris marginalibus, ftriaque retta, atra, ab apice ad apicear, Linn. o. ( 1289 .

$154^{2}$ Phalena Odora? I varietas, a'is crenatis nebulofis," Lim. 81x. 11? ( 1290.

1543- Phalena Scolopacen, Linn. O Cram. 74. D. (129:.

1544 Phalena Dolon, Limn. c. Cram IOI. D. (1448.

1545 Phalena Bubo, Linn o. Cram. 17.1. A. B. C1319.

is46 Pha!ena Crepu/cularis, alis grifeis, fascia, maculaque a!bis, Linn 81 ' 13. Cram. 159. A. (916.

1547 Phalena Crepufcularis? fo varietas præcedentis? Linn. \& Cram. I. c. ( 772 .

1549 Phalena Creprefcularis of, varietas, alia, Linn. o. (1594.

I549 Phalena .... alis fuscis, ccraceo nimbolis, macula atra lunari. Linn. O ( 445 .

1550. Phalena Militaris, Linn 81 I. 12. Cram 29. B. (1IIr. bis.

x51 Phalena Leetrix, alis atris, maculis cœruleis, albisque Havis, Linn. 834 89. Cram. 192. C. ( 708.

1552 Phalena Bajulaia, Linn. O. Crnm. I72. C. (769.

2553.Phalena Numana, Linn. O. Cram. 227. A. ( 770.

I5 4 Phalena Nodua, atra, alis fuperioribus macul's 4 fulphureis, poftica um aurantiis, marginalibus albis, ansulis abdominis auruntis, Linn $O$. Cram r92. C. (iI1I.

I555 Phalena Fusca, nrimnribus apice albo, radiatia, faccia ob'ique alba, ponticis disco albo, Linn o. ( 1597.

1556 Pnaler:a .... alis luseo fuscis, fasciis 2 revularibus, conporis nacula flava, Linn. o. ( 2288.

I557 Phalena.... alis bafilu.eis, fascia iats brunnea, Linn. c. ( 1045.

1558 Pbalena Grifro. Fusca, fasciata, macula picea aú apicem, Linn. O. ( 1373 .

1559 Phalera .... humeris cinnamomeis, fascia nebulora, Jinn o. $(14+7$

1560 Phalena Fusco.Cinerea, fascia angulata, Linn 0. ( 1449 .

I5or Palena Grifea, pofticis alis luteis, ocello atrc bipupillato, pupilla major alba, Linn. O. ( I287. 
1538 Phalene Liberia, oufemelle, Linn o. Cram. b.c. ( 1442. $15 j 9$ Phalene.... a ailes brun cendrées, d quatre ftries pales deux recolirtées, Linn. o. (12)4.

I540 Phabene .... d ail s derrieres brunes, oeil jaune, prunelie couleur de verre, Linn. O. ( 1286.

154 I Phalens..... à ailes brun pourprées, $\dot{a}$ trois ftriés noires marginales, une ftrie droite, E voire du point au point, Linn. O. ( 1289.

$154^{2}$ Phalene Odure? ou varieté, à ailes crenues nebuleufes, Linn. 811. I1? ( 1290

$15+3$ Phalene Scolopacea, Linn. O. Cram. 74. D. (1291.

r 544 Pbalene Dolon, Linn. O. Cram. 101. D. (I 448.

1545 Phalene Bubo, Linn. o. Cram. 171. A. B. (131).

1546 Phalene Crepufculaire, da ailes grifes, fajsie, Eg taches blanches, Linn 81 r. 13. Cram. 159. A. (916.

1547 Phalene Crepuiculaire? oul varieté de la precedente? Linn. Linn. E Cram. l. c. ( 772.

$154^{8}$ Phalene Crepufculaire? ou autre variete, Linn.0. (1594.

1549 Phalene .... d ailes brunes, nuancees, nebuleus jes $a$ tache noire lunaire, Linn. O.. (1445.

1550 Phalene Mi icaire, Linn. 811.12. Cram. 29. B. CIIt. bis.

I55I Phalene Lectrix, da ailes noires, taches bleues, blanches \& jaunes, Linn. 834. 89. Cram. 192. C. ( 708.

1552 Phalene Bajulaire, Linn. 0. Cram. 172 . C. ( 759.

1553 Phalene Numane, Linn. o. Cian. 227. A. (770.

I554 Pibalene Noturne, noire, les ailes fuperieures d 4 taches fouffres, les pofterieures cranges, à bordes blanches, les anneaux du ventre or nges, Linn. O Cram. I92. C. (I14I.

1555 Phalene $\mathrm{B}$ une, les $\int u$ perieures à point blanche, rayé, $\dot{a}$ bande transverje blanche, bes pofierieures à pellette blanche Linn. O. (1597.

1556 Pinlene .... d ailes d'un brun jaunes, à deux vandes rivulaires, le corps d tache jaune, Linn. O. ( 283 .

1557 Pbalene ....d ailes de la baje jaunes, de larg: bande brune, Linn. O. (1045.

I 558 Phabene Brun-zrife, bande, d'une tache noiratre arb-point,Linn. 0. ( 1373 .

1559 Phalene.... cinneliğe, do bande nebuleufe, Linn. Ce (1417.

1500 Pialıne Brun-Cendré, à bande en angles, Linn. 0. ( 1449.

156I Phabene Grife. bes ailespofterieures jaunes, à un oeil noir. $E$ deux pronelles, de prunelle grande blanche, Linn. 0 . ( 1287 . 
1562. Phalena Grifen? T. varietas, Linn. O. (ro23.

1503 Phalena Grefea? an tiailis pracedenti? Linn. O 775.

1564 Phalena Irene, Linn. O. Cram. 249. B. ( I443.

1565. Phatena Alciphron, Limo. O. Cram. 132 E. (1615.

is66 Phalena .... alis $\mathrm{f}$ scis rubentibus, Linn 0. (102r. 1567 Phalena Tricolsr, Limm. o. Lram. 203. E. (1277. bis. I568 Phalena Bicolor, an Bimaculofa? Linn. 856. 184? Cram. .... ( I102. bis.

15 19 Phalena Catalina, Linn. O. Cram.... (104r.

1570 Phalena .... alis fuscis. maculis tribus oblongis transparentibus, poftcis nigris, disco diaphano, Linn. 0 . (I) 1040

157I Phalena ..... alis atris, fascia nivea, poftcis rubra, bafi coruleo nitentibus, Linn. O. ( 1035 .

I572 Pha ena..... r. varietns pracedentis? Linn. o. (ro39.

1573 Phalena Atra, fasciis tribus hyalinis cœrulefcentibus, Linn. O. (979.

I574 Phalena . . . alis nigro viridibus, fasciis duabus macularibus albis, polticis nigris, disco flavo, Linn. o. ( 438.

1575. Phalena Fusca, alis pofticis albidis, Linn. o. ( 997.

I576 Phəlena Materna, alis deflexis grifefcentibus, Linn. 840117 . ( $: 446$ bis.

I577 Phalena Hele? an Lominula? Linn. 820. 40? f. 90? (. 548.

1578 Phalena Ammonia? cinerea, macula magna caffea, fascia obliqua interrupta, Limn. o. Cram. 250. D. ( 782.

1579 Phalena Fusca, maculis aibis, pofticis alis flavis, margine, punetoque disci fuscis, Linn. o. (II2I.

I580 Pralena Occidus, alis dentatis nebulofis, linea pallida, Linn. 812. 14. Cram. 169. A. (780., bis.

158I Phalera Occidua? f. varietas, Lim. \& Cram. l. c. ( $103 \mathrm{r}$.

I582 Phalena Strix, alis patulis, albido nigro reticulatis, Linn. 833. 82. Cram, 8. A $A_{i}$ (I 493.

1583 Phalena Odora alis crenatis nebul fis', fuperioribus uni- co ocello, Linn. 8Ir. II. Cram. r69. B. (1029. bis.

1984 Phalena Odora? f. varietas, Linn. \& Cram.I. c. (ro3o. 1585 Phalena Salamina, Linn. O. Cram 74. A. ( 1580.

1586 Phalena Materna? f. varietas, alis deflexis grifes, inferioribus llapis, Linn. 840. II ? Cram. 74. B. ( 1566. 
156.2 Ph lene Grife? ou Ja varieté, Linn. O. ( 1023.

Is63 Phalene Grife? ou Jemblable do la precedente? Linn. $(774 \cdot 77.5$.

1564 Phalene lie e, Linn. O. Cram. 249. B. (1443.

1505 Phatene Alciphron. Linn. 0. Cram. 132. E. (1615.

1566 Phalens . . à ailes brunes rougatres. Linn.c. (rozr.: I567 Phalene à Trois-couleurs, Linn. O. Cram. 263. $E_{\text {. }}$ (1277 bis.

i 568 Phalene à Deux-couleurs, ou d Deux-taches? Linn. 856. 184? Cram.... (1102. bis.

1569 Phalene Cataline, Linn. 0. Cram.... (1041.

1570 Phalene ..... di ailes brunes, trois taches allongées transparentes, les pofterieures noires, à palette diaphane, Limm. 0. (ro40.

I571 Phalene... a à ailes noires, bande blanche, les pofterieures rouge, d bafe bleue, Linn. 0. (1035.

I $57^{2}$ Phalene... ou la yarieté de la precedente? Linn. 0. ( 1039.

1573 Phalene Noire, à trois bandes hyalines bleuatres, Linn. 0. ( 979.

1574 Phalene .... à ailes noir-vertes, à deux bandes tachetées blanches, les pofterieures noires, à palette jaune, Linn. o. I 438.

I575 Phclene Brune, d ailes pofterieures blancheatres, Linn.' 0. ( 997.

1576 Phalene Materne, à ailes recourbées grifes, Llnn. 840 . 117. (1446. bis.

1577 Phalene Hebé ? ou Dominula? pertetre Demoifelle, Linn. 820. 40.? ou 90? (1548.

1578 Phalene Ammonie? cendrée, à grande tache caffée, d bande transver $\int_{e}$ interrompue, Linn. 0. Cram.250. D. (782.

1579 Phalene Bruve, à taches blanches, ailes pofterieures jaunes, le bord, Eo point de la palette, brune, Linn. O. (I I 2 I. is8o Phalene Occidua, do ailes deniées nebuleufes, à ligne pale, Linn. 8i2.14. Cram: 169. A. (780. bis.

i78r Phalene Ocridua? Line. \&o Cran. O. (103r.

1582 Phalene Str $x, d$ ailes larges, couvertes de blanc $\&$ noir, Lirn. 833.82. Cram. 8. A. (T493.

1583 Phalene Odora, $a$ ailes crenues nebuleufes, les fuperiersres à un petit oeil, Linn. 81r. II. Cram. 169. B. (I029. bis.

I584, Yhalene Odore? ou varieté, Linn. \&2 Cram. l. C. (Iс3о. 1385 Phalene Salamine, Linn. O. Cram. 74. A. (15\%0.

I586. Phalene, Materne?, ou varieté, d ailes griles inferieures jaunes, Linn. 8to. II 7 ? Cram. 74. B. (I506 
I58? Phalena .... alis grifeis fascia conco'ore, margine it. vu ar Linn. o. $\quad$ \& $48: 0$

1588 Pholena Meiicente, alis fuperioribus, lineic duahus nigris, ali- intions ftria alba margine lato a bo, Linn.o. $\mathrm{Cr}_{2}$ m. 223. C. ( 1579

I589 Phatena .... a is grife:s, noftıcisluteis, fa'ciz marginali fusca, Linn. o. (1374.

1590 Phalena .... alis tuscis, lineis rivularibus albis, Limn. 0. ( 109 )

1591 Piralena.... alis fuscis lineis tribus rivularibus, pariets \$ macuia Juna ialbis Linn o ( 785 .

I592 Phulena Grifea, macula lunari, punctoque atro, Linn. O. ( 1292.

1593 Phaiena Grifeo Fusca. puneto alro, in quaque ala, Linn. o. (13:0. 1431 bis.

I594 lha ena .... alis cinereis, triangulo fusco, Linn. o. irior bis.

1595 Paleria Fusca, fascia alba, ex pundis feptem, Linn. 0. ( 1293 .

1596 Phalena Hielvoln, fascia concolore, Limm. O. (1426.

1597 Phalena rielvoln? verietss, Linn O. ( 1425.

1598 Phaicn. Orichalea, Linn. O. ('3Ir.

' 599 Pha $\in$ a . . . alis cinereis, ftrilo vel maculis oblongis aris, Linn. o. $(786$

r 6 o Phalena.... al s cinereis, macula lunari concolore, Linn. O. (1453. bis.

1601 Pnalesa ..... alis cinereis, ftriis duabus angularibus arris, Linin. C. (15It.

1602 Ptaiena.... alis fuscis, albido marmoratis, Limn. o. $(978$.

1603 Phalena.... alis lute contibus, lunula punetoque f scis, Linn.o. (1567.

1604 Pha'ena Grijes-Fusca, marmorata, linea reeta rufa, Linn. o ( 1032 .

1605 Phalena..... alis fuscis, nigro maculatis, Linn. O. ( 788 .

1606 Phalena .... thorace alisque niveis, Li.n. o. (1295. 10,1 Phale a ..... a is fu-co-grifs is, potterioribus diaphdnis margine fu.co, Linn o. ( 789 .

16.8 Phalena.... alis paliide grifeis, fusco marmoratis. Linn. O. ( $145^{2}$.

I 6 ng Phaiena.... alis albis, margine fusco, Linn n. ( 1033 . 1610 Phaiera.... alis thoraceque ftramineis, ftris nigris obl q is, triplicique ferie punctoruin nigrorum. Lisn. o. $(784.6 i=$ 


\section{Insectes.}

5587 Phalene .... d ailes grifes, d bande de meme couleur; $d$ bord riviabire, Linn o. (I $58 \mathrm{I}$.

1588 Phalene Melicente, a ailes fuperieures, d deux lignes roires, les ailes inferieures d ftrie blanche, \& bord blanc, Linn. o. Cram. 223 C. (1579.

1589 Phalene .... ì ailes grifes, pofterieures à bande marginale brune, Linn. 0. ( 1374.

I590 Phalene . . à ailes brunes, à lignes rivulaires blanches, Linn. 0. ( 1099

I59I Phabene ....d ailes brunes, d trois lignes rivulaires, d point, EO tache lunaire, blanches, Linn. 0. (785.

1592 Phalene Grife, d tache lunaire, EO point noir, Linn. 0. ( 1292

1593 Phalene Gris-brune, d point noir, dans chaque aile, Linn. o. (1310. 1424.bis.

1594 Phalene ... d à ailes cendrées, d̀ triangle brun;' Linn. 0. (rror. bis.

I595 Phalene Brune, $d$ bande blanche, de 7 pointes, Linn. 0. ( 1293.

1596 Phalene Helvole, à bande de méme couleur, Linn. O. ( 1426 .

$x 507$ Phalene Helvole? ou variete, Linn o. : ( 1425.

1598 Phalene Orichalea, Linn. o (I3II.

1599 Phalene .... à ailes cendrées, ftries ou taches aliongées noires, Linn. O. 1786.

1600 Phalere .... d ailes cendrées, Ẽ tache lunaire de méne couleur, Linn. O. (1453. bis.

roor Phalene ..... d ailes cendries, d deux ftries angulusires, noires, Linn o. ( $151 \mathrm{r}$.

1602 Phalene. ... d ailes hrunes, martrées de blanc. Linn. $0 .\left\{9 ?^{8}\right.$. 1603 Phalene . . . d ailes jaunatres, à hune, Ẽ pointe brune, Linn. 0 (1567.

1604 Pbalene Gris-Brune, marbrée, à raye droite rouge, Linn. o. $(1032$.

I605 Phalene .... d ailes brumes, tachetées de noir, Linn. 0. (788. 1606 Phalene .... le corcelet, E ailes blanches, Linn. 0 . ( 1205.

ICO7 Phalene ... de ailes brun-grifes, les pofterieures transparantes, d lord brux, Linn. 0 . ( 789

io:8 Phalene....d ailes pales, grijes, marbrées de brun, Linn. o. $(1452$.

1609 Pralene.... dailes blanches, dbord brun, Linn. O. $\{1033$. 1610 Phalere. . . . les ailes EO corcelet, coulears de paille, d Jitries noires transverfes. d triple fuite de points noirs.. Linn. o. (784. bis. 
í I Pha'ena Colloris z thorace flavo, oprintis atris, alis fuscis, bafr flavis nigromaculatis, ad apicem maculis ditabus albis, Linn o. $C_{1634}$

I6I2 Phalena Pyralis, alis niveis, macula ferruginea, friis atris viridibu que, Linis 0. ( 791.

I612 Phalena Laducina, I Sambucaria? Linm. 800. 203? Cruin. 273. B. C. C 1027 .

1613 Phalena .... alis falcatis, pofticis margine brumneis, fascia hyalina, Linn. O. is ( 1028 .

I0I3 Phalena .... alis fuscis, fascia purpurafcente, rupra Oo notatis, Lintiv, o. (754:

i614 Phalena.... alis cinereis, pofticorum macula trigona diaphana, antennis perlongis, Limn. O. ( 1026.

I6 5 Phalena .... alis fuscis, fascia communi albida, primoribus macula albida ad marginem, Linn. O. (753.

Ioro Phalena.... alis dentatis pallidis, niveo marginatis, pofticis macula tripalmata diaphana, Linn. O. Cram. I II. E. Croro.

I6I 7 Phalena Rubro-Fuscd, macula magna viridi, Linn. o. ( 1589.

I6r 8 Phalena Ocracea; linea tenuiflima rubens, Linn. O. (r432. I6r9 Phalena Cydonia, Limn. O. Cram. 22. F. ( 753. I620 pinalena Gyges, Linn. O. Cram. 102: B. ( 1026. I62I Phalena Cribraria, Linn. O. Cram. 68. C. (76I. 1622 Phalena Cinerea, ftriis duabus fuscis, Limn. 0. (16I 3. 1623 Phalena .... alis helvolis, fascia flava, Linn. O. ( I 578.

I624 Phaiena Ocracea olivaceo marmorato, Limm. O. ( 1023. 1625 Phaiena Mori, Chinenfis, Linn. 817. 33: ' (IGQ1. I626 Phalana Nigro-Cinerea, ocello duplici, pofticorua fas. cia undulata nigra, Linn. o. ( $78 \mathrm{r}$.

I627 Phalena Tanguina, Linn. o. (I492.

I627 Phalena Lanipeda, f. Noctua? alis viridi-nigris, albido fasciatis, Linı?. O. ( 1414 .

i628 Phalena Capenris, alis helvolis, fuperioribus ftrigis duabus repandis albis, Linn. 813. 20. (1307. bis.

I629 Pbaiena Capenfis? f. varietas; magis fusca, Linn., $C_{\text {? }}$ ( I 44 I.

1630 Phalena Capenfis? f. varietas, fascia \& margine albo, Linur. O. ( 1440 .

I63 I Phalena Capenjis? f. varietas, fed minor, Linn. 0. ( 1320.

I632 Phalena Perfpicillaris, alis deflexis, Atriatis, poftice bidentatis, Linn. 849. 148. Cram. 97. A. B. (771. I633 Phalena Albo-Grifea, fáscia alba, Liann. o. ( 773. 


\section{Injectes.}

ir Phalene Collier, le corcelet jaune, d points noirs, ailes brunes, de la bafe jaunes, tachetés de noir, yers le point, de deux taches blanches, Linn. 'O. (I634.

iI 2 Phalene Pyraile, a ailes blanches, do tache de rouille, a fries noires, \& vertes, Linn. O. ( 791 :

12 Phalene Lactucine. ou Sambucaire? Linn. 860. 203? Cram. 273. B. C. (1027.

is Phalene ....a ailes courbées, ?es pofterieures à bord brun, à fascie hyaline, Linn. O. (1028.

ir 3 Phalene .... a ailes brunes, barde pourprée, en haut marquées de oo Linn. 0 . ( 754 .

is 4 Pbalene.... a ailes condrkes, les pofterienires a tache triangulaire transpdrante, les dntennes allonges, Litn. 0 . $(1026$.

ir 5 Phalene .... ḋailes brumes, à bande commdifeblanche, les premieres à tache blanchatre vers' le bord, tinn $0: ? 753$. iro Pbalere.... da ailes denteléespales, 'bordées de blanc les pofter:ezures a tache tripalmaire transparante, Linn. o: Cram. I51. E. (rolo.

it7 Phalene Rouge-Brune, do granate tacbe verte, Linn. 0. (1580.

ir 8 Phaleme Ocracee, à tres fine raye rouge, Linn. O. (1 432 .

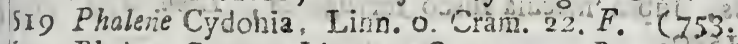

izo Phalene Gyges, Linn. O. Cram, 102. B. Crož

j2r Phalene Cribraire, Linn, o Cram. 68. C. (7t

"i22 Pbalene Cendrée, dे deux fories hrunes, Linn o. (T6 3 .

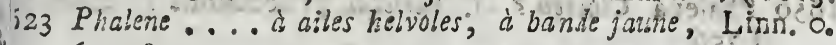
(:578.

i24 Phalene Ocracée, marbrèe d'olivatre, Linn. O. Cúnz.

i25 Phaléne Mori, de la Cinine, Lihn? 817.33. (I00r.

i26 Phalene Noir-cendrée, do double oeil, les pojterieures is bande ondée noire, Limn. O. ( $78 \mathrm{r}$.

527 Phalere Tanguine, Linn. O. ( 1492.

$\$ 27$ Phalene Pièd-à laine, ou Hibou? d ailes vertes noires, bandées de blanc, Linn. 0 . ( 1444.

528 Pbalene du Cap, à ailes belvoles, les Juperieurs d deux firies blanches, Linn 813, 20. ' (1307. bis.

\$29. Phalene du Cap? ou varieté, plus brine, Linn. O. (I $44 \mathrm{I}$.

's3o Phalene du Cap'? ou yariete, at fafcie, E' bord blanc, Linn. ○. (I 440 .

53 I Phalene did Cap? jou varieté, maisplus petite, Linn. o. (1320.

532. Phaleve Perfpicillaire, à ailes recourbees, friées, les pofterieures bidentées, Linn. 849, I 48. Crain. $97, A, B$. $(779$.

633 Pralene Blânc-grífe, a bande lahabbe, Linn. O. (773. 


\section{6 Infecta.}

1634 Phalena Capenfis? I yarietas, Linn. 813. ?0? (1376. bis 1035 Phalena Rufo - Brunnea, Atria duplici rubra, lunula fer ruginea, Linn. c. ( 788.

1636 Phalena Viridi- Pallida, fascia alba . Linn. O. ( $143 \mathrm{I}$ I637 Phalena Aprillina, Indica, lineis duabus obliquis, Linm 847.) 138 ? ( $128 \mathrm{I}$.

1638 Phalena Viridis, macula grifea, Linn o. ( 1488.

1639. Phalena Celadonis, linea oblonga alba, Linn. of (ror8.

I640 Phalena Pyralis, ftraminea unicolor, Lintn. O. (I 28c I64I Phalena Miniacea, marginibus alarum fuscis, Linn. o ( 1283 ,

1642 Phalena Miniacea, fulvo rubra, marginibus punetoqv aureis, Linn. O. (...

1643 Phalena Fulvo-Rubra, margine punctoque aureis, Lint O. (I1 22. 1508. bis.

1644 Phalena .... alis falcatis framineis, linea fulva, Lint ○. ( 1433 .

I645 Phalena Miniacea, apicibus alarum fuscis, Linn. c ( 1282.

1646 Phalena Fusca, ocello ferrugineo, Linn. o. (1015.

1647 Phalena Alftremeriana? f. varictas, Indica, alis albi dis, fupra macula fusco ferruginea, Linn. 879314 ( 1540.

I648 Phalena Grifea, linea tenui atra, Linn. O. ( Iorg. 1649. Phalena Cinerea, linea rufa, Linn. o. ( 755.

1650 Phaleca Cinerea? r. varietas pracedentis? Linn. ( 756.

I651 Phalena Pallida, Linn o. (757.

1652 Phalena Flava, maculis Atriaque aurantiis, Linn. (\% ( $75 \mathrm{x}$.

1653 Phalena Butyracea, Indica, Linn, 0. (1370. bis. 1654 Phalena Cinerea, maculis fuscis, Linn 0. ( 752. 1655 Phalena Cinerea, ftria undata, fquamulis erectis, Lin o. $(1487$.

1650 Phalena Laneftris? Indica, alis ferrugineis, Atriga alba Lisn. 815. 28? ( 1372.

1657 Phalena Allida, puncto atro, Linns. O. (1014.

r6s8 Phalena Sericea, alba, Linm. O. (1013.

I659 Phalena Sericen? f, varietas, Limn. O. ( 1222.

I 660 Phalena Flaveobata, alis nigris, maculis albis, Limis 867. 240. Cram. 88. C. ( 1285.

I66r Phalena Ammon, Linn. 0. Cram. 146. B. ( $143 \mathrm{C}$ bis.

1662 Phalena Ofiris, Linn. O. Cram. 95. E. (I284. 
34 Phalene du Cap? ou variete, Linn. o. ( 1376.

535 Phalene Roisge-Brune, d double firie rouge, a lunule cousleur de rouille, Linn. 0 . ( 788.

s36 Phalene Vert Pale, d bande blanche, Linn. O. (1431.

537 Philene Aprilline? des Indes, ddeux lignes transverfos, Linn. 847. 138 ? (1281.

538 Phalene Verte, do tache grife, Linn. O. ( 1488.

39 Phalene Celadone, d raye longue blanche, Linn. 0. (ror8.

540 Phalene Pyraile, jaunatre, d'un couleur, Linn. 0. ( 1280.

i4 I Phalene Minie, les bords des ailes brunes, Linn. 0. (1283.

$42^{2}$ Phalene Minie, brun-rouge, le bord E point d'orés, Linn. o. (...

543 Phalere Fauve-Rouge, à bord, E point d'orés, Linn.o (I122. 1508. bis.

544 Phalene.... di ciles courbées, jaunes, di raye fauve, Linn. 0 . ( 1433.

i45 Phalene Minie, les points des ailes brunes, Linn. 0. (I282.

ito Prablene Brune, do oeil rouillé Linn. O. (1015.

47 Phalene Aftromeriane? ou varieté, des Indes, à ailes blanchatres, deffus a tache brune, couleur de rouille, Linn. 879. 314 ? ( 1540.

i48 Phalene Grife, $\dot{a}$ raye fine noirc, Linn. o. (10r9.

49 Phalene Cendrée, d ligne rouge, Linn. O. (755.

iso Phalene Cendrée? ou varieté de la precedente? Linn. o. ( 756.

51 Phalene Pale, Linn. O. ( 757.

52 Phalene Jaune, à taches, E' ftries d'oranges, Linn. 0. ( 751 .

is3 Pbalene Butyracèe, des Indes, Linn. O. ( 1370 . bis.

54 Pbalene Cendrée, do taches brunes, Linn. O. ( 752.

iss Phalene Cendrée, ftrie ondée, à ecailles erigles, Linn. 0. ( 1487.

556 Pholene Laneftris? des Indes, a ailes couleur de rousille, d raye blanche. Linn. 815 . 28.

57 Phalene Blancheatre. d point noir. Linn. O. (ror4.

58 Phalene de Soye, blanche, Linn. o. (1013.

59 Phalene de Soye? ou yarieté, Linn. O. (1222.

iso Piralene Jaunatre, dailes noires, Ẽ taches blanches, Linn: 867.240. Cram. 88. C. (1285.

61 Phalene Ammon, Linn. O. Cram. 146. B. (1436. bis.

62 Phalene Ofiris, Linn. C. Cram. 95. E. (1284.

N 3 . 1603 Phalens 
1663 Phalena Agyrtis, Linm O Cram. 123. B. ( 795. 1664 'Phalena Procas, Linn. O. Cram. I7s. D. ( Io6s. I665 Phalena Interrupia, alis depreffis, linea nigra, terg rubro, Linn. 840. Iro. Crom. 185. E. ( 1577 .

f666 Phalena PJamas, Limm. o. Cram. 234. G. (100; bis.

1667 Phalena Fulvia? alis fulvis, margine atris, Linn. 830 96? Çram. 251. F. (764. I435.

I 1668 Phalena PJamas? f. varietas, an Fulvia? Limn. O. (143! 1669 Phalena Pyralis, fusco rufa, Linn. O. (762. I52! bis.

I670 Phalena Pyralis, fusca; alis polticis latioribus, Lin! o. $(763$.

I6.7. Pbalena Urticaric , flava, jubare aureo, Linm. (1020.

I 6.72 Phalena Albo- Argentea a colta marginali elevata, Lin ( 1592.

1673 Phalena Lutea, fulvo maculata, Linn. O. ('Ior6:

1674 Phalena Alba, ftriis marginalibus atris, Linn o. ( I37 bis.

I675 Phalena Nivea? Indica, alis corporeque albis, antenn tefaceis, Linn. 838. I08? ( I012. bis.

I676 Phalena Fusca, ftria macula diaphana, Linn. o. ( 159

I 677 Phalena Albida, diapbanaftriis fuscis, Linn. o. (I 59

1078 Phalena Pulchella? Indica, alis albis, fupra nigro, rubri que punctatis, Linn: 884. 349 ? (759. bis.

I679 Phalena Bella, alis flavis, fupra fex fasciis punctoru atrorum, Linn. 834. 348. ( 760 .

1680 Phalena Syringaria? alis fubrofeis, grifen flavis, pun tis duobus fuscis, Linr. 860. 200? (I576. bis,

1681 Phalena Decora, Linn. o. Cram. 219... ( I437. bis 1682 Phalena Fulia, Linn. O. Crom... (1509. bis.

I.683 Phaleria Abbida, Linm. 0 . ( 758.

I684 Phalena. . . alis diaphanis, apice fusco, corpore $r$ bro, thorace albo, nigro punctato, Linis. O. (I593.

1085 Phalena . . . alis atris, macula discoque fulvis, Lin o. ( 1065 .

I086 Phalena Tinea, alis luteis, maculis pallidis, Linn. $\left(787^{\circ}\right.$

1687 Phalena Crefus, Limm. O. Cram. 142. C. (145\%.

1689 Phalena ..... alis fuscis, porticis albis, Linn. ( 1038.

I689. Phatena . . . alis rubdiaphanis, maculis fuscis, Lin ०. (799. I439.: bis. 
1690 Phalena Micilla, Linin o. Cram. 228, G. ( 765.

1691 Pbalena Micilla? C. varietas præcedertis? Lirn. ๑. (roc8.

1692 Phalena Micilla? f. varietas, Linn. o. Cram. 1. c. (766. 767, ter.

1693 Phalena .... alis fuscis aurantio ftriatis, Linn. Q. ( 793.

1694 Phalena .... alis fuscis, apice albis; Linn. O. ( 1037. 1695 Phalena .... alis fuscis, fascia \& capite flavis, Linn. c. $(1036$.

I696 Phalend ... . alis atris, fascia nivez, Lim. O. (1403. bis.

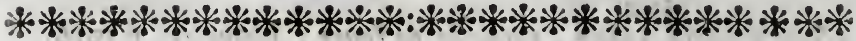

$\begin{array}{lllllllllll}N & E & V & R & O & P & T & E & R & A .\end{array}$

L I B E L L V L

1697 Libellula Vulgate, alis hyalinis, corpore grifeo, Linn. cor. 3 (455.

1698 Libellula Vulgata? f. varietas, Linn. I. c. (80g.bis.

1699 Libellula Vulgata, alis albis, inmaculatis, thoracis latera, 3 ftriis flavis, nigro marginatis, Linn. o. (945. bis.

1700 Libellu'a Capenfis? f. varietas, alis antice bafi flavis, fusco maculatis, Linn. 974. 19. (806. bis.

1701 Libel'ula.... alis aqueis, fascia brunnea, Linn. 0. (454. bis

702 Libellula .... alis puncto marginali, \& apice fusco, Linn. o. ( 807 . bis.

I 703 Libellula .... corpore lengiffimo, alarum macula fuiva, pufticis nigra, Linn U. (453. 562. b's.

$\nearrow_{704}$ Libellula Gigantea, corpore longiffimo, alarum apicibus lactea, Linn. O. ( 1002.

1705. Libellula Perlongata, corpore longifimo, alarum appicibus atra. Linn. o. ( 666.

1706 Libellula Gigantea? r. varietas, alarum apicibus flavescentibus, Linns. O. ( 565.

1707 Libellula Virgo, americana, Linn: 904. 20. (684. bis. 
I 708 Libellula Virgo? Americana, fo yarietas, Limn. 1. c. (903. bis.

i709 Libellula Trigonalis, corpore trigono, alarum margine macula fusca, Linn: o. (8ro, quater.

I 710 Libellula Trigonalis? $C$ varietas pracedentis? fusca, corpore nigro, Linn. O. (...

I7I I Libellula Trigonalis? f. parietas, alis fervicis macula marginali fusca, Linn o. ( 524 .

1712 Libellula Trigonalis? f. varietas, alarum bafi f:18ca, Linn. o. (580. bis.

I7I3 Libellula Trigonalis? f. varietas, corpore luteo, Linn.

I7I4 Libelluia Triganalis? r. varietas, thoracis lineis flavis, macula alarum atra, Linn. o. ( $\mathrm{rCO}^{\circ}$.

\section{MYR MELEONES.}

I715 Myrmeleon Caseñis, alis marmoratis, Linn. o. (42. 44. bis.

$17 \times 6$ Myrmeleon .... alis hyalinis-, axice fuscis, Linn o ( 720.

I 77 Myrmeleon Libelhaloides, alis nigro punetatis ac maculatis Linn. 913. 1. (339. bis,

1718 Myrmeleon Libelhuloides, f. Jarva. (4425.

I719 Myrmeleon Capenfis, Linn. O. (544. bis.

1720 Myrmeleon Capen/is? varietas, Limpls. o. (606 bis.

I72I Myrmeleon.... alis fusconervofis, corpore brevi to. rofo, antenuis clavatis, Limin. o. (64I.722. bis.

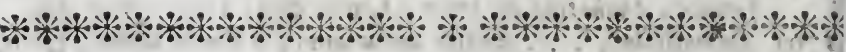

$\begin{array}{lllllllllllllllll}H & \Upsilon & M & E & N & O & P & T & E & R & A\end{array}$

\section{T E I THREDINES.}

1722 Tenthredo Punctata, abdomine atro, Linns. O. (-3.4 bis.

I723 Tenthredo Nigra, lateribus fulvo maculatis, Limn3. \& (35.

I724 Tenthredo Longiconis, thorace anogue ferrugineis, fas ciis 2 fovis, Linn. o. (... 
I708 Demoifelle Vierge? Americaine, ou varieté, Linn. l. c. (908. bis.

1709 Demoifelle Triangulaire, à corps triangulaire, le bord des ailes'd tache brune, Linn. $O$. ( 810 . quater.

1710 Demoijelle Triangulaire? ou varieté de la precedente? brune, à corps toir , Linn. o. (....

I7II Demoifelle Triangulaire? ou varieté, de ailes de foye. le bord 'a tache brine. Linn. 0 . ( $\$ 24$.

1712 Demoijelle Triangulaire? ou yariste, la baje des ailes brune, Linn. O. ( 580.

I $7 \times 3$ Demoifelle Triangulaire? os varieté , le corps jaune, Linn. o. $(\ldots$

17 I4 Demoifelle Triangulaire? ou varieté, le corcelet d geyes jaunes, les ailes d tache noire, Linn. o. (1003.

\section{MYRMELIONS.}

I7 5 Myrmelion du Cap, à ailes marbreses, Linn. 0. (43. 44 . bis.

1716 Myrmelion . . . d ailes hyalines, aupoint brunes, Linn. o. $(720$.

1717 Myrmelion Libelluloide, à ailes pointes, E' taknetées, Linn. 913. 1.. (739. bis.

I718 Myrmelion Libelluloide, ou Larye, Linn. 0. (4.25. I I7I9 Myrmelion Du Cap. Linn. 0. (544. bis.

I720 Myrmelion Du Cap? ou varieté, Linn. O. (6c6) tis.

1721 Myrlmelion.... के ailes branes nerviufes, conps petiv nigferts leux, anternes en forme de maffue, Lian. $0 .(041.722$. bis.

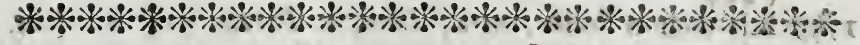

\section{$\begin{array}{llllllllllll}H & \Upsilon & M & E & N & O & P & T & E & R & E\end{array}$}

\section{MOUCHES Ä:SCIE.}

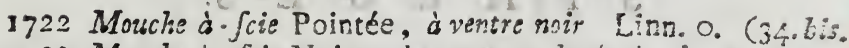

1723 Mouche a-fcie Noire, les cotes tachetés de fouve, o. ( 35 .

1724 Mouche \& fie Long-Corne. le corcelet, Es banus coisleur de rouille, do 2 fories $j$ cumes, Linn. $O$. C ... t $t$ 
i725 Tenthreço ... : abdnmine fexfili, atra, annulo primo abdominis niveo, Linn. O. (s'yg.

1726 Tenthredo Hirta, nigra, fasciis abduminis albis , Linn. D. (943. bis.

I727 Tenthredo Villosa, atra, fronte flavo, antennis clavatis, Linn. o. ( $80 \mathrm{r}$.

I728 Terthredo Lute, thorace nigro, fegmento 4 nigro, flavo marginato, Linn. o. ( 25.

\section{C H N E V MONES.}

I720 Ichneumon Corrufcator? Americanus, Linn. 934. 31 ? ( 431 .

2730 Ichneumon Ater, abdomine clavato, petiolo favo, re. dibus flavis, Linn. o." (430. bis

173 I Ichneumon Lotatarius, Linn. o. Fabric. 330. 16. ( 438.

1732 Ichneumon .... thorace luteo, alis fuscis, abdomine atro, Linn. o. $(436$.

$\times 733$ Ichneumon Luicus. capite, ano, pedibusque nigris, Linn. O (437.

1734 Ichneumon Corrufcator? f, varietas, Linn. I c. ( 995.

I735 Ichneumon Subpetiolatus, ater, alis caftaneis, Limm. 0. ( 36.

I 736 Ichneumon Drury, fronte flavo, corpore lureo, abdomine clavato, angulo nigro, Linn. O. Drury II. tab. 15. (889. bis.

1737 Ichneumon .... antennis luteis, annulo nigro, c spore brunneo, Linn. o. (442.

I 738 Ichneumon Gigas, ater, pedibus longifimis, Linn. 0. $(444$

I739 Ichneumon Scolin, atra, Linn. O. Fabric. 355. 1. (29.

I 740 Ichneumon Scolia? f. varietas, glabra, Linn. O. ( 30. bis.

174 I Ichneumon 4 Maculata, Linn. O. Fabric... (...

1742 Ichneumon .... thurace \& pitiolo luteis, abJomine \& ais fuscis, Linn. o. ( 499.

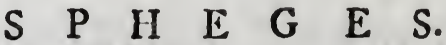

I74 Sphex Cerulea, fronte argentea, Linn. 941. 2. an 38? (796. bis.

1744 Sphex .... thorace flavo rigroque, limbo flavo, abdomine clavato, annulis 5 flavis, Linm. O. (443. 
I725 Mouched-fcie .... le ventre fix files, noire, le premier annear dis ventre blanc. Linn. O. ( 999 .

1726 Mirche a-jcie Noire, le verutre d bandes blancbes, Linn. 0. (943. bis

1727 Mouche d fcie Velue, noire. d front jaune, les antennes en mafJes, Linn. o. (80I.

1728 Mouche ḋ-fcie Jaune, à corcelet noire, la quatrieme partie, noire, borde de jarne, Linn. O. ( 25 .

\section{C H N E U M O N S.}

1729 Ichnewmon Corrufcateur? d'Amerique, Linn. 934. 3I? ( 4$\} 1$.

$173^{\circ}$ Iconneumon Noir, ventre en forme de maffue, pedicule s. $^{\circ}$ pattes jaunes Linn. $c$. (430. bis.

1731 Ichneumon Lotataire, Linn. o. Fabr. 330. 16. ( 438.

1732 Ichreumzon . . . d corcelet jaune, les ailes brunes, ventre noir. Linn. o. ( 436 .

1733 Ichnewsmon Jaune, à tette, anus, E pattes noires, Linn. o. $(437$.

1734 Ichneumon Corrufcateur? oul varieté, Linn. l. c. (995. 1735 Ichneumon Pedicuié, noir, ḋ ailes chataignées, Linn. O. $(26$.

1736 Ichnewmon de Drury, id front jaune, is corps jaunatre, be ventre en malde, angle noire, Linn. O. Drury II. tab. 15. Linn. o. (889. bis.

1737 Ichneumon .... les antennes jaunes, d anneare noir, le corps brun, Linn. 0 . (442.

1738 ichneumon Geant, noir, d pattes très longues, Linn. 0. (444.

1739 Ichmeumon Scolia, noire, Linn. 0. Fabric. 355. 1. (29.

$177^{\circ}$ Ichnewmon Scolia, ou varieté, pelé, unie, Linn. 0. ( $30, \ldots$ bis.

I 4 I Ichneumon dे 4 . Taches, Linn. o Fabric.... ( $\ldots$

$174^{2}$ Ichneumon.... le corcelet, Es pedicule jaunes, ie pen. tre, Eै les ailes brunis, Linn. O. (499.

\section{S $\quad P \quad H \quad E \quad G \quad E$ S.}

1743 Sphex Bleu-Celefte, à front argentine, Linn. 94x. 2: ou 38 ? (796. bis.

1744 sphex... corcelet jaune है noir, à tord jaune, le ventre en majjue, à cing anreaux jauries, Linn.c. ( 443 .

I 745 Spheres 
1745 Sphex Ater, abdomine petiolato luteo, Linn. O. (44 r. 1746 Sphex Longipes, ater, granulis clypeo niveis, pedibus polticis longifimis, alis fasciatis, Linn. 0 . an Tenthredo Femorata? Limm. 920. 1? (639.

1747 Sphex Lutea, capite nigro, alarum apicibus atris, Linn. o (24.

1748 Sphex Niger, alis luteis, apice albis, Linn. O. (522.

I749 Sphex Ater, apicibus antennarum, \& bafi alarum Gavis, Linn. O. ( 32.

I750 Sphex Ater, alis fuscis, thoracis lateribus, pedibusque luteis, Linn. o. ( 33 .

1751 Sphex .... capite luteo, corpore nigro, abdomine fasciculato, a 0 trifeto, Linn. 0. ( 797.

1752 Sphex Pectinipes, Linn. 944. 17. (3I. bis.

1753 Sphex Niger, collari villofo albo, Linn. o. ( 888.

I754 Sphex,.... abdomine grifeo incurvo, alis fulvis, Linn. 0 ( 28.

I 755 Sphex Ater, alis luteis, apice \& abdomine aurantio, ps. dibus teftaceis, Lim O. ( 884 .

I 756 Sphex Ater, alis luteis, abdominis apice aurantio, Lin\%. o. $(884$

I 757. Sphex Violacea, alis violaceis, apice abdominis aurantio, Linn. O. (887.

1758 Sphex Atra, alis hyalinis, corpore fubpilofo. Linn. $a_{a}$ $(800$.

1759 Sphex Atra. alis violaceis, apice \& abdomine aurantio, pedibus nigris, Linn. O. ( 857.

\section{$V$ E S P IE.}

I 760 Vespa Atra, abdominis fascia fulva, Linn. O. ( 799. bis.

176r Vespa Rubrescens, rufo fusco, alarum bafi fusca, abdomine luteo, Linn. o. ( 993.

17602 Vesps Rubrescéns, eadem fed minor, Linn. o. ( $\$ 94^{\circ}$

I $7 \sigma_{3}$ Vespa Rufo Nigra afarciis I 2 latiaribus, $3^{\circ}$ angultiore flav s, Limn 0. (798.

1764 Vespa . . . fronterubro, thorace nigro, interibus macillis 2 fascioque luteic, abdowine nigro, fascia fava, Linn. O. ( 946 . bis.

I765 Vespa .... antennis clavatis longioribus, thorace \& apice, abdonine fulvis, alis cartaneis, $\mathrm{L}_{i . n}$. C." (89r.

I 760 Vespa 
$1745^{\circ}$ Sphex Noir, 'le ventre, Es pedicute joume, Linn. O. (44r. 1746 Sphex Long-Pied, noir, pointes de l"ecuffon blanches, les pieus ou pattes de derrieres très longues, les ailes bandées. Linn.o. ou peutétre las Mouchè dcie d Grolles Cuiffes? de Linn. 920. 1.? ( 039.

1747 Sphex Jacne, à tête noire, les points des ailes noirs, Linn. o. (24.

1748 Spliex Noir, a ailes jaines, au point blanches, Linn. 0 . (522.

1749 Sphex Noir, les points des antennes. EO la baje des ailes jaunes, Li:n. C. ( 32 .

750 spinex Noir, à ailes brünes, les côtés du corcelet, fo les pattes jounes, Linn. o. ( 33 .

175I Sphex.... detete jaune, le corps noir, le ventre ban. dé d un cercle, Linn. o. ( 797.

1752 Sphex Pectinepe, Linn. 944. 17. (31. bis.

[753 Sphex Noir, à collier velu blanc, Linn. O. ( 888.

1754 Sphex .... à ventre gris', recourbé ailes jânes, Einn.

0. $(28$.

755 Sphex Noir, a ciles jaunes, le point, \& ventre dort, les pattes teftacées, Linn, o. ( 884 .

756 Spnex Noir, â ailes jaunes, le ventre d point d'orainge. Linn. O. $(834$.

757 Sphex Violet, à ailes violettes, le point du ventre orange, I.inn. O. (887.

758 Spbex Noir, $\vec{a}$ ailes hyalines, le corps veiu, Linn. 0. (300.

759 Sphex Noir à ailes violeties. le point du ventre orange, les pattes noires, Linn. 0. (857.

\section{G.U E $\quad$ E $\quad$ E S.}

760 Guépe Noire, à bande farve aw ventre, Linn. O. ( 799. kis.

76r Guépe Rouge, brune, la bafe des ailes brune, le yentre jaune, Linn. o. (9)3.

-52 Guépe Rouse, mas plas petite, Linn. O. (994.

703 Guepe Rouge - Nolre, lés I 2 bandes james laige, ba 3me plus etroite linn. O. (798.

70.4 Guepe . . a front rouge, le corcelet noir, les coies it 2 tackss jannatres, le ventre roir, à bande jaune, Linn. 0 (9g6. bis

765 Guépe. Tes artenneslongrues, en maffue, le corcelet, formet, ô ventre, jaunes, à aileschataigriees, Linn. o. (89r. 1765 cuepe 
I 760 Vespa .... fegenentis I 35 luteis, 3 fascia pedata, Linn. O. (...

\section{$\begin{array}{llllllllll}C & H & R & Y & S & I & D & E & S \text {. }\end{array}$}

1767 Chryfis ... viridi aurata, Linn. O. (30 bis.

1708 Chrylis Ignita? f. vurietas, Indica, pulcra, viridi aurea, abdomine apice violaceo, facies ignita, Linn. 947. I? ( 997 .

\section{A P E S.}

1769 Apis ..... alis violaceis, abdomine glabro, lateribus villofis, Linn. o. (841. bis.

$1770 \mathrm{~A}$ is .... r varietas præcedentis? Linn. o. (I00I.

1771 A pis.... alis bafi fuscis, abdomine fasciis 3 flavis, Linn. o. ( $44^{\prime}$ bis.

1772 Apis .... margine thoracis flavo Linn. o. ( 639 .

$I 773$ A pis . . . thorace, \& pedibus fulvis, Linm. o. ( 892 .

1774 Apis Atra, pedibus pofticis villolis, flavis, Linn. 0. (448, bis.

I 775 A pis Fulva, abdomine fulvo, fascia atra, Linn。 o (40.

1776 Apis Atra, abdomine atro, fascia fulva, Lirn. o (39.

1777 Apis Giabra, thorace fusco, abdomine glabro, pedibus villofis fulvis, Linn. C. (450.

I 278 Apis . ... thorace fulvo, valde hirfuto, abdominis feg. mentis fulvis, Linn o. $\operatorname{cros}_{3}$.

I779 Apis A:ra, parva, Linn. o. (...

I 80 Apis Atra, abdomine magno, glabro, fasciculato, Linn. ○. $(74.2$.

178 I Anis sitra, fo varietas, Linn o. (445. bis.

1782 Apis .... thoracis unargine ferrugineo, oculis albis, Linn. O, $\quad(519$.

I 783 Apis .... 1. varitias, Linn. o ( $1 \mathrm{I}$.

$17^{8}+$ Apis Fusca, alis viulaces, Linn. 0. ( 37 .

1785 A i, Nigra, alis byalinis, Linn. O ( 38 .

I $780 \Lambda_{i}$ is $N i g r a$, alis fasciis 2. \& abdomine fiavis, Linn, o ( $4 \mathrm{I}$.

1787 aps Atra, fegmentis 4. fuivo albidis, Lin?, O. ( 523 1788 A vis Fiava, thorace 3 cingulis 4 flavis, Lirm, o (622. 


\section{Injeites.}

I766 Cuépe .... d' I 35 cercles jaunes, \& 3 bandes echaladjées, Linia O. (...

\section{H R Y I D E S.}

1767 Chryfide Vert d'Oré, Linn. o. (30. bis.

I 708 Chryfade Ardence? ou yarieté des Indes, belle, verd doré. Se ventre EN joint violet, le front ardent, Liun. 947. 1? (997.

\section{A $\quad$ B $\quad$ E I L I E S.}

I769 Abeille .... d ventre pelé, les cotes velues, les ailes violettes, Linn. O. (841. bis.

I770 Abeille.... ou varisté de la precedente? Linn. O. (rosr.

1771 Aboille.... bes ailes de la bafe brunes, ie ventre at 3 bandes jaunes, Linr. 0 . (446. bis.

1772 Abeille .... le corcelet d hord jaune, I.inn. 0. (639.

I773 Abeille .... le corcelet, \& pattes fauves, Linn. O. (892.

1774 Abeille Noire, les pattes pofterieures velues, $j$ ciunes, Linn. O. $(448$

1775 Abeille Fauve, de ventre fauve, bande noire Linn. o. (40.

1776 Abeille Noire a ventre noir bande fruve Linn. 0. (39.

I777 Akeille Pelé, d corcelet brun, le verutre pelé, à pattes yelues fauves, Linn. O. (450).

1778 Abeille.. Le corcelet fauve. fort yelu, le ventre do anneaux fauves, Linn. o. (1 83 .

I779 A eille Noire petite l.inn. o.

I 750 Abeilie Noire, au grand ventre pelé, छे bande, Linn. O. $(742$

1781 Abeille Noire? ou varieté, Linn. o. (445. his.

1782 Abeille... le hord au corcelet coubleir de rouille, aux yeux blanches, Linn 0 . ( $5: 9$.

1783 Abeille ... ou varieté, Linn o. (II6.

I 784 Aheills Brune, a ailes violettes, Linn. 0 . (37.

1785 Abeille Noire, d ailes couleur de verre hyalines, Linn. C. (33.

1786 Abeille Noire, ailes d deux bandes, EN ventre jaunes, Linn. O. ( 4 I

[78? Aleille Noire, d quatre anneaux fauve binches, Linn. 0. ( 523 .

788 Abrille Jaune, à corcelet, $\mathbb{E}$ quatre cercles jaunes, Lirn. 0. (602. 
1789 Apis Fulys, alis bafi nigris, Lim. o. (j42.

I 790 Apis Fulve, abdomine fulvo, pedibus porticis atris, Linn. त. (452.

179: A is Fulva? \& yarietas, Jinn. O. ( 508.

I792 $\mathrm{A}_{\mathrm{p}}$ is Hirfuta, abdomine cingulis viridi gureis, Linn. 0. (566. bis.

I793 Apis Viridi-Aure , thorace nigro, fubtus violacea, Linn. O. (1.0co.

I794 Apis Gigas, atra, abdomine fasciculato, oculis albis, Linn. O ( 743 .

I795 Apis Gigas? א. varietas præcedentis? alis viridibus, Linn. o. ( . .

I796 Apis Meralocephahus, alis violaceis, Linn. o. (445. his

I797 Apis Megalocephalus ? I varietas, alis viridi nitidis, Linn. O. ( 944.

1798 Avis . . abdomine fasciculato, alis chalybeis, Linn. o. $(\ldots$

i 799 Apis Olivacea. Linn O. C $\mathrm{C}_{38}$.

i800 Apis Flava thorace flayo, pedibus villofis, antice flaviş, pofticis nigris, Linn. O. (74\%. bis.

180 I A pis Atra, abdomine glabro, thorace rubro, Linn. o. 7744

I802 Apis Atra, thorace flavo, Linn o. (64) ter.

1803 Ap's Cyanea, thorace cyaneo, Limn. O. (308.

I804 Apis Ocracea, thorace ocraceo, Linn, O. ( 802.

1805 Apis Alticans, tibiis delatatis albis, Linn. O. (804. 1806 A is Villofạ, tibiis a ticis, villofis albis, purticis nigris, Linn. O ( 893.

1807 A is Flava, th race flavo, Linn o. (... 3808 Apis .... tibiis delatatis albis, Linn. 0. ( 804.

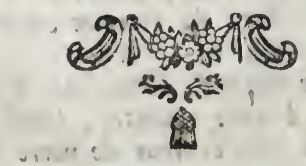


89 Abeille Fautue, d ailes de la bafe noires; Linn o ( 42 . 9o Abeille Fauve d ventre fauve, les pattes pofterieures noires, Linn. O. ( 452

-1 Abeille Fanve? ou varieté, Linn o. ( 598.

92 Abeille Velue, le ventre da cercles vertes d'orées, Linn. $\odot$. (566. bis.

'93 Abeille Vert-d'or, à corcelet noir, defJous violette, Linr. o. (iooo.

94 Abeille Geante, noire, le ventre bande, aux yeux blanches, Linn. o. ( 743 .

95 Abeille Geante? ou variete de la precedente? à ailes yertes, Linn. o. (...

96 Abeille Megalocephale, do ailes violettes, Linn. O. (445. bis.

197 Abeille Megalocephale? ou yarieté, da ailes vertes, Linn. o. (944.

98 Abeille... d ventre bandé, diles couleur d'acier, Linn. O. (...

og abeille Olivatre; Linn o. C.638.

ao Abeille Jaune, corcelet jaune, les pattes velnies, devant jaunes, les fofterieures noires, Linn: $\alpha$, (745, bis.

or Abeille Noire, le ventre jete, a corcelet rourge, Linn. O. ( 744 .

22 Abeille Noile, dy corcelat fauve, Linn. _. (640. ter.

ग3 Abeille Bleue, à corcelet bleu. Linn. O. ( 308.

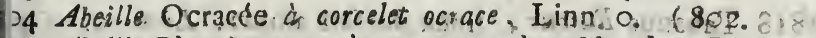

's Abeille Blancheatre, d̀ pattes etendues blunches Linn. a.

( 804.

o6 Abeille Velue à pattes anterieures velues blanches, bes poftorieures noives, Linn. o $\quad(893.2$. in

7 Abeille Jaune, it corcelet jaune, Lnn, o (

18 Abeille ..... à pattes etendues blanches, Linn o. ( 804 .

o.tini cive

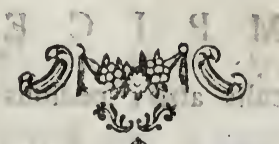

$\mathrm{O} 2$ 


\section{$\begin{array}{llllllll}D & I & P & T & E & R & A\end{array}$ \\ M V S C R:}

1809 Musca Carinata; thorace viridi, alarum nervis flavis Linn. 0. 1605 .

I810 Musca Caftaniea, capite globo'o, thorace nigro, corpn re caftaneo, alarum maculis quadratis impreffis, Lint o. (III2.

1811 Musca Capenfis, an Bombylio? Linn. roog. 3? (334 1812 Musca Helvola, capite \& corpore helvolis, Linn. (27.

18r3 Musca Atra, alis nigris, fascia alba, Linn. O. ( . . . 1814 Musca Cafar, Linn 989. 64. (503.

1815 Musca Putrida, Linn. O. Fabric. 757. 1c. (... 1816 Musca Grofla? r. varietas, Linn. 991. 75 ? (990. 1817 Musca Syrphus, niger, capite carinato, alis pullis, nigr nervolis, Linn. O. (22.

\section{T A B A N I.}

1818 Tabanus Fuscus, linea dorfali albida, Linn. o. (79: 1819 Tabanus Fuirus., fascia dorfo rubra flavefcente, Linn. i (434.

1820 Tabanus .... fasciis dorfalibus 3.. Linn. 0. (435.

I82I Tabanus... alis flavefcentibus, Linn. O. ( 604 .

1822 Conops Fasciatus, incifuris abdominis flavis, roftro Ait vo, Linn. O. ( 792.

\section{E $\quad M \quad P, I \quad C \quad E$ S.}

1823 Empis... oculis atris, pedibus teftaceis, Limm. c ( 793

1824 Empis Culici-formis, Linn. n. ( 794.

1825 Empis Signata, '. Musca Vespi-formis, villofa fusca farciis abdominalibus, geniburque flavis, Lirn. $98 t$, 44. ( $44 \mathrm{I}$.

1826 Empis Signata? vel Musca Vespi-formis, f. varietas pret. cedentis? Linn. 1. c. (449. 
18.27. Empis .... alis ferrugineis, Linn. 0. (9.92. bis.

1828 Hippobofca Media, Linn. o. (23.

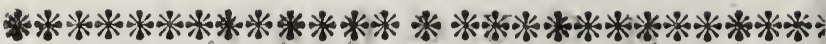

\section{$A-i \quad T \quad E \quad R \quad A$.}

\section{$A R A$ N E $\mathbb{E}$.}

1829 Aranea Avicularis, Linn. I034. 31. (385. bis.

1830 Aranea Avicularia, fimilis precedenti, Linn.1. c.-( so; bis.

I83I Aranea Aviculdria? f. varietas, Linn. 1. c. (384. 38 3.88, quater.

1832. Aranea Longifies, proxima ad luscam, oculis 6. Lif o. $\ 375$

1833 Aranea Longipes? r. varietas, Linn. o. ( 635.

1834 Aranea Rubra, an Acarus? Linn. o. Slabber, tab. (357. bis:?

I835 Arsnea Fusca, ocracea, oculis 4 \& 4. abdomine ovat peaibus fusc. fillofis, Linn o. (786. 875. bis.

1836 phrànea Fusca, epræcedenti atris is, cum ovario, Liq 0. $1(497$

I837 Aranea Afgentea, abdomine incifo basbato, Linn. (3? 3 :

I838 Aronta Argentea, fimilis prxcedenti, Linn. O. ( 378

1839 Aranea Longipes, red abdomne orba, Linn. O.- (3: 376. bis.

I840 Arano: Cancri-formis, Linn.ro37: 45. Slobier. tab. ( 538.

184I Arauca Bifasciate, Limn. O. (107I.

1842 Arane? Bifasciata? f. varietas Longipedis? thorace fu' marginatn. Linn. o. (379. 786 . bis.

I843 Aranea Bifoscians, fed minor, Linm. O. (4)6.

I844 Aranea... pedibus luteis, articulis lineis nigr: Linn. O. ( IOJ2.

7845 Arsnca Cormuta, thoracis corrua 4. abdominis Livn. o. ( 383.

1846. Aranea Globularis? abdomine globofo, thorace argente cum Nido, Linn. O. (874. ter. 
18,7 Aranea Globularis? r. varietas, Linn. O. (837. I848 Aranea urantia, aurantio fusca, Linn. o $(636$. I849 Aranea Nigra, Linn. O. ( 873 . I850 Aranea Aivicularia, Linn. 1034. 31. (... bis.

\section{A N C E R E}

185 Cancer Diogenes Linn. ro49. 58. (1079. rog6. bis. 1852 Cancer Emeritus, Linn. 1055. 79. Gronov. Zooph. II. tab. 17.f. 8. 9 (1076.

I853 Cance Emeritus, Linn. I. c Gronov.Zooph.II. n ${ }^{\circ}$ IOcI. ( $107 \%$.

18:4 Cancer Emeritus, fed parvus . Linn. \& Gronov.1 c. (1078. 185 Cancer Eremita, Linn 1049. 59. (1079.

1856 Cancer Curculioides, Linn. O. Slabber tab ultim. ( 2.

IE57 Carcer Craniolaris, Limib. I*41. 15. Fabric. 496. 7. (13

I258 Carcer Vorans, Linn. r04I. I4. Fabric. 409. I-. (29. 1859 Cancer Vocan', Linn. 1. c. Rumph. Muf. tab. 10. E. 29. (39. bis

I8ro Cancer Glnbus, Linn. O. Fabric. 7. (ro.

Ix6: Ca cet Ruher, maculis rotundis, chelis lat'fimis, Linn. - 1 is.

I862 Ca:cer . . thorace lævi, fronte quadridentato, chelis 1.t's barbatis. Linn o. ( 16 .

I863 Cancer .... thorace orbiculato quinque fpinofo, fuecn, frone tridentsto, manubus crafis, digitis parvis, pedibus mucronatis, Linn. O. ( 5 .

$186+$ Cancer .... thorace quadrato, chela altera majore compretfa, carisa crenata. Limn. o. (28.

1805 Cancer.... thorace fubtrigono, lateribus trifninofo, ancice lunato, fronte excuberante, Linn. o. Petiv. Muf. 60. 6. (22. .

1866 Cancer.... thorace quadrato, antice ircifo, chelis dentatis, Linn o. (27

186? Cancer Granulatus, Linn. o. Fabric. 501. 30. (35. ter.

186.8 Cancer Granulatus, Linn. o. Petiv Muf. 155. 2? (6. 1809 Cancer Gramulatus. Linn. o Petiv Muf. 155. 5 (14. 18 io Cancer Latro? to varietas, Linn. 1049. 56? Seb. III. tab. 22. f. 8. ( 38 .

1871 Cancer Maculatus, Linn. o. Petiv. Nuf. 1. 7. (37. 
847 Araignée G'obulaire? ou varieté, Lirn. 0. $84^{8}$ Araignee Orange-brune, Linn. $0 . \quad$ ( 036. 1849 Araignée Noire, Linn. O. ( 873. (850 Araignée Aviculaire, Linn. 1034: 31. '(... bis.

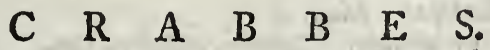

1851 Crabbe Diogene, Linn. 1049. 58. (1079. 10)6. bis. 1852 Crabbe Emerite, Linn. 1055. 79. Gronov. Zooph. II. tab 17. f. 8 9. ( 1076.

1853 Crabbe Emerite, Linn. l.c. Gronov. Zoph. II n०. $100 \mathrm{I}^{\circ}$ ( 1077.

1854 Crabbe Emerite, mais petit. Linn. E Gronov. i. c. ( 1078.

1855 Crabbe Eremite, Linn. 1049. 59. (1079.

I856 Crabbe Curculiode, Linn: o. Slabber tab. ultim. (2. I

1857 Crabbe Craniolaire, Linn. 104I. 15. Fabric. 426.7. ( 13.

1858 Crabbe Vocante, Linn. 104r. I4. Fabric. 497. 17. (29.

18 s9 Crable Vocante, ou Criante, Linn- l.c. Rumpb. Muf. $t a b$. 10. E. 29. (39. bis.

I860 Crabbe Globe, Linn, c. Fabric. 7. (ro.

I801 Crabbe Rouge, d taches rondes, à pinces tres larges, Linn. o. ( 15.

1862 Crabbe .... à corcelet poli, le front à quatre dents, $\dot{b}$ pinces larges barbues. Linn, o. ( 16.

1863 Crabbe .... à corcelet orbiculaire, à epine brumes, à front tridente, mains epais, petits doigts, pattes mucronees, Linn. O. 15.

1864 Crabbe ..... à corcelet quarré, $\dot{a}$ grande pince comprimée, Linn. o. ( 28.

1865 Crabbe .... à corcelet triangulaire, les cätes à trois epines, par devant luné. le front à tubercules, Linn. O. Petiv. Muf. 60. 6. (22.

1866 Crabbe .... à corcelet quarré, incifé far devarat, pirices dentelées. Linn. 0. (27.

1867 Crabbe Granulé, Linn. o. Fabric. 50r. 30. (35. ter.

1868 Crabbe Granulé, Linn. o. Petiv. Mur. 155.2? (6. 869 Crabbe Granulé, Linn. o Petiv. Mur. 155.5. (I4.

1870 Crabbe Latro? ou varisté, Linn. 1049. 56? Seb. III. tab. $22 . f .8$. ( 38 .

187 I Crabbe Tachete, Linn. o. Petiv. Mur. r. 7. ( 37.

O $5 \quad 1872$ Crabse 
1872 Cancer Maculatus, Linii. O. Petiv. Muf. r. 5. (3r. 1873 Canc cr Macuarus, Linm. o. Petiv. Mis ri - 3. (18.

1874 Car cer Moenas, Lina 1043. 22. Herbf Caner. KaI. 46. ( . . .

1875 Cancer Depurator, Lirn. 1043. 23. Herbf. Cancr. VII. $48 \quad(30$

1876 Carcer Latipes, Linn. o (...

I8:7 Cancer Curculioides. Linn o. Stabrer tabul. ultim.

1878 Cancer.... Linn o. Ketiv. Muf .. r. I'. ( 3.

1879. Cancer Latipes Linn. 0 an $r^{4} .875$ ? ( $\because$

1880 Cancer Feriatus, fed parvus, an no. 1.872? Linnt. k. G. (10i6

188, Cancer Grifeus, thorace conico, fustubercalato, chelis parvis cylindricis Linn. 0 ( 7 .

1882 Cancer .... thorace lato fubaculeato, roftro conico, pedibis l ngiffris : Litn. o. ( I o. bis.

I883 Cancer... thorace plan', lateribis aculeato, fronte 4 dentate Linn O. ( 2 I

1884 Cancer Feriarus Linn. I043. 25. Petiv. Muf. r. 6. (20) bis.

1885 Cancer.... . thorace conico, roftro in apicein trifidúm exeunte, oculis petiolatis, pedibus villonis, curpare triplo lorgioribus, ni tacarpis aculeatis, Linn. o. ( 39 .

I886 Cancer Moenas; fed parvus, Linn. 1043. 22 . (...

I887 Cancer Ovilis, thorace triaculeato, fronte bifido, antennis villofis, Linn. o. '( 26. bis.

1888 Carcer Quadrifpinofius, frume 4 dentato, femorious I fpinofis, Linn. O. ( 32 .

1889 Cancer..... horace mutico, fronte fpinofo, pedibus comprefis, corpare luteo rubroglte, Linns. o. ( $3^{6 .}$ Isço Cancer.... r. varieias, Linn. O. (... 1891. Cancer Eremita, Linn. 10 9 59. ( 17

1892 Cancer Macitialas; Linm. 1042. I8. Seb. III. tab. 19. f. 12. ( 41 . bis

1893 Cancer Pelagicus, Lim. Ic42. Ig. Seb. III. tab. 2 C. f. 7. 8. (4) teitx.

1894 Cincer Hajtatus, Linn. O. Fabric.... (54. 57.tefta, bis.

1895 Carcer Haftatus? ? varietas, Linn. O. (54- tefta, bis. $1896^{\circ}$ Cancer Fersatus, Línn. 1045.25. ( 55 .

1897 Carcer-Raninus? Linn 10j9. 2 ? (43. cefta. 1898 Carcer Granulatus, Linn. 1043. 26? Fabric. . . . C... tefía nivea.

1899 Cancer .... thorace quạdrifpinofo, fronte 4 dertato, Liino. c. (... iefta.

Ig0o Cancer 
1872 Crabbe Taçheté, Linn. O. Petiv. Mur. I. 5. (37. 1873 Crabbe Tacheté, Linn. o. Petiv. Muf. r. 3. ( 18. 1874 Crabbe Moenas, Linn. 1043. 22. Herbft. Cancr. VII. 46. (...

1875 Crabbe Depurateur, Linn, 1043. 23. Herbn, Cancr, VIr. 48. ( зо. ....

$1876^{\circ}$ Crabbe Large-Pied, Linn. O. (...

I877 Crabbe Curculiode, Linn. o. Slabber tab. ultinn. (3).

1878 Crabbe .... Linn. O. Petiv. Mur. 111. 1I. (3.

1879 Crabbe , ... Large pied, Linn. o aun $n$. $1875^{\text {? }}$ (... I880 Crabbe Feriate, mais petit, an nv. 1872 ? Linn. l.c. (roso.

188I Crabbe Grise, â corcelet conique, à tubercules, potits pinces cylindriques, Linn. o ( 7.

1892 Crabbe . ... à corcelet iarge pointu, à trompe contigue, E tres longues pattes, Linn. o. (r. 9. bis. $\mathrm{T}$. Is

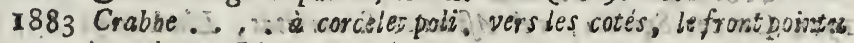
ì 4 dents, Linn. 0 . (2s.

1884 Crabbe Feriatus, Linn. 1043. 25. Petiv. MAuf. 1. 6 . ( 20 bis.

1885 Crabbe ... à carcelet coniąue, te trompe à trois pojz.tes fendu, a yeux pediculees, pattes, velsues, trois yos plus longues que le corps, Linn. O. ( 32.

1886 Crabbe Moenas, mais petit, Linn. 1043. 22. (...

1887 Crabbe Ovale, à corceled à trois pointes, le front descx. fois fendu, les antennes veizues, Lina o. ( 20 bis,

I888 Crabire à Quatre epines, front 4 fois dentete, les wuifjes epineufes, Linn. 0. (3:.

1889 Crabbe . . . à corselet Jesis barbe, front epine:ux : \& pattes comprimées, be corps d’un jaun rowge, Lim: 0 . (36.

1800 Crabbe..... qu varieté, linn. o. (...

1891 Crabbe Eremite, Linn, 1049.59. (17.

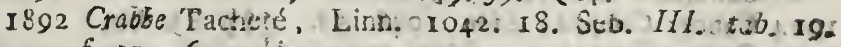
f. 12. (4I. bis.

1893 Crabbe Pclaginue, (ou de Mer) Linn. 1042 19. Sib. III. tab, 20. f. 7. 8. (4). tefte.

18.94 Crabbe Arne Linn. O. Fabric... (54.57. tejte, 3is.

1895 Crabe Arme ? ou varieté, Linn. O ( 54 tefti, bij.

1895 Crabbe Feriate, Linn. 1043. 25. (55.

1897 Crabbe Ranine? Linn. 2039. 2? (43 tefte. 1898 Crabbe Granulée, Linn. 1043.26 Fabr........ tefte
blanche.

1890 Crabbe .... de corcelet à 4 épines, le front à 4 dents, Linn. o. (... tefle. 
I900 Cancer Lanatus, Linn. I044 29. (43, tefta.

Igor Cancer Tribulus Limn. 1045. 35. (41. tefta, bis.

x902 Cancer Cornitus, Limn. 1047. 46. (56.

1903 Cancer Cornutus? f. varietas, Linn. l. c. (42.

1904 Cancer Cornusus, fed parvus, nondum pilous, Linn !a c. (48. telta.

1905 Cancer Horridus, an adultus? Linn. 1047.43. (58. tefta. 1906 Cancer Argus, maculis aurantis, albus, Linn. 8 . ( 50.

1907 Cancer Artus, Linn. rn53 75. (65.

1908 Cancer Arllus? R. varietas, Limn 1. c. (66.

x909 Cancer Homarus, Linn. 1053. 74. (69.

Igro Cancer Mantis, Linn. 1054. 76. (60.

Igl I Cancer Mantis, fed minor, Linn. J. .c. (67.

1912 Cancer Mantis? f. varietas Linn. 1. c. ( $73-75$. ter.

1913 Cancer Aftacus ; f. Squilla? Linn. 1051. 60? Fabric, 4I6. I3. ( 61 .

19:4 Carcer Squilla, vel Aftacus? א. varietas, Linn. 1051.63? (62. 08 bis.

* I915 Cancer Aflacus? Americanus, Linn. 105r. 63? (71.

IgI6 Cancer Aftacus? [ varietas, Linn. $0 \quad(72$.

I91 7 Cancer Norvegicus, maximus, Linn.ICs.3. 73. latit. tho. racis 9. chel. 9. poll. ( 75.

IgI 8 Cancer Haftatus, Linn. O ( 77 .

1919 . Cancer Raninus, Linn. 1039. 2. ( 76.

Ig:0 Cancer....thorace 5 dentato, pedibus pofticis oratis villofis, chelis prismaticis dentatis \& carinatis, Linn. o... (18I.

1921 Cancer.... f varietas pracedentic? Linn. o. ( 80.

I922 Cancer .... thorace quadrifpinofo, fronte 4 dentato femoribus I $f_{j}$ inofis, $\mathrm{Li}$ in. o. $(78$.

1923 Cancer.... fuperne fporgiofus, manubus cochliato dentatis . Linr. o (79:

1924 Cancer Calappa, Linn 1048 52. (83. 85. bis.

1925 Cancer Granulatus Linn. 1043. $26 . \quad$ (86.

$x 926$ Cancer Suberofus, as n. 1924? Linn. O. ( ...

1927 Cancer ....thorace integerrimo, lavi, pedibus aculeatis, Limn O. . ( 84.

1928 Cancer Spinofus, pedibus \& thorace fpinofis, Linn. 0.

I 29 Cancer Spinofus ? r. varietas, thorace pedibusque fpinofis, manubus cochleato dentatis, Linn o. (...

I929 Cancer Roftratus, parvus, Linn. O. ( 936. 
I900 Crabbe de Laine, ou comme couvert de laine, Linn. 1044. 29 (45. $t e f t a$

Igor Crabbe Tribule, Linn. 1045.35. (44. tefte, bis.

I 902 Cratbe Corrue, Linn. 1047 46. (56.

I 903 Crabbe Cor ue? ou varieté, Linn. l. c. (42.

1904 Crabbe C rnue, mais petit, pas encore velu, Linn. l. 6 . ( 48 tefte.

I 905 Crabbe Apre, rude, pas parfait? Linn. 1047. 43. (58. tefte.

I 906 Crabbe Argus, taches orangées, blanche, Linn. 0. (5,0. 1907 Crabbe Aretus, arcé, Linn 1053. 75. (65.

1908 Crobbe Aretus? ou varieté, Linn. b. c. ( 66.

1909 Crabbe Homarus, Linn. 1053. 74. ( 69.

rgro Crabbe Mantis, Linn. 1054 76. (co.)

IoI Crabbe Mantis, mais plus petit, Linn. l. c. ( 67.

191.2 Crabbe Mantis? ou varieté l.inn. l. c. (73-75. ter.

I9I 3 Crabbe Aftace, ou Squille? Linn. I051. 66 ? Fabric. 416. 13. (6i.

1914 Crabbe Squille, ou Aftace ? ou varieté, Linn. Io5 I. 63? (62: 68. bis.

1915 Crabbe Aftace? d'Amerique, Linn. 105 I. 63? (7I.

19:6 Crabbe Aftace? ou varieté, Linn o. ( 72 .

1917 Crabbe de Norvese, très grand, Linn. I053. 73. 20 lotit. du corcelet 9. pinc. 9 pouc. ( 75 .

1918 Crabbe Armée, Linn. o. ( 77 .

rors Crabbe Ranine, Linn. 1030.2. ( 76.

1920 Crabbe .... corcelet d 5 dents, les pattes pofferieures - vales veiues, à pinces prismatiques dentelées, Linn. 0. ( 181.

192 I Crabbe.... ou yarieté de la precedente? Linn.o. (80.

r922 Crabbe ......corcelet d 4 epines, front d 4 dent, les cuiffes di r epine, Linn. o, ( 78.

I923 Crabbe.... par en haut Spongieux, les mains dentelés, Linn. o (79.

I 924 Crabbe Chlappa, Linn. 1048. 52. (83. 85. bis.

1325 Crabbe Granulé. Linn. 1043. 26. (86.

1926 Crabbe Sub rofe, 0ut ne. 1924 ? Linn. o. (...

1927 Crabbe... d corcelet entier, poli, à pattes pointées, Linn. 0. 18 .

19:8 Crabbe Epineufe; pattes E corcelet épineufes, Linn. o. (...

1929 Crabbe Ei jneufes ou v́arieté. le corcelet. \& pattes epineufes. Les mains dentelés, en forme de cuillier, Linn. O. (... 1929 Crabbe $\frac{1}{2}$ Trompe, petit, Linn. o. (9.35. 


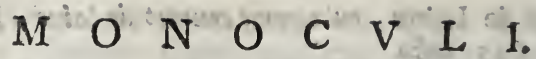

I930 Monoculus Polyphemite, maximus, Linn 1057 I. $\mathrm{C}_{3}$. 193' Monoculus Polyphemus, præcedenti affrnis, Linn. 1. c. 604

\section{$\mathrm{O} \mathrm{N}$ I S C I,}

393т Oniscus Flavus, fegmentis 12. Linn. O. (IIIG.

1932 Oniscus Viridis, qquarnis Io. Linı, o. ( 738 .

1933 Oriscus Fuscus, fegmentis 2. ftria flava, Linn. O. (871. 1934 Oviscus Pedatus, pedibus pofticis longioribus, Linm. ○. ( 1068.

1935 Oniscus Entomon? Linn. 1060. 5? Pall. Specil. VIII. ... ( 1075

$19 \div 6$ Oniscus Flavus, fcutis 14., Linn. o. (910. 920, bis. r.936 Oniscus Gigas, aquaticus, Linn. O, (717.

\section{F O R M I C E}

$\$ 937$ Formica Cephalotes. Linn. 964. I5. ( 363 .

1938 Formica Cordata, capite cordato, maxillis magnis, Limmo o. $(362$.

1939 Eormica Globularis, abdomine globofo, capite luteo, maxililis magnis, Linn $\circ . \quad(364 \ldots$ bis.

940 Formica .... capite magno, petiolo biarticulato, Linn. O. 1355.

\$941 Formica Quadrifpinasa, abdomine globolo, Limn. 0. (63r. bis.

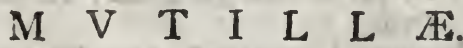

1942 Mutilla Capenfis thorace flavo, ani maculis 2 rubris, Linn C. (300. bis.

X943 Mutilla Afiatica, fascia thoracis dupla, abdominis fascia transverfa, Linn o. (198.36r.ter.

\section{S C OR P I O N E.}

I 944 Scorpio Fuscus , chelis non dentatis longis, Linn. o. (240. 


\section{O NO C L E S.}

930 Monocle Priypheme, très grand, Linn. I057. I. (6.3.

193 Monocle Polypheme, approchant au precedent, Linn. b.s. $\left(\sigma_{4}\right.$.

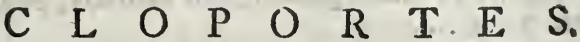

192 Cloporte Taune, 6:12 anneaux, Linn. o. (III6.

1932 Cloporte Verte à ro ecailles, Linn. o. ( 738 .

1933 Cloporte Brun, d 9 anneaux, àftrie jaune, Linn. o. (87r. 1934 Cloporte .... les pattes pofterieures longues, Linn. 0. ( 1068.

I935 Cloporte Entomon? Linn. I 060. 5? Pall. Specil.VIII.... ( 1075 .

I 236 Cloporte Jaune, dे 4 boucliers, Linn.o. (9r9. 220 . bis, 1936 Cloporte Geant, aquatique, Linn. o. (787.

\section{F. O U R M I S.}

1937 Fourmi Céphaiotes. Linn. çG. I5. (363.'

1938 Fourmi en Coeur, la tete en coeur, \& grande machoires; Linn. o ( 362.

1939 Fourmi Globulaire, à ventre rond, têtte jaune, d̀ grands machoires, Linn. o. (364 bis.

1940 Fourmi . . . c̀ grande tête, pediculée dे deux articles, o. $(355$.

194t Fourni à Quatre-epines, ventre rons, Linn. O. (631.bis,

\section{U T I L L E S.}

1942 Mutille du Cap, d corcelet jaune, d̀ deux taches rowges de l'anus, Linn. o. (360. bis.

943 Mutille Afiatique, d bande du corcelet double, le ventre à bande jaune transverfe, Lini. O. (198. 361. ter.

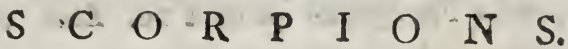

944 Scorpion Brun, pinces pas dentées longues, Linn. C. (24). 
1945 Scorpio, Cinereus, chelis compredis pilofis, Linn. ( 719.

I946 Scorpio Subluteus, thorace fusco marginato, Linn. o (178. $98:$ ter.

ig47 Scorpio Fusco-Luteus, chelis pilofis fubdentatis, Linn o. $(350$.

1948 Scorpio .... chelis glabris, non dentatis, pedibus luteis Linn. o. (...

1949 Scospio Ater, maxillis magnis iuteo fuscis, chelis pilo. fis variolofis, Linn. O. (347. 357 bis.

I950 Scorpio Piceus, digitis chela um longioribus, dentatis pilofis. masubus vario ofis, cauda quadranguiata, pulius, Linn. o. ( 348 .... bis.

ro5x Scorpio Glaber, ater, maxillis nigris, Linn. O. (1065. 1066. bis.

3952 Scorpio .... chelis teretibus, digitis incurvis, cauda hexagona, Linn. o. (353. bis.

Iو53 Scorpio..... chelis linearibus, digitis chela fesquilongionibus, pedibus nigro punClatis, Linn. o. (354. bis.

1954 Scorpio Lut:us, chelis intus gibboris, catda fubrotunda carinata, Linn. o. (351. 100. bis.

1955 Scorpio . . . chelis pyri-formibus, casda tricarinata, Linn. o. ( 351 .

2956 Scorpio Pulius, Linn. O. (352.952. bis.

1957 Scorpio Pullus? fo varietas, Linn. O. (...

I958 Scorpio Ater, maxillis magnis luteo tuscis, Linn. o:

1959 Scorpio f. cujusdan Pullus, Linn. O. (358. 359.

\section{S C O L P E N D R EE.}

I 960 Scolopendra Gigantea, Linn. 1063. 4. (633.

I961 Scclnpendra Morfitans, Linn. 1063. 5. ( 367.

×962 Scolopendra Ferruginea Linn. ion3. 6. (365.

1963 Scolopendra Ferruginea" pedihus 19. Linn. I. c. ( 368.

1964 Scolope:ndra Ferruginea? pedibus 18. Linn. 1. c. (366.

I965 Scolopendra Ferruginen? pedibu: 21. Linn 1 c (505.

I966 Scolopendra Ferrugines? pedibus 22. Linn. 1. c. (:067.

1967 Scolopendra Ferruginea? pedibus 20. Linn. 0. (306. 
1945 Scorpio Cendré, ¿ pinces comprimées velues, Linn. ०. ( 719. 1946 Scorpion Jaunatre, do corcelet brun borde, Linn. 0 ( 778. 982 ter.

947 Scorpion Brun jaunatre, d pinces yelues, dentelés, Linn. o. (350.

$19+8$ Scorpion .... d pinces glabreux, Jans dents, pattes juunes, Li n o. (...

1949 Scorpion Noir, a' grande machoires jaunes, brunes pinces velues, Linn. o. (347.357. bis

1950 Scorpion Couleur de poix, les doigts des pinces longues denteles, velues, de queue quadrangulaire, Linn. o. (343. .... bis.

95 I Scorpion Pelé, noir, à machoires noires, Lirn. o. (1005. I066 bis.

1952 Scorpion .... do pinces longues, E' rondes, doigts courbées, \& queue fexagone, Linn o. (353. bis

1953 Scorpion. . a pinces lignees, les duigts fix fois plus longues que la pince, les pattes pointees de noir, Linn. 0 . (3i4. bis.

1954 Scorpion Jaune, les pinces en dednns bof Jues, lo queus ronde cavee, Linn. 0 . (35:. W00. bis.

1955 Scorpion... a. pinces en forme de poire, la quere en trois cavées, Linn. O. (35I

1956 Scorpion Jeune, Linn. O. ( 352.952 his.

957 Scorpion Jeune? ou varietc, L.inn o. (...

1958 Scurpion Noire, d grandes maGboires dun brun joune, Linn. o. (...

1959 Scorpion . . . ou ou fon petit, Linn. o. (358. 359.

\section{S C O L O P E N D R E S.}

1960 Scolopendre Gigantesque, Linn, 1063. 4. ( 633 .

1961 Scolopendre Mordante, Linn. 1063. 5. ( 367.

1962 Scolopendre Couleur de rouille, Linn. 1063, 6. ( 365.

1963 Scoloperdre Couleur de rouille? a I 9 pattes, Linn. l. c. ( 368 .

1954 Scolopendre Couleur de rouille? $d$ i 8 pattes, Linn. l. $c$. ( 366

1905 scolopendre Couleur de roullle's à 21 pattes, Linn. $l_{0} c$. ( 505 .

1966 J'coloperdre Couleur de rouille? à 22 pattes, Linn. l. c, ( 1057.

1967 Scolopendre Couleur de rouille? a 20 pattes, Linn 0. 366. 


\section{J. V L I.}

1968 Julus Indicus? pedibus II6. Linn ${ }^{m} 1065.6$ ? ( 634.

1969 Julus Africanus, pedibus 1c0. Linn. 0. (1070.

I570 Julus sifricanus, pedibus 1wo. Linn. c. ( 369 .

1970 Notonecta Glasca, major, Linn 712. I. (167. bis. 197x Notoneda Clauca, fed minor, Linn. L. c. (843.

1972 Tenebrio Gigas, thorace oblongo ater, Linn. 674.9 I. $(287$.

I973 Ten-brio Indicus, delatato elytris ellipticis connatis, Linn. O. ( 566.

1974 Attelabus, f. Pinelia Striata, Linn. O. Fabric. Sp. I. (547. bis.

1975 Sitelabus, C. Pimelia Unicolor, Lim.n. O. Fabric. Sp. 2. ( 548 , bis.

1976 Attelabus, C. Pimelia Ciliata, Lirn. 0. Fabric. JP. 21. (070.

1977 Attelabus, f. Pimelia Triccrinata, elgtris ellipcicis cornatis tricarinatis, Linn. O. ( 565 .

1978 Attelabus, f. Pimelia Cephalotis, capite cculisgue mag. nis, ater, glaber, Linn O. (222.

1979 Artelabus, f. Pinelia Longipes, atra, ftasura dytisci, Linn. O. (221.

1980 Attelabus, f. Pimelia Miruricata, nigra, Limn. 0. 1093.

981 Attelabus, 1. Pimelia Grifea, carinis 4 muricatis, Linn. 0. (705.

1982 Attelabus, f. Pimelia Grijea? carinis 4 nigro pur:etatis, Limn. O. (223.

1983 Artelabus, f. Pimelia Cinerea, f. varietas, nigro punctam ta, Linn. O. (220.

1984 Mylabris Viridis, elytris viridi fericeis, Linn, O. ( 712. bis.

1085 Mylabris Viridis? f. varietos, Limn. O. ( 729.

1986 Mylabris Atra, humeris, anogue rubris, Linn. C. (290.

I987 Phalangium Caudatum, Linn. 1029. 8. (630. 1988 Phalangium Reniforme, Linn. 1029. 9. (029. bis. 
I989 Nidus Vesparum Indicus. ( $A$.

1990 Niảus Vesparum Americanus. ( $B$.

I991 Nidus Vesparum Americanus. ( $C$.

I992 Nidus Vesparum Arericanus ( $D$.

I993 Nidus Vesparum Americanus. ( $E$.

1994 Nidus Vesparun Europacus. (F.

1995 Nidus Vesparum Europaus. (G.

\section{A R M E.N T A R I V M}

Loculis XLII. Inftructum

in quo

\section{$I N S E C T A I N D I G E N A$.}

Lecults:

I. Muscæ, inter qua Chamelion Mori \&ic.

II. Enpis 13. Culices 4. Panornæ 1. Tipula IO. Tenebriones 37. apes 6. III. Ichneumones 59. Sphinges 2. . . IV. Ves $\approx 37$. Cmyfides 8

V. Apes.

VI Libellula.

VIr. ibellulæ (Virgines) II. Larvæ 4.

VIII. Perı 3. Ephemeræ 3. Myrmelınes 6. Panorira 3. Phryzaneæ 8.

Conopes 7.

IX. Manrides 4. Grylli 26.

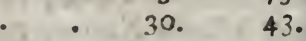

$X$. Grvili 6. Blauæ 3. Cimices 36. $245 . \quad 70$.

$X 1$. Cimices 8. Meloe 12. Siaphylin:

7. Duticl 17. Larva 3. Nepa 3.

Notoncta 4 Hepr. 3. Nonoculi I.

Spccies. Specimina.

87. 116.

$71 . \quad 86$.

61. $\quad 74$.

45. 62.

52.61 .

12. 12.

15.25.

3. 45 .

54. 89. $X I I$. Scarabc 


\section{Infeetes.}

1989 Nid de Guéspes des Indes. ( $A$.

1990 Nid de Guespes d'Arserique. ( $B$.

1901 Nid de Guéspes a'Amerique. (C.

1992 Nid de Guespes d'Amerique. ( $D$.

1993 Nid a Guespes d'Amerique $E$.

1924 Nià de Guéspes d'Europe. ( $F$.

Igy5 Nid de Guespes d'Europe. ( $G$.

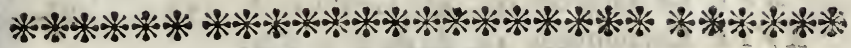

L $E$ E C A B I N E T

Avec XLII. Layettes dans les quelles fe trouvent,
D E S

INSECTES EUROPEENES.

L"yette.

I. Mouches entre les quels le Comeleon Moire. $8 \%$ 110.

11. Empis 3 Coufins 4. Panorpe r. Tipules 10. Mouches à fcie 37. Abeilles 16. 7r. 86.

III. Ichneumons 59. Spinxs 2. - - . 6: 6: $7 \%$.

IV. Guéspes 37. Chryjes 8. 8 . 45. 63.

V. Abeilles. - . 52. 61.

VI. Litellules, ou Demoifelles. - . i2. I5.

III. Demoifelies. (Vierges) II Larves. A.

VIII. Pertes 3. Ephemeres 3. Mirmelions 6.

Mouches Scorpions 3 Friganes 8. Co-

nopes 7.

1X. Mintides 4 Grillons 26.

$X$. Crillons 6. Blnttes 3. Punaifes 36.

$X I$. Punaijes 8. Profcarabés 12. Staphylines

:5. 25.

7 Ditiques 17 Larves 3. Nepce 3 Pu.

naijes à Environs 4 Scorpions áquatiques

3. Monocies.I.

$$
\mathrm{P}_{3} \cdot \text { XI\%. Scoscijes }^{54 .} 89
$$


$X I I$. Scarabei cornuti 10. inermes $6 \mathrm{I}$. Sylphæ 4. • . - 75. 121.

XIII. Lucani 6.Pelliones I9. Caffidx Ir.

Coccinella 43. Chryfomela 31. . 110. 169.

$X I V$. Curculiones $4 \mathrm{I}$. Elateres 28. inter quas Flabellicornis ruber \& alia,

Carabi 40.

$\boldsymbol{X V}$. Cicindelæ 7. Cleri 20. Attelabi 3.

Cantharides 14. $\bullet . ~ 44$.

164.

109.

164.

74 .

$X V I$. Cerambyces \&c. 46. Leptura 6. . 52. 92.

X VII. Carambyces 17. Aranea 9. Formicæ 4. Onisci 2.

XVIII. Coleoptera Siberiæ. . . 14. 14.

$X I X$. Nidi, Larva, Chryfolides \&c. 5c. 76.

$X X$. Phalenæ Geometix. ‥ 26. 35.

$X X I$. Phalenæ Geometræ. - . 56. 09.

XXII. Phalenæ Geometræ. ․ . 21. 33.

$X X I I I$. Phalenæ Pyrales. : - 22.30.

XXIV. Phalenx, Tinex, \& Tortricos, 39.

Bombyces II.

50.

63.

$X X V$. Phalenæ Bombyces \& Noetua. $\quad 36.57$.

$X X V I$. Phalena Noctux.

$X X V I I$ Phalenæ Noetux.

XXVIII. Pbalera Nodtæ

$X X I X$. Phalenæ Noctux.

$X X X$. Phalena Noftua.

$X X X I$. Phalenæ Nöetuæ.

XXXII. Phalenx Noctua.

XXXIII. Phalena Noctuz.

$X X X I V$. Sphinges.

11. 16.

$X X X V$. Sptinges \& $c$.

$X X X V I$. Papiliones.

$X \dot{X} X V I I$. Papiliones.

XXXVIII. Papiliones.

20.

34.

10, 20.

32. 35.

64. 104.

44. 00.

26. 39.

16. 29.

$32 . \quad 38$.

25. 45 .

20. 40.

$X X X I X$. Papiliones.

II. 21 .

21. 38 .

$X L$ Papilione 


\section{Injectes.}

XII. Scarabés Cornues yo. Inermes 61.Sylphes 4

75. 121 .

$\therefore I I I$. Lucaines 6. Pellions 19. Calfides II. Coccinelles 43. Chryfomeles 3 1. . II0. I69.

$X I V$. Charanfons 41. Taupins 28. entre les quels Flabsllions, rouge Eे autres, Carabes 40.

I09. I64.

XV. Cicindeles 7. Cleri 20. Ejcarbots 3. Cantharides I4.

44. 74:

XVI. Capricornes Ẽc. 46. Leptures 2.

XVII. Capricornes 17. Araignées 9. Fourmis 4. Cloportes 2.

52. 92.

32. 45.

XVIII. Coleopseres de Siberie.

XIX. Nids, Larves, Chryjolides. Ẽc. .

$X X$. Phalenes Geometres.

I4. 14-

50. 76.

XXI. Phalenes Geometres.

26. 35.

XXII. Phalenes Geometres. : . : . : 21, :33.

XXIII. Phalenes Pyrales.

XXIV. Phalenes, Teignes, Eू Tortrices, 39.

Bombyces, ou vers à Saye Ir.

22. 30.

$X X V$. Phalenes, vers d Soye \& No 0 aturnes. 36.57. $X X V I$. Phalenes Nocturnes.

II. 16 .

XXVII. Phalenes Nodurnes.

XXVIII. Phalenes Nodturnes.

XXIX. Phalenes Nocturnes.

$X X X$. Phalenes Nocturnes.

XXXI, Pbalenes Nocturnes.

XXXII. Phalenes Nodurnes.

XXXIII. Phalenes NotZurnes.

$X X X I V$. Spinxs.

$X X X V$. Spinxs E₹C.

XXXVI. Papilions.

XXXVII. Papillons.

XXXVIII. Papillons.

XXXIX. Papillons.

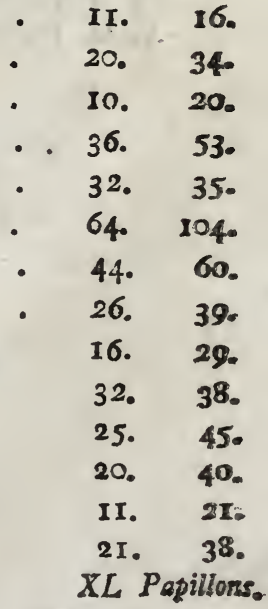


XL. Papiliones. - . $\quad$ 25. 50. XLI Papiliones. . . . 28.49. XLII. Papiliones. . . $\quad . \quad 30^{\circ} \quad 55$.

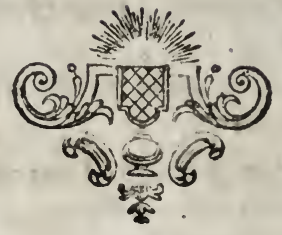

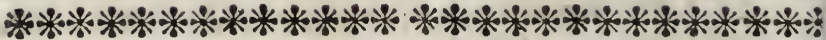
Infecites.

XL. Papillons. - . - 25. 50 , $X . I$ Papillons. - . . . 28. 49. XLII. Papillons. - . $\quad 30.55$.

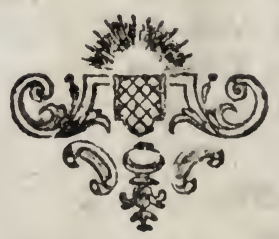




\section{CLASSISII. BIVALVIA.}

ORDO V. CARDINI FORMIA.

EN FORME DE CHARNIERE.

GENVS XXVIIT-XZX.

ORDO VI. SEMI CARDINATA.

i CHARNIERE IMPERFAITE.

GENVS XXII ET XXXI].

ORDO VII. CARDINATA.

A CHARNIERE.

GENVS XZIII - XLV.

ORDO VIII. NON CARDINATA NEC NON

ARTICVLATA.

SANS CHARNIERE ET ARTICULES.

GENVS ILVI ET XLVII.

CLASSIS III. PARASITI VALVIA.

ORDO IX. CRATERI FORMIA.

EN FORME DE SEAU.

aENVS XLYIY. 


\section{CLASSISIV. CONVEXIVALVIA.}

ORDO X. APERTVRA IN MEDIO SVPERFICIEI. OUVERTURE AU MILIEU DE LA SURFACE. GENVS XLIX. ET L.

ORDO XI. APERTVRA IN SVPERFICIE A CENTRO REMOTA.

OUVERTURE DANS LA SURFACE ET ELOIGNÉ

$$
\begin{gathered}
\text { DU CENTRE. } \\
\text { GENVS LI. }
\end{gathered}
$$

ORDO XII. APERTVRÁ IN MARGINE. OUVERTURE DANS LE BORD.

$$
\text { GENVS LII. ET LIII. }
$$

ORDO XIII. APERTVRA IN BASI A MARGINE REMOTA.

OUVERTURE DANS LA BASE LOIN DU BORD. GENVS LIV.

ORDO XIV. APERTVRA IN BASI AD MARGINEM.

OUVERTURE A LA BASE PRES DU BORD.

$$
\text { G?NOS LV-LVII. }
$$

ORDO XV. APERTVRA IN BASI A MARGINE

$$
\text { MVLTVM REMOTA. }
$$

OUVERTVRE A LA BASE LOIN DU BORD. GENVS LVIII ET LIX:

ORDO XVI. APERTVRA SVPERNE IN MARGINE. OUVERTURE DANS LE BORD. AU DESSUS. GENVS LX.

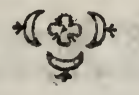

$$
\mathrm{Q} 2 \text { TESTACEA }
$$


4.

-

$\begin{array}{llllllll}T & E & S & T & A & C & E & A\end{array}$

C L A S S I S I.

$\begin{array}{lllllllll}V & N & I & V & A & L & V & I & A\end{array}$

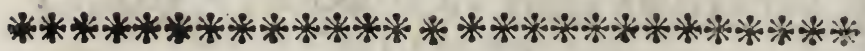

O

NONSPIRALIA.

GE NVS I. VERMIC VLI.

I V. Arenaria, Linn. 8.3 a Rumph 4 . E. Sebs III.94 in medic, long $15 \frac{1}{2}$ poll. (x specimen. 3. a.

2 V. Aranaria? f. varictas, abfque mediaftino, Linn. 803 \&. Rumph. 41. D. Lifter 1056. 3, Rircher I. 20. D. Icng. 13. pol' (I. Specim. 3 b.

3 V. Iliacus, intus fphrice claufa altera extremitate aneyta, Linn. o. Lift 105б. 5? Davilla I. 2I H? Petiv. Gazophyl 102. 23 (1. Cpecim. 2.

4 V. Anguina, Linn. SOt थ. Gualth. 10. N. Kircher I. 20. C. ( 1 rpecim 4.

5 V. Anguina? f. varietas, Linn. 804. B. Cualth. 10. N? (1. fpecim. 1021.

$6 \mathrm{~V}$. Anguina? \{. ynrietas, vel pracedenti fimillims, Linm. 804. B. (I. 'pecim. 1021 .

7 V.Vermicularis; Linm. 805. Rumph. 4T. 4. Argenville 4.C. Murici adnata, (2. Tpecim. 5. a.

$8 \mathrm{~V}$ Glomerata, Linz. 800. Ginanni II. 4. Rrmph. 4I. 3. Petiv. Mu/. 21. 18. Chamx Giganti adnata, (2. Ppecim 5 b.

9 V. Glomevcta, Linn. 80s. Kircher I. 20. F. Silici adnais, (1. Specim. 5.d.

10 V. Intricata, Linn. 796. Argenv. 4. B. Gualth. In F. Kircher. I. 20. E. Arce adnata, altera in Buccino undaco, (2. Cpecim. 5. c. 


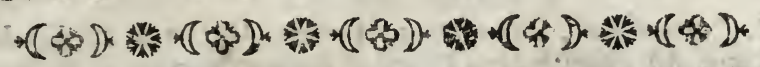
\%

$\begin{array}{llllllll}T & E & S & T & A & C & E & S\end{array}$ C L A S S E. I.

$\begin{array}{lllllllll}U & N & I & V & A & L & V & E & S\end{array}$

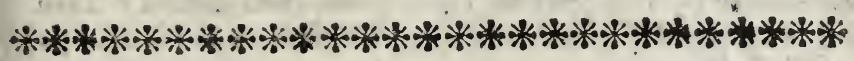

O

SA.NS SPIRALES

GENRE I. VERMISSEAUX DE MER.

I F. Arenaire, Linn. 803. „. Rumph. 4r. E. Seba III. 94. in medio, long. $15 \frac{1}{3}$ pouces, (1 piece 3.

y V. A enaire? ou varieté, fansmediaftine, Linn. \$o3. ß. Rumph " 4 I D. Lifter. 1050.3. Kircher I. 20. D. long. 13. pouc. (I. p. 3. b.

$3 V$. Iliace, en dedans à fpherique fermé, l'autre extremité ouverte, Linn. o. Lift. 10565 ? Davila I. 21. H? Petiv. Gazophyl. I02. 23. (I. p. 2.

4 V. Serpent, Linn. 804. *. Gualth. 10. N. Kircher I.20, C. (1 p. 4.

5 V. Serpent? ou varieté, Linn. 804 B. Gualth ro, $N$ ? (t.p. Ins.i.

6. V. Serpent? ou varieté, ou femblable à la precedente? Linn. $804 . \beta$ (1.p. 1021 .

$7 V$. Vermiculairs. Linn. 805. Rumph. 4I. 4 Argenville 4. C attaclice au Murex. ( 2 p. 5. a

S $V^{\prime}$. Turtilié, Linn. 80o. Ginanni II 4. Rumph 4I. 3. Periv. Mur. 2I. 18. attachée a la Chame Geante (2 p.5.b

9V Tortiile, Linn. s0o. Kircher I. 20. J. astache au Silice; (1. p. 5. d.

10 V. Intricate, Linn. 796. Argenv. 4. $B$ Gualth. 10. F. Kircher $I 20$. $E$. attachée à l'Arche, l'autre dans une Buccine vidée. ( 2, p 5.c. 
II V. Lumbricalis, Linn. 8cI. Petiver Muf. 22. I. Kircher J. 20. M. Gualth. 10. 2. (4 fpecim. 8. a.

12 V. Lumbricalis, Linn. bor. Lift. 548. 1. (3. Ppecim. 8. a.

I3 V. Lumbricalis, Linn. 80I. a Argenv. 4. I. (3. fpecim. 8. b.

14 V. Anguina, carinata, Linn. 804. Gualth. 10. V. Lift. 548. 2 ? Rumph 41. 2 ? Petiv. Muf. 22. 11? (3. Specim. 6. a. b.

I5 V. Anguina? f. varictas, carinata, Linn 804. $\gamma$. Rumph. 4 I. Hi? Kircher I. 20. M. (2. fpecim. 7. a.

$16 \mathrm{~V}$. Anastina I f. varietas, an pracedenti fimilis? Linn. 804. \%. Ginanni II.12. Petiv. Gazophyl. 153.2? (2. Tpecim. 7.6 .

I7 V. Penis, Linn. 806. Leers Muf.tab.1. f. A. Bonanni Obferv.

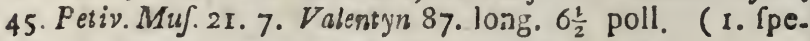
cim. I. a.

Is V. Penis, pracedenti fimilis, fed margo latior, magisque plicatus, Linn. 806. Lift. 548. 3. long. $5 \frac{1}{2}$. poll. (I. fpecim. r. b.

Ig V. Penis, hujus tantum pars anterior margo, five præputium cum glande, deeft Tubus. (I. \{pecim. I. c.

20.V. Cucurbita, Lini. o. Naturforfcher. X. tab. I. f. IO. XIII. tab. 1. \& 2. f. 1.-14. (I. Specim. I37.

\section{G E N V S II. T V E V L, I.}

2I T. Elephartinum, Linn. 783. Gunlth. 10. I. Argenv. 3. H. Lift: 747. fupra C 2. Specim. 9: a

22 T. Aprintm, Jinn. 784. Liff.547 I, Giualth. Io. G. Gi. ndni 11 . 1. (3. Ppecin. 2. b.

23 T. Entalis, Linn. 786. Argenv. 3. K K. Lift. 547. A. 2? Petiv. Gas. 65. 9? Planeus 2. I1. $A-C$. (3. fpecim. Io.

24 T. Corneura, Linn. 787. Gualth. 10. F. Giramii II. 2 ? (2. rpecin. ro. b.

$=5$ T: Politum? f. Ebumeum? lacve, Limn 788 ? an 789 ? Gralth. 10.E? Liff. 1056. 4. Girann. II. 3. (3. Tpecim. 933. 


\section{Teftaces. Ordre $I$.}

II V. Tirebourne; Linn: 8or. Petiver Muf. 22; 1 . Kircher I. 20. M. Güalth. 10. Q ( 4 p. 8. a.

$12 V$. Tirebourne, Linn. 80T. Lilt. 548. I. Knorr IV. 17.20 (3. p. 8. a.

I3 V. Tirebourne, Liřn. 8or. $\alpha$. Argenv. 4. I. (3 p. 8. b.

I4 $V$. Serpentine, carinée, cavé, Linn. 804 . Gualth. ro.' $V$. Lift. 548. 2.? Rumph. 4I. 2 ? Petiv. Mur. 22, II ? (3. p. 6. $a . b$.

I5 $V$. Serpentine? ou varieté, cavée, Linn. 804. \%. Rumph. 4I. H. Kircher I. 20, M. (2. p. 7. a.

I6 $V$. Serpentine? ou varieté, ou Semblable à la precedente? Linn. 804. \%. Ginanni. II. 12. Petiv. Gazophyl." I 53. 9? (2. p. 7. b.

I7 $V$. Arrofoir, Linn. 806. Leers. Mur. tab. I. $f$ A. Bonanni Obferv. 45. Petiv. Muf. 21. 7. Valentyn 87. long. $6 \frac{1}{2}$.pouc. (I.'p. r. $a$.

I8 $V$. Arrofoir, femblable d la precedente, mais le bord plus large, ED plus plié, Linn. 806. Lift. 548. 3. long. $5 \frac{1}{4}$.pouc. ( I. p. I. b.

Ig $V$. Arrofoir, la partie anterieure d'un arrofoir, ou le bord, ou prepuce avec lo glande, Sans Tube. (I. p. I. c.

zo $V$. Cucürbite, Linn. o. Naturforfcher X.tab. 1. f, 10.XIII. tab. I. \& $2, f$. I. -I 4. (I. p. IC 37.

GENRE IT. TUYAUX DE MER.

2 I $T$. Elephantin, Linn. 783. Gualth. ro. I. Argenv. 3. $H$. Lift. 747. Jupra, (2. p. 9. a.

$22 T$. de Cochon, Linn. 784. «. Lift. 547. I. Gualth. Io. G. Ginann. II. I. (3. p. 9. b.

23 T. Entale, Linn. 786. Argenv. 3. K. K. Lift. 547. A. 2 ? .. Petiv. Gaz 65. 9 ? Plancus 2. Ir. A-C. (3. p. 10.

$24 T$. de Chien, Linn. 787. Gualth. IO. F. Ginann. II. 2 ? (2. $p$, ro. $b$.

25 T.Polie, ou Yvoire ? Linn. 788. ? ou 789 ? Gualth. 10. E? Lift. 1056. 4. Ginann. II. 3. (3. p. 233 . 


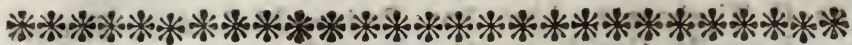

\section{O R D O}

$S P I R A L I F O R M I A V T E T$

.... A R T CVLATA.

GENVS III. SCVTIGERVLI.

26 S. Fascicularis, Linn. 4. a. Krorr IV. r7. 3. 4. long. $3 \frac{1}{2}$. lat. I $\frac{3}{4}$. poll. (1. Tpecim. 1022. a.

27 S. Squamofus, Linn. 5 *. Seb. III. 1. 14. (1. Specim. 10z2. b.

28 S. Angulatus, valvis dorlo lateribusque mucronatis, Linn. 5. B. f. Q. ( 1 . .pecim. 10.23.

29 S. Punçatus, Linn. 6. Seb. III. I. 13. (1. Specim. I024.

G E N V S IV: PA T ELI RE.

so P. Puftula, coltata, mucronata, Linn. 779. *. Lift.527.

I. ( 1. . pecim: 29.

31 P. Nimbofa, Limn.781.a. Argenv. 2. C, KircherI.3. ( 1 . fpecim. 28.

32 P. Graca, fubrubens, Zinn. 780. «. Argenv. 2. I? (2. fpecim. 949. b.

33 P. Nimbofa, 'Linn. 781. B. Lift. 528. 7? Petiv. Gaz. 80. 12. (2. Ppecim. 949. a.

34 P. Pultula, Linn. 779. Lift. 527.2? Kircher I. 6. Argenv. 2. I. Adanfon Seneg. I. 2. 7. 7. (1. Specim. 1025.

35 P. Nimbofa? 1. varietas, Linm. 781 1. d. Li/t. 528.7. Gualth. 9. T. Petiv. Gaz. 10. I1. (3. Rpecim. 948.

36 P. Nimbofa? violacea, Linn. 781 . A. Argenv. 2. E? Da. yila I. 3. C, (2. fpecim. 27. a.

37 P. Nimbofa? f. varietas, alba, radiis violaceis, Linn. 78r. B. Gualth. 9. S. (I. Specim. 27. b.

38 P. Nimbofa? 1. varietas, rubro purpurea, Linn. 781. $\%$ Petiv. Gaz. 3. 12? (I. fpecim. 30.

39 P. Cancellata, compreffa, argute radiata, purpurea, Linn. 0. Petiv. Gaz. 3. II, 85. 8. Adanfon Seneg. I. 2, 6. 7. (2. Specim. 3I. 


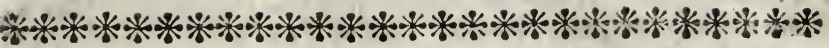

O R D R E II.

$E N F O R M E D E S P I R A L E E T$. A. RTICULE.

GENRE III. OSCABRIONS DE MER.

26 O. Bandé, Linn. 4. ๓. long $3 \frac{1}{2}$. Latit. $1 \frac{3}{4}$ pouc. (I. p. ro2?. $a$.

27 O. Plein d'ecailles, Linn. 5. « Seb. III. I. I4. (I. p. 1022. $b$.

$28 O$ Anglés, les valves au dos, Eo cotés en pointes, Linn. 5. B. f. O. (1. P. 1023.

29 O. Pointé, Linn. 6. Seb. 1II. I I3. (I. p. I024.

GENRE IV. PATELLE H.

3o $P$. Puftulé, ayant des côtés, pointue, Linn, 779. \%. Lift. 527. 1. (I. P. 29

$31 P$. Ocil de rubis, Linn. 78I,. . Argenv. 2, C. Kircher $I$.

3. (I. p. 28.

32. P. Grecque, rougeatre, Linn. 780. «. Argenv. 2. I? (2. p.: (949.6.

33 P. Oeil de rubis, Linn. 78 : . F. Lin. 528. 7? Petiv. Gaz: 80. 12. (2. p. 949. $a$.

34 P. Puftulé, Linn. 779. Lift. 527. 2 ? Kircher I.6. Argenv. 2 1. Adanfon Seneg. 1. 2. 7.7. (I. P. 10?5.

$35 P$. Oeil de rubis? ou varieté, Linn 78I. d. Lift. 528. 7. Gualt. 9. T. Petiv. Gaz. 80. Ir. (3. p 948.

$30 P$. Oeil de rubis? violet, Linn. 781 . $\beta$. Argenv. 2. E.? Davila. I 3. C (2. p. 27. a.

$37 P$. Oeil de rubis? orb varieté, blanche, $\dot{a}$ rayons violettes,

Linn. 78I. $\beta$ Guath. 9. S. (1. p. 27. b.

$38 P$. Oeil de rubis? ou varieté, rouge fourpré, Linn. $78 \mathrm{r} . \%$ Petiv. Gaz. 3. 12 ? (1. p. 30.

39 P. Cancelles, comprimé, rayonné de poutpre, Linn. o. Petiv. Gaz. 3. 11. 85. 8? A tanson Seneq. 1. 2. 6. 7. (2. p. 3I. 
40 P. Sacharina? S.varietas, fubdepreffa, I 6 angulis radiata, flavefcens maculis fuscis, Linn. 754. a f. o. Lift 538. 21 ? Kircliz I. 26? Gisaltio. 2. M? (2 fpecim 18.

4 I P. Barbara. Linn. 755. Kircher I. 32. (2. fpeciin. 19.

42 P. Laciniofa, f. Caput Medufa, Linn. 753. Rumph. 40.C? (I. ́pecim. 942.

43 P. Laciniofa, an frnilis pracedenti? Limn. 753. Argerv. 2. O? Petiv. Mir). 2. 3. (1. Specim. 943.

44 P. Selas, fulcata, cinerea, punctis fuscis, Linn. 756.. . . o. Bonam. Obferv. 35. (r fpecim. 939.

45 P. Gramularis ? f. varietas, Linn. 756. $\beta$. Lift. 534. 13. (3. \{picin. 938.

46 P. Gramularis? f. varietas, fed obfcurior tamen colore, Linss. 756. [ d. Kircher I. 30. (3. Tpecin. 937

47 P. Coftata, fusca, Linn. 756. भ. .. o. Gualth. 9. F? (2. foecim 94: $\mathrm{T}$.

$48 \mathrm{P}$. Granulnris? T. varietas pailidior quam $\mathrm{n}^{\circ} \cdot 45$. ceterum fimilis, Linn. 756. \& Kircher I. 30 ? (3. Tpecim. 940.

49 P. Granularis? r. fimilis pracedenti, fed angulato ftriata, Linn. 750. \%. Argenv. 2. G. (3. ipecim. 936.

50 P. Gramularis? f, varietas, præcedenti limilis, fed elevato, \&. margine interiore fusco punctato, Linn. 756. ร. Gualth. 9. F. Ginann. II. 17 ? (3. fpecim. I6.

5 I P. Petafus, large coftata, ftriata, viridi puncta:a, fusca, Linn. O. Argenv. 2. B? (I. fpecim. I7.

52 P. Umbella, radiata cinereo fusca, Linn. o. Gualth. 8. EL? long. $3 \frac{3}{4}$ lat. $2 \frac{3}{4}$. poll. (I. Cpecim. I3. a. b.

53 p. Umbella, præeedenti fimilis, color obfcurior, fuscus, Linn. o. ( 2 ipecin. Is b.

54 P. Umbella, prectenti fimilis, fed tenuior, fusco radiata, Lim. o. Argenv. 2. Q? (2. Pperim. I3. c.

55 P. Radiata. an pixcedenti fimilis? rufo fusca maculata, Linn. o. Petiv. Goz. 75. 3. ( 3 fpeclur. I 4 .

56 P. Caudata, radiata radiis elevatis, ftriis fuscis, Linn. 0. (I fpecion. I5.

57 P. Mucronata, apice acuto, ftriis argutis radiata, punct sque rofeis ftriatim digeltis, Limm. o. Petiv. Gaz. 85.11. (3. fpecim. 935.

58 P. Granifcre, apice albo, havefcens, ftriis rofeis, Lim. 0. (4. Trecim 934

59 P. Granifera. f. finilis præcedenti, fed pallidior, Linnio. Kircher 1. 25. (2. Specim, 934. 
40 P. Sacharine? ouvarieté, deprimée, jaune, à tacbes brunes, ED radié par 16. angles, Linn. 754. ๙. .. O, Lift. 548:2 ? Kircher I. 26 ? Gualth. 2. M. ? (2.p. 18.

4I P. Barbare, Linn. 755. Kircher I. 32. (2. p. 19.

$4^{2}$ P. Eroile, ou Tête de Medufe, Linn. 753. Rumph. 40. C? (I p. $9+2$.

43 P. Eroile, ou femblable d̀ la precedente ${ }_{2}$ Linn. $753, \cdots$. Argenv. 2. O. Petiv. Mur. 2.3. ( $p$ p 943 .

44 P. Rayon, granulé, cendré, d pointes bruzzes, Linn. 756. ж. f. O. Bonan. Ob?erv. 35. (I p. 939.

45 P. Granulée? ouv varieté, Linn. 756 . $\beta$. Lift. 534 - 13. (3. p. 938.

$46 P$. Granulée? ou varieté, mais pourtant obfcure de couleur, Linn. 756. .. o. Kircher: I. 30. (3. p. 937.

47 P. Brune, ayant des côtes, Linn. 746. \%. Guaith. 9. F.? (z. p. $94 \mathrm{I}$.

48 . . Granulée? ou varieté, plus pale que $n^{\circ} .45$. , au refte: Semblable, Linn. 756. d. Kircher I. 30 ? (3. p. 940.

49 P. Granulée? ou Jemblable à la precedente, mais à angles friés, Linn. 756. n. Argenv. 2. G. (3.p 936.

$50 P$. Granulée? ou variete, femblable à la precedente, mais

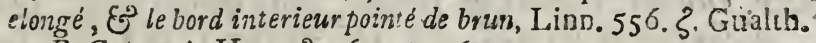
9. F. Giranni $I I .17$ ? (3. p. 16 .

sI $P$. Bonnet, ralié, ftrié, Es pointi de vert, brune, Linn.o. Argeruv. 2. B.? (I. p. I7.

52 P. Parafol, radié, bruncendré, Liun. O. Gualth. \&. E? long. $3 \frac{3}{4}$ Lat. $2 \frac{3}{4}$ pouc. (I. p. I3. a. b.

$53 P$. Paraful, femblable à la precedente, de couleur noir brun, Linn. o. ( 2 p. I3. b.

54 P. Parafol, Semblable à la precedente, mais pius mince, radis de brum, Linn. o Argenv. 2. Q.? (2. p. 13.c.

$55 P$. Radié, ou jemblable à la precedente, rouffe, tacheté de brun, Linn. 0. Petiv. Gaz. 75.3. ( 3. p. 14.

56 P En queu, à rayons elevées, of fries brunes, Linn o. (I. p. 15.

57. P. Pointée, aizue les ftries radiées, pointes couleur de rofe, diftrifués, à fommet en Jtries, Linn.0. Petiv. Gaz.85. Ix. (3. P. 9:5.

58 P. Granifere, au fommet blane, jaunatres is fries couleur de ${ }^{\circ}$ ofe, Linn. o. (4. p.034.

59 P. Ciranifere, ou Jeinhlable è la precedenze, mais plus pale; Linn. O. Kircher 1. 25. (2 p.934. 
60 P. Granatina, Linn. 75\%. Argenv. 2. H. Lift 536.15. Adansone Seneg. 1. 2. 7.7. (2. 'pecin. 2:.

6I P. Spinojula, itriis fpinclis, fu ca, Linn. o. Gualtz. 9 L. Lift 535. 14. (1. fpecin. 9+5.

62 2. Striata, albo radiata, fusco migra, radiis albiis octo, Linn. o. Gualth. 9. G? (1. fpecim. 944.

63 P. Selas, a!bo radiata, intus pullucida, Linn. o. Petiv. Muj. 3 ? (1. Specim. 21.

64 P. Coftata, fluvefcens, coltis elevatis oftu albis, Linn 0. (I. fpecim. 20

65 P. Morilles, f. Fungus, alb, thries nigro cyaneis, incus cyanea, Linn 0. Lijt. 539. 22. 23? (3. Tpecim. 23.

66 P. Teftudinaria, Limls. 77 I. Lifter 53t. 9. Kircher I. 3 I. Petiv. Muf. 16 24? (2. Specins. II. b.

67 P. Ieftudinaria, haec pulita, \& poliendn pallidior reddita, Linn 771. Argenv. 2. P. Gualth. S. B. Rumph. 40. A. Petiv. Muf. 8. 10. (2. fpecim. 11. a

68 P. Teftudiraria, pracedenti timilis, Linms. 771. (2. Specim. 1 I. a.

69 P. Stella, argute radiata, fusca, ex apice ftriæa abx, Linn. O. (2. fpecim. I2.

zo P. Oculata, granularim radiata, arice obtufo albo, $L i, n$. - Gualth. 8. G. Lift. 53\%. 10. Kircher 1. 25? (1. . Pecim. 26.

TI P. Mammillaris, Linn 763. Lift. 537. 17. Petiv. Gas. 80. IO. (1. Specim. 946.

72 P. Granatins, Linn. 75?. \& Davila 1. 3. D. Gualtl. 8. Q? altit I $\frac{1}{2}$. latit $3 \frac{3}{4}$. poll: (2. fpecin. 24 .

73 P. Granatina, albida, ftiis fuscis, Linn. 757. B. Gualtb. 8. $G$ ? (I. fpecin 25 .

74 P. Granatina? f. varietas, intus ex fufen flavoque radia. 1a, Linn. 757. \%. Gualth. 9. Q. (1. Ipecim. (947.

75 P. Albogalerus, fubrntunda grifa, annulata, incuslactea, Linn. O. G:alth. 8. P ? (I fpecim. 35.

76 P. Spheroidea, radiis trifurcatis alba, Linn o. Lift. 542. 25. (r. reccim. 855

77 P. Mammillaris, apice mammillari, pallideftriata, grifea. Linn O. Lift. 542 26. (4. ipecin. 34 a. b.

78 P. Tricarinata, deculfatim itriata, Mallide thalafina, Linn. 764. \&. Liff. 543. 28 ? (2. ppecin. 38 .

79 P. Oblizua, apice obliquo excentrico, flavefcens, argu:e ftriata, Linm. C. Gualth. 9.V? (1. f,ecim. 37. 
60 P. Granatine. Linn. 757. Argenv. 2. H. Lift. 536. 15. Adanson Seneg. I. 2. 7. 7. (2. p. 22.

6I P. kipit eure, à ftries epineufes, brune, Linn. O. Guaith. 9. Lo Lift. 535. I4. (1. p. 945 .

$02 P$. Siriée, radiée de blanc, lrun noir, à huit ra yons blanches Linn. 0. Gualih. 9. G? (1. p. 9 ts.

6: P. Rayon, fric de bianc, claire en dedens, Linn. $0_{0}$ Priv. Mur. 3. 3? (I $p$ 2 r.

$54 P$. Ayant des crites, jaunatre, is huit cotes elevies blanches, Linn. O. (1. P. 20 .

65 P. Morille ou Fanger, "Whanche, à friés noir-bleuatre, be dedans blere, Linn. O. Lift. 539. 22. 23.? (3 p. 23.

$65 P$. Ecaille de Tortue, Linn. 77 t. Lift. 53I. 9 Kircher I. 31. Petiv. Muf, 16. 24 ? (2. p. It. b.

87 P. Ecaille de Turrue, celle ci efl polie, EO par la rendus pius pale, Iinn 77 r. Argenv. ?. P Gualth. 8. B. Rumph. 40. A. Petiv. Muid. 8. 10. (2. p. 11. a.

Gs $P$. Ecaille de Tortue, fembiable do la precutente, Limn. $77 \mathrm{r}$. ( 2 p. Ir. $a$.

es P. Ecoille, fubtilement radiée. Brune. le Jonsmet du pointe os Aries haqches. Linn. O. (2. P. I 2

70 'P. Oculée, granulée, radiée à fn nmat blanc emouffé, iLinn. 0. Gualth. 8. G. Lift. 537 I6. Kircher I. 25 ? (I. p. 26.

7 P. à Mammulles, Linn. 763. I7 Petiv. Gaz. 80. I0. ( $r_{0}$ D. 946.

72 P. Granatine, Linn. 757. a. Davi'a I. 3. D. Gualth. 8. Q? altit. $I \frac{1}{2}$ latit. $3 \frac{1}{4}$ porce. (2. p. 24 .

73 P Granatine, bianche, à ftries brunes, Linn. 757. $\beta$. Gualth. $8 G$ ? (I. $p 25$.

74 P. Granatine? ou varieté, en dedans, valiée de brun, Ẽ jaune, Linr: 757. $\boldsymbol{\gamma}$. Gual:h 9. Q. (i. p 947.

$75 P$. Albogalere, arrondie, grife, a annearbx, blanche, Linn, O. Gualth. 8. P ? (x. p. 35 .

$76 P$. Spheroide, blanche, en dedans d̀ rayons trifourchées, Linn. D. Lit. $5+2.25$. (x. P. 855 .

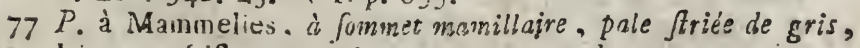
Linn. 0. rift. 54?. 26. (4. P. 34 a b.

78 P. ̀ Trois Caves ens forme de croix ftriée, paie thalafone, ou couleur de mer, Linn. 764. a. Litt. 54328 .? (2. p. 38.

$79 P$. Transv rfe, à fommet oblique en contrice, jauriatre, Jubtilement firise, Limn. v. Gualth. .. $V_{f}$ ? (1. p. 37. 
2o $\mathrm{P}$ Fiuviatilis, margine incurvo, cinereo virefcens, radiis obfcurioribus : Linn. o. (2. rpecim 33.

81 P. Compreffa, flava, Linn. 772. Lift. 54I. 25. Kircher 1. 21. (3. foecim. 950.

.82 P. Comire $\iint a$, eadem ac præceders, Linn. 772. (3. fpecim. 95I.

83 P. Ungais, Linn. 767. Rumph 40. L? Petiv. Mur. 16. 3L. Naturforfch. XVIII. tab. 2. f. 8. long. I. latit $\frac{1}{2}$ poll. (2. fpecim. 852 .

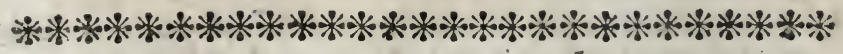 \\ O R R D \\ $S E M I S P I R A L I A$.}

GE N V S V. A N C I L I A.

81 A. Ungarica, Linn. 76r. Valent. 98.Gualth. 9.W. (r. Specim 36. is A. Ungarica, pallide rofea, Liqn. 761. Lift. 544.32. Argen\%. Suppl. I. A. (I. Ipecim. 36 .

86 A. Equeftris, Linn. 747. Argenv. 2. K. Gualth. 9. Z. Kecunda. (3. Cpecim. 39. a.

27 A. Equefiris, priori fubfimilis, fed membranaceo rugofa, albida, Linn 747. a. Gualth. 9. Z. prima, Davila I. 2. B? (1. fpecim. 952.

$8 S$ A. Equeftris, præcedenti fubfimilis, algute ftriata, Linn. 747. B. C. o. Gualth. 9. X. Lift. 546. 38 ? (!. Specim. 40.

8) A. Equeftris? f varietas, foliacea, Lim 747. भ. โ. O. Argenv. 2. S. (2. Specim. 4I.

so A. Chinenfis, Linn. 749. Gualth. 9. X? Lift. 546. 39? (2. Specim. $4^{2}$. a.

or A. Chinenfis, in nmnibus cadem, maculis incurvis fuscis, Linn. 749 \&. Lift 546. 39. (3. fpecim. 42. b.

92 A. Chinenfis? Livn.7449. B. T. O. Naturforfch. XVIII. tab.2. f. 12. a. (1. (ppecin). 953.

93 A. Caperata, rugofa, pallide fusca, Limn. o. Naturforfch. XVIII.tab.2.f. 12. (2 fpacim. 854.

94 A. Strią , concava, arcute fuicata, infundibulo interiore, Linn. 0. Naturforfch. XVIII.tab.2. f. 13. (2. fpecim. 853. 92 A. 


\section{Teftaces. Ordre III.}

80 P. Pluviatile, ou de Riviere, à bord courbé, verd cendré, tै rayons obfcures, Linn. o. (2. p. 33 .

8I P. Comprimée, jaune, Linn. 772 . Lift. 54I. 25. Kircher $I$. 21. (3. p. 950.

82 P. Comprimée, la même que la precedente, Linn. 772. (3. P. 951 .

83 P. Ongle, Linn. 767. Rumph. 40. L.? Petiv. Muf.16.3r. Naturforich.XVIII.tab. 2. f. 8. long. 1. Latit. $\frac{3}{2}$. pouc. (2.p.85\%.

\section{O R $\quad$ D $R$ R E III. \\ I SPIRALE IMPARFAITE.}

GENRE V. BOUCLIERS.

$84 B$ Bonnet de Dragon, Linn. 76r. Valent. 98. Gualth. 9. W. (x. p. 36 .

85 B. Bonnet de Dragon, couleur de rofe, Linn. 76r. Lif, 544.32. Argenv. Suppl. T. $A_{0}$ ' (. . p. 36 .

86. B. Cabockon, blanc, Linn. 747. Argenv. 2. K. Gualtb, 9. Z. Securida. (3. p. 39 a.

$87 B$. Cabochon, blanc, à pers près femblable ca la precedente, mais d'un membrane ridé, blancheatre, Linn. 747. . Gualtb. 9. Z. prima, Davilla 1. 2. B.? ( $\mathrm{x}$. p. 952 .

88 B: Cabochón, presque femblable do la precedente. EV Jubtilement ftriée, Linn. 747. B. I. 0. Gualth. 9. X. Lift. 546.38. (I. p. 4 o.

89 B. Cabochbon? ou varieté, en feuilles, Linn. $747 \%$ Argenv. 2. S. (2, p. 4I.

so B. Bonnet Chinois, Linn. 749, Gualth. 9. X? Lift. 546. 39 ? (2. P, 42, a.

9r $\vec{B}$. Bonnet Chinois, en tout le même, 'd taches courbées bru= nes, Linn. 749. a. Lift. 545. 39." (1. p. 42. $b$.

$92 B$. Bonnet Chinois? Linn. 749. $\beta$. ou o. Naturforfch. XVIII. tab. 2. f. I0. a (1. p. 953.

$93 B$. Bonnet de Matelot, ridé, d'un brun pale, Linn. $0 . \mathrm{Na}$ turforfch. XVIII. tab. 2. f. 12. (2. p. 854.

94 B. Bonnet ftrié, cavé, E̊ fubtilement fillonné dans linte: rieur, Linn, 0. Naturforfch XVIII. tab, 2, f, 13: (2, p:853. 
25 A. Nitens, apice papillofo, infundibuliformi intus, extus Ixvis grifea fusca, Linn. 0. Gualth. 9. X? (3. fpecim. 856 .

s6 A. Fornicata, Linn. 75r. Argenv. 2. N. Adanjon Seneg. I. 2. 7. 8. ( 1 . fpecim: 45 . b.

97 A. Fornicsta, gibbofa, Linn. 75 I. a. Lift. 745.33. Gualth. 69. H. O. infra. (-2. fpecim. 45.

98 A. Fornicata, alba, Linm. 75r. B. Liff. 545. 36. (1. fpecim 954.

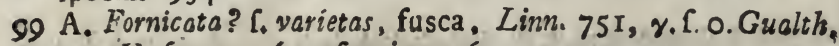
69. H. Tupra. (2. Specim, 46.

100 A. Perlucida, ftriata tranfparens, Limm. ○. Adanfon Seneg. 1. 2. 7. 9. (3. fpecim. 47 .

Ior A. Punctata, labio interiore parvo, fusco punctata, cinerea, Linn. O. Lift. 545. 34. (1. f pecim. 48.

102 A. Helvola, flavefcens; priori fimilis, parva, Linn. 0. Adanfon Seneg. 1.2.7. 10. Lift.545. 34 ? (3. fpecim. 955. IO3 A. Solea, plana, labio convexo, flavo punctata, Linn. 0. Lift. 545. 37? Adanfon Sereg. 1. 2. 7. II? Naturforfch. XVIII. tab. 2. f. 15. (I. fpecim. 956.

I04 A. Equeftris, tefta granulora, apice acuto, Linn. o. Petiv. Gaz. 21. II? Naturforfch. XVIII. tab. 2. f. 14. (2. Ipecim. 43.

ros A. Volutata, coftata, contorta, fpiris coltatis, albida, Linn. c. Argenv. 2. L. Naturforfch. XVIII. tab. 2. f. I6. (I. fpecim. 247.

rob A. Volutata. glabra, apice papillari, tefta transverfim rugofa, alba, Linn. O. Naturforfch. XVIII. tab. 2. f. I7. (I. fpecim. 44.

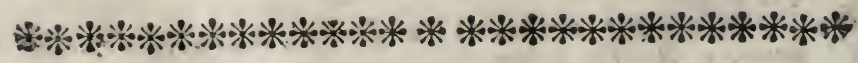

\section{O \\ $S \stackrel{P}{ } I \quad R \quad A \quad L \quad I A$.}

GENVS VI. AVRI FORMES.

I07 A. Mide, viridi rubens, Linn. 740. Gualth. 69. B. long; 6. lat. 4. poll. (1. fpecim. 53.

208 A. Striata, pallide viridis, Linn. 742. Gualth. 69. A. Lift. O11. 2. Argenv. 3. B. Kircher I. IO. (2. fpecim, 958. 


\section{Teftaces. Ordre IV.}

$95 B$. Splendide, da point papillonné, en forme defundibile endedans, Es par dehors gris.brun, Linn. O. Gualth. 9. X.? ( 3 .p. 856.

96 B. Vouté, Linr. 75I. Argenv. 2. N. Adanson Seneg. I. 2. 7.8 . (т. p. $45 . b$.

$97 B$. Vouté, bofJue, Linn. 751. «. Lift. 745. 33 Gualth.69. H. O. infra. $(2, p, 45$.

98 B. Vouté, blanche, Linn. 75r. B. Lift. 545.36. (J.p. 954.

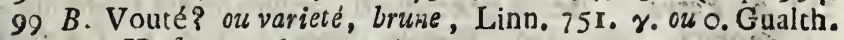
69. H. Jupra. (2. p. 46.

Ico B. Reluifant, frié, transparant, Linn. O. Adanson Seneg. I. 2.7. 9. (3:p. 47.

Ior $B$. Reluifant, pointé, la leure interieure, petite pointée de brun, cendree Linn. 0. Lift. 545. 34. (1. p. 48 .

102 B. Paillet, jaunatre, petit, femblable a la precedente, Linn. o Adanson Seneg. 1. 2. 7. 10. Lif. 545.34? (3.P. 955. 103 B. Semelle, uni, à levre recourbee, pointée de jaune, Linn. o. Lift. 545. 37 ? Adanson Senég. 1. 2. 7. I1.? Naturforfch. XVIII. tab. 2. f. Ic. (I. p. 956.

104 B. Cabochon, à ecaille granule . formet pointu. Linn. $\sigma$. Petiv. Gaz. 21, II ? Naturforfch. XVIII. tab. 2. f. 14. (2. p. 43.

105 B. Cabochon veiouté, ayant des cotes, recourbeee, ¿a spires cofées, blancheatre; Linn. O. Argenv. 2. L. Naturforfch: XVIII. tab. 2.f.16. (I p. 247.

I0б $B$. Cahochon Velouté, d Jommet papillonnè, pele, à coquille de travers ride blanche, Lisn. o. Naturforlich. Xl'lII. tab. 2.f. 17. (2. p. 44 .

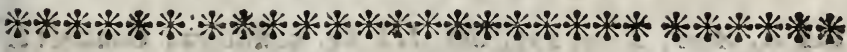

\section{O $-\mathrm{R}$ D $R$ E IV. \\ $S \quad P \quad I \quad R \quad A \quad L \quad E S$.}

GENRE VI. EN FORME D'OREILLES.

Io7 O. Midas, vert rouge, Linn. 740, Gualth. 69.-B. long. 6 lat. 4. pousc. (I p. 53.

108 O. Striée, pale vert, Linn. 742. Gua'th. 69. A. Lift. 6ị. 3. Argenv. 3 B. Kircher. I. 10. (2. . 958.

$$
\text { R } \quad \text { IOg } 0 \text {. }
$$


I09 A. Striato, rubra, Linn. 742: *. Guilth. 69 E. Argem 3. D (2. fpecim 52.

1 so A. Striata? rugofa, rugis lamellofis, Linn. $7+2$. $\beta$. C. o Gualth. 89. I ? Lifi. Anglia 3. 16 ? (1. Specim 559.

III A. Tuberculata, flavo virens, Linn. 74:. Lift. 614.6 Petiv. Muf. 16. 32. (2. ipecim. 51. 257.

112 A. Porva, margine tuberculato rufo fusco maculato Linn 746. ๓. f. o. (I fpecim. 49.

II3 A. Parva, r. fimilis precedenti, argute ftriata rubro mar morata, Linn. 746. Adanfon Serieg. 1. 2. 0. 8. (2. Spe cim. 50. a.

II4 A. Parva, in omnibus pracedenti finilis, Linn. $74 \mathrm{C}$ (3. 'pecim. 50. b.

Is A. Antiqua, cæca . Cpira elevata, foraminibus nullis, Linn o. Lift. ro56. 6? 7? (1. Specim. 54.

ir6 A. Afinins, Linn. 745. Lift. 5 10. I. Rumph. 40. E. E Petiv. Muf. 16. 34. (2. Specim. 55.

II A Afinina, in omnibus præcedenti fimilis, Linn. 745 Gualth. 69. D. (4 fpecim. 55 .

I18 A. Afinina, fimilis præcedenti, fed decorticata, Linr 745. Argenv. 3. E. (3. Ipecim. 55.

I19 A. Holiotoidea, argute ftriata, albida, Linn. 713 . A Gualth. 69. F. fupra, Rumph. 40. $R$. (3. fpecim 56.

180 A. Haliotoidea, tefta hepatica. intus fusca, Linn. 719 * Petiv. Muf. 16. 29\% ( 3 fpecim. 857.

121 A. Haliotoidea, Limn. 7r3. Gualth. 69. infra, Lift. $57 \mathrm{C}$ 21. Petiv. Gaz. 12. 4. (2. fpecim. 57.

GENVS VII. AR GONAVTR.

122. A. Argo, Linir. 27x. Seb. III. 84. 5. finiftra, Gralth. I:

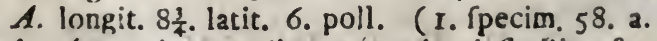

I 23 A. Argo, in omnibus pracedenti fimilis, \& magnitud eadem, Linn 27 x. Rumph. 28. $A$. (I. Specim. 58. b.

$124 \mathrm{~A}$. Argo, in omnibus fimilis præcedenti, paulo minor t: men, Linn. 27 . Argenv. 5. A. Kirclier 1. 13. ( 3 Ipe cim. 58. $c$.

125 A. Argo, fimilis pracedentibus, Limin. 271. Gualth. 1. B. Seba III. 84. 6. 7. (1. Specim. 58. d.

126 A. Argo, diffectus, Linn. 27 r. Ginann. I 39. Petiv. Mu. 10. 2. (1. fpecim. 58. e.

127 A Argo, fimilis pracedentibus, fef margine rotundato Linn. 271. a. Liff. 558. 7 \& 7. (1. f́pecim 9úo. 
9 O. Striée, rouge, Linn. 742 œ. Gualth. 69. E. Argenv. 3. D. ( 2. p 52 .

to O. Striée? Eo vidée, d lamelles vidées, Linn. 742 . B. ou o. Gualth. 69. I ? Lift. Anglia 3.16 ? (I p. 559

ir $O$. Tuberculée, verdeatre, Linn. 74I. Lift. 614.6. Petiv. Muf. 16. 32. (2. p. 51.957.

20 . Cordonné, (petite) le bord \& tubercules rougeatse, tachste de brun, Linn. 746. \& ou O. (I. P. 49

130 . Cordonnée. (petite) ous Semblable d la precedente, fubtilement friée, marlriée de rouge, Linn. 746. Adanfon seneg. 1. 2. 6. I. (2. p. 50. a.

140 . Cordonnée, (petite) en tout Semblable ì lo precedente, Linn. 746. ( 3. p. 50. b.

15 O. Antigue, à Jpire eleyé Jans trous, Linn. O. Lif. 1056. 6. 7. (1. p. 54 .

16 O. Afinine, Linn. 745. Lift. 510. 1. Rumph. 40. $E E$. Petiv. Mur. 16. 34. ( 3 p. 55.

17 O. Afinine, en tout jemblable à la precedents, Linn. 745. Gualtb. 6). D. (4. P. 55.

18 O. Afinine, jemblable d la precedente, mais decorchée, Linn. 745. Argenv. 3. $E$ (3. p. 55.

is O. Haliotode, fubtilernent ftriée, blancbeatre. Linn. 713. B. Gualth. 09. F. Jupra, Rumph. 40. R. ( $3 . p 56$.

20 O Haliotode, à coquille hepatique, en dedans brane, Linn. 713. Petiv. Mur. 16. 29 ? (3. P. 857

2I O Haliotode, Linn. 7 1 3. Guilth. 69. infra, Lift. 570. 21 . Petiv. Gaz. 12. 4. (2. p. 57.

GE NRE VII, NA U TILE S PAPIRACÉES.

:2 Nautille Papyracée, Linn. 271. Seb. III. 85. 5. Siniftra, Gualth. 12. A. long. 83 Latic 6. pouc. (I. p. 58. a.

23 Nautille Papyracée, en tout, femblante d la precedente, E' de la méme grandeur, Linn. 27 . Rumph 28. $A$. (I P. 58. b.

24 Nautille Papyracée, fernblable en tout la precedente, mais plus petite, Linn. 271. Argenv. 5. A. Kircber I. 13. ( 2 p. 58 C.

25 Nautille Papyracée, Linn. 27I. Gualth. Ir. B.Seb. III. 84. 6. 7. (1. p. 58. d.

(6 Nautille Papyracée, ouverte. Linn. 271. Ginann. I. 39. Petiv. Mur, 10. 2. (1. p. 58. e.

27 Naurille Papyracée, refjemblant aux precedentes, mais la quille ronde, Linn. 27 I. a. Lift. $55^{8} .7 .897$. (I.p. 960. IR 2 $128 \mathrm{~N}$. 
I28 A Argo, oblonga, late carinata, margine angulato, fla. vefcens, dentibus fuscis, Linn. 27r. B. f. o. Seb.1Il.84 8. 1 1. Lift. 555. 6. A. ( (1. fpecim. 60. a.

129 A. Argo, forma \& colore pracedenti fiailis, Linn. 27 I B F. 0. Rumph, 18. B. (2. fpecins. 60. b.

$130 \mathrm{~A}$. Argo, margine rotundato, ceterum pracedenti fimilis Linn. 271. भ. f. 0. Lift. 554. 5. a. Argenv. 5. B.. (2 fpecim. 962.

I3I A. Argo, precedenti fimilis, Linn: 27 r. ช.f. o. Gualth 12. C. (2. (pecim. 962 .

132 A. Argo, præcedentibus in omnibus fimilis, Linn. $27 \mathrm{I}$ \%. f. O. Seb. III. 84, 9. I0. I2. Petiv. Muf. 6. 7, 22 10. (2. fpecim. 962.

I 33 A. Oryzata, late carinata, lateribus ferlatim granulati fragilis, albo flavefcens, Linn. 27 I. $\delta$. . . o. Gualth. I: B. Rumph. 18. 1. Argenv. 5. C. longit. 7. latit. 5. altil 3. poll. ( I. Specim. 59.

133 A. Oryzata, margine rotundata, multoque minor, Lin 27I. ท. L. 0. Lift. 557.7. Seb. III. 84. 4. dextra. (2. Tpecin 961 .

\section{GENVS VIII. NAVTILI.}

134 N. Pompilius, Linn. 273. Rumph. 17. A. longit. 7. pol mingnit. 7. poll. (I. Rpecim 6r. a.

I35 N Pompilius, fimi is pracedenti, paulo minor, Lin 273. Gualth. 17. A. Kircher I. I. Hollar Icon. 3. 3. I (1. Ipecim. 6r.

136 N. Pompilius, fimilis præcedenti, fed color vividior minor, Linn. 273. Argenv. 5. E., Lift. 550. 1. (: freciu. 6I.

I37 N. Pompilins, decorticatus, Linn. 273. Gualtl. I7. S at Lift. 551. 3. 3. (1. fpecim. 61.

138 N. Pompilius, fimilis præcedenti, fed diffetus, ejusder magnitudinis, Limm 273. Gualth. 18. A. \&. 4. Brey Polytbal. I. 1. (I. Ipecim. 6r. 3.

139 N. Pompilius, diffectus, Linn. 273. Rumph. 17. C. (. fpecim. parv. 62.

$140 \mathrm{~N}$ Pompilius, fimilis ac $\mathrm{r} 34$ umbilico fubaperto, Lin .273. Argenv. 5. F. Gualth. 17. B. (2. Ipecim. 61. c

$14 \mathrm{I} \mathrm{N}$. Pompilius, umbilico aperto, Limm. 2/3. Lifl. 550. (2. Ipeciun 6r.c. 
8. Nautille Papiracée, allongée, d quille dentelée $\mathcal{E}$ व dents brungs, Lin . 271. $\beta$. ou o. Seb. III. 84, 8. 11. Lift. 555. 6. $A$ (1.p. 60. $a$.

:9 Natille Papiracée, en forme, E couleur reffemblant a la precedente, Linn. 27 I. $\beta$. ou o. Rumph. 18 B. (2.' p. $60, b$.

\$o Nautille Papiracée, à quille ronde, pour le refte le memie que la precedente, Linn. 27. $\gamma$ ou o. Lift. 554. 5. a. Argenv. 5. B. (2.p. 962 .

3 I Nautille Papiracée, Semblable d la precedente, Linn. 271. y ou O. Gualtb. 12. C. (2. p. 962.

3 a Natille Papiracée, rellemblante en à tout la precedente, Linn. 27 I. $\gamma$. ou O. Seb III. 84. 9. 10. 12. Petiv. Muf. $67,22.10$. (2. P: 962.

3 Ndutille Papiracée, en grains de Ris, d cotes gramulés, fragile d un blanc jauvatre, Linn. $27 \mathrm{r} . \delta$. ou 0 . Guaith. 12. B. Rumph. 18. I. Argenv. 5. C. long . 7. latit. 5. Altit. 3. pouc. (1. P. 59 .

13. Niutille Papiracée, en grains de Ris, à quille ronde. mais beaucoup plus petite, Linn. 271. y. ou 0. Lift. 557. 7. Seb. III. 84. 4. dextra. (2.p. 961.

GE N R E VIII. N A U T I L LES.

34 Nautille Chambréc, Linn. 273. Rumph. 17. 1. long. 7. pouc. (1. p. 6r.a.

is Nautille Chambrée, femblable à la precedente, un peu plus petite. Linn. 273. Gualth. 17.A. Kircher I. I. Hollar Icon. 3 3. $B$ ( I. p. $6 \mathrm{r}$.

16 Nautille Chambrée, refjemblant is la precedente. mais plus petite, c'un couleur plus vif, Linn. 273. Ar:env. 5. E. Litt. 550 r. (2.p. 6r.

37 Nutille Chambrée, " Jans robbe Linn. 273. Gualth: I7. A. Lift. 55I.3. a. (I.p. 6 I.

8 Nutille Chambrée, Semblable d la precedente, ouverte, de la mếne grandeur, Linn. 273. Gualth. 18. A. ED 4. B. ey: Pulythal. I. I. (I. p. 6r. a.

ig Nautille Chamb ée, ouverte, Linn. 273 . Rumph. 17.C. $(2$ p. petites 62 .

No Nautile Chambrés, Jemblable au $n^{\circ}$. 134. ouverte a Son oeil, Linn. 273. Argenv. 5. F. Gualth. 17.B. (2.p. 6i. c.

I Nautille-Chambrée, d̀ oeil ouvert, Linn. 273. Lift. 550. 3. (2. p. 61. c. 
I 42 N. Pompilius, fimills præcedenti, artifici manu floribus foliis pnlcerrime fculptus ; flores ex cortice teltz, ceterum decorticatus, cauda fcuto nobili cum galea, ornata, Petiv. Gaz. 99 9. (I. fpecim. 6r. b.

I43 N. Pompilius, fimilis præcedenti, decorticatus, in quo celeber. aitifex Belkin, triumphum Neptuni graphice ex fculpfit, magnit. 5. poll. (1. fpecim. 6I. d.

I44 N. Spirula, Linn. 274. Lift. 550. 2 Rumph. 20. I. Argenv. 5. G. Gualth. 19. E Kircher I.39. A. B. (4. Specim. B3.

145 N. Spirula, Linn. 279. Petiv. Muf. 22.4. Breyn Polythal. 2. 8. (4. Ipecim. 63 .

\section{GENVS IX. A N GARII.}

146 A. Cornis Arietis, Linn. 674. Rumph. 27. P. Argens. 8. E., Gualth 4. E E. Petiv. Mus. I2. 12 . (4. Ipecim. 64.

I47 A Cornea, fusca fragilior, Linn. 672. Gralth. $3 N$ ? 4. D. D. Lift. 137. 41. Petiv. Gaz. 50. I4, 92.5, IO4 I. (2. Ppecim 65 .

148 A Planorbis, Linn 602. Lift. 138. $42 ?$ Petiv. Gaz. 17. 6. (4. fpecim. 66.

149 A. Emirulus, ore marginato, umbilico lunato, pallide fusca, fis:cia fusca, Limn. o. Seb III 40.II. Lift. 79. 80. Petiv. Gaz 92. 10? (4, fpecim. 67.

I50 A. Diaphana, absque margine, alba, Linn. 0. Gualth. 3. G. Petiv. Gaz. 91. 11? (I Ipecim 73

$15 \mathrm{I}$ A. Ungulina? Lim 682. «. f. o. Rumph. 27. R? Petiv. Muf. 12.13? (I. Precim. 974.

is2 A Lufitanica, Linn. 084. Seb. III. 40.3-5. (2. Ipecim. 68

153 A. Citrina, Linin. 679. Gualth.3. E. Seba. III. 39.7. (4. Thecim. 69.

154 A. Citrina, fumilis præcedenti, Linn. 679. Argenv. 28. 10. Petiv. Gaz. 100. 14. (4. Ppecim. 963-965 d.

is5 A. Grifea. fimilis pracedinti, fascia fusca. Linn. 679. $\propto$. Gualth. 3. E? Seb. III 39. 6. (2 Ipecim. 850.

I56 A. Carnea, fascia alba, Linn. 679. 3. Scb.III.39. 3. (2. \{pecim. 8९9.

157 A Citrina, Linn.679.\%. Seb. III. 39.9. (4. fpecim. 958. 965.

158 A. Citrina, fasciis albis \& furcis, Linn. 679. d. Seb.III. 39. 5. Lift. 60.57. (4. Ipzcim. 965 . 2. b. 
I59 A. Aurantius, Linn. 679. \%. Seb.IIl. 39, 4? (2. Specim. 1017

I60 A P.omutia Linn.677. Gualth. 1. B. Lift. 48. 46. Argenv. 28. 3. (2. fpecim. 70.

16 A. Pomatia, fusca, Ariis albidis marmorata, Linn $677^{\circ}$ a. Lift. 48. 47. Argenv. 28. 3. Gualth. 1. D. (1. Specim. 206.

162 A. Hyalinis, lacteus, Linni. o. Lift. 46. 44? (2. Specim. 467.

$163 \wedge$. Carccolla? an fimilis præcedenti? f. $n^{\circ}$. I6I. fed globo. fior. Linns o. Lift 44 4I. ('2. Specim. 968.

164 A Striatus, citreus fasciis violaceis albi:que, an fimilis no. 162? Linn 0. RumDk. 22. I? Seb. III. 39. 18? Petiv. Gaz. 32.2?. (2. fpecim 71 .

165 A. Nucleus, transparens, albus argute ftriatus, Linm. 0. (I if ecim 72 .

160 A Viviparus, Linn. 690. Gualth 5. M Lift. 126. 26, 130. 30? Petiv. Gaz. 61. 8. Ginann. II.6. (2. Tpecim. 102: 980 .

167 A. Subovatus, gibbus, aibus, argute friatus, Linn. C. Gualth. 5. N. N. Argenv.pars. II. 27.7. prima. Lift.123. 22. (2. (pecım. 803 .

I68 A. Ianthina - cre triargulari, fubeptxrica, Linn. 689.

- Rumph. 20. 2. Aigciv. 6. 5. Gralth. 44. 0. (2. Rpecim. 101

I6g A "Ampullaceus r. Glarucus, tefta fubro:unja. fatis magna, tenuis acsminata, tantisper diapharia, viridi olivacea, lineis trancverfis fliscis diaphanis, Linn. 676. \& 678 .

4al (utiæá funt exdem, una tantum in delinatione differente) Seb. III 38.1-5. Lift. I28.28. ( 2 Tpecim. 104. a .

I70 A. Ampullaceus r. Glèrrus, in omnibus precedentifimilis, Jinn. 676 \&: 678. Lif. 129.29. 30. (2. fpecim. 104. a.

I 1 A. An:pulhcers T. Glascus ? lefta decorticata, cinerea, ac ferie linearum fuscarum fásciats, Linn. 676. \& 678. Argenv. 17. B. Rumph. 27. Q. Seb III. 38. 58.59. Petiv. Mis. I2. I 4. (2 nocim 104 b.

I $72 \mathrm{~A}$ : Ampullaceus? Coftatus, fed apice acuriors, tefta vix dia. phana, cramior, linei, transverfis coftacis, nigro-fusca. T.eimagna, Lirn 676.? 4 678? f 0. Lift. 123. 25. (I. frecith to3.

173 A Ampullaceus? C. Coftatus, in omnibus precedent fi nilis, fuerb-vridi olivaceus, ac pau:o niñor, Lim 676? \& $678 ? 9.0$. 
559 C. Orangé, Linn. 679. n. Seb. III. 39. 4.? (2. p. 1017 .

160 C. Pomatia, ou Caracol, Linn. 677. Gualth. 1. B. Lif. 48. 46. Argenv. 28. 3. (2. p. 70 .

I6r C. Caracol? brun, marbré dè Jtries blancheatres, . Linn: 677.. Lift. 48. 47. Argenv. 28. 3. Gualth. 1. D. (I. p. 966.

162 C. Hyaline, couteur de lait, Linn. o. Lift 46. 44 ? (2? p 967.

163 C. Caracol? ou Semblable à la precedente? ar $n^{2} .16$ : mais plus rond, Linn. O. Lift. 44 4\%. (2. p. 968 .

I64 C. Strise, couleur de citron, à bandes violeties, EO blanches, ou Jemblable aü $n^{\circ}$. Ió2. Linn. O. Rumph. 22 I. Sëb, III. 39. 8 ? Petiv. Gaz. 32. 2 ? (2. P. 71 .

i65 C. Cerneau, transparant, blanc, àftries fones, Linn. 0 . (i. p. 72 .

166 C. Vivipare, Linn. 690 Gualth. 5. M. Lift. I26 26. 130. 30? Petiv. Gaz. 6r. 8. Ginann. 11. 6. (2.p. roz. 980.

i67 C. Buffu, blanc, à ftries fines, Linn. o. Gualch. 5. N.N. Argenv. part. II 27, 7. prima. Lift. 123. 22, (2, p. 863 . 168 C. Janthine, á bouche triangulaire, un peu jpherique. Linn. 689. Rumph. 2u. 2. $\Delta \mathbf{r g e n v}$ 6.5. Gualtb. 44. 0 . (2.p. IOI.

IG C. Osil de Boẹf, à coquille ronde, affez graride, minte? aigue, E' tant foit peu transparante, virt-olivatre. a lignes transverfes örunes transparantes, Linn 676. E 678. (ces deux fout les mêmés, une feule differe dans la debiniation. Seb. III 38. i-5. Lift. 128. 28. (2. p. 104. a

I70 C. Oeil de Bocuf, en tout femblabled la precedente, Linis. 676. Eे 708. Lift. 12.7. 29. 30. (2. p. 104. a.

I7 C Cordon Bleu, Jans robe, cendrée, Es bandée par tune fuite de lignes brunes, I.inn. 676. E9 678. Argenv. I7. $\mathrm{H}$. Rumgh 27. 2. Seb. III. 38.58.59. Pctiv. Mur. 12.14: (3. p. 104.6

I $^{2} \mathrm{C}$ D aru-Pl:frses à fommet plus aigue, à coquible à peine diafbare, plus epriffe, à ligres dentelées transverfes, noirbrune, tres grande, Linn. 676? Ë 668.? ou 0. Lilt. I25. 25 (1. $)$ ic 3.

I73 C. D'aru Piffé, en tout Semblable a la precedente, d"un

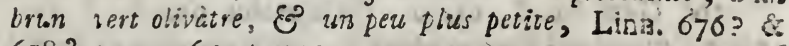
678 ? ou o. (i p. 103 . 
174 A. Carocolla, tefta rotunda fubcarinata, apice vix elevato, $\&$ inde dorfo carinato, viridi fusca, Linn.065 Lift 63. 6r. Seb. III. 40.9. Gualth. 3. I. Argenv. 8. D. (2. fpecim. 90.

175 A. Lucerna-Fasciata, fubfimilis precedenti, alba, utrinque fasciis transverfis caftaneis, Linn 065 \%. . o. Lilt. 67.60. 67? Kircher III. 333. (2. fpecim. 89.

I76 A. Lucerna-Carinata, tefta utrinque plana, acuminuta. lactea, ote carinato, trigono, Linn. o. Lift. 64.66? (r. Ppecim. 93.

177 A. Luceria-Dentata, tefta globofa, obtufe carinata, utringue convexa, ore dentibus 2. plicatis, alba lutescens, Linn. 0. Seb. III. 40. 30. Lift. 90. 90? Petiv. Ga\%. 71. To? (1. fpecim. 978.

178 A. Lucerna-Antiqua, fubrotunda, dorfo acute carinato, utrinque convexa, fusca, Linn. o. Seb. III. 40.24. 25. (r. fpecim. 9 r.

79 A. Ringens, rotundus, elongatus, fubdepreffus, apertura refupinata, flavo albicans, Linm. 664. Liff. 92. 100. Argenv. 28. 13. 14. Petiv. Gaz. 20, 9. Kircher. III $33 \mathrm{I}$. 332. (1. Specim. 92 .

180 A. Lampadaus, imperforatus, oblongus, dorfo carinato. Havicans, margine fusco, Linn.666. c. f. o. Seb. III. 40. 16. 17. Lift. 82. 83 ? (2. Specim. 87.

281 A. Spheridoideus, cinctus imperforatus, fasciis fuscis, albisque, O. Lift. 42. 43. 40. (2. fpecim. 86

182 A. Dittatus, ore oblongn, fpharoideus, vitta alba, ore carneo, Linn. o. Seb. III 40. 6. 7. (1. fpecim. 1050.

I83 A. Ellepticus, teft a convexa elleptica, ftriis fuscis, albiıque, Linn. c. Seb. III. 40. 39? Petiv. Gaz. 100. 13? (2. fpecim. 88.

184 A. Ellepticus, præcedenti affinis, Lirn o. (2. Specim. 88.

185 A. Nigriculus, ore oblongo, fusco grifeus, fasciis quatuor fuscis, ore atro, Linn. O. Lifl. 51.49 ? 1058. i.2. Seb. III. 40. 50. Ginann. II. 4. Yetiv. Gaz. 153.8. (2. Speclm. 862 .

786 A. Flavigulus, perforatus, ore oblongo oblinwo lacteas ore flavo, Linn. o Liff. 1055. 4? 'Ginam. II 8. I6. Pe. tiv. Gaz. 21. 6, 91. 9 (2. (pecim. 972.

187 A. Elongatus, apice elongato, ore marginato, Pallas Spi. cil .... (x. fpecim. 973 . 


\section{Teftaces. Ordre IV.}

374 C. Fauffe Lampe, à coquille ronde, un pers cavée, le fowmet di peine elevé, \&o carne fur le dos, yert-brunc, Linn. 605 Lift. 03. 61. Seb. III. 40. 9. Guaith. 3. I. Argenv. 8.D. (2. P. 90.

I75 C. Lampe-Bandée, fort femblable d lo precedente, blaniche, de chapue cote, d bandes transverfes chataignes, Linn. 065 .. 026 0. Lift. 67. 66. 67.? Kircher III. 333 . (2.p. 89.

I 76 C. Lampe Carinée, doquille des deux cotés polie, platte, pointée, couleur de lait, do bouche carinee triangubaire, Linn. o. Lift. 04. 66:? (I. p. 93.

I77 C. Lampe-Dentelée, à coquills ronde, cove, courbée de chaque coté, la bouche a deux derss pliées, blantjaunatre, Linn. O. Seb. III. 40. so. Lift 90. go? Petiv. Gaz.71. 10? (1. p. 978.

I78 C. Lampe-Artique, un peus ronde, lo dos carinée, cour. bée de chaque coté, brune, Liñ. 0. Seb. III. 40. 24. 25 . (I. p. 9I.

I79 C. Lampe-Antique, rond allongé, E⿱ d'un jaune blancheatre, Linn. 064. Lift 99. 100. Argenv. 28. 13. 14. Petı. Gaz. 20. 9. Kircher III. 331.332 . (1. p. 22.

180 C. Clairon? pas percée allongé le dos creux, jaznatre, to bord brun, Linn. 666. 4. 0u 0. Seb. III. 40. I6. 17. Litt. 82.83 ? ( 2 p. 87 .

181 C. Spheroide, cinglé fans trous, cinglé, à bandes brunes, \& blanches, Linn. o. Lift. 42.43 .40 (2. p. 86.)

182 C. Bandée, do bouklie allongée fphcroide, d bandeiette bianche, E bouche rouge, Linn. O. Seb III. 40. 6. 7. (I.p. IO50.

183 C. Eleptique, à coquille contrbée elettiquse, de. frries brumes, E2 blanches, Linn. O. Stb. III. 40. 39? Petiv, Gaz. 100. I3. ? (2. p. 88

184 C. Eleptique, d̀ opprochant la precedente, Lino. o. (2.ps: 88.

18 s C. Geule-noire, la bouche allongèe, d'un gris brun, \& quatre bandes brunes, la geule noire, Linn. O. Lift. $51.49 . ?$ I058. r. 2. Seb. III. 40. 50. Ginann. II. 4. Petiv. Gaz. 1538 . ( 2 p 862 .

I86 C. Genle-Jaune percḱ, d bouche longue, oblique, ED jau ne, Linn. O. Lift. 1055. 4 ? Ginann. Il. 16. Petiv. Gaz. 21. 6, 91.9. (3. p. 972 .

I87 C. Allongée, à fommet long zue, bouche bordée Pallas Spicil.... (1. t. 973 . 
188 A Undans, ore oblon nombilicatus undis cinereis piequs: Lim. 68 1. $\gamma$. T. o. Gialth. 3. F. Rumph. 27. O. Petiv: Muf. I2. H. (2. Specim. 74.

I89 A Striatus, an fimilis pracedenti? undatim punctatus.; ftriiscue longitudinalibus firscis pictus, Linn. 68 r. $\gamma$. r. o. Seb. III. 40. 48. Petiv. Gaz. 91. 10. (2. frecim. 97 r.

I90 A. Zonaria, umbilicata, ore marginato, 1. Etes. fasciis 2. longitudinalibus fuscis, Limn. 681. Seb. III. 40. 55. Gralth. 3. L. (2. Ppecim. 970.

IgI A. Zonariz? r. varietas, alb\%, friis 2. ferrugineis; Livn. 681. \%. Seb. III. 49. 53. Gualth. 3. C? Lift. IC55. 3? (2. fpecim, 950.

Ig2 A. Zonaria? f, varietas. Linn. 681. 3. Seb. III. 40. 52. Gualth. 3. C. Petiv. Ga3 65. 4? (1. Ípecim. Ic26.

193 A. Nitra, apice acuto, alba, fasciis 2. pallido fuscis, Limn. O Seb. III. 4u. 15? Petiv. Gaz. 118. 1 I? (3. fpecimin. 75 .

194 A. Umoilicus, large umbilicatus, fphæroidens, flavefcens, ftria do fi fusca. Linn o Lift. 75. 75. Petiv. Gaz. OI. 7, 76. 6., Kircher III. 409 4I م. (I. ipecim. 86I.

195 A. Pukctulatus, albus, hitc inde puretis pallide fuscis apice pullo Linn. 0. Lifl 1058. 3. 4. S૯b. III. 40. 15? (2. . . p jecim 76 .

196 A. Crenntus, anfrictibus elevatis contiguis, albus, Limn. 633. «. Petiv. Gaz. 55.7. Valent. 7 (r. Jpecim 8r.

I97 A. Clathrus, Europus, cancellatus, turritus, anfractibus contiguis ! wubus, Limn. 631. Lift. 588. 51. Plancus 57. 8. Gralth. 58. H. ( I. Fecim 80. b

I98. A. Clathrus? i. varietas Jndica, conftis magis contiguis. albus, Iorgitudinal ter fusco punetatus, Linn. 63 . $\alpha$. Rumph 29. IV Petiv Gaz 154.12. (4. fpecim. 80. a. I99: A Clathrus? firilis pracedeni, Lim 63 T. . Lift 588. 50. Gualth. 5S. H. Petiv Muf. ' 3 Io. (3. Ipecim 80. a. 200 A. Anbiguis, umbilicatus, curritus, ore marginato, Linn 632. (1. 亿pecin 79.

201:A. Scalaris, conicus, anfractihus d ftantibus, Linn. 630. Rumph. 94. A. Gualth. ro. Z. Z. Valent. ror longit. 2.

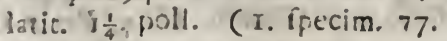

202 A. Scaliris, pracicntifimilis, rubefcens, parvus, Limnz. 630. Arzenv. II. V. Petiv. Muf, 29 (I fpecim. 77 :

203 A. Scalaris? f. variet as, rarifima, alba, tenuis clongara, flurimis contis cuntiguis, Linn. 030. $\alpha$. 1. 0. Pallas Spicil Z 0.6 Fnfa X.p. 33 tab. o. f. 5 long. $1 \frac{1}{2}$ craffit. $\frac{7}{4}$ poll. (I. f,ecim. 78 204 A. 
188 C. Ondée, à louche longue umbiliquée, peint d̀ ondes cen: drees, Linn 68I, y. 0u 0, Gualth 3. F. Rumph. 27. O. Peliv. Muf, 12. II. ( 2 p. 7.4.

189 C. Striée, ou femblable à la precedente? pointées par on tes. en ftries longitudinales trunes. Linn. o8r. $\gamma_{0}$ ou 0.Seb. III. 40. 48. Petiv. Gaz. 91. 10. (2. p. 97 I.

190 C. Zonaire, umbiliquée, à bouche bordée, blanche $\mathcal{E}^{\circ} \mathrm{d}$ deux bandes longitudinales brines, Linn. 68r. Seb. III. 40. 55. Gualth. 3. L. (2.p 970.

igr C. Zonaire? ou varieté, blanche, à deux firies couleur de rouille, Linn .081. $\alpha$. Seb. 1II. 40. 53. Gualth. 3. C.? Lift. 1055. 3 ? (2. p. 969.

192 C. Znnaire? ou varieté, Linr. 681. B. Seb. III. 40. 52. Gualth. 3 C. Periv. Gaz. 65 4? (1. p. 1026 .

I93 C Mitré de point, aigue, blanche, d̀ deux bandes pale brunes. Linn. O. Seb. 111. 40. 15.? Peiv. Gaz. I18. 11 ? (3. p. 75.

194 $C$. Umbilique, large, Jpheroide, jaunatre le dos a ftrie bruse. Linn. o. Litt. 75. 75 Petiv. Gaz. 61. 7, 76.6. Kircher 1II. 409410 . (I. p. 86r.

195 C. Pointelé, blanche, des pointes par ci far la, d'un brun pale, d̀ fomenet trun roir, Linn. u. Lift. 1058. 3. 4. Seb. III. 4 \%. 15 ? (2. p. 76.

196 C. Entaillée, à detours elevées contigues, blancke. Linn. 633. a Puiv. Gaz. 55. 7. Valent. 7 (I. p. 81.

197 C. Clatrus, d'Europe, cancellé, à detours contigues polies, Linn. 631. Lift. 588. 51. Plancus 5. 7. 3. Gualth: 58. H. (i. p. 80. b.

198 C. Clatrus ou varieté, des Indes, à cotes plus contigrues, bianche, Eo pointee en long de brun, Linn. 631. .. Rumph. 20. W. Peliv. Gaz. 154 . 12. (4. p 80. a.

I99 $C$ Clatrus, Jemólable à la precedente, Linn. 631. $\alpha$. Life. 508 50. Gualth.58. H. Putit. Muf. 13. 10. (3. p. So, a. 20n C. Ainbigue, ore Dubicux, umbiliqué, en tours, do bouche bordée, Linn. 632. (I. P 79

2or C Scalaire, ou la Scatata, conique, d detours jepares, Linn. 630 Rumph. 94. A. Gualth. 10. ZZ. Valent ror. long. 2 latit. $1 \frac{1}{4}$. pouc. (1. $p 77$.

203 C. Scalat, Semblable, au precedent, petit, rougatre, Linn. 630. Argenv. Ir. V. Petiv. Muf. 2 9. (1.p. 77

203 C. Scalata? ou varieté, très rate, blorche, allongée, à tlufieurs cotes elevies, contigues, Linn. 630. ж. ou O. Pa!. Spici! Zool. Fafc. X. p. 33. tab. O. f. 5. long. $1 \frac{1}{2}$ crafit. 3. pouc. (I. p. 78 . $204 C$. 
204 A. Elongatus, ore rotundo marginato, ftriato albidis, fafciolis fuscis, Linn. ". Lift 26. 24. Petiv Gaz r18. I1. Seb III. 39. 27? (3. Ppecim. 83.

205 A Protractus, vix umbilicatus, apice obtufo, lacteus, Linn O. Kircher. III 43. (2. Specim. 82.

206 A Uva, ore marginato, glabra, ex albo fufcoque marmorata, Linn. 636. $x$ Lift. 588. 48? (1. Specim.

207 A. Uva? f. varietas, tenuis, ftriata fufca, Linn. $336 . \beta$. Liff. 21. 18. Kircher III. 140. (I Ipecim. 976.

208 A. Uva's fo varietas altera, albida, Linn. 636. ๙. Lift. 588. 49. Gualth 58 D? (1. fpecim. 84.

209 A. Uva, Linm. 636. Gualth. 4. R. Lift 588. 47. Petiv. Gaz. 27. 2 (2. Ppecim. 975.

210 A Uva? nondum adulca, Linn. 636. f. o. Seb. III. 55. 2r. fupra. (2. fpecim. 977 .

GENVSX. RHINOCEROTES.

21 R. Auris Mida, ovali oblonga, fpira rugofa, columella bidentata, Linn 392. Lift. 1058. 6. Petiv Muf. 8. 2, (2. Specim. 96. b.

212 R. Auris Mide, decorticata, Linn. 392. Seb. III. 7I. 21. 22. (2. [pecim. 96. b.

213 R. Auris Midae, nondum adulta, Linn. 392. Seb. III. 71. 6. (1. Specim. 96. a.

214 R. Antris Mide? f. varietas Linn. 392. ๙. f. 0. Lift. 32. 30. Kircher 1I1: 4 r2. (3 fpecim. 979.

215. R. Auris Juda, Lirn. 393. \& Lijt. 1C59. 4. (2. Ipecim. 95.

216 R. Scarabaus, tefta ovats fubancipiti, apertura dentata, Linn. 655. Rumph 27.I Gualth. 4. S. Liff. 577. 3I. Petiv. Gas 4. 10. (2. fpecim. 94.

217 R. Tornatilis, tefta coasctata ovata, acutiuscula, Linn. 394. Lift. 834. 59-6I. ( I. Lpecim. 99.

$218 \mathrm{~K}$. Tornztilis? f. varietas, nigro feriatim punclata, Linn. 394 a. ( 2 f.ecim 98

$219 \mathrm{R}$ Tornatilis? f. varietas, subro undulata, Linn. O. Lift. 814. 24. (1. Specim 97.

220 R. Amarula, tefta anfractibus fpinofo dentatis, Linm. 702. Rumph 33. F. F. Lift. 1055. 8. Seba III. 33. 24. 25. (2. Ppecim. ro0.

$221 R$ Amarula, alba, Linn. 702 Lift. 133. 33. Petiv. Muf. 4. 3. (I. Ppecim. 100. a. 
$204 C$. Allongée, à bouche ronde bordée, ftriée, blanche, da bans des brunes, Linn. O. Lift. 26. 24. Petiv. Gaz. II8. II. Seb. III. 32. 27 ? (3. P 83.

205 C. Protractus. to peine umbiliqué, is fommet reboushé, cousleur de lait, Linn. o. Kircher III. 43. (2. P. 82.

206 C. Enfant Emmaillotte, pelé à bouche bordée, Eo marbre de brun, EO blanc, Linn. 636. \%. Lilt. 588. 48 ? ( $x_{\text {. }}$ (p. 85 .

207 C. Enfant Emmaillotté $?$ ou varieté finement ftriće, brune, Linn. 036. A. Lift. 21. 18. Kircher III. 140. ( $1 . p_{0}$ 976.

208 C. Enfant Emmaillotté? ou autre varieté, blancheatro, Linn. 636. *. Lift. 588. 49. Gualth. 58. D.?. (1. P. 84.

209 C. Enfant Emmaillout, Linn. 636. Gualth. 4. R. Lift, 588. 47. Petiv. Gaz. 27. 2. (2. p. 975.

$210 \mathrm{C}$. Enfant Eumaillotté? mais pas encore parfaite, Linn. 636. ow 0. Seb. III. 55. 21. Supra. (2. p. 977.

\section{GE NRE X. P OR TE COR NES.}

2:I P. Oreilles de Midas, longue ovale, a replis ridés, colus melle d derx dens, Linn. 322. Litt. Y058. 6. Petiv. Mur. 8. 2. (3. P. 96. b.

$2 \times 2$ P. Oreille de Midas, fans robe, Linn, 392. Seb. III. 7I. 21. 23. ( 2 p. 96.b.

21 3 P. Oreille de Midas, pas encose parfaite, Linn. 392. Seb. III. 71 6. (I. p. 96. a.

2r4 P. Oreille de Midas? ou varieté, Linn. 392. ※. or 0 , Lift. 32. 30. Kircher. III. 412 . (3. p. 9790

is $P$. Oreille de Judas, Linn. 393. «. Lift. 1059. 40 ( 2. P. 05 .

216 P. Scarabe, \& coquille tranchants ovale ouverture dentelée, Linn. 655. Rumph. 27. I. Gualth. 4. S. Lift. 577. 31. Periv. Gaz 4. 10 (2. p. 94.

$217 P$. Tornatile, d coquille coarctee ovale, replis ou fpire aigue, Linn. 394. Lift. 834. 59-6r. (1. p. \$9.

218 P. Tornatile? ou variete, pointee de noir, Linn. 394 . (2. p. 98.

$219 P$. Tornatile? ou autre varieté, ondée de rouge, Linn.o. Lin. 814. 24. (I. p. 97.

$220 P$. Amarule, la coguille, detours dentelés. Linn. 708. Rumph. 33. F. F. Lift. 1055. 8. Seb. III. 53. 24.25. ( 2 p. 100.

22I P. Amarule, blanche. Linn. 702. Lift. 133. 33. Petiv. Muf. 4. 3. (1, p. 100 a. 
222 R. Achatina? f. varietas ovata, apertura, \& apice fanguineis, Linn. 391. d. f. o. Seb. III. 7 I I8? 20? Lift. 23. 21 . (1. ffecim. 117.

223 R. Achatina, huic appofita ova, in quorum uno Embryon, Linn. 391. f. o. Lift. 23 21. \& Exerc. Anat. I. tab. 6. f. I. a. b. (4. fpecim. 117.

224 R. Zebra, alba, ftriis undatis fuscis, Linn. o. Argenv. Suppl 2 L. Lift. 582. 35.a. Hollar Icon. 14. 14. (2. Ipecịn. I 20.

225 R. Achatina? I. varietas, fusca, apice rofeo, Linn. 39 I. ๙. Petiv. Gaz 152 II. (2. Ipecim. IIg.

226 R. Achatina? i. varietas, Linn. 391. $\beta$. Seb. III. 7 1. 7. 8. Lift. 58 r. 35 " (2. fpecım. I'9.

227 R. Achatina, ore albo, fpira rofea Linn. 39r. Gualth. 45. B Lift. 579 34., Argenv. 10. E. (2. Tuecim. I18.

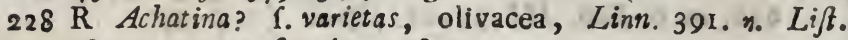
581. 35? 2. fpecim. I $184 \mathrm{a}$.

229 R. Achatina? f. varietas, fusco fiammata, Linn. 39r. $\propto$. f. o. Seb. III. 7 1. 2. 10. Adanfon Seneg. I. 1. 5. I. Lift. o. 4 ? (2. fpecim. I09. a.

230 - R Achatina? f. varietas, hepatica, Linn. 391. B. f. o. Lift. 29. 27? (2. fpecim 985.

23 I $\mathrm{R}$ Priamus, teffellata, ventricola, dorfo fubcarinato, colore grifeo, maculis fuscis quadratis, feriatim digeltis, Linn. o. Gronov Zooph. III. n०. 156r. tab. I9 f. I0. I1. Petiv. Gaz. 155. 13? ( I Tpecim. ro49.

232 R. Carinata, tefta ventricola, futcarinata, apice acuto, Lim. O. Lift. 580. 34. a. Seb. 1II. 39. 54. 55. (2. Specim. I08.

233 R. Fasciata, tefta oblonga, columella fubumbilica, alba, fascia longitudinali rofea, Linn o. Liff. I's. 8? Petiv. Gaz. I04. 6, 155 I2. (2 fpecim. I14.

$234 \mathrm{R}$ Virgines? f. varietas, tefta fubturrita erecta, columelia truncata, fanguinea, Limn 390. ๙. f. o. Lift. I 2. 7. Argenv. Suppl. I. G. Seb. 39. 62-74, Petiv. Gaz. 22. M. ( 2. frecim. 107.

235 R. Virginea? albido flava. fusco maculata, Linn. 390. ж. f. о. Argenv. 11. M. Kircher III. 66, Petiv. Gaz. 151. I. Seba III. 9 69. 70. (1. fpecim. I07.

$236 \mathrm{R}$ Virginea, tefta alba, fasciis 2. flavis, columelia rofea, Linn. 390. -Gualih 6. D. Lift. 15. 10? (2. Ipecim. 081

237 R Virginea, tefta lactea, fasciis fuscis, flavis, aurantiis violaceisquo, Limm. 390. Argenv. I I. N. ( 3 fрec.·106. $238 \mathrm{R}$. 


\section{Teftaces. Ordre IV.}

222 P. Achatine ? ou Cloche ou varieté, ovale, ouverture \&5 fommet rouges, 23. 21. Linn. 39I d. ou O. Seb III. 71. 18 ? 20? Litt. 2321 . (I. p. 117 .

$223 P$ Achatine? ou Cloche, ou varieté, avec lesoerfs, dans un des quels l'Embryon, Linn. 391 . ou o. Lift. 23. 21 ? Exerc. Anat. I. tab. 6. f. I a. b. (4. p. II7.

224 P. Zebre. blanche, à ftries ondées brunes, Linn. o. Argenv. Suppl. 2. L. Lift. 582. 35. a. Hullar Icon. 14. I4 (2.p. 120. 225 P. Achatine? ou Cloche, ou varieté, brune, à fommet rouge, Linn. 391. a. Petiv. G2z. 152. Ir. ( 2 p 119.

226 P. Achatine? ou Cloche, ous varieté, Linn. 3or. B. Seb. III. 71. 7. 8. Lift. 581.35 ? (2.p. II9.

227 P. Achatine? ou Clocne, boucbe blanche, Jpire rouge, Linn. 391. Gualtin. 45. B. Lift. 579. 34. Argenv. IO.E. (2. p. 118. $228 P$. Achatine? ou Cloche, ou varieté, olivatre, Linn. 39 r. ท. Lift. 581. 35 ? (2. p. II8, a.

229 P. Achatine? ou Cloche, ou varieté, flanmé de brun, Linn. 39r. * ou o. Seb. III, 71. 9. 10. Adanfon Seneg. I. I. 5. 1. Litt. 9.4 ? (2. P. 109 a.

230 P. Achatine? ou Cloche, ou varieté, hepatique, Linn. 321. B ou 0. Lift. 29. 27.? (2. P. 995 .

23 I P. Priam, en pieces quarrées, le dos creux, couleur gris, do taches brunes quarrées, arrangées en fuite, Linn o: Gronov. Zoøph. III. n०. 156I tab. 19. f. IO.II. Petiv. Gaz.155. I3? (1. P. 1049.

232 P. Creux, coquille is grand ventre, un peu croux, d fommet aigue, Linn. 0, Litt. 580. 34. a. Seb.III. 39. 54.55. (2.p. 108 .

$2 \hat{3}$ P. Bandé, coquille longue, columelle fubimbilique, blanche, à longze bande rouge, Linn. O. J.Ift. 13. 8 ? Peciv. Gaz. 104. 6, $155.1 \%$ (2. P. 114.

234 P. Pavilion d'Hollande? à coquille.. efpece de tour, erigée, columelle tronquée, couleur. de Jang, Linn. 390. .. ou C. Litt. 12. G. Argenv. Suppl 1.7. Seb III. 39.6274. Petiv Gaz 22. 11. (2. $p$ 107.

235 P. Pavillon d'Hollande, jasne blarcheotre, tacheté de brwn, Linn 390. क. ou o. Argenv. 11. M. Kircher III. 66 Periv. Gaz. 15i. 1. Seb III. 39.69. 70. (1. 107.: 236 P. Pavillon du Prince coquille blanche, a deux bandes jaunes, columelle couleur de rofe, Linn. 390, Gualth. 6. D. Lift. Is 10? ( 2 p. 98 r.

237. P. Pavilion du Prince, coquille blanche, d barides.trunes; jawnes, oranges, violettes, Linn.390. Argenv.21.N. (3. p. 106. 
$235 \cdot R$. Virginia apice magis rofeo, Linn. 390 . Seb. 111 。 40. 38. Gualth. 6. A. (3. Tpecim. 106.

239 R. DeprefJa, fasciis angulatis fuscis, Linn. O. Lift. Io. 5 Petiv. Gaz. 76. 5. (4. fpecim. Ic9.

$240 \mathrm{R}$ Perverfa? i. varietas, tefta fubumbilicata, ovato oblonga, flava, maculis fuscis, Linm. 688, o. f. o. Petiv. Gaz. 44. 7. (I. fnecim. III.

24 I R. Perverfa? f. varietas, fulphurea, Linm. 688: \%. f. 0. Liff. 24. 22? Kircher III. 148. (1. Ppecim. II2.

242 R. Perver $/ a$, ore finfltro, Linn, 688. Lifl. 34. 33. Argenv. 6 G. Gualth. 5. P. (2. Specim. 113.

243 R. Perverfa? \& varietas prxcedentis, alba, trinsverfim ftriata, Linn. 688. a. f. o. Lift 35. 34, Seb. III. 40. 37, Gualtb. 5. O. (2. Specim. 984.

244 R. Perver $\int a$ ? f. varietas, grifea, obfcure maculatim facciata, Linn. 688. B. .. o. Liff. 33. 3I? Kircher III. 110 ? (2 fpecim. 983 .

245 R. Perverfa? f. varietas, flava, ftriis duplicatis purpureis, Linn. 688 y. f. o. Lifl. 33. 31. (2. fpecim. I10.

246 R. Perverfa? f. varietas alia, alba fasciis longitudinal:bus fuscis, Linn. 688. 8. .o. o. Seb. III. 3i. 32? Petiv. Gaz. 105. 8 ? (.r. fpecim. 1053.

247 R Perver $\sigma_{a}$ ? f. varietas, alia, fafciis interruptis fuscis,

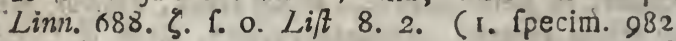

$248 \mathrm{R}$. Acuminata, ore anguftiore, tefta oblonga fubturrita alba, Lim. 0. Lift. 14. 9? (2. (pecim. 125.

249 R. Stagnalis, ovato fubulata, fubangulata, Linn. 703. Kircher III. 392. Petiv. Ga3. 10. 8. (4. fpecim. 105.

250 R. Decollatus, fpira nutilat') truncata, Linn. 695. Liff 17. 12, Gualth +4 O. Petiv. Gaz. 105. 7? (2. Specim I16. a.

251 R Decollatus, fimilis pracedenti. Limm. 695. Gualth. 4. P. Petiv. Gaz. 66 I. 105. 7.? Lif. Conch.tab. 20. f. I5 (4. fpecim. I16. b.

GENVSXI. NERITE,

252 N. Eluctifera, oblonga, Spira acuta, lactea, Iinn. Kircleer $11 I, 371$. (3. fpecim, 185 .

253 N. Guttata, coftata, alba, nigro purctata, Linm. o. Lifi 597 9. Gualth 66 P. (2. fpecim. 993.

254 N. Pitta, migro rubroque varia coftata, Linn。 o. Lij 595. 2. Kircher IIII. 214. (6, Ipecim. 147. 
238 P. Pavillon du Prince, à Jominet plus rouge, Linn. 390. Seb. III. 40. 38. Gualth. 6. A. (3. p IC6.

239 P. Deprimé, d bandes brunes en Angles, Linn. 0. Lift. 10. 5. Petiv. Gaz. 76. 5. (4-p) Iog.

240 P. Perverfe? ou varieté. ¿̀ coquille ovale longue, jonne, à tackes brunes, Linn. 688. d.0u 0. Petiv. Gaz.44. 7. ( $\mathrm{x}$. p. III.

$24 \mathrm{I} P$. Perverfe? ou varieté, couleur de Soufre, Linn. 688. ๆ. ou O. Lift. 24. 22? Kircher III: I48. (I. p. II2.

242.P. Perverfe, à bouche finiftre, Linn. 688. Lift. 34. 33. Argenv. 6. G. Gualth. 5. P. (2.p. II 3 .

243 P. Perverfe? ou varieté de la precedente, blanche, frié do travers, Linn. 688. 2. oul 0. Lift. 35.34. Seb. III. 40. 37. Gualth. 5. O: (2. p. 984.

244 P. Perverfe? ou variêté, grise, offctrement tácbetée, \&o bandée, Linn. 688, B. ou 0. Lift. 33. 31 ? Kircher III. 110 ? (2. p. 983 .

$245 P$ Perverfe? ou varieté, jaune, id doulbles ftries pourprées, Linn 688 \%. ou o. Lift. 33. 31. (2. p. rio.

$276 \mathrm{P}$. Perverfe? où autre warieté, blanche, d̀ longues bandes brunes Linn: 688. 8. ou o. Seb. III. 31. 32? Petiv. Gaz. 105. 8 ? (p. 1053.

$247 P$. Perverfe? ou autre varieté, blanche, à bandes brunes interrompues, Linn. 688. Z ou 0. Lift 8.2. (I-p.982. 248 P. Poirtée à bquche plus etroite, "coquille longue blanche, Linn. 0. Lift. 14. 9.? (2. p. 115.

249 P. Stagnaie, ovale en Angles, Linn. 703. Kircher $I I I$. 392. Petiv. Gaz. 10. 8. (4. P. 105.

250 P. Decollé, a Spire Mutilée, detronquée, Linn. 695. Lift 17. 12. Gualth. 4. O. Petiv. Gaz. 105* 7.? (2. p. II6. a.

251 P. Decollé. Semblable d la precedente, Linn. 695. Gualth. 4. P. Petiv. Gaz. 66. I, 105. 7 ? Lift. Conch. tab. 20. f. 15 . (4. 8.116 b.

\section{GE NRE XI. NERITES.}

252 N. Fluctifere, longue à jpire aigue, blanche Linn, O Kircher III: 371 . (3. P. 185.

253 N. Gouttée, dcotes blanche, pointée de noir, Linn. O. Lift. 597. 9. Gualth. 66. $P$ ( 2 p. 993.

254 N. Peinte, varié de noir, Eo rouge, a cotes, Linn. 0. Lift. 595. 2. Kircher III. 214 . (6. p. 147 .

$$
S_{2}
$$


255 N Pilata, viridi Havoque varia, Linn. o. Lifl. 6.5. 31. Pttiv. Gaz. 15.8 (2. fpecim. I45.989.

256 N. Vérficoler, alba, fasciis tribus flavis, Limn. O. Gualti. 66. M. Rumph 22.5? Petiv. MuJ. I1. 21. (3. Specim. 990

257 N. Succincta. alba viridi punctata, fafciis 2 flavis, Linn. 0. Argenv. 7. Z. Seb. III. 59. 11 ? (2. Specim 991.

258 N. Maculnja. viridi maculata, fasciis rubsis, Linn. o. Rumph. 22.L. Gualth.66. T. Lift. 595. 1. (3. Specim $992^{\circ}$.

259 N Nigella, nigro maculata, Linn. 0. Gualth. 66.X. (5. fpecim. 146 .

260 N. Exuvia, albo nigroque varia, Linn. 739. Lif. 599. 15. Rumph. 22. 3, Argenv. 7. B. (3. Rpecim. I52.

261 N. Exuvia? f. varietas, nigro maculata, Linn. 739. . Lift. 599. 15. Petiv. Gaz. I3. I2, (2. Fpecin. 15I.

$262 N$ Undata, fulcis, undata albo nigroque varia, Lim. 735 \&. Lift. 590. 4. Gualth. 66. V. Seh. III. 59. 6-9. Rumph. 22. N. Petiy.Gaz. 100.6. (6. Cpecim. 150. $\mathrm{a}-\mathrm{c}$.

263 N. Melanoleucas, profunde transverfim fulcata, nigro maculata, Linn. O. Rumph. 22. 4. Gualilz. 60. 2. Petir. Gaz. 11.20. (1. '́pecim. I48.

264 N. Melanoleucas? C. varietas, nigratota, Limn.0. Lift. 596. 5. Argenv. 7. I. (3. Cpecim I 49.

265 N. Varia, nigro alboque undulata, Linn. o. Lift. 598 13. Gualth. 66. O. (3. Ipecim. I54.

$260 \mathrm{~N}$. Petechialis, columella macula rubra notata. alba, Linn 0. Lift. 598. 12. Cualth. 66. I. Argenv. 7. E. ( 3. Trecim. 155

267 N. Gemmata, vix dentata, alba, viridi punctata, Lima. c. Lift. 598. 14 Gualth. 66. L. ( 2 fpecim. 156.

268 N. Flava, Linn. o Lift. 603. 22. Gualth. 66. B? Adaz. fon Seneg. 1. I 3. 9.5. (2. Specim. I 57.

$209 \mathrm{~N}$ Viridicata, columella punctn aurantio notata, viridi alboque undulata, Linn. 0. 'Lift. 600. 16. Wualth. 66. AA. ( 1 . Cpecim. 997 .

270 N. Pullata, dentata, ftriata, nigra, Lirn. 0. Lift. 596. 6? 10 (1. fpecim. 153.

271 N. Fasciata denticulata rugo $\Upsilon_{a}$, alba, fasciis viridibus, Linn. 0. Gualth. 66. P. Lift. 597. 10?' (3. Specim. 143. 
255 N. Peinte, varié de vert, EO jaune, Linn. 0. Lift. 605. 31. Petit Gaz: 15. 8. (2. p. 145989.

$256 N$. Verficouleur. blanche, a trois bandes jaunes, Linn. 0. Gualth. 66. M. Rumph. 22. 5.? Petiv. Muf. II. 2I.

(3. P. 990.

$257 \mathrm{~N}$. Succincte, blanche pointée de vert, à deux bandes jaunes, Linn. O. Argenv. 7. Z. Seb. III. 59. II? (2. P. 991.

:58 N. Tacheté, à bandes rouges, tachetée de vert, Linn. 0 Rumph. 22. L. Gualth. 66. T. Lift. 595. I. (3.p. 992.

:59 N. Noirette, tacbetée de noir, Linn. O. Gualth. 66. X. (5. p. I 46 .

:60 N. Exuvia , ou la Grive, variée de noir, E̊ blanc, Linn. 739. Lit. 599. Is. Rumph. 22. 3. Argenv. 7. B.' (3.p. I52. :6r N. Exuvia? ou la Grive, ou varieté, tachetée de noir, Linn. 739. *. Lilt. 599. 15. Petiv. Gaz. 13.12. (2. p.151. $62 N$. Ondée, par de fillons ondée, EO variée de blanc Ẽ noir, Linn. 738. $\alpha$. Lift. 596. 4. Guath. 66. V. Seb. III 59. 6-9. Rumph. 22. N. Petiv. Gaz. I00. 6. (6. p. I 50. $a-c$.

$\sigma_{3} N$. Melanoleucas, profonde, fillonnée de travers tachetée de noir, Linn. O. Rumph. 22. 4. Gualth. 66. 2. Petiv. Gaz. II. 20. (I. P. 148.

$64 \mathrm{~N}$. Melanoleucas? ou varieté . toute noire, Linn. o. Liff. 596. 5. Argenv. 7. I. (2. p. 149.

$65 N$. Varié, ondee de r.oir, Eo blanc, Linn. 0. Lift. 598. 13. Gualth. 66. O. (3. p. 154.

A 66 N. Petecbiale, columelle blanche, à tache rouge, Linn. 0. Lift 598. I?. Gualth. 66. I Argenv. 7. E. (3. p. I55. 67 N. Perié, à peine dentée, blanche, pointée de vert, Linn. o. Lift. 598. 14. Gualth. 66. L (2.p. 156.

$68 \mathrm{~N}$. June, Linn. C. Lift. 603. 22. Gualth. 66. B. ? Adanton Seneg. I. I 3. 9. 5. (2.p 157.

og $N$. Verdatre, columelle pointée d'orange, ondée de vert. of blanche. Linn. 0. Lift. 600. 16. ,Gualth. 66. $A A$. (x. p. 997.

70 N. Noirette, dentelée, friée, noire, Linn. O. Lift. 596. 62 10. (1. p. 153.

$71 N$. Bandée, dentelée, ridée blanche, ¿a bandes vertes, Linn. O. Gualth; 66. P. Lift. 597 . 10.? (3. P. 143. 
272 N. Deforata, a'ba, fasciis undatis rubris viridibusque labio fanguine inaculato, Limn. O. Gualth. 66. C. Z. Argenv. 7. $H$. (4. ipecim. 184.

273 N. Punctulata, alba punctis faturate viridibus, Limn. 0. Lift. 596. 4? (1. fpecim 173. a.

274 N. Cinfte, albida, fasciis viridibus, Linn. 0. Lift.596.7. (4) fpecin. I 58

275 N. Notata, alba , fasciis undatis olivaceis, Linn. o. Gualth. 66. L? (I. fpecim. I59.

276 N. Virens, maculis pallidioribus, 'Linn. 0. Argenv. 7. $N$ ? ( 2 fpecim. I60

277 N. Punctatc, viridis punetis faturatioribus, Linn. O. Lift 599. Is? (I. Rpecim. I6r.

278 N. Pallida, lutea, lineis olivaceis, Linn. O. Lift. 603. 23. (1. fpecim. 165.

379 N.Violacea, lineis purpureis, Linn. o. Lift. 603.21? Gualth. 66. C? (r. Ipecim. 162.

280 N. Carnea , f. varietas pracedentis? fasciis viridibus, Linn o Lift. 60?.20. ( 1 , fpecim. 163.

28 I N. Muftelina, citrea, albo undata, fascils viridibus, Linn. c. Gualth. 66. H? (2. Ipecim. 160. a.

282. N Prafina, albida, maculis atris, fascia lata olivacea Linn 0. Rumph. 22. 7? (r. fpecim. 166. b.

283 N. Nivea, teffa argute ftriata, alba, Limn. O. Seb. III 4I. 22. S. 24. (I. fpecim. I68.

284 N. Grifea, viridigrifea, fasciis flavis, Linn. o. Seb. III 4I. 20 ? (1. Tpecim. 169.

285 N. Purpurata, viridi marmorata, fasciis purpureis, Lim o. Seb. III 41. 27. Rumph. 22.K? Argenv. 7. K. (: rpecin. 170.

286 N. Purlurata? r. varietas, pallidior, Linn. O. Seb. II 59. 2. Petiv. Muf. I I. 6. (3. Ipecim. 170.

287 N. Purpur.ta? I. varietas, \& minor. Linq. O. Rump 22 2? Gualth. 66. E? (5. fpecim. 170.

288 N. Ramoja, fasciis untatis fuscis, Linr. O. Rumph. 2 I? Argenv 7. P. (2. \{pecim. I7I.

289 N. Marmorata alba, fasciis marmoratis viridibus, Lin o. Gualth. 66 E. Seb III. 59. 22? (4. fpecim. 172.

293 N. Marmorata, pracedenti affinis, diaphana, fasc Iuteis , maculis viridibus, Linn. Q. Seb. III. 59.2 (3. Ipecim. I72, b.

291 N. Diaphana, alba, fasciis viridibus, Linn. 0. Lift. 5! 3? Petiy. Muf, I1. 20? (4. fpecim. I 73. 
$272 N$. Quenotte Saignante, blanche, à bandes ondées, rouges E' vertes, levre tachetée de rouge, Linn. o. Gualch. 66 . C. Z. Argenv. 7. H. (4. P. 184

$273 N$. Pointelée, blanche à pointes vertes, Linn. O. Lift. 536. 4? (s. p. 173. a.

$274 N$. Cinglée, blancheatre, ì bandes vertes, Linn. O. Lift. 596. 7. (4. p. 158.

$275 N$. Marquée, blanche, da bandes ondées olivatres, Linn. 0. Gualth. 60. L.? (1.p. 159.

276 N. Verte, d̀ taches pales, Linn. o. d'Argenv. 7. N? p. 160 .

$277 N$. Pointée à plufieurs pointes yertes, Linn. 0. Lift. 599. 15.? (1.p.161.

278 N. Pale, jaune, d rayes olivatres, Linn. 0. Lift, 603.23. (I. P. 165.

279 N. Violette, d rayes pourprées, Linn. 0. Lift. 603. 2I? Gualth 66. C.? (I p. 162.

$230 \mathrm{~N}$. Couleur de Chair, varieté de la precedente, rayée à barsdes vertes, Linn. o. Lift 602.20. (I. p. Inз.

28.' 'N. Mufteline citroné, ondée de blanc, \& bandes vertes, Linn. O. Gualth. 66. H.? ( 2 p 166. a.

$282 N$. Prafine, blancheatre, it taches noires, E large bande olivatre, Linn. 0. Rumph. 22. 7? (I. p. I66. b.

$283 N$. Blanc de Neige, $a$ coquille ftriée de blanc, Linn. 0. Seb. III. aI. 22,04.24. (I. p. In8.

$284 N$. Grife, verte $\& 9$ grise, d̀ bandesjaunes, Linn. o. Seb. III. 4r. 20 ? (1. P. 169.

$285 N$ Pourprée, marbrée de vert, ¿̀ bandes violettes, Linn. o. Seba III. 4 I. 27. Rumph, 22. K? Argenv. 7. K. ( 3. P. 170 .

$286 \mathrm{~N}$. Pourprée? ou varieté plus pale, Linn. O. Seb. III. 59. 2. Petiv. Mur. II. 6. (3.p. I 70.

$287 N$. Pourprée? ou varieté, plus petite, Linn. O. Rumph. 22 2? Gualth. 66 E. ? (5.p. 170.

$2.88 N$. Brarchue, à bondes ondées brunes, Linn. o. Rumph. 22. I? Argenv. 7. P. (2. p. I 7I.

289 N. Marbrée, blanchs, à bandes Marbrées vertes. Linn.o. Gualth. 66. E. Seb. III. 59 22.? (4. p. 172. a.

290 N. Marbrée? Jenblable à la precedente, diaphane, à bandes jaunes, Eo taches vertes, Linn. O. Seo. III. 5y. $2 \mathrm{I}$. ( 3. D. $172, b$, 峞

29 İ $N$. Diaphane, blanche, à bandes vertes, Linn. o, Lift, 595. 3? Petiv. Muf. 11. 20,? (4. P. 173. 
2) 2 N. Diaphana? f. varietas, fasciis nigro viridibus, minor pracedente; Linn. o. (4. fpecim. 173.

293 N. Cinerea, apıce flavo, cinerea, fasciis faturatioribus, Linn. O. Rumph. 22. I. (5. fpecim. 174.

294 N. Ziczac, alba, maculis Havis, flriata, Limn. o. Seb. III. 4 I. 17. (1. Specim. I75.

295 N. Lutea, grifeo rubens, lineis diaphanis faturatioribus, Linn. o. (I. Specim. 869

296 N. Rubra, fasciis duabus albis, Linn. o. (i. Rpecim. 164

$297 \mathrm{~N}$ : Lineuta, thavo lutea, fasciis 3 albis, Linn: c. Petiv. Gaz. 34. 5? (2. fpecim. 167 .

$293 \mathrm{~N}$ Lineata? f. varietas, nigra, lineis albis, Linn, o. Arger.v. 7. Z? (1. fpecim. 180.

299 N. Fasciata, alba, fasciis 2 atris, Linn. O. Rumph. 22. 7. Petiv Muf. 11,23. ( I. fpecim. 179.

$300 \mathrm{~N}$ Fasciata? f. varietas, fusco flava, fasciis 2 latis nigris, Limn O, Petiv Gaz 34. 4? (1. fpecim. 178.

zor N. Viridis, olivacea, fascus 4 albis, Linn. o. Rumph. 2. $D$ ? ( 1 fpecim. $17 \%$

302 N Carinata, carnea, fasciis 4 albis, Limn "o. Rumplt. 22. 1.? 2. Petiv. Muf.22. I2. (1. fpecim. 176.

303 N. Aibicilla . Labiis fubdentatis interinre tuberculato' alba, maculis airis, Linn. 733. Seb. III. 41. 23. ( $2^{\circ}$ fpecim. 18r. a.

304 N. Livida, lineis 2 fuscis, Linn. O. Kumph. 22. 7? (2. riecin 998.

305 N. Tigrinz, alba punctis rigris, Limm. o. Petiv. Muf. 21. 10? (2 fpecim 18:.

306 N Reticulata, alba violacco reticulara: Iinn c. Lift. 541. 36, 601. 19, Naturforfch. XIII. tab. V. f. 1. a. b. (1. Specim. 183 .

307 N. Phrygia, labi, interiore edentulo, tefa fphærica, alha. lineis canillaceis vinlaceis, Linn. O. Lift. 00532 . Naturforfch. XIII tab. $V$. f 2 c-h (2. fpecim. 134.

$3 \odot 8 \mathrm{~N}$ Pbrygionia, fusco violacea. puretis albi-, fasciis 2 niveis..Linn o Lift 606. 34 ? (I '́pecim. 142.

309 N. Phrygionia? f. varietas, violacea, maculis alhis nigro ncellaris, Linn. o. Lift. Cot 24? Petiv. Gaz. 68.8. Kircher III. 2.4. (r finecim. 994.

310 N. Trahalis, violacea, fasciis albis, nigro marginatis, Linn. 0. Liff. 60u4. 25? Petiv. Gaz. 68. '4, 156. II. (2. Tpecim. 995. 
292 N. Diaphane? ou varieté, a bandes d'un vert noir, plus petite que la precedente, Linn. o. (4. p. 173 .

$293 N$. Cendrée, a fommet jaume, cenirée, àbandes remplies, Linn. O. Rumph. 22. I. (5. p. 174 .

294 N. Zic-zac, blanche friée, taches jaunes, Linn. O. Seb. III. 41. 17. (I. p. 175.

295 N. Jaune, gris.rouge, is plufieurs rayes diaphanes, Linn. o. ( 1 p. 869 .

296 N. Rouge, à deux bandes blanchos, Liñ . o ( 1 p 164.

297 N. Lignée, jaunatre, à trois bandes blanches Linp. $n$. Petiv. Gaz. 345 ? (2. p. 167.

298 N. Lignée? ou yarieté, noire, a rayes blanches, Linn, $p$. Argenv. 7. Z? ( $1, p .180$.

299 N. Bandśe, blancbe à deux basdes noires, Linn. O. Rumgh. 22. 7. Petiv. Muf. 11. 23. (i. p. 179.

$300 \mathrm{~N}$ Bandee? ou variere, a'un brus jaune, à deux bander larges noirs, Linn. O. Petiv. Gaz, 34. 4 ? (I. p. 178 .

$301 N$. Verte, olivatre, $\dot{a}_{4}$ bandes bianches, Linn. o. Rumph. 22. D.? (I. P. 177 .

302. N. Creux, rouge, à $\&$ bandes blanches, Linn o. Rumph. 22. 1.3 2. Petiv, MuC. 22.12. (1. p. 176.

$303 N$. Blancheatre, à levres dentelies, l'interieur à iulenciles, \&O taches noires, Linn. 733 . Seb. 1II. 4 I . 23. ( 2. p. $188 . a$.

$\jmath_{0} 04 \mathrm{~N}$ Livide, blanche, ì deuxrayes lrunes, Linn, o. Rumph. 22. 7.? (2. p s98.

305 N. Tigrée, blanche, à pointesnoires, Linn. C. Petiv. Mur. 2I. 10 ? (2. p. 181 .

$306 \mathrm{~N}$. Enfilets, blanc violette reticulée, Linn. o. Lift. $54 \mathrm{r}$. $3 \sigma, 601$ 1 9 . Naturforfch. XIII. ts b. V. f. I. a. b. (1, p. I $\delta 3$.

$307 N$. Phrygie, ou la Bodée, la levre interieure edentée; a coquille jpheriazue blanche à rayes violettes, en fils, Linn.o. Lift. 605.32. Naturfurfch. XIII.tab.V f.2.c-h. (2.p. (34. $308 \cdot N$. Phrygione, brun violet, pointes blanches, à deux ban. des blanches de neige, Linn. O. Lit. oc6. $34 . ?$ ( 1. p$14^{2}$.

309 N. Phrygione? ou varieté, violette, it taches blanches, moucheté de noir, Linn. O. Lift. 604. 24? Petiv. Gaz.68. 8. Kircher III. 204. (I. p. 994

3ic $N$ Traba'e, violette, $a$ bandes biarches, bordées de noir, Linn. O. Litt. 654. 25. ? Petiv. Gaz 68. 4, 156. 1 I. (3. p. 995. 
3 Ir N. Qculata, violacea, ocellis albis, Linn. o. Lift. 605. 36. "(2. Ipecim, 996 .

$3 \mathbf{2}$ N.Virgata, alba, ftriis obliquis nigris, Linn。 o. Seb. III. $4 \mathrm{I}$. in medio (I fpecim 14I.

313 N. Corona, anfractibus fpinis coronatis, Linn. 720. Rumpis.22. O. (1. Ipecim.186.

$3{ }^{4}$ N. Corona? f. varietas, nigra, Linn 720 a. f. o. Argenv. 7.2. (2. Ppeciiw, 1037.

$315 \mathrm{~N}$. Pulligera, ovalis, fisca, tuberculis oviformíbus obfita, Linn. 726. Rumph: 22. H. Gualth 4. HH? Sel. III 4I, 26. I. Cpecim 182

$316 \mathrm{~N}$. Lineata, lineis a ulatis atris, olivacea, Limn, $O, P e-$ tiv. Gaz. 156. 9? (3. '́pecim 135.

317 N. Triangularis, viridis, maculis triangularibus atris, Linn. Linn. O Lift. 604. 29. (3. F ecim. 137.

$318 \mathrm{~N}$ Angulofa, rofea, line 's angulatis nigris albo marginatis, Lir.n. O. (I. T, tecrm. 136 .

$319 \mathrm{~N}$ Plumbea, punetis draphanis slois, Linn. O. Lift.604. 28. (1. fpecim. 138 .

20 N. Fasciata, brunviea, fasciis nigris, Linn. o. (2. Ipe. cim. 139 .

$321 \mathrm{~N}$. Reticulata, glabra. fasciis alternatis albis nigrisque, rubro altog e reticulatis, Linn. o. (2. fpecim. 140 .

322 N. Granulo $/ a$, oblonga, convexa, apic acuto granuiata, Limn. o. Rimmph. 22. M. Petiv. Muf. I1.8. (2. Specim. $x 44$.

GENVS XII. NERITR FORMES.

323 N. Albrumen. albido cinerea flavefcens, Linn. 718. « Seb. III. 4I. 9. Lift 563. 10. (2 fpecin. I2r.

324 N. Allumen, pracedenti affinis, cl erea Limn. 718. $\alpha$. Grallis 67. B. I. Argenv. 7. V. (2. fpecim 121.

$325 \mathrm{~N}$. Preffa? f. varietas, umbilico fub ayice, flavefcers, Linn 718. $\beta$ f. O. Seb. 111. 41. 1c. 11. Lift. 562.9. (3. Petcim 122.

326 N. Fasciata, oblonga, ovalis, lactea, fasciis 2 fuscis, Linn. o. Seb. III 4I. 20? (2. frecim. 806.

327 N. Putamen, fuscu Alavefcens, Linn. o. Lift 566. 15. Seb III. 41.21. Gualth. 67. D. (2. Poecim. I29.

328 N. Mammilla, Hlava, Limm. 719 . Seb. III 38. 33. Lift. 566. 14? Petiv. Gaz. I52. II, (4. 'pecim 988.

329 N. Mammilla? f. varietas, aiba tota, Linn. 7 rg. a. f. o. Seb. III. 38. 9. Lift. 57 I. 22. Rumpho. 22, Fo (3. Ipecim. $: 3 \mathrm{I}$. 


\section{Teftaces. Ordre IV.}

3 II N. Oculée, violette d yeux blanches, Linn. 0. Lift. 605: 36. (2. p. 996 .

$312 N$. Virgate, blanche, \& fries obliques noires, Linn. ${ }_{0}$ Seb. III 4I. in medio. (I. p. 44 I.

$313 N$. Couronne, ou Fluviatile, au milieu les anfractes couronnées d'epines, Linn. 720 . Rumph. 22. O. ( (,$p$, 186.

$314 N$. Couronne? ou Fluviatile, ou varieté, noire, Linn. 720. ๙. ou 0. Argenv., 7. 2. (2. p. 1027.

$315 \mathrm{~N}$. Enceinte, ovale, brune, à tubercules en forme d'Oeuf, Linn. 726. Rumph. 22. H. Gualth. 4. HH? Seb. III. 41. 26. (1.p 182 .

316 Zic-zac, olivatre, rayée, à lignes angulaires noires, Linn. o. Periv. Gaz. 156. 9.? (3. p. 135 .

$3{ }_{7} \dot{N}$. Triangle, verd, à taches triangalaires noires, Lian. $a_{0}$ Lift, 60429. ( 3. P. 137.

$318 \mathrm{~N}$. Angulaire, couleur de rofe, à lignes angulaires roires? bordée de blanc, Linn. O. ('T. p. 13 '.

$3 \mathrm{r} 9 N$. de Plomb, a pointes transparantes blanches, Linn. $O_{0}$ Lift. 604. 28. (x. p. 138.

320 N. Bandée, lrune, à bandes noires, Lion. O. (2.) 139.

32. N. Reticulaire, pelé, is bandes alternatives blanches, reticulée, de rouge, È blanc, Linn. a. (2. $p, 140$.

$322 N$. Granulée, longuie, courbée, à Jonnet aigue gronulés. Linn. o. Rumph. 22. M. Petiv. Muf. I1. 8. ( 2 , $p$ 144.

GE NR E XII. NERIT OIDES.

$323 N$. Tefticule, ou Blanc d'Ozuf, blanc cendré, jaunatre, Linn. 718. a. Seb. 1II. 4 I. 9. Lift. 563. 10. (2. p. I22. $324 N$ 'Teftcule, ou Blanc d'Oeuf, Sembbaúle à la precederte cendré, Limn. $718 . \propto$ Gualth. 67. B. I. Argenv. 7. F. (2 p. 12 r. $325 \mathrm{~N}$. Tefticule, ou Blanc d'Oeuf comprimée, ou variete, l'us'sbilique fous le fommet, jasnatre, Linn. 7 I8 $8.0 u$ C. Seb. III 4I. 10. 1r. Lift. 562. 9. (3. p. 122.

326 N. Mampelon bandée, allongée, ovale, blanche à derux bandes brunes, Linn. o. Seb, III. 4T. $30 . ?$ (2. p. 866.

$327 N$. Coque d'Oeuf ou Mammelon, brun jaune, Linn. 0. Lift. 566.15. Seb. III. 41.21. Gualth. 67. D. (2. p. 129. $328 N$. Mammelle, jauns, Linn, 7 19. Seb. III. 38. 33. Lilt。 566. I 4 ? Petiv. Gaz. I52. I1. (4. p. 988.

329 N. Mammelle? ou varieté, partout blanche, Linn. 710. ou 0. Seb. III.38. 2. Lift 5iI.22, Rumph. 22. $F$. (3. p. $13 \mathrm{r}$. 
330 N. Mammilla? C. varietas, longior \& alba, Linn. 7Ig $\beta$. f. 0. Seb. III. 33. 10. Arger. 7. X. (1. fpecim $9^{\times}$.

331 N. Mammilla? if varietas, alba, punctis croce s Linn. 719. $x$. f. o. Seb. III. 38. 22 (I. fpecim 130:

332. N. Vitellus, tefta umbilicata fubglobofa, flava, fascía lutea, maculis albis, Linn. 717. Lift. 505. 12. Guabth. 67. L. Argenv. 7., $A$. (3. Tpecim 123.

333 N. Vitellus? 1. varietas, apertura umbilicali ad apicem usque pertura Linn. o. Seb IIL. 38. 33. Gualth. 07. X. Kircher III. 228. (4. Specim. 124.

334 N. Cinerea, r: flavefcens, Linn. O. Seb. III 38.8 ? Gualth. 67. I? (1. fpecim. 86i.

335 N. Punctata, favefcers, punctis fuscis, Limn. o. Seb. III 38. 60. 61. Gualth 67. Q. Argenv. 7. C. (3. fpecim. 865.

336 N. Undata, fasciis undulantibus flavis, Linn. o. Seb. III. 38 26. Lift. 567. 17. Gualth. 67. M. (2. Specim. 867 .

337 N. Fusca, fusco flua pund is faturatioribus cincta , Limn. 0. Gualth. 67. V. Seb. III. 38. 65. (2. fpecim. 125.

338 N. Fasciata, alhida flavefcens fasciis albis puncatis, Linn. O. Seb III. 38. 29. Lift. 560. 4 Gualth. 67. T. (4. Specim. 996.

339 N. Fasciata? א. varietas, cinerea fasciis 4 albis, fusco punctatis, Limm. 0. Lift 560. 3. (I. Rpecim. 1038.

340 N. Cana, flavo cinerea, 'a ciis 2 latıs fuccis, Linn. Lift. 505. 13. Argerv. 7. Z? (1. Tiecim. 868.

$34 \mathrm{l}$ N. Striata, pallida, fasciic luneisque angulatis cincta, Linn. 0. Seb. III. 4I. 15? Lift. 56r. 8. (1. Rpecim. 328.

342 N. Lemniscata, fusca, linea alba in dorfo, Linn. 0. Seb III. 4I. 14. (3. Fre im. 127.

343 N. Punctata, alba, pureti fuscis, Linn. O. Lift. 5 f́o. 5, 564. 11. Gualtis. 67. N. Adanjon Seneg. I. 13. 7.2. Petiv Gaz. 100. 9.? 10. (5. Ipccim 126.

344 N. Nigella, tita nigra, Livin. 0. Gualth. 67. F. (2. rpecim. 132.

345 N. Pifum, forma Turbinis, flavefcens, Linn. o. Gualth. 64. N. (2. fрecim. 133.

GENVS XIII. TVR BINES.

345 T. Olearius, viridis, tuberculis nitidis, Linn. 621.

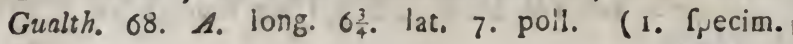
187. 


\section{Teftaces. Ordre IV.}

330 N. Mammelle? ou varieté, blanche, plus grande. Linn. 79. F. ou O Seb III. 38. 10. Argenv. 7. X. (I.p.937.

33I. N. Mammelle? ou varieté, blanche à pointes couleur de jaffrar, Linn. 719 r. ou ๑. Seb. III. 38. 22. (r. p. 130.

$332 N$ Jaur $s$ d'Oeuf, d coquille enfoncée, jaunatre, d bande jaune, El taches blanches, Linn. 717. Lift. 565.12. Gualth. 67. L. Argenv. 7. A. ( 3 p. 123 .

$333 N$. Jaune d'Oeuf? ou varietś, l'ouverture de l'umbiliqus ou nombril percé jufqua au fommet, Linn. o. Seb. III. $3^{8 .}$ Gualth. 67. X. Kircher II1. 228. (4. P. 124.

$334 N$. Cendrée, jauratre, Linn. o. Seb. III, 38. 8.? Gualth, 67. I? (I. p. 864.

335 N. Pointée, jaunatr?, a pointes bruses, Linn. o. Seb. IIT. 38. 60. 61. Gualth. 67. Q. Argenv. 7. C. (3, .365.

$336 \mathrm{~N}$. Ondée, ḋ bandes jaunes ondulées. Linn. O. Seb. III. 38. 26. Lift. 567. 17. Guath. 67.M. (2. p. 867 .

337 N. Brune, jetune, remplitie de poirts, Linn. 0. Gualth. 67. V. Seb. III. 38. 65. (2. p. 125.

338 N. Bandée, blanche, jaunatre. d' bandes blancbespointées, Linn. O. Seb. III, 38. 29. Lift, 560. 4. Gualch. 69. $T$. (4. p. 986.

339 N. Bandée? ou varisté, cendirée. à 4 bandes blanches, foina tées de brun, Linn. o. Lift. 560. 3. ( $\mathrm{r}_{0}$. . 1038.

$340 N$. Grife, cencirée, d deux larg bandes brunes, Linn. o. Lift. 565. 13. d'Argenv. 7. Z? C.r. p. 868.

34I N. Striée, pale, cerclée de bandes Eे rayes en angles, Linn. O. Seb. III. 41. 15.? Lilt. 561. 8. (I. p. 128.

342 N. Liée, brune, ligne blanche fur le dos, Linn. o. Seb. III. 4I. I4. (3.p. 127.

$343 N$. Pointée, blanche, à pointes brunes, Linn. 0. Lift. 560. $5,504.11$. Gualth. 07. N. Adanfon Seneg. I. 13. 7. 2. Petiv. Gaz. 100. 9? 10. (5.p. 126.

$344 N$ Noiratre, par tout noire, Linn. O Guath. 67. F. (2: p. 132.

345 N. Pifum, en forme de Turban, jaunatre, Linn. 0. Gualth, 64. N. (2. P. 133 .

GENRE XIIT. TURBANS.

$345 T$. Huilier, verd, at tubercules polies, Jinn. 621. Gualth. 68. A. long. 03 lat. 7. pouc. (I. p. 187 . 
346 T. Marmoratus, viridis, maculis fuscis, Linn. 619. Ruinph. 19. B? (3. Specim. 188.

347 T. Marmoratus, decorticatus, Linm. 619. (3. ippecim. 188.

348 \%. Marnoratus? f. varietas, olivacea, Linn 6r9. \&. f. o. Seb. III: 74. 1. 2. Gualth. 64. A. (2. fpecim. I89. 349 T. Marmoratus? f. varietas, minor, Linn. 619. *. f. o. Rumph. 19. A. Lift. 587. 46. (2. .pecim. I89. 350 T. Pincernatus, viridis, ftriis fuscis, Linn. O. Seb. IIIT. 74. 4. Argenv. 6. C. (3. fpecim 190.

351 T. Petholatus, anfrattibus fubangulatis, Linn. 612. Seb. III. 74. 18. 19. 26-30. Rumph. 19. D. 3.5-7. (4. fpecim. 191.

\$52 T. Petbolatus, faturatior, Linn. 6r 2. Valent. $53-56$. (4. fpecim. igr.

353 T. Petholatus, pallidior, Lim. 612. Lifl. 584. 39. (4. ipecim $19 \mathrm{t}$.

354 T. Petholatus, fimilis, Lim. or 2. Gualth. 64. F. (4. fpecim. 191.

355. T. Petholatus, fed minor, Linn 612. (5. Rpecim. 19r. 356 T. Petholatus, major, fasciis viridibus, Linn 612. «. Argenv 6. G? (3. Specin. 19r. a.

357 T. Petholatus? fovariet as, pullata, Limn. 6I2. B. f. o. (2. \{pecim. 1018.

358 T. Cocbles? f. varietas, granulata, ftriata, viridi fusco. que varia, Limm. 613 \%. f. o. Seb. III. 74. 25. Gualth. 64. D. Rumph. 19. 3. (2. fрecim. 999.

350 T. Cochlus? fo yarietas, fine granulis, maculis paucioribus, Linn. 613. S. C o. Argenv. 6. A. (2. Specim. 999. 360 T. Cochlus, granulatus \& itriatus, Linn. 613. * Seb. III. 74. 20 ? Liff. 584. 40, Valent. 80. (2. Tpecim. s92.

$36 \mathrm{I}$ T. Funicrlates, granulatus, ex albo virens, maculisque fuscis, Linn. 0. Rumph. 19. 4, Valent 80. 81. (2. fpecim 193.

302 T. Cochlus ? r. varietas. fulcatus \& maculatus, albo fla. vefcente, Linn. 613. B. f. 0. Rumph. 19. 3. (1. fpe. cim. 196.

${ }_{363}$ T. Chryfoftomus, Linn 614. Seb. III. 74. 10. II. Petiv. Muf. 5. 3. (2. fpecim. 197. a.

364 T. Chryfoftomus, flavo virens, Linn. 6r 4. Argenv. 6. D. (2. 'pecim 197. a.

365 T. Chryfoflomus, decorticatus, glaber, Linn. 614. Argenv. 6. I. (2, \{pecim. 197. b. 
$346 T$. Peau de Serpent, verd d tackes brunes, Linn. 619. Rᄂmph. I9. $B$ ? (2. p. 188.

347 T. Pau de Serpent, marbre, fans robe, Linn. 619. (3. p. 188.

$348 T$. Peau de Serpent? or varieté marbré olivatre, Linn. 6.9 ou 0. Seb III. 74. 1. 2. Gualth, 64. A. (2.p. 189. 349 T. Péau de Serpent? ou yarieté marbré, plus, petit, Linn 619 \%.0u o. Rumph. 19. A. Lif. 587.46 . (2. p. 189. $350 T$ Peau de Serpent, ou Burgeau ? verd ', flriesbrunes, Linn. - Seb. III. 74. A. Argenv. 6. C. (3. P. 190.

351 T. Petholate, ou Ruban, de replis füanglés, Linn.612. Seb. III. 74. 18. 19. 26-30. Rumph. 19. D. 3.5-7. (4.p. 191 .

352 T. Ruban, plus remplie, Lirn. 6r 2. Valent. 53-56. (4. p. $19 x$.

353 T. Ruban, plus pala, Linn, 6r2. Lift. 584. 39. (4. p. I91.

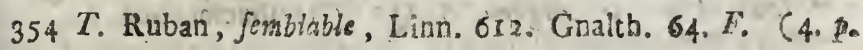
$10 \mathrm{I}$

355 T. Ruban, pius petit, Linn. 6r2. (5. p. 195. 356 T. Ruhan, plus grand, d bandes vertes, Lim. O12, Argerv. 6. G.? ( 3 p. Ior. $a$.

357 T. Ruban? ou varieté, noirstre, Linn. 612, $\beta$. ous o. (2. p. 1018.

358 T. Cochlus, ous Bouche d'Argent? ou varieté, gramulée हo? friés, de brun, E' verd, Linn. 613.y ou o. Seb. III. 74. 25. Gualth. 64. D. Rumph. I9 3. (2. p. 999.

359 T. Cochlus, ou Bouche d'Argent? ou varieté, Jans grains, as perude taches, Linn.613. D. ou 0. Argenv. 6. A (2. p. 999. 360 T. Cochlus, ou Bouche d'Argent, gramulée, Ẽ ftriee, Linn. 613 «. Seb. III. 74. 20? Lift. -58 4. 40 . Valent. 80. (2. $p .192$

361 T. Cordonné, granzulé, vert blencuieatre, $\cdot a ̀$ taches branes Linñ. O. Rumph. 19. 4. Valent 80.8r. (2. p. 193.

$362 T$. Cochlus ouBouche d'Argent? ou yarietée, tachetée de blanc, Eo jaune, Linn. 61.3. B. ou o Rumph. Ig 3. (1. p. 196. $3 \sigma_{3}$ T. Chigfoftome, ou Bouche d’Or, Linn. 6 14. Seb.III. 74. 10. Il. Petiv. Muf. 5 -3. (2. p. 197. a.

$364 T$. Bouche d'Or, jaune verd, Linn 614. Argenv. 6. D. (2. p. 197:a

365 T. Boucne d'Or, demantelé pelé, Linn. 6r4. Argenv. 6. I. $\quad(2, p, 197 . b$. 
366 T. Cochlus, ramofus, Linn. 613. Seb. III. 74. 20, Gualth. 64. B. (2. fpecim. I94.

307 T. Cochlus, C. varietas, Linn. O. Seb. III. 74. 7. Rumph. 19. 2. Kircher III. I1. Argenv. 0 I. Gualth. 64. H. (3. fpecim. 195. a. 870

368. T. Cochlus, decorticatus, Linn. O. Seb. III. 74. 7. (4. pecion. 195. b.

359 T. Diffortus, fubmucronatus, purpuratus, Linn 627. Seb. III. 59. 8. (2. Tpecin. 198.

370 T. Diftortus? f. varietas, Linn. 627. a. Lift. 608. 46. a? Seb. 111. 59. 9. Valent 97. (2. Ppecim. 199

37I T. Delphinus, ex a'bo purpuratus, Linn. 626. Lift 608. 45. Cualth. 68. C. (5. Ppecius. 200

372 T. Arion, ramis obliquis Inngis, pallide rofeas, Linn. o. Seb. III. 59. 1. 2. (I. fpecia. 1039. —— "Simile "fingulare objecrum invenicur in Mujeo SERENISS. PRIN, cip: aravsion: \&c. \&c. \&c.

373 'T. Nodofius, absque umbilicn, granulatus, ex albo griSeus, Lim. o Argenv. 6. Q? (1. fpecim. 201.

\section{GENVS XIV. TROCHI.}

374 T. Sarnaticus, nodofus \& atratus, Linn. 620. Banain. 1II. 4c6. (1. Ppecim. 208,

$\$ 75$ T. Sarmaticus? r. varietas, adhuc tunicala rubra, Lirn. 620. w. Argeriv. 8. B. (I fpecim. 209.

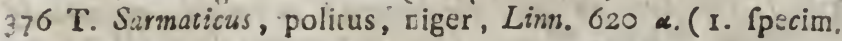
207.

377 ' $\mathrm{T}$. Rugofus, ex albo virens, decorticatus, Linr. O18. ๙. f. O. Lift. 647 4I. (2. fpecim. 210.

378 T. Rugo, fzs? fo varietas, decorticatus, Linn. 618. «. Co. Lif? 640.38. (r. fpecim. 1003.

$3: 9$ T. Caftaneres, fusco faturatus, Linn. O. Argenv. 6. T? (1. frecim. 217.

380 T. Notatus, grifeus, lineis fuscis, abruptis varius; Linn. ก. Gualth. 62. M? (1. fpecim. 216.

¿8r T. Maculofus, fusco pollutus, Linn. o. Argenv. 6. T. (4. fpcbim, 1004.

382 T. Granulatus, fuscus, punctis albis, Linn. о. Gualth. 02. N. Petiv. Gaz. 70. 11. (I. 'pecim. 221.

$383 \mathrm{~T}$. Sulcatus, grieles, Linn o. Gualth. 62. D? (I. frecin. 222.

$3: 4$ T. Pundtatus, a!bidus, maculis fuscis, Linn. o. Lift. .039 .273 ( 1 . fpecta. 213. 


\section{Teftaces. Ordre IV.}

366 T. Cochlus, ou Bouche d'Argent, branchue, Linn, 6rs. Seb. III. 74. 20. Gualtb. 64. B. ( 2 p. 194.

$367 T$. Cochius, ou Bouche d'Argent? ow varieté, Linn. ๑. Stb. III 74. 7. Rumph. I9. 2. Kirch.r III II Argenv. o. I G alth. 64. H. (3.p. I)5. a 870 .

$368 T$. Cochlus, ou Bouche d A rgent, demantelé, Linn. 0. Seb. III. 74. 7. ( 4 p. 195 . b.

$369 T$. Grimace, ou Dauphin, poinle, pourpré Linn. 627. Seb. III. 59. 8. (. 2. p. 198.

$370 T$. Grimace ou Dauphin? ou sarieté, Linn. 627. a. Lift. 608 46. a ? Seb 1II. 59. 9. Vaient 79. (2. p. 199.

37 I T. Dauphin, blanc pourpré, Linn. 626. Lift. 608. 45. Gualth. 68. C. (5. p. 200.

$372 T$. Arion, d bandes obliques longucs, couleur de refe-pale, Linn. o. Seb. III. 59 1. 2, (x) p. 1039. " Le "pareil de cet objet admirable, Je trouvedans le Cabinet de " s a s. le Prince d'orange et nassau, \&c. \&c. \&c. 373. T. Noueux ? Sans umbiique, granulé, d'un gris blaxc, Linn. 0. Argenv.0.2? (I p. 201.

GE NRE XIV- SABOTS.

374 S. Veuve noire, nesé, Linn. 620. Bonann. III. 406. (I $p 208$.

375 S. Veuve noire? ou varieté, encore vetu d'une tunique rousge, Linn. 620. * Argenv. 8. B. (1. p. 209.

376 S. Veuve noire, polie, Linn. 620 .. (1. P. 207.

377 S. Turban ridé, dun verd blame, Linn. 618. ou 0. Lift 647.41. (2. p. 20

$378 \mathrm{~S}$. Turban ridé ?ou variezé, demantelé, Linn. 6is. « ou o. Lift. 046. 38. (I. p. 100 s.

379 S. Chataigne, remplie de brun, Linn. O. Argenv. 6. T.? (I. P. 217 .

380 S. Marqué, gris, d rayes brunes, detachées, Linn. 0. Gualth. 62 M.? (1.p. 216 .

38 ז $S$. Tacheie, d taches brunes, Linn. o. Argenvms. T. (4. p. 1004

$382 S$ Granule, brun, à points blancs, Linn. 0. Gualth. 62. $N$ Petiv. Gaz 70. 1. (1. p. 221.

383 S. Bouton fillonne, gris Linn e Gualth.62. D.? (1. p. 222. 384 S. Bou on pointé, blanc, à eaches brunes, Linn, O. Lift. 639.27 ? (1. p. 213.

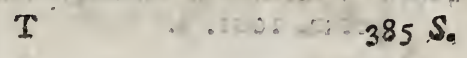


385 T. Coloratus, rofeus, enfractu nigra alboque, Limn. Lift 648 42, (1. fpecim. 220 ,

$386 \mathrm{~T}$. Candens, ore Alvo, Linn o. (T. frecim. 212.

387 T. Leinnifcatus, lineis fusco rubris, Lim?. Q. Lift, 639 28 ? (1. Rpecim. 87\%

388 T.Tenia, cirereus, lineis, abruptis fuscis, Linn. o. Lif, 642. 34? Gualth. 64. F. (2. fpecim 219.

389 T.Litteratus, glaber, niger, punctis albis, Lims. o. Lift 639. 34 ? Gualth. 63. I? (1. fpecim. 218.

$390 \mathrm{~T}$ Labio? f. varietas, rafeus, mac slis fuscis, Linn. 595 ж. f. o. Lift. 645. 2?. Gualth. 63, R. (.6. fpecim. $2<3$

39r. T. Tumidus, enta inthata lpherulued, nitida, Linn. o Petiv Gaz 57. 10. (2. Ppecim. 214.

392 T. Inflasus, rubrs ftriatus, Linn. 0. Liff. 018. 4? (2 fрecim1: 215.

393 T. Infatus? f. varietas, flavus, Linn. O. ( I. fpecim. $2 n$ C 394 T. Inflattus? C, varietas, glaber, lacteus, Linn. O. (i ili foecin. 1028.

395 T. Diadema, tefta nigra convexa, apice obturo, Lim 0. Davila, I? 5. K. (I. fpecim. 226.

396 T. Tubor, tefta oblique tuberculata, albida, virifi ma culata, Linn. 596. Seb. III. 60. 1. Argenv. 8. R? (1 Specim. 229

397 T. Tuber, albicans, virili maculatus, Limm. 596. \&. (1/i) ipecim. 1005.

398 T Tuber? ᄃ. varietas, Lim. 596. $\beta$ F. o. Seb. ill. 74. 13. 14. (1. Tpecim. 227.

$329 \mathrm{~T}$ Triber? f. varietzi, preffus, maculis olivaceis, Lin 596 o f o. Seb. III. 74. 12. ( 2 fpecim. 228.

400 T. Tuber? f. varietas:, elevatus, fardide-tavus, Linn 596. $\gamma$. . . . ( I. f, ecim. 872 .

40I T. Spinofus, pallide virens, ferie duplici fpinarum, Lin 6. Lift 57.5. 28. (I. fpecim. 202.

402 T. Glabolla tefta inflata virefcens, albn fasciat2 Einn. O. Lift. 576. 29? Valent. 35. (3. Tpecim. 2:1. 403 T. Ghabella? C. varietas, rofes, Limm. O. Gualth. 64. C (I. . Specim. 205.

40+ T, Venater, tefta glabra, ftriata \& vixidis, Linn. o. Li 576. - 9 (I fpecim. 203.

40.5 T. Venator? C. varietas, virens nebulofus, Linn. O. ( fpecim tooo.

$400 \mathrm{~T}$ Venotor? $\Gamma_{0}$ varietas, viridi rugrofus, Linn o., ( $\therefore$ fpecim. 100 r. a. 407. 
$407 \mathrm{~T}$ Venator? r. varietas, fasciis 3 rubris, Linn. O. ( $\mathrm{r}$ Specim. $100 \mathrm{r}$.

408 I. Venator? .. varietas, fusc ex flavo marmorata, Lim: 0. (1. fpecim. 10 2.

409 T. Pagodus, Linn 616. Lift. 644. 36, Seb. III. 60. 3 Petiv. Muf. 10. 8. (3. Specim. 23I. a.

410 T. Pagodus? f. varietas, fuscus, Linn. 6I6. «. Argen: 8. P. Gualth. 60. M? (2. Ppecim. 231. b.

4 II T. Pagodus? f. yarietes, minor, Linn. 616.ß. (I. (ps cim. $10 \mathrm{cos}$.

$412 \mathrm{~T}$. Zizyphinus, flavus, rubro fuccoque maculatus Linn. 599. Gualth. 61. C. Rumph. 21. 1. (4. Specin 232.

413 T Conulus, grifeus, Linn. 598. Gualti. 6r. L. N. ( fpecim. 873 .

414 T. Striatus, ex rubro marmoratus, Linn. 597. ^. Gualt. 61. G. (I. fpecim. 874.

415 T. Striatus? f. varietas, lutea, Linn. 597. B. (1. Sp cim. $100 \%$.

416 T. Nitens, ex flavo fuscus, Limm. O. Gualth. 61. (2. Specim. 234 .

417 T. Nitens? f. varietas, decorticata, Linn. o. Ruml 21. II? 2. fpecim. 233.

418 T. Ramofus, rainis longis concavis, cinereus, Linn Lift 628 14. Gualth. 60. Q. (1. Ppecim. 230 .

419 T. Pyramida, imperforata. apertura quadrata. alba, fa ciis viridibus, Linn. 0. Lift. 619. 5? 625. II, Guah no. R. (4. 'pecim. 237.

420 T. Latus, tefta a'ba viridi fasciata, Linn o. Seb.,I 60. I. infra, Gualth. 60. A. Lift. 646. 30. (3. fp cim. 40

42 I T. Nodofus, Ariis viridibus rubrisque, Linn. o. Cual 6I. D. Left 626. Ir. a. (2. Specim. 235.

422 T. Canaliculatus, anfractibus canaliculatis, Linn. Rumph. 21. A? (4. fpecim. 236.

423 T. Maculatus, àlbus, ex rofeo ftriatus, Linn. sib Gualth 61. E? Petiv. Muf. 10. 9, 21. 7. (2. fi) G. cim. 238.

424 T. Maculatus, decorticatus, Linn. 580. (3. Spec 238.

425 T. Maculatus? f. varietas, decorticatus, Linn. 580. Gralth. oI. DD. $?$ Petiv. Mru. II. 1. (I. Rpecin, 243. 


\section{Teftaces. Ordre IV.}

o7 S. Chaffeur, ou varieté, \& trois bandes rouges, Linn. O (I.'p. ICOI.

$08 \mathrm{~S}$. Chaffeur, ou variete, brun, marbré de jaune, Linn. o. (I. P. I $\mathrm{CO} 2$.

09 S. Pagnde, ou Cul de Lampe, Lin 616. Lift. 644. 36. Seb. III. 60. 3. Petiv. Muf, 1o. 8. (3. p. 231. a.

S.Pagode, ou Cul de Lampe ? ou varieté, brune, Linn 616.4. Argenv. 8. P. Gualth. 60. M? (I. p. 23I. b.

in $S$. Pagode, ou Cul de Lampe? ou varitté; plus petite, Linn. 6I6 ß. (I. p. 1006.

si2 S. Żizyphine, jaune, tacheté de rouge E? brun, Linn..599. Gualch. 6I. C.Rumph, 2I. I. (4.p. 232.

: 3 . Cone, gris, Linn. 598. Gualth. or. L. N. (1. p. 873.

$4 S$ Cone ftrié, marbré de rouge, Linn. 597. *. Gualth. 61. G. (I. p. 874.

5 S. Cone ftrié? ou varieté, jaune, Linn. 597 . B. (I. p.j1007.

$6 \mathrm{~S}$. Splendide, d'un brun jarne, Linn. o. Gualch. 61. B: ( 2.p 234 .

7 S. Sp'endide, ou varieté demantelée, Linn. o. Rumph. 21. II? (2. P. 233

8 S. Branchue, cendré, a longues branches, cavées, Linn. o. Lift. 628. I4. Gualth. 60. $Q$ (1. p. 230.

9 S. Piramide, pas perforé. d ouverture quarrée blanche, ¿d bandes vertes. Linn. O. Lift. 6r9. 5? 625. 11. Gualth. 60. $R$ (4. p. 237 .

o S. Piramide large, à coquille blanche, bandée de vert, Linn. O. Seb. III. 6I. I. infra, Gualth. 60. A. Lift. 646. 30. (3. p. 240.

I S. Piramide noúe, a ftries vertes, \& rouges, Linn. a Gualth. 61. D. Lift. 026. I1. a. (2. p. 235.

2 S." Piramide canalée, les anfractures canaliculés, Linn. a Rumph. 21. $A$ ? (4. p. 236.

$3 S$ Pirawide tachetée, blanche, ftriée de rouge, Linn. 580. Gualth.61. E. ? Petiv. Mur. 10. 9, 21. 7. (2. p. 238. it S. Piramide tachetee, $\sigma^{2}$ demantelée, Linn. 580. (3. p. 238. 5 S. Piramide tacheté, ou varieté demantelée, Linn. 580. Guaith.6I. DD. $?$ Petiv. Muf, II, I. (I. p. 243. A. 
420 T. Maculatus? f. varietas, decorticatus, Linn. 580. , Rumph. ) 3? ( 2 fpecim 242.

427 T. Niloticus? ex 10 feo \& viridi maculatus : Lin 579 Gualth. 59. B. Rumph. 21. 3. (2 Rpecim 239

428 T. Niloticus? f. varietas, Linn. 579. \& Gualth 59. Rumph. 21. A. Lift. 617. 3. Kircher. III. 102. ( rrecin. 229. a.

429 T. Niloticus? I varietas, Linn 579 B. Argenv. 8. ( Lift n2., 6 (2. Ppecim. 239. b.

430 T. Niloticus? r. varietas, viridi albaque, Linn. 579. A (3. $\{$ ecim 239 b

43 I T Asper. undique granulatus, virefcens, Linn. Rumph. 21. 6? Lift. 630. 16. Gualth. 60. A. (3. Ipt cim 10:9.

43. T Asper? f. varietas, ex rofeo virefcente, Linn. Rumph. 21. 5? ( 1 fpec m 1008

433 T. Granulatus, ex rofeo \& flavo ftriatus, Linn. o. Gualt: 60. B. 4 ppecim. $24 \mathrm{I}$.

434. T. Granulatus? r. varietas, virefcens, Linn. o Gualt, 61. DD Rumph. 21. C. 9. 10 ( 4 (ppecim. 243.

435.T Vellicatus, virefcens, Lintr. 0. Lift.632.20? Gualt. 6o. P. (2. ppecim. 242. a.

436 T. Pica . tefta glabra, nigraque, Linn. 622. AdanJon S reg. 1. 12. 8. 7, Kircher III. 30. Petiv Gaz. 70. (2. Ppecim. 211 .

437. T. Pica, p/xcedenti affinis, Lim. 622. Argenv. 8 C (2. fnecim 211 .

438 T. Pharaonius, Linn. 584. Lift. 637. 25. Petiv. Ga? ror i.t. (1. Specirn, 2.) 4 .

439 T. Pharaonius? \& varietas, grifea, rufo maculata, Lin o. Lift. 654 54. (5. fpecim 225.

$44^{\circ}$ T. Perpectivus Linn 81 . Seb. III. 40. I. 2. Lift. 63 2. Hollar Icon. 23. Gualth. $05 \mathrm{O}$ (2. Specim. 244.

44 I 'T. Perspectivus, fimilis præcedenti, Limm. 58r. Kirch III 26. 27. (3. fpecim. 244. a.

442 T. Rerspergtivus, Linn. 581. Rumpt. 27. L. Seb. II. 40. 28 . ( 3 fpecim 244 b.

443 T Perfeeativas, obfcurior, Linr. 581. Seb. III. 40. I 14. (1. poeim: $244 \mathrm{c}$.

444 T. Perfpectivas? f. varietas. grapulatus, Linn. $58 \mathrm{r}$. Co of Lif. 634. 22. Seb. III. 40. 4I? 42? (2. Sil cim. $101 \mathrm{I}$. 
426 S. Piramide tachété? ou varieté, demantelée, Linn.580. $\beta$. Rumph. 21. 3. ( 2 p. 242.

$4 ? 7$ S. Nilotique, tacheté, de couleur de roje Ẽ verd, Linn. 5ig Gualch. 59. B. Rumpi. 21. 3. (2. p. 23). a.

428 S. Nilożque? oic varieté, Linn. 579. $\approx$ Gualth. 59. C. Rumph. 21. A. Lift, 017.3. Kircher III., 102. (2. p. 239 a.

4?9 S. Nilotigue? ou varietc, Linn. 579. B. Argenv. 8. C. Lift. 620. 6. (2. p.239: b.

430 S. Nilitc que? ou variete, vert \& blanche, Linn. 579. B ( 3 . p. $239 . b$.

43 I S. $\Lambda$ ipre, par tout greinulé, verdeatre, Linn. o. Rumph. 21. 0 ? Lift. 630: 16. Gualth. 60, A. (3. p. 1009.

432 S. Afpré? ouvàrieté, d'un verd rougeatre, Linn, o. Rumph. 21. 5 ? (x. p. 1008

433 S Cone grenu, frié de rouge, Ẽ jaune, Linn. ०. Gualth. 60. B. (4) p. 241 .

434 S. Cone grenu? ou variete, verdeatre, Linn. o. Giralth. 61. DD. Rumph. 21. C. 9. 10. ( 4. p. 243.

435 S. Cone piccoté ? verdeatre, Linn. O. Lift. 632. 20? Gualth. 60. P. (2. P. 242. a.

436 S. Pie ou le Soldat. à coquille pelé, E़ noir, Linn. 622. Adanfon Seneg. 1. I2. 8. 7. Kircher. III. 30. Petiv. Gaz. 70. 9. (2. p. 211 .

$437 \mathrm{~S}$. Pie ou le Soldat, Jemblable aì precedent, Linn. 622. Argenv. 8. G. (2. p. $21 \mathrm{I}$.

$438^{\prime}$ S. Bouton de Camifole, Linn. 584: Lift. 637. 25. Petiò. Gaz. 1OI. II. (4.p. 224.

439 S. Bouton de Camifole ? ou varieté, grife, tacheté de rougé Linn. o. Lift. 654. 54. (5. p. 225 .

440 S. Perfpeatf, Linn. 581. Seb. III. 40. x. 2. Lift: 636. 24. f. Hollar IEon. 23. Gualth. 65. O. (2.p. 244. a. 44I $S$ Parpectif, femblable au precedent, Linn. 58r. Kircher III 25-27. (3; p. 244. a.

$44^{2} S$ Perfnetif, Linn. 58r. Rumph. 27. L. Seb. IIII. 40 28. (3. p. 244. b.

443 S. Perfpectif, plus obfcur, Linn. 581. Seb. III. 40. I3. I4. (I. p. $244 c$.

$414 S$. Perfpectif? ou varieté, gremu, 'Linn. $58 \mathrm{r} . \alpha_{\text {. ou }}$ o. Lift. 034. 22. Seb. III. 40. 4I? 42.? (2. p. IOII. 
445 T. Perfpoztivus? f. varietas, apice perforato, Linn. $58 \mathrm{I}$ B. $f \circ$ (. fpecim. rnio.

446 T. Perfpectivus? [. varietas, carinatus, dorfo acuto, lu. tefcens, Livin o. Gualth. 65 L? Petiy Gax. 57. 14 92. 12 ( 2 fpecim. 1 I 2

447 T. Perfpectivus? I varietas. maculatus. fasciis albis fuscisque, Linn .. Lift. 635. 23. (4. fpecim i013.

448 T. Turris, um licalus, uintilic., rorundo aperto, ore trigono, anfractibus feptem, Linn. o. Leers $M u f$. tab. i. f b, Pall. Spicil X p. 33 tab 3.f. 7 8. v, d. Mreden Muj no 156. fith romine: Bordes Trap, utrique hujus raris fima concha ampla defcriptio, (1. fpecim. 1058. _ "Singu. " lare illud ac admirandum objectum, nullis in aliis "Mufais adhuc revertum niti; Hage, in e. Dom. c "Secretis Lyonetio; inque eo. defuneti Prafidis v. D. "MIEDFN; inde perventum ad Ducem a rORT:AND: "Londini; ut et in eo Comitis defuneti a wassenaas "OBDAM; quamvis in ejus Catalogo defiderat $u r$; Bata. - vice apud defunctum Dom. I. van OORDT . fub particular. " nomine : de Kroon der Alikruiken; ultimo autum extal " exemplar Hafrice apud Dom. Direz. spenclerva.

449 T. Solaris verus, ipinofus flavus, Linn 593. Rumph. 20. $K$ latit. 4 long. $2 \frac{1}{2}$. poll. (I. frecim. 246.

$450 \mathrm{~T}$. Solaris Jpurius, ramis muricatis, flavefce's. Linn. 593. *. Seb. III. 59. 3. 4. Gualth. 65. P. Lift. 6229 (2. Poecim 245.

451 T. Calcar, Linn 617 Lift. 608.46 Rumph. 20. I. Argenv. 6. R. Gualth. 65. N. (3. Ipecim. 249.

452 T Calcar, fed millepcra obtectus, Linn. 6I7. (2. fpecim. 249.

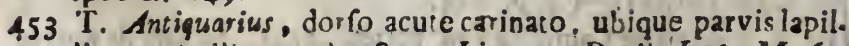
Ii. conchylisque obtetus, Linn. O. Devila I. 6. M. (1. fpecim. 248.

$454 \mathrm{~T}$ Telefcopium, Linn. 600 seb. III jo. I-4. 6. 8. Liff.

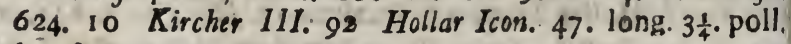
(2. fpecim. 250.

455 T. Telefcopium, fed minor, Linn. 600. Seb. III. 50. 5. 7. 9-12. (2. 'реcim. 250.) 


\section{Teftaces. Ordre IV.}

445 S. Perfpectif ? ou variete, d fommet percé, Linn. 581. B. ous o (2. p. 1010.

446 S. Perfpectif? ou varieté, cavé, d dos aigue, jauneatre, Linn 0. Gualta 65. L\% Petiv. Gaz. 17. $14,92.12$. (2. p. ICI2.

147 S. Perfpeetif? ou varieté, tacheté, \& bandes blarches $\delta$ brunes, Linn. O. Lift. ó35. 23. (4. p. 1013.

448 S. Tour, ou Cadran, umbiliqué, d nombril rond, ouvert, bouche triangulaire, $\mathbb{E}^{\circ}$ Sept anfractures, Linn. O. Leers. Muf. tab I f, 6. Pall Spicil. X p. 33. tab. 3. f. 7.8, v. d. Mieden Muf $n$. 1 56. Sous le nom de Bordes Trap, ous on trouve une trés ample defcription, de cette conchile très rare. (1. p. ross. "Cet objet fingulier, $\sigma^{\circ}$ digne d'admiration, $s^{\prime} e f t$ encore trous"vé dans aucunne Cabinet qu'd la Hayé, dans celui de $\mathrm{Mr}$.

"le Secretaire L Y OUE T ; dans celui de feu Mr. le Pre"Gdent v. D MIEDEN; $\mathcal{E}$ de lo parvenu chez la Du"cheffe de PORTIAND, d Londres; comme auffi dans le "Cebinet de feu Mr. le Comte de wassenaar obdam. "quoique pas injeré dans fon Catalogue; a Batavia, chez "feu Mr. I. VAN OORT, fous le nom particulier: ds. "Kroon der Alikruiken; un dernier enfin fe trouve a Cop"penhague, chez Mr. le Direteur SPanGLER".

449 S. Solaire verirable epineufe, Linn. 573. Rumph. 20, $K$. latit. ou long. $2 \frac{1}{2}$ pouc. (I. p. 246,

450 S. Snlaire Faux, da branches aigues, jaunatre, Linn. 593. w. Seb. III. 59. 3. 4. Gualth. 65. P. Lift. 622. 9. (2. p. 245.

451 S. Eperon, Linn. 617. Lift 608. 46. Rumph. 20. I. Argenv. 6. R. Gulath. 65. N. (3. D. 249.

$452 S$ E.seron, mais couvert d'un millepore, Linn. 6I \%. (2.p. 249.

453 S. Friperie, le dos aigue cavé. la conchile eft par tout couvertes de petit caillioux ou fils, Linn. O. Davila $I .6$. M. ( I. p. 248.

454 S. Tele'cope, Linn. 600. Seb. IiI. 50. I-4. 6. 8. Lift. 624. 10. Kircher $I I I .92$. Hollar Icon. 47. long. $3 \frac{1}{4}$ pouc。 (2. p. 250.

455 S. Telefcope, mais plus petit, Linn, 600. Sะb. III. 50. 5. 7. 9-12. (2. p. 250 . 
GENVS XV. MONOCEROTES.

456 M. Variegatus, maculis fuscis, Linn. G46. a. ᄃ. o. Seb. III. 56.26 ? (2. t, ec m \& 86 .

457 M. Siriotus, fubrofeus, Linn. 0 Adanfon Seneg. I. I’o, 4. 7 ? Petiv. Gaz. 78. 13. (2. Ppecim. $10: 9$.

458 M. Ungulinus, niger, Linn. 647. A Arreniv. it. D? Petiv Gaz. 127: 0. (1. fpecim 53.

459 M. Ungulinus? fed minor, Linn. 047. ß. f. o. Gualth. 58. A2? Argeriy. I1. T? (2. fipccim, 254. 255.

450 M. Obliquus, transverfus, ftriacus, grifeus, Linn. 647. \%. f. o. Rumph. 30. R? (2. fp.cim. 256 .

451 M. Imbricatus, Linn 640. Siv. IIi. 56. 26. 34? Kircher 1II. I12. (3. Рресіл) 25 :

4द2 IM. Albus, ore obliquo fuhplann, anfractibus approximatis biftriatis, Likno o. Petiv. Gaz. 78. I2. (1. Tpecim. 252.

463 M. Exfoletus, oblongas, carinis, duabus, Linn. 044. $\propto$. Sel. III. 56. 29. (1. fipecim. 259.

404 M. Duplicatus, flavus, Iinn 643. Argenv. 11. C? Seb. III. 56.8. Kircher III. 114. (2. Specim. 257.

455 M. Duplicatus, Linn. 613 Gulth. 58. C. 2. Specim. 257. $4^{60} \mathrm{M}$ ! Acutangu!us, Linin. 642. Seb. 111. 50. 7? Kircher, III. 117 . (1. fpecim. 875.

467 M. Terebra, ex albo fusca. Linn. 645. Ginamm. II. 53. Petiv. Muf. 13.18. Kircher 1Il.115. Adanjon Seneg. I. 10.' 4. 6. (2. Tpecim. 258. b.

468 M. Terebra, pracedenti afinis, Linn. 645. Rumph. 30. M. (2. fpecim. 258 b.

469 M. Terelra, Linn. 645. a. Gralth. 58 A (3. Specim. 258. b. 4:0 M. Polymitus? r. yrrietas, fpeciei pracedentis, Linn. 6.5. B. .. o. Set. 1II. 56. 25. (1. ffeciln. 258. a.

GENVS XVI. STROMBI.

$47^{1}$ S. Paluftris, ex atrofuscus, Lim 515 Seb III 5013. 14 Rumph. 30 Q. Lif. 837. 63? Yetiv Muf 13. 13. (2. Ppecim. 260 i.:

472 S Paluftris, non aduluc, ex grifeo fuccus, Linn. 515. Seb. III. 50. 17. 18. Lift 863.62. (2. Specim. 260 b. 473 S. Ater, alatus furcus, Limn 516. *. Rumph. 30. $T$. Lift. I021. 85. Gualth. 57. E. Adanfon Seneg. I. 10. 4. 3 ? (2. Tecins. 265 . 
GE NR E XV. L I C O R N E S.

456 L. Bariolé, à tacbes brunes, Linn. 046. a. ou O. Seb. III. 56. 26. (2. p. 876 .

457 L. Strié couleur de rofe, Linn. o. Adanfon Seneg. 1. I0. 4. 7.? Petiv Gaz. 78. 13. (2. p. 1059.

458 L. Unguline, or Teriere, noire, Linn. 647. Argenv. II. D.? Petiv. Gaz. 127.6. (1. p. 253.

459 L. Unguline? ou Teriere, mais plus petite, Linn. 647. . $_{0}$ ou o. Gualth. 58. A? Argenv. II T? (2.p. 254255 . 460 L. Oblique, Jtrie, gris, Linn. 647. $\gamma$. or 0 . Rumph. $33^{\circ}$
$R$ ? (2.p. 256 .

46I L. Grieche, Linn. 640. Seb. III. 56. 26. 34 ? Kirchet III I12. (3.p. 251 .

$462 \mathrm{~L}$. Blanche, à bouche oblique applati, les anfractires anprochantes, à deux Jries, Linn. o. Petiv. Gaz. 78. 12 .

(i. p. 252.

463 L. Exoletus, allongée, de deux caves, Linn. $644 . a$. Seb.
III. 56.29 (1. p. 259 . 454 L. Doublé, jaune, Linn. 643. Argenv. Ir. C.? Seb. 111. 56. 8. Kircber III. II 4. ( $2 . p .257$.

465 L. Doublé, Linn. 643. Gualth. 58 C. (2 p. 257. 460 L. Angle algue, Linn. 642. Seb. III. 56. 7.? Kircher III.

$467 L$. Teriere, d'un brun blanc, Linn. 645. Ginann. II. $\$ 3$.

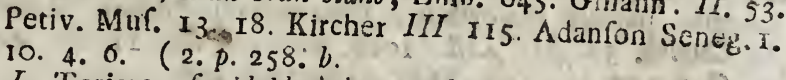

468 L. Teriere, Jemblable à la precedente', Linn. 645. Kumph., 400 L. Teriere, Linn. 645. $\alpha$. Gualth. A 58. (3. p. 258 . \%) $470 \mathrm{~L}$. Pulymite? on varieté de l'efpese precedente, Lisn. (4jo. B. ou 0. Seb. III. 56. 25 . (1. p. 258 . a.

GE NRE XVI. VIS.

$47 \boldsymbol{V}$. Faufe Telefcope, d'un brun roir, Linh. Sis. Set. IIY. 50. 13. 14. Rumph. 30. Q Lift. 837.03 ? Petiv. Mus.

472 V. Fauffe Telefcope, pas parfait d'un brun gris, Linn.

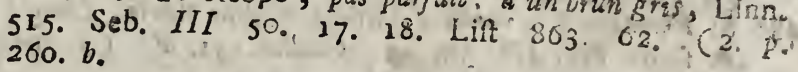

$473 V$ Noir, allé, E brun, Linn. 516 a. Rumph. 30. $T$. Lift. 1021: 85. Sualth. 57. E. Adanfon Sereg. I, I0. 43 ?
(2. p. 265. 
474 S. Ater, non adultus, Linn. 516. «. Kircher III. 8I ? (2. Specim. 266.

475 S. Nodofus, fuscus, tefta turritus nodulis five granulis in anfractibus, cincta, pullus, Linn. O. Lift. 122. 18. (I. fpecim. $2 \sigma_{3}$.

476 8. Fufcatus, nodofus Linn. 573. \%. Seb III. 50. 35. Argenv. II. \&? Gualth. 56. E. (2. Specim 262. a

477 S. Fufcatus, Linn. 573. Seb. III. 50. 32-34. Gualth. 56. $H$ (1. fpecim: 262 b

478 S. Elongatus, acuminatus, :uberculis muricatis brunneis, Linn o. Seb. III. 50. 38 ? Lift. I2I I6? Kircher III. 79. (I, fpecim. 264.

470 S. Aluco. Jacteus, fusco maculatus, Linn. 572. Seb. III. 50. 15. Rumph. 30. O. Lift. 1025. 87. Petiv. Muf. 6. 12, Adanfon Seneg. 1. 1a 4. 1. (2. fpecim. 26r.

480 S. Bubo, canaliculatus, oblique curvatus, absque dente prominente, tuberculis acuminatis, Linn. o Gualth. 57. A? Seb. III. 50. 40? 4 I ? (2. Cpecim. 1029

48 I S. Aluco? f. varietas, Linn. 572 . «. Seb III 50. 37 , 51. 22. 25. Gualth. 57. B. Rumph, 30. N? Lift. 1017. 79. (2. Tpecim. 267.

482 S Thymelus, ftriatus, cinereo fuscus, Limm. o. Seb. III. 50. $24,51.26$. Gualth. 56. L. Lift. 1019. 82. (I. fpecim. 269.

483 S. Acanthinus, ftriatus, canalicu'atus, oblique incurvatus, papillis majoribus., minoribusque - fpinis Linn. $\mathrm{O}$. Gualth. 56. M. Lift. IOI8. 80. Argeñv. II. F. Petiv. Gaz. 152. 4, 154. 13 (3. fpecim. 268.

484 S. Granulatus? f varietas. albicans, Linn. 577 . . Seb. III. 50. 20? Argenv. 1I.K? Gualth. 57. T? Lift. 1020. 84. (2. Гpecim. 270.1064.

485 S Geniculatus, fubtenuis, fuscans. Lirn. c. Lifr. 2 I. 16.? Argeny. 27. 6? Gualth. 76. E. (2. fpecim. 27I.

486 S. Vertagus, lutefcens. Linn. 571. Seb. III. 5r. 24 , Kircber III 84. Petiv. Gaz. 56 4. (2. Specim. 272.

487 S. Vertagus? f. varietas. fed tenuior punctis flavis, Linn. 57 1. a. f. o. Seb. III. 50. 42. Petiv. Muf. 14. I4. (3) fpecim. ror 4 .

488 S. Vertagus? rarietas, lineis duabus thavis, Linn. $57 \mathrm{I}$. B. C. o. Seb. III. 50. 43, Gualth. 51. F. (5. fpecim. 273. 2.

$480 \mathrm{~S}$ Vertagus? f. varietas, Linn. 57 r. भ. f. O.' Gualth. 57. H? Seb. III. 51. 34. (2. fpecim, 273. b.

S. 490 
$474 V$. Noir aile, non parfait, Linn. 516. w. Kircher III. 81 ? (2.p. 266.

$475 V$. Tour Chinoife, ou Noueux, brun, coquille en tour, cerclées les anfractures de petits noeuds, Linn. 0. Lift. I22. 18. (I. p. 263.

$476 V$. Noueux brun, Linn. 573. . Seb. III. 50. 35. Argenv. I I. EO? ? Gualth. 56. E. (2. p. 262, a.

477 V. Tenebreux, Linn. 573. Seb. III. 50. 32-34. Gualth. 56. $H$ (1. p. 262.6 .

$478 \mathrm{~V}$. Allonge, pointu, tubercules, en forme de chardjetrappe, brun, Linn. O. Seb. III. 50. 38 ? Lift. 121. 16? Kircher III. 79. (p. 264 .

$479 V$. Fauffe Thiare, couleur de lait, tacheté de brun, Linn. 572. Seb. III. 50. 15. Rumph. 30. O. Lift. 1029. $87^{\circ}$ Petiv. Muf. 6. 12. Adonfon Seneg. 1. 10. 4. I. (2.p.261.

$480 \mathrm{~V}$. Fauffe Thiare, ou Hibou, canalk, courbl de travers fans dant prominent, de tubercules aigues, Linn. 0 . Guaith. 57. A. ? Seb. III. 50. 40. ? 41.? (2. p. 1029.

$48 \mathrm{I} V$. Aluco? ou varicté, Linn. 572. «. Seb. III. 50. 37. 51. 22. 25. Gualth. 57. B. Rumph 30.N ? Lift. $1017.7 \%$ (2. p. $26 \%$.

482 V. S. Thymale, Arié, brun cendré, Linn. o. Scb. IIT. 50, $24,51.26$. Gualtn. 56. L. Lift. 1019 83. (1. p. 259 . $483 V$. Acanthine. Atrié, canalé, recourbé de travers, d grand mamillons, $\mathcal{O}^{\circ}$ petites epines, Linn. O. Gualth. so. M. Lift. TO18. 80. Argenv. I1. F. Petiv. 152. 4, I54. 13. (2. p. 268.

$484 \mathrm{~V}$. Grenu ?ouvarieté, blancheatre, Linn. 577. —. Seb, III. 50. 20.? Argenv. I1. K.? Gualth. 57. T? Lift. 1020. 84. (2.p. 270.1064.

$485 V$. Tuberculé, ou B utonné, un peu mince, brun, Linn. 0. Lift. 21. 16 ? Argenv. 27. 6.? Gualth. 76. E. (2. p. $27 \mathrm{t}$.

$480 V$. Chenille, jaunatre, Linn. 57I. Seb. III. 5r. $240^{\circ}$ Kircher III. 84. Petiv. Gaz. 56. 4. (2. p. 272. $487 . V$. Chenille? ou varieté mais plus mince a points jaumes,

Linn. 571. *. ou O. Seb. III. 50.42. Petiv. Mur. 14.14.

(3) p. $10{ }^{4}$

$488 \mathrm{~V}$. Chenille? ou varieté, d deux lignes jaunes, Linn. $57 \mathrm{I}$. B. ou O. Seb. III. 50. 43 Gualth. 57. F. (5. p. 273. a. 489. V: Chenille? ou varieté bandee, Linn. 57I. \%. ou 0 . Gualth. 57. H.? Seb: III: 5I. 34. (2. 2. 273.6 . 
490. S. Columella, cochleata, ore elongato, fasciis 2 favis, Linn o. Cualth 45. D? Rumph. 30. P.? Petiv. Gaz. 63. I 2. (6. fpecim. 274.

$49 \mathrm{r}$ S. Coactus, tefta fubventricofa, anfraetibus elongatis tuberculatis, Linn. 0. Argenv. 27. 6. f. 6. (2. Specim. 275.

492 S Elongatus, transverfe coftatus, Linn. o Kircher III. IIo. Petiv, Gaz. 152. 16. (2. Specim. 276.

493 S. Glabratus, elongatus, margine ohliquo, tefta glabra fusca, Linn. o. Lifi. I055 7? Seb, III. 56. 14. Rumph. 30. $R$ ? (2. [pecim. 277 .

494 S. FluEtuofus, columella exerta, alba, apertura marginata, tefta elorgata acuta, Linn. o. Lift. 118.14 ?' (2. 'pecim. 278.

$495 \mathrm{~S}$ Crenulatus, fuccus, Limn. 48r. Seb. III. 56. 17. Rumph. 3. E. Lift. 846. 75. (2. fpecin 280.

496 S. Strigilatus? C. varietas, Linn. 48 \& «. Seb. III. 56. B. Petiv Muf. 5,12 (2. Sp:cim 279.

427 S. Strigilatus? r. varietas, anfractuum margine nlano, flavefcens, maculis tuscis, Linn. 484. $\beta$. \{ o. Lift 845 . 72. e? 73 ? (4. fpecim 879 .

499 S. Duplicatus, maculis fivis, Linn. 485. Rumph. 30. F. Lift. 837. 64. (3. fi ecim. 877 .

499 S. Lanceatus? f. varietas, cinerefcens, Linm. 484? 486 ? f. o. (nec tamen anfractus bifidi, fed lineola alha fusco maculata circundati) Seb. III. 55. B. \&c. Lift. 979. 36. (2. Epecim. $28 \mathrm{r}$.

500 S. Lanceatus, lineis fuscis, Linr. 486. Argenv. II. Z. (2. frectm. 282

501 S Dimidiatus, glaber, flavus, albn undatus, dimidiatus. Linn. 482 ?' $.4{ }^{3} 7$ ? Rumph 30.C. Gualth. 57. M. Kircher III. 107. (4. Cpecim. 279.*

502 S. Varius, elongatus, teres, anfractibus nodulofis, fla:vus, altho maculatus Linn. o. Rumph. 30.D. Petiv. Mus. 2. 4: (2. 亿pecim. 283 .

503 S. Subulatus, glaber, anfractibus profunda ftria, flavescens, maculis brunneis, Linn. 48n. Seb. III 56. 20. 27. 28. Lift. 842. 70. Petiv. Muf. 4. 2. (4 fpecim. quorum duo diffecta 284. a.

$50 \ddagger$ S. Subulatus, fimilis precedenti, Linn. 480. (4. fpecim. 284. b.

505. S. Maculattis, Linn. 479. Lift. 846. 74. Petiv. Mus. 5. 4. Kircher III. 3I3. (3. fpecim. 285. 


\section{Teftaces. Ordre IV:}

$490 V$, Columelle, eq forme de cuillier, d bouche allongte, deux bandes jaunes, Linn. o. Gualth. 45. $D$ ? Rumph. 30. P.? Petiv. Gaz. 03. 12. (6.p. 274.

$491 V$ Tauffe Thiare de Riviere, coquille, ventrue, les anfractures elongues tuberculées, Linn. 0. Argenv. 27.6.f.6. (2. P.:275.

492. $Y$. Allongé Fluviatile, des cotes travers, brun, Linn. 0. Kircher IIL. rio. Petiv. Gaz 152. 16. (2. p. 276.

$473 \mathrm{~V}$. Pe!é de Riviere, allongé, à bord oblique, coquille pelé brine. Linn. O. Lirt ro55. 7? Seb. III. ${ }_{5} 6$. I 4. Rumpt. 30. R.? (2. p. 277.

194 V. Ondé, columelie elevée, blanche, \& ouverture bordée, coizuille allongée, aigue, Linn. c. Lilt. JI8. I4? (2.p. 278

$49 ; V$. Grenu, brun, Lirn. 48 r. Seh. III. 56. 17. Rumph. 30. E. Lift. 84 6. 75. (2. p. 230.

$496 \mathrm{~V}$. Aiguille ftriée? ou varieté, Linn. 484. «. Seb. III. 56. B. Periv. Mui. 5. 12. (2. p. 279.

$427 V$. Aiguile frié? ou varieté, le bord des anfraktures unie, jaunatre, da taches brunes, Linn. 484 . $\beta .0 u$ o. Lilt. 845. 72. e? 73 ? (4. p. 878.

$498 V$. Aiguille doubié, à taches jaunes, Linn. 485. Rumph. 3ก. F E.iी. 837. 64. (3 p. 877.

499 V Aiguille lancée, ou varieté, cendré, Linn. 484 ? ou 486? 04 \%. (l'anfracture pourtant pas fendu, mais entouné d'une ligne blanchée tachetée de brin), Seb. IIT. 56. B. Lift. 979. 36 (2.p. $28 \mathrm{r}$.

sco $V$ :Aiguile lancée, d rayes brunes, Linn. 486. Argenv. II. Z. (2. P. 282 .

501. V. Moitié . pelé, jaune, ondée de ulane, Linn. 48.2 ? ou 487? Rumb 30. C. Gualth, 57. M. Kircher $I I$ \% 107. (4. P. 279.

$502 \mathrm{~V}$. Varié, allongé, les anfractures noduleux jaunes, tacheté de blanc, Linn. o. Rumph. 30. D. Petiv. Mur.z. 4. (3. p. 283 .

$503 \mathrm{~V}$. Tigré, pele , les anfraltures a ftrié, profonde, jaunatre a taches brunes. Linn. 480 . Seb. III. 56. 20. 27.28. Lift, 842. 70. Petiv. Mur. 4. 2. (4. P. defquelles 2 font coupées, $2 R_{4}$. a.

io $V$. Tigrés, semblable ąs precedent, Linn. 480 . (4. p) 384.6

i05. $V$ Claed de mer tacheté. Linn. 479. Lift. 846. 74. Petiv. Muf, 5. 4. Kircter III. इ13. (3. P. 285 . 
506 S. Maculatus, multo minor, Linn. 479. (4. Specir 285.

GE NVS XVII. B V C CINA.

507 B. Digitale, tefta g'abra emarginata, ore obliquo, vet tricofa. elongata, flavefcens, Linn o. Lift. 977. 3. Petiv. Gax. 102. I5. Adanfon Seneg. 1. 4. 9. 2? (: fpecim. $29 \mathrm{I}$.

508 B. Digitale f. varletas, anfractibus profunde fu'catis lineoli: transverfis, Linn. 0. Lift. 977. 34. (2. (pe cim. 292.

509 B. Glans, lineis \& maculis fuscis Linn. 456. Seb. II. 39. 56. 57. 60. Petiv Muf. 13. 5 (2. fpecim. 288.

510 B. Papillofum, Linn. 455. Seb III. 49. 57-59. Guabt] 44. G Petiv. Muf. 2. 16. (2. โpecim. 290.

5 II B. Senticofum, Linn 546 Rumpb. 29. N Kircher IIJ 35. 73. Petiv, Gaz. 55. 5. ( 3 fpecim. 289 ,

$5 I 2$ B. Apex, breve, tefta ventricofa, transverfa, coftata fusca, fascia alba, Linn.0. Seb III.50, 31. Gualth. 5: G. Lift 966.21. A. (1. Ipecim 293.

513 B. Apex? f. varietas, glabrum, apice tuberculato Linn o. (1. fpecim. 294.

5 I 4 B. Pollex tefta ventricofa, apice tuberculato, fusca Linn. 0. Lift. 978 35? (2. Ipecim. 287.

515 B. Rullus, margine craffo, cefta ventricola alba, lineoli fuscis, Linn. 0. Seb. III. 54. 14-I6. Gualth. 43. X Lift. 83 I. 55. (3. Ipecim. 299.

5I 6 B. Nodofum? Linn. 472 Gualth. 48 D. Petiv. Muf. I3 4. (4. Tpecim. 300.

$5 \mathrm{I} 7 \mathrm{~B}$. Nodojum? f. varietas, minus ventricola, magis tuber calata, Linn. 472. ๙. \{. O. Seb. III. 50. 52 ? 53? Kir cher III. 7I. (3. Specim. 1015.

518 B. Comeum, tefta oblonga d.s ra, anfractuum marginibus complanatis, apice tuberculofo, Linn. 565. Gualth 46 F. Lif. 913 . 5. (1. fp cim. 1030.

sig B. Corneum, f. varietas, Linn. 565. .. f. O. Lift. 9r4f 6? (1. 'pecim. 880

520 B. Tritonium, parvim, flavefcens fasciis alhis, Lim 560. 'C. 0. Gualth. 52 I, Lift. 914. 7. Petiv Gaz I51. 6. (3. fpecim. 309.

52 I B. Tritonium? [. varietas, grifea, Limn. 560. $\gamma$ f. o Lift. 914. 7? (1. fpecim. 310. 
\$22 B. Tritoniolum 3 C. varietás, album, unsculis, brunneis, Linn. 560. D. f. 0. Lifl. 914.6? Gualth.40. F. (1 Ipecim. I060.

523 B. Ventricofum, anfractibus profundis, utrinque elongatum, coftarum, album, Linn. O. Kischer. III. 32? (4. fpecim. $30 \mathrm{r}$.

524 B. Undatum tefta oblonga, rudi, trnnsverfin ftriata, Linn. 475. Seb. III. 39.61.76-80. Lift. 962.14. Kircher III. 19 t. (2. Specim. 306

525 B.Undatum. 3 fpecimina, quorum duo, haud polita, cortice fufc. obtecta, Cancrumque Diogenem habent, tertium Oftreain plicatam continet, Linn. 575. (3. Specim 3c6. a.

326 B. Bicarinatum, lineis convexis ftriaturs, fusco lavum, Linn. 475. . . O. Lift. 1057. I. (1. Rpecim. 305.

527 B. Bicarinatum ? I varietas Linn. 475. B. fo o. Seb: III. 73. 3-6. Lift. 602. 15, gr 3 4.A. (2. fpecim. j04. b.

528 B. Bicarinatum ? C. varietas, flavefcens, Linn 475. B. C. O. (2. Ipecim. 304. a

529 B. Bicarinatum, anfraetibus finiftrofum, folfile, Linn. 475. भ. f. "o. Lift. 950. 44. a. b. ( 2 fpecim. 303.

530. B. Tritonis, Linn. 560. Seb.III. 71. Lift 959. I2. Va.

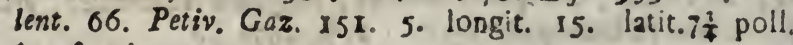
(I. Ipecim. 308.

531 B. Tritonis, fed minor, Lin. 560. Petir. Muf. 12. 15. (2. (ресіm. 308.

532 B. Tritonis, margine echinato plicatum, maculis fuscis, dorfo tuberculato, Linm. 560. «. A. O. Liff. 960. $3^{\circ}$ (I. fpecim. 307.

533 B. Tulipa, Linn. 562. Seb. IIT. 71.23-31, Lifi.910. I Bonami. Ohferv. 38. (2. frecim. 3'3.

334 B. Tulipa, minor, grifeum, Linn. 562. Kircher III. 324. (3. Speciun. $3 \div 3$.

535. B. Islardicum, album, non adeo ventricofum, Linn. 562 *. C. 0. Lift. gr5. 8? (2. Specim. 31\%.

536 B. Mediterraneun ? f. varietas, lacteum; duo fpecimina giorum alterum Vermiculis, Balanisque obfitum, Lint. 562. A. F. O. Lift. 913.-5? (2. Ipecim. 881.

637 B. Pbenifloma, ore violaceo, ventricolum, darfo cari nato, anfrattibus profundis, albo tlavefiens, Linn. c Gualth. 52. Q. (2. Specim. 316. 
122 B. Triton? ou varieté, blanche, d taches brunes, Linn. 560. d. ou o. Lif. 914. 6.? Gualth. 46. F. (1. p. 1000. 23 $B$. Pie ventrée, d detours profonds, allongé des devx coté, blanc, Lisn. 0. Kircher III. 32 ? (4. p. 30 t.

124 B. Pie ondée, à coquille allongé rude, ftrié de travers, Linn. 475. Seb. III. 39. 61. 76-80. Litt. 962 . 14. Kircher III. 191. (2. p. 306.

$25 B$. Pie ondé, trois pieces, deux desquelles pas poliés, mais l'ecorce couvert de brun; dans le quelles fe trowve le Crabbe Diogene, 60 le triofisme contient, une Hisitre plice, Linn. 475. (3. P. 306. a.

26 B. Pie creufe, ou à deux cavités, da lignes recourbẹes. Arite d'us jarne brunatre, Linn. 475. a. ou o Lilt. 1057. x. (I. P. 305 .

27. B. Pie creufe, ou à deux cavités? ou yarieté, Linn. $475 . \beta$. ou o. Seb. III. 73. 3-6. Lift. 662. 15, 513. 4. A. (2. p. 304. $b$.

28. $B$. Pie, à deux caves? ous varieté, jannatre, Limn. $475^{\circ}$ B. $0 u$ o. (2. p, 364. a.

29 B. Pie, à deux caves? les detours d̀ gaucke, NB. folfile, Linn. 475. $\gamma$. ou o. Lift. 950. 44. a. b. (2. P. 303 .

$30 \mathrm{~B}$. Triton, Linn. 560. Seb. III. 7I. Lift, y59. 12. Valent. 66. Petiv. Gaz. 151, 5. longit. 15. lativ. $7 \frac{3}{4}$ pouc. (x. (I. p. 308.

II R. Triton, mais pius petit, Linn. 560. Petiv. Mur. I2。 I5. (2. p. 308.

2 B. Triton, d bord couvert d'olguillons, plié d taches brunes, le dos a tubercules, Linn. 500. . ou 0. Lifl. 960.30 . (1. p. 307 .

3 B. Poire d'agarhe, Linn. $562 . \$ e b$. III. 7. 1.23-3r. Lift. 9ro. I. Binsnn. Osferv. 38 . (2. p. 313.

4 B. Poire d’atié, mais plus petit, gris, Linti. 562 . Kircher III. 39.4 . (3. p. 313 .

5 B. Fufeau d'Inande, blanc pasfiventru, Lian. 502. w.ow 0. Lift. 915.8 ? (2. p. 31x..

6 B. Fufeau de la Mediterrande ou yarieté, blanc; deux pieces.

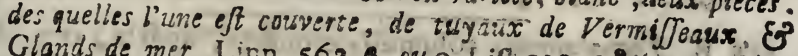
Clands de mer, Linn. 562. $\beta$. ou 0. Lift 913. 5 \% (2. p. 881.

7 B. Phoniftome, In bouche violette, ventru, le dos creux, d detours profords, d'un blenc jaunatre, Linn. 0 . Gualth, 52. Q. $(2, p, 316$. 
338 B. Gibber, tefta convexa, carinata, anfractibus elevatis planis, cinerea f foscia flava, Lirn. o. Llft. g21. I4. (2. fpecim. 3:4.

539 B. Carinatum, tefta flava, ventricola tuberculata, : berculis dorfo geminio abis, Linn. ๑. Lift. 922. Is (I. Ipecim. 314.

540 B. Molucrum, anfractibus, tuberculatis, Ariatis, flavefcer Linr. 0. Gualth.52. S? (2. Tpecim. 315.

s4I B. Evonymum, columellà abbreviarł, fubunbilicat. yedtricofa, fubtuberculata, lútea, lineis fuscis, Linn. Gualth. 48 F? Lift. 930 25? (2. [ptcim. 318.

542 B. Trapezium Linn. 567. «. Kumph. 29. G. Guaith. 5 T? (2. fpecim. 319.

543 B. Trapezium? f. varietas, decorticatum, Linn. 567. (2. Ipecim 319.

544 B. Trapezium, magis ventricofum, lineis duplicatis, alt lutefcens, Linn. 07 . Lift. 931. 26. Petiv. Muf. I3. Kircher III. 288. (2. Гpecim. 220.

545 B. Trapezium, fed mirus, Linn. 567. (2. Specim. 3

546 B. Fimbriwum, tefta bicarinata, tuberculata, angulos lute[cens, Linn. O. Argenv. I0. L. (2. fpecin. 3 I 3.

547 B. Fimbriatum, fimile precedencis, Lann. O. (2. fpeci. 3 is.

548 5. Tubicen, columellâ glabra, apertura, elongata rinata, undatim ftriata, Linn. o. Lift. 886. 7? 101 $11 \frac{1}{2}$ lat. $5 \frac{1}{2}$ pol. (I. fpecim. 823 .

549 B. Morio, nigra fasciaalba, columella rugof, Linn. 5 Lift.928.22. Kircber III. 350. Adanjon Seneg. 1. 9.2. (2. fpecim. 32 r, a.

550 B. Morio, furcus, decorticatus, fasciis albidis, Lirin. 5 (2. Specim. 32 I. 0.

551 B. Morio, flavelcens, fasciis albis, Linn. 552. [pecim. 32 I. c.

55? B. Morio, fed minor, Linn. 552. (3. fpecim. 32 1.1.

553 B. Morio, precedenti affinis, Linn 552. lor:g. 81 ? (1. Specim. 321 . e.

554 B. Cochlidium, Linn 553. Seb. III. 57. 27. 28. Arg 9. A. (3. Ipecim 322.

555 B. Tarnotanum, columella elongata, aperta, ore obi go, teft a fubventricola, glabra flava, Linn. o. Volent ( $\mathrm{x}$. fpecim. 323. a.

556 B. Tarnatanum, precedenti fimile, Linn. O. (2. cim. $323 . \mathrm{b}$. 


\section{Teftaces. Ordre $1 \mathrm{~V}$.}

38 B. Fufeau boffu, petit la coqrille recourbée creufe, les detours elevés, plattes, à bande jaune, Linn. 0, Lilt. $92 \mathrm{I}$. I4. a. (2.p. 3 I 4 .

$39 B$. Fufeau cariné, coquille jaune ventruse, tuberculé, le dosà deux tubercules blanches, Linn. O. Lift. 922 . I5. ? (I. p. 314. 40- $B$. Entlé, les anfractes à tubercules, friés, jaunatre, Linn. o. Gualth. 52. S.? (2. p. 315.

+t $B$. Evorime, ou Tulipe, à cobumelle racourci umbilique, \& ventrue, à tubercules. jaune, E' lignes brunes, Linn. 0. Gualth. 48 F? Lift. 930. 25.? (2. p 318.

$1^{2}$ B. Tapis, o? Tulipe, Linn. 567. $\propto$. Rumph. 29. IG. Gualth. 52. T.? (2.p. 319 .

13 B. Tapis, ou Tulipe? cu varieté, fans ecorce, Linn. $36 \%$. a. (2.p 319.

14 b. Tapis, ou Robe de Perfe, pluis ventru, d̀ lignes doublées. d'un jaune blancheatre. Linn. 507. Lift. 93:. 26. Petiv. Muf. I3. I. Kircher III. 288. (2. p. 320.

.5 B. Tapis, ou Robe de Perfe, mais plus petit, Linn. 56\%. (2. $p .320$.

31. $6 B$. Tulipe bordé, coquille en forme de frange, à deux ca. ves, tubercule, en angles, jaunatre, Linn. O. Argenv. Io. L. (2. p. 313 .

7 . 7 . Tulipe bordé, coquilie en forme de frange, Semblable au prece dent, Linn. o. ( 2. p. 313 .

c8 $B$. Trompette Dragon, á fut, ou columelle pelé, l'ouverture allangé, creux , Eं ftrie par ondes, Linn. 6. Lift. 886. 7 ? long. $11 \frac{1}{2}$ lat. $5 \frac{1}{2}$ pouc. (1. p. 893 .

599 B. Micresque, noire, à fascie blanche, Ẽ fut ridé, Linn. 552. Lilt. 928. 22. Kircher 1II. 350. Adanfon Seneg. 1.9. 2. 32. (2. p. 32 I. $a$.

se B. Moresque, brune, à deux vandes blanches, fans ecorce, Linn. 552. (2. p. $321 . b$.

I $B$. Moresque, jaunatre, a fafcies blanches, Linn. 552. (2. P. $321 . c$.

2 3. Moresçue, mais plus petite, Linn. 552 . (3. p. $32 \mathrm{I}$. $d$. 3 B. Mo: esque, Jemblable au precedent, LinB. 552 . long. $8 \frac{1}{2}$ pouc. (I. p. 32I.e.

+ $B$. Tulipe cordonnée, Linn. 553. Seb. III. 57. 27.28 ? Argenv. 9. A. (3.p. 322 .

$5 B$. Tarnatane, à columelle allongée ouverte, à bouche longue, coquille un peu ventru; pelk, jaune, Linn. O.Valent. 2. (x.p. 3z3. a.

i $B$. Tarnatane, Semblable de la precedente, Linn. O. (2.p: 323. $b$. 
557 B. Tarnatanum? f. varietas, anfraetibus acuminato tuber. culatis, elevato lineatis, Linn. O. Li/f. 892. I2. ( 3 . fpecim. 324.

558 B. Perverfum, Linn. 557. Lift. 907. 27. 28. Argenv. 15. F. (2. fpecim. 325 .

559 B. 'Ficus, fpinofus, fusco ftriarus, Linn. 557. f. o. Liff. 830. 3. b. Seb. III. 68. 19?-22? Petiv. Gaz. 78. 11. (2. fpecim 326.

s60 B.Ficus, major, albus, Linn. 557. f. o. long. $8 \frac{1}{2}$ latit. 5. poll. (2. Ipecim. 894.

561 B. Canaliculatum, Linn. 555. Lift. 878. 2. Valen. $92^{\circ}$ (2. fpecim. 32.

562 B. Spirillus, Linn. 554. *. f. o. Seb. III: 68. 19-22. (2. Specima. 882.

563 B. Spirillus? f. varietcs, magis angulatus, ventre tuberculofo, albus, fusco maculatus, Linn. 554. B. .o. (2. ) pecim 328.

504 B. Babylonicum, tefta turrita, cingulis acutis meculatis, Linn. 549. Lift. 917. I1. Petiv. Muf. 4. 7. Valent. 8. (2. fpecim. 330.

565 B. Babylonicum, maculis flavis, Linn. 549. (2. Specim. 330. a.

560 B. Babylonicum, maculis fuscis, Linns. 549. a. Lift.917. 10? (2. Rpecim. 33s.

567 B. Babylonicum? i varietas, album, Linn. 550. ※. f.o. Argenv. Zoomorph. 4. B. (2. fpecim. 332.

568 B. Tuberculatum, tuberculis obliquis, argute ftriatum, Linn. 550. B f. o. Seb.1II. 79 ? infra, Petiv.Gaz.56.6, I02. I6. (2. fpecim. 333.

569 B. Muricatum, ventricofum elongatum, acuminatum, anfråtibus acuminatis, transverfe tüberculacum, album, Linn. O. (r. fpecim. 335.

570 B. Aculeatum, columella, truncata, oblongum aculeis truncatis, obliquis in dorfo, Linn. O. Seb. III. 60. 49. (2. Specim. 302.

571 B. Coftatum, oblongum, lineis elevatis cinetum, fuscum, Linn, 0. (2. Ipecim. 336.

572 B. Cinctum, tefta oblonga, acuta, trabsverfe tuberculatum albidâ, Linn 0. (2. Specim. 883.

573 B. Striatum fimile pracedenti, transverfe undatum ftria. tum, Alavelcens, Linn. o. (1. Ppecim. 334. 


\section{Teftaces. Ordre IV:}

557 B. Tarnatane? ou variete pointu, $d$ anfractures, tubercislëes, elevée en lignes, Linn. O. Lift. 892. 12. (3. p. 324.

$558 B$. Figue d bouche gauche, , Linn. 557. Lilt. 907.27 .28$. Argenv. 15.F. (2.p. 325

559 B. Figue epineufe, ftrié de brun, Linn. 557. or 0. LiR. 880. 3. b. Seb.IIII. 68. 19 ? -22 ? Petiv. Gaz. 78. II. (2. p. 326 .

560 B. Figue epineufe, plus grand, blanc, Linn. 557. ou 0. long. $8 \frac{1}{2}$. latit. 5. pouc. (2. p. 894 .

561 B. Figue canalé, Linn. 555. Lift. 878. 2. Valent. 92. (2. p. 327 .

562 B. Navette, d longue quers, Linn. 554. a. ou 0. Sob. III. 68. 19-22. (2. P. 882 .

363 B. Navette? d longue queue, ar variete, plus anglèe, d ventre tuberculeux, blanc, tachete de bren, Linn. 554. B.ou O. (3. P 328.

564 B. Tour de Babel a coquille en forme de tour, d cercles aigues tachetées, Linn. 549. Liift. 9r7. I1. Petiv. Mur, 4. 7. Valent. 8. (2.p. 330.

565 B. Tour de Babel, d taches jaunes, Linn. 549. (2. p. 330.

566 B. Tour de Babel, d taches brunes, Linn. 549. ๓. Lift, 917. 10? (2.p.331.

$567 B$. Tour de Babel? ors variete, blanche, , Linn. 550, w. ow o. Argerv. Zoomorph. 4. B. (2. p. $33^{2}$.

$568 B$. Tubercu'é, à tubercules transyerfes, \& ftries fines, Lina. 550. $\beta$. ou 0. Seb III. 79.? infra, Petiv. Gaz. 56. 6, 102. IG. (2. P. 333.

$569 B$. Tour pointu, blanc, ventrus allongt, bes anfrodtures twberculés de travers, Linn. 0 . (I. p. 335.

$570 \mathrm{~B}$. Tour piquant, d columelle tronqués, tres long, le dos à pointes tronguées trensverjes, Linn. 0 . Seb. III. 60. 49. ( 2 p. 302.

$57 \mathrm{I} B$. Tour à coles, à courte queue, entoure de lignes devées brus, Linn. o. (2. p. 336 .

$572 B$. Tour entoure, d coquille allongéc aigue, tubercule de travers, blanc, Linn. O. (2. p: 883 .

$573 B$. Tour rayé, femblable au precedent, jaunatre \& ftrite de trayers par ondes, Linn. 0. (1. P. 334. 
574 B. Tuberculatum, linea elevata cinctum, \& transverfé tuberculatum in anfractibus, flavefcens, tuberculis rufis, Linn. o. Lift. 92518 ? (2. fpecim. 1031.

575 B. Scavolum, minimom, brunneo albidum, ftriatum, Linn o Naturforfch XII. tab. 3.f. 2. a. b. (2. fpecim. 340 . 576 B. Sulcatum, tefta elongata. columella longa plicata, transverfe fulcata, lineis elevatis cincta, fufca, Linn. o. Gualth. 52. P? Seb. III. 50. 54? Lift. 921. 14. (I. fpecim. 338.

577 B Tubicen, elongata, tereti accuminata, anfractibus tuberculatis. albida, Linn. o. Valent. 6 ( I. Specim. 339. 578 B Colus, tefta currita, carinata, labro crenulato, Linn. 55 т. Lift. 91 8. I1. Kircher III. 88. Petiv. Gaz. 73. 2.. Muf. 6. 5. lon:. 6. poll. (2. fpecim. 34I.

579 B. Colus? fo varietas, tuberculatus, Linn 551. Seb.III. 79. infra, long 9. poll. (2. Specim. 342. a.

580 B. Colus? fo varietas. Linn. 55I. «. long. 10. poll. (I. fpecim. 342. b.

581 B. Colus? f. varietas, ventricofius, magis tuberculatum,

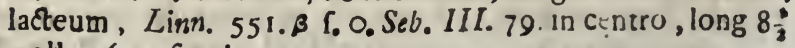
poll. (1. fpecim. 343 .

582 B Glomeratum, dorfo tuberculato, anfractibus carinatis, pallide fuscum. Linn. 0. Gualith. 52. L. Argenv.9. B. Seb. III. 79. Liff. 917.10 ? (2. Tpecim. 337.

$58_{3}$ B. Tibicen Aruanus, cauda longa, dorfo tuberculato carinata anfractibus cuberculatis, Linn. O. Rumpb. 28. A.Kircher III. IOI. lorg. 5. poll. (2. Tpesim. 329.

584 B. Lineatum. tefta ventricofa, ad apicen truncata, tuber- culis transverfis, ftriatis, alba, tuberculis rufis Linn. O. Seb. III 50. 55. 56. Li,t. 925. 17? (3. fpecin. 889.

585 B. Vitiliginofum, tefta vencricofa, elongata, acuminata, transverfa, tuberculata, anfractibus decu $f a$ is. Linn 0 . Rumph. 49. G. Liff. 1022. 86. Seb. IIl. 51. 20. 2 1. (2. fiecim. $\$ 85$.

586 B. Aiuncum, albido brunncum, Linn. O. Liff. 926.19? (I. fpecim. 886.

537 B. Aduncum? [ varietas, fed minor, Linn. 0. Lift.926. 19. ( 3 fpecim. 887 .

588 B. Digitus, in quoque anfractu tuherculum, Linn. O. Rumph. 29. 2. (2. '́pecim. 888.

589 B. Rude, tefta oblonga, ventricofa, acuminata, in quoque anfractu tuberculum, undique granulatum, Linn. o. Seb. JII. 56. 36. Gualth. 44. H. Lift. 949. 44 ? (1. Spe(1) cjø. 349 . a. $590 \mathrm{~B}$. 
$574 B$. Tour tuberculé, entouréd'une ligné elevée, E tubercule de travers dans les anfractures, jaunatre, d tubercules rou. ges, Linn. O. Lift. 925. 18 ? (2. p. 103 I.

575 B. Scœvole, ou gauche, très petit, ftrié de brun, Linn. ○. Naturforfch. XII. tab. 3. f. 2. a. b. (2. p. 340 .

576 B. Fuleau tillonné, à coquille allongé, brune, à colunelle longue pliée, fillonné de travers, Ẽ entouré de lignes elevéss, Linn. O. Gualtb. 52, P.? Seb. 1II. 50. 54 ? Lift. 92 ? I4. (i. p. 338 .

$577 B$.'Trompette ou Fufeau, à coquille allong', blanche ronde, $\delta$ aigue, anfractures à tubercules, Linn. o. Valent. 6. (1.p. 330 .

$578 \mathrm{~B}$. Quenouille, à coquille elevée creufé, à levre crenu, Linn. 55r. Lift 918. II Kircher III. 88. Petiv. Gaz. 73. 2. Muf. 6. 5. long. 6. pouc. (2.p 34I.

$579 B$. Quenouille tuberculée? ou varieté, Linn. 55 1. «. Seb. III. 79. infra, long..9. pouc. (2 p. 342. a.

$580 \mathrm{~B}$. Quenouille tuberculée ? ou varieté, Linn. 55 I. a. long. Ic pouc. (I. p. $3 \dot{4} 2 . b$.

58 I B. Quenouille? ou varieté, plus ventru, EO plus tuberculée, couleur de lait, Linn. 55I. B. ou O. Seb. Ill. 79. in centro, long $8 \frac{1}{2}$ pouc. (1. p. 343 .

582 B. Fufeau, ou Globe, le dos d itubercules, les anfractures cavés, d'un brun pale, Linn. o. Gualth. 52. L. Argenv. 9. B. Seb. III. 79. Lift. 917. 1C.? (2. p. 337.

$583 B$. Trompette Aruane, à queue longue, le dos tubserculé: cavé, EO les anfractures tuberculés, Jinn. o. Rumph. 28. A Kircher III. 10I. long. 5. pouc. (2. p. 329.

$584 B$ Fureau rayé, d coquille ventrue, tronquee vers le fommet, à tubercules transverjes, ftrié, blanc, à tubercules rouges. Linn. 0. Seb. III. 50. 55. 56. Lilt. 925.17 ? (3. p. 889.

585 R. Tacheté, à coquille ventruse, allongée, pointue tubercuiée. de travers, les anfrabtures l'un fur l'autre croijes, Linn. 0. Rumph. 49. G. Lift. 1022. 86. Seb. III. 51. 20. 21 . ( $1 . p 885$.

586 B Tortu, brun blancheatre, Lisn. o. Lift. 926. Ig? ( $x_{0}$ p. 886.

587 B. Tortu? ou varieté, plus petit, Lirn. O. Lift. 925. 19. (3. P. 887

588 B. Doigt tortu, dans cbaque anfracture eft us tubercule, Linn. O. Rumph. 29. $Q$ (2. p. 888.

589 b. Rude, coquille allongé, ventre aigque, un tubercule dans chaque onfracture, partout grenulé. Linn. O. Seb. III. 56. 30. Gualtb. 44. H. Lift. 949: 44 ? ' (I. p. 349. a. 


\section{5}

590 B. Granulatum, tefta oblonga, ventricofa, acuminata, granulis, elevatis, rufo cinerea, Linn. O. Gualsh. 49. A. Séb. III 60. 21. 23. Lill. 939. 34 ? ( I fpecim. 349. b. 591 B. Granulatum? f. varietas, red granulis levioribus, Linn. o. Petiv. Gaz. 79 9. (1. Ipecim. 349. c.

592 B Celibare, tefta elongata, apice acuto, cœruleo nodofo, carinata, alba, fusco punctulata. Linn. o. Liff. 1024. 91? (2. Specim. 350.

593 B. Cranulatum, tefta oblonga, ventricofa, acuminata, anfraetibus tuberculatis, granulata, Limn. O. Seb. III. 55. in num. 21. ad marginem. ( $x$. Ppecin 352.

594 B. Pterophotum, telta ventricora, acuminata, transverfe undatim tuberculata, alba. Linn. O. Gualth. 44. Ny (x. fpecim. 353.

595 B. Scrobilator, tefta transverfe nodola, lata obtufa, alba, fasciis flavis, Linn. 537. «. f. O. Seb. III. 60.26. 27. Gualth 49. E. Lift. 939. 34 'Petiv. Gaz. 102. 14. (3. fpecim. 35I.

596 B. Olivator, tuberculis granulisque ftriatum, Liwn. O. an 538 ? Seb.III. 57. 34, 60. 25? Gualth. 49. M. (I. fpecim. 348.

597 B. Rana, Linn. 527. Lift. 992. 58? Kircher III. 346? Petiv. Mus. II. 15. (4. Specim. I051.

598 B. Rana? f. varietas, fed aculeis elongatis, magisque gra. nulatum, Linn 927. \& f. O. Seb III. 60. 18. 19 ? Lift. 949. 44. Rumph. 24. I. Petiv. Gaz. 100. 12. (2. Specim. 346.

599 B. Rane? f. varietos, aculeis fulcatis, tuberculis torofis in anfractibus, fusco, lutefcens, Linn. 527. $\beta$. . . o. Seb. III. 60. 14. 20. Argenv. 9. R. (2. Ppecim. 347.

600 B. Rana? r. yarieias, magis compreffum, dorfo tuberculo, gibbofum. grannlatum, luteum, Linn. 927.y f.o. Adanfon Seneg. 1. 8. 2. 13 ?' (I. Ppecim. 884.

Gor B. Rubecula, Linn 536. Seb. III. 57. 32.34. Lift 934. 29. (4. fpecim. 354 .

602 B. Cincticulum, tella ventricofa, caudata, apice obtufo, lineis elevatis cineta, Linn. O. Lift. 940-36? (2. Specim. 357.

603 B. Cinaticulum, fed minus, Linn o. (3. fpecim. 357. 604 B. Hebetatum, cauda aperta, tefta oblonga, apice obtufo, anfractibus tuberculatis, Gavefcens, Linn. o. Liff. 958. II ? Valent. 93. (2. Specim. I06r.

605 B. Cofratum, anfraetu primo transverfe tuberculato, Linn. 0. Seb. III. 57. 30. Rumpin. 29. 148 (2. fpecim. 358. $606 \mathrm{~B}$. 
590 B. Grenu'é, \& coquille allongte ventrue, aigue d grains elevées, d'un rouge cendré Linn. o. Gualth. 49. A. Seb. 1II. 60. 2\%. 23. Lift. 939. 34. I - (1. p. 319 b.

s9r $B$. Grenulé? ou varieté, mais d grains plus polies, Linn. 0 .' Petiv. Gaz. 79. 9.? (1. P. 349.

592 B. Aiguille, d coquille allongte, \& formet aigu bleu, avec de noeux, carinée, blanche, pointé de brun, Linn.o. Lift, 1024. 91.2 (2. p 350.

593 B. Grenule, d coquille allongte, ventrse aigue, les en fradures d tubercules, Linn. 0. Seb. III. 55. in nim. 2I. ad marginem. ( I. P. 352.

594 B. Pterophote, d coquille ventrue pointuse, blanche, tuber-; culé de travers, par ondes, Linn. O. Gualth. 44. N.? (I. p. 353.

$595 B$. Scrobilateur, ol coquille noue de travers, large, emou $\iint^{e}$. blanche \& bandes jaunes, Linn. 537. . ous O Seb. III. 60. 26. 27. Gualth, 49, E. Lift. 939. 34. ? Petiv. Gaz. 102. I4. (3. p. 351 .

596 B. Olivateur, frié de tubersules, \& grains, Linn. o. ous 538 ? Seb. III. 57. 34,60. 25 ? Gualth. 49. M. (I. pv $34^{8}$.

597 B. Crapaud, Linn. 527. Lift. 995. 58.? Kircher III. 346 ? Petiv. Mur. II. 15. (4. P. 1051.

598 B. Crapaud? ou yariete, mais digrillons allongées, E plus grenues, Linn. 527. \%. 0u 0. Seb. III. 60. I8. 19? Lift. 949. 44. Ruwph. 24. I. Petiv. Gaz. 100. 12. (2. p. 346.

599 B. Crapaud? ou varieté. à aiguillons fillonnes, tubercus les cbarnues, dans bes anfrodures, d'un brun jaunatre, Linn. 527. B. 026 O. Seb. III. 00. 14 20. Argen v. 9. R. (2. p. 347. $600 B$. Crapaud? ou variete, plus comprime, le dos baffu. d tubercule grenue EO jaune, Linn. 527.\%. ou 0. Adanton Seneg. 1. 8. 2. 13.? (1. p. 884 .

601 B. Livree, Linn. 536. Seb. III..57. 32 34. Lift. 934. 29. (4. P. 354.

$602 B$. Bandelette à boffages, d coquille verstrue, en queue. dे Soramet emouffé, entourée do lignes elevées, Linn. O. Lift. 940. 36 ? (2.p. 357 .

603 B.Bandelette à bofrages, mais plus, petit, Linn. O. 3. \$. 357 . $60_{4} B$. Emoufié à boflages da queue ouvert de coquille allongée, ¿ Sommet emond Jé, les anfractures tubersulés, jaunatre , Linn. c. Lift 958. 11 ? Valent. 93. (2 p. ro6́r.

$605 B$ à Cotes, la premiere anfracture tuberculé de travers. Lina. O. Seb. III. 57. 30 Rumph. 29. H ? (2. p. 358 . 
606 B. Pileare, Linn. 534. «. Seb. III.52.3. Gualth.49. G?

(2. Ipecim. 359.

607 B. Pileare, præcedenti affine, Linn. 534 थ. (2. Ppecim. 359.

608 B. Pileare, multo minus, Limn. 534. B. Lift. 933. 28? (3. Ipecirr. 355. 356.

609 B. Lampas, tefta varicibus oppofitis, Linn. 529. Seb. III. 57. 29? 31? Rumph. 28. C. (I Tpecim. 345. a.

610 B. Lampas, tuberculis varicofis, flavum, Linn. 529.. Kircher III. 103. Rumph. 28. D. Lift. 1023. 88. (r. fpecim. 345. b.

6ir B. Lampas, fed minor, Linn. 529 (2. Specim. 345. b. 612 B. Olearium, Linn. 5 0. Rumph. 49. I. Lift. 935. 30.

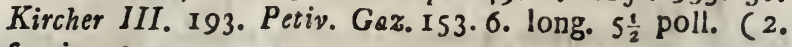
fpecim. 344.

613 B. Femorale, Linn. 531. Gualth. 50. B. C. Lift. 941.37. Seb. III. 63. 7-10. Kircher III. 29I. long. 6 poll. \& ultra. (2. fpecim. 302 .

614 B. Femorale, fed minus, Linn. 531. (2. Ipecim. 362. b.

615 B. Pyrum? f. varietas precedentis, Linn. 531. «. ᄃ. O. Rumph. 26. B. Argenv 10 B. Petiv.Muf.1 2.8.(2.fpecim 361.

616 B. Pyrum ficcatum, Linn 535. «. f. o. L. Lift. 942. 38 ? Gualth. 37. F. Rumph.26.E.Petiv.Gaz, 1 2.4.(2. (pecim.300. 617 B. Pyrafter, oblongo ventricofum, anfraetibus nodofis, fulco feparatis, apice breviore. granulatum, flavefcens, Linn. O. Argenv. 10. M? (2. fpecim. 890.

618 B. Coftatum. oblongum vix convexum nodolo coftatum, rufum, Linn. o. Li:f. 935. 29. a ? (1. Specim. 360.b.

619 B. Pyrum, Linn. 535. Argenv. 10. O. ( I. Cpecim. 8?1. 620 B. Congylis columella aperta, longiffina, labro dentato anice obtufo, coftis tenuibus ad apicem nodofis, tefta albida, Linn. o. (3. 〔recim. 892.

GENVS XVIII. PURPUR E.

621 P. Hiftrix, magnus, cauda corpore duplo longiore corpus ventricufum, fpinæ concavæ, curvæ ad apicem, Iongiffimæ, trifariam pufiı interjectis minoribus, Linn. $5: 9$. ๙. f. О. Rumph. 26.3. Gualth. 3I. A a. Argenv. I6. A. Hollar Icon. 22. long. 6. lat. 3. poll. (1. ipecim. 363. 622 P. Hiftrix oræcetenti fimilis, fed minor, Linn. 5 '9. «. f. ०. Seba III. 78. 1-3. long. $4 \frac{1}{2}$ lar. $2 \frac{1}{4}$ poll. (1. $\int_{F}$ ecim. 363. b. 


\section{Teftaces. Ordre-IV.}

606 B. Pileare, ou Huilé à boflages, Linn. 534, w. Seb. III. 52. 3. Gualth. 49. G.? (2. P. 359.

607 B. Pileare, ou Huilé à boffages, approchant au precedent, Linn. 534. \%. (2. $p 359$.

608 B. Pilteare, ou Huilé à bofrages, plus petit', Linn. 534. 8 . Lift. 933.28.? ( 3 p. 355. 350

609 B. Truité, à coquille, à varices oppofées, Linn. 529. Seb. III. 57. 29 ? 31.? Rumph. 28. C. (I. p. 345. a.

610 B. Truitté, jaune, atubercules enfflees, Linn. 529. a. Kircher III. 103. Rumph. 28. D. Lift. F'23 3. 88. (I. p. 345. b.

6 II B. Truité, mais plus petit, Linn. 529. (2. p. 345. b.

of 2 B. Huiler à boffage; $\tilde{E}^{\circ}$ ris de lait, Linn. 530 . Rumph. 49. I. Lift. 935. 30. Kircher HII. 193. Petiv. Gaz. 153. 6. long $5 \frac{1}{2}$. pouc.

613 B. de Boffe, ors Piediforme, Linn. 531. Gualth. 50. B.C. Lift. 94 I. 33. Seb.III. 63. 7-10. Kircher III. 291. long. 6. pouc. Eं plus. ( 2 p. 362 .

6rt B. de Boffe, ou Rieditorme, plus petit , Linn. 53r.(2.p.362.8. ois $B$. Poire raboteufe, ou variete de la précédente, Linn 53 I. a. ou ๑. Rumph. 26. B. Argenvil. IC. B. Petiv. Muf. I 2 8. (2.p. 361 .

$6: 6$ B. Poire fectê, Linn. 535. a.0u 0. Lift. 942.38 ? Gualth. 37. F. Rumph. 26. E. Petiv. Gaz. 12. 4. (2. p. 360.

617 B. Py̆ráte, ou Pcire feché, ventru à long, les anfractures di neutuds, Separé d'un fillon, à Jommet court, grenu jalinatre, Linn O Asgenv. 10. M? (2. p. 890 .

$618 B$. Cofté, allongé, à peine recourbé, coté par des nesuds rouge, Linn. O. Lift. 935. 29. $a$ ? (I. p. $360^{\prime} b$.

619 B. Poire, Liniz. 535. Argenv. 10. O. (I. p. 89r.

020 B Navet, à longue queue, à columelle ouverte très lon-

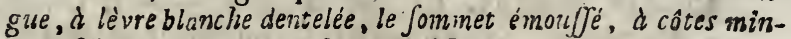
ces, Ef noueux vers le Sommet, छ ì coquille blanche, Linn. ○. (3. p. 892 .

GE NR E XVIII. POURPRES.

621 P. Porc-épic, ou double Becaffe épineufe grand, la queue deux fois plus grande que le corps, le corps ventrue. les Jpires concavées. E' recourveés vers le formmet, très longues, E pofét en trois maniere, Liun. 519. a. ou o. Rumph. 26.3. Gualth. 3r. $A$ C. Argenvo 16. A. Hollar Icon, 22. long. 6. latit. 3 pouc. (1. P. 363.

622 P. Porce épic, ou double Becaffe épineufe. Jemblable à la précédente, mais plus. petit, Linn. 5 I9. * ou, o. Seb. III.

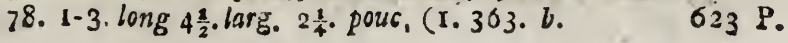


623 P. Hiffrix, pracedenti affinis, Linn. 5I9. 6. f. o. ( I. fpecim. 363. c.

624 P. Tribulus, Linn. 519. Rumph. 26. G. Gualth. 3r. A.b. Lift. 902. 22. Petiv. Gas. 101. 16. (3. Ppecion. 364.

625 P. Cornuta, Linn. 520. *. Lift 901. 21. Kircher III.284. long . 5. lat. 4. poll. ( I. Ipecim 366.

626 P. Cornuta. aft connatis margine \& labro, alba, Linn. 520. . Seb.III. 78. 9-II. long. 4. latit. $3 \frac{1}{4}$ poll. ( I. Ipecim. 366. 2.

\$27 P. Cornuta, aculeis uninoribus minusque curvatis, Linn. 520. (2. Lpecim. 366. b.

628 P. Cornuta, aculeis nodulofis obtufis, Linn. $520 R u m p h$. 26. 4. Gualth. 30. F? Petiv. Gaz 68. I2. Seb. III.78.8. (2. Ipecim. 367 .

629 P. Cormuta, multo xinor, Lin 520. (3. [pecim. 367.

630 P. Spirillus, Linn. 554. vid. n०. 503. fupra. (3. Ipecim. $3 \% 0$

G3I P. Cornesia, tefta, cauda Innga fulcata, nodofa, nodis aculeatis, lutea, Linn. o. Lift. 900 20? (I fpecim. 365. 632 P. Harftellum, Linn. 518. Lift. 303. 23. Hollar Icon. 37. longit. 7 latit $2 \frac{1}{2}$ poll. (I. Ppecim. 37 r. a.

633 P. Hauftellum, minor, fusce!cens, Linn. 518, Seb. III. 78. 5. 6. Kircher III. 270. longit. $7 \frac{1}{3}$ poll. (2. Ipecim. $371 . b$.

634. P.Houfteilum, brevior, Linn. 518 . longit. 4 poll. (3. Cpecim. 37 I. c.

635 P. Scorpio, Linn. 524 Seb. III. 79. 13-16. Valent.35. 37. Lift. 906. 25. (2. fpecim. 372. - Unum, fed , giganteum. Anflellodani invenitur in praftanti Mu", fro, feduli Coltectoris I C. BRANDT II.

636 P. Scorpio, alba, Linn. 524. Argenv. I C.D (2. fpecim. 372. 637 P. Scorpio, præcejenti aftinis, Linn, 524. (2. fpecim.372. 638 P Volans, tefta convera ftriata, aculeis curvis abbreviatis, inter fe adnatis, alba, ramulis piceis, Linn. o. Adanfon Seneg: I. 9 2. 21 ? Davila 16. P\& (1. fpecim. 373.

639. P. Sceleton, oris margine dentato, tefta oblonga, contata, convesa Atriala, imulis curvatis, fusca, coltis albis, Linn. 0. (2. fpecim 374.

640 P. Dams, entricola acuminata, ramis latioribus fulcatis plicatis, pulcerrima, Linn. 523. F. 0.Seb.IIl.77. 1 ? longit. $3 \frac{1}{4}$ lacil. 3 poll. (r. fpecion. 384. 


\section{Tefaces. Ordre IV.}

623 P. Porc épic, ou double Becaffe épineufe, approchant la precedente, Linn. 519. a. ou o. (I, p*363.c.

624 P. Becaffe épineufe, Linn. 519. Rumph 26. G. Gualth: 31. A.b. Lift. 902. 22. Petiv. Gaz. IOI. 16. (3. p.364a 625 P. Maffuée, ou Navette épineufe, allongée Linn. 520 . \%. Lift. 901. 21. Kircher 111. 284. long. 5. lat. 4. pouc. (1. p. 366.

626 P. Mafruée, ou Navette épineufe, blancbe, Semblable à lo précédente, excepté que dans cetre le bord. É la levre fone jointes enjemble, Linn. 520. a. Seb. III.78.9-1 r. long. 4. lat. $3 \frac{3}{4}$ porce. (1. p. 366. a.

627 . Cornue, ou Navette épineufe, a aiguillons plus petits EO moins courbés, Linn. 520. थ. (2. p. 366. b.

628.P. Cornue, ou Navette épineufe, a aiguillons noueuse imoufJés, Linn. 520. Rumph. 26. 4. Gualth. 30. F ? Petiva Gaz. 68. 12. Seb 1II. 78. 8. (2. p. 367.

629 P. Cornue, ou Navette épineufe, beaucoup phis petit, Linn. 520. (3. p. 367 .

630 P. Spirille, ou Navette d longue queue, Linn. 554. yoyez Supra $n^{\circ} .563$. (3. P. 370 .

631 P. Becaffe à tête épineure, coquille d queue longue. fillone, noue, d neuds aigues, jaunatre, Linn. O. Lift. 900.20 ? (1. P. 305.

632 P. Becaffe, Linn. 518. Lift. 303. 23. Hollar Icon. 37. a. long. 7. larg. 2 pouc. (I., p. 37 I. a.

633 P. Becaffe, brunatre, mais plus petit, Linn. 518. Seb. III. 78. 5. 6. Kircher III. 270. long. $7 \frac{1}{2}$ pouc. (2. p. 37r. b: 634 P. Becaffe, plus court, Linn. 5 88. long. 4 pout. (3. P. 37 I $c_{\text {. }}$ 635 P. Scorpion, Linn. 524. Seb. III. 79. 13-16. Valent. 36 37. Lift. 906. 25. (2. p. 372. "Un, mais tres "grand fe trouve a Amfterdam, dans le Cquinet renommé, "du diligent Collecteur I. C. B R A D.

6.36 P. Scorpion, blanc; Linn S24. Argenv. 16.D. (2. p. 372. 037 P. Scorpion, approchant d la précedente, Linn. 524. (2 p.372. 638 P. Scorpion volant, d ftries, courbees a a aiguillons courbées courtes, jointes enfembles, \& coquille iblanche, rameaux crabless de poix. Linn. O. Adanfon Seneg. 1. 9. 2. 21? Tzavila 16. P? (I. p. 373 .

$6_{39} \cdot P$. Scelette, le bord de la bouche denté, d coquille longue coftee. E firits, courbele, drameaux courbes, bran, à cotes blanche., Linn. o. (2 p. 374.

640 P. Daine, ou Chaufre Trappe, ventrue, aigué d rameaux plus barges, fillonnes plies, trés. belle, Linn. 523. a. ow O.'Seb. III. 77. I? long. 34. larg. 3. pouc. (I. p. 384 . 
G4 P. Ramofa, Linn. 523. Seb. III. 77. 4. (2. Specim. 385. 642 P. Ramofa, præcedenti affinis, Lin?. 523. (2. Specim. 385.

643 P. Saxatilis, ramis parvis, fafciis fuicis \& albis, Linn. 525. Seb. 11I. 77. 5. Gualth. 37. G. (2. Lpecim. 386 .

644 P. Saxatilis, fimilis pracedenti, Linn. 525 . (2 fpecim. 386 .

645 P. Saxatilis, ramulis brevioribus, alba, Linn. 525. Seb. III. 77. 6\% (1. Specim. 387.

646 P. Ignifer, tefta oblonga acumirata, frondibus cencavis curvatis nodofic, fusca, Livin. 523. *. f. O. Seb. III. 77. 9, 10. Rumph. 26. 2. (2. tpecim. 380 .

647 P. Ignifer - Iinilis præcedenti. Linn. 523. «. . o. Valent. - 42. Petiv. Gaz. ror. 17. ( 2 fpecim. 380.

648 P. Ignifer? r. varietas, nodis tribus frondofis brevibus, duobusque longioribus, fusca, Linn. 523. ß. C. O. Liff. 448,73 ? (2. ipecin. 377 .

649 P. Argyna, tefta oblonga, convexa, acuminata, trifariam frordola, frondibus transverfis rofeis, fusca, Linn 523.

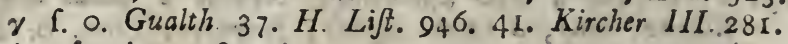
(2. Specim. 378 .

650 P. Argyna, præcedenti affinis, Linn. 523. \%. C. ०. Ar.

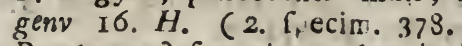

651 P. Argyna? [ varietas, fronde breviori, Linn 523. $x$. f. c. Seb. 1II. 73 11. Lift 945. 40 ? Petiv. Gaz. 99. 13. Vaient. 5. (2. fpecin. 379.

652 p. ArRyna? fo varietus, Limn. 523. भ. .0 0. (2. (pecim. 379.

653 P. Curnu Cervi, flavefrens, trifariam frondofa, nodisque

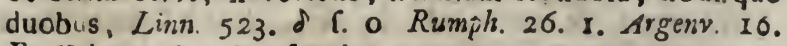
E. Valent. 39. ( $\because$. Specim. 375 .

654 P. Cornu Cervi. timilis præcedemi, Limn. 523. 8. T. C. (2. 'pecim 375.

655 P. Calcatrippa, tefta longa acuminata, nodofa frondofa, fronde minuri, Linn. O Gualth. 37. D? (2, fpecim.

656 P. Ventricola, tefta acuminata trifariam frondofa, nodora, fucca, Linn. 523. F. 0. Seb. III. 77. 7. (x. fpecim. 381.

$657 P$ Coflatn, tefta rb'onga, ventricofa coftata, trifariam frondola, lutea, Linn. 523. 乡. f. ०. Seb. III. 77. 1. 8. Gualth. 37. 1. Argenv. 16. C. Valent. 85. (2. Specim. 382. 


\section{Teftaces. Ordre IV.}

64. P. Cichorée, Linn, : 523. Seb, III. 77. 4. (2. p.

642 P. Cichorée, approchante da la précédente, Linn. 523.
(2. p." 385 .

643 P. Brulée, d petites branches, à bandes brunes, $E^{0}$ blanches, Linn. 525. Seb. III. 77. 5, Gualth. 37. G. (2.
p. 386 .

644 . Brulée, fémblable à la précédente, Linn. 525. (2. P. 386.

645 . Brulee ad rameaux plus courts, blanche, Linn. 525.

Seb. 1II. 77, 6? (I. p. 387 .

646 P. Chicore-brulé, à coquille allongé, aigut a feusilles cayes courbees, noulés, brune, Linn. 523. a. au o.. Seb. III. 77. 9. 10. Rumph. 26. 2. (2. p. 380 .

$647 P$. Chicore-brulee, Semblable d la precedente, Linn: 523 .

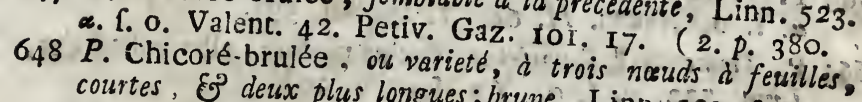
courtes, E' deux plus longues; brune, Linn. '523. A. ot o. Lift. 448. 73 ? (2. p. 377.

$649 P$. Chicoré Argyne à coquille longue, recourbee, aigue. froindee de trois maniere, id frondes transverfes couleur de rofe; brune, Linn. 523. Y. ou o. Gualth. 37. H. Lift. 946. 41.
Kircher III. 28 L. (2. p. 378 .

$650 P$, Chicore Argyne, approchant d la precedente, Linin. 523. y. ou o. (2.p. 378 .

65I P. Chicoré Argyne? ou varieté, a fronde plus courte, Linn. 523. r. ou. O. Seb. III. 73. I1. Lift. 945. 40.? Petiv.
Ga. 99. 13. Valent. 5. (2. P. 379.

652 P. Chicoré Argyne? ou variete, Linn. 523 . \& on 0. (2. p. 379.

553 . Bois de Cerf, jaunatre, frondé de trois manieres, di deux

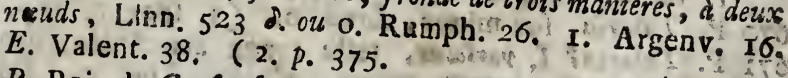
54 P. Bois de Cerf, femblable a la precedente, Linn. 523. d.
ou o, (2 p. 375 .

55 P. Calcatrippa, coquille longuie aigue, fronde, à fronde plus petite, Linn. o. Gualth. 37. D.? (2.p. 376 . 56. $P$. Chicore brulée, à coquille acuminee, pointue, feuilhu de trois manieres, nouleux, brune Linn. $523, \mathrm{n}$ ou $\mathrm{O}$. Seb. III. 77. 7. (I. p. $38 \mathrm{r}$.

57 P. Chicoré coftée, à coquille longue vextrue, a coftes, feuil-

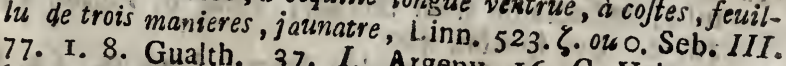
(2. p. 382. 
658 P. Coflata, fimilis pracedenti, Linn. 523. ל. f. O. (3. fpecim. 382.

659 P. Ramofa, labio rofeo, Linn. 523. Gualtb. 38. A. longit. 8. latit. 5. poll. (2. \{́pecim. 385 .

660 P. Ramoja, tefta margine angulato, ore elongato, coftis octo, fusca, Linn. O. (2. '́pecim. 388.

66r P. Trunculus, tefta oblonga, medio carinata, fexfariam nodofa . nigra, Linn. 522. «. f. o. Ginann.II. 64? Kircher III. 272. (2. fpecim. 392.

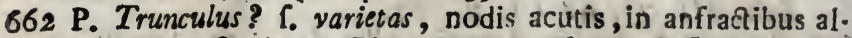
ba, fusco fasciata, Linn. 522. B. T. O. Liff. 947: 42? Kircher III. 277. (2. Ipecim. 389.

663 P. Trunculus? P. varietas, fexfariam nodofa, Linn. 522 , a. To 0. Ginan. II. 64. (r. fpecim , jor.

664 P. Trunculus? f. varietas, decies nodofa, alba rubefcens, Limr. 522, भ. . . o. (2. fpecim. 390,

665 P. Nodulo $\sqrt{a}$, teitta lata, gibbofa, acuminata, trifariam nodofa, Linn. 0. Adamfon Seneg. 1. 9.2.22\% (2. fpecim. 393 . 666 P. Volatilis, tefta oblonga, nodola, Havefcens, Linn. 0. Adonfor Sereg. I. 9. 2. 21 ? (2. Pिecim. 394.

667 P. Proctra, tefta anfractibus tumidis nodofis. elongata nodis, Limn. 0. Lift. 948. 43 ? Davila I. 16. N. O. ( (I. Ipecim. 395 .

668 P. Procera, pracedenti affinis, Linn. O. (2. fpecim. 395.

669 P. Talaria, tefta, fubtriquetra, anfraetibus longatis, trifariam nodofa, nodis delatatis, rofea, Linn. o. Davila

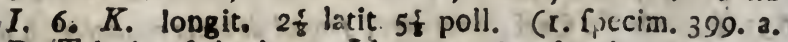
670 P. Talaria, fed minor, Linn. o. (1. fpecin. 399.

$67 \mathrm{i}$ P. Talaria? f. varietas, tefta longa acurt inata, quaque anfraclu tribus ramis ornata, albida, Linn. O. Davila 16. $L$ ? (2. fpecim. 398 .

672 P. Talaria?f.varietas, fimillina pracedenti, flavefcens, Linn. O. (2. fpecim. 398.

673 P. Talaria, paulo minor, Linn. O. (2. Specim. 397.

674 P. Otliquata, tefta acuminata, anfractibus nodofis, Linn. o. Davila I. 16. M. (1. Ppecim. 396.

675 P. Grana punctulata, tefta convexa, granulata, apice obtufo, anfraetaum nodi feptem, Lim. o. Valent. 14. a18. e. (2. fpecim. 400 .

676 P. Grana punclulata, albido fusca, Linn: o. (2. Specim. 400 . 
658 P. Chicoré cofté, Semólable la precedente, Linn. 523. 3. ou o. ( 3. p. 382 .

$659 P$. Chicoré, ou Chauffe-trappe, à levre couleur dé refe, Linn. 523. Gualth. 38. A. long. 8. Latit. 5. pousc) (2. p. 385 .

$660 P$. Branchu, à coquille le bord en angles, la bouche allongé, छ. huit cotes, brune, Linn. 0. (2. p. 388.

$66 \mathrm{I} P$. Troncon, à coquille longue carine au milieu, noueux de fix manieres, noire, Linn 522. a. ou o. Ginann. II. 64.? Lift. 947.42 ? Kircher $I I I .272$. (2. p. 392 .

$662 P$. Troncon? ou varieté, d-noeuds pointues, dans les anfractures blanche, rayé de brun, Lino. 522. B. O3s o. Kircher III. 277 .? ( 2. p. $3^{30}$.

$603 P$. Troncon? ou varieté, nouse d fix manieres, Linn. 522; * ou o. Ginann. II. 64. (1. P. 391 .

604 P. Troncon? ou varieté, dix fois noué, d'un blanc rougecs tre, Linn. 522. \%. r. o. ( $2 . p .390$.

o6s $P$. Plein de Noeux, à coquille large, boffu pointze, nout de trois manieres, Linn. O. Adanfon Seneg. 1.9.2. 22.? (2. p. 393.

$666 P$. Ailée. à coquille longue, nouerue, jaunatre, Linn. o. Adonfon Seneg. I. 9. 2. 21? (2.p. 394 .

$667 P$. Branchue tuberculé, coquiille, les anfracturès enflées noueux alongé, à trois noeuds, Lion. 0 . Lift. 648. 43 ? Davila I. 16. N. O (1. p. 395 .

$668 P$. Branchue tuberculée, Sembilable di la precedente, Linn. O. (2. P. 395 .

609 P. Talaire ai ée, da coquille, les anfractures elongée, nouée de trois manieres, de noeux, dilatées, couleur de roje, Linn. 0. Davila. I. 16. K. long. 25. lat. $5 \frac{5}{8}$ pouc. (1. p. 399. a.

670 P. Talaire ailée, mais plus petit, Linn. o. (r. p. 399.

$671 P$. Talaire membranée ? ou varieté, à coguille longue, pointue chaq̨ue anfracture orne de trois branckes, blanche, Linn. ه. Davila 1. 16. L? (2. p. 398 .

G72 P. Talaire membranée? ou varieté , très femblable da la precedente, jaunatre: Linn. o. (2. p. 398.

673 P.Talaire membrané ? un peuplus petit, Linn o. 2.p. 397.

$674 \mathrm{P}$. Oblique, à coquille pointue, les anfractures nouersfes, Linn. o. Davilla I. I6 M. (I. p. 326 .

675 P. Grains pointus, ou Chagrinée, à coquille recourbée, grenue, à fommet emoulfoe, à fept noerix davis l'anfratture, brune Linn. o. Valent. r4. a- 18.e. (2.p 400.

676 P. Grains pointus, ou Chagriné, 'brun blancheatre, Linn.o. (2. p. 400 . 
GENVS XIX. MVRICI FOR MES.

677 M. Mofcatus, tefta ventricofa , delatata, arf:actibus obliquis, dorfo fubaculeato frondofo, Linn. O. Lift: 894 . i4? long. \& latic. 4. poll. (1. Tuecim. 40r.

678 M. Radix, columella longiore, tefta umbilicata, anfractibus fulcatis dilatatis, apice brevi', fusco flavefcens,

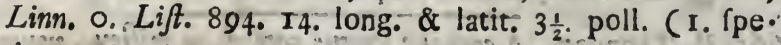
cim. 402 .

679 M: Radix, albefcens, fed minor, Linn: o. (2. Ipecim. 402.

$680 \mathrm{M}$. After, tefta ventricofa, anfractibus aculeatis, aculeis curvatis, bicarinata, carinis aculeatis, Linn. $0 .{ }^{3 e b}$. III. 60. 30? ( 1 . fpecim. 1040.

68I M. Coftatus, coft is quacuor aculeatis, rufo fuscis, Linn. 0. Seb. III. 60. 3I ? (r. fpecim. 893.

882 M. Bimuricatus, tefta dupliciter aculeata, Limm. o. Seb. III. 60. 32? Lift. 958. 11 ? (3. fpecim: 411.

683 M. Trifoliata, tefta fulcato coftata, trifariam frondofa, fronde obtura, Linn. o. Dayila I. II.E. (2. Tpécim. 403.

684 M. Nigrita, tefta alba oblonga, undecim frondofa, fronde crifpa, nigra, Linn. 0. Argenv: Suppl. 2. $K$. long. \& latit. $2 \frac{1}{2}$. poll. (2. Ipecim. $404 .-$, Si", milis fpecies egregii objecti, invenire licet Parifis, ", in celeberrimo Mufeo prænob. Matronz Prafid. de " BANDEVILle, de quo Argenv. affirmat. pro eo $f_{1700}$ "f. gall. efle folutum ":

685 M. Granatus, anfractibus depreflis, frandolo, flave. fcens, ore rubro fusco, Linn. o. Lift. 952. I ? $\mathrm{Da}_{-}$ vila I. Jo. b. d. (I. fpecim. 405.

686 M. Granatus? א. varietas, cre rubro fusco, Linn. c. (I. frecim 405.

687 M. Pinus, tefta oblonga, lactea, anfractibus frondora, Linn. 473. ๙. f. 0. Lift. 895. 15? Argenv. 15. G. Seb." III 52. 20. Rumph. 24. 4. (1. Specim. 406.

688 M. Larix, tefta oblonga, lactea, aníractıbus ramofis, Linn. 473. $\beta$ f. o. Seb. III 67. c. c. Rumph. 23. D? Lift. 895. 15? (2. ípecim. 410.

689 M. Larix, flavefcens, Lirn. 473. B. f: o. (2. Ipecim. 410.

690 M. Hippocaftanum, tefta albida, anfractibus echinatis, Lirn. 545. ๙. T. O Gualth. 3 I. F. Rumph. 43. D. Seb. III. $4980-82$. Lift. 895. 15 ? (r. ́pecim. 407.

69I M Hippocaftanum?? S.varietas,Linn. 545. . . 0. (2. (pecim. 408. 
GENRE XIX. EN FORME DE ROCHERS.

$677 R$. Noix de Moscate, a coquille ventrue éténdue, les anfroctures obliques, le dos un pers feuillé, \& pointue, Lip. 2. o. Lift. 894. I4? long. E larg. 4. pouc. (t. p. 40r.

$678 R$. Maffuée, ou Navette. à columelle longue, coquilie umbiliqué, les anfractures fillonnés étendues, ¿̀ fommet court, d'un brun jaunatre, Linn. 0. Lift. 894. 14. long. E larg. 3i. pouc. (I. p. 402.

$6: 9 R$. Maffuée, ou Navette, blanche, mais plus petite" Linn. 0 ( 2. p. 402.

$680 R$. Etoile, $\dot{a}$ coquille ventrue les anfractures pointzes. pointes recourbées, a deux caves, les carines pointues, Linn. o. Seb. III. 60. 30? (I. p. 104).

$68 \mathrm{I} R$. Etoile coftée, à quatre cotes pointues, d'un brun rouge, Linn. c. Seb. III. 60. 3I ? ( (. p. 898.

$682 R$ Ernile double, d coquille deux fois pointue, Linn, o. Seb. III. 60. 32? Lift. 958. II? (3. 4 II.

$683 R$. Navette à trois feuilles, $d$ coquille, dont les cotes font fillonnés, frondiu de trois maniere, d'un fronde obfcure, Linn. o. Davila I. Ir. E. (2. p. 403.

$68+R$. Noirette, ou Miafluée, d̀ coquille blanche allongée, onze fois frondu, d'une feuille crepue noire, Linn. O. Argenv. Suppl. 2. $K$. long. EO larg. $2 \frac{1}{2}$. pouc. (1. p. 404. - "Une - Pemblable piece de cet excellent objet, Se trouve a Paris ", dans le tres celebre Cabinet de Madame la Prefidente de "BANDEvíle, pour lequel Argenv. alJure qu'on a payé " $f$ I 700 livr. de fr.

$685 R$. Granatin, ou Pomme de Granate, les anfrabtures de primés, à feuilles jaunatre, $l_{2}$ bouche brun rougeatre. Linn. o. Lift. 952. I 'Daviia, I. 1o.b.d. (r. p. 405.

$686 R$ Pomme de Granate? ou varieté, à bouche d'un rouge foncé, Linn. O. (I. P. 405 .

68) $R$. Pomme de lir, a coquille allongé, blanche, les anfraEtures frondues. Linr. 473. $\alpha$. ou 0. Lift. 895. 15 ? Argenv.15.G. Seb III. 52.20. Rumph. 24. 4. (1.p.406. $688 R$. Pomme de Larix, ou de Pin, à coquille allongée, blanche. les anfraçures branchues, Linn. 473. $\beta$. ou O. Seb. III 67. c.e. Rumph. 23. D? Lif. 895. 15? (2. p. 410.

$689 R$. Pomme de Larix, jaunatre, Linn. 473.040. (2. p. 410. 690 R. Faux Moscate couronné, d̀ coquille blanche, les anfračures couverte d'aiguillons, Linn. 545. $.0 u$ o. Gualth. 3r. F. Rumph. 43.D. Seb.1II.49.80.82 Lift. S95. 15 ? (1.p.407: 691 R. Faux Mioscate couronné, ou varicté, Linn. 545. Q. ous O. (2. p. 408 . 
692 M. Cornutus tuberculatus, tefta oblonga, acuminata, anfractibus planis, tuberculis obfita, Limm. o. Seb. III. 60. 9 ? Petiv. Muf. 8. II. (2. Ppecim. 409:

693 M. Pyrus, tefta oblonga, rotundata, glabra, fusco linea. tà, Linn. O. Lift. 897. I7? (2. 'pecim. 429 .

694 M. Ficus Tarnatanus, tefta oblonga fubtrigona, feriebus duabus, muricatis fupra ramolis, Linn. 556. \%. f. O. Davila I. 15. G? Lift. 885. 6. b. (i. Ipecim. 895.

695 M. Tarnatanus, teft oblonga, ventricofa, acuminata, anfractu echinato, Linn. 556. $\beta$. F. O. Lift. 884. 6. a. (3. Fpecim. 896.

696 M. Tannatanus? fo varietas, Linn. 556. B. f. o. (2. fpecim. 896.

697 M. Nyeleris, f. Tarnatanus, tefta oblonga, lævis acuminata, vix convexa, columella ftriata, Linn. o. Lift. 896. 16 ? (2. Tpecim. 428.

698 M. Clava muricata, tefta oblónga, trifariam ramofa, flava, ramis albis, Linn. 0. Gualth. 37. C? (2. Specim 412. 2.

699 M. Clava muricata? if varietas, alba, fusco, maculata, Linn: 0: Seb. 1II. 60. I2. Petiv. Gaz. Ior. 18. (2. fpecim: 412 . b.

700 M. Prolefcens, terta anfractibus ramolis acumine obtufo, rugis, clathrata, albo nigroque varia , Linn. o. Lift. 956. 8 ? Seb. 1II. 52. 27. Gualth.43. V? (2. Specim. 413.

701 M. Prolefcens? f. varietas, Linn. 0. Lift. 811. 20? ( 2. fpecisin. $4 \mathrm{r} 4$.

702 M. Melongina, Linn. 547. Seb. III. 72. 2. 6. 7. Lift. 905. 24 a. Rumpir. 24. 2. Kircher III. I86. Hollar Icon. 39. Jong. $4 \frac{2}{2}$. latit, $3 \frac{3}{4}$ ( I. fpecim. 424. a.

703 M. Melongina, fasciis fuxcis, Lim. 547. Seb. III. 72. r. 3. 8. 9. Gualth. 26. F. (2. Epecim. 424. b.

704 M. Melongina, fasciis nigris, fuscisque, Linn. 547. Liff. 904. 24. Seb. III. 72. 4. 5. Petiv. Mirf. 21. 8? (2. fpecim. 424. C.

705 M. Armata, tefta fubrotunda acuminata, bifariam fpinofa, longitudinaliter itriata, fusca, apice albo, Linn. 0. Seb. III. 60. II. (2. fpecim. 426 .

$706 \mathrm{M}$. Armata, anfractibus fuperioribus albis, Linn. 0 . (2. Ipecim. 415 : 426 .

$707 \mathrm{M}$ Gericulatus, tefta oblonga, ramis obtufis, varicofa, trifarian cuberculata, Linn. 0. Lift. 991. 52? (2. Specirp. 425 , 
$092 R$. Faux Mofcate tuberculé, d̀ coquille allongée pointue; les anfractures plattes, couverte de tubercules, Linn. 0 . Seb. III.60. 9 ? Petiv. Muf. 8. II. (2. p. 409.

$093 R$. Poire, à coquille allongée, ronde, pelé, royée de brun, Linn. 0. Lift. 897. 17? (2. p. 429 .

$694 R$. Figue Tarnatane, à coquille allongée un peu triangu-

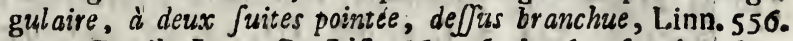
ou O. Davila I. 15. G? Llft. 885. 6. b. (I. Rpecim. 895. $695 R$ Tarnatane, ou Chauve-fouris, is coquille allongée, ventrue, pointue, anfradture à aiguillons, Linn. 556. $\beta$. ou o. Lift. 884. 6. a. (1. p. 896 .

$696 R$. Tarnatane, ou Chauve-fouris? ou varieté, Linn. 556. B. ou e (2. p. 895 .

$697 R$. Nyctere, ou Tarnatane, à coquille allongke, unie, poine tue, da peine recourbée, $\dot{a}$ columelle ftrié, Linn, 0 . Liff. 826. 16 ? (2. p. 428.

$698 R$. Herifion, ou Cheval de Frife, à coquille allongée. branchue de trois manieres, jaume, à branckes blanches, Linn. O. Gualth. 57. C ? (2. p. 412.a.

$692 R$. Heriffon? ois varieté, blanche, tacheté de brun, Linn. O. Seb. III. 60. I2. Petiv. Gaz, IOI. I8. (2. p. 4I $2 . b$.

$700 R$. P rolefcent, ou Heriffon, à coquillle, à anfrątures branchues, à fommet tmoulfé, ridé, varié de blanc Es de noir, Linn. 0. Lift. 956. 8? Seb. III. 52. 27. Gualth. 43. $V ?$ (2. P.4I3.

zor $R$. Prolefcent, ou Heriflon? ou varieté, Linn. O. Lift. 811.20 ? (2. p. 414.

$702 R$. Melongene, ou Coutis, Linn. 547. Seb. III. 72. 2. 6. 7. Lift. 905.24 . a. Rumph. 24. 2. Kircher III. 186.

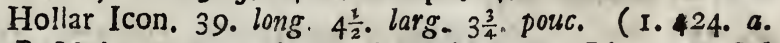

$703 R$. Melongene, ou Coutis, $a$ bandes brunes, Linn. 547. Seb. III. 72. I. 3. 8. 9. Gualth. 26. F. (2. p. $424, b$.

$704 R$. Melongene, ou Coutis, à bandes noires, E brunes, Linn. 547. Lift. 9־4. 24. Seb. III. 72. 4.5. Petiv. Mur. 2I. 8 ? (2. $424 . c$.

$705 R$. Armé, ou Singe, à coquille ronde pointée, épineuse a deux maniere, à ftries longitudisales, brunes, à fommet blanc, Linn. O. Seb. III. 60. II. (2. p. 426.

$706 R$. Armé, ou Singe, les anfrattures fuperieures blanches; Lirn. O. (2. p. 415.426 .

$707 \cdot R$. Heriffon variceule, à coquille allongée, branches émous. fées, variceux, tuberculé de trois manieres, Linn. 0. Lift. 991. 52 ? (2. p. 425 . 
708 M. Tuberculatus, tefta oblonga, convexa, tuberculis obfita, fufcefcens, Linn. O. (I. fpecim. 899.

7c9 M. Hamaftama? flavefcens, Linn. 466. a.f. o. Seb. III. 52. 27? Rumph. 24. D? Argenv. 17. H. (2. Pрecim. 417. 1033.

710 M. Mancinella, flavefcens, columella transverfim ftriata, Linn. 544. a. f. 0. Seb. III. 52. 27. Lift. 957.9? (2. fpecim. 418.

III M. Ricinus, apertura dentata, labro dentato, Linn. 540. Seb. III 60. 37. 47. (2. Ppecim. 420.

712 M. Ricinus? f. varistas, Linn. 540. $\alpha$. Seb. III. 52. 28. 29. Gualth 28. R. Rumph. 24. C. Argenv. 14. A. ( 3. fpecim. 419.

713 M. Varicofus, tefta oblonga, columella lævi acuminata, varicofa, fulcata, flavefcens, fasciis fucescentibus, Limm, ○. (3. fpecim. $4 \mathbf{3}^{2}$.

714 M. Luteus, tefta oblonga, convexa, coftata, Linn. O. (1. Specim. 1052.

715 M. Aulumiceus, tefta rotundata, ventricofa, anfractubus frondofis, ore pallide rofeo, Linn. o. Seb. III. 79. 7 I ? Lif6. 990. 51. b. (2. Specim. 416.

716 M. Dolariuns, Linn. 564. Seb. III. 52. 11. Kircher III. 347. Petiv. Gaz. 55. 4 ? (I. Specim. $42 \mathrm{I}$.

717 M. Planatus, tefta oblonga, anfractubus compreffis, fulcatis, tuberculatis, grifea, Linn. o. Lift. 1059 2. Davila I. 8. V. Petiv. Gaz. IOr. 4. (2. Ipecim. 422 .

718 M. Plaratus, fed minor, Linn. o. Lift. EO Petiv. I. c. (2. fpecim. 423.

Jrg M. Argus, tefta oblonga ventricola, convexa, feriebus tuberculoruin quinque, margine uberculato, Limb. O. Rumph, 49. B. Petiv. Gaz. 6. 6. (2. Ipecim, 433.

720 M. Fasciatus, tefta ventricola, convexa, acuminata, gla: bra, alba, fasciis tribus fuscis, Linn. ०. Petiv. Caz. I8. 5. (2. fpecim. 430.

721 M. Violaceus, tefta oblonga, tumida, acuminata, granulis ftriata, violacea, Linn. O, (2. Гpecia. 43 I.

722 M. Rufticus, telta oblonga, acuminata, ftriata, albo maculata, Linn. 0. Lift. 986.45 . (2. Ipecim. 435.

$723 \mathrm{M}$ Calcaratus, f. Perficus, tefta ventricola, rotunda, anfractibus tumidis, coftatim ftriata, fusca, Linn. $4 \sigma_{4} . \alpha$. f, O. Davila I. 9. B b. (2. \{pecins. 436 . 
$708 R$. Heriffun tuberculé, ì coquille allongée, recourbée remplie de tubercules, brunatre. Linn. o. (r. p. 899.

709. $R$. Hæmaftame, ou Conque Perfique, jaunatre, Linn. 465. a ou O. Seb. III. 52. 27? Rumph. 24. D? Argenv. I7. H. (2. p. I 7. 1033 .

7ro R. Mancinelle, ou Conque Perfique, jaunatre, à colnmelle friée de travers Linn. 544 a. ou U. Seb. III. 52, 27. Lift. 957.93 (2. p. 418.

7II $R$. Meure, à ouverture, E levre dentée, Linn. 540. Scb. III. 60. 37. 47. (2. p. 420 .

7I2 R. Meure? ou varieté; Linn. 540. a. Seb. III. 52. 28. 29. Gualth. 28. R. Rumph 24. C. Argenv. I4. A. (3. p 419 .

$.713 R$. Variceufe entlés, à coguille allongée, la columelie po: lie, pointue, enflée, fillonné, jaunatre, dे baindes brunes, Linn. O. (3. P. 432 .

7I4 $R$. Varieure jaune, à coquille allongée, recourbée à cotes, Linn. 0. ( I. p. IOS2.

II5 $R$. Alume, coguille ronde, ventrus, les anfrabtures en feuilles, ta bouche d'un rouge pale. Linn. O. Seb III. 79. 71 ? Lift. $990.51 . b 0^{\circ}$ (2. ${ }^{\circ} .416$.

716 R. Dolaire, ou Faux Escailler, Linn. 564. Seb. III. 52. II. Kircher III. 347. Petiv. Gaz. 55. A? (2. p. 42 r.

7I 7. R. Applati, ou Faux Cadran, à coquilile allongée, les anfrattures comprimés, fillonnés, tubercules, grife, tinn. o. Lift. I059. 2. Davila I.8. V. Petiv. Gaz 1GI - 4. (2. p. 422.

718 R. Applati, ou Faux Cadran, le pareil, mais plus"petit, Linn. o. Lift. EO Petiv. Gaz.l. c. (2. p. 423 .

$719 R$. Argus fafcie, la coquille allongée, ventruc recourbees à cinq rangs de tubercules, le bord tubercule, Linn. 0 . Ruinph. 42. B. Petiv. Gaz. $\sigma$ б. (2. p. 433 .

$720 R$. Bandé, à coquille ventrue, recourle é pointus pelé, blanche, à trois bandes brumes, Linn. O. Pettv. Gaz. I8. 5. (2. p. 43 .

$721 R$ Violetis, a coquille longue, exfle, pointue, Strix de grains, couleur violette, Linn. C. (2 p. $43 \mathrm{r}$.

722 R. Päira Batave, ou Champetre, d coquîlle longuc, pointue friée, tacineté cieblanc, Linn o. Lilt. $985.45 .(2 . p .435$. $723 R$ Perfigue eperonné, à coquitlle ventrue ronde: bes an-

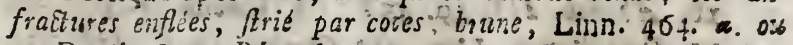
0. Davila 1. 9. B b. (2. p. $430^{\circ}$.

$$
\text { X } 5
$$


724 M. Perficus, tefta oblonga dilatata, acuminata, argute ftriata, fasciis fuscis, albisque, Linn. 464. Seb. III. 72. 12-16. Lift. 987. 46. 47. Argenv. 17. E. Petiv. Muf. 12. 7 ? (2. fpecim. 437. a.

725 M. Rudolphus, f. Perficus, pracedenti affinis.Linn. 464. Gualth. 51. L. (2. Specim. 437.a.

726 M. Patrlus, fimilis præcedenti, fed profundius ftriatus, \& magis granulatus, grifeus, Limn. 465. «. Seb. III. 72. 10. II. Gualth. 51. H. Rumpb. 27. E? ' (2, ppecim. 437 .

GEN VS XX. MVR ICES.

727 M. Urna, tefta obovata, rugofa, ftriata, Linn. 431. «. Fo O. Lift. 810. 19. Rumph. 24. 1. Gualth.

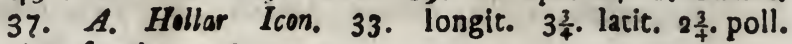
(2. fpecim. 438.

728 M. Urno? f. varietas, Linn. 431. «. f. o. Kircher III. 285. (2. Ppecim. 438.

729 M. Capitellum, Linn. 43I. Seb. III. 49. 77. Gualth. 49. H. Argenv. 15. K. (2. Specim 439.

730 M. Ceramica, Linn. 432. Seb. III. 52. 22 ? 23 ? Lift. 829. 51. Argenv. 15. E. Kircher III. 287. Hollar Icon. 38. longit. 4. poll. (2. Specim. 44I.

731 M. Ceramica, Linn. 432. Rumph. 24. A. Pétiv. Muf. II. I3. (2. Ipecim. 44I.

732 M. Turbinellus, Linn. 430. Seb. III. 60. 8. Petiv. Muf. Gualth. 26. L. Rumph. 24. B. Argenv. 14. P. Petiv. Muj. 4. 12, 11. 12. (2. Specim. 440 .

733 M. Turbinellus, Linn. 430 . (2. '́pecim. 440.

$734 \mathrm{M}$. Alatus, tefta rocundata obliquata . nodofa, ftriata, ramis crípis alata, albida, Linn, 0. Lift. 804. 12 ? ( 1 . fpecim. 443.

735 M. Neriteus, tefta tumida, oblonga acuminata, feriebus nodorum feptem, Linn. 542. Seb. III. 60. 42. 43. 46. 47. Angenv. 14. A. (2. 'pecim. 442.

736 M. Memacifon, Linn. 542. Seb. III. 60, 33. 37. 39. 4I. 42. Gualth. 28.N. Lift. 804.12, Rumph. 24. E. (3. ipecim. 444.

737 M. Cancellatus? f. varietas, tefta ovata, plicata, reticulata, columella fub umbilicata, Linn. 413. «. Lift. 965. 18. A? Seb. III. 49. 45 ? (2. fpecim. 445.

738 M. Caniellatrus. Linrs. 413. Gualth. 48. C. Lift. 830.54? 965. 19? Kircleer III. 343 ? (3. Specim. 446. 
$724 R$. Perfique, à coquille allongée étendue $\Xi 2$ pointue , d friés fines, \& fascies brunes \& blanches, Linn. 464. Seb.IIr. 72. 12-16. Lift. 987. 46. 47. Argenv. 17. E. Petiv. Muf. 12. 7 ? (2. p. 437. a.

$725 R$. Rudolphe, ou Perfique, approchant au precedent, Linn. 464. Gualth. 51. $L$. (2. p. 437. a.

$726 R$. Gcule Perfique, femblable au precedent, mais à ftries plus profondes, EO plus granulées, gris, Linn. 455 a Seb. 1II. 72. 10. 11. Gualth. 5I. H. Rumph. 27. E? ( 2 . p. 437 .

GE NRE XX. MURICES.

727 M. Urne, à coquille ovale, ridèe, flriée, Linn. $43 \mathrm{I}$. $\alpha_{\text {. }}$ ou 0 . Lift. 5 io. 19. Rumpn. 24. I. Gualth. 37. A. Hol-

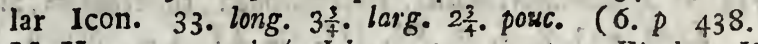

$728 M$. Urne? ou varieté, Lirn. 43I. \&. ou o. Kircher III. 285. (2. p. 438.

729 M. Vafe, Linn. 43r. Seb. III. 49. 77. Gualth. 49. H. Argenv. 15. K. (2. p. 439.

730 $M$. Heriffon, ou Cheval de Frife, Linn, 432. Seb. III. 51. 22 ? 23 ? Lift. 829. 51. Argenv. i 5. E. Kircher III. 287. Hollar Icon. 38. long. 4. pouc. (2.p. $44 \mathrm{~J}$.

73r $M$. Heriffon; ou Cheval de Frife, Linn. 432. Rumph. 24. A. Petiv. Mur. Ir. 13. (2. p. 44 r.

732 M. Culotte de Suifre, Linn. 430. Seb. IIT. 60. 8. Gualth. 26. L. Rumph. 24. B. Argenv. 14. P. Petiv. Mur. 4. 12, II. 12. (2. p. 440.

733 M. Culotte de Suiffe, Linn. 430. (2. p. 440.

$734 M$. Eponge ailé, à coquille ronde, oblique, nouenfe friée, a branches crepues, ailé, blanche, Linn. o. Lift. 804. 12? (2. p. 443 .

$735 M$. Fraife tuberculée, àcoquille enflée, allongèe appointue, à fept rayes de noeux, Linn. 542. Seb, III.60. 42.43. 46. 47. Argenv 14. A. (2.p. 442 .

736 M. Fraife pointée, Linn: 542. Seb. III 60. 33. 37. 39. 41. 42. Gualch. 28. N. Lift. 804. I2, Rumph. 24 E. ( $3 . p 444$.

$737 M$ Cancellé, ? ou varieté, à coquille ovale, pliée, reti. culé, à columelle umbiliquée, Linn. 4r3.a. Lift. 965. I8. $A$ ? Seb III. 49. 45 ? (2. p. 445 .

$738 M$. Cancellé Linn. 4I3. Gualth. 48. C. Lift, 830. 54 ? 965. 19? Kircher III. 343 ? (1. p. 476. 
739 M. Nigritus, tefta oblonga, tumida acuminata, acumu ne nodofo fusco, nigra, Linn. o. Valent. 20? (1. Specim. 465.

$740 \mathrm{M}$. Mucronatus, tefta oblonga, tumida, convexa, acuminata, rugofa, Linn. 413. $\beta$ f. 0 . Argenv. 17. M? Petiv. Gaz. 64. 8. Lift. 830. 52? (I. Specim. 434.

74 I M. Sjanko perverfus: tefta oblonga obliqua, medio convexa, coftaca, tunica olivacea adhuc tecta, Linn. o. Naturforfch, XII. tab. 3.f.r.a.b. longit. $4 \frac{1}{4}$. poll. (I') fpecim. 448.

742 M. Sjanko ramofus, tcfta oblonga, anfractu primo emarginato alba, apex poliendo detritus, Linn. 433. \&. f. o. Naturforfch. XIX, tab. I. f. 2. longit. $5 \frac{1}{2}$. latit. 3. poll. (I. fpecim. 450.

743 M. Sjankotuberculatus, tefta oblonga, tuberculata, albida, Linn 433. \%. C. O. Seb. III. 52. 4 ? (2. fpecim. 447.

744 M. Pyrum, tefta oblonga, nedio tumida, tuberculata, convexa, Linn. 433. a. Rumph. 46. 7 \% Lift, 816. 36. 27. (4. . Ppecim 452 ,

745 M. Sacrificator, tefta tumida, fubfphxroidea, accminata, glabra, lactea, Linn. 433. B. .. o. Gualth. 46.C.E? L ift 815. 25. Petiv. Gaz. 77. 6. longit. $6 \frac{\frac{1}{2}}{2}$. latit. $3 \frac{1}{2}$. poll. (I. Specim. 449.

$746 \mathrm{M}$. Sacrificator? f. varietas, margine columellam tegente, monftrofus, \& maxime docilis, Linn. 433. ..f. o Naturforfch. $X I X$. tab. 1. f. a-c, a-o. long. 3. latit. $2 \frac{3}{4}$. (1. Specim. $45 \mathrm{I}$.

747 M. Sacrificator, fed arrificiofe politus, \& excavatus, long. $7 \frac{1}{2}$. latit. $3 \frac{3}{4}$. poll. (1. Rpecim. 442.

748 M. Sacrificator, præcedenti fimilis, fed multo minor, (I. fpecim. 4.49.

749 M. Vespertilio, Linn. 428. Seb. III.67. \&c. Gualth. 28. T. V. Rumph. 32. I. Lift. 807. 16. Valent. I. 62, Petiv. Gaz. 70. 10. (4. fpecim. 453.

750 M. Vespertilio, delineatiore, Linn. 428. Kircher III. 294. Petiv. Muf. I5. I2. (6. Rpecim. 453.

75 I M. Vespertilio, fed dorro acute ramofo, anfractibus fpinofis luteis, Linn. 428. Seb. III. 67. \&c. Gualth. 28. G. I. M. Rumph. 32. H. Lift. 808. 17. Kirciner $11 I$. 295.306. Petiv Muf. 15. 8. (3. Specim. 454.

752 M. Vefpertilio, fed colore differens, \& minur, Linn. 428. ( 4 fpecim. 454

753 M. Velpertilio, pracedenti fimilis, Linn. 428. (4. Cpecim. 454. 
739 $M$. Moresque, d coquille allongée, enflée, pointue, d Jommet noueux, brun noir; Linn. O Valent:'20? (I. p. 405. $740 . M$. Pointu, à cóquille allongée, enflée, recourbée, ridée E pointés, Linn. 413. ß. ou O. Argenv. 17. M? Petiv. Gàz. 64. 8. Litt. 830. 52 ? (1. p. 434.

74I M. Sjanko pervers, on gauche, à coquille allongée oblique, recourbée au milieu, à côtes, encore couverte d’une tunique olivatre Linn. o. Naturforfch. XII.tab. 3. f. I. a.b. long. $4 \frac{1}{4}$. pouc. (I, $p$. $44^{8}$.

$742 M$. Sjanko branchu, d coquille allongée, la premiere an. froaure ébordee, blanche, la pointe eft froiffé, Linn 433. $\delta$ ou 0. Naturforich. $X I X$ tab. 1, f.: 2 long. $5 \frac{1}{2}$. larg. 3. pouc. (i.p 450 .

$743 M$. Sj nko tubercule, à coquille allongée, trbercubée, blanche, Linn. 433. $\gamma$ : ou o. Seb. III. 52. 4? (2. p. 447.

$744 M$. Poire, ou d'Offrande, à. coquille allongée, enflee aus milieu, tuberculée, recourbée, Linn. 433. «. Rumph. 26. 7 ? Lift. 816.26 .27$. ( $4 . p 452$.

745 M. D'Offrapde, coquille enflie, fuhfpheroide pointue, pelée, couleur de lait, Linn. 433. $\beta .016$ o. Gualth. 46. C. E?

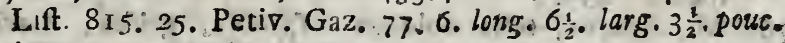
(.T. p. 449 .

746 M. D'Offrande? ou vavieté, le bord couvrant la columelle, - 2. monftreux, \& fort inftrudt if, Linn. 43j. $\beta$ : ou o. Naturforicb. XIX.tab. T. a-c, a-o. long. 3. larg. $2 \frac{3}{4}$. pouc. (1. p. 45 I. 747 M: D'Offrande, le pareil, mais artificiellement polie, $\mathcal{E}^{*}$ excavé, long. $7 \frac{1}{2} \cdot$ larg. $3 \frac{3}{4}$. pouc. (1. p. 44).

748 M. D'Offrande, femblable au precedent, mais beascous plus petit $\therefore$ ( $\mathbf{x}$. p. 449 .

749, $M$. Chauve fouris, ou Foudre, Linn, 428. Seb. IIT. 07. \&c. Gualth. 28. T. V Ramph. 32. I. Lift. 807. 16. Valent. I. 62 Petiv. Gaz. 70. 10. (4, p, 453.

750 $M$ Chauve fouris , ou Foudre, delinie, Linn. 428 . Kircher III 294 Petiv. Mor 15. 12. (6. p. 453.

$75 \mathrm{r} M$. Chauve fouris, mais le dos plus branchue, les anfradzures épineufes, jaune, Linn. 428. Seb. III. 67. \&c. Gualth. 28. G I $M$ Rumph. 32. H. Lift: 808 . 17. Kircher III. 295. 3c6. Petiv. Mur. 15. 8. (3. p. 454.

$752 M$. Chauve rouris, mais different de couleur E plus petit, Linn. 428. (4. $p .454$.

753 M. Chauve fouris, Jemblable au precedent, Linn. 428. (4. p) 454 . 
754 M. Luteoftama, tefta oblonga, anfractubus fpinolis, al$\therefore$ ba, reticulatim punctata, ore luten, Linn. 428. a. f. o. Spengler Icon: II. G. Naturforfch XIX. tab. I. fig. 3. \&.... 3. a. (2. ipecim. 900 .

755 M. Muficus, Linn. 427. Seb. III. 57.7-20. Lift. 805. 14. Graltih. 28. Z. tertia, Kircher III. 298. Hollar Icon 27. Spengler Icon. II. G? (2. Ipecim. 460 .

756 M. Muficus , fed minor, margine craffiore, albidus, Linn. 427. Argenv. 14. F. (3. fpecim 460.

¡57 M. Muficus? fed loco linearum fasciæ fuscre, Linn. 427. 6. F. O. Gualth. 28. T. (3. Ppecim. 460.*

758 M. Muficus granulatus, tefta granulato triata, flavefcens, Linn. 427: B. f. O. Seb. III. 57. 21. Lift. 806.15,812. 21 ? Gualth. 28. Z. prima. (3 fpecim. 463.

759 M. Contilenofus columella multi-plicata, tefta oblonga, fo flàvefcens, fusco punctata, fasciis oiivaceis, Linm.427. x. C. O. Seb. III. 57. 22. Spengler Icon. I. F? (I fpe. cim 456.

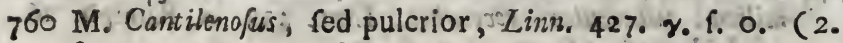
fpecim. 456.

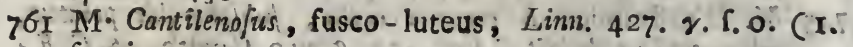
fpecim 457 .

762 M. Amrifus, tefta oblonga . Ppinofa, grifea, fasciis, fuscis. Linr. 429. a. C. o. Seb. III. 57. 3 ? 6. Valent. - 63 ? (x. pecim. 450 .

763 M. Ebraus, tefta oblonga, acumine obtufo, tubercu- Citlata, flavescens, fasciis fuscis. Linn. 429. Seb. III. 57. 3. 6? Lift. 809 i 8. Kircher III. 297. 298. D. Gualtla. 28. $Z$ in medio. (2. recim 458 .

764 M. Ebratus, fed major, Linn. 429. Seb. III. 57. 1. 2. long. $4 \frac{3}{4}$. latit. 3. poll. (I. Cpecim. 458.

765 M. Araufiacus, tefta nblonga, apice acuto, extenfo parvo; alto, aurantio diftinele fasciata', Linn. 434. * f.o.

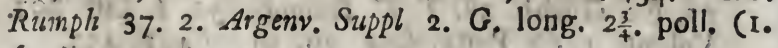
fpecim. 455 .

766 M. Lapponicus, Linn. 434. Rumph. 37. 3. 4? Seb. III. 57. 25. 26. Spengler Icon. II. A. long. 3ร. poll. (I. Epe. cim. 462 .

767 M. Metallicus, tefta oblonga, tuberculata, glabra, tunica metalli inftar. fulgente obtecta, Linn $\bullet_{0}$ longit. $2 \frac{1}{2}$, poll. (I. fpecim. 463 .

$768 \mathrm{M}$. Mamilla, teftánb!onga, ventricofa, gibbofa, glabra, apice marnillato, alba, lineis angulatis fuscis, Linn. 429. $\beta$. ' $^{\circ}$ c. Seb. III. 67. 5. 6. Gualth. 28 T. Lift. 799.6. longit. 5. latit. $3 \frac{1}{2}$. poll. (I. Specim. 464. 
754 M. Luteoftama, ou Cbauve fouris, it bouche jorme, a co. quille allongée, les anfractures épineufes, blanche, pointée, a bouche jaune, Linn. 428. \%. ou o. Spengler Icon. II. G. Naturforfch. $X I X . t a b .1 . f .3$ \& 3. a. (2. p. 900 ,

755 M. Mufique, Linn. 427. Seb.1II. 57. 7-20. Lift.805: 14. Gualth. 28. Z. tertia, Kircher III. 298. Hollar Icon. 27. Spengler Icon. II. G ? (2. p. 460 .

756 M. Mufique, mais plus petit, le bord epais, blancheatre, Linn. 427. Argenv. 14. F. (3. P. 460 .

$757 M$. Mufique? mais au lieu de lignes, d bandes brunes; Linn. 427 . a ou o. Gualth. 28. T. (3. p. 460.*

$758 \mathrm{M}$. Mufique granulée, d coquille grenue frie, jaureatre. Linn 427. $\beta$. ou o. Seb. III. 57.21. Lift.806.15, 812.
21 ? Gualth, 28. Z. prima (3. p. 46r.

$759 \mathrm{M}$. Cantilene, ou Mufique verd, columelle à plufieurs plies, à coquille allongée, jauneatre, pointée de brum, d fascies olivatres, Linn $427 . \%$ ou o. Seb. III. 57.22
Spengler lcon. I. F? ( 1. p. 456 .

$760 \mathrm{M}$. Cantilene, ou Mufique verd, le pareil, mais plus bears; Linn. 427. \%. ou 0. (2. p. 456 .

7бI M. Cantilene, brun-jaune, Linn. 427. $\gamma_{0}$ ou 0 . (I. p. 457.

$762 M$. Mufique, ou Bois vens, d coquille allongée, épinerufe, grife, à bandes brunes, Linn. 429, a. ou o. Seb. III. 57. 3 ? 6. Valent. 63 ? (1. p. 459 .

$703 M$. Hebraé, ou Bois vene, d caquille allongée, au fommet émouffé, tuberculé, jaunatre, a bandes brunes. Linn. 429. Seb.III. 57. 3. 6 ? Lift. 809. 18. Kircher III. $297^{\circ}$ 429. Gualth. 28. $Z$ ? in medio. (2. p. 458 .

$764 . M$. Hebræé, ou Bois vene, mais plus grand, Linn. 429. Seb. III. 57. I. 2, long. $4 \frac{3}{4}$. larg. 3 pouc. ' ( 1. p. 458 . 7.65 M. Pavillon d'Orange, do coquille allongée, à fommet aigue,
en longeur petit, blanche, d'orange diftinctement bande, Linn.
434. a.ou o. Rumph. 37.2. Argenv. Su 434. a. ou o. Rumph. 37.2. Argenv. Suppl.2. G. long. $2 \frac{3}{4}$.
pouc. ( 1 . P. 455.

200 M. Lapponée. Linn. 434. Rumph. 37. 3. 4 ? Seb.III. 57. 25. 26. Spengier Icon. II. A. long. 3ร. poruc. (I. p. 462. $767 \mathrm{M}$. Pavillon Metallique, de coquille allongée, enflée, tubberculée, pelé, couverte comme dinne tunique brillante de me-

$708 \mathrm{M}$. Mammelle, ou Perfane. (I. p. 463. bosfue, pelé , à fommet mannmelléblance àle allongée, ventrue, Zizac angles, Linn. $429 . \beta_{0}$ ou o. Seb. III. 64 s brines, É en 28. T. Lift. 799.6, long. 5. ou O. Seb. III. 64. 5. 6. Gualth. $769 \mathrm{M}$ : 
769 M. Mamilla, fed-minor, pulcrior, Linn. 429. B. f. O. (i. Speciu. 464.

770 M. Momilla, f. Perfus, frimilis pracedenti, non adultus, Linn. 439.2 C. o. Seb. III. 57.4 . 5. KircherIII. 10. Iongit. 2. tatic. is. poll. (2. Specim. rorg.

271 M. Perfictilus, teíta ovata tumida, apice depreffo, alb3, rubro friata, Limi. 404. a. Gualth 28. B. Lift.8n3. 9. Adna Jon Seneg. 1. 4. 10. 4. Petiv. Gaz. 8. 10. Kir- cher III. 238. Valent 78 ? (4. Tpecim. 406.

772 M. Perficulus, albus, punetis luteis Limn. 404. Gualih. 28. C. D. E. Lift, 803. 10. Petiv. Gaz. 8. 2. (2. Ppe.
cim 467.

773. M. Monilis? f. varietas, tefta ovata cumida, vis acuminata, alba, fasciis punctatis circumdata, Linn. 403. *.

f. o. Lift. 803. ir. (1. Specim. 47 I.

274 M. Pallidus, Linn 405. Gualth. 74. B. Lift 818. 32. (4. Specin. 901.

775 M. Pallidus? f. varietas, transverfim maculis grifeis pal:lidis ornata, Linn. 405. \%. C. o: Gualth. 43. H. Adanfon Seneg. I. 4. 10. I. (2. Ipecim. 474.

776 M. Miliarius? \& varietos, tefta ovaßa oblongata, acuminata, nitida, grifea, Linn. 402 . a.f.c. Gualth. 28. $A$ ? (1. Tpecim: 472 .

777 M. Arabicus, tefta ovata marginata, flavescens, luteo undatim fasciata, Lirm. 407. $x_{0}$ f o. Lift: 81 S. 31. (4.fpecim. 470 ,

378 M. Marginellus, tefta ovata, acuininata, gibbofa, margine tuberculat, flavefcens. Linn. 407. $\beta$. f. o. Lift. 818. 29 ? Gualth, 28. 1. Adanfon Seneg. 1. 4. 10. j. (i. Specim. 473.

779 M. Pyrulus, tefta ovata fubconvexa, acuminata, a'bus, flavefcens, Linn. 407 r. f. O. Lift. 818.29. Kircher III 329. (3. Firecim 469 .

780 M. Faba, tefta livi fubplicata, labro crenulato. Linn. 406. Petiv. Gaz. IO. 5. 102. 10, Lift. 812. 22 ? (4. fpecim. 468.

$28 \mathrm{I}$ M. Leptocaryon, tefta cylindrica, acuminata, friata gri-

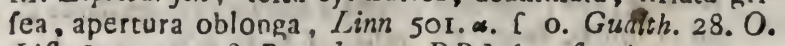
Lift. 813.23. a ? Rumph.33. DD? (2. Tpicim. 477.

782 M. Nux Seleri, tefta ovata, acuminata, alba, rugofa, fusco maculata, Limn. O. Lift. 813. 23, a. Rumph. 33 . CC? (2. Specim. 476 . 


\section{Teftaces. Ordre $I V$.}

769 R. Mammelon Perfane en Ziczac, plus petit, mais plus beau; Linn. 429. $\beta$. ou O. ( I. p. 454.

770 R. Mammelon Perfane en Ziczac, femblable au precedent, mais l'aile lui manque, Linn. 429. $\gamma_{0}$ ou o. Seb. III.57. 4:

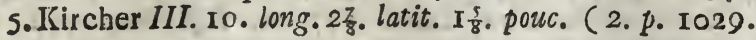

77 I $R$. Perficule ftriée, à coquille ovale, tumide, d fommet deprinae, blancbe, Jtrié de rouge, Linn 404.a. Gualth. 28 . B. Lift. 803.9. Adanfo Seneg. 1. 4. 10. 4. Petiv. Gaz.8. I0. Kircher IIII. 238. Valent. 78 ? ( 4. p. 466.

$772 R$. Perficule tigrée, blanche, à pointes jaunes, Linn. 404: Gualth. 28. C. D. E. Lilt. 803. 10. Petiv. Gaz. 8. 2. (2: p. 487 .

$773 R$. Chinoife? ou varieté, à coquille ovale, enflée, à peins pointue, blanche, entourée de bandes pointées, Linn. 403. c. ou o. Lift. 803. II. (I. p. 47 I.

$774 R$. Virgine pále, Linn. 405. Gualth. 73. B. Lift. 818. 32. (4. $p$. 901 .

$775 R$ Virgine Hammé? ou varieté, orné de travers par des taches grifes, E2 pales, Linn. 405. a. ous o. Gualch. 43. H. Adanfón Seneg." I.4. IO. I. ( I. P. 474.

$776 R$. Poire lazuré ou varieté, d̀ coquille ovale, allongée, pointre d'un beau gris, Linn. 402 a. ou 0 . Gualth. 28. A? (I. p. 472.

$777 R$. Arabique tigré, à coquille ovale, bordée jaunatre, fafcié de jaune, en ondes, Linn. 407. a. ou o. Lint. 818. 31. (4. p. 470 .

$778 R$. Marginelle, ou Bordée, à coquille ovale pointue, à boffe, à bord tuberculé, jaunatre, Linn. 407. $\beta$. ou o. Lift. 818. 29 ? Gualth. 28, $A$. Adanfon Seneg. I. 4. IO. 3o (I. P. 473 .

$779 R$. Poirette jaune, á coquille ovale un peu courbée, pointue jaunatre, Linn. $407 \cdot$ y.0u 0. Lift. 818.29. Kircher III. 329. (3. p. 469 .

$780 R_{3}$ Feve pointu, à coquille polie, un peu pliée, de levré crenue, Linn. 406. Petiv, Gaz. 10. 5, 102, 10. Lift, 812.22.? (4. p. 468 .

$78 \hat{\mathrm{r}} \cdot R$. Aveline feneftrée, à longue ouverture, coquille cylindrique aigue, ftriée de gris, Linn. 40 r. w. ous 0. Gualth. 28 . O. Lift. 813. 23. a? Rumph. 33. DD? (2. p.477.

$782 R$. Noirette Selderie, $d$ coquille ovale aigue, blanche, vidée, tachetée de brun, Linn. 0. Lift. 813. 23.a. Rumpho 33. CC.? (2. p. 476 . 
783 M. Vetula, tefta convexa ftriata, lineis longitudinalis

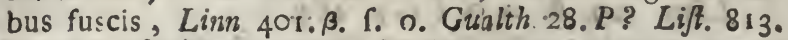
23. Rumph. 33. cc: (2. fpecim. 475.

$784 \mathrm{M}$. Coff tuss, tefta parva ovata, obtufo apice, caftanea, fascia unica alba, Linn. 397. ※. Liff. 824. 43 ? (I. fpecim. 1065 .

$785 \mathrm{M}$. Tornatilis, minima, ovata, acuminata, oblique antrotfum Itriata, fusco alboque maculata, Linñ. 394. $\alpha$.

f. O. Gualth 43. D. E. Litt. 824. 41 ? (2. fpecim. 902, 880 M. Pauperculus, Linn. 411. Lift. 819.35 Petiv.Gaz. II. 3. (2. fpecim. 500 .

287 M. Zebrula, tefta parva elongata, convexexa, acuminata ftriaca, flava, fasciis tuscis, Lirn. 4II. ๓. f. o. Lift. 8 I9. 33 ? ( 5 . fpecim. $50 \mathrm{I}$.

88 M Papalis, Linn. 426. Seb. III. 51. 1-5. Gualtb 53: I. a. Li/t. 839. 67. Kircher III, II9 Petiv. Muf. I3. 12. Hollar Iconi.43. long. 12. la' it 4. boll. (2. Ipecim. 99. 789 M.Cinetus, tefta magis ventricofa, anfractibus tuberculatis. alba, maculis quadratís rubris, Linn 426. « f. O. Seb. III. 50. 29. 30, 5I, in centro. Lift. 840, 68. Rumph. 29. I ? Valent. 45: (3. fpecim. 498.

790 M. Cinctus? I. varietas, tefta flava, fusco maculata, Linn. 426. 3. f. o. Seb. III. 50. 28. (2. '́pecim. 490.

791 M. Cinclus? fo varietas, tefta oblonga, ventricofa, granulora, fusca, albo guttata, Linn 426. \%. f. o: Gualth 53. L? Lift. 81 g. 36. ( 2 fpecim. 495.

792 M. Plicarius, Limn 423. Seb. III. 49.3I.32. Lift.820. 37. Kircher III. 65. Petiv. Gaz. 50. I. (4. Ipecim. 478.

793 M. Plicarius? f. varietas, tefta tuberculato coftata, flav. vefcens, ftriis fuscis, Linn. 423. $\alpha$. f. ค. Seb.'III. 49. 35. 38. Gualth. 54. E. Rumph. 29. S. Petiv. Muj.: 13. 7. (4. Specim. 479.

794 M. Undatus, tefta fubconvexa, tuberculata albida, fasciis fuscis albis, flavisque ftriata, Linn. 422. a. א. O. Seb. III 29. 30. Gualth. 54. B. (5. fpecim. 480.

795 M. Tuberculatus, tefta fubglabra, anfractibus tuberculatis, flava, olivaceaque, Linn. 422 . $\beta$ f. ०. Seb. $I I I .49$. 43. Gualth 54. D. (2. Ppecim. 481. a.

$796 \mathrm{M}$. Geniculatus, tuberculis acutioribus albida, flavo fasciata, lineis fuscis marginata, Livin. 422. $\boldsymbol{\gamma}$. ᄃ. O. Seb. III. 49. 27. 28. Grablth. 54. D. (2. Tpecim. 48I.

797 M. Vulpecula Linn. 422. Seb. III. 49. 33. 34. Petiv. Mus. 13. 6. Kiroher III, 78. (5. Specim. 482. 


\section{Teftaces. Ordre IV.}

$\$ 83$ R. Vieille ridée, do coquille courbée, ftriée, à lignes longitudinales brunes, Linn. $40 \mathrm{I}$. $\beta$. ou 0 . Gualth. 28. $P$ ? Lift 813. 23. Rumph. 33. cc. (2. p. 475.

$784 R$. Caffé, 1 petite coquille avale, fommet emouffé, cbatcigne, \& une bande blanche, Linn. 397. .. Lift. 824. 43? (I. p. IC65,

$785 R$. Tornatile, tres petit, ovale, aigue, endedans ftriée, obliques, tacheté de brun Eं blanc, Linn 394. \%.ouo. Gualth. 43. D. E. Lift. 824. 44 ? (2. P. 902.

$786 R$. Pauvrette, ou d'Ane rayé, Linn. 4II, Linto 81》. 35. Petiv. Gaz.ir. 3. (2. p. $5 \mathrm{co}$.

$787 R$ Zebrula, ou d'Ane jaune, 'd coquille petite allongée, courbée pointué, ftrièe, jaune, d̀ bardes brunes, Linn. 411 . \%. ou o. Lift. 819. 33.? ( I. p. 50 r.

$788 R$. Thiare, ou Couronne Papale, Linn. 426. Seb. III. 51. I-5. Gualth. 53. I. a. Lift. 839. 67. Kircher III. 119. Petiv. Muf. 13. 12. Hollar Icon. 43. long. I2. latit. 4 pouc. (2. p. 499.

$789 R$. Thiare cinglé, plus ventrue, les anfractures tuberculées, blanche, à taches quarrées rouges, Linn. 426. a. ou o. Seb. III 50. 29. 30,51. in centro, Lif. 840. 68. Rumph. 29. I? Valent. 45. ( 3.p. 498.

$790 R$. Thiare cinglé? ou varieté, à coquille jaune, tachetés de brun, Linn. 426. 3. ou o. Seb.III, 50. 28. (2: p. 490.

79I $R$. Thiare cinglé? ou varieté, d coquille allongée, yentrue, granulée, brune, gouttée de blanc, Linn. 426. \%. ou ○. Gualth. 53. .L ? Lift. 819.36. (2.p 495.

$792 R$. Chenille, ou Vis ridé, Linn. 423. Seb. III. 49. 3 I. 32. Lift. 820 37. Kircher III.65. Petiv.Gaz. 56. I. (4. p. 478.

$793 R$. Chenille, ou Vis pliffé? ou varieté, d coquille coftee par tubercules, jaunatre, d̀ ftries brunes, Linn $423 . \alpha_{.0 u}$ o. Seb. III. 49. 35. 38. Gualth. 54. E. Rumph. 29. S. Petiv. Muf. 13. 7. (4. p. 47 \%.

$794 R$. Chenille, ou Vis ondé, à coquille un peu courbée, triberculie, blancheatre, d bandes brunes, friees de blanc, ED de jaune, Linn. 422 \%. 0u 0. Seb. III. 29.30. Gualth. 54. B. (5. p. 480 .

$795 R$. Chenille, ou Vis tuberculé à coquille un peu pelée, les anfractures tuberculés, jaune, E olivatre, Linn 422. $\beta$. ou 0 . Seb. III. 49. 43. Gualth. 54. D? (2. p. 48 I. a.

$796 R$. Chenille, ou Vis pointu, à tubercules plus aigues blan. cheatre, fafcié de jaune, marginé, ou bordé de deux lignes brunes, Linn, 422. \%. ou O. Seb. III. 49. 27. 28. Gualth. 54. D. (2.p. $48 \mathrm{r}$.

797 R. Minaré, ou Vis jaune, Linn. 442. Seb. IIII. 49.33. 34. Petiv. Muf, I3. 6. Kircher III. 78. (5. 2. 482 . 
793 M. Fasciatus, tefta anfractubus tuberculatis, fasciis latis flavis, albis, fuscisque circumdata, Linn. 0 . Valent. 12 : (2. Specim. 483.

799 M. Succinctus? f. varietas, alba, lineis flavis, Linn. o. Seb. III. 49. 42 ? Valent. 12 ? (2. fpecim. 9)5.

800 M. Morio, Linn. 421. Seb. III. 49. 21.22. Gualth. 53. E. a. (4. fpecim. 484 .

801 M. Striatus, tefta vix tuberculata, antice ftriata, fasciis flavis fuscisque, Linn. 421. *. Seb.III. 49.4I. Gualth. 53. E. b. (I. fpecim. 904.

802 N. DecudJatus, tefta tenuis oblorga, acuminata, ftiata; alba, flavó maculata, Linn. O. (I. fpecim. 489.

803 M. Sanguifugus, Linn. 419. Seb. III. 49. I I-ro. Liff. 821. 38 Petiv. Gaz. 4. 5. (4. fpecim. 487.

804 M. Sanguifugus? f. varietas, albo ftriata, punctis fan-

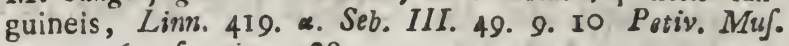
13. 9. (2. Ipecim. 488.

805 M. Circumplicatus, tefta longa acuminata, tuberculata grifea, Linn. o. Lift. 821.39? (1. Ppecim. 1066.

806 M. Coftatus, tefta oblonga tumidula, acuminata, fulcata, Linn. O. Gualth. 53. H? Rumph. 29. X? (I. Rpecim. 502.

807 M Ruffinus, tefta granulata, alba, coltis fulvis, Limn. 418 a. Seb. III. 50.1 47? 48? Gualth. 53, F. Petiv. Mus 9. 18. (4. Tpecim. 486.

808 M. Ruffinus? C. varietas, tefta fulcata alba, transverfim flavo fasciata, Linn. 4I8. א. Seb. III. 50. 47. 48. Rumpha 29. T. Argenv. 9. V. (3. fpecim. 485.

809 M. Scabriculus? f. varietas, alba, maculis flavis acutis, Lin?. 417. ๙. f. O. Gualth. 53. D? (2. fpecim, 491 .

8 ro M. Asceta, tefta fulcata, fusco tlammata, Linn. 424: ๓. F. O. Lift. 819. 34 ? (2. fpecim. 492 .

8 I M. Asceta? f. varietas, Linn. 424. B, \{. 0. Liff. 819. 33 ? Gualth. 54. H? (2. Ipecim. 493.

812 M. Sulcatus, tefta oblonga, fuscis pertufis longitudinaliter iulca'a, transverfim undatim ftriata, Linn 424. $\gamma$. f. o. Seb. III. 50. 49. Liff. 822. 40. (1. fpecim. 494

8 I 3 M.Mitra Cardinalis, ftriata, maculis fuscis punetatis, Linn. 425. *. . . . Seb. III. 50. 50. 51. Gualth, 53. C. b. Lift. 838. 65. (1. Specim. 486. 
798 R. Minaré, ou Vis bandé, d̀ coquille les anfractures tuberculées, entouré, de large bandes jaunes blanches E̊ bru. nes, Linn. O. Valent 12. (2. p. 483.

799 R. Minaré, ou Vis falcié? ou varieté, blanche, à lignes jaunes, Linn. O. Seb. III. 49. 42 ? Valent I2? (2. p. 905.

800 R. Negre, Linn. 42 r. Seb. III. 49. 2I. 22. Gualch. 53. E. a. ( 4. P. 484 .

$801 R$. Minazé, ou Vis ftrié, d peine tuberculé, ftrié par dedevant, à bandes jaunes, EO brunes, Linn. 421 . a. [Seb: III. 49 4I. Guath. 53. E. b. (I. p. 904

$802 R$. Minaré ou Vis croifé, de coquille inince, allongée, pointue, Jtriée, blanche, tachetée de jaune, Linn. C. (I. p. 489.

so3 $R$ Pavillon d'Hollande, ou Ruban, Linn. 419. Seb. IIT. 49. I-I9. Lift. 821. 38. Petiv. Gaz. 4. 5. (4. p. $48 ?$.

$\mathrm{SO}_{4} R$. Pavillon d'Hollande? ou varieté, ftrié de blanc, dpointes couleur de Jang, Linn. 4I9. Seb. III. 49. 9. 10. Petiv. Mur 13. 9. (2. p. 488.

$\$ 05 R$ Minaré, ou Vis circumplié, à coquille longue pointue, tuberculée grife, Linn. o. Litt. 821. 39? (1. p. 1066.

$806 R$. Minaré, ous Vis cofté, d coquille allongée, enflée, pointu, fillonnée, Linn. O. Gualth. 53. H.? Rumph. 29.X.? (I. p. 502 .

$807 R$. Ruffine, ou Vis granulé, d̀ coquille blanche, à cotes fauves, Linn. 418.. Seb III. 50, 47 ? 48 ? Gualth. 53. F. Petiv, Mur. 9. 18. (4. p. 486.

308 R. Minaré, ou Vis fillonné? ou varieté a coquille fillon née, blanche, bandée de travers de jaune, Linn. 418. B. Seb. III. 50. 47. 48. Rumph. 29. T. Argenv. 9. V. (3. p. 585 .

$809 R$. Minaré, ou Vis fcabriculé? ou varieté, blanche, ¿i taches jounes, aigues, Linn 417. a. ou 0 . Gualth. 53. D? (2. p. $49 \mathrm{I}$.

810 $R$. Moine brun, do coquille follonnee, enflammée de brun, Linn. 424. *. ou O. Lift. 8 i 9.34 ? (2. p. 492 .

8 II $R$. Moine brun? ou varieté, Linn. 424 . $\beta$. ou 0 . Lift. 819. 33.? Gualth. 54. H.? (2. p. 493 .

$812 R$. Moine fillonné, d coquille allongée, difillons rompus fillonné en long, Es friée de travers par ondes, Linn. 424. $\gamma$, ou o. Seb. III. 50. 49 Lift. 822. 40. (I. p. 494 .

$\$ 13 R$. Mytre de Cardinal, frié, a taches brunes pointées Linn. 425. a.0u 0. Seb. III. 50. 50. 5I. Gualth. 53, G b. Lift. 838. 65. (1. p. 496 . 
814 M. Mitra Cardinalis, Linn. 425. «. f. O. (3. Ipecim. 496.

815 M. Mitra Epifcopalis, Linn. 435. Seb. III. 51. 7-16. Gualth. 53. G. a. Lift. 839. 66. Kircher III. 120 . Petiv. Muf. I3. I I. Hollar Icon. 44. A. longit. 5. poll. (2.) fpecim. 49\%.

816 M. Mitra Epifcopalis, minor, præcedenti affinis, Limm. 425. (2. Specim. 497.

817 M. Mitra Epifcopalis, fed minor, fimilis præcedenti, Linn. 425. duo fpecim. quorum alterum apertum. (2. fpecim.497.

\section{GENVS XXI. A L A T E E.}

818 A. Terebella, tefta alba, ftriis undatis fuscis, Linn. 388: a. Lift. 736. 3r. (I. Specim. 5I4.

819 A. Terebella, tefta punetata, lineolisque picta, Linn. 388. Gualth. 23. O. Lift. 736. 32. Argenv. II. G. Pe tiv. Muf. 13. 24. Kircher III. 57. (2. Specim. 515.

820 A. Terebella, pallidior, Linn. 388. (3. Ipecim. 515.

821 A. Fufus, Linn 489. Seb. III. 56.1. Lift.854.11.12. Spengler Icon. III. B. longit. 7. craffit. $2 \frac{1}{2}$. poll. (1. fpecim. 503.

822 A. Fufus, fimilis præcedent1, Linn. 389 . longit. $6 \frac{1}{2}$. craft. 2. poll. (2. fpecim. 503 .

823 A. Lacinia, longa, anfractibus ad apicem granulofis, Linr. 489. . . F. O. Seb. III. 56. 2. Lift. gr6. 9. Argenv. IO. D. Kircher III. $12 \mathrm{I}$. Hollar lcon. 4 I. Jongit.

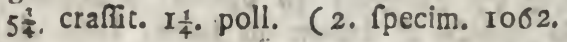

324 A. Lacinia, nondun adulta, Linn. 489. «. . O. Sob. III. 56. 3. Argenv. 10. A. (I. Specim. 504.

825 A. Pes Pelecani, fusco albida, Linn. 490. Lift. 865: 866. 20. 21. b. Kircher III. 85. 87. (2. Specim. 505. 826 A. Pes Pelecani, Linn. 490 Ginann. II. 58. 60. Petiv. Gaz: 79. 6, 127. II. (3. Ppecim. 505.

827 A. Vittata, flava fasciis tribus albis, Linn. 508. Seb. III 62. 20. Argenv. 9. F. Lift. 852. 8. Petiy. Muf. 7. 9. (2. Specim. 508.

128 A. Vittata, Linn. 508. Putiv. Gaz. 98. 12. tres fpecim. una Bava bondum adulta. (3. Tpecim. 508. 2. 
814 R. Mytre de Cardinal, Linn. 425. *. ou O. (3. P. 496. $815 R$. Mytre, ou Thiare Epifcopale, Linn. 425. Seb. III. 51. 7-16. Gualth. 53. G. a. Lift. 839. 66. Kircher III. 120. Petiv. Muf. I3. II. Hollar Icon. 44. A. long. 5. pouc. $(2, p .497$.

$816 R$. Mytre, ou Thiare Epifcopale, approchant au precedent, plus petit, Linn 425. (2." p. 497 .

\$17 R. Mytre ou Thiare Epifcopale, Semblable au precedent, mais plus petit, Linn, 425. deux pieces, dont l'uné eft ouverte? (2. p. 497.

GENRE XXI. A IL E'ES.

8 I8 A. Tariere ftrié, d̀ coquille blanche, à ftries ondées brunes, Linn 388. a. Lift. 736. 3I. (I. p. 514.

8 I9 A. Tariere pointu, mais la coquille pointée, $E^{2}$ peinte do lignes, Linn. 388. Gualth 23. O. Lift. 736. 32. Argenv. II. G. Petiv. Muf. I3. 24. Kircher III. 57. (2. p. 5 I5. 820 A. Tariere pointu, plus pale, Linn. 388. .. (3. p.515. \$2I A. Etoile, ou Fureau grande, dे queue courte, Linn. $489^{\circ}$ Seb. III. 56 I. Lift. 854. II. I2. Spengler Icon. III. $E$. long. 7. craff $2 \frac{1}{2}$ jouc. (I. p. 503.

82. A. Etoile, ou Fureau grande, a queue courte, le pareil de la precedente, Linn. 489. longit. $6 \frac{1}{\frac{1}{2}}$ cra $\iint^{2}$ 2. pouc. (2, p. 503 .

823. A. Etoile, ou Fufeau grande, à quere allongée, les anfractures vers le fommet granules, Linn. 489. \&. ou 0. Sej. III. 56. 2. Lift. 916. 9. Argenv. 10. D. Kircher III. I2I. Hollar Icon. 4I, long. $5 \frac{3}{4}$ crafJ. I $\frac{1}{4}$, pouc. ( 2 . p. 1062.

824 A. Etoile, ou Fufeau grande, \&̀ quene albongée, pas encore parfaite, Linn. 489. *ou 0. Seb. III. 56. 3. Argenv. Io A. (I. p. 504 .

825 A. Pied de Pelecan, ou petite Scorpion, brun blancheatre, Linn. 490. Lift. 865. 866, 20. 21. b. Kircher III. 85. 87. (2. p. 505 .

826 A. Pied de Pelecan, ou petite Scorpion, Linn. 490. Ginann. II, 58. 60. Petiv. Gaz.79. 6, I27. II. (3. p. 505.

827. A. Mifaine roullee, jaune, d trois bandes blanches, Linn. 508. Seb. III. 62. 20. Argenv. -9. F. Lift. 852. 8. Petiv Mur. 7. 9. (2. p. 508.

828 A. Mifaine roullée, Linn. 508. Petiv. Gaz. 98 12. trois efpeces, dont une of jaune, pas encore parfaite. ( 3. p.508.a. 
829 A. Vittata? r. varietas, fed tuberculata, Linn. 508. $\%$ f.' o. Lift. 852. 8, 855. 12. b. Seb. III. 62. 18. 19, (3. fpecim. 506. 7 .

830 A. Epidromis, Linn. 506. Seb. III. 62.21.26. Lift. $853^{\circ}$ 10. Petiv. Mus. 14. 18. (2. Specim. 509.

831 A. Epidromis, præcedenti affinis, Linn. 506. (2. fpecim. $5 \mathrm{c} 9$.

832 A. Epidromis? \{. varietas, flava, Linn. 506. \&. Rumpti. 36. P. Seb. III. 61. I6 ? Gualth. 32. G. (2. Ipecim. 5 ro.

833 A Epidromis? f. varietas, flava albo punctata, tuberculata, Linn. 506. B. f. O. Gualtb. 31. L. Kircher III. 342. (I. Rpecim. 1034.

834 A. Epidromis? f. varietas, macula quadrata, in anfractu quafi inulta, Linn. 506. $\%$. f. O. (1. Specim. 546.

835 A. Succincta? fo varietas, Linn. 509. ' $\%$. א. o. Seb. IIL 61. 15? Lift. 859. 16? Rumph. 37. X? (4, fpecim. $5 \mathrm{II}$.

836 A. Succintta? f. varietas, fed minor, Linn. 509. a. f. o. Seb. III.61. 15. Argenv. 10. C. (7. Specim. 511.

837 A. Succincta, Linn. 5c9. Gualth. 33. B. Rumph.36. X. (2. fpecim. 512.

838 A. Canaritm, Linn. 507. Seb. III 62. 23-25. 28. 29. Petiv. Gaz. 98. II, 100. 7 ? Kircher| III. 146. ( 4. fpecim. 513.

839 A. Canariun, nondum adulta, Linn. 507. (4. Specim? 513. a.

$840 \mathrm{~A}$. Canarium ? $\mathrm{f}$ variet is fasciis 2. fuscis, Linn $507 . \propto$. f. o. Petiv. Muf. 14. 17? (3. fpecim. I035.

84. A. Samar, tefta tumida convexa, transverfim nodofa in primo arfractu, Linn. O. Seb. III. 61 34.4I-46. Lift. 358 I 4. Rumph. 37. T? Gualth. 33. C.D. (1. Ppecim. 516.

842 A. Urceus, tefta alba, fasco guttata, Linn. 512. \%. Seb. III. 61. 30. 31. Gualth. 32. E. Lift. 857. 13. Valent. 65. (3. fpecim. 517:

843 A. Urcerus, Linn. 512. Seb. III. 6r. 23. 24. \&c. Lift. 848. 2. Petiv. Gaz. 98. 14. Muf. 14. 21. (6. fpecim. 5 I3.

8.4 A. Urceus, fed nondum adultus, Linn. 512. Seb.III.61. 49. 55. 56. (4. fpecim 555. 1016.

845 A. Gibbofus, fasciata ex flavo alboque, Linn.5I2. B. f. a: Seb. III. 6r. 54? Lift. 848. 3. Rumph. 27. W? (G. fpecim. 519.

346 A. Melaftoma, tefta oblonga nodofa, oblique acuminata, fauce nigro, Linm. 495. \&. . . C. Lift. 959. 15. (1. Rpeaim. 968. 


\section{Teftaces. Ordre IV.}

829 A. Mifaine roullée? ou varieté, mais à tubercules, Linn. 508. a ou 0. Lift. 852. 8,855. 12. b. Seb. III. 62. I8. 19. (3. p. 506. 7.

830 A. Epidrome, ou Mifaine deployée, Linn 506. Seb.III. 62.21.26. Lift. 853. 10. Petiv. Muf. 14. 18. (2. p. 509.

\$3I A. Epidrome, ou Mifaine deployée, le pareil de la precedente, Linn. 506. (2. p. 509.

$8_{3} 2$ A. Pigonneau? ou yarieté de la precedente, jaune, Linn. 506, «. Rumph. 36. P. Seb,III. 61, 16 ? Gualth. 32. G. (2. p. 510 .

833 A. Pigonneau? ou varieté, jaune, pointée de blane, tuberculée, Linn. 506. $\beta_{0}$ ou 0 . Gualth. 31. L. Kircher III. 342. (1. p. 1034.

$834 A$. Pigonneau brulé? ou varieté, d̀ tache quarrée, comms inuré dans l'anfrą̧ure, Linn. 506. \%. ou u. ( . p. p. 546.

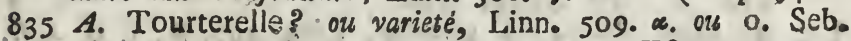
III. 6r. 15? Lift. 859. I6 ? Rumph. 37. X? (4. p. 51 r. 836 A. Tourterelle? ou varieté, plus petite, Linn. 509. ๓. f. o. Seb. III. 6I. 15 Argenv 10. C. (7.P. 5 Ir.

837 A. Tourcerelle ou Canarie carinée, Linn. 509. Gualth. 33a B. Rumph. 56.X. (2. . . 512 .

838 A. Boffette, Linn. 507. Seb. III. 62. 23-25. 28. 29. Petiv. Gaz. 98. II , I00. 7 ? Kircher III. I46. (4, p. 5 I3. 839 A. Boffette, la mbme, pas encore parfaite, Linn. 507. (4.p. $513 . a$.

840 A. Boffette? ou varieté, dd deux bandes brunes, Linn. $50 \%$ \%. ou 0. Petiv. Mur. I 4. 17? (3. p. 1035.

845 A. Samare, ¿ coquille enflée, recourbée, noude de travers dans la premiere anfrafture, Linn. 0. Seb. III. 61. 34. 4146. Lift. 858. 14. Rumph. 37. $x$ ? Gualth. 33. C. D. ( 1, p. 516 .

842 A. [Urcée, ou Tourterelle boflue, d coquille blanche, gouttée de brun, Linn. 512.. Seb. III.61. 30. 31. Gualth. 32. E. Lift. 857. I3. Valent. 65. (3. p. 517 .

843 A Urcée, ou Tourterelle bolfue, Linn. 512 . Seb. III. 61. 23. 24. \&z. Lift. 848. 2. Petiv. Gaz. 98. I4. Muf, 14. 21 . (6. p. 518.

844 A. Urcée, ou Tourterelle boffue, mais pas encore parfaite, Linn, 5r2. Seb. III. 6r. 49. 55. 56. (4. D. 555. Ior6.

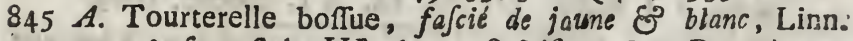
512. B. f. O. Seb. IIT. 61. 54.? Lift. 848. 3. Rumph. 27. $W$ ? (6.p. 519.

$346 \mathrm{~A}$. Grenouilie, à bouche noire, d coquille allongée, nouke, oblique pointue, is bouche noire, Linn. 495, \%. ou 0. Lift. 959. Is. I. P. 908. 
847 A. Melaftoma? f. varietas, dorfo tuberculis fasciatr. ore carneo, Linn. 495. \& C. 0. Seb. III. 61. 7? Lift. 850. 5 ? (I. fpecim. 9)7.

848 A Flavigula, lineolis fuscis cincta, ore flavo, Limm. 495. ข. 1. O. (2. Specim. 523.

849 A. Lentiginofa, albida, maculis fusco olivaceis, Linn 495 : Seb. III. 62. 11. 30. Kircher III. 3c0. Lift. 861. 18. (4) fiecim. 524. 5.

$\$ 50$ A. Lentiginoja, fimilis pracedenti, Linn. 495 . ( 3. \{реciur $\{26$.

85 I A. Lentiginofa, imperfecta, Linn. 495. Seb. III. 62, 37. 40 Argenv 14. I? (4. Specim. 520*,

852 A. Auris Diane? f, varietns, tefta oblonga, convexa, trifariam nodola, flavo grifea ; Linn. 497. B. \& O. Lift. 872 27. (2. Rpecim. 529.

853 A. Auris Diana? I varietes, Limm. 497. A. C. O. Liff. 871. 26? (2. fpecim 529.

85 A. Auris Diance, Linn. 497, Seb. III. 62. 13. Gualth. 52. D Lift. 871.26 Kircher III. 302 3. (4. fpecim. 528 . 855 A Auris Diane? 1. varietas, Linn. 497. ๓. f. O. Seb.III. 61. 1-4, 62, 16. Petiv MuS. 14. II, Lift. 872. 28. (4. fpecim 527.

856 A. Auris Diance? f. varietas, Linn 497. «. . o. Lift. 893. 12 a? (4. fpecim. $527^{*}$.

857 A. Gallus, Linn. 496. «. Lift. 873, 29. 30. longit. $4 \frac{1}{2}$. poll ( $\mathrm{r}$. fpecim $53 \mathrm{r}$.

858 A. Gallus? I varietas, tefta albido rubroque flava, Linn. 496. «. f. O. Seb. III. 62. 4, 63. 6. Kircher III 321 (2. fpecin. 531 .

859 A. Gallus, fed lutous, Linn. 496. Seb. III. 62. 36. (2. fpecim. 53़t.

860 A. Marginata, Linn. 499. *. C. O. Seb. III. 62. 9. 10. 3. \& c. Gualth 32. F. Kircher III. 308. (2. fpecim. 533.

86r A. Marginata, grifea, Linn. 499. ๙. 1. O. (2, fpecim. 5 ?3.

862 A. Marginata? f. varietas, coftata, tuberculata, Linn. 499 B. Y. o. Seb. III 62. 4. 5. I2. (2. fpecim. 530 .

803 A. Marginata? fo varictas, ex tlavo rubefcens, Linn. 499. B f. 0. Seb III. 62. 14. (1. fpecim. 1063.

864 A. Latifima tefta flavefcente, labio recurvo, Linn. 505.

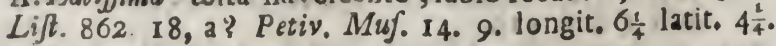
po:. ( 4 ipecim. 542.

865 A. Latifjoma, labio recurvo, ejusdemque magnitudinis, Lirn. 505. (I, fpecim. 542. 
847 A. Grenouille bandée, ou varioté , le dos à tubercules bandés, la bouche rouge, Linn. 495. $\beta$. ou O. Seb, III. 6r.' 7. ? Lift. 850. $5 . ?$ (1. p. 907.

848 A. Grenouille? ou Jaune Gorge, entourée, de lignes brunes, la boucho jarne, Linn. 495. $\gamma$. ou O. (2.p. 523.

849 A. Grerouille tuberculée, blancheatre, a taches brunes olivatres, Linn. 495. Seb. III. 62. TI. 30. Kircher III. 300. Lift. 86r. 18. (4. p. 524. 5.

$\$ 50$ A. Grenouille tuberculée, pareille de la precedente, Linn. 495 (3. $p$ 526.

851 $A$. Grenouille tuberculé, imparfaite, Linn. 495. Seb. III. 62. 37, 4. Argenv. I4. I? (3. p. 529 \%.

852 A. Oreille, ou Cadran à bord noir, d coquille allongte; recourbé, nouée de trois manieres, d'un gris jaune, Lina. $497 \beta$ ou o. Lift 872.27 . (I.P. 529 .

$853 A$. Oreilie, ou Cadran à bord noir? ou variete, Linn. 497. $\beta$. ou o. Lift. 871. 26 ? (2. p. 529.

$854 A$ Oreille d'Ane, Linn. 597.Seb. III.62. 13. Gualth. 52. D. Lift. 87 I. 26 ? Kircher III. 802. 3. (4. P. 528 .

855 A. Oreille d’Ane? au varieté, Linn. 497. a. ou o. Seb. III. 61. 1. $-4,62.16$. Petiv. Muf, 14. 11. Lift, 872. 28. (4. P. 527 .

$856 A$. Oreille d'Ane ? ou variete, Linn. 497. \&. ou ०. Liit. 893. 12. a? (4. P. 527 .

857 A. Cocq, ou aile faillante, Linn.496. «. Lift. 873.29.30, long. $4 \frac{2}{2}$. pouc. (I. p. 531 .

858 A. Cocq, ou aile faillante? ow varieté, \& coquille blancheatre, jaune EO rouge, Linn. 496 \&. ou o. Seb. III. 62. $4,63$. 6. Kircher IIII. 321. (2. P. $53 \mathrm{r}$.

859 A.Cocq, maisjaune, Linn. 496 . Seb III.62.36. (2. p. 53x.

860 A. D’Amerique bordée, Linn. 499 \%. ou o.Seb.III. 62. 9. 10. I 3. Gualth. 32. \&c. F. Kircher III. 308. (2. p 533. 861 A. D'Amérique bordee, grife, Linn. 499 . «. ou ๑. (2. p. 533 .

862 A. D'Amérique bordée? ou varieté, d côtes tubercules. Linn. 499. B. ou o. Seb. III. 62. 5 . I2. (2. p $530^{\circ}$.

863 A. D'Amerique bordée? ou variete, d'un rouge jounatre Linn. 499. B. or O. Seb. III. 62. 14. (1. p. 1063.

864 A. Tres large, ou Levre recourbée, d coquille jaunatre: Linn. 505. Lift. 862. 18. a? Petiv. Mur. 13. 9. long:

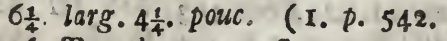

865 A. Tres large, au Levre recourbée, de la méme grans: deur, Linn. 505. (I. P. 542. 
866 A. Latiflma, nondum adulta, Limn. 505. Seb. III. 83. 12-14- longic. 5. poll. (2. fpecim, $542^{*}$.

867 A. LatiJima, præcedenti affinis, albo maculata, Linn, 505. longit. $4 \frac{z}{2}$ poll. (2. Specim. $642 \%$.

868 A. Millepeda, Capenfis, tefta oblonga, dilatata convexa, dorfo tuberolo, ore fusco, labro convexo, Linn. 494. $\alpha$. f. 0. Seb.III.62.3, Spengler Icon. III. A ?, (2. Specim. 54I-

$\$ 69$ A, Chiragra, Iabro bexadactylo . digitis curvis, Linz. 49r. Seb III. 62. \&c. Gualeh. 35. B. Petiv. Muf. I 4. 3 Hollar Icon.6. duo fpecimina, longit $8 \frac{1}{2}$. latit. $5 \frac{3}{2}$. alterum $7 \frac{2}{2}$. Lat. $4 \frac{z}{2}$. poll. (2. Specim. 540 .

870 A. Chiragra, nondum adulta, Linn. 49r. Seb. III. 82. \&c. Lift 875. 31. Rumph. 36. G. Kircher III. 317. 318, lon. git. $7 \frac{2}{2}$ latit. 5. poll. (2. Specim. 340*.

87 I A. Chirsgra, perfecta \& nondum adulta, Linn.49x longit. 5. lat. $3 \frac{1}{2}$. poll. (2. fpecim, 540 .

872 A. Chiragra, præcedenti affinis, lactea, Linn.49r. Ion. git. 6. latit. 4. poll. (I. fpecim. 540*.

873 A. Chiragra, Capenfis, affinis $n^{\circ} .869$, alba, fusco fascia-

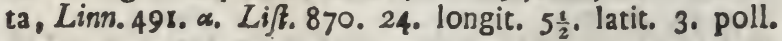
(2. (pecim. 9 ro.

874 A. Chiragra, nondum adulta, absque digitorum indicio, ex albo flava, fuscoque varia, Linn. 49r. Seb.III. 62. 34, 83. I. 2. Rumph. 35. C. (3. fpecim. 540**.

875 A. Chiragra, præcedenti affinis, Linn. 491. (2. Specim. $540^{\text {k*. }}$.

876 A. Congylis, f. Lambis, Linn. 493. . five Gigantea? Linn. 504. ๙. โ. O. Seb.III.63.3. Lift. 931. A. Davila I. 13. A. Gualth. $36 . B$ ? longit. $8 \frac{1}{2}$. latit. 5. poll. (I. fpecim. 1032.

377 A. Congylis, f. Lambis? flava alboque varia, Linn. 493. *. f. O. Gualth. 30. A (2. fpecim. 539.

878 A. Lambis, Linn. 493. Seb. III. 82. \&c. Gualth. 35. C, 36. A. B, Rumph. 35. H. Lift. 866. 21. longit. $5 \frac{1}{2}$. latit. 3. poll. (I. Specim. 534.

879 A. Lambis, aliquanto minor, Linn. 493. (2. Specim. 534.

880 A.Lambis, nondum adulta, Linr. 493. Seb.'III. 82. \&c. Rumph. 36. G. Lift. 883. a. 5? (2. Ipecim. $534^{*}$.

881 A. Lambis, nondun adulta, flavefcens, Linn.493. (3. fpecim. $534^{*}$. 


\section{Teftaces. Ordre $I V$.}

866 A. Tres large, ou Levre recourbée, pas encore parfaite, Linn. 505. Seb. III. 83. 12-I 4. long. 5. pouc. (2: p $542^{*}$.

807 A. Tres large, ou Levre recourbée, la méme, tachetée de blanc, Linn. 505. long. $4 \frac{2}{2}$. ponc. (2. P. 542*.

868 A. Millepied, du Cap, do coquille allongée étendue recourbée, d dos tubercule . bouche rouge, E' levre recourbée, Linn. 494. *. ou o. Seb. III. 62.3. Spengler Icon. III. A. (2. p. $54 \mathrm{r}$.

869 A. Grife du Diable, le levre à fix doigts recourúes, Linn. 491. Seb. III, 62. Eंc. Gualth. 35, B. Petiv. Mur. I4. 3. Hollar Icon. 6. deux pieces, l'une long. $8 \frac{1}{2}$. latit. $5 \frac{1}{2}$, l'autre long. $7 \frac{1}{1}$. latit. $4 \frac{1}{2}$. pouc. (2. p. 540 .

870 A. Grife du Diable, imparfaits, Linn. 491. Seb. IIT. 82. \&c. Lift. 875. 3I. Rumph. 36 G. Kircher III. 317 . 318. long. $7 \frac{3}{3}$. latit. 5. pouc ( 2 p $540^{*}$

871 A. Grife du Diable, parfaite, E imparfaite, Linn. 49r, long. 5. latit $3 \frac{1}{3}$. pouc. (2. p. 540 .

872 - A. Grife du Diable, Semblable dे la precedente, couleus de lait. Linn. 491. long. 6. latit. 4. pouc. (1. p. 540*:

873 A. Grife du Diable, du Cap approcbant d $n^{\circ} .869$. blanche,

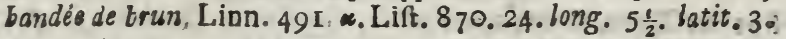
pouc. (2. p. gro.

$874 A$. Grife du Diable, imparfaite, fans marque ds' points, d'un jaune blancbeatre, varié de brun, Linn. 49 \%. Seb. IIL. 62. 34,83 . I, 2. Rum h 35. C. (3. P. $540^{* *}$.

$875 A$ Grife du Diable, famblable d la precedente, Linn. 49r. (2. P. $140^{* *}$.

876 A. Lambis émoufré, Linn. 493. a. ou Gigantesque? Linn: 504 a. ou 0. Seb. III. 63. 3. Lift. 93 I. A. Davila $I_{\text {: }}$ I3. A. Gualth. 36. B ? longit. $8 \frac{1}{2}$. latit. 5. pous. (I. P. 1032.

877 1. Navette, or Lambis rebouché? varie de blanc; $\mathcal{E}^{\circ}$ jaune, Linn. 493. «. or6 0. Gualth. 30. $A$. (2. p. 539. 878 A. Crocbet, ou Crapaud, Linn. 493. Seb. III. 82. \&c.? Gualth. 35. C, 36. A.B..Rumph.35.H, Lift. 806.21. long. $5 \frac{1}{8}$ : latit. 3 pouc. (1. p. 534 .

879 A. Crochet, or Crapaud, tant Joit peu, plus petit, Linn. 493. (2. p. 534 .

880 A. Crochet, ou Crapaud, imparfaite, Linn. 493. Seb. III. 82. \&c. Rumph, 36. G, Lift. 883. a. 5? (2. p. $534^{*}$ :

881 A. Crochet, ou Crapaud, imparfaite, jaunatre, Linn. 493. (3. p. $534^{*}$. 
882 A. Lambis, glabra, alba pullaque, Linn. 493. Scb. IIT. 62. 33 ? (2. fpecim. $544^{*}$.

883 A. Lambis, præcedenti affinis, ftriata, alba, fusco maculata, nondum adulta, Linn.493. Seb. III. 62.35, 3i. Lift.883. 6 . Rumph. 35. D. : (3. Cpecim. 534**

884 A.Lambis, pracedenti affinis, Limn. 493. (3. [pecim. $534^{* *}$. 885 A. Millepeda, Capenfis, late marginata, Linn. $494 . \propto$. . . ०. Liff. 868. 23 ? longit. $4 \frac{\frac{1}{2}}{2}$ latit. $2 \frac{1}{2}$. poll. (I. fpecim. 909.

886 A. Millepeda, fauce alba, fusco friata, exius flava, Linn. 494. Seb.III.82. \& c. Lift. 868.23. Kircher III.315. Petiv. Muf. 14.7. (2. fpecim. 535.

887 A. Scorpio, ore purpureo ftriato, Linn. 492. Seb. III. 82. âc. Lift. 867. 22. Petiv. Muf. 3.2. Valent. 28. longit. $4 z_{2}^{t}$ poll. (2. fpecim. 537 .

888 A. Scorpio, pracedenti affinis, Linn. 492. duo fpecimina, una cuin ron adulta, digitis carente. (2. ppecim. 537. 889 A. Securiclata, ore rufefcente, glabro, Linn. 492. « f.o. long. $5 \frac{2}{2}$ latit. $2 \frac{1}{2}$ poll. (1. fperim. 533 .

890 A. Securiclata, præcedenti fimilis, Linn. 492. a. f. O. (2. fpecim. 538.

891 A.Gigas, flava, apice albo. Linn.504. Gualth. 34. A. Lift. 856. 12. c ? 862. 18. a. Kircher III 384.385. 386. longit. ro. lat. $7 \frac{1}{2}$. poll. (r. Ppecim. 543. a.

892 A. Gigas, pracedenti fimilis, Linn 504. longit. 9. la. tit. 7. poll. (I) fpecim. 543. a.

893. A. Gigás, nondum adulta, Linm. 504. Gualth. 54. M. Argenv. I4. I. Kircher III. 32 I. longit. 5 poll. ( 2. fiecim. 543.

894 A. Gigas, præcedenti fimilis, fed revelata, Linn. 504. (2. Specim. 543.

895 A. Lucifer, tefta alba, fusco punctata, Linn. 503. Argenv. 14. K. Petiv. Gaz. 74. 1. Lift. 883. 6 ? 886-888. 7. 8. 9. Hollar Icon. 32. (3. Specim. 544.

896 A. Lucifer, finilis præcedenti, fed minor, Linn. 503. (3. Epecim. 544.

897 A. Pugilis, Linn. 498. Gualth. 32. B. Kircher III. 229. (2. fpecim: 549.

898 A. Pugilis. fimilis præcedenti, fed major, Linn. 498. (2. fpecim. 549 .

899 A Coronalis, Giganti affinis, tefta anfractibus femituberculatis, Limn. 504. «. ᄃ. O. Gualth. 54. M? longit. 4. poll. (3. fpecim. 550.

900 A. Coronalis, fimilis pracedenti, Linn. 504. \%. F. O. (2. fpecim. 550. $901 \mathrm{~A}$. 


\section{Teftaces. Ordre IV.}

882 1. Crochet, ou Crapaud, pelé, blanche, E̊ noire, Linn. 493. Seb. III. 62. 33 ? (2. p. 544*.

883 A. Crochet, ou Crapaud, imparfaite, frice, tacketée de blane, Eे brun, Linn. 493. Seb. III. 62. 35. 37. Lift, 883. 6. Rumph. 35. D. (3. p. 534 .

$884 A$. Crochet, ou Crapaud, femblable a la precedente, Linn. 493. (.3.p. $534^{* *}$.

$885 A$ Millepied, du Cap, ¿ bord large. Linn. 494. Ou Ou O. Lift. 86823 ? long $4 \frac{3}{2}$ latit. $2 \frac{1}{2}$. pouc. ( 1.0909.

$\$ 86$ A. Millepied, gorge blanche, ftriée de brun, jaune par dehors, Linn. 494. Seb. III. 82. \&c. Lift. 858. 23. Kircher III. 3is. Petiv. Mur 14. 7. (2.p. 535.

887 A Scorpion, d bouche pourprée ftrié, Linn. 492. Seb. III. 82.\&c. Lift. 867. 22. Petiv. Muf.3. 2. Valent. 28. long. $4 \frac{3}{2}$. porc. (2. P. 537 .

888 A. Scorpion, approcbant d la precedente, Linn. 492, deus pieces, jointe a une imparfaite Jars doigts. $(2, p, 537$.

889 A. Crochet de Matelot, à bouche rougeatre, pelé, Linn. 492. ou o. long. $5 \frac{t}{2}$. latit $2 \frac{t}{2}$. pouc. ( I $p .538$.

890 A. Crochet de Matelot, fermblable dे la precedente, Linn. 492 a. $0: 4$ O. (2. P 538.

291 A. Geant, ou Coronale, jaune, d fommet blanc, Linn. 504. Gualth. 34. A. Lin. 85612 c? 862 . 18. a. Kircher III. 384.385. 380.long. 10 latit. $7_{2}^{2}$. pouc. ( 1. p. 543. a.

$892 A$ Geant, ou Coronale', Jémblable à la presedente, Linn. 504. longit. 9. lat. 7. pouc. ( (1. p. 543.

893 A. Geant, ou Coronale, pas encore parfaite, Linn 504 5. pouc (2. p. 543.

$\$ 94$ A. Geant, ou Coronale, femblable au meme, mais rivis.

895 A Chameau bariole, coquille blanche, pointée de noir,
Linn. 503 . Argenv. Linn. 503. Argenv. 14. K. Petiv. Gaz. 74. I. Liff.883. 896 A. Chameau bariole, jemblable à la precedente, $p .544$. petit, linn. 503. (3 p 544 a precedente, mais plus 897 A. Oreille dechiré, Linn. 498. Gualth. 32. B. Kircher $898 \mathrm{~A}$. Oreille dechiré, femblable de la precedente, mais plus grand, Linn. 498. ( 2 , p. 549. a demitubercules, Linn. 504. \%or o. Gualth. 53. M?
long. 4. pouc. (1. p. 550.

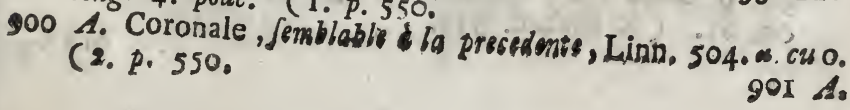


$901 \Lambda$. Coronalis? f. varietns, Linn. 504, ß. ..- O. Seb. III. 61. 8? 83. 1I? Gualth. 26. N. Hollar Icon. I5. (2. fpecim. $550 *$.

902 A. Torofa, tefta alba, fasciis carneis, membrana flavá tecta, Linn. 504. $\gamma$. T. o. Seb. III. G2. 6. 8. Lift. 860 . 17. 18. (2. fpecim. 548.

903 A. Torofa, præcedenti affinis, Linn. 504. $\gamma$. f. О. Seb. III. 62. 7. (3. Specim. 548*.

204 A. Cibberula, Linn, 50r. Seb.III. 62. 48 ? 49? Gualth, 32. C. Valent. 64. (2. fpecim. 520.

905 A. Gibberula, præcedenti fimilis, Linn. 50r. Seb. III 61. 17-19. Lifl. 847. 1. Petiv. Muf. 14. 13. Argenv. 14. N. (I. fpecim. 521 .

906 A. Gibberula, præcedenti affinis, fed fasciata, Lirr. 5or. (3. fpecim 521.

907 A. Luhuana, Linn. 500.' Gualth. 3r. H. I. Lift. 85r.6. Petiv. Gaz. 98. 10. Muf. 14. 12. (3. fpecim. 522.

908 A. Luhuana, fimilis præcedenti, Linm. 500.' (2. fpecim. 906.

909 A. Luhuana, eadem, vix aduita, Linn. 500. Seb.111.6r. I3? Liff. 849. 7? (3. Ipecim. 907\%.

gro A. Lahiabana, fusco maculata, ore albo, Limı. 500. Seb. III. 62. 31? 32? Petiv. Gaz. 98.13, (1. fpecim. 90 .a.

GENVS XXI1. C O N I.

9II C. Geograplius, tefta glabra, alba, fusco ftriata, Linn. 324. Seb. III. 42. I-4. Lift. 747. 4r. Petiv. Gaz. 75. r.

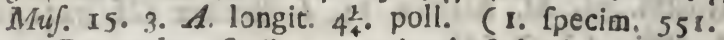

912 C. Geographus, fimilis præcedenti, fed-minor, Linn. 324. (4. Ipecim. 551.

Q13 C. Tulipa, Linn. 323. Seb. III. 42. 16-21. Gualtb. 26. C. G. Lift. 760. 6. Adanfon Sereg. 1. 6. J. 8. (3. \{pe-3 cim. 552 .

$9{ }_{4}$ C. Tulipa, fimilis pracedenti, Linn. 323. ( 3 , Specim. 552.

9rs C. Tulipa, fimilis precedenti, decorticatiore, violacea, Litno. 323. (3. Tpecim. 552.

916 C. Araufiacus f. Bullatus, albo luteoque varia, Linn. 322. «, f. 0.Seb. III.42, 15. Argenv. 13.H. (2. Ipecim. 553.

917 C. Striatus, maculis pallide fuscis, Linn. 318. Seb.III. 47. 22. 23. Lift. 755. 7? Gualth. 26. D. Petiv. Gaz. 98. 9. Muif. 15. 4. Adanjon Seneg. 1. 5. 1. 2. Valent. 60. 61. (2: fpecim. 554.

$918 \mathrm{C}^{\prime}$ 
g0r A. Coronale? ou varieté. Linn. 504. $\beta$. ou o. Seb. IIT. 61. 8 ? 83. II ? Gualth. 29. $N$ Hollar Icon. 15. (p. 2.550* $902 A$. Torofe, ì coquille blanche, à bandes en couleur die rofe, memurane jaune couverte, Linn. 504. \%. ou O. Seb. III. 62. 6. 3. Lift. 860. 17. 18. (2. p. 548.

903 A. Tornfe, fennblable à la precedente, Linn. 504. भ.ox 0: Seb. 1II. 62. 7. (3. $548^{*}$.

$904 A$. Gueuie blanche, ou Tourterelle, Linn. 50I. Seb. III. 02. 48 ? 49 ? Gualth. 32. C. Vaient. 64. (2. p. 520. $905 A$. Gueule blanche, ou Tourterelle, Semblable ì la precea dente, Linn. 50 r. Seb. III. 6r. I 7-19. Lift. 847. I. Petivo: MuC. I4. I3. Argenv, I4. N. (I. p. 52 I.

906 Gueule blanche, ou Tourterelle, Jemblable à la precedente, mais bandé, Linn. 50r. (3. p. $52 \mathrm{r}$.

907 A. Luhuane, ou Gueule noire, Linn. 500, Gualth.3T. H. I. Lift. 851. 6. Petiv. Gaz. 93. 10. Muf. 14. 12. ( 3 - p. 522 .

908 A Luhuane, ou Gueule noire, Senblable à la precedente;" Linn. 500. (2. p. 9 (1).

$900 A$. Luhuane cordonné, ou Gueule noire, la pareille, a peine parfaite.Linn. 500. Seb. III. 01. 13 \& Litt. 849.7? (3. $p$ 907*.

910 A. Luhuane, ou Gueule noire, tachetée de brun, a bouche blanche, Linn. 502. Seb. III. 62. 3I ?'32? Petiv。 Gaz. 98. I2. (I. p. 906. $a$.

\section{GE NRE XXII. VOLL T E S.}

9ir $V$ Brocard de foye d'agatée, coquille pelée, blanche, ftriée de brun, Linn. 324. Seb. III 42. 1-4.Lift. 747. 4r. Petiv. Gaz. 75. I.Muf. 15. 3. A. long. $4 \frac{3}{4}$ pouc. (I, $p$. 55 I.

$912 V$. Brocard de foye d'agatée, femblable à la precedente, Linn. 324. (4. p. 55 I.

91 $3 V$.Tulipe, ou Brocard de foye d'agatée, Linn. 323 . Seb. III. 42, 16-2I. Gualth. 26. C. G. Lift, 760. 6. Adanfon Seneg. 1. 6. I. 8. (3. p. 552 .

$914 V$.Tulipe, on Brocard de foye d'agatée, jemblabled la precedente, Linn. 323. (3. p. 552 .

$915 V$. Tulipe, ou Brocard de loye d'agatée, Semblable cila prew cedente, plus decorchée, violette, Linn. 323." (3. p. 552. 916 $V$. Tulipe d'orangée, ou Bullate, varieté de blanc, Ẽ de jaune. Linn. 322. a. ou o. Seb. III. 42. 15. Argenv. 13. H. (2. p. 553 .

91 $7 V$. Ecorchée, $d$ taches pales brunes, Linn.318. Seb. III. 470 22. 23 .Lift.755.17 ? Gualth. 26 D. Petiv Gaz. 98.9. Mui. 15.4. Adan fon Seneg. 1.6. I. 2. Valent. 60.61. 2. 2. p. 554 . 
918 C. Striatus, pracedenti fimilis, ex atro \& fusco miculatus. Linn. 318. (3. Specim. 554.

919 C. Striatus, fimillimus præcedent, fed nigricars, Linn. 318. (3. fpecim. 554.

920 C. Striatus, fimilis pracedenti, fed minor, labro plicato, Linn. 318. (3. fpecim. 554.

92 I C. Stercus Muscarum, Limn. 3 r r. Sel. III. 55. I. in medio, Liff. 757. 9. Argenv. 13. E. Petiv. Muf. I 5. 2I. (3. Ppecim. 555.

922 C. Stercus Muscarum, præcedenti affinis, Linn. $3 \mathrm{II}$, (3. . pecim. 555.

223 C. Ammiralis fummus, fasciis hicce quinque, Linn. 298. «. Set. III. 46. 4. Petiv. Muf. 15. 14. Spengler Icon. I. E. (2. Specim. 556.

924 C. Ammiralis fummus, pracedenti fimilis, fed colore fortior, Linn. 298. $\beta$. (2. Specin. 556.

925 C. Ammiralis funmus, præcedenti fimilis, colore caftaneo, fasciis tamen quatuor, Linn. 298. $\%$. Seb. III. 48. 5. 6. Argenv. Suppl. 1. O.P? Petiv. Muf. 15. II. (2. fpecim. 556. a.

926 C. Ammiralis ordinarius, precedenti affinis, fed bifasciatus; Linn. 298. D. Seb. III. 46. 2 f. \&c. Rumph. 34. B. Petiv. Muf. 15. 18. (I. fpecim, 556. b.

927 C. Perfonatus, pracedentí quoad figuram fimilis, fasciis nullis, Limn. 298. ». Argenv. Supplo I. V. Petiv. Muf. 15. 19. (2. Tpecim. 631.

928 C. Ammiralis granulatus, maculæ albæ minores, lineis punctatis , fasciis 2 flavis, lineolæ granulate, Limn. 292. ๖. f. O. Argenv. Suppl. I. M. (I. fpecim. 557.

229 C. St. Oma, tefta alba, lineis anguftis brevibus, luteis transverfis, Linn. 293 y.f. O. Argenv. Suppl. I. T. longit. $2 \frac{1}{2}$. poll. (I. fpecim. 59r.

930 C. Generalis, Linn. 293. Seb. III.'54. I3. dextra, Lift. 785. 33. Rumph 33. $\Upsilon$ ? Petiv. Muf. 3. 9, Valent. 47. (2. fpecim. $561 *$

931 C. Generalis, varietas precedent1, tria Ipecimina, quorum alterum fascia I. latiore, Linn. 293. «. Liff. 786. 35. Valent. 9. (2. Specim. 56r.

932 C. Generalis, pracedenti fimilis, fasciis flavis, lineis undatis fuscis, Linn 293. (3. Tpecim. 561.

933 C. Generablis, præcedenti affinis. tefta Alava, vel grifea , fasciis ex fasco punctatis, Linn. 293. (3. '́pecim. $56 \mathrm{I}$. 


\section{Tefaces Ordre IV.}

9r8 V. Ecorchée, Semblable di la precedente, tachetée de brun,

Eo de noir, Linn. 318. (3. p. 554 .

919 V. Ecorchée, très Jemblable de la precedente, mais noiratre, Linn. 318. (3. p. 554.

$920 V$. Ecorchée, femblable à la precedente, mais plius petit. di levre plié, Linn. 318. (3. p. 554.

92I $V$. Drap d'argent, Linn. 3Ir. Seb. III. 55, I. in medio, Lift. 757.9. Argenv. I3. E. Petiv. Mur. 15. 21. (3. p. 555 .

$922 V$. Drap d'argent, approckante ì la precedente, Linn. 3 I r. (3.p. 555 .

$923 V$. Grand Amiral, de cinq bandes, Linn. 298. «. Seb. III. 46. 4. Petiv. Muf. I5. I4. Spengler Icon. I. E. (2. P. 556.

$924 V$. Grand Amiral, Semblable da la precedente, mais d'un couleur plus fort, Linn. 298. $\beta$. " (2. p. 556.

$025 V$. Grand Amiral, Semblable à la precedente, d'un couleur chataigne, mais poustant à quatre bandes. Linn. 298. $\%$ Seb. III. 48, 5. 6. Argenv. Suppl. I. O. P? Petiv. Mur。 I5. II. (2. p. 556. a.

$926 V$. Amiral, femblable d̀ la precedente, mais d̀ dęsx bandes, Linn. 298. 8. Seb. III. 46. 21. \&c. Rumph. 34. B. Petiv. Muf. 15. 18. (1. p. 556. b.

$927 V$. Amiral marqué, Semblable de la precedente, quant à la figure, fans bandes, Linn. 298. ฑ. Argenv. X. V. Petiv. Muf. 15. I9. (I. p. 63I.

$928 V$. Amiral granulé, taches blanchespetites pointées de lignes; \&' deux bandes jaunes, EO lignes granulée, Linn. 298. З. 02s ○. Argenv. Suppl. I. M. (I.p. 557.

$929 V$. St. Omar, dे coquille blancbe, à petites lignes étroites.

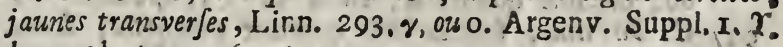
long. $2 \frac{1}{2}$. pouc . (I. p. 591.

$930 V$. Flamboyante, Linn. 293. Seb. III. 54. I3. dextra. Lift. 785. 33. Rumph. 33. $\Upsilon$ ? Petiv. Muf. 3.9. Valent. 47. (2. p. $561^{*}$.

931 $V$. Flamboyante, verieté de la precedente, trois pieces; done l'une eft $a \dot{a}$ bande large, Linn. 293. ${ }^{\circ}$. Lift. 786.35. Valent. 9. (2.p.56I.

$332 V$. Flamboyante, femblable à la precedente, do fascies jarsnes, EO lignes ondées brunes, Linn. 293. (3. p.56r. $933 V$ Flamboyante, approchant à la precedente, do coquille jaune, ou grife, les fascies pointées de brun, Linn. 293. (3. P. 561 . 
934 C. Generalis, pracedenti fimilis, fasciis punctatis, fusce maculatisque, Limm. 293. \&. 1. O. Gualth. 20. F? (4 ipecim. 9ri.

935 C Generalis, pracedenti affinis, fed punctis lineatis Linn. 293 a. f. o. (2. fpecim. 9 Ir.

936 C. Generalis fed in medio fascia lata alba, Linn. 293 a. f. o. (2. fpecim. 9 Ir.

937 C. Generalis, præcedenti îmilis, tefta flavefcente trans verfim fusco maculata, fasciis 3 flavis, Linn. 293. A f. O. (I. fpecim. 9II. a.

938 C. Gernuanus, Linn. 302. Seb.III. 44. 5. 7. Gualth. $2:$ G. Argenv. 12. Q. Lift. 707. 16. Kircher III. 132. (: fpecim. 559.

939 C Genuanus, præcedenti fimilis, Linn. 302. (2. Ppe cim. 559.

940 C. Genuanus, præcedenti affinis, fed minor, Lim 303. (2. fpecim. 559.

94 I C. Papilio, fasciis flavis maculis quadratis fuscis Linn. 302. B. f. O. Seb III. 48. I-3. Argenv. 12. Lift. 769 17. Kircher III. 336. (2. fpecim. 558.

942 C, Papilio, fimilis præcedenti, fed multo major, Lim 302. $\beta$. f. o. (I. fpecim. 558.

943 C. Lithoftrotus, tefta alba. maculis quadratis coccinei fasciatim difpofitis, Linn. 0. Seb. III. 55.4-9. Gualt: 21. $H$. (2. Ppecim. 562.

944 C. Lithoftrotus, fed maculis minoribus, Linn. O. (1) fpecim. 562.

945 C. Lithoftrotus, fed minor, Linn. O. (2. Specim. 56: 946 C. Lithoftrotus, maculis irregularibus aurantiis, $\&$ in $q u$ bus maculx quadratæ, Limn o. (3. Tpecim. 562*.

947 C. Fucatus, fasciis latis quatuor aurantiis, Linn. 292 ๙. f. O. Li/t. 774. 20? Argenv. 12. I? (1. Ipecin $563^{*}$.

948 C. Fucatus? C rietas, fasciis flavis, Linn. 292. B.f. o.Se III. 44.23\} Gualth. 22.F? Argenv. I 2.I (2. \{pecim. $563 .:$

949 C. Fuctus? \{. varietas, maculis piceis, Linn. 292. f. o. Seb. III 44. $23,45.6$. Gualth. 22. H. L? (.) fpecim. 563 . b.

$950 \mathrm{C}$ Oxygalus, tefta alba, punetis fuscis diftantibus, Linn. 308 ๙. ศ. O. Seb. III. 44. IO. 11. Gualth.42. 1 (3. Ipecim. 564*.

95I C. PunEatus, tefta glabra, alba, punetis fuscis approx matis, Linn. a. Seb. III. 47. 25? (I. fpecim. 9! 2. 


\section{Teftaces. Ordre IV.}

$934 V$. Flamboyante, Semblable di la precedente, da bans pointées, Ẽ tachetées de brun, Linn. 293. a. ou 0. Gualihde. 20. F? (4. P. 9 II.

$935 V$. Flamboyante, approchante d la precedente, mais à pointes lignées, Linn. 293. a ou O. (2. p. 9 II.

$936 V$. Flamboyante, mais au milieu une large bande blanche, Linn. 293. os ou o. (2. p. grr.

$937 V$. Flamboyante, femblable da la precedente, à coquille jaunatre, tacbetée de travers de brun, à trois bandes jaunes, Linn. 293. $\beta$. ou O (I. p. grr. a.

$938 V$. Faux aile de Papillon, Liun. 302 . Seb III. 44. 5.7: Gualth. 22. G. Argenv. 1s. Q. Lift. 767. 16. Kircher III. I32. (2. p. 559 .

$939 V$. Faux aile de Papillon, Semblable à la precedente, Linn." 302. (2. p. 559.

$940 V$. Faux aile de Papillon, approchante d la precedente, mais plus petite, Linn. 302. (2. p 559.

94I $V$. Aile de Papillon, $a$ bandes jaunes, E taches quarrées brumes, Linn. jo2. $\beta$ ou O. Seb III. 48. I-3. Argenv. I2a $V$. Lift. 769. 17. Kircher $I I I$ 236. (2. $p-558$.

$942 V$. Aile de Papillon, femblable à la precedente mais bears: coup plus grande, Linn. 302. B. ou O. (I. p. 558.

$943 V$. Mofaique, cu Pavé ltalien, $\grave{a}$ coquille blanche, d̀ taches quarrées rouges difpofées par bandes, Linn. O. Seb. III. 55. 4-9. Gualth. 2 I. H. (2. p. 562.

$944 V$. Mofa que, ou Pavé Italien, mais à taches plus petites, Linn. o, (I. p. 562.

$9+5$ V. Mofaique, ou Pavé Italien, mois plus petit, Linn. o: (2. p. 562 .

$946 V$. Mofaique, ou Pavé Italien, d taches irrégulieres orangées, $\mathcal{E}^{\circ}$ dans les quels font des taches quarrées, Linn. 0. (3. p. $562 *$.

$947 V$. Mouchetée, à quatre bandes larges quarrées, Linn. 292. a ou O. Lift. 774. 20? Argenv. 12. I? (1. p. 563*.

$948 \mathrm{~V}$. Mouchetée ? ou varieté. à bandes jarnes, Linn. 292. $\beta$ ou o. Seb. III. 44. 23 ? Gualth. 22. F ? Argenv. I2.I. (2. 503. a.

$919 \mathrm{~V}$. Mouchetée? ou varieté, \& taches couleur de poix , Linn: 292. \% ou O. Seb. III. 44. 23, 45. 6. Gualth. 22. H. L?. (4 P. $563 . b$.

$950 V$. Mouchetée, ¿̀ coquille blanche, pointes brunes Separées: Linn. 308. a. ou o. Seb. III 44. IO. 11. Gualth. 42, L.' (3. p. $564^{*}$.

95 I $V$. Pointée, à coquille palé blanches, à pointes brunes approchantes, Linn, O. Seb. III. 47. 25? (I. P. 9I2. 
952 C. Leo, tefla alba, maculis oblongis irregularibus, obliquis rubro fuscis, Linn. O. Seb. III. 46. 24? 25? Gualth. 21. P? Argerv. I 2. C? Rumpl. 34. M? Valent. 48. (3. fpecim. $506^{*}$.

953 C. Leo, maculis fuscis, interfperfis punetis, Linm. O. Seb. III. 44. 25. 27. 23. Gualth. 21. D. P. Petiv. Mus. 4. 15. (3. fpecim. 566.

954 C. Leo? f. varietas, maculis luteis, trigonis paucioribus . Liniı. O. Gualth. 21. C? (2 Ipecim. 567.

955 C. Fluct ifer, tefta alba, flammulis fuscis undata, itriata, punctata, Linn. o. Gualth. 22. B. Lift. 772. 18. (2. fpecim. 560.

956 C. Fludtifer, tlavelcens, ftriis latioribus, Linn. O. Seb̆. III. 45. 8. 9. (I. Ipecim. $560 \%$

257 C. Fluctifer, tefta flava, Linn. o. Seb. III. 73. 27. 28. Lift. 77 1. 17. Gualth. $21 . F$ ? long. $6 \frac{1}{4}$. latit. $3 \frac{1}{4}$. poll. (1. Ppecim. 560**.

958 C. Glaucus, Linn. 303. Seb. III. 43. 32? Lift.757.19. Petiv. Muf. 9. 10. (3. fpecim. 568.

959 C. Betulus, tavefcens, maculis parvis fuscis, Linn. 308. B. f. 0 Seb. III. 44. 6? long. 3. poll. (I. fpecim. $565^{\circ}$.

960 C. Betulinus, albido flava, ftriis parallelis maculisque fuscis. Limn. 308. Seb. III. 44. I-3. Lift. 762. II. Petiv. Muf, 15.2. Spengler Icon. II. D. long. $3 \frac{1}{4}$. poll. (2. Ppecim. 564 .

961 C. Betulinus, fed minor, Linn. 308 (2. Specim. 564: 962 C. Figulinus. Linn. 309. Seb. III. 54. I-4. 8. Gualth. 20.E. Lift. 785.32. Argenv. 12. A Rumpb. 33. 1. Valent. 34. long. 3. crafl, 2. poll. (2. fpecim. 569.

963 C. Figulinus, fed minor, colore fortior, Linn. 309. Petiv. Muf. 5. 7. (2. โpecim. 569.

964 C. Figulinus, fed fascia dilatiore circtus, multoque major, Linn. 3c9. (2. Tpecim. 569.

965 C. Litoglyphus, tefta fusca, ftriis transverfalibus fuscis, fasciis albis, Linn. o. Seb̆. III. 42. 41. $42,54.9 .10$. Gualth 20. I. Valent. 47. (2. Specim. 570.

965 C. Litoglyphus, fed minor, Linn. O. (4. fpeciun. 570. 967 C. Litoglyphus, luteus, absque fasciis, Linn. ๑. ( 4. rpecim. $57 \mathrm{r}$.

968 C. Epiftomium teftá glabra, lutea, fascia maculari alba, Linis. O. Seb. III. 44. 8-II, 45. 16. 17. Gualth. 20. M. Rumpho 31. 5. Petiv. Gaz, 153. 10? (2. Specim. 572. 
$\$ 52 \Gamma$. Lion combattant, à coquille blanche, taches allongées irrégulieres, obliques, rouge brines, Linn. 0 . Seb.III. $46_{*}$ 24 ? 25? Gualth. 21. P? Argenv, $12 C$ ? Rumph. 34. $M$ ? Valent. 48. (3.p. $566^{*}$.

$953 V$. Lion combattant $\dot{a}$ taches brunies, à pointes entresparSées, Linn. o. Seb.III. 44. 25.27. 28. Gualth. 2I.'D. $P_{\text {a }}$ Petiv. Mur. 4. I5. (3. p. 506.

954 V. Lion combattant? ou varieté, à taches jaunes triangulaires, Linn. o. Gualth. 2 r. C? (2. p. 567.

$955 V$. Ondée, à coquille blanche, à flammes brunes ondées. friée, pointée, Linn. 0. Gualth. 22. B. Lift. 772.18. (2. p. 560.

$956 V$. Ondée, jaunatre à ftries plus larges, Linn. o. Seb.IIT. 45. 8. 9 (1. $560^{*}$.

$957 V$. Ondée, a coquille jaune, Linn. O. Seb. III.' 73. 27. 28. Lift. 771 . 17. Gualth, $21 . F$ ? long. $0 \frac{T}{4}$. latit. $3 \frac{1}{4} \cdot$ pouc. (1. p. $560^{* *}$.

958 V. Chat de Cypre, Linn. 303. Seb. III. 43. 32? Lift. 757, 19. Petiv. Mur. 9. 10. (3. p. 568.

959 V. Tinne de Beurre, Linn. 308. B. ou 0. Seb. III. 442 6 ? long. 3. pouc. (I p. $5655^{\circ}$

960 V. Tinne de Beurre, d'un jaune blancheatre, à fries pas ralelles. E taches brunes, Linn. 308. Seb. III. 44. I-3. Lift. 762. 11. Petiv. Mur. 15.2. Spengler Icon. II. D. long. $3 \frac{1}{4}$. pouc. (2.p. 564 .

961 $V$. Tinne de Beurre, mais plis petit, Linn. 308. (2a' p. 564 .

$952 V$. Minime, Linn. 309. Seb. III. 54. I-4. 8. Gualth. 20. E. Lift. 785. 32. Argenv .12. A. Rumph. 33. I. Valent: 34. long. 3. crađJ. 2. pous, (2. p. 569.

$963 V$. Minime, mais plus petit, \&ं plus fort de couleur, Linn 309. Petiv. Mur. 5. 7. (2. p. 569.

$954 V$. Minime, mais cerclè à'une bande plus étendue, \& beauscoup pless granide, Linn. 309. (2. p. 569.

$965 V$. Lptoglyphe, ou Cornet de Buis, à coquille brune, E ftries transverfes brunes, à bandes blanches, Linn. o. Seb. III. 4 2. 4 I. 42,54 . 9. 10. Gualth. 20. I. Valent. $47^{\circ}$ (2. p. 570 .

956 V. Lytoglyphe, ou Cornet de Buis, mais plus petit, Linn. o. (4. p. 570 .

$967 V$. Lytoglyphe, ou Cornet de Buis, jaune, Jans bandes, Linn. o. (4. p. $57 \mathrm{I}$.

$968 \mathrm{~V}$. Bouchon, à coguille pelée, jaune, a bande tachetée blanche. Linn. O. Seb. III. 44. 8-I1, 45. 16. 17. Gualth. 20. M. Rumph. 3I. 5. Petiv. Gaz, 153. IO? ( 2, p. 572 . $\mathrm{Z}_{4}$ $969 D_{i}$ 
969 C Epiftomium, pracedenti affinis, refta fusca, fascia maculari alba, Linn. o. Lift. 777 23? Argenv. 1 2. D? Petiv. Muf. 21. 12? longit. 3, poll. (2. fpecim. $573^{*}$. 970 C. Epifomium? f. varietas præcedentis, fascia ad apicis bafin, \& in medio, Linn. o. Seb. III. 42. 37. (1. Spe: cim. 913.

27 C Miles, Linn. $296^{\circ}$ Lift. 736. 34. Petiv. Muf. 8. I. Gualth. 20. N. (2. Rpecim 573.

972 C. Miles, fed minor, fascüs anguftioribus, Linn. 296. Argenv. 12. L. (3. fpecim. $5>3$.

973 C. Capitaneus, Linn. 295. Grialth. 20. 2 Lift.780. 27. Rumph. 33. X. Kircher 1II. 354. Valent. 49. (2. Cpecim. 574 .

274 C. Capitaneus, pracedenti affinis, unum viride, alterum fuscưm, Linn 295. Petiv. Muf. 2. I I. (2. Specim. 574. 975 C. Capitaneus? r. varietas, tenuior, viridi flavefcens, fasciis albis, Linn. 295. *. Seb: III.42.31. 35. Kircher III. 139. (2. fpecim. 574.

976 C. Capitaneus, politus, inde violaceus, fasciis albis, Linn. 205. B. Seb. III. 42. 37-39: Lift. 758. 3? (2. fpe. cim. 578.

977 C. Capitaners, idem ac pracedens, una cum duobus fuscefcentibus', Linn 295. $\beta$ (4. fpecin. 578.

978 C. Dultor, fuscus, fasci's obfcurioribus, Linn. O. Gualth. 25. F. (5. Ipecim. 575*.

979 C. Leopardus, apice fubdeprefto, rufefcens, fasciis repandis caftaneis, rarus, Linn。 o. Lift. 78r. 28, ( 1 . fpecim. 576 .

980 C. Bifafciatus, téfta fusca, albo transverfim ftriata, fasciis longitudinalibus duabus albis, Linn. 0: Seb.III. 59. II? 23? Lift. 78r. 28 ? (2. Rpecim. 575.

981 C. Bifafciatus, fed minor, Linn. 0. (2. fpecim, 575 . 982 C. Granifer, tefta alba, guttis five punetis feriatim digeftis fasciisque duabus fuscis, Linn. 0. Lift.755.7? Petiv. Muf. 6. 10. (2. 亿pecim. 577.

983 C. Granifer, pracedenti fimilis, Linn. o. (I. Specim. 577.

984 C. Ammiralis occidentalis, tefta fusca, maculis parvis albis, reriatim digeftis, Linn. 298. o. f. O. Seb.III. 42. 22, 44. 13. Lift. 565. 14 ? Grialth. 25. R. Rumph. 34. E? $K$ ? (2. fpecim. 579.

285 C. Ammiralis occidentalis, maculis plurimis majoribus, Linn. 298. D. f. 0. Petiv. Mus. 4. 13? (3. Specim. 579. 


\section{Teftaces. Ordre $I V$.}

$969 V$. Bouchon, Semblable de la precedente, $a$ coquille brurte, bande tachetée blenche, Linn. 0 . Lift. 777. 23 ? Argenv. 12. D? Petiv. Muf, 21, 12 ? long. 3. pouc. (2. p. $573^{\circ}$.

$970 V$. Bouchon? ou varieté de la precedente, mais une bande la baze du Jommet, de même qu'au milieu, Linn. o. Seb. III. 42. 37. (1. p. 913.

$971 V$. Navet, ou faux Amiral, Linn, 296. Lift. 736. 34. Petiv. Muf. 8. I. Gualth. 20. N. (2. p. 573.

$972 V$. Navet, ou faux Amiral, mais plus petit, $\grave{a}$ bandes étroites, Linn. 296. Argenv. I2. L. (3. p. 573.

$973 V$. Fromage verd, ou l'Onix, Linn, 295. Gualth. 20. 2 . Lift. 780. 27. Rumph. 33. X. Kircher III. 354. Valent. 49. (2.p. 574 .

$974 V$. Fromage verd, ou l'Onix, Semblable à la précedente, l'une verd, l'autre brun, Linn. 295. Petiv. Muf. 2. I r. (2. P. 574 . $975 V$. Fromage verd? ou l'Onix, ou varieté, plus mince, d'un verd jaunatre, à bandes blanches, Linn. 295. «. Seb. III. 42. 31. 35. Kircher III. 130. (2. p. 574.

$976 V$ : Fromage verd, ou l'Onix, polie, de la couleur de violette, $\grave{a}$ bandes blanches, Linn. 295. $\beta$. Seb. III. 42. 37-39. Lift. 758. 3? (2. p. 578.

$977 V$. Fromage verd, oul'Onix, comme la precedente enfem. blable, avec deux brunes. Linn. 205. $\beta$. (4. p. 578.

978 V. Conducteur, brun, $\dot{a}$ bandes plus obfcunes, Linn. 0. Gualth. 25. $F$. (5. p. $575^{*}$.

979 V. Leopard, à formmet un peu deprimé, rougeatre, à bandes chataignes, rare, Linn. O. Lift. 781.,28. (I. p. 576.

980 $V$. Faux Flamboyante, à coquille brune, ftrié de travers de blanc, à deux bandes longitudinales blanches, Linn. 0 . Seb. III. 54. II? I 3 ? Lift. 78 I. 28 ? (2. p. 575.

98I V. Faux Flamboyante, mais plus petite, Linn. 0. (2. p. 575.

$982 V$. Chagriné, ou Grenu, à coquille blanche parfemé de gouttes, ou points, à deux bandes brunes, Linn. U. Lift. 7557 ? Petiv. Mur. 6. 10. (2. p. 577.

$983 V$. Chagriné, ou Grenu, Jemblable à la precedente, Linn, c. (I. p. 577 .

$984 V$. L'Amiral de l'Occident, à coquille brune, à petites taches blanches, difperfés par Juites, Linn. 298. $\delta$. ou 0. Seb. III. 42. 22 , 44. I3. Lift. 565. 14? Gualth. 25. 5 . Rumph. 34. E? $K$ ? (2. p. 579.

$.985 V$. L'Amiral de l'Occident, à plufieurs grandes tachis, Lina. 298. \& ou O. Petiv. Muf, 4. I3? (3. P. 579.

$$
\text { Z } 5
$$


986 C. Ammiralis occidentalis, precedenti fimillimus fed nigro fuscus, maculis albis minimis, Linn.298. 2. f.o. Liff. 784. 31? ( 1 , fpecim. 579*.

987 C. Ammiralis granulofus, tefta tumida abbreviata, apice obtufo, alba, flammulis fuscis, ultra dimidium granulata, Linn. O. Lift. 784. 31. 789. 42? (2. fpecim 580. 988 C. Ammiralis gramulojus, fed dilatior, Linn. O. (2. ipecim. 580.

989 C, Capucinus, tefta abbreviata, tumida, fubcrocea, granulata, Linn. o. Lift 759. 4. (2. Specin. 914.

990 C. Capucinus, magis flavefcens, Limn. O. (3. Specim. 914.

991 C. Hebreus, tefta obata alba \& magna, fasciis nigris ex maculis transverfis, Limı. 3 10. Petiv. Gaz.99. 12. Muf. 9. 12. Kircher III. 132. Valeut. 94. Sping ler Icon. II. $F$. long. I $I_{4}^{\frac{\pi}{4}}$ craff. I. poll. (2. Specim. 58I.

992 C. Hebreus, fed minor \& maculatus, Linn. 310. Ar. genv. 12. G. (3. .pecim. 58I.

993 C. Hebreus, fasciis obliquis, linea longitudinali alba , Linn. 310. a. Seb III.47.30.31. Lift. 779. 26. Gualth. 25. O. Argenv. 12.B. Kircher III I38. (3. fpecim. 5826 294 C. Fafcialzs, fuscus, fascia lineari alba, Limn. O. Lift. 786. 36? Argenv. 12. D. (1. fpecim. 585.

095 C. Africanus, tefta rufo grifea, maculis parallelis ap. proximatis albis, fuscisgue, Linn. O. Lift. 763. 12. Petiv. Gaz. 15. 11. (2. fpecim. 583.

996 C. Mercator, tefta albida fasciis articulatis flavis., Linn: 307. Seb. III. 54. \&et. Lift. 788. 4I. Kircher III. 136. Valent. 65. B. (2. Specim. 584.

997 C. Monachus, tefta gibba, fusco carulea, nebulata, bafi ftriata, Iinn. 304. Seb. III. 43, 23? 24. Kircher III. I26. (4. fpecim. 586.

998 . C. Monachus. red colore rubro vel fusco, maculis guttatis albis, Linm. 5c4. ※. Seb. III. 43. 28? (4. Specim. 587.

999 C. Achates, tefta lutea, nebulis albis, rubro marginatis, Linn. 304. B. F. o. Seb. III. 43. 34? Lift. 778.24. b? Gralth. 25. R? (r. Ppecim. 588.

I000 C. Achates, argute granulata antice, tefta alba, olivaceo nebulata, Limm. 304. \%. .. O. Seb. III. 43. 36. (5. fpecim. 589.

IOoI C. Achates? f. varietas, tefta magis elongata, grifeo cœrulefcente, asgute ftriata, màculis luteis fubfasciata, Lim. 304. d. T. O. Séb. III. 43.31, 44. 30? Lift. 767. 13. Rumph. 34. L. Pctiv. Caz. 102.1\%. (3, R Pecim.590. $1002 \mathrm{C}$. 


\section{Teftaces. Ordre $I V$.}

$986 \%$. L'Amiral de l'Occident, tres Semblable ars precedent, mais d'un brun fonce, à petites taches blanches, Linn. 298 . $^{-}$ d. ou o. Lift. 784 31? (1. p. 579*.

$987 V$. Amiral granulé, à coquille enflée, racourcie; à fomimet rebouché, blanche, à flammes brunes, grenulé pliw de la moitié, Linn. 0. Lift. 784. $31,789^{\circ} .42$ ? (2.p 58c). $988 V$. Amiral granulé, mais plus étendiue, Linn.o. ( $2 . p$. 5815 . $989 V$. Capucin, à coquille racourcie enflée, reffemblant un peu au Saffran, granulé, Linn. o. Lift. 759.4 (2. p. 9I/4. $990 V$. Capucin, plus jaunatre, Linn. O. (3, p. gr 4 .

99I $V$. Hebraique, $a$ coquille grande, ovale, blanche, $\grave{a}_{1}$ ban des noires, par des taches traverfos, Linn. 310. Petiv. Gaz. 99. 1 2. Mur. 9. 12. Kircher III. I 22. Valent. 94. Spengler Icon. $I I_{0} F$. long. I $\frac{3}{4}$. craff. 1. pouc. (2. p. $58 \mathrm{r}$.

$992 V$. Hebraique, mais plus petit, E tacheté, Linn. 3 ICl. Argenv. I2. G. ( $3 . p .58 \mathrm{I}$.

$993 V$. Hebraique. à bandes obliques, $\mathcal{E}$ ligne longitudinale blanche, Linn. 3 Io. $\alpha$. Seb. MII. 47. 30. 31. Lift. 779. 26. Gualth. 25. Q. Argenv. 12. B. Kircher III 138. (3. P. 582 . $994 V$. Païfan bandé, brune, $\dot{a}$ bande lineaire blanche, Linn. ๑. Lift. 786. 36 ? Argenv. I2. D. (I. p. 595 .

$995 V$. Africain, $\dot{a}$ coquille rouge grife, $\dot{a}$ taches paralelles approchantes blanches, $\mathcal{E}^{\circ}$ brunes, Linn. O. Lift, 76,3.12. Petiv. Gaz, 15. II. (2. p. 583.

$996 V$. ̀̀ Rezeau picotté, ou d'araignée, à coquille blarcheatre, à bandes articulées jaunes, Linn. 307. Seb. III. 54. \&C. Lift, 788. 4I. Kirchér III. I36. Valent. 65. $B_{0}^{\circ}$ (2. p. 584 .

$927 V$. Moine, ou d'Agate, a coquille bofjue, d'un bleu foncé nebuleufe, à bafe ftriée, Linn. 304. Seb. III. 43.23? 24. Kircher III. 126. (4. p. 586.

$998 V$. Moine. ou d'Agate, mais d'un couleur rouge, ou brum, à taches gouttees. blanches, Linn. 304. a. Seb, III. 43. 28 ? (4. P. 587.

$999 V$. Achate, à coquille jaune, mues blanches, bordés de rouge, Linn. 304. $\beta$. orb o. Seb. III. 43. 34? Lift. 778. 24. $b$ ? Gualti. 25. $R$ ? ( 2 \$. 588 .

I000 $V$. Achate, finement grenulé par devant, ì coquille blanche, olivatre, nébuleufe, Linn. 304. $\gamma$. ou .0. Seb. III. 43. 36. (5. p. 589 .

ICor $V$. Agathe? ou varieté, à coquille plus élongée, d'un gris bleuatre, finement ftriée, un peu bandée à taches jounes. Linn. 304. ס. ou o. Seb. III. 43. 31, 44. 30? Lift. 704. 13. Rumph, 34: L. Petiv, Gaz. 102. 17. (3. p. 590. 
roo2 C. Achates? r. varietas, maculis colore variantibus in variis, Linn. 304. d. f. O. (4. fpecim. 590.

2003 C. Achates? f. varietas, tefta alba, maculis grifeis, vel rufo fuscis, coeuntibus, Limn. 304. ฑ. f. o. Seb. III. 43. 24 ? 25? 35? (3. fpecim. 598 .

$I_{0} 4$ C. Achates? f. variet as, tefta lactea, punctulis feriatis, maculisque piumbeis confluentibus, Linn. 304. ท. $\{$. (2. fpecim. 598.

1005 C. Mafan, tefta elongata, apice acuto, alba, fusco punctata, \& maculata, Linn. O. Seb. III. 4t.30. AdanJon Seneg. X. 6. I. 4 (2. Ppecim 594.

1006 C. Guttatus, tefta ftriis elevatis, maculis flavo viridi. bus, nebulatis, punetatim fasciata, Linis. O. Seb.III. 43. 26. (4. (pecim. .5)6.

rooz C, Guttatus, præcedenti fimilis, tefta alba fasciis 2. pallide flavis, Linı. O. Seb. III. 44, 26? Lift.755, 7?

( 3 Specin. 595.

1008 C. Guttatus, præcedenti affinis, fasciis oblique maculatis, Linn. 0. (4. Specim. 595.

Icog C. Guttatus, tefta alba, coloris hepatici, maculatim fasciata, punctulis obícurioribus ftriata, Linn. o. Valent. 50. (3. Specim. 597.

Ioro C. Guttatus . tefta caftaneo maculata, lineolis tenuibus albo punetatis, ftriata, Linn. O. Seb. III. 44. $29,48$. 41. 44. Argerw. Suppl. 2 C. (3. Ipecim. 593.

101 I C. Guttatus, maculis undatis clivaceis fufcisve, Limm. 0. (3. Specim 593.

$x 012$ C. Geographus, an Granulatus? granulis paralle. lis ftriatus, caitaneus, apice \& medio maculis albis, Linn. 315. a. f. O. Lift. 775. 21, Argenv. Suppl. I. X. ubi vera effigies reperitur, longit. $2 \frac{1}{4}$. poll. Ipecimen politum, decorticatum (I. fpecim. 599.

IOI 3 C. Geographus, tefta fulphurea, maculis aliquot albis, 315. a. f. O. Gualth. 20 F? 2I L? (r. Ipecim. 599. 1014 C. Geographus, minor, flavus, maculis majoribus, Lim. 315. «. f. O. (2. fpecim. 599.

xors C. Geographus, fed paulo minor, Linn. 3I5. «. f. O. (2. 'pecim. 599.

1016 C. Cinclus, tefla fusco grifea, fascia lineari grifea, Linn. o. Gualth. 20. R? 25. D? (2. Ipecim. 602.

ror 7 C. Lama, tefta lutea, lineis punctatis longitudinalibus, hinc $\&$ inde albo maculata, Linn. O. Seb. III. 4421 , 48. 20.23 ? 25 ? (3. โpecim. 601. 
I002 $V$. Achate? ou varieté, à taches de differentes couleurs: Linn. 304. d. ou o. (4. $p$ 59ว.

I003 V. Achate? ou varieté, ì coquille blanche, taches grifes? ou d'un brun rougeatre, qui s'afJemblent, Linn. 304. v'. ou o. Seb. III. 43. 24 ? 25 ? 35 ? ( 3 p. 598.

Ico $V$. Achate? ou varieté, dे coquille couleur de lait, of poirites de Joye, Eे taches couleur de plomb, confluantes: Linn. 304. *. ou O. (2. P. 598.

$1005 V$. Vice-Amiral, ou Mafan, d coquille allongée, le fons: met aigue, blanche, pointée, Eे tachetée de brun, Linn. o. Seb III. 44. 30. Adanfon Seneg. I. 6. 1. 4. (2. p. 594.

1006 $V$. Gouttée, ou Agate bandée, coquille d fries élevées: ¿t taches jaun vertes, nebuleu/es, Eे bandées de points, Linn. O. Seb. III. 43. 26. (4. p. 596.

$1007 V$. Gouttée, ou Agate bandée, femblable à la preceden: te, d coquille blanche, E doux bandes pale-jaunes, Linn. ०. Seb. III. 44. 26 ? Lilt. 755. 7 ? ( 3. p. 595.

$1008 \mathrm{~V}$. Gouttée, ou Agate bandée, approchonte de la precedenté, à bandes tachetées obliques, Linn. O. (4. p. 595. 1009 V. Gouttée, ou Agate bandte, à coquille blanche, de couleur de foye, bandée par taches, EO friée de pointes obfcures, Linn. c. Valent. 50. (3. p. 597.

Ioro $V$. Gouttée, ou Aqate bandée, d̀ coquille chataigne, tachetée, d̀ lignes minces, pointés de blanc, Ẽ friées, Linn. o. Seb. III. 44. 29, 48. 41. 44. Argenv. Suppl. 2. C. ( 3 p 593

Io i I $V$. Gouttée, ou Agate bandée, d taches ondées olivatres: ES brunes, Linn. O. (3. P. 593.

I012 V. Geogıaphe, ou Granulé? d grains paralelles, ftrié cha: taigne, le fommet, $\varepsilon^{2}$ milieu ì taches blanches, Linn. 3 I 5. $\boldsymbol{\alpha}_{0}$ ou 0. Lift. 775. 21. Argenv. Suppl. I. $\boldsymbol{X}$. ous fe trouve le veritable effigie, polie, \&2 Jans robe, long. $2 \frac{2}{4}$. pouc. (I. p. 599.

IOI 3 . Geographe, à coquille couleur de foufre, Ẽ quelques taches blanches, Linn. 315 . x. 0w 0. Guath. 20. F? 21. L? (1. p. 599:

$1014 V$. Geographe, plus petit, jaune, d taches plus grandes, Linn. 315. *. ou o. (2. p. 599.

I015 $V$. Geographe, un peu plus petit, Linn. 315. *. ore O. (2. p. 599 .

Iо16 $V$. Cerclée, d̀ coquille brun-grife, \& bande ligniale grife, Linn. 0. Gualth. 20. R? 25. D? (2. p. 602.

Ior $7 V$. Lama, ou Ravine, d coguille jaune, d lignes pointées lon. gitudinales, parci par la tachetée de b̆lanc, Linn. o.Seb. III. $44.21,48.20 .23$ ? 25 ? (3.p. 60I. 
1018 C. Geomantiatus, tefta grifea, fasciis duplicatis flavis, longitudinaliter punctulis ftriata, Limn: O. Seb. III. 48 . 28. Berlin. Befchaeft. I. tab. 9 f. 6 . (2. (pecim. 600 . aorg C. In/ulatus, tefta alba, maculis concatenatis hepaticis. rufo marginatis, punctulis ftriata, polita, Linn.o.Seb.III. 44. 19, 48. 25 ? (2. (pecim. 600*.

Io20 C. Infulatus, tefta longitudinaliter punctata, alba, maculis transverfalibus albis, Linn. o. Seb. III. 44. 15? Argenv. Suppl. 2. B ? (1. Ipecim. 690*.

IO2I C. Vicarius occidentalis, tefta elongata glabra, anfractibus nodofis, alba, maculis rufis, argute ftriata, Linn $\mathrm{C}$. Seb.III. 44. 14, 16. Lift. 777. 24. (2. Specim' 603.

1022 C. Vicarius occidentalis, minor, maculis majoribus, Linn. o. (4. fpecim. 603.

1023 C. Profe.Jus, tefta flavo rofea, fascia lineari alba, Linn, 355. a. f. O. (I. Fpecim. 607.

I024 C. Sterculeus, tefta brevi ventricofa, anfractibus nodô̂s, apice acuto, granulato, Atriata, aibo grifea, ore fusco, punctulis fuscis ftriata, Linn. 305. B. I. O. (3, fpecim. 606.

I025 C. Minimus, tefta anfractibus nodofis, prima nodis acutis, viridi fusca, albo fasciata, Linn. 305. Liff. 759. 4. Petiv. Pterigr. 12. 7 (5. Ppecim. 605.

I026 C. Varius, tefta oblonga, apice acuto, granulata, albo fuscoque maculata, Limn. 312. a. Argerv. 12. R? (4. 'pecim. 6I 2.

IO27 C. Stercus Culicum, tefta elongata, tumida, anfractibus nodofis. apice brevi, alba, fusco undata, punctulis minimis guttata, Linm. 3 Ir. a. f. o. Rumph. 33. AA. (3. fpecim. 609.

1028 C. Stercus granulatus, tefta alba, maculis fuscefcentibus, punctulis fuscis, granulata, Linn. 3rr. $\beta$. ศ. o. Gualth. 25. O. Petiv. Muf. 15. 20 ? Valent. 24. (2. fpecim. 610.

ro25 C. Stercus Culicum coronatum, tefta elongata, dilatata, anfractibus nodofis, glabra, alba punctulis atris, Limn. 3i t. भ. f. O. Seb. IIII. 55. r. Gualth. 25. P, Argenv. 12 S. (2. Ppecim. 608.

Io3o C. Stercus $P$ ulicum, tefta tumida, elongata, dilatata, anfractibus acute nodofis, alba, maculis ex punctulis nigris, Linn. 311. 8. Co O.Rumph. 33. 2. Lifl. 761. 10. (2. โреcim. GIt. gI6.

do3t C. Stercus Muscarum varium, tefta apice acuto, glabra, albo rufefcers, fusco punctata, transverfim lineata,

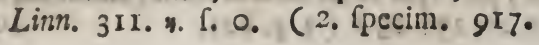


jor 8 V. Faux Cedo nulli, à coquille grife, da fafcies doublées jaunes, Atriés ex longitude par des pointes, Linn. O. Seb. III. 48. 28. Berlìr. Befchaef. .I. tab. 9. f.6 (2. p. 600: Iorg $V$. Infulée, à coquille blancbe, $a$ taches enchainées couleur de foye, bordée de rouge, E' Jiriée de pointes, $8^{\circ}$ polie, Linn. o. Seb. III 44. 19, 48. 25.? (2. p. $60^{*}$.

$1020 V$. Infu'ée, ḋ coquille pointie en longeur, b!anche, d taches transverfes blanches, Linn, o. Seb. III 44. I 5.? Argenv. Suppl. 2. B.? (1.p.600.*

ro2I $V$. Vicaire de l'Occident, d coquille elongée pelée, les anfractures noueufes, blanche, a taches rougeatres, finements ftriée, Linn. 0. Seb. III. 44. 14. 16. Lilt. 777. 24. (2. p. 003.

I022 $V$. Vicaire de l'Occident, plus petit, ì taches plus gran des, Linn. O. (4. $p$ 6ว3.

$1023 V$. Petit Moine, d coquille jaune, couleur de rofe, at fafcie liniaire blanche, Linn. 305. a. ou o. (1. p.607.

$1024 V$. Moire ventrue, à coquille courte ventrue, les anfractures en noeuds, à fommet aigue, granulé ftrie, d'un blanc gris, d bouche rouge, E' firiee de pointes brunes, Linn 305. . . . . . (3. p. $60 \%$.

$1025 V$. Minime, $\ddot{a}$ coquille les anfrainures nouteufes, la pre. miere à noeux aigues, brun-yertes, bandée de bline, Linn. 305. Lift. 759. 4. Petiv. Pterigr. 12. 7. (5.p 605.

$1026 V$. Lion combattant granulé à coguille allongée, form. met aigue, grenu, tachetée de blanc, EN de brun, Linn. 312. «. Argenv. 12. R.? (4. P. 6I2.

$1027 \mathrm{~V}$. Woire, à coquille allongée, enflie, les anfrac: tares en naux, à fommet court, onduiée de blanc, 8 brun, ED gouttée de très petits points, Linn. 31r. $\%$ ou o. Rumph. 33. $A A$. (3. p, 609.

$1028 V$. Moire granulé, d̀ coquille blencbe, granulé, d taches trunatres, Eं de pointes brunes, Linn 31я. B. ou o. Gualth. 25. O. Petiv. Mur. 15.20.? Valent. 2 ( (2 p. 6ro. $1029 V$. Moire couronré, d coquille allongée, etennué, - les anfractures noueufes, pelée, blanches à poirts noirs. Linn. 3 II. y. ou 0. Seb. III. 55. 1. Gualth. 25. P. Argenv. 12. S. (2. p. 603.

I0зо V. Chiure de Puce, à coquille erfiée, allongée, etendue, les anfractures is nouds aigues, blanche, ì taches de poin. tes noires, Linn. 3Ir. d. ou o. Rumph. 33. 2. Lift. $76 \mathrm{I} .10$. (2. p. $6 \mathrm{II}, 916$.

I03 $V$. Drap d’Árgent varié, d̀ coquille à Sormmet pointu, pelée d'un blanc rougeatre, pointée de trun, Eे lignée de travers, Linn. 3is. n: ors 0. (2. p. 917 . 
I032 C. Vicarius gramulatus, tefta apice obtufo, transverfim granulata, fusca, maculis albis cincta, Linn. 290. «. f.o. (I. fpecim. '613.

1033 C. Vicarius fafciatus, tefta conica depreffa, apice obtufo, atra, maculis trigonis albis, fascia lineari flava, Linn. 290. ․ f. O. Seb. III. 46. 5? 6? (I. fpecim. $104 \mathrm{I}$.

1034 C. Vicarius, tefta atra, maculis albis. Linn. 290. Y.f. o. Seb. III. 46. 7-12, 47. 5. 6. Valent. 10. (2. Ipe. cim. 6r4.

x035 C. Vicarius, præcēdenti affinis, Linn, 290. \%. ค. o. (2: fpecim. 614.

s036 C. Vicarius, paulo minor, Linn. 290. \%. f. o. (2. fpecim. 6r4.

1037 C. Marmareus granulatus, tefta picea, maculis cordiformibus albis, minute granulata, Limn. 290. D.f. O. Valent. 25. (1. Specim. 616.

I038 C. Marmoreus, tefta conica elongata, anfractibus nodo. fis, apice obtufo atra, macisis cardiformibus albis, Linn. 290, Seb. III. 46. \3-19. Lift. 787 39. Argenv. 12. M Petiv. Gaz. 47. II. long. 4. craffit. 2. poll. (I. Ppecim. 617.

1039 C. Marmoreus, fimilis præcedenti, fed minor, Linn. 29c. (2. fpecim 617.

1040 C. Marmorews, fed minor, Linn. 290. (3. Specim. 617.

104I C. Marmoreus, magis ventricofus . Linn. 290. Seb. III. 47. 4. (2. fpcim. 617. a.

1042 C. Marmoreus, tefta lutea, maculis albis, Linn. 290. Argenv. 12. O Valent. 43. "Hac a Cono Cervulo, in " $\mathrm{n} \cdot 1056$. caute diftinguenda, dum in hacce color tla", vus, non a natura, fed vel a caufa externa ortus, haud ", vero arte". (2. fpecim. бr 7. b.

1043 C. Cadjos, tefta anfractibus canaliculatis, alba, lineolis. fuscis reticulata . fasciisque duabus fuscis cincta, Linn. 0.

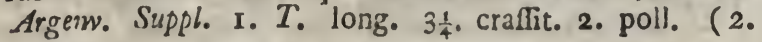
fpecim. 615.

1044 C. Imperialis, tefta albida, fasciis longitudinalibus lividis, ungulis linearibus albo fuscoque articulatis, Linn. 291. Ssb. III. 47. 18-21. Lift. 766. I5? Argenv I2. E. Petiv. Muf. 6. 6. Hollar Icon. 48. Spengler Icon. II. I. (r. 'pecim. 618.

I045 C. Imperialis, paulo minor, Linn.29i. Rumph.34H. (2. fpecim. 618. 


\section{Teftaces. Ordre IV.}

I032 V. Vicnire, ou Contre-Amiral granulé, coquille di Sommet emouffé granulé de travers, brune, cerclée de taches blanches, Linn. 290. «. ou a. (1. p. Or 3 .

$1033 V$. Vicaire, ors Contre-Amiral bandée, $d$ coquille conique, deprimée, à Sommet emou[Jé, noire, a taches trian. gulaires blanches, E bande ligniaire jaune, Linn. 290.

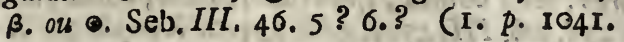

$1034 V$. Vicaire, ou Contre-Amiral, d coquille noire, $\delta^{2} t a-$ ches blanches, Linn. 290. $\gamma$. ou o. Seb. III. 46. 7-12. 47. 5. 6. Valent. 10. (2. p. 6I4.

I035 $V$. Vicaire, ou Contre-Amiral, approchant ar precedent, Linn. 290. \%, 0u O. (2. p. 6I 4.

I036V. Vicaire, ou Contre-Amiral, plus petite, Linn. 290. r. ou o. (2. p. 6r 4 .

I037 V. Leopard tigré, coquille couleur de poix, $d$ taches en forme de coeur blanches, un peu granulée, Linn. 290. dou o. Valent. 25. (I. p. бrб.

$1038 V$. Leopard marbré, d coquille conique elongée, anfraitrures noueufes, a fommet emoulfé, noire, à taches blancbes. en forme de coeur, Linn. 290. Seb. III. 46. 13-19. Lift. 787. 39. Argenv. 12. M. Petiv. Gaz. 47. IR long. 4. cralf. 2. pouc. (I. p. 017 .

$1039 V$. Leopard imarbre, Semblable di la precedente, mais plus. petite, Linn. 290. (2. p.617.

1040 V. Leopard marbre, plus petite, Linn. 290. (3.p.617\% I04I $V$. Leopard marbré, plus ventrue, Linn. 290. Seb. III. 47. 4. (I. p. 6I7. a.

1042 V. Leopard marbré, coquille jaune, à tacbes blanches, Linn. 290. Argenv. 12. O. Valent. 43. "Il faut pru", demment diftinguer celle ci de la Volute Damier, in " $n^{\circ}$. 1056. par ce que dans celle ci la couleur jarne, ne "previent pas de la nature, mais de quelque cause exter"ne, Ẽ non pas de l'art. (2. p. 617.b.

I043 $V$. ESpladian. ou Toile d'A raignée, coquille à anfractures canalées, blanche, à lignes brunes, en forme de refearux, E cerclée, ou entourée de deux bandes brunes, Linn. 0 . Argenv. Suppl. I. T. long. $3 \frac{1}{4}$. cra J. 2. pouc. (2.p.6r5. $1044 V$. Couronne Imperiale, d coquille blancbatre, $d$ bandes longitudinales livides, couleur de ploimb, à lignes articulées de blanc-brun, Linn. 29I. Seb. JII. 47, I8-21. Lift. 756. 15 ? Argenv. I2. E. Petiv. Mur. 6.6. Hollar Icon. 48. Spengler Icon II. I. (1.p. 618 .

$1045 V$. Couronne Imperiale, tant foit peus plus petite, Linn. 291. Rumpb. 34. H. (2. p. 618 . 
1046. C. Imperialis, fed fascix anguftiores, nec fere lineata maculis fuscis confluentibus, Linn. 291. \%. Petiv. Muf́. 15. 17. (2. fpecin. 6ig.

1047 C. Imperialis, fed tunicatus adhuc, atque in apice $\mathrm{Mit}$ lepora Pumicofa, Linn. 291. (2. fpecim. 018.*

1048 C. Virgo, tefta conica, fpira tuberculata, apice violaceo, Linn. 294. Seb. III. 47:9. Lift. 754.2. Rumph. 3r. E long. $3 \frac{1}{4}$. poll. (1. Specin. $620 . *$

5049 C. Virgo, paulo minor, Linn. 294. (2. Specim. 620.

2050 C. Litteratus, tefta conica alba, maculis parvis quadratis, punctisque coeruleo fuscis maculata, Linn. 292.. Seb. III. 45. 1-3. Lift.772. 18. Kircher III. 132. (2 fpecim. 62I.

I05I C. Litteratus, maculis numero minoribus, Linn, 292. (2. Tpecim. $62 \%$

Ie52 C. Tigrinus, apice deprefto, tefta fasciis tribus flavis, maculisque fuscis, Linn. $292 \propto$ f. O. Seb. III. $4 \mathrm{I}$. 4-7. 10-1 5. Lift. 770, 17. c. Kircher III. 131.357?

- 1 (2. ipecim. 62z.

1053 C. Tigrinus, præcedenti affinis, Linn. 292. «. f. o. (2, fpecim. 622.

105.4 C. Tigrinus, fasciis rofeis, maculis minoribus, Linns. 292. a. f. O. (I. fpecim. 624. a.

I055 C. Tigrinus, fasciis tribus rubris interruptls, Linn. 292. B. r. O. (1. Tpecin. 622*.

1056 C. Cervulus, tefta oblonga, non corotata glabra, an. fractibus canaliculatis, acuie nodofis, flava, maculis cordiformibus albis, Linn. O. Seb.III. 43. I3 14. (2. . pecim.623. 1057. C. Cervulus, pracedenti affinis, fed minor, Linn. 0 . (2. . pecim. 623.

1058 C. Spectrum, tefta tumida elongata, apice acuto fla. to, nebulofa, pundtis ftrisque, albo luteis, Linn, 321 . Lift. 783. 30. Valent. 39. 40. (2. Ipecim. 624.

1059. C. Spettrum, pracedenti affinis, Limn. 321. (3. Ipecim. 624.

1060 C. SpeEtrum, ftriatum, tefta alba, maculis longitudinalibus luteis, Linn. 32I. «, Seb. III. 43. 32? (2 ipecim, 625.

1061 C. Specarum, tefta glabra, maculis fuscis flammata, Linn. 32 r. B. C. O. (2. Specim. 627.

1062 C Ruficus, tefta oblonga, tumida, cinerea, fasciis longitudinalibus, flavis punetatis, Linn. 306 . Lift. 765. 14. Petiv. Muf. 15. 6. (2. Specim. 629. 


\section{Teftaces. Ordre IV.}

$1046 V$. Couronne Imperiale, mais dont les bandes font plus etroites, Ė a peine lignées, à tacbes brunes confluentes, Linn. 291. a. Petiv. Muf, 15. 17. (2. p. 619.

$1047 V$. Couronne Imperiale, mais encore en tunique, $\mathcal{E}^{2}$ au jommet une Millepore Poncée, Linn. 291. (2. p. $618 *$

1048 V. Cierge, \& coquille conique, fpire tuberculèe, di Sommet violet, Linn. 294. Seb. III. 47. 9. Lift. 754. 2.

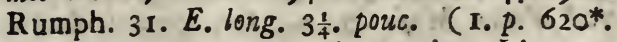

ro49 V. Cierge, un peu plus petite, Lian. 294. (2. p. 620.

1050 V. Lettrée, ou Ecriture Arabique, d̀ coquille conique blanche, à petites taches quarrées, E tachetée de points brunbleuatres, Linn. 292. Seb. III. 45. 1-3. Liit. $77^{2}$. I8. Kircher III. 132. (2. p. 62I.

I05I $V$. Lettrée, ou Ecriture A rabique, ou Hebraique, do taches moins en nombre, Linn 292. (2. p.621.

$1052 V$. Tigrée, a fommet deprimé, coquille à trois fajcies jawnes, E taches brunes, Linn. 292, «. ou O. Seb. III. 4I. 4-7, 10-15. Lift. 770. I7. c. Kircher III. 13I. 357. (2. p. 622 .

I053 V. Tigrée, Semblable de la precedente, Linn. 222 o ou 0. (2. p. 622 .

$1054 V$. Tigrée, a fafcies couleur de rofe, EO petites taches, Linn. 292- a. 0ul o. (1.p. 622.a.

I055 $V$. Tigrée, à trois fafcies, interrompues rouges, Linn. 292. B. 0u O. (I. p. 622*.

$1056 V$. Damier, à coquille allongée, fans couromne peliee, les anfractures caneles, $a$ nouds, jaune, $d$ taches blanches en forme de coeur, Linn. 0. Seb. III. 43. 13. 14. (2. p. 023.

1057 V. Danı.er, approchant au procedent, mais plus petit, Linn. O. ( 2. p. 623.

$1058 V$. Spectre, à coquille enfiée elongée, à fommet aigue, jaun nebuleufe, à points, Ẽ Stries blanc jaunes Linn. 321. Lift. 783. 30. Valent 39. 40. (2. p. 624.

Io59 $V$. Spectre, femblable au precedent, Linn. 321 . (3. $p$. 624.

$1060 V$. Spectre, frité, d coquille blanche, taches longues jawises, Linn. 321 . '. Seb. III. 43: 32.? (2. p.625.

Ic6r $V$. Spectre, ou Moftillaire, d coquille pelée, enflammée de taches brunes, Linn. 32I. $\beta$ : or o. - (2. p.627. $1 \otimes \sigma_{2} V$ Ruftique, ou d'Agathe cendrée, do coquille allongée, enfiée, cendrée, à fajcies longitudinales, jarnes pointées, Linn. 306. Lift. 765. 14 Petiv. Mur. I5.6. (2. 1.629. $\triangle 22$ $1063 P_{0}$ 
1063 C. Rufticus, luteus, fasciis longitudinalibus fuscis, albo punctatis, Linn. 306. a.' Seb. III. 44.30? Va!ert. 50? (1. Ipecim. 630.

1064 C. Rufticus, tefta alba, lineis flavis, nubeculisque ro. feis \& purpureis fasciata, Linn. 306. $\beta$. (I. fpecim. 626.

1065 C. Coftareus, tefta fusca, albo punctata. fasciis lineatis, albo maculatis, Linno 306. \%. f. o. Lift. 776. 22 ? Valent. 76? Petiv. Muf. 15. 5. (2. Specim. 628.

1066 C. Amadis, tefta anfraetibus depreffis, apice elongato. fusca, reticulatim albo maculata, longitudinaliter linesta, punctulis niveis, Linn. o. Seb. III. 47. 24 ? Liff. 785, 33 ? Argersv. Suppl. I. S. (2. fpecim. 634. 1067 C. Amadis, paulo minor, Linn o. (2. (pecim. 634. 1068 C. Amadis, magis flavefcens major, Limm. o. longit. 3. poll. (2. Ipecim. 634.

ro69 C. Amadis, anfractibus tumidioribus, tefta aiba, undatim ftriata, Linn. 0. (2. Specim. 633.

1070 C. Amadis, punctatus, punctulis caftaneis, Liras o. Ar. genv. Suppl. 1. $S$ ? (1. Ppecin. 632.

Io7I C. Araufiacus, tefta alba, laevi fasciis incarnatis, an: fractuum fummis canaliculatis, Linn 316. Argenv. Suppl. I. I. Petiv. Mus.7.7. Valent. 100. Spengler Icon.II. E. (1. fpecim. 592.

1072 C. Cynorrhodonus, tefta incarnata, fulcata, fasciis dustbus pallidioribus, Linn. 316. \&. f. o. Rumph. $32 T$ ? Lift. 760. 5. (2. fpecim. 645.

1073 C. Sipmanus, fortius coloratus, \& fafciatus, fed minor, Linn. 316. ß. .. 0. (2. fpecim. 645.

1074 C. Coccinatus, craffior, tefta incarnata, fasciis tribus rofeis. Linn. 316. \%. f. o. Li/f. 760. 6? (2. Specim. 644.

1075 C. Terebra, terta alba, prope apicem craffiore, antice. cœrulefcente, Linn. O. (2. (pecim. 646.

1076 C. Terebra, fed minor, Linn. o. (2. fpecim. 646.

1077 C. Terebra, tefta elongata, ftriata, fasciisque flavis, Linn. O. Valent. II. long. $2\{$ poll. (I. Specim. 647.

1078 C. Terebra, eidem affinis, Linn. O. (2. Specim. 647. 1079 C. Terebra, tefta tava, fasciis interruptis, maculisque fuscis, Linn. O. Valent. 70. ( 1. '́pecim. 647*.

3080 C. Terebra, tefta violacea, fasciis flavefcentibus, maculis albidis, caftaneo punctatis, Linn. C. (1. Ipecim. 64?. 


\section{Teftaces. Ordre IV.}

Ic $\sigma_{3} \nu$. Ruftique, ou d'Agathe cendrée, jaune, a fascies iongitudinales brunes, pointées de blanc, Linn. 300. $\alpha$. Seb. III. 44. 30? Valent. 50? (1. p. 630.

ro64 $V$. Ruftique ou d'Agathe cendré, à coquille blanche, Elignes jaunes, Ẽ bandée de petites nues couleur de roje, Es pourprées, Linn 306. $\beta$. (i. p. 626.

$1065 \mathrm{~V}$. Chaitaigne, à coquille brune, pointée de blanc, a fafcies lignées, tachetées de blanc, Linn. 306 y. ors 0. Lift. 776. 22. ?. Valent. 76.? Petiv. Muf. 15.5. (2. p.628. $1066, V$. Amadis, caquille d anfractures detrimees, d fommet elongué, brune, tachetée de blanc, en forme de refears lignées en long, à pointes blanches, Linn. O. Seb. III. 47. 24 ? Lift. 785.33 ? Argenv. Suppl. I. S. (2. p. 634. V. Anadis, un peu plus petit, Linn. o. (2. p. 634. 3 pouc. (I. p 634.

I060 V. Amadis, les anfractures plus enflées, d coquille blanck friée par ondes, Linn. O. (2. p. 033.

$1070 V$. Amadis pointée, à points chatcignes, Linn. 0 . Argenv. Suppl..1. S.? (1.p.632.

ro71 $V$. Amiral d'Orange, d̀ coquille blanche, polie, $\dot{d}_{\mathrm{q}}$ fafcies coulentr de chair, les anfraetures, trés grandes, canalés Linn. 316. Argenv. Suppl. I. I. Petiv. Muf. 7.7. Valent. roo Spengler. Icon. II. E. (2. p. 592.

$1072 V$. Faux Amiral d'Orange, $\dot{a}$ coquille incarnée, fillonnee, dे deux bandes pales, Linn. 316. a ou 0. Rumph. 32. 7. Lift. 760. 5. (2.p. 645 .

ro73 $V$. Faux Amiral d'Orange, de Guinée, pluis fort coloré, E bandée, phus petit, Linn. 3 I6. B. ou o. (2. p. 645 .

jo74 V. Faux Amiral d'Orange, de Guinée, d coquille in. carnée, à trois bandes couleur de rofe, Linn. $316 . \beta^{\prime}$. ou $\odot$. Lift. 760, 6.? (2. p, 644.

$1075 V$. Tariere, aे coquille blanche, plus epais vers le formmet, devant blevatre Linn. o: (2. p. 646.

ro76 V. Tariere, mais plus petit, Linn. O. (2. p. 646. $1077 V$. Tariere, 's coquille elongé, Jtriee, E fafcies jaunes, Linn, O. Vaient, I1. long. 2 \& pouc. (1. p 647.

Io78 V. Tariese, asprochant d la precedente, Linn. O. (2.p. 647.

ro $79 V$. Tariere, d coquille jaune, a fafcies interrompiues, $\mathbb{E}^{2}$ taches brures, Linn. O Valent. 70. (i. p: $647^{*}$.

1080 $V$. Tariere, a coquille violette, $d$ bandes jaunatres, \& taches blancheatres, pointée de chataigne, Linn. 0. (1. p. 048. 


\section{Teftacea. Ordo IV}

1081 C. Teretra, tefta alba, apice \& anfractu primo, antice. que violaceo, fusco maculata, Linn, O. Lift. 745. 36. (1. 'pecim. 650.

1082. C. Nux Sativa, tefta ventricofa, tumida, longitudinaliter rugola, rude ftriata, lutea, fasciis tribus violaceis, Linx. o. Argenv. Suppl. 2. D. (1. Tpecim. 642.

1083 C. Nux Sativa, tefta fulcata, granulata, violacea, Linn. 0. Rumph 33. DD. Petiv. Muf. 15. 16. (1. Ppecim. 643.

1084 C. Terebra granulata, teftà alba, maculis thavo fuscis parvis, Linn. 314. Gralth. 25. H. L. Lift. 744. 35 . Rumph. 33. EE. Argenv. 13. P. (2. Ipecim. 651.

Io85 C. Terebra granulata, pracedenti affinis, Linn. 3 I4. (4. fpeciom. 65I.

1086 C. Terehra, telta lutea in medio albo fuscoque marmorata, Linn o. Rumph. 31. D. Seb. III. 43. 35? (3. fpecim: 9:9-921.

1087 C. Gloria Maris, tefta valde elongata alba, lineolis fla. -14. vis, fe decusfantibus \& anaftomofantibus reticulata, in medio obfcurioribus, Linn o.long. $3 \frac{3}{4}$. poll. "Eadem fpe" cies extar etiam in Collectione Dom. a Secretis Lyo"o NETIO; nec non in Mufeo Excell. Confil. Intim. Comi", tis A MOLKTE, Hafnic; cetera defcriptio videatur "in Berlin. Befchaeft. 1II. tab. 8. f. A. p. 32 I-33I."

(I. fpecim. 635 .

$1088 \mathrm{C}$ : Reticulatus, tefta argute ftriata, iransverfim flavo maculata, lineisque obfcurioribus reticulata, Linn. 319. «. . O. Gualth. 25. X? Rumph. 32. O? (3. Tpecim. 049.

1089 C. Reticulatus, tefta albo rofea, maculis caftaneis, lineis. que longitudinalibus atris, Linn. 319. B. f. O. Petiv. Muf: 15. 9? (2. Ipecim. 637 .

1090. C. Aulicus, tefta caftaneo fusca, lineis longitudinalibus, \& transverfalibus, fusco reticulatis . Linn. 320. Seb. III, 47. 10. II. Rumph. 33. 3. (3. Rpecim. 033. rogr C. Aulicus, pracedenti affinis, Limn. 320. (3. Ppecim. 053.

1092 C. Aulicus, fed minor, Linn. 320 . (3. Specim. 653. 1093 C. Brunneus, tefta oblcure fusca, maculis albis cordiformibus, fusco marmoratis, Linn. $320 . \alpha$. . . O. Seb. III. 43. I' 2? 47. 13. Liff. 790. 43. Rumph. 33. 4. (2. Ipecim. 652 .

109.4 C. Brunneus, præcedenti fimilis, Linn. 320. «. ケ. o. (3. Specim. 652. 


\section{Teftaces. Ordre IV:}

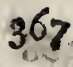

IOS\& $V$. Tariere, à coquille blancbe, le - Jammet, E' premiere anfracture, E devant violette, tachetee de brun, Linn. 0 . Lift. $745 \cdot 36.61 . p 650$.

I082 V. Noix Sative à coquille ventrue, enflé, 80 vélu on long, rudement friée, jaunatre, id trois fafcies violettes, Linn. O. Argenv. Suppt. 2. D. ( $x$. p. 642.

IC83 V. Noix Sitive, d̀ cogrille granuté fillonnée, violette, Linn. o. Rumph. 33. D D. Petiv. Mur. 15.-16. (I. p. 643.

$1084 V$. Tariere granulée, à coquille blanche, do petites tachss brune jaunatres, Linn, 314. Gualth. 25. H. L. Lift. 1085 V. Tariere granulée, Semblable do la precedente, Linn. 31 I
io (4. p. $65 \mathrm{x}$.

x086 $V$. Tariere, à coquille jaunatre, au milieu, marbrée de blanc, E2 brun, Linn. O. Rumph, 31. D. Seb. III. 43. .35? (3. P. 9ro-92 I.

$1087 \mathrm{~V}$. Gloire de Mer, a coquille fort elongée, Bblanche, $d$ lignes jaunes: fe croiffantes. .E anaftomifantes en forme de refear, au miliew plus ob fcures, Linn. o. long $3 \frac{1}{4}$ pouc. 3. Une Semblable espece fe trouve dans la Collection de "Mr. le Secretaire LyoNeT; conme auff dans le Cabi" net du Confeiller Intime le Comte de molrTe, d "Coppenhague : la defcription eft contenue dans les Berlin. ". Befchaeft. III. tab. 8. f. A. p. $321-331$ ". (r. p. 635.

$1088 V$. Brunette en forme de refeau, d coquille fubtilement ftriée, E. de travers tachetée de jaune, $\mathcal{E}^{\circ}$ à lignes obfcures en forme de refeaty, Linn. 3I9. a. ou o. Gualth. 25. X. ? Rumph. 32. O? (3. p. 649 .

jos $\mathrm{V}$. Bruntte en forme de refeau, coquille blanche, couleus de rofe, à taches chataignes, à lignes longitudinales noires, Linn. 319. B. ou o. Petiv. Muf. 15. 9.? (2. . .637. iogo $V$. Brunette, it coquille chataigne brune, a lignes bongitudinales, Es traverfes, brines, en forme de refear, Linn. 320 . Seb. III. 47. 10. II. Rumph. 33. 3. (3. p. 633 .

iogr $V$. Brunette, jemblable d̀ la precedente, Linn. 320. (3. p. 653 .

1092 V. Brunette, mais plus petit, Lijn. 320 . (3. p, 653 .

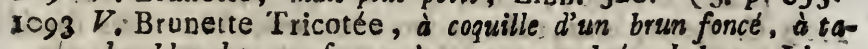
ches blanches en forme de coeur, marbrées de brun, Linn. 320. a. ou 0. Seb. III. 43. I, 2 ? 47. I3. Lift. 790. 43. Rumph. 33. 4 ( 2 p. 652 .

$1.094 V$. Brunette Tricotée, ous Jemblable à la precedente, Linn. $320 . \alpha .04$ 0. (3. p. 652 .

$$
\text { A } 24 \text { I } 2095 \text {. }
$$


Iogs C. Brunneus? f. varietas, brevior tamen, fusco venofus, maculis cordiformibus albis, Linn. 320 . $\beta$. f. 0 . Seb. III. 43. 3. 7. 8, 47. 14. 15. (1. Ipecim. 640.

I096 C. Cylindricus, pulcerrimus, tefta alba, transverfim luteo undatim ftriata, \& reticulata, Linn. 319. भ. f. O. Seb. III. 43. 9. Gualth. 25. I? (3. Ipecim. 641.

1097 C. Cylindricus, tefta alba, transverfion lineolis fuscis maculata, reticulatim venofa, Linn. 319. d. f. O. Seb. III. 43. I1. 12. Gualth. 25. V? (1. Ipecim. 639. 1098 C. Cylindricus, præcedenti affinis, Linn. 319. \&. .. 0. 4. Rumph. 32. P. (3. fpecin. 639.

I099 C. Cylindricus, tefta alba, reticulatim, flavo venofa, ma: culis luteis fafciata, Linn. 319. ^. T. 0. Argenv. I3. $\boldsymbol{E}$. (3. Ipecim. 636.

I 100 C. Cylindricus, pracedenti affinis, Linn. 3I9. थ. .. ०. (3. fpecim. 636.

Iror C. Textile tefta venis reticulatis luteis, maculis lu. teis, fuscisque, Linn. 319 . Seb. III. 43. 7. 8. Lift. 788, I4. Kircher III. I35. Petiv. Mus. I5. 3. longit. $3 \frac{1}{2} \cdot$ poll. " (2. fpecim. 638.

1102 C. Textile, fimilis, Linn. 3 ig. (2. fpecim. 638.

IIO3 C. Textile, pallidior, Linn. 3 19. (3. fpecim. 638. $\mathrm{Iro}_{4}$ C: Textile, féd minor, Linn. 319. - (4. Specim. 638.

\section{GE N V SXIII. C YL INDRI.}

I105 C. Ifpidula, tefta oblongata, anfractibus approximatis; apice acuto, cinerea fusco fasciata, ore grifeo . Linn. 400. a. Seb. III..53. P. Q. Gualtb. 23. R. Lift.722. 9: Rumph. 39. 9. " (2. Specim. 654.

I106 C. IJpidula? r. yarietas, fed major, Linn. 400. a. ( 2. ppecim $654^{*}$.

I107 C. Bifafciatus, tenta laevi hepatica, fasciis 2. angulato undatis fuscis cincta, purpurafcente, Linn. 400. b. Seb. III. 53 R ?... (2. fpecim. 655 .

I 108 C. Vannillus, tefta flava, fuscis punctis confluentibus pisa, ore albo, Linn. 400. c. (1. fpecim. 1056.

1109 C. Leucophaus, tefta grifeo alba, maculis pallidis fuscis fasciata, ore cinereo, Linn. 400. d. (2. Specim. 922.

IIIo C. Aranea, tefta flavo grifea, lineolis obfcurioribus reticulata, fusco longitudinaliter ftriata, ore cinereo, Linr. 400. e. Seb. III. 53.W.? (6. fpecim. 656. 
ICg5 $V$. Brunette Tricotée? ou varieté, pourtant plus courte? tachetée de brun, à taches blanches, en forme de coeur, Linn. 320. B. ou 0. Seb. III. 43. 3. 7. 8, 47. 14. 15. (I. p. 640 .

Iog6 V. Drap de mille beautés, tres belle, à coquille blanches de travers jaune, friée par ondes, EO en forme de refeau,

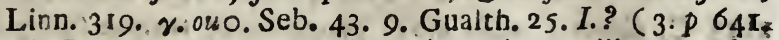
$1097 V$. Brunette à natte Tricotée, ì coquille blanche, de travers à lignes brunes tachetée, en forme de refeau. Ẽ remplie de veines, Linn. 319. d. ou 0. Seb. III. 43. II. I2. Gualth. 25. V.? (I. p. 639 .

rog8 $V$. Brunette à natte Tricotée, approchante à la precedente. Linn. 319. d. ou o. Rumph, 32. Y. (3. p 639.

1099 $V$. Drap d'or cylindrique, à coquille blanche, en forme de rejearb, à veines jarmes, Ẽ bandée de taches jaunes. Linn. 319. भ. ou 0. Argenv. 13. F. (3. p. 636.

I $100 V$. Drap d'or cylindrique, femblable à la precedente, Linñ. 319. x. ou o: (3 p. 636.

Iror $V$. Drap d'or, coquille $\dot{a}$ veines en forme de refeas jaunes, $a$ taches jaunes, E brunes, Linn. 319 . Seb. III. 43. 7, 8. Lift. 788 I4. Kircher $I I I$. 135. Petiv. Muf. I5. 3. long. $3 \frac{1}{2}$, pouc, (2. p. 638 .

I102 $V$. Drap d'or, femblable, Linn. 319. (2.p. 638.

$1103 V$. Drap d'or, le pareil, plus pale, Linn. 319 . (3.p.638. ${ }_{1104} \mathrm{~V}$. Drap d'or, mais pius petit, Linn. 319 . (4. p. 638.

GE NRE XXIII. R O ULEA UX:-

I105 $R$. Ifpedule, ou Olive de Vafe, d coquille allongée, les anfractures approchantes, à fommet aigue, cendrée, bandee de brun, d bouche grije, Linn 400 . a. Seb. IIII. 53.P. $Q$ Gualth. 23. $R$ Lift. 722 9. Rumph. 39. 9 . (2. p. 654 .

n106 $R$. Ifpedile, ou Oiive de Vafe? ou varieté, allongée, plus grant, Linn. 400. a. (2. p. 654*

$1107 R$. Olive de Vafe, à coquille polie, coulesur de foye, $a$ deux bandes, cerclée en anglées, ondées: "brunes, à bousche pourprée, Linn. 400.. b. Seb. III. 53. R.? (2. p. 655 .

$1108 R$. Vanneau, $\dot{a}$ coquille jaune, $\dot{a}$ points bruns confluents, à bouche blaxche, Linn. 400 c. (

$1109 R$ Gris, à coquille grife-blanche, Eे bandée de taches pales trunes, à bouche grife, Linn. 400 . d. (2.p.9z2.

Ir $10 \quad R$. Toile d'Araignée. $\dot{a}$ coquille pale grife. à lignes oúfcures en forme de refeau, Eै firiée de brun en long, is bouche cendrée, Linn, 400. e. Seb. III. 53. WV? ( $\sigma^{\circ}$. p. 656. 
III. C. Fecusculum, tefta hepatica, maculis trigonis albis, oro rubefcente, Linn. 400. f. Gualtk. 24. G? (4. fpecim. 657.

IrI2 C. Undatus, tella grifeo fusca, fasciis angulofis fuscis, ore flavefcente, Linn. 400. g. Seb. III. 53. c ? Gualth. 24 L? Lift. $72 C .4$ ? (2. Specim. 665.

yrr3 C. Collaris, tefta alba, prope apice torque fusco angulofo, ore faturate puniceo, Linn. 400. h. Rumph. 39. 7 ? Argenv. 13. R? Pctiv. Gaz. 59.8, 150. 19. (3. Ppecim. $66 \mathrm{r}$.

Irr4 C. Collaris, pracedenti affinis, Limn. 400. h. (5. fpecim. 66r.

III5 C. Litteratus, tefta hepatica flava, vel grifea, lineis angulofis ftriata. Linn. 400, h. Seb. III. 53. e? Gualth. 23. I. Kircher III. 363 . (3. Cpecim. 658 .

II 16 C. Litteratus fimilis pracedenti. Linn. 400. b. Seb. III. 53. X? Gualth. 23. $R$. (5. Fpecim. 060 .

xii 7 C. Litteratus, fimilis præcedenti, $t \in$ fta tamen alba, Linn, 400. h. Gualth. 23. I. (4. Specim. 658.

IrI8 C. Litteratus - præcedenti affiris, tefta flavefcente, fusco punctata, Linn. 4co. h. Seb. III. 53. m. Gualth. 23. Q Li/t. \$21.7? Argenv. 13. R. (6. fpecim. 064.

Irig C. Punctatus, flavus, Linn. 400 . h. Seb. $111.53 . X$. Gualth. 23. N? (8. Ipecim.659 663.

Irzo C. Tigrinus, an fimilis pracedenti? tefta flavefcente \& fusco maculata, Linn. 4co, h. Gualth. 23. N. Lift.721. 7:Rumph. 39. 8. (3 fpecim, 662 .

II2I C. Fuscus, tefta caftanea, ventre \& vertice grifeis, ore fusco, Linn. 4co. i. Seb. III. 53. d. (2. Specim. 668. 923.

I1 22 C. Albus, tefta \& ore albo, Linm. 40c. k. Argenv. I3. 2? (2. fpecim. 666.

rr23 C. Helvus, tefta flava, ore albo, Linm. 400.1. ( 2. 'pecim. 924.

1124 C. Meloftomus. tefta grifea corulefcente, ore fusco, Linn. $400 \mathrm{~m}$. (3. Specim 067.

1125 C. Salicinus, tefta caftanea, Linn. 400. n. Seb. III. 53 . $X$ ? Gualth. 23. $R$ ? (4. Ipecim. 600.

1126 C. Gibbofus, tefta albo lutefcente, Limr. 4c0. 0, Lift. 717 . I. (2. fpecim. 672 .

1127 C. Emicator, tefta flavefcente, maculis violaceis, ore Juteo, Linn 400. p. Gualth 23. L? Lift. 720.4 ? Rumph. 39. 6. (4. Ipecim. 670. 


\section{Teftaces. Ordre IV:}

IIII R. or Olive Flammée, d̀ coquille hepatique, ì tachestriangulaires blanches, at bouche rougeatre, Linn. 400.' $f$. Gualth 2 . . G.? (4. p. 657.)

III2 $R$ Orà̉é en Ziğ-zag, à coquille gris-brune, d fa/cies angulaires brunes, à bouche jaunatre, Linn. 400. g. Seb. III 53.c ? Gualth. 24. L.? Lift. 720.4 , ? (2. p. 605. III3 $R$. Collier, à coquille blanche, vers le fommet en collier brun, en forme de coins, à bouche pompadoure, Linn. 400. h. Rumph. 39. 7 ? Argenv, 13. R.? Petiv. Gaz. 59. 8, 156. 19. ( 3 p. 661 .

1114 . Coliier, approchant au precedent, Linn. 400, h. ( 5 . p. 66r.

III5 $R$. ou Olive Lettrée, à coquille hepatique blanche, ou griz fe, a lignes angulaires ftrie, Linn., 400. $h$. Seb, III. 53. $e$ ? Gualth. 23. I. Kircher III, 303. (3. p. 658. IIt6 R. ou Olive Lettrée, femblable au precedent, Linn 400 h. Seb. IIl. 53. X, ? Gualth. 23. R. (5. p. 660 .

III $R$. ou Olive Lettrée, Semblable au precedent, mais à co. quille blanche, Linn. 4c0. h. Gualth. 23. I. . (4. pe 658.

1.118 . Lettrée, ou Ecriture Grecque, Semblable au precedent: di coquille jaunatre, pointée de brun; Linn. $400 . h$ Seb. III. 53. m. Gualiti. 23. Q. Lift. 72 I. 7.? A grgenv. 13. R. (6. p. 064 .

IIIg $R$. Pointé, jaune, Linn. 400.) h. Seb. III. 53. $\bar{X}$. Gualth. 23. N.? (8. p. 659. 603.

I $120 R$. Tigré, ou femblable au precedent ? à coquille jaunatre, $E^{2}$ tachetée de brun, Linn. 403. h. Gualth. 23. $N$. Liff: 721. 7. Rumph. 39. 8. (3. p. 662 .

II2 I $R$. Brun, à copuille chataigne ventrue, $\mathcal{E}^{2}$ fonmet grife. a bouche brune, Linn. 400 . $i$. Seb. III. 53. $d$, (2) to (668.923.

I $22 R$. Blanc, à coquille, $\mathcal{E}^{2}$ bouche blanche, Linn. 4co. $k$, Argenv. 13. Q? ( 2 p. 606.

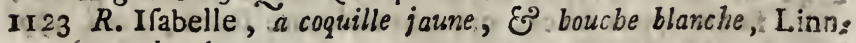
400 . l. (2. p. 924 .

$1124 R$. Meloftome, ou Cendré, à coquille gris -bleuatre , à bouche brune, Linn. $400 \mathrm{~m}$. ( 3 p. 667 .

II25 $R$. Bois de Chene, à coquille chataigne. Linn. 400. ns Seb. III. 53. X.? Gualth. 23. $R$ ? (4. p. 660 .

II $26 R$. Boffu, $\dot{a}$ coquille blanc-jaunatre, Linn. 400. O. Lift, 717 . I. (2.p. 672 .

II $27 R$. Transparence, $\grave{d}$ coquille jaunatre, à taclies violettes. a bouche jarne, Linn. 400. p. Gualth. 23. L.? Lif. 720. 4. ? Rumph. 39.6. (4. P. 670. 
xi28 C. Verficolor, tefta fufcefcente punctata, maculisque parvis fuscis obfcurioribus, ore aurantio, Linn. 400. q. Seb. III. 53. w. Gualth. 23. F? (2. Ipecim. 671.

Iir29 C. Extuberatus, tefta alba, transverfim angulolo fasciata, Linn. 400. r. Seb. III. 53. Y? (2. Cpecim. 673. II 30 C. Striatus, tefta alba, friis longitudinalibus maculatis, Lirn. 400. s. Seb. III. 53. m? (I. Ipecim. 083. II31 C. Fasciatus, tefta fasciis transverfalibus augulofis, Linn.400. t. Seb. III. 53. $\Upsilon$ ?. (x. fpecim. 677.

1132 C. Fludtifer, tefta olivacea, fasciis undatis, Linn. 400.

u. Gualth. 24. N? (1. fpecim." 678 ."

Ir33 C. Varius, tefta alba, maculis flavis viridibusque, fasciis maculofis nigris, Linn. 399. v. Sel. III. 53. F? (3. Ipecim. 674.

II34 C. Maculatus, tefta virefcente transverfim undata, ma-: culisque fuscis, Linn. 399. w. Seb. III. 53. $R$ ? ( 5. fpecim. 676.

I135 C. Chryfoftomus, tefta alba, lineis undatis fuscis, ore flavo, Linn. 399. X. (I. Rpecim. 675.

II36 C. Tricolor, tefta flavefcente, olivaceo undata, macualis obfcuris fasciata, Linn. 399. y. Gualth. 24. I? Petiv. Gaz 102. I9. (3. Cpecim. 926.

Ir37 C. Tricolor, præcedenti affinis, fed magis expreflus, Limn. 399. y. (3. Tpecim. 925. 926.

I138 C. Tricolor, fimilis præcedenti, Linn. 399. y.) Lift. 728. 15 ? (2. Tpecim. 714. 715.

Ir39 C. Undrubatus, tefta flavefcente, punctis flavis viridibusque undata, Lim. 399. y. Lifk. 732. 21 ? (3. fpecim. 679.

3140 C. Flammatus, tefta albida, ftriis, triangularibus viridibus, flavisve, Linn. 399. Y. Gualth. 23. PP. (7. \{pecim. 681.

II4I C. Guttatus, tefta flavefcente, maculis punetatis piceis, fascia apicis fusca, ore coerulefcente fusco, Linn. 399: z. (2. 'pecim. 705 .

Ir42 C. Maculo/us, tefta cinerea, purctis cinereo fuscis, ore cærulefcente, Linn. 399. a a. (2. fpecim. 927.

Ir 43 C. Ventricofus, tefta flavefcen'e, lineolis angulatis fuscis ftriata, ore albo, Linn. 399 b b. (I. fpecim.680.

Ir44 C. Albus, tefta alba, lineolis angulatis fuscis, ore puniceo, Linn. 399. c c. (1. Ppecim. 682.

1145 C. Olivaceus, terta olivacea, lineis obfcurioribus angulatis, ore cinereo, Limm. 399. dd. (I. Specim. 1055. 


\section{Teftaces Ordre IV.}

II28 $R$. Varié, d coquille brunatre, pointée, d petites tacher brunes, plus objcures, d bouche orange, Linn. 400. 2 . Seb. III. 53. w. Gualth. 23. E'? (2. p. 671.

I $29 R$. Enfle, d coquille blanche, fafcie de travers, en angles, Linn. 400. r. Seb. III. 53. $\Upsilon$ ? (2.p. 673.

1130 $R$. Strié, à coquille blanche, \& longues ftries, tachetéts, Linn. 400. s. Seb. III. 53. m.? (1. p. 683.

II I $R$. Fafcié, coquille dे fafcies longues traverfes anglkes, Linn. 400. t. Seb. III. 53. X? (I. p. 67?.

I132 R. Vagué, à coquilie olivatre, à fofcies ondées, Linn.400, u. Guath. 24. N.? (1. p. 678 .

II33 R. Varié, à coguille blanche, d taches jaunes, \&o vertes, à fafcies tachetées noires, Linn, 399: ข. Seb. III. 53. F. ? (3. P. 674 .

$1134 R$. Tacheté, do coquille verdeatre onciée de travers, à taches brunes, Linn. 399. W. Seb, III. 53. R.? (5. p. 676 .

$1135 R$. Chryfoftome, a coquille blanche, à lignes brunes, boukhe jaune, Linn. $399 x$. (r. p. 675 .

I136 R. a Trois Couleurs, a coquille jaunatre, ondée d'olzvatre, E' fafcié de taches obfcures, Linn. 399. y. Gualth. 23. I. ? Petiv. Gaz. 102. 19. (3. p 926.

$1137 R$. à Trois Couleurs, le pareil, mais plus expresfif. Linn. 399. y. (3. P. 925.926.

II $38 R$ aे Trois Couleurs, fermblable au precedent, Linn. 399; $y$ Lift 728. 15.? (2. p. 714. 715

II $39 R$. Ondé, d coquille jaunatre, ondée de points verdeatres, Linn. 399. y. Lift. 732. 21.? (3. p. 579.

140 R. Flanme, i coquille blancheatre, d ftriés triangulaires vertes, EO jaunes; Linn. 399. y. Gualth. 23. P P. (7. $p$ 68I.

II 4I R. Goutté, a coquille jaunatre, d taches pointéos coulewr de poix, le fommet d bande brune, bouche bleus - celeffe, Linn. 399. z. (2. p. 705.

Ir $42 R$ 'Tacheté. d coquille cendréo, a points brun cendrés, la bousche bleu-celefte, Linn. 399. a a. (2. p. 927.

ir $43 R$. Ventrue, a coquille jaunatre, ftriée de lignes anglés. brunes, à bouche blanche, Linn. 399. b b. (1. p. 680.

$1544 R$. Blanc, à coquille blanche, a lignes anglées brunes, in bouche rouge, Linn. 309. cc. (1. p. 682.

II45 $R$. Olivatre, d coquille olivatre, d lignes objcures en angles, la bouche cendréc, Linn. 399. dd. (I. p. 1055. $1346 R$. 
1146 C. Lineatus, tefta flavefcente, lireolis angulatis fus cis, ore fusco purpurafcente, Linn 399 ee. Seb.III. 53. f. Lift. 731. 20? Argenv. Suppl. 2. A? Petiv.Gaz. 156. 18. (3. fpecim. 684.

1147 C. Flavefcens, tefta flavefcente, punetulis fusco viridibus, ore cinereo, Linn. 392. ff. Seb. III. 53. h? Lift. 734. 24? (3. Ipecim. 685.

II 48 C. Flavejcens, præcedenti affinis, magis ftriatus, Linn. 399. ff. Gualth 23. Q2. (4. Ipecim. 686.

II49 C. Flammans, tefta tlavefcente, flammis latis viridi fuscis, ore albo, Lim. 399. g g. Seb. III. 53.N? Gualth. 24. $L$ ? (7. Cpecim. 692 .

II50 C. Reticulam, tefta alba, viridi fusco reticulata', fusco

Aasciata, ore albo, Linn. 399. h h. Seb. III. 53. H. I.
. Cualth. 24. M? Lift. 739. 28. (5. Specim. 601.

fasciata, ore albo, Linn. 399. h h. Seb. III. 53. H. I.
. Cualth. 24. M? Lift. 739. 28. (5. Specim. 69r.

1151 C. Reticulum, fimilis præcedenti, Linn. 399. h b. (4. fpecim. $69 \mathrm{r}$.

1152 C. Funambulus, tefta carina, elevata, alba in dorfo, Lirn. 399. h h? (I. Rpecim. 1042.

1153 C. Rctis, tefta alba, fusco ftriata, fasciata, ore albo, Linn. 399. h h. Seb. III. 53. O ? Lijt. 739. 26. (3. fpecim. 1054.

1154 C. Ziczac, tefta fusca, ftriis fusco viridibus, angulato undatis, Linn. 399. i i. Gualth.23. M? (2. fpecim.690. I155 C. Ziczac, tefta flava, Linn. 399. i i. Gualtb. 23. PP: (3. Specim. 1043.

Ir56 C. Strictus, tefta Cordide alba, Linn. 399. i i. Lift. 728. 16? (2. 'pecim. 688. 694.

$1 \times 57$ C. Flucticulus, tefta flava, ftriis fuscis, ore purpurafcente, Linis. 399. kk. Gualth. 33. $R$ ? Lift. 731.20. (3. . pecim. 687.

2158 C. Multicolor, tefta pallide viridi obfcure punetata, ore albo, Linn. 399. 11. Lift. 734. 34? (I. Cpecim. 689.

I59. C. Multicolor? f. varietas, tefta alba, punctulis flavis, ore albo, Linn. 399. ai m. (4. Specim. 693.

I60 C. Bombacynus, tefta alba, viridi fasciata, punetis thavo viridibus, Linn. 399. m m. (5. Specim. 695.

Iror C. Netulofus, tefta oblonga flavefcens, nebulis obfcurioribus, fasciis 2 luteis, ore flavo, Linn. 39\%. n n. Lift. 726. 12 ? (2. Ipecis. 700.

1162 C Trifasciatus, tefta flava, viridi punctata, fasciis tribus latis viridibus, ore flavo albefcente, Linn. 399. a o. Seb. III. 53. E? longit. 3. crafl, $1 \frac{2}{2}$. poll. (I. Ipecim. $69 i^{*}$. 
Ii 46 R. Ligne, à coquille jaunatre, d lignes brunes en angles, la bouche brune, pourprée, Linn. 399. ce. Seb. III. 53. f. Lift. 731. 20.? Argenv. Suppl. 2. A. ?. Potiv. Gaz. I56. 18. (3. p. 684.

$1147 R$. Jaune, à coquille jaunatre, d̀ points brun-vertes, bouche cendrée, Linn. 399. ff. Seb. IIl. 53. h.? Lif 734. 24 ? (3.p. 685 .

$1148 R$. Jaunatre, le meme que la precedente, mais plus ftrï, Linn. 399. ff. Gualth. 23. 2Q. (4. p. 636.

Ir $49 R$. Flammant, à coquille jauratre, d larges flammes versbrunes, dे bouche ölanche, Linn. 399. gg. Seb. III. 53. $N$ ? Gualth. 24. L.? (7. P. 692.

$1150 R$. Filet, à coquille blanche, d'un vert-brun en formet de refeau, bandé de brun, d bouche blanche, Linn. $396 \%$ $h$ h. Seb. III. 53. H. I. Gualth. 24. M. ? Lift.739.28. (5.p. $69 \mathrm{I}$.

II5I $R$. Filet, le pareil, Linn. 399. lh h. (4. p.69r.

I52 $R$. Cerclé, coquille d carine elevee, blanche fur le des; Linn. 399 hh ? (1. p. 1042.

$1153 R$. Filer, d̀ coquille blanche, ftriée dc brun, bandke, bor he blanche, Linn. 399. $h$ h. Seb. III. 53. O.? Liat 739. 26: (3. p. 1054.

$1154 R$. Zig - zag, à coquille brune, d ftriés brun.vertes, ondés par angles, Linn. 392. $i i$ Gualth. 23. M.? (2. p. 69a.

II55 R. Zig-zag, à coquille jaure, Linn, 399. $i i$. Gualth 23. P.P. (3. p. $10+3$.

$1156 R$. Rayé, à coquille d'un blans fale, Linn. 399. ii. Lift. 728. 16 ? (2.p. 688. 694.

$1157 R$. Ondoyante, d̀ coquille joune, ftries brunes, $\mathbb{O}^{\circ}$ bouche pourprée, Linn. 399. kk Gualth. 33. R.? Lift. 73 r. 20. (3. p. 687.

Is $58 R$ Panaché, $\dot{a}$ coquille pale verte ob curement pointée, bouche blanche, Linn. 392. li. Lift. 734 . 34? (I.P. 689.

II59 R. Panaché ? ou varieté, \& coquille blanche, pointes jors-

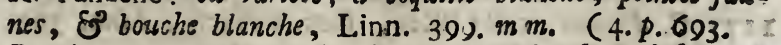
II60 Bombacine, ou Marbré, do coquille blanche, fafciée de vert, ì points jaun-vertes, Lirn. 399.. m m. (5. p.695. I16I $R$. Nebuleux, $d$ coquille allongée, jaunatse à nues plss -bfoures, d deux fajcies jaunes, la bouche jaune, Lipn: 399. $n$ n. Lift. 725. 12 ? (2. p. 700

$1162 R$. a Trois bandes, d coquille jaune, pointée de verd, d trois fafcies larges vertes do bouche jaun-blancheatre, Linn. 399.

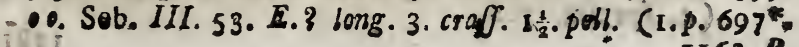
$1163 \boldsymbol{R}$. 
I163 C. Areolatus, tefta albida, ineis fuscis fe decuffantibus, Linn. 399. 00 ? Gualth. 24. B? (4. fpecim. 697.

ix64 C. Leucoftoma, tefta albida, viridi punctata, fasciis fuscis, ore albo, Limm. 399.p p. Seb. III. 53. D ? V.? Gualth. 24. C? Rumph. 39. 3? (2. Rpecim. 930.

1165 C. Flovrus, tefta flava, fasciis caftaneis, ore cinereo, Linn. 399. q q. Seb. III. 53. C. F. Gualth. 24. H? Lift. 724. 11. Argenv. 13. L. (2. Ppecim. 928.

sio6 C. Erythroftomus, tefta albido flava, ftriis fasciisque fuscis, ore aurantio, Linn. 399. $\mathrm{rr}$. Seb III. 53. A. B. Gualth. 24 Q. Argenv. 13. N. Lift. 727. 14? (2. fpecim. 098.

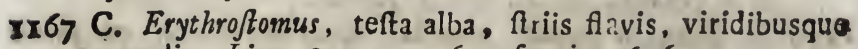
maculis, Linn. 399. $r$ r. (2. Specim. 626.

₹168 C. Erythroftomus, fasciis viridibus \& fuscis, Linn• 399. rr. (2. fpecim. 696.

I169 C. Porphyrio, tefta fusco carnea, reticulata, maculis caftaneis, Linn. 398. s s. Argenv. 13. K. Kircher III, 143. longit. $3 \frac{3}{2}$. craff. $1 \frac{3}{4}$. poll. (1. Specim. 699.

xi 70 C. Porphyrio, paulo minor, Limn. 398. s s. Gualth. 24: P. (2. fpecim. 699.

i17x C. Porphyrio, paulo minor, Linn.398. ss. Rumph.39. 1. (3. Ppecim. 609.

II 72 C. Funeralis, tefta tumidiore, olivacea, ftriis longitudinalibus caftaneis, Linn. 399. t t. Seb. III. 53. M. Rumph. 39. 4. Lift. 735.25. Gualt to 24. E. (2. Rpecim. 712 .

173 C Funeralis, tefta incarnata, ftriis caftaneis, Lirn. 399. t t. Pctiv. Muf.2. I. (1. fpecim. 712 .

II 74 C. Funeralis, tefta olivacea, ftriis fuscis, Linn. 399. tt. Seb. III. 53. a. (2. fpecim. 712.

1175 C. Maculofus, tefta olivacea, fasciis maculofis fuscis, Linn. 399, u u. (4. Specim. 710.

ii 76 C. Qtercinus, tefta fusco virente, maculis obfcurioribus rásciáta, Linn 399: u u. (3. Specim. 7II.

II 77 C. Fuscus, tefta lutea, fasciis fuscis, Linn. 399. u u. (4. Ipecim. 709

I178 C. Brunneus, tefta grifeo fusca, maculis brunneis fas. ciata, Linn. 399. u u. (4. Ipecim 702.

I179 C. Ifabella. tefta tlavefcente. Linn. 399. vv. Seb.III. 53. K. (3. โpecim. 703.704.

I180 C. Nigellus, tefta fusco nigra, ore albo, Linn. 399: พw. Seb. III. 53. L. Lift. 739. 27. Rumph. 39. 2. (4. Specim. 708 . 


\section{Teftaces. Ordre IV.}

II63 $R$. Tricoté, " ¿̀ coquille blancheatre, ¿̀ lignes brunes quż Je croifJent, Linn. 399. 00 ? Gualth. 24. B? (4. p.697. II64 $R$. Leucoftome Moire, à coquille blancheatre, pointée de verd, $\dot{a}$ bandes brunes, $\dot{a}$ bouche blanche, Linn. 399. $p p$. Seb III. 53. D? V? Gualth. 24. C? Rumph. 39. 3? (2. p. 930.

$I 165 R$. Jaunatre, a coquille jaune, $d$ bandes chataignes, $i$ bouche cendrée, Linn. 399. qq. Seb. III. 53. C. F. Gualth. 24. H. ? Lift. 724. I I. Argenv. 13. L. (2. p. 928.

II66 R. Erythroftome Moire, à coquille blanche jaune, a ftries, EO fafcies brunes, à bouche orange, Linn. 399. $r r_{\text {. }}$ Seb. III. 53, A. B. Gualth. 24. Q. Argenv. I3. N. Lift. 727. I4.? (?..p. 698.

II67 R. Erythroftome Moire, à coquille blanche, ftries $j a u-$ nes. Es taches vertes, Linn. 399. rr. (2. p. 696.

II68 $R$. Erythroftome Moire, le pareil, $\dot{a}$ bandes vertes, $E^{2}$ brunes, Linn. 399. rr. (2.p. 696

II69 $R$. Porphyre, coquille brun-rouge 'à refeau', à taches chas' taignes, Linn. 398. ss. Argenv. 13. K. Kircher III. I43. long. $3 \frac{1}{2}$. craff. I $\frac{3}{4}$. pouc. (I. p. 699 .

II70 $R$. Porphyre, plus petit, Linn. 398. s s. Gualth. 24. $P$. (2. P. 699.

II 7 I R.Porphyre,plus petit, Linn.398.ss.Rumph.39.I. (3.P.699:

II72 $R$ Funerale du Prince, $\grave{a}$ coquille plas enflée olivatre, les ftries chataignes en long. Linn. 399. t t . Seb. III. 53. M. Rumph. 39. 4. Lift. 735.25 . Gualth. 24. $E_{\text {a }}$ (2. p. 7 I2.

I1 $73 R$. Funerale du Prince, d̀ coquille ronge, à ftries chataignes, Linn. 399. tt. Petiv. Mur. 2. I. (I. p. 7I2. II $74 R$. Funerale du Prince, à coquslle olivatre, ftries brunes, Linn. 399. $t$ t. Seb. III. 53. a. (2. P. 712.

II $75 R$. l'Ane rayé, à coquille okivatre, Eे fafcies tachetées brunes, Linn. 399. u u. (4. p. 710.

II $76 R$. Bois de Chene fascié, coquille brun.verte, bandée, \& taches plus obfcures, Linn. 399. u u. (3. p 7 71 .

I 77 : Brun-jaune, coquille à fofcies brunes, Linn. 399." uи. (4. p. 709.

II78 $R$. Brun grife, à coquille gris-brune, bandée de taches brunes, Linn. 399. uи (4. p. 702 .

I179 $R$. Ifabelle, $\dot{a}$ coquille jaunatre, Linn. 399. vv. Seb. III. 53. K. (3. p. 703. 704 .

I180 $R$. Noir, d coquille brun-noire, \& bouche blanche, Linn. 399. w w. Seb. III. 53. L. Lilt. 739. 27. Rumph. 39.2. (4. p. 708. 
I18I C Nigellus, pracedenti affinis. Linn 1399. W W. (4a fpecim. 708:

I182 C. Cinnamomeus, tefta rufo lutea, Linn. 399. x x. (2. fpecim. 7or.

1183 C. Striatzs, tefta tenuis, magis flavefcens, pur.et:lis fuscis, Linn. 399. y y. (I. Specim. 706.

1184 C. Globulus, tefta tumida, alba, fusço ftriata, Linn. 399. z2. Gualth. 23 B. Lifl. 708. 2? (2. ipecim. 707.

2185 C. Interruptrus, tefta olivacea, fascia maculofa obfcu. riore, Linn. 399. u u ? Seb. III. 53. L? (2, fpecim. 929.

1186 C. Maculofus, tefta flava, virefcente, Limm.390. u u? Lift. 74C. 29? ( 4 Tpecir. 713.

1187 C. Maculofus, precedenti affinis, Linn. 399. u u? (3. [pecim. 713.

1188 C. Tumidus, tefta lactea, fasciis transverfalibus, angulofis fuscis, Linn. 399, z 2 ? Seb. 1II. 53. y? Gualth. 23. V? (1. fpecim. 716 .

1189 C. Turgidus, tefta cinerea, fascia maculisque flavis, Linn. 399. a a a. Lift. 723. 10. Petiv. Gaz. 19, 9. (2. fpecim. 717.

1190 C. Turgidus. decorticatus, inde fuscus, albo punctatus, Linn. 399. a a a. (3. Specim. 717 .

IIgI C. Turgidus, pracedenti fimilis, Linn. 399. a a a. (3. fpecim. 717 .

1192 C. Labiatrs. Sed tenuior, tefta flava, antice fascia fusco maculo\{a, Linn. 399. bbb. (2. Specim. 931.

1193 C. Labiatus, decorticatus, Linn. 399. b b b. (2. Specim. 931.

1194 C. Elongatus, tefta cinerea, fascia ftava, pallide maculofa, Linn. 399 b b b? Gualth. 23. S S. Lift. 729.17. Petiv. Gaz. 102. 18. (3. Specim. 718.

1195 C. Elongatus, decorticatus, Linn. 399. b b b? (2. Spe. cim. 718.

3796 C. Nux Avellana, tefta oblonga, tumida, flavefcente, maculis fusco violaceis, Linn. 399. c c c. Gualth.23. $E$. Rumph. 39. 8 ? ( . Specim. 932.

1197 C. Nux Avellana, tefta iutea, fascia media alba, Linn399. c c c ? Seb. III. 53. K? (3. fpecim. 720,

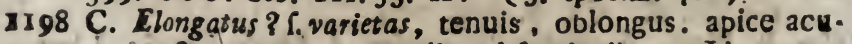
to, tefta cinerea, maculis obfcurioribus, Linn. 399. ddd. Lifs.725.x3 ? Potiv. Gaz. 152. 6. (2. fpecim. 719. 


\section{Teftaces. Ordre $1 \mathrm{~V}$.}

18r R. Noir, Jemblable au precedent, Linn. 399. w w. (4. P. $7<8$.

(I82 $R$. Cinnamomne, ou Canelle, d̀ coquille d'un jausne foncée.' Linn. 399. $x x$, (2. p, 701 .

us $R_{0}$. ou Olive ftrié, jaune, à coquille mince, plus jaznnatre; d pointes brunes, Linn. 399. y y. (1. p. 706.

[184 R. Globule, à coquille enflee, blanche, friée de brun, Linn. 399. zz, Gualth. 23. B. Lift. 708. 2.? (2. p. 707.

I185 $R$. Interrompue, d̀ coguille olivatre, da fafcie tachetée, plus obfcure, Linn. 399. u u.? Seb. III. 53. L? ( 2 p. 929.

$1186 R$. Verd-tacheté, d coquille jaune, verdeatre, Linin. 399. $u$ ? Lift. 740. 29? (4.p. 713 .

$1187 R$. Verd-tacheté, approchant au precedent, Linn. 329. u $u$ ? . (3. P. 713 .

$1188 R$. Entlé en Zig-zag, à coquille couleur de lait de fafciss traverfes, en angles brunes, Linn. 399. $2 \approx$ ? Seb. III. 53. $y$ ? Gualth. 23. V? (1. p. 716 .

I189 $R$. Vafe, à coquille cendrée, ¿a bande 80 taches jaumes, Linn. 399. a a a. Lift. 723. 10. Petiv. Gaz. 19. 90 (2. p. 717 .

$1190 \quad R$. Vaie, fans robe, par la brun, pointé dé blanc, Linn. 399. a a a. (3. p. 717 .

II9I $R$. Vafe, en tout Jemblable au precedeint, Linn. 399. aba. (3. p. 717 .

$1192 R$.Vafe levré, mais plus mince, d coquille jaune, dovant do bande jaune, tachetée de brun, Linn. 399. b bb: (2. p. 93 I.

II 93 R. Vafe levré, fans robe, Linn. 399 b b b. (2. p. 931 .

Iig4 $R$. Vafe allongée, à coquille cendrée, à fafcie jaune, \& $d$ taches pales, Linn. 399. $b$ bb? Gualth. 23. S S. Liff, 729. 1 7. Petiv Gaz. 102. 18. (3. p. 718.

Ir 95 . Vafe allongé, fans robe, Linn. $399 . b$ b b ? (2.p. 718.

II96 R. Noifette, à coquille allongée, enflée, jaunatre, ¿ tachés brun-violettes, Linn. 399. ccc. Gualth. 23. E. Rumph. 39.8 ? (1. p. 932 .

$1197 R$. Noifette, à coquille joune, à fafcie au milieu blanche, Linn. 399. c c c? Seb. III. 53. K? (3. P 72c.

1198 . R. Vafe allongée? ou variete, dे fommet aigue, coquille cendrée, d taches plas obfcures, Linn. 399, ddd. Lift. 725. 13 ? Petiv. Gaz. 152. 6. (2. p. 719. 
1199 C. Megalofromus, tefta ventricofa, ore fubviolaceo, fuscn-cinerea, Linn. 399. è e e. Lift. 730. 18. (3 \{pecim. IO20.

I 200 C: Lacteus, apice rufo, dorfo pallide rufefcente, Linn. 399. ff f. Seb. III. 53. e. Lift. 730. 19? Petiv. Gaz. 69. 3. 4. (2. fpecim. 1057.

x201 C. Torojus, tefta fubrugofa flava, Linn. 399. $\mathrm{g} \mathrm{g} \mathrm{g.} \mathrm{Lift.}$ 746. 40 . (I. Ipccim. 721 .

\section{GE N V S XXIV. GLOBOSA.}

1202 G. Nusfatella, tefta rugofa, oblonga, acuminata, alba, Linn o. Davila $I$.: 8. S. longit. $6 \frac{3}{4}$ craft. $2 \frac{3}{4}$. poil. (1: ) fpecim. 1036.

1203 G. Oenophorum, tefta apice plano, depreffo, flava, tunica adhuc verrucofa veftita, Linn 436. $\beta$. f 0 . Seb.

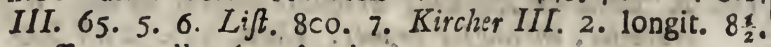
craft. 4. poll. ( 1 . fpecim. 722.

2204 G. Oenophorum, fimilis præcedenti, Linn. 436. B. f. ○. longit. $7 \frac{3}{4}$. craff. $3 \frac{3}{4}$. poll. (2. Ipecim. 722 .

1205 G. Cynabium, tefta emarginata ; ventricofa, anfractibus canaliculato marginatis, apice papillari ; columella biplicata, Linn. 436. Seb. III. 66. 13. 14. 16, 64. 9. (2. 1pecim. 723.

I 206 G. Cymbium, tefta giabra, ore flavo, coftis duobus, tenuibus elevatis, Linn. 430. $\alpha_{\text {. }}$ (3. Specim. 723 .

1207 G. Gondola, tefta glabra, albo fuscoque varia, Linn. 436. . . . o. Seb. III. 65. 9. longit. 5. crail. $2 \frac{1}{2}$, poll. (1. fpecim 724.

I $208^{\prime}$ G. Gondola, præcedenti fimilis, Linn. 436. y. f. ०. (2. fpecim. 725 .

1209 G.Nevofa, tefta ventricola, apertura flava, fasciata, guttata , Linn. O Seb.III,65.3 Gualth, 27.A Lift.802.8. long. 8. cratff $5 \frac{1}{i}$ poll. (1. Specim. $73 \mathrm{c}$.

1210 G. Nevoja, præcedenti affinis, maculis transverfalibus fuscis, Linn o. Seb. III. 64. 3, 66. 4. longit. 6. craff $4 \frac{1}{2}$. poll. (I. Specim. 730.

I2II \& Navo $\int a$, fimilis pracedenti, tefta flava ; fasciatim fusco maculofa, Linn o. long. $5 \frac{3}{4}$. crafr. $3 \frac{3}{2}$. poll. ( fpecim. 730.

1212 G. Nevoja, prxcedenti affinis, tefta \& ore luteo mi befcente, Linn. 0. (1. Rpecim. 730.

1213 G. Marginata, anfractibus acute marginatis, canaliculatis, fusca, Linn, 0. S6b. III. 65. 7. ' (2. fpecim. 729. 
Irg9 R. Megalaftome, ou grande Gueule, à coquille ventrue, la bouche un per violette, d'un brun cendré, Linn. 399. e e.e. Litt. 730.18. (3. p. 1020.

$1200 R$. Blancheatre, à Sommet rouffe, le dos pale brunatre, Linn. 399. $f f f$. Seb. III. 53. e. Lift. 730. 19.? Petiv. Gaz. 69. 3. 4. (2.p. 1057.

$\$ 201 R$. Large-Gueule, à coquille un pen ridée, jaune, Linn. 399. $g \mathrm{gg}$. Lift. 746. 4 O. (I. p. 72 亿.

GENRE XXIV. TONNES.

$1202 T$. Nuffatelle, ou Tariere, à coquille ridée, allongée, pointue, blanche, Linn. O. Davila I. 8 S. long 6 $\frac{3}{4}$. crafJ. $2 \frac{3}{4}$ pouc (I. p. 1036 .

1203 T. Cruche de Jaqueline, coquille à Sommet platte, deo primée, jaune, encore couverte d'une tunique verruceufe. Linn. 436. $\beta$. ou 0. Seb. III. 65. 5. 6. Lift 800. 7. Kircher III. 2 long $82^{3}$. craff. 4. pouc. (1. p. 722.

$1204 T$. Cruche de Jaqueline, le pareil du precedent, Linn:" 436. $\beta$. ou o. long. $7 \frac{3}{4}$. cralf $3 \frac{3}{4}$. (2.p. 722 .

$1205 T$. Cruche de jaqueline, ou Gondole, à coquille emarginée ventrue, les anfraetures bordés par canalicules, Sommet \& mamillons, Es columelle à double plie, Linn. 436 . Seb. III. 66. I3. I 4. 16, 64 9. (2. p. 723 .

\206 T. Cruche Perfanne, ¿̀ coquille pelée, bouche jaune, deux cotes minces, elevees, Linn 436. «. (3. p. 723 .

1207 T. Cruche Perfanne, à coquille pelée, variée de blanc $\mathrm{E}^{2}$ brun. Linn. 436.\%. ou o. Seb. III. 65. 9. long. 5.cralf: $2 \frac{1}{2}$. pouc. (I. p 724 .

$1208 \mathrm{~T}$. Cruche Perfanne, Jembiable ì la precedente, Linn. $436 . y$ ou 0 . (2. p. 725 .

I209 T. Mamillon moucheté, à coquille ventrue, ouverture jaure, bandée, gouttée, Linn: o. Seb.1II. 65. 3. Gualth.27. A? Lift. 802.8. long. 8.cralf $5 \frac{3}{2}$. pouc. ( 1. p. 7306.

I210 T. Mamillon moucheté, approchant d̀ la precedente, ḋ tacbes traverfes brunes, Linn. 0 . Seb. III. 64. 3, 66. 4. long. 6. cralf. $4 \frac{1}{2}$. pouc. (I. p. 730 .

1211 T. Mamillon moucheté, femblable à la precedente, d coquille jaune, en bandes, tachetée de brun, Linn. O. long. $5 \frac{3}{4}$. craff $3 \frac{1}{2} \cdot$ pouc. (1. p. 730 .

$1212 T$. Mamillin moucheté, femblable à la precedente, \& coquille, \&2 bouche jaun-rougeatre, Linn o: (r.p.730:

I2 I $T$. Mammillon cordonné, brume, les anfractures aigue bordées, canalées, Linn. 0. Seb. III. 65. 7. (2. p. 729. $\mathrm{Bb} 3_{1214} \mathrm{~T}_{\text {: }}$ 
1214 G. Ampullata, apicis loco tuber, anfractibus nullis fusca, albo punctata, Linn o. Seb. III. 64. 10. II 66. 17. Lift. 796. 3. Argenv. 17. G. (3. xpecim 728. \&*.

q215 G. Olla, tefta emarginata ventricofa, fpira levigata, apice papillari, columella quadriplicata, flava, Linn

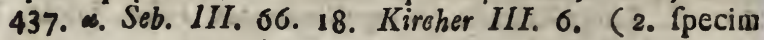
727.

1216 G. Flammeola, tefa ventricofa tumida . transverfim ftriata, fusco maculata, Linn. 437. א. f. O. Seb. III. 65. 8, 66. I 2. Lift. 795. 2. Argenv. Suppl. 2. H. Hollar Icon. 17. Spengler Icon I. N. (2. (pecim, 726.

1217 G. Flammeola, tefta floribus ramulisque exfculpta. (I. [pecim. 726.

1218 G. EEthiopica, tefta ventricofa, fpira coronata, fpinis fornicatis, apice papillari, columella quadriplicata, Linn. 435. Seb. III. 04. 2. 4. Lift. 80I. 6. 7. longit. $8 \frac{3}{4}$. craff. $5 \frac{1}{2}$. poll. ( I. fpecim. 732 .

1219 G. It thiopica, flavo fusca, fed minor, Linn. 435. Rimph 3 I. A. Hollar Icon. 9. (2. Recim. 732.

$7220 \mathrm{G}$ Withiopica, pracedenti affinis, tefta fusca, $\alpha$ fusca al. bo fasciata, Limm. 435. ๙. Seb. III. 66. 12. Petiv.Gaz. 156. 17. (4. โpecim. 733. a. b.

I22I G. Submerja, tefta albo flava, maculis fuscis fasciata, columella tri- \& quadruplicata, Linn. 435. $\beta$. f. o. Seb. III. 66. 6. 7. Lift. 797. 4. Kircker IIII. 1. (2. Ipecim. $73 \mathrm{r}$.

1222 G. Submerfa, præcedenti fimilis, tefta fusco alba, abs-

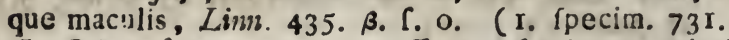

1223 G. Submerfa, pracedenti affinis, fascils maculatis Linn. 435. 6. f. O. (2. Specim. 731.

1224 G. Ramofa, tefta flava, dentata, transverfim undata, Linn. 435. y f. o. Seb. III. 65. 12, 66. 1. 3. 10. Gualth. 29. H. longit. $4 \frac{3}{2}$. craff. $2 \frac{1}{2}$ poll. (I. Specim. 734.

1225 G. Ramoja, pracedenti affinis, Linn. 435. \%. f. o.Gualth? 29. $H$ ? (2. fpecim. 734.

1226 G. Ramofa, præcedenti fimilis, tefta alba, flavo reticulata, fusco maculofa, Linn. 435. \% C. o. Seb. III. 65. I. 2. 10. Rumph. 3 I. B. longit. $4 \frac{1}{1}$ poll. (1. fpecim. 735.

$\$ 127$ G. $R$ a mofa, præcedenti affinis, flava, albo macu'ata. Limb. 435. भ. T. $\bullet$, longit, $5 \frac{1}{2}$. craft. $3 \frac{1}{4}$. poll. (I. Specim. $734^{*}$. 
I2T 4 T. Mamillon à tubercules, ayant au licu, d'un fồmet, un trbercule, fans anfräaures, brune, \& pointée de blanc, Linn. O. Seb. III. 04 10. I1, 66. 17. Lift. 796. 3. Argenv. 17. G. (3. p. 728. 89*.

1215 T. Mamillon ventrue, à coquille bordée ventrue, d̀ fpire polie, le fommet à mammillons, la columelle $\dot{a}$ quatre plies, jaune, Linn. 437. «. Seb. III. 66. 18. Kircher III. 6. (2. p. 727 .

2216 $T$. Mamillon flamboyante, d̀ coquille ventrue, enflée, Jtiéé de travers, tachetée de brun, Linn, 437. ß. ou c. Seb. III. 65 8, 66 I2. Lift. 795. 2. Argenv. Suppl. 2. H. Hollar Icon. I 7 . Spengler Icon. I. N. (2. p. 726. T. Mamil!on panaché, la coguille ex/culpté en fleurs, \& petites branches. (1. p. 726 .

I2 I\$ T. Couronne d'Ethiopie, à coquille ventrue, Spire couronnée, dè Spires voutées, fommet à mammillons, columelle d quatre plies, Linn. 435. Seb. 1II. 64. 2. 4. Lift. 801.6. 7. long. $8 \frac{1}{2}$. craff. $5 \frac{1}{2}$. pouc. (I. p. 73 ?

I2I $T$. Couronne d'Ethiopie, plus petite, jaune brune, I Lint. 435. Rumph. 3 I. A. Hollar Icon. 9. (2. p. 732 .

I2zo $T$, Couronne d'Ethiopie, approcbant à la precederite, if coquille brune, Eo bandée de blanc, Linn. 435. «. Seb. III. 66. 12. Petiv. Gaz. 156. I7. (4. p. 733. a. b.

I22I T. Couronne d'Ethiopie mouchetée, à coquille blantjaune, fafciee à taches brunes, columelle d trois है quatre plies, Linn. 435. B. ou o. Seb. III. 66. 6.7. Litt. 797. 4 Kircher III. I. (2. P. 731 .

12227 . Couronne d'Ethiopie mouchetée, femblable da precedente, à coquille brun-blanche, fans taches, Ling. 435. B. ou O. ( I. p. $73 \mathrm{I}$.

$1223 T$. Couroine d'Ethiopie moucheté, la pareille, \& bandes tachetées, Linn. 435. B. ou o. (2. p. 731.

$1224 \mathrm{~T}$. Couronne d'Ethiopie agathée, da coquille jasise, dentelée, ondée de travers, Linn. $435 \%$. ou o. Seb. III. 6 s. 12, 66. 1. 3. 10. long. $3 \frac{1}{2}$. crafl. $2 \frac{1}{2}$. pouc. (I. p. 734. $1225 T$. Couronne d'Ethiopie agathée, femblable d la precedente, Linn. 435. \%. ou 0. Gualth. 29. $H$ ? (2. p. 734 . I226 T. Couronne d'Ethiopie agathée, Jemblable à la precedéente, à coquille blanche, 85 jaurie en forme de refear. tachetée de brun, Linn. 435. \%. ou 0. Seb. III. 65. I. 2, 10, Rumph: 3 I. B. tong. $4 \frac{2}{2}$-pout. ' I. p. 735 .

$1227 T$. Couronne d'Ethiopie agathée, la pareille, jaune.

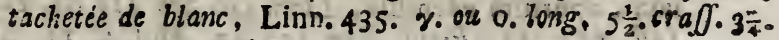
porc. (I. P. $734^{*}$. 
I228 G. Ficus, tefta obovata, clavata, reticulato ftriata; cauda exferta, fpira obliterata alba, fusco maculata, ore violaceo, Linn. 382. Seb. III. 68. I-6. Lift. 750. 46:\& a. Argenv. 17. O. Petiv. Muf. 6.9, 7. 5. Kircher III. 15. (2. fpecim. 736.

1229 G. Ficus, fimilis præcedenti, Linı. 382. (4. fpecim. 736.

I 230 G. Rapa, tefta rotundato turbinata, fubftriata, cauda curva, fpira exquifita, alba, Linn. 383. Seb. III. 38. 13. 14. 16. 18-21. Lift. 377.1? Argenv. 17. K. (1. pecim. 737.

I23I G. Rapa, fimilis præcedenti, Linn. 383 Rumph. 27.F. Gualth. 26. H. (2. Ppecim. 737.

1282 G. Harpa, tefta varicibus aqualibus, longitudinalibus, interftinctis, mucronatis, columella lævigata, cinerea, varicibus rubris, ventre fusco, Linn. 462. Gualth. 29. C. Rumph. 32. K. Argenv. I7. D. Hollar Icon. 42. longit. $3 \frac{1}{2}$. craff. $2 \frac{1}{2}$. poll. (I. fpecim. 739 .

1233 G. Harpa, præcedenti fimilis, Linn. 462. '(2. Ipe: cim. 739.

I234 G. Harpa, præcedenti affinis, fed coltis anguftioribus, Linn. 454. a Gualtb. 29. D. Lift:992.55. Petiv. Gaz. 78. 13. Muf. 7 8. (2. [pecim. 740.

I235 G. Harpa. fimilis præcedenti, Linn. 462 . a unum fpe. cimen inter coftas apertum, ut ftructura interna pateat. (2. fpecim. 740.

1236 G. Harpa, præcedenti affinis, fed pallidior, Linn. 462. ๙. ( 3 '́pecim. 740.

I 237 G. Harpa, rubro maculata, Linn. 462; $\beta$. Gualth. 29. E. Petiv. Muf. 2. 2. (4. Ppecim. 74 \%.

$I 238$ G. Harpa, fimilis præcedenti, Limn. 452. B. (4. fpecim. $74 \mathrm{I}$.

1239 G. Harpa, præcedenti affinis, Linn. 462. \%. (O. Ipe: cim. 741 .

$124^{\circ}$ G. Harpa, coftis tenuioribus. cinerea, roleaque, fasciis flavis, fuscisque, Linn. 462. \%. Gualth. 29. G. Rumph, 32. L. Lift. 993. 56? (3. Specim. 742.

1241 G. Harpa Virginea, coftis nigro ftriatis, fulcis favo fuscoque maculatis, Limn. 462. d. Lift. 994. 57. (6. fpe. cim. 743 .

$\mathbf{1 2 4 2}$ G. Harpa, tnta alha . Tinn. 46 2. B. (2. fpecim. 741.a.

1243 G. Perdix, tefta ovata inflata, fubfulcata, albo undulata, apertura edentula, Linn.440. Seb. III.68. I4-I6. Lift. 984. 43. Petiv. Gaz 153.13.Muf.4. II. Kircher III. 189. Hollar Icon, 18. (2. Specim, 746. 


\section{Teftaces. Ordre IV.}

1228 T. Figue tachetée, à coquille un peu ovale, clavée, ftriée en forme de refeau à longue queue, E̊ Spire lettrée blanche, tachetée de brun, à bouche violette. Lins. 382. Seb. 11I. 68. 1-6. Lift. 750 , 45 Eे a. Argenv. I7. O. Petiv. Muf. 6. 9, 7. 5. Kircher III. 15. (2. p. 736.

I229 T. Figue tachetée, pareil, Linn. 382 . (4. p. 736.

I230 T. Navet pasiracée, à coquille arrondie turbinée, ur peu ftriée, la queue courbée, la Spire exquifite blancibe: Linn 383. Seb. III. 38 13.14.16.18-21. Lilt 877. 1 ? Argenv. 17. K. (I. p. 737.

123 T. Navet papiracée, Semblable à la precedente, Linn. 383 Rumph. 27. F. Gualth. 26. H. (2. p. 737.

$1232 T$. Harpe à large cotes, coquille $\grave{a}$ varices egales longitudinales, diftinctes. mucronées, ou pointées, à colvmelle polie, cendrée, à verices ringes, Ẽ ventre brun. Linn. 462. Gualth. 29 C. Rumph. 32. K Argenv. 17. $D$. Hollar Icon. 42. long. $3 \frac{1}{2}$. crall $2 \frac{1}{2}$. pouc. (I. p. 739 . I $233 T$. Harpe d larges cotes, Semblable ḋ la precedente, Linn. 462. (2. p. 739 .

1234 T. Harpe à étroites cotes, la pareille: mais à cotes plus etroites, Linn. 452. a. Gualth., 29. D. Lift. 992. 55. Potiv. Gaz. 78. 13. Muf. 7. 8. (2. p. 74).

$1235 T$. Harpe à étroites cotes, femblabie, Linn. 462, $\alpha$. deux pieces, dont une eft ouverte entre les cotes, pour que ba Arulture, intrinfique pliffe mieux paroitre. (2.p. 740. $1236 T$. Harpe détroites cotes, pareil de la precelente, mais plus pale, Linn. 462 a. (3. p. 740 .

$1237 \%$. Harpe Noble, tachetée de rouge, Linn. 462. 3. Gualth. 29. E. Petiv. Muf. 2. 2. (4. p. $74 \mathrm{I}$.

$1238 T$. Harpe Noble, pareille de la precsdente, Linn. $452 . \beta$. (4. p. 74x.

1239 T. Harpe N ble, la pareille, Linr. 46 ?. $\beta$ (०. p. 74 r. $1240 T$. Harpe Noble bandée, à cotes plus minces, cendrée, couleur de rofe, É fafcies jaunes, $10^{\circ}$ brunes, Lim. 462. \%. Gualth. 29. G. Rumph. 32. L. Lift. $903.5 \pi$ ? (3 p. 74\%.

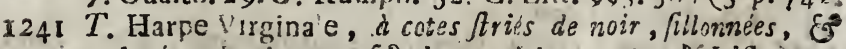
tachetées de jaune, E brun, Linn. 462. 8 Lift. 994. 57. (6.p. 743 .

1242 T. Harpe, par tout blancbe, Linn. 452. $\beta$. (2, p. 741. a.

I243 T. Perdrix, à coquille ovale, enflée, un peu fillonnée. ondée de blanc, ouverture fans dens, Linn. 440. Seb.III. 68. 14-16. Lift. 984. 43. Petiv. Gaz. 153. 13 Muf. 4. I1. Kircher III. 189. Hillar Icon: 18. (2. p. 746. 
1244 G. Perdix, fimilis pracedenti, fed minor, Linn. $44^{\circ}$ Rumph. 24. C. Gualth. 52. F. (3. fpecim. 746.

$\$ 245$ G. Olearium, tefta cincta, fulcis obtufis, lineola elevata interftinetis, apertura edentata, Linn. 435. Seb. III. 70. 5. 6. Kircher III 25. Rumph. 27. D. Adanfon Seneg. I. 7. 2. 7. Ginann. II. 93. (3. Specim. 745.

1246 G. Dolium, tefta cincta, fulcis, obiufis remotis, cauda prominuta, Linn. 442. Seb. III. 68. s-11. Argenv. 17. C. Petiv. Gaz. 99. 11. Muf. 12. 5. Adanjon Seneg. I. 7. 2. 6. Hollar Icon. 19. (2. ipecim. 747. 1247 G. Dolium, precedenti affinis, fed major, Linn, 442. Rumph. 27. A, Gualth.39 E. (I. (pecim. 747.

1248 G. Dolium, fimilis pracedenti, fed minor, minusque maculata. Linn 442 . (2. Specims. 747.

1249 G. Dolium, affiris precedenti, fed minor, pulcerrime maculata, Linn. 442. Liff. 899. 19. (4. Rpecim. 747. I250 G. Galeator. fulcis antice geminatis, Linn 442 a. 1. o. Seb. III. 68. 17. Liff S98. 18. Petiv. Muf. 9. 7. Kir. cher III. I7. (2. Specim. 748. a.

I25I G. Galeator? f. varietas:, coftis approximatis, Linn. $44^{2}$. ๘. S. o. Ginann. 11. 45. (2. ffecim 748. b.

\section{G E N V S XXV. CASS I DES.}

1252 C. Pomum, tefta fulcis obtufis, apertura dentata, fiavo alboque maculata, Linn. 44I. Seb. III. 70. 34. Lift. 792. 45. Argenv. 17. L. Gualth.5x.C. Petiv. Muf. 12, 6. Kircher 1II. 22. (2. [pecim. 741.

1253 C. Pomum. fed minor, Linn. 441. (3. Specim. 74 r. $\$ 254$ C. Echinophortum, tefta cingulis quatuor nodofis, cauda prominente, Linn. 443. Seb. III. 68. 18. Lift ICO3. 08 ? Gualth. 43. 3. Argenv. 17. P. Kircher III. 19. Ginann. II. 43. (3. (pecim. 750 .

2255 C. Echinophorum, fed major, colore fortior, Limn. 443. (2. Ppecim. 750.

2256 C. Sulcata, cortis elevatis approximatis, a'ba Litrz. 443. a. C. O. Guaith. 59. B, 43. 2? Ginann. II. 44. Kircher III. 163. (2. Tpecin. 74 ).

$\$ 257$ C. Anus, tefta varice, labiisque dclatato membranaceis, gibbofa reticulaio tuberculata, apertura finuofa, cauda erecta, Linn. 539. Seb. 1II. 60. 6.7. Lift $83 \%$ 57. Petiv. Gaz. jo. 10. Muf. 9. 21. Argenv. $9 H$. Gualth. 27. B. Kircber III. 278.9. Spengler Icon. III. G. (3. fpecim. 75 I. 


\section{Teftaces. Ordire $I V_{\text {. }}$}

2244 T. Perdrix, la pareil de la precedente, mais plus petite. Linn. 440. Rumph. 27. C. Gualth. 5 I. F. (3. p. 746.

I245 T. Pelure d'Oignon, à coquille cerclée, ou entourée, de fillons emou[jez, diftinguée d'une ligne elevée, a ouverture Sans dens, Linn. 438. Seb. III. 70. 5. 6. Kircher III. 25. Rumph. 27. D. Adanfon Seneg. x. 7. 2. 7. Ginann II. 93. (3. P. 745.

$2246 T$. Cordelet canalée, d coquille entourrée, de filtons Jep̧orés. d queuc prominente, Linn. 442. Seb. III. 68. 9-11. Argenv. 17. C. Petiv. Gaz.99. Ix. Muf. 12. 5. Adanfon Seneg I. 7. 2. 6. Hollar Icon. 19. (2. 747.

I247 T. Cordelet canalée, be femblable de la preced cnte, mais plass grande, Linn 442.Rumph. 27.A. Gualth 39. E., (t.p.747.

$1248 T$. Cordelet canaiée, pareil de la precedente, mais plus petite, Ẽ moins tachetée, Linn. 442 . (2. p. 747.

$1249 T$. Cordelet canalée, la pareil, plus petite, trés joliwent tachetée, Linn. 442. Lift. 890. 19. (4. p 747.

$1250 T$. Cordelet fafciée, à fillons devant doublés. Linn. 442. «. ou 0, Seb. III. 68. 17. Lift. 893. 18. Petiv. Muf. 9. 7. Kircher III. 17. ( 2 p. 748 a.

I25 I T. Cordelet bandée? ou varieté. da cotes qui s'approcbent, Linn. $44^{2}$ ๘. I. o. Ginann. II. 45. (2. P. 743. b.

GE NRE XXV. CAS Q UE S.

1252 C. Groffe levre, ou Cordelet, coquille of fillons emonfiee, ouverture dentelée, tachetée de jaune, Ef blanc, Linn 441 . Seb. III. 70. 3. 4. Litt. 792. 45. A / env. I 7. L Gralth. 51.C.Petiv. Mur. 12. 6. Kircher III. 22. (2. p. 741 .

1253 C. Groffe levre, ou Cordelet, muis plus petit, Lina. 44. I, (3. p. 74 r.

1254 C. Cordelet tuberculée, ccquille à quatre cercles ḋ nousuds. à queue prominente, Linn. 443. Sủb III 68. I8. Lift. 1003. os? Gualih. 43. 3 Argenv. 17. P. Kircher III. 19. Ginann. II. 43. (2.p. 750.

1255 C. Cordelet tuberculée, la pareille, plus grantde, go plus forie de couleur, Linn. 443. ( 2 p. 750 .

$1256 \mathrm{C}$. Cordelet canalée. d cotes elevées approchantes, blaz. che. Linn. 443 a. ou O. Gualth. $39 . B, 73.2$ ? Ginann. II. 44. Kircher III. 163 . ( 2 p. 749

$1257 \mathrm{C}$ Grimace, do caquille, EO levies membranacées bo flut, E tuberculée en refecu ouverture tortillée Es pueve urgée , Linn. 539. Seb. III. 60. 6. 7. Lift. 833.57. Pedir. Gaz 99. Io Mur 9. 21. A rgenv. 9. H: Guatth. 27. B. Kircher HII.278. 9. Spengler Icon. III, G. (3, p. 75I. 
I258 C. Penita, flavefcens, Linn. 539. a. r. o. Seb. III. 60. 5. Gualth. 3r. D. (2. fpecim. 752 .

125.9 C. Glabrata, tefta glaberrima, anfractıbus obfoletis infimo fubcanaticulato bafi producto, alba, luteo maculata, Lirin. 470. Liff. 974. 29. Argenv. 9. G. Gualth. 43. T. Kircher 1II. 149. (2. fpecim. 755.

1260 C. Coacta, columella umbilicata, tefta ventricola, acttminata, alba, maculis hepaticis. Linn. 469. ๙. ๔. o. Seb. III. 73. 24? 25? Lift. 982. 42 ? Rumph. 49. E? (2. 'pecim. 756.

261 C. Coacta? f. varietas, brevior maculis fuscis, fasciata, Linn. 459. B. f. o. Seb III. 73. 23 ? 26 ? Lift. 981. 41. Petiv. Muf. 9. 20. Kircher III. 362. (2. fpecim. 757.

1262 C. Spirata, tefta lævi, anfractibus canali plano, diftinctis, columella abrupta perforata, alba, undis interruptis, rufo fuscis maculata, Linn. 469. Seb. III. 73. 21. 22. Gualth. 51. B Lift.883.42. c.? Rumpl. 49.D. Argenv. 17. N Petiv. Gaz. 101. 13. (2. Specim. $758^{\circ}$.

1263 C. Cuneata, tefta oblonga, tumida, acuminata ftriata, ftriis elevatis, forlide alba, anfractibus fusco ftriatis, Linn. o. Seb III 53. 43. Gualth. 44. C? Lift. 966. 21. A. (2. fpecim. 295.

1264 C. Cuneata, coftis friisque acuioribus, tefta albida, Linn. O. Gualth. 51. 1? Lift. 965. 20. B? (1. Tpecim. 296.

1265 C. Chryfoftoma, ore \& labio columeli: aurantiis, Linn. C. Lift. 268. 22 b. (I. fpecim. 297.

I266 C. Glabra, tefta glabra, albido flava, anfractu primo fasciato, Linn. c. Gualth. 43. O? Rumph. 29. V. Lift. 968. c ? Kircher III. 03. (1. Specim. 298.

¿267 C. Pollicaris, tefts labio columell xullo, tumida, gla - . bra, grifeo fusca, Linn. 460. «. Seb. III. 53. 38. 39. Gualth. 44 A B. Lift. 770. 25, 775.30. (2 'pecim. ro 44 . a. b.

1268 C. Vellicata, tefta glabra, ftriata, porcata - fusco grifea, Linn. 457. 8. Seb. III. 53. 28? Gualth. 44. D. Kircher III. 59. (3. Ipecim. 1044. c.

1269 C. Arcularia, tefta anfractibus nodofis, alba, vel lutea, Linn, 457 B. Sel. III. 53. 35. 37. Liff. 970.24. (3. fpecim. 759 a.

$1270 \mathrm{C}$. Arcularia, glabra, alba. longitudinaliter fusco ftriáta, Limbn. 457. y Seb. III. 53. 34. Gualth. 44. O. (1. Tpecim. 752,5 . 
127x C. Arcularia, pallide fusca, fasciis longitudinalibus ala bis, Linn. 457. *. Seb. III. 53. 28. Gualth. 44. $R$. Argenv. I4. C. Kircher III. 175. (3. fpecim. 750. c.

1272 C. Arculcria, transverfim oblique nodofa, lactea, Linn. 457. \%. Seb. III 53. 40-43. Gualth. 44. 2. (2. ipecim. 759. d.

1273 C. Plicata, tefta labio plicato, ventricofa, oblonga, unduiatim coftata, fusca, ftria alba longitudinali, Linn. O. Gualth. 44. I. Lift. 97 1.20. Rumph.27. N. (3. Ipecim. 7 ro.

1274 C. Clatrata, tefta tumida, acuminata, transverfim granulata, flavo fasca, Linn. O. Seb. III. 53. 33. Gualth. 44. N. Lift. 965. 18. A? Kircher III. 62. (1. Specim. 1045.

1275 C. Perlata, tefta gibba acuminata, transverfim tuberculata, fusca, Linn. 458. a. f. C. Seb. III. 53. 44? 45? Gualth. 44. M. Lift. 972, 27. (1. Specim. 761. 1276 C. Gibbofula, tefta gibba tuberculata, ftriata, flava, fascia longitudinali fusca, Limm. 459. a. Seb. III. 53. 44. 46. Lift. 973, 28. Rumph. 27. N? (I. Ipecim. 762 .

2277 C. Erinaceus, tefta fubplicata, papillis coronata, labro poftice muricato, grifea, undis longitudinalibus fuscis, Linn. 452. Seb. III. 53. 3. 16. 29. 30. Gualth. 39. D. Lift. 1002. 67. Petiv. Muf. 9. 9. (4. (pecim. 765.

$\$ 278$ C. Erinaceus, fimilis pracedenti, Linn, 452. (4. Ipecim. 765.

1.279. C. Erinaceus, præcedenti affinis, Linn. 452. Rumph. 25 7. (4. Specim. 705.

1280 C. Vibex . tefta glabra, labro poftice muricato, Linn. 454. Seb III. 53. 3-8. 17. 26. 27. Gualth 39. F.I. L. Lift. 1015. 73. Argenv. 14. G. Petiv. Muf. 4. 9. ( 7 fpecim. 763 .

228 I C. Geminata, tefta ovata, dorfo fubcarinato, anfractibus fubrodofis, grifea undata, margine maculis 4 piceis, Linn. 452. ^. C. O. Seb. III. 53. I. 2. (1.Rpecim. 766 .

1282 C, Geminata, dorfo carinato - carina elevata, grifea margine maculato, Linn. 452. ๙. P. O. Seb. III. 53. 18-21, Rumph. 25. 9. Argenv. 14. H. (5. Ipecim. 767

$\$ 2 S_{3}$ C. Maculata, tefta gibba, dorfo fubnodofa, glabra, alba, labro fusco maculato, Linin. 451. ※. f.o. Seb.III. 53. 9-11. Rumph. 25. 6? Lift. 1013. 77. (4. Ipecim. $7^{6}+$ 


\section{Teftaces. Ordre IV.}

3271 C. Arculaire, ou Coffrette bandée, d’un brun pale, fascies longues blanches, Linn. 457. «. Seb. III. 53. 28. Gualtb. 44. R. Argenv. I4. C. Kircher III. 175. (3. p 759. $C$

1272. C. Arculaire, ou Coffrette feneftrée, obliquement routé de travers. cou eur de lait, Linn 457. $\gamma$. Seb. III. 53. 40-43. Gualth. 44 Q (2.p. 759 d

1273 C. Pierre pointée, coquille à levre pliée, ventrue allongée. coftée par ondes, brus:e, à longue ftrie blanche, Linn. O. Gualth. 44. T. Lift. 97 I. 26, Rumph. 27. N, (3. p. 760 ,

1274. C. Pierre fereftrée, à coquille enflée, pointue, granulée de travers, d'on brun jaunatre. Linn. O. Gualth. 44.N. Lift. 965. 18. A ? Kircher III. 62. (1. p. 1045.

1275 C. Pierre perlée ous Tuberculée, à coquille boffue, point té tuherculée de travers, brisne, Linn. 458. $\alpha$. ous o. Seb. III. 53. 44 ? 45? Gualth. 44. M. Lift. 972. 27. (I. p. $76 \mathrm{r}$.

\$276 C. Boffetre, à coquille bo:fue, tuberculée, ftriée, jaune; d longue fascie brune, Linn 459 a. Seb. III. 53. $44-$ 46. Lilt. 973. 28. Rumph. 27. $N$ ? (I. p. 762.

1277 C. Bezoar pointée, à coquille un peu pliée, couronnée de mamillons, de levre parderriere pointée, grije, d longue ondes brunes, Linn. 452 Seb. III. 53.13.16.29 30. Gualtb. 39. D. Liff. I002. 67. Petiv. Muf. 9. 9. (4. p. 765.

1278 C. Bezoar pointése. la pareil, Linn. 452. (4. p. 765 .

1279 C. Bezoar pointée, approchant d la precedente, Linn: 452. Rumph. 25. 7. (4. p. 765 :

1280 C. Vibec, ou Bezoar pointée, à coquille pelée, la levre par derriere pointue, Linn. 454. Seb. III. 53. 3-8. 17.26.27. Gualth. 39 F.I.L. Lift. 1015. 73. Argenv. 14. G. Petiv. Muf. 4. 9. (7. P. 763 .

128 I C. Bezosr à deux Biurreleis, à coguille ovale, le dos un peu cavés, les anfractures norverfes, grife, ondée, le bord a 4 taches noiratres, Linn, 452. * ou 0. Seb. III. 53. I. 2. ( I. p. 766.

1282 C. Bezoar à deux Bourrelets, à dos creux, ou cavé, \& carine élevié grife, le bord tacheté, Linn. 454. . ouz $^{\circ}$ o. Seb. III. 53. 18-21. Rumph. 25. 9. Argenv. I4. $H$. (5. p. 767.

1.283 C. Bezoar d'agathe, à cogrille boffue, le dos a noezds pelée blanche, d levre tachetée de brun, Linn. 45I. a. or o. Seb. III, 53. 9-11. Rumph. 25. 6 ? Lift. I0I3.77.. (4. p. 764 . 
I284 C. Geometrica, tefta gibbora ftriata, maculls quadratis, flavo luteis, Linn. 45 I. $\beta$. f. o. Rumph. 25. C? Lift. 1012. 76 ? Adanfon Seneg. I. 7.2.8 (4. Ipecim. 769. 770.

5285 C. Areolus, fubltriata, maculis quadratis, quadrifariam cincta. apertura dentata, cauda curva, Linn. 451. Seb. III. 70. 8. 9. Gualth. 39. H. Li/t. 1016. 75 ? Rumph. 25. I. Argenv. 15. I. Petiv. MuJ. 2. II. (2. fpecim. 773.

1286 C. Areolus, præcedenti affinis, Linn. 451. (3. Spe. cim. 773 .

1287 C. Alveolus labro elongato \& corrupto, f. lufus Natura, Linn. 451. $\gamma$. (1 fpecim. $773^{*}$.

1288 C. Deculfata, tefta decuffatim ftriata, lavigata, fquamulis quadratis, apertura dentata, cauda recurva, Linm. 450. Gualth. 39. G Lift.1000. 65. Rumph. 25. B ? Kir. cher. III. I57. (I. Specim. 774. a.

I289 C. Striata, tefta grifea, undis fuscis ftriata, Linn. 450. \&. r. O. Argenv. 15. D. Lift. ror 4. 78. Rumph. 25. 2. Petiv. Muf. 2r. Ir. (2. fpecim. 774.

ז290 C. Cicatricofa, ventrico $\{$, anfractibus nodofis, tefta macuilis quadratis impreffis, Linn. 453. a. f. o. Gronov. Zooph. III. I9. I. 2. Petiv. Muf, 7. 4. (4. Ipecim. $772^{*}$.

I29I C. Cicatricofa, præcedenti affinis, Linn. 453. «. ᄃ. o. (4. Specim. 772.

I3.92 C. Glauca, tefta lævi, papillis coronati, labro po?tice muricato, Linn 453 . Seb.III. 7 I I1.12.13.16. Lifl. 996. 50. Petiv. Muf. 11. 18. (1. Ipecim. 771.

I293 C. Glauca, pracedenti finilis, Linn. 453. (2. Specim. 775 .

1294 C. Glauca, tefta cirerea, flavo fusco undata, Linn. 453. (2. fpecim. 771 .

1295 C. Tuberculata, tefta ventricofa elongata, apice acuminato, dorfo nodofo echinata, maculis triangularis fasciata, Linn. O. Seb. III، 73. 1 2 ? 13? Lift 997.62. Rumpl. 25. 3. longit. $7 \frac{1}{4}$ craft. 4. poll. (1. Specim.

1296 C Tuberculata, pallidior, Linn. o. long. $5 \frac{2}{2}$. pol'. ( 1 . fpecim. $i 70^{*}$.

1297 C. Ziczac, r. Plicata, tefta ftriis undatis, cinereo fus. coque varia, Linn. 444. ๙. Seb.III. 73. 10.11. 14. I5. 19. 20. Lift. 1004. 09, 1005. 72. Petiv. Gaz. 133. I. Muf. 10. 10. (2 ipecim. 777.

1298 C. 7iczac, f. Plicata, pracedenti limilis, fed minor, Limn. 444 . $\alpha_{\text {. (2. Specim. } 777 .}$ 


\section{Teftaces. Ordre IV.}

1284 C. Bezoar feneftré, ou truité, d coquille bodrue, ftriée, as taches quarrées.jaunes, Linn. 451. $\beta$, ou O. Rumph. 25. I? Lift. 101 2. 76 ? Adanfon Seneg. 1.7, 2. 8. (4. p.769. 770. $128{ }_{5} \mathrm{C}$. Bezoar d'agathe, un per ftriée, is taches quarrées cerclée de quatre maniere; ouverture dentelée, la queue courbée; Linn, 451. Seb. III. 70. 8. 9. Gualth. 39. H. Lift. I016. 75 ? Rumpb. 25. I. Argenv. 15. I. Petiv. Muf. 2. Ir. (2.p. 773 .

I286 C. Bezoar d'agathe, approchant d la precedente, Linn. 451 . ( $3 . p$. 773 .

1287 C. Bezoar d'agathe, d levre allongée, E corrumpée, ou jeil de la nature, Linn. 451. $\gamma$. (1.p. $773 *$.

1288 C. Bezoar truitée, d coquille ftrié par croix, polie, des ecailles quarries, ouverture dentelée, la queue courbée, Linn. 450 . Gualth. 39. G. Lift. 1000. 65. Rumph. 25. B ? Kircher III. 157. (1. p. 774. Q.

1289 C. Bezoar ondé, à coquille grife, ftriée par ondes brunes; Linn. 450. a. ou o. Argenv. 15. D. Lift. 101 4. 78. Rumph. 25. 2. Petiv. Mur 2I. II. (2. p. 774.

$\$ 290$ C. Bezoar verolé, ventrue, les anfractures à neuds; coquille, à taches quarrées imprimées, Linn. 453. a. ous 0. Gronov. Zooph. III. 12. 1. 2. Petiv. Muf. 7. 4. (4. p. $772^{*}$.

1291 C. Bezoar verolé, approchant d la precedente, Linn. 453. a. ou o. (4. P. 772 .

1292 C. Bezoar cendré, à coquille polie, couronnée de Mamillons, la levre, de derriere pointue, Linn. 453. Seb. IIT. 7I. II. 12.13.16. Lift. 996. 60. Petiv. Muf. II. 18. (I. p. $77 \mathrm{x}$.

1293 C. Bezoar cendré, Jemblable à la precedente, Linn. 453 . (2: $p .77 \mathrm{I}$.

1294 C. Bezoar cendré, a coquille cendrée, ondée d'un brun jaune, Linn 453 . (2. p. 77 r.

$1295 C$. Tuberculee, $\dot{a}$ coquille ventrue, elongée, de fommet pointu, le dos à nceuds, EO fafciée dé taches triangulaires, Linn. 0. Seb. III. 73.I2 ? I 3 ? Lift. 997. 62. Rumph. 25. 3. long. $7 \frac{1}{4}$. cralf. 4 . pouc. (1. p. 776 .

I296 C. Tuberculée, E plus pale, Linn. o long. $5 \frac{\hbar}{2}$. poric. (i: p. $776^{*}$.

1297. C. Zig-zag, ou Pliée flammée, à coquille variée de gris-brun; d ftries ondées, Linn. 444. a. Seb. III. 73. 10. II. I4. 15. 19. 20. Lift. 1004. 69. 1005. 72. Petiv. Gaz. 153. I: Mur. 10. 10, (2.p. 777.

1298 C. Zig-zag, ou Pliée flammée, Semblable à la precedenté mais plus petite 3 Linn. 444. \%! (3. p. 777 .

$$
\text { C c } 1299 \text { C. }
$$


1299 C. Chartoprates, tefta inabfoluta, antice fubplicata, dee cuffatim fubftriata, apertura dentata, cauda recurva, Linin. 444. B. F. O. Seb. III. 73. I. Lij., 1007. 71? Rumph. 23. C. (2. Ipecim. 777. a.

${ }_{3} 300$ C. Chartoprates, pracedenti affinis, fed imperfecta \& major, Linn. 444. ß. I. O. (2. Specim. $777^{*}$.

I 301 C Chartoprates, præcedenti fimilis: nondum adulta, Limn. 444. $\beta$. f, o. (1. 'pecim. $777^{*}$.

1302 C. Rufa. tefta decuffatim Itriata, cingulis nodofis interftinctis, linea gemina, apertura dentata, cauda re* curva, Linn. 446. Seb. III.73.3-6. o. L.ift. 1004. 69. Kircher III. 160. 1. Petiv. Múf. 5. 5. Hivblar Icon. II. Spengler Icon. III. D? (2. fpecim. 778.

I 303 C. I ibberofa, tuberculis majoribus, grifeo alba, rufo fusco flammea, Linn. 447. Gualth. 4I. $A$. (1. Specim. $778 *$

I3C4 C. Tuterofa, præcedenti fimilis, Linn. 447. Kircher IIT. 327. (2. fpecim. $778^{*}$.

I305 C. Tuberofa, pracedenti affinis, Linn. 447. (2. Ipecim. $778^{*}$.

1300 C. Tuberofa, pracedenti fimilis, Linn. 447. (2. Ipecim. $778^{*}$.

I 307 C. Cormuta, teffa turbinata, fcrobiculis punctata, co. ronata, apertura dentata, cauda recurva, Linn. 445. Lift. 1006. 70 , 1009. 7 I. c. Gualth. 40. C. Kircher III. 155. 322. Petiv. Muf. 7. I4. (2. Ipecim. 775.

iso8 C. Cormuta, Linn. 445. Hollar Icon. 10. tria fpecimina, quorum partim defciffum ut ftructura interna pa. teat. (3. Specim. 775 .

1309 C. Cornuta, fed folito major, Limm. 445. Petiv. Gaz. 15I, 9. longit. $6 \frac{3}{4}$. craff. $5 \frac{3}{4}$. poll. (I. Specim. 775 .

1310 C. Cominta, fed magis tuberculata, matgineque apicis longe ramofo, planus, color albidus, labio fusco macu. lato, Linn. 445. Lift. 1008. 71.b. Petiv. MuJ.7. 10, 11 : Ic. Kircher III. 326. 7. long. 9. craff. $6 \frac{1}{2}$. poll. (I. [pecim. $775^{*}$.

I3I I C' Cornuta, præcedenti finilis, Linn. 445.-Rumplo.23.

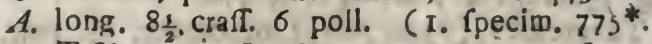

I3I 2 C. Tefficulus, tefta fubovata, cinerea, flammis fuscis circumdata, Linn. 449. Set. III.72. 17-21. Lift. 1001. 66. Rumph. 23. 2. Petiv. Gaz. 152. 17. Kircher III. 163. (2. Specim. 780. 
1299 C. Papier marbré, à coquille imparfaite, par devant un peu plicee, un peu frriée, en forme de croix, ḋouverture dentelée, la queue recourbée Linn. 444. $\beta$ ou o. Seb. III. 73. ¿. Lilt. 1C07.7I? Rumph. 23. C. (2, p. 777. a.

I300 C. Papier marbré, la pareille, imparfaite, Es plus grande, Linn. $444, \beta$ ou o. (2. p. $777^{*}$.

I301 C. $\mathrm{Pa}$, ier maıbré, femblable à la precedente, pas encore parfaite, Linn. 444. B. ou o. ( 1 p. $777^{*}$.

1302 C. R uifle flammé, à coquille friée en forme de croix dise tingués, par des cercles nousufes, double ligne ouverture dentelée, la queue recourvée, Lisn 446. Seb. III. 73. 3-6 9. Lift. 1004 69. Kircher III. 160. I.Petiv. Mur. 5. 5, Hollar Icon. I 4 . Spengler Icon. III. D? (2. p. 778

1303 C. Epaiffe, d̀ tubercules plus grandes, gris-blanche, flam. mée de brun-rouge. Linn. 447. Gualth. 4I. $A$. (1.p. $\left(77 \delta^{*}\right.$.

1304 C Epaiffe, femblable à la precedente, Linn. 447. Kircher III. 327. ( 2 p. $778^{*}$.

I305 C. Epaife, la pareille de la precedente, Linn. 447. (2. p. $778^{*}$.

I306 C. Epaife, ou Tuberculée, Semblable d̀ la precedente, Linn. 417. (2. p, 778*.

I307C. Triçotée à coquille turbinée, a fcrobicules pointée, couronnée, à ouverture dentelée, la queue recourbée, Linn. 445. Lift $1006.70,1009.71$. C. Gualbu.40. C. Kircher III I 55. 322 Petiv. Mur. 7. I4. (2. p. 775.

1308 C. Tric tée, Linn. 445. Hollar Icon 10, 3 pieces defquels Jont dejchire afin que la Jtructure interieure puif]e paroitre, (3. p. 775

1309 C. Tricotée, mais plus grande, Linn. 445. Petiv. Gaz, 151. 9. long. $6 \frac{3}{4}$. craff. $5 \frac{3}{4}$. (1. p. 775 .

T3ro C. Tricotée mais phus tuberculée, le bord du fommet dongue, branchue, platte, à couleur blanche d levre brune, tacket ke, Linn, 445. Lift. 1008. 71. b. Petiv. Mur. 7. ro, I1. 10. Kircher III. 326. 7. long. 9 craf 6 $\frac{1}{2}$ pouc. (I. p. $775^{*}$.

3ri. C. Tricotée, ferrblable d la precedente, Linn 445. Rumph. 23 A. long. $8 \frac{\pi}{2}$, cralf. 6. poll. (1. p. $775^{*}$.

$\$ 312$ C. Plume, ou $B$ nnet de Pologne, d coquille an peu ovasle cendrée, entourée de flammes brunes, Linn. 449. Seb. III. 72.17-21. Lift. ICOI.66. Rumph. 23. 2. Petiv. Gaz。 i52. 17. Kircher III. 163. (2. p. 780. 
GENVSXXVI. BVLL IE S. SEMI-PORCELLANAF

I313 B. Lignaria, obovata; oblongiufcula transverfe ftriata, vertice fubumbilicato, Linn. 379. Lift. 714. 71. Kircher III. 406. $3^{*}$. Spengler Icon. III. H. (2. fpecim. 78I.

I3I4 B. Lignaria, præcedenti affinis, Linn. 379. (2. fpecim. $78 \mathrm{r}$.

I315 B. Lignaria, fimilis, fed minor, Linn. 379. (2. fpecim. 781 .

I3 6 B. Ampulla, tefta rotundata, opaca, vertice umbilicato, flava, plumbeo gattata, Linn. 378. Seb. III. 38. $34-40.42$. Lift. $713.69,1056.8$. Kircher III. 4. Petiv. Gaz. 99. 14. Muf. 9. 19. (4. fpecim 782.

I317 B. Ampulla, rufo fusco punctata, Linn. 378. (4. fpecim. 782 .

33I8 B. Ampulla? I. varietas, fasciis oblcurioribus circumdata. Linn. 378. «. Seb. III. 38. 41. 43. Rumph. 27.0. (3. ppecim. 782 . a.

I3I 9 B. Ibyx, f. Ampulla, magis, cylindrica, Linn 378. f. o. Seb. III. 38. 44. Lift. 714. 72. (3. Rpecim. 783

1320 S. Phy $/ 6 s$, tefta rotundata, glaberrima pellucida, lineis crifpata, fpira acuta, Linn, 380. Argenv. I7. I? Lift. 715. 75. Kircher III. 403. (2. fpecim. 784 .

132 I B. Phy/is, præcedenti fimilis, Linn. 380 . Seb. III. 38. 47-50. (2. Specim. 784.

1322 B. Ampluftre, tefta fubrotunda, fpira elevata, obtufa alba, fasciis incarnatis, nigro marginatis, Linn. 381 . Valent. 19. (2. Ipecim. 738.

I323 B. Narucum, tefta rotundata, pellucida, transverfim fubftriata, utrinque umbilicata, alba, Linn. 375. Lift. 714. 73. Bonann. Obferv. 14. (4. Ipecim. 785.

I324 B. Naucum, præcedenti fimilis, Linn. 375. Argenv. I71 Q. Gualth. I3. G G. ( 5. Ipecim. 784 .

1325 B. Aperta, tefta fubrotunda, intlata, pellucida, tota hians; Linn. 376. Petiv. Gaz. 97. 23. Planc. II. F.G. Gualth. I3. $E E$. ( 3 fpecim. 786 .

3326 B. Volva, tefta biroftri roftris elongatis ftriatis; acutis, Linn. 370. Seb. III. 55.13-16. Lift. 7 I I 66\% Spengler Icon. I. C. long. 3. poll. (2. fpecim. 787.

I327 B. Panus, tefta biroftri, margine extus incraffato, ro: ftris elongatis lavibus, Linn. 371 . . . . . Gualth. I5. 4. Kircher III. 390, Bonann. Obfery, 21. (1. fpecim: 787. 2. 


\section{Teflaces. Ordre $1 \mathcal{V}$.}

GENRE XXVI. VESSIES OU DEMI-PORCELLAINES.

I313 V. Gofre roulée, ovale, un peu longue, ftriée de travers ă Sommet un peu umbiliquè, Lin n. 379. Lift. 714. 7I. Kircher, III. 4 66. 3*. Spergler Icon. III. H. ( 2 p. 78 I, $1314 V$. Gofre roulée, approchant d̀ la precedente. Linn. 379. (2. p. $78 \mathrm{I}$.

$1315 V$. Gofre roulée, Jemblable, mais plus petite. Linn. 379. (2. p. $78 \mathrm{r}$.

I3I 6 . Gondo'e, de coquille ronde. opaque, à fommet umbiliqué, jaune, gouttée de couleur de plomb, Linn. 378. Seb. III. 38.34-40. 42. Lift. 713.69 1056.8 Klrcher III. 4. Petiv. Gaz. 99. 14. Muf. 9. I9. (4. P." 782.

$1317 V$. Gondole, pointée d'un brun rouge, Linn. 378. (4.) p. 782 .

I $318 \vec{V}$. Gondole fascié? ou varieté, entouré, de fascies plus obfcures, Linn.378. a.Seb.III. 38.41. 43.Rumpb 27. G. (3.p.782. $a_{\text {. }}$. I319 $V$. Gondole allongée, plus cylindrique. Linn. 378. $\beta_{1}$ ou O. Seb. III. 38. 44. Lift. 7I 4.72 . (3.p. 783 .

$1320 V$. Gondole émouffée, à coguille arrondée, tris pelée. reiuifante, crepée de lignes, à tour, ou Jpire aigue, Linn. 380. Argenv. 17. 1 ? Lit. 715. 75. Kircher III. 403. (2. p. 784 .

'I32I V. Gondole émoufrée, Semblable à la precedente, Linn.' 380. Seb. III. $38 \quad 47-50$. (2. p. 784 .

$1322 V$. Pavillon d'Hollande, $\dot{a}$ coquille un peu ronde, a jpire devée, émouffée, blanche, à fascies rouges, bordées de noir, Linn. 381. Valent. 19. (2.p. 738.

I323 V. Gondole papiracée, à coquille ronde, reluijante, un peu friée de travers, umbiliqué de chaque coté, blanche, Linn. 375. Lift. 71 4. 73. Bonann. obferv. 14. (2.p. $785^{\circ}$ $1324 V$. Gondole papiracée, Semblable d̀ la precederte, Linn. 375. Argenv. 7. Q. Gualth. I 3. G G. (5. p. 784.

I325 V. Ouverte, à coquille un peu onde, enflée, reluifante, tout à fait ouverte, Linn. 376. Petiv. Gaz. 97. 23. Planc. I 1. F. G. Gualte 13. $E E$. (3. $p$ 786.

$1326 V$. Navette de Tifferant, Oriental, d̀ coquille à double bec, les bécs allongées ftriées, aigues, Linn. 370 . Seb. III. 55 . 13-16. Lift. 711 1. 66? Spengler lcon. I. C. long. 3. pouc. (2. p. 787 .

I327 $V$. Navette de Tifferant, Occidental, d coquille d̀ dous: ble bec, le bord épaiffé, a bec élongées, polies, Linn 375 . *. ou O. Gualth. Is. 4. Kircher III. 390. Bonann. Obferv. 21. ( $., p .787, a$. 
1328 B. Gibbofa, tefta elongata glabra, angulata, angulo elevato, Linn. 374. Lift. 7II. 6t. Kircher III 249. 338. 339. Petiv. Gaz. 15. 5, 156. 21. (2. fpecim. 792.

1329 B: Verrucofa, tefta glabra transverfe angulata, acuta utrinque punet, often, Linn 373. Lift. 712. 68. Seb. III. 55 I7 Kircher III. 250. Petiv. Gaz. 97. 22? Mus. Ir. 23. (? . Specim. 791 .

1330 B Columbula, refta gibnofa, iventricofa - margine crasfiore alba, Linn. 0. Valent. '32? (2. f,ecim. 790.

1331 B. Ovum, tefta tubtenuis ovata, obtufa hiroftr, la= bro dentato, ore albo, Linn. 369 Gustth. 15. B. Argenv. 18. A. (3. fpecim. 789.

1332. B. Ovum, præcedenti affinis, fed ore fusco, Linin 369 , Gualth i5 A. Lift 711. 65. Kircher III. 251. Petiv, Gaz 97. 7. Mus. 8.6. (3. (pecim. 789.

G E N V S XXVH. P OR C E L L A N IE.

3333 P. Tigrea, tefta fubtenuis ovata, elevata glabra, alba fusco punctata, Limai 3 i6. \& r. o. Gualth: 16.S. Lift. 672. 18. Argenv. 18. F? Kircher III 255. (2. Specim. 793.

1334 P. Tigrea, non adulta; Linn. 336 . «. C. o. Lift. 748. 4\%. 43. (2. fpecim $793^{*}$.

1335 P. Tigrea, nond im adulta, minor, Linn. 336 * C. o. (3. fpecim. 79$)^{\%}$.

1336 P. Enervis, tefta fragilis, ore ftriis dentato caftanea tribus lineis longitudinalibus albis, Linnı. 338. «. Lift.: 663. 7? 675.22. (2. Ipecim. 794.

1337 P. Enervis, pracedenti affinis, dentibus minoribus, Linn. 338. «. (2. fpecim. 794.

1338 P. Enervis, præcederti fimilis, nondum adalta, Linn. 338. *. (4. \{pecim. 794. a.

7339 P. Amathyftea, tefta violaceo cinerea, undis fuscis, margine albo, fusco punctato, Linn. 334. qui decortatam exprimit, Gunlth. 26.-C? Lift. 662. 6. Argeny. 18. G? Petiv. Mus 16. 5. (4. Specim 795.

1340 P. Amathyftea, pracedenti limilis, denticulis albis, Linn. 334, -s. (3. fpecim. 795 .

I34I P. Amathyftea, nondum adulta, Linin. 334. $\alpha_{0}$ Lift. $7420^{\circ}$ 38. (5. Ppecim. $795^{*}$.

1342 P. Zetra, tefta glabra turbinata, cinerea fasciis 4 . fuscis, Linn. 333. Gualth. 16. O. Rumph. 38. J. Argenv. 18, G. Kircher III. 265 . (1. fpecim. 726. 


\section{Teftaces. Ordre IV.}

I328 V. Boffue, à coquille élongée, pelée en angles, à cercle élevée. Linn. 374. Lift. 7II. 64. Kircher III 249.338. 339. Petiv. Gaz. I5. 5, 156. 21. (2. p. 792.

I329 V. Jambofin, or Boffue, de coquille pelée, anglée de travers, aigue, à chaque coté un point rouge, Linn. 373. Litt. 71 2. 68. Seb. III. 55. i 7. Kircher III. 250.' Petiv. Gaz. 27. 22 ? Mur, 16. 23. (2. p. 79 r.

I330 V. Oeuf de Pigeon, à coquille boffue, ventrue, $d$ bord plus épais, blanche, Linn. O.Valent. 32 ? (2. p. 790.

I33I $V$. Oeuf de l'Oie, à conzille un peu mince, ovale, do double bec émouffé, à levre dentelée, $\mathcal{E}^{2}$ bouche blanche, Linn. 369. a. Gualth. 15. B. Argenv, 18. A. (3, p. 789 . 1332 $V$. Oeuf de l'Oie, Semblable à la precedente, mais la bouche brune, Linn. 369. Gualth. 15, A. Lift. jri. 65. Kircher III. 25I. (3. P. .789.

\section{GE NRE XXVII. POR CELAINES.}

\$333 P. Mince tigrée, di coquille mince ovale, élevée, pelée, blan. che, pointée de brun, Linn. 336; \& ou O. Gualth. I5. S. Lift. 672. 18. Argenv. 18. F ? Kircher III. 255. (2 p. 793. I334 P. Mince tigrée, imparfaite, Linn. $336 . \infty$, ou o, Lift. 748. 42. 43. (2. p. $793 *$.

I335 $P$. Mince tigrée, pas encore parfaite, plus petite, Linn. 336. a. ou O. (3. p. $793^{*}$.

I336 P, Mince bandée à coquille fragile à ftries, bouche dentelée, chataigne, à trois lignes en long, blanches, Lino. 338. \&. Lift. 663. 7 ? 675. 22. (2. p. 794.

I337 $P$. Mince bandée, approchant à la precedente, wais à dens plus petites, Linn. 338. \&. (2. p. 794.

1338 P. Mince tandée, Semblable à la precedente, mais pos encore parfaite, Linn. 338. \& (4. p. 794. a.

1339 P. Mince, ou Papier marbré, à coquille violette, cendrée à ondes brunes, à bord blanc, pointé de brun, Linn. 334. la definition de Linné, exprime la decorchée, Gualtb. 26. C? Lift. 662. 6. Argenv. 18.G? Petiv. Mur. I6.5. ( 4. p. 795.

I340 P. Mince, ou Papier marbré, femblable d la preceden. te, a' petits dents, blanches, Linn. 334. a. (3. p. 795. 134I $P$. Mince, ou Papier marbré, la pareille, pas encore parfaite, Linn. 334. a. Lift. 742.38 , (5. p. $795^{*}$

1342 P. Taupe cendrée, à coquille pelée, turbinee, cendrée is quatre fascies brunes, Linn. 332 . Gualth. I6. O. Rumph. 38. I. Argenv, 18. G. Kircher III. 265 . (1. $p$. 796.

C c 4

$1343 P$. 
I343 P. Zebra, præcedenti fimilis, nondum adulta, Linn 332. Gualth. 16. P ? Liff. 741. 37. (2. Specim, 796.a.

1344 P. Talpa, tefta teftacea, fasciis duabus fuscis, fubtus incraffata, Linn. 333. Argenv. 18. H. Rumph. 38. K. (1. Specim. 797.

I 345 P. Talpa, præcedenti fimilis, Linm. 333. (2. Specimi 797.

1346 P. Carneola, tefta fubturbinata, pallida, fasciis incarnatis, ore ftriis purpurafcentibus, Linn. 331. Rumph. 38. K? Petiv. Gaz. 80. 8. (4. fpecim. 798.

1347 P. Carneola, pracedenti affinis, fed minor, Linn. 33 I. (4. 'pecim. 798.

\$38 P. Carneola, pracedenti affinis, fed non adulta, Limm. 331. (3. '́pecim. $798^{*}$.

1349 P.Grifea, tefta fragilis cinerea, fasciis grifeo fuscis, Limm: 33r. a. f. O. Lift. 649.44? 670. I6.? quatuor f́pecimina, unum nondum adultum. (4. fpecim. $8 \mathrm{co}$.

$\times 350$ P. Succincta, tefta ventricofa, fasciis 2. albis, Linn. $338 \%$ B fo. o. Lift. 667. II ? 749.45'? Petiv. Gaz. 156. 22. tria fpecimina, quorum 2 nondum adulta, ( 3 . Ppecim. 818.

235 I P. Succincta? f. varietas, lineis quatuor fuscis, glabra; craffa, Linn. o. Gualth. I6. L, (3. ffecim. 816.

1352 P. Succincta, præcedenti fimilis, fasciis 3. media latiore, Linn. 0. Rumph. 38. P? Lift. 678. 25? (1. Ppecim. 826.

1353 P. Cincta, tefta veatricola alba, fusco fasciata, Linn. o. Argenv. 18. N? (1. fpecim. 804.

1354 P. Amygdala, tefta elongata tumida, viridefcente, dorfo flavefcente, Linn. O. (I. fpecim. 799.

1355 P.Violafcens, tefta glabra, oblonga, cincta, grifeo viola fcente, Limn. O. Seb. III. 55. in n8. 19. Lift. 656. I, Argexv. 18.1? Gualth. 16. A? B? Petiv. Gaz. 97. 10. (6. fpecim. 801-803.

1356 P. Cinerea, præcedenti affinis, tefta oblcure cinerea, albo purctata, parva, Lim. o. Seb. III. 55. in $n^{\circ}$. 19. Lift. 686. 33 ? (7. fpecim. 835. 836.85 I.

$\$ 357$ P. Ziczac, tefta elongata, alba, flavo undatim picta, ore a tergo flavo. punctis fuscis, Linn. 349 Seb. III. 59. in $n^{\circ}$. 12. Lift, 66 I. 5 \%, Argersv. 18. N. Kircher III. 260. (4. - pecim. 837.

1358 P. Cribraria, tefta elongata, marginata lutea, punctis rotundis albis, Linn. 353. Lift. 695.42. Argenv. 18. X. Petty. Gaz. 8. 3. (4. fpecim. 83I. 


\section{Teftaces. Ordre IV. $\quad 40 \mathrm{I}$}

1343 P. Taupe cendrée femblable à la precedente, pas encore par.faite, Linn. 332. Gualth. I6. P? Lift. 741 . 3\% (2. P. 796. $a$.

$1344 P$. Taupe bandée, à coquille teflacée di deux fascies brbnes,'defous épaîfJée, Lien. 333. Argenv. 18. H. Rumph. 38. K. (I. p. $76 \%$.

3345 P. Taupe bandée, Semblabie de la trecedente, Linn. 333. (2.p. 797.

I346 P. Taupe bandée rouge, à coquille un peu turbinée, pale, à fascies incarnées, la boucbe de fries pourprées, Lion. 331. Rumph, 38. $K$ ? Petiv. Gaz. 80. S. (4. p. 798.

$1347 \stackrel{P}{P}$. Taupe bandé rouge, Semblable dे la precedente, mais. plus petite, Linn. 331. (4. p 798.

1348 P. Taupe bandée rouge, approchant à la precedente, mais pas encore parfaite, Linn 33 r. (3.p. $798^{*}$.

1349. $P$. Taupe bandée grife, fragile, d coguille cendree d fascies gris-brunes, Linn. 331. a. ou o. Lift. 64944 ? 670. 16.? 4. pieces, dont unen'eft pas encore parfaite. (4.p.800.

$1350 P$. Taupe, ou Succinte, à coquille ventrue, $a \dot{a}$ deux bandes blanches Eg brunes, Linn. 438. $\beta$ or 0 . Lift. 607. I i? 749. 45? Petiv. Gaz. 156. 22, 3 pieces, deux desquelles. ne font pas encore parfaites. (3. p. 818 .

I 351 P. Taupe, ou Succinte ? ou varieté, à quatre lignes bras: nes, pelée, épaiffe, Linn o. Gualth. I6.L. ( $3 . p 816$.

I352 $P$. Taupe, ou Succinte, femblable à la precedente, aufso ì trois fascies, dont celle du milieu, eft pluslarge, Linar O. Rumph. 38. $P$ ? Lift. 678. 25 ? (I. p. 826.

$1353 P$. Taupe cerclée, à coquille ventrue, blanche, fasciée de brun, Linn. O. Argenv. 18. $N$ ? (I p. 804 .

1354 P. Amande ventrue, d coquille allongée, enflee, verdia: tre a d dos jaunatre, Linn. o. (1. p. 799.

ذ355 P. Lien bleue, à coquille pelée, allongée gris violotte, Linn. O. Seb. III. 55. in $n^{\circ}$. 19. Liff. 656. I. Argepva 18. I ? Gualth I6. $A$ ? $B$ ? Petiv. Gaz. 97. 1C. (6. p. $801-803:$

$\$ 356$ P. Cendrée, Jemblable d̀ la precedente? à coquille centcrée, pointée de blanc, petite, Linn. O Seb. III. 55. in n̊. 19. Lift. 686. 33 ? (7. p. 835. 836. 85i.

1357 P. Zig-zag, à coquille allongée, blanche, peinte de junie, par sndes, la bouche par derriere jaune, id points iruns. Linn. 349. Seb. III.59 in $n^{2}$. I9. Lift. 65I. 5 ? Argenv. I8. $N$. Kircher III. 260 . ( 4 p. 837 .

I358 P. Argus, ou Crible, à coquille jaune, allongée, bordée, a pointes rondes blanches, Limn. 3 ; 3. Lilt. 695. 42. Argenv. 18. X. Putiv. Gaz. 8. 3. (4. P. 83 I. 
I359 P. A ellus, teffa elongata alba, fasciis 3. fuscis, Linn. 351. Lift. 606. 10. Argenv. 18. T. Rumph 39. M Pe. tiv. Gaz 97. 11. Kircher III. 236. (6. fpscim. 832.

I360 P. Hirundo, tefta elongata, fupra coerulefcente, extremitatibus maculis 2 fuscis, Linn. 350 . Seb: 111.55. in $\mathrm{n}^{\circ}$. 19. f-k. Gualth. 15. DD. Lift. 674.20. 21. Petiv. Gaz.30.3. Kircher III. 235. (3. Fpecim. 833. 834. I361 P. Phasma, tefti cylindrica, marginata, grifea, dorfo maculis fuscis, Linn. O. Seb. III.55. in no. 19. Rumph. 39. F. Petiv. Gaz. 97. 21. Muf. 16. 10? (4. Ppecim. 829.

I362 P. Ifabella, tefta obtufa fubcylindrica, flavo grifea, dorfo vigro ftriata, extremitatibus luteis, Linn. 345 . Lift. 660. 4. Argenv. 18. P. Petiv. Gaz. 97. I6. Muf, 16. I6. (3. fpecim. 1048.

I363 P.. Trifofcia, tefta oblonga, glabra grifea, fasciis tribus viridi flavis, Linn. 0. Gualth. 13. Q? Lift. 668. 13. a. Adanfon Seneg. 1. 5. II. E. (4. 1pecim. 815. 1364 P. Trifafciata, an pracedenti fimilis? fasciis tribus fuscis, punctatis, margine maculato, Limm. O. Lift. $67 \mathrm{I}$. 37 ? (5. fpecin. 827.

1365 P. Trifalciata, pracedenti affinis, fasciis oblcurioribus, Linm. 0. Lift. 678. 25? Rumph. 38. R? (4. Tpecim. 826.

1360 P. Tefindinaria, tefta obtufa fubcylindrica, extremitatibusque depreflis, albo fcabinofo pun tata, maculis magnis fuscis, Linn. 329. Lift. 689. 36. Petiv. Maj. 8. 7. (2. Specim. 820 .

I 367 P. Atheroma, tefta oblonga elevata fusca, fascia longitudinali alba, punctisque majoribus albis, Linn. 325. ๙. F. O. Liff. 69\%. 44. (2. fpecim. 821.

I36s P. Atheroma, pracedenti fimilis, fed minor, Linn. 325. ๙. f. o. Lift. 699.45. Kircher III.366. dua fpecimina, quorum unum lineis obfcuris fasciatum. (2. fpecim.822.

I369 P. Exantheme, tefta fubturbinata, ferruginea, maculis albis, fuscisque, rotundis adfperfa, linea longitudinali fubramofa, Linn. 325. Lift. 699. 46. Petiv. Gaz. 153. II. (2. โpecim. 823 .

1370 P. Exanthema, præcedenti fimilis, fed minor, Linn. 325. duo fuecimina, quorum alterum fasciis obfcurioribus, (2. fpecim. 823.

237 P. Amarata, tefta cylindrica, glabra, fusco flava, ncellis flavefcentibus, extremitatum maculis fuscis, Limn.o. Rumph. 39. I1. (2. Specim. 825. 


\section{Teftaces, Ordre $I V$.}

I359 $P$. Ane rayé, tres petit, d' coquille allongée, blanche, trois bandes brunes, Linn. 351. Lift 006. 10. Argenv. 18. T. Rumph 39: M. Peciv. GaZ. 97. I1. Kircher III. 236. ( 6 p. 832

I360 P Hirondelle, a coquille allongée, deffus bleu celefte, les extremités ont deux taches brunes, Li $\mathrm{n}$ 350. seb. IIT. 55. in $n^{\circ}$. I 9. $f-k$. Gualth. 15. DD Lift 674.20. 21. Petiv. Gaz. 30. 3. Kircher III. 235. (3. P 833. 834. I36I P. Spectre, ou Phantome, $\dot{a}$ coquilile cylindrique bordee, grife, le dos à taches branes, inn. O. Seb. IlI. 55. in n\&. 19. Ruinph. 39 F. Pctiv Gaz. 97, 21. Mul. Io 10? (4. p. 829 .

1362.P Ifabelle, di coquille emouffée, un peu cylindrique, gris. jaunes, Linn. 345. Litt. 60.4. Arge v, 18. P. t'etiv.

Gaz. 97. 16 Mui 16.16. (0.p. 1048 .
1363 P. Bà de cendiée, d.coquille allongée, pelée, grifes do trois bandes yert-jaunes, Lin. O. Gualth. 13. Q Liff. 668. 13. a. A Janfon Senes. I 5 it E. (4 P. 815 .

r364 $P$. Bande brune tigrée, ou femblable à la precedente? a bandes trunes pointées, à bord tachete, Linn. O. Lift. $67 \%$. I7? ( 5. p. 8. 7 .

I $365 \mathrm{P}$. Bande brune tigrée, approchant à la precedente d bandes plus obfcures, Linn. 0. Lift 678. 25? Rumph. 38. $R$ ? (4. p. 826 .

I366 P. Ecaille de Tortae d coqiulle émnudée, un peu cylindrique, les extremitès deprimées, pointée de blanc, a grande taches brines Linn. 329. Lift. 639 36 Putiv. Mur. 8. 7. (2. $p, 820$.

I $_{3} 67$ P. Rougeoles blanches, à coquille allongée, élevié brune, a fafcie longue blanche, it points pliss gra ds blancs; Linn. 325. a. ou 0. Lift. 697. 44. (2. p. 82 r.

I358 P. Rougeoles blanches, femblable $a$ la precedente, mnis pius petite, Linn. 325. a ou 0. Lift.698.45. Ki cber IIT. 366. deux pieces dont l'une eft fofciée par des lignes obfcures. (2. p. 822 .

I369P. Argus, à coquille un peu turbinée., couleur de rouille, à taches blanches", EO brimes rondes, à ligne longue run peu branchue, Linn. 325. Lift 699. 46. Peciv. Gaz 153. Ir. (3 p 82?.

1370 P. Argus, pareille, 'mais plis petite, Linn. 325. deux picces dont wne à bandes plus objcures. (2. p. 823 .

137r P. Petit Argus, ou Arlequin, à coquille cylindrinzse, pelée brun-jaune, à yeux jaunatres, les extremites à taches brunes, Linn. O. Rumph. 39. H. (2.p.825. 
1372 P. Argus, tefta fubturbinata cylindrica, adfperfa ocel. lis, fuscis, fubtu: fasciis quatuor fuscis, Linn. 328. Lift. 705 54. Argenv. 18. D. Kircher III. 203. Petiv. Gas. 97. 6. Muj. 5. 9. (3. fpecim. 824 .

1373 P. Stolida, tefta marginata flavo cinerea, in dorfo maca. la fusca, Linn. 360. Rumph 39 E. Argenv. 18. X. Petiv. Gaz. 97. 18. (2. fpecim. 830.

1374 P. Granata, tefta oblonga fubfpherica, antice flava, albo tuberculata, Linn. 365 . f: O. Seb. III. 55. in ñ. 22. Gualth. 14. 2. R. Lift. 708. 58. (4. Specim 838.

I375 P. Nucleus, tefta alba, utrinque, marginata, fubroIt ata rugofa, fupra punctata tuberculis, Linn. 365. Lifs. 710. 61. Argetiv. 18. V. Petiv Gaz. 97.12. (3. Ipecim. 839.

$\$ 376$ P. Staphylea, tefta fubroftrata, grifea tuberculata flava, antice flavo marginata, Linn. 366. $\%$. Gualth. 14. T. Lift. 7 10. 60. Rumph. 39. K. Petiv. Gaz. 97. I3. (2. fpecim. 845.

1377 P. Globulus, tefta utrinque roftrat?, lavi alba, ad mar inem flavo maculata, Linn. 368. Seb. III. 55. in $\mathrm{n}^{\circ}$. 22. Petiv. Gaz. 97. 4. Rumph. 39. L. N ? (4. Ipecim. 840. 841 .

1378 P. Ocellata, tefta fubemarginata, araufio lutea, ocellis albis, in medio, ore purpureo nigris, Linn. 362. Gualth. 15. B B ? Liff. 996. 43. Petiv. Gaz 9. 7. Kir. cher III. 332. (3. Fpecim. 814.

1379 P. Arabica, tefta fubturbinata, characteribus infcripta, punctis fuscis marginata, macula longitudinali fimplici, Linn. 327. Lift. 058. 3. Gualth. 16.V, Petiv. Gaz. 96. 6. 7. (2. fpecim. 806.

I380 P. Arabica, præcedenti fimilis, Linn. 327. Rumph. 38.M. quatuor fpecimina, quorum duo flavefcunt, (4. Ipecim. 806:

1381 P. Hiftrio, elevatior, tefta maculis atro fuscis, albida, fusco reticulata Linn. $327 . \%$. f O. Seb. III. 55. in no. 23. Lift 659. 3. a. Argenv. Suppl. 2. I Rumph. 39. R? Petiv Gaz. 53. 6. (I. Cpecim. 805.

3382 P Tigris, tefta glabra, obtufa ovata, antice rotundata, linea longitudinali teftacea, maculis nigris punctata, Linn. 343. Gualth 14.G I. L.Lift. 682. 29. Kircher III. 23I. 232. (3. fpecim. 810.

1383 P. Tigris, præcedenti fimilis, Linn. 343. Gualth. I4. H. Lift. 681. 28. (x. fpecim. 810, a. 


\section{Teftaces Ordre $I \hat{D}$.}

$1372 \mathrm{P}$. Grand Argus, d̀ coquille un peu turbinée, cylindrig̨ue, Sparfée par des yeux, deffous à quatre bandes brunes, Linn. 328. Lift. 705. 54. Argenv. 18. D. Kircher III. 263. Petiv. Gaz. 97.6. Muf. 5.9. (3. p.824.

I373 P. Taupe, ou Tête de Dragon, à coquille bordée, jaune cendrée, Jur le dos une tache brune, Linn. 360. Rumply. 39. E. Argenv. 18. Y. Petiv. Gaz. 97. 18. ( 2 p. 830. $P$. Cloporte, ou Grelée, à coquille allongke, un pers Spherique, joune par devant, Eे tuberculée de blanc, Linn. 305. a. ou o. Seb. III. 55. in $n^{\circ}$. 22. Gualth. I4. $Q$. R. Lift. 708.58 , '4. p. 838.

1375 P. Cloporre, ou Tarée, da coquille blanche, bordée de chaque coté, pointu, ridée, Eo pointée en deffus de tubercules, Linn, 365. Lift. 710. 61. Argenv. 18. V. Petiv. $\mathrm{Gaz}$ 97. 1 2. ( 3 p. 839.

I376 P. Oscabrion, ou Punaife de mer, \& coquille un pers pointue, grife, tuberculée, par devant, bordée de jaune, Linn. $366 . \alpha$. Gualth. I4. T. Lift, 710. 60. Rumph. 39, K. Petiv. Gaz. 97. 13. (2.p. 845.

I377 $P$ Bouton ventrue, $a$ coquilile roftué de chaque coté, polie hlanche, vers le bord tacheté de jaunne, Linn. 368. Seh. III. 55. in $n^{\circ}$. 22. Petiv. Gaz. 97.4 Rumph 39.L.N? (4.P. $840.8+$ I.

I378 $P$. Verole, ou en oeil, $d$ coquille un peu émarginée, jaune, au milieu, des yeux blancs Eo noirs, la bouche pour. prée, Linn. 362. Gualth. I5. B B? Lift. 996. 43. Petiv. Gaz. 9. 7. Kircher $I I I .332$. (3: p. 8I4.

1379 P. Ecriture Arabique, d coquille un peu turbinée, écrite en caratteres, EO bordée de points bruns, à fimple tache longue, Linn. 327. Lift. 658, 3. Gua'th. 16. V. Petiv. GaZ 96 6. 7. (2. p. 806 .

1380 P. Ecriture Hebraique, femblable d la precedente, Linn: 327 Rumph. 58. M. quatre pieces, dont deux font jaunes, (4. p. 806.

1381 $P$ Arlequin, plus élevée, la coquille à taches noir-blanche, brun, en forme de refeau, Linn. 327. a. ou 0 . Seb. III. 55. in ne. 23. Lift. 659. 3. a. Argenv. Suppl. 2. $I_{\text {. }}$ Rumph. 39. $R$ ? Petiv. Gaz. 53. 6. (1. p. 805 .

1382 . Epaifre tigré, de coquille pelée, 'émouffèe, ovale, arrordie par devant,$\dot{a}$ longue raye teftacée. EO paintée de points noirs, Linn. 343. Gualth. 14. G. I. L. Lift. 682. 29. Kircher III. 23I. 232 . (3. p. 810.

\383 P. Epaiffe tioré, Jemblable d la precedente, Linn. 343. Gualtb. 14. H, Liit. 081. 28. (1. p. 810. a) 
1384 P. Mappa, tefta elevata fubturbinata, caracterlbus in fcripta linea longituduali ranofa, Linn. 326 Argenv. 18. B. Rumph. 38. B. (I. fpecim. 809.

2385 P. Mappa præcedenti affinis, fed miror, Linn. 326. Petiv. Muf. 16. 2 (2, fpeciti. 809.

I386 P. Mappo, pracedenti finulis, Limm. 336 una magis ruber te, (2.-'pecim. sog.

I387 P. Caftanea, tefta fub urbinata ovata, gibba atrn caftanea, dorfo albidiore, Limm. O. Gualth. 15. N. (2. $f_{s}$ ecim. 819

C388 P Mcrio, tefta fubturbinata, ovata, fusco nigra, ore margine;ue violacea, fasciis 2. alb dis longitudinalibus. Liriz. O. Lift 66\%. ri? Kircher III. 250. (I. Specim. 818.

I389 P. Oliva, tefta ovata, olivacea fusca, ore rubro circumdata, fasciis 2. longitudinalibus albidis, Linn. 0 . (2. fpecim. 817.

I390 P. Vitellus, lefta , biura livida, maculis albis, dorfo non fasciato, Limn.-34 l. Gualth. 13.T V. Lift. 676.23? Rumph. 38. II Pctiv Muf. 310 (3. 1 prcim. $8 \mathrm{Ir}$. I39I P. Vitellus, pracedenti fimilis, fed minor, fusca \& fásciata, Linn. 341 . (4. ipecim $8 \mathrm{il}^{\circ}$

I392 P. Salinator, tefta glabri Havo aurania, albo maculata, Lim 34.... f. O. Gualth. 13.V. Lift. 676.'23. (I. fpecin 1046.

I393 P. Petechifans, tefta glaura alba , fusco punetara, Linn. O. Seb. III 55 in no 20. Gualth. 16 R? Rumph. 38. N. Lift. 684. 31. Petiv. Gaz. 80, 9. Kircher III. 242 . (5. roecim. 813 .

1394 P. Variolans, refta glabra, crafta cinered, fusco maculata, ore albo, denticulic Havis, Linn. O Gualth. I4. A? Lif. 633. 3c? Argenv. 28. E. Kircher III. 252. (4. fpecin. 812 .

I395 F Mauritiana, tefta globofa, gibba, poftice depreffa, craff, brunnea. margine atro lato, Linn. 340 . Gualth. 15. S'T T? Lift 703 52. Petiv. Gaz. 96. 8. (2. Specim. 807.

1596 P. Mauritiana, przcedenti fimilis, Lin»s. 340. Rumpls. 38. E. Kircher III 26I. (2. frecim. 807.

I397 P. Gibber, teła gुibbufa, gibso elelato fubacuminato, crafra, flavefcente, pallide fusco maculata, Linn. 340. a. I. o. Lift. 687. 34? Kircber III. 262? (1. Ipe cim. 808. 
${ }_{3384} P$. Carte Geographique, d̀ cogrille élevée, un peu turbinée, marquée, par des carcicteres à longue. ligne brancbue. Linn. 326. Argenv 18. B. Rumph. 38. B. (1. p. 809. I385 P. Carte Geographique, approchant à la precederte; mais phus petite, Linn. 326. Petiv. Mur. 16. 2. (2. p. 809.

I386 P. Carte Geographique, femblable a la precedente, Linn. 326. dont l'une eft plus rouge, (2. p. 809 .

I $387 P$. Chataigne crevaffée, d coquille un peu turbinée, ovale, boffue, d'un noir chiataigne, le dos plus blanc, Linn.o: Gualth. 15. N. (2.p.810.

I $388 P$. Negre bandé, à coquille un peu turbinée, ovale, branz. noire, la bouche,. $\mathcal{E}^{3}$ le bord violette, $d$ deux bandes longues blanchatres, Linn. O. Lift. 067 . It? Kitcher $11 I$. 250. (I. P. 818 .

I.389 P. Olive bandée, à coquille ovale, olivatre, brune, ds bouche rouge, entourée de deux bandes longues blancheatre, Linn. O. (2. $p=817$.

$\$ 390$ P. Jaune d'oeuf, ou Varioles blanches, d̀ coquille émousSée, livide, à taches blanches, be dos Jans bande, Linn. 341. Gualtb. 13. T. V. Lift. 676. 23 ? Rumph. 38. II. Petiv. Muf. 3.10. (3.p. 8Ir.

I $39 \mathrm{I}^{\circ} \boldsymbol{P}$. Jaune d'oeuf, ou Varioles blanches, femblable da la precedente . mais plus petite, brune, \& bandée, Linn.34I. (4. p. 8II*.

\392 P. Rougeoles brunes, à coquille pelée d'un orange pale. tachetés de blanc, Linn. 34I.a. ou o. Gualth. I3.V. Lifi. 676. 23. (1. p. 1046.

$\$ 393$ P. Rougeoles brunes, à coquille pelée, blanche, pointée de brun, Linn. 0: Seb. III. 55. in $n^{8}$. 20. Gualth. I6. $R$ ? Rumph. 38. N. Lift. 684. 31. Petiv. Gaz. 80.9. Kircher III. 242. (s. p. 813 .

1324. P. Rougeoles noires, à coquille pelée, épaiflee, cendrée, tachetée de brun, à bouche blanche. $\mathcal{E}^{\circ}$ dens jaunes, Linn. o. Gualth. 14. $A$ ? Lift. 683. 30. Argenv. 18. E. Kircher III. 252. (4. p. 812 .

1395 P. Tête de Serpent, à coquille globuleufe fronde, bo/Jue, deprimé par derriere, épailfe, brunatre, a bord large noire, Linr. 340. Gualth. 15. S? T? Lift. 703. 52. Petiv。 Gaz. 96. 8. (2. p.807.

$1396 P$. Tête de Serpent, Jemblable à la precedente, Linn, 340. Rumph. 38. 6. Kircher III. 26r. (2. p.807.

1397 P. Boffe tigrée d coquille boffue à boffe élevée, un per pointue, épaiffe . jaunatre, palement tachetée de brus, Linn. 340. a. 0u 0. Lift. 087.34? Kircher III. 262? (1. p. 803. 
1398 P. Gibber, pracedenti affinis, Lim. 340. a. C. O. C2. fpecim. 808.

I399 P. Mus, tefta tuberculata preffa, retufa, gibba, cinerea, fusco punctata, denticulis nigricantibus, Lints. 342. Rumph. 39. $\$$ ? (2. fjecim.' 846.

I400 P. Mus. fimillima, fed non recufa, Linn. 342. a. Seb. III. 76. 33. 34. Rumph. 39. S. Petiv. MuS. 8. 8, 16.4. (2. ppecim. 847.

I40I P. Cafut Serpentis, tefta obtufa, triqueftre gibbora, poftice obtufiuscula, craffa fusca, oceilis albis, Linn. 339. Seb. III. 55. in n. 20. Lift. 702.50. 53. Petiv. Gaz. 96. 9. Muf. I6. 7. Adanfon Seneg. I. 5. Ix. G. (7. fipecim. 843 .

I402 P. Caput Serpentis, pracedentl fimilis, linea transverfa albida, margine punetaro, Linn. 339. \&. Lift. 707.48 ? Rumph. 38. F: (1. Specim. 842.

${ }^{4} 403$ P. Radiaris . refta oblonga, glabra, fusca, ftellulis radiantibus punftata margine aurantio, Linn c. Gualth. 15. Z Lift.691. 38 ? Petiv. Gaz.95, 10. (8. fpecim. 844.

1404 P. Erofa, tefta parva, dorío marginato, flava, albo radiatim punctata, albo guttata, lateribus macula fusca, Linn. 357. Seb. III. 55. in no.20. Lift. 69I. 38. 39. Gualtb. 15. I. Rumph. 38. P. (6. fpecim. 828.

I405 P. Ocellata, tefta glabra flava, ocellis flavefcentibus, linea transverfa flaveicente, Linn. O. Seb. III. 55. in n. 20. Lift. 701. 48. Gualth. 15. G? Rumph. 38:O? (2. fpecim. 1047.

1406 P. Monita, teita quadrangulata, marginato nodola, flava, Linn. 5.54. Gualth. 14. I. 3. 4. 5. Lift.709.59: Argenv. 18. K Petiv. Muf. 16. 14. (3. Tpecim. 850.

1407 P. Annulus, tefta quadrangulata marginata, cinereo flava, dorfo anvulo flavo cincta, Linn. 355. Gualth. 34. 2. Rumph.39. D. Petiv. Gaz.6. 8 ? (3. fpecim. 84 9. I408 P. Anmulus, pracedenti affinis, dorfo violaceo, Linn. 355. Rumpli。 38, G. (2. fpecin: 848 .

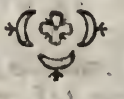




\section{Teftaces. Ordre I V.}

I398 P. Boffe tigrce, approchant, à la precedente, Linn 340. a ou o (2. P. 808 .

$\$ 399$ P. souris, ou boffe Carthagenois, a coquille tubercule; emouffée, boffue , cendrée, pointée de brun à dens noiratres, Linn. 342. Rumph 39 . S ? (2. p 846.

I400 $P$. Souris, ou Carthagenols, femblable, mais pas émous. fée Linn. 342. \& Seb. III 76. 33. 34. Rumph 39. S. Petiv Muf. 8. 8, 16, 4. (2. p. 84\%.

I40I $P$. Tête de Serpent, tres petit, d coquille émoulfée. triangulaire boffue, par derriere émoífJée, épaifje brune, a yeux blancs, Linn. 339. Stb. III 55. in $n^{2}$. 20. Lift. 702 50. 53. Petiv Gaz. 96. 9. Muf. 16. 7. $\Delta$ danfon Seneg I. 5. II. G. (7 p. 843 .

$1402 P$. Tête de Serpent tigrée, Semblable de la precedente, d ligne de travers blanche le bord pointé, Linn. 339. $\alpha^{\circ}$ Lift. 701 48? Rumph. 38 F. (I p. 842 .

${ }_{403} P$. Rayonné, ou d'Orange ventrée à coquille allongée, pé lée, brune, pointée en forme de rayons, a bord orange, Linn. 0. Gualth. ' 5.Z Lift. 091.38 ? Petiv. Gaz 96 10.(8.p.844. $1404 P$. Ambre, à raches blanches, à coquille petite le dos bordée, jaune, blanche, pointée en forme de rayons, ondée dé blanc, lés cotes à tache brune, Linn. 35 \%.Seh. III. 55 in $n^{\circ}$. 20. Lift. 69138.39 Gualth. 15. I. Rumph. 38. P. (6.p.828,

I405 P. Ambre, à taches pourpres, à coquille pelée jasne, yeux jaunatres, à ligne transverfe jaune, Linn o. Seb IIL. 55 in $n^{\circ}$. 20. Lift. 701. 48. Gualth.15. G ? Rumph. 38. 0 ? (2. p. 1047 .

I406 P. Colique de Chene, ou Cauris jaune, d coquille quadtangum laire bordee, rouenfe, jaune, I.inn. 354. Gualch. 14. 1.3 4.5. Lift. 709.50, Argenv. 18. K. Petiv. Muf. 16 I4 (3 p.850.

$1407 P$. Colique grife, on Torax, d̀ coquille quadrangulaire bordée, cendrée. jaunne, le dos entouré d'un anneau ou cercle jaune, Linn. 355. Gualth. 14. 2. Rumph. 39 D Petiv. Gaz. 6. 8 ? $\bigcirc$ ( 3. p 849.

$\$ 408 P$. Colique de Guiné, ou Cauris bleue, Semblable d la precedente, le dos violet, Linn. 355. Rumph. 38. G. (2. P. 848.

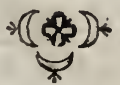




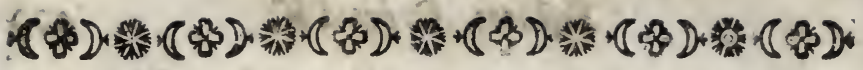
C L A S S I S I I.

$\begin{array}{llllllll}B & I & V & A & L & V & I & A\end{array}$

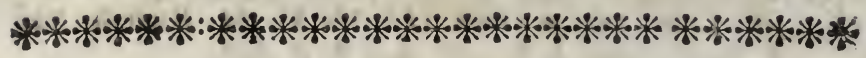

O R D O V.

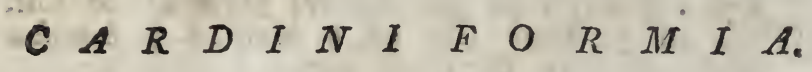

GENVS XXVIII. PINN

I409 P. Rudis, tefta fulcata, fquamis fornicatis, per feries digeftis, Linn.263. Seb.III. 91. I. Cualth. 78. I. A. Lif. 374. 2 15. Argenv. 22. B. Ginan. II 165. long. $16 \frac{1}{2}$. lat. 7. poll. (I fpecim. I.

I4lo P. Pectinata, tefta dimidia, longitudinaliter ftriata, latere alcero transverfim, a coftis ruzofo minoribus, Zinn. 264. Seb. III. 91. 3. Gualth. 79. A. Lift. 370.210. Rumph. 46. I? (1. fpecim. 2.

1411 P. Pettinata, præcedenti fimilis, Linn. 264. (4. Ppecim. 2.

I4r2 P. Nobilis, terta undatim ftriata, fquamis canaliculato tubulofis, fubimbricatit, Linn. 265. Gualth 78. B. Lift. 372. 213. Ginann. II. 166. (2. fpecim. 3.

I4I3 P. Muricata, tefta longitudinali ftriata, fquamis concavis, ovaris cbtufis, Linn. 266. Lift. 373. 214. Ar. genv 22. F. Petiv. Muf 19. 14. long. IO poll. (I. Specim. 4.

1414 P. Alata, teßa ovata convexa atra, fquamis brevioribus, Linn o. Seb III. 91. 2. Gualth 81. A. Rumph. 46. $L$ ? long. 9. lat. 6 . poll (i. Ppecim 5.

1415 P. Alata, præcedenti affinis, Linn o. (2. fpocim. 5. $1416 \mathrm{P}$ Alata, prxcedenti fimilis, fed parva, Linn. o. duo fpecimina, alterum muricatum. (2. fpecim. 4. 5.

1417 P. Saccata, tefta tumidata, lævi, erectiuscula, faccata; Linn 208. Seb. III. 92 in medio. Rumph. 49. N. Pe. tiv. Mul 19. 7. (I. fpecim. 6.

7418 P. Saccata, pracedenti fimilis, magis incurva, Linn' 268. (1. Ppecim. 6. 


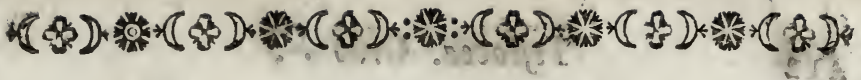
C L A S S E I I.

\section{$B$ I V A L V E S.}

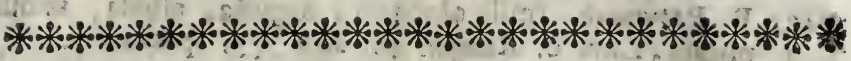

O R D R E V.

EN FORME DE CHAR NIERE:

GENRE XXVIIL. QUEUES DE CHEVAL OU PINNES MARINES.

I409 P. Jaínbonneau, de coquille fillonnée, à écailles voutées, difperfées, rangées en fuites, Linn. 203. Seb. III. 9r. I: Gualth: 78. 1. A. Lift. 374.215. Argenv. 22. B. Ginann. II. 165. long. 16 $\frac{1}{2}$. lat. 7. pouc. (I. piec. I.

iqto $P$. Jambonneau peiené, la coquille de moitie, friées en long l'autre coté ridé de travers, $\dot{a}$ cotes plus minces, Linn. 264. Seb.III. 9I/3. Gualch.79. A. Lift. 370. 2 I0. Rumph. 46. I? (I: p. 2 .

I 4ir $P$. Jambonneau peigné, Semblable da la precedente, Linn. 264. (4. P 2.

ír2 $P$. Jambonneau écaillé, ou Noble, de coquille ftriée par ondes, à ecailles tubereufes caralée, un peu pointues, Linn. 265. Gualth. 78. B. Lift. 372. 213 . Ginann II. 166. (3.p.3. I 413 . Jambonneau pointué, $i$ coquille friée en long, d écailles concaves, ovales émorffés', Linn. 266. Lift. 373. 214. Argenv, 22. F. Petiv, Muf, 19. 14. long. 10 pouc. (1. p. 4 .

$\mathrm{I}_{4} \mathrm{I}_{4} P$. Jambonneau ailé, à coquille recourbée, ovale, noire; les écailles plus courtes, Linn. O. Seb. III.9r 2. Gualth. 81. A Rumph. 40. L? long. 9. lat. 6. pouc. ( I: $p$ 5. $1415 P$. Jambonneau ailé, Jemblable d la precedente, Linn. 0. (2. $p .5$.

1416 P. Jambonneau aile, Semblable d la precedente, mais petite, Linn. o. deux pieces, dont une eft pointue. (2. p. 4.5.

I 417. $P$. Jambonneau papiracé \& ailé, d coquille enflée, ünie érigée, coulée, Linn. 268. Seb.IIII. 92. in medio, Rumph. 49. N. Petiv Muf. 19. 7. (1. p. 6.

$4418 P$. Jambonneau papiracé \& ailé, femblable de la préced’́nte, plus incourbée, Linn. 268. (I, l. 6. 
1419 P. Saccata, pracedenti affinis, fed minor, Linn. 2 f 3. (2. fpecim. 6,

I420 P. Saccata, precedenti fimilis, magis plicata, purpu. rafcens, Linn. 268. (I. Specim.6.

1421 P. Papyracea, teftis elongata, angufta, albida, minus plicata, Linni o. Rumph. 46. K? (3. Tpecim. 7.

\section{GENVS. XXIX. TAMARIND-I F ORMES.}

1422 T. Lithophagus, tefta fubtenuis cylindrica, utrinque rotundata, \& transverfím ftriata, fofca, Linn. 248. Gualth.90.D. Lift. 47. 268. Rumph. 46. F. Kircher III. 27. (1. Tpecim. 8.

1423 T. Lithoglyphris, f. Lithophagus, pracedenti fumilis, fed minor, Linn. 248. (2. fpecim. 8

$1424 \mathrm{~T}$. Dendrites, tentà elongata, poftice eleva, antiçe plana, glabra, flavefcente, fusco maculata, utrinque marulis pieta ad dimidium; Linn. O. Davila 1. 19. Z. (1: Ypecim. 9.

1425 T. Pileatus, tefta natibus convesis, oblonga, antice planiufcula, transverfim rugofa, caftanea, Linn. o, Rumph. 46. C? (I. fpecim. 10 .

I 426 T. Striazus, tefta natibus convexis, rugofa cinerea, fasciîs fuscis L Linin D. Liff. 365. 205. Petiv. Gaz. 153. 3 ? (1. fpecim. II.

1427 T. Ziczac, tefta natibus convexis, antice plana, fusca, undatim ftriata, Linn. O. (1. fpecim. I2.

I428 T. Fafciatus, tefta natibus elevatis acutis, medio inflata, antice planior, fascia maculofa, fusca, Linn. o. Lift. 365. 205 ? (1. fpecim. I3.

1429-T. Rugofus, tefta, natibus inflatis, acutis, antice ungulata, planiore rugofa, rubra, Linn. O. Rumph. 46. C? Lift. 359. 198. (2. Specim, 14.

I 450 T. Phyrropecillus, tefta umbonibus fornicatis, colore oblique albo, vel purpureo, Linn. 256... f. o. Rumph. 46. B ? Petiv. Gaz. 7r. II ? Knory Conch. I. 15. 3. (3. ipecim. 15.

343 T. Modiolus, tefta lævi, margine anteriore carinato, natibus gibbis, Linn. 256. Lif6. 356. 195? 302. 200 , A. (1. Ppecim. '16.

$1432 \mathrm{~T}$. Arenarius, tefta in medio elevata, violacea albida, obliqua, Linn o. Lif. 1055. 0? Rumph. 46. B. Ar. genv. 22. C. (2. Tpecim. 17.

I433 T. Aremarius, fimilis pracedenti, Linn. O. Ellis tab. $\mathbf{T}$ $f: B$. duo fpecimina, quorum alterum tunicatum, tunica atra, Tubutis, Fucisgine obfitum. (2. Epecim: 17. 
I419 P. Jambonneau papyracé \& ailé, approckant à la precedente, mais plus petit, Linn 268 . (2. p. 6.

1420 P. Jambonneau papyracé $\&$ ailé, Semblable de la prece. dente, mais plus pelée, Ẽ pourprée, Linn. 268. (x.p. 6. $422 P$. Jambonneau papyracé ; $a$ coquille allongée, étroite. blancheatre, moins pliée, Linn 0. Rumph. 46. K? (3.p. ?.

GENRE XXIX. EN FORME DE TAMARINDE.

$\$ 422$ T. Date, à coquille, un peu mincs, cylindrique, de chaque coté ronde, E' ftriée de travers, brume, Linn:248. Gualth. 90. D. Lift. 427. 268. Rumph. 46. F, Kircher II. 27. (I. p. 8.

I423 T. Date, Semblable do la precedente, mais plus petite. Linn. 248. (2. p. 8.

- $224 T$. Agathée arborirée, de coquille élongée, par derriege - élevee, devant platte, pelée, jaunatre, tachetée de brun, pein. te de chaque coté jar des petites branches, vers la moitié. Linn. O. Davila 1. I 9. Z. ( I. p 9.

I425 T. Cabochon, coquille à fefjes recourbées, allongée, deyant platte, ridée de travers, chataigne, Linn.o. Rumph. 46. C? ( I. p. ro.

I 426 T. Striée, coquille à fe Jes recourbées, ridée, cendrée, a bandes brunes, Linn. O. Lift. 365. 205. Petiv. Gaz. 153. 3 ? (I. p. II.

$I_{427} T$. Zic-zac, coguille d fefes recourbèes, devant platte, brune, ftriée par ondes, Linn. O. (I. p. I2.

I4 428 . Bardée, coqulle à féfles élevees aigues, au milieu enflee, devant plus platte è bande tachetée, brune, Linn.o. Lift. 365. 205? (1. p. 13.

I429 T. Ridée, coquille à fe Jjes enfièes aigues, à devant en angles, ridée, rouge, Linn. 0. Rumph. 46. C? Lift. 359. 198. (2.p. 44 .

I430 T. Bahama rouge, coquille d boutons voutées, a couleur blanc, ou pourpré, Linn. 256. a. ou o. Rumph. 46. B? Petiv. Gaz. 71. I1 ’ Knorr Conch. V. 15. 3. (3. p. 15.

I43I T. Poire de Papoës, à coquille polie, le bord anterieur carine, les felfes boffues, Linn. 256. Lift. 356.195? 362. 200. $A$ ? $\quad(I: p .16$.

$\$ 432$ T. Sablonnier de Papoës, coquillé élevée au milieu, violette, blancheatre, oblique, Linn, 0. Lift. 1055.9? Rumph. 46. $E$. Argenv. 22. C. (2, p. 17.

I 433 T. Sablonnier de Papoës, Semblable d̀ la precedente. Linn. o. Ellis tab. I.f.B. deux pieces, dont l'une eft tuniquée. d tunique noire, couvert de petit Tuyaux de mer, $\varepsilon^{\circ}$ Branches, (2. p. 17 . 
\$434 P. Unbriculum, tefta valva, altera alba, glabra, intus a centro ftiiis elevatis, altera hepatica, ftriis obfcurioribus, Linn. 190. . f. ०. Gualth, 73. B. long. \& lar. 5. poll (I. fpecim. 18.

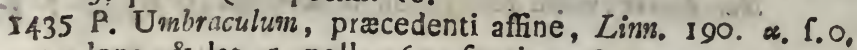
long. \& lat. 5. poll. (r. fpecin. I8.

I436 P. Anufium, f. Pleuronectes, fimile præcedenti. hepatica, fed minor; hic margo iliterius flavus, Linn. 100. a. Gualth. 73. B. Rumph. 45. AB. Argenv. 24. G. long. \& lat. $3 \frac{1}{2}$. poll. (2. ipecim. 19 .

I437 P. Amufium, præcedenti fimile, fed tefta valva altera pulcre coccinea, Linn, 190. $\beta_{\text {if. }}$ o. Kircher II. 108. (1. fpecin. 20.

3438 P. Amufium, fimile precedenti, fed tefta valva altera intenfior coccinea, ftriisque per paria digeftis, diaphanis; Limn. 192. \%, 1. O. Petiv. Gaz.49. 13 Muf. 17. 14. Knorr I. 20. 3? 4? (2. Specin. 21.

1439 P. Rutilus, tefta, valvis magis tumidis, utroque rofea. Linn. 0. Lift. 173. 10? Gualth. 73. C? Knorr $\dot{V}$. 21. 6. (2. fpecim. 22.

1440 P. Zicrac, tefta valvis fulcatis, ftriatis, maculatis; fubtus fusca, fupra pallidior, radiis obfcurioribus, Linn. 187. «. Knorr II. 12. 3, 20. I. (I. Specim. 23.

I44I P Ziczac, præcedenti affinis, interne magis grifea, fusca, \& minor, Linn. 187. a. ( 2 fpecim. 23 .

2442 P. Schediarius, tefta radiis rotundatis, fulcatis, color carneus, lineolis transverfalibus ftriata, Linas, o. Lift. 168.5? Petiv. Gaz, 153. 7. Knorr I. 14.3. (3. fpecim. 24.

I443 P. Maximus, tefta inæquivalvi, radiis rotundatis lóngitudinaliter ftriatis, coccinea fuperne, inferne fasciata, Limm. 185. Lift. 163. I, 167. 4. (I. Specim. 25.

I444 P. Facobers, tefta inæquivalvi, radiis I6. . plurimis angulatis, longitudinaliter Atriatis, valva coccinolla, Linn. 186. Lift. 165. 2. Kircher II. 3. (1. Specim. 26.

1445 P. Facobeus, præcedenti fimilis, fed minor, Linn. I86. Gualth,.99. A. B. (2. fpecim. 26.

I446 P. Nodofus, tefta inæquivalvi, radiis plusve minus 9. nodoro veficularibus, coccineo fusca, fubtus grifeo rubente, Linn. 194. Seb. III. 87. I-5. Lif. 186. 24. Argenv. 24. F. Rumph' 48. 7. 8. Kircher II. ${ }_{132,}$

is long. \& lat, 5. poll. (1. fpecim. 27. $\quad 1447 \mathrm{~Pa}$ 
GENREXXX. MANTEAUX。

$1434 M$. Parapluie Chinoife, à coquille, l'une valve blanche pelée, en dedans du centre à Jtries elevées, l'autre, couleur de foye, à ftries plus obfcures, Linn. 190. \&. 0u 0. Gualth. 73. $B$ ? long. Eे lat. 5. pouc. (I. p. 18.

$1435 M$ Parapluie Chinoife, approchant au precedent, Linn. 190 \&. ou O. long. EO lat. 5. pouc. (I. p. 18.

2436 M. Eventail farnée, femblable au precedent, hepatique, mais plus petit, le bord interieur jaune, Linn. 190. «. Gualth. 73, B. Rumph 45. A. B. Argenv. 24. G. long. E lat. $3 \frac{1}{2}$, pouc (2. p. 19.

$1437 M$. Evertail carmofin, femblable au precedent, mais la coquille à valve rouge, Linn. 19o. B. ou O. Kircher II. I08. (r. p. 20.

I438 M. Eventail carmolin, Semblable au precedent, mais la coquille ì valve, plus rouge, à firies diaphanes, rangès a deux, Linn. 190. y ou 0. Petiv. Gaz. 49. I3. Mur. 17. I4. Knorr I. 20. 3? 4 ? ( 2. p. 2I:

2439 M. Eventail pourpré. coquille plus enflée, chaque cous leur de rofe, Linn. o. Lift. I73. 10? Gualth. 73. C? Knorr V. 2 I. 6 (2.p. 22.

$\times 44^{\circ} M$ Benitier en Zic-zac, coquille d̀ valves fillonnées. Stries tachetées. deffus brune deffus plus pales à rayons, plus obfcures, Linn. 187. a. Knorr II. 19.3, 20. I. (I. "p. 23.

$144 \mathrm{I} M$. Benirier en Zic-zac, approckant au precodent, er dedans plus gris, brun, ED plus petit, Linn. 187. a. (2. p.23. $2442 M$ St. Jaques en Zic-zac, do coquille, t̀ rayons rondes, fillonnés, couleur de clair, ftriée de lignes transverfes, Linn 0. Lift. I68. 5 3 Petiv. Gaz. 153. 7. Knorr I. I 4.3. (3.p. 24.

I443 M. St. Jaque ployée, coquille d̀ valves, d̀ rayons rondes ftries, en long en haut rouges, en bas fascit, Linn.185. Lift. $163.1,167.4$. (2. p. 25.

$1444^{\circ} M$. St. Jaque pliffe, coquille is valve inégale d 16 rayons ou plufieurs ers angles, ftriés en long, à valve rouge, Lina. 186. Lift. 165.2. Kircher II. 3 (1. p. 26.

$1445 M$. St. Jaque pliffé, Jemblable au precedent, mais plus petit, Linn. 186. Gualth. 91. A.B. (2. p. 26.

$2446 M$. Coralline, à coquille d valve inégale, à 9. rayouns noeuez dे veficulaires, d'un brun rouge, defous gris roug eatre. Linn. 194. Seb. III. 87. I - 5. Lilt. 186.24. Argenv. 24. $F$. Rumph, 48.7.8. Kircher II. 132, long. 5. pouc. (I. P. 27. Dd 4 $1447 \mathrm{Ma}$ 
1447 P. Nodefus, fimile precedenti, Linn 194. Gualtb. 99. C D. long, \& iat. 5. poll. (I. fpecim. 27.

1448 P. Nodofus, fed verfus auriculam aurantius, Linn. 194. long. \& lat., $3 \frac{2}{2}$ poll (I. Specim 27 .

1449 P. Nodofus, fed rodis majoribus, Linn. 194, long. \& lat 3. poll. (I. fpecim. 27.

$1450 \mathrm{P}$. Radula, tefta fub æquivalui radiis circa 13 . convexis, ftriis decuffatis, crenatis, auriculis æqualibus, albicans, maculis fuscis, Linn, I9I Lift. x 75. 12. Argenv. 24.D. Gualth 74. L? Petiv. Mu/. 17. 1. (2. fpecim. 28.

I451 P Radula, præcedenti affinis, fed pulcrior maculata; Linn. 191. Knorr.V.9. 2. (2. fpecim. 28.

I452 P. Raduba, præcedenti fimilis, maculis varia, fupra \& infra, Linn. I9I. (2. fpecim. 28.

1453 P. Radula, præcedenti limilis, valvis atrofuscis, Linn. 191 Gualth 74. L? Lift. 170. 7. (1. Specim. 31

I454 P. Plica, latior, radiis pallidioribus 6. convexiusculis læviusculis, decuffato ftriata, maculis caftantis, Linn. 192. Gualth. 74. B ? C? Argenv. 24. C. Petiv. Muj. 2. 10. (3. Cpecim. 29.

1455 P. Venatrix, $\{$ varietas pracedentis, auriculis uno verfu concavis, radiis I 2. fusco $\mathrm{g}$ if a , flammis albis, Linn I I 9.2.

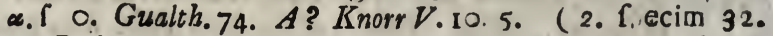

1456 P. Radulamus, radiis five plicis, 12 valvis albidis, fusco maculatis, Linn. 191. $\beta$ f.o Gualth. 74. E? Knorr. II. 10. I. V. 10. 6. (2. Specim. 33.

$I 457$ P. Glaber, radiis Ir. vix elevatis, citrea, Linn. 20 . Lift. 183 26. Gualth. 73. H. (1. fpecim. 30.

$1458 \mathrm{P}$. Cilicinus, tefta radiis pluribus quam 20 fulphurea, Linn. 197. B. f. O. Seb. III. 87. 13 ? Lift. 182. 19. Knorr. V. II. 3.VI. 19. 4. (3. fpecim 34.

1459 P. Cilicinus, radiis rudioribus, flavefcens, vel fangulneus, Linn. I97. \&. Rumph. 44. C? Gualth. 74 H? Argenv. 24. H? Petiv. Gaz 94. 2. (3. fpecim. 35.

$\$ 460$ P. Cilicinus, pracedenti fimilis, Linn. 197. *. (3. fpecim. 35.

I46I P. Obliteratus, radiis pluribus echinatis violaceo fuscus, Lixn. 197. Gualth. 74. G? Liff. 192. 29? (3. Spe. cim. 36.

1462 P. Obliteratus, præcedenti affinis, radiis pallidioribus, Striatus, fasciatus, Linn 197. (2. fpecim. 36.

${ }_{3463}$ P. Varius, tefta convexa, radiis ultra 20. echinatis alba, flammis majoribus fuscis, Linn. 199. «. Seb.III. 87. 6 ? Gualth. 73, G. Lift: 179. 16. (3. Ipecim. 37. 
1447. M. Coraline, Semblable au precedent, Linn. 194. Gualth; э9. C. D. long. E lat. 5. pouc. ( I. p, 27.

$1448 M$. Coraine, mais vers b'oreille plus orange, Linn. 194 long. Eे lat. $3 \frac{1}{2}$. pouc. (1.p 27 .

$1449 M$ Coraline, mais à roeuds plus grands, Linn. 1940: long. E lat. 3. pouc. (I $p 27$

I $450 M$. Gibeciere, coquille $a$ valves égales, ayant environs $\mathbf{r 3 .}$ rayons recourbées ftries croiffées, crenues, les oreilles èga-. les, blancheatre, à taches brunes, Linn. I9r. Lift. 175. 12. Argenv. 24. D. Gualth. 74. L? Petiv. Muf. 17. I. ( 2 p. 28.

I45I $M$. Gibeciere, approchant auprecedent, mais plusjolimens tacheté Linn. 19i. Knorr V. 9.2 (2. p. 28.

I4.52 $M$. Gibeciere, Semblable au precedent, variée de taches

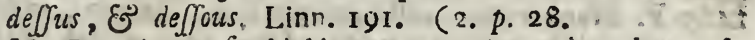

I 453 M., Gineciere, Semblable alb precedent, $d$ valves noirbrines, Linn. 191. Gualth. 74. L? Lift 170.7 (1.P 31. I $454 M$. Bandouiliiere, plus large, $a ̀$ 6. rayons plus pales, recourbées, polies, ftriée par croix, à taches chataignes, Linn. 192. Gualth. 74. B ? C P Argenv. 24. C. Petiv. Muf. 2. I0. (3. p. 29.

I455 M. Bandouilliere? ou varieté de la precedente, d oreilles ereufes, da 12 . rayons brun-grife à flammes blanches, Linn. 192 a. ou o. Guath. 74. $A$ ? Knorr V. In. S. (2.p.32. I450 M. Bandouilliere dे 12 . rayons ou plies, valves blancheatres, tachetées de brun, Linn. I9!. $\beta$. ou o. Gualth. 24. $E$ ? Knorr II. 10 I. V. :0.6. (2. p. 33.

$1457 M$. Peigne, da I I. rayons dे peine élevées, couleur de citron. Linn. 20r. Lift: 183. 26. Gualth. 73. H. (I. p. 30.

I $458 \mathrm{M}$. Velue . coquille à plus de 20 . rayons, couleur de fous: fre. Linn. 197. a. ou $\cap$. Seb. III. 87. I3 ? Lift. 18\% 19. Knorr. V. II. 3. VI 19. 4. (3.p. 34.

$1459 M$. Velue, à rayons plus rudes, jaunatre, ou couleur de fang, Linn. 197. \& Rumph. 44. C? Gualch. 74. H? AE. genr. 24, H? Petiv. Gaz. 94 . 2. (3. p. 35.

$1460 M$. Velue, femblable au precedent, Linn $197 \cdot \alpha .(3, p \cdot 35$.

$146 \mathrm{I} M$. Rayonné, à plufieurs rayons . couvert d'aiguillonse tran-violet, Linn. 197. Gualth. 74. G? Lift. 192. 29?

( 3 p. 36.

I462 M. Rayonné, d̀ rayons plus pales, ftrié, Ẽ bandé, Linq: I 97 . (2. $p$. 36 .

\$463 M Varié, de coquille recourbée, à plus de 20. rayons cour vert d'aiguillons, blanche, d feammes plas grandes brunes. Linn. I 99 \%. Seb. III. 87. 6? Gualth. 73. G. L.ịt.179. 16. (3. p. 37 . 
4464 P. Darius, præcedénti fimilis, fed minor, Linns. 199: «. Knorr V. I3. 2. (3. Specim. 37.

I465 P. Varius, fimilis præcedenti, fed major, Linn. 199. «. (3. Ipecim. 37 .

I466 P. Varius, præcedenti affinis, tefta flava, Linn. 199. $\beta$. Gualtb. 73. L Lift. 189. 23. (I. Tpeecim. 38.

$\$ 467$ P. Varius, præcedenti affinis, tefta rofea, obfcure violaceo flammata, Linn. 199. d. Gualth. 74. X. Lift. 185. 22?. (I. Specim 40.

468 P. Varius, precedenti fimilis, flavefcens, fasciis fuscis, Linn. 199. \& Gualth. 73. P. Lift.169. 6. Petiv. Muf. 17. 3. (2. โpecim. 39.

2469 P. Marmoratus, tefta convexiore, latiore, radiis ultra 20. violacea fusco varia, Linn. 199. ท. Seb. III.87. 9. Lift. 180. I>? Gualth. 74. G? Petiv. Muf. 17. 2. Valent. 26. Knorr II. 18. 3. (2. Specim. 42.

470 P. Marmoratus, præcedenti affinis, Linn. r99. ท. (3. fpecim. 42.

I471 P. Varius, tefta uniaurita, radiis 20. feu 30 fcabris compreffis echinatis, rubra, Linn. 199. Seb. III 87. 15? Liff. 175 ? infra, Rumph. 44. C. Argenv. 24. H. (2. ppecim. 4I.

$\$ 472$ P. Varins, pracedenti affinis, fed minor, Linn. 199. (2. fpecim. 4r.

I473 P. Varius, fimilis præcedenti, Linm. 199. (3. Specim: $4 \mathrm{I}$

$\mathbf{1 4 7 4}$ P. Opercslaris, tefta altera convexiore albida, Vermiculis obfita, Linn. 202. \&. (I. fpecim. 43.

I475 P Ruber, radiis granularis plus minus 30 . obfcure ruber, Linn. 199 и. Gualth. 74. A A? Lift. 181.18? Valent 26? Knorr I. 18.2. (3 rpecim. 44.

I476 P: Gibbus, tefta radiis ultra 20 , glabris, gibba, fanguinea Linn. 203. Gualth. 74. O? 77. D? Rumph. 1 44. C? Lift 182.19. Knory II 5.4, 17.2. (3 โpecim. 50. 477 P. Tslandicus, tefta radiolis innumer is, coccinea, Limn. 197. ๙. f. O. Seb. III. 87. 7. Gualth 74. T? long. \& lat. 4 poll. (1. rpecim. 49 .

478 P Tslandicus, pracedent1 fimilis, Linn. 197. \&. ᄃ. 0. Knorr I. 4. 1, 5. 2. V. 15. I. (I. fpecim. 49.

$1479 \mathrm{P}$. Ofercularis, tefta fuperiore, radiis pius 20 . glabris, rubro tlavo, fuscoque marmorata, altera flava, fasciis albis, Linn. 202. \& Gualth. 73. F? Seb. III 87.15? Valent. I. 2. Knorr II.3. 2.3,19.4. (I. Specim 48. $1480 P$. 


\section{Teftaces. Ordre $D$.}

464 M. Varié, Jemblable au precedent, mais plus petit, Linn, 199. «. Knorr $V$. 13. 2. (3. p. 37.

1465 M. Varié, Semblable au precedent, mais plus grand, Linn: 199. . $(3, p .37$.

I466 M. Varié, approchonit aus precedent, à coquille jaune, Lina: 199. B. Gualth. 73. L. Lift. I89. 23. (r. p. 38 .

$I_{467} M$. Varié, approchant au precedent, coquille couleur de rofe, flammee de violette, Linn. 199. d. Gualch. 74. $\boldsymbol{X}$. Lift. 185. 22 ? (1. p. 40 .

I468 M. Varié, femblable au preceitent,$j$ aunatre, $a$ bandes brunes, Linn. 199. \%. Gualth. 73. P. Lift. 169. G. Petiv: Muf. 17. 3. (2, . 3 .

1469 M. Marbré, à coguille plus courbée, plus large, à pluss de 20. rayons, varié de bruns violet, Linn I99. III. 87. 9. Lift. I80. I7? Gualth. 74. G ? Petiv. Mur. 17.2. Valent. 26, Knorr II 18.3 (2. p. 42 ,

I470 M. Marbré, Jemblable au precedẹnt, Linn. 199.:. (3.' p. 42.

$147 \mathrm{I} M$. Varié, ou Manteau rouge, coquille, à une oreille, d rayons au dela 20 . ou 30 . rudes comprimées, as aiguillons, rouge, Linn. I99 Seb, III:87. I5? Lift. 175? ixfra, Rumph. 4t. C. Argenv. 24 H. (2.p. 4x. $\mathbf{1} 472 \mathrm{M}$. Varié, approchant au precedent, mais plus petite, Lian. 199. (2. p. 41 .

I473 $M$. Varié, rouge, Jemblable au precedent, Linn. 199; (3. p. $4 \mathrm{I}$,

I474 M. Uperculé à coquille pilus recourbée, blancheatre, conzvert de Vermiffeaux, Linn. 202. $\alpha$. ( $:$. p. 43 .

I $475 \mathrm{M}$. Rouge, de plus ou moins de 30 . rayons granulées: d'un rouge foncé, Linn 199. \%. Gualth. 74. A A? Lif, I81. I8? Valent. 26 ? Knorr I. 18. 2. (3. p. 44.

I476 M. Adama, coquille à plus de 20 , rayons pelés, boffu, rousge, Linn. 203. Gualth. 74. O ? 77. D ? Rumph.44. C? Lift. 182. 19. Knorr II. 5.4, I 7.2. (3. P. 50.

$1477 \mathrm{M}$. d'Islande, Goquille rouge, \& rayons innombrables, Linn. 197. a. or 0. Seb. III. 87. T. Gualth. 74. T? long. EO lat 4. pouc. (I. p. 49

1478 M. d'Islande, femblable au precedent, Linn.197. \&. ou O. Koorr I. 4. I, 5. 2. V. I5. I. (r. p. 49.

1479 $M$ De Davids, coquille Juperieure, qui a plus de 20. rayons pelés, eft rouge, marbrée de jozune, EO brun, l'azstre efl jaune à bandes blanches. Linn. 202. $\alpha$. Gualth. 73. F? Seb. III. 87. 15 ? Valest. I. 2. Knorr II. 3. ? 3, 19. 4. (I. P. 48 . 
2480 P. Opercularis, fimilis pracedenti, glabra, carnea linels latis rubris, Linn. 197. \%. 202.. . Knorr I. 5. 2 V. 15. I. duo fpecimina, quorum alterum Ptet. Yslandicus, (2. fpecim. 48. 49.

I48I P. Pallium, tefta radiis I 2. f. I4. convexis, ftriata, fcabra, fquamis imbricata, undata, Limn. 193. Seb. III. 87. 8-10. Lift. 178. 15? Rumph. 44. B. Petiv. Gas. 62. 5. (2. Ipecim. 45 .

1482 P. Pallium, præcedenti affinis, Linn. 193. Argenv. 24. I. Gualth. 74. F. (2. Ipecim. 45.

148, P. Pallium, fimilis præcedenti, Linn 193. (2. Specim. 45. 1484 P. Pallium, præcedenti affinis, magis maculatus, Linn. 193, ( 2 fpecim. 45.

I485 P. Pallium, præcedenti affinis, fasciis transverfalibus, Linn 103. (3. fperim. 45.

1486 P. Pallium. præcedenti iffinis, radiis I2.glabris, inter quos ftriæ granulatæ. Linu. 193. *. Seb. III 87. 16. Lift 185. 22 ? Gualth. 73. N. Petiv. Gaz. 94. I. (I. fpecim. 46.

$\times 487$ P. Pallium, radiis I 4. fquamis erectis, albidus, maculis rubentibus, Linn. 193. $\beta$. f. O. Seb. III. 87. I 7, Lift. 183. 20. (1. Specin. 47.

1488 P. Lima, tefta æquivalvi alba, radiis plus ultra 22. im. bricatis fquamatis, altero margine rotundato, auriculis oblitceratis, Linn. 206. Lift. I 77 I 4 ? Kircher 1I. 69. Petiv. Muf. 8. 4. longit. $3 \frac{1}{4}$. latit $2 \frac{1}{2}$. poll. (I. Specim. $5 \mathrm{r}$.

1489 P. Lima, fimilis pracedenti, fed minor, Linn. 206. Rrmph. 44. D. Argenv. 24. E. (2. fpecim. 5i.

1490 P. Fasciatus, refta æquivalvis tumidula, radiis fcabris interftitiis ftriatis, auriculis æqualibus exoletis, Linn. 205 Gualtb. 74. EE, 88. FF. Lift. 176. 13. Knorr. VI. 38. 5. (2. โрecim. 52 .

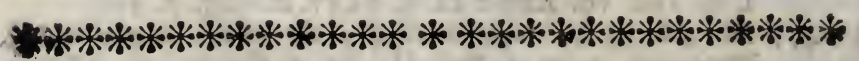

\section{O}

\section{$S E M I C A R D I N A T A$.}

G E N V S XXXI. OSTRE

1491 O. Amplettens, tefta alveolata, figura varians baculo inharente, Linn. 0. Gualth. 77. B. long, 9. poll. (1. โpecim. 55. 
I492 O. Maxillaris, tefta adhærens incurva angufta valva plana, echinata, alba, ftriis fordide violaces, Lin. .. (1. fpecim. 56.

4493 O Edulis. tefta inæquivalvi ferniorbiculata, membra. nis imbricatis, unduatis, valva alcera plana, integerrima, Linn. 211. Lift. 193. 30, 202. 36. A. Adanfon Seneg. 1. 14. 1. 4. (3. ípcim. 53.

च494 O. Edilis, cnngeries, Hippo jodis Cordi adnata, Linn. 211. «. Gualth. 102. A. D? (1. Cpecim. 54.

$\$ 495$ O. Philastropos, tefta fuborbiculata rugofa, plicata, planiore erfurata, Linn. 2:8. Lift. 204. 28. Argenv. I9. C. Kircher II. 5j. Ginanz. II. 17. (3. Specim. 57. $\$ 4960$. Cepa, tefta lævi obovat, inæquali, violacea, fuperiore convexa, inferiore perforata, Linn. 219. Liff. 205. 39. (2. fpecim. 58.

497 O. Placenia, tefta orbiculata plana, nitide pellucida, cardinum callis linearibus $\Lambda$ binis, teftæa adıatis ,Linn. $24 \mathrm{I}$. Seb.III. 9c. fupra ớ infra. Knorr II. 24.I. (2. f́pecim. 59.

\$498 O. Ephippium, non adultum, tefta tenuis xquivalvi, orbiculata compreffa minus incurvata, violacea, Linn. 215. «. Seb. III. 90. \&c. (2. Specim. 60.

$x_{499}$ O. Ephippium, tefta æquivalvi orbiculata, curva compreffa membranacea, cardium callis linearibus crasfi: $\Lambda$ binis, Linn.215. Seb.III 90. \&c. Gualth. IO2.B. Lift. 225. 60. Petiv. Muf. 20. 3. long. 6. lat. $7 \frac{3}{4}$. poll. (r. Ppecim. 6r.

$\mathbf{5 0 0}$ O. Ephippium, præcedenti affinis, fed non ita incurva, Lim 215. long. ultra plus 4. lat. minus 6. poll. (I. specim. 6I.

r5Or O. Foliata, tefta valvi laurifo!iis plicata, cinerea, intus fusca, Linn. o. Cusalth. 104. A. (1. fpecin. 62.

$\mathbf{1 5 0 2}$ O. Foliata, binx fibi adnatx, intus alba, Linn. O. ( 1 . fpecim. 62.

$I_{503}$ O. $\mathcal{J}_{w b a}$, fimilis pracedenti, rubefcens, Linn. o. Gualth. r04. F. Davila I. I $r$. continens duas adnatas in Aftroite. (1. Ipecim. 63.

1504 O. Gubcrnacula, fimilis præcedenti, fordide violacea, Linn. o. Gualth. 104. G? (2. Specim 64.

X505 O. Citrifolia, teft 3 valva convexa, g'abra, rugofa, cinesea, hepa:ica, Linn. O. Argenv. 19.F? Rumph. 46. O (1. Specim. 65. 


\section{Teftaces. Ordre VI.}

$1492 H$. Groffe Dent, coquille adherant, recourbée, étroite valve platte, unie, en aiguillons blanche, à ftries fale, violettes, Linn. 0 . (I. p. 56.

$1493 \mathrm{H}$. Ordinaire, coquille $\grave{a}$ valve inégale, demironde, membranes, en forme de tuilles, ondées, la valve platte entiere, Linn. 211. Lift.193.30, 202. 36. A. Adanfors Seneg. I. 14 , I. 4 . (3. p. 53 .

1494 H. Ordinaire, en Groupe, aljemblage de huitres, Baljin Capochon attaché, Limn, 2II, Gualth. 102. A. $D$ ? ( I. p. 54.

$2995 \mathrm{H}$. Pelure d'oignon, à coquille un peu ronde, ridée, plites platte, percée, Linn. 218. Lift. 204 28. Argenv. xs. C. Kircher II. 55. Ginnan. II. 17. (3. p. 57.

$1496 \mathrm{H}$. Pelure d'oignon, à coquille polie, un peu ovale, inégale, violette, la fuperieure recourbée, E' l'inferiears perforé, ou percée, Linn. 219. Lift. 205 39. (2. p. 58.

$1497 H$. Tranfparente, à coquille ronde, platte, reluifante. lescharnieres à deux lignes $\Delta$ innées. Linn. 24r. attaclec à ls coquille, Seb.1II.90./upra Ẽ infra, KnorrII.24.I.(2.p 50. $1498 \mathrm{H}$, Selle Angloife, imparfaite, à coquille mince, d̀ valve, égale, ronde, comprimée. moins recourbée, violette, Linn. 215. \& Seb III. 9o. Eंc. ( 2. p Go.

I $499 \boldsymbol{H}$. Selle Polonaife, coquille à valve égale, un peu row de, courbée, conrprimée, à membrane la charniere do 4. lignes, 2. epaifJes $\mathbf{A}$. Linn. 215 Seb. III. 90. \&rc. Gualth. 102 B. Lift. 225. 60. Petiv. Muf, 20. 3. long. 6. lat. $7 \frac{3}{4}$. pouc, (1. p. 6i.

I500 H. Selle Polonaife, approchant au precedent, mais pas $\mathrm{f}$ incourbée, Linn. 215. loing. plus de 4. large moins de 6. pouc. (I. D. 6r.

I50I $H$. Feuilleté, coquiile, $\dot{a}$ valves, en forme de baurier plie, cendré, en dedans brune, Linn. o. Gualth. I04. A. ( I p. 62.

$1502 \mathrm{H}$. Feuilleté, deux joints enfemble, blancbe en dedans Linn. 0. ( 1 , p. 62.

I503 H. Juba, ou Crin de Cheval, Semblable au precedent. rougeatre, Linn. O. Gualth. 104. F. Davila I. I9. X. une piece, qui contient deux autres preces attachées, y jointes, dans l'Aftroide. (I. P. 63 .

$1504 H$. Artaché au gouvernail, semblable au precedent, mais fale violette, Linn. o Gualth. 104. G? (2. p. 64

$1505 \mathrm{H}$. Feuille de Citron, l'une valve de la coquille eft re courbée, pelée, ridée, cendrée, couleur de foye, Linn. 0: Argenv. 19. F? Rumph. 46. O? (1. p.63. 
I506 O. Tranftra, tefta valvis ad dimidium rugofis /ceterum -planis, alba, Linn. 245. «. C. o Lift.197. 32. Argenv. 19. F. Knorr IV 30.4 quatuor ligno adnatæ. (I. fpicim 66.

$\$ 507$ O. Frons, tefta valvis granulatis, utrinque acuminatis antice rugofis petiolata, Linn.2 45. Rumph. 47. A. Pctiv. MuS.20. I.SpenglerIcon.I.H.Knorr I. 23. 2. (2 fpecim. 07.

c508 O. Crifta Galli, tefta plicata, labro atroque fcabro, Linn. 243. Gualth. 104. D. Argenv. 20. D. (I. Kpecim. 68.

7509 O. Crifta Galli, plicata purpureo cinerea, Linn, 243. Lift. 198. 32. a. Davila I. I9. X. (I. Specim. 68. a.

isıo O. Crifia Galli, præcedenti fimilis, Linn. 243. Gualth. 104. C ( I. Specim 68. b.

15II O, Gifta Galli, præcedenti fimilis, fed major, duß̂ connata, Linn. 243. Gualth. IO4. E. (I. fpecim. 69.

3512 O. Crifta Galii, fimilis antecedentibus, fed minor, Strombis dua adnatre Linn. 243. (x. fpecim. 69. a. I5I3 O. Crifta Galli, pracedenti fimilis, tres contuata, Linn. 243. Rumph. 47. D Argenv.20. H. (1. Ipecim. 70. 1514 O. Crifta Galli, pracedenti afti is, plicis altioribus, tres magna concretæ, Linn. 243. Peitiv. Muf. 20. 2. long. 5. lat. 3. poll. ( I fpecim. $7 \mathrm{I}$.

2515 O. Crifta Galli, præcedenti fimilis, fed minor, quatuor concre $\boldsymbol{x}$, Linn. 243. Knorr.V. 17. 1-3. (I fpecim. 71, a.

15I6 O. Crifla ramofa, an Hyotis, bubulis concavis, Linn. 244. \& 1. O. Argenv. Suppl. 3. F. Gualth. 103. C. long. 5. lat. 4. alt. $2 \frac{3}{2}$. poll. ( $\mathrm{r}$. Specim. 72.

$\mathbf{5} 5 \mathbf{1 7}$ O. Hyotis, tefta plicata imbricata, fquamis compresfis, patulis, labro Iævi, Linn. 241. a. Rumph. 47. ¿C. Inorr I.29. 1.2.IV.10.3-5.V:16. I. (I. Ipecim.72.a.

\section{GENVS XXXII. A R C E.}

1518 A. Tortuofa, tefta alba, parallellipeda, ftriata, valvu. Ia oblique carinata, natibus recurvis margine integer. rimo, Linn 168. Kircher II. I22. I23. Petiv. Muf. 20. 9. Argenv. 19. I. Knorr I. 27. 3. long. 3. lat. I $\frac{3}{4-}$ poll. (I. fpecim. 73.

r519 A. Tort:6ofa, pracedenti affinis. fed minor Linn. 168. Cualth. 95. B 1.2.3.Rumpir.47.K. (r. Rpecim. 73. a.

1520 A. Barbata, teita oblonga, ftriis barbata, natibus incurvis approximatis. ferratis, margine integerrimo claufo, albicans, Limm. 170. Argenv 22. M. Kircher II. 18. Knerr 11. 2. 7. ( I, Tpecim. 74. 


\section{Teftaces. Ordre $V I$.}

$1506 H$. Feuille de Laurier, coquille blanche, dont les vah ves à demi ridés, au refte, unies, Linn. 245.. ou o. Lift. 197. 32. Argenv. 19. F. Knorr IV.30.4. quatre font collés ou attackée au bois, (I. p. 66.

I507 $\mathrm{H}$. Feuille de Laurier, coquille à valves grenues, pointés de chaque coté, par devant rités, Linn. 245. Rumph. 47. A. Petiv. Muf. 20. I. Spengler Icon. I. H. Knorr I. 23. 2. . (2. p. 67 ,

I508 $\mathrm{H}$. Crete de Cocq, à coquille plicée, levre raboteux violet Linn. 243. Gualth. 104. D. Argenv. 20. D. (r. p. 68.

I509 $H$. Crete de Cocq, plicé, pourpré, cendré, Linn. 243. Lift. 198. 32. $a$. Davila $I$, 19. $X$. (I. p. 68. $a$.

$1510 H$. Crete de Cocq, Semblable au precedent, Linn. 243" Gualth. 104. C. (1. p. 68. b

I5 I $H$. Crete de Cocq, Jemblable au precedent, maisplus grand, Linn. 243. Gualth. ro4. E. deux joints enfembles. (1, p. 69.

$1512 \mathrm{H}$. Crete de Cocq, Semblable au precedent, mais plus petit, deux joints enfemble au le Vis, Linn 243. (I. p 69.

I5I3 H. Crete de Cocq, femblable au precedent, trois joints enfembles, Linn. 243. Rumph.47.D. Argenv.20. H. (1. p. 70.

$1514 H$. Crete de Cocq, Semblable au precedent, d plies plus profondes, Linn.243. Petiv. ivir. 20.2. trois grands collés enfemble; long. 5. lat. 3. pouc. ( 1. p. $7 \mathrm{I}$.

I 515 H. Crete de Coc4, femblable au presedent, mais plus petit, quatre joints enfemble, Linn. 243. Knorr. $V .17$. I-3. (1. p. 7 r.a.

I5 $16 \mathrm{H}$. Crete de Cocg, branchue double, pointée, avec des cavités, Linn. 244. $\alpha$. ols O. Argenv. Supp. 3. F. Gualch. 103. C. long. 5. lat. 4. altit. $2 \frac{1}{2}$ pouc. (r.p. 72 . I5I $H$.Crete de Cocq, à fimple branche, à coquille pliée, demironde, à ecailles comprimés, larges, d̀ levre unie, brune, Linn. 244. «.Rumph. 47.C.Knorr I.29.I. 2.IV.I0.5.V.I I I.I.(I. P.72 a.

\section{G. E N R E XXXII. A R C HES.}

1518 A. Torfe, à coquille blanche pararelle friée, la valve cariné oblique, à felJes recourbées, le bord entier. Linn. 168. Kircher II. 122. 123 . (Petiv. Muf. 20. 9. Ärgenv.

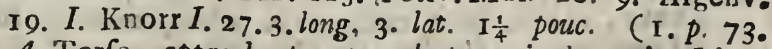
1519 A. Torfe, approchant au precedent, mais plus petit, Linn. 168. Gualth. 95. R. I. 2. 3. Rumph. 47.K. (1.p.73.a.

520 A. Barbué, à coquille allongée, blancheatre, barbue par des Atries, hes fefles recourbés, s'approchantes, ferrés, EO bord entier \&o fermé, Linn. 170. Argenv. 22. M. Kircber II. 28, Knorr $11.2 .7 \cdot\left(2 . p .74 . \quad \mathrm{E} \mathrm{e}\right.$ I52I $A_{0}$ 
I521 A. Angulata, an Modiolus? tefta antice angulata, oblon. ga firiata, albefcente, Linn. 17 I \%. .. O. Lift. 233. $67,367.207$. Adianfon Seneg. I. 18. 6. 9. (I. Specim. 75 .

I522 A. Gramulata, tefta oblonga, tumida transverfim gra. nulata, fusca, Linn o. Seb. III. 88. 13. Lift. 231.65, Davila I. 7. R. $(2$ fpecim. $i 6$.

1523 A Gramulata, fimilis pracedenti, fascia obliqua alba, Linn. O. Gualth. 90. B. Ginnen. II.. 160. (2. Specim. 76 .

1524 A. Noë, tefta oblonga, ftriara, apice emarginata, natibus ferratis, incurvis remotiffimis, unargine biante. Linn. 169. Rumph. 44. P. L? Argenv. 23. G. Liff. 368. 208. Petiv. Muf. 17. 6. 10. (2. fpecim. 77.

1525 A. Nö̈, fimilis præcedenti, fed minor, Linn. 169. Kircher II. 3r. (2. Specim. 77

1526 A. Antiquata, tefta oblique cordata, multifulcata, fulcis muticis, natibus recurvis, margine, crenato, Linn. I 74. Lift. 230. 64. a, 232. 66. Rumph. 44.I. (I. Ipecins.78.

1527 A. Granofa, tefta fubcordata, fulcis muricatis, natibus recurvis, margine crenulato, lactea, Linn. I $76 . L_{2} / l_{0}$. 234. 68, 242. 79. Argenv. 23. C. Rumph. 44. K. (1. ipecim. 79.

$\mathbf{I 5 2 8}$ A. Granofa, præcedenti fimilis, fed minor, Linn. $\mathbf{1} 76$. Kircher II. 74. (2. fpecim. 79.

1529 A. Senilis . telta oblique cordata, octofulcata lavi, natibus recurvis, margine plicato, Linn. 175. Argenv. 23. K. Rumph. 44. N. Lift. 244. 75. (1. fpecim. 81. 1530 A. Cor, tefta trigona, altero latere tumida, altera angulata, fulcato ftriata, alba, Linn. 175. a. f. O. Gualth.87. D. Lift. 238. 72. Knorr IV.14. 2, 24.2. (2. fpecim. 80.

1531 A. Flammata, an Undata? tefta orbiculata, natibus approximatis, glabra, a!ba, flavo undulata, Limn. I 79. «. Gualth. 72. G. Petiv. Gaz. 79. 7. Knorr V. 30. 3 ? VI. 12. 4. (2. Cpecim. 82.

1532 A Coftata, an Pefsunculus? tefta orbiculata, vix convexa, coftis, plus minus 16. alba, maculis fuscis, Linn. 18 * Gualth. 72. H. Lift. 229. 75' Kircher II. 129. Knorg I. 21. 4. II. 23. 6. NI. 12.4, I4. 3. (I. Ipecim. 83. 
7521 A. Angle, ou Modiole, \& coquille par devant, en 40 gles, ftriée, blancheatre, Linn. I7 I. a. ou O. Lift. 233. 67. 367.207. Adanfon Seneg, I. 1 8. 6. 9. (i. p. 75.

$1522 A$. Granulé, d̀ coquille allongée, enflee, granulé de trayers, brune Linn. O. Seb. III. 88. 13. Lift. 231. 65. Davila. I: 7. R. (2. p. 76 .

$1523 A$. Granulé, femblable au trecedent, à faccie oblique blanche, Linn. O. Gualth 90. B. Ginanni II. 160. (2. p. 76,

$1524 A$. de Noë, a coquille, Jtrié, Sommet ebordé, les feffes recourbés. fort feparé l'un de l'autre, $a$ bord entre ouvert, Linn. 169. Rumph. 44. P. L? Argenv. 23. G. Lift. 368. 208. Petiv. Muf, 1 7. 6. I0. (2.p. 77.

1525 A. de Noë, Semblable aus precedent, mais plus petit. Linn. 169. Kircher II. 31. ( 2 p. 77,

I526 A. Antique, ou faux Arche, coquille oblique, en forme de coeur, à plufieurs. feuilles, fillons fans barbes fe fes recourbées, bord crenus, Linn. I 74. Lilt. 230. 04. a. 232. 06. Rumph. 44. I. (1. $p 78$.

$1527 A$. Corbeille grenu, coquille prefque en coeur, fillons pointées, felfes recourbèes, le bord crenule, blancheatre. Linn. 176. Lift. 2 34. 68, 242. 79. Argenvv. 23. C. Rumph. 44. K. (1.p. 79.

1528 A. Corbeille grenue, Jemblable au precedent, mais plus petit, Linn. 176. Kircher. II. 74 . (2. p. 79.

$1529 A$. Pliffé large, ou petit Coeur de Boeuf, $d$ coquille oblique en coeur, a huit fillons, polie, les felfes recourbées. le bord plié, Linn. 175. Argenv. 23. K. Rumph. 44. N. Lift. 244. 75 . (1. p. 81 .

$1530 A$. en Coeur, à coquille triangulaire, de l'autre coté, enfiée', l'autre en angles, fillonne, ftrié, blanc, Linn. 475. a. ou O. Gualth. 8 7, D. Lift.238. 72.Knorr IV. 1 4. 2,24.2. (2.p.80.

1531 A. Flammée, ou Ondée? à coquille orbiculaire (ronde) les felfes approckantes, peiée, blanche, ondée de janne, Linn. 179 \& Gualth. 72 G. Petiv. Gaz. 79. 7. Knorr. V. 30. 3? VI. I2. 4. (2. p. 82 .

I532 A. Coftée, ou faux Arche pliffée? du coquille orbiculaire, d peine recourbée, da 16 cotes, plus ou moins, blanche, taches brunes, Linn. 180. a. Gualth. 72. H. Lift 229. 75 ? Kircher II. I29. Knorr I. $21: 4 . I I .23,6 . V I .12$. 4. 14. 3. (1. p. 83 . 


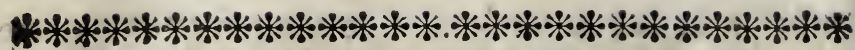

O

$\begin{array}{llllllllll}C & A & R & D & I & N & A & T & A \text {. }\end{array}$

GE N V S XXIH. HI P P O PODES.

$1533 \mathrm{H}$. Cor, tefta fubrotunda, lævi, natibus recurvis, rima hiante, flava, Linn. I 54. Rumph. 47. 10? Lift. 275. III. Argenv: 23. K. long. \& lat. 3. poll (I. Ppecim. 84 .

1534 H. Cor, pracedenti affinis, fed poliendo alba, Linn. 154. Gualth. 71 E. Ginann. II. 129. (I. Specim. 84.

$1535 \mathrm{H}$. Cor, timilis pracedenti, fusca, Vermicubis obfita, Linn. 154. Kircher II. 92. (1. fpecim. 84.

1536 H. Lacteus, tefta orbiculata, convexa, lactea, natibus incurvis, fine nota cardinis, Linn. 65. a. Lift. 261.97. Gualth. 7i. D. Valent. 27. (2. Tyecim. 85.

1537 H. Semiontrulatus, tefta femiorbicularis compreffa, rudi, decuffatim ftriata, caftanea, a natibus oblique alba, Linn. I59 Lift I057. 5. Valent. 27. 28. long. 3. lat. 2. poll. (r. fpecim 86.

1538 H. Semiorbiculatus, præcedenti affinis, fed polita, Linm. 159. Knorr II. 23. 7. (1. Specim. 86.

$1539 \mathrm{H}$. Calyculatus, tefta oblonga, fulcis imbricatis antice retufa, alba, fuscis maculis punetata, Linn. 160. Lift. 347. 184. Gualth. 90. F. Adanfon Seneg. I. 15. 3.8. (2. Specim. 87.

$1540 \mathrm{H}$. Glans, an præcedenti affinis ? tefta oblenga convexa, oblique carinata, ftriata, fulphurea, Linn. I6c. «. f. o. Lift. 347. 185? Krorr II. 20. 3. (1. [pecim. 88.

I54I H. Antiguatus, tefta fubcordata, itriis transverfis, fulcis longitudinalibus, fusca, farciatim albo maculata, Linn. I 57. a. Gualth. 83. D. Liff. 348. I8. infra, Valent. 30? Knorr VI. 14. 2. ( I. Specim. 88*.

1542 H. Equimus, tefta plicata, muricata, ano retufo claufo, alba, ftriis coccineis, Linn. 156. Lift.349. 187,350. 188. Argenv. 23. H. Petiv. Muf. 18. 15. Hollar Icon. 49. (2. Tpecim. 89

1543 H. Equinus, præcedenti affinis, fed minor, Linn. 156. Rumph. 42. C. (2. f́pecim. 89. 


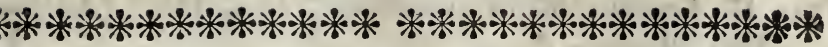

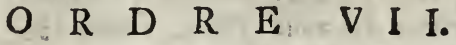

$\begin{array}{llllllllll}A & C & H & A & R & N & I & E & R & E \text {. }\end{array}$

GENRE XXXIII. BASSINS OU LAVOIRS.

533 B. Coeur de Boeuf, ou Cabochon, à coquille un pere ronde, polie, les feffes recourbeses, is fente entre ouverte, jaune, Linn. 154. Rumph. 47. 10? Lift. 275. I I I. Argenv. 23. $K$. long. E lat. 3. pouc. (I. p. 84.

534 B. Cabochen, approchant au, precedent, mais blanche, polie . Linn. 154. Gualth. 7t E. Ginann. II.129. (1 p. 84. $B$. Cabochon, femblable au precedent, brun, couvert de Vermiffeaux, Linn. 154 Kircher II.92. (I.P. 84.

536 B. Blanc, à coquille orbiculaire, recourbée, blanche, les felfes recourbêes, fans marque de charniere, Linn. 65. Lift. 261. 97. Gualth. 71. D. Valent. 27. (2.p.85.

$537 B$. Feve, ou Haricot brulée, à coquille demi orbiculaire, comprimée, rude, friée en forme de croix, chataigne, \& feffes obliques, blanche, Linn. I59. Lift. IO57.5. Valent. 27. 28 . long. 3. lat. 2. pouc. (1. p. 86.

538 B. Feve, ou Haricot brulée, approchant au precedens, mais polie, Linn. 159. Knorr II. 23. 7. (1. p. 86 .

$539 B$. Gland pliffe, de coquille allongée, d̀ fillons en forme de tuiles, par devant emou/fée, blanche, pointée, à taches brunes, Linn. 160. Lift. 347. 184. Gualth. 90. F. Adanfon Seneg. I. I5. 3. 8. (2.p 87 .

$540 \mathrm{~B}$. Gland ftrié, approchant au méme? à coquille allongée, recourbée, carinée de travers, ftriée, couleur de foufre, Linn. I 60. $\propto$, ou 0. Lift. 347. 185 ? Knorr II. 20. 3 . (I. p. 88.

54 I $B$. Noix de mer, à coquille en coeur, d ftriés transverJes, fillons longues, brune, E tacheté de blanc, par bandes, Linn. 157. $*$ Gualth. 83.D. Lift. 348. 18. infra, Valent. 30? Knorr VI. 14. 2. (I. p. $88 *$.

¡42 B. Faitiaire, ou feuille de Choux, à coquille pliée, pointée,' l'anus emouffé \&o fermé, blanche, d ftries rouges, Linn. I56. Lift 349. 187, 350. 188. Argenv. 23. H. Petiv. Mur. 18. 15. Hollar Icon. 49. (2. p. 89.

$543 B$. Faitiaire, ou feuille de Choux, approcbant au precedeat, mais plus petit, Linn. 156. Rumph. 42.C. (2. p. 89: 
1544 H. Equinzss, fimilis præcedenti, Linn. 156. (2. Cped cim. 89.

1545 H. Gigas, tefta plicata, fornicata fquamofa, ano hiante crenato, rubra fquamis albis, Linn. 155. Gualth. 22. E? (2. Tpecim 90 .

$1546 \mathrm{H}$. Gigas, pracedenti affinis, fed multo major, albus, 1 Linn. 155. Gualth. 93. B. Lift. 352 189 b. Petiv. Muf. 7. 13. Argenv. 13. E, (I. fpecim. 9,0. c.

$1547 \mathrm{H}$. Gigas, fimilis præcedenti, fed minor rubro albi-l cans, Lirn. 155. Gualth. 92. C. F. Lift. 351. 189. Kircher II. 82. (2. Ifecim. 90. d.

$1548 \mathrm{H}$. Gigas, præcedenti affinis, fed minor \& latior, Linn. 155. (2. fpecim. 90, e.

1549 H. Gigas, fimilis, aliquanto latior, brevior, flavo al- I? befcens, Linn. 155. a. Gualth. 92. A. B. (2. fpe. cim. 90. f.

$\$ 550 \mathrm{H}$. Scutra, latior, plicis pluribus, albus, Linn. $\times 55 . \beta^{\text {is }}$ f. O. Gualth. 92. E. Lift. 354 I9I. (2. fpecim. 90.a

$3 \$ 5 \mathrm{IH}$. Scaphe, triqueter, antice rotundatus, ftriatus. fqua mis nullis, Limn. I55. भ. f. O. Gualth. 92. G? Lifl 353. 190. Rumph. 42. B ? (2. fpecim. 90. b.

GENVSXXXIV. MACEROPHYLLA.

I552 M. Gryphoides, tefta orbiculata, muricata, val vula al tera planiore, altera nate productiore, fublpirale, qua tuor connatæ, purpurefcens, Linn. 165. Seb. III. 89 12. Gualth. 101. C.D. Lift. 217. 53. (1. [pecim. $9 \mathrm{r} / \mathrm{IS}$

I553 M. Gryphoides, precedenti affinis, purpurafcens, tri horum Arco connata, Linn. 165. Lift. 212. 47. Knor I. 8. r. (1. fpecim. or.

I554 M. Gryphoides, fimilis præcedenti, continens duas $\mathrm{Ma}$ drepore innatas, cum Vermiculis, \& Wltrea Eduli, Linn. 165. (1. Ipecim. 91.

1555 M. Gryphoides, pracedenti affinis, alterum Madreport adharens, Linis. 105. (2. ipecim. 91.

$3556 \mathrm{M}$. Gryphoides, fimilis, in ramulis Madrepora Afroti Linn. 165. (3. Tpecim. 9r.

1557 M. Gryphoides, fimilis pracedenti, fquamis anguftior bus, longioribus, fulphurea, Linn. 165. Seb.III. 88. \& Liff. 216. 50-52. Knorr 1. 21. 2. ( 1. fpecim. 92.

$7558 \mathrm{M}$. Gryphoides, præcedenti affinis, fquamis longior bus, fulphurea, a nate alterum Lapillo per extenfionen Linn. 165. Knorr V. 14. I. (2. Specim. 92. 


\section{Tefaces. Ordre VII. $\quad 43 \mathrm{I}$}

544 B. Faitiare; ou feuille de Choux, femblable au prece dent, Linn. 156. (2.p 89.

:545 B. Tuilé, d̀ coquille plicée, vouté en ecailles, l'anus entreouvert, cremu, rouge, d ecailles blanches, Linn. IS5. Gualth. 92. E.? (2. p. go.

i546 B. Tuilé, opprochant au precedent, mais beaucoüp plus grand, blanc, Linn. 155. Gualth. 93. B. Lift. 352. 189. b. Petiv. Mur. 7. r3. Argenv. 23. E. (1. p 90, $c$.

[547 B. Tuilé, Semblable au precedent, mais plus petit, d'un rouge blancheatre, Linn. 155. Gualth. 92. C. F. Lift.351. 189. Kircher II, 82、 (2. p. 90.d.

1548 B. Tuilé, femblable au precedent, mais plus petit, E plus large, Linn. 155. (2. p.90, e.

i 549 B. Tuilé, Semblable, tant foit peu, plus largè, Ẽ plus courte, d'un jaune blanchèatre, Linis. 155. $\alpha$, Gualch.92. A.B. (2. p. 90. $f$.

$1550 B$. Tuilé ventrue, beaucoup plus large, blanc, à plufieur's plies, Linn. 155. $\beta$ ou 0. Gualth. 92.E. Lift. 354.191. (2. p.,90.a.

I55I B. Platte triangulaire, par devant arrondie, Strie, faǹs ecailles, Linn. 155 $\gamma$. ou 0. Gualth. 92. G.? Lift. 353. 190. Rumph. 42. B.? (2. p.-90. b.

GENRE XXXIV. GATEAUX FEUILLETÉS. 1552 G. Griffe, ou Bec Crochu, à coquille ronde, pointée, l'une valveplus platte, l'autre à fe fje plus prominente, un peuspirale, quatre joint enfemble, pourpré, Linn. 165. Seb. III. 89. 12. Guatth. 101. C. D. Lift. 217. 53. (1. p. 41. 1553 G. Griffe, ou Bec Crochu, approchant au precedent, pourprée, trois Aiches Sont joints enfemble, Linn. 165. Lift. 21 2. 47. Knorr I, 8. I. (I. p. 91 .

1554 G. Griffe, ou Bec Crochu, Semblable au précedent, contenant deux Madrepores, ayec de' Vermifeaux \& $l^{\prime}$ Huitre Ordinaire, Linn. 165 . (1. p. 9I.

I555 G. Griffe, ou Bec Crocbu, approchant auprecedent, deuse pieces, dont l'une eft attachée au Madrepore, Linn. 165. (2. p. gr.

1556 G Griffe, ou Bec Crochu, Semblable au precedent., deux dans les rameals du Madrepore Aftroide. Linn. 165. (3.p.91. $1557 \mathrm{G}$ Griffe, ou Bec Crochu, Semblable auprecedent, a ecail. les plus etroites \& plus longes, couleur de foufre, Linn. I65. Seb. III. 88. 8. Lift. 216. 50-52. Knorr I. 21.2。 (I. p. 92.

$1558 \mathrm{G}$ Griffe, ou Bec Crochu, decailles plus longues, cous leur de foufre, aux felfes attachée, accrocbue d une pierre par extenfion, Limn. 165. Knorr. V. 14. T. (2. p. 92.

$$
\text { E e } 4
$$


1559 M. Acanthina, fquamis aculeatis triquetra, rufa, Linn: 165. a. f. O. Gualth, 101, L. Lift. 213. $48,320.157$. (3. Specim. 93.

I560 M. Acanthina, oblonga, alba, aculeis rubris, Linn. 165. ß. f. о. Gualth. 10I. E. F. Knorr VI. I6. I? (2. specim. 93. a.

1561 M. Lazarus, tefta imbricata, ramofa, lamellis laceris, nate obliqua, fublpirali, flavefcens, Linn. 164. Seb.III. 89. 6. Valent. 4. long. lat. $2 \frac{1}{2}$. poll. (I. Ipecim. 94. a. 1562 M. Lazarus, pracedenti affinis, Linn. I64. Seb. III. 88. 12. Rumph. 47. C. Argenv. 30. F Petiv. Muf.20. 5. long. \& lat. 3. poll. (I. fpecim. 94. b.

1563 M. Lazarus, pracedenti fimilis, luteo carnea, Linn. 164. Seb. III. 89. II. Rumph. 48. 3. long, $2 \frac{i}{2}$. poll. (I. fpecim. 94. d.

I564 M. Lazarus, præcedenti affinis, lamellis tenuibus, Linn. I64. Set. III. 89. 9. Valent, 22. long. $2 \frac{1}{4}$. poll. (1. fpecim. 94. c.

1565 M. Lazarus, fimillima pracedentibus, fed minor, val- Vula una latifolia, altera anguftifolia, alba, ftriis coccineis. Linn. 104. Argenv. $20 . K$. Valent. 22. (2. Ipe94. e.

1566 M. Lazarus. pracedenti affinis, foliis dilatatis, Linm 164. long. $2 \frac{3}{4}$. poll. (I. fpecim. 94. f.

$2567 \mathrm{M}$. Arcinella, tefta fulcata, muricata, excavato punctata, alba, Linn. 167. Davila I.17. T. t. KnorrVI. 36.1.2. (I. fpecim. 95.

\section{GE N V S XXXV. S P O ND Y L I.}

1368 S. Gaderopus, tefta convexa, longitudinaliter plicata, feriebus ramulorum fex carmineis Linn. 151. a. Seb. III. 87. I I. Gualth. IOI. A. Argenv. 19. G. Rumpb. 48. 2. Valent. 24. (2. Ipecim. 96

1569 S. Gaderopus. finilis præcedenti, ramulis albis, valvis carmineis, Linn. I51:-a. Seb. III. 88. 11. Gualth. 99. G. Li/t. 2C6. 40 ? Knorr VI.9. 2. (1. Rpecim. 108.

1570 S. Gederopus, affinis pracedenti, plicis ftriatis, ramis albis. Linn. I5I. a. Sebv III. 87. 10. Knorr I. 2. 2. (3. Specim. 98.

157 I S. Surculofus, foliis linearibus maci fimilibus, flavis, valvis purpureis, Linn. 151. b. Seb. III. 89. 5. Rumph. 47 E. Argenv. 20. M. Petiv. Muf. 20. 6. (2. fpecim. 97. 


\section{Teftaces. Ordre VII.}

1559 G.Acanthine a ecailles aigues triangulaires, rorige, Linñ. $165-$ a. ou o. Gualth . 101. L. Lift. $213.48,320.157$. (3. p. 93.

$1560 \mathrm{G}$. Acanthine, allongé. blanc, à aiguillons rouges Linn. 165. B. 0u 0. Gualth. 101. E. F. Knorr VI. 16. 1? ( 2 \% p. 93 a.

1561 G. Lazare, coquille, en forme de tuilles, branchue, \& lamelles deckirées, la felfe oblique, un peu fpirale jaunstre, Linn. I64. Seb. III. 89. 6. Valent. 4. long. 4 lat. $2 \frac{2}{2}$. pouc. (I. p. 94. a.

I562 G. Lazare, Semblable au precedent . Linn. 164. Seb III. 88. I2. Rumph 47. C. Árgenv. 20. F. Petiv. Mür. 20, 5. long. Eg lat. 3 pouc. (1. p.94. b.

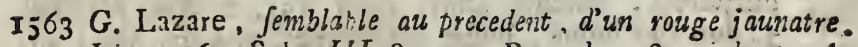
Linn. 164. Seb. III. 89. 11. Rumph. 48. 3. long. $2 \frac{1}{2}$, pouc. (I. p. 94. $d$.

1564 G. Lazare, femblable au precedent, à lamelles minces Linn. 164. Seb. III.89. 9. Valent. 22. long. $2 \frac{1}{4}$ pouc. (x. p. $94 . c$.

I565 G. Lazare, tres Jemblables aux precedens, mais plus petit, l'une valve à large feuille, l'autre à etroite feuille, blexs? che, a ftries rouges, Linn. 164 . Argenv, 20. $K$ V'alent? 22. ( 2.' p. 94, e.

I $506 \mathrm{G}$. Lazare, fort Sembiable au precedent, à feuilles eters

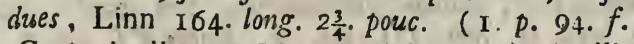

$1567 \mathrm{G}$. Arcinelle, or Marron epineux, à coquille fillannée. pointue encavé, blanc, Linin. 16\%. Davila I 17. T. Knorr VI. 36. I. 2. (I. p. 95.

GENRE XXXV. CLIQUETTES.

1508 C. Feuilleté, à coquille recourbée, plicẻe en loigeur. à fise fuites de branches carmoifies, Linn. 151.a. Seb. III. 87. 11. Gualtb. 101. A. Argenv. 19, G. Rumph. 48. 2. Valent 24. (2. p. 96.

I569. C. Feuilleté, femblable au precedent, d brancbes blanches Eo valves rowges, Linn. 15i. a. Seb. III. 88. 11. Gualth: 99. G. Lift 206. 40 ? Knorr VI. 9. 2 (1. p. 108.

$1570 \mathrm{C}$. Feuilleté, approchant ais precedent, à plies friées, branchesblancbes, Linn. I I I. a.Seb. III. 87. 10. Knorr $\boldsymbol{F}_{3}$ 9. 2. (3. p. 98 .

$157 \mathrm{I} C$. Feuilleté, ou Branchue, à feuillies lignées, fèmblable au maci, à valvespourpiées, Linn.151.b. Seb.III.89. 5. Rumph. 47. E. Argenv. 20. M. Petiv. Mur. 20. 6. (2.p.97. E e 5 
1572 S. Fimbriatus, ramulis, acutis pluribus, valvis purs pureis albisque, Linn. I5I. C. Seb. III. 87. 7 ? Knorr $V I$ 9. 2. (2. fpecim. 102.

5573 S. Fimbriatus, præcedenti affinis, ramulis albis paucioribus, Linn. 151. c. Seb. III. 87. 7. (2. Specim. 104.

I574 S. Fimbriatus, fimilis præcedenti, Linn. 151. c. Seb. III. 89. 7. (2. fpecim. I05.

2575 S. Fimbriatus, præcedenti affinis, valvulis albo fuscoque punctatis, Linn. 15I. C. Argenv. 20. 1 duo Ariftroite adhærent, Knorr V. 13. 1. (3. Specim. 106.

$1576 \mathrm{~S}$. Fimbriatus, præcedentibus fimilis, valvis albis, ftriis purpureis, ramulis albis, Linn. 15I. c. Seb. III. 88. 5. duo fpecimina, unum Madrepora, alterum Silici adharens. (2. fpecim. 107.

4577 S. Araufracus, feriebus ramulorum, plus minus quin. que, totus aurantius, Linn. 15I. c. d. Seb.III. 87. 3. 4. 7. Lift. 206. 40. Argenv. 23. B ? conftans ex triga, quorum duo colore! variant, Silicique inhserent. ( $\mathrm{I}$. Ipecim. 100.

1578 S. Araufiacus, precedenti affinis, Linn. 151. d. Seb. III 87.5. Rumph. 48. I. Valent. 24. Knorr I.6.3 , 7.2 ? III. 6. 1. (2. Ipecim. IOr.

2579 S. Araufiacus, fimilis pracedenti, fed paulo minor. Linn. 15 1. d. Seb. III. 87. 5. Knorr VI. 12. 3. (2. fpecim. IOI. a.

I580 S. Princeps, tefta valvis gibbis, convexis, echinatis plicatis, plicis elévatis, ramis longis incurvis, fubtus concavis, purpureis, Linn. 15r. e. Rumph, pag. 156. $n^{\circ}$ 8, Petiv. Gaz. 23. I2? ad extremitatem ramorum. long. \&lat. $7 \frac{1}{4}$ poll. alt. $3 \frac{1}{2}$. poll. (1. \{pecim. 126.

I58I S. Depreflus, tefta depreffiore echinata, ramis longioribus, Linn. I I r. f. Petiv. Gaz. 23. I 2. Knorr V. I. 9. long. \& lat. 4. five $3 \frac{2}{2}$. poll. (I. fpecim. 127 .

1582 S, Ramofus, tefta ramis longioribus incurvis, fubtus concavis, valvula fuperiore rubra, inferiore flava, Linn. 151, g. Gualth 101. B Rumph.48. I? Argenv. I9. H? Spengler Icon. II. B. long. $5 \frac{1}{2}$. latit. $4 \frac{1}{2}$. poll. (I, Specim 114.

7583 S. Ramofus, præcedenti affinis, ramis linearibus longis, valvula cornea, inter ramos, ramuli Madrepora cernuntar, valvula inferiore thava, Linn. 151. g. Seb. III. 87. 3. Gualth, 101. B. long, \& lat. $4 \frac{1}{2}$. poll. (1. fpecim. I03. 


\section{Teftaces. Ordre $P$ f.}

$\mathrm{I572}$ C. Frange, ou Applatti, approchant aus precedent, \& plufieurs branches aigues, valves pourprées E blanches, Linn. 151. c. Seb. 1II. 87. 7.? Knorr VI. 9.2. (2.p. 102.

I573 C. Frangé, ou Applatti, Jemblable au precedent, à quelques branches blanches, Linn. 15I.C. Seb.III, 87.7. (2.p. 104

1574 C. Frangé, ou Applatti, femblable au precedent, Linn. 151.c. Seb. III. 89. 7. (2. p. 105.

I575 C Frangé, ou Applatti, approchant au precedent, les valves poirtées de blanc, E brun, Linn. I51. C. Argenv. 20.I. trois pieces, dont deux Jont attachée d l'Aftroite, Knort $V_{0}$ 13. 1. (3. p. 106.

I576 C. Frangé, ou Applatti, fermblable? aux precedens, id valves blanches, ftries pourprées, branches blanches, Linn. 15t. c. Seb. III. 88. 5. deux pieces, l'une attachee, wi Madre: pore, l'autre ar Silice. (3. p: I07.

1577 C. Orangé, de plus ou moins cing fuites de rameaux, pay tout orange, Linn. I5I, c. d. Seb. III. 87. 3. 4. 7. Lift. 206. 40. Argenv. 23. B. ? confiftant, en trois pieces, donc deux different de couleur, EO font attache ax Silice. (1. p. 100.

1578 C. Orangé. Jemblabic au precedtent, Linn. 151.d. Seb. III. 87. 5. Rumph. 48. I. Valent. 24. Knorr. I. 0. 3, 72 ? III. 6. 1. (2.p. Ior.

1579 C Orangé, Jemblable au precedent, mais un pew plus petit, Linn. I5I. d. Seb. III. 87. 5. Knorr VI. 12. s. ( 2 p. ror. a.

1580 C. Royal, coquille d valves boffues, recourbées, pointués. pliées, d plies elevées, d longues branches incourbées: defous caves, pourprées. Linn. I5I e. Rumph. p. 156. $n^{8}$. 8. Petiv. Gaz. 23. 12 ? long E lat. vers l'extremité des brancbes $7 \frac{1}{4}$. alt. $3 \frac{3}{2}$. pouc. (1. p. 126 .

is81 $C$. Royal deprime, d̀ coquille deprimée, poirtue, d branches longues, Linn. 151, $f$. Petiv, Gaz. 23. 12. Knorr, V. 1. 9. bong. \& or $3 \frac{1}{2}$. pouc. (x. p. I27.

I582 C. Branchue, coquille, d branches longues recourbes, defous concaves, la yalve fuperienre rouge, El l'inferieure jaune, Linn. 15 1. g. Gualth. Ior B. Rumph. 48. ' 1 ? Argenv. 19. $H$ ? Spengler ICon. II. B. long. $5 \frac{1}{2}$. lat. $4 \frac{3}{2}$ : pouc. (1. P. 114 .

$\$ \$^{83}$ C. Branchue, opprochant ar precedent, coquille, \& brañches lignées longues, ba valve rouge, entre les rameaux, on appercoit des petite branches dus Madrepore; la valve inferieure joane. Linn. 151. g. Seb III. 8\%. 3. Gualth. 101. B. long. E lat. $4 \frac{1}{4}$, fout. (I. H. 103. 
I584 S. Ramofus, fimilis precederti, rubefcens natibus $\mathrm{ru}-$ bris, Linn. 15r. g. Seb. III. 88, 7. Argenv. 20. G? long. \& lat. $4 \frac{1}{2}$. poll. (2: fpecim. 112.

1585 S. Ramofus, pixcedenti affinis, fed minor, valvis ra. misque albis, Linn. I5t. g. Gualth. 101. B. (2. Tpecim. II 3 .

1586 S. Ramofus? r. varietas pracedenti , natibus rubris, Linn. I51. g. Seb. 1II. 88. 6. Argeny. 20. B ? ( 2. cim 115.

i587 S. Ramulofus, tefta a!ba, rubraque, Linn. 15I. h. Gualth. 101. B? Rumph. 40. I? Argerv. 19. H? (1. fpecim. II4. $a$.

1588 S. Taleolus, ramis brevioribus, valvula altera alba, altera rubra, Linn. 151. i. Seb III. 88.2. Gualth. 100. A. Argeny. 19. E. Ginann. II. 125. Kircher II. 21. (2. Ipecim. Iog.

$\$ 589$ S. Hebes ramis anguftis obtufis, araufiacis, flavis, fusscisque, Linn. 151. k. Seb. III. 87. 2. Argenv. I9.H? (2. fpecim. 99.

$1500 \mathrm{~S}$. Hebes, fimilis præcedenti, tertis flavis, ramis flave. fcentibus, Linn. 151. k. Seb. III. 89. 7. (2. fpecim. III.

I59r S. Hebes, pracedenti fimilis, fed minor, Linn. 151. k. Seb. III. 88. 5. (2. Ipécim. IIO.

$1592 \mathrm{~S}$ Hebes, ramis brevioribus flavis, valvis fuscis purpureisve, Linn. 15t. k. Seb. III. 89. 3? Gualth. 99 F? Lift. 208. 43 ? (2. fpecim. I16

t593 S. Rhamnus, pluriffimis ramis armatus, flavefcens, Linn. 151.1. Seb. III. 89. 8. long. \& lat. 4. à 3. poll. (I. fpecim. 118.

1594 S. Rhammus, præcedenti affinis, natibus albis, Linn. 15I. l. Seb. III. 89. 8 ? long. \& lat. 4. poll. ( $I$. 117.

1595 S. Rhamnus, finilis pracedenti, valvulis albis, Linn. 151. 1. Seb. III. 89. 1. Argenv. 20. E. long. \& lat. 4. poll. (r. fpecim. irg.

$\$ 596$ S. Rkannus, precedenti fimilis, tefta fusca.ramis flavis, Limm. I5 1. 1. Seb 1H. 89. 8 ? Gualth. ror. $A$. prima. (2. Specim. 120.

$\times 597$ S. Rhammus, tefta alba, ramis flavis, duo fpecimina, alterum fubtus foliis aurantium, Linn. I 5I. I. Seb. III. 89. 3. (2. 'pecim 121 .

1598 S. Xanthium, tefta pluriffimis Cpinis armata, flava, Linn. 151. m. Seb. III. 89. 4. Knorr VI. 9. 2. (2. fpecim 124. 


\section{Teftaces Ordre VI:}

1584 C. Branchue, femblable au precedent, rougeatre à feffes rouges, Linn. I5 I. g. Seb. III. 88. 7. Argenv. 20.G? long. Eे lat. $4 \frac{1}{2}$. pouc. (2. p. 112

1585 C. Branchue, opprocbant au precedent, mais plus petit; a valves, $\mathcal{E}^{2}$ tranches blanches, Linn. I5I. g. Gualth. 10r. B. (2. P. II 3 .

1586 C. Branchue, Jemblable as precedent, à fefjes rouges: Linn. 15I. g. Seb. III. 88. 6. Argenv. 20. B? (2\&) p. 115.

1587 C. Branchue, à coquille blanche, E rouge, Linn. 15I. $k$ : Gualth. 101. B? Rumph. 40. I ? Argenv. 19. H? (2. II4. $a^{\circ}$

1588 C. Taleole, d branches plus courtes, l'une yalve blanche, l'autre rouge, Linn. I $51, i$. Seb. III.88,2. Gualth: 100. $A$. Argenv. I9 E. Ginann. II. I25. Kircher II. 2I. (2. p. ico.

1589 C. Emouffé, à branches étroites, émouffées, oranges, है brunes, Linn. 15I. $k$ Seb. III. 87. 2. Argenv. I9. H? (2. p. 99.

1590 C. Emoufté, fembilable au precedent. \& coquille joune; branches jaunatres, Linn. 15i. k. Seb. III.89. 7० (2. p. III.

I59I C. Emouffé, Sernblable au precedent, mais plus petit, Linn. 15 t. $k$ Seb. III. 88. 5. (2. P. I 10.

1592 C. Emcufté, d̀ branches plus courtes, jaunes à valves brunes, ED pourpres, Linn. 15I. k. Seb. III. 89. 3? Gualth. 99. F? Lift 208. 43 ? (2. p. II 6.

1593 C. Rhamnus, armé de plufieurs branches jasunatres, Linn. I 5 bo l. Ssb. III. 89. 8. long. E lat. 3. dे 4. pouc. (I. p. II8.

1594 C. Rhamnus, femblahle au precedent, à feffes blanches, Linn. 15I. l. Seb. III.89. 8 ? long. 'E lat. 4. pouc. (r. p. 117.

1595 C. Rhamnus, Semblable au precedent, à valves blanckes. Linn. 15 r. l. Seb. III 89. I. Argenv. 20. E. long. E' lat. 4. pous. (I. p. IIy

1596 C. Rhamnus, femblable au precedent, d coquille brune, branches jaunes, Linn. 151. l. Seb. III. 89. 8 ? Gualtha 10I. A prima. (2. p. 120.

1597 C. Rhamnus, d coquille blanche, branches jarnes, deux pieces, une des quelles eft deffous orangée, Linn. I5r.l. Seb. III. 89. 3 ? (2. p. I 21 .

1508 C. Xanthium, coquille armée de plufieurs épines jaunes, Lim. 15I. m. Seb. III, 89. 4. Krorr VI. 9. 2, (2. p. 124. 
I599 S. Xanthium, precedenti affinis, Linn. I5 I. m, Seb. III. 89. 3. Knorr V. 6. 2, 7. 2. 3. (2. Ipecim. 1 22. I600 S. Xanthium, fimilis precedenti, fed minor, ramis albis, Lirn I5I.m. Seb. III. 89' 2. Argenv. 20. L. (3. fpecim. I 24.

E60r S. Xanthium, fimilis præcedenti, albefcente fusco, tria fpecimina, unum in Silice, Linn. I 51.m. Seb. III .88, 5, 89. 2. 3. Gualth. IO1. A. fecunda. (3. fpecim. 125.

1602 S. Plicatus, tefta iuaurita plana, mutica, plicata, Linn. 453. n. Gualth. 99. E. minima, Lift. 210. 44 . (2. Ipecim. 128.

\section{GE N V S XXXVI. LA MPADES.}

I603 L. EleEtrica, tefta fubrotunda, convexa gibbofa, plicata, ftriata, rubefcens, Linn. 220 Lift. 450. 8. Gualth. 66. B? Petiv. Muf. 20. 8. Davila I. 20. G. (2. Ipecim. 129.

1604 L. Rubus, tefta convexa, rotunda, margine ult:o \& citro plicata, alba, Linn 220. «. 〔 0. Lift. 453. 1I. Davila I. 20. A. (I. fpecim. I30.

$\$ 605$ L. Rubus, fimilis pracedenti, glabra fusca, Linm. 220. ๙. f. 0. Lift. 457. 16. b? Davila I. 20, $A$ ? (1. fpecim. $13 \mathrm{r}$.

2606 L. Terebratula, tefta obovata, glabra, convexa, valvula altera triplicata , altera biplicata, margine incurvato, Linn. 237. Lift.41 3. I2 Argenv. Suppl. 3. E Petiv. Gaz.93. 19. Davila I. 20. E. Knorr IV. 30. 4 (I. fpecim. I32.

G E N V S XXXVII. CAR DIA.

1607 C. Cardiffa, tefta cordata, valvulis compreffis, dentato carinatis, natibus approximatis incurvatis, Linn 74 Rumph. 42. B. Gualth. 84. C. Lift. 318. 155. Kircher II. 110. (2. Tpecim. 133.

2608 C. Cardiffa, pracedenti affine, minus punetis rubris, Linn. 74. Knorr VI. II. I. (2. fpecim. 133.

1609 C. Tenue, pracedentis varietas, ftriis elevatis gibbolis, Linn. 74. «. Cualth. 7I. D. (I. Specim. 134.

1610 C. Tumidum, precedenti affine, fed magis tumidum, Linn. 74. B. C. O. Guabth. 84. B. Argenv. 23. I. ( 2. fpeciun. I 35 . 


\section{Teftaces. Ordre VII.}

2599 C. Xanthium, approchant au precedent, Linn. 15T.

Seb. III. 89. 3. Knorr V. 6. 2, 7. 2. 3. (2. P. I 22.

I600 C. Xanthium, Semblable au precedent, mais plus petit, d branches blanches, Linn. 15I. m. Seb. III. 89. 2. Argenv. 20. L. (3. P. 124 .

$160 \mathrm{r} C$. Xanthium, femblable eu precedent, d'un brus blan chatre, trois pieces, dont wne eft dans le Silice, Linn. 15r. m. Seb. III. 88. 5, 89. 2. 3. Gualth. IOI. A. Jecters da. (3. P. 125 .

I602 C. Plicé, à coquille fans oreille, dechiré, pliée, Linan 453. n. Gualth. 99. E. minima, Lift.210. 44. (2. P. 128 .

GE NRE XXXVI. I. A M P S.

1603 L Electrique, coquille un peu ronde, recourbé, boffue; pliee ftrié, rougeatre, Linn. 220. Lift. 450. 8. Gualth. 96. B ? Petiv. Muf. 20. 8. Davila I. 20. G. (2. Po 129.

I004 L. Rubus, ou Flambofe, d coquille recourbée, ronde, te bord plié, Linn. 220. a. ou O. Lift. 453. II. Davila I. 20. $A$.' (I. P 130 .

1605 L. Rubus, ou Flambore, Semblable au precedent, pelse, brune, Linn. $220 \propto .0 u$ o. Lilt 457 i $6 . b$ ? Davila I. 20. A? (1. p. 13 r.

1606 L. Coq \& Poule, à coquille un peu ovale,"pelée, recossebée. l'une valve, à trois, l'autre d deux plies, bord recourbé, Linn. 237. Lift. 453 r2. Argenv. Suppl. 3. E. Petiv. Gaz. 93. 19. Davila I. 20. E. Knorr IV. 30. 4. (I. p. 132 .

GE NR E XXXVII. C OE UR S.

I $\mathrm{CO}_{7} \mathrm{C}$. de l'Homme, ou Bourrelet. à coquille, en coeur, à yol ves comprimés, par dens carinee, feJfes s'approchantes, Linn. 74. Rumph. 42. E. Gualth. 84. C. Lift. 313. 155. ISircher II. 2 10. (2. P. 133

$1608 \mathrm{C}$. de l'Homme, ouBourrelet approchant au precedent, moins de pointes rouges, Linn 74. Knorr VI. I1. I. (2.p. 133. 1609 C. Mince, Semblable au precedent, d fries ellevées boffues, Linn. 74. «. Gualth. 84, D (1. p. 34 .

$1610 C$. de Venus, approchant ars precedent, mais plus enflee, Linn. 74. B. 04 O. Gualth. S4. B. Argenv. 23. I. (2. p. 135 . 
Iorr C. Gibbofum, tefta carnea, maculis obfcurioribus, Linin. 74. भ. א. O. Lift 319. 156. Argenv. 23. D. Kircher II. 48. (2. fpecim. I36.

I6I 2 C. Hemicardium, tefta cordata, latera, valvulis carinatis, natibus diftantibus lactea, Linn. 76. Lift. 315152. Argenv. 23. L: Petiv. Muf. 17. 4. (3. Ipecim. 137.

I613 C. Granulatum, an Fragum? fimile præcedenti, album gra:ulacum, Linn 76. a. א. ०. an 83 ? Gualth. 83. $E$. Lift 315. 152? Rumph. 44. G. (2. fpecim. 138.

$16 \mathbf{I}_{4}$ C. Mediun, tefta fubcordata, fubangulata, valvulis angulatis, fulcatis lavibus, Linn. 77. Gualth. 83 . A? Rumph. 44. G? Petiv. Muf. 18. 21. Kircher II. 127. Knorr V. 20. 5. (I. Specim. I39.

I6I5 C. Unedo, tefta fubcordata, lunulis coloratis, Linn. 84 . Seb, III. 86. 12. Lift. 315. 15I, 153. Argenv. 23. N. Petiv. Muf. 18. 19 Valent. 23. Spengler Icon. I. $K$. Knorr II. 29. 3. 5. (2. f́necim. 540.

I610 C. Gargadia, fubrotundum compreffum, antice rugofum rima dentata. corruleo purpurefcente fusca, Linn. 44. Rumph. 42. F. 43. N. Petiv. Gaz. 19, 11. Muf. 18.12. (3. Ipecim. r41.

ICI 7 C. Rimula, tefta natibus acutibus recurvis, trigona, rima cordata, margine ferrato, alba, Linn. o. Rumph. 44. $M$ ? Gualth. 83. H? (1. Ppecim. I 42.

I6ıs C. Prunum, convexius, magisque coftatum, flavum, Linn 0. (1. frecim. I43.

1619 C. Coftatum, tefta gibba, æquivalvi, conis elevatis, carinatis concavis, membranaceis, Linn 73. Argenv. 23 A. Adarson Seneg. 1. 18. 4. 2. long. 3. lat. $2 \frac{1}{2}$ poll. ( fpecim. I44.

1620 C. Coftatum, præcedenti affine, Linn. 73. Petiv. Gaz. jo. 1 ? Knorr I. 28. 2. (I. fpecim. 144.

102 I C. Ciliare. teft r rutunda'a cultala, ancice tuberculata, fasciat?, fusca. Linn. 8c. «. Seh III 86. 7. Lift 340.177. Petiv. Gaz. 156. 24. Ginann. II. 13r. (I.fpecim. 145. 1622 C. Ciliare? $\mathrm{f}$. pracedentis varietas, tefta coftata, tuberculata, echinata, Linn. 80. B. Lift. 321. 158. Argenv. 23. C. (I. Rpecim. 1.16.

$1623 \mathrm{C}$ Echinatum. teita fubcordata, fulcis exaratis, flava, lirea ciliata, aculeis inflexis nlurimis, Linn. 79. Seb. III. 86. 8. Lift. 324 161. Rusnph. 48. 9. Kircher II. 95. Ginan. II. 130. (I. Specim. I47. 


\section{Teftaces. Ordre VII.}

YGII C. Boffue, à coquillele couleur de chair, do taches plus ob- Seures, Linn. 74. $\gamma$ ou 0. Lift. 3 19.156. Argenv. 23. D. Kircher, II. 48. (2. p. 136.

I6i2 $C$. Triangulaire canalée, coquille en coeur, d̀ valves ca. rinées, blanches, les fefles diftantes, Linn. 76. Liff. 315. 152. Argenv. 23. L. Petiv. Muf. 17. 4. (3. p. 137.

16I3 C. Triangulaire granulé, ou Fraíe, femblable au precedent, blanc gramulé, Linn. 77. «. ou o. ou \$3? Gualth. 83: E. Lift. 315. 152 ? Rumph. 44. G. (2. p. 138.

$161_{4} C$. Triangulaire frie, $d$ coquille pres qu'en coeur. anglé, à valves anglées, fillonnées, polies, Linn. 77. Gualth. 83. $A$ ? Rumph. 44. G? Petiv. Muf. I8. 21. Kircher II. 127. Knorr V. 20. 5. (I. p. r 39 .

1615 C. Fraife, a coquille presqu'en coeur, ä lumiles colerées, Linn. 84. Seb..1II. 86. I 2. Lift. 31 5. 151. 153. Argenv. 23. N. Petiv. Muf. 18. I9. Valent. 23. Spengier Icon. I. K. Knorr II. 29.3. 5. (2. p. I40.

I6I6 C. Triangulaire pointé, un per rond, compprimé, ridé par devant, à fente dentée, d'un brun pourpré, Linn, 44. Rumph. 42. F. 43. N. Petiv. Gaz. 19. Ix. Muf. 18. I2. (3.p. I4I. IGI7 C. Ouvert, ou Coupure violeite, coquille, les feffes aigues recourbées, triangulaires, $\dot{a}$ fente en coour, le bord ferré, blarc, Linn. O. Rumph. 44. $M$ ? Gualth.. 83. $H$ ? (I. P. 142.

1618 C. Ouvert, ou Cerneau de Prane, plus recourbée, \& plus cofté, Linn. o. (I. p. r 43 .

16Ig C. Exotique, ou Venus Orientale, à coquille bolfue, a valve ézale, les cotes élevées, carinées, cavées, ¿' comme en membranes, Linn. 73. Argenv. 23. A. Adanfon Seneg. 1. 18, 4. 2, long. 3. lat. $2 \frac{1}{2}$.pouc. (.I. p. 144. I620 C. Exotique, ou Venus Orientale, approchant au precedent, Linn. 73. Petiv Gaz. 30.1 ? Knorr I.28.2 (1. P. 144.

I62 I C. Marron, ou Corbeille, ì coquille ronde, coftée. tuberculée par devant, bandée, brune, Linn: 80 ๓.Seb. III.86.7. Lift. 340 . I77. Petiy. Gaz. 156. 24. Ginann. II. I3I. (I.P.145. 1622 C. Marron, ou Corbeille? varieté de la precedente, is coquille coftée, tuberculée, couverte d'aiguillons, Linn.80. ß. Lift. 32r. I58. Argenv. 23. C. (I. p. I46.

iб23 C. Chataigne épineufe, à coquille, en forme de coeur, flllons élevces, ligne ciliè, à plufieurs aiguillons recourbées, jaune, Linn. 39. Seb. III. 86.8. Lift. 324. $16 \mathrm{~L}$ Rumph. 48.9. Kircher III. 95. Ginann. II. 130. (I. p. 147. a. 
I624 C. Aculeatum, aculeis inflexis, fulcis convexis, lines exaratis, flava fusca, Linn. 78. Seb.III. 86.4. Argenv. 23. B. Petiv. Gaz 54. 5. (1. Ppecim. I47.

1625 C. IJocardia. tefta cordata, fulcis fquamis fornicatis, imbricatis, alba, maculis luteo fuscis, Linn. 82. . Seb. III. 86. 2. 5? Lift. 323. 160, 33 I. 168. (, I. Ipecim. 148.

1626 C. Tuberculatum, tefta fubcordata, fulcis obtufis nodofis transverfim ftriatis, Linn. 81. Lift. 328. 165.166, 333. I7C. Petiv. Gaz. 52. 8. Mus. 17. 7. (2. fpecim. 149.

1627 C. Tuberculatum, pracedenti affine, Linn. 81. Kircher II. 90. (2. [pecim. 14 \%.

1628 C. Rubus, tefta alba coftata, fasciis! purpureis, Linn. o. Seb. III. 86. 14. Lift. 322.159. Petiv. Gaz. 93. I0. (I. Rpecim. 150.

1629 C. Albefcens, T. varietas præcedentis? tefta fubrotunda, fulcata, fubfquamata, flavefcens. Linn. 87. . . [ O. Gualth. 71. G. Lift. 326. 103. Petiv. Muf. 17.5. (1. Specim. 151. $1630 \mathrm{C}$ Pragum granulatum, album, purpureo varium, Linn.0. Seb. III. 86. 13. Gualth 7I. N. Lift.334.17r. (2. fpecim. 152.

163x C. Dijpar, tefta fubrotunda dilatata, ftriis approximatis longitudinalibus, contra alias diftantes trausverfales pofitis, alba, purpureo varia, Limn. O. Lift.314. 150. Kircher II. 89.Knorr V. 26. x. 2, 27. 3. (I. fpecim. 153.

1632 C. Dijpar, precedenti affine, purpureo varium, Linn. $\odot$. (I. Specim. 153.

I633 C. Serratum, tefta obovata lævi, ftriis obfoletis margine interiore ferrato, Limn. 89. «. f. 0. Gualth. 82. A. Knorr. II. 20. 4. (3. fpecim, 154.

1634 C. Hiatus, tefta levi, ftriata, natibus purpure is, Linn, - Gualth. 85. H. (2. Specim. 155.

1635 C. Hiatus? f. varietas procedentis, flava, Linn. o. Rumpls. 44. N? Knorr IV. 3. 3? (1. Specim. 156.

1636 C. Prunus, tefta natibus acutis approximatis, remotis a centro, coltata margine ferrata, alba, luteo fasciata, Lim, O. Gualth. 85. G? KnorrVI. 6. 1 ? (2. Specim. 157.

1637 C. Spinofum, tefta fpinis coftata, flava, Linn. ๑. Lift. 342. 179. Knorr VI. 7. 6。 (1. Ppecim. 157. a. 
1624 C. Gris à piquants, les aiguillons courbès endans, fil lons courbés, ligne jaune, Linn. 78. Seb. III. 86. 4. Argenv. 23. B. Petiv. Gaz. 54. 5. (1. p. T47.

x625C. Ecailleux, d coquille en coeur, fillons d ecailles vorstées, en forme de tuilles, blanche, ì taches brunes, Linn. 82. «. Seb. III. 86. 2. 5 ? Lift. 323. 160, 33r.' 168. (1.p. 148,

1626 C. Feytama, ou Carthagene, d coquille en forme de coeur, d' fillons emoufJés, ftrié de travers, Linn. 81. Lift. 328. 165. 166, 333. 170. Petiv. Gaz. 52. 8. Muf. 17. 7. (2.p. 149 .

I627 C. Feytama ou Carthagene, approchant au precedent, Linn. 81. Kircher II. 90. (2. p. 149.

1628 C. Flambore, a coquille blanche, à cotes, \& fafcies paure pres, Linn. O. Seb. III. 86. 14. Lift. 322. 159. Petiv., Gaz. 93. 10. (1. p. 15c.

I620 C. Fraife blanche, ou varieté duprecedent? à coquille un peu ronde fillonnée, un peu ecaillée, jaunatre, Linn. 87. a. ou 0. Gualth. 7 I. G. Lif. 326. 163. Petiv. Muf. 17. 5. (1. P. 151 .

I630 C. Granulée, ou Fraife panachée, blanche, variè de pour. pre, Linn. o. Seb. III. 86. 13. Gualth. 71. N. Lift. 334. 17r. (2. P. 152 .

r63r C. Bifarre, ou Strié à deux Zones, d coquille un peu ronde, etendue; $\grave{a}$ Jtries s'approchantes longitudinales, qui Jon pofées contre d'autre diftantes transverfales, blancles, varié de pourpre, Linn. O. Lift 314. I50. Kircher II. 8\%. Knorr $V$. 26. 1. $2,27.3$. (1. p. $153^{\circ}$

1632 C. Bifarre, ou Strié à deux Zones, approchanst au precedent, yerié, pourpre, Linn. O. (1, p. 153.

I633 C. Bignet Jaune, à coquille ovale, polie, de fries obfolées. le bord interieur ferré, Linn. 89. a.ou O. Gualth. 82. $A$. Knorr II. 20. 4. (3. p. 154.

1634 C. Bignet, à coquille polie, ftriee, les fefles pourpres, Linn. o. Gualth. 85. H. (2. p. I55.

I635 C. Bignet? ou varieté de precedent, mais jaune, Linn. 0. Rumph. 44. $N$ ? Knorr IV. 3. 3 ? (1.p. 156 .

$1 \sigma_{3} 6 C$. Prune, coquille a feffes approchantes, eloignés du centre à coftes, EO bord ferré, blanche, bandée de jaune, Linn.

o. Gualth. 85. G.? Knorr VI. 6. I? (2. p. 157 .

1637 C. Prune pointee, coquille coftée d'epines, jaune, Linn. o. Litt. 342. 179. Knorr. VI. 7. 6. (I. p. x57.a.

$$
{ }_{6} \text { Ff } 2 \text { 1638C. }
$$


I638 C. Reticulatum, tefta fubcordata, ftriis elevatis decusfatis, ano cordato, margine integro, Linn. 134. Gualth. 75. C. Rumph. 43. F. Lift, 335. I72. Bonann. Obferv. 3. 10. Knorr VI. 39. 4. (I. Specim. 157. b.

GENVS XXXYiI. C II A M E F R M S.

I639 C. Erycina, tefta cordata transverfim paralelle fulcata, fulcis obtufiffimis, valva glabra, ano ovato, cinerea, fusco fasciata, Linn. 122. a. Gualt h. 75. Q. Lift. 251. 85? Rumph 42.I. Valent. 20, KnorrV.15.2, (2. fpecim.15E. 1640 C. Triquetra, tefta oblique triquetra, Itriata, citrea, Linn. O. Gualth. 85. D? Lift. 273. I09? Knorr VI.8. 2 ? (I. (pecim. $\div 159$.

${ }_{64}$ I C. Selata, pracedenti affinis, flavefcers, Linn. O. (I. fpecim. 160.

$\mathbf{1 6 4 2}$ C. Striata, precedenti affinis, flavefcens albo radiata, Linn: O. Lift. 401. 245. (r. Ipecim. r6r.

I043 C. Meretrix? \& varietas, fuscum tranfparers. Linn. I2\% क. f. O. Lift. 306. I 39 ? Knorr VI. 63 (1. fpecim. I62. 1644 C. Radiata, tefta orbiculata, natibus recurvis, fusca, radiis albis, Linn. O. Lift 401. 245? (1. fpecim. T63. I6 45 C. Guttata, tefta alba, luteo feriatim punctata, Limiz o. Lift: 255. 90. Adanjon Seneg. 1. 8. 5. 5. (4. Specim. 164.

$\mathrm{IG}_{4} 6$ C. Bifafciata, tefta triquetra, antice rotundata, natibus acutis approximatis, grifea, fasciis 2. fuscis, Linn, 0. Lift 306. 130. (2 ipecim. 164. 8.

1647 C. Ziczac, tefta flavefcente, fusco undata, Linn. 0. Lift. 256. 91. 93, 380. 223. (2. Specim 164 b.

1648 C. Meretrix. tefta cordata fubrugofa, valva fusca, gibba, nimphis biantibus, Limn. 127: Gualth. 76. C. Lift: 300. 137. Argenv. 21. F. Rumplo 42. G. (2.fpecim. 165.

r649 C. Meretrix, præcedenti affinis, polita, irtus aurata, pißtaque, Linn. 127. (I fpecim. 165. a.

1650 C. Punctata , triquetra, Eaiba, lineolis punetata, picta, Linn. 127. a. א. 0. Lift. 252. 86 ? (1. Specim. 166.

1651 C. Maculofa, præcedenti affinis, fusco flava, Linn. 127. 1. 1. O. Adanfon Seneg. 1.18.5.4 ? Valent. 20. (2. pecim. 167.

I652 C. Marginata, tefta tumida triquetra, natibus diftantibus, craffa, margine albo, Linn. O. Adanfon Serzeg. 1. 16. 4. 7 ? Potiy, Muf. 18.20. (2, fuecim. I68. 
7638 C. Reticu'é, ou Coeur a Refeaux, coquille presqu'sn coeur, à ftries elévées croilfées, l'arus ent coeur, à bord entier, Linn. I 34. Gualth. 75. C. Rumph 43, F. Lift.335. I72. Eonann. Obferv. f. 10. Knorr VI. 39) 4. (I. p. 157.b.

GENRE XXXVIII. EN FORME DE CAMES.

1639 C. Quacre, coquille en coeur. pararellement, à fillons tres emousJés, valve pelé, l'anus ovale; cendrée, fafciée de brun, Linn. 122. «. Gualth. 75. Q Lift. 251.85? Rumph. 42. I. Valent, 20. Knorr V.15.2. (2. p. 158.

I640 C. Pomme jaune, coquille oblique triangulaire, Jtriée, couleur de citron, Linn. o. Gualth. 85. D? Lift. 273 . 109 ? Knorr VII. 8. 2.? (1. p. I59.

I64 I C. Prune jaune, jemblable au precedent, jaunatre, Linn. $o_{2}$ (I. $p$ I 60 .

I642 C Strié, approchant au precedent, jaunatre rayonné tle blanc; Linn. O. Lift. 40I. 245. (1. p. 16I.

I6 43 C. Coupure brune? ou varieté, dun bruntransparent, Linn: 127. a. $0 u$ O. Lift. 306 I 39 ? Knorr.VI 6. 3. (I.p.163. 1644 C. Prune rayonnée, à coquille orbiculaire, felfes recourbées, brune, à rayons blancs, Linn. O. Lift. 40I. 245? (I.p. 163. 1645 C. Prune pointée, or Chatadoa, d coquille blanche, pointeé, en ordre, de jaune, Linn. O. Lift. 255. 90 Adanfón Sentg. I. 8. 5. 5. (4. p. 164.

I6 46. C. Prune bifafciée, à coquille triangulaire, devant arrondie, les feffes aiguses s'approchantes, grife, à deux bandes: brures, Linn. 0. Lift. 306. 130. (2. p. 164. a.

I047 C. Prune en Zigg-zag, à coquillejaunatre, ondée de brun 2 Linn. o Litt. $256.91 .93,380.223$. (2.p. 1́́4. $b$. I648 C. Gourgandine, coquille en coeur, un peu ridée, polie à valve brune, boffue, à nimphes ouvertes, Linn. 127. Gualth. 76. C. Litt. 300. I37. Argenv. 21. F. Rumph. 42. G. (2. p.: $: 65$.

I649 C. Gourgandise, Jemblable au precedent, polie, peinte, $\mathcal{E}^{\circ}$ doré en dedans, Linn. 127. (r.p. 165. a.

1650 C. Gourgandine pointé, triangulaire, blanc, peinte avec des pointes, EO des lignes, Lirn, 127. \&. ou O. Lift. 252. 86 ? ( I. p. I 66.

I5 I C. Gourgandine jaune tachetée, approchant au precedent, d'un brun jaune, Linn. 127. $\beta$. ou O. Adanfon Seneg. I. 18. 5. 4 ? Valent. 20. (2.p. 167 . $^{\circ}$

1652 C. Gourgandine bordée, à coquille enflée, triangulaire, feffes diftantes, epaifle, à bord blanc, Linn. o, Adanfon Sctseg. I. 16. 4. 7 ? Petiv. Muf, 1820. F $f 3$

(2. p. 168. I653 $C$. 
1653 C. Marginata, tefta fimilis præcedenti, albo radlata; Linn. 0. Lift. 306. 139. (2. Ipecim. 169.

1854 C. Violacea, tefta triquetra, antice rotundata, albo violacea, rima purpurafcente, Limin. 127. भ. ᄃ. O. (I. fpecim. 170.

1655 C. Biradiata, tefta ut pracedens cinerea, radiis duobus albis, Linn. 127. d. \{. o. Lift. 403. 247,? Gualth. 77. $D$ ? (2. Ipecim. 171 .

1656 C. Biradiata, fimilis præcedenti, flava, triliniata, ru. bro fusca, Linn. 127. \&. ᄃ. o. Gualth.77. E. Lift. 299. 136. Knorr IV. 24. 2. (3. Ppecim. 172.

1657 C. Tela Aranea, tefta alba, ftriis fe decuffantibus fuscis , Linn. 129. «. f. o. Gualth. 76. A. Lift. 246. 80. (I. fpecim. 173.

1058 C. Striatä, præcedenti fimilis, Havefcens, Linn. I29. B. ᄃ. O Gualth. 75. G? Lift. 254.89, 259. 95. Adanfon Seineg. I. 17. 4. 5. (2. Ipecim. 174.

I659 C. Striata, præcedenti affinis, maculis \& Atriis fuscis, Linn. 129. B. I. 万. (2. \{pecim. 174.

1660 C. Undata, tefta flava, fasciis undatis, fuscis, Linn. 129. \% r. o. Gualth. 76. A. Lift. 255. 90. Valent.16. (I. Ppecim. 175.

I66I C. Stativa, tefta alba, lineis triquetris fuscis, Limm. I29. D. C. O. Gualth. 75. O. Lift. 257. 93. Kircher II. 78. (2. Lpecim. 176 .

1662 C. Stativa, præcedenti affinis, maculis majoribus, Linn. 129. 8. .0 0. (2. .pecim. 176.

I663 C. Flanmeola, tefta alba, maculis flammeis fuscis, Linn. 129. . . O. Gualth. 82. Y. Lift. 258. 94 ? (I. fpecim. 177.

1664 C. Caftrenfis, tefta triangulo rotundata, gibba, glaberrima, caraeteribus angularibus infcripta, Linn. 129. Lift. 287. 123. a. Argenv. 21. M. Petiv. Muf. 5. 2. Kivcher II. 60. (2. Lpecin. 78.

1605 C. Caftrenfis, pracedenti affinis, Linn. 129. Rumph. 42. K. ( (. Specim. 178 .

I666 C. Violacea, poliendo alba natibus violaceis, Linn: 126. ๙. 1. O. Adanfon Sereg. 1. 17, 4. I7? (2. Ipecim, 180.

1667 C. Maculata, tefta cordata, fasciata, lavi, maculis exoletis [parfis, Linn. 126. Argenv. 21. H. Kircher II. 126. Adanjon Seneg. I. 17. 4. 12. (2. Specim. I8r.

1668 C. Maculata, pracedenti fimilis, Linn. 126. Lift. 270. 106. (2. Гpecim. 181 . 
8653 C. Rayonné, coquille femblable d la precedente, rayonnée de blanc, Linn. o. Lift. 306. 139. (2. p. 169.

1654 C. Coupure Violete, à coquille triangulaire, devant arrondie, blanc violette, à fente pourprée, Linn. 127. $\gamma_{\text {. }}$ ou o. ( I. p. 170.

1655 C. Rayon gris, coquille comme la precédente, mais cendrée, d deux rayons blancs, Linn. 127. d. ou 0. Lift. 403. 247.? Gualth. 77. D.? (2. p. $17 \mathrm{I}$.

I656 C. Rayon gris, Semblable ars precedent, jaune, d trois lignes, d'un brun rouge, Linn. 127. d. ou o. Gualth. 77.

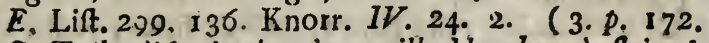

1657 C. Toile d'Araignée, à coquille blanche, \&े ftries brunes qui Se croidfent, Linn. 129. a. ou ๑. Gualtb. 76. A. Lift. 246. 80. (I. p. I 73.

i658 C. Strié, ou Cotan, jemblable au precedent, jaunatre, Linn. 129. B. ou 0. Gualth. 75. G? Lift. 254. 87, 259. 95. Adanfon Seneg. I. I7. 4 5. (2. p. 174 .

1659 C. Strié, femblable au precedent, à taches \& ftries brunes, Linn. I29. $\beta$. ou o. (2. P. I 74.

I600 C. Ondé, à coquille jaune, à fascies ondées brunes, Linn: I 29. $\gamma_{0}$ ou 0 . Gualth. 76. A. Lift. 255. 90. Valent. 16. ( 1. p. 175 .

$166 \mathrm{I}$ C. Camp tacheté, coquille blanche, d̀ lignes triangulaires brunes, Linn. 129. $\delta$. ou o. Gualth. 75. O. Lift. 257. 93. Kircher II. 78. (2. p. I 76.

1662 C. Camp tacheté, Semblable au precedent, mais a taches plus grandes, Linn. 129. d. ou o. (2. p. I76.

1663 C. Camp flammé, ¿̀ coquille blanche, taches flammées brunes, Linn. 129. *. ou O Gualch. 82. T.Lift. 258. 94? (I. p. 177 .

1664 C. Ecriture Arabique, ou Point d'Hongrie, coquille en triangle ronde, boljue, tres pelée, marquée par des caracteres angleés, Linn. I29, Lift. 287. 123. a. Argenv. 2I. M. Petiv. Muf. 5. 2. Kircher II. 60. (2. p. 178.

1665 C. Ecriture Arabique, ou Point d'Hongrie, approchant au precedent, Linn. x29. Rumpb. 42.K. (2. p. 178.

1606 C. Orpheline pourprée, ou Gatan, polie, blancke, les fefes violettes, Linn. 126. *. ou o. Adanfon Seneg. I. 17.4 . 17 ? (2.p. 180 .

1667 C. Tigré radié, ou Pegan, coquiile en coeur, falcié, polie à taches difperfées, Linn. 126. Argenv. 2t. H. Kircher II. 126. Adanfon Seneg. 1. 17. 4. 12. (2. p. I8 I.

2668 C. Tigré radié, Semblable ar precedent, Linn, I26. Lift. 270. 106. (2, p. 181 . 
1609. C. Lapidea, tefta ${ }^{\circ}$ transverfe fubrugofa laví, caro dinis dente pofteriori lanceolato, poliendo alba, Linn.' 125. \&. f. O. Lift. 274. I IO? (1. Specim. 182.

- 670 C. Radiata fusca longitudinaliter ftriata, Linn 125. भ. . ०. Giralth. 86. A. Lift 269. I05. Argenv. 21. C. Adanjon Seneg. 1. 17, 4. 16 ? long. $2 \frac{3}{4}$. lat. $3 \frac{3}{4}$. poll. (2. fpecim. 183 .

I671 C: Radiata, præcedenti affinis, Limn. 125. $\%$. f. ๑. (2. Tpecim. 183.

1672 C. Cedo mulli, tefta ftriata, paliide fusca, radiis obfcurioribus, Linn. 125. *. f. o. Lift. 268. 104. Kircher II. 63.64 ? long. $2 \frac{5}{8}$. lat. $3 \frac{3}{4}$ poll. (2. fpecim. ' 184 . 1673 C. Cedo mulli, præcedenti affinis, Linn. 125. a. f. 0. (2) fpecim. 184 .

1674 C. Cedo nulli, pracedenti affinis, fed minor, lutea, ftriis duabus, Linn. 125. a. ᄃ. O. (2. ́pecim. I85.

1675 C. Soloris, tefta oblonga magna, transverfim ftriata, hepatica, grifea, ftriis obfcure grifeis, Linn. 147. $\beta$. f. O. long. $3 \frac{1}{4}$. lat. $6 \frac{3}{4}$. poll. (I. fpecim. 186.

I 676 C. Ziczac, ovata, compreffa, $1 \mathfrak{a}$ vi, fcripta lineis coccineis undatis, intus purpurea, rima acuta, marğinibus crenu. latis, Linn. IC 9. a. r. O. Lift. 378. 221. Argenv. Suppl. 3 B. Petiv. Gaz. I3. 8. Kircher II. I34. (4. Ppecim. 187.

1677 C. 'Ziczac, fimilis præcedenti, Linn. 109. a. f. o. (3. fpecim. 187.

1678 C. Flammen, rima . macula violacea, Limn. Iog. . . . O. Gualth. 75. P. Lift. 381. 224. Adanfon Seneg. 1.17.4. I3. (4. fpecim. 188.

I579 C. Litterata, tefta alba, ftriis angulofis rubris, margi . ne cnerulefcente. Linn. I09 a. Lift. 379.222. Rumph. 43. L. (5. Гpecim. 189.

1680. C. Arachne, tefta alba lavis, ftriata rofea, intus violacea, Linn rog $\gamma$ f, o. Lift. 330. 2.23. Rumph 43. -M:Petiv. Gaz. 16. 9. Kircher II. 42.: (3. Ipecim. 190. 168 r C. Hebes, tefta fusca, maculis fuscis, Linn. 105: $\alpha$ f.o. Lift. 384. 227 ? 392. 231 ? Gualth. 88. O? (2. Ipe. cim. 19I.

1682 C. Scripta, tefta lentiformi compreffa, flavefcente fusca, ftriis angulofis fuscis, Linn. I 45. Lift. 253. 94. Rumph.43.C. Petiv. Muf. I8.3. Adanfon Seneg. I. 16.4.4? (2. fpecim. I 92 . 
1669 C. Noyau, à coquille de travers, un peu ridée, blantche, polie, le dent pofterieur de la charniere, eft poin. tue, Linn. 125. o. ou o. Lift. 274. 110 \% í I. p. 182. I670 C. Rayonné, on Lifon, brune, ftrié, en longeur, Linn. 125. \%. 026 o. Gualth. 86. A.: Lift 209. 105. Argenv. 21. C. Adanfon Seneg. I. 17. 4. I6? long. $2 \frac{3}{4}$ lat. $3 \frac{3}{4}$. (2.p. 183 .

IG7I C. Rayonné, approchant au precedent, Linn. I25. y.ou o.? (2. p. 183 .

1672 C. Cedo nulli, ou Rayonnante de Ceilon, à coquille frié, pale brune, à rayonis plus obfcures, Linn. I25. * ort o. Lift. 268. I0 4. Kircher 11.03 .64 ? long. $2 \frac{5}{8}$. lat. $3 \frac{3}{4^{\circ}}$ (2. P. 184 .

I673 C. Cedo nulli, ou Rayonnante de Ceilon, approchants au precedent, Linn. 125. a. ou o. (2.p. 184.

I6 74 C. Cedo nulli, ou Rayonnante de Ceilon, Semblable aus precedent mais plus petit, jaune, d̀ deux ftries, Linn. 125 $\propto$ ou o. (2.p.185.

$1675 C$ Solaire, à coquille allongée, grande, ftriée de travers, couleur de foye, à ftries grifes, Linn. 14.7. $\beta$. Ou o bong. $3 \frac{1}{4}$. lat. 6 $\frac{3}{4}$. pouc. (I. P. I86.

Io 60 C. en Zig-zag, coquille ovale, comprimée, polie, à lignest rouges, ondlees, en dedans pourpre, à ferite aigue, les bords crenues, Linn. 109. \& ou O. Litt. 378. 221. Argenv. Suppl. 3. B. Petiv. Gaz. 13. 8. Kircher II. 134\% (4 p. 187.

1877 C. en Zig-zag, Semblable au precedent, Linn. 109. «. ou o. (3.p. 187 .

1678 C. Flamboyante. ou le Sunet, fente à tache violetie. Linn. 109 B. ou o. Gualth. 75. P. Litt. 33r. 224Adanfon Seneg 1. 17.4. :3. (4. P. 188.

16/9 C. Ecriture Xulanefe, a coquille blanclie, les ftries en angles rougres, le bord bleu celefte, Linn. 1Og. $\alpha$. Lift:379. 222 . Kumph 43. L. (5. P. 189.

1680 C Arachnte Xulanefe, ou Elpladian, $\dot{a}$ coquille blanche polie, is frries rouges, en dedans violette. Linn. 1. $22 \gamma$. oul O. Lift. 380. 223. Rumph. 43, M. Petiv. Gaz. 16. 9. Kircher II. 42. (3. p. I90.

168I C. Emoufté, d̀ copuille brune, E taches brunes, Linn. 105. a. oul c. Lift. 384. 227 ? 392. 231 ? Gualth. 8et 0 ? (2. p." Igt.

$1682 \mathrm{C}$ Fauffe Corbeille, ou Ecriture Chiroire, a coquille comp̀rinaée, juznatre, brune, $\dot{a}$. Atries anglées brines. Lina. 145. L.R. 258. 94 Rumph 43 C. Petiv. Mur. I8. 3. Adanfon Seneg. I. 16. 4.4 ? (e.p. 102:

$$
\text { Ff5 } 1683 \mathrm{C} \text {. }
$$


1683 C. Cultrata, tefta albida, lineolis maculisque fuscis, Linn. 135. «. f. O. Gualth. 85. E? (I. fpecim. 193.

1684 C. Pectinata, tefta fublentiformis, fulcis longitudinalibus rugofis, pube antrorfum ramofa, flava, fusco punctata, Linn, 144. Lift. 312. I48. Gualth. 75. A. Petiv. Muf. 18. 14. (2. fpecim. 194.

1685 C. Thafia? i. varietas præcedentis, tefta compreffa, flavefcens maculis fufcis, Linn. 144. a. f. 0.Lift. 313 . 149. Gualth. 72. E. Argenv, 21. P. (2. Ppecim. 195.

$1686 \mathrm{C}$. Amygdala, an fimilis pracedenti? ftriis elevatis ru. gofis, maculis fuscis, Lin?. 44. B. f. O. Lift. 404. 249. ( I. fpecim. I g6.

r687 C. Perficites, tefta globulora, alba, maculis \& "ftriis fuscis, Linn. 144. \%. ᄃ. O. Gualth. 75. H? Lift. 310. 146. (I. fpecim 197.

I688 C. Exoleta, tefta lentiformi, alba, transverfim ftriata, pallida, obfoleta, radiata, ano cordato, Linn. 142. $\alpha$. Gualth. 75. E. R. Lift. 261. 97, 292. 128 ? Adanfor Seneg. I. 16. 4. 5. (I. fpecim. I98.

1689 C. Obfoleta, lactea, maculis ftriisque fuscis, Linn. 142. B. Lift 295. 13 I. Petiv. Gaz. 93. 15. Kircher II. 44. Adanfon Seneg. I. 16. 4. 4. (2. Ipecim. 139.

3690 C. Borealis, tefta lentiformi, ftriis transverfim membranaceis, erectis remotiffimis, grifea, fusco maculata, rima violacea, Linn. 143. a. Gualth.75. N. Lift. 297. 134. Petiv. Gaz. 76. I. (I. Specim. 200.

1691 C. Cancellata, tefta ftriis longitudinalibus transverfalibusque grifea, rima violacea, Linn。 O. Gualth. 75. B. Lift. 338. 175. (ז. Specim. 201 .

1692 C. Dione, tefta fubcordata carina transverfe fulcata, pube, fpinis finuantibus, Linn. 112. Lift 307.140. Petiv. Gaz. 31. 9. Argenv. 21. I. Kircher II. 121. (2. fpecim. 202.

\section{GEN V S XXXIX. C HA M}

¿693 C. Apluftre pacis, tefta fubcordata craffa, fulcis transverfis coftatis reflexis, margire crenulato, ano cordato, rofeo violaceo, alba, Linn. II5. a.f.o. Valent.21. long. $2 \frac{2}{2}$. lat. $2 \frac{3}{4}$. poll. (I fpecim. 203.

1694 C. Apluftre pacis, precedenti affinis, Linn. II5.๘, I. O. (I. fpecim. 203. 


\section{Teftaces. Ordre VII.}

1583 C. Aigue, à coquille blanchatre, à lignes, \& taches brunes, Linn. 135. \& ou 0. Gnalth. 85. L ? (1.p. 193

1684 C. l'Amande peignée, à coquille un peu en figure de Leritille, ad fillons longitudinales ridés. la pube en dedans branchue, jaune, pointée de brun, Linn. 144. Lift. 312. 148. Gualth. 75. A Petiv. Mluf. I8 144. (2.p 194.

1685 C. Amande platte? ou varieté de la precedente à coquille comprimée, jaunatre à taches brunes, Linn. I44: $\infty_{0}$ ou 0 . Lift. 3 I3 - I49. Gualth. 72. E. Argenv. 2 I. P (2. p. 195.

1686 C. Amande, ou femblable à la precedente? a Jtries elevées ridées, taches brunes, Linn. 144. B. 0us o. Lift. 4C4. 249. (I. p. I.96.

1087 C Cerneau de Peche, à coquille ronde, blanche, à taches. Eo ftries brunes, Linn. 144. $\%$. ou o. Gualth. 75. H? Lift. 310.146 (J.p. 197.

1688 C. Coquette, coquille en figure te lentille, blanche: friée, pale, radiée, l'anus en coeur, $L$ inn. 142. $\alpha_{\text {. }}$ Gualth. 75. E. R. Lift. 261. $97,292.108$. Adanfon Seneg. I. 16. 4. 5. ( I. p. 108.

3689 C. Coquette, ou Cotan, coquille. ¿̀ taches Ef fries brunes, Linn. 142. $\beta$. Lift. 295. 13 1. Petiv. Gaz. 93. 15. Kircher II. 44. Adanfon Seneg. I. 16. 4. 4. ( 2. p. 199.

$1690 \mathrm{C}$. 'Thetis, coquille en figure de lentille, frie de travers en membranes, erigées, treseloignées, grije, tachetée de brast, à fente violette, Linn. 143. «. Gualth. 75.N. Lift. 297. r34. Petiv. Gaz. 76. I. (I. p. 200.

I60I C. en Treilles, coquille, grife $\dot{a}$ ftries longitudlnales, $\xi^{2}$ transverfes, ì fente violette, Linn. O. Gualth. 75. $B$. Lift. 338. 175. (I. p. 201.

1692 C. de Venu', coquille un peu en forme de coeur, $\sqrt{2}$ lonnée de travers, la pube à epineus courtées, Linn. 112. Petiv. Gaz. 3I. 9. Argenv. 21. I. Kircher II. 12I, Lift. 307.140. (2. p. 202.

GE NRE XXXIX. CAMES.

1693 C. Pavillon de Paix, à coquille en forme de coeur, epaiffe. à fillons transverfes, coftées recourbés, à bord crenus. l'anus en coeur, rofe violette, blanche, Linn. I I 5.\&. ou O. Valent. 2I. long. $=\frac{3}{2}$. lat. $2 \frac{3}{+}$. pouc. (I. p. 203.

2694 C. Pavilion de Paix, fernblable au precedent, Linn. Ir5. a. 0u O. (I. P. 203. 
I695 C. Vexilluim pacis, tefta fubcordàta craffe, fülcís trañs verfis remotis, margine crenulato, flava grifea, Linn.115. B. f. O. Gualth 75. F Lift. 286.I23. (I. Tpecin:204. 1696 C. Caperata, tefta transverfim lavis, fulcata, margine

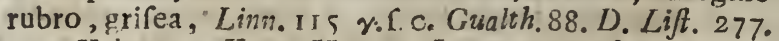
II 4. Valent. 29. Knnorr II 28.3. IV . 24.3. (3. fpecim. 205.

1697 C. Cancellata, tefta fubcordata, ftriis transverfis membranaceis remotis, matgine lævi crenulato, ano cordato, alba, maculis caftaneis, Linn. I18. c. Liff. 278. II5: Argenv. Suppl.3. G. Peitv. Gaz. 156. 25. Knorr VI. 10. 2. 4? (2. -pecim. 206.

1698 C. Paphia, tefta futcordata, rugis incraftata, pube rugis attenuatis, labris complicatis, alba, fusco maculata, Linn. I13. Lift. 279. Is8. Argenv.21. B. Rumph. 48. 5. Kircher II. 72. Spengler Icon. I. I. Kn:orr VI. 6. 2. (I. fpecim. $20 \%$.

r69.9 C. Verrucofa, telta fubcordata, fulcis membranaceis, ftriatis, reflexis, antice imprimis verrucofis, margine violaceo, crenulato, Linn. 116. Lift. 284. I22.'Petiv. Ga\%. 93. I 7. (I. fpecim. 208.

I700 C. Perfica, tefta lentiformi tumida, longitudinaliter ful. cata violacia, fusco varia, Jimm。 140 a. Gualth. 75. S?" Lift. 285.122.1 ? Petiv. Muf. 18. 10. KnorrVI. I0. 3. (I. Recim, 20 ).

I70I C. Fimbriata, tefta ovali, f. fubrotunda, gibba, longitudinaiiter ftriata, transverfe fulcata, margine crenulato, incus violaceo, Hava, fusco punctata, Linn 133 , Gualt k. 76 G. Lift. 336.173. Kumph.43.F.G.K. Argenv. 2r. G. (I. Rpecim. 2 II.

$I_{7} 02$ C. Marginnta, fefta fubrotunda, oblonga, dilatata, ftriata, margire aurantio, Linn. o. Lift. 271.107. 108. Guath. 83. F? (I. 'pecim. 210.

I703 C. Remies, teft a fuborbiculata, compreff, lævis ftriata, lactea, Linn. 62 Lift. 288. 124. Petiv. Giz. 93. 18. longit. $3 \frac{1}{2}$. latit. 4. poll. (1. Rpecim. 212 .

ro4 C. Tigerina, tefta lentiformi, ftriis crenatis decufatis, ano impreffo ova:o, lactea, Linn. 1 $36 . \& 144$. Seb.III. S6. 16 Lift. 357.174. Gualth 77.A. Petiv. Miuf. 18. 6. 7. lorg. - lat. $3 \frac{7}{8}$. poll. (i. Specim. 2I3.

ryos C. Tigcrina, pracedenti iffinis, polita, margire cecci. neo, Linn 1 $36 . \& 141$. Kircher 11. 7c. long. 3. lat. $3 \frac{1}{2}$. poll. (1. Specin. 214 .

I 705 C. Tigerina, frailis præcedenti, polita, margine coccinco, Limo. :35. \& i41. (2. Rpecim. 214. 
rogs C. Pavillon de Paix, à coquille en forme de coeur, epriffe, d. fillons de travers feparées, à bord crenu, grife, Linn. III5. . o. ou 0.' Gualth. 75. F. Lift. 286. I23. (1. p. 204 $1096 \mathrm{C}$. Vierge, or Pucells ridée, d̀ coquille polie, fillonnèe de travers grife, $\dot{a}$ bord rouge, Linn. II $\gamma$. ou 0 . Gualth. 88. D. Lift. 277. I 14. Valent. 29. Knorr. II. 28. 3* IV. 24.' 3. (3. p. 205.

1697 C. Cancellé, ort Veitle ridée, is coquille en forme de coeru, Atries transverfes, membranacées feparées, le bordcrena, polie. lanus en coeur, blanche, à taches chataignes, Linn. 118. * Lift. 278. I15. Argenv. S .ppl. 3. G. 156. 25. Knore VI. I0. 2. 4? ( $2 p=06$.

I698 C. Veille ridée, $i$ coquille un per en cosur epaifée par des rides, hevres comprimées; blanche, tachetée de brun. Linn: Ir3. Lilt. 279. Ir8. Argenv. 2r. B. Rumph. 48. 5. Kircher II. 72. Spengler Icon. I. I Knorr VI. 6. 2. (I. p. 207. I099 C. Verroceufe, à coquille un peru en coèur, à fillons, en forme de membrane, à Aries recourbées, devant fur tom verruceufes, d̀ bord creviz, violet, Linn. 116. Lift. 284. 122. Prtiv. Gaz. 93. 17. (i. p. 208.

'1700 C. Peche, à coquille en figure de lentille, enflée, fillonate en long. yariée de brum, Linn. 140. Gualth. 75. S.? Lint. 285. I 22.a.? Petiv. Muf.18.10. Knorr VI. IO.3. (I.p.200 -170I C. Tricotée, ou Corbeille, d̀ coquille ovale, ou un pers ronde, boffuse. Poriée en long, fillonnée de travers, à bord crenu, en dedans violet jaune, pointée de brun, Linn. $\mathbf{x}_{33}$. Gualth. 76. G. Lift. 336. 173. Rumph. 43. F. G. $\bar{K}$. Argenv. $21: G$. (1. p. 2 rr.

I702 C. Bord, ou Panache Orange, d coquille un peu ronde, allongée etendue, firiée, à bord oỵangé, Linn, O. Lift. 271. 107. -108. Guath. 83. F.? (1. P. 210.

$\$ 703$ C. Refeau platte, à coquille un peru ronde, comprimbe, polie, Jtriée, blanche, Lirn. 62. Lift 288. 124 . Petiv. Gaz. 93. 18. long. $3 \frac{1}{2}$. lat. 4. pousc. (I. P. 212 .

1704 C. Tour de Gorge de Venus, coquille en forme de lentille, à ftries crenues, croifjées, blanche, l'anus imprimé ovale, Linn. 136. E9 141. Seb. III. S6. 16. LiR. 357. 174. Gualth. 77. A. Petiv. Mur. I8, 6. 7. long. 25. it. 3. pouc. (I P . 213.

.1705 C. Tour de Gorge de Vcrus, approchant de la prece. dente, à bord rouge, polie, Limn. 136 ơ $14 \mathrm{I}$. Kircher II. 7o. long. 3. lat $3 \frac{1}{2}$. pouc. (1. p. 214.

syos C. Tour de Gorge de Vepnus Semblable d la precederste, folie, à Eord rusg, Linno $30.0^{\circ} 141$. (2. p. 214 . 
1707 C. Tigerina . præcedenti afinis, margine coccineo, Linoso 136. \& I4I. (2. Specim. 214.

1708 C. Tigerina, fimilis præcedenti. Red lutea, Linn. ' 136. Eீ 14 I. (2. Specim. 214.

1709 C. Glabra, tefta triquetra dilatata. glabra, lactea, Linn.

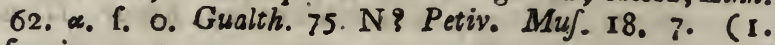
fpecim. 215.

1710 C. Trianguburis, tefta triquetris elongata, dilatata, natibus remotis, fulcata . flavefcente, radiis fuscis, Linn. 0 . Liff. 295. 131 ? 377. 220 ? Petiv. Gaz. 80. 6. (1. Ipecim. 216 .

I7II C. Sqramo $\int_{a}$, tefta fubcordata, tumida, reticulato ftriata, retrorfum (quamofa, flava, punctata, Linn. 135, Gualth. 83. I. Petiv. Gaz. 25. 8. Muf. 18. 17. (2. fpecim. 217.

2712 C. Hebes, tefta triquetris, obtufo ftriata, margine crenulato, lactea \& flava, Linn. 105. $\alpha$. f. o. Gualth. 88. N. (I fpecim. 218.

1713 C. Radiata, tefta triquetra fubcordata, lactea, radiis violaceis. Linn 105. $\beta$ I. O. Gualth. 88. Q Petiv.Gaz. 18. 4. Kircher II. 46 (I. Specim. 219.

$17 \mathrm{r} 4$ C. Catapetasma, tefta triquetris fubcordata, ex plumbeo fusca, lacteoque varia, Linn. 105. \%. f. 0. Gualtb. 88. S. (I. Tpecim. 220.

1715 C. Striata, tefta triquetris globofa, rotunda, lævis ftriata, flavefcens, radis plumbeis, Linn 147. ร.f. O. Gualth. 75. R? Liff. 403. 247? (I. Ípecim. 221.

1716 C. Undo $\int_{a}$, tefta flavefcens, lineis angulofis, rufo fuscis. Limn. I 47. A. f. o. Gualik 75. S. Lift. 402. 248? Petiv. Gaz. 75. 12. (2. Ipecim. 222.

1717 C. Coffata, tefta precedenti affinis . fed radiata, flava, radiis ciriereis, Linn. I47. r. I, O. Lift. 294 130? Petiv. Gaz. 94. 8. (I. Specim. 223.

T718 C. Rigate, tefta ovalis rotunda, in angulo patens, lineolis grifeis undata, maculis fuscis radiata, Linn. I47, d. f. O. Gualth. 86, E. Knorr II. 28. 4. (I. Specim. 224.

1719 C. Textilis, tefta precedenti affinis, fed glabra, lineis angulofis fe decuffaptibus purpurafcentibus, Linn. 147. y. . . O. Gualth. 86. H. Liff. 400. 239, Kircber II. 66. (2. pecim. 225.

1720 C. Textilis, pracedenti fimilis, Linn. 147 , n, . O. Knorr. 11. 18. 4. (2. fpecim. 225. 


\section{Teftacea. Ordo VII.}

I707 C. Tour de Gorge de Venus, approckant d la precedente, polie, d bord rouge, Linn. 136 E 142 . (2. p. 214

1708 C. Tour de Gorge de Venus, femblable il la preceden te, mais jaune, Linn. I36. EO I4I. (2. p. 214

I709 C. Refeau unie, iे coquille triangulaire, ettendue pelè, coulcur de lait, Linn. 62. $\alpha$, ou $0^{\circ}$ Gualth. 74. N? Potiv. Muf. 18. 7. (I.p.215.

17 to $C$. Triangle, à coquille triangulaire, allongée, étendue,

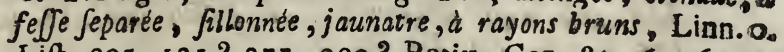
Lift. 295. 131 ? 377. 220 ? Petiv. Gaz. 80. 6. (I. P 216.

1711 Feffe ftriée, ds coquille un peu en coetir, enflée, reticalée, ftriée, écailleufe en derriere, jaune, pointée, Linn. 135. Gualth. 83. 1. Petiv. Gaz. 25. 8. Muf. 18. 170 (2. p. 217.

1712 C. Feffe jaune, d coquille triangulaire, ftriée, émouffés; de bord cremu, couleur de lait. E jaune, Linn. IO5. ou o. Gualth. 88. N. (x. p. 218 .

I713 $C$. Rayonnée, d coquille triangulaire, un peu en coessr coulewr de lait, $\dot{a}$ rayons violettes, Linn. 105. $\beta_{0}$ ou $C_{0}$ Gualth. 88. Q Petiv. Gaz. 18. 4. Kircher II. 46. (I.P219.

1714 C. Masque, à coquille triangulaire, un peu en coeur, yoriée de couleur de plomb, EO lait, Linn. 105. $\gamma$. ox 0. Gualth. 88. S. (I p. 220 .

17 I 5 . Plomb ftrie, d coquilie triangulaire, ronde, polie, jarr matre, $\grave{a}$ rayons couleur de plomb, Linn. 147. そ. ou ०. Gualth. 75. $R$ ? Lift. 403.247 ? (I. p. $22 \mathrm{I}$.

1716 C.Zig zag rouge, jaunatre a lignes anglees, brunes, Linn: I47. 8 ou o. Gualth, 75. S. Lift. 402. 248 ? Petiv. Gas. 75. 12. (2. p. 222.

I, I 7 C. Rayon cendré, coquille femblable à la precedente, mais jaune, $\mathcal{E}^{2}$ d coftes, EO rayons cendrés, Linn. 147. T. our 0. Lift. 294. 130 ? Petiv. Gaz. 94. 8. (I p. 223.

1718 C. Ondée brune, à coquille ovale ronde, en angle ouververte, ondée de lignes grifes, 8 rayonnée de taches brusnes, Linn. 147. $\delta$. ou $0 .{ }^{\circ}$ Gualth. 86. E. Knorr II. 28. 4. (I P. 224.

zig C. Corbeille noue, à coquille, femblable da la precedente. mais pelée, d lignes en angles pourprées, ¿o qui $\int e$ croisJent Linn. 147. ฯ. ou o. Gualth. 86. H. Lift. 400.23\%. Kircher II. 66. (2. p. 225.

1720 C. Corbeille noué, Semblable da la precederte, Linn.147. y. ou o. Knorr II. 18. 4. (2. p: 225. 
I72r C. Lacinia, tefta piæcedenti affinis, fed ftriata, pallida; maculis fuscis, margine cœruleo, Linn. 147, $\gamma$. . 0 . Lift. 404. 248? Argenv. Suppl. 3. D. (2. fpecim. 226.

1722 C. Lacinia, pracedenti fimilis, fed minor, Linn, I47. $\gamma$ f. O., (2. fpecim. 226.

I723 C. Litterata, tefta ovata, antice angulata, ftriis transverfis, fubundulatis, albeicens, atro lineata, Linn. 147. Lift. 402. 246. Argenv. 2r. A. Petiv. Muf. 18. 2. Valent. 6. 13. (2. fpecim. 227.

I724 C. Teges? C. varietas præcedentis, fed fusca, maculisangulofis, Linm. 147. a. Valent. 7. 10. I I. I4, I7. (2. Ipecim. 228.

I725 C. Teges, tefta præcedenti affinis, fed obfcurior ftriata, Linn. 147. $\alpha$.Gualth. 86.F. Rumph. 43. B. (2. fpecim. 228 .

I726 C. Guttata, tefta fimilis pracedenti, lutea, maculis fascis, Linn. 147. B. 1. O. Adanjon Seneg. 1. 17. 4. 12? Valent. 19. ( 1 . Ipecim. 229.

- 727 C. Guttata, precedenti affinis, fed minor, Linn. 147. B. f. O.: (4. fpecim. 229.

1723 C. Guttata, tefta fimilis præcedenti, carnea, fava, ra. diis obfcurioribus, Linn. 147. 1. f: o. Valent. 18. (1. ipecim. 230.

1729 C. IEteriç, tefta , an ptæcédenti affinis? Havefcens, Linn. 147. $x$, f. 0. Valent. 18 ? (I. Specim.-23I.

I 730 C. Medulla, tefta natibus acutis, reflexis, approximatis, rhomboidea, olivacea, Linn. O. Gualth. 89, H? (2. fpecim. 232.

873r. C Oliva, tefta natikus fubremotis oblonga, dilatata, ftriata, alba, lineis f. flammis olivaceis, Linn. $\bigcirc$. Gualth. 89. H. (2. fpecim. 233.

1732 Fafelus, tefta natibus remotis -incurvis, approximatis, oblorga, decuffata, Linn. 0. Gualth. 85. E. (1. (pecim. 234.

7733. C Transverja, tefta natibus elevatis, acutis, recirvis, oblonga, dilaiata, coftata, lactea, Linn. o. (I. fpe. cim. $234^{*}$.

\section{G E N V S XL. T E L L I N IE.}

1734 T. Doliclius, tefta rotundato oblonga, natibus elevatis, violacea, Linn. O. Lijt 388.235? 395. 242 ? I056.I? long. ' $1 \frac{3}{4}$. lat. $2 \frac{1}{4}$. (I. f pecim. 235 . 


\section{Teftaces. Ordre VII:}

T721 C. Panache brune, coquille Semblable do la precedente, mais friée, \&O pale, d taches brunes, E lefte, Linn. 147. $\%_{0}$ ou 0 . Lift. 404. 248? Argenv. Suppl. 3. D. (2. P. 226.

I 722 C. Panache brune, fermblable d̀ la precedente, mais plus petit, Linn. 147. r. ou o.' (2. p. 226.

1723 C. Ecriture Arabique, à coquille ovale, devant en angles; blanchatre, lignée de ftries traverJes, un peus ondées, Linn. 147. Lift, 402. 246. Argenv. 2I. A. Petiv. Muf. 18. 2. Valent. 6. 13. (2. p. 227.

I 24 C. Natte d'ECpagne, approchant d la precedente, mais à coquille brune, à taches anglées, Linn. I47. $\propto$. Valent. 7. 10. II. 14. 17. (2. p. 228.

1725 C. Natte d'Efpagne. Semblable d la precedente, mais plus obfcure, ftrié, Linn. 147. *. Gualth. ${ }^{26}$. F. Rumph. 43. B. (2..p. 228.

I726 C. Pavé d'Épagne, ou Ecriture Chinoire, ou Pagan, femblable à la precedente, à coquille jaune, à taches brubnes. Linn. 147. $\beta$ ou $0^{\circ}$ Adanion Seneg. 1. 17.4. 12? Valent. I9. (I. p. 229.

1727 C. Ecriture Chinoire, mais plus petit, Linn. 147. $\beta$, ou O. (4. p. 229.

1728 C. Ecriture Chinoire, femblable d la precedente, à cor quille couleur de chair, jaune, à rayons plus obfcures, Linn. I 47. 6. 0u o. Valent. 18. (1. p. 230.

I729 C. Feve jaune, coquille approchant $\dot{a}$ la precedente ? jaus: natre, Linn. 147. x. 02u o. Valent. I8? (1.p. $23 \mathrm{I}$.

I 730 C. Noyau de Prune, coquille à feffes recourbées, approchantes, en forme de cylindre, olivatre, Linn. O. Gualih. 89. $H$ ? (2. p 232.

I73I C. Rayon d'Olive, coquille a fefJes un peu separées: allongée. etendue; ftriée, blanchatre, di lignes, ou flam: mes olivatres, Linn. 0 . Gualth. 89. H. (2 p.233.

1732 C. Haricot, coquille d fefles Separées, recourbées, approchantes, ailongées, croifjee, Linn. O. Gualtb. 85. E. (I. p. 234.

733 C. Ligne therfe, coquille d feffes elevées, aigues, recourbées, allongée, etendue, do coftes, E couleur de lait, Linn. O. (I.p. $234^{*}$,

G E N R E XL. TE L L I N E S.

X734 T. Feve, ou Haricot grande, d coquille un per ronde allon: gée, violette, les fefjes elevées, Linn. O. Lift. 388235 ; 395.242 ? 1050. 1.? long. J条. lat. $2 \frac{3}{4}$. pouc. (1.p. 235 . $\mathbf{G} \mathbf{g}$ 
3735 T. Gari, tefta ovali, ftriis transverfis recurvaris, den. tibus láteralibus obfoletis, alba, margine violaceo, Linm. 48. Seb. III. 86. 17. Lift. 395. 242 ? Rumph. 45. C. Argenv. 21. D. (1. f́pecim. 236.

I736 T. Gari, fimilis præcedenti, polita, Linn. 48. ( 2. fpecim. 236.

1737 T. Gari, fimilis præcedenti, tefta violacea albida, ftriis transverfalibus rugofis, Limn. 48. Seb. III. 86. I I. (2. \{pecim. 236. a.

1738 T. Gari, præcedenti fimilis, fed major, alba \& viola. cea, Linn. 48. ( 1 . Specim. 236. a.

1739 T. Gari, pracedenti affinis, fed minor, alba, rofeaque, Linn. 48. Gualth. 86. B? (3. 'pecim. 236. b.

I740 T. Lutea, fiavefcens, fasciis obfcurioribus, margine crenúlato, Linn. 58. \%. C. 0. Gualth. 88. M. Knorr VI. 7. 7. (2. Ipecim. 238.

17.4I T. TeEtoriola, an fimilis pracedenti? alba, Limm. $58 . \beta$ f. O. Gualth. 88. I? $N$ ? (I. Specim. 239.

7742 T. Violacea, an præcedenti affinis ? alba, ftriis violaceis radiata, Linn. 59. «. 1. O. Gualth. 88, H? Lif. 384. 227. (I. Specim. 240.

7743 T. Canufina, tefta ovali, ftriis transverfis, fasciis elongatis, rofeatris, recurvatis, antice angulata, dentibus la: teralibus prominulis, Limn. 46. B.f.o. Gualth.89. I. Lifo. 393. 240. Rumph. 45. D. Argenv. 22. A. Valent. 5. (2. Ipecim. $23 \%$.

I744 T. Canufina, præcedenti affinis, alba, natibus rofeis, Linn. 46. B. f. 0.Knorr I. 19. I. (2. fpecim. 237.

1745 T. Canufina, fimilis pracedenti, Hava, fascia transver. fali alba, rubro radiata, Linn. $46 . \beta_{0}$. . . o. Knorr IV. 2. 4. (2. Ipecim. 237.

I746 T, Favana, tefta oblonga fulphurea, antice angulato, roftrata, angulis fubdentatis, Linn. 55. «. f. c. Lift. 382. 225 ? Rumph . 45. L. (I. Gqucim. 24I.

3747 T. Favana, præcedenti affinis, fed minor, Linn. 55. a. S o. (2. fpecim. $24 \mathrm{I}$.

1748 T. Roftrata, an pracedenti affinis? t" ta nitens rolea: fasciata, Linn. 55. Lijt. 382.225. Argenv. 22. O ? Petiv. Muf. 17, 20. (2. Specim. 242.

1749 T. Roftrata, fimilis prxsedenti, Linn. 55. (2. Specim. 242.

I750 T. Radiata, an pracedenti affinis? rofea sadiis elongatis albis . Linn. 55. 3. . . O. Knorr IY. 2. 3. S2. Spe(im. 243 . 


\section{Teftaces. Ordre VII.}

I735 $T$. Bacaffan, d coquille ovale, fries traverfes recourbées, les dens laterales obfolées, blanche, d botd violet, Linn. 48. Seb. III. 86. 17. Lift. 395. 242? Rumph. 45. C. Argenv. 21. D. (I. p. 236.

1736 T. Bacaffan, Jembbable à la precedente, polie, Linn. 48. (2. P. 236.

I 737 T. Bacaffan, approchant da la precedente, d coquille vio. lettes blancheatre, à ftriestransverfales, ridées, Linn. 48. Seb. III. 86. II. (2. p. 236. a.

T 738 T. Bacaffan, Semblable di la precedente, mais plus grande, blanche, $\mathbb{E}^{2}$ violetse, Linn. 48. (I. p. 236.a.

I739 T.Bacaffan, Semblable d la precedente, mais plus petit, blanche, couleur de rofe, Linn. 48. Gualth. 86. B.? 3.p. 236.b.

I740 T. Vierge jaune, jaunatre, d fafcies plus objcures, a bord crenu, Linn. 58. \%. ou 0. Gulalth. 88. M. Knorr VI. 7. 7. (2. p. 238 .

774 $T$. Vierge blanche, ou Semblable d la precedente? blanche, Linn 58. $\beta$. ou o. Gualth. 88. I? $N$ ? (I. p. 239.

$1742 T$. Radiéepourpré, ou femblable d̀ la precedente? blanche rayonné de fries violettes, Linn. 59. "*. ou $0_{\text {. Gualch }}$ 88. $H$ ? Lift 384. 227. (1. p. 240.

I743 $T$. Soleil levant, d coquille ovale, d ftries traverfes re courbées, it fafcies allongées, rouges, par devant en an gles, a dens laterales prominentes, Linn, 45. $\beta$, ou o. Gualth. 89. I. Lift. 393.240. Rumph. 45. D. Argenv. 22. A. Valent. 5. (2.p. 237.

I744 T. Soleil levant, approchant $\dot{a}$ la precedente, blancbe, feffes couleur de rofe, Linn. 46. $\beta$. ou O. Knoir, I. I9. I. (2. p. 237 .

1745 ' T. Soleil levant, femblable da la precedente, jaune, a fafcie transverfale blanche, rayomné de rouge, Linn. 46. B. ou o. Knorr IV. 2. 4. (4. p. 237.

1746 T. Pincette jaune, d̀ coquille allongèe, couleur de foufre, par devant en bec anglé, de angles un peu dentelées, Linn. 55. a ou O. Lift. 382. 225 ? Rumph. 45.'L. (1.p. 24I, 1747 T. Pincette, approchant à la precedente, mais plus petit, Linn. 55. * : ou 0. (2. P. $24 \mathrm{I}$.

$7748 \mathrm{~T}$. Pince rouge, ou approchant d la precedente? à belle coquille couleur de rofe bandée, Linn. 55. Lift. 382. 225. Argenv. 22. O ? Petiv. Mur. 17. 20 (2. p. 242. 1749 T. Pince rouge, femblable à la precedente, Linn. 55. (2. fpeciem. 242

7750 T. Pince rayonnée, ou Jemblable à la precedente? couleur de rofe, da rayons allongés blencs, Linn. 55. $\beta$. oz o. Knorr IV. 2. 3. ( 2. p. 243.

I751 $T_{s}$ 
I75I T. Meandrata, ina juivalvis, oblongo roftrata, transverfim ftriata, valvula altera plana, angulo agerata, vulva aculeata, lactea, Linn. 56. \& ᄃ. o. Gunlth 88. T. Lift. 398. 237. Knorr IV. 2.'5. (I. Ipecim. 244.

I752 T. Fucata . tefta ovata, angulata, compreffa, transverfim ftriata, lævi, marginibus acutis, pube fubtomentofa, lactea, natium macula carnea, Linn, 52 \% f. $。$. Rumph. 45. I? Petiv. Mirs. 17. 18 ? (I. Specim. 246.

1753 T. Radiata, tefta ovali, ftriis transverfis recurvatis, antice an; $013 t a$, oblonga, violaceo punctatim radiata, Linn. 46. a f. O. Lift. 399. 238. Knorr IV. 25. I. long. $3 \frac{1}{4}$. lai, rs poll. (r. fpecim. 245.

1754 1. Virgata efta o ata, transverfim ftriata, angulata, citrna, radiis icfels, Linn 46. Gualth. 86. G. Lift. 387. 234. Peiv. Mul 17. I2. Argenv. 22. G. long. $3 \frac{1}{4}$. lat, 2. p.ll. (I. foecin. 247.

1755 T. Virgata, præcedenti affinis, Linm. 46. (2. Specim, 247.

I756 T, Virgata, fimilis præcedenti, alba, radiis rofeis, Linn. 46. (2. Specim. 247. a.

1757. T. Pallidula, alba, rofeoque fasciata, Linn. 52. $\alpha$. Gualth. 89. G Lift. 397. 236. (r. Fpecim 248.

I758 T. Foliacea, tefta ovali, pube feshra, rima ferrata, lu. teo aurantia, Linn 51. Petiv Muf. 17.'17. Argenv. 22. E. Knorr V.29.2. long, 3 $\frac{3}{4}$. poll. (2. fpecim. 249.

1759 T. Angulata, tefta fubovata, ftriis transverfis recurvatis, antice angulata, a'ba, Linn. 47. Petiv. Muf. I7. I4. (I. fpecim. 250.

$1760 \mathrm{~T}$. Lingua felis, tefta fubovata, fcabra, fquamulis ilnatis, alba, lineis rofeis, Linn, 45. Gualth. 76. B. Petiv. Muf.17.16. (2. 'pecim.'251.

I761 T. Muftelina, tefta radiata, flavefcente ftriata, Linin. 52. भ. T. O. Gualth. 89. C? Li/f. 386 233. (2. Speeim. 252.

1762 T. Muftelina, præcedenti affisis, fed major, Linn. 52. y f. O. (2. fpecim. 252.

1763 T. Cruentata . tefta levigata, pube ftriata fcabra, alba, natibus fanguineis, Linn. 53. a. Lift. 4=8.253. Rumph. 45. I. ( I. Spacim. 253.

I764 T. Cruentata : fimilis pracedenti, fed minor, Linn. 53. «. (2. Specim. 253.

1765 T. Rofea, an pracedenti affiris? tefta rolea, natibus

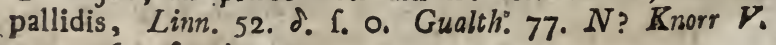
9. 3. (I. Ipeçim. 254. 
175I $T$. Pince traverfe, d̀ valve inegale, allongé ou bec ftriée de travers, l'autre valve platte. couleur de lait, Linn. 56. «. or o. Gualth. 88. T. Lift. 398. 237. Knorr IV. 2. 5. (I. P. 244 .

:752 $T$. Feffe fardée, à coquille ovale, en angles, comprimée, ftriée de travers polie à bords aigues, la pube charnue, couleur de lait, les fefes à tache rouge, Linn. 52. $\beta_{0}$ ou 0. Rumph. 45، I. ? Petiv. Mi,. I7. I8 ? (1. p. 246.

:753 T. Rayon, al coquille ovale, Jtries traverfes recourbées, par devant anglée, allongée, É rayonnée par des points. violette, Linn. 46. a. ou o. L ft. 399 . 238. Knorr. IV. 25. 1. long. $3 \frac{1}{4}$. lat. is. pouc. (I. p. 245 .

:754 T. Rayon rouge, à coquille ovale, ftriée de travers en angles, couleur de citron, à rayons couleur de rofe, Linn. 46. Gualth. 86. G. Litt. 387. 234. Petiv. 1Vuf. 17. 12. A rgenv. 22. G. long $3 \frac{1}{4}$. lat. 2. pouc. (I. P. 247.

:755 T. Rayon rouge, approchant à la precedente, Linn, 46. (2. p. 247.

i56 T. Rayon rouge, femblable d la precedente, blancke, rayons couleur de rofe, Linn. 46 . (2. p. 247. a.

1757 T. Rofe bleme, bandée de blanc E̊ de couleur de rofe, Linn. 52. \& Gualth. 89. G. Lift. $397^{\circ} 236$. (I p. 248.

$1758 T$. Langue d'Or, ì coquillle ovale, la pube raboteufe, la fen-: te dentelé, jaun orange, Linn. 51. Petiv. Muf. 17. $17:$ Argenv. 22. E. Knorr. V.29.2. long. $3 \frac{3}{4}$. pouc. (2. p. 249. $T$. Langue de Chat, à coquilie ur peu ovale, à ftries traverfes recourbées, par devant anglée, blanche, Linn. 47. Petiv. Mus. 17. 14. (I. p. 250.

1700 T. Langue de Chat chagriné, à coquille un peu ovale; raboteufe, à ecailles lunées, blanche, à lignes rouges, Linn. 45. Gualth. 76. B. Petiv. Mar. 17. 16. (2. p. 251

I76I T. Aurore, ou Rayon jaune, à coquille rayonnée, jaunatre

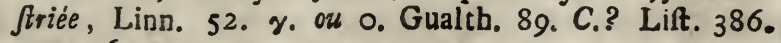
233. (2. p. 252 .

$1762 T$. Aurore, ou Rayon jaune, approchant à la precedente, mais plus grand, Linn. 52. $\gamma$. ou a (2. p. 252.

1763 T. Feffe faignante, d coquille polie, à pube frié, rabo-: teufe, blanche. dे fefes faignantes, Linn. 53. a. Lift. 408. 253. Rumph. 45 I. (I. p. 253.

1764 T, Feffe faignan:e, Semblable $d$ la precedente, mais plus petit, Linn. 53. $\alpha_{0}$ (2. p. 253.

$1765 T$. Feuille de rofe, or -femblable d lo precedente? de co. guille rouge, fefles pales, Linn. 52. D. ow ०. Gualtb. 77. N. ? Knorr V. 9. 3. (.. p. 254 .

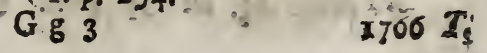


I766 T. Rojes pallida, tefta ovali lavi, nymphis prominentibus, flava rofea. Linn. 50. \&. Gualth. 89. C? ${ }^{2}$ ( 2. fpecim. 255.

1767 T. Exilis, an fimilis pracedenti? tefta plana, rofea nitens, Linn. 52 . .. .0. Gualth. 77.L? (2.fpecim. 256. 1768 T.Cluniculus, tefta ovata, flavefcens, gibba ftriis transverfis, natibus flavefcentibus, Linn. 49. «. Cualth.77. $H$ ? 1 3. Ppecim. 257.

1769. T. Rofacea, tefta fuborbiculata, utrinque incarnata, oblique ftriata, ftriis hinc reflexis, albo rofeo, flavoque fasciata, Linn 66. «. .. o. Gualth. 89. B? Lift. 405. 250? Petiv. Gaz. 94. 6. (3. Specim. 258.

7770 T. Scobinata, tefta ampullacea, fenticulari fcabra, fquamis lunulatis, alba, Linn. 64. Rumph. 43. H. Petiv. Mus. 18. 9. (2. fpecim. 259.

I77I T. Cryfoftoma, tefta natibus approximatis, margine gib. bofo, membranaceo ftriata, intus aurea , Linn. o. Lift. 260. 96. Gualth. 38. B. Rumph. 43. I. Knorr IV.14. 4. (1. Specim. 260.

1772 T. Triquetra, telta aliquo modo triargularis, denuo rotunda, rugofa, compreffa, lactea. Linn. 62. $\propto$. f. 0 . Lift. 406. 252. Rumph. 43. I. (2. Specim.' 261.

1773 . T. Nucleus Acacia, glabra, Jaetea, Linn. 62. B. f. O. Grolth 89. E. Lift. 407. 253. (I. Specim. 262.

1774 T. Nucleus Acacia, fimilis præcedenti . alba, glabraque, Linn. 62. B. 1. O. (2. Ipecim. 262. a.

I775 T. Coaxata, tefta trigonia, convexa, rotundata, alba, glabra, Linn. O. Rumpk. 42. H. Petiv. Muf. 18. 18. (1. fpecim. 263.

GENVS XLI. CAPIS TERIA.

1776 C. Spengleri, tefta triangulo cardato, lavi, vulva plana. rima lunula hiante transverfa, lutea, Linn. 94.

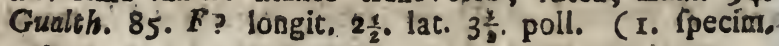
264 .

ryi7 C. Scortum, tefta triangularis cordata, Itriata, glabra, vale vaplana, alba, Limn. 102. Gualth. 85. F. Argenv. 21. B. Pe. tiv. Gas 156.35.36. Kircher II. I24. 125. Knorr VI.34. I. long. 21. lat. $3 \frac{t}{2}$. poll. (I. fpecim. 264 .

7778 C. Globulata, tefta triangulart cordats, lavi, diaphanz, friala, umbonibus lavillimis, vulva anóque Ariatis, albida', fusco radiata, Linn, 97. \& Gualth. 7 I B? long. 23. lat. $3 \frac{\%}{2}$ poll. (2. fpecim. 266. 
\$766 T. Feuille de rofe pale, à coquille ovale, polie, les nimphes prominentes, jaune rouge, Linn. $50 . \alpha$. Gualth. 89. C? (2. p. 255 .

2767 T. Feuille de rofe perlé, ou pareille de la precedente? coquille platte, couleur de roje, Linn. 52. n. ouo. Gualth. 77. L.? (2. p. 256 .

$1768 \mathrm{~T}$. Ferte jaune, ds coquille ovale, jaunatre, bo/Jue, ftries traverfes, $\dot{a}$ feffes jaunatres. Linn. 49. «. Gualth. 77. H.? (3. p. 257 .

E769 T. Bandée traverfe, a coquille un per ronde, rouge de chaque coté , ftrié oblique, à fries recourbées, bandée de rofe pale, Eे jarne, Linn. 66. «. ou 0. Gualth. 89. B.? Lift. 405. $25 \mathrm{c}$ ? Petiv. Gaz. 94. 6. (3. p. 258.

E77̧o T. Langue d'Ecaille, à cóquille fenticulaire glabreujes à ecailles lunu!ées, blanche, Linn. 64. Rumph. 43. H. Petiv. Mur. 18. 9. (2. p. 259.

I77I T.Toifon d'or, coquille à feffes s' approchantes, a bord bagru, ftrie en némbrane, en dedans dorée, Linn. 0 . Lift. 260. 96. Gualth. 88. B Rumph. 43. I. ? Knorr IV. I 4. 4. (I. p. 260. $277^{2} T$. Triangle traverfe, a coquille tant foit peu triangulaire, pour le refte ronde, ridée, comprimée, couleur de lait, Linn. 62. «. ou o. Lift. 406. 252. Rumph. 43. I. (2. p. 26r.

I773 T. Pepin de Tamarinde, pelée, coulerir de lait, Linn.62., ou o. Gualth. 89. E. Lift. 407.253 (r. p. 262 .

$1774 T$. Pepin de Tamarinde, Semblable dे la precedente, blasse che, pelée, Linn. 62. B. ou O. (2. p. 262.

$5775 T$. Quacre ventrue, di coquille triangulaire, recourbée, ronde. blanche, \& pelée, Linn. O. Rumph.42. H. Petiv. Mur. 18. 18. (I.p. 263.

GE NRE XII. TINET TES.

$1776 T$. de Spengler, or Fente, à coquille triangulaire en coeur, polie, d pube platte, a fente ouverte transverfe, jaunatre, Linn. 94. Gualth; $85 . F$ ? long. $2 \frac{1}{2}$, lat. $3 \frac{1}{2}$. pouc, (I. p. 264 .

17 i7 T. Putain, oú Fente levré, de coquille triangutaîre eñ coeur, ftriée, pelée, à pube platte, blanche, Linn.102 'Gualth. 85. F. Argenv. 21. L. Petiv. Gaz, 156. 35. 36. Kircher 11. 124.125 . Knorr VI. 34. I. long. $2 \frac{1}{4}$. lat. $3 \frac{1}{2}$. poive. (1. p. 265 .

$778 \mathrm{~T}$. Rayon pale, coguille triongulaire en coetus, polie: transparante, ftriee, a pointes tres polies, la pube, \& l'anus ftriés, blancheatre, radiée de brun, Linñ.' 97. a. Gualth. 7I. B.? long. $2 \frac{3}{4}$. lat. $3 \frac{1}{2}$. pouc. (2. p. 266 . $\mathbf{G}_{\mathbf{g}} 4$

$1179 \mathrm{~T}_{\mathrm{i}}$ 
I 779 C. Ferruginosa, tefta triangulari cordata, levis, diaphana, umbonibus fubftriatis, vulva lavi imprefla, carina circumfcripta, alba, ferrugineo maculata, Linn. 96. «. Gualth. 85. D? (I. fpecim. 207.

I780 C. Ziczac, tefta triangulari fubovata, flavefcente, ftriis angulofis caftaneis, Linn. O. (I. Specim. 268.

I78I C. Erugata, tefta triangulari formis cordata, opaca; læviufcula fubantiquata, albida, maculis fuscis, Linn. I00. «. .. O. Kircher II. 50? (I. Ppecim. 269.

I782 C. Radiata, f. Stultorum, tefta fubdiaphana, ovali, lævi, ob-i foleta, flava, radiata, intus purpurafcente, vulvagibba?

Linn. 99. Gualth. 71. C. (1. Specim. 270.

I783 C. Glabrata, tefta triangulari cordata, læui, diaphana; umbonibus fubftriatis, vulva lævi, impreffa, carina cir. cumfcripta, grifeo fusca, ftriis flavefcentibus, intus violacea, Linn. 97. Lift. 25388 ? Gualth. 7 1. B. Knorr VI. 5. I. (2. fpecim. $27 \mathrm{I}$.

178 C. Corallina, tefta triangulari cordata, lavi, alba, fasciis ftriis fuscis, vel cyaneis maculata, Linn. 98.' Lifto 253.87.? Petiv. Gaz. 94. 7. Kircher II. 52. Knorr VI, 1. 5. 2? 8. 5. (2. fpecim. 272.

\section{GE N V S XLII. M Y T VL I.}

I785 M. Edulis, tefta læviufcula, elongata, valvulis antice carinatis, poftice retufis, natibus acuminatis, violacea, albo ftriata, Linn. 253. Lift. 362. 200. Kircher II. 29. Ginann. II. 168 ? (2. fpecim. 273.

x786 M. Edulis, pracedenti affinis, tefta nigro violacea, natibus albis, ftriis transverfalibus, Linn. 253. a. 'Knorr IV. 15. 1. (2. fpecim. 274.

1787 M. Edulis; fimilis præcedenti, Linn. 253. «. (2. fpecim. 274.

1788 M. Eduslis, præcedenti affinis, teita glabra, in extremo alba, Linn. 253. $\beta$. Lift. 360. 199. finiftra. (I. fpecim. 275.

1789 M. Edulis, fimilis præcedenti, artificiofe fculpta, arbores fylvamque referens. Linn. 275. $\beta$. (1. Spe cim. 276.

1790 M. Emicatrix, tefta parva, lrvi granulari ftriata, caftanea, pulcerrimo ultramarino renitens, Linn. 250. f. O. Lift. 362. 201. Rumph 46. D. Gualth. 91. H. fupra, Argenv. 22. H? Petiv. Mus. I9. 3. (I. Ípecim.' 277. 


\section{Teftaces Ordre VII.}

779 T. Rouillée, à coquille triangulaire en coeur, polie; transparante, 'à pointes un peu'ftriées, à pube polie, insprimée, à carine entourée, blanche, tachetée de rouilile. Linn. 96. «. Gualtb. 85. D.? (I. p. 207.

780 T. Zic-zac, d́ coquille triangulaire, un peu ovale, jaura:tre , d fries chataignes en angles, Linn. O. ( I. p 208.

78I $T$. Tachetée, à coquille triangulaire en forme de coeur.; obfcure, un peup polie, blanchatre, à taches brunes, Linn. 100. $\alpha .0 u$ 0. Kircher II. 50. ? (1. p. 269.

$782 T$. Rayon brune, ou Marotte, à coquille un peu transparante ovale, polie fale, jaune, rayonnée, pourpre en dedans. à pube bolfue, Linn, 9y. Gualth. 71. C. (1. p. 270... $783 T$. Feffe brune, coquille triangulaire ens, forme de coeur. polie, transpar ,nte, à points un peu ftriés, à pube polie. imprimée, carine, entourte, gris-brune, à ftriés jaunm tres, violette en dedans, Linn. 97. Lift. 253.88. ? Gualth. 7 r. B. Knorr. V'I. 5. I. (2.p. 271.

784 T. Coralline, 'ou Marbré ftrié, de coquille triongulaire on forme de coeur, polie, blanche, bandes \&o frites brunes. ou bleues tachetées, Linn. 98. Lift, 253.87? Petiv. Gaz. 94. 7. Kircher II. 52. Knorr VI. 5. 2 ? 8. 5. (2.p.2\}2. I

GENRE. XLII. MOULES.

$785 M$. Ordinaire, à coquille polie, eiongée, les valves carinęer: par devant, emouffjees par derriere, à felfes pointues, vio. lette, friée de blanc, Linn. 253. Lift. 362. 200. Kircher. II. 29. Ginanni II. I68? (2.p. 273 .

$786 \mathrm{M}$. Ordinaire, ou Violette, approchant à la precedente, a coquille noir-violctte, à feffes blanches, E Jtrues traverfes; Linn. 253. $\alpha$. Knorr IV. 15. I. ( 2 p. 274 ..

787 M. Ordinaire, femblable da la precedente, Liñ. 253. (2.p. 274 .

$788 M$. Ordinaire, approchant dà ĺs precedente, à coquille pelée, blanche au bout, Linn. 253. $\beta$, Lift. 360. 199. fintifra, (2. p. 275 .

$789 M$ Ordinaire, femblable d̀ la precedente artificiellement Jculptée, reprejentant, des arbres, E' un bois, Linn. 275. B. (I. p. 276.

$790 \mathrm{M}$. Changeant, à coquille petite, polie, ftriées en grains: chataigne, reluifante d'un tres teau ultramarin, Lims. 250. a. ou o. Lift. 362 201. Rumph. 46. D. Gualth. 92.

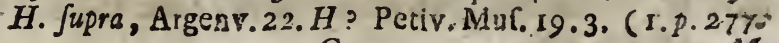
Gg 5 
x79r M. Renitens, tefta, an pracedenti fimilis? gibbofidr, ? nitida caftanea, Linn. 251. . f. 0. Lift. 362. 202. (1. fpecim. 278.

2792 M. Gibber, tefta gibbola, dilatata, caftanea, cyaneo : diaphana, Linin. 250. B. f. 0. Lift. 357. 195. b? Adanjont Serieg. I. 15. 3. 4. (I. Kpecim. 280.

I793 M. Teffera, tefta dilatata, margine denticulato, in miedio oblcure ftriata, plumbea, Linn. O. Lift. 366. 206. Adanfon Seneg. 1. 15.3. 3. (1. fpecim. 279.

1794 M. Candens, tefta pracedentis varietas? caftanea nitens, Linn. O. Kntrr VI. 4. 3? ( r. Ipecim. 279.

1795 M. Tuber, tefta tuberculato gibbofa, margine lavi, fusca, purpurea, Lins. o. Lift. 356.194? Knorr VI. 13. 6. (1. Ppecim. 281 .

x796 M. Magellanus, tefta oblonga coftata, friis transverfalibus rugola, fubcurvata, margine pofferiore inflexo, rubro violacea, Lirn. 255. «. 1. O. Lift. 358. 196. Argenv.

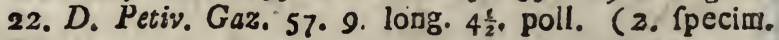
282.

1797 M.Pumicatus, teftalevi, fubcurvate, margine polteriore inflexo, alba, viridefcens, circumflexa fusca, Linn. 254. «. C. O. Gualth. 91. E. fupra, Lift. 105\%.

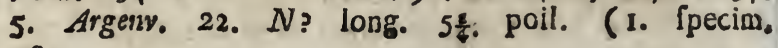
283.

2798 M. Pumicatus, pracedenti attinis, Limn. 254, ๔. ᄃ. C. (2. fpecim. 283.

I799 M. Talaris, tefta oblonga acuta, rugofa, purpurea, Linn, 254. B. f. O. Gualth. 9r. E. infra. (2. 'pecim. 284.

$2800 \mathrm{M}$. Ignifer, tenta glabrata, lineis angulofis teftaceis fusco flavis pieta, Linn. 254. d. 1. O. Gualth, 91, $H$ ? infra, Argenv. 22. N. (2. Specim. 285.

1801 M. Ignifer, tefta pracedenti affinis, grifeo rufeleens, lineis angulofis tlammeis, Limmo 254 . d. T. O. (2. fpecim. 285.

1802 M. Ignifer, fimillimus pracedenti, dao fpecimina, alterum flava, Linin. 254. o. . . O. (2. (pecim. 285.

$\$ 803$ M.Smaragdylus, an finilis pracedenti? viridis, angulofis Itriis, luceus, Linn. 254.y. fo O. Li/f. 363. 204. long. 4. poll. (I. Specim. 286.

1804 M. Smaragdylus, tefta pracedenti fimilis, fed paulo minor, Linn. 254. y. ศ. O. (2. fpecim. 286.

2805 M. Smaragdylus, pracedenti atinis, Lim. 254. y. L.० (3. ipecim. 286. 
I79I $M$. Boffe brune, ou . Reluifante, coquille ou jemblabtén d la precedente ? plus boffue, d'un beau chataigrie, Linn. 25I. «. ou O. Lift, 302. 202. ( J.p.'278:

I792 M. Elargé, à coquille bofrue, etendue, chatcigne, d'ure bleue diaphane, Linn. 250. $\beta$. oib o. Lift. 357. 195. b? Adanfon Seneg. 1. I 5. 3. 4. (I. p. 230.

1793 M. Dolel, à coquille etendue, le bord dentelé, couleur de plomb, ftriée obfcurement au milieu, Linn. 0. Lift. 366. 206. Adanfon Seneg. I, 15. 3. 3. (I. p. 279.

x $794 M$. Brulant, coquille ou varieté de la preceilente? couleur de beau chataigne, Linn, O. Kinorr VI. 4. 33 (1. P. 279.

1795 M. Boffue. ou de Magellan, d coquille bofue par tuber. cules, à bord polie, brun pourprée, Linn. O, Lift, 356: 194 ? Knorr $\nu_{\text {. }}$ 13. 6 . (I. p. 281.

$1796 M$. de Magellan, coquille allongée, coftée, à ftries traverfes, ridée, un peu courbee, le bord pofterieur. recourbé, rouge. violette, Linn. 255. a. ou O. Lift. 358. 196. Argen甲. 22. D. Pctiv, Gaz. 57. 9. long. $4 \frac{1}{4}$. pouc. (2. p. 282 .

$1797 M$. Grande de Magellan, a coquille polie; un peu coussbée; blanche, le bord poferieur recourbe, verdeatre, courbee brun, Linn. 154. a. otu 0. Gualth. 91. E. Supra, Litt.

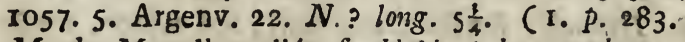

I 798 M. de Magellan plié, Semblable d̀ la precedente, Lina 254. $\alpha$. ou 0. (2. p. 283.

1799 M. Rougeatre, d coquille allongée, aigue, pourprée. Linn. 254. $\beta$. ou o. Gualth. 91. E. infra. (2. p. 284. $1800 M$. Flamboyante, de coquille pelée, peinte de lignes en angles teftacées, brun-jaunes, Linn. $254 \therefore$ ou o. Gualit. 91. $H$ ? infra, Argeuv. 22. N. ( 2 p. 285 .

I80r $M$. Flamboyante, de coquille approcbant di la precedente. gris-rougeatre, à lignes en angles, enflammées, Lines 254. d. ou o. (2. P. 285 .

$1802 M$. Flamboyante, tres femblable a la precedente; deus pieces, dont Pune eft jaune; Linn. 254. 2.0u o. (2.p. 285.

180,3 M Verte, ou Emeraude, on Semblable au precedent? yerte. E jaune, frié en angles, Linn. 254. $\boldsymbol{\gamma}_{\mathrm{i}}$ ou 0 . Lift, 363. 204. long. 4. pouc,... (x. p. 286.

$1104 M$. Verte, ou Emeraude, is cogaille femblable a la prece dente, mais un pers plus petite, Linin. 254. \%. ors a (2. p. 286 .

I805 $M$. Vente, on Eneraude, approchiont fla prebederte, Linn. 294. $\%$. ou o. (3. p. 286 . 
r806 M. Violaceus, tefta fublongata glabra, poftice recurva: ta, Linn. 254. ฑ. f. o Gualth. 9r. E ? infra, Sift. 364. 203. Knorr V. 25. 1. (2. Ipecim. 287.

GE NVS XLIII. RIPARI庍。

Y807 R. Anatina, tefta ovall compresfiufcula, fragilifima, margine membranaceo, dente coftato natibus nodulo. fis, Linn. 258. Gualth. 7. E. F. Lift. 146. I, 151.6.

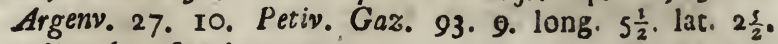
poli. (3. 'pecim. 290.

1808 R. Hirundo, tefta lævi, valvults bilobis, lobo cardinali longiore, tenuioreque, Linn.262. Gualth 94. A. Lift. 220. 55. Adanjon Seneg. I. 15. 3. 6. Kircker 11.

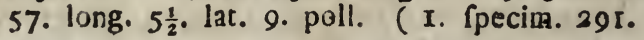

4809 R. Hirundo, præcedenti affinis, decorticata, Linn. 262. Knorr VI, 2. I. long. 4. lat. $4 \frac{3}{4}$. poll. (1. Specim. 29 I.

1810 R. Hirundo, fimilis præcedenti, fed minor, flava, radiis rufo fuscis, Linn. 262. a. Gualth 94. B. Petiv.

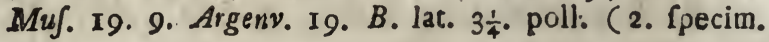
292.

I8x I R. Hirundo, præcederiti affinis, Linn, 202. «. Knorr IV. 8. 5. V. 10.1. 2. (2. Ppecim. 292.

1812 R. Hirundo, fimilis præcedenti, fed minor, crufta cellulofa obtecta, an Efchara? Linn. 262. B. Ginann. II. 115. (4. Ipecim. 293.

$\times 813$ R. Malleus, tefta æquivalvi plicata, triloba, lobis transverfis, natibus affinis pracedentibus, violacea atra, Linn. 207. Lift. 219. 59. Petiv. Mu/ 20. 10. Argenv. 19. A. Kircher II. 130. Spengler Icon. I. A. Jong. 6. lat. 9. poll. (1. Ipec:m. 294. a.

1814 R. Malleus, præcedenti affinis, fed minor, Linn. 207. Knorr 11I. 4 I. long. $4 \frac{i}{4}$. lat. $7 \frac{1}{4}$. poll. (r. fpecim. 294. b.

1815 R. Malleus, fimilis præcedenti, fed minor, \& rubro

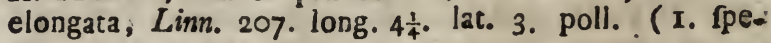
cim. 294. C.

m16 R. Mallers, præcedenti fimilis, Linn. 207. long. 4. lat. $3 \frac{1}{2}$. poll. (I. Ipecim. 294. d.

'1817 R. Malleus, tefta curva plana, fere non fquamofa, Linn. 207. w long. $3 \frac{3}{2}$. lat. $2 \frac{1}{4}$. poll. ( I. Ppecim, 294. e. 
I8cs $M$. Violette, d coquille un peu allongée, pelée par derviere recourbée. violette, Linn. 254. ฑ ou O. Gualth. 9 I. E.? infra, Lift. 364..203. Knorr V.25. 1. (2.p.287.

GE NR E XLIII. H I R ONDELLES.

$1 \$ 07$ H. Bec de Canard, à coquille ovale, un peu comprimée; tres fragile, à bord membrané, dent coftée. à felJes noueufes, Linn. 258. Gualth. 7. E. F. Lilt. I46. I. 151. 6. Argenv. 27. 10. Petiv. Gaz. 93. 9. long. 52. lat $2 \frac{2}{2} \cdot$ pousc. (3. P. 290 .

1808 H. de Mer Grande, 'd coquille polie, d valves bilobes la lobe cardinale plus longue, EO plus mince. Linn. 262: Gualth. 94. A. Lif?. 220.55 Adanfon Seneg. 1. 15. 3. 6. Kircher 11. 57. long. 52 : lat. 9. pouc. (1. p. 29r.

$1809 H$ de Mer Grande, approchant a la precedente, fans robe, ou polie, Linn. 202. Knorr VI. 2. r. long. 4. lat. $4 \frac{1}{4}$ pouc. (I. p. $29 \mathrm{r}$.

1810 H. de Mer, Jemhlabie d̀ la precedente, mais plus petit. non polie, jaune, à rayons rouge brunes, Linn. $262 . \alpha_{\text {, }}$ Gualth. 94. B. Petiv. Mul. 19. 9. Argenv. 19. B. lat.

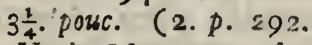

I8r I H. de Mer, approchant, d la precedente, Linn. 262., Knorr. IV. 8. 5. V. 10. 1. 2. (2. p. 292.

I8I 2 H. da Mer, jemblable a lis precedenis, mais plus petite, couverte d'une croute celluleufe, ou Effcara? Linn. 262, Ginanni II. II5. (4. p. 293.

1813 H. Marteau-Polonais, à coquille d valve egale, pliée trilobe, à lobes traverses, a felfes approchantes aux precedentes, violette, noire, Liinn. 207. Lift. 219. 59 . Petiv. Muf. 20. 10. Argenv 19. A. Kircher II. 130 . Spengler Icon. I. A. long. o. lat 9 pouc. (1. p. 294. a

I8I 4 H. Marteau Polonais, approchant d is precedente, mais

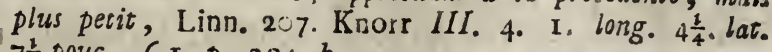
$7 \frac{1}{2}$ pouc. (1. p. 294. b

$1815 H$. Marteau Polonais Jemblable à ha precedente, mais plus petit, ou d bras plus court, rsuge, allongée, Linn.

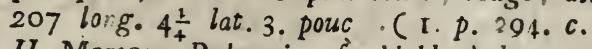

$1816 \mathrm{H}$. Marteau Polonais, Temblable d la precedente, Linn.' 207 long 4. lat. $3 \frac{2}{2}$ pouc. (I.p $94 . d$.

1817 H. Marteau Pulonais, le pareil courbee platte, pres. que Sans ecaille, Linn. 207. a long. $3 \frac{1}{2}$. lat. $2 \frac{\frac{1}{4}}{4}$. pouce. (I. p. 294. e. 
I818 R. I Jogonsy, tefta æquivalvi, Jobo laterali majore, cardine multoties fulcato, atro rufefcente, Linn. 214. Lift. 227. 62 ? Petiv. Muf. 2c. Ir. Valent. 3. long. $6 \frac{1}{2}$. lat. $3 \frac{1}{2}$. poll. (r. fpecim. 295. a.

1819 R. Ifogonum, pracedenti affinis, fed minor, Linn. II4. Seb. III. 91. 6.8 .Knorr VI. I3. 1. long. $4 \frac{1}{2}$. lat. $2 \frac{3}{4}$. poll. (I. Rpecim. 295. b.

1820 R. Ifogonum, fimilis præcedenti, natibus decorticatis, Limn. 2 2 4. Rumph. 47. I. Knorr IV. xO. I. 2, long.

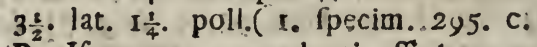

182I R. Ifogonum, præcedenti affinis, magis concava, Linn. 214. long. 3. lat. $2 \frac{3}{4}$. poll. (I. fpecim. 295. d.

1822 R. IJogoalata, tefta angulata, rotunda, plana, luteo qlivacee, intus nitens, margine lato fusco, Linn. 214. a. f. O. Sel. III. go figura extrema, linize fecunda, Knorr VI. 2I. I. long. 5. lat. $4 \frac{2}{2}$. poll. (I. fpecim. 296. a.

1823 R. Ifogoalata, fimilis pracedenti, tefta flavefcente alba, Linn. 2 i4. \&. f. 0.Lift.227. 62. long. $1 \frac{3}{4}$. lat. $1 \frac{3}{4}$. poll. (I. Rpecim. 296. b.

1824 R. Margaritifera, tefta compreffo plana, fuborbiculata, bafi transverfa, imbricata, cardine finiiil pracedentibus. tunicis dentatis, deverffata, Linn. 246. $\alpha$. Gualth, 84. F. Petiv. Muf. 20.4. lopg. 5. lat. 6. poll. (I. 'pecim. 297. b.

1825 R Margaritifera, fimilis pracedenti, decorticata, Linn。 246. a. iong. 3. ar. $3 \frac{t}{2}$. poll. (I. Cpecim. 297. c.

2826 R. Margaritifera, pracedenti affinis, olivacea, imbricata, radiis aibis, Linn. 246. Gualth. 8I. G. Lift. 223. 57. 58. Rumph. 47. G. Argenw. 20. A. Petiv. Muj. 20. 7. long. \& lat. $3 \frac{1}{2}$. poll. (I. fpecim. 298. e.

2827 R. Margaritifera, finilis pracederti, decortata, Linna. 246. (2. fpecim, 298 d.

1828 R. Margaritifera, precedentifimilis, fedminor, radiis albis vel rubris, Linn. 246. (4. Fecim. 298. Ca

1829 R. Margaritifera, fimilis præcedenti, fed valvula una. intus perlata, Linno 246. Gualth. 84, E. Liff. 221. 56. Kircher II. I. ( I. Specim. 299. b.

1830 R. Margaritifera, piæcedenti affinis, intus magis perlata Limn 246. (2. fpeciun. 298. a.

- 1830 R. Margaritifera, una valvula, perita manu celeb. Bel. kin ex'culp!a , fugam Maria in Aegyptumn reprefentans, cum multis figuris elevatis Seb III. $3 \%$. I. ubi veria effizies reperitur, compacta in regula quadrati. deaurata; alto G: lat 7. poll. (1. Speciu. 2,98. 1. GENVS 
I818 H. Equerre, da coquille da valve egale, lobe laterale phes grande, à charniere plufseurs fois fillonnée, noire-brunatre

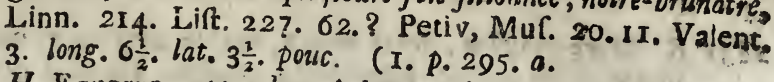

$819 \mathrm{H}$. Equerre, approchant d la precedente, mais plus petite. Linn. 214. Seb. III. 9r. 6-8. Knorr VI. 13. x. long. $4 \frac{1}{2}$. lat. $2 \frac{3}{4} \cdot$ pouc. (I, p. $295 \cdot b$.

820 H. Equerre, jemblable d ba precedente, a fefJes de: corchées, Linn. 21 . Rumph. 47. I. Knorr. IV. IO. I.2. long. $3 \frac{3}{2}$. lat. $1 \frac{\mathrm{T}}{4}$ pouc. (1. p. 295.c.

$82 \mathrm{I} H$. Equerre, approchant d la precedente, olus concave, Linn. 214. long. 3. lat. $2 \frac{3}{4}$. pouc. ( 1. p. 295. d.

822 H. Equerre ailé, d̀ coquille en angles, ronde, platte; jaun-olivatre, luifante en dedans, à bord large, bruns, Linn. 2 I 4. $\alpha_{\text {. ou }}$ o. Seb. III. les 90 . figures finales du Jecond rang, Knorr VI. 21 . I. long. 5. lat. $4 \frac{\frac{2}{2}}{2}$. pous. C $I_{\text {. }}$ p. 296. $a$.

$823 H$. Equerre ailé , femblable d la precedente, a coquille

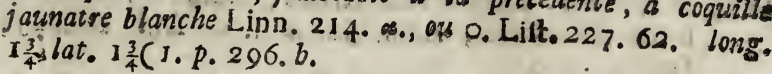

$824 H$. Pintade nacrée, \& coquille comprimée, platie, un peu ronde, à bafe traverfe, en forme de tuile, charniere femblable aux precedentes, dituniques dentelees, decorchee, Linn. 246 . * Gualth. 84. F. Petiv. Mur. 20.
4 long. 5. lat. 6 . pouc. (I. p. 297. b.

825 $H$. Hintade nacrée, Semblable a la precedente, Sans robe,

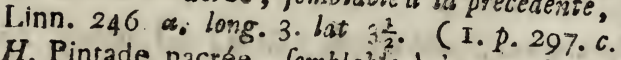

$826 \mathrm{H}$. Pintade nacrée. Semblabbe d ia precedente. d coquille olivatre, en forme de tuille, à rayons blanches, Linn. 246. Gualth 8I. G. Lift. 223.57. 58.Rumph. 47.G. Argenv. $20^{\circ}$ A. Petiv. Mur. 20. 7. long \& lat. $3 \frac{3}{2} \cdot$ pouc. ( 1. p 298. $\varepsilon_{\text {. }}$ 327 H. Pintade nacrée, femblable d la precedente, fans robe,
Linn. 246 (1. p. 298. d.

$328 H$. Pincade nacrée, femblable di is precéente, mais plas: petite, à rayons blanches, ou rouges, Linn. 246 . (4.p. $298 . c$. perlée, manque une valve. Linn. 246. Gualib. 84. E.
Lift. $22 \mathrm{I} .56$. Kircher II. I. (I. p. 298. b.
$H$. Pintade nacrée $330 \mathrm{H}$. Pintade nacrée, une valve manque, perlée, en dedans
Linn. 246 . (2. p. 298 a.

$330 \mathrm{H}$. Pintade nacrée une valve, en fouipture en camajeu, par la main du celebre Belkin, reprefentant la fuite de Marie, en ou je trouve le veritable effigie; en demi horfe. encadrée en cacipe roir, a fraije dorée. long. $6 \frac{1}{4}$. bat. 7. pouc. (I. p.293.f. 
I\&3r B. Margaritifera, telta ovata, antice coartata, cardinis dente primixio \& fecundario conico. natibus decorticatis, atra fuscens, itcus perlata Linn. 29. Gualth. 90. A ? fupra, Lift. 147. 2. A. Kircher II, 50. long. 2. lat. $4 \frac{1}{2}$. poll. ( I. fpecim. 288.

I832 B. Pidtorum, tefta ovata, cardinis dente primario cre. nulato, laterali longitudirali, alterius duplicato, olivacea flava, Linn. 28. Lift. 149. A. Kircher II. 39. (I. fpecim. 289.

2833 B. Piftorum, præcedenti affinis, Linn. 28. (2. Ipecim. 289.

i834 B. Solaris, tefta ovata, oblonga, antice depreffa, vio. laceo grifea, radiata, Linn:0. Lift. 394.241. A. Rumph. 45. D. Petiv. Gaz. 94. \%. (4. fpecim. 302.

$\mathbf{8} 35 \mathrm{~B}$. Fucata . tefta ovali ftriata, albida: natibus rofeis,' intus flsvefcens, Liinn. 50. «. r. O. Lift. 408.254? (r. fpecim. 300.

I836 B. Planata, tefta ovata, comprefla, transperfim ftriata, lævi, marginibus acutis, pube fubtomentofa, Linn. 52. œ. Gualth. 59. G? (1. fpecim. 30r.

I837 B Crerulefcens, tefta ovata oblonga, lineata, hians, ccrulefcenti violacea ftriata, Linn. O. Liff. 4 I 7. 26r ? 421. 265. Adanjon Seneg. 1. 19. 7. 2? (I. Ipccim. 303.

1838 13. Carulefcens, pracedenti affinis, fed minor, Linn. 0. Kircher IL. 106? (1. Ipecim 303.

I839 B. Mirio, tefta oblonga, lavi ovali, utraque patens cardini dente albo, Limn 101. ๙. r. o. Gualth. 90. A. infra, Lift. 415. 259. Rumph. 45. N? (1. Ipecim, 307.

1840 B. Vulfella, tefta linguiformi, tenuis lavi, rugofa, cardine rerminali, depreffo femiorbiculato. Iuteo fufcoque ftriata, Linn. 31. Lift. 1055. 10. Pečiv. Muf.19.1. Kr.orr V.2.1-3. (2. fiecim. $3=8$.

184ז B. Pala, telta arce;s orbiculata, valvula alcera plata, altera concava, ftriata, rubefcens, Linı. o. Lift. 202. $36 ? \cdot 203.37 ?$ (2. Ipecim. 299. 


\section{Teftaces. Ordre VII.}

GENRE XLIV. TEINTURIERS.

183I $T$. Mante à perles, d coquille ovale devant comprimée, is premier, Es second dent de la charniere, conique, d feJjes decorchées, noir brunatre, en dedans d perles, Linn. 29. Gualth. 9c. $A$ ? Jupra, Lift. 147. 2. A. Kircher II. 50. long. 2. lat. $4 \frac{2}{4}$. pouc. (I. p. 288.

$1832 T$. Moule des Peintres, d coquille ovale, le premier dent de la charniere eft crenue, laterale, longitudinale, l'artre duplicée, doublée, jaun-olivatre, Linn. 28. Lift. 149. A. Kircher II. 39. ( 1. p. 289.

I833 T. Moule des Peintres, approchant d la precedente, Linn. 28. (2. p. 289 .

I834 T. Solaire, ¿̀ coguille ovale, allongée, deprimée par devant, violette grife, rayonnée, Linn. o. Lift. 394. 241: A. Rumph. 45. D. Petiv. Gaz. 94. 9. (4.p 302.

¿835 T. Feffe fardée, à coquille ovale, friée; blanche, d feJjes couleur de rofe, jaunatre en dedans, Linn. 50. $\alpha_{0}$ ou o. Lift. 408. 254? ( 1, p. 300.

$1836 T$. Feuille de rofe, a coquille ovale comprimée, ftriée de travers, polie, à bords aigues, à pube un peu bouré, Linn. 52. $\propto$. Gualth. 89. G? (1.p.30I.

I837 T. Langue bleu celefte, d̀ coquille ovale, allongée, lignée entre-ouverte, de bleu violette ftriée, Linn. O. Lift: 417. 261 ? 421.265 ? Adanfon Seneg. I. I9. 7. 2? (1. p.303.

$1838 \mathrm{~T}$. Langue bleu celefte, approchant d̀ la precedente, mais plus petite, Linn. 0. Kircher. II. 106.? ( I. p. 303.

I839 T. Bec d'Oye, d coquille allongée polie ovale, ouverte de deux cotés, à charniere denteiée, blanche, Linn. Ior. .. ou 0. Gualth. 90. A. infra, Lift. 415. 259. Rumph. 45. $N$ ? ( т. p. 307 .

I840 T. Pince tronquée, à coquille en forme de langue, polie; ridée, à charniere demi ronde, Linn. 31 .' Lift. I055. I0: Petiv, Muf. I9. I. Knorr V. 2. I-3. (2. p. 308.

I84I T. Pele, à coquille orbiculaire, dont une valve eft platte, l'autre cavée, ftrié, rougeatre, Linn. ○. Lilt 202. 36 ? 203. 37.? (2, p. 299. 


\section{i $\pi$ G E N S XLV. S OLE NES.}

2842 S. Anatimus, tefta ovata, tenuatin inflata membranas cea, pilo \{a, cardinis cofta falcata, alba, Linn. 40. Gualth. 91. D. Petiv. Muf. 17. 6. Argenv. 22, R. (2. Specim. 306.

I843 S. Radiatus, tefta fubovali, recta, lævi, cofta trans. verfali, adnata, depreffa, violaces, ftriis 4 . albis trans. verfis, Linn. 38. Lift. 422. 266. Petiv. Muf. 21. I6. Argeny. 22. P. (I fpecim. 304.

1844 S. Radiatus, præcedenti affinis, fed minor, Linn. 38. (2. Tpecim. 304 .

I845 S. Strigilatus, tefta ovali rotunda, obliq̨ue ftriata, carnea, Linn: 39. Lift. 4I6. 260. Kircher II. 76. (2. Ipecim. 305.

I846 S. Strigilatus, pracedenti aflinis, fed minor, flavefcens, Linn. 39. (2. fpecim 305.

1847 S. Cultellus, telta ovali, oblonga, fubarculata, grifei rubro punctata, Linn. 37. Liff. 4II. 257 A? Petiv. Mus. 17. 13. T. fpecim. 399.

1848 S. Cultellus, fimilis præcedenti, fed minor, Linn. 37. (2. fpecim. 309.

1849 S. Vagina, tefta lineari, fularcuata, cardine altero bidentato, oliveo albefcens, Linn. 35. a. Lift. 409. 255? 413. infra, Argern. 24. L. Ginanib. II. 170.

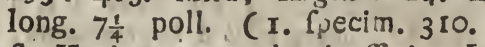

1850 S. Vagina, præcedenti affinis, Linn. 35. $\alpha_{0}$ (I. Specim. 3 IO.

İ85I S. Deliquia, C. Vagina, tefta lineari recta, extremitate alcera marginata, cardinibus unidentatis, rubro fla: voque albida, Linn. 33. Lift 4io. 255, Petiv. Muf. 17. 22. Argenv. 24. $K$. (2. fpecim. 3 Ir.

I\$:52 S. Deliquice, f. Dagina, fimilis præcedenti, fed minor, rubefcens, Linn. 33. (2. fpecim. $3 \mathrm{rr}$.

$\$ 853$ S. Siliqua, tefta lineari recta, cardine altero bidenta. to, coccineo, ftriata. Linm. 34. a. Lift. 4ro.250́.412? fupra, Argenv. 24. M. (2, fpecim. 3 12.

854 S. Siliqua pundata, an varietas pracedentis? albefcens. ftriis \& punctis carmineis, Linn. 34. $\beta . \int_{0} .0$. Lift. 413 ? infra. (2. Specim. $3 \times 3$. ), 


\section{Teftaces. Ordre VI}

GENRE. XLV. MANCHES DE COUTEAU.

I842 M. Lanterne papyracée, à coquille mince ovale, enfíe membranacée, velue, la cote de lá charniere, blanche, Linn. 40. Gualth. 91. D. Petiv. Muf. 17. 0. Argenv. 22 R (2. p. 306

I843 M. Rayon blew, d coquille un pen ovale, droite, polie, d cote traverfe, deprimée, violette, d 4 Siries traverfes blanches, Linn. 38. Lift, 422, 266. Petiv. Muf, 2r. 16. Argenv. 22. P. ( I. p. 304.

$1844 M$. Rayonné, approchant à la precedente, mais plus petite, Linn. 38. (2. p. 304 .

1845 M. Lanterne fillonné, à soquille ovale, ronde obliquement ftrice, couleur de chair, Linn. 39. Lilt. 416. 250. Kircher II. 76. (2. p. 305.

$1846 M$. Lanterne fillonné, approchant d̀ la precedente, mais plus petit, jaunatre, Linn. 39. (2. p. 305.

$1847 M$ Pois, $d$ coquille ovale, allongée, un peu arquée, pointée de gris rouge, Linn. 37. Lift: 411. 257. A? Petiv. Muf. 17. 13. (ब. p. 309.

$1848 M$. Pois, femblable $\grave{a}$ la precedente, mais plus petit; Linn. 37, (2. p. 309 .

1849 M. Sabre Hongrois, $a$ coquille lineaire, un peu arquée, l'une charnier, bidentée, d'un bianc olivatre, Linn. 35. A Lift. 402. 255.? 4r3. infra, Argenv. 24. L. Ginann. II. 170 . long. $7 \frac{1}{4}$. (I. p. 3 io.

1850 M. Sabre Hongrois, opprockant di la precedente, Linn.' 35. $\alpha$. (I. P. 3 IO .

I85 I M. 'Tuyas, à coquilie lineaire, droite, blanchatre, l'une extremité bordée, rouge Eे jarne, à charnieres d'une den. te, Linn. 33. Lift. 410. 256. Petiv. Muf. 17.22. Argenv. 24. K. (2. p. 3II.

I852 $M$. Tuyau, Semblable à la precedente, plus petite, rougeatre, Linn. 33. (2, p. $3 \mathrm{Ir}$.

I853 Gaine de Couteau, a coquille lineaire, droite, une charniere bidentée, Atriée de rouge, Linn. 34. a. Lift. 410. 256. 4I2? Jupra, Argenv. 24. M. (2. p. 312.

I854 M. Gaine de Couteau pointé, ou varieté de la precedente? Blancheatre, à friés, \&o pointes rouges, Linn. 34. $\beta_{0}$ or o. Lift. 413. infra. (2. p. 313 . 


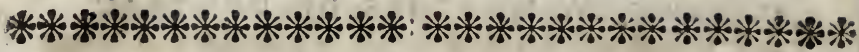

\section{$\begin{array}{lllllllll}O & \mathrm{R} & \mathrm{D} & \mathrm{O} & \mathrm{V} & \text { I I I. }\end{array}$}

$N O N$ C A R D I NATA NEC

I O N A R T I C ULATA.

G E N S XLVI. P H O A DES.

1855 P. Coftatus, tefta ovata, articulata, coftis granulatis, elevatis, ftriata, alba. Linr. 21. Lift. 434. 277. Argenv. Zoomorph.

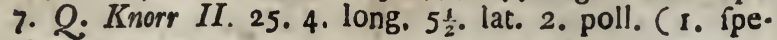
cim. 3r 4 .

I856 P. Dactylus, tefts oblonga, reticulato ftriata, Linz: 20. «. Gualth. 105. D. Lift. 433. 276. Argenv, 26. H. Petiv. Gaz. 79. IO. (I. fpeciin. 315.

1857 P. Striatus, tefta ovata, multifariam ftriata, alba, Linn. 22. Seb. III. I6. 6. 8. Gualth. 105. E? Lift. 435. 278. Rumph. 40. H? (I. fpecim. 316.

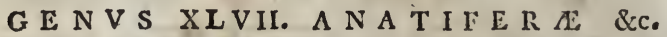

1858 A. Pedata, tefta comprefla, quinque articuli, lævi, inteftino infidente, ftriata, transverfali rugofa. alba, Linn. 18. Seb. III. 16. 1. Gualth. 106. D. Lift. 439. 282. 283. Argenv. 26. E. F. Append. 7. r. Ginann. II. I8I-183. (3. fpecim. 317 .

1859 A. Luzona, tefta oblonga, unguiformis lavi . ftriata, inteftino infidente, prafina, Linn. O. Seb. III. 16. 4? ¿. Petiv. Gaz. 32. 9. (2. Specim. 318.

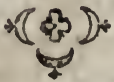




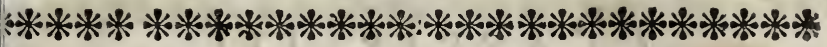

O R D R E V I I I.

$S A N S C H A R N I E R E$ ET ARTICULES.

GE NRE XLVI. P.HOLADES.

855 P. Coftée, à coguille ovale, à cotes grenues, elevies, ftrié, blanche Linn. 21. Lift. 434. 277. Argenv, Zoomorph. 7. Q. Knorr II.25.4 long. $5 \frac{2}{2}$. lat. 2. pouc. (I. p. 314.

$856 P$. Dail tricoté, à coquille allongée, par articles frié, Linn. 20. \& Gualth. 105. D. Lift. 433. 276 . Argenv. 26. H. Petiv Gaz. 79. IO. (r. p. 315 .

857 P. Dail ftriée, à coquille ovale, ftriée à plufieurs manie. res, blanche, Linn. 22. Seb. III. 16. 6.8. Gualth. 105. $E$ ? I.ift. 435. 278. Rumph. 46. H? (1. p. 316 .

GE NRE XLVII. ANATIFERES.

858 A. ou Conque Anatifere, à coquille comprimée, do cinc valves, ou articles, polie, aux inteftins infedente, ftriée, traverfe ridée, blanche, Linn. 18. Seb. III. I6. I. Gualth. 106. D. Lift. 439. 282. 283. Argenv. 26. E. F. Appendix 7. I Ginann. II, 181-183. (3. P.317. 859 A. Luzon verd, d coquille allongée, en forme d'angle polie, friée, verte, attaché aux inteftins, Linn. O. Seb. III. 16. 4? Petiv Gaz. 32. 9. (2. p. 318.

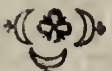




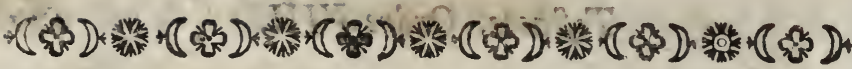

\section{LASS IS II I.}

\section{PARASITIVALVIA.}

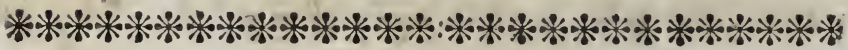

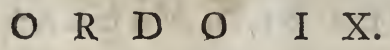

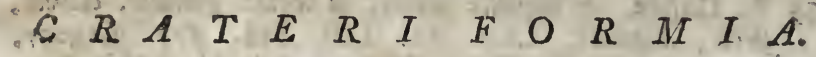

G E N V XLVIII. B A L A N I.

1860 B. Tintinnabulum, tefta conica, rugofa, fixa, purpurafcens, Linn. 12. Seb III. 94. \&ic. Argenv. 26. A. Gialth. 106. H. Rumph. 4I. A.Lift. 443. 285. Inorr II. 2. 6. V.30. I. (I. fpecim. 3I9. a.

1861 B. Tintinnabulum, præcedenti fimilis, Linn. I2. Gualth. I06. E. I. Lift. 443.285. Knorr IV. 21. I. (2, Specim. $319 . b$.

I862 B. Balanoides, tefta conica, truncata, lrvi, fixa, operculis obtufis, in margine aperturæ, nembrana cum 4. articulis confpicitur, Lïnn. II. Seb. III. 94. infra, Lift. 444. 287. Argenv. 26. C? Kircher I. 35. Ginanno. II. $\times 76$. (I. fpecim. 3ig. c.

1863 B. Disderna, tefta polythamia, fubrotunda, fulcata, fixa, Linn. 13. a. f. O. Gualth. 106. 2. Lift. 445. 288." Naturforch VIII. t. 4. f. $A-C$. (i Ipecin. 320.

1804 B. Cucullus planus, præcedenti forte afinis, fed minor. alba, ftriata, Linn. 13. Lift.442. 284? Rumph. I 4. H. Petiv. Gaz. I33. I 2 ? Muf. I. II, 3. II. Naturforfch. $X$. t. I. f. II. I2. (I. fpecim. 321.

1865 B. Teftudinaria tefta plano convexa, fixa, radiis fex excavatis ftriatis, albefcens, Linn. I4. Gualth. Io5. M. N. O. Rumpls. 40. K. Petiv. Gaz. 9 9. optima . Ginann. II. 175. Kircher I:36.Planc. 5. II. III. KnorrIII. 30. 3. 4. Naturforfch, XII. t. 4. f. 9. A.B. (I. Rpecim. 322.

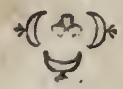




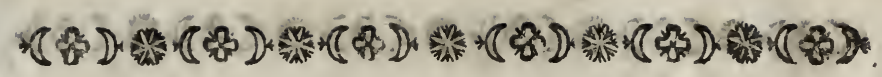

$$
\text { C.L' } \Lambda \text { S S E III. }
$$

PAR:ASITE VALVES.

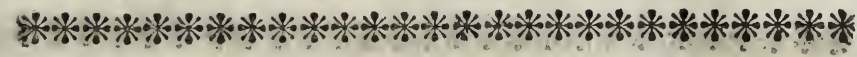

O. R D R E E I X.

C CRATERI FORMES. A

GENRE XLVIII. GLANDS DE MER.

$\$ 860$ G. Tulipe de Mer, à coquille conique, ridée, fixe, porisprée, Linn. 12. Seb. III. 94. \&c. Argenv. 26. A. Gualth. 105.H. Rumph. 4 I. $A$. Lift. 443. 285. Knorr II. 2. 6.V. 30. T. (I. p. 3I9. a.

I86I G. Tulipe de Mer, femblable d la precedente, Linn. I2. Gualth. IO5. E. I. Lift, 443. 285. Knorr IV. 2I. I. (2. D. 3I I. b.

I862 G. Balanoide, à coquille conique, tronquée, polie, fixe, à opercules emoufjés, dans le bord de l'ouverture, fe trouvis zin membrane iे 4 articles, Linn. Ix. Seb. III. 94: infra, Lift. 444. 287. Argenv. 26. C? Kircher I. 35. Ginann. II. 176. (1. p. 3rg.c.

$\$ 863$ G. Turban de Mer, coquille polythalame, un peu ronde, $f_{i b}$ lomnés, fixe, Linn. I 3. a ou 0. Gualth. 106. Q. Lift. 445. 288. Naturforfch. VIII. t. 4.f. A-C. (I. p. 320 .

1864 G. Turban de Mer platte, approchant de la precedente: mais plus petit. blanc, firié, Linn. I3. Lift. 442. 284.? Rumph. I4. H. Petiv. Gaz. 132. I2? Muf. I. I1, 3. II. Naturforfch. X. t. I. f. I1. I2. (I. p. 321.

$1865 \mathrm{G}$. Tortue, à coquille platte recourbée, fixe à $\hat{x} x$ rayons excavées, firiés, blancheatre. Linn. I4. Gualth. ro6: $M$. N. O. Rumph 40. K. Petiv. Gaz. 9. 9. optima, Ginann. II. I75. Kircher I 36. Planc. 5. II. III. Knorr III. 30. 3. 4. Naturforfch. XII. t. 4.f. .. A. B. (1.p. 322 .

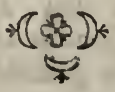

$\mathrm{Hb}_{4}$

C L A S S E 


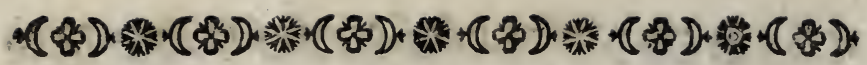

\author{
C L A S S IS I V. \\ CONVEXIVALVIA..
}

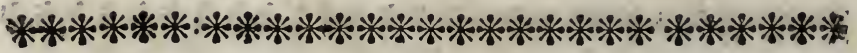

O $\mathrm{R}$ D

APERTVRA IN MEDIO SVPERFICIEI.

GE N V S XLIX. A N A D E ME。

1866 A. Cedaris . hæmisphærico depreffa, ambulacris quinis . repandis linearibus, lineis alternatim bifariis, Linn. 8. ๙. f. a. Klein 7. A. C. D. Breyn r. 2. Seb. III. 13. 12. Gualth. 108. E. Argenv. 25.E Kircher I. 18. (2. fpecim.I. 1867 A. Regia, an præcedenti affinis? Linn. 8. «. .. b. Klein 7. B. Breyn I. 3. 4. Seb. III. 13. 13. Rumph. 13. 3. Gualth. I10, D. E. Petiv. Gaz. 126. I1. A. Mus. 7.2? (1. Ipecim, 2.

1868 A. Turcica, hæmifphærico depreffa, tuberculis minoribus, Linn。 o. f. c. Klein 3. C. D. Breyn 3, 4. Seb. III. 10. 5. (1. fpecim. 4.

1869 A. Arabica, fimilis præcedenti, figura magis angulofa, Linn.o. f. d, Klein 2.F. Seb. III. I I. 8. diam. $4 \frac{1}{2}$. poll, (I. fpecim. 3.

1870 A. Fudaica, coccinea, tuberculis milii granulis xqualibus, Linn. I. a f. e. Klein 1. A. B 2. E. Seb.III. II. 4 ? Rumph. 13. B. Lift. Angl. 3. 18. altit. 5, latit. $5 \frac{2}{2}$. poll. (I. Ipecim. 5.

$187 \mathrm{r}$ A. Fudaica, pracedenti affinis, Linn. I. a.f. e. altit. 4. diam. $4 \frac{1}{3}$. poll. (I. Specim. 5 .

1872 A. Fudaica, fimilis præcedenti, Linn. I. B. f. e. diam.4. poll. (I. Specim. 5.

1873 A. Bardana maxima, fphxroidea uftulata, fubtus plana, tuberculis minoribus, Linn 1. B. . f. Rlein 9. A. B. Io. A.49.2.? Seb.III. I 4. 2 ? Ginann. II. I 73. (r. Specim.6. 1874 A. Depre $\int J_{a}$, fubtus alba, Linn. O. r. g. Klein 10 $D . E$. Seb, III. II. 7. Gualth. 107. A. A. (1. fpecim. 7. $\times 875$ A. Deprefa, fimilis pracedenti, Linn. O. f. g. (2. Ipecim. 7. 
(6)

C L A S S E I V.

C O NVEXE VALVES.

********** $* * * * * * * * * * * * * * * * * * * * * * * * * * *$

O R D R E X.

OUVERTURE AU MLLIEU DE LA SURFACE.

GENRE XLIX. DIA DEM ES.

I866 D. Couronne Imperiale, hemispberique deprimée, à cinq ambulacres recourbés en lignes, à lignes à deux aiternatí. ves, Linn. 8. a ou a. Klein 7. A.C. D. Breyn. I. 1. 2. Seb. III. 13. 12. Gualth. 108. E. Argenv. 25. E. Kirche I. 18. ( 2 piec. '.

1867 D. Couronne Royale, ou approchant au precedent? Linn. 8. $\beta$ ou b. Klein. 7. B. Bregn. t. 3. 4. Seb. III. I3.13. Rumph. 13. 3. Gualth. 110. D. E. Petiv. Gaz. $126.11 \%$ A. Mur. 7. 2 ? (I. p. 2 .

I8:68 D. du Grand Vifir, hemispherique deprimée, à tubercules plus petites, Linn. O. ou c. Klein. 3. C. D. Breyn 3.4. 1569 D. de Baffa, femblable auprectdent. mais la figure plus anglée, Linn. O. ou d. Klein. 2. F. Seb. III. Ii 8. dian. $4 \frac{1}{2}$ pouc. (I. p. 3 .

$1870 \mathrm{D}$. Judaique, rouge. $\dot{a}$ tubercules, $\dot{a}$ grains egales, Linn. I a.e. Kleyn I.A.B.2.E.Seb. III. I I. 4?Rumph. I $3: B$. Lif. Anglo 3. 18. air. 5. lat. $5 \frac{1}{2}$. pouc, (I. $p 5$.

I $37 \mathrm{I}$. Judaique, approchant aub precedent, Linn. I. a ou $e_{\text {. }}$ altit. 4. diam, $4 \frac{2}{2}$. pouc. (I. p. 5 .

1872 D. Judaique, Jemblable aus precedent, Linn. I. a. ou $e_{0}$ diam. 4. pouc. (I. $p$ s.

ז873 D. Glouteron de mer, fphraroide uftulée, deffous plette, $\dot{a}$ tubercules olus petites. Linn. T. $\beta$. ou $f$. Klein 9 . A B. Io. A. 49.2 ? Seb.III. 14 2. ₹ Ginann. II. r73 (I. P. 6. 1874 D. Preffe, defJous blanc, linn. O. ou g. Klein. IO. D. E. Seb. III I I. 7. Gua'th. I07. $A: A$. (I. p. 7. I875 D. Preflé, Semblable au precedent, Linn. o. oul g.
(2. P. 7.

Hh 5

$18 ; 6 \mathrm{D}$. 
I876 A. Depre $\int a$, præcedenti affinis, fed minima, Linm. o. f.g. (6. fpecim. 7 .

I877 A. Saxatiilis, hamifphrico depreffa, ambulacris denis, poribus approsimatis, areis transverfe punctatis, verrucofis, Linn 6. 3.f. h. Klein 5. a a. c c? Seb. III. 10.12, II. 5. (4. Specim. 8.

1878. A. Globula, an fimilis pracedenti? granulis minimis; Linn 6. ๙. \{. i. Klein 2. A.B? C. D? Seb. III. 10. 20. Gualth. I 7. L? (4 ipecim 9.

879 A. Divifa, tuberculis minimis, Limn. 6. \%. f. k. Klein 10. a. I. C. Rumph. 14. A. Seb. III. 10. 15 ? Petiv. Muf. 22. 6. (2. fpecim. 10. a.

I880 A. Plana, minus gratulata, Linn. 6. भ. . k. Klein 37. I? 492 ? Seb.III. 10. a. (2. fpecim. s०. b.

I88I A. Striata, f. varietus præcedentis? areis una ferie granulorum, Limn. 6. d.f. I. (5. fpecim. 10. b.

I882 A. Striata, fimilis præcedenti, Limn. 6. d. f. 1. (5. Ipecim. Io b.

I883 A. Diadema, hæmifphærico depreffa, ambulacris 5 . longicudinalibus, recurvis, areis laneolatis, Linn. 7.6 . m. Klein 46, 1. Seb. III, 10. 10. Rumph. 14. B. Petiv. Muf. 8. 5. ( 1 . Grecim, Ir.

I884 A. Mamillatis, hæmifphærica, ambulacris denis flexuofis, areis muricatis verrucofis, anguftioribus abbreviatis, Linn. of r. n. Klein 3. A. B. Seb. III. 10. 14, 11. 9. Gualth. 107. $N$ (2. Specim. I2.

1885 A. Filura, an fimilis pracedenti? viridis, granulis fuscis, Linn. о. f. o. Klein 10. B. C? I1. E? F? Seb. III. I0. 13. Gualth, 107. F? (2. Specim. I3.

1886 A. Pentapetala, rotunda, quinque fectoribus divifa, gra. nulata $i$. verrucofa, grifea, Linn O. f. p. Thlein 11.G? Guath. 108. A. (I. fpecim. 14.

2887 A. Succinatorium, quinquareolata viridis, fasciis transverfalibus cincta, Linn. o. I. q. Seb. III. I r. 6. Gualth. 107. M. Argenv. 25. H. (1. fpecim. I5.

$$
\text { GENVS L. C I D ARES. }
$$

1888 C. Maroccana, tefta ovali convexa, mamillata granulata, Linn. C. f. r. Klein 6. A. B? Breyn r. 5? Seb. III. IO. I7? Gualtho 108. C. Argenv. 25. D? (I. fpecim. I6.

1880 C. Tunetana, an fimilis pracedenti? paucioribus mamillis, fluvercens, Linnz. o. f. s. Klein 6. C. D. Seb. III. 10. 16? Rumph. I3. I. (1. fpecim. 17. 


\section{.IIT Teftaces. Ordie $-X_{0}$}

1876 D. Preffe, approchant ani precedent, mais trespetit, Linio 0. ou g. (6. p. 7.

$1877 D$. Noeud de Batelier, hamispberique deprimée, ambir lacres deux $a$ pores s'approckantes, ì aires traverfes poinstées, plein de verrues, winn. 6. $\beta .0 u$ h. Klein. 5.a.a.c.c.? Seb. III. IO. 12 , II. 5 . (4. p. 8.

$x 878$ D. Bouton de Pailfan, ou femblable au precedent? a tres petits grains, Linn. 6, 2. ar $i$ Klein. 2. A. B ? C. D.? Seb. 10. 12, II. 5. Gualth. 707. L? (4. p. 9).

$1819 \mathrm{D}$. Noeud divife, tres petit, tuberculée, Linn. $\sigma . y$ ouk. Klein io. a. II. C Rumph, I4. A. Seb. III. IU.' Is Petiv. Muf. 22. 6. (2.p. Io. a.

1880 D. Noeud divifé, platte, moins gremu, Linn. 6. \% ou k. Klein, 37. 1 ? 49. 2 ? Seb. III. xо. a. (2.p. 10. $b$.

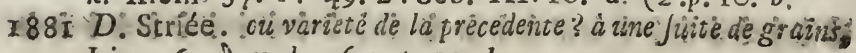
Linn. 6. d. oul. ( 5 p. ro. b.

1882.D. Striée, femblable aus précedent, Linn. 6. d. ou $l$. (5. p. I0. b.

1883 D. de Mahonet, liémisplierique deprimé, à ciñq ambu」a. cres longitudinales, pleindeverrues, $a ̀$ aires anceolés, Linn. 7.0u m: Klein 46. I. Seb. III. IO. 10. Rumph. I4. B. Petiv. Mur. 8.5. (I. p. II.

1884 D. à Grands Mamillons, bemispherique, "ci ambulacres, $\dot{a}$ aires pointubs, plein de verrues, plus étroites racurr:cies, Linn. 9. ou n. Klein. 3. A. B. Seb.III.10. 14\% II. 9. Gualth. $107 . N$. (2. p. 12.

$1885 \mathrm{D}$. Verd, ou femblable au precetient? a grains bruns, Linn. o. ou o. Klein Ic. B. C? II. E? F.? Seb. III. I0, 13. Gualth. Io7 F? (2. p. I3.

I\$866 D. Divifé, ronde, divifée en conq coins, grenue, plein da ysrrwes, grife, Linn. o. ou p. Klein II. G? Gualth: 108: A. (I. P. I 4 .

$1887 D$ Bouton falcié, verdí, entouré par des bardes traverJes, Linn. C. ou q. Seb. III. II. 6. Guaith. 108 M. Argenv. 25. H. (I. p. Is.

GENRE L. DIADEMES OVALES.

$1888 D$. de Marocque, à coquille ovale, recourlice, d̀ visomilb lons, gresiue, Linn. O. $\int$ r. Klein $6 . A$. B.? Jireyn. $1-5$ ? Scb. III. 10. 17? Gualth. 108. C. Argenv. 25. D.? (I. p. 16.

1889 D. Du key de Thunis, ou femblable au precedent? mais pas tant de mamillons, jaunatre, Linn. o. ou s. Kiein 6. C. D. Seb. III. :0. I6 ? Rumph. I3.r. (1. p. 17. 
1890 C. Tunetana, præcedenti fimilis, Linn. O. I. s. (2. fpecim. 17.

I891 C. Lucunter, bæmifphærico-ovalis, ambulacris denis flexuofis, areis muricatis, anguftioribus longitudinalibus, Linn. 10. ๔. t. Klein 4. C-F. Breyn 1.6. 7. Seb. III. 11. II. Gualth. 107. I. (2, fpecim. 18 .

1892 C. Lucunter, præcedenti affinis, Linn. IO. C. t. (3. fpecim. 18.

1893 C. Lucunter, fimilis præcedenti, Linn. ro. «. f. t. Klein 5. b. b. Seb. III. 10. 18. (2. fpecim. I9.

1894 C. Maura, an pracedenti affinis? magis tamen globo. fus, flavefcens, Linn. o. f. u. Klein 4. A. B. Gualth. 107. C. (2. Ipecim. 20.

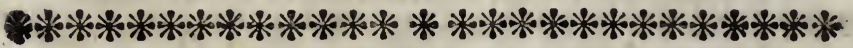

O

APERTVRA IN SVPERFICIE

A CENTRO REMOTA.

GENVS LI. VMBILICI MARINI FORMES.

1895 Culeola, f. Faba Coffec, apertura remota, tefta convexa, ovalis, fubtus piana, petalis quinque, ore in bafi. Linn. O. .. v. Klein 49. 10. I1. Breyn 6.1?-3? (4. fpe. cim. 2 I .

„, Huc referri polfunt: Klein 38. 4, 53. 5. 6. 7 .

************************************

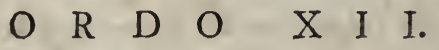

APERTVRA IN MARGINE.

GE N V L LIJ. COR DIFORMES.

1896 C. Marimum, apertura marginali, cordiformi, con* vexum, flore pentapetalo pictum, granulatum, coc. cineum, Linn. 12.\%. f. w. Klein 23.x. C? 45. 5. Breyn. 5. 5. 6? Seb. III. 10. 19? Rsumph. I4. 3. Argenv, 25. K. Gualth. 109. B. (2 fpecim. 22.

1897 C. Marinum, præcedenti affinis, Lim. I2. \% f. W. (2. fpecin. 22 . 


\section{Teftaces. Ordre XI. XII.}

$1890 D$ du Bey de Thunis, femblable au precedent, Linn. ou s. (2. p. 17 .

189 I $D$. d'Ethiophie, hemispherique ovale, d dix ambulacres, recourbées, à aires pointues, etroites longitudinales, Linn. 10. ou t. Klein. 4. C-F. Breyn. I. 6. 7. Seb. III. II. i I. Gualth. I07. I. (2. p. 38.

1892 D. d'Echiophie, approchant au precedent, Linn. 10. ou to: (3. $p .18$.

1893 D. d'Ethiophie, Semblable au precedent, Linn. Io. «. ou $t$. Klein. 5. b. b. Seb. III. 10. 18. (3. p. 19.

1894 B. Moresque, approchant aus precedent? mais pourtant plus rond; jaunatre, Lirn. o. ou u. Klein, 4. A. B. Gualth. 107. C. (2. P. 20.

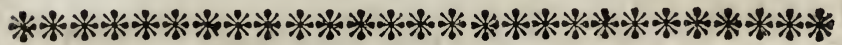

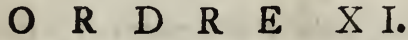

OUVERTURE DANSLASURFACE ELOIGNE DU CENTRE.

GENRE LI. FORMES D'UMBILIQUE MARINE.

$1895 U$. Feve de Caffé, ouverture eloignée, '̇ coquille recourbée, ovale, defJous platte, à cinq petales, la bouche dans la bafe, Linn. o. ou v. Klein. 49. 10. II. Breyn. 6. $1 ?-3$ ? (4. $p$ 2I.

Avec ceux ci ont peut joindre, Klein 38. $4,53.5 .6 .7$.

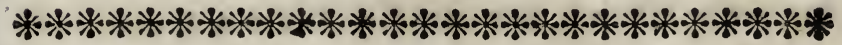

$\begin{array}{llllllll}\mathrm{O} & \mathrm{R} & \mathrm{D} & \mathrm{R} & \mathrm{E} & \mathrm{X} & \mathrm{I} & \mathrm{I}\end{array}$

OUVERTUREDANS LEBORD.

GLNRE LII. EN FORME DE COEURS.

1896 C. Marin, ouverture marginale en forme de coeur, recourbée, peinte d'une fleur à cing peticules, grenue, rouge, Linn. 12. \% ou w. Klein 23.x. C? 45. 5. Breyn s. 5-6? Seb. III. 10. 19 ? Rumph. J4. 3 Argenv. 25. K. Gtalth. IC9. B. ( 2 p. 22.'

1897 C. Marin, approchant au frecedent, Lisn. 12. y. ouw. โ2. p. 22 . 
I898 C. Neptuni, f. yarietas pracedentis? ftella prefru coccineo grifeum, Linn. 12. \&. '. $\mathrm{x}$. Klein. 24. A. B. Seb. IIt. 10. 22 Gualth. 109. A. Rumph. 14. 2. Petiv. Gaz. 12 z. 3. Muf, 22. 9. (2. fpecim. 23.

1899. C. Oyrum Marinum, ovale granulatum, album flore pentapetalo pictum, Linn. I 2. $\beta$. . y. Klein. 25.B. C. Brayn 5. 2 ? Seb III. 15. 3. 4. (โpecim. 24.

1900 C. Lacurofum, ovale, gibbum, ambulacris quinis depreffis, Linn. 13. א. z. Klein 24. c. e. 38. 5. Brey! 5. 1. 2. Seb. III. 10. 21. Gualth. 109. C. D. Argenv. 25. I. Lift. Append. 1. 13 (2. fpecim. 25.

Ig0I C. Pifum, ovatum, fubconvexum, ambulacris quinis, flavercens, Limn. o. 1. aa. Klein. 49. 12. I3. Seb. III. 15. 28. ( I. fpecim. 26.

\section{GE $N$ N V S LIII: G A L E IE:}

Ig02 G. Gibbum, tefta ovali-convexa, ambulacris quinis, granulis ftellatis; obfitum, Linn. o: fo bb. Klein 20. a. b. $c$ ? $d$ ? Breyn. 4. I. 2. Seb. III. 10. 23. Rumpli. r4. 3. (r. іресіm. 27.

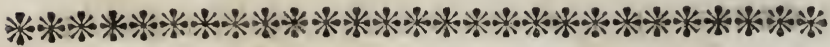

\section{O R D O X X I I I.}

APERTVRAINBASIA MARGINE REMOT $A$.

\section{G E N V S LII V: O L I V TE F O R M E S.}

1903 O. Orches, convexa, ambulacris quinis ad os uf̧̧ue elongatis, granulofa, alba, Limn. O. f. cc. Klein 37. 4-6. 49. 8. 9. Breyn 2.5. 6. Se3 III. 10. 7, I5. 30. 33-38. Rumplo i4. D. Petiv. Gaz. 5. 3. C( fecim. 28.

I0?4 O. Nucleus, an fimilis precedentis? minor, Iinn. ०. . dd. Klein 48. x-3. Petiv. Gaz. 3r. 10. Phelfum x. $1-35,2$. I-35. (30. (pecim. 29.

3905. O. Nucleus, præcedenti affinis, Lim, o. ᄃ. dd. (30 fpecin. 2y. 


\section{Teftacea. Ordo XIII.}

1598 C. Marin preffé, ou varieté de la precedent? à étoile prelfes rouge grife, Linn. 12. a. olb $x$. Klein 24. $A$. B. Sel. 111. i0. 22. Gualth. Iog. A. Rurnph. 14. 2. Petiv. Gaz. I27. 3. Mur. 22. 9. (2. 2.23.

I899 C. Oeuf Marin, ovale, gremu, blanc, à fleur, dे cin petales, Lin 12. B. ou y. Klein 25. B. C. Breyn 5. 2? Seb. III. I5 3. 4. (3. p. 24.

I 900 C. Tête de Mort, ovale, bolfue, à cinq ambulacres deprimés, Linn. 13. 0u z. Klein 24. c. e, 38. 5. Breyz 5. I. 2. Seb. III. 10. 21. Gualth. ró. C. D. Argenv. 25. I. Lift. Append. I. I3. (2. P. 25.

rgor C. Feve, ou Haricot Angloire, ovale, un peu courbée, a cing ambulacres, jaunatre, Linn. $O$ ou a a. Klein 40 12. 13. Seb. III. 15.28. (1. P. 26.

GENRE LIII. POTS E N TE TE:

$\$ 902 P$. Oeuf ventrue, à coquille ovale, recourbée, à cing awo bulacres, couverte de grains étoillées, Linn. 0 . ou $b b_{\text {a }}$ Klein 20. a.b. c. ? d.?Breyn 4. I. 2. Seb. III. 10. 23. Rumph. I4. 3. (I. P. 27.

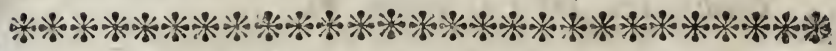

\section{O R D R E X I I I.}

OUVERTURE DENS LA BASE

$$
\text { LOIN DU BORD. }
$$

GE NRE LIV. EN FÓRME D'OLIVES.

I903 O. Grenue, recourbée, ¿d cinq ambulacres allongées jufqu'd̆ la boucke, grenue, blanche, Linn. O. ou cs. Klein 32 4-6, 49. 8.9. Breyn 2. 5. 6. Seb. IIT. 10. 7, 15.30. 33-38. Rumph. 14. D. Petiv. Gaz. 5. 3. (I. p. 28. $19040^{\circ}$ Pepin, ou semblable di la precedente? plus petit, Linn. 9. or dd. Klein 48. 1-3. Petiv. Gaz. 3r. ro. Phelfum r. I $-35,2$. I-35. (30. p. 29 .

1905 O. Pepin, approchant dे la precedente, Linn. O. oudds ( 30. p. 29. 


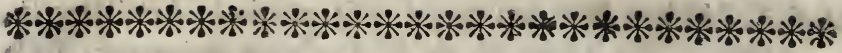

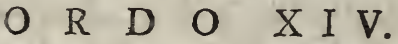

APERTVRA IN BASI AD MARGINEM.

G E N V S L V. A R T O C R E \&.

Ig06 A. Minutal, planiuccula ovata, fubrotunda, ambulacris ovatis quinis punctata, Linn. 34. r. ee. Klein 17. A. 18. B. Seb. III. I I. 2. 3. Gualth. A. I I O. iong. 5. lat. $4 \frac{1}{2}$. (1. fpecim. 30 .

I907 A. Minutal, præcedenti affinis, Linn. I4. r. ee. long. $5 \frac{2}{2}$ lat. 4. poll. (1. fpecim. 30.

I908 A. Minutal, fimilis piæcedenti, Limn. 14. f. ee. long. 4. $\frac{x}{2}$ Iat. $3 \frac{z}{2}$. poll. ( I fpecin. 30 .

I909 A. Clibanites, an pracedenti affinis? figuris hexagonis picta, Linn. 14. «. f. ff. Klein. 19. A. B. Seb. III. 15. I3. I4. (2. frecim. $3 \mathrm{I}$.

rgro A. Clibonites, an varitas pracedentis? Linn. r4. $\beta . \Gamma$. gg. Klein. 19. C. D. 45. 8? 9? Seb. III. 15. 23. 24. Gualth. IIO. D? (2. fpecim. 32 .

G E N V S LVI. S T R I B I L I T

Igr $\$$. Paftilla, planiufcula ovata integra, ambulacris

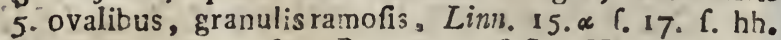
Klein 22. a-c, 45. 6? 7? Breyn 7. 1? 2? Seb. III. 1 5. 25. 26. Gualth. I10. B. C. C, Rumph. 14, E. Petiv. Muf. I. 10. (2. Ipecim. 33 .

\section{G E N V S L VII. L A G A N I.}

Igr 2 L. Stribilite, planus fuborbiculatus, ambulacris, foraminibus, oblongis, 2. quinis ovalibus, $\operatorname{Lim}$ 17. $\beta$. f. ii. Klein 21. A. B. Seb. III. 15. I-4, Rumph, I4. F. Petiv. Muf. 4. I. (I. fpecim. 34. 


\section{Teftaces. Ordre XIV.}

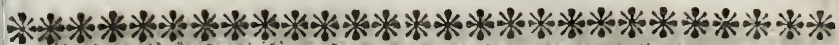

\section{O R D ROE X I V.}

OUVERTURE A LA BASI PRES DU BORD.

GE NRE LV. PA TE S.

1906 P. Pavois de Mer, platte, ovale, un peu ronde, à cinq ambulacres ovales, pointée, Linn. I4. ou e e.Klein I 7.A. $18 . B$. Seb. III. II. 2. 3. Gualth. IIO. A. long. 5. lat. $4 \frac{3}{2}$. (I.P. 3C. 1907 P. Pavois de Mer, approchant als precedent, Linn. 14. ou ee. long. $5 \frac{1}{2}$. lat. 4 pouc. (I. p. 30 .

1908 P. Pavois de Mer, Jemblable au precedent, Linn. I4 ou e e. long. $4 \frac{1}{2}$. lat. $3 \frac{\frac{1}{2}}{2}$. pouc. ( $1 . p$. 30 .

1909 P. Pavois platte, ou approchant als precedent? peinte de figures $\ddot{a} f i x$ coins, Linn. 14. a. ou $f f$. Klein 19. A. B. Seb. III. 15. 13, 14. (2. p. 31 .

rgro P. Pavois platte, ou femblable au precedent? Linn. 14. B. ou g g. Klein 19. C.D. 45.8? 9? Seb. III. 15. 23. 24. Gualth. I10. D? (2. p. 32 .

GE NRE LVI. G A.LE T TES.

IgI I G. Petit gateau, platte, ovale, entiere, à cing ambulacres ovales, à grains branchues, Linn: 15 . * ou 17 ou $h h$. Klein. 22. a-c. 45. 6 ? 7 ? Breyn 7. 1. 2. Seb. III. 15. 25. 26. Gualth. I10. B.C. C. Rumph. 14. E. Petiv. Muf. I. 10. $(2 . p .33$.

GE N R E LVII. B I G N E T S.

zor 2 B. Percé, platte, un peu ronde, à detxx ouvertures allon. gées, E cing anabulacres ovales, Linn. - 17. $\beta$ ou $i i . \mathrm{K}$ ein 21. A. B. Seb. III. I5. I-4.Rumph. I 4. F. Petiv. Muf. 4. I. (I. p. 34 . 
*******************************1

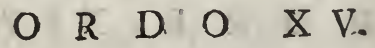

APERTVRA INBASI A MARGINE $M V L T V M R E M O T A$.

GENUS LVIII. DISCI TRA N S F X I.

Igr3 D. Orbis, fuborbiculatus, ambulacris quinis ovalibus, foraminibus 5. Linn. I7. $\gamma$. f. kk. Klein 2 I. C? $D$ ? 50. 3. 4. Seb. III. I5. 7.8. 9? I0? Gualth. IIO, EE? Argenv. Pars 11. 7.C? (1. rpecim. 35.

Ior 4 D. Orbis, præcedenti affinis, Linn. I7. $\gamma_{0}$ f. kk. (2. fpecim. 35 .

IgIs D Obiculatus, an pracedenti affinis? foraminibus 6. quorum 2. ad marginem laceris, Linn. o. $\{1$ : Klein 50. 5. 6. Seb.'III. 15. 5. 6. (1. Ipecim. 36.

$$
\text { G E N V S LIX. R O T V L I. }
$$

IgI6 R. After, foraminibus duobus nerviis, lobatus, Linn. I7 ß. I. mm Klein 22. L?-D? Breyn 7. 5? 6? Seb. III 15. I7. I8. Gualth. 110. FF? Argenv. Pars. II. 7. $D$ ? (2. fpecim. 37 .

IgI7 R. After. præcedenti affinis, fed incifuris longioribus, Linn. 17 B. . . nm Klein 22. A. B. 49. 6. 7. Breyx' 7. 3? 4 ? Seb III I 5. I5? 16? Gualth. I10.H. Rumph. 14. 1 ? (2. fрecim. 38 .

I918 R. After, timilis præcedenti, fed foraminibus nullis, pervius, lobatus, Linn. 17.œ. L. nn. Klein 22.E.F. Seb. III. 15. I 9 ? 20? Rumph. I4. I. Petiv. Ga\%. I. I2. (2.1pecim, 39.

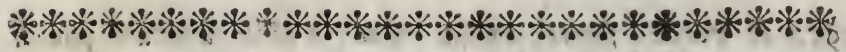

\section{O R D O. X V I.}

$A P E R T V R A S V P E R N E$ IN $M A R G I N E$. G E N V L X. B I I T E.

2919 B. Placenta, plana, olbiculata, ambulacris quinis, biparticis, ano marginali, Linn. I6. r. no. Klein 20. A. B. Breyn 7. 7. 8. Gualth. IIn. GG, Rumph. I4. G. Pegtiv. Muf.3. 5. (2) ifecim. 40. 


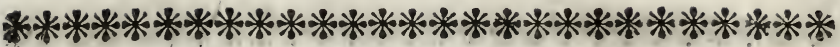

O $\quad R \quad D \quad R \quad E \quad X \quad$ V.

OUVERTURE A LA BASE $L O I N D U$ BORD.

GENRE LVIII. PALETS TRO UE S.

Igr3 $P$ Orbiculé, ou un. peu rond, à cinq ambulacres, ovales, \& cinq trous, Linn. 17. $\gamma$. ou kk. Klein 21. C? D? 50 . 3. 4. Seb. III. 15. 7. 8. 9? I0? Gualth. IIO. EE? Argenv. Part. II 7. C? $\quad$ (I. p. 35 .

Igr 4 P. Orbiculé, femblable au precedent, Linn. I : $\gamma$. ou $k k$. (2, p. 35 .

IgIs P. Gateau rompu, ou approchant au precedent? dे 6 trous, deux desquels font dechirés, vers le bord, Linn. 0. ou ll. Kiein 50.5. 6. Seb. III. I5. 5.6 (I. p. 36 .

GE N R E L I X. R O U E S.

Igr 6 $R$. Dentelé, lobé, à deux trous nerveux, Linn. I7. B.ou $m$ m. Klein 22. $A$ ? $-D$ ? Breyn. 7. 5.? 6? Seb. III. 15. 17. I8. Gualth. I 10, $F$ ? $F$ ? Argenv. Part. II. 7. D? (2. P. 37.

- IgI7 $R$. Dentelé; approchant d la precedente, mais ayant les incifures plus longues, Linn. 17. $\beta$. ou $m m$. Klein 22. A. B 49.6. 7. Breyn 7. 3 ? 4 ? Seb. III. I5. 15? 16 ? Gualch. II $\mathrm{H}$. Rumph. I4. I? ( 2 p. 38 .

IgI $8 R$ Etoile, femblable d la precedente, mais fans trous. lobé, ouvert, Linn. I 7. *.ou nn. Kleın 22. E.F. Seb. III. 15. I9?'20? Rumph. I4. I. Petiv. Mur. I. 12. ( 2 p. 39.

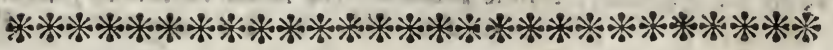

$\begin{array}{llllllll}\mathrm{O} & \mathrm{R} & \mathrm{D} & \mathrm{R} & \mathrm{E} & \mathrm{X} & \mathrm{V} & \mathrm{I} .\end{array}$

OUVERTURE DANS LE BORD $A U D E S S U S$.

GE N V L L. BON NETS.

Igrg B. Gaufre, platte, orbiculaire, ou ronde, d cinq amis. lacres, partegés en deux, l'anus marginal, Linn. 16. ou 00. Klein 2c. A. B. Breyn 7. ?. 8. Gualth. IIo. GG. Rumph. I4. G. Petiv. Muf. 3. 5. (2. p. 40. 


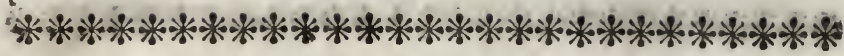

\section{$E C H I I M A R I N I$ \\ S $P I N I S A R$ A A T I.}

I 920 E. Scoiymus tefta ovali, aculeis canaliculatis, purpureis Klein 47. I. 2. Argenv. 25. G. (2. fpecim. 4I. Ig2 $I^{\circ}$ E. Hyftrix, tefta ovali, acuieis triquetris obtufis, lividis, albo annulatis, fulca, Klein 39. I. Seb. III. 13. I. 2. 3. long, 6. latit. 4 poll. (I. Ipecim. 42.

1922 E. Armiger, aculeis teretịbus trigonis, minoribus intermixtis, fufco lutefcens, Set. III. 13. 4. Rumph. 13. 2. Argenv. 25. A. long. 91. latit. 8. poll. (fpecim. 43.

1923 E. Erinaceus, teftaglobulofa rotunda, longiffimis tenuibus aculeis granulatis, per feries digeftis, innumerisque aliis fitif rmibus, flavo fufcus, Klein 39.2. Seb. IIT. 15. 5. Rumph. 13. 4? Gualth. 108. D. long. \& lat. $7 \frac{1}{38}$ poll. ( $\mathrm{r}$. Specim. 44.

1924 E. Evinaceus, pracedenti affinis, aculeis adharentibus aliis corporibus, Klein 37.3 ? 'Seb.III. 13.5? Kircher 1. 17. long. \& lat, 7. poll. (I. fpecim. 45.

1925 E. Aculeatus, tefta globulofa rotunda, aculeis longioribus granulatis, aliisque filiformibus, atra, Klein 37. 2 ? 39. 3. Seb. III. 13. 7. Rumph. 13. 5. Petiy. Muf. 3. 8. long. \& lat. $4 \frac{1}{2}$. poll. (r. Rpecim. 46.

J26 E. Pufillus, aculeis brevioribus filiformibus nullis, nigrefcens Kleyn 30. A. B. Seb. III. 13. 6. Gualth. 108. $B$. (r. Rpecim. 47 .

1927 E. Pufillus, precedenti affinis, pallide purpureus, Klein 38.2? 3 ? Seb.1II.12.5? 6? Rumph. 13. C. (2.fpecim. 43.

1928 E. Puffllus , fimilis præcedenti, Havo violaceus, Klein 30. C. D. 38. 2. 3. Sẹb. III, 12, 8 ? Argenv. 25. F. (2. (pecim. 49.

1929 B. Pufillus, fimilis præcedenti, fufco virefcens, Klein 38. 1. Seb. III. I2, 2. 3. 9 ? Petiv. Muf. 2. 8. ( 2. (fpecim. 50.

3930 Spimus Surauenfis, vel Aculei tenuiffimi, longiffimi , albi, Seb. III. 114. 2, long. $13 \frac{1}{2}$. poll. (4. Ipecim, 5i.

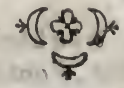




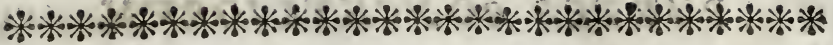

\section{OURSINS MARINS}

$A R \quad M \quad E \quad S \quad D E$ L'P I $N$ E $S$.

1920 O. Artichot, d̀ coquille ovale, d̀ aiguillons canelées, pourprées, Klein 47. I. 2. Argenv. 25. G. (2. p. 4I.

I921 O. Heriffon armé, à coquille ovale, brune, da aiguillons triangulaires, emou [jés, livides, annulés de blanc, Klein 39. I. Seb. III I3.1.2. 3. long. 6. lat. 4.pouc. (1. p.42.

1922 O. Soldat, à coquille ovale, à aiguillons triangulaires, plus petites, entremelées, brun-jaunatre, Seb. III. I 3.4. Rumph. 33. 2. Argenv. 25. A. long. $9 \frac{3}{2}$. lat. 8. pouc. (I. p. 43 .

1923 O. Cochon, de coquille oyale, globuleufe, ronde, à aiguillons treslong ues grenues, minces, rangées en fuite, innombrables, EO autre, en formes de poils, brun-jaunatre, Klein 39. 2. Seb. III. 13 5. Rumph. 13. 4? Gualth. 108. D. long. E' lat. $7 \frac{1}{2}$. pouc. (I. p. 44 .

1924 O. Cochon, approchart aus precedent, à aiguillons adkerentes à des autres corps, Klein 37.3 ? Seb. III. 13. 5\% Kircher I. I 7, long. EO lat 7. pouc. ( I. p. 45.

1925 O. Pointé, à coquille globuleufe ronde, noire, dे aiguillons plus longues, granulées, E autres filiformes; Kiein 37. 2 ? 39. 3. Seb. III. I3. 7. Rumph. I3. 5. Petiv. Mur. 3. 8. long. Eீ lat. $4 \frac{\pi}{4}$. pouc ( T. p. 46.

i 1926 O. Petic Heriffon, d aiguillons plus coustes fans filiformes, noiratre. Klein 30. A. B. Seb. III. 13. 6. Gualth. 108. B. (I. 0. 47.

I 927 O. Petic Herifion, approchant au presedent ; pale pourprés; Klein 38, 2 ?3 ? Seb. IlI. I 2. 5 ? 6? Rumph. 1 3. C. (2.p. 48.

1928 O. Petit Heriffon, femblable au precedent, jaun-yiolette, Klein 30. C. D. 38. 2. 3. Seb. III. 12.8? Argenv. 25. F. (2. P. 49.

1920 O. Petit Herifion. Semblable au precedent, brun-verdeatre, Klein 38 I. Seb.III. 12.2.3. 9? Petiv. Mur. 2. 8. (2.p. 50.

I 930 Fleche de Surau, ou Epines, tres minces, treslongues, blanches, Seb, III.

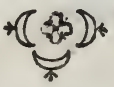




\section{PARTES CONCHYLIORVM.}

I93r Umbill. Cyclopis, fupra convexus, fpiraceus, infra planus, rubro fufcus, Rumph. I9. F. 20. A. Gualth. 70.A.

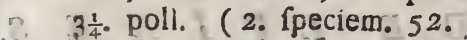

1932 Umbil. Veneris, fupra convexus, infra concavus fpira. 1is, Rumph. 20. C. G. Gualth. 70. E. Petiv. Muf. 10. 4. 5. (2. fpecim. 53:

2933 Umbil. Veneris, fupra convexus, infra planus, oblongus, Rumph. 20. E. Cualth. 70. F. Petiv. Gaz. 65. 5-6. (2. Ppecim. 54.

2934 Umbil.Veneris, luteus, fubcus viridis, Rumph.20.B.D. Gualth. 7c. L. M. Petiv. Mus. 10.3.6.7. (5. 1pecim. 55. 3935 Umbil Granulatus, partim convexa granulofa, Gualth. 70. H. I, Petiv. Gaz. 18. 12 ? 57. 12 ? I3. 'Knorr. VI. 32 5. (2. fpecim: 56.

I936 Blatta Byzantina, cornea, ovalis concava marginata, fufca, Rumph..20. 3. Gualth. 70. B? Petiv. Gaz. 4. 9. (2. rpecim 57.

1937 Blatta Byzantina, cornea, ovalis, convexa, margine foliaceo, ftriata, fufca, Gualth.70.C.D. (2. fpecim. 58. 1938 Blatta Byzantina, fimilis præcedenti, Gualth. 70. P ? (6 fpecim. 59.

1939 Blatta Byzantina, tenuis, lævis, ftriata, fuperne cofta glabra, lanceolata, fufca, Gualtb. 70. G. Petiv. G๘z. 4. 8. (4. fpecim. 60.

T940 Ovarium Concharum Univalvium, oblongum, inteftini forme, Lift. Exercit. Anat. Altera tab. 6. Knorr. IV. 19. 2. ( 2 fpecim. 6r.

294 Ovarium Concharum Bivalvium, globofum, in Oftrea Foliacea, Kr.ort. IV. 19. 3. 5. long. 7. lat. 6. poll. (1. . pecim.62.

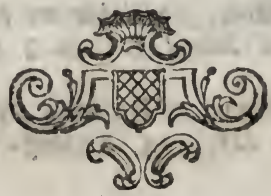




\section{PARTIES DES COQUILLES.}

I93i Un'bil. ou Nombril de Venus, defus recourlée, fpiracḱ, del(Tails plaite, bruir foncé, Rumph. 19. F. 20. A. Gualth. 70. A. 3. pouc. (2. p. 52.

1932 Umisil, ou Nombril de Venus, deffus recourbé, Jpirace, delforis concave, Jpirale, Rumph. 20. C. G. Gualch. 70." E Petiv. Mur 10 4 5.:2, p. 53.

1933 Unbil, or $\mathrm{N}$ mmtoril de Venus, deffus recourbé, defous platie, allongé, blanc. Rumph. 20. E. Gualth. $70 . F$. Petiv. Gaz. 65. 5. 6. (2. p. 54.

1934 Uinbil. ou Nrimbre de Vents, junne, deffous verte, Rumph, 20. B D. Gualth. 70. L. M. Petiv. Muf 10. 3 . 6. 7. (5. p. 55 .

I235 Uiquil. ois Nushabril granulé, en partie, fecouirlée granulée, Gualth. 70. Il. 1. Peii:.Gaz. 18. 12.? 57. 12 ? 13. Knore VI:32, 5." 2. p. 56 :

1936 Blatte Byzantine, cornée, ovale, concave, bordée, uruns, Rumph. 20. 3 Gualth. 70 B? Periv. Gaz. 4 2. p. 57. 1937 Blatte Byzantine, cornée, recourbée, ouale, d bord feuilleté, friée, brume, Guatth. 70 C. D. 2. p. 58.

1938 latte liyzantine, Semblable do la presedente, Gualth. 70. $P$ ? 6, ,, 59.

1939 Blatte Byzantine, mince, polie, Atrié, de/fus à cote pelée, en forme de lances, brume, Gualth. 70. G. Petiv. Gaz. 4. 8. (4, P. 60 .

2940 Nids d'Geufs, de Conques Univalves, ollongé, en forme d'inteftins, Lift. Exercit. Anatom. Altera, tab. 6. Knorr IV. 19. 2. (2. p. 6r.

94 I Nids d'Oeufs, de Conques Bivalves, globibleux, dans zane Huitre Feuilletée, Knorr IV. 19. 3. 5. long. 7. lat. 6 polic. (I. p. 63.

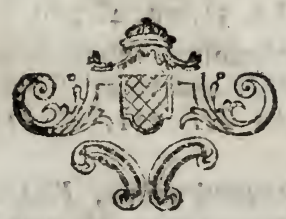




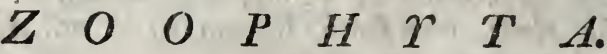

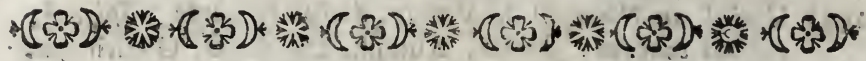
MA D R E P R F.

I M. Fungites, ftella convexa, lainellora, cui fubtus adnata Madr. Faftigiata, Pallas Elench. Zoopizyt. Spec. 165. a. \& I75. Linn. p. $1273.8, \& 34$. Seb. III. 109. 3 6, 111 . I. 2. ( 6 .

2 M. Fuingites, concava, permagna, Pall. \& Linn. l. c. (. .

3 M. Fungites, Pileiformis, minutiffina, Pall. \& Linn. 1. c. (13I:-

4 M. Birito, Fungitiformis, fubplana, \& acaulis, lamellis ferratim dentatis, approximatis, in medio ad marginem, quafi infra convexum, nullibi apud auchores deforip. ta, pulcerrima, Fail. o Linn. o. Seb. III. 111. I? diam. 7 . poll. ( 2. .

.5 M. Birita, Fungitiformis, pracedenti aflinis, minor, $P$ all. \& Linn. o. (16.

6 M. Birita, Fungitiformis, an vurietas præcedentis? Pail. \& Livin. 0.: (18

7 M. Pileus? f. varietas, pulcra, \& cavata, vel media inter Fungtum, \& Pilezm, Pall. \& Linn. o. (8.

$8 \mathrm{M}$. Pileus? T. varietas altera, Pall. \& Linn. O. (10.

9 M. Pileus Neptani, pulcerrimus, concatenata, acaulis gibba, ftellis confertis, Pall. 166.Linn. 1273.9. Seb. III. III. 3. 5. long. I 2. Iat. $5 \frac{1}{2}$. alt. 4. poll. ( I 56.

Io M. Talpa, Pileiformis, futura longicudinali, ftellis pinnata, fubtus leviter excavata, fupra convexa, Pal!.j66. b. Linn. ०. Seb. III. III. 6, II2. 31. long. 9!. lat. 3! poll. ( 4 .

II M. Talpa, pracedenti affinis, Pall. ), c. (20.

I2 M. Talpa, finilis pracedenti, Pall. I. e. (I 35 .

I3 M. Talpa? f. variens, dentibus lamellarnm rotundis, $p$ all. 166. (160. 
I4 M. Cymba, five Pileus Neptuni elongatus, Pall. 166. \% Linin. o. (....

15 M Foliacea, an rarietas pracedentis? Pall. \& Linn. o. $(87$.

I6 M. Foliacea, foliis truncatis, craffis, lamellis dentatis, Pall. * Liní O. (3.

I7 M. Fotiacer, precedenti affinis, Pall. \& Lim. o. ( 16.

I8 M. Foliacea, fimilis precedenti, Pall. \& Linn. 0. (I8.

Io M. Fulicicea? f. varietas, Pall. it Linn. 0. 19.

$20 \mathrm{M}$. Agiricites, ftellis per fulcos ciebris, concatenatis, Pall. 167. Livir. 1274. 13. Seb. III. 110. 6. CCC. long. G. alto 7 poll. 94.

2I N. Agaricites, valde màgna, planis perpendicularibus, aliisque minoribus, anatomifantihus, exerrplar puletrrimim, \& rarifinum, cum Madr. Fivites, Paill. 167. \& 187. Linn. 1274. 13. \& 14 long. \& lat. 17. alt. 12 poll. 1167.

22 M. Agaricites? f. varietas, Pall. \& Linm I. c. (IC4.

23 M. Laituca, ftellis magris, confertis, frondefcestibus, frondibus lacinofis crifpatis, Pall 168. Linn. O. (164.

24 M. Lactuca, fimilis præcedenti, fed pulcrior. Falb. I. c. ( 120.

25 M. Lactuca, pracedenti aftinis, Pall. L. c. ( 136 ,

$26 \mathrm{M}$. Laciuca, frimilis pracedenti, fed pulcerrima, Pall. l.c. (14r.

\% 11. Lactuca, pracederiti affinis, Pall. ô Linn. 1. c. 22.

28 M. Lailuca, fmilis præcedenti, Pall. \& Linn. I.-c. (\&c.

29 M. Elephantotus, lamellis granulcfis, ftellisque laceroproninulis frarfis, Fall. 168. $\beta$. א. O. Linia O. long. $7 \frac{1}{2}$ lat. 4 alt. 5 poll. $(42$.

$20 \mathrm{M}$. Elephantobus? I. varietas prxcedentis, Pall. 1. c. (oc.

31 M, Exefa? f. varictas, an Cruftacea? brachia humana gicanteaformis, ftellis anfractuofis, fuperficie planara, ftellara', radiis concatenatis, Pall. 16o. r. 170. Linn. O. Helenflreit Muf. Richter. t. 14. f. E. 3 ? long. 28. Latit. $\sigma_{2}^{\prime}$ craft. 2 poll.. ( 69 .

32 M. Macandritts, ftellis anfractuofis. Iamellis derticulatis, Pall. 171. Linn. 1274. 11. Seu. III. 111. 7. G:calth. 29 b. $(83$.

33 M. Maeandrites? f, varietas, pulcerrima, Pali. \& Lirn ?. . . . 0, (105. 
I4 $M$. Nafrelle, ous Bonnet de Neptune, allongée, Pall. 166. $\because$ Linir. o. ( $\therefore$. .

$15 M$. Levilletée, ou varieté de la prosedente? Pall. ER Linn. $0 . .(87$.

16 $M$. Feuilletée, à feuilles tronquées, epaiffes, d̀ lamelles dentelées. Pall. EO Linnio ( 3 .

$17 M$ Feuilletée, approchant à la precedente, Pall. Ẽ Limn. 0.16 . 16

$18 M$ Feuilletee, Semblable da la precedents, Pall. ED Linn. o. $(\$ 8$.

19 M. Feuilhetée? ou varieté, Pall. E̊ Linn. O. (19.

$20 \mathrm{M}$. Morillon, à etoiles enchainés, par des fillons. Pall. 167. Linn. 1274. 13. Seb III. :10. 6. CCC. long.9, alt. 7 pouc. (94.

21 M. Mrillon, très grand, à perpendiculaires plattes, $\mathbb{E}$ a al-

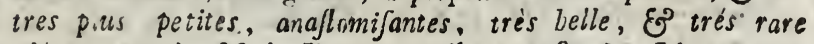
piéce, avec la Malr. Tiavite, Pall. 167. \& 187. Linb. 1274. 13 \& 14. long. E Iat. 17. alt. 12. pouc. (167.

22 M. Morillon? ou varieté, Pall. E Linn. I. c ( 104.

$23 M$. Laicue de iner, remplie de grandes étoiles feuilletées, do feuilles crevues, É pliées Pall- I68. Linn. O. (164.

24 M. Laitue de mer, jemblable it la precedente, mais plus belie, Pall. l.c.. ( 120.

$25 M$. Laitue de mer, approclimt di lo precedente, Pall. l.c. ( 136.

26 M. Lailue de mer, femblable à la precedente, mais très belle, Pail. l.c. (14I.

27 M. Laitue de mer, approchant à la precedente, Pall. E Linn. l.c. $(32$.

28 M. Laitue de mer, Semblable à la precélente, Pall. EO I.inn. l. c. ( \& c c.

$29 M$. Oreille d'blephant, à lamelles grenues, \& étoiles prominentes, Jparfëes, Pa!l. 168. B. .. O. Linn. O.long. $7 \frac{2}{2}$ lat. 4. alt. 5 fortc. ( 42.

$30 M$. Oreille d'Elephant? ou varieté de la precedente, Pall. l. c. $(60$ )

31 $M$ Rongée? on vasieté, ou Cruftacée? en forme d'un bras kubswain gigartesque, d étoiles anfracticeufes, la furfuce platte, étoilée, à rnjons enchainée, Pall. 169. ศ. 170. Linn. Hebeuftreit Muf. Richter. t. 14. f. $E$. 3 ? long. 28. Lat. $6 \frac{1}{2}$ crall. $2:$ pouc. 69.

$32 M$. Cerveau marii, de etoiles anfractenfes, \&o lamelles dentelées. Pall. 17I. Linn. 1274. I1. Seb. III. III. 7. Gualth. 29. b. 83 .

$33 M$. Cerveau marin? ots varibte, trie belle, Pall ED Linn. b.6. C. O, (105. Kk $3^{\text {34. M.Cer; }}$ 
34 M. Meandrites? fo varietas, permagna, Pall. \& Linn. '. c; f. o. lor g. 13. lat. 12. alt. 8 poll. (\&c. 35 : M: "Manidrites, fo varietas precedentis? minor, Pall. it Linn. 1. c. (\&c.

${ }_{36}$ M: Maeandrites, præcedenti affinis, Pall. \& Linn. 1. e. ( $6 \mathrm{r}$.

37 M. Areo!ata, ftella crifpato laciniofa, lamellis crenulatis, Pall. 171. a. Linn, 1274. 12. Séb. III. 112. 23-27. (140.

38 M. Arenlata, fimilis precedenti, fed minor, \& alia varia, Pall. \& Lina. 1.c. Sej. III. 108. 5. ( I 2 ppecin. $18 \mathrm{I}$.

39 M. Labyrinthica, ftellis anfractuofis, fimilis, la mellis cras. fiufculis integris, in qua Madr. Aftroites, Damisornis, \& Pumila, Pall. 156. 172. 188. 197. Linn. "1274. 10. 21. 32. Seb. III. 109. 9. Gualth. 51. b. long. L. !at. 15. alt. 12. poll. ( 1.

$40 \mathrm{M}$. Labyrinthica, precedenti fimilis, permagna, cui acnata. Madr. Damicsrnis, Pall, \& Limn. 1. c. lung. 15. lat. I3. alt. 12 poll. $(23$.

4r M. Labyrinthica, fimilis pracedenti, Pall. \& Limn. 1. $2,4: c_{0}(63$.

42 M. Labyrinthica, an precedenti finilis? permagna Pail, \& Linn: 1. c. long. 8. lat. 6. poll. $\{: 39$.

43 M. Labyrinthic ? varietas precedentis, Pall. \& Linn.!. c. (15I.

44 M: Labyrinthica, pracedenti affinis, pulcra, fed minor, Pall. \& Linr. J. c. ( 150.

45 M. Lacera, ftellis inxqualibus, lacera dentatis, Pall 173. Linn. O. Seb. III. 108. 2. 4. ( 32 .

46 M. Lacera, præcedenti affinis, Pail: 1. c. ( 46.

47. M. Lacera, fimilis præcedenti, Pall. 1. c. (184?

48 M. Angulofa, ficllis rurbirato - angulofis, lamellis dentatis; Pall. 174. Linz?. O. (i62.

4.9 M. Anguloja, precedenti affinis, Pail. I. c. ( 174 .

50 M. Angulofa, finilis pracedenti, Pall. I. c. (157.

5I M. Faftigiata, ftellis fubturbinatis, lamellis incegerrimis; Pall. 175. Limn. I280. 34. S'es III. 109. I. (6.

52 M. Faftigiata? fo verietas pracedentis, Pall. \& Linn. 1. c. $(44$.

53 M. Faftigiata, eadem, fed pulcerrima, Pall. \& Linn. I. c.: (Is9.

54 M. Faftigiata, in Spondylo, Pall. \& Linn. I. c. (119. 55 M. Faftiginta, fimilis precedeuti, cum Spondylo accedente; 5) Pall, \& Linn, 1. c. ( 149. 
34 M. Cerveau marin ? ou varieté, très grande, Pall. EF Linit. l. c. f. o. long. 13. lat. 12. alt. 8 pouc. (\&c.

$35 M$. Cerveau marin, ou varieté de la presedente? plus petite, Pall. Eश Linn. $l$ c. (\&c.

36 M. Cerveau marin, approchant à la presedente, Pall. $\&$ Liun. 1. c. (6r.

$37 M$. Anarainthe de mer, de étoile crepue, plicée, à lamelles crenues. Pall. I7r. a. Linn. I274. I2. Seb. III. II2。 $23-2 \%$. ( 140 .

$38 M$. Amarunthe de mer, femb!alle at li precedente, mais plus petite, EO differentes autres: Pall. EO Linn 1.c. Seb. 111. 108. 5. (12. piéc. 181.

$39 M$. 'Labyrinthifornt, $a$ ètoiles anfracteufes, fiffles, à la. melles craffes, entieres, dans la quelle fe trouve la Madr. Aftroite, E la Corne de Dair, \& Pumile. Pall. 156.172. 188 197. Linn. 1274. 10. 21. 32. Seb. III. 109.9. Gualth. 5r. b. long. ou lat. 15. alt. I2. pouc. (I.

4o $M$. Labyrinthiforme, femblaile à la precedente, très grande. 'à laquelle eft attachée, Madr. Corne dè Daim, Pall. 'छे Linn. l. c. long. 15. lat. 13. ait. 12 pouc. (23.

4t $M$. Labyrinthiforme femblable d la jrecedente, Pall. Es Linn. l. c. ( 63.

42 M. Labyrinthiforme, ou femblable $\dot{a}$ la precedertse? très grande, Pall. E Lian. l. c. long 8. lat. 6. pouc. (180.

43. M Lauyrinthiforme? varieté de la precedente, Pall. E̊ Linn. l. c. '́ I5I.

44 M. Laliyrinthiforme, approcinani $a^{\prime}$ la preceden'e, belle, nais plus petite, Pall. E Linn, l c. (150.

45 M. Lacera, ou Mutile à etoilles ineegales. ED mutilee, E' dientelées, Pall. 173. Linn. 0. Seb. IIT. 108.2. 4. (82.

$40 M$. Lacera, Mutilée, approchant à la frecedente, Pall. l.c. ( 146. 47 M. Lacera ou Mutilée Semblable à laprecedente, Pall. l.c. 6 I84. -48 M. Anglée, (plicée, ) à etoiles ang'eés en pointes, à lamel- les dentelées, Pall. 174 . Linn. 0. ( 162.

$49 M$. Anglée, approchant à la precellente, Pall. l. c. (174.

-50 M. Ang'ée. Se mblable à la precedente, Pall. l.c. ( I57.

SI M. Rofe do mer, à étciles unz peu pointées, à lanelles ens. tierres, Pall. 175. Linn. 1280. 34. Seb. III 109. 1. (6. 52 M. Rore de iner ? ou varieté de lu precedente, Pall.Ẽ Linn.l.c. (r 44.

$53 M$. Rofe de mer, pareille, mais fort bille, Pall. EO Linn. l. c. ( 139 .

$54 M$. Rofe de mer, fur un Spondyle, Pall, EO Linn. b. c. (iig. 55. M. Rofe de iner, Semblable à la precadense, aviec un Spondyle, Pall. E Linn. l. c. (149. Kk 4 56 M. Rofe 
$56 \mathrm{M}$. Foftigiata, an precedenti afinis? f, Rsmea? Pall. \& Linn. 1. c. f. o. (16r.

57 M Ramea, ftellis terminalibus cylindraceis, Pall. 76 , Linn. $128 \bullet \quad 35.64$.

58 M. Ramea, precedenti affinis, Pall. \& Linn. 1. c. $(68$.

$59 \mathrm{M}$. Raunea, fimilis praecedenti, maxima, bipedalis, Pall. \& Linn. 1. c. (.70.

бo M. Ramea, præcedenti afinis, fed paulo minor, Pall \& Linn. 1. c. ( 169.

6I M. Ramea, fimilis pracedenti, Pall. \& Linn. 1. c. (I 73 .

M. Ramea? I. varietas, nigra, in Ajtroite, Pall. \& Limn. 0. (143.

63 M. Prolifera, ftellis turbinatis, margine proliferis, Pall. 178. Jinn 1281, 38. 605 .

64 M. Prolifera, piacedenti affinis, Pall. \& Li:n\}, 1. c. (66.

65 M. Prolifera, fimilis pracedenti, fed pulcerrima, Pall. \& Linn 1 c bong \& lat 7. a 8. poll 793 .

66 M Prolifera? fo varictas alia, Pall. \& Limm. 0. (97.

$67 \mathrm{M}$ Oculata, flava, magna, rariffina, mullibi defcripta, Pall: \& Linin o. an Millipora? (....

$68 \mathrm{M}$ Oculata? f. varietns, 1el Lirhenöides? flavescens, pulcer. rima, reriffimaque, Pall \& Linn.0.Jong. 5. lat. 8.poll (1 10.

$69 \mathrm{M}$. Virginea. Itellis alternatim fparfis prominulie, in $M a$ : cerophylla, Pall. :80: Linib. 1281. 37. 100.

$70 \mathrm{M}$. Virginia precedenti affinis, in Spondylo, fpinis longifine is, jall \& Linn. I. c. I 26.

$71 \mathrm{M}$. $R_{0} \int_{\mathrm{e}}$. Atellis minutis; paffim congeftis eftorescentibus; Pall. 18 Linn o. (115.

$72 \mathrm{M}$, Rofea? f. varietas pracedentis, Pall. I. c. (103.

$73 \mathrm{M}$. Caryoplyllites, pulcerrima, ftellis diftinctis cylindraceis, urbinatis, lamellofis, Pall. 183. Linn. 1278. 30. Seb. III. 108: y. Gualth. 106. G. 26.

74 M. Caryophylittes, pracedenti affinis., Pall. \& Linm. 1. c. 127.

75 M. Caryophyllites, fimilis pracedenti, \& pulcra, Pall. \& Linra I. c. I 32 .

76 M. Caryopliyllites, pracedenti ñmilis, pulcerrima, Pall. \& Linn: 0. 1. 1154

77. M. Flexwofa, cylindris friatis, apice truncatis, Itellatisque Pall. I 4. Limb. 1278, 28, ( 92. 
56 M. Rofe de mer, on Jemblable â la precedenie? ou Corall te Cadix? Pitll. Eo L inn. l.c. T. o. '10 r.

5? M. Cotail de Cadix, de toiles terminantes, cilindriques", Pail. 176. Linn. 1280. 35.:04.

$58 M$. Corail de Cálix, aptrochant de ta pretedente, Pall. E Linn. l. c. ( 68 .

$59 M$. Corail de Cadix, femblable d la precedente, très grande, de deux pieds. Pall. Eo Linn. l. c. ( خo.

$60 \mathrm{M}$. Corail de Cadix, apsroctzant d ta precederite, mais plus petite, Pall. EO Linn. l. c ( 169.

61 M. Corail de Cadix, fembläle à la prectedente, Pall, $\varepsilon^{2}$ l.i 11. l c. ( 173 .

$62 M$. Corail de Cadix? ou varieté noire, dans l'Aftroite, Pall." Eे Linn. 0. 143.

63 vl. Prolifere, à etoiles pointices, au bord proferentes. Pali, 178. Linn. 1281, 38. 105.

64 M. Prolifere, approckant is la precetente, Pall. E L Linn. l. c. (6r.

${ }_{55} M$. Prolifere, femblable d la precedente, Pall. EO Linu. l. $c_{0}$ long. Eे lat. 7. à 8 pouc. ( 93 .

so $M$. Prolifere? ou atstre varieté. Pall. fo Linn. o. (97:

57 $M$. Oeilleté, joune, grande, très rare, nullepart decrite Pall, EO Linn, n. or Millepora?

;8 M. Oeilleté ? ou varieté, ou Lichenöide? jaunatre, trss belle "s eft très rare, Pall. EO Linn. 0. long. 5. lat. 8 pouc. (I10. is $M$. Vierge, à étoiles, alternativement disperfées, prominsm. tes, en Macerophylle, Pall 180. Linn. 128x. 37. 1 100.

- $M$. Vierge, approcizant de la precedente, fur un Spondyle, epines très longues, Pall. हo Linn. l. c. 126.

I $M$. Coulcur de Rofe, ì etoiles memues, amafjes par ci par la, effenrantes, Pall. 181. Linn. O (115.

$2 M$. Couleur de Rofe, an varieté de la precedente, Pall.l c. (103b 3 M. Caryophillite, très bel.e, à etoiles diffinguees. Cylindriques, pointées, lamelleufes, Pail. 183. Linn, 1278. 30 . Seb. 1il. 108. 9 Gualth. 106. G: 26.

li $M$ Caryophillite, approchant à la precedente. Pall Ẽ Linh. 1. c. $(27$

M. Caryophillite, Semblable di la preceilente, beile, Pall. Eפ Linis. l. c. (132.

11.4, M. Caryophillite. Semblable i la precedente, tres belle, Pall E Linn. l. c. (154.

M. Courbées, à cylindres ftriés, an sommet tronquices, étow bées, Pall: 134. Linn. 1278 28. 92. 
78 M. Organum, ftellis cruftaterminali, connexis prominulis, Pall. 185. Limn. 1278. 27. (121.

7y M. Favites, ftellis angulofis cavis, intus lamellofis, Palls 187. Linn. 1275. 14 Seb. 111 111. 8. 10. 18. (178.

80 M. Favites? $\int_{0}$ varietas precedentis, Pall. \& Linn. 1. c. f. o. (2 fpecim. 177.179 .

8I M. Fuvites, precedenti affinis, fed permagna, pulcraque, Pall. \& Linn. 1. c. Irt.

82 M. Favites, fimilis frrecedenti, Pall. \& Limi. 1. c. (III.

83 M. Favites, pracedenti fimilis, fed permagna, Pall. \& Linn. 1. c. lorg. 19. alt . 16. lat. 12. poll. (12.

84 M1. Favites? f. varietas alia, Pall. \& Linn. O. ( 179.

85 M. Favites, f. varietas, ramofiffima, permagna que, Gualth. XX. b? Pall. \& Limn. I. c. f. o. long. 11, alt. 7. poll. ( I3.

86 M. Favites? f. varietas, precedenti affinis, paulisper minor, Pall.\& Linn. I. c. f. o. long. 12. alt. 3. poll. ( I I.

87 M. Favites? f varietas, fed minor, fupra Maúr. Maandri. tes, Pall. \& Linn. I. c. f. o. ( 168 .

88 M. Afroites, grifea, magna in pede, ftellis cavis lamello. fis, ambitu radiatis, Pall. 188. Limn. 1276. 21. long. 17. lat. 13. poll. ( 52 .

89 M. Aftroites, fimilis pracedenti, Pall. \& Linn. 1. c. (or. 90. M. Afroites Talpa, f. media inter Talparn \& Aftroitem, Pall. \& Linn. O. (158.

or M Stellaris, five Aftroites, in Buccineo Morioni, Pall. 188. f. 19:. Linis, 1276. 21. (....

22. M Radians, centri poro radiante, ftriis fcabriufculis, pulcra, Pall, 190. Lini. I276. 19. Sol. III. I12، I2. I6: 77 .

93 M. Rndians, pracedenti affinis, fed micor, \& alia varia, Pall, \& Linn. 1. c. \&c. Seb. 1II. I12. I4. 18. (12. fpecim. 180 .

94 M. Por ites, polymorpho fubratnofa, minutiffimis, fellis punctato fcabris, Pall. 192. Linn. 1279. 31. Gualth. 104. b? -17 .

95 M. Porites, pracedenti fimilis, Pall. \& Linn. 1. c. (7. a.

96 M. Porites, fimilis precedenti, fed pulcrior, Pall. \& Linn. 1. c. (159.

97 M. Porites, fimilis precedenti, fed minor, Pall. \& Linn. J. c. $(15$. a.

$98 \mathrm{M}$. Porites, precędenti affinis, Palles \& Linn. 1. c. (15, b, 
$78 M$. Flute de Pan, détoiles, prominentes, liées par une croute terminale, Pall. 185 Linn. 1278. 27. $12 \mathrm{I}$.

$79 M$. Rayon à miel, étoiles anglées, cavées, en dedanis lamelleus Ses, Pall. 187. Linn. 1275. 14. Seb. 1II. I1 1. 8. 10. 18. 178 80 M. Rayon à miel ? ou varieté de la precedente, Pall. E Linn. l. c. ou 0. (2. piec. 177. 179.

8I $M$ Rayon à iniel, approchant a la precedente, mais trés grande, EO trés belle, Pall. EO Linn. l. c. II

82 M. Rayon à miel, Semblable à la precedente, Pall. \& Linn. l. c. ( 11 I.

$83 M$. Rayon à miel, femblable à la preredente, mais fort grantde, Pall. E Linn. l. c. long. I0. alt. I6. lat. 12 po:c. (12. $84 M$. Rayon à iniel ? ou nutre varieté, Pall. EP Linn. o. (I 79 . $85 M$. Rayon à miel? varieté fort branclute, Eo très grande, Pall.EP Linn. lc. ou o. Gualth xx. b? long. I I.lat. 7 poucc. (13: 86 M.Rayon à miel? ots varieté, approchant à la precedente, tant foit perl plus petite, Pall. E Linn. 6 c on o. long I 2 alt. 8. pouc. (II.

$87 M$. Rayon à miel? ou varieté, mais plus petite, fur la Madr. Mraneirite, Pall. E Linn.-l.c. ou o. ( 168 .

$88 M$. Aftroite, au Etoilée, grife. grande, d̀ étoiles caves, lamelieufes, rayonnées en circuit, Pall. 188. Linn, 1276.21. long. 17. lat. 13. pouc. ( 52.

$89 M$. Etoilée, Semblable $i$ ín precédente. Pall. E Linn l.c. (or. $90 M$. Taupe de mer Etoiléc. ou moyene entre la Taupe de mer, Eे l'Etoilée, Pall. E Linn. O. (158.

9I $M$. Etoilée, nu Aftroite, dans un Buccin Moresque, Pall. I 88. f. I91. Liñn. 1276. 2 1.' ...

92 $M$. Rayonnce, $a$ pore du centre rayonnante, is ftries raboteufes. belle, Pall. 190. Linn. 12;6. 19. Se b. III. 112. 12. $16 .(77$.

$93 \cdot M$. Rayonnée, approchant a la precedente, mais plus petite, E autres differentes, Pall. EO Linn. l.c. Eंc. Seb. III. I12. 14. 18. 12. piéc. 180 .

$94 M$. Porites, ou Poincillée, "un per Uranchue, i-fort petites étoiles raboteufes. Pall, 192. Linn. 1279. 3I: Gualth. 104. $b$ ? 7 .

$95 M$. Pointillee, femblable à la precedènte, Pall. É Linhio l. c. (7. $a$.

$96 M$. Pointillée, approcbant à la precederite, mais plus bslle; Pall. EO Linn. l.c. ( 159.

$97 M$. Pointillee, Semblable d la precedente, mais plus petice, Pall, ES Linn. l.c. (15. a.

98 M. Pointillée, approchant la precedente, Pall. E Linn. b. c. (15.b. 
99. Marrites, finilis pracedenti, Tall. \& Limn.1. \& 150.

100 M. Parites, præcedenti affinis, Pall. \& Linn. 1. c. i $14 \hat{3}$.

Ior M. Parizes, finilis pricedenti, Pall. \& Linn. 1. c. ( 5 I.

102 M. Parites, prectedemi afruis, Pall. \& Linn. I. c. ( 17.

J०3 M. Parites? f. variotas præcedentis, pulcrior, \& major, Pall. \& Linn. I. c. 17.

304. M. Porites'? f: varietas, permagna, \& pulcrior, 'Pall. \& Linn. 1. c. 0.177 . a.

joj M. Porites? f. raxietas, ramońllima, echinaque, pulcer rima, Pall. \& Linn. O. aht. 5. lat. 6 poll. ( I33?

106 M. Porites? f. varietas, an precectenti finilis? Pall. \& Linn. o. long. 6. lat. 4 poll. . 113.

107 M. Muricala, ftellis crebtrrimis cylindriacis, prominulis, pulcra, Pall 194. Linn. 1279. 33. alt. 7. lat 6 poll. ( I 48, 108 M. Viuricata, præcedenti affinis, pulcerrima, Pall. \& Linx. ). c. ( 345 .

Iog M. Muricata, an varietas precedentis? minor, I'alt. \& Linn. 0. 79.

I10 M. Mrtricata, fimilis præcedenti, Pall. \& Linn. 1. c. 175 .

IIf M. Muricata? f. varietas precedentis, Pall. \& Linn. o, (116:

112 M. Muricata? f. varietas, pulccrima, rara, fpinulis fulratis diạphaniș, Pall. \& Limn. o. alt. . lat. 7. à 8. pol!, (142.

II M. Muricata, minutifima, Pall. \& Linn 1. c. ( I 45. İ 14 M. Muricata, pracedenti afininis, Pall. \& Limn. I. c. (92.:

j15 M. Crater, ftellis obliquis, pulcerrima, \& permagna, Pall. 395. Linn. 3282.39 . f. o. long. 9 lat $7 \frac{1}{2}$ ait. $5 \frac{1}{2}$ poll. ( 163. $916 \mathrm{M}$. Crater, fimilis procedenti, fed minor, Kall, \& Linn. l. c. alt. 4. lat. 6 i poll. ( 155 .

ijs M. Faliaja? f, qarietas. polyinorpho laciniofa, Pall. 196 ? Lin?. o. Gronov. Znophyt. n". I747. Seb. III. I10.7. (13. 318 Ni. Pamiconis, poris crebris, cæcis, crenatisque, I'abl. I97 linn. 1279.32 ( 71 .

M. Damicornis, piaceitenti affinis, I alb. \& Tinm. I. c. (20.

120 M. Damicarnis, fimilis pracedenti, Fall, \& Linn, 1. c. 


\section{Zoophytes.}

90. M. Pointillée, femblable à la precederite, Pall E Linn. lo ca 50.

100 M. Pointillée, aptorncliant ä la precedente, Pall. E Linn: l. c. 48.

$101 M$ Pointillée, Semilable do la precedente, Pall. Ẽ Linn. b.c. $5 \mathrm{I}$.

102 M. Pointillée, approchant à la precedente, 1'all. É Linn?. I. c. $(47$.

I03 M. Pointillée? au varicté de la precedente, plas belle, $\&$

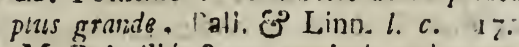

$104 M$ Pointillée? nu varieté, très grande, \& plus belle, Pall. EO Linn. l. c. ort 0. (17. a. .

$105 M$ Pointillée? ou varieté, fort branclue, très belle, Pall. ED Linn. o. alt. 5, lat. 6 , pauc. I 33 ?.

soo M. Pontillée? ou varieté, ou Semblable à la precedente? Pall. 'E Lisn. o. lang. 6. lat. 4. pouc. 1 is 8.

$107 M$. Epineufe, ì btoiles cylindriques, prominentes, belle; Pall. 194. Linn. 1279. 33. alt. 7. lat. 6. pouc. I 48 . $208 M$. Epineufe, approchant d la precentente, très belle. Pall. E̊ Linn. l. c. : 145 .

tog M. Epineufe, ou varieté de la precedente? flus petite;" Pall. Eे Linn. O. $(79$.

510 M. Linineufe, femblable d la precedente, Pall. \& Linn: i.c. 175 .

in $M$. Epineufe? ou varieté de la precedente, Pall. EO Linn o. isio.

I12 $M$. Iipineure? au varieté, très belle, rare, à epines file bonnées, diaphanes, Pall. E Linn. o. alt. ou lat. 7. à 8. pouc. $(: 42$.

I18 M. Epineufe, trés merna, Pall. Eg I.inn. l. c. (145:

ij4 M. Epineufe, approchant di la precedente, Pall. E⿺ Linni. l.c. $(99$.

IIS M. Gobelet, a étoiles obliques, très belle, Etres grande, Pall. 195. Linr. 1282 39. ous o long. 9. lat. $7 \frac{1}{2}$. alt. $5 \frac{1}{2}$ peuc. 163.

I16-M. Gobelet, femblable an precedent, mais plus petit, - Pall.

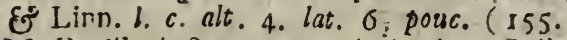

ir 7 . Feuilletée? ou varieté, à plies, Pall. igs? I.inn, o. Gronnv. Zocphyl. $n^{\circ}$. 1747. Seb. III. I10. 7. (13.

II\& $M$. Corne de Dain, $a$ plufieurs pores cremues, Pall. 197. Linn. 1279 32. ( 71 .

$119 M$. Corne de Daim, approchant ì la precedente, Pall. EO Linn. l. c. $\{29$

120 $M$. Corne de Daim, femblable à la precedente, Pall. E2 Linn. l. c. (84. 
72 M. Damicornis, r. varietas pracedentís? Pall. \& Linn. 1. c. ᄃ. O. ( 152.

122 M. Damicornis? f. varietas alia, Pall. \& Linn. 1. c. f. o. long. r. alt. 8. lat. 15 poll. ( 54 .

123 M. Damicornis? f. varietas præcedentis, Pall. \& Linn.1. c. f. o. alt. Ir'. lat. 19 poll. ( 55 .

I24 M. Damicornis. fimilis præcedenti, fed permagna, \& ramofis fimá, Pall \& Linn. 1. c. a t. I2. poll. ( $\leqslant 6$.

125 M. Damicornis, præcedenti affinis, fed ramofiffina, cum Vermiculis, Pall. \& Linn. I. c. alt. 12. Jat. I3 poll. $(57$.

I26 M. Damicornis? f. varietas alia, Pall. \& Linn. l. c. f. o. alt. 11 . lat. 15 poll. ( 58.

127 M. Damicornis, fimilis precedenti, Pall. \& Linn. 1. c. ( 152.

I28 M. Damicornis, pracedenti finilis, fed permagna, \& - pulcrior, Pall. \& Linn I. c. alt. 8. Jat. I2 poll. (59.

I20 M. Damicornis, pracedenti affinis, fed minor, plurimis Macerophylis, Strombis \& Vermiculis, Pail. \& Linn. 1. c. $(59$ b.

I30 M. Damicornis, fimilis precedenti, Pall. \& Linn. I. c. $($...

131: M. Damicornis, minutiffima, fupra Macerophilla, Palbo \& Linn. I. c. ( 78 .

132 M. Seriata, poris ftellaribus longitudinaliter feriatis, pul: cra, Pall. 198. Linn. 1283. 46. alt. 5. lat. 6 poll. ( 127.

33 M. Seriata? r. a arietas, minutiffima, Pa!l. \& Linn. I. c, $\therefore$ ( 3 Specin 81. 84.89.

I34 M. Rosunito fellata, ftellarum diftantium margine tevato, \& ad ambitum ftellatum, Pall. ए̦ Liniz. o. (1 30.

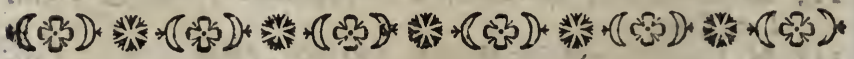

$$
\text { M I L L E P O R F. }
$$

235 M. Frondipora, ramulis porofiffin is, Jall. I 47 . Linno 1284. 48. Marjigli, Hift. de Mer.t 34. $f$ i65. 166. (... $136 \mathrm{M}$. Retepora, infundibiliformis, crispa, Pall. I48. Linm. 1284. 49. d'Aubeton, t. 23. (II3.

337 M. Retepora, pracedenti affinis, F'all. \& Linn. 1. c. (II 4 .

I38 M. Retepora, fimilis pracedenti, Pall. \& Linn. !. c. ( 106.

$\$ 39$ M. Retepora, praecedenti affinis, Pall, \& Linn.-1. c. (1) 


\section{Zoophytes.}

$\$ 2$ I $M$. Corne de Daim, ou varieté, de la precedente? P.ll. E Linn. l.c. f. o. ( 152 .

$122 M$. Corne de Daim? ou autre varieté de la precedente, Pall. Eे Linn. l. c. ou o long. ou alt. 8. lat 15 pouc. ( $54^{\circ}$

$123 M$. Curne de $D_{\text {aim }}$ ? ou varieté de la precedente, Pall. E? Linn. l. c. oulo. alt. 11 !. lat. 19 pouc. ( 55.

$\$ 24 M$. Corne de Daim, Senzllable à la precedente, mais fors branchue, E très grande, Pall. \& Linn. l. c. alt.12 pouc. 56.

I25 $M$. Corne de Dain, approchant d la precedente. mas très branchue, avec Vermiffeaux, Pall. غं Linn. l. c. alt. 12. lat. I.3 pousc. ( 57 .

$126 M$. Corne de Dain? ou autre varieté, Pall Eे Linn. l.c. ou 0. alt. 11. lat. 15 pouc. ( 58.

$127 M$. Corne de Daim, Jembiable is la precedente, Pall. E Linn. l.c. ( 152.

$128 M$ Corne de Daim, femblable d̀ la precedente, mais très gras. de. EO plus belle, Pall. EO Linn. l.c alt. 8. lat. 12 pouc. 59.

$129 M$. Corne de Daim, approchant à la precedente, mais pius - petit, Jur plufieurs Maccropyic's, Strombes, EVVermifeaux, Pall. EO Linn. J. c. ( 59 b.

I30 M. Corne de Daim, Semblable do la precedente, Pall. E Linn. l. c. ( ...

13r $M$. Corne de Daim, fort menue, fur une Macerophile, Pall Es Linn. l. c. ( $7 \%$.

I32 M. Striée, à pore ftriés en long par étoiles, belle, Pall. 198.

$\therefore$ Linn. 1283. 46. alt. 5. lat. 6 pouc. $12 \%$.

I33 $M$. Striée? ou varieté, très petite, Pall. Eे Linn. l.c. (3.) piecc. 8r. 84, 8.9.

$134 M$. Sphærique, le bord elové des étoiles Separées, etoilé ver's le circuit, Pall. \&ं Linn. o. ( 130 .

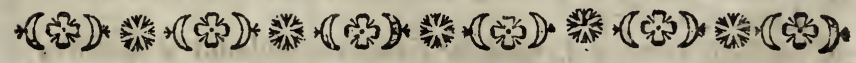
M L L L E P O R E S.

I35 $M$. ̀̀Feuilles large, à rameaux, ou petites. brancbes très poreuz Jes, Pall. 147. Linn. 128448. Marfigli, Hift. de mer, t. 34 . f. $165.166 .(\ldots$

I36 M. Manchette de Neptune, en forme d'infundible, crepue, Pall. 148. Linn. 1284. 49: d'sibeton t. 23. (I13.

$13.7 M$. Manchette de Neptune, approchant à lo precedente, Pall. Es Linn. l.c. II 4 .

I $38 M$. Manchette de Neptune, Jemblable à la precedente, Pall. E. Linn. l. c. ( 106 ,

${ }_{3} 3 \mathrm{M}$. Manchetie de Neptune, la meme déla precedente, Pall. ED Linn. H. c. ( 117 . 140 MiManch: 
140 M. Retepora, fimilis precedenti, Pall. \& Linin. 1. c. Long 6न alt. 4. poll. (II 8 .

I4I M. Retepora, pracedenti finilis, Pall. \& Limb. I. co ( 108 .

$14^{2}$ M. Retepcra? T. varietas, Pall. \& Linn. I. (129.

I43 M. Retepora, pulcerrima, Pall. \& Linit. 1. c. v'Auteton, t. 3.. \&c. ( 138

I.44 M. Clatrata, reticulata, ramulis dechotomis anaftonifantibus, Pall. 149. Linni. o. (128.

I $45 \mathrm{M}$ : Licberiöides, $\mathrm{f}$ Oculata? ftellic ad fexuras mannum concấvis, margine ftriatis, pucra, Pall. I50.1 199? Linn. $1281^{\prime \prime}$ 36. S. 1283. 45? (112.

146 M. Lichenöides, f. Ocuhala? an varietas praccdentis? cum Spongia lapidea alba, foliacea, fiorilis pucerrima, haud. communis, in $n^{\circ}$. 182 . defiripta, Pall. E Linn. I. c. (107:

I47 M. Pumila, poris undique fcabriucculis, Pall. 155. Limn. o. Marjigli, Hift. de Mer. t. 32. f. 57. 5.S. ( I 47 .

$148 \mathrm{M}$. Fumila, fimtis prxcedenti, cum Sertulatia Lichenafiri, I'all. I56. \& 85 l.inn. o. \& $1313.27 .(\ldots$ э 49 M. Pumila? S. vartetas, in patina, Pall. I. c. (... 150 M. Alcicornis, puris minutiffunis obfoleris, fparfis, P'all. 161. Limi 1282. 40. Gronve. Zoo byt tal. 20.f.5. ( 5 .

$15 I$ M. Alcicornis, piacedenti aftinis, Pall. \& Linm. I. c. ( 9 .

I52 M. Alcicornis, maxima, pulcra, \& ramofifina, Pall. \& Limn. I. c long, 19 alt. 16. lat. 12. poll. ( 12.

153 M. Alcicornis, fimilis, fed minor, Pall. \& Linn. 1. c. ( 74 .

154 M. Alcicarnis, fimilis precedenti, fed major, Pall. \& Linn. I. c. ( 37 .

$155 \mathrm{M}$. Lileicomis? f. qarietas, ramis circulatim dispofitis, Pall. \& Linn. I. c. lorg. 16. latt. Is. pull. (30.

$156 \mathrm{M}$ Alcicurnis, piacedenti afliris, Pall. \& Linn. 1. c. (37.

157 M.: Alcicornis. Iimilis pracedenti, fed pulcrior, Pall. \& Limu l. ce lens. 14. lat 1r. poll. \& 123 .

$158 \mathrm{M}$. Aicicornis? 1 varietas prixcedentis, Pall. \& Linn. 1.: 159 M. Filciconis, fimilis pircedenti, Pall. \& Linn. I. c. (36. 160 w. -trecornis, pracetenti affinis, fed pulcrior, haud crnuli.ls, Pall. \& Linn. I. c. ( 96.

$161 \mathrm{M}$ Alcicurnis, finilis precedenai, red major, Pall. \& Linn. 1. c. (37. 
I40 M. Manchette de Neptune, femblable d la precedente, Pall. EO Linn. b. c. long. 6. lat. 4 pouc. ( 118.

I4I M. Nanchette de Neptune, Semblable de laprecedente, Pall. Eo Lina. l. c. ( 108.

I42 M. Manchste de Neptune? ou varieté, Pall. EOLinn.L.c. I29. I43 M: Manchette de Neptune, très belle, Pall. EO Linn. $\%$ c. d'Aubeton. t. 13. \&c. 138.

I44 $M$. Clathrate, reticulée, de petite branches dichotones, ana. ftomijantes, Pall. 149. Linn o. 128.

I45 M: Lichenoide, ou Oeilleiée? belle, d etoiles concaves. vers le combinement des branches, auliord ftriées, Pall. I50. 179 ? Linn. 128 I. 36.041283 . 45 ? ( I 2 .

$146 \mathrm{M}$. Lichenoide, ou $\mathrm{O}$ silletée? ou varieté de la precedente, avec nne matière fpongieufe, picrreule blanche. à fibrilles, pierre, tris belle, pas commune, Pall. \& Linn. l. c. $1 \mathrm{IO} 7$.

147 M. Naine, a pores par tout raboteufes, Pall. 156. Linn. Q. Marfil. Hift, Mer. t. 32. f. 57.58 . I 47 .

I48 M. Ndine, Sembiable à la precedente, avec Sertulaire Lichenaftri, Pall. 156. E5 85. Linn. 0. \&c. I313. 27. . . .

I49 M. aise? ou varieté, en une affette, Pail. l. c. .

I50 M. Corne d'Elan, à pores très menucs, disperfés, Pall. I6r. Linn. 1282. 40 Gronov, Zoophyl tab. 20. f. 5. s."

I5I M. Corne d'Elan, apprachant à la precedente, Pall. of Linn. l. c. $(9$ :

152 M. Crne d'Elan, très grande, trés belle, Eo très brancluue, Pall. \& Linn'l. c. long 19. alt. Io lat. 12. ponic 12 .

I53 M. Corae d'Elan, Semblable, mais plus patite, Pall. ED Lints. l. . . ( 74

154 M. Corre d'Elin, Semblable a la precedente, mais plus grande, Palt \&o Linn. l.c. ( 37.

I55 Corne d'Etan, ¿a branchies, dispojes par cercles, Pall. $\mathcal{J}^{5}$ Linn. l. c. long. 16. hat. II pquc. ( 30 .

\$5 M. Corne d’Elas, approchant de la precedente, Pall. ED Linn。 l.c. 37 .

I57 M. Corne d'Elan, feinblable a la precedente, mais phis bel. le, Pall. E Linn. l. c. long. I4. lat Is pouc. I23.

$158^{\prime} M$. Corne d'Elan? ou varieté de la precedente, Pall. $8^{5}$ Linn l. c. ( 31 .

I59 M. Corne d Lian, Jemblable d la precedente, Pall. E Linn, lc. $(36$.

$160 M$ Corne d'Elan, approcliant d la precedente, mais plus belle, pas commune, Pall. Ẽ Linn. 3. c. 96 : ? 361. M. Corne d'Elan, la méme que $N^{0}$. 95. mais plus grancle, Pall. É Linn, b.6. (37. Lll $162 \mathrm{M}$. Corne 
$\$ 62$ M. Alcicumis, pracedenti affinis, Pall. \& Limn, 1. c. long. f. lat. 16. poll. ( 38.

163 M. Alcicornis, pracedenti fimilis, Pall. \& Linn. 1. c. (49.

764 M. Alcicornis? f varictas, Fall. \& Linn. l. c. ( 72 .

I65 M. Alcicornis? fo varietas praecedentis, Pall. \& Linm. 1. c. ( 75 .

166 M. Alcicornis, fimilis pracedenti, Pall. \& Linn. I, c. $(88$.

167 M. Alcicornis? f. varietas, pulcerrima, maxima, Pall. \& Limn. l. c. lang. 12. alt. 0. pol. ( 122.

$168 \mathrm{M}$. Alcicomis? f. vurietas, precedenti affinis, Pall. \& Linn. 1. c. long. S. lat. II. poll. (I 24.

I09 M. Alcicornis? f. varietos, permagna, foliacea, Pall. \& Linn. I. c. f. o. ( 149 .

7io M. Alcicornis, pracedenti affinis, Pall. \& Linn. I. c. ( 79.

I7I M. Rezta, ramis teretibus, tubulis rectis, Pall. \& Linn. o. ( $3 \mathrm{I}$.

$372 \mathrm{M}$. Cylindrica, ramis longis, teretribus, tubulis cylindricis, permagna, Pall. \& Linn. o. long, f. alt. 18. lat. 16. polk, (43.

\73 M. Cylindrica, fimilis pracedenti, Pall. \& Linı. 0. 43.

374 M. Cylintrica, precedenti affinis, Pall. \& Linn. o. Long. 19. alt. 15. lat. 17. poll. (45.

175 M. Cylindrica, finilis præcedenti, Pall. \& Linn. O. (45.

I76 M. Cylinlirica? f. varietas, ramis brevioribus dichotomis, Pall. \& Linn O. long, 35. lat. 14. alt. 10 poll. (44,

I77 M. Compreffa, teres, compreffa, Pall. \& Linm. o. (... Ij8 M. Ocracea, pertufa, ramofa, ocraçea, Pall. \& Linn. o, ( 160.

$\mp 79$ M. Bifide, ramis obtufis, bifidis, Pall. \& Linn. 0. ( $\cdots$. .

ב80 Ai. Verrucefa, ramis compreflis, verrucofis, Pall, \& Linn, o. long. f. lat. s. poll. (33.

38 I M. Verrucnfa, fimilis præcedenti, fed pulcrior, Pall. \& Linn. 0. ( $3 \mathrm{I}$.

382 M. Lanuginofa, f. Lithophyton rariffimum, fpongian at bido Lanofam in $\mathbf{N Q}$. 355. infra referens, fubftantiarn lanam mentiens, ?edlapidea, duriffima, nullibi apud Authores defcripla, Poll. \& Lit:n. o. long. 11. poll. (125. 


\section{Zoophytes:}

$162 M$. Corne d'Elan, approchant da la precedente, Pall. E Linn. l. c. long. ou lat. 16 . pouc. ( 38.

163 M. Corne d'Elan, Jemblable à la precedente, Pall. EO Linn: l. c. 49.

164 M. Corne d'Elan? ou varieté, Pall. ED Linn. l.c. ( 72 . $165 \mathrm{M}$. Corne d'Elan, ou varieté de ta precedente, Pall. \&? Linn. l.c. 75.

I66 M. Corne d'Elan, Sernblable d la precedente , Pall. EO Linu. i. c. (88.

I67 $M$. Corne d'Elan? ou varieté, très belle, très grande, Pall. है Linn. l. c. long. 12. alt. 9 potuc. ( 122 .

$168 M$. Corne d'Elan, ou varieté, approckant d la precedente," Pall. Co' Linn. l.c. long, bu lat. 11 : pouc. ? 24.

$169 M$ Corne d'Elan? ou varieté, trés grande, feuilletée, Pall:' Ef Línin. l. c. oll o. 149.

$170 \mathrm{M}$. Corne d'Elan, approchant de la precedente, Pall.' $\mathcal{E}^{2}$ Lim. l. c. 79

I I $M$. Droite, à branches longues, minces, oupores, tububles droites, Pail. Ë Linn 0. (3I.

$172 M$. Cylindrique, à branches longues, mirces, tubules cylin:driques, très grande, Pall. E' Linn. o. long. 18. lat. 16pouc. $(43$.

173 M. Cylindrique, ferablable d la precedente, Pall. EO Linn:$0 .{ }^{\gamma} 43$.

1.4 M. Cylindrique, approchant ì la precedente, Pall. E Lima - long 19. alt. 15. lit. 17. poile. 45.

$175 M$. Cylindrique, semblable de la precedente, Pall. E Lina. o. 45.

I76 M. Cylindrique? ou varieté, $a$ ì brancbes phus courtes, dichotomes, Pall: Eे Linn. O. lóng. I5. lat. 14, alt. 10. pous. 144 a

177 M. Comprimée, mince, Pall. Ẽ Lisn. o. (....

I78 M. Ocracke, rompue, ou percée, branchue, achrace, Pallis: E Linn. 0. 166.

179 M. Fendue, à branskes, emougdes, fenducs, Pall. Eo Linnde

$180 M$. Verruceufe, d branches comprimces, plein de verrues: Pall. Ef Linn o. long. ou las 9. pouc. 33 .

181 $M$. Verruceufe, Semblable, à la precedente, mais pius belle Pall. \& Linn. 0. 31 .

182 M. Mouftée, ou Lythophite très rare, reffemhlant à laine blanche, tant en couleur, qu'en fubftance, nais pierreafe, हga, tres dure, ale fe trouse nulle part decrite par les Autheurs: Iallo E⿱ L Linn. 0. long. Ir. pouc. (125. 


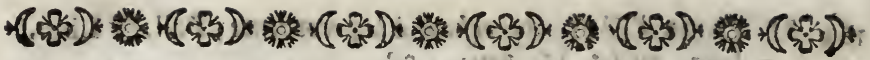 a M}

283 I. Nobilis cortice cattilagineo, fparfin papillofo, Pall. I 42 ; Linn. 1288. 6. Knorr. Delic. Nat, tab. 2A. f. I. 2. 6.62.

184 I. Nobilis, fimilis præcedenti, pulcerrima, Pall. \& Linn\%。 $1 \mathrm{e}=85$

$185 \mathrm{~J}$ Nobilis, precedenti affinis, Pall. \& Limq. 1. c. alt. 9. (8.605

I86 J. Nolilis, fimilis præcedenti, pulcerrima, Pall. \& Lins. i. c alt 9. lat. 6. 137.

$187 \mathrm{~J}$. Nobilis, precedenti affinis, Pall, \& Limn . il c. alt 7. "lat 5 poll? 1.59 .

$188 \mathrm{~J}$. Nobilis, pracedenti affinis, in matrice, Pall, \& Linn. 1. c. 67.

189 T. Nobilis, f. Orramentum, Pectorale, frellaeforme, Tiara, \& Torques, ; artificiofe ac transparenter fculptice excufum, Tiarae pluma, conftat 40. verrum Ornamentun pectorale fugam in Egyptum reprefentans, ex 34 . conftat membris, ita invifibiliter junctis in -1 mellam argenteam, ut tancum unum: videatur fpecimen; Mônile, Torques f. Catena 37. conftat neinbris ; pervent un, ex rapina Vigos; ac verofinnile eadem. artificiofa manu facta,qua Currus Triumphalis, in Mufeo sare:NISS:PRINCIP: A RA A S I O N : confervatus; magnitudino juxta proportionem, (. . .

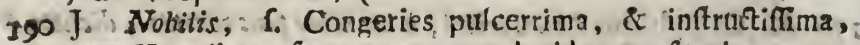
cum Vermibus, fupra concretum lapideum, fjecimen permagnum,. Poll. \& Linn. I. c. long. I6. alt. 9. lat: I r. polt. Hit 782

Igr J. Ocracea, pulcerrima articulis discorticata, cortice papilloro, Pall. 144. Lima, 1287. 3. Seb. IIt. 104. Ia: ( 17 I.

192 J. Hipputis, pulcerrima, cortice crafto, lavi porofo, P'all. 145. Linn. 1287. I. Edve Av. f. 93. s 170.

193 J. Hiţruris, fimilis pracedenti, Pall. \& Linn.'1. c.

194 J: Hipporris, pricedenti aftinis, Pall \& Linn. l. E. 93.

$95 \mathrm{~J}$. Hippruris, articulato diffufa, damofa, $P$ all. \& Linn. l. c. ( 172 .**. 's is] 


\section{(C) +}

\section{S I D E S OU, CORA UX.}

1837 . Corail rouge, lecorce cartilegineale, par.ci, par-la mamilleufe, Pall. 142. Linn. 1288. G. Kuorr Delic. Nat. tab. A.f. r. 2. (62.

184 Fo Corail rouge, femblable à la precedente, très bolle, Palla द्? Linn. l. c. 85.

185 F. Corail rouge, approclaant di la precelente, Pall. E Linn. i.c. alt. 9. (\$6.

$186 \widetilde{F}$. Corail rouge, femillable à la precediente, très belle, Pall. Eg Linn. l. c. alt 9. lat. 6. pouc. (I37.

187 . . Corail rouge, approchant ì laprecedente, Pall. E Einn. i. c. ált. 7. lat. $5 \frac{1}{2}$ pouc. ( 159 .

$188 \mathcal{F}$. Corail rouge, npprochant il la precelense, dans $\int_{\sigma}$ matrio ce, Pall. E Linn. l. c. ( 67.

189 7. Corail rouge, ou Ornement de Poitrine, en forme d'etoilla: Thiare, $\&^{\circ}$ Chaine de Col, ou Coilier, travailiez avec art, $\mathrm{g}^{\circ}$ transparent, la Plume du Thiare, efu rompofáe da 40 oièces, mais $l$ ' Ornement de Poitrine, qui reprefente la frite en Egypte, confifte en 34. pièces, le tout fi inperceptiblement joint enfemble. qu'il ne paroiffe quiune feule piece; le Collicr eft compofé de 37. meinbres, Eo originehe du pillage de Vigos; Ẽ vraifemblablemente. faite sar l'inventive main, qui a conftruite le Chariot de Triomplie, guti fe troure dans le Cabinet de S. A. S. LE PRINCE D'ORALGE LTNASSAU; la grandeur de cette piece eft felon fa proportion.

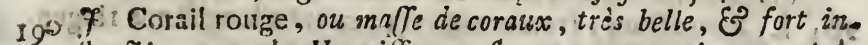
jtructive, avec des Vermiffeaux, fur un concrete pierreux, trets granide piece, Pall. E Linn. i. c. long. 16. alt. 9. lat. II. pouc. ( 182.

19I $\mathcal{F}$. Corail orangé, très belle, decorchée par articles, \& ecorce mamilterfe, Pall, 144. Lirn. 1287. 3. Seb. III. I04. r. ( 171 :

192 . . Corail Hippuris, ou Articulé, très belle, de ecorce, esaiffe, polie, pareufe, Pall. 145. Linn. 1287.1. Edw. Av. f. 93. 170 .

193. F. Corail Articulé, Sernblable à la precedente, Pall. Ẽ Linus' i. c. 172 . 17.

124 f. Corail Articule, approchant à la precedente, Pall. Es Linn, l. c. 93.

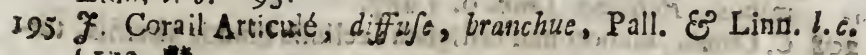
(J) $2, * *$. 
516

Zoophyta.

296 J. Hippuris, an varietas precedentis? Pall. \& Linn. L. e. (2. )pecim. I72.*.

- (59)2)

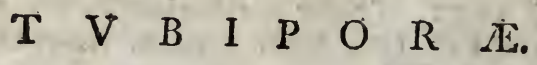

197 T. Purpourea, f. Muifica, tubulis linearibus, articulatis parallellis. Pall. 199. Linn. 1270 . I. 173 .

Ig8 T. Purpurea, f. Mufica, tubulis brevioribus, Pall.\& Linn. 1. c. $(73$.

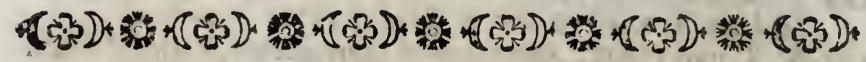

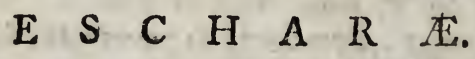

199 E. Ciliata, an Cellularia Pallafit? an Sertularia Linnei? Pill. \& Linn. o, alt. 6. lat. 12 poll. ( 329.

200 E. Fascialis, lamellora, frondofa, laminis conglomeratis, 25 utrinque poris, pulcerrima, Pall. 9. Linn. 1283 47. (100. zoI E. Fascialis? f. varietas, permagna, Pall. \& Linn. I. c. f. o. Seb III 100 12. long. 7. lat. 6. poll. ( 102.

202 E. Fascialis, pracedenti affinis, fed minor, Pall. \& Linn. 1. c. K. 0.98.

203 E. Lat iformis, f. Folincea, cellulis ringentibus arcuatis, utrinque fpirula auritis, Pall. 16 Linn. I 300. I. (326.

$20_{4}$ Eecurifrons, fronde radicata, cellulis longifimis ringen. tibus, Pail 19. Linn. 1300. 2. lat. poll. ( 227.

205 E. Securifrons, pracedenti affinis, Pall. \& Linn. 1. c. lato $5:$ joll. $(328$.

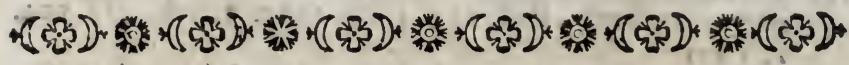
C I L L V L A R I E.

200 C. Neritina,cellulis alternis, mucronatis, interjeet is galericis k. labiatis, P'all. 25, Linn. i3i5 34. Ellis tab.. 19. ( $3: 8$.

207 C. Fioccofa, ramofifima faftigiata, articulis fubcuneifor1. mibus, Pall. 27. Jinn. o. (287.

208 C. Ciliato, dichotona, faftigiata, cellulis binatis aketnis, turLi. binatis, Palb. 32. Linp. I316.28. (333.. 
196. F. Cortil Articule, ou varieté de la precedente? Pall. Em Limn. l. c. 2. piece. 172 . *.

-(S3) $\begin{array}{lllllllll}T & \mathrm{U} & \mathrm{B} & \mathrm{I} & \mathrm{P} & \mathrm{O} & \mathrm{R} & \mathrm{E} & \mathrm{S} \text {. }\end{array}$

I $97 T$. Rouge, dt tubes ligniaires, artioulées, paralelles, Pall. 192: Linn. 1270. 1. $(73$.

$198 T$. Rouge, a tubes p'us courtes, Pall. Eg Linn. b. c. ( 73.

4(ç)

\section{E S C H A R E S.}

I99 E. Velué, ou la Cellulsire de Pallas? ou la Sertulaire de Liuné? Pall. EQ Liwn. o. alt. 6 . lat. 12. pouc. 329. $200 \mathrm{E}$. Fascié, lamellerufe, feuilletée, d̀ lamelles rondes, pores de chaque côté, très belle, Pall. 9. Linn. 1283. 47. (100. $201 E$. Fascié? ou varicte, très grande, Pall. EO Linn. l. c. or o: Seh. III. 100. 12, long. 7. lat. G. pouc. 102.

$202 E$. Fascie, approchant à la precedente, mais plus petite, Pall, Ẽ Linn. l. c. ou a. ( 98.

$203 E$. Large feuille, orb Feuilletée, du cellules ouvertes nrquées, d chaque coté epinzenfe, Pall. I6. Linn. 1300. 1. ( 326. $204 E$. Tronquée, à ferille en racine, cellules très langues, oú vertes, Pall. I9. Linn. 1300 . 2. lat. 6 pouc. $(327$.

$205 E$. Tronquée, approchant de la precedente, Pall. E Linn. l. c. lat. $5:$. pouc. ( 328 .

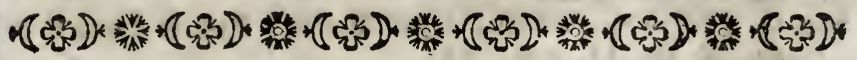
C E L L U L A I R E S.

206 C. Neritine, d cellules, pointues, entremeleses, de petites tours - de poils levrées, Pall. 25. Linn. 1315. \$4. Ellis tab. 19. 318. 207 C. à Floccons, trés branchue, en pointe, غ̀ articles un peu en forme de cuin, Pall. 27. Linn. o. (287.

208 C. à longs poils, dichoome, en pointe, à cellules, deux di deux, turbinées, Pall. 32. Linn. I316. 38 . ( 333. 


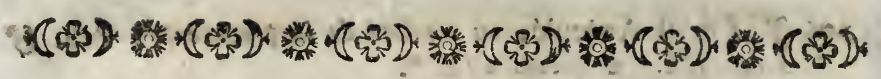

\section{$S$ E}

209 S. Nigellastrum, alterne ramofa, calyculis tuhulofis, ovariis truncatis, Pall. 78 . Linn. 1300. 1. 322.

210 S. Usntöides, dichotoma, calyculis oppofitis bidentatis, ovariis operculatis; Pall. 80. Lini. 7307.3 . ( 332

2 I S. Nigra, f. varietas Purpurec? Pall. 82. f. o. Linm. o. ( 3 Ig, 212 S. Purpurea quadrangulari, calyculis fubiubricatis, ovariis erectis, Pall. 87. Linn. I314. 29. 1320.

213 S. Purpurea? f. vnrietos praecedentis, Pall. \& Linn, I. c fo o. $(42 I$.

214 S. Anteninina, furculis fubfimplicibus verticillatis, ovaris pedunculatis, obliquetruncatis, Pall. 9 I Linn.1310 14. $324^{\circ}$ 2 I5 S. Antemine, finilis praecedenti, Pall. \& Linn. 1. c. (323.

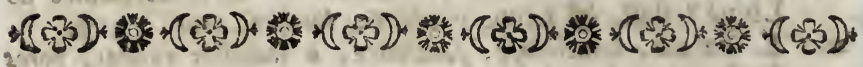
G $O R \quad G \quad O \quad N \quad I$ E.

216 G. Ventilabrum, flavum, maxime lacerum, cum Madr. Angaricite, in bafi, Pall. 101. Linn, 1293. 15. alt. 15. lat. 7. poll. ( 256.

217 G. Flabellum, reticulatum rubrum, f. parpureum, Pall, 104. Linn. 1293. 16. (241.

218 G. Flabellum, purpurcum, Pall. \& Lim. 1. c. (244.

219 G. Flabellum, fimile prxcedenti, duo in cadem bafi, una, cum Spongia Fiftulofa, pulcrum, Palt. \& Linn. 1. c. alt. f. lat. Ir. poll. ( 246 .

220 G. Flabellum, rubrum, ramolifimum, Pall. \& Linn. I. c; alt. 18. lat. 12. poll. 284 .

221 G. Flabellum, pracedenti affine, fẹd magnum, Pall. \& Limn. l. c. ait. I8. lat. 27 . poll. ( 250.

222 G. Flabellum? f. varietas, Pall.\& Linn. 1. c. r. o. (243. 223 G. Flabillum, grifeum, Pall. \& Linn. 1. c. alt. 1. lat. 36. poll. ( 253.

$224 \mathrm{G}$. Flabellum, fimile præcedenti, grifeo purpureum, $\mathrm{Pall}$ \& I I inn. I ¿. (239.

225 G. Flabellum, flavum, minutifimum, puicrum, Pall. \& Linn. 1. c. alt. 7. lat. 9. poll, ( 249. 
226 G. Flabelium, fimile præcedenti, flavum, Pall. \& Lirrr. l. c. $(240$.

227 G. Flabellum, præcedenti affine, flavum, Pall. \& Linn. 1. c. $(238$.

228 G. Flabellum, fimile præcedenti, flavum, Pall. \& Linn.l. c. alt 27. lat 18. poli. ( $8 \mathrm{c}$.

229 G. Flabellum, forma arboris, fupra Mill. Agaricites, Pall. \& Linn. 1. c. ( 256.

229 G. Pinnato, depreffa, ramofa, pinnisque fuboppofitís, Pail. 106. Linn. 1292. 11. d' Aubeton 48. alt. 27. lat. J7. poll. (251.

230 G. Pinnata? f. varietas, pulcerrima, rubra, cui adhæret Efchaia Securifrons, \& Sertularia Lichenaftrum, Pall. \& Linn. I. c. f. 0. alt. J9. lat. 13. poll. (259.

231 G. Pinnata, præcedenti affinis, Capenjis, Pall. \& Linn. 1. c. (...

232 G Pinnata, fimilis præcedenti, Pall. \& Linn. 1. c. (290.

233 G. Pinnata, affinis pracedenti, fed rubra, Pall. \& Linn. 1. c. $(258$.

234 G. Pinnata, fine cortice, permagna, Pall. \& Linn. o. 3 c6.

235 G. Yinnata? f. varietas pracedentis, absque cortice, Pall. \& Linn. o. (304.

236 G. Violacea? f. Purpurea, pinnata, Pall. 108. f. o. Linns.' o. \& alt. 13. lat. 6 . poll. 26 Linn. ( 288.

237 G. Violacea, deprefiuscula, ramofo pinnata, cum Verruco: fa, Pall. 108. \& 126. Linn. o. (. . .

238 G. Violacea, r. Purpurea? fo an varietas pracedentis? Pall. \& Linn. I. c. .. o. 265.

239 G. Verticillatn, teres, longa, pinnata, flava, Pall. rog. Limm. 1289. 2. Ellis tab. 26. S. S. T.V. (., .

240 G. Verticillata, cum appendicibus, five lobis, Palb. \& Linn. I. c. ( . . .

e 41 G. Verticillati, fimilis pracedenti, Pall. \& Linn. 1. c.' (298.

242 G. Peatinata, teres, pinnis adlcendentibus, parallelis longiff; mis acutis, permagna, Pall. 110. Linn. 1292. I4. (279.

243 G. Anceps? f. varietas, fubdichotoma, diffufa, permagna; Pall. II5 f. o. Linn. I292. 10. f. o. Caresby II. tab. 34. (2)6.

$244 \mathrm{G}$. Anceps, praecedenti affinis, in bafi Madr. Agaricites, Pall. \& Lim, I. c. ., O. (...,

\section{G. Ancegs?}




\section{Zoophytes.}

226 A. Eventail de Mer, femblable d la grecedente, jaune, Pall. Eे Linn. $l$ c. : 240.

227 A. Eventail de Mer, approchant ì la precedente, jaune, Pall. EO Linn. l. c. $(238$.

228 A. Eventail de Mer, femblable à la trecedente, jaune, Pall. s.? Linn l. c. alc. 2 ? lat. 18 pouc (\&c.

229 A. Eventail de Mer, en forme d'arbre, fur un Mill. Agaricite, Pall. \& Linn. l. c. ( 256.

229 A. Arbriffeau du Cap, deprimée, branciune, d pointes un pen oppofée, Pall. ICG. Linn. I292. II. d'Aubeton, 48. ait. 27. lat. 17 . pouce. $25 \mathrm{~T}$.

230 A. Arbrifieau du Cap? ou varieté, trés belle, rouge, au quel adihere un Efcare à Feuilles tronquées, EO Sertulaire Lichenaftre, Pall Eo Linn. l c. 043 o. alt 19. lat. 13. pouc. 259. 23I A. Arbriffeau du Car, aptrochant ì la precedente, Pall. E' Linn. l.c. (. . .

232 A. Arbriffeau du Cap, femblable da precedente, Pall. E2 Linn. l. c ( 290.

233 A. Arbriffeau du Cap, apprachant à la precedente, mais rous: ge, Pall. E Linn. l.c. (258.

$234 A$ Arbriffeau du $C_{a p}$, jans ecorce, très grande, Pall. $\mathbb{E}^{2}$ Linn. 0. ( 306.

235 A. Arbriffeau du Cap? ou varieté de la precedente, fans ecorà ce, Pall. Eे Linn. O. ( 304.

236 A. Violette? ou Pcurprée, o. Pall. 108 ou. o. Linn. o. alt: I3. lat. G. poulc. ( 288.

237 A. Violette, un per deprimée, branchue, en pinnes, avec la Verruceufe, Pall. 108. E9 126. Linn. o. (...

238 A. Violette, on Pourprée? ou varieté de la precedonte? Pall. l. c. oul 0. $(265$.

239 A. Vericillé, jaune, longue, mince, à pinnes, Pall. 109. Linn. 1289. 2. Ellis $t a b .20$ f. S. T.V. ( . .

$240^{\circ}$ A. Verticille, avec des appendices, ou lobes, Pall.EंLinn. l.c.( $\because$ :

$241 A$. Verticillé, approcbant a la pracedente, Pall. EO Linn. $l$. c. (298.

242 A. Arbrifreau de Cap, or Peigne de Venus, longue Ë min$c e, a$ pinnes allcendentes, paralelles trèslongues aigues, trés grande piece, Pall. 110. Linn 12y2. I 4. (279.

$243 \AA$. Eventail bordé? ou varieté, un pert feparée, diffufe très grande, Pall. I1 5. ou 0. Linn. 1292, 10. ou 0. Catesby II. tab. $34 . ~\{276$.

$244 A$. Eventail bordé, approchart do la precedente, en $\int a$ bafe 2m Madr. Agaricite, Pall. E Linn. b. c. (. .

245. A. Eves, 
245 G. Ahceps? ramis teretibus, porofis, dichoromis', Pall. \&. Liniz. O. lat. 16. alt. $5 \frac{1}{2}$. poll. 277 .

246 G. Ancets? f. varietas, purpureo cinerea, permagna, fupex Agaricites, Pall. \& Linm. 1. c. Г. o. ( 278 .

247 G. V'iminalis, deprefra, ramis divaricatis, fetaceis, Pall, I16. Linis. O. ( 289.

248 G. Viminalis? præcedentis varietes, major, \& ramis minutifimis, Pall. 1. c. f. 0. alt. 13. lat. 9. poll. 207.

249 G. Cera:opby: a, depreffiuscula, dichotoma, ramis adfcen. dentibus, Pall. 11 7. Linn. 1290. 6. ( 296.

$250 \mathrm{G}$ Purpurea, fubdichotoma, ramis divaricatis, virgatis, Pall. II 8 . Limin, o. alt. 23. lat 12. poll. (260.

25 I G. Purpurea, pracedenti affinis, f. Violacea? Pall. I. c. (29r.

252 G. Purpurea, finilis p:æcedenti, Pall. 1. c. (293.

$253^{\circ}$ G. Purpurea, precedenti affinis. Pall. 1. c. (294.

254 G. Purpurea? f. varietas, rofea, Pall. 1. c. f. 0. (269.

255 G. Purpurea? f. varietas altera, Pall. o. (27. I.

256 G. Purpurea? f. varietas, præcedenti affinis, Pall. O. (2. fpecim. 271

257 G. Purpurea, fimilis precedenti, Pall. 1. c. f. o. alt. 9. lat. 7. Poll. (26r.

258 G. Safappo dichotoma, teres, ramis divaricatis virgatis, Pall II L. Limi. o. $(292$.

259 G. Coralloides, ereeta . fubdichotoma difformis, cum Mill. Retepora, Pall. 123 . Linn: 0. (330.

260 G. Coralloides, fimilis præcedenti, cum Eschara, Pall. 1 , c. $\{273$.

261 G. Coralloides, precedenti affiris, cum E fchara, Pall.1. c. $(274$.

262 G. Coralloides, fimilis praecedenti, Pall. 1. c. (282.

263 G. Coralloides, afinis pracedenti, Pall. I. c. (285.

204 G. Coralloides, fimilis pracedenti, Pall. 1. c, ( 268.

265 C. Coralloides, dichotoma albida, ramis fubverrucofis, Pall. 1. c. (286.

206 G. Coralloides, pracedenti aftinis, Pall. 1. c. ( 288.

257 G. Coralloides? fo vorietas præcedentis, Pall. 1. c. $(28 \%$

268 G. Antipathes, erecta, ramis alternis divaricato ad?cendentibus, Fall. r24. Linn, 129r 9. long. 39. poll. (203.

20,9 G. Antipathes, pracedenti affinis, Pall. \& Limm. I. c, 2ิt. 26. Lat. 20. poll. (314. 
245 A. Eventail bordé? à branches longues, E् minces, poreufes. Separées e. deux, Pall. 89 Linn. o. lat. 16. nlt. $5 \frac{1}{2}$. powc. (277. 246 Eventail bordé? ous varieté, pourpré centrée. fort grande, fur un $\mathrm{A}$ garicite, Pall. ES Linn. l.c. ou o. ( 278.

$247 A$-Saule de Mer, deprimé, d̀ branches devariquées, feta. cées, Pall. ru 6. Linn. 0. $(289$.

248 A. Saule de Mer? varieté de la precedente, plus grande. Pall. l. c. ols 0. alt. 13. lat. 9. pouc. ( 267.

249 A. Mahony Marin, deprimée, Jeparée, à branches adjcen. dentes, Pall. II7. Linn. 1290.6 . ( 296.

250 A. Pourprée, un peu feparée en deux, ¿̀ branches devariquées, vergetées, Pal!. 118. Linn. 0. alt. 23 lat 12. jouc (260."

$25 \mathrm{I} A$. Pourprée, approchant de la precedente, ou Violetie? Pall.l.c. ( 291.

252 A. Pourprée, femblable à la precedente, Pall. l. .. (293.

253 A. Pourprée, approchant ì la precedente, Pall l.c. (294.

254 A. Pourprée? ou variete, couleur de rofe, Pall. l. co ou 0. 269.

255 A. Pourprée? ou autre varieté, Pall. o. (27:.

256 A. Pourprée? ou varieté, approchant à la precedente, Pall. 0. (2. piec. $27 \mathrm{r}$.

257 A. Pourprée, Semblable d la precedente, Pall. l. c. ouso. alt. 9. lat. 7. pouc. ( $26 \mathrm{I}$.

$258 \mathrm{~A}$. la Verge rouge, Jeparćs en deux, longue $\mathcal{E}^{2}$ mince, ḋ branches devariquées, vergetées, Pall. IIg. Linn. 0. (292.

259 A. Coralloide, erigée, un peu Setrarée en deux, avec un Mill. Marchette de Neptune, Pall. I23. Linn. 0.(330.

$260 A$. Coralloide, Semblable d̀ la precedente, avec un Efcare, Pall. l. c. $(273$.

261 A. Coralloide, approchant is la precedente, avec un Iifcare; Pall. l.c. (274:

262 A. Coralloide, pareille, Pall. $l$ c. ( 282 .

263 A. Coralloide, approchant à la precedente, Pall. l.c. (285. 264 A. Coralloide, jemblable is la precedente, Pall. b. $c_{\text {. }}$ (268.

$265 A$. Coralloide, Separée en deux, blancheatre, ¿̇ brancǩes un peu verruceufes, Pall. l.c. ( 286.

266 A. Coralloide, approchant d la precedente, Pall. $l$. $c_{\text {. }}$ ( 288.

267 A. Coralloide? ou variete de la precedente, Pallo l. c. $(287$.

208 A. Antipathes, erigée, i branches ecartées, adfcenders. tes, Pall., 124. Linn. 1291. 9. long - 39. pouc. (203.

R60 A. Antipathes, approchant $\dot{a}$ la precelente, Pall. E? Linn. b. 6, alt. 26. lot. 20, Poutc. ( 314. 
270 G. Antipathes? f. varietas, Pall. \& Linn. 1. c. 1. ก. alt. \& lat 24. poll. ( 315 .

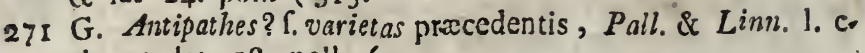
alt. 23. lat. 18. poll. ( 312 .

272 G. Antipathes, fimilis præcedenti, fed minor, Pall. \& Liniv. I. c. $(303$.

273 G. Antipathes? f. varietas piacedentis, Fall. \& Limn. 1. c. I. O. (305.

274 G. Antipathes, B. f. Savalia? nigra, Pall. \& Linn. 1. c. f. o. alt. 57 . poll. $(183$.

275 G. Antipathes, B. r. Savalia? præcedenti affinis, Pall. \& Linn. 1. c. T. o. alt. 60. poll. ( 184 ,

276 G. Antipathes, B. f. Savalia? fimilis pracedenti, Pali. \& Linn. I. c. f. o. alt. 54. poll. (184.

277 G. Petechizans, teres, ramofiffima, diffufa, Pall. 125. Linn。 1292. 13. (275.

278 G. Petechirans, pracedenti affinis, Pall. \& Linn. 1. c. 279 G. Petechizans? f. varietas, Pall. \& Linn.l c. f. o. (298. 280 G. Varioloja, an præcedenti affinis? Pall. \& Linn. O. (297.

281 G. Verrucofa, teres fubdichotoma, divaricata, magna, cum Afteria Meilufe, Pall. 126. Linn. 1291.8. Seb. I11. 10\%. 3. alt. 57. poll. ( 185 .

282 G. Verrucofa, major, cum quatuor Afteriis Medufa, Pall, \& Linn. 1. c. long. 27. alt 30. poll. ( 186.

283 G. Verrucosa, pracedenti affinis, cum Arteriis Medufa, Pall. \& Linn. l. c. ( I $\delta 6 . *$

284 G. Verracofa, fulva, Pall. \& Limn. 1. c. (249.

285 G. Verrucofa, fimilis præcedenti, Pall. \& Linn. l. c. (264.

286 G. Verrucofa, fulva, an pracedenti affinis? Pall.\& Linn. 1. c. $(262$.

287 G. Verrucofa, fimilis prasedenti, cum 3. sifteriis Redufa, Pall. \& Linm. 1. c. (186**

283 G. Verrucofa? f. varietas, aurantia, Pall. \& Linn. l. c. (257.

289 G. Verrucofa? f. varietas, rofei forma, fupra Mill. Retepora; Pall. \& Linn. 1. c. (. .

200 G. Verrucnfa? f. varietas, prxcedentis, fed foliacea, Pall. \& Linn. 1. c. alt. 7. Jat. \%. poll. ( 266. 


\section{Zoophytes.}

270 A. Antipathes? ou varieté, Pall. \& Linn. l. c. ou o.alt. E lat. 24. pouc. ( 315 .

27 A. Antipathes? ou varieté de la precedente, Pall. E Linn. l. c. alt. 23. lat. 18, pousc. 312.

$272 A$. Antiparhes, fermblable da la precedente, mais plus pețte, Pall. E Linn l. c. (303.

273 A. Antipathes? ou varieté de la precedente, Pall. EO Linn: l.c. 026 0. (305.

274 A. Antipathes, $\beta$ ou Savalie? noir, Pall. EO Linn. l. G. ou o, alt. 57 . potic. ( 183

275 A. Antipathes, $\beta$ ou Savalie? approchant de la precedenre, Pall EO Linn. l. c. ou o. alt 60. poute. (184.

276 A. Antipathes, $\beta$ ou Savalie, femblable d la precedente, Pall: EO Linn. l.c. ou. o. alt. 50. porse. ¿ 184 .

27: A. Eventail pointillé, mince, três branchue, diffufe, Pall. 125. Linn. 1292. 13. ( 275 .

278 A. Eventail pointillé, approcbant à la precedente, Pall. E Linn. l.c. (...

279 A. Lventail pointillé? ou varicté, Pall. \& Linn. l. c. o:2 0.298. 280 A. Variolé, ou approchant d̀ la precedente? Pall. ED Linn. 0. $(297$.

281 A. Verruceufe, ou Granule, longue Es mince, un peus feparée en deux, grande, avec l'Etoile de mer de Medufe, Pall. 126. Lirn. 1291. 8. Seb. IlI. 106. 3. alt. 57. pouc. (185.

282 A. Verruceufe, ou Granulé, thlus grande, arec quatre Etoiles de mer de Medufe, Pall. ED Linn.l.c.long 27.alt. 30. pouc. (186. $283 A$ Verruceufe, ou à tubercules, approchant à la precedente, avec des Etoiles de mer de Medufe, Pall. EO Linn. l. $c$. ( $186 . *$

$284 A$. Verruceure, ou à tubercules, fauve, Pall. EO Linn. l.c. ( 249.

285 A. Verraceufe, ou à tubercules, femblable à la precedente; Pall. EO Linn., l. c. 264.

$286 \mathrm{~A}$. Verruceufe, ou à tubercules, fauve, ou approchant is la precedente, Pall. E Linn. l. c. 262.

$287 \mathrm{~A}$. Verruceufe, on à tubercules, Jernblable do la precedente; avec 3. Etoiles de Medure, Pail. 'O Linn. l. c. ( 86 .**

288 A. Verruceufe, ou à tubercules? ou varieté, orange, Palle' Eं Linn. l.c. 257.

289 A. Verruceufe, ou à tubercules? ou variete, en forme de rofe, Jur un Mill. Manchette de Neptune, Pall. ES Linn.' i. c. (....

290 A. Verruceufe, ou à tubercules? eu varieté do la preceden' te, mais feuilletée, Pall. EO Lino. l. 6. alt. 7. lat. 9. pouce. 206 291 A. Verri: 
29 I G. Derrucofa? f. varietas, Pa!l. \& linn. 1. c. (295.

292 G. Verrucofa? f. varietas, ve! Cerathophyton Boerlaciii, Pall. \& Linn. 0. alt. 23. lat. 17. poll. ( 252.

293 G Placomus, teres lignola, dichotoma, Aava, Pall. 129. Linn. 3290. 3. alt. 36. lat 27. poll. ( 245.

294 G. Refeda, alterne ram fa, diffura, Pall. 131. Linn. o. $(247$.

295 G. Refeda, præcedenti affinis, fed magna, atra, Pall. 1. c. alt. \& lat. 36. poll. ( 253.

296 G. Refeda, funilis præcedenti, p̧ermagna, Pall. I. c. ( 255 .

297 G. Compreffa, ramis flexuolis, cortice albo, Capenfis, 1'all. \& Linn. o. alt. I4. lat. 16 poll. ( 230.

298 G. Compreffa, præcedenti aflinis, Capenfis, Pall. \& Linn. o. alt. 23 , 1at. 9. poll. ( $28 \mathrm{r}$.

399 G. Compreffa, aurantia, fulva, Capenfis, Pall. \& Linn. o. (...

300 G. Ariorea, magna apud nullos Aubores defcripta, Palb. \& Limn. 0. alt. 51. lat. 36. poll. ( 299.

$301 \mathrm{G}$. Lateritia, permagna, Pall. if Lirn. o. alt. 36 . lat. 16!. poll. ( 299 .

302 G. Lateritia, Variolofa, Pall. đ̃ Limn. O. ( 272.

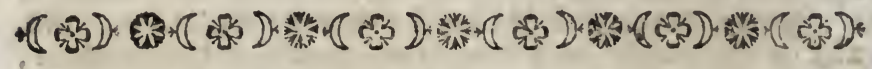

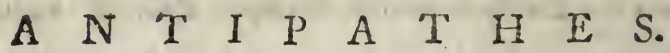

303 A. Ericoides, ramofiflma, hispidiflima, atra, Pall. I33. Linn. o. (...

304 A. Pennacea, f. Acabar nigrum, ramofa fubincurva, Pall 134 . Limn. O. ( 308.

305 A Pernacea, an varietas præcedentis? minor, cum Mill. Frondipora, Pall. \& Linn. 1. c. ᄃ. O. (33r.

306 A. Clathrota, ramofifima, incricata, Pall. 137. Linn. 0. ( 308 .

807 A. Cuprefina, trunco fimplici longifimo flexuofo, ranulis confertis ramofis, \& paniculato, Pall. 138. Linin. 1290.4., alt. I1. lat. 18. poll. ( 307.

308 A. Dichotoma, longiffima, dichotoma erceta, Pall. 140. Lintr. O. alt 18. lat 16. poll. 310.

309 A. Stiralis, fimplicifima, attenuata flexuofo-fpiralis, cum Madr. Croter, \&ialia, Pall, I4 I, \& I95.Linn. 1290. 4. $\beta$. alt 22. poll. ( 300 , 
310 A. Spiralis, polita, absque aculeis, Pall. \& Linn. l. c. (301.

3 II A Spiralis, precectenti afintis, cum Gorgonia, Pall. E? Linn. 1. c. ( 302 .

312 A. Atra, ranis incurvis, apice bifidis, I'all. \& Lim. o. alt. 12. poll. $(313$.

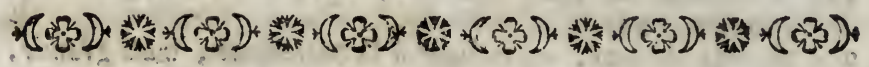

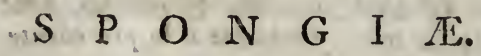

313 S. Flalielliformis, rigida, rotundata, tlabelliformis plana, pulcerrima, Pall.226. Linn. 1296. 2. alt. 7. lat. 8 poll. (213.

314 S. Febrillofa. fibris divergentibus contextis, poris fuarfis, rparfis dentatis, Pall. 228. Linn. o. (232.

315 S. Fulva, amorpho fubramola, rigidiffima, fulva, Pall. 230. Liniv. $0 .(224$.

316 S. Fiffuicris, tubulora attenuata rigida, extus ruberçuln. fa , pulcerrima, longifima, Pall. 232: Linn. 129?. 4. (301.

317 S. Fiftularis, fimilis-precedenti, fed minor, fupra diffec. ta, Pall. \& Linn. 1. c. !org. 16 poll. ( 204.

318 S. Fiftularis? f. rarietas precedicritis, Pall. \& Linn. I. c. f. o. long. 19 poll. (205.

319 S. Fiftularis, præcedenti affinis, tubulis duplicatis, Pall. \& Linn: 1. c. long. I's. lat. II poil. (206.

320 S. Fifularis, fimilis nræcedenti, minor, Pall. \& Linn. I. c. (20\%)

321 S. Officinalis, fublobata, tomentora, porulenta, Pall. 234. Linis. 1298.8 . (235.

322 S. Officrnalis? i. varietas, Pall. \& Linn. o. Seb. III. 97.4 ? (225.

323 S. Fanacea, aniorpha, mollis, tenerrima, fubtilifinna, porofa, perinagna, Pail. 235. Linn. 1299. 12 ? (...

324 S. Cerricornis, tenax, ramis teretibus fubvillofis, Pall. 235. Linn. 1299. 14 alt. 6. lat. 4 poll. ( 227.

325 S. Cervicornis, finilis præcedenti, Pall. \& Linn. 1. ć, (220.*

$326 \mathrm{~S}$. Cervicornis, ramofifitma, teres, ramis teretibus, Pall. \& Linn. I. c. (230.*

327 S. Rubens, moilis, poris majusculis fparfis, Pall. 238 . Linm. 1298. 15. ( 237. 
310 A. Corde de Mer, polie, Sans aiguillons, Pall. EO Linn. i. c. $(301$.

3 I $A$ : Corde de Mer, approchant d la precedente, avec un Aröre de Mer, Pall. Eg Linn. l. c. ( 02 .

$312 A$. Ebene marin, ¿̇ branche, courbées, au fommet four: chues, Pall. EO Limn 0. ait. 12. pouc. ( 313.

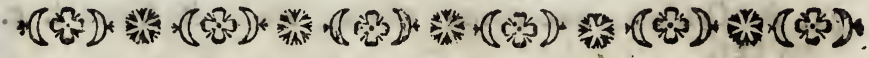

\section{E $\quad P \quad O \quad N \quad G \quad E$ S.}

3ı 3 E. Eventail de Nimphe, rigide, ronde, platte, tris bely le. Pall. 226. Linn. ing6 2. alt. 7. lat. 8. pouc. (213.

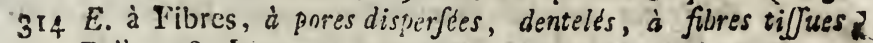
Pall. 228. Linn, 0. 232 .

315 E. Orangée, un peu branchue, trés rigike, fauve, Pall. 230. Linn. 0. ( 224 .

316 E. Trompe d'Elephant, d tubule, mince, rigide, de hors tuberculée, tries belle, très longue, Pall. 232. Linn. 1297. 4. $(301 \cdots$

317 E. Trompe d'Elephant, ferrblable la precedente, mais pla pesite, romput par de fus, Pall. EO Linn. l.c. long. Io pouc. (204. 318 E. Trompe d'Eiephant? ou varieté de la precedente, Pall. Eg Linn. l. c. ou o. long. 19. pouc. (205.

3Ig E. Tompe d'Elephant, approchant do la precedente, tubales duplickes, ou doublees, Pall. EO Linn. b. c. long. I8. lat II pouc. ( 266 .

320 E. Trompe d'Elephant, Semblable d la precedente, Pall. E J.inn. b. c. $\{20 \%$.

32! E. Vulgaire, un peu lobt, bourrue, poreufe, Pan. \&3 st5 Linn. 1 298, 8. , 235.

$322 E$. Vulgaire? ou variete, Pall. Eश Linn. o. Seb. III. $97 ;$ 4.? ( 225.

$323 E$. Panacee, molle, trés mince, $E^{2}$ très fubtile, porsufe. très grande, Pall 235. Linn. 1299. 12 ?

$324 E$. Branchue, gluant, d branches longues, \& $0^{\circ}$ ninces, peu velues, Pall. 236. Linn. 1299 . 14. alt. 6. Lat. 4 pouc. $(227$. 325 E. Branchue, Semblable d la precedente, Pall. El Linn. i. (220.*

$326 E$. Branchue, \& brancies très longues, \&2 minces, Pall. \&2 Linn. b. c. (230.*

327 E. Rougeatre, molle, à pores plus grandes disperftes, Pall 38. Ling. 1298. 11. (237. 
- 328 S. Rubens, fubramora, poris majusculls, Palt. \& Litth. 1. c. $(237 . a$.

329 S. Rutens, fimilis, pracedenti, Pall. \& Linn. 1. c. ( 2 I 8 .

330 S. Oculata, ramofifima, mollis, ramis teretiusculis, potis prominulis, fparfis, pulcerrina, Pall. 239. Linn. I298. 9. alt. $7 \frac{1}{3}$. lat. 8 poll. ( $214 . *$

331 S. Oculata, pracedenti affinis, Pall. \& Linn. I. c. ( $22 \%$.

332 S. Oculata, fimilis præcedenti, Pall. \& Linn. 1. a (235. a

333 S. Uculata? varietas pracedentis, Pall. \& Linn. 1. c. (222.

334 S. Oculata, finilis Pall. \& Linn. 1. c. ( 23 r.

335 S. Faftigiata, tubulofo-ramofifima, ramis faftigiatis, truncatis, Pall 241. Linn 1297. 6. (205

336 S. Faftigiata, fimilis pracedenti, Pall. \& Linn. I. c. ( 220.

337 S. Villofa, tenacifima cava, extus muricata, intus lae. vis, Pall. 242. Linn. 1297. 5. long. 5 lat. 4 poll. (21 2 : 338 S. Sinnofa, cruftacea, fuperficie finubus creberrimis cavernofa, Pall. 243. Linn. O. ( 217.

339 S. Cavernofa, fifilis cavernofa, crebris papillis promisulis, Pall. 244. Linn. O. (...

$340 . S$. Foliascens, tenax laciniata, altero latere exefo fcabra, pulcerrima, Pall. 246. 1296. 3. Pailas Plant-Dicren, cura Boddaertii , tab. 13. f. 3. alt. 7 . . lat. 8 poll. ( 2 I $4 .{ }^{*}$ $34 \mathrm{I}$ S. Foliascens, pracedenti affinis, Crateriformis, Pall. \& Lijnn. I. c. alt. 18. lat. II poll. (216.

342 S. Elephantoti, an Membranofa? fub-ramofa, cellulofi, pulcra, Pall. 250? Linn. o. alt. 13. Iet. 7 poll. (2 15. 343 S. Tomentofa, porofa, fubaculeato tomentola, Palb. 0. Linn. 129g. 12, a.t. 6. lat. 12 poll. (229.

344 S. Tomentofa, fimilis precedenti, Pall. o. Linn. 1. c. (229. 2.

343 S. Tomentofa? f. varietas, Pall. o, Linn. 1. c. alt. 10. lat. 9: poll. (229.*

346 S. Tomentofa, f. varietas pracedentis? Pall. ๑. Linn. I. c. (22\%. b.

347 S. Cancellata, tubulofa foraminulata, fibris capcellatis, Pall. O. Lin. 1297. 7. ( ...

348 S. Lanoja, atra, $r$ Plunioja, ad plumam Galez referens, Pab. \& Limz. 0. (210. 
328 F. Rougeatre, un perb branclune, \& pores plus grandes, Pall. \& Linn. 1. c. (237. a.

329 E. Rougeatre, Semblable d̀ la precedente, Pall. ED Limn. $k_{0}$ c. $(213$.

$330 \mathrm{~F}$. Oeilletée, très brancluue, molle, à hranches plus longues E minces, pores prominientes, disperfées, très belle piéce, Pall. 239. Linn. 1208 . 9. alt. $7 \div$. lat. 8. pouc. $\left(214 *^{*}\right.$.

331 E. Oeilletée, approchant d la precedente, Pall. Eं Linr. l.c. $(22 \mathrm{I}$.

332 E. Oeilletée, Semblable la precedente, Pall. E Linn. $l$. c. (235. a.

333 E. Oeilletée? ou varieté de la precedente, Pall. Ẽ Lina. l. c. i 222.

$334 E$. Oeilletée, Semblable. Pall. E Linn. l. c. (23 $3^{\mathrm{T}}$.

335 E. Eitagée, très brancliule en tubule, les branches ebe. vées tronquées, Pall. 241. Linn. 1297. 6. (205.

$33 \sigma E$. Etagée, femblable is la precedente, Pall. Ẽ Linn. $l_{\text {. }}$ c. $(220$.

337 E. Veloutée, trìs creufe, delisrs pointée, en decians polie. Pall. 242. Limn. I297. 5. long. 5. lat. 4. pouc. (212.

338 E. Dechiquetée, cruftacée (en croute) la furface, par des continuelie cavites, caverizufe, Pall. 243. Linn. O. (217. 339 E. Caverneufe, fedile, caverneufe, à mamillons proninentes, Pall. 244. Linn. 0: ( . .

340 E. Fcuilletée, tenace, plein de plis, l'antrecôté vermoulue: raboteufe, trés belle, Pall. 246. Linn. 1296.3 . Pallas Planto Dieren, Boddaert, tab.13.f. 3. alt. 7. lat. 8. fouc. (214. 34r $E$. Fetuillerée, aptrochant a la precedente, en forme de gos. belet. Pall. EO Linn. l. c. alt. 18. Iat. I I. pouc. 1216.

342 E. Oreille d'Elephant? ou Membraneufe? un peu branchue, celluleufe, belle, Pall. 250 ? Linn. 0. alt. 13 . lat. 7 pouc. $\left(2150^{\circ}\right.$ $3+3 E$. Tomentofe, ou Charnue, porevfe, un peu aigue, Pall. 0. Linn, 1299. 12. alt. 6. lat. 12. poiuc. 229.

344 E. Tomentofe, ou Charnue, Jemblable d la precedente, Pall : o. Linn. I. c. (a) a.

$345 E$. Tomentofe $\{0 u$ Charnue, ou varieté, Pall. o. Linn. i. c: alt. 10. Iai. 9' pouc. 1229 . *.

346 li. 'Tomentofe, ou Charme, varieté de la preceilente?" Pall. EO Linu. l. c. 62:9. b.

\$47 E. Cancellé, en Mailles, ou à Treilies, tubuleutfetroué, de fitres cancellées, Pall. 0. Linn. 1297. 7. ( . .

i48 E. Plunet d'Auphitrite, reffembialit a la pirme d'Amphes: trite o loine, noira, Pald, है Lina o. (210. 
349 S. Intinata, erecta, ramis incurvis, obtufis, muricata, 3cts. leis longiusculis, rara, Pall. \& Linn. o. ( ० 6 .

350 S. Echinata? f. varietas, ramis teretibus, Pall. \& Linn. o. alt. 6 . lat. 4 poll. $(22 \%$.

35 I S. Echinata, pracedenti affinis, fupra Madr. Damicornis, Pall. \& Linn. o. $\$ 228$.

352 S. Sinto/a? f. varietas precedentis, Pall. o. ( 33.

353 S. Folitum Quercus, exacte folium Quercus referens, Palt. \& Lina. o. ( 219.

354 S. Gerdinia, ramis obtufis incurvis, Pall. \& Linn. 0. (2:3.

355 S. Larofa, albido flavescens; fibrofa, foraminulenta. maxima, analoga n`. 182. fupra, Pall. \& Linn. o. (211.

350 S. Scyphus, ex fubfantia Spong. Aoulenta, figura fcy: phi, in quo Tubuli duo, Spong. Fiftularis, rariffima, ex Curacao, apud Authores nonduns defcripta, Pall. \& Linn. 0. long. 6. lat. 9. alt. 7 poll. ( 208.

357 S. Scyphus, pracedenti affinis, in quo cjusdem Tubula ; \& Spong. Cavernofa, pulcerrima, rarilfinaque, Yall. \& Linin. 0. long. 7. lat. 6. alt. 10 poll. ( 209.

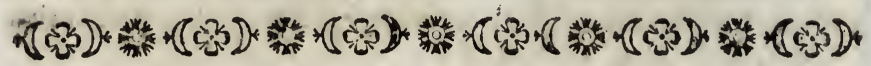

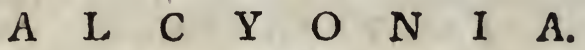

इ5 A. Arboreun, ramofum, poris papillaribus congeltis, $P_{a} l l$. 202. Linn. 1293. 1. ( 236 .

359 A. Arboreun, præcedenti affine, Pall. \& Linn. 1. ๔. 236.

360 A. Ficus, tubiforme pupofum, papillis crebris ftellatis, Poll. 209. Linn. 1295. 10. (3I6.

$36 \mathrm{r}$ A. Manus Diaboli, protulscrantiis obtufis, perforatis, Pall. 0. Linn. 1296. 12.?:317.

((3))+ -

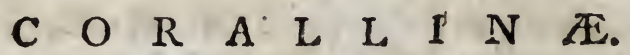

$\$ 62$ C. Ofuntia, articulis planis reniformibus, Pall. 2. Linnt 1304. I. (210!' 
363 C. Officinalis, bipinnata, articulis fubovatis fuperioribus compresfis, Pall. 4. Linn.1304. 2. (19I.)

$36+$ C. Oficinalis, fimilis precedenti, Pall. \& Linn. I. C. ( 199 .

365 C. Officimslis, pracedenti affinis, \& alia, fupra Patella, Pall. \& Linn. l. c. ( cr.

306 C. Offrcinalis? f. varietas, pall. \& Linn.1. c. (107.

367 C. Officinalis, pricedenti fimilis, pall. \& Linn. 1. c. ( 198.

368 C. Oficinalis, pracedenti aftanis, Pall. \& Linn. l. c. ( 190.

369 C. Articulata, ケ. Barlata? articulis cylindricis, Pall. Q?

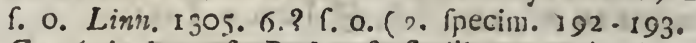

370 C. Articulata, r. Barbata? fimilis præcedenti, Pall. \& Linn. 1. c. (2. fpecim. 194. 200.

$37 \mathrm{r}$ C. Articulata? fo varietas, alba, Pall. \& Linn. l. c. 'I 88.

372 C. Articulate, pracedenti affinis, alba, Pall. \& Linn. 1. c. ( 189 .

373 C. Androface, tulsulofa, pel:a radiata, Pall. 13.? Linn. o. ( 196.

374 C. Androface, fimilis precedenti, Pall. 1. c. Linin. o. (195.

375 C. Sertularice varie, in lapidis fuperficiei, ( 325.

376 C. Pars Olla, f. Uma, Serpulis, f. Vermiculis, ac Tubulis adfcerdentibus obfita , permagna, ( 1 . fpecim, ..

377 C. I'ars Vafis Vitrei, præcedentibus obfita, (1. Ipecima (352.

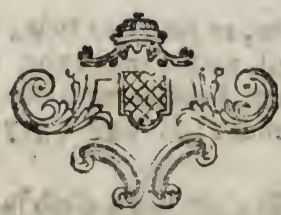


83 C. Commune, is deux plumes, articles ovales, les $\int_{L}$ rieurs comtrimées. Pall. 4. Loinn. 1304. 2. 1 19r. $35+$ C. Commune, Seinblable à la precedente, Pall. E Linn. la c.) ( 199.

305 C. Commune, pprocbant à la frecedente, Pall. Eg Linn. l. c. (20I.

366 C. Commune? ou varieté. Pall. \& Linn. l. 6. (197.

367 C. Cunmune, Semblabie à la precedlente, Pall. EO Linn. c. $(198$.

368 C. Commune, approchant d la precedente, Pall. E Linn.' l. c. $(190$.

369 C. Articulée, ou Barbue ? d articles cilindriques, Pall. 9. 04 0. Lirn. $1305: 6 \%$ ou 0. (2. piec. 192-193.

370 C. Articulée, ou liarbue, fernblable d̀ la precedente, Pall. E० Linn. l. c. r 2. piéc. 194. 200.

37 I C. Articulée? ou varieté, blanche, Pall. E Linn.l.c. 198. 372 C. Articulée, approchant ì laprecedente, blancbe, Pall. Es Linn. l.c. $(189$.

373 C. Parạfol de Mer, tubuleufe, d bouclier rayonné, Pall. 13 ? Linn. O. (196.

374 C. Paraful je Mier, Semblable da la precedente, Pall. Eจ Linn. l. c. ( 195.

375 Sertulaires diver fes, dans le furfface d'une pierre, $(325$.

376 Morceau d'une Vafe, convert de Serpules, Vermilfeaux, ET Tubules, très grande piéce. I. piec....

377 T. Morceau d'une Vafe de Verre, couvert coinme la pres sedente, (I. piec. 352.

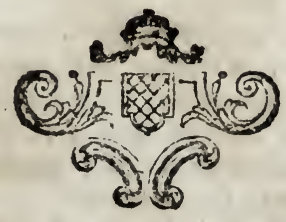




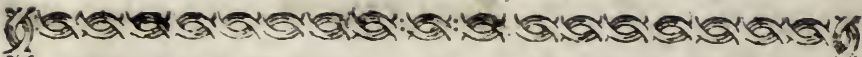

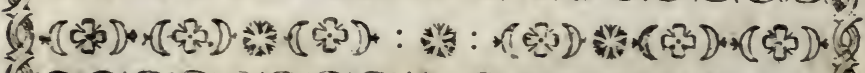
4.5.

\section{$V E G E T A B I L I A$.}

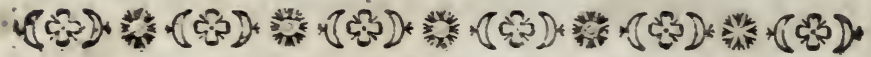

\section{PLANTESEMINA \\ F R V C T V S \&. .}

I Cartaphoram, cum varils plantis magnis, ac multis eorum, fructibus, ac femini')us adhesentihus. omnia in charta nagna impreriali, in qua corum nomina addita, ex Ceilnnia, ( 39. fpecim.

2 Cartophorum, chin fimilibis plantis variis, eodem modo aff. zis, ac cuin eartm nominiuus defcriptis, ex Ceiloria, ( 3 1. fpecim

s Colletzio irgers, Seminum ac Frugtuun, ex fere omnibus rezionibus, tam Europxis, qquan Indicis, ommes in lageris site confervatis, additis fere ombibus cormu nominibus, ( 3 IC. Specin.

4. Semina, ac Trudus, varii, ex Celloria, (50. Ppecim.

5 Semina, ac Frufus, varii ut fupra, ex Ceibunia, ( 32. fpe. cin.

- Fruatus, ac Semina, a pracedentibus divern, ex Capo Bon. Siei, (50. fpecim.

y Fatre limrarie, fenticofo, intus ferrinibus repleto, valdo aiatis, \& Tamarindis frucus, ex America, ('3 fpecim.

8 Nux Maldiva medicinalis, haud conmunis, ex India Oriens. Inrg. 13. lar. ro craf. $6 \frac{1}{2}$ poll. ( J. fpecira.

9 Cortex ligni fibriliofus, infar denticuli, cum tali fegmen. tato, ex America, (2. Specim.

30 Eignam ferpentiforme, fimile Anitpathes Spirali, pro quo olim habitum, vide vera hujus efligies apud Seb. III. I04. 3. Paliaj Zcopb. p. 259. ex Irdia Orientab. long. $23 \frac{1}{2}$. Lat. $3 \frac{1}{6}$ - (1. freçim. 


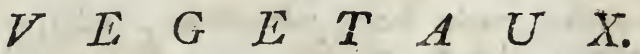

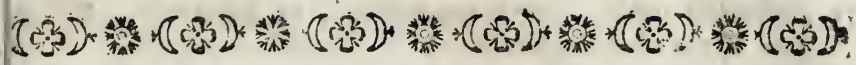

PLANTES SEMENCES F R U I T S \&.

I Portefenille, avec differentes grandes tlantes, plufieurs desz quels adlutrent d leairs fruits, E fênences; tous fur par, pier imperial, ou font joints leur noms, de Ceilon, ( 3 . piec.

2 Portcfeuille, avec femblables parailles plantes, attachts de In même maniere, "È avec leurs noms ecrites, de Ceilon, 34 piec.

3 Grande Collection de Semences, \& Fruits, de presque to:s les pays taint d'Europe, que des Indes, tous bien conferes of dans des petites bouteilles, arec presque tous leur noors? (3ic. piec.

Semences. E2 Fruits differentes, de Ceilon, (50. piec. Fruits, 80 Semences differentes, comme defus de Ceilon, (30 piec.

Semences, $\&$ Fruits differentes desprecedentes, du Cap d Homne Eifperarce, 50. piec.'

Feves epineufes, en dedars remplies de Jemences, fort ais lées, Eे fruis de Tamarinde, de l'Ametique, (3. piec. Noix Maldive, medicinal, pas commune, des Indes Oriers tales long. I3. lat. 10. craf. 6!. pouc. (1. piec.

Ecorce de bois fibrillenfe, rejeenblant la dentelle, arieo une pièce de celle, comme broché, de l'A merique, (2.tiec: Bois, en forme de Terpentin, pareil refJemblant id l'Autipa. thes Spirale, pour le quel ors" l'a pris autrefois, ta vrais effigie, voyez Seb. III. 104. 3. Pall. Zooph. p. 219. des Irde. Drientales, long. 21. $\frac{1}{2}$. lat. $3 \frac{1}{2}$ pouc. (s. piec. 


\section{8}

iz Planta, cum foliis, ac rubris ficcatisque fructibus, ex qut: bus varia, ac parva objecta artificiofe conficiuntur, ex Capo Bon Spei, (2. Ipecim.

12 Infantilia, varia \&c. minutifima, facta ex follis pracedentis plabta, ex Capo Bori. Spei, (plür. Specins.

I3 Infantilic varia, fimilia precedenti, ac variantia, ex Capo Bon. Spei, (plur. fpecim.

I4 Flores aftroides ficcoti, in aqua fe aperientes, ex Capo Bors. Spei, (3. fpecim.

I5 Pileus Venatoris, inftar retis, f. tegmentum cujusdam plan. tæ, ex America, (1. fpecim.

s6 Annuli Nigritini, ab iisdem mirifiot facti, ex cernibus, es America, (40 fpecim.

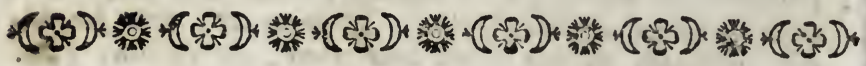

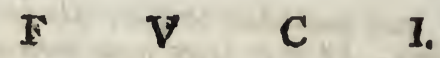

Y7. Fomus Plantæ Marine, figura haud communi, niger, duobus ramis echinatis longis, connatis, longe diltantibus, propter fingularitem a $\mathbf{P}$ allafio defcriptus, . - long. 30 . alt. 16. poll. (1. Specim.

I8 F. Fulia duo, adherentia longa, tenuia, long. 5o. polt. (1. ipecim.

19 F. Rapa, f. Fruétus plantæ marinæ, crufta tentuis, cxcavatus, niger, sapæformis, a parte craffiore sugasus, long. 18. poll. ( 1 . fpecim.

20 F. Folia, varia figura ac coloris, in Charta polita, (I2. fpecim.

2】 F. Folic, varietas precedentiun, ac pofita in Charta,

(12. fpecim.

22 F. Folia, fere inftar pracedentium, \& a pracedentibus variantia, in Charta pofita, ( 14 . fpecim.

9) F. Folia, varia, que ob ficcatitem, ac foliortm rigidita. tem definiri non potuerunt, ( plurima fpecim. $334-357$.

24 F. Cartilaginezs, arborescens, hagelliformis, variz magni cudinis, pulcri, tam rubri, $甘$ lavl, virides, quau vari colo rati, is Chaita pofita, (x2. Specisa. 
11 Plante, avec Jes fectilles, ED fruits rouges, feches des quele fe font fas urt differess petits objezs, du Cap de Bonne Efperance, (2. piec.

12 Panicr au langes, ous diforentes ha' illemens d'enfans. comme petits manchoos gro. tris patites, zous faites des feuilles de la predite, ou precellente plante, du Cap de Bonne Efperance, (plufielirs tieces.

13 P'anier au langes, Semblable aus precedent, \&o un peu differema te, du Cap de Bomne Leperance, (phifieurs pieces.

I4 Fleurs feches, en forme d'eisiles, quis souvrent dans locan, dib Cap de Bonne Épernice, (3. piec.

I5 Bonnet de Chafieur, comme uni filet, ou le tegment $d^{3}$ wne cem taine plante, de l'Anerique, ( I, piec.

so Amneauz de Negres, ingenieufemen: fair des cernausx, de l'Anerique, (4. jiec.

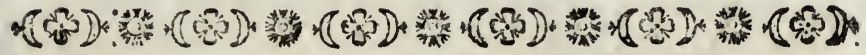

\section{IIIRBES MARINES.}

17 IT. Rameau, d'une Pinte de Mer, d"une figure pers comb munze, noir, avec deatx hranches efineides, pointues, Inngues, à cotes ciiftantes, Eo pour leur fingularité decrite par $\mathrm{Pal}$ las, .... long. 36. alt. 26. pouc. (1. piec.

I8 11 . deux Feuilles jointes enfenvile, longues, minces, longa' 30. pouc. (I piec.

Ig H. Navet, ou Fruit dine plante morine, d'une croute mince crenfé, voir, en forme de navet, du coté epais ridé, long. is. poucc. ( I. piec.

$20 \mathrm{H}$. Fenilles de differente figure, Es grandeur, mifes ere Enveloppi. (12. piec.

$21 H$. Feuilles ou varieté des precedentes, mifes dans un Enveloppe, (12. piec.

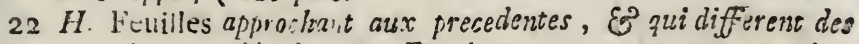
precedentes, mijes lins zune Enveloppe, ou couverturc, (It piec.

$23 \mathrm{H}$. ou Feuilies qu $u^{3}$ on n'a jat diftinguer à caufe de leur de. fefchement. (pirufieurs piects. $334-\cdots 35 \mathrm{r}$.

$24 \%$. Cartilagincule, arlorifée, eniforme d'eventoil, dz differintes grandeurs, trés beiles, tant jaunes, vertss, que कis differente coideurs, mijes dars une Enveloppe, (12. piec. 
25 F. Cartilagineus, varix figure, \& coloris, precedentilus affines, in Charta pofita, $(10$. fpecim.

- 6 F. Cartilagineus, pracedentimi varietates, \& a praceden. \& tibus vaiantes, in Charta polita, (20. fpecim.

$=7$ F. Cartilagineus, aboorecens, flugelliformis, varii colorait, pulcerrimi, permagni connpaçi in regulis q̣uadratis nigris, vitro tectis, \& pottice, fecundun objecta pictis, alt. 23 . lat. $1 \sigma_{2}$. poll. 2 . fpecim.

\$8 F. Cartilaginerus, arborescens, fimiliter varii, compscti, picti, ac pofiti, tit pracedentes, alt. 23. lat. $I \sigma_{2}$. poli. (2. fpecim.

\$. F. Cartilagineus, arborescens, précedentibuts fere fimiles, alt. 23. Lat. $I \sigma ! 2$ poll. $(2$. fpecim.

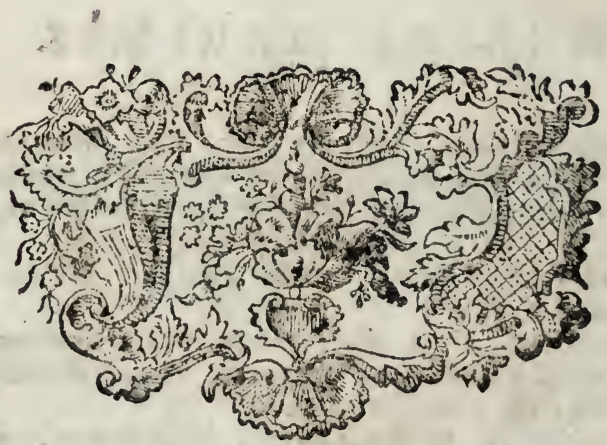


25 H. Cartilagincufe arlorifée, de differente figure, Eo couleur des precedentes, dans une Enveloppe, (10 piec.

$26 \mathrm{H}$. C.artilagineufe ariorifée, varieté des precedentes, dans une Enveloppe, (20. piec.

$27 H$. Cartilagineure arborife, en forme d'eventail, differem: ment colorées, bolles $89^{2}$ grandes, maprimée. en cadres noires, couvert de vitre, Es peintespar derviere jelon les objects, alt. 23. lat. $16 \frac{1}{2}$. pouc. (2. piec.

28 H. Cartilagineure arborifese en forme d'eventail, pareilles, differentes, comi,rimices, peintes, EO en cadres, comme les precedentes, alt. 23. lat. $1 \sigma_{2}^{1}$, pouc. (2. piec.

29 H. Cartilagineule arborifée, en forme d'eventail, do perb pres femblable aux precedentes, abs. 23 . bat. $1 \sigma_{2}$ pouc. (2. piec.

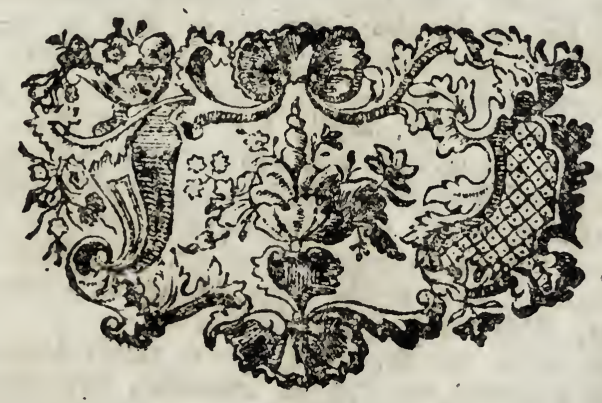




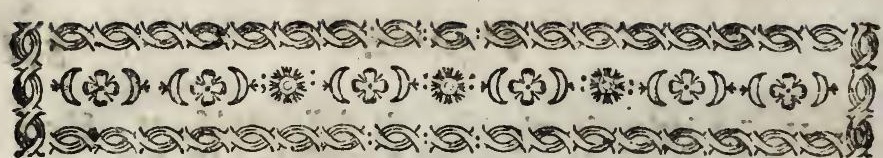

$\begin{array}{lllllllll}F & O & S & S & I & L & I & A .\end{array}$

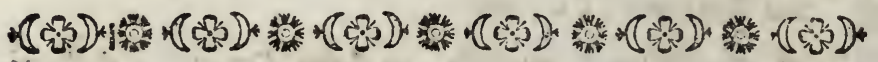
$\begin{array}{cccccccc}C & L & A & S & S & I & S & \text { I. } \\ & T & E & R & R & A E .\end{array}$

I Humus atra, rubra, Umbra, arenofa, grifea, fusca, Caspēs, Lac Lune, Eic. ex 66 Colonia, 102, ex. . i19. ex Norvegin, 85. 86. ex Lipfra, 99 sс6. ex Gertrudenberga, \& Breda, 1 10. ex Merfeburgo, 1087. ex Frifia, I20. ex Witten. berga, 8r. ex Gerinania, Wallerins Syftem. Mineral, ed. 1. Spec. I. 2. 3. 6. 9. II. (13. frecim.

2 Argilla, grifea, nigra, flava, fufca, rubra, plumbea, \&c. omnes figillatæ, ex Saxonia, Wall. 16.. 18. ?.0. 22. ( 53. fpecim.

3 Argilla, grifea, nigra, plumbea, fusca, flava, rubra, \&c. 67. \& 69..ex Berbices, 70: 71. ex Anterica, 73. 79. eะ Meliffe, 80, ex Germania, 89 ex Ofterfold, or. ex Amme berga, 92. ex Lipfia, 96 ex Halla in Saxon. 105. ex Hafja. 1821. ex Geervlieda, Wall, l.c. (It. fpecin.

'Bolus, flavus, grifeus, fuscus, niger, ruber, quidam figilla ti, 55. 58. ex Bayruthun, 54-57-59.62. ex Saxonia, 60.64 65. ex Colberga, 71. ex America, 72. ex Meliffe, 75 ex..95 101. 103.117. ex Norvegia, Wall. 23. (17. Ppecim.

5 Porcellana, \& Leucargilla, alba, 87. 88. ex Misnia, I12. c:s Colonia, Wall. 26.27. (3. fpeciun.

6 Margh, aiba, venofa, rubra, fusca, rubro dendritica, occ\$ 74. ex Lapponia, 76・-78. ex Armenia, 107. ex Saalfelda 113. 114. ex Chemnitio, 10S. II5. ex Norvegia, 150. e Saxonia, Wall. 30. 31. (10. fpecion.

7 Marga, r. Smectis, cinereus, ruber, \& Creta Venetia, 560 ex Saxonia, 1044. ex China, 1045. ex Norvegia, I16. e. Venetia, Wall. 29. (15. Tpecim.

\& Arena, flava, mixta, Micacea, \& Tripla, 94. ex Norvegic I21-I25. ex Bayrutho, 88. ex Anglia, 93. ex Clzemn tio, 100 ex Coburgo, Wall. $33-36$. (9. Specim.

(*) Numeri in guogue articulo, diffgnant fpecies cuigue miner (" otjesto additas, idtoque paulum ante allegationem Waller. ad def. gut nandá loca, xnde originens iraluunt, additi Sunt.

CLASSI 


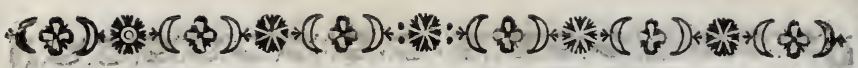

$$
\begin{aligned}
& \text { C L A S S I S II. } \\
& \therefore \quad L A P I D E S .
\end{aligned}
$$

9 Lapis Calcareuis, albús, flavus, grifeus, imbricatus, 93. ex Coburgo. 104. ex Saxonia, 126. ex Hemmeren, 136. ex Grefenthal, 138. ex Querfurth, 141. ex SondersharsJen, I48. 308. 3II. ex Lipfia, 30y.312.313. ex Suecia. 3 1o. ex Statua Apollinis, 1337. ex Bohemia, Wall.41-43. ( I4. Ipecim.

10 Marmar Spathofum, Give Ludus Helmontii, politum 1010. ex Coburgo, Wall. 45. ( I. Ipecirn.

II Marmor, viridi-fuscum, ex quo Lapides AEtites, politum. 1020. ex Coburge, Wall. l. c. (I. Tpecim.

12 Marmor, $C$, Theca tabacaria, cum operculo. ex marmo. re rubro grifeogue, operculum Jonam ex Pifce exeuntem, exprimit, 1596. ex Bayrexth, Wall, 46. (r.' 'pecim.

13 Marnor, f. Laminx Marmorex polita, 208. ex Saxonian. 299. 300. ex Blanckenburg, 302. ex Suecia, 306. ex Voigtlandia, Ir22. ex Norvegia, 1518. ex Zweybrucken, (Biponte,) Wall. 45 . (8. fpecim.

I4 Marmor, f. collectio Marmorum variorum, \& Lapidum Flo. rentinorum, quadratorum, polito um omnia, $1541-1591$. ex Baden-Durlach, Wall. 45. 46. (5 I. fpecim.

15 Marmor, f. Laminx Marmorum variæ polita, 266.267. 277. ex Blanckenburg , 268-270. 274. 275. ex Saxonia, Wall. 44. 45. (8. fpecim.

I6 Marmor, f. Lamine magnx Marmorum polita, varii coloris, 271. ex Voigtlandia, 272. 291-295 ex Bayreith, 273. 276. ex Saxonia, 295. 297. ex Blanckenburg; I119-I 121. ex Norvegia, Wall. l. c. (14. fpecim.

37 Marmor, f. Laminz politx, ftriatæ, variæque, $1294-1307$. ex Blanckenburg, 1774. ex Bayreuth, Wall. l. c. (I5, fpecin.

18 Marmor, f. collectio Marmorum, Alabaftrorum, Gypfse rum, Serpentinorum, Granito-Porphyrorum, Italicorum politorum \&c. 156-265. ex Italia, Wall.45-48. (I09.fpecim.

- $M$ Mrmor, f. collectio Marmorum politorum Italicorum, aliorumque, 279. 280 282. 314-316. ex Italia, 281. 284-287i 289. ex Saxonia, 283. ex Anglia, 288. ex Blanckenburg, Wall. l. o. (15. fpecin. 


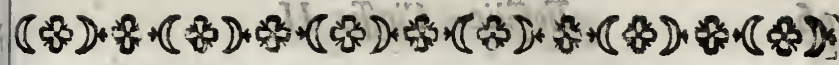

\section{L A S S E I I.}

\section{$P \quad I, E \quad R \quad R \quad E$ S.}

9. Pierre dे chaud, blanc, jaune, gris, tuile, 90 . de Cobourg, ro4. de Saxe, 126. de Hemmeren, 136. de Grafenthal, 138. de Querfurth, I 4 I. de Sondershaufen, 1 48. 3c8. 3 II. de Lipfienne, 309. 3 I2. 313. de Suede, 310. de Statue fruftule d'Apollon, 1337, de Boheme, Wall. 41-43. (13.piea:

- Marbre Spatherix, ou Jeu d'Helmont, polie, roro. de Cobourg, Wall. 45. (I. piec.

1 Marbre, verd-brun, polie', dont font les pierres d'aigle, 1020. de Cobourg, Wall. l. c. (I. piec.

2 Marbre, ou Boite à tabac, avec fon couvert de marbre rouge; E̊ gris, le couvert reprefente Fonas Sortant du Poijfon, 1596 de Bayreuth. Wall. 46. (I. piec.

3 Marbre, ou Plaques de Marbre polies, 298 . de Saxe 299. 300. de Blanckenburg, 302. de Suede, 306. de Voigtland. Ir22. de Norvegue, 1518. de Zweybrucken, (Deuse Ponts), Wall. 45. (8. piec.

4 Marbre. ou collection de differentes Plaques de Marbre, E9 pierres de Florence, quarrées, polies de I54I-I59I. tous de Baden-Dourlach, Wall. 45. 46. (5I, piec.

5 Marbre, ou differente Plaques de Marbre polie, 266. 267 277 . de Blanckenbourg. 268-270.274.275. de Saxe, Wallo 44.45. (8. piec.

6. Marbre, ou grandes Plaques de Marbre polie, de differente couleur, 271. de Voigtland, 272. 29I 295 . de Bayreath, 273. 276, de Saxe, 296. 297. de Blanckenbourg, III9II II, de Norvegue, Wall. l. c. (14. piec.

; Marbre, ou differentes Plaques de Marbre ftrié, $1294-130 \%$ de Blanckenbourg, 1774, de Bayreuth, Wall. l. c. (15. piee.

8 Marbre, ou collection de Marbres, a' Albatres, de Gipfe, Serpentines, Granates, E Porphyres, Ecc. d'Italie, polies $156-265$. d'Italie, Wall. $45-48$. Eं $c$. (109. pies.

9 Marbre, ou collettion de Plaques de marbres polies, a"Italie, E9 d'autres, 279. 280. 282. 314-316 d'Italie, 281. $284-287.289$, de Saxe, 283. d'Angleterre, 288. dt Blanckenbourg, Wall. l.c. (I 5. piec. 
20 Marmor, variegatum magnum, ex Italia, cum fimili ntgro, 278. 307. cx Italia, 304. ex Germania, Wall. l.c. (3. fpecim.

2 I Alabaftrum, f. collectio Laminum Alabaftri \& Gypfi , variorum colorum, politorum, 1021 . ex Hellingen, 1050-1083. ex Hohenftein, Wall. 47. 48. 53? (35. 1pecim.

22 Alabaltrum, rude nitens, Gyp/um ftriatum, filamentofum, \& Alumen plumofuni, I37. 149. ex Naumburg, 46 . ex Schwaben, 142. ex fena, 147. ex Saxonia, Wall. 52.53. (5. " Cpecim.

23 Spathum cryftallinum, lamellofum, Selenites, \& Glacies Maria, 573.ex Norvegia, 772 ex Suecia, $732 . ., 567$. ex Gallia, I654. ex Cap. Bon. Spei. Wall.49-51. ( 5. (pecim.

24 Spathum duplicans, valde diaphanum, magnum fpecimen 1027. ex Islandia, Wall. 60. (1. Specim.

25 Spathum cubicum, amethyftinum, magnum, I770. ex Saxonia, Wall. 56. (1. Ipecim.

25 Spaihum cubicum, colore hyacinthi, drufi forme, Iorg: 1787. ex Hartz, (Hercynia) Wall. l. c. (2. fpecim.

26 Spathum lamellatum, diaphanum, opacum, album, I095. ....., I I 58.ex Saxania, I785. ex Clausthal, Wall. 57.59. (3. ipecim.

27. Spathum cryftallifatum ; 694. ex Hifpania, I77I. ex Saxonia, Wall. 6r. (2. fpecim.

28 Spathum hexædrum, polyedrum, planum, cryftallifatum; If56. ex Saxonia, 1339, ex Hispania, Wall. 61. (2. [pecim.

29 Spathum Hyacinthinum, Amethyftinum, Smaragdinum, \& Lapis Violacers, 571. ex Saxonia, 719. 720. ex Hartz, Wall. 62.63. (4. Ipecim.

30 Spathum cryftallifatum, ramofum, album, ramis craffiori. : bus. magnum, 1510. ex Norvegia, Wall.6r. (1. fpecim: 3I Spathum cryltallifatum, albicans, minoribus ramis, 1772. ex Monte St. Andrea, Hartz, Wall. b. c. (I. Ifecim.

32 Fifllis, r. Schiftus, virefcens, rudis, niger, aluminolus ; \& Creta folida, mollisque nigra, I633. ex Spa, I 39. ex Sonnenberg . I40. ex Bayreuth, I 43. ex Osnabruck, I293. ex Brabantia, Wall. 68-71. (5. Specin.

33 Saxum, f. Lapis Arenareus, micaceus, albus, globofus, flavo friatus, talcolus, rubefcens, cinerefcens, flavus, friabilis, marga lapidea mixtus, ochraceus, imbricatus \&c. 82. I 32. I5 1 . ex Lipfia, I27. I 33.1 35. ex Coburgo, I 28. I30. 133. 760. ex Saxonia, I3I. ex Querfurth, I34. ex Hartz, ivall. $77-79$. (13. fpecim. 


\section{Fofjiles. Claffe II.}

9o Marbre Panaché, tres grand d' Italie, avec une femblable plaque noire 278307 d'Italie, 304 d'Allemagne, Wall I.c. (3.piec. 2x Albatre, ou collection de Plaques d' Albatre, $\mathcal{E}$ de Gypfe, de diverfes couleurs, polies 1021. de Hellingen, 1050-1083. Hohenftein, Wall. 47. 48. 53 ? (35. piec.

22 Albatre, non poli, luijant, Gypre Jtrie, fibreux. E Alun à plumes, 137.149 . de Naumburg, 146 de Schwaben, 142. de Jene, 147. de Saze, Wall 52. 53. (5. piec.

23 Spath criftallijé, feuilleté, Selenite, E Glace de Marie; 573. de Norvegue, 772 . de Suede, $732 . . .567$ de la France, 1654. du Cap de Bonne Efperance, Wall. 49-51. (5. piec

24 Spath doublant les objets, fort diaphane, grand, 1027.d'Islande, Wall. 60. ( $x$. plec.

25 Spath cubiquee amethyfte, grand 1770. de Saxe, Wall. 56. (I. piec.

25 Spath cubique, couleur d'hyacinte, en forme: de drufes; ro19. 1787. de Hartz, Wall. 1. c. (2. piec.

26 Spath feuillieté, tranjparent, opaque, blanc, 1095.... . II58. de Saxe, I795. de Clausthal, Wall. 57.56. (30) piec..

27 Spath, cryftallifé, I694. d'ERpagne, 177 I. de S2re, Wall. 6I. (2. piec

28 Spath, is $\sqrt{2} x$, E plufieurs pans, fini Jant en pointe, arfallifé, Ir56. de Saxe, 1339. d'ESpagne, Wall. 6r. ( 2 piec.

29 Spath, Hyacinthe, Amethyfte, Emeraude, \& Pierre Violette, 57 I. de Saxe, 719.720. de Hartz, Wall. 62. 63. (4. piec.

30 Spath, cryftallife, oudrufe fpatkeufe, grande, d grofjes brans: ches, 1510 . de Norvegue, Wall. 1. c. (1. piec.

3x Spath, cryftallifé blancheatre, en branches plus minces; I772. du Mont St. André, de Hartz, Wall. 1. co (
piec.

32 Ardoife groffiere, ou Schifte, verdatre, rude, noire, alu mineux, ED Craye noire, en bloc, EO en poudre, 1633. de Spa, 139. de Sonnenberg, 140.de Bayreuth, 143. d'Os. nabrug, I293. de Braband, Wall, 68. $\rightarrow 7$ I. (5. piec.

33 Grais Grofier, ou Pierre Arenaire, melée de mica, blanc, en boule, ftrié de jaune, rougeatre, gris, friable, melé de marge, de lochre, feuilleté, 82. 132. 151. de Lipfienne, 127: 133. I35. de Cobourg , 1281 I 30. 1 33. 760 . de Saxe, I $3 K_{2}$ de Querfurth, 134. de Hartz, Wall. 77. 79. (13. piec. 
34 Silex venofus, politus, $677-680$. ex Germania, Wall. 80. (4. fpecim.

35 Silex fasciatus, albus, diaphanus, 673. ex Brafilia, 674. Rofenthal, 675. 676. 68I. ex Zweybrucken, Wall.8I. (8. Ipecim.

36 Silex Igniarius, niger, Bafaltes niger, Lava nigra, fcintillans, ad chalyb: 687. ex Anglia, 1016. ex Islandia, 1343....,Wall. 82. (3. Tpecim.

37 Silex Igniarius, grifeus, \& alii, 685.692. ex India Orient. 695. ex Germania, Wall l. c. (14. Specim.

38 Achates Cacholonius, uviformis, Cornalinus, Sardonix, achates olivaceus, Globulus achatinus intus cavus, 633. ex India Orient. 684. 688. ex Germania, I752. ex Frey. berg, 1383. ex Zweybrocken, Wall. 83. 84. (5. fpecim,

39 Achates quartzolus amethyftinus, politus ..., ex Zwey brucken, Wall. 89. (i. Specim.

40 Achates rudis, una parte politus, lacteus ftriis quartzo(1) rubris, 1344. ex Zweybrucken, Wall. 86. (2. ipecim.

41 Achates fimilis, ftriis tenuioribus, 1345, ex Zweybrucken; 16r8. 16rg. ex Veldenz, Wall. l. c. (3. Specim.

42. Achates : ex grifeo, rubro alboque varius, politus, $65 \mathrm{r}$ : 652. ex Saxonia, 1346. 1347. 1350. 1355. ex Zweybrucken; Wall. l. c. (6. fpecim.

43 Achates, zonis latioribus, granulis rubris, aliæque quartzofx, 633. 658. ex Saxonia; 1351. I352, ex Zweybrucken, Wall. b. c. (4. Ipecim.

44 Achates Cornalinus, olivaceus, altera parte politus, quartzofus, 660. ex Saxonia, 690.1348.1353, 1354. 1617. ex Zweybrucken, Wall. l. c. (7. Specim.

45 Achates, zonis latis, nebulofus, amethyltinus, Cornalinus, rudis \& politus, 654-657. ex Cunnersdorf, Wall. l. c. (4. fpecim.

46 Achates, flavus, carneus, olivaceus, \&cc. politus \& rudis. 1500. ex Veldenz, Wall. 89. (I. fpecim.

47 Achates fimilis \& achates quartzofus, rubro venofus, politus, I 499. ex Zweybrucken, x6II. ex Veldenz, Wall. l.c. (2. fpecim.

48 Achates, ex albo rubroque annulatus, politus, I349. ex Zweybrucken, 16i2-I6i6. ex Veldenz, Wall. $l$ ci ( 6 . ipecim.

49 Achates, f. Chelidoners, in lapide calcario, Oculi Cati inItar, nitens, 315. ex Gormania, Wall. 90. (1. fpecim. 


\section{Folfiles. Claffe It:}

34 Caillou opaque, vené, polie, 677-680. d'Allemagne, Wall. 80. (4. piec.

35 Caillou, bandé, tlanc, tranfparent . 673. de Brafil 674. de Rofenthal, 673 676. 681. de Zweybrucken (Diux Ports) Wall. 81. (8. piec.

36 Caillou, ou Pierre a feu, noir, Bazalte noir, Lava noire: Eo etincelante, avec l'acier, 687. d'Angleterre, ror6 d'lslande, 1384. . Wall. 82, (3. piec.

37 Caillou, ou Pıerde à feu, gris, Ej autres, 685. 692. dess Indes Orientales, 695. d'Allemagne, Wall. 1. c. (4.) piec.

3s Achate Cacholon, on grappes, Cornaline, Sardoine, agan te verd d'olive, bouie encavé d'agathe, 633. des Indes Orien. tale. 684. 688. d'Allemagre, 1752 . de Freyberg, 1383. de $Z$ weybrucken, Wall 83. 84. (5. piec.

39 Achate, ou un grand morceau d'agathe, quartzeufe, amethyfte, polie, ... de Z weybrucken, Wall. 89. (r. piec.

40 Agathe, rude, couleur de lait, do lignes rouges quartzenjes. polie d'un coté, 1344. de Zweybrucken, Wall. 85. (2. piec.

4 I Agathe, pareille, à fries plus minces, 1345 . de Zwegbrucken, 1618. 1619. de Veldenz, Wall. 1. c. (3. piec.

42 Agathe, melé de gris rouge, É blanc polie, 651. 652. de Saxe, 1346.1347. 1350. 1355. de Zweybrucken; Wallo l. c. ( 6. piec.

43 Agathe, is zones larges, a grains rouge, E̊ autres quartzexres, 633. 658. de Saxe, 1351. 1332. de Zweybrucken. Wall 1. c. (4. piec.

44 Agathe Cornalin, quartzeufe, couleur d'olive, polie d'un cote. 660. de Saxe, 690. 1348. 1353. 1354. 1617. de Zwegbrucken (Deux Ponts) Wall. I. c. (7. piec.

45 Agathe, $\dot{a}$ zones larges, nebuleux, couleur d'amethyfte, Cornaline, polie, Ev rude, 654-657. de Cunnersdort, Wall. I. c. (4. piec.

46 Agathe, jaune, couleur de chair, couletir d'olive, 890 . lie, E' rude, 1500. de Veldenz Wail. 89 (I. piec.

47 Agathe. Semblable, E' agate guartzeux, vené de rouge, polie, 1499 de Zweybrucken, 1611 . ie Veldenz, Wall. I c. (2. piec.

48 Agathe, annulé de blane, E de rouge, polie, 1349 de $Z$ weybrucken, 1612-16r6. de Veldenz, Wall. I. c. (6.) piec.

49 Agathe, ou Pierre de Safienage, ou Hirondelle agatiée, dans une pierre calcaire, reinijant cumme un Oeil ae Chat, 715. d'Allemagne, Wall. gn: (1. fiec. 
50 Achates, olivaceus, fuscus, flavo venofus, rubro ma culatus, 635. 647. ex India Orient. 636.050. ex Italia, 645 ex Zweybrucken, 1161, ex Dresda, Wall. 89. (7. fpe cim.

5x Achates, rubro nebulofus, flavus, cinnamomeus, albus nigro punctatus, mufcofus politus, $612.6: 5$. ex India Orient. 037. 639. 645. ex Zweybrucken, Wall. l. c. ( $\sigma$. fpecim.

52 Achates, ruber. olivaceus, fuscus, maculatus, politus,

- 623. 627. ex India Orient. 638. 640. ex Zweybrucken, Wall. l. c. (4. Rpecim.

52 Achates, valde tranfparens, albo ftriatus, rubro varie maculatus, cyanefcens, politus, in laminis, 619. ex India Orient. 1513. 1620. ex Zweybrucken, Wall, l. c. (4. fpecim.

53 Achates, f. Laminæ achatinæ, flavæ, fuscæ, rubræ, \&c. polita, 641-643. ex Zweybrucken, Wall. l. c. (3. fpecim.

54. Achates graniticus , fusco flavefcens, punetis nigris, in lamina oblonga, 1375. ex Zweybrucken, Wall. b. c. ( $\boldsymbol{x}^{\circ}$ ipecim.

55 Achates, i Laminæ amethyftin achaticæ, politæ, or $\delta$. ex India Orient. 646. ex Zweybrucken, Wall. l. c. (2. ipecim.

56 Achates Cacholonius. diaphanus, politus in laminis quadratis, vel roturdis, 574. 575. 586. ex India Orient. Wall. 83. (3. fpecim.

57 Achates, I Thecula tabacaria, ex Cacholonio achate, cum zona Onichis, inclufa zinco cuprifato, (Pinsbec)... ex Zweybrucken, Wall. l. c. (1. Ppecim.

58 Achates, r. Thecula fimilis, ex Cacholonio achate quartzofo, .., Wall. l. c. (1. fpecim.

59 Achates, $\mathrm{r}$ poculum $\mathrm{T}$ tieæ, cum disco fuo, ex agathe diaphano, fusco maculato, 1512. ex India Orient, Wall.

- 89 (2. Specim

60 Achates. . Scyphus \& Difculus, ex agathe oiaphanoar-

- borifante, 1705 . ex India Orient. Wall l. c. (2. fpecim.

6I Achates, f. Thecula tabacaria ovalis cum operculo, ex agathe quartzolo albo, fuscoque friato, 1609. ex Saxo: ria. Wall. $l$ c. (2. If pecim.

62 Ackates, r. Thecula fimilis, ex achate flavicante, \& vafculum ex achate rubello, 629. 1610. ex India Orient. Wall. l. c. (3. Tpecim.

63 Achates f. Thecula finilis octogona, ex Cacholonio diaphano, 6I6. ex India Orient. Wall.' 83. (2. \{pecim.

64 Acliztes 


\section{Fafjiles. Clafle Il.}

50. Agathe, coulear d'olive, brune, do veines jarnes, à taches rouges, 635. 647. des Indes Orientales, 636. 650. d'Italie, 645. de Zweyorucken, II6I. de Dresde, Wall. 89. (7. piec.

5x Agathe d nuées rouges, jaure, coulcur de canelle . blanc, ¿ points noirs, mouffé, polie, 612.625. des Indes Orientales, 637. 639. 645. de Zweybrucken, Watl. I. c. (6. piec.

52 Agathe, rouge, couleur d'olive, bruse, tacbeté, polie, 623. 627. des Indes Oriencales, 638.040. de Zweybrucken, Wall. I. c. (4. piec.

52 Agathe, en plaques, fort tranjparent, ftrie de blanc, tachetê de rouge, bleuatre, Ẽ polie, o19. des Indes Orientales, 1513. I620. de Zweybrucken, Wall. I. c. (4. piec.

53 Agathe, ou Plaques d'agathe, jounes, brunes, rouses, polies, 64I-643. de Z weybrucken, Wall. I. c. (3. piec.

54 Agathe granatique, jaune brunatre, à points noirs, exs plaque allongée, I375. de Zweybrucken, Wall. I. c. (I. piec.

55 Agathe, ou Plaques d'agathe, omethyftines, polies, 618. des Indes Orientales, 646. de Zweybrucken, Wall. I.. (2. piec.

56 Agathe Cacholong, tran/parant, polie, en plaques quarrées. ou rondes, 574. 575. 586. des Indes Orientales, Wall.83. (3. piec.

57 Agathe, ou Tabatiere d'agathe de Cacholong, à banded'Onyx. montée en Pinsbec, ... de Zweybrucken, Wall.1. c. (I. piec.

58 Agathe, ou pareille Tabatiere d'agathe Cacholong quartzert: fe, .., Wall.1. c. (1, piec.

59 Agathe, ou une talfe $a$ The, avec Ja foucoupe, d'agathe. transparente, à tacbes brunes, 15 I $^{2}$. des Indes Orientales, Wall. 89. (2. piec.

60 Agathe, ou un petit Gobelet, E' une Soucnupe, d'agathe tran." Jparent, arborifant, I 765. des Indes Oricntales. Wall 1.c. (2. piec.

6I Agathe, ou ane Tabatiere ovale, avec fon convert d'agathe quartzeux, ftrié de blanc, Ë de brun, 1609. de Saxe. Wall. 1. c. (2. piec.

62 Agathe, cu pareille Tabatiere d"agathe jounntre, 80 un petit baquet d'ngatbe rougeatre, 629. 1610. des indes Orientales, Wall. 1. c. (2. piec.

o3 Agathe, ou femblable Tabatiere à huit pans, de Cacholong diaphane, ou tres pur, 6r6. des indes Orientales, Wall. 83. (2. piec. 
64 Acbates, f. Cultrorum mabubria ex achate arborifanto $\&$ allo albo lineato, 648. 1248 . 1242 . ex India Orient. WWall. 89. (4. Specim

65 Achates, f. Conorum lufus cum globulis fuis, ex achate ftriato, 649. ex Zweybrucken, Wall. l. c. (II. fpecim.

66 Achates, f. Monile globulis albis Cornalinis, 639. ex Zweybrucken, Wall 84. (46. fpecim:

67 Achates, f. Lami $æ$ achatum varii coloris rubri, flavi, lutei, virides, fusci, \&c. 1337-1374, ex Zweybrucken, Wall. 89. (18. fpecim.

68 - Achates, C. Lamina permagna, cblonga, polita, achates Cacholonio, lactei in medio ftriata, 1486 . ex India Orient. Wall. 89. long. 8. lat. $4 \frac{1}{4}$. poll. (1. Specim.

69 Achates, f. Thecula forpicata, ex achate arborifante fusco, argento inftricta, $\ldots$ ex India Orient. Wall. 89. long $4 \frac{1}{4}$ lat. $2 \frac{1}{2}$. altit. $3 \frac{1}{2}$. poll. (I. Specim.

7o Achates, r. Theca tabacaria fumatoria, zinco cuprifato, (Pinsbec) ornata, ex achate Cacholonio, ex Zweybrucken, Wall. 83. long. $5 \frac{3}{4}$. lat. $3 \frac{1}{2}$. altit. $2 \frac{1}{2}$. poll. ( I. Ipecim.

71 Achates, f. Lamina ovales achatinæ, fusco maculatæ, poliı 577. 579. ex India Orient. Wall. 89. (2. fpecim. 72 Achates, f. Laminæ fimiles, 611. 614. ex India Orient. Wall l. c. (2. fpecim.

73 Achates, f. Laminæ fimiles, ovales, multo minores, 590 , 592-594. 596. 599. 003 . ex India Orient. Wall. l. c, (7. Tpecim.

74 Achates, f. Laminæ octngena \& oblongæ achatinæ, rubro venofx, 580. 620. ex India Orient. Wall. l. $c^{\circ}$ ( 2. fpecim.

75 Achates, f. Lamire fimiles, mirores, ovales, (000. 628.632. 1524: ex India Orient Wall. l. c. (4. rpecim.

76 Achates, f. Laminæ fimiles, trigonæ, ovalesque majores, 578. 583. 584 ex India Orient. Wall. l c. (3. Ipecim.

77 Achates, f. Laminæ lactex, vel fuscæ, ovales, valde diaphanx, 589. 598. 601. 624. 626. 1525. ex India Orient. Wall. l. c. ( 6 . ipecim.

78 Achates, f. Lamina fimiles, ovales, nitidifimx , in una harum arbor, arte pietus, 576.595. 615. ex India Orient. Wall. l. c. (3. Specim.

79 Achates, f. Lamin $x$ varix, parvæ, nitidiffimx, achatina albx, rubro ftriatx, laetex, fusco punctatx, flavx \&c. I519-1521. 1256. 1603-1606. ex India Orient. \& Ger. mamia, Wall. l. e. (21. Ipecim. 


\section{Foliles. Claffe II.}

64. Agathe, ou manches de couteau d'agathe arborife, ED autrea a fries blanches, 648. 1248. 1249. des Indes Orientales, Wall. 89. (4. piec.

65 Agatie, ou jeu de quilles avec fes boules, d'agathe ftrie, 649. de Zweybrucken, Wall. I. c (I1. pier.

66 Agathe, onc un Collier a grains blancs, en forme de Perles, de Comaline 639. de Zweybrucken, Wall. 84. (46.p. ou grains. on giobules.

67 Agathe, ou Plaques d'agathe de differentes couleurs, comme rorge, jaune, couleur de lait, verd, brun; $1337-1374$. de Zweybrucken, Wall. 89. (18. piec.

68 Agathe, ou tres grande Ploque d'agathe Cacholong, allongé, polie, blanc de lait au miliers, 1.986 des Indes Orientales, Wall. 89. long. 8. lat $4 \frac{1}{4}$ pouc. (I piec

69 Agathe, ou urie Coffrette, d'agathe arborifée, brune monteze en argent,.... des Indes Orientales, Wall. 89 long. $4 \frac{1}{2}$. lat. $2 \frac{3}{2}$, haut $3 \frac{3}{2}$, pouc. (I. piec.

70 Agathe, ou Boête à tabac d fumer, d'agathe Cacholong, montée en Pinslec, ... de $\mathrm{Z}$ weybrucken, Wall. 83. long. $5 \frac{3}{4}$. lat. $3 \frac{1}{2}$. bautu $2 \frac{1}{2}$. pouc. (I. piec.

II Agathe, ou Plaques ovales polies, d'agathe à taches brunes; 577. 579. des Indes Orieniales, Wall. 89. (2 piec.

72. Agathe, ou pareilles Plaques, 6ri. 614. des lrdes Orientales, Wall. 1. c. ( 2. piec.

73 Agathe, ou pareilles $P$ laques ovales, plus petites, 590. 592-594. 596.599. 603. des Indes Oriertales, Wail. 1. c. ( 7 piec:

74 Agathe, ou Plaques ovales à huit cotés, d'agathe veinée de rouge, 580. 620. des Indes Orientales, Wal.1 c. (2.piec. 75 Agathe, ou pareilles Plaques, plus petites, ovales, 600. 628. 632. 1524 des Indes Orientales, Wall 1. c (4. piec

76 Agat he, ou pareilles Plaques d'agathe, triangulaires, oxales, EO plus grandes, 578. 583. 584. des Indes Orientales, Wall: 1. c. ( 3 piec.

77 Agathe, fou Plaques d'agathe, blanches 89 brunes, ovales, fort tranjparentes 589.598 .601 .624 .626 . 1525. des In: des Orientales, Wa!l. 1 . c. ( 6 piec.

73 Agathe ou pareilles Plaques, ovales, Etres belles dans une des quelle un arborifears, fait por art, 576.595. 615. des Indes Orientales, Wall. I. c. (3. piec.

79 Agathe, ou diverfes petites plaques d'agsthe, tres belles; blanches, couleur de lait . Stries de rouges. jarnes, pointés de brun Esc. 1519-1521. 1256.1603-1606. des Indes Orienuales, go d'Allemagne, Wall. 1. c. (2 I. piec. 
80 Achates, f. Globus politus, ex agathe Sardonio, I292. ex Germania, Wall. 86. 89. diam. $2 \frac{3}{4}$. poll. (I. fpecim-

8r Achates, f. Laminæ nitidiffimæ, ovales, octogonæ, ex achate nigro arborifante, 597. 606.613.63i. ex India Orient. Wall. 89. (4. Tpecim.

82 Achates, f. Lamina fimiles, ovales, arborifantes, una cum w lamina achato Sardonia, 588. 605. 610.020. Ex India Orien. Wall. 86. 89. (4. Specim.

83 Achatès. f. Laminæ octogonæ, nitidifimæ, achatinæ, 1603 .

5 ex India Orient, Wall, \&y. (2. (pecim.

84 Achates, f. Laminx oblengæ octogonæ, minores, 584 . ex

- India Orient. Wall. l.c. (2. Cpecim.

85 Ackates, I. Laminæ nitidifima . fusco arborilantes, ovales achatinæx, è... India Oriênt. Wall. l. c. (2. Specim.

86 Athates, f. Laminæ Calcedonicæ \& Cacholoniæ ovales; - 58 r. 582. ex Inaia Orient. Wall. l. c. (2. fpecim.

87 Achates, f. Lamina octoedra fusca, in media Onjxece, 15 26. 1692. ex India Orient. Wall. 86. (2. fpecim.

38 Achates? $\uparrow$. Laminæ ovales ,diaphanæ, rubro maculatæ, cum alia rubella transparente, ex Oryza facta, 621. 1523. ex India Orient. Wall. 89. (2. Ipecim.

89. Achates pellucidus vitri inftar, arborilans, fylvaticam re. gionem referens, nitidiffimum fpecimen. ac inter omnes arboifantes, princeps, 1690. ex India Orient. Wall.l.c. греcim.

go Achates, haud communis, fat magna rotunda, lactea, rubro arborifans, 1597. ex India Orient. Wall. l. c. (r. (x. fpecin

or Achates, fimilis nracedenti : convexa, rubro arborifante , 1598. ex India Orient. Wall. l. c. (I. Tpecim.

92 Achates, præcedenti affinis, ovalis, fusco albefcens; 1250. ex India Orier.t. Wall. l.. c. (1: fpecim.

93 Achates, oblongæ, fufco arborifantes, altéra diaphana, altera lactea, 1251. 1503. ex India Orient. Wall. l.c. (2. fpecim.

94 Achates, fimilis ovalis, in annulo aureo, 602. ex India Orient Wall. l. c. (x. Ipecim.

95 Aclates, pracedenti affinis, fed major, 602. ex India Orient. Wall.l.c. (I. fpecim.

96 Achates granfparens, fusco arborifans, ovalis, 1342. ex India Orient. Wall. l c. (1. fpecim.

97 Achates, fimilis arborifans fortior, rotunda, 1504. ex India Orient. Wall $l$. c. (I. Specim.

98 Achates, affinis, in medio fusco arborifans, 1502. ex India Orient Wall. $i, c$. (I. f fecim. 
80 Agathe, eu une Globe polie d'agathe Sardoine, 1292. d'Allemagne, Wall. 86. 89. diam. $2 \frac{3}{4}$. pouc.. (I. piec.

\$I Idgathe, ou de tres jolies Plaques ovales, à huit cotes, d"aga. the noire, arborifée, 597.606. 613.631. des Indes Orien. tales, Wall. 89. (4. piec.

82 Agache, ou pareilles Plaques d'agathe arborifée, avec uns autre plaque dagathe Sardoine. 588. 605. 610.620. des Indes Orientales, Wall. 86.89... (4. piec.

83 Agathe, cil Plaques octogones, d'agathe tres luijante, 1608. des Indes Orientales, Wall. 89. ( 2 "piec.

84 Agathe, ou Plaques d'agathe oftogones, plus petites, $584^{\circ}$ des Indes Orientales, Wall. I. c. (2. piec.

85 Agathe, ou Plaques d'agathe, tres blanches, ovales, arbo vifées de brun ... des Indes Orientales, Wall. I.c. (2. piec. 86 Agathe, ou Plaques d'agathe ovales de Calcedon, Eे CachoJong, 581. 58 2. des Indes Orientales, Wall. 1. c. (2. piec:

87 Agathe, ou Plaques diagathe oetogones brunes, Onyx эnilieu, I526. 1692. des Indes Orientales, Wall. 36. (2. piec. 88 Agathe? ou Plaques d'agathe oviales, diaphanes, tacheté de rouge, avec une autre rougeatre transparente faite de $\mathrm{Riz}_{\text {}}$ 621. 1523. des Indes Orientales, Wall. 89. (2. piec.

89 Agathe, extraordinaire, tranjparente comme de verre, arboris fée, reprefentant unpayjage, la meilleture des pieces arborifées, 1690. des Indes Orientales, Wall. 1. c. (x. piec.

90 Agathe, tres rare, affez grande, fonde, couleur de lait, arborifé de rouge, 1597. des Indes Orientales, Wall. 1. $c_{\text {. }}$ ( I. piec.

9r Agathe, femblable a la precedente, convexe, arborife de rouge, 1598. des Indes Orientaies, Wall 1. c. (1. piec. 92 Agathe, opprochante da la precedente, ovale arborifée de brun, 1250. des Indes Orieriales. Wall. I. c. (1. piec.

93 Agathe, allongées, arborifées de brun l'une diopiane, l'autre couleur de lait, 1251. 1503. des Indes Orientales.t. Wall. 1. c ( 2 piec.

94 Agathe, pareille, ovale, dans un anneau d'or, 602. desi Indes Orientales, Walt, I. c (r. piec.

95 Agathe, approchant a la precederte, mais plus giande, 602. des Indes O:ientales. Wiall, 1. c. (r. piec.

.96 Agathe, tranjparant arborifée de brun, ovale, 1342 , des Indes Orientaies, Wali. I. c . (1. piec.

97 Agathe, pareille, ronde, phus fort arborifée, 1504. des: Indes Orier:tales, Wall. I. c. T) 1.ppiec.

98 Agaihe, pareille, ronde, nuborifée de brun dans le milieu, I502. des Indes Orientales, Wall. L. ci (1. piec. 
99. Achates, fimilis pracedenti, fed ovalis; I34I. ex India Orient. Wall. l. c. . (1. Ipecim.

100 Achates arboilfans, præcedenti affinis, 1600. ex Indis Orient. Wall. l. c. ( I. fpecim.

IoI Achates, rotunda convexa, rufo fusco Gorgonix Flabelli inftar picta, 1599. ex India Orient. Wall. l. c. ( 1 . fpecim.

I02 Achates, fimilis, alba, fusco arborifata, 1340. ex India Orient. Wall. ll c. (1. Specim.

103 Ailates. f. Lamina plana, ovalis, arborifans, 630. ex India Orient Wall l. c. (1. Ppecim.

104 Achates, rotunda, aliquot minores, flavæ, albæ, vel fus* cx arborifantes, II23. II24.: I1 26-II28. ex India Orient. Wall $l$ c. (5. fpecim.

I05 Achates, fimiles convexa, ovales, fed minores, 1505. 1506. ex India Orient. Wall. l. c. (2. Specim.

Io6 Achates, fimiles rotunda, ovales arborifantes, 1029. ex India Orient. Wall. l. c. (8. Specim.

o7 Achates, pracedentibus affines, fed minores, 1254. ex India. Orient. Wall. l. c. - (6. fpecim.

208 Achates, præcedentibus affines pallidiores, 1252. ex India Orient. Wall. l.c. (4. Ipecim.

109 Achates, fimiles pracedentibus, ovales \& rotunda, 1253. ex Germania, Wall. l. c. (8. Tpecim.

IIO Achates, arborifantes, rubra parva, II25. I601.ex Indir Orient. Wall. l. c. (2. fpecin.

III Achates, fimiles precedentibus, rotundæ, 1507. ex Indie Orient. Wall. b. c. (3. fpecim.

112 Achates, f. Carneolus, magnus, rotundus, convexus, lac. teus arborifans; 1130, ex India Orient. Wall. 84. (1. fpecim.

I1 3 Achates, f. Cameolus, varii polyhedri parvi, 609. ex India Orient. Wall l. c. (Io. Specim.

Ir4 Achates, f. Lamina Carneoli, ovalis, rubra, 688. ex India Orient Wall. l.c. (I. Specim.

I15 Achates, f. Carneolus, obliqui, ftriati, ovales, minores, 1602 ex India Orient. Wall. l. c. (5. Ipecim.

116 Achates. pracedentibus affines, lacte 2 \& rubræ, II3I. ex India Orient. Wall. l. c. (7. Ppecim.

II7 Achates, f. Carneoius, nitide lacteus, 1256. ex India India Orient. Wall. l. c. (5. Specim.

Is Achates, r. Carneolus, Hivus, ovalis, 162 I. ex.India Oriezt. U' ${ }^{\top}$ all. l.c. (I. fpecim. 


\section{Eoffiles. Claffe II.}

99 Agathe, femblable à la precedente, ovale, 1341. des Indes Orientales, Wall. b.c. (1. piec.

100 Agathe, arborifée, approchant dे la precedente, 1600. des Indes Urientales, Wall. l.c. is. piec.

IoI Agathe, ronde, convexe, avec un arbriffeau roux-brun, peinte en Eventail de mer, 1599. des Indes Orientales, Wail. l.c. (I. prec

102 Agathe. pareille, blanche, arborifée en brun, I340. des Indes Orientales, Wall. l. c. (r. piec.

IC3 Agathe, ou Plaque d'agathe platte, ovale, arborifée, 630. des Irdes Orientales, Wall. l. c. (I. piec.

104 Agahe, ou Pliques d'agathe plus petites, jaunes, blanches, ozs brames, arborifees, I123. II24. I126-II28. des Indes Orientales, Wall, $b$. c. (5.piec.

I05 Agathe, pareille, recourbées, ovales, mais plus petites, r505. I506. des Indes Orientales, Wall $l \boldsymbol{c}$. (2. piec

106 Agathe, p.reilles, rondes, ovales, arborifées, 1029. des Indes Oienrales, Wall. l. c. (8. piec.

I07 A2a: he, pareilles, approchantes aux precedentes, mais plus petites I 254. des Inde's Orientales, W 11. . l. c. (6. piec. I08 Azathe, pareilles, approchantés aux prec dentes, masis plus pales, 1252; des I ides Orienrales, Wall. l. c. (4. piec. Iog Agathe, femblables aux precedentes, ovales, Eo rondes, I253. d'Alleuagne, Wall. b. c. (8. piec.

IIo Agathe, arborifées, rouges, petites, II 25.1601 . des Indes Orientales, Wall. $l$ c. (2. piec.

II Agathe, pareilles rondes, 1307. des Indes Orientales, Wall. $l$ c. (3 piec.

I1 2 Agathe, ou Cornaline, grande, ronde, arborifée, recourhée. couleur de lait, iizo des Indes Orientales, Wall. 84. (I. piec.

II 3 Agathe, ou plufieurs Córnalines à facettes, petites, 609: des Indes Orienta es, Wali, l. c. (ro. piec.

II4 Agathe, ou Plaque de Cornaline ovale, E rouge, 688, des Indes Orientales, Wall $l$. c. (I piec.

II5 A athe, ou Cornalines. friées, ovales, plus petites, I602. des Indes Orientales. Wall. l. C. (5. piec.

216 Agathe, approchant d la presedente, ou pareilles Cornalines, couleur de lait, E rouges, II 3 I. des Indes Orientales, Wal'. l. c. (7. piec.

II 7 Agathe ou Cornalines, tres belles, couleur de lait, 1256. aes Indes Orientales. Wall. l. c. (5. piec.

tI8 Agathe, ou Co naline jaune, 1621 , des Indes Orientales, Wall, l. c. (I. piec.

O० 4 Ag Agatho 
IIg Acbates, f. Sardomyx? convera rotunda, Havefcens, oculo fanguineo, aurantio Alavove, 1357. ex India Orient. Wall. 86. (4 rpecim.

I20 Achates, f. Onyx, magna, lactea, ovalis, femidiaphana, fusca, una cum alia flavo circulata, excavara, operculata, fpecimen nitidiffimum, \& inter omnes rarifimum, 1091. 1693. ex India Orient Wall. 86.l. c. (2. fpecim.

321 Achates . Gmilis pracedenti, convexa, parva, diapha. na, alba, flava, circulo albo, $6 c \%$. ex India Orient. Wall. $t$. c. (2. Specim.

122 Achates, $\mathrm{f}$. Onyx, fusca, duplici circuleo albo', $\mathbf{2} 259^{\circ}$ ex India Orient. Wall, l. c. (3. Specim.

123 Achates, fimilis præcedenti, fusca, circulo albo, $126 x$. ex India Orient. Wall.' l. c. (2. Specirn.

124 Ahates, f. Sardonyx, rubra, circulis luteis albisque, I258. ex India Orient. Wall. l. c. (3. Rpecim.

I25 Achates, f. Onyx, fusca, circulis five ocellis duplicatis, flavis, 1267. ex India Orient. Wall. l. c. (3. fpecim.

126 Achates, f. Sardonyx, fusea, ocellis circellatis albis, 1264. 1265. ex India Orient. Wall. l. c. (3. Fpecim

127 Achates, f. Sardonyx, albicans, parva, circulis variis rubris, \& alia, I607. ex India Orient. Wall. l. c. (3. fpecim.

128 Achates, f. Onyx, fusca, circulis duplicrtis albis, ocellisque fuscis, 1262. ex India Orient. Wall. l. c. (4. fpecim.

129 Achates, f. Onyx fusca, ocellis albo circinnatis, I 260. ex India Orient. Wall. l. c. (4. fpecim.

I30 Achates, f. Sordonyx, quartzozus, fuscus, ocellis tribus flavis, rubellisque circulatis, .... ex India Orient. Wall. l. c. (r. fpecim.

131 Achates, f. Onyx, fuscus, ocellis albis, rubris, fuscis, maculatis, 15I1. ex India Orient. Wall. l. c. (4. fpecim.

$\$ 32$ Achates, f. Oculus Cati, fuscus, viridi aureo reflectens, ovalis, convezus, in annulo aureo, $7 \mathrm{I}_{4}$. ex India Orient. Wall. 87. (1. 'pecim.

133 Achates, f. Oculus Cati, viridi reflectens, 1266 . ex India Orient. Wall l. c. (I. fpecim.

134 Achates, f. Oculum Cati referens, partm refiectens. parvus, nitidus, ocellis albis, 1267. ex India Orient Wall. l. c. (5. Ipecim.

135 Acbates, r. Caput Imperatoris cujusdam Romani convexam, in onyce alba, 791. ex.... (I. fpecim. 


\section{Foliles. ClaJje II.}

II9 Agathe, ou Sardoine? recourbée, ronde, jaunatre, oẹillete de rouge orange, ou jaune 1257 . des Indes Orientales, Wall.86. (4. piec.

120 Agathe, ou un grand Onyx à couleur de lait, ovale, demi transparent, brun, avec un aytre à cercles jaunes. convave avec Sor couvercle, tres belle E rare piece. 1691. 1693 des Indes Orientales, Watl. l. c. (2. jiec.

I2 I Agathe, Semblable dे la precediente, ou Onyx pareille, convexe, petite, tranjparente, blanc, jaune, avec un oeil, ou cercle blanc, 607. des Indes OtientalesWall.l.c. (2. piec.

I22 Agatie, ou Onyx brum, à double cercle blanc; I259. des Indes Orientales, Wail. l. c. (3. piec.

I23 Agathe, on pareille Onyx brun, à cerche blanc 126x. des Indes Orientales, Wall. l.c. (2. piec.

I24 Agathe, ou Sardoine, rouge, à cercles jeunes $E^{2}$ blanches; I258. des Indes Orientales, Wall. l. c. (3. piec.

I25 Agathe, ou Onyx brun, à cercles, ou yerux doubles, jaunes, 1263. des Indes Orientales, Wall.l. c. (3.piec.

I26 Agathe, ou Sardoine brune, $\dot{a}$ yenx cerclées blancs, 12.54. $x 265$. des Indes Orientales, Wail. $l c$. (3. piec.

I27 Agathe, ou Sardoine blanchatre, petite, ḋ cercles variées. rouges, ¿े un autre, 1607. des Indes Oriencales, Wall. l. $c$ (3. piec.

128 Agathe, ou Onyx- brun, à cercles äorbles, blanches $\&^{\circ}$ yeux bruns, 1262. des Indes Orientales, Wall. l. c. (4. piec.

129 Agathe, oul Onyx brun, de yeux cerclés de blance. I260: des Irdes Orientales, Wall. l. c. (4. piec.

I 30 Agathe, ou Sardoine quartzeux, brun, à trois yeux jaunes; bordés de rouge,..., des Indes Orientales, Wall, t. $_{\text {. }}$. (I piec.

I3I Agathe, ou Onyx brun, a yeux blancs, rouges, brunes; tachetés, I5I . des Indes Orientales, Wall. l.c. (4) piec.

I32 Agathe, ou Oeil de Chat, brun chatoyant, en yerd dore, ovale, recourbée, montée en bague d'or, 7í. des Indes Orientales, Wall. 87. (1. piec.

133 Agathe, ou Oeil de Chat, chatoyant en verd, 1260. des Indes Orientales. Wall. l. c. (I. piec.

I34 Agathe, reffemblant, à un Oeil de Chat, \& un per cha: toyant petit propre, à yeux blancs, 1267. des Indes Orientales, Wall l. c. (5. piec.

I35 Agathe, ou Camajeu, la tete d'un Empereur Romaing wo courbe, dans un onyx blanc, 79, I. ex... (I. piec. 
I36 Achates, f. Caput Petri I. Ruffi Imperatoris, eodern modo ac fupra, ... (I. fpecim.

137 Achates, f. Caput Feminæ R mar æ, cum manibus, eodem modo fculptum,.... (I. Inecim.

I38 Achates. fo. Cleopatra, cum Cane, in lapide onyce flavefcente \& rubra,... (2. fpecim

139 Achates, f. Caput Philofophi in Jasp-achate flavelcente, ... (I. Specim.

I 40 Achates, f. Caput laureatum, in lapide flavo,... (I. fpecim.

14I Achates, 1. Capita antiqua, Mafcula \& Feminea, una cum Mercurio concaive fculpta, in carneola achateque,... (4. Ipecim.

142 Achates, in quo Cupido rofas defiendentes collegens, cum Junone Corru copiæ tenente, altera manu clavem, in lamina ovali concave fculptum, ... ex India Orient. (I.fpecim.

I43 $\mathcal{F}$ aspis, albus, lineatus, intermixtis granatis minoribus, ex monte Nanifter, lamina octogona, IO. poll. diametri , Irc8. ex Moravia.Wall, 94. (I. fpecim.

I44 Faspis grifeus, fiftens Thecam tabacarian, 591. ex Moguntia, Wall, l. c. (r. Specim.

145 Fafpis, albus, grifeusve, rubro maculatus, 601. ex Saxonia, Woll. l. c. (1. fpecim.

146 Fajpis carneus, politus, 1018, ex Norvegia, Wall. l.c. (1. fpecim.

477 Faspis filiceus, ruber, rudis, 1632. ex India Orient. 1788. ex Surinamo, ubi Nigritæ eo utuntur ad poliendum, Wall. l. c. (I. fpecim.

I\$8 Faspis. f. Laminæ ovales rubro fuscæ viridesque, ad thecam conftruendam, 1626. ex Germania, Wall. l. c.

.3.) (2. fpecim.

$\$ 49$ Faspis, f. Lamina ligni petrificati, in Jafpidem rubrum nigro flammatum, 1017. ex Bohemia, Waill. l.c. (1.fpecim.

150 faspis, r. Lamina parvæ, politæ, ex Jafpide rubro viridi maculato, I625. ex Germania, Wall.l.c. (2. fpecim.

151 Fafpis. C. Laminæ fimiles, ex Jafpide rubro flavo: que, 1624. ex Germania, Wall. b. c. (2. fpecim.

$151 \mathcal{F}$ a fpis grifeus, $\mathrm{f}$. Theca tabacaria pulcerrima, operculo cinereo fusco, in quo ab utraque parte Cignus albus confpicitur, fubtus achate-jápideo, cinereo lacteo, cetogonooblongo, auro inclufa,...ex ..., Wall l.c. (r.fpecim.

152 Gospis, $\mathrm{C}$ Theca tabacaria oblonga, cum operculo ex Jafpide favo, 1623. er Moguntia, Wall. l. c. (1. Ipecim.

152 Jaspis, f. Lamina utrinque politæ, ex Jarpide flavo rubro, ftriato, 697. ex Germania, Wall. l. c.. (2. cpecim. 


\section{Foffiles. Clafje II.}

36 Agathe, ou pareil Camajeu, reprefentant la tete de Czar Pierre I. fait de la mêrue maniere,..... (1. piec.

37 Agathe, ou Camajeu, reprefentant une Femme Romaine, avec des mains, fculpté de la sneme maniere,.... (I piec. 38 Agathe, ou Camajeu, reprefentant Cleopatre, avec un Chien dans une pierre d'onyx jumntre, Eo rouge,... (2 piec. 39 Agathe, ou Tête d'un Fhilojophe, on Camajew, en jafpd'agathe jaunatre,.... (r. piec.

40 Agathe, ou Camajeu d'un Tête ceinte de laurier, en pierre jaune, .... (r. piec.

4I Agathe, ou Têtes antiques. d'Hommes Ẽ Fernones, un de Mercure, fculptez en creux, de Cornaline E' d'Agathe, ... (4.\%.

42 Agathe, ou Plaque d'agathe ovale, Jculptée en creusc, dans la quelle on voit l' kmour rafemblant des rofes, dans un panier, à coté 'Funon, avec la Corne d'abondance, E' de l'artre main elle montre un clef, $\mathrm{r}$. des IndesOrientales, ... ( $\mathrm{r}$.piec.

43 Jafpe blanc, à lignés, entremelés de petits grains, de la Montagne Nanifter, en une plaque octogone longue de ro. pouc. diam. I 108, de Moravie, Wall. 94 (1. piec.

44 Jafpe gris, formant une Tabatiere, 59r. de Mayance, Wall. l.c. (r. piec.

45 Jafpe blanc E gris, tacheté de rouge, 66r. de Saxe, Wall. l.c. (I. piec.

46 Jalpe, polie, couleur de chair, ror8. de Norwege, Wall. l. c. (r. piec.

47 Jarpe cailloux, rouge, rude, 1632. des Indes Orientales 1788. de Suriname, ou les Negres en font ufage pour polir, Wall. l.c. (I. piec.

48 Jafṕe, ou Plaques ovales de jafpe rouge brun, Ë zerd, pour enfaire yne Tabatiere 1626. de l'Allemagne, Wall.l.c.(2.p. 49. Jafpe, ous Plaques de bois petrifié en jafpe rouge, à flammes noires, 1017. de Boheme, Wall. l. c. (I. piec.

50 Jafpe, ou petites Plaques, polies de jafpe rouge, ED verd', tachetéde verd, 1625. d'Allemagne, Wall. l. c. (2. piec.

5 I Jaipe, ou pareilles Piaques de jajpe rouge, EO jause, 1624. d'Allemagne, Wall. l. c. (2. piec.

5 I Jalpe grife. ou tres belle Tabatiere, dont le couvert brun cendivé, reprefenté áe chaque coté naturellement une Cigné blanche, la defjus eft d'a gathe-jappe, cendrée ciuleur dez zait, octogene abiongée, informé dans un cerclé d'or, ... de..., Wall. l.c. (ז. piec.

52 Ja pe, ou Tabatiere, allongée, avec fon couverle, d'un jajpe jaune 1623. de Mayence. Wall. l. c. (X piec.

52 Jafpe, ou Plaques polies de jaspe, jaune da ftries rouge; 697. de l'Allemegne, Will. l. c. (2. piec:

$$
\mathrm{O} \circ 3
$$




\section{Fofilia. Clafis II.}

ـ $\mathcal{F} a \int p i s$, f. Silex Eggyptius, fuscus, ovalis, ab altero latere Militem armatum referens, 163i. ex Africa, Wall. $l, c$. (I. 亿pecim.

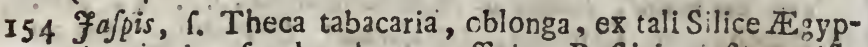
tiaco, in fundo theca effigies Ruftici poftice viff, zinco cup:eato, inclufa, 1811 . ex Africa, Wall. l. c. ( I. fpecim.

I5s $\mathcal{f}$ afpis, $\mathrm{r}$. Theca tabacaria fimilis ovalis, inclufa zinco cupreaio, 1812. ex Africa, Wall. l. c. (1. I'pecim.

156 Jajpis, f. Silex fuscus, rudis, \& politus altera parte, 693. ex IEgypto, Wall. l. c. (1. fpecim.

$157 \mathfrak{F}$ aspis, f. Lamina Silicis flavo-fusci, fimilis polita, $166 \%$ ex Atgypto, Wall. l. c. (1. Iperim.

I $58 f a / p i s$, f. Lamina fimilis, cum Silice olivaceo, nigro, ftriato, Jaf́ídoo, 602. 682. ex ...W Wall. l. c. (2. rpecim.

159 Faspis, nigro vel fusco virefcens, in laminis ovalibus politis, 729. 1093. ex .... Wall. l. c. (2. \{pecim.

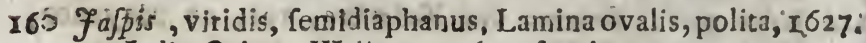
ex India Orient. Wall. 95. (2. Specim.

I6r Fafpis, f. Laminæa timiles, oblongæ, quadrata, rubro veno?æ, vel punctatæ, I628. ex India Orient. Wall. l. $c$. (2. fpecim.

162 Fafpis, f. Laminx affines femidiaphanæ, 694.730. I629. ex...,Wall. l.c. (3. fpecim.

${ }^{6} 6_{3} \mathrm{~F}$ ajpis viridis, albo ftriatus, rubro punetatus, lamina polita, г6зо. ex India Orient. Wall. 97. (I. fpecim.

164 Jajpis viridis, albo rubroque punctatus, politus, 696. ex India Orient. Wall. l. c. (1. Specim.

105 Faspis, f. Lapis Lazuli, Cespita antiqqua, \& aliz figuræ, concave fculpta, I091. $1287 . \mathrm{ex} \ldots$...Wall. 96. (3. foecin.

I66 Jaspis, r. Lapis Armenius, viridi cyaneus rofeo mixtus, politus, 668. ex .....Wal!. l.c. (I. fpecim.

I67. Fopis, f. Porphyrials, \& la'pis campeftris, laminx polite, Iogo. IOg2. ex Germania, Wall. 99. (2. fpecim.

168 Fa/pis, f. Granites, viridis, albo maculatus, lamina ovalis nolita, 1322. ex ....W Wall. l c. (I. fpecim.

169 Quartzum. fragile f. ficcum, (fic dietum) aggregatum, ocbra ferri teetum, 1385. "ex Zw:ybrucken, Wall. 100. (2. rpecim.

\$70 Quartzum, fragile aggregatum, album, i 786. ex Hartz, Wall. b. c. (I. fpecim. 


\section{Fogfles. Clafje II.}

I53 Jafpe, ou Cailiou d'Egypte, brun, ovale, de l'autre coté reprefentant un Soldat armé, 163x. d'Afrique, Wall.l.c. (1.piec.

154 Jafie, ou Tabatiere, oblonge, d'un tel Caillou d'Egypte, dans le fond, un Paijan vu ḋ dos, montée en pinsbec $181 \mathrm{I}$. d'Afrique, Wall. l. c. (I. piec.

I55 Jafpe, ou pareille Tabatiere ovale, montée en pinsbec, I8I2. d'Afrique, Wall. l.c. (I. piec.

156 Jafpe, ou Caillou de jafpe, b*un, rude, E poli del'autre coté, 693. d'Egypte, Wall. i. c. (1. piec.

157 Jafpe, ou pareille Plaque d'un Cailiiou, brun polie, 1667. d'Egypte, Wall, l. c. (I. piec.

158 Jafpe, ou pareille Ploque, avec un Cailliou de jajpe olive. à lignesnoires, 602.032. de ...WWall. l. c. (2. piec.

159 Jafpe, ou Plaques ovales, de jaspe verdeatre, noir, ou brun 729. 1093. de .... Wall. l. c. (2. piec.

160-Jarpe, ou Plaques ovales, polie, de jaspe verd presque transparant, IO27. des Indes Orientales Wall. $l_{\text {. }} c$. (2. piec.

I5r Jaipe, ou pareilles Plaques, quarrées, oblonges tachetée, ta veines rouges, I628. des. Indes Orientales, Wall. $l . c$. (2. piec.

162 Jarpe, ou Plaques pareilles deini transparantes 694.730. 1629. de..., Wall. l. c. (3. piec.

I63 Jarpe verd, ftrié àe blamc, is pointsrouges, plaque polie, 1630. des Indes Orientales, Wall. 97. (r. piec.

I64 Jafpe verd, polie, pointillé de blanc, E de rouge, 696, des Indes Orientales, Wall. $l$. c. (1. piec.

165 Jaípe, ou Pierre de Lazur, dans lequel Tetes antiques, E० autres finures gravées en creux, 109I. 1287. de .... Wall. 96. (3. piec.

166 Jarpe, ou Pierre d'Armerie, polse, couleur de celadon. melé de couleur de rofe, 668. des .... Wall. l. $c_{0}$ (1. piec.

I67 Jafpe, ou Pierre Porphyre, $\mathcal{E}^{\circ} \mathrm{J} a \int p e$ rude, plaques polies, rogo. I092. d'Allemagne, Wall. $99 . \quad$ (2. piec.

I68 Jarpe, ou Granit, verd, tacheté de blanc, plaque ovale polie, 1322. de....W Wall. l. c. (I. piec.

169 Quartz friable, en drufes, fec, couvert d'ocre brun, 1385. de Zweybrucken, Wall. roo. (2. piec.

I70 Quartz friable, blone, en drufes, 1786 de Hartz, Wall: i. c. (1. piec. 
171 Quartzum pingue, $f_{0}$ folidum, cryftallis pulcris tectum; i, cermixtis plumbi cubis, fpecimen magnum planum, 1509. ex Norvegia, Wall IOI. long. 16 lat. 15. poll. (1. fpecim.

I72 Quartzum pingue, f. folidum, pellucidum, cryftalliforine, 749. ex Helvetia, 1 484. ex India Orient 1487. ex Ceilonia, Wall. l. c. (8. ' pecim.

I73 Quartzum folidum, pellucidum, fpatho lamellofo, uviformi brunneo obfitum. pyrite, \& zinco penetratum, 743. 746. 747. ex ..., Wall. 102. (3. Specim.

174 Quartzum folidum, fubdrufiforme album . pyrite obfitum; 736. 739, 740. 741.745. ex ..., Wall. İI. (5. foecim.

I75 Quartzum amethyftinum, 718. ex Saxonia, 722.ex Helvetia, Wall. 104. (5. Ppecim.

I76 Quartzum molare, reticulatum, exefum, album, I386. ex Zweybrucken, Wall' 106. (1. Ipecim.

I77 Criftallus, C. rami criftallini, tenues, drufiformer, a'bi, 737. 284-1286. ex ..., Wall. rog. (4. fpecim.

I78 Criftallus. F. rami diaphani, craffi, drufiformes, 748 . ex He vet:a, Wall. l. c. (1. fpecim.

179 Criftallus, ? rami fimplices lactei vel diaphani criftallini, 744. 752 753.1282. 1283. ex Helvetia, Wall. l. c. (4. Specim.

180 Criftallus, f. ramus criftallinus craflws valde tranfparens, cum aliis minoribus; I 764. ex Blaanwenberg in Ameriea, Wall. $l$ c. (I. Specim.

I81 Criftallus, f. rami longi, craffique, puriffimi in drufa quartzofa, 748. ex Helvetia. Wall l. c. (I. Specim.

182 Criftallis, f. ramus criftallinus valde craflus, 751 . ex Helvetia, Wall. l. c. (I. fpecim.

183 Criftallus, f. ramus criftallinus, co'oris Topazii, 750. ex iaxonia Wall. 112. (1. Specim.

184 Criftallus obfcura, fere nigra, brevi acuta, in quartzo \& agathe, 1733. ex Veldenz, Wall. Ir4. (I. Pptcim.

185 Criftallus obfcura, affi is, fed absque achate, 734. 735. ex Germanta, Wail b. c. (2. Fipecim.

I86 Criftallus ubfcura, fuscus, brevi acuta in quartzo, cortice achatino, 1015. ex-Zweybrucken, Walb. l. c. (I. fiecim.

187. Criftallus obfcura. in globo achates, femi aperto, ... ex Germania, Wali. $l$ c. ( r. fpecim.

188 ' r.f. llus obfcura, in achate ovoideo, intus criftallis bievi acutis, in forman Thecæ tabacarie, inciufa zinco eupreato, 356. 6x Veldenz, Wall. b. 6. ( т. fpec m. 


\section{Fofiles. Claffe II.}

I7I Quartz gras, folide, couvert de beaux crifteaux, entremele. de prufieurs cules de plomb, grande E platte piece, 1509. de Norvegue, Wall. sor. long. 16, lat. i5. pouc. (1. piec

I72 Q partz gras, folide, transparant, cryjtallifé, 749 de Surfe 1 484. des Indes Oitentales, 1487. de Cellon, Wall. l. c (s.pec.

173 Quartz Solide, transparant, ou couvert de Spath feuilletés. brwn, Eे penetre de pyrite, E de zinc, 7+3. 746. 747. de ... Wali. 1oz. (3. piec.

174 Quartz folide, en drufes, ulanc, avec des pyrites 736. 7.9. 740. 74I 745 de..., Wall: 101 (5.picc.

175 Q artz couleur d'amethifte, 718. de Saxe 722. de Suiffe, if all. IC4. (5. piec.

176 Quirtz vermoulı, peticulé, blanc, I386. de Ziweybracken, Wal. 106. ( 1 piec.

177 Criftal, ou tranches criftalliwes, minces, en drufes, blanches, 737. I 284. I 286. de ..., Wall. 109. (4. piec.

198 Criftal, ous grolfes branches transparentes, en drufes, 743 . de Suiff Wail $l$ c. (1. piec

179 Criltal, ou fimples branches criftalines, conleur de lait, ou diaphanes, 744. 752 2. 753. 1282. 1283. de Suiffe, Wall. l. c. (4. piec.

I80 Criftal, ou branche criftalline tres transparente, avec des. autres pluss petites I764. de Blaauwe berg, en Amerique, Wall, l. c. (I. piec.

I8 I Criftal, ou branche crifuallines, tres groffes है longues, fur une drufe quartzerfe, 748. de Suife, Wail. L c. (x. piec. 182 Criftal, ou une branche criftalline tres groffe, 75I. de Suifre, Wall. $b$ c. (r. piec.

I83 Criltal, ou branche criftalline couleur de Topazs, 750. de Saxe, Wall. II2. (1. piec

I84 Criftal obfcure, presque noir, d pointe courte, fur quartz EO agate, 1733. de Veldentz, Wall. Iis. (1. piec.

185 Criftal, le pareil, obfcure, mais fans agate, 734. 735. d'Alleonagrie, Wii.b.c. (2 piec

186 Criftal obfcure, bruis à couste pointe, fur quartz à ecorce agatize, Ior5. de Zweybrucken, Wail. l. c. (I. piec

I87 C iffal obfure, dans une boule d"cgathe, à demi ouverts, d'A leinagne, Wall. l. c. (1. piec.

888 Criftal obfoure en agathe, en forme ovoide, en dedans decriftaux pointus, en forme de Cabatiere, montée en pins. bec, I356. de Veldenz. Wall. l. c. (1. piec.

$$
\text { O } 5 \text { I } 5 \text { Crifla }
$$


189 Criflallus obfcura, in globo permagno, ex achate aperto, in qua patent criftalli breves, acuta, Amethiftæ, Hyacinthæque, cum columna bexagona fpatbofa alba, politum, valide rarum, ... ex Velderiz, Woll. b. t. (I. Specim. 190 Criftallus obfcura, in giobo rusi achate, in quo criftalli brevi acuta, rubra, columnaque hexagone fpathora rubra fuperficiata, 1247. ex Veldenz, Wal!. l. c. (I. rpecim.

Igr Criftallus, f. ramus criftalligus, intus tubulis concavis, fpecimen rarifimum; 1595. ex...W Wall. l. c, (I. Specirn:

192 Criftallus, C. ramuli value diaphani, crifalilini, tetrædi, ntrinque apice donati, r635. ex Irdia Orient. Wall.l.c. (6. Specim.

193 Criftallus, f. Silex aqueus valde tranfparens, 1229. ex Ceilon, Wall. l. c. (3. Rpecim.

194 Criftallus, f. majores, minoresque criftalli Bohemica; 698. ex Bohemia, 099. ex Helvetia, Wall. l. c. (20. fpecim.

I05 Criftallus, crafa, tranfparens, in qua ochra veneris flofa, mascum mertiens, 1514. ex Helvetia, Wall. l.c. (1. Specin.

196 Criftalius, f. Pferdo Adamas utrirque acutus valde tran. fparens, in globo depreffo lapideo mineralifato, 1046. 1047. ex ..., Wall. 109. (I. fpecim.

197 P feudo Adamas, ipurius, tetrædrus, utrinque acuminatus, parvus, 700. ex India Orient Wall. 115? (2. Ipecim. 198 P feudo Adamas, precedenti afficis, ... ex Europa, Wal!. $l$ c. (10. fpecin.

I99 Rubinus, rudis, polyæedros, I493. ex India Orient. Wall, no. ( Io. Ceciun.

200 Rubinus, præcedenti fimiles, tranfparentes, minores, I 275. ex India Orient. Wall. $i$. c (multa fpecm.

20r Rubinqs, affines præćedentibus, nitidi minores, $7 \mathrm{cr}$. ex India Orienit. Wall. l. c. (multa fpecim.

202 Rubinus, politus nitidifimus, $12 \%$. ex India Orient. Wall. b. c. (4. rpecim.

203 Rubinus, f. Rubini Balaffi, minores, 701. ex America, IVall. l. c. (6. rpecim.

204 Rubinus Spinellus, refeo aibus, 1377. ex India Orient. Wall. . . c. (I. Specin,

zo5 Sapprirus palidus, rudis, 1439. ex Irdia Orient. Wall. II \%. (20. Tpecim.

200 Sapphirus, intenfior, polyalro parvus, 1329. ex India Orient. Wall. l. c. (1. Rptcia. 
189. Criftal obfcure, dans -unie grande boule d'agathe ouverte, dans laquelle on voit des criftaux Anethifte, Hyacintes, d courte pointes, arec une calomne hexangulaire, de spath blanc, poli, pas commune, ... de Veldenz, Wall. l. c. (I. piec.

Igo Criftal obfcure, dans une boule rude, d'agathe dans le quels font des criftaux, à courte pointes, rouges, EJ colonne hexagone, E' Spath rouge fuperficielle, 1247 . de Veldenz, Wail. l.c. (1. piec.

Igr Criftal, ou branche criftalline, à petits tubes concaves en dedans, tres rare piece, 1595. ex ..., Wall. l. c. (. . pice.

192 Criftal, ou branches criftallines, tres diaphanes, quayrées, d pointes, 1635 . des Indes Orientales, Wall. l. E. (6. piec.

193 Cfiftal, ou Cailiou aquerax. tres transparant, 1229. de. Ceilon, Wall, l.c. (3. piec.

I94 Criftal, ou grands, Ef petits criftanx de Boheme, 698. de Boheme, 699. de la Suifre, Wall. l. c. (20. piec.

I95 Criftal, gros, transparant, dans le quel de l'ocre cuirrenfe verte, eir forme de monlfe. 1514, de la Suiffe, Wail. l. c. (I. piec.

Ig6 Criftal, ou Diamant brut, pointue, tres transparant, dans une boule comprimée, pierseufe mineralifée, 1046. 1047. de ..., Wall. 109. (I. pic.

197 Diamant batard, à quatre pans, petit, dे deux pointes, 700, des Indes Orientales. Wall. ri 5? (2. piec.

I98 Diamant batard, pareille de laprecedenti, ... de l'íurope, Wall. i.c. (Io. piec.

199 Rubis, brut, polyadire, I493. des Indes Orientales, Wall. no. (10. piec.

200 Rubis, pareilles, transparentes. plus petites, 1275. des Indes Orientriss, Waill. $l \quad c$. (plufiarts's piec.

$20 r$ Rubis Jemilables au precedentes, pesiteș, $70 \mathrm{r}$. deo Indes Orientales, Wall. l c. (phifieurs piec.

202 Rnbis poli, tres beau, 1274 . des Indes Orientutes, Wall. l. c. (4. piec.

203 Rubis, ou Balais, très petit, 7or. de l'Amerique, Wall. I.c. (6. jiec.

204 Rubis Spinel, couleur de rofe pale, 1377. dés Indes Orien: tales, Wall. l. c. (I. piec.

205 Saphir pale, brut, 1489. des Indes Orientales, Wall. II7. (20 piec.

206 Saphir, beau bleu, d facette, petite, I329. des Indes Orientales, Wall. l. c. (s. pier. 
207 Sapphirus pallidus, 1272. ex India Orient. Wall. l. e. (2. Ipecim.

208 Sapphirus, colore celeftino, 1330. ex India Orient.Wall. b. c. (4. Specim.

209 Sapphirus, pallidifimus, 333 I. ex India Orient. Wull. l.c. (3. Ipecia.

210 Sapphirus, aqueus, 1273. 1488. ex India Orient. Wall. l. c. (2. fpecim.

'2II Topazius, tetrædus, \& polyædris angulis truncatis, $127 \mathrm{I}$. 1381. es Schnecken/tein, Wall. 118. (6. fpecim.

212 Topazius, coloris intenfioris, polyzdrus, politus, I $629 . \mathrm{ex}$ Brafilia, Wall. l. c. (ז. Ipecim.

213 Topazius, affinis, fed multo major, 18\%3. ex..., Wall. l. c. (I. Tpecim.

214 Topazius, pallidi varix magnitudinis,704. I270. I3781380. ex Schneckengtein, Wall. l. c. (5. fpecim.

215 Hyacinthus, rubro luteus, polyædrus, 7II. 712. ex India Orient. Wall. 123. (3. fpecim.

216 Hyacinthus, rudis, luteus, 1490. ex ...,Wall. l. c. (12. Ipecim.

217 Hyacinthus, rudis, luteus, I49r. I492. ex . . , Wall. l. c. (25. Rpecim.

218 Granati, incertæ figuræ rudes, 708. ex Bohemia, Wall. 122. ( 25 fpecim.

2 Ig Granati, affines, fed minimi, I278. ex Bohemia, Wall. l. c. (per multa fpecim.

220 Granati, penta, \& hexadri amorphi, in \& absque matrice, 703. 709. ex India Orient Wall. b. c. (4. fpecim.

221 Amethyfius, rudis, atque politus, 707. ex India Orient. Wall. I2I. ( I2. rpecim.

222 Amethy/lus, palidus, 1332. ex India Orient. Wall. l.c. (3. fpecim.

223 Aqua Marina, r. Berillus . pallide thalaffinus, polyxdré politus, 1276. ex India Orient. Wall, 124. (1. Specim. 224 Chryjolithus, colore prafino, polyædrus, 127\%. ex India Orient. 120. (1. fpecin.

225 Chryfolitlius, rudis, parvus, r494. ex India Orient. Wall. l. c. (15. fpecim

226 Smaragdus, rudis inzqualis, cui ineft marcafita, 706. 1382. ex America, Wall. I19. (4. Specin.

227 Smaragdus, rudis, in matrice, 7I6. ex Brafilia, Wall. b. c. (I. fpecim. 
207 Saphir pale, 1272. des Indes Orientales, Wall. 1.c. (2. piec.

208 Saphir, conleur celeftin, 1330. des Indes Orientales. Wall. I. c. (4 piec.

209 Saphir. tres pale, I33r. des Indes Orientales, Wall. I. c. (3. piec.

210 Saphir aqueux, 1273. 1488, des Indes Orientales, Wall. l. c. (2. piec.

211 Topaze, $\ddot{a}$ quatre cotez, $\mathcal{E}^{2}$ un autre $a ̀$ plufeurs angles tronquez, 1275. 138.i. de Schnetkentein, Wall. 248. (6. piec.

212 Topaze, ì couleur foncée, en facetter, polie, 1629 . de Brafille, Wall. 1. c. ( . piec.

213 Topaze, pareil, mais beaucoup plus grand, 1813. de..., Wall. 1. c. (1. piec.

214 Topazes, pales, de diverfe grandeur, 704. 1270. 13781380. de Schneckenftein, Wall. 1. c. (5. piec.

215 Hyacinthe, jaunatre. rouge, en facette, 7 11.712 . des Indes Orientales, Wall. I23. (3. piec.

216 Hyacinthe, rude, jaune, 1490 de..., Wall. 1.c. (I2. piec.

217 Hyacinthe, brut, jaune fonce, 149r. 1492. de..., Wall: l. c. (25. piec.

218 Granates, \& cotez inegaux, rudes, 708. de Boheme; Wall, 122. (25. piec.

210 Granates, pareilles tres petits, 1278. de Boheme, Wallb 1. c. (phufieurs piec.

220 Granates, do cing, E० fix cotez des autres d cotez inegaux, dans, EO hors de leur matrice, 703. 709. des Indes Orientalss, Wall. 1. c (4. piec

221 Amethifte, brut , \& polie, 707 . des Indes Orientales; Wall. 12I. (12. piec.

222 Amethifte, pale, 1332. des Indes Orientales, Wall. 1.c. (3. pies.

223 Berylle, pale, couleur celadon, ì facettes, polie, 1276 . des Indes Orientales, Wall. I 24. (I. piec.

224 Chryfolite . couleur verte de herbe, $\dot{a}$ facette, 1277 . des Indes Orientales, Wall. I20. (I. piec.

225 Chryfolite, brut, petit, 1494. des Indes Orientales, Wall. I. c (15. piec.

226 Emeraude, brute, d cotez inegaux, avec marcafite, 706. I382. de l'Amerique, Wall. II?. (4. piec.

227 Emeraude, brut, dans fa matrice, 716, de Brafil, Wall. 1. c. (1. piss. 
228 Smarggins, pulcerrimus, valde tranfparens, faturate viridis, in matrice, cum fpatho \& pyrite, $128 \mathrm{I}$, ex.Bra. falia, Wall. l. c. (I. Specim.

229 Turmalimus, flavefcens, rudis, parvus, 1496. $1497 . \mathrm{ex}$ Ceylonia, Wallo. (25. fpecim.

230 Turmalinus, rudis obfcutior, parvus, 1495. ex Cey. lonia, Wall. o. (15. fpecim.

231 Turmalinus. fuscus politus polyædro, generofe vigens, 13-6 ex Ceylonia, Wall. o. (1. Specim.

232 Turmalimus, niger, rotundus polyædros, generofus, 1034. Ex Ceylonia, Wall. O. (I. Specim

233 Turmalinus? cubicus, opacus, fuscus, I280. ex . . . . , Wali. O. (1. Specim.

234 Beryllus? f. Silex aqueus, tranfparens, 713. ex Saxonia, Wall. 124? (I. Tpecim.

235 Toposius? f. Silex aqueus, flavefcens, vitri inftar tranfpa. rens, 1479. ex India Orient. Wall. rc9. (2. Specim.

336 Amethyftus quartzofus, f. Theca tabacaria, zinco cuproque (pinsbec) cincta, 1734. ex Vienns, Wall. 102. (1. Specim.

237 Vitrum Muscoviticum. f. Glacies Marice, in laminis, \& in quartzo, 568. ex Siberia, 73I. ex Rufia, Wall. 125. (3. fpecim.

$23^{8}$ Mica alba, five Argentum Cati, cum quartzo fpathofo, \& Molybdana, f. Mica Pittorum, 5II. ex Salfeld, I 816. ex Hortz, Wall I 26. \& I3I. (3. fpecim.

239 Mica Aurea, granatulis infperfa, in lapide quartzolo, 569. 5;0. ex Germania, Wall. I 27. (2. 'pecim.

240 Talcum Micaceum Aureum, in lapide fquamofo, I44. 145. ex Soxonia; Wall. 133. (2. Ipecim.

24 I Serpentinus, viridis, ruber, aliusque coloris, laminæ ovales, quadratave polita, 302. 303. 721 . ex Suecia. 710. 722. es Saxonia, 1698. ex Anglia, Wall. 136. (6. fpecim.

242 Serpentinus, vario colore marmorati, laminæ quadratæ, 1028-1043. ex Tóplitz. Wall. l. c. (16. Tpeeim.

243 Serpentinus, in forma ovi, $7_{23}$. ex Típlitz, Wall.l. c. (2. Spec m.

244 Saxum Corneum, rubrum glau cumque, cum quart zo, 059. 6or. ex Saxonia, Wall. r42. \& 156. (2. Specim.

245 Saxum Corneum, nigruin, lineolis a'bis, five quartzo Jafpideo penetratum, politum, 663. 654. ex AEgypto, Wall. l. c. (2. fpecim. 


\section{Fofiles. Claffe II.}

228 Emeraude, tres beau, tres trenfparant, d'un beaze vert, dans $\int a$ matrice, avec fpath, EO pyrite, 128r. de Brafile, Wall. I. c. (r. piec.

229 Tourmalin, jounatre, rude, petit, 1496. 1497. de Ceil lon, Wall. O. (25. pirc

230 Tourmalin, brut, plus foncé, fetit, I495. de Ceilon, Wall. O. (15. piec.

23i Tourmalin. brum, poli, à facettes, tirant tres bien, 13760 de Cerlon, Wall. o. ( I. piec.

232 Tourmalin, noir, rond $a$ facettes, tirant tres bien, 1634. ie Ceilon, Wall. o. (1. piec.

233 Tourmalin? cubique, opaque, brun, 1280 de ..., Wall. o. (I. piec.

234 Berylle? out Caillou aq̧ueux, tran fparant, 7r3. de Saxe, Wall. 124? (1. piec.

235 Topaze? ou Caillou aqueux, jarn.tre, transparant, comme du verre, 1479. des Indes Orientales, Wall. 109. (2. piec..

236 A machifte, quartzeive, ou Tabatiere, monté de pinsbec, I 734 : de Vienne, Wall, .c2. (x. pies.

837 Verre de Muscovie, en feuilleté, É en quartz, 568. 줗 Syberie, 731. de Ruffie, Wall. I25. (3. piec.

238 Mica blanc, ou Argent de Chat, avec quartz Spaterx, \& crayon de plomb, 5 11. de Sasifeld, 18:6, de Hariz, Wall. 126. E $13 \mathrm{~L}$. (3. pies.

239 Mica doré, parfemé de petites granates, en pierre quare zeufe, 569.570. d'Allemagne, Wall. 127. (2. piec.

240 Talc micacé, doré, dans une pierre écailleufe, 144. 145. de Saxe, Wall. 133. ( 2 piec.

$24 \mathrm{I}$ Serpentin, verte, róuge, Ed d'autre coubeur, plaques ovales, quarrez, polies, 302. 303. $72 i$ de Suede, 710.722 . de Saxe, 1098. d'A ngleterre, Wall. 136. (6. piec.

242 Serpentin, marbrée de diver $\int e$ couleur, plaques quarrées 1028-1043. de Tórlitz, Wall. 1. c. (I6. piec.

6. 243 Serpentin, eiz forme d'oerif, 723. de Tóplitz; Wallo l. c. (2. piec.

244 Pierre Corněe, rouge Eo verl, avec quartz, 659. 69r. de Saxe, Wall 142. Oे is6. (2. piec.

24.5 Pierre Cornée, noire, à petites lignes blanches, penetré de quartz jafpé, polie, 663. 664. d'Egypte, Wall. 1. c. (2. piec. 
245 Sexuin $\mathcal{F}_{\text {afpo-corneum }}$ rubrn fusc mque, nornhyrum mentiens, politum, 1162. ex Germaria, Wall 156." ( $\mathrm{I}_{\text {a }}$ fpecim.

247 Saxum Corneun granulofum ex quartz? fnatho, \& faxocorneo uritum, 758. ex Italia, Wall. 160. ( 1 . rpec m

248 Saxum Corneum arenaceum, rchra martis criftallisi, ue quartzofis, pinetratum, \& of fitum, 1388. ex Veldenz. Wall ing. long. 14 poll (1. $f_{\text {, ecim. }}$

249 Saxum Cornetim arenaceum, grifeum, cum crifta lis quartzofis, 89. I 390. c $x$ Veidenz Will l. c. (2. Specim. 250 Saxum Corneum, rubro alnuque vatium - arenace, (riftallinum, lamina quadraı, 1364 . ex Veldenz, Wall. l. c.. ( $\mathrm{I}$. $\left\{\mathrm{p}=\mathrm{c}^{\mathrm{i}} \mathrm{m}\right.$.

25x Lapis Serpentinus antiọuus ,f. Saxum petrofum (Puddingftone) affi e, lamina ovali, magna, 1697. (x Avglia, Wall. 172. (1. fpecim.

252 Lapis Serpentinus antiquus, f. Lamira ovales obfcure virides, al is que culoribus mix æ, 1695. 1696. ex Anglia, Wall l. c. (2. fecim.

253 Amiantus, f. Linum Montanum, in lapide grifeo As beftino, 1391. ex Norvegia, Wall 145. (I fifcim.

254 Amiantus, f. L:num Montanum, fibris longioribus, I193. ex Norvegia, Wall. b. c. (1 -fpecim.

255 Amiantus, f. Linum Montanum flavum, fbris Ingioribus, 1707. ex Hurgari?, Wrill. l. c. (1. Ipec:m.

256 Amiantus, f. Linumi Vontanum, tortum f. nectum, 555. 566 ex Germania Wall l. c. ( 3 Specim.

257 Amiantus, C. Aluta Montana imbricata, fusco-lutea, 1775. ex.Germania, Wall. 46. (I. Specim.

258 Amiantus. F. Suber Montanum, fuscum, 563. ex Ger-2 mania, Wall 148. (1. Specim.

259 Amiantus if Caro Montana, imbricata, flavefcens, I154. 1204. ex Saxo.ia Wall. 147. (3. fpecim.

260 Asteftus. of Abvfinus, maturus, \& immacurus, 564. ex Nova Hollandia, 5,5. ex ..., Wall. 149. 150. (¿.ipecim. 26I A. beftus immaturus,... ex Germania, Wall. I50. (I. fpecim.

202 Asteftus, f Papyrus . ex asbefto vel amiante arte factus, 20 s049. ex Germania, folium $8^{\circ}$. furma, Wall. b. c. ( $x_{\text {. }}$ fpecim. 


\section{Fofsles: Claffe It.}

:46 Pierre, ou Jaspe Corné, rouge, Ẽ brun, imitant le porphyr, polie, 1162. d'Allemagne, Wall, 156. (I. piec.

-47 Pierre Cornéégranulée, ou plaque de quartz, jpath, É pierre cornée, 758. d’Italie, Wall. I60. ( 1 . piec.

348 Pierre Cornée arenaire, ou fablonneufe, penetré, d'ocre de fer, \& crijzaux quartzeux, 1388. de Veldenz, Wall, 169. long. 14. pouc. (I. piec.

249 Pierre Cornée arenaire, Eे un autre gris, avec des criftaux quartzeux , 1389. I390. de Veldenz, Wall. l. c. (2.piec.

850 Pierre Cornée, yarié, de rouge, Ẽ blanc, Sablonneufe, criftalline, plaque quarrée, 1384. de Veldenz, Wall. 1. c. (r. piec.

25I Serpentine antique, reffemblant do un Pondingue vert, plas que grarde, ovale, 1697. d'Angleterre, Wall. 172. (1. piec.

252 Serpentine antique, ou pareilles plaques, ovales vertes, \&2 rouges, 82 melées d'autres couleurs; 1695. I696. d Angleterre, Wall. 1. c. (2. piec.

253 Amiante, ou Lin de Montagne, dans une pierre Asbeftine grife, 139x. de Norwege, Wall. 145. (r. piec.

254 Amiarte, ou Lin de Montagne, d̀ longues fils, II93. de Norvegue, Wall. 1. c. (I. piec.

255 Amiante, ou Lin de Montagne, jaisne, d longues fils, I 767. de Hongrie, Wall. 1. c. ( $I$, piec.

256 Amiante, ou Lin de Montagne, tortu, 565. 566. d'Allemagne, Wall. 1. c. (3. piec.

257 Amiante feuilleté, ou Cuir de Montagne, en forme de tuiles, brun jasnatre, 1775 . d'Allemagne, Wall. I46. (I. piec.

258 Amiante, ou Liége fofile de Montagne, brun, 563. d'Allemagne, Wall. 148 . (r. piec.

259 Amiante, ou Chair de Montagne, jaunatre, II 54. 1204. de Saxe, Wall. I47. (3. piec.

60 Asbefte, mur, EO non mur, 564. de la Nouvelle Hollande, 565 de ...., Wall. 149. 150. (2. piec.

6r Asbefte non mur, ... d'Allemagne, Wall. 150. (r. piec.

62 Asbefte, ou feuilles, in 89. de Papier fait d'asbefie, ow d'amiante, 1049. d'Allemagne, Wall. I. c. (1. piec.

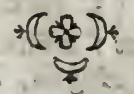




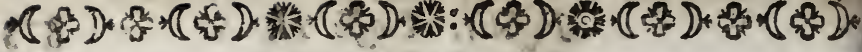

\section{L A S S I S III $M I N E R \quad R E$.}

"263 Yitriolum eupri, viride coeruleumque fuperficiale, 768 ex Roma, 769. ex Sriecia 773. ex Dalecarlia, Wall. 173 . (20. 'pecim.

204 Vitriolum ferri, viride, 770. 771. es Suecia, 774. ex Ungat ria Wall. 174. (8. fpecim.

265 Vitriolsm ferri, viride, 775. ex Ungaria, Wall. l. c. (I. fpecim.

266 Vitriolum ferri, viridi, 773. ex Anglia, 776. ex Dalecarlia, 778. ex Hartz, Wall. l. C. (3. fpecim.

267 Vitriolum żinci, album, 779. ex Gosbarienfi, Wall. 175. (2. ) pecim.

268 Vitriolum mineralifasum, C. Lapis Atramentarius, ruber, flavus, grifeus, 782-784. ex Goslar, Wall. 178. (5. fpecim.

269 Vitriolum mineralifatum, rubrum, fimile precedenti, 780 。 781. 785. ex Goslar, Wall. l.c. (3. Specim.

270 Alumen nativum, in quo alumen plumofum, flavefcens, 1675. ex Saltzburg, Wall. I 79. (I. Specim.

271 Alumen nativum phumofum, fibrillis albis, 1822. ex Stahlberg in Polatin, Wall. l. c. (I. Specim.

272 Alumer purificatum, Ir64. ex Germania, Wall. l. c. (i. Specim.

273 Nitrum, II r. ex Bamberg. Ir65. ex . . ., Wall. 183. (3. Specim.

274 Sal gemme, criftallifatum , \& ftalaciticum, 155. ex Halle; 724. ex Polonia, Wall 184. E9 186. (3. Tpecim.

275 Borax crudus, 1073. ex Indis Orient. Wall. 200. (2.) (2. Epecim.

276 Afphaltum, f. Bitumen Gudaicum ... ex Aegypto, Wall 204. (1. Ifpecim.

277 Bitumen, f. Terra mineralifata, gagatica, vel libera, 1735. 1820. ex Nova Hipania, Wall. 205. (2. Specim. 278 Bitumers, f. Lithantrax, 319. 320. ex HaJia, 318. ex Anglia, Wall. 206. (3. Ipecim.

279 Bitumen, f. Lithantrax, fifflis, ligni foffilis inftar, cun marcafita, I1gr. ex Anglia, Walb. b. c. (I. fpecim. 280 Bitumes 


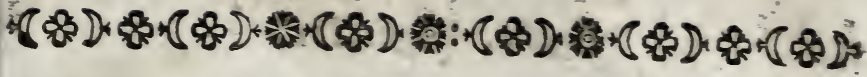

\section{L A S S E III. $M I N E R A U X$.}

263 Vitriol vert, E' blen fuperficiel, 768. de Rome, 769. de Suede, 772. de Dalecarlie, Wall. 173. (20. piec.

254 Vitriol yerd, 770. 77 r. de Suede, 774. d'Hongrie, Wall. 174. (8. piec.

265 Vitriol yard, pareille, 775. d'Hongrie, Wall. b. ce (I, piec.

266 Vitriol yerd, pareille, 773. d'Angleterre, 776. de Dalecar. lie, 778. de Hartz, Wall. 1. c. (3. piec.

267 Vitriol de zinc; blanc, 779. de Goslare, Wall, 175.
(2, piec.

208 Vitriol mineralife, on Pierre Atramentaire, rougs \& jaune, grife, 782-784. de Goslar, Wall, 178. (5. piec.

269 Vitriol mineralire, ots Pierre Atramentaire, femblable ons precedent, rouge, 780. 785. 785. de Goslar, Wall. 1. c. (3. piec.

:70 Alun natif, dans lequel alun en plumes, jaunatre, 1675. de Saltzabourg, Wall. 179. (I. pies.

7I Alun natif en plumes, d plamettes blanckes, 1822. de Stahlberg en Palatin, Wall I. c. (I. piec.

72 Alun roffine, 1164. d’Allemagne, Wall. 1. c. (I, piec.

73 Nitre, inr. de Bamberg, I165. de ..., Wall. I 83 . (3. piec.

$74 \mathrm{Sel}$ foffile, criftallife, \&o falactise, iss. de Halle, 724. de Pologne, Wall. 184. \& I86. (3. piec.

75 Borax crud, 1073. des Indes Orientales, Wall. 200. (2. piec.

76 Asphalte, ou Bitume des Juifs, ... de l'Egypte, Wall. 204. (I. piec.

77 Bitume, o: Terre minéralife, gagaté, 80 en poudre. 1735. 1820. de la Nouvelle Efpagne, Wall. 205.
(2. piec.

IS Bitume, ous Charbon fofile, 319,320 . de Hartz, 348. d'Angleterre, Wall. 2є6. (3. piec.

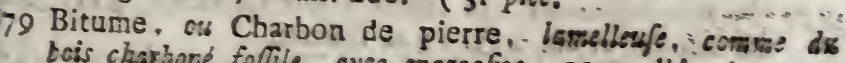

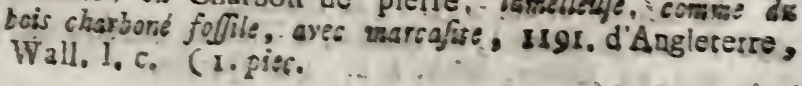


280 Bitumen, f. Lignum follile, 1022. ex Bergssand, 1196. ex Anglia, Wall. l. c. (2. Specim.

281 Bitumen duriffimum . . Gagates, ater, cum ligno forfili, in gagatem mutato, I1 57. ex Regensburg, 1636. ex Saxo. nia, Wall. 207. (6. fpecim.

182 Succinum, f. Elętrum, flavum tranfparens, 342. ex Borufjo, Wall. 208. (8. Specim.

283 Succinum, f. Electrum, diaphanum, Infeeta includens; 1288. ex Borufia, Wall. l. c. (2. Specim.

284 Succinum. C. Electrum flavum lacteumque, Infecta continens, 1789. ex Borugba, Wall. l. c. (5. fpecim.

285 Succinum, f, Electrum. luteum, \& albo flavefcens, opacum, in globulos artefactum, . . ex BoruJia , Wall. 209. (38. fpecim.

286 Succinum, C. Electrum, albo flavum, opacum, 1790. ex Borwilia, Wall. l. c. (2. fpecim.

287 Succinum, f. Electrum, flavum, opacum, lacteo veno. fum, manubrium Enfis, 341. ex Borufia, Wall, l. c. (i. Specim.

288 Gummi Copal, tranfparens electrum fimulans, 1823. ex America, Wall. o. (1. Specim.

289 Gummi Copal, flavefcens opacum , electrum fimulans, 1824. ex America, Wall. O. (1. Cpecim.

290 Ambra grifea, 336. ex India Orient. Wall. 211 . (1. Ipecim.

291 Sulphur nativum, tranfparens, 777. ex Hijpania, Wall. 213. (1. fpecim.

292 Sulphur nativum, in lapide atramentario, 76r. ex Saxonia, 762. ex Goslar, Wall. l. c. (3. Ipecim.

293 Sulphur nativum, in fcoria lapidea vulcani, 339. ex Monte Vefuvio, Wall. l. c. (I. Ppecim

294 Sulphur fufum, ftalactitiforme, 347. 348. ex Germania, Wall. o. (3. Tpecim.

295 Sulphur fufum, criftallifatum, fpecimen magnum. 346. ex Germania, Wall. O. (1. fpecim.

296 Sulphur aureum, ita dietum, 763. ex Ungaria, Wall. 0. ( $\mathrm{r}$. 'pecim:

297 Pyrites fulphurezs, in lapide nigro fchiftofo, 321. 322 ex Hartz, Wall. 215. (4. Specim.

298 Pyrites fulpherreus, in (chifto, 2 Nicolao penetratus, 325 326. ex Hartz, Wall, l, c. ( 2 , fpecim. 


\section{Fofiles. Clage III.}

80 Bitume, ou Bois Charbonné. 1022. de Berglànd, I196. d’Angleterre, Wall. I.'c. (2. piec.

81' Bitume, tres dur, ou Gajate, noir, avec du bois folfle gagatifé, 1157. de Regensburg, 1636, de Saxe, Wallo. 207. (6. piec.

282 Succin, ou Ambre jaune, transparant, 342. de Pruffe, Wall. 208. ( 8 piec.

83. Succin, ou Ambre, transparant, avec des Injeetes, 1288. de Pruffe, Wall. 1. c (2. piec.

284 Succin, ou Ambre, jaune, opaque, avec des Infectes, 1789. de Pruffe, Wall. I c. (5. piec.

285 Succin, ou Ambre, jaune, opaque, en grains de chapelet, … de Pruffe, Wall. 209. " (38. piec.

286 Succin , ou Ambre, blanc-jaunatre, opaque, I 790. de Pruffe, Wall. 1. c. (2. piec.

287 Succin, ou Ambre jaune, opaque. a veines blancheatres. fait en manche d' Esée, 341. de Pruffe, Wall 1. c (I. piec.

288 Gomme Copal, transparant, reffemblant d l'ambre, 1823 . de l'Amerique, Wall. o. (I. piec.

28, Gomme Copal, jaunatre, opaque, refJemblant à l'ambre, 1824. de l'Amerique, Wall. 0 . (1. piec.

290 Ambre gris, 336. des Indes Orientales, Wall. $2 \mathrm{II}$, (r. piec.

29r Soufre natif, transparant, 777. de l'Efpagne, Wall. 213. (I. piec.

292 Soufre natif, en pierre Atramentaire, 76r, de Saxe, 762. de Goslar, Wall. l.c. (3. piec,

293 Soufre natif, en fcorie de pierre du vulcan, 339. du Mont Vefuve, Wall. 1. c. (I. piec.

294 Soufre fondu, en forme de ftalactite, 347.348. d'Allemagne, Wall, l. c. (3. piec.

295 Soufre, fondu, criftallife, une grande piece, 346. d'Allemagne, Wall. o: (r.piec.

2,06 Soufre d'oré, ainfi nommé, 763. d'Hongrie, Wall. o. (I piec.

297 Pyrite fulphureux, dans un pierre de Schift noir, 321.322. de Hartz. Wall. 215. (4. piec.

298 Pyrite fulphureux, en (chife, avec mine d'Arfenic rougeatre, 325. 326. de Hartz, Wall. 1. c. (2. piec. 
299 Pyrites sulphureuss granularis, cum quartzo criftalliatce? albo, 328. ex Hartz, i113. 1532.1645. ex Norvegia, Wall. l. c. (4. Specim.

300 Pyrites, drufi formis, cum alia verficolore, in quartzo, \& Spatho, 333. ex Hartz, 334. 335.337. ex Saxonia, I660. ex Nafjovia, Wall. l. c. (5. fpecim.

301 Pyrites, f. globi pyriticofi uvaformes, in fpatho albo, \& Glacie Märix , 1700:170r. ex Anglia, 1791, ex Goslar s Wall. 216. (3. f́pecim.

302 Pyrites, reniformis, apertus politus, r. Lapis fanitatis antiquorum, 1992. ex Coburgo, Wall. l. c. (2. Rpecim:

303 Pyrites, in faxo corneo, utrinque polito, 1393. ex Gold-Cronach, Wall. l. c. (I. Specim.

304 Pyrites, fo renis pyriticofi, aperti, politi, vel lapides fanitatis, II 49. eX Turnar, Wall. $l$. c. (2. Ipecim.

305 Pyrites, fo reni pyriticofum, durum, oblongum, 324. ex Germania, Wall. l. c. (I. Ipecim.

306 Pyrites, f. Marcafita, 331. ex Saxonia, Wall. 217. ( $I_{\text {, }}$ Ipecim.

307 Pyrites, f. Marcafita, polyædra, drufformis, in pyrite;du a ro, 332. ex Saxinia, Wall. b. c. (1. Ipecim.

308 Pyrites, f. Marcafita, fimilis, angulis páucioribus, III4, ex Norvegia, Wall. l. c. (I. ipecim.

jog Pyrites, $f_{0}$ Marcafira, pentagonus, \& alius in Spatho al. bo, 329. ex... 1291. ex Norvegia, Wall. b.c. (2. fpecim.

310 Pyrites, f. Marcafita, polyædra, cum fpatho albo, \& quartzo, .. ex Noryegia, Wall. b. c. (1 . Ipecim.

3i 1 Pyrites, $f$. Marcafita, drufiformis, polyædra, cum quartzo criftallifato, galena plumbi, galena fterili, \& lapide corneo grífeo, 1702. ex Anglia, Wall. l. c. (I. foecím. 3I 2 Pyrites, C. Marcafita, in Spatho drufiformi fusco, in lapide corneo flavefcente, 307. ex Germania, Wall. $l_{\text {. }} c_{\text {. }}$ (I. Ipecim.

313 Pyrites, f. globus Marcafite, fpatho albo penetratus; 330. ex Saxonia, Wall. l. c. (1. Specim.

344 Pyrites, f. Marcafita, dodecadrica, 338. ex Anglis, Wall, l. c. (4. Ipecim.

315 Pyrites, C. Marcafite, polyædra, lamellata, \& quadrata " 336. 343. ex Saxonia, Wall: l. c. (4. Specim.

3I6 Hydrargyrum nativum, in minera Mercurii, \& in lapide.

corneo quartzozo, drufiformi, 349. ex Ungaria, Wall. 219. (I. fpecim. 
299 Pyrite fulphureux, en grains, avec du quartz, blane criflalité, , 328. en Hartz. III3. 1532. 16 45. de Norvegue, Wall. I. c. (4. piec.

300 Pyrite, en forsse de drufe, $E^{\circ}$ autre à queue de pan, fir quartz, Eo Spath, 333. de Hartz, 334. 335. 337. de Saxe, 1660. de Naffau, Wall. 1. c. ( 5 , piec.

301 Pyrite, ou bosles pyriteufes, en forme de grappe, en Jpath blanc, \&ं Glace de Marie, 1700. 1701. d'Angleterre, 1791 . de Goslar, Wall. 216. (3. piec.

302 Pyrite, en forme de reins, ouvert. Ẽ polis, ou la Pierre de fanté des anciens, 1992. de Cobourg, Wall. 1. c. (2. piec.

303 Pyrite, dans une pierre a corne, polie de deux cotez, 1393. de Gold-Cronach, Wall. 1. c. (I, piec.

304 Pyrite, ou reins pyriteux ouverts, polies, ou pierres de Janté, Ir 49. de Turnau, Wall. 1 c. (2. piec.

305 Pyrite, ou rein pyriteufe, dure, oblongue, 324. d'Alle. magne, Wall. I. c. (I. piec.

so6 Pyrite, ou Marcafite, 33I. de Saxe, Wall. 217. (I. piec.

307 Pyrite, on Marcafite, en drufe, d beaucoup d'angles daris un pyrite dure, 332. de Saxe, Wall. 1. c. (I. piec.

$\$ 08$ Pyrite, ou Marcafite, pareil, à moirdrés angles, II 14 : de Norvegue, Wall. I. c. (r. piec.

309 Pyrite, ou Marcafite, pentagone, \& un autre, en Spath blanc, 329. de.... 129r. de Norvegue, Wall. 1. c. (2. piec.

3Io Pyrite, ou Marcafite, à plufieurs angles, avec Jpath blanc, Eo quartz .... de Norvegue, Wall. I. c. (I. piec.

II I Pyrite, ou Marcafite, en drufe, à plufieurs angles, avec quartz criftallifé, galene de plomb, de Zinuc, 'E pierre cornée grije, 1 702. d'Angleterre, Wall. l. c. (1. piec..

3i2 Pyrite, ou Marcafite, en drufe fpatheufe, brine, fur une pierre cornée jaune, 307. d'Allemagne, Wall. 1. c. (I. piec.

iI Pyrite, ou boule de Marcafite, penetré de Spatb blane, 330. de Saxe, Wall. 1 c. (I. piec.

II 4 Pyrite, ou Marcafite, à douze cotéz, 338. d'Angleterre, Wall. 1. c. (4. piec.

3is Pyrite, ou Marcafite, de plufeurs angles feuilleté, \& quarrée, 336. 343. de Saxe, Wall. 1. c (4. piec

156. Mercure Vierge, dans une mine de Mercure, en pierre cormée quartzeûfe, en forme de drufes, 349. d'Hongrie, Wall, 219. (I. piec. 
317 Hydrargyrum nativum, cum pyrite cupreo graniformi, marcafitam mentiens, in lapide arenaceo, 351 , ex Un: garia, Wall. l. c. (1. Tpecim.

s18 Hydrargyrum nativum, cum Cinnabare, in lapide fer reo, 350. ex Ungaria, Wall. l. c. (I. Ipecim.

319 Hydrargyrum nativum, in lapide ferreo, Cinnabare inmiztum, 352. ex Ungaria, Wall. l. c. (1. Specim.

320 Hydrargyrum nativum, in lapide ferreo, venis quartzofis, 355. ex Ungaria, Wall. l. c. (1 fpecim.

321 Hydrargyrum nativum, cum Cinnabare, lapide inmixtum, 1396. ex Tyrol, Wall. l. c. (1. fpecim.

322 Hydrargyrum nativum.. in fpatho albo, aut corneo quart. zofo inmixtum, cum Cinnabare, 354. ex Ungaria, 356. ex Bohemia, 361. ex Saxonia, 786, ex ..., Wall.l.c. (3. . fpecim.

323 Hydrargyrum nativum, in Spatho globofo, \& marcafita drufiformi inmixtum, 353. ex Ungaria, Wall. l. c. (2. Specim.

324 Cimmataris, in lapide corneo quartzozo, aut cum fpatho, 357. 365. ex Saxonia, 364. ex Bohemia, jo88. ex .... Wail. 220. (4. '́pecim.

325 Cinnabaris, cum Hydrargyrio, in fpatho, \& quartzo, 1246. ex Palatina, Wall. l. c. (r. r pecim.

326 Cinnabaris, in quartzo, cum Hydrargyrio ', 363 . ex Saxonia, Wall. l. c. (i. fpecim.

327 Cinnabaris, fubdrufiformis, cum pyrite radiante, 1395. ex Carinthia, Wall. l. c. (3. Ppecim.

328 Cinnabaris, druffiformis, in quartzo albo, granulato, fpecimen pulcerrimum, 362, ex Zweybrucken, Wall. $l$. : (I. Tpecim.

329 Cimmabaris, cum Hydrargyrio, \& aurum continente pyo rite \& quartzo, fpecimen pulicerrimum, 1394 . ex $\mathcal{f}_{\text {apan }}$ ( Wall. b. c. (I. fpecim.

330 Arfenicumn nigrum, in cobalto teftaceo, 1398 .ex Saxonia, Wall. 223. \& 225. (I. fpecim.

33I Arfenicum, f. Auripigmentum luteum, \& rubrum, cum Lithargyrio, 152. ex Saxoriza, 767. en Schnebergia, 1687. Ungaria, Wall. 223. \& 224. (4. Ipecim

332 Arjenicum teftaceum, cum galena plumbi, \& Ipatho, 1792. ex St. Andreasberg Hartz, Wall. 225. (x. Tpecim.

333 Arfenicum tefaceum, in lapide arfenicali, cum Cobaits Scorieformi, 1138 . ex Annaberg, Wall. l. c. \& 233 . 334 Arfenicum, r. Teffera arfenicalis, lamellora, femidiapha. na, fusco lutea, cum quartzo albo, I7/2. ex Stolberg, Wail. 226?. (I. fpecim. 


\section{Folfiles. Claffe III,}

317 Mercure Vierge, avec pyrite cuivreux, en grains, comme marcafite, dans une pierre arenaire, 351. d'Hongrie, Wall. l. c. (I. piec.

318 Mercure Vierge, avec Cinnabre, dans sne pierre ferreufe, 350. de Hongrie, Wall. I. c. (I. piec.

319 Mereure Vierge, avec Cinnabre, dans une pierre ferrease, 352. d'Hongrie, Wall. 1. c. (I, piec.

320 Mercure: Vierge, en pierre ferreufe, veinée de quarts, 355. d'Hongrie, Wall. 1. c. (I. piec.

321 Mercure Vierge, melée avec Cinnabre, 1396. de Tyro!, Wall l. c. (I. piec.

322 Mercure Vierge, en Spath blanc, ou en cornée quartzesse? avec du Cinnabre, 354. de Hongrie 356. de Boheme, 36r. de Saxe, 786. de ..., Wall. 1. c. (3. piec.

323 Mercure Vierge, en fpath globuleux, \& marcafite drufz forme, 353. de Hongrie, Wall. 1. c. (2. piec.

324 Cinnabre, en pic ornée quartzeuse, ou avec $\int p a t h, 357$. 365. de Saxe, 364. de Boheme, 1088, de ...., Wall: 220. (4. piec.

325 Cinnabre, \& Mercure Vierge, Jur Jpath, E quartz, 1246. de Palatin, Wall. I. c. (I. piec.

326 Cinnabre, en quartz, avec Mercure Vierge, 363. de Sare, Wall. I c (x. piec.

327 Cinnabre, en forme de drufe, avec pyrite radieux, 1395. de Carimbe, Wall. 1. c. (3. piec.

328 Cinnabre, drufi forme, en quartz granuleux, blanc, tres belle piece, 362. de Zweybrucksn, Wall. 1. c. (I piec.

329 Cinnabre, \&o Mercure Vierge, avec du pyrite, \& quartz contenant de l'or, une tres belle piece, 1394, de Japan, Wall. J. c. (1. pisc.

330 Arfenic noir, en cobalt teftacé, 1393. de Saxe, Walt: 223. 82225 . (I. piec.

3.31 Arfenic, ou Opriment, jcune, \& rouge, avec Lithar: gyne, 152." de Saxe, $76 \%^{\circ}$ de Schneberg, 1087. de Hongrie, Wall. 222, 224 . (4. piec.

332 Arfenic, teftacé, avec galene de plomb, E Spath, r 792 de Mont St. André, Hartz, Wall 225. (4. piec.

333 Arfenic teftacé. en pierre arfenicale, avec Cobalt fcorie forme, Ir 38 d'Annaberg. Wall. I. c. Ë233. ( I piec.

334 Arfenic, orb Mine d'arfenic cubique, lamelleufe, à demi transparante, brun-joune, avec guartz blanc, 1712 . de Stolberg, Wall. 220. (I. piec.

$$
\text { P } 5 \text { 335 Arínic }
$$




\section{Fofjicia. Clafis IIt.}

335 Aifenicum, r. Tefera areenicalis, nigra, drufformi, puls cra, cum fpatho amethyltino, in quartzo albo, 771 . ex Anglia, Wall. l. c. (1. (pecim.

\$36. Arjenicum ferro mineralifatum, T. Pyrites arferiealis albuss? marcafitam mentiêns, \& criftállino druffiformi, ut \& bfeudogalena picea', cum quartzo, 426: 1397. ex Schneherg, Wall. 228. ('2. rpecim:

337 Arfenicum, , C. Pyrites arfenicnlis, in quartzo drufformi, 426. 1142, ex Schreberg, Wall l. o, (2. Specim.

338 Avfenicum, f. Pyrites albus. criftallifatus, cum fpatho pyritofo, Ir 35. ex Schneberg, Wall. 227. (I. fpecim:

339 Arfenicum, fulphure \& cupro mineralifatum, f. Cuprum Nicolai, nec non Pyrites albuis, in Spathn, fpecies pulcerrima, 1666, ex Schneberg, Wall. 227:\& 220. (1. . pecim.

340 Arfenicum, f. Cuprim Nicolai, cum cobalto, \& fpatho arenofo, 1036. ex Saalfeld, 1208. ex Freiberg, Wall: l. c. (2. fpecim.

34t Atfenicum, r. Pyrites albus, in Spatho carnoo, I328. ex Wall. 227 . (r. fpecim.

342 Arfenicum, I Cuprum Nicolai, pulcerrimum, haud commune, drufforme, in corneo quartzofo, cum cobalto chalybeæ, \& flore cobalti viridi; 1781. St. Andreaberg , Wall, 229. \&c. (I. fpecim.

343 Cobaltum dendriticum, in lapide corneo, cum quartze, una cum Cupro Nicolai, \& Pyrite albo inmixtum, politum, I 40r. ex Saalfeld, Wall. 234. (2. Specim.

344 Cobaltum criftallifatum, cum 5patho, 1682, ex Hanau, Wall. l. c. (1. Specim.

345 Cobaltwim criffallifatum, drufiforme, haud commune, pulcerrimum, in lapide corneo, \& quartzo albo, criftalli fpathofo, \& criftallis fpathofis, amethyltinis, I400. ex Saalfeld, Wall. $l$ c. (I. Specim.

346 Cobalium criftallifatum. cum quartzo \& $\&$ patho, 486.488. ex Saalfeld, 1399. ex Freiberg, Wall, l. c. (3. Ipecim.

347 Cobaltrum fpeculare, cum cobalto chalybea, \& floribus cobalti, 489. ex Saxonia, 1206. ex Annaberg, 1207. ex Georgenjfad, Wall. 232. (3. Specim.

348 Cobabtum cirereum, chalybeum, 469. ex Saxonia, Wall. 231. ( 1 . Specim.

349 Cobaltum arfenicale, chalybeum, cun fpatho , 424. ex Saxonia, 470. es Wurtemberga, cum fcoria ad pigmentum coeruleum, inte:viente, Wall. l.c. (3. Specim.

350 Cobaltum arfenicale chalybrum, drufiformis, cum fpa: tho albo, \& flore colorato, 477\% ex Saalfeld, 1213. ex Kunitz, Wall. b. c. (3. Specim. 


\section{Fogiles. Claffe IIT.}

35 Ârfenic, ou Mine d'arfenic cubique, noir, tres bears, ets forme de drufe, avec Jpath amethifte, छे quartz blanc, I ? I 1 . d'Angleterre, Wall. 1. c. (I. piec'.

36 Ar[enic mineralife, ou Pyrite arienicale, refjemblant mas. cajite, avec faux galene, $\S^{2}$ quartz druf forme, 426. 1397. de Schneberg, Wall. 228. (2. piec.

37 Arfenic, ou Peryte arfenical en quartz drufi forme, 426. II42. de Schneberg, Wall. I. c. (2. piec.

38 Arfenic, ou Pyrite blanc, criftallifé, avec jpath pyriteux. II 35. de Schneberg, Wall. 227. (I. piec.

39 Arfenic, de foufre, Eo cuivre minheralifé, ou Cuivre Nico. lai, \& Pyrite blanc, \& en Jpath, tres belle piece, 1666 . de Schneberg, Wall, 227. $0^{2} 229$. (I. piec:

40 Arfenic, ou Cuivre de Nicolai, avec cobalt, \& Spath fablonneux, 1036. de Saalfeld, 1208. de Freiberg, Wall,
1. c. (2. piec.

4 I Arfenic, ou Pyrite blanc, en Jpath cornéé, 1328. de ..., Wall. 227 . (I. piec.

12 Arfenic, ou Cuivre de Nicolai, non commune, $\&^{2}$ tres bear, en drufe, avec cobalt chalybé, E. fleurs de cobalt yert. en quartz corné, 1781. du Mont St. André, Wall. 229:

3 Cobalt arborife, en pierre corntes, melée avec quartz, $\mathrm{E}^{3}$ Cuivre Nicolai, ou mine d'arfenic rouge, \& Pyrite blanc. I 40r. de Saalfeld, Wall. 234 . (2. piec.

14 Cobalt criftallife, ayec Spath, i682. de Hanau, Wall. 1. c. (I. pieć.

5 Cobalt criftallise en forme de drufe, en pierre corrice, है quartz blanc, Spatheux, avec des criftaux vinolettes, Spatheujes.

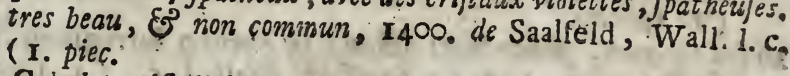

16 Cobalt criftallifé avec quartz, E Spath, 486. 488. de Saalfeld, 1399. de Freiberg, Wall. 1. c. (3. piec.

7 Cobah speculaire, avec le cobalt en forme d'acier, E' fleurs de cobalt, 489 . de Saxe, I 206 . d'Annaberg, $120 \%$. de Georgenftad, Wall: 232 . (3 piec.

8 Cobalt ceridré, en forme d'acier, 469. de Saxe, Wall. 23 r. (I piec: Saxe, 470. de Wurtemberg, avec la' fcorie fervant pour la peinture, Wall. l. c. ( 3 piec. Cobalt arfenical, en forme d'acier, en drufes, avec Jpath
blanc, Es ficursvariez, 477. de Saalfeld, 12 3. de Kunitz, Wall. I. c. (3. piec. 
351 Cobaitum cinereum, chalybeum; cum ipatho albo, \& to ribus cobalti. 476. ex Saalfeld, 1680. ex Stolberg, Wall." l. c. (2. Epecim.

352 Cobaltum, f. flores cobalti virides, in lapide corneo, obfcure arborifans, 1211. ex Saxonia, Wall. 235. ( 1 . fpecim.

353 Cobaltum, f. flóres cobalti violacei, rufi, viridesque, cum Spathis albo, \& pyrite cupreo 484. 487. ex Saalfeld, 1423. ex Saxonia, Wall. l. c. (4. frecim.

354 Cobaltum, f. fiores fimiles, virides, purpurei, atri , \& ochracei, cum fpatho \& arena, 474. ex Saxonia, 47948r. ex Saalfeld, Wall 6 . c. (6. Tpecim.

355 Cobaltum, flores, flavi, cum Auripigmento \& pfeudo galen 2 arfenicail, cum fparho. in lapide c) rneo, 87. ex Meinungen, 475.485. I220. ex Saalfeld, Wall. l. c. E0 236. (4 fpecim. 356 Cobaltum, f. flores, rubri, virides, azurei, in lapide arenario. 478. I224. ex Saalfeld, 1218.ex Saxonia, Wall. l. c. 3. fecim.

357 Cobaltum flavum, nigrum, cum foribus viridibus, rubrisque, in fpatho albo, \& lapide corneo, una, cum terra cobalti fuliginea, 1137.0 II39. ex Saalfeld, I210. ex Saxonia, Wall l.c. (3. Specim.

358 Cobaltum galenæ, cum cobalto drufiformi, \& floribus ruit is, \& fpacho albo, 1412. ex Freiberg, Wall. 235. (1. Specim.

359 Cobaltum galenx, reticulatum, floribus pulcerrime roleis drufiformibus, in corneo cinereo, II4I، ex Saalfeld, Wall l.c. ( 1 . Specim.

360 Cobaltum I flores cobalti, ftellæformes, in lapide ccbalti flavo, \& nigro, 1403. ex Georgenftadt, Wall. l. c. ( I. fpecim

36 i Cobaltum, pracedenti affine, in corneo ferrugineo, cum ipatho albo, 472 ex Saalfeld, Wall. l. c. (1. Ipecim. 362 Cobaltum, $c$. flores fimiles, rubri, flavi nigrique, in cobalto arenofo, 480.483. ex Saalfeld, Wall. l. c. (4; fpecim.

$\$ 63$ Cobaltum. f. flores fimiles, carminei, rofeique, in corneo flavo arenofo, 1405. ex Freiberg, \& Saalfeld, Wall. l. c. (2. fpecim.

364 Cobaltum, f. flores cobalti, rubri, flavo viridesque, in corneo grifeo, 1212. ex Saalfeld, 1223. ex Ungaria, Wall l. c. (2. Specim.

$\$ 65$ Cobaltum, f. flores cobalti, rubri, fuperficiales, cum fpatho rubro, drufiformi, in lapide grifeo, flavoque, 1217. 1404. ex Freiberg, Wall. l. c. (2. Ipecim. 


\section{Fogfiles. Claffe IIt.}

15i Cobalt cendré, en forme d'acier, avec Spatb blanc, EO fleurs de cobalt, 476. de Saalreld, I680. de Stolberg, Wall. 1, c. ( 2 piec.

152 Cobalt, ou fleurs de cobalt verds, en pierre cornée obfcurta ment arborifé, 1211. de Saze, Wall. 235 . (1. piec.

353 Cobalt ou fleurs de cobalt violettes, vertes, roufes, avec: Spath, E9 pyrite cuivreux, 484.487. de Saalfela, 1423. de Saxe, Wall. I. c. (4. piec.

354 Cobalt, ou fleurs pareilles, vertes, pourpres, noires, ocres, avec Jpath, Ẽ Jable, 474. de Saxe, 479-48I de Saalfeld, Wall. I c. (6. piec.

355 Cobalt, ou fleurs de cobalt, jaunes, avec Oprimext, Eं cua bique arfenicale, ou blende, avec Spath, en pierre cornée. 87 de Meinungen, 475. 485. 1220. de Saalfeld" Wall. l. c E 236 . ( 4 piec.

356 Cubalt. ou fleurs de cobalt, rouges, vertes, bleus, en pierre arenaire . 478. 1224. de Saalfeld, 1218. de Saxe, Wall. 1. c. (3. piec.

357 Cobalt jaune, EO noir, avec fleurs vertes, \& rouges, ex Spath blanc, $\mathbb{E}^{\circ}$ pierre cornée, avec de la terre de cobabta fuligineufe, I137. I139. de Saalfeld, I210. de Saxe? Wall. l. c. (3. piec.

358 Cobalt cendré, Ė en forme de drufes, à fleurs rous: gies, en. fpath blanc, I412. de Freiberg, Wall.235. (I. piec.

359 Cobalt cendré, reticulé, à fleur's de belle couleur de roje. ens drufes, fur pierre cornée grije, II $4 \mathrm{I}$, de Saalfeld, Wall. 1. c. ( $:$ piec.

360 Cobatt, ou fleurs de cobalt, en etoile, en pierre de cobalt jaune, EO noir, 1403. de Georgenttadt, Wall. 1. c. (1. piec.

30I Cobalt, Semblable au precedent. en pierre cornée, brun-jaus' notre, aves Spath blanc, 472. de Saalfeld, Wall. 1. c. (1. pisc.

362 C balt, ou fleurs de cobalt, pareilles, rouges, jaunes, nai, res, en cobalt fablonneux $x$ 480. 483. Saalfeld, Wall. 1. c. (4. piec.

363 Cobalt, out fleurs de cobalt, carmins, EO couleur de rofe: en cornée jarne fablonneufe, 1405 . de Freiberg, EO Saal. feld, Wall. 1. c. (2. piec.

364 Cobalt, ou fleurs de cobalt, jaunes, \&O vertes, en pierre cornée grije, I212. de Saalfeld, 1223. d'Hongrie, Wall. 1. c. (2. piec.

305 Cobalt, ou fleurs de cobalt, rouges, fuperficielles, äveé Spath rouge, drufforme, èn pierre grife, ED jarine, 1217 . If04, de Ereiberg, Wall, 1. c. (2. piec. 
366 Cobaltum, f. flores virides, rubri, azurei, in fpatho albo, \& minera cobalti nitente, 47.1. 1214. ex Saalfeld: Wall.l c. (2. Specim.

367 Cobaltum, r.flores in cobalto duro \& fpatho albo - 12rg: ex Saalfeld, I221. ex Cranach, Wall. I c. (2. Ppecim.

368 Cobaltum. f. flores fimiles, I425. ex Saxonia, 1440.ex Hartz, Wall. l. c. (2. Tpecim.

369 Cobaltum, f. flores fimiles, præxcedentium variantes, 1215. ex Saalfeld, 1222. ex Ungaria, Wall. l. c. (2. ipecim.

370 Cobaltunn, f. flores corrulei, virides, flavique, in Spatho albo, r406. ex Georgenfadt, Wall. l. c. (I. fpecim.

37 I Cobaltum, f. flores ccerulei, azurei, cum viridibus, flavisque in fpatho, 1209. 1210. ex Saalfeld, Wall $l . c$. (2. fpecim.

372 Cobaltum, f. flores azurei, viridesque , in fpatho drufiformi albo, 1699. ex Saalfeld, Wall. l. c. (1. Specim.

373 Cobaltum, f. flores fimiles, cum cobalto galena, \& correo arenofo, 473 ex Saalfeld, Wall. l. c. (I. fpecim.

374 Antimonium ftriatum, quafi nativum, in lapide corneo quartzofo, 1408. ex Saxonia, Wall. 237 ? (I. fpecim.

375 Antimonium rude, \& arguce friatum, cum fulphure in lapide corneo, 416. ex Hartz, 417. 788. ex Saxonia, Wall. 238. (3. Specim.

375 Antinsonium rude, \& tenue ftriatum, ftellatumque, cum regulo antimonii, 427. 789. I145. ex Ilmenau, I205. ex Anglia, Wall. l. co. (4. Tpecim.

377 Antimonium rude friatim, of granulatum, cum fulphure, in corneo quartzofo martiali, 787.790 . ex Saxonia , 1737. ex Bohenia, Wall. l. c. (3. Ipecim.

378 Antimonium fitriatum, f. plumofum, ftriis latioribus, tenuioribusque, cum antimonio colorato, purpureo rubro, in quartzo fpathofo albo, 419. ex Bohemia, Wall. 239? (1.) fpecim.

379 Antimonium, finile , cüm minera antimonii folida, 4Is. ex Bohemia; Wall. b. c. \& 240. (1. Ipecim.

380 Antimonium tenue frellatum, \& ftriatum, criftallifatumque, cum fulphure in quartzo criftallifato, 1407 . ex Saxomia, Wall. 24 r? (r. Ppecim.

380 Antimonium, $\therefore$ flores antimonii colorati, rubri, fibrillofi, in minera, cuin fu'phure, in quartzo, I4c9. 1410. ex Saxonia, Wall. 239? 242. (2. Specim.

SBI Antimonium tenue, ex rubro flavo ftriatum, pavoninum, I100. ex Braunsdorf, Wall. b. c. (2. fpecim.

382 Wismuth- 


\section{Folfiles. Clafle III.}

366 Cobalt, ou fleurs de cobalt, vertes, rouges, bleues, en Spath blanc, avec cobalt mineralifé, $47 \mathrm{I}$. 1214. de Saalfeld, Wall. 1. c. (2. piec.

367 Cobalt, oufleurs de cobalt, pareilles, en cobalt dur, $8^{\circ}$ Spath blanc, 1219. de Saalfeld, I221. de Cranach, Wall. 1. c. (2. piec.

368 Cobalt, ou pareilles fleurs, 1,425. de Saze, 1440. du Wartz, Wall. I. c. (2. piec.

369 Cobalt, ou pareilles fleurs, varieté des precedens, 1215. de Saalfeld, 1222. de Hongrie, Wall. 1. c. ( 2 piec.

370 Cobalt, ou fleurs de cobalt bleues, azurées celadons, \& jaunes, en Jpath blanc, I406. de Georgenftadt, Wall. 1. c. (I. piec.

37r Cobalt, ou fleurs de cobalt bleues, azurées, celadons, jaunes, en jpath blanc, 1209. 1210. de Saalfeld, Wall. 1. c. (2. piec.

$37^{2}$ Cobalt, ou fleurs de cobalt azurées, vertes, en $\int p a t h$, blanc, drufe forme, 1699. de Saalfeld, Wall. 1. c. (2. piec.

373 Cobalt, ou pareilles fleurs, avec cobalt de galene, \& cornée fablonneufe, 473. de Saalfeld, Wal!. 1. c. (1. piec.

374 Antimoine, $a$ grandes ftries, comme natif, en pierre coro née quartzeufe. 1408. de Saxe, Wall 237? (1. piec.

375 Antimoine rude. E finement ftriee, avec foufre, en pierre cornée, 416. de Hartz, 417. 788. de Saxe, Wall, 238. (3. piec.

376 Antimoine groffe, \& à ftries fines, en etoile, avec le roitelet d'antimoine, 427. 789. I I 45. de limenau, I 205. d'Angleterre, Wall. I. c. (4. piec.

377 Antimoine rude ftrie, Eo granulé, avec Soufre, en cornée quartzeufe, contenant du fer, 787.790. de Saxe, 1737. de Boheme, Wall. 1. c. (3. piec.

$37^{8}$ Antimoine ftrié, d ftries larges, \& fines, avec antimoine colorée, pourpré, rouge, en quartz jpatheux blanc, 419 . de Boheme, Wall. 239.? 240. (r. piec.

379 Antimoine pareil, avec mine d'antimoine, en acier, 4I 4 : de Boheme, Wall. I. c. E\$ 240 . (1. piec.

380 Antimoine, mince, frie, en etoile, criftallifé. avec foufre, en quartz criftallife, 1407. de Saxe, Wall, 241? (I piec.

380 Antimoine, ou fleurs d'antimoine rouges, fibreufes, dans $\int \frac{6}{6}$ mine, avec joufre, en quartz, I 409. I410. de Saxe, Wall. 239? 242. (2. piec.

38I Antimnine. \& fries fines, joun-rouge, d queuse de pan, i100. de Braunsdorf, Wall, l. c. 52. piec. 


\section{$\$ 88$}

Folfilia. Clafis III.

382 Wismuthum nativum? granulis infperfis in wismutho, ope lampadis liquefacto, 1200. ex Saxonic, Wall. 243 ? 244. (1. Ipecim.

383 Wismuthum cinereum, folidum, cum wismuthi regulo furo, 419 . 420. ex Saxonia, Wall. 244. (2. Specim.

384 Wismuthunn cinereum, cum floribus, \& pyrite aurifero, in quartzo, I:43. ex Sclhineberg, I4Ir. ex Saxonia, Wali. 244. 245. (2. fpecim.

385 Wismuthum cinereum, arborifatum , in corneo rubro jarpideo, polito, I I 47. ex Bayreuth, Wall. 244. (I. ipecim.

386 Wismuthum cinereum, arboilfatum . in Spatho albo: polito, 418. ex Saxonia, Wall. l. c. (I. f́pecim.

387 Wismut thuni cinereum, granulofum, cum pyrite granulofo, in Spatho albo drufiformi, haud communi. 1783. ex St. Andreasberg, Wall. l. c. (I. Specim.

388 Zincum, fquamorum ferreum, quafi tlores fuperficiales antimonii azurei mentiens, 1736. ex Suecia, Wall. 247. (I. ́pecim.

389 Zincum, galeneticum, ferrum continens, in quartzo, cum fpatho, \& pyrite cupreo, 50I. ex Saxionia, 539. ex Hartz, Wall. l. c. (2. fpecin.

390 Zincum, C.PSeudogalena, obfcura, fquamulis tenuioribus, cum quartzo a!bo, I677. ex Stolberg, Wall. 249. (I. Ipecim:

391 Zincim, f. P feudogalena rubens. cum fpatho, \& zinco fufo, 42r. I68I. ex Stolberg, Wall, 250. (2. fpecim.

392 Zincum, f. Pfeudogalena, nigra, picea, drufiformis, in $\mathrm{p}$ patho criftallino, hexar gulari truncato, 1710. ex Anglia, Wall. 249. (x. Specim.

393 Zincum fufum, 422. ex Saxonia, Wall. l.c. (I. fpecim. 394 Ferrum teffulatum, quadratum, \& cubicum, magnum fpecimen, 1637. ex Cap. Bon. Spei, Wall. 252? (2 fpecim.

395 Ferrun teffulatum, f. fimile, \& multa minora, varix magnitudinis, ex 1825 . Cap. Bon. Spei, Wall. l. c. (...

396 Ferrum criftallifatum, angulofum, ex viridi azureo, rubroque nitens, pulcerrimum, 1743. ex Lotharingia, Wall. 252. (I. Specim.

397 Ferrum criftallifatum, precedenti affine, cum mica ferrea, \& criftallis dinfi formibus, apice subris, haud commune, 1.742. ex Lotiaringia, Wall. b. c. ( I. fpecim.

398 Ferrum criftallifatum, fimile, drufformis, in hamatita nigro. cum f́patho alto, \& minera ferrea livida, 1778 . ex Iblefeld, Wall, l.c. E' 266 . (I. fpecim.

399 Ferrum fquamofo criflallinum, rufo fusco nitens, pulcerritmum 1655 . ex Naffovie, Walli l. c. (r. fpecim. 402 Ferrun: 


\section{Fofiles. Clafe III.}

382 Bismuth natif? a grains infperfés, en bismuth fondu d la lampe, I200. de Sdxe, Wall. 243 ? 244. (1. piec.

383 Bismuth cendre, Solide, avec bismuth le roitelet fondru, 419: 420. de Saxe, Wall. 244. (2. piec.

384 Bismuth cendré, E quastz, avec des feurs, EO pyrite cons tenant d'or, I143. de Schneberg, I4IJ. de Saxe, Wall. 244. 245. (2. piec.

385 Bismuth cendré, arborifé, Ẽ pierre jaspe cornée, rouge polie, 1147. de Bayreuth, Wall. 244. (I. piec.

386 Bismuth cendré, arborifé, en Jpatb blanc, posi, 418. de Saxe, Wall. I. c. (I. piec.

387 Bismuth cendré, granulé, avec pyrite granulée en dirife. en Jpath blanc, une grande pioce, 1783. de Mont St, André, Wall. I. c. (I. piec.

388 Zinc ecaillewx, comme du fer, reprefentant des fleirs azurés d'antinoine, 1736. de Suede, Wall. 247. (1. piec,

389 Zinc à galene, contenant du fer, en quartz, avec Spath, E pyrite cuivreufe, 501. de Saxe, 539, de Hartz, Wall. 1. c. (2. piec.

390 Blende, à ecailles plus minces, avec quartz blanc, 1677 . de Stolberg, Wall. 249. (I. piec.

91 Blende rouge. ou faux Galene, avec Spath, \& zinc fondu, 42I. I08I! de Stolberg, Wall. 250, ( 2 piec.

392 Blende ou faux Galene, noire, en drufe, Jur Spath criftallifé, hexangulaire tronquée, 1710. d'Angleterre, Wall. 249, piec.

93 Zinc fondu, 422. de Saxe, Wall. 1. c. (I. piec.

94 Fer cubique, quarré, 1637. du Cap de Bonne Efperance; Wall 2s3.? (2. piec.

95 Fer cubique, ou plufieurs autres pieces de diverfe grandeur, 3825. du Cap de Bonne Elperance, Wall. I. c. ( .

96 Fer criftallife, anguleux, chatoyante, en verd bleu, E rouge, tres beau, I743. de Lorraine, Wall. 252. (I. piec,

27 Fer criftallijé, approchant d la precedente, avec mica de fer, EO criftaux blancs, pointe rouges, non commun, 1742. de Lorraine, Wall.I c. (I. piec.

98 Fer criftallifé, pareil, micacé en drufe, avec hématite. noire, 1778. d'lhleveld, Wall. 1. c. \& 266. (I.: piec.

99 Fer criftallifé, rouge decailles, tres beaus 1085. de Nase fau, Wall. 1, c. (I. piec. 
400 Ferrum, f. fpathum martiale, criftallinum, drufiformen nigro fuscum, 1413. ex Schmalkalden, Wall 254? ( I. Kpecim.

401 Ferrum fquamofum, \& tenue granulatum, cum guarizo, \& fpacho drufiforme, pyriteque, sta. ex Saxonia, 1233. ex Wa!lis, Wall. l. c. (2. Ipecim.

402 Ferrum rubrum, tenue granuiatum, ad fpathum pellucido fuscum, albumque, criftallifatum, 1:94. ex llilefild, Wall. b. c. (I. Specim.

403 Ferrum, vel mineta ferri albi germinans, intra duo Itrata, 'pecimen pulcerrimum, I+19. ex Steyermarck, Wall. 253 (I. Speçio.

404 Ferrum album, germinans, cum ramis tenuioribus, in hamatite druieo, 515. ex Steyermarck, Wall. b. c. (I. ipecim.

405 Ferrom fquamofum, plumbum, \& antimonium mentiens, in asbefto pyriticalo, permugnus, 1439. ex . ., Wall. 254. ( $\mathrm{r}$. pecin.

406 Ferrim fpatbiforme nigrum, in (patho albolameliofo, \& flavo ferreo, 726. ex...1703. ex Camsdorf, Wall 253. \& 255. (2. rpecim.

407 Ferrum nigrum, tenue granulatum, cum pyrite, \& quartzo ferreo, 407. ex Suscia, 507. ex Hartz, Wall. 254. (2. Specim.

408 Ferrum giifeo nigrum, granulatum, compactum, I 4 I 4. ex Siberio, Wall. l. c. ( $\mathrm{r}$ : fpecim.

fog Ferrum, præcedentl affine, in hamatite nigro, \& lapide ferre?, ochraceo, 1777. ex St. Antreasberg, Wall. l. c. (I fpecim.

410 Ferrum mineralifatum, 1. Magnes, I 202. ex Suecia, Wall. 259. (I. fpecim.

4II Ferrum mineralifatum, f. Magnes, fimilis magnitudinis, I4I2. ex Suecia, Wall. l. c. (1. fnecim.

412 Ferrum chalybeatum, nitens, granulofum, 495. ex Schmalkalden, Wall. 254. (I. Specim.

413 Ferrum nitens granulofum, rubefcens, cum fcoria, 1743. ex Leodio, Wall. l. c. (I. Specim.

414 Ferrum fpeculare, compactum, II 46 . ex Graefenthal, Wall. 257. (1. Specim.

415 Ferrum fpeculare, cum hæmatite globolo rubro, 1240. ex Hartz. Wall. 257. 258. (3. โpecim.

416 Ferrum, r. Hamatites, \& ferrum granulare, 493. 498. ex Bohemia, Wall. 258. (2 Tpecim.

417 Ferrum, f. Hamatizes niger, nitens, 490. ex Germania, Wall. l. G. (I. Specim. 
100 Fer, ou jpath ferreux, criftallifé, en drufe, brun foncé, 1413. de Schmalkalden, Wall. 254? (1. piec.

401 Fer ecailleux, mince, en grains, avec quartz, \& Spath err drufe, Ej pyrite, 510 de Saxe, 1233. de Wallis, Wall. 1.c. (2. piec.

402 Fer rouge, fin en grains, fur Spath brun, E blanc, criffallifé, 1794. d'Ihlefeldr, Wall. 1. c. Cir. prec.

403 Fer blanc, vaineau tres grand, entre deux couches, $1419^{\circ}$ de Stiermarc, Wall. 253. (I. piec.

404 Fer blanc, avec branches plus fines, fur hèmatite noir, 515. de Stiermarc, Wall. 1. c. (I. pisc.

405 Fer ecailleux, osı relfemblant, au plomb, E? antimoine, fur asbefte pyriteufe, 1439. de .... Wall. 254. (I. piec.

405 Fer Jpathi forme, noir, en Jpath, blanc lameilleux, \& fer jaune, 726 .de... 1703. de Camsdorf, Wall. 253. E3255. (2. piec.

$407 \mathrm{Fer}$, gramuleux, noir, avec pyrite, Eq quartz de fer ,407. de Suede 507. de Hartz, Wall. 254. (2. piec.

408 Fer granule gris, noir, compadie, I4I 4. de Syberie, Wall. I. c. (1. piec.

409 Fer, Semblable au precedent, en hẻmatitè noiré. \&2 pierre de fer, penetrée d'ocre, I777. du Mont St. André, Wall. 1. c. (1. piec.

q10 Fer mineralifé, ou Pierre d'Aiman; grand, 1202. dé Suede, Wall. 259. (r. piec.

fII Fer mineralife, ou Pierre d'Aiman, de pareil grandeur, 1412. de Suede, Wall. 1. c. (I. piec.

fí 2 Fer fplesdide granilé; 495. de Schmalkalden, Wall. 254, (I. piec.

II3 Fer Jplendide, rougeatre granulé, avec Jcorie, I643. de Liege, Wall. 1. c. (I. piec.

14 Fer fpeculaire, compacte, II 46 . de Græfenthal, Wall. 257. (x. piec.

I5 Fer fpeculaire, avac hématite globuleufe fanguine, 1240.d6 Hartz, Wall, 257. 258. (3. piec.

II F Fer, ou Hénatite, E fer granulé, 493. 498. de Boheme, Wall. 258. (2. piec.

17 Fer, ou Hératite, noir, brillant, 490. d'Allemagne Wall. I. c. (I. piec. 
418 Ferrum, fo. Hcematites, globulofus niger, 492. ex Bohemia :-Wall. l. c. (I. Ipecim.

4 I9 Ferrum, f. Hematites fimilis, 494. ex Bohemia, 495. ex Saxonia, Wall. l.c. (2. Specim.

420 Ferrum, f. Hematites, nitens, globulari nigrum, 49! ex India Orient. Wall. l. c (2 Specim.

42t Ferrum, f. Hematites niger fatiscens, 1415. ex Nafjavia, Wall. l. c. (1. fpecim.

422 Ferrum, f. Hamatites, braeteatus cellularis, i237. ex Suecia, 1656. ex Cap. Boin. Spei, 'Wall. l. c. (2. fpecim.

423 E'errum. f. Hamatites niger, finilis pracedenti, $1657^{\circ}$ ex Nafjovia, Wall l. c. (I. Rpecim.

424. Ferrum, f. Hamatites niger, arenam fimulans, cum lamina chalybea, 6r4. 1658. ex Nalfovia, Wall. l. e. (2. fpecim.

425 Ferrum quafi nativum, cum ochra viridi, \& ferrugi. nea, 504. ex Hartz, Wall. 25t. (2. Kpecim.

426 Ferrum mineralifacum micans, cum Homatite pigro, Lithomarga, \& lapide ferreo, 359502 . ex Bohemia, 1793. ex Ihlefeld, Wall. 255. (3. Specim.

427 Ferrum. f. Hamatites, fatiscens, \& cchra ferri, 505. ex Bohemia, 1236. ex Scotia. 1238. ex Hartz, 1239, ex Anglia. Wall 258. (5. Specim.

428. Ferrum, f. Lapis martinlis, cum fpatho drufiformi albo, \& Hematite faticente, 1520. EX Norvegia, 1738. ex Daventria, 490. 1741. ex Germania, Wall. l.c. (3. fpecim.

429 Ferrun, r. Lapis martialis ruber . cum Hematite brac. teato, ochra tecto, 1410. ex Wolffinhous, 1641. ex Aquisgrano, Wall.'l. c. (2. fpecim.

$43^{\circ}$ Ferrum, f. Arena ferri nigra, 728. ex Italia, Wall. 260. (I. Specim.

43 I Ferrum, marcafitam numismalem mentiens, 512. ex Saxonia, I417. ex Tyrol, Wall. 26, . (2. Ppecim.

432 Ferrum, lacuftris, numismalis, 1638. ex Cap. Bon. Spei, Wall. l. e. (plurima rpecim.

433 Ferrum, fimile præcedenti, fed pifformis, in minera ferri fubaquofa, cuin lapidibus quadratis, $154.508 \mathrm{ex}$ Saxonia, 509. 510.513. ex Osnabrugo, Wall. l. c. (plurima fpecim.

434 Ferrum, fubaquofum, 503. 506. ex Bohemia, 1656. ex Cap. Bon. Spei, Wall. l. c. (4. fpecim.

435 Ferrum paluftre \& ochra, II33, ex Sylva Ducis, I4I8. ex Cypro, Wall. 201. E 262. (2. fpecim. 


\section{Foffles, Clafe IIL.}

A218 Fer, ou Hématite, globuleufe noire, 492. de Boheme, Wall. 1. c. (1. piec.

frí Fer, ou Hématite, pareille, 494. de Boheme, 405. de Saxe, Wall. I. c. (2. piec.

720 Fer, ou Hématite, brillant, globulaire, noire, 49r. des Indes Orientales, Wall. I. c. ( 2 piec.

121 Fer, ou Hématite, tombant en poudre, 1415. de Naffau, Wall. I. c. (I. piec.

122 Fer, ou Hématite en forme de tubes, I237. de la Suede, 1656. du Cap de Bonne Efperance, Wall. 1. c. (I. piec.

123 Fer, ou Hématite, noire, pareille, 757. de Naffau, Wall. 1. c. (r.piec.

124 Fer, ou Hématite, noire, relfemblant le fable, avec lamelle d'acier, 614. 1658. de Naffau, Wall. 1. c. (2. piec.

.25 Fer, comme natif, avec ocre, verd, \& ferrugineux, 504. de Hartz, Wall. 25r. (2. piec

$.26 \mathrm{Fer}$, mineralifé, brillant, avec Hématite , noire, Lithemarge, ED pierre ferreufe, 350. 502. de Boheme, 1793. de Jhlefeld, Wall. 255. (3. pirc.

.27 Fer, ou Hématite, Eo ocre de fer, 505. de Boheme, 1236. de i'Ecofre, I 238. de Hartz, I 239. d'Angleterre, Wall, 258. (5. piec.

.22 Fer, ou Pierres ferreufes, avec fpath, blanc en druje, $E^{\text {s }}$ Hématite, 1529. de Norvegue, 1738. de, Deveater; 490. 1741. d'Allenagne, Wall. i. c. (3. piec.

29 Fer, ou Pierre ferreux, rouge, avec Hématite, tubuleusfe, couverte d'ocre. 14:6. de Wolfenhaus, I64I. d Aix la Chapelle, Wall. 1. c. (2. piec.

so Fer, ou Sable de fer noir, 728. d'Italie, Wall. 260. (2. piec.

31 Fer, lenticulaire, reffemblant du marcafite, 512 . de Saxe, 1717. de Tyrol, Wall. 26r. (2. piec.

$32 \mathrm{Fer}$, limonneufe, lenticulaire, en forme de feve, 1638 . dus Cap de Bonne Eiperance, Wall. l. c. (plufieurs pieces.

$33 \mathrm{Fer}$. Semblable au precedent, dans mine de fer limonnerufe, ou bourbeufe, avec pierres quarrées; 154. 508. de Saxe, 909. 510.513 . d'Osnabrug. (pirfieurs pieces.

34. Fer, ou mine de fer bourbeufe, 503. 506. de Boheme, 1656. du Cap de Bonne Efperance, Wall. l. c. (4. piec.

35 Fer, ou mine de fer bourbeufe, EO ocre. 1133. dus Bois le Duc, I4I8. de Cypre, Wall. 26r. Eं262. (2. piec. Q 93 
594

436 Ferrum fatiscens, cum fpatho, \& Hamatite nigro, I 234 ex Wallis, 1235. ex Hartz, Wall. l. c. (2. Ppecim.

437 Ferrsm lapideum, cum ochra, 1739. 1740. ex Tranjil. vania, Wall. l. c. (2. fpecim.

438 Ferrum exefum, luteum, I 639. ex Aquisgrano, Wall. b.c. (r. Specim.

439 Ferrum exefum, retiforme, 1640. ex Gquisgrano, Wall. b. c. (1. fpecim.

440 Eerrum fubaquofum, ochra arenacea penetratum, 725 . ex ..., Wall. $261 ?$ ( 1 . Specim.

44r Ferrum, f. Ochra, rubra cretacea, \& flava, 83 84. ex Coburgo, Ir 8. ex Chemnitz, Wall. 262. (4. Specim

442 Ferrum f. Lapis martialis ochraceus, Conchis petrificatis repletum, 1642. ex Aquisgrano, Wall. l. c. (1. rpecim.

443 Ferrum, f. Lapis martialis ochraceus, cum præcedentibus Conchis, 1642. ex Aquisgrano, Wall. b. c. (2. Ppecim.

444 Ferrum, r, Magnefa, fquamofa pyramidalis lamellofa, in lapide arenofo calcareo, flavo, 1779. 1780. ex Osta. bruga, Wall.264. (2. Ppecim.

445 Ferrum. C. Magnesia, ftriata, in quartzo, 1678. ex Thuringo, Wall. I. c. (I , rpecim.

446 Ferrum, f. Magnefia, ex centro ftriata, 1704. ex Thus ringo, Wall. l. c. (r. ṕpecim.

447 Cuprum nativum, granulatum, in Ipatho albo foliaceo, fpecimen dives \& magnum, 1420. ex Marck-Bayreuth, IVall.267. (I. Specim.

448 Cuprum nativum dendroites, \& granulatum, ramofum, in lapide arenaceo, 1683. ex Suecia, Wall. l. c. (I. foecim.

449 Cuprum nativum dendroites, in quartzi albi drufa, cum ochra cupri viridi, \& azurea, 530. ex Marck-Bayreuth, Wall. l. e. (I. Specim.

450 Cuprum nativrum granulatum, in fpatho quartzofo, cum ochra viridi fuperficiali, 352. ex Marck-Bayreuth, Wall. l. c. (2. Tpecim.

451 Cuprum nativum dendroites, cum viridi fuperficiali, 555. ex Suecia, Wall. b. c. (I. Specim.

452 Cuprum nativum foliaceum. \& granulatum, in corneo fiffili, Ir48. ex Eislecen, Wall. b. c. (1. Ipecim.

453 Cuprum notivum granulatum, \& foliaceum, in corneo, cum ochra in guartzo deufiformi. cum viridi montano, \& flores plumbi albi, I 7C5. ex Càmsdorf, Wall. l. c. (I. Ipecion. 


\section{Fofliles. Clafle III.}

436 Fer fondu, avec Spath, \& Hématite noire, 1234. de Wallis, I 235. de Haria, Wall. 1. c. (2. piec.

337 Fer pierreux, avec ocre, 1739. 1740. d'Overrisfel, Wall, i. c. (2. piec.

438 Fer vermoulu, our rouge Ẽ jaume, I'339. d’Aix la Chapelle. Wall. I. c. (r. piec.

439 Fer vermoulu, ou rouge, en refeau, 1640 d'Aix la Chapelle. Wail. I. c. (I piec.

440 Fer limonneux, penetrie d'ocre, en fable, 725. de .... Wall. $26 \mathrm{I}$ ? (1. piec.

$44 \mathrm{I}$ Fer, au Ocre rouge, $\mathcal{E O}^{\circ}$ jaune craye 83. 84. de Cobourg, 118. de Chemnitz, Wall. 262. (4 piec

442 Fer, ou Pierre martiale d'ocre, remplie de Coquilles petrifiées, 1642. d'Aix la Chapeile, Wail. 1. c. (1. piec.

443 Fer, ou Pierre martiale d'ocre avec de precedentes Coquilles, 642. d'Aix la Chapelle, Wall. I. c. (2. piec.

414 Fer, ou Magnefie, écailleufe, pyramidale, lamelleufe, en pierre calcaire jaune, EO grife 1779. 1780. d'Osnabrug, Wall. 264. (2. piec.

445 Fer, ou Magnefie, ftriée, en quartz, 1678. de Thuringe, Wall. I.c. (r.piec.

$445 \mathrm{Fer}$, on Magnefie, pareille, en etoile, ou frice, 1704: de Thurirge, Wall, I. c. (I. piec.

447 Cuivre natif gramuleux, en Jpath blanc, feuilleté, grande, $\mathrm{c}^{3}$ riche piece, 1420. de Marck-Bayreuth, Wall. 26\%. (r. piec.

448 Cnivre natif grarnuleux, branchue, en pierre arenaire 1683. de Suede, Wall. I c. (r. piec.

449 Cuivre natif branchue, en drufe. de quartz blanc, avec ocre de cuivre, vertes, E̊ bleues, 530. de Marck-Bay. reuth, Wall. i. c. (r. piec.

450 Curvre natif granuleux, en fpath quartzeux, avec ocre yerd fuperficielle, 35.2. de Marcts-Bayreuth, Wall, I. c。 (2. piec.

451 Cuivre natif brarchue, avec ocre verd fuperficiel, 555. de Suede, Wall 1. c. (2. piec.

452 Cuivre natif feuilleté, Ė granuleux, en pierre cornée fisfile, Ir 48. d’Eisleben, Wiail 1. c. (r. piec.

453 Cuivre natif, granuleux. EO featilleté, dans pierre cornée. avec ocre, en quartz drufiforme, avec verd de gris, ED fleurs de plomb-blanc, 1705. de Camsdorf, Wall. I. c. (1. piec. 
454 Cuprum nativum, dendroides, \& Precipitatum, cum viridi montano, \& galena plumbi micante, in quartzo, 538. ex Saxonia. Wall. 267. \& 268. (I. fpecim.

455 Cuprum prcecipitatum, 554. ex Neufol in Ungaria, Wall. 208. (1. 'pecim.

456 Cuprum precipitatum, affine, 543. ex Ungaria, Wall. l c. (3. rpecim

457. Cuprum precipitatum, in corneo grifeo quartzofo, 543. ex Nerfol in Ungaria, 550. ex Saxonia, Wall. $l_{\text {. }}$. (2. fpecim.

458 Cuprum, 'fufum flavum, Zinnopolum dietum, I422. ex Srxoria, Wall. 3. (1. Specim.

459 Cuprum f. Malachites, faturate viridis, 1431 ex Saxo. nia, Wall. 269 . (I. fpeclm.

460 Cuprum, f. Malachites palide virens, five ftalactites cupreus, II5I. ex Freiberg, Wall. l. e. (I. fpecim.

461 Cuprum, r. Malachites, faturate \& dilute virentes, 522. 15:5. ex Hartz. Wall l. c. (.5. fpecim.

462 Cuprum, r. Esugo nativa terrea, cum ochra \& fpatho, 523. ex Hartz, 1430. ex Nieww-York, Wall. l. co (3. ipecim.

463 Cuprum, f. Errugo nativa terrea, velutina, in Spatho drufif rmi, \& quartzo ochraceo, 532. ex Saxonia, 533. ex Ungaria, Wall l. c. (2. fpecim.

464 Cuprum $f_{0}$ Erugo nativa terrea, fuperficiale, in guart: 20 martiali nchraceo, cum floribus plumbi albis, 407. ex Hartz Wall. l. c, (1. Frecim.

465 Cuprum, f EErugo montana, in quartzo fpatholo granulato 52^. ex Hartz Wall l. c. (I. Ipecim.

466 Cuprum, $f$. Erugo nativa, fuperficiale, in cupro fufo, in criftailis niridis prismaticis, 1229. ex Anglia, 1684. ex Ungaria, Wall. l. c. 2. Ppecim.

467 Cuprum, f. Elrugo nativa, \& Ceruleum montanum, in minera cupri flava. 548 ex Saxonia, 1228. ex Anglia, Wall. 269. 270. (2. Ppecim.

468 Cuprum, $\mathrm{f}$. Erugo nativa, cum ochra flava, in lapide corneo, cuprum continente, 528.551. ex Saxonia, Wall 269 (5. Rpecim.

469 Cuprum, I Cerruleum lazureum, in fpatho, cum ochra, \& aliud drufiforme, in malachice polito, 529. ex MarckBayreuth, 1796. ex Claustbal, Wall. 270. \& 271. ( 2. fpecim.

470 Cuprum, f. LErugo nativa, \& Ceruleum montanum, in quart20 granulofo, \& minera cupri alba, 531. ex Saxonia, 1326. Ex Scotio, Wall. I. o. (2. Epecim. 


\section{Fofiles. Clafe III.}

454 Cuivre natif, branchue, E Precipité, avec verd de gris \$. E' galenie de plomb trillant, en quartz, 538 . de Suxe, Wali. 267.8268 . (1. piec.

455 Cuivre preci,ité, 554. de Neufol, en Hongrie, Wall. 268. (3. piec.

456 Cuivre precip.té, pareil, 543. d'Hongrie, Wall.'1. ca (1. piec

457 Cuivre precipité, en pierre comée grife, quirizun/e, 543. de Neufo en Hongrie, 550. de Saxe, Wall. 1.c. (2. piec. 458 Cuivre fondu, jaune, ou Zinrople, 1422. de Saxe, Wall. o. (1. piec.

459 Cuivre, ous Malachite, verdfoncé, $143 \mathrm{I}$. de Saxe, Wait. 269 (1. piec

460 Cuivre, 0:6 Malachite, verd pale, ous ftalaciite cuivreux, 1:51. de Fre berg, Will 1 c. ri. piec.

461 Cuivre. ou Malachite pareilles. d'un verd pale, Ė objcast, 522 1515. de Hartz, Wa!l 1. c. (5. piec

462 Cuivie, ou Verd de Montagne, veloute, avec spath, $8^{\circ}$ ocre, 523. de Hartz, 1430. de Nieuw York, Wall, 1. c. 3 piec.

$\Delta 63$ Cuivre, ors Verd de Montagne, velouté, fur Jpath, en forme de drufe, Ẽ quartz d'ocre, 532, de Saxe, 53j. ae Hongrie, Wal. 1. c. (2. piec.

464 Cuivre, ou Verd de Montagne, Juperficiel. Jur quartz martiale docre, EO fleurs blanches de plomb, 407. de Hartz; Wall. I, c. (I. piec.

465 Cuivre, ou Verd de Montagne, en quartz Jpatheux, granuleux, s24. de Hartz, Wall. 1. c. (x. piec.

466 Cuivre. ou Verd de Montagne, Superficiel, Jur cuisre fondu, en criftaux prismatiques, transparans, 1220. d'Aa. gleterre, 1684. d'Hongrie, Wall. 1. c. (2. pec.

467. Cuivre, oti Verd de Montagne, Ẽ B'eu de Montagne: en mine de cuivre jaune, 548. de Saxe, 1228. d'Angleterre, Wall. 269270. (2. piec.

468 Cuivre, ou Verd de Montagno, avec ocre jaune, en fierre. cornee, de cuivre contenant du cuivre, 528. $55 \mathrm{I}$. de Sase, Wall 209. (5. piec.

469 Cuivre, ou Bleu de Montagne azuré, en Jpath, avec ocre, Ẽ au're, en forme de drufe, fur zus malachite polie, 529. d: Marck-Bayreuth, I 756. de Clausthal; Wall. 270. छٔ 27i. (2. piec.

$47^{\circ}$ Cuivre, ou Verd de Montagne, Ẽ Bleu de Montagne, en quartz granuleux, E⿱ mine de cuivre blanche, $53 \%$. dé Saxe, 1226. d'Ecorte, Wall. 1. c. (2. piec. 
171 Cuprum, f. Ceruleum lazureum, drufiforme, in fate quarizuro, cum galena plumbi, 406. ex Saxonia, 535. ex Saalfeld, Wall. l. c. (2. f,ecim.

472 Cuprum, r. Caruleum montanum, \& EErugo nativa, cum. floribus plumbi, \& ochra cupri drutitormi, 1432. ex Ungaria. Wall, l. c. (I foecim.

473 Creprim, fo. Exrago rativa, cum ochra cupri, 534. ex Saxonia, Wall. l. c. (2. Cpecim.

474 Cuprum, f. Creruleum lazureum, \& Erugo nativa, in la. pide lameliari arenufo, \& pyrite auico, 557. I $456 . \mathrm{ex}$ Germania, Wall. l. c. (2. Tpecim.

475 Caprum, f. Lapis Lazuli, cum pyrite aureo, ... ex India Orient Wall. $9^{\wedge}$. (6. Specin.

476 Cuprum flsvum, diverficolor, vulgo cauda pavonina violacea fuperficiale, in quartzo drufiforosi, \& lapide corneo, $1744^{\circ}$ ex Bergia, Wall 276. (1. Ppecim.

$4 i 7$ Cuprum grifeum, diverficolor, five cauda pavonina, azurea, in quartzo albo, 1717. ex Norvegia, Wall. 273. (x fpecim.

$4 i 8$ Cuprum flavum, diverficolor five cauda pavonina violacea, ru'raque, in quartzo albo, 1230. 1424 ex NafJovia, Wall. 276 . (2. Ppecim

479. Cutrum vitrean, cum cupro flavo, 1225. ex Scotia, 1428. 1434. ex Śrecia, Wall 272 . (3. Specim.

480 Cuprum vitreum, \& ftavran, cum ærugine nativa, \& ochra, in lapide arenario, 1132. ex Suecia. Wall. l. c. ( $\mathrm{r}$. fpecim.

48r Cupram vitreum, \& diverficolor flavum, in quartzo, 1426 ex Palatino, Wall l.c. (I riecim.

482 Cuprsm grifeum, \& flavum, in quartzo, I115. III6. ex Norveria, Wall 273. हु) 276 . (2 fpecim.

483 Cutrum hepaticlom, in minera, cum arugine nativa, fuperficiali. \& cusum thavum, reguloque cupri fufo, 526. 527. ex Saxonia, Wall. 274. (5 f pecim.

484 Cuprum hepaticum, \&fhavum, cum arugine nativa, 5:6. 549 ex Saxoria, Waib. l. c (3. F, ecim.

485 Cupmun hejaticum, cum aruginte nativa, \& cuprom radiatum, in fpatho, 553. ex Saxoria, 1427. ex Suecia, Wall. $l$ c (3. Rpecin.

\&s Cuprunx hepatickm, cumochra, \& ærugine nativa, drufiformi, in fpatho, 516. 525.*es Saxonia, Wall. l. c. (2. fpecim.

487 Cup rum album, \& flavum, cum fpatho, \& quartzo albo, in corneolamellnio, 537. Ex Hartz, $54 \mathrm{I}$. Ex Saxonia, 542. ex Breybach, Wall, 275. \& 276 . (3. Specim. 488 Cuprum 


\section{Foliles. Clasfe III.}

47r Cuivre, ou Bleu de Montagne azuré, en forme de drufe. en pierre quartzeufe, avec galenze de plomb, 406. de Saxe, 535. de saalfeli, Wall. 1. c. (2. piec.

472 Cuivre, ou Bleu, EO Verd de Montagne, avec des fleurs de plomb, ED ocre de cuivre, en forme de drufe, I432. de Hongrie Wall. i. c. (1. piiec.

473 Cuivre, ou Verd de Montagne, avec ocre de cuivre, $534^{\circ}$ de Saxe, Wall, I c. (2. piec.

474 Cuivre, ou Bieu lazuré. So Verd de Montagne, en pier re arenaire lamelleufe, do pyrite d'or, 557. 1456. d'Alle? magne, Wall. l.c. ( 2 piec.

475 Cuivre, ou Pierre de Lazur, cvec pyrite d'or, .... des Indes Orientales, Wall. 96 . (6. piec.

476 Cuivre jaune, de differentes coule rs, nommé commanement à queue de pan, violette. en Jur quartz, en forme de drufe, en pierre, connée, 1744 de Berg, Wall. 276. (r. piec.

477 Cuivre gris, de diverfes coulerss, à queue de pan, azyrée, en quartz blanc, III7. de Norvegue, Wall. 273. (I. piec.

478 Cuivre jaune, de differentes coulerurs, à quese de para violette, E9 rouge, en quartz blanc, i230. 1424 , de Nasfau, Wall. 276. (2. piec.

479 Cuivre vitreux, avec cuivre jaune, 1225. d'Ecolfe, 1428. 1434. dé Suede, Wall. 273. ( 3 piec.

480 Cuivre vitreux, ¿2 jaune, avec verd de montagne, 8 ocre, en pierre arenaire, II32. de Suede, Wall. I. c. (r. piec.

48r Cuivre vitreux, E à queue de pan jaune, en quartz; I426. du Palatine, Wall. 1. c. (I. piec.

482 Cuivre gris, E2 cuivre jaune, en quartz, III5. III6. de Norvegue, Wall. 273. E9 27\%. (2. piec.

483 Cuivre hé acique, en mine, avec verd de montagne fus - perficiel, \& cuivre jauns, avec le roitelet fordu, 526 . 527. de Saxe, Wall. 274. (5 piec.

484 Cuivre hépatique, Eे jaune, avec verd de montagne; 546.549 de Saxe, Wall. I. c. (3. piec.

4.85 Cuivre hepatique, avec verd de montagne, Eo cuivre etoilé, en Jpath, 553. de Saxe 1427. de Suede, Wall.l.c. ( 3 piec

486 Cuivre répatique, avee ocre, Ẽ verd de montagne, en forme de drase, en Spath. 5:6. 525. die Saxe, Wall. 1. c. (2. piec.

487 Cuivre blanc, \& jawne, avec Spath. E quartz blane, en pierre cornée lamelleufe, 537. de Hartz, 54I. de Saxe, 542. de Breybach. Wall, $275.8^{2} 276$. (3. piec.

488 Cuivre 
488 Cuprum hepaticune, \& flavum, cum ærugine nativa, \& e ochra, 679. tx Bergia, 1232. ex Saxonia, 1795. ex Brunsuico, Wall. 274. \& 276 . (3. Specim.

489 Cuprum flavum, diverficolor, \& xugine nativa, ochraque cupri, 153. 5:6 es Saxomia, 1227. ex Bergia, 1435. ex Suecia, Wall. l c. (3. Tpecim.

490 Cuprum flavum, diverficolor, in quartzo albo, 536. ex Bergia, 1644. ex Norvegia, Wall. l. c. (2. Specim

491 Cuprum flavum. diverficolor, vulgo pavonina, violacea, in fpatho, 5I 7. ex Saxonia, 52I ex Hartz, Wall.l.c. (2. Ipecim.

492 Cuprum fubflavum, diverficolor, in quartzo albo, 518 . ex Haffia, 1201. ex ..., Wall. $276 . E^{2} 277$. (2. Specim. 493 Cuprum fulflavum, absque mixtione, politum, 520 ex Y.. Saxonia, Wall. 277. (2. Ipecim.

494 Cuprum viridefcens, cum ochra cupri, 323. ex Suecia, 1429. es Norvegia, Wall. 278. (2. fpecim.

495. Cuprum viridefcens, in Cpatho, \& corneo, 547. 1433. ex Norvegia, Wall. $l$ c. (2. Specim.

496 Cuprum, ouafi nativum, una cum bacillis fufis, 540. ex Saxonia, Wall. O. (5. Specim.

497 Cuprum fufum, in bacillis, 545. ex Saxonia, Wall. \%. (5. fpecim.

498 Cuprum alchymice fufum, cum bacillo, 559. ex ..., Wall o. (2. fpecim.

499 Cuprem fufum in bacillis, ex Faponia, Wall. o. (I. Specim.

500 Cuprum, f. fpuma, vel fiores cupri, ... ex ..., Wall.. . (3. Specim.

50r Plumbum, f. Galena, teffulis majoribus micans, pulcrum, cum fpatho lamellofo flavo, 1679. ex Bergia, Wall. 282. (I. fpecim

502. Flumbum, f. Galena, cefulis majoribus, \& minoribus . in fpatho cubico drufitormi, a!bo, 17 7. ex Anglia, Wall. l. c. (1. Specim,

503 Plumbun, f. Galens, affinis, cum fpatho cubico, marcafita, \& plumbagine tecta, 1708. ex Anglia, Wall. 282. \& 283. (I. fpecim.

${ }_{504}$ Plumbum, r. Galena, particulis majoribus, \& minoribus micans, cœrulea granulata fuperficiali, cum quartzo drufofo, \& cupro verficolore, 23 I. I533. ex Norvegia, 1797. ex ..., Wall. 232. (3. Specim.

505 Plumbum, f. Galena, teffulis majoribus, micans, 387. 388. ex Suecia, Wall. l. c. (5. ́́pecim. 
488 Cuivre hépatique, E jaune, avec verd de montagne, E ocre, 519 de Berg, 1232. de Saxe, 1795. de Brunsvic, Wall. $274 \& 276$. (3. piec.

489 Cuivre jaure, $\mathcal{E}^{2}$ à queu de pan. vird de montagne, $\mathcal{E}^{8}$ ocre de cuivre, 153. 536. de Saxe, 227. de Berge, 1435. de Suede, Wall. 1. c. (3. piec,

490 Cuivre jaune, Ẽ cuivre dे queu de pan, en quartz blanc; 536. de Berge, 1044. de Norvegue, Wall. I. c. (2. piec.

49I Cuivre jaune, à queue de pan, violet, en $\int p a t h, 517$. de Saxe, 52 I de Hartz, Wall. I. c. (2. piec.

492 Cuivre bleme, $d$ queue de pan, Eश quartz blanc; 518. de Hefre, I20I. de .... Wall. 276. \& 277. ( 2. piec.

423 Cuivre pa'e, polie, fans melange, 520 . de Saxe, Walle: 277. (2. piec.

49+ Caivre verd, avec ocre de cuivre, 323. de Suede. 1429: de Norvegue, Wall. 278 . (2. piec.

495 Cuivre verd, en Spath, EO pierre cornée, 547. 1433. de Norvegue, Wall. I. c, (2, piec.

496 Cuivre, comme natif, avec des lingots fondus, 540. de Saxe, Wall o. ( 5 piec

497 Cuivre fondu, en lingots, 545. de Saxe, Wall. O: (5. piec.

498 Cuivre fonda, d la maniere des alchimiftes, avec un lin. got, 559. de..., Wall. O. (2. piec.

490 Cuivre, ou lingot de cuivre, de Japan, Wall.o. (I. piec. 500 Cuivre ecusze, ou fleurs de cuivre, Wall. O. (3. piec. 50I Plomb, ou Galene, à culiques plus grandes, tres bear. brillant, aigues, avec Spath bamelleux jaune, I679. de Berge, Wail. 282 (I. piec.

502 Plomb, ors Galene, à cubes grands, Ë petits, "en Spathcubique, en forme de drufe, blanc, 1707. d'Angleterre, Wall 1. c. (I. piec.

503 Plomb ou Galene, pareil, avec Ppath cubiqué, couvert de marcafite, \&o plumbage, x 703. d'Angletterre, Wall. 282. E2 283. (I. piec.

504 Plomb, ou Ga'ene, à gros, $\delta$ petits grains, brillant, bleuatre fupaficielle, avec quartz, dir faforme, Eo cuivre jaune de different couleurs. I231. I533. de Norvegue 1797. de ..., Wall. 282 . (3. piec.

505 Plomb ou Galene, d cubes grands, brillant, 387. 388. de Suede, Wall, l. \&. (5. pies. 


\section{2}

506 Plumbum; r. Galena, pracedenti affinis, in fpatho albo, 391. 392. ex Hartz, II10. ex Norvegia, Wull. l.c. (3. Specim.

507 Plumbum, f. Galend, fimilis, in quartzo drufiformi, al bo, \& cupro flavo, 396. eX Hartz, I531. ex Norvegia, Wall: l. c. (2. fpecim.

508 Plumbum, f. Galena, in quartzo fpathofo, \& correo la. mellato, 390. 394. ex Bergia, 399. 400. ex Hartx, Wall. l. c. (4. Tpecim.

509 Plumbum, f. Galena, fimilis, in fpacho, \& pyrite albo, 393. 397. ex Hantz, Wall. l. c, (2. fpecim.

5ro Plumbum, f. Galena, tefrulis minoribus, micans, cum oupro flavo, \& quartzo, \& Plumbagine drufiformi, 389. ex Germania, 403 ex India Occident. 404. 405. ex Hartz, Wall. l. . . (4. fpecim.

511 Plumbum, f. Galena, affinis, in quartzo, cum ejus plumbo fufo, 40r. 402. ex Hartz, Wall. l.c. (2. fpecim.

512 Plumburs, f. Galena, præcedenti finvilis, cum quartzo, fpatho, \& corneo argentifero, 398. ex Hartz, I!II. ex Norvegia, Wall. l. c (2. Tpecir.

513 Flumbum fpachaceum, cum qquartzo, \& ochra ferri, 4 Ir.ez Hartz, Wall. 284? ( 2. fpecim.

554 Plumbrum thavum, \& viride folidan, in fpatho quartzofo albo, \& flavo montano, 1437. ex Bolemia, Wall. 285. (I. fpecim.

515 Plumbun viridi ramofum, in fpatho albo, 1438. cx Zfopkairs, in Boliemia, Wall. 285. ( $\mathrm{r}$. Specim

516 Plumbum viridi ramofum, fimile; $408-410$. ex Saxoria, Wall. l. c. (4. fpecim.

517 Plumbum fpatnaceo-viride, criftallifatum, cum galena, in quartzo criftallino , drufformi, 1747. ex Bohemia, Wail. 285 ? f. o. (I. fpecim.

518 Plumbum fpathacen-fiavum fuserficiale, in guartzo granulofo, isig ex Freiverg, Wall. l.c. (r. fpecim.

sig Plumbum foathaceo-flvum. "ps æcedenti affine, 18I8. ex Freiberg, Wall. l.c. (1. Specim.

520 Plumbim fpathaceo-viride, fuperficiale, in fpatho ouartzofo albo, cum flavo montano, 1783. ex Bohemia, Wall. l. c. (r. fpecias.

521 Plumbum fpathaceo-viride, in minera plumbi exefa, 1817. ex Freiberg. Wall. l. c. i, Ifpecim.

522 Plumbum ipathacen-viside, ramofum, in fpatho ferreo excfo, 1750. ex Bchemia, Wa!l. l. c. ( $x$, fpecim. 
506 Plomb, ou Galene pareil, en Jpath blanc, 39r. 392. de de Hariz, II:o. de Norvegue, Will. I. c. (3 piec.

507 Plomb, ou Galene, Jemblable, en quartz druffiforme blanc, pyrite blanc ơ cuivre jaune, 396. de Flaxiz, 153\% de Norvegue, Wall. I. c. (2. piec.

508 Plomb, ou Galene pareil, en quartz Jpatheux, Eo connée lamelleufe. 590. 394. de Berge, 39\%. 400. de fiurtz, Wall. 1. c. (4. piec.

50) Plcmb, ou Galene pareil, en Jpath, E̊ pyrice blanc, 393. $3 \vee 7$. de Hartz, Wall, 1. c. (2. piec.

5:0 Plomb, ou Galene. à cubes petits, brillmat, avec cuivre jaune, \& quart\%, \& Plumbage drufforme, 389 Allemagne, 403 des Indes Occidencales, 404. 475. de Hartz, Wall. 1. c. (4. piec.

5 II Plomb, ou Galene, pareil, en quariz, arec fon plomb fondu, 401. 402. de Hartz, Wall. 1. c. (2. piec.

512 Plomb, ou Galene pareil, avec quartz, Eे pierre cornée, contenant de l'argent, 398. de Hartz, IiII. de Norvegue, Wall. 1. c. (2. piec.

513 Plomb, ou spath de plomb, avec quartz, Eo ockire de fer, $4 \mathrm{Ir}$. de Hartz, Wall. 284? (2. piec.

514 Plomb jaune, \& verd folide, en Jpath quartzeux blanc, \& jaune de srontagne, 1437. de Boheme, Wall. 285. I0. ( I. piec.

515 Plomb verd branchue, en fpath blanc, 1438. de Zfopkau, en Boheme, Wall. 285. (x. piec.

516 Plomb verd branchue, pareil, 408-410. de Saxe, Wall: 1. c. (4. piec.

517 Plomb fpatheux yerd, criftallifé, avec galene, en quartz criftallife, en forme de drufe, 1749. de Boheme, Wall. 285 ? 04 O. ( I. piec.

51 8 Plomb Spatheux jaune fuperficiel, enquartz granuleux, 1819. de Freiberg, Wall. I. c. (I. piec.

519 Plomb jpatheux jaune, pareil de la precedente, 1818. de Freiberg, Wall. I. c. (r. piec.

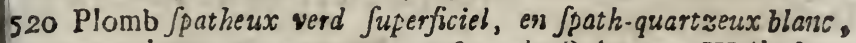
avec-jaune de montagne, 1783. de Boheme, Wall. 1. c, (r. piec.

521 Plomb Spatheux verd, en mine de plomb vermoulu, 1817. de Freiberg, Wall. I. c. (x. piec.

522 Plomb $\int p a t h e u x$ verd, branctu, en fpath de fer, yerrnosslée I750. de Boheme, Wall, l.s. (I. piec. 
523. Plumbum Spathaceo-album criftallifatum, $f$. flores, in minera ferri exefa, cun quartzo critiallino, \& intra ftra. ta, puicerrimum . 340. ex Hartz, Wall. O. (I. Cpecim.

524 Plumbum fpathaceo-album, criftallifatum, f. Hores, I nge ratiaturn, ramis tenuibus, album, r44r. ex Hartz, Wall. O. (1. fpecım.

525 Plumbum fpathaces album criftallifatum, quafi exefum, curs galena, teffulis majoribus micante, 1443. ex Schlac. kenw?ald, Wall. O. (I fpecim

526 Piumbum fpachaceo-album crittallifatum, fimile, in $\mathrm{Ppa-}$ tho exefo, rog9. ex Hartz, Wall o. (r. Ipecim.

527 Plumbum fpathaceo-album criftallifatum, in quartzo fal vo fuperficiali, drufiformi, 1745. ex Hartz, Wall. O. (1. fpecim.

528 Plimbum fpathaceo-album, criftallifatum, cum marga lapid fica, \& Spatho tlavo, 412.1442. ex Hartz, Wall. o. (2. f́ecin.

520 Kumbum fpathaceo-a'bum, tenue, xrugine nativa cerrea teclum, in quartzo forreo exefo, 1447. ex Hartz: Wall. o. (I. Specim.

530 Pinmbums frathaceo-album, præcedenti fimile, III2. 1444. I\$45 tx Hartz, Wall. O. (3. fpecim.

53 I Phumbur foathaceo-album, cum ramis craffioribus xrugine, nativa terrea, denfe tectum, 1706. ex Zellerfeld \& Hartz, Wall, O. (. f pecim.

532 Plumbum fpathacco-album, terue, cum foatho, \& xrugine nativa terrea, fuperficiali, r446. ex Ungaria, Wall. o.

(r.. pecim.

533 Phumbum frathaceum, criftallifatum, ramis tenuibus olivaceis, drufifurmi, in Tpatho savo quartzofo, exefo, I 42 I. ex ...., Wall. O. (I. Tpecim.

534 Plambum fasthaceo-album, tenue, fusco tedum, in lapide corren, cum galena, 1746. ex Hartz, Wall. 284. ( I. 'pecim.

535 Plumbum fpathaceo-album, fuperne nigrum, cum galena, in lapiúc corneo, 1448. ex Hartz, Well. l. c. ( $\mathrm{r}$. frecim,

536 Plumlum fpathaceo-album, fimile, 1747.ex Herta, Wall. ․ c. ( $\boldsymbol{x}$. fpecim.

537 Stanrem crifal'ifatum album , fubpeilicidum, drufiforme, in ipath) al'so, ris9.ex Freiberg, W'all 239. (r. Ppecim. 538 Stannum crifta!lifatum album, I460. ex Ehanfriedersdorf. Wall l. c. (I. rnecim.

539 Stammbn crift illifaum, f crittaili nigra, 356. ex Bohemia, 367. ex Saxoinia, 308. ex Anglia, Wallob.c. (4. Specim. 540 Stannum 
23 Plomb fpatheux blanc, criftallife, ou fleurs de plomb en mine de fer vermoulu, avec quartz criftallifé 'E entre bandes, un tres beau morcear, 1440. de Hartz, Wall. O. (I. piec.

24 Plomb Spatheux blanc, criftallife, ou fleurs d rayons allorsgés, É branches fines, 144 i. de Hartz, Wall. o. (1. piec.

25 Plomb Jpatheux blane, criftallife, comme vermoulu, avec galene à cubes grands, briblant, 1443. de Schlackenwald, Wall. o. (1. piec.

26 Plomb Spatheux blanc, criffallife, parei!, en Spath ferreux vermoulu, 1099. de Hartz, Wall. O. (1. piec.

27 Plomb spatheux blanc, criftallifé, en quartz jaune Suster. ficiel, en forme de druse, 1745. de Hartz, Wall. O ( 1 piec.

28 Plomb Jpatheux blanc, criftallife, avec lithomarge, छ? Spath jaune, 4I2. I 442. de Hartz, Wall. O. (2. piec.

29. Plomb Spatheux, mince, couvert de verd de montagne, $e^{93}$ quartz ferreux vermoulu, 1447. de Hartz, Wall, O. C. I. jiec.

30 Plomb fpatheux blanc, comme le precedent, iriz. 1444. t 445 . de Hartz, Wall. O. (3. piec.

3 I Plomb Jpatheux blanc, à groffes branches, tres couvert de verd de montagne," 1706 . de Z (1. piec.

;2 Plowb spatheux, mince, blanc, avec verd de montagne fus. perficiel, $x_{446}$. de Hongrie, Wall. O. (1. piec.

33 Ilomb fpatheux, criftallifé, d̀ branches fines, olivatres, en forme de drufe, dans la cavite de Spath quartzeux, yermoulu, $142 \mathrm{r}$. de ..... Wall. o. (x. piec.

4.Plomb spatheux blanc, mince, couvert de brun fuperficiel, en pierre curnée, avec galene, 1746 . de Hartz. , Wall. 284. (r. piec.

5. Plomb fpatherx blanc, couvert de noir, avec galene, en pierre cornée, 1448 . de Hartz. Wall. l. c. (I. piec.

6 Plomb Jpatheux blanc, pareil, 1747. de Hartz, Wall. l.c. (r.piec.:

7 Etain criftallifé blanc, luifant, en forme de drufe, en Spatk blanc, I 459' de Freiberg. W.all, 3 .89. (I. piec. 8 ktain crifiallife blanc, 1460... d'Ehrenfriedersdorf, Wall. I. c. (r.piec.

9 Etain criftallife, ou criftaux noirs 366 . de Boheme, $367^{\circ}$ de Saye, 368. d'Angleterre, Wall. l. c. (4.piec. 
540 Stannim criftallifatum, f.criftalli nigre, of fusce, in maft concreta, 360. ex Anglia, 370. ex Saxonia, 371. ex Bohemia, Wall. l. e. (3: 1́pecim.

54I Stanmum criftallifatum, f. criftalli fimplices, \& drufiformes, 359. ex Bohemia, 372. 376. ex Saxonia, Wall, t. Q, (3. fpecim.

542 Stannum criftallifatum, f. criftalli albæ, nigraque, drufio formes , \& fimplices, magne, 380. 381 , ex Saxonia, 1448 . 1530. ex Anglia, Wall. l. c. (4. fpecim.

543 Stannum criftallifatum \& granulatum, in quartzo albo 374. ex Anglia, 375. ex Saxonia, 378. ex Bokenia, Wall. 289. \& 290. (3. Ipecim.

544 Stanurm granulatum, in quartzo, \& lapide corneo, 377 379. 382. ex Saxonia, Wall. 290. (3. Rpecim.

545. Stannum granulatum, antimoiiale. \& criftalli ftanni parva , cum fpatho, \& quartzo, is i 50 . ex Fichtellerg, i 45 i. 1452. ex Saxonia; Wall. l. c. (3. Specim.

346 Stansum granulatum, \& fusco criftallifatum, in lapide corneo, \& quartzo, 373. ex Schlackenwali, 383. ex Saxonia, Wall. l. c. (2. Specim.

547 Stasnum criftallifatum, nigro compactum, \& drufiforme, II 44. ex Anglio, Wall. 289. (I. Tpecim.

548 Stannum criftallíatum, nigro compactum, cum quaftzo albo, 17c9. ex Anglia, Wall. l. c. (I. '́pecim.

549 Stannem criftallifatum, nigrum, in ftanno granulato, \& corneo, 1734. ex Saxonia, Wall. l. c. (I. fpecim.

550 Stannum criftallifatum, permagnum, nigrum, in drufa criftallina alba, \& in lapide corneo, 1457. ex Freiberg. Wall. l. c. ( $\mathbf{r}$. r pecim

55I Stannume criftallifatum, permagnum, nigrum, cum pyri te arfericali albo, in quartzo \& corneo albo, duplicatum \& politum, 1458. ex Saxonia, Wall. 1. c. (2. Specim. 552 Stannum criftallifatum nigrum, \& albefcens, cum ftannd granulato, fpatho , \& floribus cobalti, I449. ex Saxonia, Wall. b. c. ( r. Specim.

533 Stamus fguamofo criffallifátum, imbricatum, \& granu dofum, cum quartzo, 384. 1453. ex Bohemia, Wall. l.c (2. fpecim.

354 Stanmum granulatum, tenue, cum ochra ferri, in quartze corneo, 1455. ex Cornwailis, 1456. ex Margenberg, Wall l c. (2. fpecim.

555 Siennusm granulatum, cum cobalto, \& arugine nativa vi ridi fuperficiali, 385. 1458. ex Soxonia, Wall. $l, 1$ (3. Specim. 


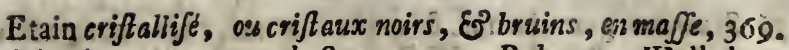
d Angleterre, 370. de Sare, 37x. Bobeme, Wall. 1. c。 (3. piec.

Etain criftallife, ou criftaux, fimples, en drufes, 359. de Boheme, 372. 376. de Saxe, Wa!!! 1. c. (3. piec.

Etain criftallife, ou grands criftaux d'etain blone, EN noir, fimples, en drufes, 380.33r. de Saxe, 1448.1530. d'Angieterre, Wall. $l$ c. (4. piec.

Etain criftallifé, \&o granulé, en quartz blarc, 374. d'Angleterre, 375. de Saxe, 378. "de Boheme, Wall. 289. E? 290. (3. piec.

Etain gramule, en quartz, $\oint^{2}$ pierre cornée, $377.3790^{\circ}$ 382. de Saxe, Wall. 200. (3. piec.

Etain granule, antimonial, \& criftaux d'etain petits, avic Jpath, E? quartz, 1150 . de Fiçhtelberg, 1451. 1452. de Saxe, Wall. 1. c. (3. piec.

Etain, granulé, E criftallifé de brun. en pierre cornée, E quartz. 373. a'e Schlackenwald, 383. de Saxe, Wall. 1. c. (2. piec.

Etain criftallifé, noir. compacte, $E^{2}$ en forme de drufes, II 44. d'Angleterre, Wa:1. 289 . (1. piec.

Etain criftallifé, noir, compacte, avec quartz blanc, 1709. d’Ang!eterre, Wall. i. c. (I. piec.

Etain criftallijé, noir, avec étain gramulé, Ẽ corné, 1734. dc Saxe, Wall. I. c. (1. piec.

Litain criftallifé, tres grand, noir, en drufe criftalline, blanche, \& en pierre cornée, 1457. de Freiberg, Wall:1. c. (x. piec.

Etain criftallifé, tres grand, noir. avec pyrite arfenicale, blanche, en quartz, È cornée blanc, folie , 1458 . de, Saxe, Wall. 1. c. (2. piec.

Etain criftallifé noir, Es blanchatre, avec étain granüle, Spatb, EO fleurs de cobalt, 1449. de Saxe, Wall. I. c. (1. piec.

Erain criftallifé, lamelleux, \& granuleux, avoc quartz, 384. I 453 tle B heme, Wall.1. c. (2. piec.

Etain gramulé, mince, avec ocre de fer, en groartz cornée, r455. de Carnwal, 1456. de Margenberg, Wall. I. c. (2. piec.

Etain gramule avec cohalt, Eg yerd de montagne fuperciel, 385. 1458. de Saxe, Wall. I. c. (2. piec. 
556 Stanmum granulatum, \& criftallifatum, nigro compaetum, 1454. ex Bernftein, Wall. l. c. (1. fpecim.

557 Stannum fpathofum, album, criftallifatum, \& granulatum, 386. 1686. ex Saxonia, Wall. l. c. (2. pecim.

558 Argentum nativen granulatum, valde compae?um, in fpatho rubro, 1464. ex Peru, Wall. 293. pond. 8. unc. 2. engl. ( $\mathrm{x}$. Specim.

559 Argentum nativum granulatum, compectum, cum minera argenti nigra, in fpatbn albo, \& amethyftino,... ex Kongsberg in Norvegia, Wall. 293. 8298 . pond. 3. unc. $7 \frac{1}{2}$. engl. ( 1 . '́pecim.

560 Argentum nativum dendroide \& dentatum, fpatho gyp. feo albo penetratum, $\times 768$. ex Freiberg, Wall.2293. pond. 12. unc. (I. Ipecim.

365 Argentum nativum dendroides, in quartzo albo, fpathofo, I ir 3. ex Freiberg, Wail.l c. pond. 4. unc. I4. engl. (I. Ipecim.

562 Argentum nativum dendroides, in fpatho albo, cum galenæ teftulis majoribus, \&c, I594. ex Kongsberg, W Wall. l. e. pond. 4. unc. 3. engl. (4 epecim.

503 Argentum notis um dendroides, ramofum, ramis tortis, in fpatho albo, pulcerrimum, II18. ex Kongsberğ, Wall. l. $c$, pond. 2. unc. I6. eng. (I. fpecim.

504 Argentum nativum bracteatum, dendroides, \& granuld. fum, in fpatho albo, cum minera vitrea fuperficiali, \& marcafitis aureis, \&c. 1098. ex Kongsberg, I199. ex Ambe rica Wall. l. c. pond. t. inc. I3. engl. (2. [pecim.

565 Argentum nativum dendroides, tenue, \& crafum, it fpatho albo, femidiaphano, i198. cx America, Wall. l. c pond. I. unc. 13. engl. (1. fpecim.

506 Argentum nativum dendroides, minera vitrea tum in fpatho albo, femidiaphano, 1097 . ex Kongsberg, Wal. l. c. pond. I. unc. 4. engl. ( $\mathrm{I}$. fpecim.

567 Argentum rativum dentatum, fpatho granulato, hic in 6 ? de obfitum, in fpatho pyrite tecto, ... ex Saxonia, Wal l. c. pond. 1. unc. 1. engl. (1. 'pecim.

508. Argentum nativum dontatum, pulcerrimum, in quartz $68 \mathrm{~A}$ albo criftallino, \& rpatho albo, cum minera argen nigra, \& lapide cornen, 1593. ex Kongsberg, Wall.l. pond. 17. unc. 5. engl. (I. fpecim.

569 Argentum nativum, craffius dentatum, in quartzo, cu argento rubro, nigro, vitreo, minera mercurii, \& $æ r$ gine viridi, 443. ex Saxonia, Wall. l. 6. pond. 10.ur. IO. engl. (r. fpecim. 


\section{Fofiles. Claffe III.}

36. Etain granulé, \& criftallifé, noir, en mafje, 1454 . de Bernftein, Wall. 1. c. (1. piec.

57 Etain Spatheux, blanc, criftallifé, \& granule, - 386. I686 de Saxe, Wall: I. c. (2. piec.

;8 Argent natif, ou Vierge, granulé, tres compact, en fpath rouge, I 464. de Peru, Wall. 293. pefant 8. onc. 2 engl. (I. piec. 59 Argent natif gramulé, tres maJlif, avec mine $d^{\text {'argen }}{ }^{t}$ noire, en Spath blanc, E् amethy/tine, .... de Kongsberg en Norvegue, Wall. 292. E. 268. pef. 3. onc. $7 \frac{1}{2}$. engl: (x. piec.

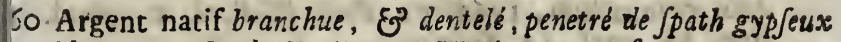
blanc, I 768. de lireiberg, Wall. 293. pef. 12. onc. (I. piec.

5r Argent natif branchue, en quartz blanc, Spatheux, 1713. de Freiberg, Wall. I. c. pef. 4. onc. I4. engl. (1. piec.

52 Argent natif branchue, en Jpath blanc, avec galene, \&o cubique grands, E'c. 1594. de Kongsberg, Wall. I. c. pef. 4. onc. 3. engi. (1. piec.

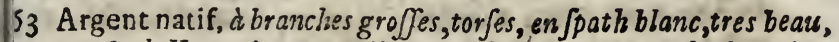
1 1 18. de Kongsberg Wall. 1.c. pef.2. onc. 16 engl. (1. pieq.

54 Argent natif branchue, feuillete, granule, en Spathblanc. avec mine vitreufe fuperficielle, Eु marcafites d'or, E'c. 1098. de Kongsberg. I 99. d'Amerique, Wall. I. c. pef. r. onc. 13. angl. (2. piec.

55 Argent natif trancliue mince, $\&^{2}$ gros, en $\int p a t h$ blanc demidiaphane, I 98 . de l'amerique, Wall. 1. c. pef. x. onc. 13. engl. (I. piec.

56 Argent natif branchue, couvert de mine vitreufe, en Jpath blanc, presque diaphane, ro97. de Kongsberg, Wall. 1. c. pef. I. onc. 4. eng. (I. piec.

57 Argent natif dentelé, parfemé de fpath granuleux, en jpath couvert de pyrite, ..... de Saxe, Wall. I.c. pes. 1. onc. I. engl. (x. piec.

j8 Argent natif dentelé, tres bear, en quartz blanc criłtallifé, E' Spath blanc, avec mine d'argent noire, ED pierre cornée, 1593. de Kongsberg, Wall. 1. c. pef. 17. onc. 5. engl. ( I. . (piec.

59 Argent natif branchue, dentele, en quartz, avec argent rouge, noire, E゚ vierée, mine mercurielle, Ẽ vesd de montagne, 443. de Saxe, Wall. I. c. tef. I0, onc. 10. engl. (1. piec. 
570. Argentum nativan, fimile, in quartzo, mercurium ferrum. que continente, 444. ex Ungaria, Wall. l. c. pond. 3. unc. 17. engl. (1. Specim.

571 Argentum nativum dendroides, \& filofum, in quartzo, cum lapide argenti corneo, 445. ex Saxonia., Wall. lo e. pond. 4 unc. 6. engl. (I. pecim.

572 Argentum nativum, bracteato foliaceum, dentatumque, pul. cerrimum in quartzo fragili, cum argento quafi corneo fuperficiali, 1463. ex India Occid. Hijpan. Wall. l.c. pond. 14. unc. 3. engl. (1. fpecim.

573 Argentum nativum, foliaceo bracteatum, fimile ob́ritum compactum, r462. ex Peru Wall. b. c. pond. 2. unc. $8 \frac{1}{2}$. engl. (1. Specim.

574 Argentum nativum bracteatum , cum minera argenti vitrea, 435. ex Mexico, Wall. 293. \& 294. pond. I unc. $7 \frac{1}{2}$, engl. (1. Ipecim.

575 Argentum nativain capillare, cun minera argenti vitrea . 100r. ex Freiberg, Wall, l. c. pond. 5. engl. (r. fpecim.

576 Argentum rativum capillare, \& filofum , cum quartzo, \& corneo mercurlum tenente, 446. ex Saxonia, Wall. 293. pond. 7. unc. 2 engl. (s. f́pecim.

577 Argentum nativam capillare, cum minera argenti alba, in tpatho drufiformi, 1468. ex Hartz, Wall. l. c. pond. 6 unc. 6. engl. ( 1 . fpecim

578 Argentuin vitreum, \& rubrum, in quartzo drufiformi, 456. ex Saxonia. Wall. 294. \& 296. pond. 1, unc. 16. engl. (I. f́pecim.

579. Argentum nativum capillare, cum minera argenti nigra, in lapide corneo pyritofo, 448. 44y. ex Saxonia, Wall, 393. \& 298. pond. I. unc. 6. engl. (2. Specim.

580 Argentrum vitreum, cum minera argenti nigra, in fpatho granulofo, \& galena argentifera, 455. ex Hartz, Wall. 294, \& 298. pond. 1. unc. 9:. englo (2. Tpecim.

581 Argentuin vitreum, cum minera argenti alba, \& granulis auri, in fpatho quartzofo, 453. ex Saxonia, Wall. 294. \& 297. pond. 4. unc. 13. engl. (I. F ecim.

582 Argentuin vitreum, compactum, \& minera argenti grifea alba, cum fpatho, $45^{2}$ ex Saxonia, Wall. l. c. pond. 9. unc. I5. engl. (2. Tpecim.

583 Argensum vitreum \& minera argenti, cœrulefcens alba, cym fpatho, \& pyrice, 400. ex Hartz, Wall. l. c. pond. 11. unc. 17. engl. (I. Ipecim.

584 Argentum rubrum cum pyrite aureo, in quartzo albo, 1467. ex Hartz, Wall, ag6.pond 3. unc.7. engl (r. fpecim. 585 Argentum 


\section{Folfiles. Claffe III.}

590 Argent natif, pareil, en quartz, contenant mercure, E? fer, 444. de Hongrie, Wall.!. c, pef. 3, onc. 17, engl. (1. piec.

57I Argent natif branchue, E en fils, en quartz. avec pience argentine cornée 445 . de Saxe, Wall. I. c. pef. 4. onc, 6. engl. (I. piec.

572 Argent natif, en lames dentele, tres beaux, en quarts fragile, avec argent comme corneté fuperficielle, 1463 . des ln: des Occident., erz Hispagne, Wall. I. c. pef. 14. onc. 3. engl. (I. piec.

573 Argent natif, en lames, ou feuilleté, pareil, compaitte, 1462. de Peru, Wall. 1. c. pef. 2. onc. $8 \frac{1}{2}$. engl. (I. piec.

574 Argent natif, en lames, avec mine d'argent vitrd, 435. de la Mexique, Wall.293. \&2 294. pef. I. onc. $7 \frac{1}{2}$. engls. piec.

575 Argent natif capillaire, avec mine d'argent vitrée, 100r. de Freiberg, Wall. l. c. pef. 5. eng. (1. piec.

576 Argent natif capillaire, Eक en fils, avec quartz, E3 cornée contenant mercure, 446. de Saxe, Wall. 293. paf. 7. onc. 2. engl. (1. piec.

577 Argent natif, capillaire, avec mine d'argent blamche, en jpath druffiforme 1468. de Harts. Wall. 1. c. pef. 6. onc. 6. engl. (I. piec.

578 Argent, vitré. E rauge, en quartz draffforme, 456. de Saxe, Wall. 294. E2 296. pef. x. onc. 16. engl. (1. piec,

579 Argent natif sapillaire, avec mine d'argent noir, en pierre cornée pyriteuje, 448. 449. de Saxe, Wall. 293. E2 298. tof. 1. onc. 0. engl. (2. piec.

580 Argent vitré, avec mine d'argent noir, en.jpath gramuleux, $0^{\circ}$ galene contenant argent, 455. de Hartz, Wall. 294.

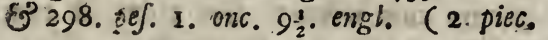

581 Argent vitré, avec mine d'argent blanchie, $\mathcal{E}^{2}$ grains d'or, en Jpath quartzeux , 453. de Saxe, Wall | 294. E 297. pej. 4. onc. 13. engl. (1.piec.

582 Argent vitré. compacte, E̊ mine d'argent gris, \&' blanc,

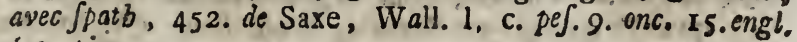
(2. piec.

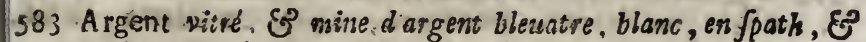
pyrite, 460. de Hartz, Wall. I. c. pef. I I. onc. I 7. engl. (x. piec.

584 Argent rouge, avec pyrite d'oré, en quartz blanc, 1467. de Hartz, Wall. 296 pef. 3. onc. 7. eng. (1. piec. 
$585^{-A r g e n t u m ~ r u b r u m ~ g r a n u l a t u m, ~ c u m ~ f e r r o ~ m i c a n t e ~ m e r c a-~}$ - rifero, in fpatho quartzofo, 450. ex Saxonia, Wall.l.c. pond 10. unc. 3. engl. (I. Specim.

586 Argentum rubrum, \& album, cum pyrite aureo, cuprum ferente, in corrseo quartzofo, 45ז. ex Freiberg, Wall. 296. \& 297. pond. 9. unc. I3. engl. (I. Specim.

587 Argentum rubrum, \& album, cum granulis aureis. in cornea quartzofo, I 244.ex Freiberg, Wall.I c (I. fpecim.

588 Argentum rubrum, vitreum, \& nativum dendroides, cum nvrite globulofo, in quartzo drufiformi, 458.ex Saxonia, Wall. 293. 294. 296. (I. fpecim.

589 Argentum rubrum, cum minera cinnabaris, pyrite, \& -galena tefrulata, in corneo, 360. 454.ex Hortz, Wall. 22c. \& 295. (4. Tpecim.

590 Argentum rubrum, crifallifatum, cum galena argentea teffulata, miserx rubrx, \& pyrite, in fpatho drufiformi, 457. $1245^{\circ}$. ex Hartz, Wall. 296. (3. Specim.

59I Argentum album, dendrificaum, cum minera argenti nigra, in corseu quartzolo, 1800. es Hartz, Wall. 297. (I. ipecim.

592 krgentum alhum, fubcriftallifatum, in quartzo, 1798. ex Hartz, Wall. i. c. (I. fpecim.

593 Argentum album, \& nigrum, cum fpatho, \& giartzo, \& argento nativo filofo, 45I. 46\%. ex Hartz, Wall. 293. 297. 298. (2. fpecim.

591 Argentum album. cum galena teffulata argeniffera, \& nivite. in fpatho drufformi, fubquartzofo. I799. ex Zcllerfeld. Wall. 297. pond. ir unc. (1. Specim.

595 Argentum rubrum \& album, pulcerrimum, cum vitreo fuperficiali , \& cum quartzo crifta lifato, in corneo, $1 ; 84 . \mathrm{ex}$ Andreasberg, Wall. 296. is 2y7. pond. 33. unc. 10. engl. ( $\mathrm{i}$. Rpecim

596 Argentum nigrum, cum arfenico inmixtum, \& cum pyrite, in Spatho albo, I465. ex Bratinsdorff, Wall. 293. ( 1 ipecirn.

597 Argentum nigrum pracedenti affine, 1460. ex Freiberg, Wall. l c (1. fpecin.

598 Argentum nigrum, cum argento rubro fuperficiali, in quartzo, Iz+1. ex Saxonia, Wall. l. c. (1. fpecim.

599 Argentran album, \& nigrum, cum argento vitreo funerficiali . in quartzo drufiformi, 1409. ex Hartz, Walb. 297. \& 298. (1. Ppecim.

600 Argentum album, rubro tectum, in quartzo, \& corneo, 465. ex Hastz, 1242, ex Saxonia, Wall. l. c. (2. ipecim. 


\section{Foffiles. Clafe III.}

585 Argent rouge gronulié, avec de fer relui jant, contenant mercure, en Jpath quartzenx, 450. de Saxe, Wall. 1. c. pef. 10. onc 3 engl. (i.piec.

586 Argent rouge, Es blanc, avec pyrite d'or; contenant duc cusvre, en pierre cornée quartzeufe, 451. de Freiberg. Wall. 290. Eं 297 pef. 9. onc. 13. engl. (1. piec.

587 Argent rouge, Ė blanc, en grains dior, ens roche de corns quartzeux, 1244. de Freiberg, Wall. I. c. (I piec.

588 Argent, rouge, vitreux, Ẽ natif. ou vierge, ramifié, avec pyrite globuleux, en quartz drufiforme 458. de Saxe, Wall. 293. 294 296. (I. piec.

589 Argent rouge, avec mine de cinnabre, pyrite, E galents cubique, en roche de corne, 360. 454. de Hartz, Wall. 2 20. Eं 296. (4. piec.

590 Argent rouge, criftallifé, avec galene d'argent cubique,

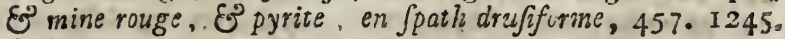
de Hartz, Wall. 296. (3. piec.

59I Argent blanc arborifé, avec mine d'argent noire, en roche de corne quartzeufe, 1800. de Hartz, Wall. 297. (I. piec.

592 Argent blanc, un peu criftallifé, en quartz, 1798 . de Hartz, Wall. I. c. (r. piec.

593 Argent blanc, Eे noir, avec fpath, Eे quartz, E argent natif. ens fils, 46I. 464. de Hartz, Wall. 293. 297. 298. (2. piec.

594 Argent, blanc, avec galene contenant d'argent, Es yrite, en Spath, un peu quartzeux, en forme de drufe, 1799. de Zellerfeld, Wall. 297. pef. Ir. onc. (r. piec.

595 Argent rouge. EO blanc, avec vitreux fuperficiel, tres bedv, avec quartz criftallifé, en cornee, 1784. da Mont St André, Wall. 296. Ë 297. pef. 33. onc. 10. engl. (1.piec.

506 Argent noir, melé d'arfenic, avec pyrite, en fpath blaric. 1465. de Braunsdorf, Wall. 298. (I. piec.

597 Argent noir, approchant au precedent . 1466. de Freiterg, Wall. 1. c. (I, piec.

598 Argent noir, avec argent rouge fuperfociel, en quartz, $124 \mathrm{r}$. de Saxe, Wall. I. c. (I. piec.

599 Argent, blanc, \&o noir, avec argent vitrensx fuperficieh, en quartz draffforme, I 409. de Hartz, Wall.297. $0^{2} 29 z^{\circ}$ (I. piec. .

600 Argenc blanc, couvert de rouge, elz quartz, E piessz corné, 465. de Hartz, 1242 , de Saxe, Wail.1.c. (2. tiec. 
601 Argentzm rubrum, album, \& nigrum, cum pyrite, in 1pa. tho, \& quartzo, 358. ex Hartz, 1243. ex Comubia, Wall. l c. (3.' '́pecim.

602 Argentum album, \& nigrum, cum arfenico, in fpatho, 423. 463. ex Hartz, Wall. l. c. (2. Specim.

603 Argentum album \& nigrum, cum argento vitreo fuperficiali. pyrite, \& Spatho, 395. ex Hartz, 466. ex Saxonisa, Wall. $l$ e. (3. Tpecim.

604 Argentum plumofum, rum quartzo argentifero, granu!o10, 415. ex Hart3, 1661. ex NoDovia, Wall. 300 ? ( 2. Ipecim

605 Argentum nigrum, plumofum, ftriatum, compactum, cum Spatho, 1290. ex Hartz, Wall l. c. (2. Specim.

606 Argentum album, :adiatum, cum tpatho, 1659. ex Nasfovia, Wall. 297. (I. Soecim.

607 Argentum nativum, fubvitreum, in carbone fiffili, cum arugine nativa fuperficiali, 447.459 . ex Minera Carbonaria Heffia. Wall. 302 ? (2. Specim.

608 Argentum, f. Argilla colore plumbeo, argentifera, 467. ex Saxonia, Wall. 30 r. (r. Specim.

60) Argention, fo flores, live fpuma lunæ, ex feparatione argenti a plumbo orta, 4 is. ex Saxoria, Wall. O. (3. Ipecim

6ro Aurum rativum, fingulare. rarumgue, in fpatho albo quartzofo, in capitate drufa, minera vitrea arfenicalis, \& pyrites vitriolatus, 1815. ex Sumatra, Wall. 303. 3. unc. 8. engl. 10. az. (1. (pecim.

6 I Aurum rativrom, præcedenti fimile, 1485. ex Sumatra, Wall. $l$ c pond. 2 unc. 27. az. (I. Tpecim.

012 Aurum nativum, in Cpaths albo, quartzofo, \& pyrite albo, 1289. ex Sunatra, Wall l.c. pond. 3. unc. 9. engl. 8. az. (r. Specim.

613 Aurum nativusn, in ipatho quartzolo, albefcente, cum pauco pyrite arienicali, \& pyrite quadrato, I177. ex Madagascar, Wall. l. c. pond. 3. unc I2. eng. (1. fpeciin.

$61_{4}$ Aurum nativum, cym minera vitrea arfericali \& pyrite quadrato, in quartzo, ir $76 .^{\circ}$ ex Malacca, Wall. l. $t$. pond. 7. unc. 19. engl. (I. Ppecim.

615 Aurum nativum, cum parvis marcafitis, \& minera vitrea arfenicali, in quartzo albo, 1179 . ex Sumatra, Wall, l. c. pind. 2 unc. 4 . engl. ( $r$. Specim.

6I6 Aurum nativum, fpumo-granulofum, in quartzo albo, cum pauca minera vitrea arfenicali, 1197. ex Malacca, Wall. b. c. gond. I1. engl, 10. az. (I. rpecim. 


\section{Folfies, Clafse III.}

Gor Argent rouge, blanc, $\mathcal{E}^{2}$ noire, avec pyrite, en ppath \& quartz, 358. de Harte, 1243. de Curnube, Wali. 1. c. (3. piec.

602 firgent blanc, E5 noir, avec arfenic, en Spath, 423.46.de Hariz, $W$ all, I. c. (2. piec.

603 Argent blanc, E noir, avec argent vitreux fupariciel. pyrite, E Jpath, 395. de Hartz, 460. de Saxe, Wall. 1. c. (3. piec.

604 Argent à plumes, avec quartz, contenant d'argent granssleux, 4is. de Hartz, 1661 de Nasiau, Wail. 300? (2. piec.

605 Argent noir, a plumes, frié, compact, avec fpatk, I29a. de Hartz, Wall. I. c. (2. piec.

606 Argent blanc, rayonné, avec Jpath, 1659 de Nasfad. Wall 207. (x. piec.

607 Argent natif, un peru vitreux, en charbon. couvert de verd de montagne fuperficiel, 447. 459. de la Mine Charbonniere de Hefre. Wall: 302? (2. piec.

608 Argent, ou Argille couleur de plomb, contenant argent. 467 de Saxe, Wall. 301 . (I piec.

609 Argert, ou fleurs, ou ecume dargent, de la feparation de l'argent du plomb, 413. de Saxe, Wall. O. (3. piec. +

610 Or natif, ou vierge, tres fingulier, \& rare, en spath blanc quartzeux. dans les cavité du drufe, on voit une onine vitreufe arfenicale, ED pyrite vitriclije, 1815. de Sumatrs? Wall. 303. pef. 3 onc. 8. engl. :0. afen. (1. piec.

6II Or natif, fembiable au precedent, 1485, de Sumatra. Wall. 1. c. pef. 2 onc. 27. as. (I. piec

612 Or natif, en fpath blanc, quartzeux, ED pyrite blanche. I 289. de Suinatra, Wall, 1. c. pef. 3. onc. 9. engl. 8. as. (I. piec.

6:3 Or natif, en fpatis quartzeux, bianchatre, aves an pers de pyrite arfenicalc, $\mathbf{B}^{5}$ Pyrite cubique, I177. de Madagafcar, Wall. L c. pej. 3. onc. 12 engl. (I. pisc.

$6 \mathrm{I}_{4}$ Or natif; aves mine vitreufe arfenicale, Eo pyrite cubique. en quartz. I176. de Malacca, Wall. 1. c. pej. 7. onc. 99. eng. (I. pisc.

Grs Or nacif, avec petits marcafites, E9 mine virveufe, arjenicale, en qrattz blarc, II79. de Sumatra, Wall. 1. C. pef. 2. onc. 4 exgl. (I. piec.

616 Or natif, comme écume gramuleux, en qusartz blane, avec zin peu de mine vitreufe arfenicale, Ir97. de Malacca, Wall. L c. pef. 11. engl. Io. as." (1, piec. 
Gy Aarum natirum, præcedenti affine, in quartzo, quafi minerz mercurii fimillimo, cum pyrite, $175 \mathrm{~J}$. ex Sumatra, Wall. l. c. pond. I. unc. 10. engl. 26. az. (I. ipecim.

618 Aurum nativum, foliaceum, cum lithomarga, \& mine. ra vitrea fuperficiali arfenicali, in quartzo eodem, ac fupra, 1c96. ex Chennitz Wall l. c. pond. 14. engl. 18. az. (1. fpecim.

6 Ig Aurum nativum, cum minera vitrea arfenicali, in quartzo eodem, 430. ex Ungaria, Wall. l. c. pond. I2.engl. 4. 'az. (r. fpecim.'

620 Aurum, nativum, granulatum, \& foliaceum, fpumofum, cum minera eadem, in quartzo arenofo, 1180 . ex $U_{n:-}$ garia, Wall. l. c. prind. 12. engl. 3.az. ( I. fpecim. . $\sigma_{21}$ Aurum nativum, foltaceum, \& ramoin, cum teffulisarfenicalibus, \& pyrite in quartzo, $429.0 \times$ Ungaria, Wall. l. c. pond. 7. eng!. 6.22. (1. fpecim,

622 Aurum nativum, cum minera arfenicali vitrea, \& argenti albi, in fpatho drufiformi, I689 ex Sumatra, Wall. l.c. pond. 10. engi. 16. az. (1. fpecim.

623 Aurum nativum, cum minera argenti nigra, in quartzo albo pingui, I 752 . ex Sumatra, Wall. l. c. pond. 3. engl. 10. az. (r. ipecim.

624 Aurum nativum, cum minera argenti vitrea, \& nigra, in quartzo albo, pyritofo, 1461. es Sumatra, Wall. l..c. pond. I. unc 7. engl. II. az. (I Tpecim.

625 Aurum nativum, artefactum? in minera arfenicali vitrea implantatum, cum f parho cubico, pyrite, \& marcafita, in quartzo, 431. ex Saxonia, W Will. l. c. pond. I. unc. 15. engl. 22. az. ( I ípecim.

636 Aurum notivum, $r$ Pyrites auratus, \& radiato globulofus, cum granulis auri, inter fcorias mentientia, ftrata, 434. ex Saxonia, Wall. 304. pond. II. engl. 4. az. (I. fpecim.

627 Aurum nativam, granulatum, \& foliaceum, fpumofum, cum minera nigra, in cartzo, \& corneo, 428 . ex Ungaria, Wa!l. l. c. pond. x. unc. 6. engl. 22. az. (I. fpecim.

$\$ 28$ Aurum nativum, in pyrite arfenicali, minera argenti vitrea, \& quarizo exefo, $432 . \mathrm{ex} . .$. Wall. $294.80^{2} 303$. pond. 3. unc. 2. engl. 3. az. (r. rpecim.

629 Aurum rativun, foliaceurs, granulofum, cum minera argenti nigra, pyrite arfen:cali exefo, in quartzo, \& corné, 1688. ex Sumatra, Wall. 3 3, pond. 7. unc. 12. e.gl. 4. az. (?. Speciur. 
бIy Or Natif, approchant aus precedent, en quartz, fort feirb: blable au mine de mercure, avec pyrite, $175 \mathrm{I}$. de Sumatra, Wall.1. c. pef: I. onc. 10. engl. 26. as. (1. piec.

618 Or natif, lamelleux, avec lithomarge pierreufe, E mine vitreufe fuperficielle arfenicale, dans un quartz pareil, de la precedente, 1096. de Chemnitz, Wall. 1. c. pej. 14. engl. 18. as. (1. piee.

619 Or natif avec mine arfenicale vitreufe, en quartz pareil, 430. de Hongrie, Wall. J. c. pef. I2. engl. 4. as. (I. piec.

620 Or natif, granule, \& ferilleté, ecumeux ovec mine pareil, en quartz Jablonneux, II80. de Hongrie, Wall. 1. c. pef. 12. engl. 3. as. (1. piec.

621 Or natif, lamelleux, feuilleté, branchue, avec galene, o6s teffules arfenicale, Es pyrite en quartz, 429. de Hongrie, Wall. 1. c. pef. 7. engl. 6. as. (1. piec.'

$C_{22}$ Or natif, avec mine arfenicale vitreufe, E d'argent blanc; en Spath drufiforme, 1689. de Sumatra, Wall. 1. c." pef. 10. engl. 16. as. (1. picc.

623 Or natif, avec mine d'argent noir, en quartz blanc, gras, 1752. de Sumatra, Wall. 1. c. pef. 3. engl. 10. as. (i. piec.

624 Or natif, avec mine d'argent vitreufe, \&o noire, en quartz blanc, pyriteux, I46r. de Sumatra, Wall. 1. c. pef. 1. one. 7. engl. Ir. as. (1. piec.

625 Or natif, en mine arfenicale vitreuse, artificiel? avec Spath cubique, pyrite, \& marcafite, en quartz, $43 \mathrm{I}$. de Saxe, Wall. I. c. pef. I. onc. I5. engl. 22. as. (1. piec.

¿26 Or natif, ou Pyrite dore, en boules radieufes, avec des, grains d'or, entre couches, en forme de Scories. 434. de Saxe, Wahl. 304. pef. Ir. engl 4. as. (1. piec.

627 Or natif, granule, E̊ fetilieté, en ecume, avec mine, noire, en quartz, \& roche de come, 428. de Hongrie. Wall. 1. c. pej. 1. onc. 6. engl. 22. os. (I. piec.

628 Or natif, en pyrite arfenicale, $\mathcal{E}^{2}$ mine d'argent vitreufe, Ë quartz vermoulu, 432. de .... Wall. 294. E\$ 303. pef. a. onc. 2. engl.3. as (1. piec.

929 Or natif, feuillete, EO en grains, avec mine d'argent' noire, pyrite arenicale vermoulu, en quartz, E roche de corne, 1688 de Sumatra, Wall, 303.pef. 7o onc.12.englo: 4. as. (I. piec. 
630 Aurum nativum, artefaçum, quartzo drufiformi implantatum. cum minera argenti nisra, marcaficis, \& pyrite cupreo, 436 es Saxonia, Walk. l. c. pond. 7, unc. 6. ergl ( I pecim.

631 Aurum nativum, f. granula auri, cum márcafita, \& py. rite aureo, in quarizo albo, Ii/8. ex Malacca, Wall. 304 pond. 7. unc. 2: engl. 20. az. (I. fpecim.

632 Burum nativum, r. granula auri, cum marcafitis, in marmere grifeo, arfenicali, 717 ex Afia, Waill. l. c. pond. 4. unc. 14. engl, 10. az. (1. fpecim.

633 Aurum nativum? f. Pyrites auti, cum pulcris marcafitis, in lapide curneo, aurifero, lameilofo, 433. ex Saxonia, Wall $I$ c. pond. 5. unc. Ig engl. I6. az. (I. fpecim. 634 Aurum nativum, f. ğranula auri, in argilla, 439. 440. ex Bohemia, Wall. 304. (2. Ipecim.

635 Aurum nativum; f. Terra argillofa, \& cinerea, aurifer?, 437. ex ..., 44 r. ex India Orient, Wall. l.c. (2. (pecim. 636 Platina del Pinto, granulata, mixta, \& feparata, 422. 438 ex Pinto, $f$. Quinto, in America, Walb. o. $(2$. - rpecin.

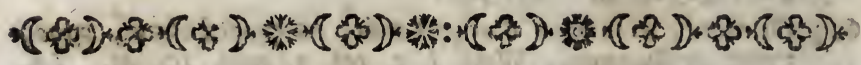
.

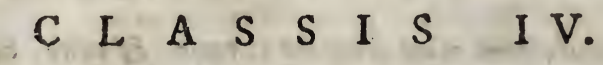

$\begin{array}{lllllllllll}P & E & T & R & I & F & I & C & A & T & A\end{array}$

637 Incruftatusn. 1. Nidus avium, crufta tectum, ... ex Hartz, Wall. 30\%. (1. Specim.

638 Incruftatum, r. Ramus, cum nucibus, foliisque avellanis, crufta teẼa ochracea, 131\%. ex Cialsbad, Wall. l. c. ( I. f ceciul.

B39 Incruftatum, f. Rarus cardui, crufta teclus, 1318. ex Carlsbad, Wall. $b$ c. (1. fpecim.

640 Incruftatum, f. Pyri aviculares, crufta teeli, 828. ex Carlsbad Wall. l. c. (1. fpeciun.

$\sigma_{4}$ r Incruftatusn. C. Cancer fluviatilio, crufta tectum, 1316 , ex Carlsbad. Wall. l. c. (I. Specim.

$6_{42}$ Incruftatum, f. Cancer brachyurus, lapidificatione grifea tectus, gi4. ex .... Wall l. c. ( I. Epecim.

043 Incruftatum. . . Nidus avintm, concretione grifea tectus, 999. ex Sonciershouten, Wall. i. c. (1, fpecim. 
630 Or natif, artificiel, joint en quartz drufiforme, avec mins d'argent noir, fmarcafites, E pyrite cuivreufe, 436 . de Saze, Wall. 1. c. pef. 7. onc. 6. engl. (I. piec.

$6_{31}$ Or natif, ou grains d'or, avec marcafite, ED pyrite d'or, en quartz blanc, II 78. de Malacca, Wall. 304. pef. 7. onc 2. engl. 20. as. (1. piec

632 Or natif, ou grains d'or, avee marcafite, en marbre gris. arfenical, 717. d'Afie, Wall. I. c. pef. 4, onc. 14. engl. 10 as. (I. piec.

633 Or natif? ou Pyrite d'or, avec beaux marcafites, en roche de corne Jchiffeufe, contenant d'or, 433. de Saxe, Wall. 1. . . pef. 5. onc. 19. engl. 16. as. (1. piec.

634 Or natif, ou grains d'or, en argille, 439. 440. de Boheme, Wall. 304, (2. piec.

635 Or natif, ou Terre argilleuje, E cendrée, contenant de l'or. 437. de... 44r, des Indes Orientales, Wall. $1 . c$. (2. piec.

636 Platine de Pinto, gramuleux, lavée, $E^{2}$ non lavée, $422:$ 438. de Pinto, ou Quinto, en Amerique, Wall, O. (2. piec.

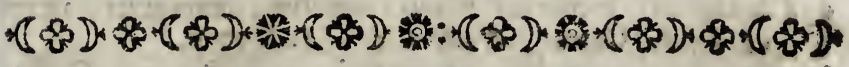

$$
\begin{aligned}
& \text { C L A S S E IV. } \\
& \text { PETRIFIC ATIONS. }
\end{aligned}
$$

637 Incruftation, or Nid d'oifecas, incrufte, de Hastz, Wall. 307. (1. piec.

638 Incruftation, os Branche, avec des noix, EO des feuilles: incruffée, couvert d'ochre rouge, 1317. de Larisbad, Wall. 1. c. (1. piec.

639 Iucruftation, ou Branche de chardon, incruftée, 1318. de Carlsbad, Wall. 1. c. (1. piec.

640 Incruftation, ou Poires d'oijeau, incruftée, 828. de Carlsbad, Wall. I. c. (r. piec.

642 Incruftation, ou Ecriviffe de riviere, incruftée, 1316. de Carlşbad, Wall, l. c. (r. piec.

042 Incrufation, ou Crabe, couvert d'une concretion pierreuse, grife gr 4. de ....WWall. I. c (1. piec.

643 Incruftation, ou Nid d'oifears, couvert d'une concretion pierreufe, 8.9. de Sondershaufen, Wall. 1. c. (I. piec 
644 Incruftatum, Concretio ramofa, tubulofa, 1002. 1006. d Saxonia, Wall. l. c. (2. Specim.

645 Incruftatum, \& Calcareum, vulgo Rindeftein, dictum, 10c0. ex Rackanje ad Brielam. Wall. l. c. (4. fpecim.) 6.46 Incruftatum, f. Calcareum, fimile, 1534. ex Saxonia, Wall $l$ c. (3. fpecim.

647 Stalactites,, . Porus aqueus, ramolus, 1005. Ior 4 ex Saxonia. Wall 308. (2. fpecim.

648 Stalaftites, fimilis præcedenti, fed tenuior, permagrus, ..: Ex. Hartz, Wall. l. c. long. 1\%. lat. 6. polı. ( $\$$ fpecim.

649 Stalactites craffus, $147 \mathrm{I}$. ex Antro Baumann:ano, 1651. - 9 ex Fodinis Argentiferis Saxonice Wall. l. c. (3. Ipecimi. 650 Stalactites albicans, ramis tenuibus, pulcerrimus, 1326 . ' ex Infula Gallia Wall. l. c. (r. Specim.

-51 Stalaetites, ramofo tenuis, 1325. ex Infula Gallia, Wall. l. c. ( (. R Recim:

652 Stalactites pulcerfimus, ramis planis, coronam mertientibus.; $10 \mathrm{r}$. ex Infula Gallia, Wall. $l . c$. . ('r. Ipecim.

653 Stalarites tophofus, ramofus, planus, Ior 3. ex Infula Gal. lia, Wrall. l. c. (I. fpecim.

654 Stalactites tophofus plano-ramofus, aurantius, 1327. ex Infula Gallia, Wall; l. c. (1. Rpecim.

655 Stalactites tophofus, drufiformis, 826.827. ex Gallia, Wall. l.6. (2. Epecirn.

656 Stalactites albus, 1ophofus, 1003. ex Germatia. Wall h.c. (3) fpecim.

657 Oolithes, albefcens, crufta tophacea, in qua oculi lapidai, forme Pifi, Irg?. ex Carlsbad, Wall. 309. ( 1 . frecim.

658. Oolithes, fimills procedenti, pifis wuitis exitructis : 976 . ex Carlsbad, Wall la (2. Tpecim.

659 Oolithes, fuscus, lamina polita, 1194. ex Mansfeldia, Wall, l. c. (I. Specim.

600 Tophus globularis, porofus, rojI. ex Rackanje ad Brielam, Wail. 310. (I: rpecim.

601 Topbus, f. Laminx rolundx rufæ, 832.833.ex Gcrmania, Wall. l. c. (2. fpecim.

662 Phytolitus, 1: Filix convesus, in fihilto, permagnus, 1195. ex Mannsfeld, Wall. 3 I r. long. 9'-poll. ( 1 . ipecim

663. Phytolitzls, præcedenti affinis, in fchifto calcareo, gri: feo, 17 I5. ex Anglix. Wall. i. c. (2. Specim.

664 Phytolitus, i. Carbonaria Arbor, forfilis, niger gagatiaus, 1195. ex Anglia, W'all, 325. (1. [pecim. Alspial in. 605 Phytolo 
44 Incruftation, ou Concretion branchue; en tuyau, 1002 1006. de Saxe, Wall. I. c. (2. piec.

45 Incruftation, ou Calcaire, nommé Rindeftein, Ic00. de Rakanie, aupres de Briel, Wall. I. c. (4. piec. 46 Incruftation, ou Calcaire fareil, 1534. de Saxe, Wall. I. c. ( 3 piec

47 Stalactite, ou Pure aqueux, branchue, 1005. Tor4: de Saxe, Wall. 308. (2. piec.

48 Stalactite, fort grande, Semblable au precedent, mais plus mince, .... de Hartz, Wall. 1. c. long. 17. lat. 6. pouc. (I. piec.

49 Stalactice, epaiffe, i47I. de la Grotte de Bauman, 1651 . des mines Argentines de Saxe, Wall, I.c. (3. piec.

so Stalaetite blarche, d branches minces, 1326, de l'ine de France, Wall. I. c. (t. piec.

5I Stalactite blanche, d branches minces, 1325. de l'ifle de France, Wall I. c. (i piee.

52 Stalaetice pareil a branches plattes, en forme de couronne, Io i2. de l'ine de France, Wall. 1. c. (1. piec.

53 Stalactite topherse, branchue, platte, I013. de l'Ine de France, Wall. 1. c. (1. piec.

54 Stalactite, topherse, platte, branchue, couleur d'orange, en forme de grappe, 1327. de l'une de France; Wall. I. c. (I. piec.

55 S alactite, topheufe. drufiforme, 826. 827. de France Wall. 1. c. ( 2 piec.

;5 Stalactite blanche, topheufe, roo3. d'Allemagne, Wall. I. c. (1. piec.

17 Oolithe blanchearre, en crocte topheufe, dans lequel des yeux pierrenfes, en forme de Pife, I I 92. de Carlsbad, Wall. 309.(1.p.

8 Ooithe, femblable do la precedente, $d^{\prime}$ ou l'on a siré beaucoup de pijes, 970 de Caristad, Wall 1. c. (2. piec.

9 Oolithe, brune, plaque, polie, I194: de Mansfeld, Wall. 1. c (1. piec.

- Tuf, en boule, poreux, Iоэr. de Rackanje, aupres le Briel, Wall. 310. (1. piec.

I Tuf, ou Plaques rondes, roufes, 832.833 . de l'Allemgne, Wall 1.c. (2. piec.

2 Pytholite, ou Fougere, tres grande, en relief fur l'ardoife, IrD5. de Mancfeld, Wall. 311 . long $9 \frac{1}{2}$, pouc. (I. piec.

3 Pythoiite, femblable da la precedente, en fchift calcaire, gris, 17r5. d'Angleterre, Wall. 1. c. (2. piec.

4. Pytholite, ou Bois foffile, noir, changé en gagate. I ig5. d'Angleterre: Wall, 325 . (r, piec. 
-665 Lithoxylon, f. Lignum fofile, fere naturale, ex cryptis fcopulorum, 1023. Ex Islandia, Wall. 326: (1. fpecim.

666 Lithoxylon, f. Lignum Nucis, petrificatum, politum, I 769. ex Coburgo, Wall. 3i2. (I. Rpecim.

607 Lithoxylon, f. Lignum Quercus, fuscum petrificatum, politum, \& non politum, \$334.1472. ex Scheuerfeld, Wall. b. c. (2. fpecim.

608 Lithoxylon, f. Ligmum Quercus, petrificatum, discus ma. gnus, ex trunco politus, 827. ex Coburgo, Wall. l. c. (1. fpecim.

669 Lithoxylon, f. Lignum Betule, petrificatum, discus fimilis, rufo fuscus, politus, 1025. ex Birckenfeld, Wall. l. c. ( . fpecim.

670 Lithoxylon, f. Lignum Fagi, vel discus, grifeo niger, politus, 1335. ex Dambach, Wall. l. c. (1. fpecim.

671 Lithoxylon, f. Radix Querci, petrificata, lamina quadrata polita, 804.1100. ex Ungaria, Wall. b. c. 2. rpecim.

672 Lithoxylon, 1. Lignum Quercus, \& Nucis . petrificatum, nigrefcens, laminx ovales, \& rotundx, politæ, 84I. 1474. ex Bohemia, Wall l. c. ( 2 fpecim.

673 Lithoxylon, f Lignum yiride, pecrificatum, \& politum, haud commune, 838. ex Furchheim, Wall. l. c. (I. Ipecim.

674 Lithoxylon, C. Lignum viride, fimile, una cum rude ra. mula, altera polita, 839. ex Furchloim, Wall. l. c. (2, specim.

675 Lithoxylon, f Ligna petrificata, polita, 2uercus, Fagi \& Pini, 846. ex Saxonia, 842. 843. ex Bohemia, 1473. ex Coburgo, Wall. l. c. (4. Ppecim.

676 Lithoxylon, f. Lignum Fagi, petrificatum, discus, es trun co fusco nigro, lamina polita, 1333. ex Dambach, $W_{\text {a }} h_{i}$. b. c. (r. Specion.

677 Lithoxylon, f. Lignum Taxi, petrificatum, ramus poli tus, 1024. ex Coburgó, (1. Ipecim.

678 Lithoxylon, I Lignum Abietis, petrificatum, \& magnum, 13ig. ex Saxonia, Wall. l c, (r. fpecim.

679 Lithoxylon, f. Lignum Pini, petrificatum, grifeum, not politum, 847. 1320. ex Saxonia, Wall.l.c. (2. Rpecim

680 Lithoxylon, f. Ligna petrificata, varia frufta, Quercus Fagi, \&c. aliarumque arborum, 846. ex. Saronio 845. 848. 849.) ex Coburgo, Wall. l. c. (5. frecim. 681 Lithoxylon, f. Ligna petrificata, varia, frufta conto nata, 850-852. ex Vismusa, Walb. l. c. ( 5. fpecim. Aat?..? 082 Oflear 


\section{Fofiles. Clafe IV.}

65 Lithoxylon, ou Bois folfile, presque natuzel des rochers, 1023. d'Islande, Wall. 326. (I. piec.

66 Lythoxylon, ou Bois de Noix, petrifie, polie, I769. de Cobourg, Wall. 312. (I. piec.

57 Lythoxylon, ou Bois de Chene, petrifié, palie, Ẽ non polie, 1334. 1472. de Scheurfeld, Wall. 1. c. (2. piec.

58 Lythoxylon, ou Bois de Chene, petrifie, noir, en grande plaques, 827. de, Cobourg, Wall. l. c. (1. piec.

5o Lythoxylon, ou Bcis de Bouleau petrifié, disque pareille, rouge, brun, polie, 1025. de Bircenkfeld, Wali. 1.c. (1. piec.

7o Lythoxylon, ou Bois d'Huitre, ou disque, gris. noir, polie, I335. de Ombach, Wall. I: c. (I. piec."

II Lythoxylon, ou Racine de Chene, petrifie, plaque quarree, polie. 864. 1160 . de Hongrie, Wall. 1 c. (2.piec.

12 Lythoxylon, ou Bois de Chene, Eे Noix, petrifié, ou pareilles plaques ovales, E rondes, polies, 841. 1474. de Boheme, Wall. 1. c. (2. piec.

3 Lythoxylon, ou Bois verd, petrifie, \& polie; pas commun, 838. de Furchheim, Wall. 1. c. (I piec.

4 Lythoxylon, ou Bois verd. petrifié, avec une branche, polie, "Ẽ non polie, 839. de Furcheim, Wali. 1. c.' (2. piec.

5 Lythnxylon, ou Bois de Chene, d'Hêtre, E̊ de Pin, petrifié, polie, 840. de Saxe, 842. 843. de Boheme, I473. de Cobourg, Wall. 1. c. (4. piec.

(o Lychoxvlon, ou Bois d'Hêtre, petrifié, plague de tronc noir, E2 brun, polie, I333. de Dambach, Wall. 1. c. (1. piec.

7 Lythoxylon, ou Bois de Taxe, petrifie, branche, polie, 1024. de Cobourg. (1. piec.

8 Lythoxylon, ou Bois de Sapin, petrifié, tres grand morcear, I319. de Saxe, Wall. I. c. (2. piec.

9 Lythoxylon, ou Bois dé Pin petrifie, gris, non polie, 847. I320. de Saxe, Wall. 1. c. (2. piec.

Lythoxylon, ou diverfe pieces de Bois Chenes, d'Hêtres. 'E c. $\mathcal{F}^{2}$ autres arbres, 846 . de Saxe, 845. 848. 849. de Cobourg, Wall. I. c. (5. piec:

fr: Lythoxylon, ou Bois petrifié, divers pieces, travailles ard tour, 8sc-852. de Vienne, Wall. 1. c., (s. piec.: 
682 Ofteccolla, f. Radices petrificatæ, $1470.0 \times$ Palatino, Wall. 3I3. (5. Tpecim.

683 Lithocalamus, ut \& Phytotypolithus, caules plantarum petritrificatx, in fchifto grifeo; 223.924 . ex Saxonia, Wall. 3:4. \& 318. (2. Specim.

684 Carpolithus, quafi fructus Nucem Mofchatum, referens, duplicacus, \& politus, 990. ex Coburgo, Wall. 3 16. ( 2 . fpecim.

685 Phytotypolithus, f: Folia Lnuri, in lapide calcario imprefra, 229. ex Helvetia, Wall. 319: (1. Ipecim.

686 Phytotypolithus, f. Folia Filicis, impreffa, in fchifto nigro, 925. ex Suhla, Wall. b. c. (1. Ppecim.

687 Phytotypolithus, f. Folia Filicis, pracedentibus fimilia, 926. ex Suhla, Wall l. c. (1. Specim.

688 Phytorypolithus, f. Folia fimilia, 927.' ex. Suhla, Wail. l. c. C $\mathbf{x}$. fpecim.

689 Phytotypolithus, f. Folia fimillima, 928. ex Suhla, Wall. l. c. (1. 'pecim.

690 Phytotypolithus, f. Folia permulta Populi, in calcario to phofo, 915. ex Colurgo,Wall. l. c. (I. Specim.

Cor Phytot ypolithus, f. Folia Querci, \& Ulmi, 91 7. ex Coturgo Wall. l. c. (1. Ppecim

692 Phytotypolithus, C. Folia Ulmi, 9:6. ex Coburgo, Wall. l.c (1. rpecim.

693 Phytotypolithus, f. Folia Querci, Fagi, \& Ulmi, 91 9.920 923. ex Coburgo, Wall. t. c. (3. Rpecim.

694 Phytotypolithus, f. Folia varia, nervis elevatis, $918.92 \mathrm{I}$ ex Coburgo, Wall. $l$ c. (2. Ppecim.

695 Lithophytum. f. Corallia, nec non Conchylia varia, minu tiffima, in afferculo fixa, ... ex Monte St. Petri, Wall. \&c. Cplurifima f́pecim.

696 Litbophytum, 1. Corallia', Madreporites, Milleporites, Alcyo nia, \& alia, in lapidibus arenofis, ..., ex Maaftricht Wall \&c. (30. fpecim.

697 Lithophytum, f. Corallia fimilia,..., ex Monte St. Petri Wall. \&c. (30. fpecim.

698 Litbophytum, r. Corallia fimilia, majora, ... ex $M a$ fricht, Wall. \&cc. (18. fpecim.

699 Lithophytum, f. Corallia fimilia, adtuc majora, ... e Maaftricht, Wall. \&c. (18. fpecim.

700 Conchytes, 'f. Uni- \& Bi-valvia, multa, ... ex Maaftricht Wall. \&c. (plurima fpecim.

701 Concbytes, r. Pedinata, \& Echenita,... ex Maaltricht Wall.\&ic.) ( $\sigma$. specim. 


\section{Fogiles. Clafse IV.}

2 Offeocolle, ou Racines petrifiés, r470. de Palatin, Wall. 313 . (5. piec.

3 Tiges, EO Empreintes petrifiées, en Jchift gris, 923. 924 . de Saxe, Wall. 314. E 318. (2. piec

4 Carpolite, ou fruit petrifié. rel(Jemblant au Noix de Mufcat, doublé, Es polié, 990. de Cobourg, Wall. 316. (2. piec.

5 Phytotypolite, ou Feuilles de Laurier, imprimée, en pierre calcaire, 209. de Suiffe Walli.319. (1. piec.

6 Phytotypolite, ou Feuilles de Fougeres, imprimée en : Jchit noir, 925, de Subla, Wall. 1. c. (I. piec.

7 Phytorypolite, ou pareillesFeuilles, 926. de Suhla, Wall. : 1. c. (1. piec.

3 Phytotypolite, out pareilles Feuilles, 927. de Subla, Wall. 1. c. (I. piec.

Phytotypolite, ou tres pareilles Feuilles, 928. de Subla, Wall. I. c." (i. piec.

Phy totypolite, ou Feuilles de Peuplier, imprimées en pierre calcaire, tophsufe, 915. de Cobourg, Wall. 1. c. (I. fiec. Phytotypolite, ou Feuilles du Bois de Chene, $E$ d'Ormeau, 917. de Cobourg, Wall. 1. c. (I. piec.

: Phytotypolite, ou Feuilles d’Ormeau, 9r6. de Cobourg, Wall. l. c. (I. piec.

Phytotypolite, out Feuilles de Chene, d'Hétre , छ d'Ormeau, 919.920. 922 . de Coburg, Wall. I. c. (3: piec. Phytotypolite ou differentes Feuilles, à nervures elevées. 918. 92 I. de Cobourg, Wall. I. c. (2. piec.

Lychophice, ou Corallites, E? différens Coquillages petits, dans une layette, ... du Mont St. Pierre da Ma ftricht, Wall. \&c. (phufeurs piec.

Lythophite, ou Corallines, Madreporites, Milleporites. Alcyones, Ẽ autres, en pierres fablonneufes, .... de Maftricht, Wall. \&c. (30 piec.

Lythophire, ou Corallines pareilles, ... du Mont St. Pierre. Wall. \&e. (30. piec.

Lythophite. on Cora'lines pareilles, plus grandes; .... de Maftricht, Wall. \&c (i8. piec.

Lythophite ou Corallines pareilles, encore plus grandes. .... de Maftricht, Wall. \&c. (18. piec.

Conchyte, ou d Univalves, \& Bivalves, en quantité. petrifies, .... de Maftricht. Waill. \&sc. (plufeeurs pieces. Conchyce, ou Manteaux, E Echinites ... de Maftricht, Wall. ¿c. (6. piee. 
702: Conchytes, f. Pettita \& alia, ex ... Trajecti at Mofoxn, W.all. \&c. (7. Ipecim.

703 Conchytes, f. fimilia precedentibus, ... ex Trajezti ad Mo. Jam, Wall. \&c. ( 6 fpecim.

704 Conchytes f. varia, fimillima, ... ex Maaftricbt, Wall \&c. (ro. Гpecim.

70s Aftacolithes, fo brachia, Cancrorsm, vel Eremitorum, \& Be. lemnitis, .. ex Maaftricht, Wall. $354 . \&$ 355. ( 9 . fnecim.

$700 \mathrm{Xyloftea}$, f. Os magnum diffecatum, in lapide arenaceo.... ex Maaftricht, Wall. 3+2: (I. fpecin.

707. Lishophytum, 1. Entrochite, Corallite, Terebratulite \&c. permulta, ... ex Monte St. Pieter, Wall. \&c. ( plurima ipecim.

908 Lithophytum, f. Fungite, in lamina marmorea, grifea, polita, 816. 817. ex Blankenburg, Wall. 335. (2. Specim.

709 Lithophytum, C. Fungite, firniles, laminæ rubra, politx, 1308. 1309. ex Blankenburg, Wall. h, c (2. Ipecim.

710 Lithophytim, f. Fungite limiles, rubra, \& grifeæ, polita 1310. 1313. ex Blanckenburg, Wall. b. c. (2. Tpecim. 7 ir Lithophytum, f. Fungite, præcedentibus fimiles, rubræ \& polite. I3rr. ex Blanckenburg, Wall. l. 6. (2: Speeim, 712 - Lithophytum, f. Fungite, cum Entrochitis, in lamina rub bra, grifeaque po'ita, 1313. 1314. ex Blanckenburg, Wallo l. e. (2. fpecin.

7 I3 Lithophytwin, f. Madreporites, \& Tubiporites, ogr. ex Sue yia, 1756. ex Groninga, Wall. 328 . \& 330 (2 Tpecim

${ }_{714}$ Lithophytum, f. Madreporites, \& Tubiporites, 1758. 1760 1762. ex Groninga, Wall. l. c. (3. Ppecim.

7is Lithophytum, S. Madreporites, \& Milleporites, 1536 ?

Trajesti ad Mofam, 1757 1759. I 763. ex Groninga, Wall 328. \& 329. (4 frecim.

786 Lithophytum, f. Madreporites \& Milleporites, 992. rool 16 ex Norvegia, 1755 ex Groninga. Wall. I c. (3. fpecia

717 Lithophytum, I Madreporites, \& Milleporites, minores, 99 924: eX ....,Wall l.c. (3. fpecim.

718 Lithophytum, f. Fungite, varise, polite, \& non polite, 72 N18 8i5. ex Pomerania, 995. ex Helvetia, Wall. 335. fpecim.

7Ig Lithophytum, f. Zosphytholithus Madrepore, vel Monticul Veneris, fimplex fubovatus, acaulis, lamellis æqualibu tenuis fubcrenatis inflexis, hand commune, I809. Monte St. Pieter, vel ex ....W Wall.328. (I. fpecim 780

720 Zoolithus, fo. Ebur foflibe molare. fat magnum, 1007. Pommerana, Wall. 343 . (I. fpecim. 


\section{Foffiles. Clafle IV.}

702 Coohyte, ou Manteaux, \& autres .... de Maftricht, Wall. \&ac. (?. piec.

703 Conchyte, ou pareilles aux precedentes,.... de Maftricht, Wall. \&ic. ( 6 . piec.

704 Conchyte, ou differentes, tres Jemblables... de Maaftricht, Wall \&c. (10. piec.

yos Aftacolithe, ou Pinces d'Ecrivifes, ou d'Heremite, E' Belemnites, .... de Maftricht, Wall. 354. E 355. (9.piec. 706. Ofteocolle, ou grand Os divift en deux, en pierre fablonnerife, .... de Maftricht, Wall. 342. (I, piec.

707 Lythophite, ou Entrochites, Corallites, Terebratulites, $E^{\circ}$ d'autres, .... du Mont St.Pierre, Wall. E'c. (plufseurs piec. 708 Lythophite, ou Fongites, en plaques marbré, grife, polie, 8 i6. 8i 7. de Blankenbou!g, Wall. 335. (2. piec. 700 Lithophyte, ou Fongites, pareiiles plaques rouges, polies, 1308, I309. de Biankenbourg, Wall. I. c. (2. piec. 7 IO Lithophyte, ou Tongites, pareilles plaques rouges, E' grijes, polies, I310.13!3. de Blankenbourg, Wall. 1 , c. (2. piec.

7Ir Lithophyte, fou Fongites, plaques femblables aux precedentes, rouges; I3II. de Blankenbourg, Wall. 1. c. (2. piea.

712 Lithophyte, or Fongites, avec Entrochites, en plaques rous ges, grijes, E' polies, 1313. 1314. de Blankenbourg, Wail. 1. c. (2, piec.

7I 3 Libophyte, ou Madreporites, E2 Tubiporites 991. de Suevie, 1 756. de Groningen, Wall. 328. 85 330. ( 2. piec.

7r4 Lthophyte, ou Madreporites, \& Tubiporites, 1758 . 1760. 1 762 . de Groninge, Wall. 1.c. (3. piec.

715 Lithophyte, or Madreporites, 8 Milleporites, 1336. de Mlaftricht, 1757. 1759. 1763. de Groninge, Wall. 328 58 529. (4. piec.

216 Lithophite, ou Madreporites, EO Milleporites, 992.1008. de Norvegue, 1755. de Groringe. Wall.1.c. (3.piec.

2I7 Lithophite, ou Madreporites, E Milleporites, plus petites, 993 994. de ...WWall. I. c. (3. piec.

718 Lithophite, 0r Fongites, polies, E rodes, 727. 815. de Pommeranie, 395. de la Suife, Wall: 335 . (4 piec.

7×9. Lithophyte, ou Zoophythoilte Madrepore, ou Monticule de Venus, fimple ovale. fans queue, à lamelles egales minces, un peu crenues, incourbées, pas commune, de MaAtricht. ou de .... Wall. 328. (I. piec.

720 Zoolithe, ou Y voir foffile, piece grande, platte, 1007. de Pommeren, Wall. 343 . ( $\mathrm{r}$ piec. 
721 Zoolithus. C. fragmenta Odium, 3753. ex Groninga, * TrajeCti ad Mofam, Wall. 342. (3. Specim.

722 Zoolithus. f. Turcoides, virides, 1315. ex Gallia, Walls 344 (5. fpecim.

723 Zoolithus, f. Turcoides, in matrice, 854. ex Gallia, Wall. l. c (2. rpecim.

724 Zoolishus, fo oficulum Avis, petrificatum, in lapide calcareo, 1476. ex Hartz, Wall. 346. (I, fpecim.

725 Zoolithus, C. Dentes quadrupedum, 1009. ex..., Wall. 343? 2. (2pecim.

7:6 Zoolithus, f. Glofopetra, varialormes, II 89. ex Hartz, .2 Wall. 349. (3. fpecim.

727 Zoolithus, r. Glagfopetra, pracedentibus affines, ... ex Monte St. Petri, Wall. l c. (plurima fpecim.

728 Zoolithus of Buffoniti varii, in matrice, 853. ex Mis. nia, Wall. 350. (7. Ppecim.

729 Zoolithus, f. Aftacolithus, vel fragmenta Cancri, 1498. ex India Orient Wall. 354. (9. Specim.

730 Zoolithus, [. Affacolithus vel brochia maj ra, Cancri Ere-

mite. in caicareo-Havo, 1535. ex Monte St. Petri, Wall. l. c. (3. Ipecim.

$731^{2}$ Zoolithus. Aftacolithus, five brachia fimilia, ,.. ex Manfricht, Wall. l. c. (5. Specim.

732 Z.oolithus, vel ejus Nidus Verminum, quafi caput Medus-

-D fa referens, in lamina calcarea, duplicata, 1646. ex Eichftrd, Wall. 354. a (2. ipecim.

.33 Zoolithus. f Vermiculi minores, in lapide calcạreo, 1804. ex Bafilea, Wall. i. c. (I. Specim.

734 Zoolithus, r. Vermiculi fimiles minores, in lapide calcareo grifen, I805. ex Baden, Wall. l. c. (3. ipecim.

735 Zoolithus, f. Vermiculi, , in calcareo, 1801-1803. ex Italia, Wall. $l$ c. ( 9 , fpecim.

736 Zoolithus, f Belemniti, magni, 1540. 1647. ex Monte St. Petri, Wall. 355 . (2. Ppeciun.

737 Zoolithus, r. Belemniti, fimiles, 930. ex Saxonia, Wall. l. c. (3. Specim.

738 Zoolithus, f. Belemniti, fimiles pracedentibus, $1647.10 \times$ Moaftricht, Wall. l. c. (5. Specim.

739 Zoolithus, f. Belemniti vasii, in lamina dendritica fusa ca, utrumque polita, 822. ex Brunsuigia, Wall. l. c. (r. Specim

740 Zoolithus, r. Belemnitus fimilis, in lamina olivacea, ab altera tantum parte polita, 823. ex Altorf, Wall. $l$ c.

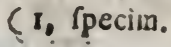




\section{Folfiles. Cladje IV.}

7et Zoolithe, ou fragmens d'Os, 1753. de Groninge \& $\mathrm{Ma}$ ftricht, Wall 342 . (3. piec.

722 Zoolithe, ou Turquoifes, vertes, 1315. de la France, Wall. 344. (5 piec.

723 Zoolithe, ou Turquoires, dans leur matrice, 854. de la France, Wail. il. c. (2. piec.

724 Zoolithe, ou petit os d'Oifeau, petrifié, en pierre calcaire, 1476 . de Hartz; Wall. 346. (1. piec.

725 Zoolithe, ou .Dens petrifiés de quadrupedes, 1000. de Wall. 343 ? (2. piec.

726 Zoolithe, ou Gloffopetres, ou dens de Serpens, de differente forme, 1189 de Hartz, W 11.349 (3. piec

727 Zoolithe, ou Gloffopetres, ou dens de Serpens, fembla-: bles aux precedens, ... du Mont St: Plerre, Wall i. c. (plufieurs pieces.

728 Zoolithe. ou Crapaudines, differentes, dans leur matrice, 853. de Meilfen, Wail. 350. (7. piec.

729 Zoolithe, ou Altacolithe, fragmevs ou pates d'Ecriviffe petrifies, 1498. des Indes Orientales, Wall. 354: (9.piec.

730 Zoolithe, ou Aftacolithe, ous pattes d'Ecriviffe Heremite, petrifices, en pierre calcaire jatme, 1535 . du Mont St. Pierre, Wall. '. c. (3. piec.

73I Zoolithe, ou Attacolithe, ou pareilles pates d'Ecriviffe, fetrifiées, de Maftric 7 , Wall. 1. c. (5. piec.

732 Zoolithe, ou Vermiffeaux, ou leur Nid. reffemblant d ls tete de Medufe, dans une plaque calcaire, doublée, $16+6$. d'Eichltad, Wall. 354. (2. piec.

733 Zoolithe, ou Vermiffeaux de mer, plus petit, dans une. pierre calcaire, 1804 de B.lle, Wall. I. c. (1. piec.

734 Zoolithe, ou Vers de mer, petits, en pierre Jablonneufe; grife, 1805. de Bade, Wall 1. c. (3. piec.

735 Zoolithe, ou Vers de mer, pareilles, en pierre, calcaire. 1808-1803. d'Italie, Wall. I. c. (9 jiec

i 236 Zoolithe, ou Belemnites, grandes, 1540. 1647. du Mont Sr. Pierre, Wall. 355 . ( 2 piec.

137 Zoolithe, ou Belemnites, pareilles, 930. de Saxe, Wall. I. c. (3. piec.

38 Zoolith , ou Pelemnites, Semblables aux precedentes, 1647. de Maftricht, Wall. I. c. (5. piec.

3) $Z$ olithe, ou Belemnites, differentes, en plaque arlorifée brune, polie de chaque côtś, 822 de Brunsvic, Wall. I.ca (I. piec.

$40 \mathrm{Z}$ 'oititie, ois Belemnites, en pareille plaque, cculeur d'olive, feulement polie d'un coté, 823. de Alttorf, Wall. I. $c_{\text {. }}$ (I piec.

$$
\text { S25. } 741 \text { Zoolith. }
$$


74. Zooththus, f. Belemnitus, affinis pracedenti, fed major, 824. 'x Altorf, Wall l. c. (r. Pocim.

742 Zoolithus, r. Trochite, \& Entrochite, in Lamina quadratopolita, marmorea cinerea, 825. ex Sandarshaufen, Wall. 356. \& 357. (I. fpecim.

743 Zoolithus, 1. Trochita, \& Entrochita, in lamina polita, mulcis replita, 829. ex Coburgo, Wall. l. c. (1. Kpecim.

744 Zoolithus, f. Entrochita ramoiæ, \& Afteria lapides, 1190. ex Germanic, 1717.ex Anglia, 1754.ex Groninga, Wall. l. c. (3. Specim.

745 Zoolithus, t. Encriniti poiygoni, 997. ex Hartz, Wall. 360. (I. Tpecim

246 Zootypolithus, I. Apus polyphemi Schaefferi, in marmore grifeo, 17:6. ex ... Wall 363. (I. fpecim.

747 Ichthyotypolithis, f. Pleuronedies, convexus \& impreffus, pulcerrimus, in fchilto albo, filfo, duplicato, $: 062 \ldots$, Wall. 36r. (2. Specim.

748 Ishtyotypolithus, f Pleuronedtes, vel Plateffa minoris, impreffa, in fchitto albo, 895. ex Paleftina, Wall. b. $c$. (1. rpecim.

249 I6bthyotypolithus, f. impreffiones 4. flava Pifciculorum, in fchifto albo, calcareo, ab altera parte, figurre Metufarum parvarum, 1516. ex Sidon, Wall. l. c. (1. Tpecim.

750 Ichthyotypolithus. f. impreffiones varix minores, in fchifto præcedenti, 897.895. 900. ex Pappentheim, Wall. l. c. (3. Specion

751 Ichthyotypolithus, f. impreffiones fimiles, 809. 201 . ex Pappenheim, W'all. l. c. (3. Specim.

752 Ichthyotypolitbus of impreffiones convexe, \& impreffe, in fchifto albo, 902-yot. ex Pappenhein, Wall. l. c. (3. fpecim

753 Ichthyotypolithus, pracedentibus fimiles, in fchifto albo, 905. yos. tx Pappenkeim, Wall. l. c. (3! '́pecim.

254 Ielithytyopolithus, f. nracedenti affinis, in marmore grireo, ... ex Sidon, Wall l c. (1: rpeciun.

755 Ichthyotypolithus, f. imprefiones fimiles majores, in marmore grifeo. 1587. ex Sidon, Wall l. c (I. Specim.

756 Ichthyotypolithus, fo impreffiones finiles! latiores, ... ex Sidon, Wall. l. c. (r. rpecin.

257 Ichthyotypolithus, magous. qquamis pyriticofis, in fchitto nigro. 9c9. ex Ilmenare Wall. l. c. ( 1 . Ppecm.

758 Ichthyotypolithus . præcedenti affinis, 'fed non pyriticolus, g1o, ex Suhla, Wall. l. c. (I. Tpecim.

759 Ichthyotypolithus, pracedentis varietas, cum pyirte, in

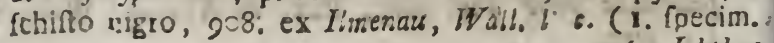
700. Ichthyo- 


\section{Foffiles. Clafse IV. I}

741 Zoolithe, ou Belemnite, approchant ì la precedente, mais nhus grande 824. d'Altorf, Wall 1. c. (1. piec.

742 Zooirinc, ou Trochites, Es Entrochites, en plaqué de marbre quarré, Ė grife, s25. de Sondersnaufen, Wall. 3. 5. है 357. (1. piec

743 Zuu! e. ou Troctites, E Entrochites, ou pareilles ptaques joliss, tres chargées, 829 . de Cobourg, Wall. 1. $\mathrm{c}_{g}$ (I. prec

744 Zoolithe, oss Entrochites branchues, "है Aferies pierres, 1190. d'Allemagne, 1717. d'Argleterre, 1754. de. Groningue, Wall I c. (3. piec.

745 Zoolithe, ou Encrinites, pobygones, 29\%. de Hartz, Wall, 36o. (1. pies.

740 Emareinte d'Tnfeete, ou Monncle de Schaeffer, en marbre gris, 1716 . de .... Wall. 363 . (I piec.

747 Empreinte de Puifion, ou Sole grand Eo tres beau, courbé, Ė imprimé en Schifte double., blanc, Ẽ ouvert, 1562. de..., Wall. $36 \mathrm{r}$. (2. piee.

748 Empreinte de Poiffon, ou a'uze Sole, plus petit, en fthiffe. blanc, 895. de lo Paleftine, Wall. 1. c. (1. piec.

749 Empreinte de Poiffon, jounes ou de quatre petits Pois. fons, en Jchife bbanc calcuire, de laautre cotés les figures. de petites Medufes, 1516. de Sidon, Wall. I. c. (1. piec.:

750 Empreinte de Poiflons, pareilles aux precedentes, petites, en Jchif 897. 9)8. 900. de Pappenheim, Wall. 1 c. (3. piec.

75I Expreinte de Poiftons, pareilles, 899.9 ir. de Pappenheim: Wall. I. c. (3. piec.

752 Empreinte de Poifrons, courties, ED imprimées en fchift blanc, 902-904. de Pippentieim, Wall. I. c. (3. piec.

753 Empreinte de Poiflons, Semblables aux precedentes, em fchift blanc, 905. 906. de Pappenheim, Wail. 1. c. (3. piec.

754 Empreinte de Poiffons, approchantes aux precédentes, en marbre grâs, .... de Sidon, Wall. I. c. (1. piec.

755 Empreinte de Poiffions, pareilles, thuis grandes, en marb.e gris. 1517. de Sidor, Wall I c (1. piec.

756 Empreinta de Poifors, pareilles, p'us larges,... de Sidon, Wall. 1. c. (I. piec

757 Empreince de Poiffon, grand à eezitles periticenfes, en Schifte noir, 909. de llmenau. Wall. I. c. (I pi.c.

758 Empreinte de Poiffon, femblable à la precédente, mais fans pyrites, gro. de Suhle, Wall. l. c. (r. piec.

759 Empreinie de Poiffun, varieté de la precedente, avec pys sites, en Jehift noir, gos. de llmenau, Wa I l.c. ( - piec. 700 Emprein. 
760 Ichthyotypolithus, fimilis precedentibus, in fehifto nigro, gir. y12. ex Ilmenar, Wall. l. c. (1. Tpecim.

Tor Ichthyotypolithus, fimiles, in fchifto nigro, $907.913 . \mathrm{ex}$ Ilmenar, Wall. l. c. (2. Ipecim.

762 Ichthyotypolithus, \& Cauda quafi Murana, in lapide calcareo, cinereo, 896 ex Sidon, Wall. 347? (1. Ppecim. $7^{6} 6_{3}$ Aftacolithus, vel Cauda Cancri permagnt, in fchifto nigro imprefi, 1673 . ex :.., Wall. 354. (1, \{pecim.

264 Aftacolithus, r. Cauda Aflaci magni, convexi, \& impreffi, in fchifto nisro lamellofo, duplicato, 1671. 1672. ex Wall. 254? (2. ipecim

765 Cachlites, r. Cormua Ammonis, permagnus, politus , duplicatus, inde patent concamerationes, 857. ex Coburgo, Will 387. (2. fpecim.

266 Cochlites, f. Cornua Ammonis, finilis fiffus, extus coftatus, duplicatus, politus, 1477. ex Coburgo, Woll $l$. $c$. (2) pecim.

267 Cochlites, 1. Cornua Ammonis, cum fragmento articulatiore dendritico, 856. 872v ex Saxonia, Wail. l. c. (2. fpecim.

768 Cochlites, f. Corrua Ammonis, fimile coftatus, intus arti. cularifatus, mag us, 87í. ex Saxonia, Wall. l. c. (I. fpecim.

769 Cochlites, f Cornua Ammonis, in lamina nolita ammo. nitis, fpacho repleta, 866. ex Coburgo, Wall. l c. (I. friecim.

270 Cochlites, f. Cornua Ammonis, in lamina finiii marmorea, olivace? rubra, 821. ex Rayreuth, 857. 808. ex Altdorff, Wall l. c. (3. Ppecim.

271 Cochbites, f. Cornua Ammonis, vel Theca tabacaria oblongo quadrata, ex maimure ammonitico, 865. ex Aitdorff, Woll. \&c. (I. Ipecim.

$77^{2}$ Cuchlites, f. Cornus Ammonis, in globo argillaceo polito, defciffo repleto, 858 859. ex Coburgo, Wall. l. c. ( 2. Ipecim

773 Coch!ites, f. Cornua Ammonis, fimilis, in giobo argilla cen, pyriticofo, 860. 861. ex Cósurgo, Wall. l. c. (2. ipecitn.

774 Cochiites, f. Cornua Ammonis, parvus, totus mineralifetus, \& duo alii fpatnofi, 8ก̃2. ex Turnau, 863. 8๕4. ex Solsthal, Wall l. c. (3. Specim.

775 Cochlites, f. Cornula Ammonis, permulia, in globo ar. gillacen, valde cofta'o, cum crufta caicarea, 875.876 . ex Soxonia, Wail l. c. (2. Specin. 


\section{Fofjles. Clafje IV.}

760 Empreinte de Poiffon, ou pareilles, en - fchift noir, 911 r. 913. de Ilmenau, Wall. I. c. (i. piec.

761 Empreinte de Poiffon, ou pareilles, en Jchift noir, 907. 9r3. de llmenau, Wall. I. c. (2. piec.

762 limpreinte de Poiffon; ou queue ae Murene, dans uns pierre calcaire cendrée, 896. de Sidon, Wall. 347 ? (x. piec.

763 Aftacolite ou Empreinte d'Ecrivice, ou impreffion de la Queue d'une Crabe, tres grande, enl Jchift noir, I673. de Q.... Wall, 354. ( 1 . piec.

$7 \sigma_{4}$ Aftacolite ou Empreinte d'Ecrivice, ou grande Queue d'Rcrivice, convexe, EO tmprente, en Jchift noir lamelleux, double, 1671. 1672. d'e ..., Wall. 354 ? (2. piec.

$7^{65}$ Coquille, ou Corne d'Amm in, tres grand polie, en deux, pour voir les chambrettes, 857. de Coboung, Wall. 387. (2. pies.

766 Coquille, ou Cnrne d’Ammon, pareil, cofté, divife, Ė polié, à grande cotes, 1477. de Cobourg, Wall. i. c. ( 2 piec.

767 Coquille, ou Corne d'Ammon, avec un fragment arbe. rifé, dans l'articulation, 856.872 . de Saxe, Wall. I. c. (2. piec.

768 Coquil'e, ou Corne d'Ammon, pareil, à cotes, articuld en dedans, grand, 871. de Saxe, Wall. I. c (1. piec.

769 C quille, ou Corrie d'Amunon, or plaque polie, chargée d'anmnonites Jpathenfes, 866. de Cobourg, Wall. 1. c.

(1. piec.

770 Conuille, ou Corne d'Ammon, ou plaques parsils, de marbre rouge, \& olivatre, 821. de Bayreuth, 867.868 . de Altdorf, Wall. I c. (3. piec.

77 r Coquille, ou Corne d’Ammon, or Tabatiere en querré longue, olivatre, de marbre ammonite, 865. de Alrdorf, Wall. \&c. ( I. piec.

772 Coquille, or Corne do Ammon, ou boules argilleujes remplies d'ammonite, 858. 859. de Cobourg, Wall. I. c. ( 2 piec.

773 Coquille, ou Corne d'Ammon, ou pareilles boules argilleufes, pyriteufes, 800. 86r. de Cobourg, Wall. 1. c. ( 2 piec.

774 Coquille, ou Corre d'Ammon, ou petit ammonite, tous mineralife, E 8 deux autres Jpatbeux. 862. de Tournaa, 803. 86 1. de Salsthal, Wall. I. c. ( 2 piec.

775 Coquille, ou Corne d'Ammon, ou plufieurs boules d' le d'ammonites, a grandes cotes, couverte d'une crouse calcaire, 875: 876. de Suxe, Wall. 1. c. (2. piec. 
776. Cochlites, f. Cornua Amnonis, fimiles, intusque Belemmi tes, 877. 878. ex Saxomia, Wall l. c. (2. Specim.

777 Cocklites, 1. Cornra Ammonis, fimiles, 879. 880 ex Saxonia, Wall. l. c. (2. Specina.

778 Cochlites, f. Cornug Ammonis, planus, mineralifatus, cum capite ferpentis, artefactus, 874. ex ...,Wall l.c.

ai (I. Ipecim.

779 Cochlites, f. Corma Ammonis, fimiles, mineralifuta, parva, 873. ex Saxania, Wall. l. c. (4. Specim.

280 Cocoblites, C. Cornua Ammonis. fimi'es, in calcareo transmutaio, 88i. ex Saxonia, $W$ all. b. c. (4. Tpectis?.

281 Cochlites, f. Cornua Amronis, Havefcentes, minores, argute collaix, \& alix, 882. ex. Bimberg, 884. ex ..., Wall. l. c. (4. Specim.

782 Cochbites, r. Cornua Ammonis impreffi, in fchifto albo, \& nigro, 883. ex Ho【ja, 894. ex Pappenheim, Walk. b. c. (2. 'pecim

783 Cochlites, r. Nerititi, alixque Cochlitæ, \&c. 888889. 1102. ex Gallia, I158. ex Stevice, 1719. ex Angtia, IWall 375. Eं 376. (8. rpecim.

784 Coclulites, r. Nerititi, cum aliis, in matrice ochracea, folida, y 4. ron3. ex Aquisgrans, Wall. I. c. (2. fpecim。

785 Cechlites, f. Nerititi, pl æcedentibus affines in calcareo grifeo mutati, 836. 887. ex Hartz, Wabl. h.c. (3 fpecin.

786 Cochlites, r. Nerititi, aliique fimulis, elongati, 1670. ez ...,Woll. b. c. (1. Specim.

787 Cochlites, f. Nerititi fimiles pracedentibus, valde elongati, in matrice, 1669. ex ..., Walb. l. c. (2. fpecim.

788 Cochlitcs, f. Nerititi, fimiles, valde parvi, \& aliæ, in matrice, 89r. ex Suevia, Wall l. c. (4. Ppecim.

789 Cochlites, f. Bucciniti glabri, 887. ex Hartz,... ex Gallia, Wall: 378 , (4, fpecim.

795 Cochlites, f. Bucciniti, coftati, varii, ..., ex Bononia, Woll b. c. (8. fpecim.

79r Cochlites, f. Strombiti, contorto elongati, permulti, in tapide calcareo, permagno, plano, 893. ex Saxonia, Wall, 379. long. $9 \frac{1}{2}$. lat. 8. poll. (I. fpecim.

792 Coclibites. S. Strombiti Neritaque, in lapide achate, plurimi, 892. ex Ganpanic, Wall, lic $c$. (x. Specim. 
776 Coquilte, ou Corne d'Ammon, pareil, avec Belemnites en dedans, 877. 878. de Saxe, Wall. 1. c. (2. piec.

777 Coquille, ou Corne d'Ammon, pareil, 879. 880. de Saxe, Wall. 1. c. (2. piec.

778 Coquille, ou Corne d'Ammon, plat, minsralifé, arac une tête de Serpent, fait par art, 874, de ..., Wall 1. c. ( I. piec.

779 Coquille, ou Corne d'Anmon, pareilles, mineralifés. petites, 873. de Sixe, Wall. 1. c. (4. piec.

780 Coquille, oa Corne d'Ammon. pareilles, changes ess pierre calcaire, 881 . de Saxe, Wall. 1. c. (4. piec.

78r' Coquille, oul Corne d'Ammon, ou pareilles jaumatres, plus petites, d cotes fines, Eo autres; 882. de Bambers? 884. de .... Wall. 1. c (4. piec.

782 Coquille, ou Corne d'Ammon, ou empreinte d'ammonenite, en fchift blanc, $\mathcal{O}^{2}$ noir, 883. de Heffie, 894. de Pappenheim, Wall. 1. c. (2. piec.

783 Coquille. ou Neritites, E autres Cochlites, 888. $88 \%$, I102. Le la France, ris3. de Snevie, 1719. d'Angleter. re, Wall. 375 \& 376 . (8. piec.

784 Coquille, ou Neritites, pareilles, avec autres, dans was? matrice d'ochre, folide, 974. Iro3. d'Aix la Chapelle? Wall. J. c. (2. piec.

785 Coquille, or Neritites, partilles des precedentes, changées en pierre calcaire grife, 886 . 887 . de Haitz, Wall. I. Co (3. piec.

780 Coquille, on Neritites, pareille, allongée, 1670. de .... Wal'. 1. c. (I. piec.

787 Ccanille, ou Neritites, femblables aux precedentes, tres allongée, dans fa matrice, 1609. de ..., Wall. 1. c. (2. piec.

788 Coquille, ou Neritites, pareilles, tres petites, $8^{0}$ artroz dans fa matrice, 891. de Suevie, Wall. 1. c. (4 piec

789 Coquille, ots Buccinites telées, 887. de Hartz, ... de la France, Wal. 378 . (4. piec.

790 Coquille, ou Buccinites differentes, \& cotes, Es...., de Bononia, Wa!l. 1. c. (8. piec.

791 Coquille, ou Strombites, allongés, on Jpirales, daris un: pierre calcaire, platte, 893. de Saxe, Wall. 379. bang. 9!. lat. 8. pouc. (I fiec.

792. Coquille, ou Strombites, \& Neritites en pisrre ng?. the, $8 y^{2}$, de la Chaingagne, Wall. 1. c. (r. poce. 
793 Cochlites, f. Strombiti, \& alii parvi, in achate, \& liberi, \& in lapide arenario, 975. ex Pedemontio, \& Galo Iia 1537. ex Monte St Petri, Wall. 1.c. (3. Specim. 704 rochlites, f. Turbiniti \& Muriciti, 885. ex Hartz,890. ex Galbla, Wabl. 380. 383. (2. Ppecim.

795 Cnchlites, f. Volutiti, I649. ex India Orient. \& Gallia, W Il. 3\%r. (2. fpeciin.

796 Cochlites, f. Ovaria Bucciniti Undari, 1810. ex Angerburg, Wall. o. "non ovum ovo fimilius, quam hic lapis , ovario hujus rariflimi petrifacti, Gronov. MuS. P. 232. , $n^{2} .2658 . "$ (1. Ip cim.

797 Conchites, f. Oftraciti, pernagna, cum operculo, comple us, 1323. 1324. ex Suevia, Wall. 388. (2. f́pecim. 298 Conchites. r. Oftraciti, fimiles, absque operculo, cum a ia mirore fere naturali, in matrice. ex variis conchitis conft inte, 997. ex Juevia, 9.9. ex Hartz, Wall. b. c. (2. fpecim.

790 Conchites, f. Margaritifere, vel Nacra Perli, cum operculo, in lapide calcareo, 93I. 956. cx Helvetia, Wall. 390. ( 3 fpecim.

800 Canchites, $f$ Oftraciti completi, cum Mytulite Criftagalli fimplici, i 188. ex Helvetia, 1322. ex Suevia, Wall. 380 \& 390. (3. rpecim.

801 Conchites, f. Mytuliti Crifta Galli, \& Oftraciti completi, 932. ex Helvetia, 1321. ex Suevia, Wall. l. c. (4. fpecim.

802 Conchites, f. Oftraciti parvi, \& Lazare, in auris com. pletx, cum numino Brandenturgico, 94r. 965. ex ..., Wall. l.c. ( 6 . fpecim.

803 Conchites, f. Lamina permagna, rubra, ¿ polita marmorea, cum multis Conchitis, 830. ex Coburgo, Wall. b.c. ( I. fpecim.

804 Conchites. f. Lamina fimilis, utrinque polita, 83 r, ex Coburgo, Wall. Ẽc. (I. Specim.

805 Conchites, frlamina fimiles, minores, quadratæ ovales, \&c. 818-820. ex Hartz,800 ex Halberfad, Wall. E\%c. (4. 'pecim.

806 Corchites, f. Lamina fimilis, rudis, cum Tellinitis, \& aliis, 83 . ex Coburgo, Wall. 392. (I. Specim.

$8 C 7$ Conchites. f. Chamiti, parvi, in mafta, $966 \rightarrow 969$. ex Coburgo, Wall. 389 . (4, rpecim.

808 Conchites, f. Mytuliti coltati, \& aiii, 952. 958. 959. ex Hartz, 260. ex Colurgo, Wall. 390. (4. fpecim.

809 Conchites, f. Chamiti taves, 948. ex Barbaria, 962.964. ex Haitz, Wall. 389. (4. Tpecim. 
93 Coquille, or Strombites, E autres petites, an agathe, libres, Es en pierre fallomenfe, 975. de Piemont, .... de la France 1537. du Mune St. Pierre, Wall. 1. co (3. piec.

94 Coquille, ou Túrbinites, \& Muricites 885 , de Hartz, 890. de la France, Wall: 300 . 383 . (2 piec.

95 Coguille, ou Voluties, 1649 des Indes Orientales,... dc la France, Wall. $38 \mathrm{r}$. (2. piec.

96 Coquille, ou Nid, on Ovalie cé Ruccin Ondé, 1810. d'An,

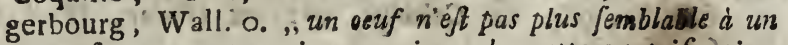
" oeuf, que cette pierre ovaire, de cette spetrification, ", voyez Gronos. Mur. p. 232 ne. 2658 . (I. piec.

97 Conchite, ou Oftracites, complet, avec fon couvercle, tres grande; $1323 \cdot 1324$. de. Suevie... Wall. 383. (2. piec. 38 Conchite, ou Oftracite pareille, fans convercle, ave uns autre petite, coinme naturelle, en matrice, compofée de plufecurs conchites, 997. de Suevie, 949. de Hartz, Wall? 1. c. (2. piec.

19 Conchite, ou Margaritiferes, ou Nacre de Perles, avec fon couvercle, en pierre calcaire, 931. 956. de ta Suiffe, Wall. 390. (3. piec

10 Conchite, ou Oitracite, complet., avec Mytulites, ह? Crete de Cocrifimple, I1885i de la Suife, 1322. ds Srevie, Wall. I c. (3.piec.

I Conchite, ors Mvtulites, Crete de Coca \& E Oftracites complettes, 932. de ia Suifre, 1321. de Suevie, Wall. I. c. (4. piec.

2 Conchite, olb Oftracites, petites, avec Lazares, \& autres, 94I. 955. de .... Wall. 1. c. (6. piec.

3 Conchite, ou Plaque de màrise, tres grandé, polie, avec quantité de coquillages, 83o de Cobourg, Wrall. i.c. (I. piec. - Conchite, ou pareille Plaque, polie, $83 \mathrm{I}$. de Cobourg, Wall, 1. c. (r. piec.

; Conchite, ou Plaques pareilles, plus petites, quarrées', ovales, EOc. 81 8-820. de Hartz 869. de Halberftad, Wall. 1. c. $(4 ;$;iec.

Conchite, ou Tellinites, sj autres, en plaqule, non políe, 834. de Cobourg, Wall. 392. (I. piec.

Conchite; ou Chamites, fetites, en mafe, 966-969. de Cobourg, Wall. 389. (4 piec.

Conchite; ou-Mytulices, à cotes, छ autres, 9\$2. 958. 959. de Hartz, 960. de Cobourg, Wall. 390 . (4. piec. Conchite, ous Chamites. liffe; 948. de Barbarie, 9,62. 964. de Hartz, Wall. 359 . $(4$, piec.. 
810 Conchites, f. Chamiti majures, 945. 946.954. ex Harte W.all. b. c. (3. Tpecim.

Ir Conchites, f. Chamiti, pracedentibus fimiles, 943. 944. ex Hartz, Wall. l. c. (2. Ipecim,

812 Conchites, T. Chamiti parvi, \& Pinniti, in matrice arenofa, 949-952. ex ..., 1758. ex Suevia, 1759. ex Rimtni, Wall. 389. \& 39r. (8. Specim.

813 Conchites, f. Mytuliti varii, in matrice, cum Amnonisis, Octaliis, 969. ex Coburgo, 972. ex Goslar, Wall. 390 . (3. fpecim.

814 Conchites, f. Bucarditi laves, \& alii, 942. 955. 956. ex Hartz, Wall. 393. (3. Specim.

815 Conchites, f. Bucarditi varii, leves, \& coftati, 936. ex ..., 937. I185. ex Helvetia, Wall. l. c. (3. Specim?

Ir6 Conchites, r. Buccarditi minores, coftati, in matrice, 938. 940. 957. ex Saxonia. 951. ex Hartz, Wall. b. c. (6. fpecim.

817 Conchites, f. Bucarditi echinati, \& coftati, in lapide cretaceo, 1720. 172 1. ex Anglia, Wall.b.c. (2. fpecim.

8 is Conehites, f. Bucarditus, magnus, lævis, Mactram Stultorum referens, 1186. ex Goslar, Wall.l.c. (I. Specim

819 Conchites, f. Peßzenitus, perfeetus, permagnus, pulcre coftatus, 933. ex Ortenburg, Wall. 394. $3 \frac{1}{2}$. poli. dia meter. (I. Ypecim.

820 Conchites, r. Pestiniti varii, coftati. cum Amurso, it lapide cretaceo, 935. 939. ex Suevia, Wall. 1. c. (3) specim.

22x Conchites, f. Gryphiti varii, 973. ex Coslar, 1655. e Cap. Bon. Spei. Wall. 396. C 4 . ipecim.

822 Conchites, f. Hyfterolithus, \& alius, 970. ex Helvetia 1648. ex Nafjovia, Wall. 432. (2. Ppecim.

823 Conchites, f. Terebratuliti glabri, \& alii, in lapid. cretaceo albo, 977. ex Aquisgrano, 1722. ex Anghia Wall. 398. (4. fpecim.

224 Conchites, f. Terebratuliti plicati, coftati, varii, 97 ex Hartz, Wall. l. c. (10. Specim.

225 Conchites, f. Vermiculiti permagni, in lapide ar. naceo, 1807. ex Injula Melitis, -Wall, 403. long. 6 poll. (I. Specim.

226 Conchites, f. Balaniti, (Lepaditi) \&c. fuper conchar 939. ex Hartz, Wall. 405. ( 1 . Ipecim.

227 Conchites, f. Echiniti Cydares, 980. ex Helvetia, Wi 399. (2. Specim. 


\section{Foftles. ClafJe IV.}

3ro Conchite, ou Chamites pareilles, plus grandes, 945. 946. 954. de Hartz, Wall. 1. c. (3. piec.

3 ar Conchite, ou Chamites pareilles, 943. 944. de Hartz, Wall. 1. c. (2. piec.

112 Conchite, ou Chamites pareilles, petites, avec Pinnites. en matrice, fablonneufe 949-952. ex ... 1758. de Suevie, II 59. de Rimini, Wall. 389. EO 391. (8. piec.

13 Conciite, or Mitulites diverfes, dans leur matrice, avec Ammonites, Es autres, 969. de Cobourg 972. de Goslar, Wall. 390. (3. piec.

14 Conchite, ou Bucardités liffes, \& autres, 242. 955. 956. de Hartz. Wall. 393. (3. piec.

15 Conchite, ou Bucardites differentes, lifjes, Es coffees, 936. de.... 937. I185. de la Suiffe, Wall. 1, c. (3.piec.

16 Conchite, ou Bucardites pareilles, plus petites coftees. en matrice, 938. 940.957. de Saxe, 961 . de Hartz, Wall. 1. c. (6. piec.

17 Conchite, ou Bucardites epineufes, El coftes, en pierre de craye, 1720. 1721. d’Angleterre. Wall. I. c. (2.piec. 18 Conchite, ou Bucardite grand, liffe imitant le Bonnet d'Hongrie, 1186 . de Goslar, Wall. I. c. (r. piec.

19 Conchite, ou Peetinite, tres grand, beau, parfait, \& cotes; 933. de Ortenbourg, Wall. 394. $5 \frac{1}{2}$, pouc. de diam. (r. piec.

20 Conchite, ou Pectinites differentes, d cotes, avec une coquille d'Eventaille, en pierre de craye, 935. 939. de Suevie, Wall. I. c. (3. piec.

I Conchite, ou Gryphites differentes, 973. de Gosiar, 1655. du Cap de bonne Efperance, Wall. 1. c. 396. (4. piec. 32 Conchite, ou Hyfterolite, \& un autre, 970. de la Suife, 1648. de Nalrau, Wall. 432 . (2. piec.

:3 Conchite, ou Terebratulites pelées, \& autres, en pierre de craye blanche, 977. d'Aix la Chapelle, 1722. d'Angleterre, Wali. 328 . (4. piec.

4 Conchite, ou Terebratulites plicées, El d cotes differtentes, '971. de Hariz, Wall. I. c. (10. piec.

5 Conchite, ou Entale, tres grand, en pierre arenaire. 1807. de l'10 Melite, Wall. 403. long 6 $\frac{3}{2}$. porc. (I. piec.

6 Conchite, ou Balanites, (Lepadites) Jur une coquille, 989. de Hartz, Wall. 405. (I. piec.

7 Conchite, ou Echinites Diadernes 980 . de la Suiffe, Wall. 399. (2. piec. 
828 Conchites, f. Ex liniti fimiles, alhi, in creta, a pyro. macho nigro, 1723. 1724. ex Anglia, Wall. l. c. (s. fpecim.

829 Conchites; f. Echiniti, in creta alba, 1725.1726 . ex Anglia, Wall. l. c. (3. '́pecim.

830 Conchites, f. Echiniti cordifnrms, arenlis hexagonis, 982. 983. ex Pedemortio, Wall. l. c. (2. fpecim.

831 Conchites, f. Echiniti giabri, 930 ex Aquisgrano, 978. Trajecti ad Mofam, 981. ex Ginglia, Wall. b. c. (3. fpecim.

832 Conchites, f. Echiniti plani, convexi, 979 . ex Trajecti ad Mofam, Wall b. c. (2. fpecim.

833 Conchites ir Echinitus, permagnus, convexus, I763. ex Maftricht, Wall. l. c, (I rpecim.

834 Conchites, f. Errhinitus, profunde fulcatus, achatinus, 1538. ex Anglia. Wall l. c. ( 1 fpilin.

$\mathbf{8 3 5}$ Conchites, f. Echiniti globulofi, convexi, plani, in achatem mutati, 984. ex Pedenontio, Wall. b. c. (3. fpecim.

836 Conchites, f. Echiniti varii, minus fulcati, glabri, 985. ex Anglica, 987. ex Pedemontio, Wall. $i, c$. (4. fpecim.

337 Conchites, r. Lapides Fudaici, 886. ex Hartz,Wall. $\$ 00$. (12. Ipecim.

838 Conchites. f. Lapides Fudaici fimiles, 1650. ex Trajęti ad Mosam, Wall l. c. (r z. rpecim.

839 Conchites, f. Lopides Fudaici, cylindroides, \& alii, in creta alba, 1203: 1727, ex Anglia, Wrall. 40r. (4, fpecim.

840 Conchites? f. Mamilliti, five lapilli regulariter bexago. ni, vel pentagoni, vel quadrati, afterifon notati, cum lapidibus Judaicis caudatis, in creta aloa, 1718. 1728. ex Anglin, Walb. \&c. (3. fpecim.

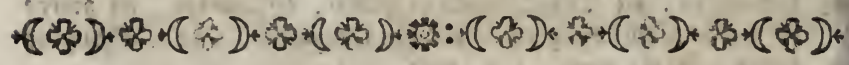
LAPIDES FIGVRATI.

34 r Lithomorphus, f. Lapis Florentinus, muros antiquos, ca. lumrac oebulofum referens, ro84. ex Fiorentia, Wall. 449. in quadro aurato, altit. $8 \frac{8}{2}$. Latit. II poll. (I. Ipecim.

342 Lithoniorpins, r Lapides Florentini, oblongi quadrati, 7v2. 793. ex Fierentia, Wall. b. 6. (3. Specim. 


\section{Fufiles. Clafe IV.}

828 Conchite, ou Echirites pareilles, en craye blanche, E? pierre d feu noir, 1723. 1724 . dingleterre, Wall. I. c. (2. piec.

829 Corchite, ou Echinites pareilles, en craye blanche, 1725. 1726. d'Angleterre, Wall 1 c (3. oiec.

830 Conchite, ou Echisites, en forme de coeurs, avec des ftries hexagones, 982. 983. de Piemont, Wail. 1. c. (2. piec.

83r Conchites, ou Ecrinites pareilles, pelées 936 . d'Aix la Chapelle, 978 de Maftricht, 981. d'A gleterre, Wall. 1 c. (3. piec.

332 Conchite, ou Echinites pareibles, EO convexes, 979. de Maftricht, Wall 1. c. (2. piec.

333 Conchite, ou Echinite pareil, elevée, tres grande, 1763. de Maftricht, Wall. I. c. (I. piec.

334 Conchite, ou Echinite pareille, tres grande, à ftries tres profondes agatkifées, 15,8. d’Angleterre, Wall. I. c. (I. piec.

335 Conchite, ou Echinites globuleufes, convexes \&2 applaties, changées en agathe, 984. dé liemont, Wall. 1. c. (3. piec.

36 Conchite, ou Ecbinites pareilles, diverfes, pas tant fillonnés, pelées, 985. d’Angleterre, 987 de Piemont, Wall. 1. c. (4. piec.

37 Conchite, ou Pierres Judaiques, 886. de Hartz, Wall. 400. (12. piec.

38 Conchite ou Pierres Judaiques, pareilles, $\mathcal{E}^{\circ}$ autres, 1650. de Maftricht, Wall. I. c. (12. piec.

39 Conchite, 0u Pierres Judaiques, cylindriques, EO autres, en craye blanche, 1203. 1727. d'Ang eterre. Wall. 401. (4.p.

40 Cosctite. ou Mamellites, ou pierres regulierement hexagones, ou pentagones, ou quarrées, marquées de petites étojles, ayec pierres gindaiques aे queue, fur craye blaneñe, 1718. 1928. d'Angleterre, Wall. \&c. (3. piec.

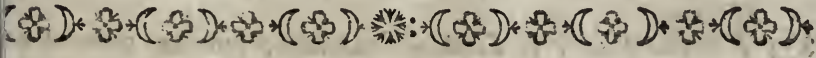 PIERRES FIGUREES.}

11 Lithormorphe, ous Pierre Florentine, reprefentant des murs antiques, Ë un ciel en nues, 1084. de Florence, Wall. 449. encadré d'une bordure d'orée, altit. $8 \frac{1}{2}$. batit. $11 \frac{l}{2}$. pouc. (r. piec.

I Lithomorphe, ou Pierres Florentines, pareilles, oblongues, quarrés, 792. 793 de Florence, Wall. 1.c. (2. piec. 
843 Lithomorphus, f. Lapides Florentini, minores, quadrati 794-796. ex Flarontia, Wall. b. c. (3. fpecim.

844 Lithomorphus, f. Lapides Florentini, oblongi, 797-799. ex Florentia, Wall. l. c (3. Ipecim.

845 Lithomorphus, vel Phytomorphus, 1. Lapides dendritici, in laminis dendriticis oblongis, fuper fchiftum album, rubro nigroque arborefcentibus, 802. .803. ex Pappentieim, Wall. 448. (2. fpecim.

846 Lithomorphus, f. Lapides dendritici, in haminis fiffis dendriticis, permagnis, arbufculis rufis, nigrisque, utrinque conficicuis, 1663. ex Pappenheim, Wall. l. c. long, 123. lat $7 \frac{2}{4}$. (2. Cpecim.

847 Lithomorphus, f Lapides dendritici, in laminis fimiles dendriticis minoribus, in fchifto albo, 1181. ex Pappenheim, Wall. l. c. (2. Ipecim.

848 Lithomorphus, f. Lapis dendritica, in lamina utrinque dentrica, in fchifto albo, 1184. ex Pappenheim, l. c. (I. f́pecim.

849 Lithomorphus, f. Lapis dendriticus, in lamina alta, 805. ex Pappenkeim, Wall. l.c. (I. fpecim.

850 Lithomorphus, f. Geographicus, figuris grifeis, in fchifto albo, 807. ex Pappenheim, Wall. 449. (I. rpecim.

350 Lithomorphus, c. Lapides figurati, ab una parte florem afterilantem, ab altera lunara crefcentem referentes, $\mathbf{1} 182$. 1183. ex Papperheivo, Wall. 445. \& 449. (2. Ipecim.

851 Lithomorphus, f. Lapis dentriticus, , adjectis (arte) adibus, margine marmoreo, 814. ex ..., Wall. l. c. ( $\mathrm{r}$. Ipecim.

852 Lithomorphus, f. Lapis dendriticus, arbufculis utrinque punctatis, in lamina flava, 808. ex Florentia, Wall, l.c (I. \{pecim.

353 Lithomorphus, f. Lapides dendritici, f. lamine fimiles, a minores, 8i1-813. ex Brunfuicia, Wall. l. c. ( 3 ipecim.

854 Lithomorphus, f. Lapides dendritici, laminæ precedenti bus. \& variz craffiores, 809. 810 . ex Sandershaufen Wall.t.c. (2. Ipecim.

855 Lithoinorphus, f. Lapis dendriticus, in lamina fimili, fe major, 1732. ex Blankenburgo, Wall. l. c. (3. Specim.

856 Lithomorpbus , f. Lapis dendriticus, in lamina preceder te variante, 1731. ex Blankenburgo, Wall.l.c.c. (1. fpecin

857 Lithomorphus, I. Lapis dendriticus, in lamina magna utrii que dendritica, 3729 . ex Blankenburgo, W.all. i. c. ( Ipeciss. 
843 Lithomorphe, ou Pierres Florentines, pareilles, plus petites, quarries, 794-796. de Florence, Wall. 1. C. (3. piec.

844 Lithomorphe, ou Pierres Florentines pareilles, oblongues, 797-799. de Florence, Wall. 1. c. (3. piec.

845 Lithomorphe, ou Pierres dendriticées, oblongues, en $\int c h i \beta$ blane, darbres rouges, $\mathcal{E}^{2}$ noires, 802.803 . de Pappenheim, Wall. 448. (2. piec.

846 Lithomorphe, ou Pierres dendriticées, ou grandes plaques dentritique, devifée, en arbres roufjes, \& ${ }^{2}$ noires, 1663. de Pappenheim, Wall. 1. c. long. $12 \frac{3}{4}$, latit. $7 \frac{3}{5}$. pouc." ( 2. piec.

847 Lithomorphe, ou Pierres dendriticées, or pareilles plaques, plus petites, en Jchift blane, 1181. de Pappenheim, Wall. 1. c. (2. piec.

848 Lithomorphe, ou Pierre dendriticke, ou pareille plaque dentritique, en fchift blanc, Ir84. de Pappenheim, Wall.

1. c. (x. piec.

849 Lithomorphe, ou Pierre dendriticée, ou paseille plaque dendritique, en fchift blanc, 805. de Pappenheim, Wall. I. c. (1. piec.

850 Lithomosphe, ou Pierre Geographique, en Jchift blane, à figures grijes, 807. de Pappenheim, Wrill. 449. (1. piec. 850 Lithomorphe, ou Pierres figurées, d'un coté, \& fleur en etosile, $\mathcal{E}^{\circ}$ de l'autre coté, reprefentant une dennie lune. 1182. I183. de Pappenheim, Wall. 445.\& 449. (2.piec.

85 I Lithomorphe, ou Pierre dentriticée, ou l'on a adjouté par art des maifornettes, en bordsre de marbre 814. ex .... Wall. 1. c. (I. piec.

852 Lithomorphe, ou Pierre dentriticée, darbres pointillée, en plaque jaune, S08 de Florence, Wall. l. c. (I. piec.

853 Lithomorphe, ou Pierres dentriticées pareilles, phus petites, 8II-8r3. de Brunsvic, Wall, I. c. (3. piec.

854 Lithomorphe, ouPierres dentriticées, pareilles plaques, differentes, plus epaifjes, 809. 810 . de Sondershaufen, Wall. 1. c. (2. piec.

855 Lithomorphe, ou Pierre dendriticée, pareille plaque, plus grande, I732. de Blankeobourg, Wall. I. c. (I. piec.

356 Lithomorphe, ou Pierre dendriticée, ou pareille plaque, autrement figuree, 1731. de Blankenbourg, Wall. I. c. (1. piec.

857 Lithomorphe, ou Pierre dendriticée, ou grande plaque de chaque coté dendritique, 1729. de Blankenbourg, Wall. I. c. (I. piec.

$$
\text { Ti } 4 \text { 858. Litho- }
$$


858, Lithoworphus, f. Lápis devidriticus, in lamina, fimtlis magnitudinis, 1730. ex Blankenburgo, Wall. l. c. (I. Ipecim.

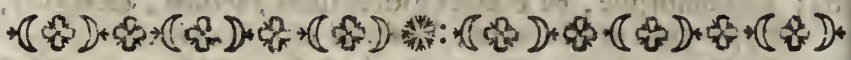

$$
\begin{gathered}
L A P I D E S W A R I I \\
C A L C V L I Q V E .
\end{gathered}
$$

859 Lithoglyphus, an Lithomorphus, f. Ludus naturce, vel Helmontii, five lapis cairaréus, extus ochraceus, intus giifeus, planus. firuc politus, venis fpathofis nigris . 1478. ex Coburgo Woll 454. (2. Specim.

860 Lithoglyphus, r. Ludus Helmontii, politas, cum venis fpathofis nigris, 1011. ex Coburgo, Wall. l. c. (I. fpecim.

861 Lithoglyphus, f. Glabuius calcareus, (Veldkogels) fiffus politus, pyrite dendrito-minerallfatus, originem REtitarum elucidans, 870. ex Bambergia, Wall. l. c. (2. it Wfpecim.

802 Lithoglypkus, f Lapis Malleiformis, vel Fulminans fic dictus, 1604 ex .... Wall. 455. (I. Ipecim.

863 . Lithoglyphus, r. Gloius, fimilis præcedenti, rubens, 1665. ex ....Wall. l. $c$, (1. Specin.

864 Lithoglyphus. C. Tefferce parva. ad I00. orgyas effolf , 1652, ex Helvetio. Wall l. c. (2. Ppecim.

865 Lithotomus, r. AEtites oblongus, cum lapillo inclulo, 1025. ex ..., Wall. 457. (I. Tpecim.

866 Lithotomus of. Etites magnus, converus oblongus, cum arena, \& lapillis intus.... ex ..., Wall.l.c. ( 1. rpecim. 867 Calculus, f. Lapis Malaccenfis verus, vel Pedra del Porco, cinereus, pinguis magnus, fifura in medio, filo aureo inclufus, catena aurea appendente, ... ex $\mathbf{M a -}$ lacca, Wall. 48r. pond. lapidis, dragm. V. gran. IX. pond, auri, 1 $3 \frac{1}{2}$. engl. (I. Specim.

808 Calculus, f. Pedra del Porco, grumulatus, pond. lapidis: gran. XXVII. ( $\mathrm{x}$. Tpecim.

869 Calculus, f. Lapis. Ceilonenfis, vel Pedra del Porco 3 filelog aureo inclufus, \& ex catena aurea dependens, 1668 ex Ceilon, Wall 48r? (x. Specim.

8;0 Calculus, f. Lapis Equi, five Hippolithus, r107. ex .... Wall.483, (I. Ipecim.

871 Calculus', '. Lapis Bradypi, coloris hepatici, 1666, es India Orient. Wall. O. (I. Specim. 
858 Lithomorphe, ou Pierre dendriticée; au pareille plaque, de la mesne grandeur, 1730. de Blankenbourg, Wall. 1, c. (1. piec.

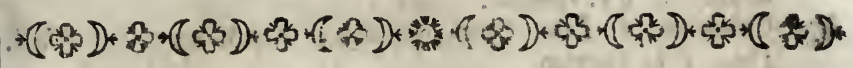

PIERRES, TANT D'ANIMAUX

QUE DAUTRES.

859 Lithoglyphe, ou Lithorrorphe, ou Jeu de la Naturo, ou de Helmont, ou pierre caicaire, ochracé en dehors. grife en dedans, platte, fendu, polie, d veines jpatherfes noires, 1478. de Cobourg, Wall. 454 . (2. piec.

860 Lithoglyphe, ou Jeu de Helmont, polie, avec veine de Spath noiratre, 1011. de Cobourg, Wall. 1. c. (I, piec. 86I Lithoglyphe, ou Boules calcaires, Separies en deux, polie. mineralifées de pyrite, demonftrant l'origine de la pierred' Aigle, 870. de Bamberg, Wail. I. c. (2. piec.

862 Lithoglyphe, ou Marteau de Pierre, ou Pierre de Foudre; 1664 de.... Wall. 455. (I. piec

863 Lithoglyphe, ou Boule partille, rougeatre, I665. de.... Wall. 1. c. (I. piec.

354 Lithoglyphe, ous petits Dez, à 100 . toifes de profondeur, 1652. de Suifre, Wall. l. c. (2. piec.

365 Lithotome, ou Pierre d'Aigle, oblongue, avec petites pierres en dedans, 1026. Wall.457. (1. piec.

36 Lithotome, ou Pierre d'Aigle, pareil tres grande, convexe, oblongre, avec fable, Es petites pierres en dedans, ... de... Wall. I. c. : (I. piec.

307 Calcule. ou veritable Pierre de Porc de Malacque, cendréz, graffe, grande, au milieu une fente, enchainté par dès fils d'or, une chairie dor.... de Malacca, Wall. 411. pef. de la pierre, dragm. V. grains IX pef.d'or I $3 \frac{1}{2}$. engl. (1. p.

68 Calcule, ou Pierre de Prrc, en prudre, ou grumulé, pej. de la pierre grains $X X \boldsymbol{X} I I$. (I. piec.

69 Calcul ou Pierre de Porc? entouré de fils d'or, pendant 4 une chaine d'or, I668. de Ceilon, Wall. 48I? (I. piec.

70 Calcul, ou Pierre de Cheval, trouvé dans l'eftomac, 1107. Wall. 483. (I piec.

$77^{1}$ Calcu! , ou Pierre d'Unau, couleur de foye, :666. des Indes Orientales, Wall. o. (r. piec.

$$
\text { T t } 5+872 \text { Cated }
$$


872 Calculus, f. Lapis Bezoar, olivacuus, 1048. ex Indie Orient. Wall. 478 . (1. Specim

$\$ 73$ Calculus, f. Lapis de Goa, non auratus, 1653. ex India Wall. O. (1. fpecim.

$\$ 74$ Calculus, r. Pila Cervi, fusca, Iro6. ez ..., Wall. 479. (1. fpecim.

875 Calculus, f. Pila perməgna, intus villofa, fusca, .... Wall. 487. (1. Ppecim.

876 Calculus, f. Pila magna, intus \& extus fusca, villofa, 1674. Wall. l. c. (r. Specim.

877 Celeulus. F. Pile, minora, fimilia, $804.805, \ldots$ Wall. b. c. (2. fpecim.

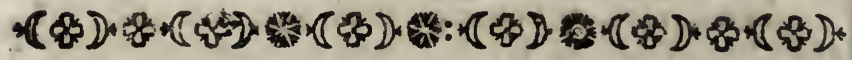
$A R$ T $E$ E F A C C T $A$.

I Magnes, Orichalco inclufus, fatis validus, ac. (I. fpecim.

2 Magnes, fimilis, cum Anchora, fortior. (x. fpecim.

3 Bacilliferrei, magnetici, conjuncti. (3. Specim.

4 Caput Philofophi, caput ex lapide fiavo, tunica peetoris, ex alabaftro, in pede lapidis lydii, antiquum? (I. Ipecim.

5 Caput Philofophi, more antiquo. (1. Cpecim.

6 Caput Sileni, ex marmore albo, in pede ferpentino. ( $\boldsymbol{I}$. Ipecim.

7 Bufta Pontificialis, femi convera, ex alabaftro, in qua. dro aurato. ( $\because$. ppecim.

- Caput Imperatoris, v. Ducis Romani, femi convexum, es alabaftro, in quadro rotundo deaurato. (r. fpecim.

9 Sanea Mater, cum Infante, valde convexa, in lamina ebur. nea ovali. (r. Speciss.

10 Podephare Uxor, in leculo, fugientis fojephi tunicam tf it nens, in parva tabella, oblonga, ebursea, opus femicor vexum. (I. fpecim.

II Bacchonale, fiftens Silenum, \& alium, cum uvis, \& is in lamira eburnea, opus rersiconvexum. (I. Specir

12 Bacchanale, fimilis, cum tribus figuris, \& aliis rebus, op! 12 femiconvexum, in eburne ovali (1. (pecim.

x3 Mandragore (Áardmannetjes) corpus ex radice, capi ex cranis avicularum, manus ex earum pedibus, pedt ex pedibus rararom (2. fpecim.

I4 Gummi Maflix, frufta magna, diaphana, ex Guinea. $\left.1\right|_{4}$ Specim.

15 Indige, ex Guirea. (2. fpecim. 
$87^{2}$ Calcul, ou Pierre de Bezoar, olivatre, 1048. des Indes Orientales, Wall. 478 . (I. piec.

873 Calcul, ou Pierre de Goa, fans dorure, 1653. Whes Indes, Wall. o. (1. piec.

874 Egagropile, ou Boule de Cerf, trun, II 66 . Wall. 479. (1. piec.

875 Egagropile, ou Boule de Poil, tres grand, velu en dedans brun, .... de Wall. 487. (1. piec.

876 Egagropile, ou Boule pareille, grande, velu, en dehors EO dedans, brun, 1674. Wall. I. c. (I. piec:

877 Egagropile, ou Boules, pareilles, plus petites, 804. 805. Wall. 1. c. (2. piec.

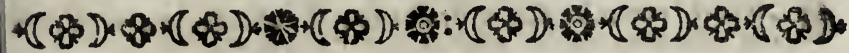

\section{$A R T I F I C I E L L E S$.}

I Pietre d'Aiman, monté en cuivre, affez fort, (1. piec.

2 Pierre d'Aiman, pareil, monté de meme, avec un Ancre; plus fort . (1. piec.

3 Batons ferreufes, magnetiques, jointes, petites. (3. piec.

4 Tete, ou Bufte d'un Philofophe, en maniere antique, fur un piedeftal de pierre de toucke, à tete de pierre jaune, E\$ tumique d'albatre. (r. piec.

5 Tete, d'un Philofophe, ou Bufte pareil. (I. piec.

6 Tete de Silene, de marbre blanc, fur un piedeftal Jerpentir. (1. piec.

7 Bufte d'un Pape, en bas relief, d'albatre, en cadre d'ore. (r. p.

8 'Tete d'un Empereur Romain, en bas relief; d'albatre, en cadre ronde, d'oré. (I. piec.

9 Sainte Mere, avec l'Enfant, en bas relief, tres convext, en plaque d'yvoire ovale. (I. piec.

10 Femme de Pothiphar, couchée fur un fopha. tensant le martear de Jofeph fuyant, tres relief, fur une petite plaqus oblongue d'yvoire, ( $x$. piec.

I I Bacchanale, reprefentant Silene, Eo une autre figure, avec des raifins, en bas relief, en yvoire ovale. (x. piec.

12 Bacchanale, pareil, d trois figures, Es beaucoup d'autses chojes, en basrelief, fur yvoire ovale (x. piec.

3 Mandragores, le corps de la racine, les tetes de celle des oifeaux, les mains des pattes de l'oifeau, les pieds ceux d'une grenouille. (2. piec.

(4) Gummi Maftix, grand, E transparent morcears, de de Guinée. ( 2 piec.

5 Indigo, de ls Guinée. (2. piec. 
16 Tegmen, f. Ornamentum pedroris, ex plumis rubris flavis, cyareis, albis, pfittaceic, ex America. (I. fpecim.

17 Tegmen, f Ornamentum pę̈oris,'pracedenti varians. (I.fpec.

18 Tegmen pridoris, ex globulis vitreis verficoloribus. ( 2 , Specim

19 Tegmen pudoris, f. alia finilia. (3. Ipecim.

20. Leêus penfilis, ex cuttoneo albo, fatis craffo, in quo fo. minæ Amelicar infante gerunt. ( 1 : fpecim.

21 Zona, lata, ex cottnneo albo, five tegmen corporis virilis Americi. (I ip cim.

22 Arma lignea, ex America. (2. Specim.

23 Collarium, ex nucleis, corallisque, in catenam effec. tis, gau bus milites Americani utunrur. (2. \{pecim.

24 Arnubli rachiales \&c. cruralesve, \& collaria, ex fruetibus vel conchilis juico adpiicatis. (3. fpecim.

25 Collarivm, ex fruclu Tamarindi, cottoneo jubeto. (I. Specim.

26 Collarium, fimile pracedenti. ( $\mathrm{r}$. fpecim.

27 Ornamentum, tx fricku, vel ex rucleo Tamarindi, valde fonorum, quo in Caltando utuntur Nigritz ( $\mathrm{r}$. fpecim.

28 Oriamentuns, fin le sliud. (I fpecim.

29 Fifru! Nigritarum, ex arundine fachari, \&̌ ex ofle. (2. fpecim.

30 Pocula colata, ex nuce cocor. (4. fpecim.

31 Pocula crata, mirora. (6. fpecim.

32. Idolizusculum, fervorum nigritarum, cum duplici campana, pro pontifice, ex ligno. (2. Specim.

33 Idoliusculum. fimile cotonatum, ex ligno. (1. fpecim.

34 Lectus penfilis, ex foliis arundinaceis, ex America. ( 1 . fpecim

35 Scapha parva, ex ligno a'bo, inqua infantes cunarum loco imponuntur. ( 1 . fnecim.

36 Vagine longæ, expanfiles, in qquibus paftum ad panem condunt Anericani. (4. Specim.

37 Calathi, texti Americanorum. (3. Tpecim.

38 Calathi viatorii, five capfule quadratæ, planæ, ex junco texto. (2. 1pecim.

39 Calatbi viatorii, fimiles, minores. (2. Ppecim.

40 Calatbi viatorii, fimiles, minimi. (4. Trecim.

4I Crepitacula, ex nuce cocos, fripitibus adfix?. (2. fpecim.

42 Crepitacula, fimilia, minora, aliter fabrefacta. (C. Specim.

43 Fasciculi graminis, (Zeilgras) haud torti fila, una cum filis tortis, noftris cordis agiva'entibus, (2. Ipecim.

44 Pasciculi gram nis. finiles, ac præcedentes. (3. fpecin. 45 Utemfilia, $\int$ varia fievilia Nigritarum. (plurima fpecim. 
26 Ornament de poitrine, quarré, de plumes rouges, jaunes,blewes, Blanches, vertes, de l'amerique. '(1."piec.

I7 Orn tre nt de poitrine. Sersblable au procedent, mais d'as outro defein (1. piec.

18 Tabliers, pour l'ujage des femmes, de coraux ou boules de verre, de diverje couleurs (2. piec

ro Tabliers quarréz, pareilles, \& autres. (3. piec.

25 Hamac de coton blanc, afez epais, dans lequel les femmes d'Amerique, portent leurs Enfans (". piec

- Ceinture, large, de coton blanc, que bes hommes portentas Amerique (1. piec.

22 Armes de Bois, de l'Amerique. (2. piec.

23 Colliers, faits de noix \&o de coraux de verre, en forme de chaine, que les foldats ae negres d'Amerique, mettent quand ils yunt en guerre, (2. piec

4 Anneaus de Bras, EOc. de jambes $E$ colliers faitsdes fruits, ou petites conques attachez au jonc. (3, piec.

is Collier, ou le memes du fruit de Tamarinde, attache as coton. (1. piec.

6 Collier, pareil du precedent. (x. piec.

17 Ornament, du fruit ou dis Noyau de Tamoriside, ou mollóes tres Jonare, que les Negres Se metsent dans leur dances. (I: $\mathbf{p}$.

8 Ornament pareil. ou autre. (1. piec.

- Flutes des Negres. faites des os, EO de la canne du fucre. (2.piet.

- Vafes, du noix de cocos, (4. piec.

I Vafes pareilles, plus petites. ( 6 . piec.

2 Idole, fait de bois, pour les efclaves negres, avec une doss. ble clochette pour le pietre. (2. piec.

3 Idole pareille, couromé, de bois, ( 1. piec.

4 Hamac, fait des feuilles de canne, on de jonc. (I. piec.

5 Bateau de bois blanc, dans les quels ils mettent leurs enfans, comme dans un berceau, petite. (I. piec.

6 Etuis long:, qui pexvent s'allonguer, dans lesquels les Americains mettent la plie de leurs pain. (4 piec.

7 Corbeilles, des Americains. (3. piec.

8 Corbeilles de voyage, ou etuis long's quarrée de jonc leck, (2 piec.

- Corbeilles de voyage. pareils. (2. piec.

3 Corbeilles de voyage, tres petits. (4. piec.

1 Grelots, de noix de cocos, attaches d des batons. ( 2 piec.

2 Grelots parilis, plus petits, EO d'urse autre figure. (6. pies.

3 Paifceau de fils non tordus, d'une eppece de gramen, avec wis faifce au de fils tordis, en cordes fi fortes que nos cordes communes. (2. pies.

(1) Faifceau pareil. (3.piec.

s Utenfiles. de terre cuire des N'egres, de diverfo forme. Ef figure (plufeetrs pieces. 


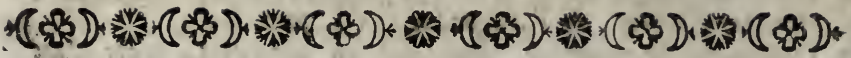 $A R M E N T A R I A E \odot C$.}

I Een Taafel van Mahonie hout, gedekt met een onge: meen fchoon blad van drie breedens, en gecombineerd uit agt, door fchroeven onınerkelyk aan elkander fluitende Itukken; langwerpig vierkant, met boogsgewys uitIpringende hoeken; hebbende wederzyds in de lengte vyf colommen, en in ieder 7. ladens, de vier hoeken een gelyk getal, dus te zaamen 98. ladens; daar nevens in de wederzydifche breedtens vier colommen, met in jeder 10. Ladens, dus 40 . of in ' $t$ geheel 138 . vierkante Ladens, zynde de eerftvoorfz. lang $16 \frac{1}{4}$. breed $14 \frac{2}{2}$, ea hoog $3 \frac{1}{4^{*}}$ die van de vier hoeken, zyn lang en breed 15. en de laatft voorfz. zyn breed 19 lang

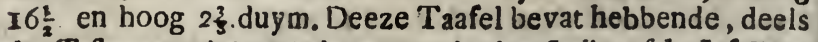
de Teftacea, gelyk ook het vervolg der Indiaan/che Infeeten, heeft een lengte van 10 . ende breedte van 7 . en eene høogte van 3. voet.

2. Een Glaaze Kabinet, van Noteboome hout, met eene boogsgewyze kap, hebbende van boven twee deuren, en van onderen, twee doorgaande Laden. De deuren bevatten 6 . gefleepe fpiegel-ruiten, met 4 . derzelven wederzyds, van binnen wit, verdeeid, met geningerde, en op de kanten vergulde planken, in zes vakken, op welke de Steenagtige Zoophyten zyn geplaatft geweeft: zynde hoog 8. voet, breed van agteren 5. v. 5. d. door de fchuintens van vooren 3. v. 3. d. en diep 18. d.

3 Een Kabinet van Amboins-wortel hout, met eene doorloopende lyft, van booven, gelyk ook van onderen met geflingert lyftwerk gedekte deuren; hebbende van booven in twee colommen 36 . en mede van onderen, behalven eene regt doorloopende, 8. Laden, dus 45. Laden, van verfchillende dieptens, alle welke voor het grootfie gedeelte met groen fatyn bekleed $2 \mathrm{yn}$; zynde voorts hoog 6. v. $7 \frac{1}{2}$, d. breed 4. v. 4. d. en diep 19. aे $22 \frac{1}{2} \cdot \mathrm{d}$.

4 Een Kabinet-kaft op het zelve geplaatf, hebbende een doorgaande, en met groote glaaze ruiten gedekte deur, breed en diep als het. Kabinet hier vooren, en boog $\mathrm{r}, \mathrm{v}, \mathrm{IC} \frac{1}{\mathrm{~s}}$. d. 
5 Een Kabinet, amboinswys gecoloreerd, gepiaatft a2n de eene zyde van voorfz. Kabiret $n^{2} .3$. hebberide vant booven twee, met fyne glaaze ruiten gedekte deuren, en van onderen in vier colommen $3 \kappa$. Laden, die $18 \frac{1}{2}$. lang, en 14. d. diep zyn; het Kabinet is van binnen wit, en de geningerde planken der vakken zyn op de kanten verguld, hoog 6 v: $7 \frac{1}{2}$. d. breed 6. v. I. d. en diep ontrend $16 . d$.

Een Kabinet, gelyk het voorgaande, geplaatf aan de andere zyde van $n^{n}$. 3. zynde in allen de wedergaa, welke beide bevat hebben de Hoorn- en Houtagtige, gelyk mede de Spon Foljilia.

7 Een Kabinet, gecoloreerd als beide de voorgande, en tot een vleugel van dezslve geplaatft, hebbende van booven eene deur, en van onderen 6 . Ladens van verfchillende dieprens, hoog 6 . v. $7 \frac{1}{2}$. d. breed 20 , en r diep $23 \frac{\mathrm{k}}{2}$, d.

8 Een Kabinet, zynde cen diergelyke vleugel, gelyk en in allen als het voorgaande, zynde dus de wedergaa.

9 Een Kabinet, gecoloreerd gelyk amboins - worrelhout waar in, in twee colommen, van booven 22. en van onderen 16. dus te zamen 38. Laden, welke, door Stulpen, bevat hebben het begin der in de Taafel $n^{\circ} . \mathbf{r}$. door 40. Laden, mede in Stulpen vervolgde Indifche Infecten, zynde hoog 6. v. $7 \frac{t}{2}$. d. breed 3. v. en diep $T \delta \frac{i}{2}$. duim.

ro Een Kahinet, zynde de wedergaa, hebbende van booven in twee colommen 28 . en van onderen 18. of gezaamentlyk 46. Laden, welke, mede door Stulpen, benooten hebben de Europaifche Infecten.

II Een vafte $K a / t$, noteboomsgewys gecoloreerd, van agteren, gelyk ook alle volgende, befchooten, van binnen wit en de randen van de doorgaande planken, verguld, verdeelc in zeeven vakken, die de Uitlandifche Vogelen in glaaze Klokken bevat hebben, hoog 6. v. $7 \frac{3}{2}$ of met het dek ftuk 8. v. 6. d. breed 7. v. II. d. en diep II $\frac{k}{2}$. d.

12 Een vafte Kaft, in allen op die zelfde voorgaande vize met agt vakken, waar op de Gediertens in glaaze fles fen zyn geplaa:ft geweeft, hoog 6. v. $7 \frac{1}{2}$. a B. v.6. d.bree 7. v. Ir. d. diep $11 \frac{l}{2}$ d.

33 Een vafte $K a f t$ daar an fluitende, in allen met de voos gaande evenaarende, dog zonder verdeelingen, doo dien de glazze Kaften van de Vogels, \&c. aan elkand $\epsilon$ 
5 Un Cabinet, de couleur d'ambibine, pofe d coté du Cabinet $n^{\circ}$. 3. ayant, en haut, 2. portes a carreaux de vitres fines, $E^{\circ}$ en bas, en quatres colomnes, 36. tiroirs chaque long 18. large 14 pouc. blanc, en dedans 'E les bords des planches qui divifent les fonds, Sont dorés, haut 6 , pieds $7 \frac{3}{3}$. pouc. large 6. pieds I. pouc. profond environs 16. pous.

6 Un Cabinet, comme le precedent, fooé, à loautre coté de no. 3. en tout femblable, dans les deux dits Cabinets ont été gardés en haut les Zoophites de Bois, Cornées, E' Spongieufes, E2 leurs tiroirs ont contena les Foffiles.

7 Un Cabinet, de couleur comme le precedent, ayant Jervi d'aile au Cabinet $n^{\circ}$. 5. ayant en haut une porte $E^{\circ}$ en bas 6. tiroirs, de differentes profondeurs, haut 6 . pieds $7 \frac{1}{2}$. pous.

8 Un Cabinet, Jemblable au precedent, étant pofé, à coté dus Cabinet de $\mathrm{n}^{\circ}$. 6 . en tout le pareil.

- Un Cabinet, comme de couleur d'amboine, dans lequel en deux Colomnes en kaut 22 E en bas 16. ainji, en/emule 38 tiroirs, qui ont contemu, be commencement des Infectes Indienn s. dont on à fait mention en la Table de $n^{2}$. I. haut 6 pieds $7 \frac{1}{2}$, pouc. large 3 pieds, profond $18 \frac{1}{2}$ pouc.

, $\dot{U}_{n} \vec{C}_{\text {abinet, de la meme maniere, ou le pareil du precedent }}$ mais ayant en haut en deux colomnes, 22 . Ẽ en bas 18 . ous enfemble 46. tiroirs, dans lefquels ont été les Infectes Europeelunes.

Un Armoire fixe, de couleur de bois noyer, cloifonne par derriere, comme tous les fuivans, en dedans blans, divifé en 7 fonds, dont les bords de planches font dorés dans lefquisls ont été mis les Oifeaux Indiens., fous des Cloches de verre. haut 6 pieds $7_{2}^{1}$ pouc. avec couvert 8. pieds, 6. pouc large 7. pieds 18 pouc. profond $\mathrm{I} / \frac{3}{2}$. pouc.

Un Armoire fixe, en tout femblable au precedent, de hus divifions, fur lefquels ont ete mis les Animaux dans lewrs bouteilles, haut 6 . pieds $7 \frac{3}{2}$ pouc. profond $I \frac{1}{2}$ pouc.

Uu Armoire fixe, y ottenant, en tout pareil au precedent. mais fans divifions, țuisque les. Oifeaux, Ėc. y ont été place 
fluitende, zyn geplaatit geweeft, hebbende ter zyden een aanfluitende boogsgewyze kaftje, waar in op veele verdeelingen, waar van de randen der planken mede verguld $2 y n$, geftaan hebben de Zaaden, en Vrugten in glaaze flesjes, hoog 7. v. $7 \frac{1}{2}$. ̀े v. 6. d. breed 12. v. 12. diep I2 à I4. d.

14 Een Pyramidaal Opfel, trapsgewys, van drie hoogtens op voorfz. Taafel $n^{2}$. I. geplaatft, zynde de regt doorgaande, en op de hoeken gellingerde witte planken derzelver kanten verguld, lang 6 . v. breed $3 . v_{0} .4 . d$. boog 12. d.

15 Twee glaaze Klokken. zeer ruim en groot, met derzelver gedraayde platte voetftukjes, gelyk alle volgende.

I6 Vyf glaaze Klokken, iets laager en minder van ruimte.

I7 Vyf glaaze Klokken, in allen als de vuorgaande.

18 Agt glaaze Klokken, omtrent van gelyke hoogte, alfchoon iets naauwer.

I9 Agt glaaze Klokken, in alle als de voorgaande.

20 Zes glaaze Klokken, alfchoon iets laager, dog veel ruimer.

21 Zes glaaze Klokken, in allen als de voorgaande.

22 Agt glaaze Kilokken, mede gelyk de voorgaande.

23 Ronde Tobbetjes, van wit hout, zeer dun gedraaid, met groene randen, van zesderhande grootens, (een veel. tal van dozynen.

24 Ovaale Tobbetjes, gelyk de voorgaande, van zesderhan. de grootens, (een veeltal dozynen.

\section{O M I S S A.}

Pag. 28. $n^{\circ}$. 83. \&c. Zoogende Dieren, Visfchen, Dieren va beiderley leever, Vogelen, Injęten \&c. i Flesichen met geeftig Vogt.

P. 228. $\mathrm{n}^{\circ}$. 1996. \&c. Indifche Infecten, of Torren, Dag- e Nagt-Vlienders, \&c. alle verfchillend in Doozen.

P. 494. $\square^{\circ}$. 1942. \&c. Hoorens \& Schulpen \&c, alle van elkar der verfchillende,

P. 534. n². 378. \&c. Koraal Gewasfen, meett al onderfche dene Voorwerpen.

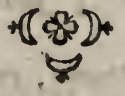


dans les cotfjes de verre, ayant d coté un petit armoire en forme d'arc attache, dans le quel, en plufieurs divifions, dont les bords des planches augli doré, ont eté placé, les Semences, Fruits, dans des petites bouteilles de verre, haut 6 . pieds $7 \frac{1}{2}$. pouc. $d 8$ pieds 6 . pouc. long. 12. pieds ix. pouc. profond 12. a 14. pouc.

14 Une Machine Pyramidale, en forme de degré, pres du miliers de la table $n^{\circ}$. I a trois hauteurs, paljant outre, E fur les coins font les planches, en ziczac, ou rondes, blanches, dont les bords dorés, long 6.pieds, large 3. pieds. 4. pouc. haut I 2. pouc.

I5 Deux Cloches de verre, tros larges, E hautes, avec piedelle contournéz, EO plattes, comme font tous le Juivantes.

16 Cinq Cloches de verre, plus bafjes, \&o moins larges.

17 Cing Clorhes de verre, en tout jemblables aux precedentes.

18 Huit Cloches de verre, \& pers pres de la meme hauteur., quoique un peu plus etroites.

19 Huit Cloches de verre, en tout, comme les precedentes. 20. Six Cloches de verre, un peu plus bafjes, mais plus larges.

2I Six Cloches de verre, en tout comme les precedentes.

22 Huit Cloches de verre, en tout femblables aux precedentes.

23 Des petites Cuves rondes, de bois blanc, d bords verte, de fix differentes grandeur, finement tournés, (une quantité de douzaines.

24 Des petites Cuves ovales, femblables aux precedentes, de fax differentes grandersr, (une quantité de douzaines.

\section{O M I S.}

Pag: 28, nº 83. \&c. Animaux à Mamelles, Poiffons, Amphibies, Oifeaux. Infectes $E^{2} c$. dans. des Bouteilles en Ligieur.

P. 228. no. 1996. \&c. Infectes Indiennes, ou Scarabees, $\mathrm{Pa}$ pillons diffirentes Eंc. variées; dans des Boites.

P. 494. $\mathrm{n}^{\circ}$. 1942. \&c. Coquillages, \&c. tout differentes,

P. 534. 0 \% 378. Q C. Coraux, ou Zoophytes, confistant en quelques objets differens,

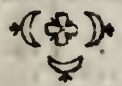




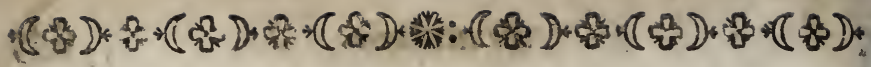
ADDENDA ET EMENDANDA.

No: Pag. $2-\mathbf{r}$. MELLES.

ro. Loris Volant, ou Maki,

10. 4I. 45. 50. Sarigue

89. alt. Lagein 36. poll.

97. Callaneus,

ros. deleatur: varieté

Pag. $18-23$.

19. capite permagno, Indicus,

I9. des Indes,

48. adpofitus

84. Balifte Velu,

Pag. 22 - 29.

4. Linn. 748. 20.

82. Aurantius

10. fitisformis,

$$
\text { Pag: } 28-35 \text {. }
$$

xo. nageoire ?

39. Chatoton tachetée

$$
\text { Pag. } 33-3 \% \text {. }
$$

ultimo loco: 5. Roftrum

in primo loco, \& feyq. Oifeaux Ec. Pag. $38-67$.

in Capite: MAMMELLEZ.

7. Ceilonenlis, Naturforfcher XIII. t. 4 .

8. 19. Naturforsch. 1. c.

39. deleatur: ou varieté du meme,

47. Buff. 84.

67. Caranche doré,

82. pennularum

97. Linn. 0.

I15. Linn. 338. 1.

Ir6. Edw. II. t. 264 . f. 2.

Buff. t. 34: f. x. E.

136. Fringilla

I37. les ailes

34S. Linn. O.

177. Todús Plumatus, Natuyfor fih.

XVII. t. $x_{0}$

I95. Currucaria,

2I2. longicaudatus,

212. la iete noire, les ailes \& queue brunas, les reitrices latérales tres langues,

222. les ailes, \& ' la queue brunes, d fommet blane,

943. rectricibus,

353. intcrompws, at fommet do\%ble, \&f poitrine blaizches.

¿66. finvefcentibus,
No.

267. fafcia alarum,

$27 x$. albo,

285. Edw. 79 .

309. deleaiur: 112.

324 . trics belle,

346. les ailes d tache blanche, desfous ceindré,

357. tete, \& cou noir, deffous blanche, 377. dc fous fauve, à ligne collaire bianc,

385. da ailes noires, tache blanehe glomark,

387 . deleatur: tuche blanche glonore,

390. Tetrao

405. de poitrine noire, tachete de blanc,

431. Linn. 3I5. 9. f. 6.

436. epaules blanches, diailes bleues, Pag. 68-232.

56. an non Cimex?

208. Fabric 198. 29.

373. Capricorne Brun,

393. Liinn. O.

396. de Geer IV. t. 3. fo 2 .

423 Fabric. 273. 2.

445. Carabus Ruber,

447. Fabric. 24 I. 27.

45\%. Carabus Sacharivorus,

508. Fabric. 274. 3:

540. les cuiffes

543. paintes, à pattes rouges,

597. tachictie,

608. fubcarinato,

607. ailes'brunzes,

624. de Geer III tr. 4t. f. 4 .

685. jaunes, à collier "orange,

840. Papilio

970. Papillon Erato?

roo9. f. yarietas præcedentis?

I026. Linn. 778. I68?

Roes. I. t. 5 ?

IC4I. Cram. 58. D.E,

II68. aे ailes brimes,

T267. Papillon Belie,

1295. cn dedans

1343. alis fufcis,

1418. ccrochues,

1479. ultimo loco: 1469, Sphinx I503. Cram. VI!. 8. F.

I549. mancees mebalouges, IEI2 


\section{Addenda et Emendanda.}

No.

I6I2 Phalene Pyrale, I7Ir. alis fcriceis, I72r. Myrmeleon r764. fasciisque 1767 . \& abdomine 1835. aे 4. \& 4. d yeux, ventre ovale, 808. L pattes brun,

42. Knorr III. 30. 495

56. Knorr II. 26. 3 .

80. Knory II. 20. 2 .

80. Fluviatile,

84. Knorr VI, x6. 3 .

114. Knorr I. 20. 5.

II5. Naturforfch. XVIII.t. 2. f. I8. II9-I2I. ou Impelforéc, 18r. Linn. 0.

200. Knorr VI. 34. 2.

20I. Spengler ICon. I. B.

206. Rnorr VI. 32. 3 .

220-22I. Ou. Thiare fluviatile, 223. Naturforfch. XII. t. I. f. I-3. 225. Hollar Icon. I4.

39. Seb. III. 39. 62-68. 7I-74.

313. Naturforfch. IV. t. I. f. I. 2 .

370. Valent. 79.

373. Kizorr. IV. 6. 5.

75. Knorr I. 3. I.

89. Lift. 639. 33 ? Knorx. VI.28.8

17. Knorr III. 14. 3 .

40. Link. 58r. Lift. 636. 24.

48. ubi hujus rarifimse

49. n'eft encore trouvé

49. Linn. 593. A. .. O.

71. Knorr III. 18. 1.

74. Knor, V. I3. 8.

9S. Lift: deleatur : 73 ?

o7. debordes.

18. cauda elevata,

20. Linn. 560. Bi, f. o.

2r. Knorr IV. 2I. 6.

18. Tubicen, r. Dimachi,

5. Knorr VI. 26. I.

53. Knorr VI. 24. 3.

Spengler IiOn. III. EE.

33. Hollar Icon. 8.

I. Knorr VI. $24: 6$.

०. $\operatorname{Linn} .527 \cdot \boldsymbol{\gamma} \cdot 1.0$.

2. Fiolzar. Icon. 3r.

2. Huiliè a boirfage , "ay

8. Kinorr II 7. 2. 3 .

2. Hullar licon. 22. n'zorr TI
No.

630. Spengler Icon.-III. EE. Knorr V1. 28.

63r. Spino $\int a$,

636. spengler Icon. II. C.

64I. Spengler lcon. III. I.

666. Devila. T. I6. P ? Knorr III. 29.5.

678. Spengler lion. III. C. 683. Spengler Icon. $11 . \mathrm{H}_{\text {. }}$ 685. Knotr IV. 30.2. 629: Knorr VI. 24. 2 . 689. Kinorr V. 2I. 20. 706. Knorr VI. 22. r。 7ro. Knorr V. 3. 3. 723. Knorr IV. 3I. I. 744. Knorr VI. 27. 2. 754. Spengler Icon. II. A A. 750. Spengler Icon. I. G. 765. Spengler Icon. I. F F. Knorr V. I. I. 766. Knorr VI. Ir. 2. 788. Mitra Papalis, long. 4. polli 818. Knorr II. 4. 4. 822. Knorr V. 6. I, 7. I. 846. Knorr III. 26. 2. 3 .

889. Knorr V. 4. 3 . 916. Knorr V. Ix. I. 924. Spangler Icon. I. E? 925. Spengler Icon. 1. E. 928. Linn. 298. द. 1. ०. 937. Knorr III. 6. 3. 95I. pelée blanche, 959. jaunatre, it petites taclies brisines,

969. Knorr III. IX. 2. 998. Linn. 304. Qh. roo\%. Knorr VI. I6.5. ro13. Kinorr I. 8. $4,24.50$ I0I8. Knort VI. to I.

ro19. Knorr V. 24. 3 . ro3r. Finor V. 9. 6?

1038. deleatur. Argeny. I2. AR.

1042. Knorr IV. I7. I.

ro43. Knor VI. 4. 4.

I055. Knors J. I6. 3?

cos6. Aigenv. 12. M.

106r. Knorr V. 24. 4.

1067. Knotr VI. I. 5, 5. 3.

roI. Seb. III. 48. ?. Knorr I. 8. $3, V_{0} 24.1$.

ro72. I'nory III. 6. 5 .

ro73. Krorr V. I 7. 5, 24. 2. ros8. Kinorr V. Ir. 5.

IIos. Inorr, $\dot{V}, 18,2$. 
No.

II32. Kritr III. 2. 4 .

II52. Krorr V. I9. X.

II66. Gualth. 24. O.

II73. Knorr III. $17 \cdot 3$.

I182. Knarr I. 15. 7, V. $2 \% \cdot 4$.

II90. Knorr V. 4. 4.

1202. Knorr IV. 29. I. 2.

1257. E' querse erigée,

I295. triangularibus

1325. un peu ronde,

1386. Keorr V. I. 2. 3.

343. Knorr VI. 4. 5.

1381. Knorr II. 16. I.

I397. Knorr IV. I3. x.

I402. Lift. 701. 48 ?

1440. deffous brune,

1497. innées . attache \& la coquille, Linn. $24 \mathrm{I}$.

1532. deleatur: 12.4 .

T544. F aitiaire,

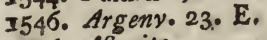

7556. Aftroite,

$156 \%$ excavé,

1602. Linn. I53.

1006. Lift. 453. 12.

I6ri. à coguille

1638. Bonainn. Objerv. f. 10.

36 48. vulva fufca,

I648. à pube brune,

1697. Vieille ridéc,

1795. Knorr V. 13. 6.

1802. alterum flavum.

1814. Rumph. 47. H. Gualth. 96. D. E.

1815. Seb. III. 91. 4. 5, 95. 1. 2. 1819. Linn. 2. 14.

1836. Gualih. 89. G ?

I85I. Lift. 410.256, 413. fupra,

1866. Cidaris,

xS72. Linn. т. ๙. f. e.

189.5. Ii on peut

anie 1903. in capite: DANS ante 1906 . In capite: BASE ante 1920. in capite: $D^{\prime} E P I N E S$. 1934. ou Nombril de venus,

8. efpece

$$
\text { Pag. 496 - } 535 \text {. }
$$

46. ou Mutilée,

62. M. Ramée?

65. mais tres belle,

85. A. Fayites?

90. ou moyenne

9r. in Buccina Marioni,
No.

r29. Macerophiles,

I35. ou petites branches

143. d' Alubeton f. 13. \&c.

162. ult. loco. M. Alcic-

145. Pall. I50. ou 179?

146. avec une

I47. Marfigli

I82 reffemblant d̀ Laneufe blancke celle de $n^{\circ} .355$.

226. ult. locn: G. Fiabel-

243. Catesby.

285 de Medufe,

294. Linn. 1289. $\mathrm{I}$.

295. 296. Puill. \& Linn. 1. c.

303. noire,

$317 \cdot$ diffecta,

339. feffilis

343. alt.

355. maxime analogia

357. Tubuli,

363. les fuperieurs

6. diverfa,

Pag. $536-54$ r.

7. \& fruit de Tamarinde,

27. par derriere

Pag. " $542-649$.

r. ed. I. efpec. I. 2. 3. 6.9. II

9. fruftule ftatue d'Apoilon,

II. Pierres d'Aigle.

26. Spath ferillete,

38. 39. Agathe

47. agathe

78. curbriffeau,

152. couvercle,

176. reticule,

185. d'Allemagne,

x98. precedente,

212. en facettes,

225. ex Uingaria,

280. ex Berglandia,

300. cum alio

305. f. reni pyritofus, durus, obl gus,

33r. Lithargyre,

349. inferviente,

354. f. flores

368. du Hartz,

372. druje forme,

378. Wall. 339 ? 340 .

385. en pierre

405. tres grand,

436. Fer cn haut en poudre, 


\section{Addenda et Emendanda.}

458. Wall. .

468. deleatur: de cuivre

480. \& flavum,

500. Cuivre, ou ecume, ,

5x4. Wall. 28s? f.o.

520. verd,

522. yermoulu,

53t. "rugine nativa terrea,

540. \& bruns,

544. Stannum granulatum,

546. Etain granule,

551. pyrite arfenicale blanche,

572. tres beau,

572. en Efpagne,

628. 3. onc. 2. eng. 3. as,

64r. Cancer fluviatilis,

5so. tres belle, 67o. d'Hêtre,

670. de Dambach,

686. Feuilles de Fougere,

7Q2. 1. Pectinata,

7०5. r. Brachia Cancrorum,

740. de Altorf,

742. ex Sondershousfen,

749. en Schift blanc,

786. fimiles,

792. Neritique,

803. tres grande,

832. Echinites plattes,

840. Judaiques.

848. Lapis dendriticus

864. trouvé d 100. toifes

32. le pretre.

"Si qua menda irrepferunt, ant oculo effugierune, B. L. difficili ac
, cium corrigat." literarum forma benigne adfcribas, ac lecundum judjo

"L'Ami Lecteur eft prit, d"excuser les erreurs qui pouront etre echape ", a locil, fur tout dans une impiefion \& difficile, "\$ de les corriger fidon 


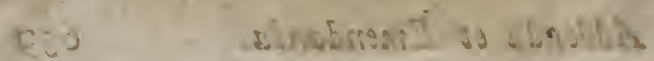
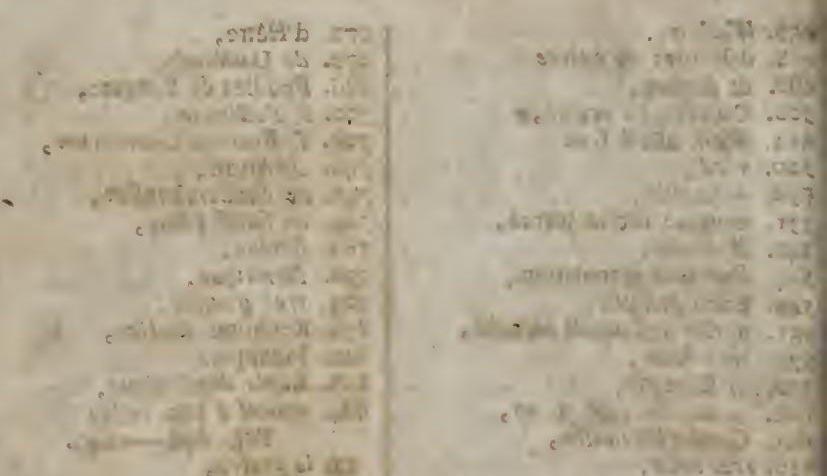

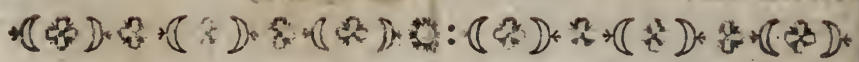
$A \quad L A \quad H A R E$, I $\begin{array}{llllllllllll}M & P & R & I & M & E\end{array}$

CHEZ JAQUES VAN KA R NEBEK, Imprimbeur de la.Ville, E du petit Sceau de la Province d'Hollande.

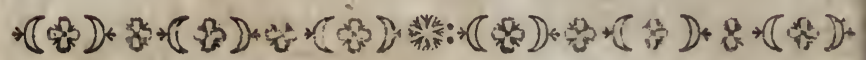




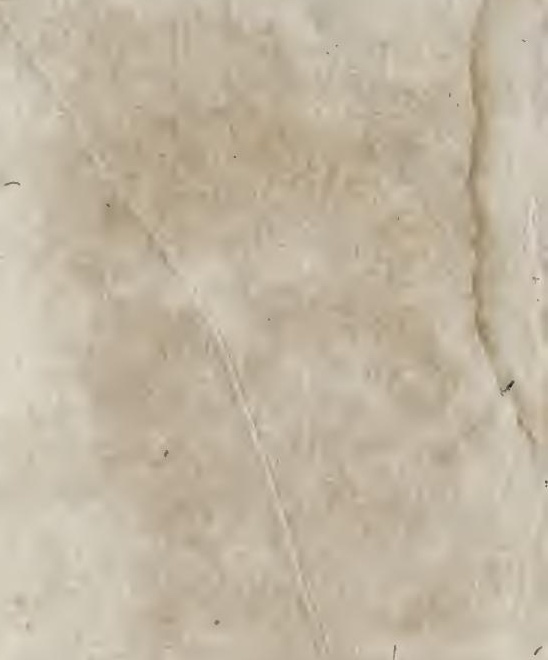






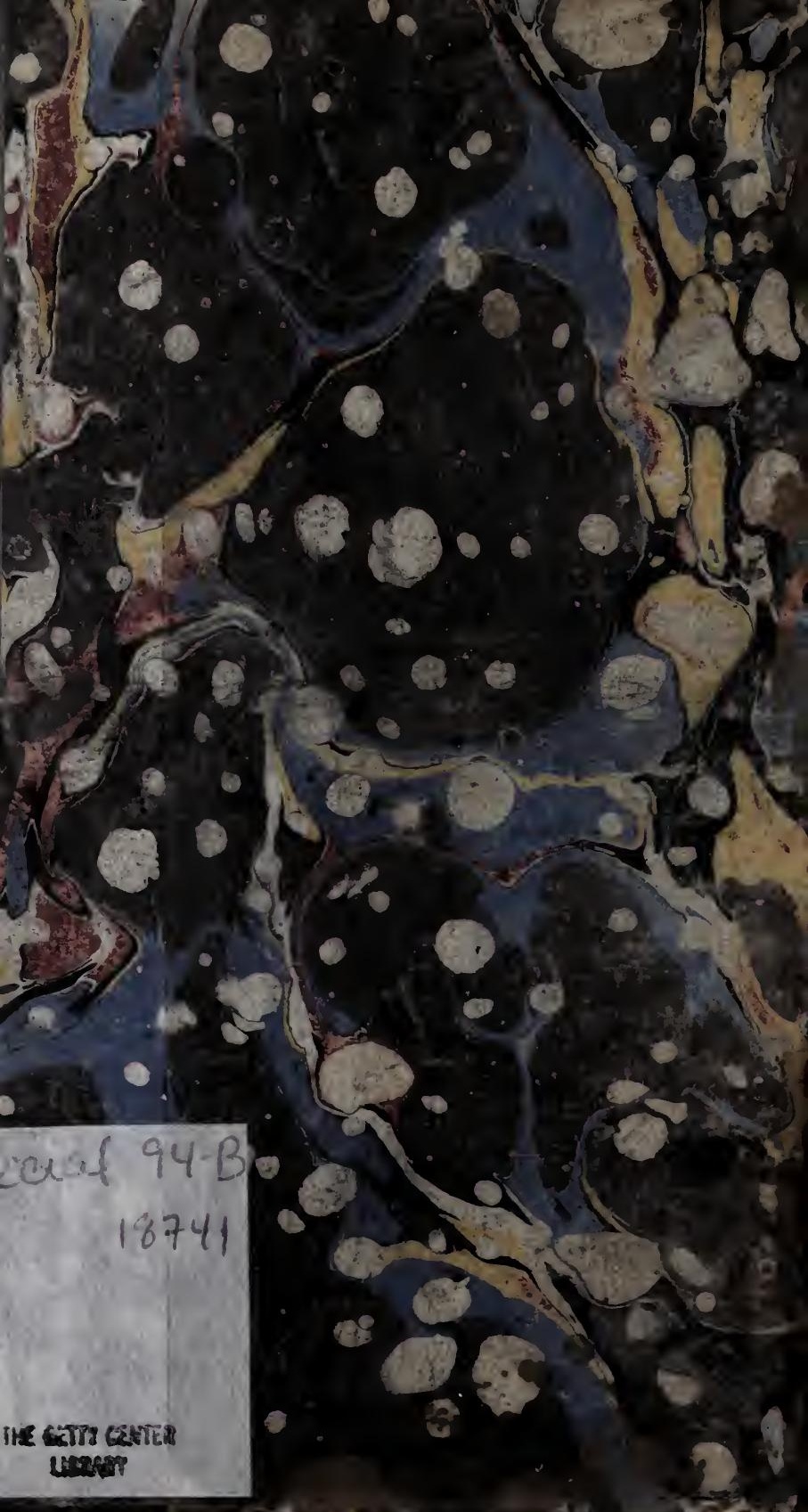



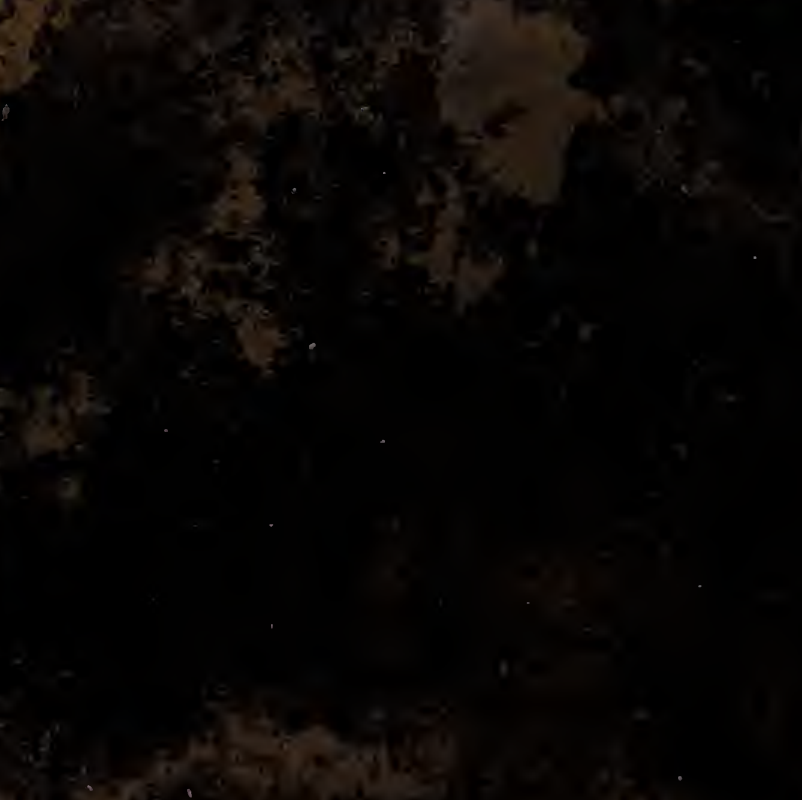

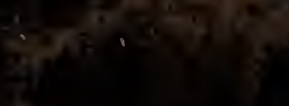

aptant
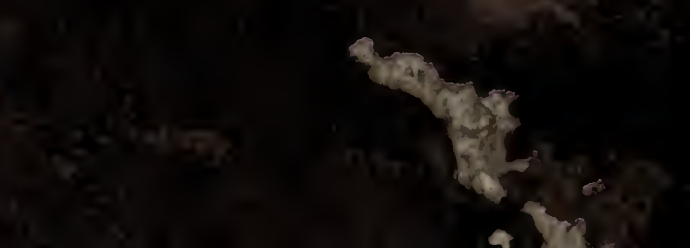

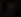

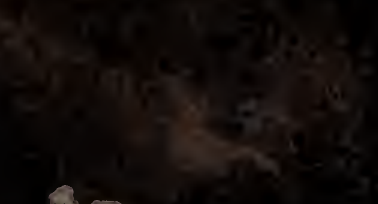

